\title{
Abstracts of the MASCC/ISOO Annual Meeting 2018
}

Supportive Care
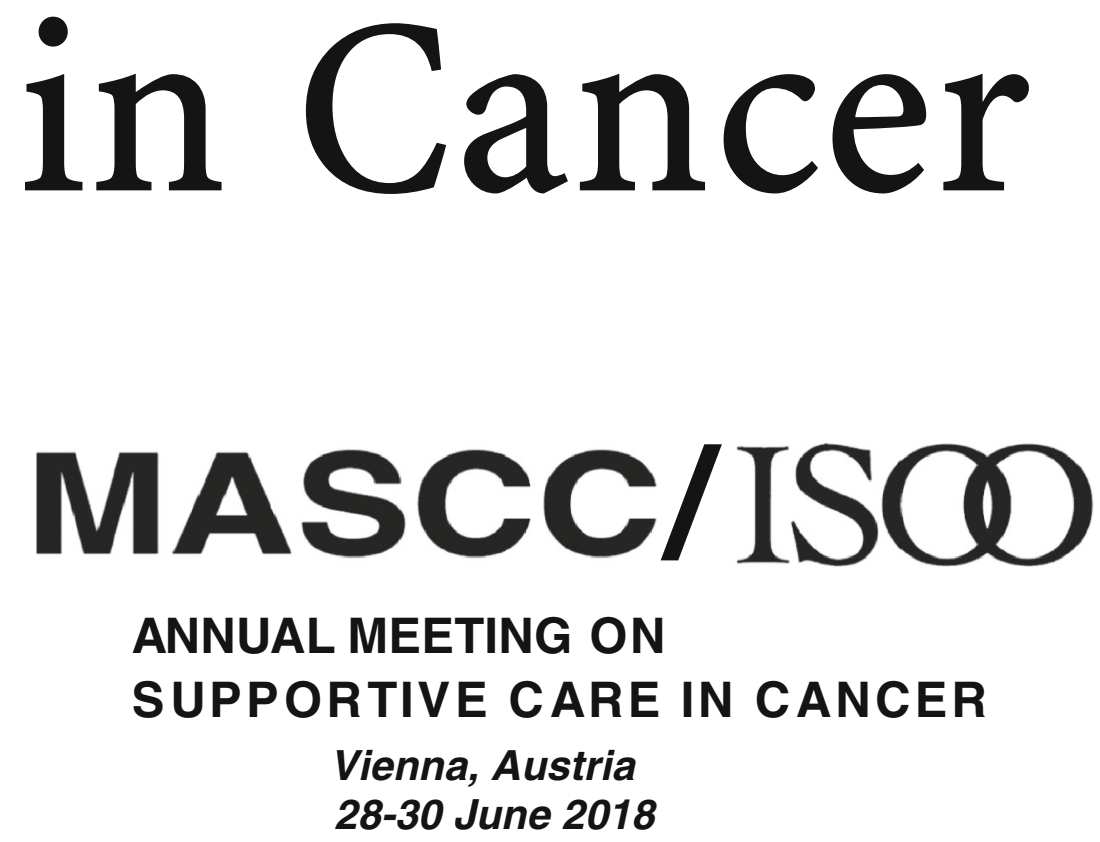

ANNUAL MEETING ON SUPPORTIVE CARE IN CANCER

Vienna, Austria

28-30 June 2018
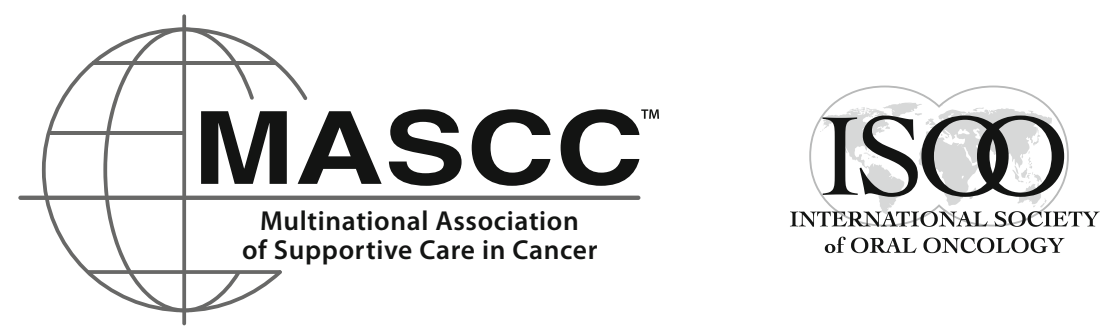

Supportive Care Makes Excellent Cancer Care Possible

This supplement was not sponsored by outside commercial interests. It was funded entirely by the publisher and the Societies 
Explanation of the Abstract Coding System

Each abstract type is assigned a special code:

- eP: e-Poster

- PL: Plenary

- PS: Parallel

- W: Workshop

The abstracts are presented in the following order with the below codes:

\begin{tabular}{|c|c|c|}
\hline EP & Antiemetics & eP001-eP023 \\
\hline EP & Bone & eP024-eP036 \\
\hline EP & Cancer Pain & eP037-eP069 \\
\hline EP & Complementary and Alternative Medicines & eP070-eP090 \\
\hline EP & Education in Supportive Care & eP091-eP133 \\
\hline EP & Fatigue & eP134-eP156 \\
\hline EP & Financial Toxicity & eP157-eP161 \\
\hline EP & Geriatrics & eP162-eP167 \\
\hline EP & Mucositis & eP168-eP208 \\
\hline EP & Neurological Complications & eP209-eP229 \\
\hline EP & Neutropenia-Infections and Hematologic Toxicity & eP230-eP238 \\
\hline EP & Nutrition and Cachexia & eP239-eP264 \\
\hline EP & Oral Care & eP265-eP281 \\
\hline EP & Pediatrics & eP282-eP302 \\
\hline EP & Palliative Care and End-Stage Disease & eP303-eP385 \\
\hline EP & Psychooncology & eP386-eP446 \\
\hline EP & Skin Toxicity & eP447-eP453 \\
\hline EP & Toxicities of Immunotherapy & eP454-eP466 \\
\hline EP & Toxicity of Targeted Anti-Cancer Therapy & eP467-eP476 \\
\hline EP & Quality of Life & eP477-eP542 \\
\hline EP & Rehabilitation & eP543-eP565 \\
\hline EP & Survivorship & eP566-eP621 \\
\hline EP & Other & eP622-eP706 \\
\hline PL & Post Chemotherapy Cognitive Impairment & PL001 \\
\hline PS & $\begin{array}{l}\text { ISOO Session 01: Bone Necrosis: A Joint ASCO/MASCC/ISOO Consensus on } \\
\text { Management and Prevention Strategies for the Oncologist and Dentist }\end{array}$ & PS001 \\
\hline PS & $\begin{array}{l}\text { EONS/ONS/MASCC Joint Session: Etiology and Management of Sleep Disruption, } \\
\text { Fatigue and Related Symptoms Across the Cancer Trajectory in Patients and Caregivers }\end{array}$ & PS002-PS003 \\
\hline PS & Emergency Supportive Care & PS004-PS007 \\
\hline PS & Oral Care in Pediatric Oncology: Needs and Risks & PS008-PS010 \\
\hline PS & Young Investigator Awards & PS011-PS020 \\
\hline PS & $\begin{array}{l}\text { Navigating the Twittersphere - How the Supportive Care Community Can Best Use Social } \\
\text { Media to Further Our Health Intelligence }\end{array}$ & PS021-PS024 \\
\hline PS & Challenges of Hemostasis in Cancer Patients & PS025-PS027 \\
\hline PS & How to Prevent Cardiotoxicity - A Real Challenge & PS028-PS030 \\
\hline PS & Cannabinoids for Symptom Management & PS031-PS034 \\
\hline PS & JASCC/MASCC Joint Session - The Evolving Approach to Management of Cancer-Cachexia Syndrome & PS035-PS036 \\
\hline PS & Regimen Related Oral Mucosal Injury - New Age Anti-Cancer Therapies & PS037-PS039 \\
\hline PS & Education for a Better Chemotherapy Experience & PS040-PS046 \\
\hline PS & Chemotherapy-Induced Peripheral Neuropathy: 2018 Update & PS047-PS050 \\
\hline
\end{tabular}


(continued)

PS Current and New Drugs to Maintain Bone Health, Oral Clinical Issues and Emerging Bone Health Issues in Cancer Patients

PS Never Too Old - The Important Role of Exercise in Older Adults with Cancer

PS Educational Session: How to Write a Manuscript for Supportive Care in Cancer

PS051-PS054

PS055-PS058

PS059

A "Good" Death - The Role of Supportive and Palliative Care

PS060-PS062

PS Dyspnea: Novel Strategies for Evaluation and Treatment

PS Recent Advances in the Management of Infections in Cancer Patients

PS Advances in Oncodermatology: Prevention and Management of Dermatologic Toxicities PS070-PS072

PS063-PS066

PS067-PS069

PS Supporting Patients and Family Caregivers Throughout the Cancer Journey

PS073-PS076

Side Effects of Targeted Drugs in Oncology

PS077-PS080

PS Managing Challenging Issues Among Adolescent and Young Adult Cancer Survivors

PS Developing Supportive Care in Eastern Europe: Different Models and Similar Challenges

W Mucositis Study Design: What Do We Need to Know?

W What Does the Future Hold? Prognostication in Advanced Cancer and Clinical Decision Making

PS081-PS084

PS085-PS088

What Does the Future Hold? Prognostication
Challenging Communications in Oncology

W001

W002

W003

\section{PL001}

OVERVIEW OF THE EFFECTS OF CANCER TREATMENT ON COGNITIVE IMPAIRMENTS IN CHILDREN

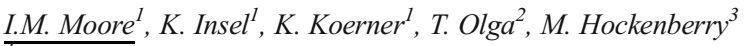 \\ ${ }^{T}$ University of Arizona, College of Nursing, Tucson, USA \\ ${ }^{2}$ Baylor College of Medicine, Pediatrics, Houston, USA \\ ${ }^{3}$ Duke University, School of Nursing, Raleigh, USA
}

One of the most challenging treatment-related sequelae experienced by children receiving central nervous system (CNS)-directed treatment is decline in cognitive abilities, manifested as problems with memory, attention, processing speed, visual spatial skills, and academic abilities. Despite considerable evidence for long-term cognitive problems, knowledge about the mechanisms of neurologic injury is lacking, and greatly needed in order to develop novel neuroprotective interventions. This presentation will summarize current knowledge proposed mechanisms of neurologic injury associated with CNS-directed therapy and cognitive consequences.

Children with newly diagnosed acute lymphoblastic leukemia (ALL) were enrolled and followed for 3 years. Cognitive abilities were assessed as soon as the child was medically stable and then every 12 months for 3 years. Biomarkers of oxidant defense (glutathione) and apoptosis (caspase $3 / 7,8$ and 9 activity) were measured in cerebrospinal fluid samples collected in conjunction with diagnostic and therapeutic lumbar punctures. Changes over time in cognitive abilities and biomarkers were analyzed using general linear model for repeated measures. Stepwise multiple linear regression analysis was used to identify neurocognitive predictors of academic outcomes.

Performance on measures of visual motor integration, working memory, and some measures of attention were significantly below normative data for healthy peers. Significant changes in oxidant defense and apoptosis biomarkers were found across ALL treatment phases; caspase 3/7 activity was significantly and negatively associated with performance on measures of cognitive abilities. Visual motor integration, verbal short-term memory, fine motor dexterity, and visual working memory abilities predicted academic math and reading abilities

Findings suggest that decreased oxidant defense and apoptosis may be involved in neurologic injury associated with CNS-directed treatment and biomarkers of apoptosis may identify children at risk for decline in cognitive abilities. Monitoring cognitive changes during ALL treatment may be used to screen for children at risk for academic problems and who could benefit from intervention programs.

Funding for this project was received from the National Institute of Nursing Research at the National Institutes of Health (NR010889) National Institutes of Health and Alex's Lemonade Stand Foundation Discovery Award.
PS001

OSTEORADIONECROSIS OF THE JAW: PAST AND PRESENT. CLINICAL AND ECONOMIC IMPACT IN ONCOLOGY

\author{
$J . W u^{l}$ \\ ${ }^{T} B C$ Cancer Agency- University of British Columbia, Radiation \\ Oncology, Vancouver, Canada
}

Head and neck cancers, particularly those of the oral cavity, are primarily treated by radiation and/or surgery. These treatments are associated with significant acute and chronic side effects. As treatment techniques and local control rates improve, many of our cancer survivors must live longer with these side effects. Osteoradionecrosis (ORN) is an example of a devastating result of cancer therapy. The probability of developing ORN depends on many risk factors including radiation technique and dose, tumour size and location, the volume of mandible or maxilla treated, and the presence of tissue trauma after therapies. In our session, we will discuss how improvements in radiation techniques combined with our understanding of bone toxicities have resulted in decreasing rates of ORN for our patients.

\section{PS002}

THE LATEST ADVANCES IN TECHNOLOGY IN DIAGNOSIS AND MANAGEMENT OF SYMPTOMS IN CANCER PATIENTS AND CAREGIVERS

\author{
A. Charalambous ${ }^{1}$ \\ ${ }^{T}$ Cyprus University of TEchnology, Nursing, Limassol, Cyprus
}

\section{Introduction}

Sleep disturbance, cancer related fatigue, and depressive symptoms are commonly reported by patients diagnosed with and living with various malignancies. These symptoms co-occur in symptom clusters, exacerbating one another, decreasing the quality of life (QOL) and making the management of such a cluster a complex and demanding task. Effective and cost-effective management calls for the integration of technological advances in combination with the traditional interventions.

\section{Objectives}

To present the latest advances in technology that have been introduced in diagnosis and management of sleep disturbances, CRF and depression in cancer patients and caregivers

\section{Method}

Based on a selective literature review, evidence is provided on the technological breakthroughs in diagnosis and management of sleep disturbances, CRF and depression 


\section{Results}

The results showed the complexity of managing these symptoms in cancer patients throughout the cancer care continuum. Effectively and costeffectively dealing with these symptoms presents as a challenge for patients and caregivers (formal and informal). Smartphone technology, virtual environments, actigraphy, video games (e.g. kinetic exercise) energy and sleep enhancement, internet-based and phone-based (apps) are only some of the technological interventions used to manage these symptoms. These interventions also utilize older techniques such as guided imagery and relaxation therapy to optimize symptom management.

\section{Conclusions}

sleep disturbances, cancer related fatigue, and depressive symptoms are a prevalent and persistent issue for patients with cancer before the start of the treatment and even a year following its completion. The findings stressed the need to implement various technological interventions in combination to more traditional ones as a means to manage these symptoms effectively. As most of these symptoms are managed at home, it is important for these interventions to promote patient's empowerment and personalized care.

\section{PS003}

\section{IMPACT OF SYMPTOMS ON THE CAREGIVERS' WELL- BEING}

\author{
E. Papastavrou ${ }^{l}$, A. Charalambous ${ }^{1}$ \\ ${ }^{T}$ CYPRUS UNIVERSITY OF TECHNOLOGY, NURSING, LIMASSOL, \\ Cyprus
}

\section{Introduction}

The increasing number of cancer patients worldwide combined with the chronicity and progressive nature of the disease, has increased the caring role of the family throughout the different stages of the cancer trajectory. Symptom management and response to side effects of treatment is a stressful experience that may have a negative impact on family caregiver's life.

Objectives

To discuss family care givers experiences in symptom management through an exploratory literature review and to briefly describe a comparative study exploring the caregiver coping, burden and depression in three different groups of care givers.

Method

An exploratory literature review to answer the question of the impact of caring on family care givers of patients with cancer and a descriptive and correlation design.

\section{Results}

The results through the literature review showed that family caregivers provide emotional support, physical help including administration of medicines and feeding through "tubes", wound dressing, monitoring and assessment of symptoms and side effects. The one-way analysis of variance in the comparative study showed significant differences $(\mathrm{P}=$ $.008, \mathrm{~F}=4.85$ ) between the 3 care-giving groups in terms of depression, with the highest depression levels being for cancer caregivers. Conclusions: Family carers are burdened to providing support to their relative and deal with the complexities of symptom and side-effect management that may result in negative outcomes for both the patient and the relative. The study findings support the necessity of family education to the management of cancer care.

\section{PS004}

\section{EVALUATION OF IMMUNOTHERAPY TOXICITY FROM THE EMERGENCY STANDPOINT}

\author{
T. Cooksley ${ }^{I}$ \\ ${ }^{T}$ The Christie NHS Trust- Manchester- United Kingdom, Department of \\ Acute Medicine and Critical Care, Liverpool, United Kingdom
}

Checkpoint inhibitors have significantly improved outcomes for patients in a number of malignancies. These treatments are associated with toxicities which stem from increased activity within $\mathrm{T}$ cell lineage similar to that observed in autoimmunity. These immune-mediated toxicities can affect virtually any organ system and are potentially fatal. The timing of the onset of the adverse events is dependent on the organ system affected and can be delayed significantly after initiation or completion of therapy. The critical first step in the management of immune-mediated toxicities is their recognition. The increasing indications for, and usage of, checkpoint inhibitors means that more patients on these therapies will present to emergency settings. Determining if these patients have an immunemediated toxicity requires careful clinical workup and necessitates education of health care professionals working in acute care settings. Early recognition and intervention can reduce the duration and severity of the complications. This necessitates that consideration is given to the models of acute care utilised in the emergency management of patients on checkpoint inhibitors.

Guidelines for the acute management of immune-mediated toxicities have been developed and published. The basics of treatment are to withhold the checkpoint inhibitor, supportive treatment of the organ system affected and potentially the use of immune modulating medications, such as corticosteroid therapy.

This presentation will consider the emergency management of immune-mediated toxicities, the clinical workup of acute presentations in patients on checkpoint inhibitors and the challenges of ensuring safe care for these patients in various emergency oncology models.

\section{PS005}

VALIDATION OF CLINICAL INDEX OF STABLE FEBRILE NEUTROPENIA (CISNE) MODEL CAN IT GUIDE EMERGENCY PHYSICIANS TO A REASONABLE DECISION ON OUTPATIENT VS. HOSPITALIZATION?

H. Moon ${ }^{1}$, S.H. Sim ${ }^{2}$

${ }^{T}$ National Cancer Center, Department of Internal Medicine, Goyang, Republic of Korea

${ }^{2}$ National Cancer Center, Center for Breast Cancer, Goyang, Republic of Korea

\section{Introduction}

Advances in modern oncology enable physicians to treat a subset of febrile neutropenia (FN) patients at low risk in outpatient settings instead of hospitalization.

\section{Objectives}

This study aimed to validate CISNE model for Korean FN patients presenting to emergency department (ED) and identify the best triage strategy to guide emergency physicians to an evidence-based decision on discharge $v s$. admission.

\section{Methods}

We retrospectively enrolled 400 adult febrile neutropenia patients presenting to ED of National Cancer Center, Korea who had been treated by cytotoxic chemotherapy for solid tumors. The primary outcome was the frequency of any serious complications (ASC) that occurred during the duration of illness.

\section{Results}

Apparently stable patients (ASP) accounted for 299 of 400 (74.8\%). 56 patients (18.7\%) belonged to CISNE I (low risk); 124 (41.5\%), CISNE II (intermediate) and 119 (39.8\%), CISNE III (high). ASC, the primary outcome, occurred in $10.7 \%, 19.4 \%$ and $33.6 \%$; the composite of ASC or bacteremia occurred in $16.1 \%, 25.0 \%$ and $38.7 \%$ as per the triage. Compared with MASCC low risk group, CISNE I stratum had significantly lower sensitivity ( 0.22 vs. 0.95 of MASCC low risk) but higher specificity ( 0.91 vs. 0.17$)$ to predict no occurrence of the primary outcome. 

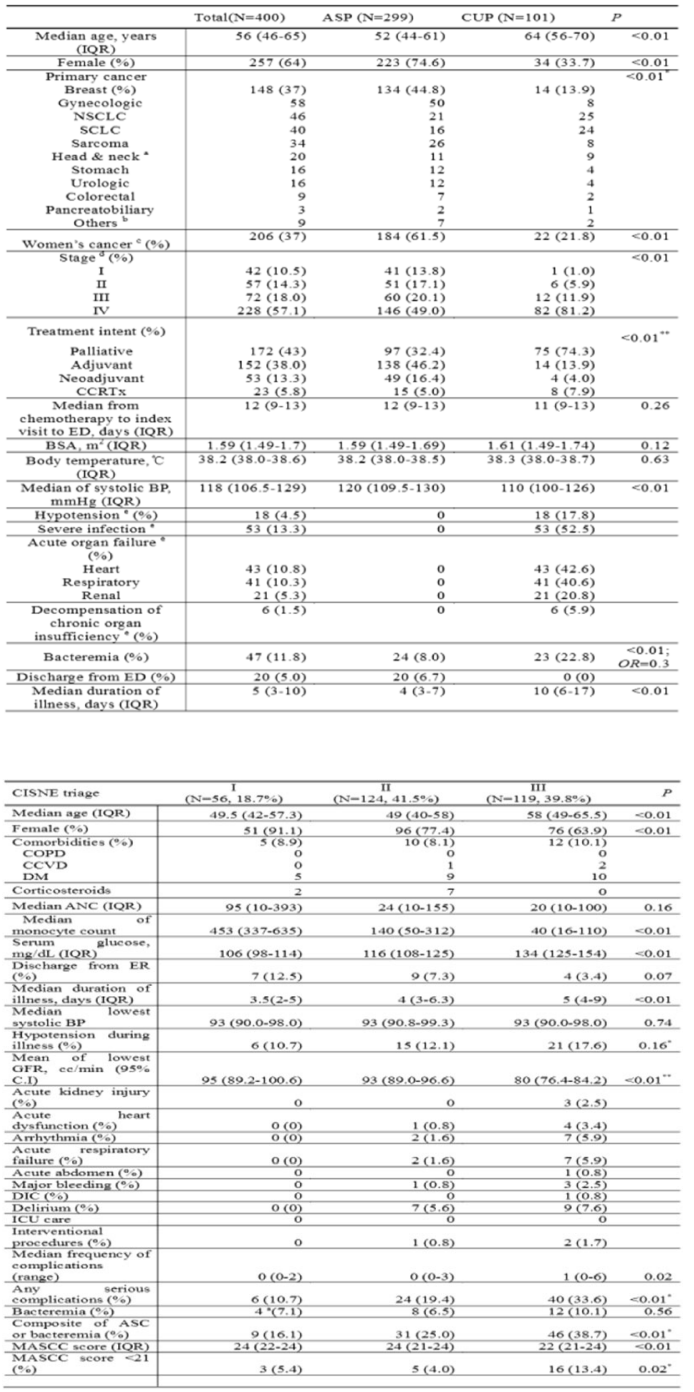

\section{Conclusions}

MASCC and the CISNE model are complementary and likely to be a winning combination to select FN patients at low risk. While MASCC is suitable for quick screening, the CISNE model proved to be a good confirming test because of its high specificity and low false positive rates to predict no occurrence of ASC.

\section{PS006}

COST REDUCTION IN HOSPITALIZATION AND EMERGENCY ROOM VISITS ASSOCIATED TO EARLY PALLIATIVE CARE INTERVENTION AMONG BREAST CANCER PATIENTS.

R. Ramirez-Morales ${ }^{1}$, C. Arce-Salinas ${ }^{2}$, L. Mendoza-Galindo ${ }^{2}, S$. Allende-Perez, ${ }^{3}$, E. Monreal-Carrillo ${ }^{3}$, E. Verastegui-Aviles ${ }^{3}, C$. ArzateMireles $^{3}$, D. Perez-Camargo ${ }^{4}$, D. Flores-Diaz ${ }^{2}$, J. Matus-Santos ${ }^{2}$, L. Lopez-Rojas ${ }^{1}$

${ }^{1}$ Nacional Cancer Institute - Mexico City INCan, Clinical Research, Mexico City, Mexico

${ }^{2}$ Nacional Cancer Institute - Mexico City INCan, Medical Oncology, Mexico City, Mexico
${ }^{3}$ Nacional Cancer Institute - Mexico City INCan, Palliative Care, Mexico City, Mexico

${ }^{4}$ Nacional Cancer Institute - Mexico City INCan, Nutrition, Mexico City, Mexico

\section{Introduction}

Earlier palliative care attention is associated with reduction in costs related to hospitalization days and emergency room consultations (ER).

\section{Objectives}

To analyze the cost of ER consultations and hospitalization days in patients with an early palliative care intervention in contrast with patients treated only with oncological intervention

\section{Methods}

Metastatic breast cancer patients with recent diagnosis were included, they were randomized to standard care given only by oncology team (control arm) or intervention adding palliative care (experimental arm). Patients were stratified according to ER/PR, and HER2 status (positive vs negative). Local ethical committee approved protocol. The number of ER consultations, hospitalizations (yes/no) and number of hospitalization days were evaluated.

\section{Results}

Total of 53 patients were included, $62 \%$ were assigned to experimental arm and 38\% to control arm. Control group had greater number of hospitalization days and ER visits. Median cost of ER consultation was \$21.99USD for experimental group vs. \$46.35USD for control group; there were found a trend in the cost reduction per visits, $p=0.081$. There was a significant reduction in the cost of hospitalizations days, with a median cost of \$167.57USD for the experimental arm vs. \$295.05USD for the control arm, $\mathrm{p}=0.015$.

\section{Conclusions}

Reduction in hospital stay costs was statistically significant in patients with early palliative care intervention in comparison with patients with only oncological intervention. There is also a trend to lower cost associated to ER consultations for the experimental group. It is necessary to increase the sample size and include an evaluation of costs related to interventions during ER visits and hospitalization.

\section{PS007 \\ INCIDENCE AND MORTALITY OF SPONTANEOUS INTRA- CEREBRAL HEMORRHAGE IN PATIENTS PRESENTING TO THE EMERGENCY DEPARTMENT OF A COMPREHENSIVE CANCER CENTER}

\author{
A. Wechsler ${ }^{1}$, A. Qdaisat ${ }^{1}$, Yeung $^{1}$ \\ ${ }^{T}$ UT MD Anderson Cancer Center, Emergency Department, Houston,
} USA

\section{Introduction}

Spontaneous intra-cerebral hemorrhage (ICH) is a dreaded complication of both cancer and its treatment. In the general population, ICH has a reported case fatality ratio of $36.6 \%$ at 7 days and $59 \%$ at 30 days. There is limited information on how the higher bleeding risks of the oncologic population effect the incidence or fatality of ICH.

Objectives

To differentiate the incidence and mortality of ICH by cancer type.

Methods

We performed a retrospective cohort study of all patients presenting with spontaneous ICH to the Emergency Department (ED) of The University of Texas MD Anderson Cancer Center from 9/1/2006 to 2/16/2016. Patients were identified using all ICH related ICD-9 codes in a billing database.

\section{Results}

Within the 10-year study period, 77,925 unique cancer patients made 204,464 ED visits, of which $0.33 \%$ (678) were for ICH. Patients with CNS tumors, melanoma, or leukemia had the highest incidence of ICH. Overall mortality in the initial 7 days after diagnosis of ICH was $11.9 \%$; $24.3 \%$ at 30 days and $63.4 \%$ at 1 year. Mortality was significantly higher 
in patins with liquid versus solid tumors at 7,14 , and 30 days $(\mathrm{p}=0.007$, $\mathrm{p}=0.003$ and $\mathrm{p}=0.003$, respectively), but not at 1 year $(\mathrm{p}=0.377)$. ICH was fatal in only $10-25 \%$ of patients with solid tumors in the initial 30 days. Conclusions

Spontaneous ICH is a rare finding even in the cancer patient with an incidence lower than previously reported. More than three quarters of cancer patients with ICH lived beyond the first month. Mortality varied by cancer type.

\section{PS008}

\section{PREVALENCE OF PATIENT-REPORTED DYSPHAGIA AND ORAL COMPLICATIONS IN CANCER PATIENTS}

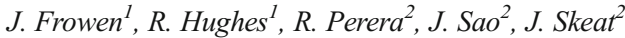

${ }^{T}$ Peter MacCallum Cancer Centre, Nutrition and Speech Pathology, Melbourne, Australia

${ }^{2}$ University of Melbourne, Department of Audiology and Speech Pathology, Melbourne, Australia

\section{Introduction}

Swallowing problems (dysphagia) and complications within the oral cavity are common following treatment for head and neck cancer, however research investigating these problems in other cancer patients is limited. Objectives

To determine the prevalence of patient-reported dysphagia and oral complications in all cancer patients across all treatment settings and to examine relationships between cancer types, oral complications and dysphagia. Methods

A cross-sectional study was conducted at a specialist cancer centre in Australia. Participants were either admitted patients or outpatients currently receiving treatment, who completed the Vanderbilt Head and Neck Symptom Survey (version 2.0) to report on dysphagia and oral complications experienced.

Results

In total, 239 patients, receiving treatment for 14 cancer types, participated. The proportion of patients who reported symptoms were: dysphagia for solids $(46.4 \%)$; dysphagia for liquids $(20.1 \%)$; any dysphagia (54.4\%); weight loss $(48.1 \%)$; xerostomia $(56.1 \%)$; mouth/throat pain (19.7\%); thick phlegm (33.5\%); taste changes $(61.8 \%)$; and voice changes $(36.8 \%)$. There were no significant differences in the symptoms reported across cancer types. Significant correlations were found between dysphagia and: xerostomia $\left(r_{s}=.707, p=<0.01\right)$; mouth/throat pain $\left(r_{s}=.756\right.$, $\mathrm{p}=<0.01)$; thick phlegm $\left(\mathrm{r}_{\mathrm{s}}=.663, \mathrm{p}=<0.01\right)$; taste changes $\left(\mathrm{r}_{\mathrm{s}}=.860\right.$, $\mathrm{p}=<0.01)$; and voice changes $\left(\mathrm{r}_{\mathrm{s}}=.466, \mathrm{p} \mathrm{p}=<0.01\right)$.

\section{Conclusions}

This is the first known set of comprehensive data on dysphagia and oral complications across all cancer patients. It identifies that dysphagic symptoms - which have implications for quality of life and function - are common in patients receiving cancer treatment. Additionally, dysphagia is associated with common oral symptoms of cancer treatment. Implications for monitoring, assessment and management are discussed.

\section{PS009}

DECREASE IN ORAL CARE UTILIZATION AMONG PEDIATRIC CANCER SURVIVORS IS MEDIATED BY LOWER EDUCATION AND HOUSEHOLD INCOME

\section{Smith ${ }^{1}$, B. Murphy ${ }^{2}$ \\ ${ }^{T}$ Vanderbilt University Medical Center, Oral Maxillofacial Surgery, Nashville, USA \\ ${ }^{2}$ Vanderbilt University Medical Center, Medical Oncology, Nashville, USA}

\section{Introduction}

Survivors of pediatric cancers face a number of oral health-related challenges. However, there is little data on oral health maintenance among pediatric cancer survivors or the mediators thereof. We hypothesized that pediatric cancer may influence dental utilization later in life.

\section{Objectives}

Data from a nationally representative cross-sectional study in the United States (National Health and Nutrition Examination Survey) were used to examine the dental health care utilization of pediatric cancer survivors and investigate social factors which may mediate this relationship.

\section{Methods}

Data from surveys conducted between 2007 and 2016 were obtained from the National Center for Health Statistics. The data were analyzed using surveyweighted linear and logistic regression and bootstrap mediation analysis.

Results

Patients who reported having a cancer diagnosed prior to age 20 were substantially less likely to report having visited a dentist in the past year $(\mathrm{OR}=0.459$ (95\% CI: $0.226,0.935))$ after adjusting for age and gender. They also reported lower household income $(\mathrm{p}=0.036)$, and there was a trend toward less education that failed to attain statistical significance $(\mathrm{p}=0.17)$. A mediation analysis demonstrated that the decrease in dental care utilization was mediated by lower education and household income $(\mathrm{p}<0.01)$, which mediated $48.5 \%$ of the effect. Of patients who did not receive dental care, $86.6 \%$ of patients endorsed cost as a barrier.

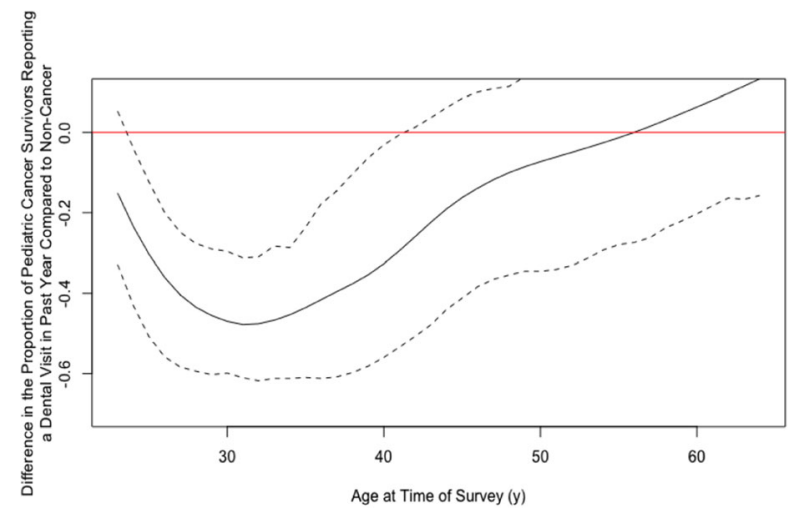

\section{Conclusions}

The data suggest that pediatric cancer survivors are dramatically less likely to see a dentist over their lifetime compared to non-cancer patients. A substantial percentage of the association was mediated by lower education and income.

PS010

ORAL LATE EFFECTS AND TASTE FUNCTION IN LONGTERM SURVIVORS AFTER TREATMENT OF MEDULLOBLASTOMA AND SUPRATENTORIAL PRIMITIVE NEUROECTODERMAL TUMOR DURING CHILDHOOD OR ADOLESCENCE - PRELIMINARY RESULTS

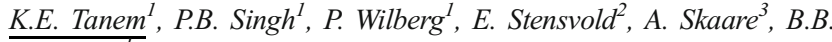
Herlofson ${ }^{1}$

${ }^{1}$ University of Oslo, Department of oral surgery and oral medicine, 0317 Oslo, Norway

${ }^{2}$ Oslo University Hospital- Rikshospitalet, Department of Pediatric Medicine- Women and Children's Division, Oslo, Norway

${ }^{3}$ University of Oslo, Department of Pediatric Dentistry and Behavioural Science, 0317 Oslo, Norway

\section{Introduction}

Oral late effects in long-term survivors (LTSs) after treatment of medulloblastoma $(\mathrm{MB})$ and supratentorial primitive neuroectodermal tumor (CNS-PNET) during childhood or adolescence have hardly been studied. Like other malignancies in the central nervous system, these patients 
undergo comprehensive treatment; surgery in combination with irradiation, chemotherapy or both.

\section{Objectives}

The aim of this study was to investigate oral late effects including taste function in LTSs after treatment of MB/CNS-PNET.

Methods

All LTSs treated between 1974-2013 at Oslo University Hospital for MB/CNSPNET before the age of 20 and with $>2$ years follow-up time, were invited to this multidisciplinary study. Data were collected through i) an interview regarding oral health conducted by a dentist, ii) an oral examination including registration of dental developmental disturbances, iii) bitewing and panoramic $\mathrm{x}$-ray and iv) taste test using taste-strips (Burghart, Wedel, Germany).

\section{Results}

Out of the 65 LTSs, 48 (74\%) were included in this study. Complete oral examination was undertaken in 46 participants. Fourteen (30\%) of these had trismus $(\leq 35 \mathrm{~mm})$. Impaired taste perception was registered in 16 (39\%) out of 41 subjects, of which seven had ageusia and nine hypogeusia. Dental developmental disturbances were registered in 13 of the 17 participants treated before or at the age of 5 years; microdontia was registered in 6, hypodontia in 10 and hypoplasia in 4.

Conclusions

The preliminary results indicate that LTSs after treatment of MB/CNSPNET may experience several oral side effects, including trismus, dental developmental disturbances and reduced taste function. This may impact their oral health negatively.

\section{PS011}

\section{THE ASSOCIATION OF CANCER-RELATED FATIGUE WITH} ALL-CAUSE MORTALITY OF COLORECTAL AND ENDOMETRIAL CANCER SURVIVORS: A POPULATIONBASED STUDY

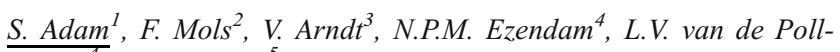
Franse, M.S.Y. Thong ${ }^{5}$

${ }^{1}$ University of Zurich, Division of Chronic Disease EpidemiologyEpidemiology-Biostatistics and Prevention Insitute, Zuerich, Switzerland ${ }^{2}$ Tilburg University, Center of Research on Psychology in Somatic Diseases- Department of Medical and Clinical Psychology, Tilburg, The Netherlands

${ }^{3}$ German Cancer Research Center DKFZ, Unit of Cancer SurvivorshipDivision of Clinical Epidemiology and Aging Research, Heidelberg, The Netherlands

${ }^{4}$ Netherlands Comprehensive Cancer Organisation IKNL, Netherlands Comprehensive Cancer Organisation, Utrecht, The Netherlands

${ }^{5}$ Amsterdam Medical Center University of Amsterdam, Department of Medical Psychology-Amsterdam Public Health Research Institute, Amsterdam, The Netherlands

\section{Introduction}

Cancer-related fatigue (CRF) is prevalent among cancer survivors and is associated with outcomes such as depression and poorer health-related quality of life. However, the mechanisms underlying CRF and its association with all-cause mortality are still unclear.

Objectives

To assess whether CRF is associated with all-cause mortality in cancer survivors.

Methods

The study sample comprised 2941 short-term cancer survivors $(<5$ years post-diagnosis) from four PROFILES registry studies conducted in 20082011. Survivors diagnosed with colorectal or endometrial cancer were identified from the Netherlands Cancer Registry. Fatigue was assessed with the Fatigue Assessment Scale. Cox proportional hazards models, adjusted for several sociodemographic and clinical characteristics, were performed to assess the influence of CRF on all-cause mortality. Additionally, we adjusted the models for survivorship bias by adding a variable with the left-truncation time (time between diagnosis and study invitation) and time of study invitation was set as entry time. Date of censor was February 12017.

Results

Around $1 / 3$ of survivors were fatigued and $5 \%$ very fatigued. Fatigued and very fatigued cancer survivors experienced a significant increased risk of death when compared to non-fatigued cancer survivors (HRadjusted $(\operatorname{adj})=1.40: 95 \% \mathrm{CI}[1.26-1.57], \mathrm{HRadj}=2.53: 95 \% \mathrm{CI}$ [2.26-2.85], respectively). This effect remained strong after excluding depressed survivors $(n=551)(\mathrm{HRadj}=1.42$ : 95\%CI [1.29-1.56], HRadj=2.38: 95\% CI [2.13-2.66], respectively).

Conclusions

Our study found that CRF is a predictor for all-cause mortality in cancer survivors, irrespective of potential confounders. Current results suggest further research into whether lowering burden of CRF in cancer survivors reduces mortality risk is recommended.

\section{PS012}

CANCER CARE-RELATED SOCIAL MEDIA (SM) AND INTERNET USAGE DIFFERENCES BETWEEN AGE GROUPS AMONG PATIENTS WITH CANCER

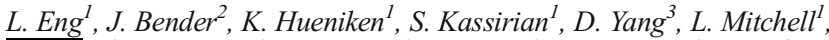

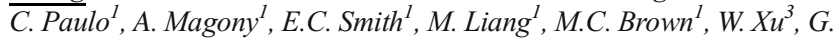 \\ Liu $^{1}$, A. Guptal \\ ${ }^{1}$ Princess Margaret Cancer Centre, Medical Oncology and Hematology, \\ Toronto, Canada \\ ${ }^{2}$ Princess Margaret Cancer Centre, Wellness and Cancer Survivorship \\ Centre, Toronto, Canada \\ ${ }^{3}$ Princess Margaret Cancer Centre, Biostatistics, Toronto, Canada
}

\section{Introduction}

Internet and SM provide important information and support to cancer patients. Evaluating how patients use these resources is important as it can impact decision-making.

\section{Objectives}

We evaluated associations between patients' age, confidence in computer-use, and use of Internet/SM for cancer care.

\section{Methods}

Cancer patients completed a cross-sectional survey of cancer-related SM/ Internet use and self-confidence using these resources. Multivariable logistic regression evaluated factors associated with Internet/SM use.

\section{Results}

Among 320 patients, 127 were AYA (age 18-39), 127 were adult (40-64) and 66 were elderly (65+). Most (>95\%) had a smartphone/tablet/computer and used the Internet daily. Compared to AYA, non-AYA were less likely $(\mathrm{P}<0.001)$ to own a data plan $(77 \%$ vs $92 \%)$, have a SM account (72\% vs $95 \%$ ) or feel confident using computers (76\% vs $98 \%$ ). $75 \%$ used Internet and $43 \%$ used SM for cancer care; $58 \%$ felt confident using online information for decision-making. AYA were more likely than nonAYA to use the Internet $(\mathrm{aOR}=1.60,95 \% \mathrm{CI}[0.93-2.81], \mathrm{P}=0.10)$ and $\mathrm{SM}$ $(\mathrm{aOR}=1.75[1.04-2.95], \mathrm{P}=0.04)$ for cancer care. Confident computer users were more likely to use Internet $(\mathrm{aOR}=5.36[2.67-11.00], \mathrm{P}<0.001)$ and $\mathrm{SM}(\mathrm{aOR}=4.61[1.98-12.14], \mathrm{P}<0.001)$ for cancer care and were more confident using this information in decision-making $(\mathrm{aOR}=5.12[1.92$ $17.81], \mathrm{P}<0.001)$. Age was not associated with self-confidence using online information for decision-making $(\mathrm{P}>0.10)$.

Conclusions

Despite higher use of internet/SM for cancer care, AYA did not feel more self-confident evaluating online cancer information. Confidence in computer use was associated internet/SM usage and confidence evaluating online information. Patient education programs should focus on improving patients' confidence in using online resources so they can better evaluate online information for cancer care. 
PS013

THE EFFECT OF PREHABILITATION ON PREOPERATIVE FUNCTIONAL CAPACITY, SYMPTOM BURDEN, AND POSTOPERATIVE COMPLICATIONS IN ESOPHAGEAL CANCER SURGERY

D. Makiura ${ }^{1}$, J. Inoue ${ }^{1}$, R. Ono ${ }^{2}$, Y. Sakai ${ }^{1,3}$

${ }^{T}$ Kobe University Hospital, Department of Rehabilitation, Kobe, Japan

${ }^{2}$ Kobe University Graduate School of Health Sciences, Department of Community Health Sciences, Kobe, Japan

${ }^{3}$ Kobe University Graduate School of Medicine, Division of

Rehabilitation Medicine, Kobe, Japan

\section{Introduction}

Previous studies have shown that prehabilitation improve preoperative functional outcomes and decrease postoperative complications in abdominal cancer surgery. However, the effect of prehabilitation in esophageal cancer surgery remains unclear.

Objectives

The objective of this study was to clarify the effect of prehabilitation on preoperative functional outcomes and postoperative complications in esophageal cancer surgery.

Methods

This study was a prospective cohort study. Among the 155 patients who underwent esophagectomy, 73 patients received hospital-based prehabilitation consisting of inspiratory muscle training, aerobic exercise, and resistance training. Prehabilitation was carried out for 40-60 minutes daily on weekdays under the supervision of physiotherapists. The 6-min walk test and the MD Anderson Symptom Inventory (MDASI) were assessed as indicators of preoperative functional outcomes. Postoperative complications were defined as Clavien-Dindo grade $>=2$ within 30 days after surgery. Paired t-test and logistic regression analysis were performed in statistical analysis.

Results

Patients in the prehabilitation group were significantly older and showed poorer performance status. The mean 6-min walk distance increased significantly from $421 \mathrm{~m}$ at baseline to $439.1 \mathrm{~m}$ at the end of the preoperative period $(\mathrm{p}=0.01)$. The mean MDASI score decreased significantly from 2.60 to 1.62 $(\mathrm{p}=0.03)$. The incidence of postoperative pulmonary complications was lower in the prehabilitation group (17.8\%) than in the control group $(31.7 \%)$. Multivariable logistic regression analysis showed that prehabilitation was effective in the prophylactic treatment of postoperative pulmonary complications (odds ratio $0.38,95 \%$ confidence interval $0.17-0.89$ ).

Conclusions

Prehabilitation may improve preoperative functional capacity and symptom burden and decrease postoperative pulmonary complications in esophageal cancer surgery.

\section{PS014}

EARLY INDEPENDENT PREDICTION OF ANTHRACYCLINEINDUCED CARDIOMYOPATHY BY N-TERMINAL PROBRAIN NATRIURETIC PEPTIDE

\author{
V. Poroch ${ }^{1}$, A.M. Pascu ${ }^{2}$, A. Bisog ${ }^{3}$, M. Radoi ${ }^{2}$ \\ ${ }^{T}$ Grigore T. Popa University of Medicine and Pharmacy Iasi, Palliative \\ Care-Surgery, Iasi, Romania \\ ${ }^{2}$ Faculty of Medicine- Transilvania University of Brasov- Romania, \\ Pathophysiology, Brasov, Romania \\ ${ }^{3}$ Faculty of Medicine- Transilvania University of Brasov- Romania, \\ Cardiology Department, Brasov, Romania
}

\section{Introduction}

Early prediction of anticancer therapy cardiotoxicity is essential for applying proper preventive and supportive therapeutic strategies.

Objectives

Evaluation of plasma N-terminal fragment of pro-brain natriuretic peptide(NT-proBNP) in predicting cardiac dysfunction assessed by transthoracic 2D echocardiography(2D-TTE) in patients with cancer and early onset asymptomatic anthracycline-induced cardiomyopathy(AIC).

\section{Methods}

Prospective study of 68 patients with cancer treated with anthracyclines, followed up for 6 months. Diagnosis of AIC was set at 6 months by decreasing of left ventricular ejection fraction(LVEF) below $50 \%$ or with more than 10 units or $20 \%$ from baseline. NT-proBNP and 2D-TTE were assessed at enrolment, and thereafter at 3 and 6 months.

\section{Results}

Fifteen (22.1\%) patients developed AIC at 6 months of anthracycline treatment (group 1), and 53 (77.95\%) patients did not evolve with AIC (group 2). At 3 months, in patients from group 1 NT-proBNP was significantly higher compared to group $2[121.0(119.8 ; 140.8) \mathrm{pg} / \mathrm{mL}$ vs. $97.7(75.5 ; 111.7) \mathrm{pg} / \mathrm{mL}, P=0.0001$, values expressed as median $\left(25^{\text {th }} ; 75^{\text {th }}\right.$ percentiles)]. NT-proBNP at 3 months proved accurate in predicting asymptomatic AIC at 6 months [area under the receiver operating characteristic curve (AUC) $=0.845,95 \%$ Confidence Interval (CI): 0.735-0.954, $P=0.0001]$. Left ventricular diastolic dysfunction(LVDD) was significantly more frequent in group $1(93.3 \%) v$. group $2(37.7 \%), P=0.0002$. New-detected LVDD at 3 months had $60 \%$ sensitivity, and $77 \%$ specificity in predicting AIC at 6 months. NT-proBNP assessed at 3 months above a cutoff $=118.5 \mathrm{pg} / \mathrm{mL}$ was an independent predictor of AIC at 6 months.

\section{Conclusions}

Plasma NT-proBNP at 3 months of anthracycline therapy proved to be an early independent predictor of asymptomatic anthracycline-induced cardiomyopathy.

\section{PS015}

INTRAVENOUS FOSAPREPITANT FOR THE PREVENTION OF CHEMOTHERAPY INDUCED VOMITING IN CHILDREN, A RANDOMIZED, PHASE 3 TRIAL

\author{
V. Radhakrishnan ${ }^{1}$, A. Joshi ${ }^{1}$, J. Ramamoorthy ${ }^{1}$, S. Rajaraman ${ }^{2}$, P.

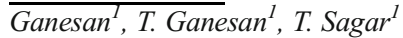 \\ ${ }^{1}$ Cancer Institute W.I.A, Medical Oncology, Chennai, India \\ ${ }^{2}$ Cancer Institute W.I.A, Epidemiology and Biostatistics, Chennai, India
}

\section{Introduction}

Fosaprepitant, is a NK-1 receptor antagonist approved in adults for the prevention of chemotherapy induced vomiting. However, its safety and efficacy in pediatric patients is not known.

\section{Objectives}

Evaluation of efficacy and safety of fosaprepitant as an antiemetic in pediatric patients receiving moderate to highly emetogenic chemotherapy Methods

The study was a phase 3 , double-blind, randomized, placebo controlled trial. Patients aged 1-12 years with cancer, scheduled to receive moderate to highly emetogenic chemotherapy, were randomly assigned to fosaprepitant or Placebo arms. Patients in both arms received ondansetron plus dexamethasone and these were continued for 48 hours after completion of chemotherapy. Primary end-point of the study was the proportion of patients who achieved a complete response (defined as no vomiting, no retching) during the 25-120 hours (delayed phase) after administration of fosaprepitant or placebo. Secondary end-point was proportion of patients who achieved complete response during the acute ( $0-24$ hours) and overall phases after administration of fosaprepitant or placebo.

\section{Results}

135 patients were analyzed (68 in fosaprepitant arm and 67 in placebo arm). Complete response rates were significantly higher in fosaprepitant arm compared to placebo arm during acute phase ( $84 \%$ vs. $57 \%, \mathrm{p}<0.001)$, delayed phase $(79 \%$ vs. $51 \%, \mathrm{p}<0.001)$ and overall phases $(69 \%$ vs. $42 \%, \mathrm{p}=0.0014)$. Three (4\%) patients in fosaprepitant arm and fourteen $(21 \%)$ patients in the placebo arm required rescue anti-emetics $(\mathrm{p}=0.004)$. No fosaprepitant related grade 3-4 adverse events were observed.

\section{Conclusions}

Fosaprepitant is safe and effective for the prevention of chemotherapy induced vomiting in pediatric patients receiving moderate to highly emetogenic chemotherapy. 


\section{PS016}

PHOTOBIOMODULATION THERAPY PREVENTS SEVERE ACUTE RADIODERMATITIS: A RANDOMIZED, PLACEBOCONTROLLED TRIAL IN BREAST CANCER PATIENTS WITH CLINICAL AND OBJECTIVE OUTCOME MEASURES

J. Robijns ${ }^{1}$, S. Censabella ${ }^{2}$, S. Claes ${ }^{3}$, L. Pannekoeke ${ }^{3}$, L. Bussé $e^{1}, I$.

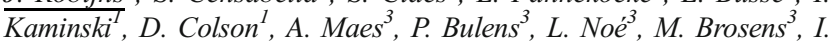
Lambrichts ${ }^{1}$, A. Timmermans ${ }^{4}$, V. Somers ${ }^{1}$, J. Mebis $^{2}$

${ }^{1}$ Hasselt University, Medicine and Life Sciences, Hasselt, Belgium

${ }^{2}$ Jessa Hospital, Medical Oncology, Hasselt, Belgium

${ }^{3}$ Jessa Hospital, Limburg Oncology Center, Hasselt, Belgium

${ }^{4}$ Jessa Hospital, Dermatology, Hasselt, Belgium

\section{Introduction}

Acute radiodermatitis (ARD) occurs in up to $95 \%$ of the patients undergoing radiotherapy (RT). No general consensus on the prevention and management of ARD is currently available.

Objectives

To investigate the efficacy of photobiomodulation therapy (PBMT) in the prevention of ARD in breast cancer patients undergoing RT.

\section{Methods}

A randomized, placebo-controlled trial (RCT) with 120 breast cancer patients that underwent RT was performed. Patients were assigned to a control $(n=60)$ or laser group $(n=60)$. PBMT or placebo was applied $2 x /$ week, from the first until the last day of RT. The MLS ${ }^{\circledR}$ M6, a scanning laser, consisting of two synchronized laser diodes in the infrared range (808-905 nm) was used. Skin toxicity was clinically evaluated by the Radiation Therapy Oncology Group (RTOG) criteria and objectively by measuring the transepidermal water loss (TEWL) and erythema. Measures were collected at baseline (before the start of RT), a RT dose of 40Gy and the end of RT (66Gy).

\section{Results}

At the end of $\mathrm{RT}$, the incidence of acute $\mathrm{RD} \geq \mathrm{G} 2$ was significantly higher in the control than in the laser group ( $\mathrm{p}=0.004$, Fig. 1$)$. The objective measures confirm these clinical results by showing that the mean percentage change from the baseline TEWL and erythema value was significantly higher in the control than in the laser group at the end of RT (ps<0.05, Fig. 2).

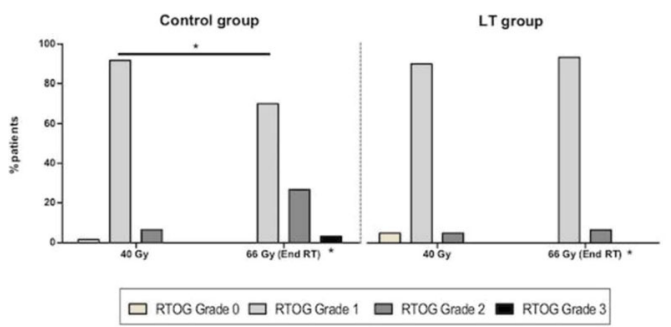

Fig.1: Clinical evaluation of skin toxicity. Severity of ARD expressed in RTOG grades for the control and LT group at a RT dose of 40Gy and at the end of RT (66Gy). *Significan difference within the control group between the two time points and between the two groups
at the end of $R T$ ( $p<.0 .05$; Chi-square test, two-tailid). ARD, accte radiation dermatitis; $G y$, Gray; LT, laser therapy; RT, radiotherapy; RTOG: Radiation Therapy Oncology Group
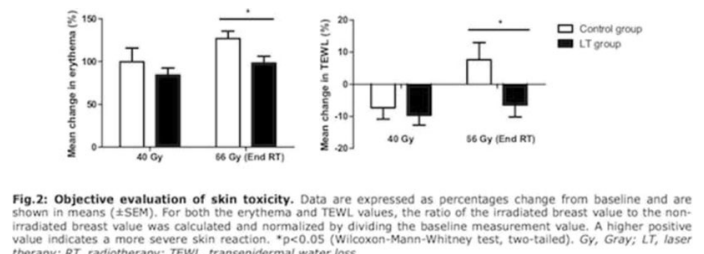

Conclusions

This is the first RCT demonstrating by both a clinical and objective approach that PBMT based on laser diodes is effective in reducing the incidence of high grade ARD in breast cancer patients.
PS017

NASAL COLONIZATION WITH STAPHYLOCOCCUS AUREUS BEFORE RADIOTHERAPY PREDICTS RADIATION DERMATITIS: A PROSPECTIVE STUDY

A.Rzepecki ${ }^{1}$, M. Birnbaum ${ }^{2}$, N. Ohri ${ }^{3}$, J. Fox ${ }^{3}$, R. Kabarriti ${ }^{3}$, W. Bodner ${ }^{3}$, K.Mehta ${ }^{3}$, M. Garg, J. Daily, S. Kalnicki ${ }^{3}$, B. McLellan ${ }^{2}$

${ }^{1}$ University of Michigan Medical School, Dermatology, Ann Arbor, USA

${ }^{2}$ Albert Einstein College of Medicine, Dermatology, New York City, USA

${ }^{3}$ Montefiore Medical Center, Radiation Oncology, New York City, USA

${ }^{4}$ Albert Einstein College of Medicine, Microbiology, New York City, USA

\section{Introduction}

Acute radiation dermatitis is a common side effect of radiation therapy (RT). Radiation-induced skin changes may increase the risk of secondary skin infection. Little is known regarding the baseline incidence of microbial colonization prior to RT and whether it is associated with development of radiation dermatitis.

\section{Objectives}

Our goals are to 1) characterize the incidence of baseline bacterial colonization in patients undergoing RT, and 2) examine the association between radiation dermatitis severity and baseline bacterial colonization. Methods

We are presenting preliminary results from a prospective trial in which cancer patients undergo bacterial culture swabs from the nares prior to RT initiation. Patients are evaluated weekly during RT, and dermatitis is graded using CTCAE version 4. Descriptive statistics and odds ratios are utilized to present associations between culture results and skin toxicity.

\section{Results}

Forty-eight subjects with cancers of the breast (48\%), head and neck (44\%), and anus $(8 \%)$ completed RT. Baseline bacterial cultures in the nares were positive for Staphylococcus aureus in 10 subjects (21\%). Twenty-eight patients (58\%) developed grade 1 dermatitis, 15 (31\%) developed grade 2 dermatitis, and $5(10 \%)$ developed grade 3 dermatitis. Positive culture for S. aureus at baseline was associated with increased risk of grade $2-3$ dermatitis $(80 \%$ v. $32 \%, \mathrm{OR}=8.7$, $\mathrm{p}=0.012)$ and with receipt of silver sulfadiazine $(70 \% \mathrm{v} .29 \%, \mathrm{OR}=5.7, \mathrm{p}=0.025)$.

\section{Conclusions}

Bacterial colonization with S.aureus prior to RT initiation is a risk factor for developing high-grade radiation dermatitis. Further studies are needed to understand this association and to explore antimicrobial therapy as a strategy to prevent radiation dermatitis.

\section{PS018}

PROTECTIVE EFFECT OF BRAIN-DERIVED NEUROTROPHIC FACTOR (BDNF) GENETIC POLYMORPHISM (RS6265) AGAINST CHEMOTHERAPY-RELATED COGNITIVE DECLINE IN EARLYSTAGE BREAST CANCER PATIENTS: A VALIDATION STUDY

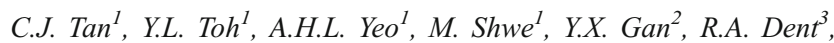
R.C.H. Ng ${ }^{3}$, Y.S. Yap ${ }^{3}$, K.W.J. Loh $^{3}$, G.E. Lee ${ }^{3}$, T.J.Y. Tan ${ }^{3}$, S.Y. Beh ${ }^{3}$, M. Wong ${ }^{3}$, C.C. Khor ${ }^{4}$, H.K. Ho ${ }^{1}$, A. Chan ${ }^{1}$

${ }^{I}$ National University of Singapore, Department of Pharmacy, Singapore, Singapore

${ }^{2}$ National Cancer Centre Singapore, Department of Pharmacy, Singapore, Singapore

${ }^{3}$ National Cancer Centre Singapore, Division of Medical Oncology, Singapore, Singapore

${ }^{4}$ Genome Institute of Singapore, Human Genetics, Singapore, Singapore

\section{Introduction}

We have previously observed that a $B D N F$ single nucleotide polymorphism (rs6265) leads to substitution of valine by methionine at codon 66 of BDNF peptide (BDNFVal66Met) and protects against cognitive decline in patients undergoing chemotherapy $(n=145)$, specifically in the multitasking $(\mathrm{OR}=0.37$; 95\%CI:0.15-0.91) and verbal ability $(\mathrm{OR}=0.34$; 95\%CI:0.10-0.90) domains (Ng et al., Neuro Oncol 2016). 


\section{Objectives}

In a temporally separate cohort, to validate the associations observed between $B D N F$ Val66Met polymorphism and cognitive function.

Methods

This was a prospective, longitudinal study conducted between February 2014 to November 2017. Early-stage breast cancer patients were recruited and assessed before, during and after chemotherapy. Cognitive function was evaluated using validated FactCog version 3.0, which comprises 6 cognitive domains, with cognitive decline defined as $>15 \%$ reduction from pre-chemotherapy scores. Genotyping was conducted using Sanger sequencing. Associative relationships were investigated with logistic models, adjusting for potential confounders of cognition including age, education level, ethnicity, anxiety, insomnia, fatigue, chemotherapy regimen and menopausal status.

Results

189 patients were successfully genotyped. Mean age \pm SD was $51.8 \pm 9.0$ years and 123 patients $(65.1 \%)$ underwent anthracycline-based chemotherapy. 86 patients $(45.5 \%)$ reported cognitive decline in at least 1 domain. After adjusting for confounders, odds of cognitive decline were lower among Met allele carriers in 3 domains: multitasking $(\mathrm{OR}=0.34$; 95\%CI:0.15-0.73; $\mathrm{p}=0.006)$, memory $(\mathrm{OR}=0.25 ; 95 \% \mathrm{CI}: 0.10-0.63$; $\mathrm{p}=0.004)$ and mental acuity $(\mathrm{OR}=0.45 ; 95 \% \mathrm{CI}: 0.21-0.99 ; \mathrm{p}=0.046)$.

Conclusions

In a temporally separated cohort, carriers of $B D N F$ Met allele have demonstrated to be at lower odds of developing multitasking problems postchemotherapy. These results validate our previous findings that $B D N F$ polymorphism confers varying degrees of protection in different cognitive domains.

\section{PS019}

THE ASSOCIATION BETWEEN GLUCOCORTICOID RECEPTOR SENSITIVITY AND FATIGUE IN PATIENTS WITH HEAD AND NECK CANCER

C. Xiao ${ }^{1}$, R.C.Eldridge ${ }^{I}$, J. Beitler ${ }^{2}$, K.A. Higgins ${ }^{2}, J$. Felger $^{2}$, E.C. Wommack $^{2}$, N.F. Saba', D.M. Shin ${ }^{2}$, D.W. Bruner ${ }^{1}$, A.H. Miller ${ }^{2}$

${ }^{I}$ Emory University, School of Nursing, Atlanta, USA

${ }^{2}$ Emory University, School of Medicine, Atlanta, USA

\section{Introduction}

Most head and neck cancer (HNC) patients experience significant fatigue during and after cancer treatment. Although our previous studies have shown a positive link between inflammation and the development of fatigue, the mechanisms of this association are not well-understood.

Objectives

Glucocorticoids binding to their receptor play a primary role in the negative regulation of inflammation. The purpose of this study is to explore whether low glucocorticoid sensitivity was associated with fatigue.

Methods

Consented patients were followed pre-, and one-month post-radiotherapy. Fatigue was assessed by the Multidimensional Fatigue Inventory-20. Glucocorticoid receptor (GR) sensitivity was calculated based on the relative (percentage) in vitro inhibition of interleukin- 6 by increasing concentrations of the synthetic glucocorticoid dexamethasone $\left(10^{-9}-10^{-}\right.$ ${ }^{5}$ ). Using linear regression, we examined whether the change in the GR sensitivity between pre- and post-radiotherapy was predictive of fatigue at one-month, adjusting for fatigue pre-radiotherapy, age, sex, race, body mass index, human papillomavirus, smoking status, alcohol consumption status, marital status, and chemotherapy.

\section{Results}

Seventy-four patients were enrolled into the study; the majority were male, white, and married. Sixty-percent had smoking history and half had alcohol history. Fifty-five percent had oropharyngeal cancer and half were HPV positive. Majority received concurrent chemoradiotherapy. Decreased glucocorticoid sensitivity was associated with higher fatigue at one-month post-radiotherapy ( $\mathrm{p}=0.031$ ), after controlling for covariates and pre-radiotherapy fatigue.

\section{Conclusions}

Our findings support our hypothesis that lower glucocorticoid sensitivity might be linked to high inflammation, which may in turn lead to fatigue. More data, including inflammatory markers, are needed to verify this hypothesis

\section{PS020}

LEPTIN AS A PREDICTIVE BIOMARKER FOR THE ONSET OF CANCER-RELATED FATIGUE (CRF): A PROSPECTIVE COHORT STUDY

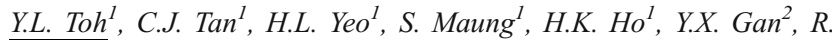
Dent, C.H. Ng ${ }^{3}$, Y.S. Yap ${ }^{3}$, W.J. Loh $^{3}$, G.E. Lee ${ }^{3}$, S.Y. Beh ${ }^{3}$, J.Y. Tan ${ }^{3}$, M. Wong ${ }^{3}$, A. Chan ${ }^{1,2}$

${ }^{I}$ National University of Singapore, Department of Pharmacy, Singapore, Singapore

${ }^{2}$ National Cancer Centre, Department of Pharmacy, Singapore, Singapore

${ }^{3}$ National Cancer Centre, Division of Medical Oncology, Singapore, Singapore

\section{Introduction}

Leptin is known for its metabolic effects, ranging from appetite suppression to increasing energy expenditure. Being pro-inflammatory, leptin is postulated to have a role in predicting onset of Cancer-Related Fatigue (CRF).

\section{Objectives}

To evaluate the association between leptin and CRF in early-stage breast cancer patients receiving chemotherapy.

\section{Methods}

In a prospective cohort study, patients completed assessments at baseline (T1), during (T2) and after chemotherapy (T3). At each time point, plasma leptin level was measured using Luminex bead-immunoassay and the validated Multi-Dimensional Fatigue Symptom Inventory-Short form (MFSI-SF) was utilized to measure CRF. Data was longitudinally analysed using a Generalized Estimating Equation model, incorporating clinically relevant parameters and pro-inflammatory cytokines that were statistically associated with total MFSI-SF score.

\section{Results}

136 patients were recruited (mean age $\pm \mathrm{SD}=51.5 \pm 8.8$ years; $69.1 \%$ receiving anthracycline-based chemotherapy). More patients experienced CRF at T3 $(22.1 \%)$ than at T2 $(13.2 \%)$ compared to baseline. Mean $( \pm$ SD) leptin level was $5.44 \pm 4.24 \mathrm{ng} / \mathrm{mL}$. Leptin inversely correlated with total MFSI-SF score $(\beta=-0.56, p<0.01)$, general $(\beta=-0.16, p<0.01)$, emotional $(\beta=-0.11, p<0.01)$ and mental sub-domains $(\beta=-0.06, p=$ $0.025)$. In the final model, leptin was significantly associated with total MFSI-SF score $(\beta=-0.22, p<0.01)$ after adjusting for anxiety, depression, insomnia, age, menopausal status and chemotherapy.

\section{Conclusions}

This is the first study to demonstrate leptin as a biomarker to predict the onset of CRF over time. Future studies are required to validate the findings.

\section{PS021}

USING A SYMPTOM HEURISTICS IPAD APP TO IMPROVE SYMPTOM SELF-MANAGEMENT IN ADOLESCENTS AND YOUNG ADULTS WITH CANCER

\author{
S. Ameringer $^{1}$, J.M. Erickson ${ }^{2}$, R.K. Elswick- Jr. ${ }^{1}$, L. Linder ${ }^{3}$, C.F. \\ Macpherson $^{4}$, K. Stegenga ${ }^{5}$ \\ ${ }^{I}$ Virginia Commonwealth University, Family and Community Health \\ Nursing, Richmond, USA
}


${ }^{2}$ University of Wisconsin-Milwaukee, College of Nursing, Milwaukee, USA

${ }^{3}$ University of Utah, College of Nursing, Salt Lake City, USA

${ }^{4}$ Seattle Children's Hospital, Cancer and Blood Disorders, Seattle, USA

${ }^{5}$ Children's Mercy Hospital, Division of Hematology/Oncology and Patient Care Services, Kansas City, USA

\section{Introduction}

Adolescents and young adults (AYAs) with cancer experience multiple distressing symptoms from their disease/treatment and voice the need to better self-manage their symptoms. The Computerized Symptom Capture Tool (C-SCAT) is an inductive, heuristic-based resource for AYAs to communicate their symptom experience by creating an image of their symptoms and perceived relationships between symptoms. This study pilot-tested the efficacy of the C-SCAT as a resource to support symptom self-management.

\section{Objectives}

To examine the effects of using the C-SCAT on perceived self-efficacy for symptom self-management and patient-provider communication of AYAs receiving chemotherapy.

\section{Methods}

The study used a single-group pretest-posttest design to enroll 85 AYAs with any cancer, ages 15-29 years, at five U.S. sites. Data were collected at baseline and two subsequent clinic/inpatient visits. AYAs completed the C-SCAT prior to a provider visit and used the image to discuss symptoms during the visit. Measures of self-efficacy and perceived patientprovider communication were completed following the visits. A linear mixed effects model containing fixed effects of site, visit, and site $\mathrm{x}$ visit interaction, and a random effect for subject was used to analyze these longitudinal

\section{Results}

Of the 85 participants who completed the baseline visit, 81 completed Visit 1, and 77 completed Visit 2. Mean (SD) age was 20.9 years (4.93), $54 \%$ were male, and $72 \%$ were White. Self-efficacy for symptom selfmanagement improved significantly over time $(\mathrm{p}=0.03)$. Patient-provider communication did not improve significantly $(\mathrm{p}=0.27)$; however, scores were high across visits.

\begin{tabular}{|c|c|c|c|c|c|c|}
\hline \multirow[b]{2}{*}{ Variable } & \multicolumn{2}{|c|}{ Baseline } & \multicolumn{2}{|c|}{ Visit 1} & \multicolumn{2}{|c|}{ Visit 2} \\
\hline & Mean & SE & Mean & SE & Mean & SE \\
\hline PROMIS S-E & 102.22 & 2.124 & 103.52 & 2.150 & 107.01 & 2.182 \\
\hline PPCS & 3.62 & 0.055 & 3.63 & 0.056 & 3.71 & 0.057 \\
\hline
\end{tabular}

\begin{tabular}{|c|c|c|c|}
\hline & df & $F$ & $p$ value \\
\hline \multicolumn{4}{|l|}{ PROMIS S-E } \\
\hline $\begin{array}{l}\text { Main enlects } \\
\text { Site }\end{array}$ & \multicolumn{3}{|c|}{ Main effects } \\
\hline Visit & 149 & 3.50 & $0.033^{*}$ \\
\hline \multicolumn{4}{|c|}{ Interaction effects } \\
\hline Site $\times$ Visit & 149 & 1.10 & 0.364 \\
\hline \multicolumn{4}{|l|}{ PPCS } \\
\hline \multicolumn{4}{|l|}{ Main effects } \\
\hline Site & 80 & 1.54 & 0.198 \\
\hline Visit & 149 & 1.30 & 0.275 \\
\hline \multicolumn{4}{|c|}{ Interaction effects } \\
\hline Site $\times$ Visit & 149 & & 0.677 \\
\hline
\end{tabular}

PROMIS S-E twenty-eight item Patient Reported Outcomes Information System Self-Efficacy Scale (range, 28-140; higher scores indicate greater self-efficacy), PPCS four item PatientProvider Communication Scale (range 0-4; higher scores indicate better communication)

${ }^{*} \mathrm{p}<.05$

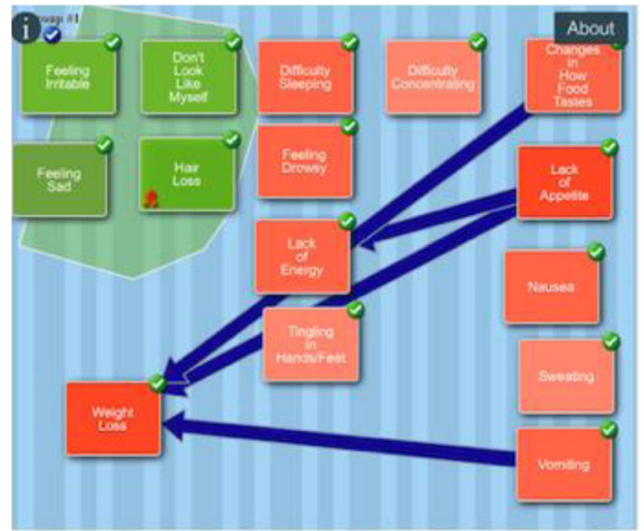

Figure 1 Image created on the C-SCAT by a 17-year-old male with acute lymphocytic leukemia. The AYA chooses symptoms, draws lines between symptoms they perceive are related, groups symptoms that occur together, and identifies priority symptoms.

\section{Conclusions}

The C-SCAT shows promise for improving self-efficacy for symptom self-management in AYAs with cancer.

\section{PS022}

\section{THE EFFECT OF A CULTURALLY TAILORED INTERNET CANCER SUPPORT GROUP ON SUPPORT CARE NEEDS OF ASIAN AMERICAN BREAST CANCER SURVIVORS}

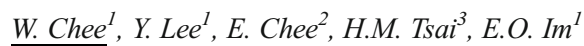

${ }^{T}$ Duke University, Nursing, Durham, USA

${ }^{2}$ North Carolina State University, Biomedical Engineering, Raleigh, USA

${ }^{3}$ Chang Gung Univ. of Science and Technology, Nursing, Taipei, Taiwan R.O.C.

\section{Introduction}

Internet cancer support groups (ICSGs) effectively provide emotional support, information, and interactionswith peers and health care professionals for cancer survivors, including those with breast cancer. Although racial/ethnic minorities reportedly have much greater benefits (compared to Whites) from participating in ICSGs, the use of ICSGs by ethnic minorities including Asian Americans is still minimal.

\section{Objectives}

The purpose of this study was to explore the preliminary efficacy of a culturally tailored ICSG on support care needs of Asian American breast cancer survivors.

\section{Methods}

This was a pilot study adopting a randomized controlled pre-test and post-test design. The participants included 65 Asian American breast cancer survivors. Multiple instruments were used to collect the data, which included the Support Care Needs Survey, the Memorial Symptom Assessment Scale, the Brief Pain Inventory, and the Functional Assessment of Cancer Therapy Scale-Breast Cancer. The data were analyzed using inferential statistics including the repeated ANOVA.

\section{Results}

Significant positive changes were found in support care needs of the control group from the pre-test to the post-test $(p<.01)$. Also, significant positive changes were found in physical and psychological symptoms of the control group from the pre-test to the post-test $(p<.05)$. When background and disease factors were controlled, the intervention group had significantly greater improvements than the control group in physical and psychological symptoms and quality of life from the pre-test to the posttest $(p<.10)$. 


\section{Conclusions}

The findings support that ICSGs have positive effects on support care needs of Asian American breast cancer survivors.

\section{PS023}

CAN CANCER ADVOCACY BE INFLUENCED BY SOCIAL MEDIA?

A. Charalambous

${ }^{T}$ Cyprus University of TEchnology, Nursing, Limassol, Cyprus

\section{Introduction}

Throughout the history of mankind there has been a single factor that remained unchanged. That is the need to adapt to a constantly changing environment in order to be part of the evolution. In the beginning, the adaptation was necessary for survival but nowadays it's about adapting to aspects such as the social media and optimizing these to shape politics, business, world culture, education, careers, advocacy and innovation to name a few. The dilemma presents itself as "Adapt or Perish" to a digital era.

\section{Objectives}

To present ways that the soft power of social media can be utilized in influencing advocacy within the cancer context.

\section{Method}

The discussion will be based on successful examples on how social media have influenced policy and advocacy within the cancer context. Relevant research on the value of specific social media (i.e. twitter) will also be discussed.

Results

Social media have not changed the impact of influence on cancer related topics but they certainly changed the way such influence is introduced. Existing models of influence and traditional hierarchical power structures have been challenged by the soft power of social media. Social media offer an accessible mean to mass networking and a platform where political ideas can be consistently advocated by ways such raising awareness (of the public) or providing evidence (and placing pressure) to relevant policy makers. In any case, social media can be a driver of change and here lies the reason for the tremendous increase of social media's influence in political campaigns.

\section{Conclusions}

Social media is seen as the latest arena in the global struggle for influence. Government and non-government stakeholders demonstrate a growing appreciation of its power to influence large numbers of people around the world. Research in this field shows that social media have value to cancer stakeholders such as family members, friends of cancer patients, journalists, cancer patients/survivors and policy makers. Therefore, there is a real opportunity for these stakeholders who may have traditional issues of access to cancer information and support to become actively engaged with social media.

\section{PS024}

CONVERSATIONS AT END-OF-LIFE: THE USE OF SIMULATION TO TARGET COMMUNICATION SKILLS IN END-OF-LIFE CARE

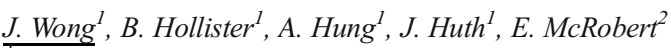 \\ ${ }^{T}$ Sunnybook Health Sciences Centre, Veterans Centre, Toronto, Canada \\ ${ }^{2}$ University of Toronto, Factor-Inwentash Faculty of Social Work, \\ Toronto, Canada
}

\section{Introduction}

In this long-term care facility,the distinction between "palliative" and "longterm" care has become increasingly blurred due to the continued aging of current and potential residents. Due to increased aging and frailty, two of our units have experienced increased turnover and decreased length of stay, increasing the need for quality end-of-life care. Effective communication is known to impact the quality of care at end-of-life, and published work supports the use of simulation to target communication skills at end-of-life. Objectives

The aim of this study is to pilot a simulation-based education program targeting end-of-life communication practices.

Methods

1. Pre-education surveys: Baseline data was gathered to assess staff self-ratings of comfort, knowledge, skill, and attitudes towards communication with patients/families at end-of-life.

2. Education program: A four week education program was piloted to provide staff with opportunities for team-based experiential learning. Key education strategies included simulation and debriefing.

3. Post-education surveys: Repeat surveys will be conducted following the education program and at six month follow-up to evaluate the effect of the simulation-based education program.

\section{Results}

This project commenced in November 2017 and will conclude in July 2018. The education simulation program is to be piloted January 2018. Findings from pre-/post-staff surveys will be shared at this conference.

Conclusions

Simulation-based education has the potential to positively impact communication practices at end-of-life. Interprofessional learning opportunities also provide teams with the ability to learn from, with, and about each other's roles in providing end-of-life care.

\section{PS025}

A NOVEL APPROACH TO IMPROVING AMBULATORY OUTPATIENT MANAGEMENT OF LOW RISK FEBRILE NEUTROPENIA: AN ENHANCED SUPPORTIVE CARE (ESC) CLINIC

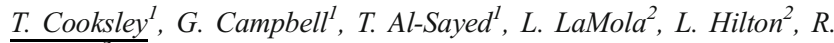
Berman

${ }^{1}$ The Christie-Manchester- United Kingdom, Acute Medicine and Critical Care, Manchester, United Kingdom

${ }^{2}$ The Christie-Manchester- United Kingdom, Supportive Care, Manchester, United Kingdom

\section{Introduction}

Outpatient management of low risk febrile neutropenia patients (LRFN) identified by the MASCC score is a safe and effective strategy. Early supportive care has been shown to improve outcomes in patients with care. We developed an innovative ambulatory outpatient "enhanced supportive care" (ESC) clinic combining emergency oncology and supportive care through which we incorporated the management of patients with LRFN.

\section{Objectives}

To assess the feasibility and effectiveness of the ESC clinic as a setting for the ambulatory outpatient management of patients with LRFN.

Methods

An ESC clinic was started in January 2017 at a tertiary cancer hospital in the North West of England. An integral part of the clinic was an ambulatory pathway for patients presenting with LRFN. Patients with a MASCC score $\geq 21$ and an Early Warning Score $\leq 3$ were potentially eligible for the pathway. Suitable patients were managed with oral amoxicillin/clavulanic acid (500/125mg TDS) and ciprofloxacin (500mg BD) or moxifloxacin $400 \mathrm{mg}$ OD if penicillin allergic. All patients had one dose of intravenous meropenem on arrival.

\section{Results}

In its first year 68 patients with LRFN were managed through the clinic. Table 1 shows the demographic data of the patients. $6(8.8 \%)$ patients had a 7 day readmission. There were no serious complications in the cohort. 


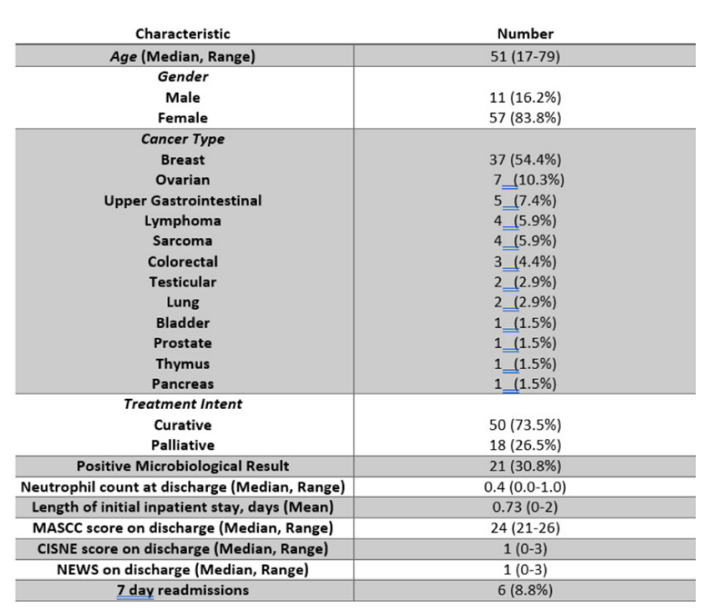

Table 1: Characteristics of patients with low risk febrile neutropenia managed through the ESC clinic

\section{Conclusions}

The ESC clinic appears to be an innovative and effective method for outpatient ambulatory management of patients with LRFN.

\section{PS026}

EFLAPEGRASTIM IS SAFE AND EFFECTIVE IN REDUCING SEVERE NEUTROPENIA IN PATIENTS RECEIVING MYELOSUPPRESSIVE CHEMOTHERAPY IN A PHASE 3 RANDOMIZED, CONTROLLED TRIAL COMPARED TO PEGFILGRASTIM (ADVANCE)

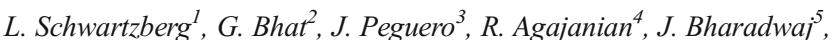

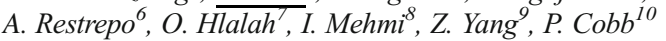

${ }^{1}$ University of Tennessee Health Sciences Center, Division of Hematology/Oncology, Memphis, USA

${ }^{2}$ Spectrum Pharmaceuticals-Inc., Biostatistics-Data Management- and Medical Writing, Irvine, USA

${ }^{3}$ Oncology Consultants PA, Department of Research, Houston, USA

${ }^{4}$ The Oncology Institute of Hope and Innovation, Hematology/Oncology, Downey, USA

${ }^{5}$ Pacific Cancer Medical Center, Hematologic Oncology, Anaheim, USA

${ }^{6}$ Texas Oncology, Medical Oncology, McAllen, USA

${ }^{7}$ Bond Clinic, Oncology and Hematology, Winter Haven, USA

${ }^{8}$ Edwards Comprehensive Cancer Center, Hematology/Oncology, Huntington, USA

${ }^{9}$ Spectrum Pharmaceuticals- Inc., Clinical Development, Irvine, USA

${ }^{10}$ Frontier Cancer Center, Hematology/Oncology, Billings, USA

\section{Introduction}

Eflapegrastim is a novel investigational biologic comprised of recombinant human G-CSF covalently linked to the human immunoglobulin G4FC fragment using proprietary LAPSCOVERY ${ }^{\mathrm{TM}}$ technology.

Objectives

This study was a randomized, Phase 3 study to demonstrate the noninferiority (NI) of eflapegrastim to pegfilgrastim in patients receiving chemotherapy for breast cancer.

Methods

Patients with Stage I to Stage IIIA breast cancer were treated with a single subcutaneous dose of either eflapegrastim $13.2 \mathrm{mg} / 0.6 \mathrm{~mL}$ or pegfilgrastim $(6 \mathrm{mg})$ in a 1:1 ratio on Day 2 of each of four cycles following adjuvant/neo-adjuvant docetaxel and cyclophosphamide (TC) on Day 1. The primary endpoint was to demonstrate noninferiority of eflapegrastim as measured by the mean duration of severe neutropenia (DSN) in Cycle 1 with NI margin of $<0.62$ day.

\section{Results}

In a total of 406 intent-to-treat patients (randomized to 196 eflapegrastim; 210 pegfilgrastim), median age was 61 years (range 24 to 84 years); mean (SD) DSN was $0.19(0.478)$ days for eflapegrastim and 0.34 (0.668) days for pegfilgrastim, demonstrating the non-inferiority (95\% CI of $\triangle \mathrm{DSN}$ : [$0.260,-0.035] ; \mathrm{p}<0.0001)$. The non-inferiority of eflapegrastim for DSN was maintained across all 4 cycles. The adverse events observed in $\geq 10 \%$ of patients were similar across both arms and were mainly hematologic including neutropenia, decreased lymphocytes, anemia and leukopenia.

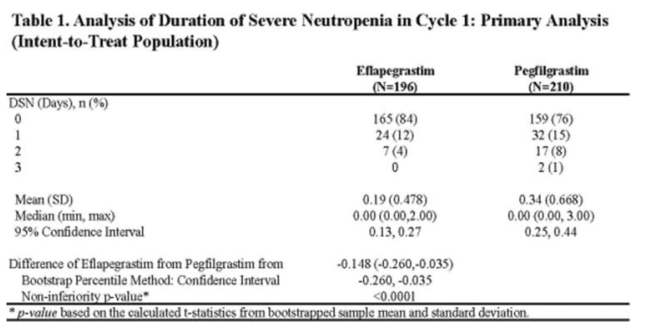

\section{Conclusions}

Eflapegrastim, a novel long acting G-CSF was non-inferior to pegfilgrastim in the reduction of DSN in Cycles 1-4, in breast cancer patients treated with TC. Eflapegrastim was safe and well-tolerated with a similar safety profile to pegfilgrastim.

PS027

TIMING OF EMPIRICAL COMPUTERIZED TOMOGRAPHY IMAGING OF CHEST IN CHILDREN WITH HIGH RISK FEBRILE NEUTROPENIA- SHOULD DELAYED IMAGING BE PREFERRED?

J. Ramamoorthy ${ }^{\text {, }}$ V. Radhakrishnan ${ }^{2}$, T.G. Sagar ${ }^{2}$

${ }^{T}$ Assistant professor-Cancer Institute, Department of Medical Oncology, chennai, India

${ }^{2}$ Cancer institute- Adyar-Chennai, Department of Medical Oncology, Chennai, India

\section{Introduction}

Isolation of the organism and site of infection is a vital task during treatment of high risk febrile neutropenic episode (HRFNE). It is recommended in adults to perform CT chest after 5 days in HRFNE. Pediatric data is limited and still there is no clear recommendation on timing of $\mathrm{Ct}$ chest evaluation.

Objectives

To determine the ideal timing for performing of empirical computerized tomography imaging (CTI) in children with HRFNE.

\section{Methods}

Children treated for acute lymphoblastic leukemia (ALL) from January 2013 to December 2014 were analyzed retrospectively. Those who underwent CTI of chest for evaluation of high HRFNE were enrolled. The timing of the CTI of chest was decided at the discretion of treating physician. Patients were classified as non-empirical CTI if the CT scan was performed after positive localizing symptom or screening imaging or serum galactomanan. Those without these were classified as empirical CTI.

\section{Results}

Fifty CTI of chest including 23/50 empirical CTI were performed in 42/ 194 children. Abnormality in CTI was identified in 36/50 (72\%) scans including 16/23 empirical CTI. The abnormalities seen in empirical CTI were: pulmonary nodules- $11 / 23$, consolidation- $2 / 23$, cavity, lung abscess and air crescent sign in $1 / 23$ each. Normal and abnormal empirical CT imaging was performed on 6 (IQR 5-10) and 10.5 (IQR 7.5-12.25) days of FNE respectively. There was significant association between abnormal empirical CTI and performance of CTI $>7$ days of FNE ( $\mathrm{p}=0.048$ ). 
Conclusions

Performance of empirical CTI of chest after 7; days of FNE has the highest probability of identifying an abnormality.

\section{PS028}

SYSTEMATIC REVIEW AND META-ANALYSIS: THE PREDICTIVE VALUE OF SERUM BIOMARKERS IN THE ASSESSMENT AND MANAGEMENT OF FEVER DURING NEUTROPENIA IN CHILDREN WITH CANCER

\author{
T. Arif ${ }^{\text {}}$, R. Phillips ${ }^{2}$ \\ ${ }^{T}$ Sheffield Children's Hospital, Paediatric Oncology, Sheffield, United \\ Kingdom \\ ${ }^{2}$ University of York, Centre for Reviews and Dissemination, York, United \\ Kingdom
}

\section{Introduction}

Clinical decision rules for risk stratification in pediatric febrile neutropenia (FN) perform more reliably in the country they originate than internationally. Biomarkers have been used in 3 out of 25 existing clinical decision rules. Understanding biomarkers as predictors for adverse outcomes in FN could help formulate a clinical decision rule that can be applied internationally.

Objectives

To assess the sensitivity and specificity of biomarkers in predicting bacteraemia, clinically documented infections, microbiologically documented infection, severe sepsis requiring intensive care or high dependency care, and death. To explore the role of biomarkers in reducing length of stay in hospital or community based treatment.

\section{Methods}

The review protocol was registered with the international register of systematic reviews database (March 2016) and updates two previous systematic reviews. No language restrictions were applied. Electronic databases were searched with further articles identified through checking reference lists and contacting authors of unpublished data.

Results

This review collates 7675 episodes from 4508 patients. Pooled sensitivities and specificities analyses show PCT to be the most specific and IL-8 the most sensitive biomarkers for predicting adverse events (Table 1).

\begin{tabular}{|c|c|c|}
\hline Blomarker & $\begin{array}{l}\text { Pooled Sensitivity (95\% } \\
\text { Confidence Interval) }\end{array}$ & \begin{tabular}{|l} 
Pooled Specificity (95\% \\
Confidence Interval)
\end{tabular} \\
\hline $\begin{array}{l}\text { C-reactive Protein (CRP) } \\
\text { Procalcitonin (PCT) }\end{array}$ & $\frac{0.45(0.15-0.75)}{0.60(0.35-0.81)}$ & \begin{tabular}{|l|}
$0.62(0.31-0.87)$ \\
$0.78(0.53-0.90)$
\end{tabular} \\
\hline Interleukin-6 (IL-6) & $\frac{0.0(0.35-0.01)}{0.62(0.24-0.90)}$ & \begin{tabular}{|l|l|}
$0.76(0.53-0.90)$ \\
$0.73(0.32-0.94)$
\end{tabular} \\
\hline Interleukin-8 (IL-8) & $0.75(0.42-0.91)$ & $0.57(0.27-0.82)$ \\
\hline
\end{tabular}

CRP, IL-6, IL-8 and PCT reliably predict bacteraemia and sepsis but other outcome subgroups do not consistently fit within $95 \%$ confidence prediction regions. Biomarker performance at different thresholds is better understood (Table 2). A PCT $>0.5 \mathrm{ng} / \mathrm{mL}$ possibly offers the best compromise between sensitivity and specificity.16 studies describe the use of serial biomarkers with PCT potentially having the greatest discriminatory role.

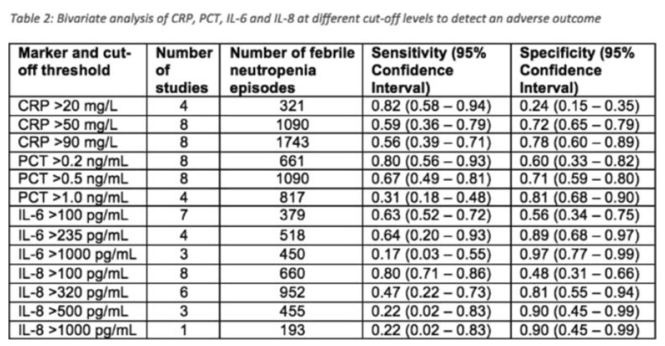

\section{Conclusions}

Biomarkers reliably predict adverse outcomes and their role in risk stratification is promising.

\section{PS029}

NUTRITION SUPPORT IN A PAEDIATRIC STEM CELL TRANSPLANT UNIT

L. Devereux ${ }^{1}$, C. Carroll ${ }^{2}$, A. Kennedy $y^{3}$, M. Capra ${ }^{4}$

${ }^{T}$ Dublin Institute of Technology and Trinity College Dublin, Human Nutrition \& Dietetics, Dublin, Ireland

${ }^{2}$ Our Lady's Children's Hospital Crumlin, Clinical Nutrition \& Dietetics, Dublin, Ireland

${ }^{3}$ Dublin Institute of Technology, School of Biological Sciences, Dublin, Ireland

${ }^{4}$ Our Lady's Children's Hospital Crumlin, National Paediatric Haematology \& Oncology Centre, Dublin, Ireland

\section{Introduction}

The intensive conditioning treatments used in stem cell transplantation (SCT) result in many significant multi-systemic side effects which impact on nutritional status. Nutrition support (NS), either enteral nutrition $(\mathrm{EN})$ and parenteral nutrition $(\mathrm{PN})$, plays an important role in the supportive care provided to this patient cohort. Consensus has yet to be reached on the optimum type and timing of NS in this setting.

Objectives

To assess NS use in a paediatric SCT unit and to investigate if transplant type influenced type of NS utilised or BMI-for-age $\mathrm{z}$ score $\left(\mathrm{BMI}_{\mathrm{z}}\right)$ change from admission to discharge.

\section{Methods}

A retrospective review of paediatric SCT patients treated between the $1^{\text {st }}$ June 2014 and $31^{\text {st }}$ December 2016. Ethical approval was obtained. Data was collected from medical and dietetic records.

\section{Results}

50 patients (median age 60.5 months, $54 \%$ female) were eligible for inclusion ( $\mathrm{n}=35$ allogeneic, $\mathrm{n}=15$ autologous). $30 \%$ were admitted on NS ( $n=13 \mathrm{EN}, \mathrm{n}=2 \mathrm{PN}) .92 \%$ required NS during their admission $(54 \%$ $\mathrm{EN}, 8 \% \mathrm{PN}, 30 \%$ combined $\mathrm{EN} / \mathrm{PN}$ ). $50 \%$ of patients were discharged on EN. There was a median $\mathrm{BMI}_{\mathrm{z}}$ change of $-0.19 \pm 0.88$ from admission to discharge. No statistically significant difference was found between transplant type and type of NS utilised $(P=0.166)$ or $\mathrm{BMI}_{\mathrm{z}}$ change $(P=0.560)$. Conclusions

NS plays a significant role in this paediatric SCT centre. Earlier initiation of NS may help to minimise changes in BMIz. A larger sample size is required to establish if a statistical difference can be found between transplant type and type and duration of NS delivered.

\section{PS030}

IS USAGE OF COLISTIMETHATE SODIUM IN PEDIATRIC ONCOLOGY PRACTICE RATIONAL OR RAMPANT? - A RETROSPECTIVE SELF AUDIT OF TERTIARY CARE CENTER

J. ramamoorthy ${ }^{1}$, V. Radhakrishnan ${ }^{2}$, V. vijayakumar ${ }^{3}$, T.G. Sagar ${ }^{4}$

${ }^{T}$ Assistant Professor, Pediatric Oncology Unit- Department of medical oncology-Cancer Institute- Adyar- chennai, chennai, India

${ }^{2}$ Cancer institute- Adyar-Chennai, Pediatric Oncology UnitDepartment of Medical Oncology, Chennai, India

${ }^{3}$ Cancer institute-Adyar-Chennai, Department of medical Microbiology, Chennai, India

${ }^{4}$ Cancer institute- Adyar-Chennai, Department of Medical Oncology, Chennai, India

\section{Introduction}

In developing country setting resistance to beta-lactam/beta-lactamase inhibitors and carbapenems is not uncommon in gram negative bacterial (GNB) isolates. The only lifeline left in such situation is colistimethate sodium (CS). 


\section{Objectives}

To audit the frequency, indication and impact on outcome of febrile neutropenia episode (FNE) by CS administration.

Methods

FNE in children during the period from January 2016 to July 2017 in our centre were audited retrospectively.

Results

Of the 924 FNE, CS was administered in 116 (12.3\%) episodes. These FNE were secondary to treatment for acute myeloid leukemia in 62, acute lymphoblastic leukemia in 42 , lymphoma in 9 and other malignancies in 3 . The median days of FNE \& absolute neutrophil count at the time of CS initiation was 4 (3-5) and 200 $(100-400) / \mathrm{mm}^{3}$. CS therapy was initiated following clinical sepsis in 46 episodes, microbiologically documented infection (MDI) in 39 episodes, previous MDI by MDR isolates in 14 episodes, clinically documented infection in 8 episodes and severe sepsis in 9 episodes. MDR GNB were isolated in 33/39 episodes with MDI (Escherichia coli- 19 episodes, Klebsiella pneumonia- 12 episodes and pseudomonas aeruginosa 2 episodes). After initiation of CS, deferevescence was achieved in 102 FNE $(87.9 \%)$ after an interval of $10.9 \pm 5.7$ days. Defervescence was not achieved in 14 episodes (12.1\%) resulting in death.

Conclusions

CS was administered in 60/116 FNE without a suggestive culture report. CS initiation will be a necessary evil until clinical risk stratification scores and robust biomarkers become available to guide antibiotic escalation and response assessment

\section{PS031}

TRANFORMING SYMPTOM MANAGEMENT IN CANCER PATIENTS: IS MEDICAL CANNABIS A NEW PARADIGM?

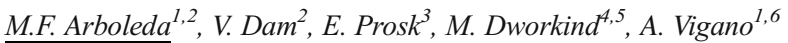

${ }^{T}$ McGill Univeristy, Oncology-Supportive and Palliative Care, Montreal, Canada

${ }^{2}$ Santé Cannabis, Research, Montreal, Canada

${ }^{3}$ Santé Cannabis, Director, Montreal, Canada

${ }^{4}$ Santé Cannabis, Medical Director, Montreal, Canada

${ }^{5}$ McGill Univeristy, Family Medicine and Palliative Care, Montreal,

Canada

${ }^{6}$ Santé Cannabis, Research Director, Montreal, Canada

\section{Introduction:}

Unrelieved symptom burden creates significant challenges for cancer patients, including functional impairment, social isolation and emotional distress. Poor symptom control and/or intolerance of other medications (i.e. opioids) support a need for other therapeutic options such as cannabinoid-based medicines (CBM). Objectives

To provide initial evidence on the safety and efficacy of CBM and recommendations for product and dose selection to achieve improved symptom control in a cancer population.

Methods

A retrospective chart review of cancer patients followed between March 2016 and February 2018 at a medical led cannabis clinic. The revised Edmonton Symptom Assessment Scale (ESAS-r) at baseline and 3-month follow-up was the primary outcome measurement.

Results

112 patients were identified (mean age 58.7 years, $\mathrm{SD} \pm 14 ; 45.5 \%$ male). Gastrointestinal (22.3\%), breast (21.4\%) and hematological (13.4\%) cancers were the most frequent diagnoses. Cannabis products with 1:1 ratio of Tetrahydrocannabinol (THC) and Cannabidiol (CBD) (36.6\%); and oral administration (i.e. cannabis oil) $(62.5 \%)$ were most frequently prescribed. Significant symptom relief was confirmed for pain (from $3.0 \pm 2.9$ to $2.0 \pm$ 2.7; $\mathrm{p}=0.01$ Wilcoxon signed-rank test), depression (from $3.0 \pm 2.8$ to $2.0 \pm$ 2.6; $\mathrm{p}=0.04$ ) and anxiety (from $3.0 \pm 3.1$ to $2.0 \pm 2.9 ; \mathrm{p}=0.02$ ); likewise, trend towards improved nausea ( $\mathrm{p}=0.05$ ). Finally, $25.9 \%$ of patients experienced at least one side effect, all classified as mild to moderate. Product and dosage adjustments were utilized to overcome all side effects.

\section{Conclusions}

CBM prescribed by physicians within an interdisciplinary team with supportive care and medical cannabis competencies, appear a promising and safe tool to improve symptom management in cancer.

PS032

THE EFFECT OF CANNABIS AND MAIN CANNABINOIDS USE ON TUMOR RESPONSE TO NIVOLUMAB IN PATIENTS WITH ADVANCED MALIGNANCIES

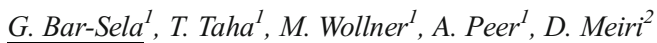 \\ ${ }^{T}$ Rambam health care campus, Oncology, Haifa, Israel \\ ${ }^{2}$ Technion-Israel Institute of Technology, Rappaport Faculty of Medicine, \\ Haifa, Israel
}

\section{Introduction}

Immunotherapy has come into wide clinical use recently, as has the use of cannabis in oncology patients, mainly for palliative indications which has potential immune-suppressive effects.

\section{Objectives}

The aim of this study was to evaluate the influence of cannabis use during immunotherapy treatment on response rate (RR) as primary outcome and also progression-free (PFS) and overall survival (OS).

\section{Methods}

In this retrospective, observational study, data was collected from the files of patients treated with nivolumab in 2015-2016 at Rambam Health Care Campus in Haifa, Israel. Nivolumab was given to 140 patients (89 nivolumab alone, 51 nivolumab plus cannabis) with advanced melanoma, non-small-cell lung cancer, and renal clear cell carcinoma. The groups were homogenous regarding demographic and disease characteristics.

\section{Results}

In a multi-variant model, cannabis was the only significant factor which reduced RR to immunotherapy $(37.5 \% \mathrm{RR}$ in nivolumab alone compared to $15.9 \%$ in the nivolumab plus cannabis group $(p=0.016, \mathrm{OR}=3.13$, CI95\% 1.24-8.13((. Cannabis use was not a significant factor for PFS or OS. In the cannabis users group, full reliable data on cannabis type, including THC and CBD, was evaluable for 36/51 (70\%) patients. RR was 56\% in patients using $\mathrm{CBD}>1 \%$ compared to $18 \%$ for $\mathrm{CBD} \leq 1 \%$ ( $\mathrm{p}=0.077$ ).

\section{Conclusions}

The use of cannabis in combination with immunotherapy decreased RR to treatment. High percentages of $\mathrm{CBD}$ may not interfere with response to immunotherapy. This information can be critical for a large group of patients and requires caution when starting immunotherapy. Considering the limitations of the study, further prospective study is needed.

\section{PS033}

\section{CANNABINOIDS IN TASTE AND FLAVOR FUNCTION}

\section{J. Epstein ${ }^{l}$ \\ ${ }^{T}$ Cedars Sinai Health System, Samuel Oschin Comprehensive Cancer Institute, Los Angeles, USA}

Taste, Appetite and Cannabinoids

Taste changes and appetite are key issues in cancer patients. Taste and flavor are complex senses and changes or loss are common during active cancer therapy and may continue in survivors, in some cases indefinitely. Taste change or taste loss can impact flavor and thereby affect appetite, and oral intake. In those affected, dietary adaptations and maladaptations may occur impacting quality of life, the ability to maintain or increase weight and general health.

Cannabinoids may have a role to play by affecting chemosensory changes, appetite and interest in food. Further pain effects may affect oral comfort and taste function. Therefore, cannabinoids may play a role in management of chemosensory disorders. Limited studies to date in oncology care and future directions will be presented. 


\section{PS034}

CANNABIS-BASED MEDICINES SHOW MORE PROMISE FOR IMPROVING APPETITE IN CHRONIC DISEASES

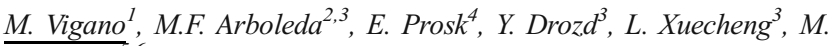 \\ Dworkind $^{5}, 6$ \\ ${ }^{1}$ Lower Canada College, Department of Science, Montreal, Canada \\ ${ }^{2}$ McGill University, Oncology-Supportive and Palliative Care, Montreal, \\ Canada \\ ${ }^{3}$ Santé Cannabis, Research, Montreal, Canada \\ ${ }^{4}$ Santé Cannabis, Director, Montreal, Canada \\ ${ }^{5}$ Santé Cannabis, Medical Director, Montreal, Canada \\ ${ }^{6}$ McGill University, Family Medicine and Palliative Care, Montreal, \\ Canada
}

\section{Introduction}

Cannabinoids are widely claimed to improve appetite in both cancer and non-cancer patients, however, the evidence for their orexigenic effect is still weak and very few studies have evaluated the impact of cannabis on weight.

\section{Objectives}

To determine the impact of cannabinoids on appetite and weight maintenance.

\section{Methods}

We conducted a retrospective chart review of all adult patients who were assessed at Santé Cannabis Clinic (SCC) between August 2016 and October 2017, and who had "increase of appetite" as a treatment goal. SCC is the only medical cannabinoid clinic in the province of Quebec, Canada. The "lack of appetite" item $(0=$ no lack of appetite to $10=$ complete lack of appetite) from the revised Edmonton Symptom Assessment System (r-ESAS), and weight (kg), were considered our primary outcomes.

\section{Results}

Fifty four patients suffering from anorexia from cancer (43\%) and non-cancer $(57 \%)$ were analyzed. The r-ESAS score for lack of appetite significantly improved between baseline $(5.07 \pm 3.21)$ and follow up $(3.56 \pm 3.15)(\mathrm{p}$-value $=0.0026)$. Weight remained stable between baseline $(70.74 \pm 14.63)$ and follow up $(71.02 \pm$ 14.76). The use of nabilone $(\mathrm{p}=0.0521)$ along with type of administration (inhaled and oral plus inhaled vs. oral) $(\mathrm{p}=0.0142)$ impacted the difference in appetite. Overall, $18.5 \%$ of the subjects reported only mild side effects such as anxiety, fatigue, dizziness and dry mouth.

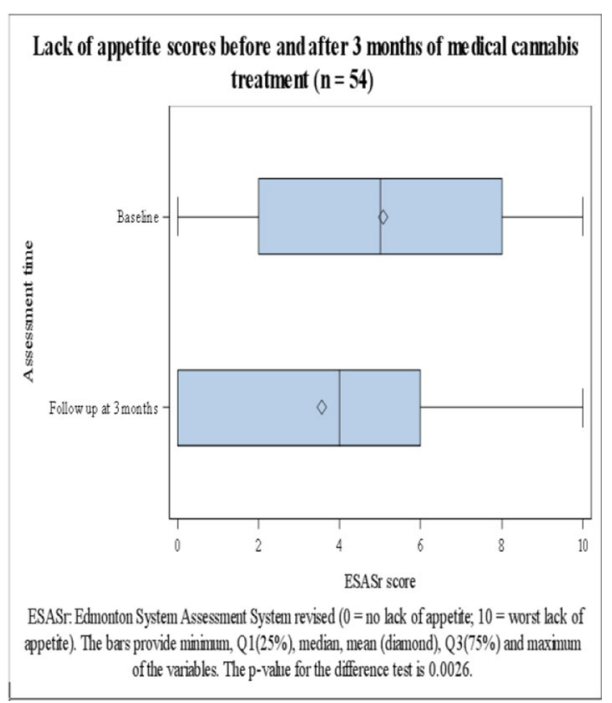

\section{Conclusions}

Administration of cannabis-based medicines within an individualized and supportive monitoring program may safely improve appetite and weight maintenance in patients suffering anorexia from cancer as well as nonmalignant chronic diseases.

\section{PS035}

\section{MOLECULAR MECHANISM OF CANCER-CACHEXIA} SYNDROME

J.M. Argiles $^{1}$

${ }^{T}$ Universitat de Barcelona, Bioquimica i Biomedicina Molecular, Barcelona, Spain

\section{Molecular Mechanisms associated with the Cancer-Cachexia Syndrome \\ Josep M. Argilés}

Cancer Research Group, Departament de Bioquímica i Biomedicina Molecular, Facultat de Biologia, Universitat de Barcelona, Barcelona, Spain.

In spite of the fact that muscle tissue represents more than $40 \%$ of body weight and seems to be the main tissue involved in the wasting that occurs during cachexia, recent developments suggest that tissues/organs such as adipose (both brown and white), brain, liver, gut and heart are directly involved in the cachectic process and may be responsible for muscle wasting. This suggests that cachexia is indeed a multi-organ syndrome. However, none of the numerous existing definitions of cachexia include the fact that cachexia is a "multi-organ" syndrome. Indeed, many different cell types are involved in cachexia. Current definitions put special emphasis on skeletal muscle and adipose tissue but we now know of the uttermost importance of other organs/tissues in the wasting syndrome. The implications of this are important since, on the one hand, the metabolic alterations affecting all cellular types may be very relevant for the understanding of the cachexia syndrome and, secondly, the development of new therapeutic approaches may benefit from this knowledge: for instance it may be relevant to interfere with lypolysis of with acute-phase protein synthesis to block muscle proteolysis. Therefore, future studies on this field are needed and should concentrate on unrevealing the different mediators released by non-muscle tissues that may influence muscle metabolism and, therefore, wasting. Furthermore, another important aspect that future research should contemplate is to establish the chronological involvement of the different organs/tissues in cancer cachexia. 


\section{PS036}

CURRENT STATUS CONCERNING CANCER-CACHEXIA SYNDROME IN JAPAN

\author{
T. Naito ${ }^{1}$ \\ ${ }^{T}$ shizuoka cancer centar, The division of thoracic oncology, Shizuoka, \\ Japan
}

Muscle wasting caused by a variety of chronic inflammatory diseases has been known since ancient time in Europe and Asia. In our time, the importance of cancer cachexia is increasing because number of elderly patients living with cancer is increasing as a result of the aging of the population and progress in medical oncology. Cachectic cancer patients have poor physical function, quality of life, tolerability to cancer treatments, and prognosis. Although several investigational agents improve appetite or lean body mass, they fail at improving physical functions. One possible reason may be a lack of concurrent nutritional and exercise interventions. However, there is no standard multimodal intervention combining with these investigational agents.

In this review, we summarized history, epidemiology, physician's awareness, and the results of recent clinical researches in this area in Japan. We will also introduce the NEXTAC (Nutrition and Exercise Treatment for Advanced Cancer) -one and two studies which were designed as a national, prospective, multicenter study to assess the feasibility, safety, and efficacy of non-pharmacological multimodal interventions for elderly patients with advanced cancer receiving chemotherapy. We hypothesize in these studies that early induction of NEXTAC program might maintain physical function and prevent disability in elderly patients with advanced cancer who are at considerable risk of cancer cachexia.

\section{PS037}

GC4419, SMALL MOLECULE SUPEROXIDE (SO) DISMUTASE (SOD) MIMETIC: RANDOMIZED TRIAL TO REDUCE CHEMORADIOTHERAPY (CRT)-INDUCED MUCOSITIS (OM) IN ORAL CAVITY (OC)/OROPHARYNGEAL (OP) CARCINOMA (OCC) PATIENTS (PTS)

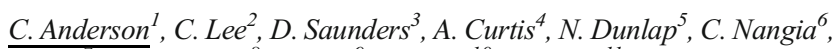
A. Lee', J. Holmlund ${ }^{8}$, J. Brill ${ }^{9}$, S. Sonis ${ }^{10}$, J. Buatti ${ }^{11}$

${ }^{1}$ University of Iowa Hospitals and Clinics, Radiation Oncology, Iowa City, USA

${ }^{2}$ Cancer Care Northwest, Radiation Oncology, Spokane Valley, USA

${ }^{3}$ Northeast Cancer Centre of Health Sciences North, Department of Dental Oncology, Sudbury, Canada

${ }^{4}$ Spartanburg Medical Center Gibbs Cancer Center, Department of Radiation Oncology, Spartanburg, USA

${ }^{5}$ University of Louisville James Graham Brown Cancer Center, Department of Radiation Oncology, Louisville, USA

${ }^{6}$ UC Irvine Medical Center Chao Family Comprehensive Cancer Center, Department of Hematology/Oncology, Orange, USA

${ }^{7}$ Hope Cancer Center of East Texas, Tyler Hematology-Oncology, Tyler, USA

${ }^{8}$ Galera Therapeutics Inc, Chief Medical Officer, Malvern, USA

${ }^{9}$ Galera Therapeutics Inc, Director Clinical Development, Malvern, USA

${ }^{10}$ BioModels, Chief Scientific Officer, Boston, USA

${ }^{11}$ University of Iowa Hospitals and Clinics, Department of Radiation Oncology, Iowa City, USA

\section{Introduction}

CRT-induced SO contributes to OM initiation. GC4419 specifically mimics SOD's dismutation of $\mathrm{SO}$ to $\mathrm{H}_{2} \mathrm{O}_{2}$, and previously attenuated severe OM (SOM) in a published Phase 1b/2a open-label trial.

Objectives

Determine whether GC4419 reduces duration, incidence, \& severity of SOM.

\section{Methods}

Pts with locally-advanced OCC; definitive or post-op intensity-modulated (IM)RT (approx. 70 Gy [ $>50$ Gy to $>2$ oral sites]) plus CDDP (weekly or q3wk) were randomized (stratification: tumor HPV status, CDDP schedule) to 30 or $90 \mathrm{mg}$ of GC4419, or placebo (PBO), 60-minute IV infusion, M-F, ending < 60 minutes before IMRT. WHO Grade OM was assessed by trained evaluators biw during IMRT \& qwk for up to $8 \mathrm{wks}$ after IMRT. Primary endpoint: duration of SOM. Efficacy was tested for each active dose vs PBO (ITT population) by a sequential, conditional approach (2-sided alpha 0.05).

Results

223 pts (44 sites): $90 \mathrm{mg}(\mathrm{n}=76), 30 \mathrm{mg}(\mathrm{n}=73)$, or PBO ( $\mathrm{n}=74)$. Baseline patient \& tumor characteristics, \& treatment delivery, were balanced. Safety: comparable across arms; no significant GC4419-specific toxicity; other known toxicities of IMRT/CDDP were not increased. Efficacy: At $90 \mathrm{mg}$ GC4419 vs PBO, duration of SOM was significantly reduced (median, 1.5 vs $19 \mathrm{~d}, \mathrm{p}=0.024)$. SOM incidence ( $43 \%$ vs $65 \%, \mathrm{p}=0.009$ ), Gr 4 incidence ( $16 \%$ vs $30 \%, p=0.045) \&$ time to onset (median 61 vs 39 days) also improved. There were intermediate improvements with $30 \mathrm{mg}$.

Conclusions

GC4419 produced a significant, clinically meaningful reduction of SOM duration, and dose-dependent improvements in other SOM parameters, with acceptable safety.

\section{PS038}

PHOTOBIOMODULATION EFFECTS ON SQUAMOUS CELL CARCINOMA IN AN ORTHOTOPIC ANIMAL MODEL OF SQUAMOUS CELL CARCINOMA OF THE ORAL CAVITY

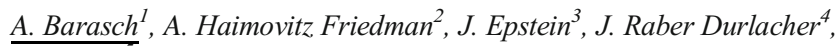 \\ J. Carroll ${ }^{5}$ \\ ${ }^{1}$ Weill Cornell/Rockefeller/Sloan-Kettering, Medicine, New York New \\ York, USA \\ ${ }^{2}$ memorial sloan kettering cancer center, Radiation Oncology, New York, USA \\ ${ }^{3}$ City of Hope Hospital, Oncology, Duarte, USA \\ ${ }^{4}$ ACTA, Periodontology, Amsterdam, The Netherlands \\ ${ }^{5}$ THOR Laser LTD, na, London, United Kingdom
}

\section{Introduction}

Photobiomodulation (PBM) has shown efficacy in preventing and treating cancer therapy-induced mucositis and/or dermatitis. However, there is a dearth of information regarding the effect of PBM on (pre)malignant cells, which has led to questions regarding the safety of this technique. We address this issue in an orthotopic mouse model with human squamous cell carcinoma of the oral cavity.

\section{Objectives}

We hypothesized that PBM will have no protective effects on oral tumors treated prior to exposure to ionizing radiation.

\section{Methods}

Animals with actively growing tumors (Cal-33) were divided into 4 groups: control; PBM only; radiation therapy (RT) only; and PBM + RT. We performed three experiments: 1. PBM @ $650 \mathrm{~nm} 6 \mathrm{~J} / \mathrm{cm}^{2}$ and 5 RT doses of 4 Gy delivered daily; 2. PBM @ $650 \mathrm{~nm} 6 \mathrm{~J} / \mathrm{cm}^{2}$ and one RT dose of $15 \mathrm{~Gy}$; and 3. PBM @ $650 \mathrm{~nm}+810 \mathrm{~nm} 3 \mathrm{~J} / \mathrm{cm}^{2}$ and one RT dose of $15 \mathrm{~Gy}$. Mice were weighed daily and tumor behavior evaluated with fluorescence. Survival time was also evaluated.

\section{Results}

Animals treated with RT survived significantly longer and had significantly less fluorescence scores when compared to control and PBM only groups. No significant differences were noted between the RT alone and $\mathrm{PBM}+\mathrm{RT}$ groups in any of the experiments.

\section{Conclusions}

Our results suggest that $\mathrm{PBM}$ at the utilized parameters does not stimulate tumor growth and does not provide protection to the tumor from the killing effects of RT. 


\section{PS039}

PERSONALIZED SUPPORTIVE CARE FOR PATIENTS RECEIVING 5-FLUOROURACIL (5-FU): INTERIM ANALYSIS OF MULTIVARIATE SNP RISK PREDICTION FOR GI TOXICITY

S.K. Korver', J.M. Bowen', I.A. Ball, R.J. Gibson', J. Tuke ${ }^{3}$, R.M.

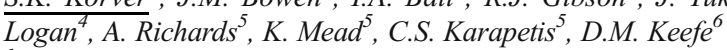

${ }^{1}$ University of Adelaide, Adelaide Medical School, Adelaide, Australia

${ }^{2}$ University of South Australia, Division of Health Sciences, Adelaide, Australia

${ }^{3}$ University of Adelaide, School of Mathematical Sciences, Adelaide, Australia

${ }^{4}$ University of Adelaide, Adelaide Dental School, Adelaide, Australia

${ }^{5}$ Flinders Medical Centre, Flinders Medical Centre, Bedford Park, Australia

${ }^{6}$ Royal Adelaide Hospital, Royal Adelaide Hospital, Adelaide, Australia

\section{Introduction}

Severegastrointestinal (GI) toxicity following 5-FU-based treatment is highly prevalent and negatively affects therapy. No effective predictors for severe GI toxicity risk have been identified. Our pilot study $(\mathrm{n}=34)$ reported TLR2 and $T N F$ genetic variants and cancer type predicted severe toxicity risk (receiver operator characteristic (ROC) area under the curve (AUC) 87.3\%).

Objectives

To validate our pilot study observations in an independent patient cohort. Methods

One hundred and three participants whom previously received 5-FU-based treatment were recruited. GI toxicity data (toxicity: symptoms Grade $\geq 3$ NCI's CTCAE v4, or requiring treatment cessation or reduction), demographics and treatment parameters were mined from clinical records. Genetic variability in the following genes was determined using a customised Sequenom MassArray: IL1B, IL2, IL6, IL6R, IL10, TNF, TGFB, TLR2, TLR4, MD2, MYD88, CRP, ICE and OPRM1. Multivariate logistic regression produced a prediction model of severe GI toxicity risk, with ROC AUC assessing model performance.

Results

Twenty-two participants experienced severe GI toxicity. Colorectal and gastric cancer and $I L 1 B$ variants (rs16944 and rs1143634) were significantly associated with severe GI toxicity risk with a ROC AUC of $82 \%\left(\mathrm{P}=3.4 \times 10^{-5}\right)$.

Conclusions

Compared to the pilot study, different genetic variants were identified as predictive of severe GI toxicity risk. This may reflect the shift in cancer type between the pilot and current study that favored breast cancer regimens. Nonetheless, we have identified genetic markers related to inflammatory responses as being predictive. Further patients are being recruited to expand study cohort to 150 to meet a-priori power calculations.

\section{PS040}

WHAT IS ACCURATE TO TEACH PATIENTS AND FAMILIES, REGARDING NEW EVIDENCE, ON MEDICAL MARIJUANA AND E-CIGARETTES?

T. Austin

${ }^{T}$ Cancer Care of Western North Carolina, USA

What is needed during the initial educational session?

Teaching is necessary prior to beginning chemotherapy and or radiation treatment. Patients and families wish to know what they can do to help manage their new situation. Psychosocial and financial issues are often of great need, therefore this needs to be addressed. Side effect management of the cancer and the treatments is necessary.

What resources are available in the community? Clotting disorders are higher in this population, so what should we teach? Sexuality and fertility are of great concern, what can be done and what are the risks? Many treatments are given orally, what are safe handling instructions? What should the young patient do to preserve fertility?

All of these issues will be discussed during this session.

PS041

HOW TO DEAL WITH THE PSYCHOLOGICAL AND FINANCIAL ISSUES REGARDING THE NEW DIAGNOSIS OF CANCER

P. Fernandez-Ortega ${ }^{1,2}$

${ }^{T}$ Institute Català d'Oncologia, Nursing Research Coordinator, Barcelona, Spain

${ }^{2}$ University of Barcelona, Salud Publica- Mental i Materno Infantil, Barcelona, Spain

At the time of an initial diagnosis of cancer, patients and their family members often feel the acute need for additional information. Patients often have questions regarding the journey ahead and family members often want to know how they can be of most assistance. This time also provides an opportunity to educate on the importance of healthy lifestyle measures such as nutrition, exercise, and tobacco cessation.

This session will equip healthcare providers with useful information that can be used during these important teaching moments. There will be a discussion on topics such as symptom management, incidence and prevention of thrombosis, psychosocial issues, and the wealth of available information due to technology. Finally, due to advances in cancer care many of our cancer patients will become survivors and hence the importance of addressing healthy lifestyle measures will be discussed.

\section{PS042}

SYMPTOM EXPERIENCES AND SYMPTOM MANAGEMENT AMONG PERSONS WITH LUNG CANCER IN BANGLADESH

B. Halder ${ }^{1}$, A.P.D.P. Phornphibul ${ }^{2}$, P.D.A. Klunklin ${ }^{3}$, A.P.D.P. Soivong ${ }^{3}$ ${ }^{T}$ PhD candidate-Chiang Mai University-110/406 Inthrawaraoros RoadSriphum District- Chiang Mai-50200- Thailand- +66 063-531-7811, Oncology Nursing, Chiang Mai, Thailand

${ }^{2}$ Chiang Mai University- Thailand, Faculty of Nursing, Chaing Mai50200, Thailand

${ }^{3}$ Chiang Mai University, Faculty of Nursing, Chaing Mai-50200, Thailand

\section{Introduction}

Lung cancer is a leading cause of death. Various symptoms can result from the disease itself and treatments, better understanding of symptom experiences and management is needed to design effective health-care services.

Objectives

This study was done to identify symptom experiences and management strategies among persons with lung cancer in Bangladesh.

\section{Methods}

A descriptive study utilizing combined methods was conducted, using the Dodd Symptom Management Model. The 382 participants were purposively selected from four tertiary hospitals to identify symptom experiences, using the MD Anderson Symptom Inventory Lung Cancer (MDASI-LC). Semi-structured in-depth interviews were conducted of 16 participants on symptom management. Quantitative data was analyzed by descriptive statistics, and qualitative data by content analysis.

\section{Results}

Participants experienced 4-16 symptoms, with a mean of 13.64. Pain was the most frequently reported and most severe; mood disturbance was the area of top life interference. Reported symptom severity had a median of 3-7 and interference range of 2-4 (/10). Qualitative data revealed 5 categories of symptom management: preventive actions; taking prescribed medications; cultivating mind-body balance; distraction from symptom 
focus; use of adjunct therapies. Locally available resources and traditional health-care practices were used by most participants, including herbal medicine, prayer, meditation, massage, and deep breathing.
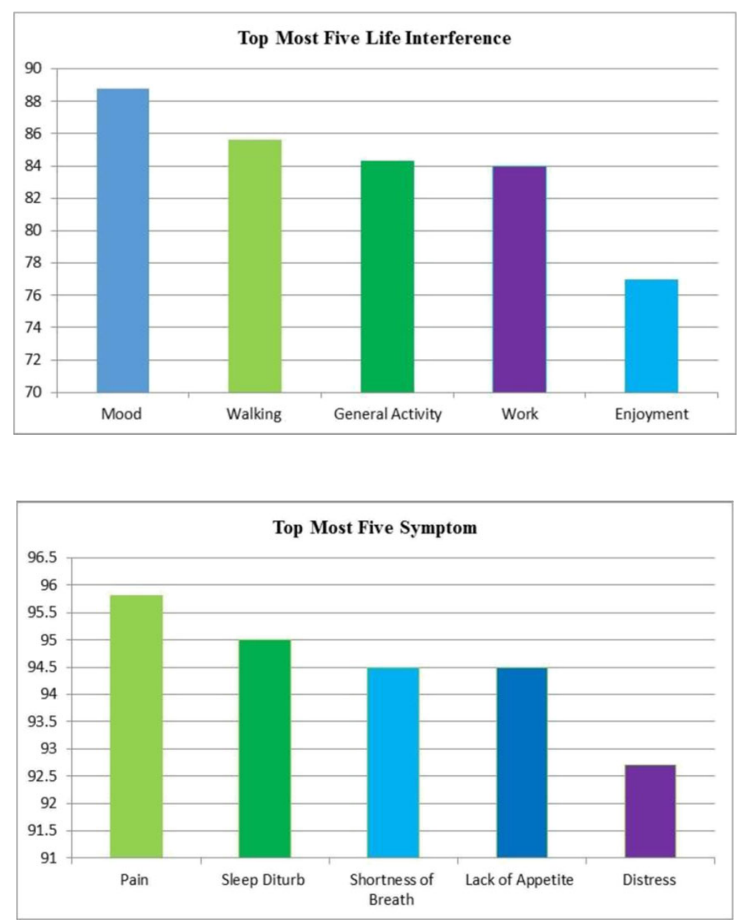

Conclusions

These knowledge can contribute to nursing education and practice. Health-care workers should conduct integrated assessments of symptoms including symptom frequency, severity, and interference to provide complete pictures of experiences. People can utilize locally available resources as added management strategies. It is necessary to identify the most effective model of care and symptom management.

\section{PS043}

MULTIMEDIA PSYCHOEDUCATION FOR CANCER PATIENTS ELIGIBLE FOR CLINICAL TRIALS: A RANDOMIZED CLINICAL TRIAL

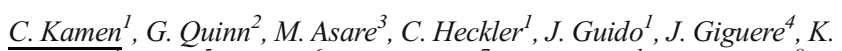

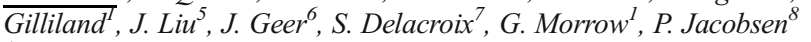

${ }^{1}$ University of Rochester School of Medicine, Surgery, Rochester, USA

${ }^{2}$ New York University Medical Center, Obstetrics and Gynecology, New York, USA

${ }^{3}$ University of Rochester Medical Center, Surgery, Rochester, USA

${ }^{4}$ Greenville Health System Cancer Institute, Medicine, Greenville, USA

${ }^{5}$ Heartland NCORP, Medicine, Decatur, USA

${ }^{6}$ Metro-Minnesota NCORP, Medicine, St. Louis Park, USA

${ }^{7}$ Louisiana State University Health Sciences Center, Medicine, New Orleans, USA

${ }^{8}$ National Cancer Institute, Healthcare Delivery Research Program, Bethesda, USA

\section{Introduction}

Supporting patients' decision making about clinical trials may enhance trial participation. To date, few theory-based interventions have been tested to address this issue.
Objectives

In this study, we aimed to evaluate the effect of a multimedia psychoeducation (MP) intervention, relative to print education (PE), on patients' decision support needs and attitudes about clinical trials.

\section{Methods}

Patients with cancer who were eligible for participation in an National Cancer Institute (NCI) therapeutic cancer clinical trial were recruited through the nationwide University of Rochester Cancer Center (URCC) NCI Community Oncology Research Program (NCORP) from 20142016 and randomized to the MP or PE intervention. Assessments at baseline (before intervention), post-intervention, and at a two-month follow-up included patients' decision support needs, attitudes regarding clinical trials, and clinical trial participation.

\section{Results}

We recruited 418 cancer patients (ages 26-89, various cancer types). Relative to the PE condition, the MP condition did not significantly affect decision support needs. However, patients in the MP condition reported significantly more positive attitudes about clinical trials and were more likely to participate in a clinical trial than those in the PE condition $(69 \%$ vs. $62 \%, \mathrm{p}=0.01)$. Furthermore, improvement in attitudes about clinical trials significantly mediated the effect of the intervention on participation in clinical trials.

\section{Conclusions}

The MP intervention was able to improve patient attitudes toward clinical trials when compared with $\mathrm{PE}$, and this improvement led to increased rates of participation in trials. The MP intervention could be disseminated to improve attitudes about clinical trials among cancer patients.

\section{PS044}

PREVENTION AND EDUCATIONAL TEACHING FOR THE NEWLY DIAGNOSED ONCOLOGY PATIENTS, REGARDING THE PATIENT'S RISK OF BLOOD CLOT DEVELOPMENT

\section{E. Manzullo ${ }^{1}$ \\ ${ }^{T}$ General Internal Medicine, The University of Texas MD Anderson Cancer Center, Houston, USA}

At the time of an initial diagnosis of cancer, patients and their family members often feel the acute need for additional information. Patients often have questions regarding the journey ahead and family members often want to know how they can be of most assistance. This time also provides an opportunity to educate on the importance of healthy lifestyle measures such as nutrition, exercise, and tobacco cessation.

This session will equip healthcare providers with useful information that can be used during these important teaching moments. There will be a discussion on topics such as symptom management, incidence and prevention of thrombosis, psychosocial issues, and the wealth of available information due to technology. Finally, due to advances in cancer care many of our cancer patients will become survivors and hence the importance of addressing healthy lifestyle measures will be discussed.

\section{PS045}

SUPPORTIVE CARE STARTS WITH COMMUNICATION AND COLLABORATION: AN AUSTRALIAN PERSPECTIVE

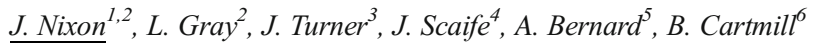

${ }^{T}$ The University of Queensland, School of Health and Rehabilitation Sciences, Brisbane, Australia

${ }^{2}$ Princess Alexandra Hospital, Occupational Therapy Department, Brisbane, Australia

${ }^{3}$ The University of Queensland, Faculty of Medicine, Brisbane, Australia ${ }^{4}$ Princess Alexandra Hospital, Division of Cancer, Brisbane, Australia

${ }^{5}$ The University of Queensland, QFAB Bioinformatics, Brisbane, Australia

${ }^{6}$ Princess Alexandra Hospital, Speech Pathology Department, Brisbane, Australia
} 


\section{Introduction}

To provide quality care all health professionals need appropriate skills in recognising and responding to a variety of patient psychosocial concerns. Objectives

This clinician-led exploratory study compared an eight-hour inter-professional communication skills training (CST) workshop "CARE", with a modified two hour workshop "CARE Express".

Methods

Recruited participants were partially randomised to control $(n=50)$; CARE $(n=49)$; CARE Express $(n=48)$. Measures included perceived skills and confidence by self-report, physician's belief scale (PBS), and responses to clinical vignettes at baseline (T1) and three-month post training (T3).

\section{Results}

There were no changes in confidence in identifying or responding to emotional cues between $\mathrm{T} 1$ and $\mathrm{T} 3$ in the control group. CARE and CARE Express participants demonstrated improvement from T1 to T3 in both recognising and responding to emotions $(\mathrm{p}<.001)$.

There were no differences in PBS between the 3 groups at baseline. A decrease in the score from baseline to T3 for CARE $(p<.001)$ and CARE Express $(p<.001)$, demonstrated increase in psychosocial priority. No difference was observed in the control.

The control demonstrated no changes in response to the clinical vignettes from $\mathrm{T} 1$ to $\mathrm{T} 3$. The CARE and the CARE Express participants demonstrated improvement in acknowledging responses from $\mathrm{T} 1$ to $\mathrm{T} 3(\mathrm{p}<.001)$.

Conclusions

CARE and CARE Express are training packages that increase psychosocial focus, sustained improvements in confidence, and the ability to acknowledge emotions. CARE has become core training for all clinicians employed in this cancer centre and is a driving factor in new interprofessional research collaborations.

\section{PS046}

THE NECESSARY INFORMATION TO INCLUDE IN PRECHE M O T H E RA P Y EDUCATION : NUTR I T I O N, MEDICATION, AND EXERCISE TO IMPROVE SYMPTOM MANAGEMENT AND SURVIVAL

\author{
J. Phillips ${ }^{1,2}$ \\ ${ }^{T}$ Cancer Care of Western North Carolina, USA \\ ${ }^{2}$ Lenoir Rhyne University
}

At the time of an initial diagnosis of cancer, patients and their family members often feel the acute need for additional information. Patients often have questions regarding the journey ahead and family members often want to know how they can be of most assistance. This time also provides an opportunity to educate on the importance of healthy lifestyle measures such as nutrition, exercise, and tobacco cessation.

This session will equip healthcare providers with useful information that can be used during these important teaching moments. There will be a discussion on topics such as symptom management, incidence and prevention of thrombosis, psychosocial issues, and the wealth of available information due to technology. Finally, due to advances in cancer care many of our cancer patients will become survivors and hence the importance of addressing healthy lifestyle measures will be discussed.

\section{PS047}

\section{MEASURING CIPN, IN PRACTICE AND RESEARCH SETTINGS}

\section{E. Lavoie Smith}

${ }^{T}$ University of Michigan, School of Nursing, Ann Arbor, USA

The presentation will begin with a brief summary of the incidence and clinical manifestations of chemotherapy-induced peripheral neuropathy (CIPN). The presenter will review basic nerve fiber physiology and neurotoxic drug-specific pathophysiologic mechanisms underlying painful and non-painful symptoms. CIPN leads to several unfortunate outcomes, and the presenter will describe how patient and clinician-related assessment barriers lead to under-assessed and poorly managed CIPN symptoms. The presenter will describe three general measurement approaches and provide examples of each. These approaches include (1) objective assessment approaches such as physical examination, nerve conduction studies, and quantitative sensory testing, (2) functional measures such as the Timed Get Up \& Go test, and (3) subjective patientreported outcome measures such as the European Organisation for Research and Treatment of Cancer (EORTC) Quality of Life Questionnaire (QLQ) Chemotherapy-Induced Peripheral Neuropathy Scale (CIPN20). The presenter will review the available evidence supporting the strength of all these measures, including composite measures such as the Total Neuropathy Score, and Grading Scales. This discussion will include a review of the most cogent information regarding measurement reliability, validity, responsiveness, sensitivity, and feasibility. The presentation will conclude with recommendations for how to best measure CIPN in both research and clinical practice settings.

\section{PS048}

GABAPENTINOID USE FOR CHEMOTHERAPY NEUROPATHY: THE GOOD AND THE BAD

\author{
C. Loprinzi $i^{1}$ \\ ${ }^{T}$ Mayo Clinic, Oncology, Rochester, USA
}

Gabapentinoid Use for Chemotherapy Neuropathy: The Good and the Bad

\section{Charles L Loprinzi}

Chemotherapy-induced peripheral neuropathy (CIPN) is a prominent clinical problem caused by multiple cytotoxic agents. Given that gabapentinoid medications have been commonly utilized in clinical practice for treatment of CIPN, it is appropriate to critically look at the oncology literature regarding the utility of gabapentin and/or pregabalin for prevention and/or treatment of CIPN.

The first known report regarding the use of gabapentin for chemotherapyinduced neuropathy came from Italian authors at the 2000 ASCO annual meeting, entitled 'Oxaliplatin-induced Neuropathy: Could Gabapentin be the Answer?'. This report described the use of gabapentin in 7 patients who developed neuropathy while receiving oxaliplatin. With the initiation of neuropathy, gabapentin was given, at a total daily dose of 200-300 mg per day; this remarkably low dose reportedly led to the disappearance of neuropathy symptoms, even with the ongoing use of up to 14 total oxaliplatin doses. This work is not available in manuscript form.

Subsequent uncontrolled trial reports have not, on the whole, provided convincing evidence of gabapentinoid benefit for prevention/treatment of CIPN. Additionally, 3 randomized placebo-controlled reported trials have not provided good evidence of benefit for gabapentinoids.

Despite a lack of good evidence that gabapentinoids are helpful for preventing/treating CIPN, arguments supporting the use of gabapentinoids for treating CIPN include: 1) they are beneficial in other neuropathy states, based on phase III data supporting use in such situations; 2) a few reports of clinical practice experiences and unblinded clinical trials suggest that they might be helpful for CIPN in some cases 3) anecdotal use in individual patients in clinical practice suggest that they appear to provide benefit in some individual patients; and 4) there are anecdotal reports of worsening neuropathy symptoms when gabapentinoids are discontinued (understanding that gabapentinoids can cause withdrawal symptoms when they are stopped). 
Unpublished surveys of oncology clinicians illustrate a large range for the enthusiasm and utilization of gabapentinoids in clinical practice for treatment of established CIPN

In total, there is limited scientific basis for the widespread utilization of gabapentinoids for prevention and/or treatment of established CIPN.

\section{PS049}

\section{LONG-TERM OUTCOMES OF PACLITAXEL-INDUCED} PERIPHERAL NEUROPATHY FOR CANCER SURVIVORS

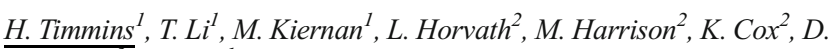 \\ Goldstein $^{3}$, S. Park ${ }^{l}$ \\ ${ }^{1}$ University of Sydney, Brain and Mind Centre, Camperdown, Australia \\ ${ }^{2}$ Chris O'Brien Lifehouse, Oncology, Sydney, Australia \\ ${ }^{3}$ University of New South Wales, Prince of Wales Clinical School, Sydney, \\ Australia
}

\section{Introduction}

Paclitaxel-induced peripheral neuropathy (PIPN) is a dose-limiting side effect producing paraesthesia, numbness and functional impairment. Despite a high incidence, patient impact and long term outcomes remain poorly understood.

Objectives

To examine long term neurological deficits using objective clinical assessments and patient reported outcomes (PROs).

Methods

Prospective assessment was undertaken in 22 female breast-cancer patients (Age:48 \pm 3.8 years) initially prior to paclitaxel treatment, on treatment completion and at follow-up utilising a validated PRO (FACT-GOG-NTX13) and neurological examination (TNSc) comprising assessment of pinprick, vibration-sensibility, power and tendon reflexes. Objective neurophysiological testing recorded the amplitude of the compound sensory action potentials (CSAPs).

Results

At completion of treatment $\left(891 \pm 28 \mathrm{mg} / \mathrm{m}^{2}\right.$ paclitaxel), the majority of patients reported PIPN in both hands and feet $(85 \%)$. On neurological examination, $80 \%$ had $\geq 2$ abnormalities, predominately recorded as reduction in pin-prick sensibility and depressed tendon reflexes. Amplitude of CSAPs was significantly reduced $(36.9 \pm 5.2 \mu \mathrm{V})$ compared to baseline $(55.9 \pm 7.1 \mu \mathrm{V}, \mathrm{p}<.05)$, and objective neuropathy scores increased (TNSc baseline $1.1 \pm 0.3$; follow-up $4.6 \pm 0.4, \mathrm{p}<.05$ ), confirming the development of significant nerve dysfunction. At long term follow-up ( $10 \pm 0.6$ months), fewer patients reported symptoms compared to final treatment $(\mathrm{p}<.05)$, although residual neuropathy remained in $63 \%$ of patients. However, there was no improvement at follow-up in neurological examination (TNSc 3.1 $\pm 0.5, \mathrm{p}=.97$ ) or neurophysiological parameters(CSAP $38.6 \pm 5.2 \mu \mathrm{V}, p=.92)$, suggesting a degree of adaptation to these sustained deficits.

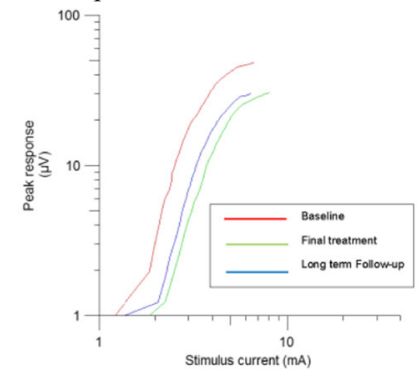

Figure 1. Stimulus response curves of median CSAP amplitude $(\mu V)$ at baseline, final treatment and long term follow-up, demonstrating reduction in amplitude following

Conclusions

Paclitaxel produces persistent neuropathy at long-term follow up, with discrepancies between the level of objective and patient-reported neuropathy. Incorporating objective measures and patient reported outcomes will better inform treatment strategies to improve long-term quality of life.
PS050

CRYOCOMPRESSION VS CONTINUOUS-FLOW COOLING: OPTIMAL METHOD TO DELIVER LIMB HYPOTHERMIA IN PREVENTING CHEMOTHERAPY-INDUCED PERIPHERAL NEUROPATHY

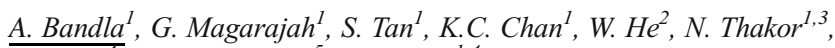
S.C. Lee ${ }^{4}$ E. Wilder-Smith ${ }^{5}$, R. Sundar ${ }^{1,4}$

${ }^{1}$ National University of Singapore, Singapore Institute for Neurotechnology, Singapore, Singapore

${ }^{2}$ Agency for Science- Technology and Research ASTAR, Singapore Institute of Manufacturing Technology SIMTech, Singapore, Singapore

${ }^{3}$ Johns Hopkins University, Biomedical Engineering, Baltimore, USA

${ }^{4}$ National University Health System, Haematology - Oncology, Singapore, Singapore

${ }^{5}$ National University Health System, Neurology, Singapore, Singapore

\section{Introduction}

Chemotherapy-induced peripheral neuropathy (CIPN) is a debilitating and dose-limiting toxicity of commonly used chemotherapeutics with no treatment. Recent studies suggest that hypothermia is neuroprotective in CIPN. Currently used methods such as ice/gel packs are unable to achieve deep and continuous delivery of hypothermia, limiting efficacy. Objectives

Assess optimal method for delivery of limb hypothermia - continuousflow cooling (CF) vs cryocompression (CC) (with cyclic pressure). The study was designed to simulate a chemotherapy infusion, with the eventual goal of administering these techniques on cancer patients receiving neurotoxic chemotherapy.

\section{Methods}

Healthy subjects underwent limb hypothermia for three hours. CF was administered through Blanketrol-III (Cincinnati Sub-zero) and CC through Game Ready 2.0 (CoolSystems, Inc.) at low cyclic compression (5-15 $\mathrm{mmHg}$ ). Skin-surface temperatures and tolerance scores were monitored.

\section{Results}

In total 51 subjects $(15 \mathrm{CF} ; 36 \mathrm{CC})$ underwent limb hypothermia, with the only adverse event of transient erythema. CF was tested between coolant temperatures $25-21^{\circ} \mathrm{C} ; \mathrm{CC}$ between $21-10^{\circ} \mathrm{C}$. Lowest tolerable temperature was $22^{\circ} \mathrm{C}$ for $\mathrm{CF}$ and $11^{\circ} \mathrm{C}$ for $\mathrm{CC}$. There was strong correlation between coolant temperature and skin temperature. $\mathrm{CC}$ at $11^{\circ} \mathrm{C}$ achieved the lowest mean resultant skin temperature (arm: $17.92 \pm 1.86^{\circ} \mathrm{C}$, leg: $18.08 \pm 5.72^{\circ} \mathrm{C}$ ). Mean skin temperature decreases achieved by both $\mathrm{CF}$ and $\mathrm{CC}$ at $21^{\circ} \mathrm{C}$ were similar $\left(4.49 \pm 3.11^{\circ} \mathrm{C}\right.$ for $\mathrm{CF}$ and $4.42 \pm 0.92^{\circ} \mathrm{C}$ for $\left.\mathrm{CC}\right)$.

\section{Conclusions}

Cryocompression permitted hypothermia delivery at significantly lower temperatures compared to continuous-flow cooling with similar safety profile. Cryocompression may provide greater efficacy in preventing CIPN, with clinical trials currently ongoing (NCT03248193).

\section{PS051 \\ OCCURRENCE OF SKELETAL-RELATED EVENTS (SRES) IN PATIENTS WITH SOLID TUMORS AND BONE METASTASIS (ST/BM): EARLY VERSUS LATE INITIATION OF SRE PREVENTATIVE AGENTS (SPA)}

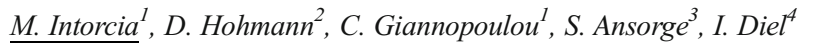
${ }^{7}$ Amgen Europe GmbH, Global Health Economics, Zug, Switzerland

${ }^{2}$ Amgen GmbH, Health Economics, Munich, Germany

${ }^{3}$ Arvato Health Analytics, Data Scientist, Munich, Germany

${ }^{4}$ Schwerpunktpraxis für Gynäkologische Onkologie SPGO, Gynäkologie und Geburtshilfe, Mannheim, Germany

\section{Introduction}

SREs are common in patients with ST/BM. SREs have been associated with poor survival and increased costs. 


\section{Objectives}

To estimate the time from diagnosis to occurrence of first and subsequent SREs in patients with early versus late treatment initiation with SPA.

\section{Methods}

We retrospectively analyzed a German Sick-Fund claims database including data from 2007 to 2015 . Patients $\geq 18$ years old, with ST coded with $\geq 2$ outpatient or one inpatient diagnoses and newly diagnosed with BM after 07/2011 were included. Patients received SPA within 9 months from study inclusion. Outcomes were: time from BM to occurrence of first and subsequent SREs in patients with early treatment ( $\leq 3$ months $[\mathrm{m}])$ versus late treatment $(4-9 \mathrm{~m})$ initiation with SPA. The two cohorts were adjusted for baseline characteristics with matched-pairs, randomly drawing 3:1 matches in early vs late patient.

\section{Results}

Out of 1,144 patients included 949 initiated treatment early and 195 late. Baseline characteristics in early versus late initiators: mean age (70.1 versus 69.9 years), SRE present (25.7\% versus $24.6 \%$ ), osteoporosis (20.7\% versus $12.8 \%$ ), renal disease (17.1\% versus $23.1 \%$ ). After matching, baseline characteristics were balanced: 444 started treatment early and 148 late. The median $(95 \%$ confidence interval) time to first SREs was $19 \mathrm{~m}(12 ; 33)$ and $7 \mathrm{~m}$ $(4 ; 20)$ for the early and late initiators, respectively; the median time to second SREs was $39 \mathrm{~m}(33$; NA) and $21 \mathrm{~m}(13$; NA), respectively.

Conclusions

Time free from SREs was longer in patients with ST/BM starting treatment with SPA earlier than late.

\section{PS052}

DENTAL EXTRACTIONS, MANAGEMENT OF ONJ AND DRUG INTERRUPTIONS: RESULTS ON THE BSG SYSTEMATIC REVIEW

O. Nicolatou-Galitis ${ }^{1}$

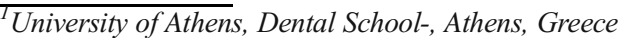

\section{Introduction}

Questions of clinical importance, such as risk of osteonecrosis after dental extraction, best management, and characteristics of ONJ related to nonantiresorptives remain unclear.

Objectives

To address the above research questions.

Methods

A systematic review of the literature between 2009-2017 was conducted. 6,249 titles/abstracts/articles were reviewed. Four articles were included in the 1st research question, reporting 255 dental extractions, 38 articles were included in $2^{\text {nd }}$ question for the management of ONJ and 31 articles, describing 39 cases of $\mathrm{ONJ}$ related to non-antiresorptives were identified. Results

(1) All dental extractions were performed due to periodontal/dental disease. ONJ developed in 14 patients (11.2\%), while over $85 \%$ of extractions did not lead to ONJ. (2) When ONJ developed, different types of management were used. (3) Antiangiogenics, tyrosine kinase inhibitors, mammalian target of rapamycin inhibitors, immune checkpoint inhibitors and conventional chemotherapy were reported as related to ONJ. In comparison to bone targeting agents (BTAs), radiology, histology and management were similar. Important differences included earlier time to ONJ onset, absence of trigger event, greater likelihood of healing and shorter healing time as compared to BTA-related ONJ. Gastrointestinal cancers predominated, followed by renal cell carcinomas in contrast to breast, followed by prostate cancers in BTA-related ONJ, reflecting the different treatments.

Conclusions

(1) Extractions are not completely contraindicated in such patients, with adequate precautions. (2) A trend towards surgical resection of necrotic bone to achieve complete clinical healing at all MRONJ stages was observed. (3) ONJ related to "non-antiresorptives" may have better prognosis compared to the BTA-related.

\section{PS053}

CHARACTERISTICS OF OSTEONECROSIS OF THE JAW (MRONJ) IN CANCER PATIENTS RECEIVING ANTIRESORPTIVE AND/OR TARGETED AGENTS

E. Papadopoulou ${ }^{I}$, O. Nicolatou-Galitis ${ }^{I}$, M. Kouri ${ }^{I}$, E. Vardas ${ }^{I}$, M. Demiri $^{2}$, D. Tryfonopoulos ${ }^{2}$, M.C. Kyrtsonis ${ }^{3}$, A. Megalakaki ${ }^{4}$, F. Antoniou $^{5}$, P. Repousis ${ }^{4}$, M. Dede ${ }^{2}$, A. Ntokou ${ }^{2}$, A. Ardavanis ${ }^{2}$

${ }^{1}$ National and Kapodistrian University of Athens, Dental School, Athens, Greece

2'Agios Savvas' Hospital, 1st Pathology/Oncology Department, Athens, Greece

${ }^{3}$ National and Kapodistiran University of Athens, "Laiko" Hospital-1st Department of Propaideutic Clinic of Internal Medicine-Medical School, Athens, Greece

${ }^{4}$ Metaxa Cancer Hospital, Clinic of Hematology, Piraeus, Greece

5 'Helena Venizelou' Hospital, Oncology Department, Ahens, Greece

\section{Introduction}

MRONJ is an uncommon complication associated with antiresorptive and/or targeted agents.

\section{Objectives}

To present the characteristics of MRONJ in our Clinic, 2015- 2017.

\section{Methods}

Two-hundred-forty-three cancer patients were evaluated. Underlying main diagnoses were breast cancer $(44.9 \%)$, multiple myeloma $(22.6 \%)$, prostate cancer $(11.5 \%)$. One-hundred-fifty-four patients received antiresorptives alone (63.4\%), or combined with targeted agents $(42,17.3 \%)$. Nineteen received targeted agents alone $(7.8 \%)$.

\section{Results}

Osteonecrosis was diagnosed in 76 patients. Mandible was affected in 43/ 76 cases $(56.6 \%)$, maxilla in 20/76 (26.3\%) and both jaws in $13 / 76$ $(17.1 \%)$. Osteonecrosis was exposed in 54 cases $(71.1 \%)$ and nonexposed in $22(28.9 \%)$.

Osteonecrosis was related with antiresorptives in 57 cases $(75 \%)$, with antiresorptives and targeted agents in $18(23.7 \%)$ and with targeted agents alone one $(1.3 \%)$

Dental extraction preceded osteonecrosis in 29 patients (38.2\%), followed by denture trauma $(14 / 76,18.4 \%)$. Painful, mobile tooth was the reason for extraction in 21 patients $(27.6 \%)$, while self-tooth exfoliation was reported in $10(13.2 \%)$.

Patients were managed with antibiotics and ozone. Six patients $(10.5 \%)$ healed, $25(43.9 \%)$ are stable and $19(33.3 \%)$ remain on intermittent antibiotics. ONJ worsened in $7(12.3 \%)$.

167 patients were referred for MRONJ prevention; prior to (28/167, $16.8 \%)$ and after the initiation of medications $(139 / 167,83.2 \%)$. Dental extractions were performed in 19 patients receiving medication; 13 $(68.4 \%)$ due to pain and/or tooth mobility. All healed.

\section{Conclusions}

ONJ group: Periodontal disease/infection (painful mobile teeth/ self-exfoliation), preceded extraction and ONJ in most cases (40.8\%), supporting its association with ONJ. Prevention group: ONJ risk following extraction performed by an expert clinic was limited.

\section{PS054}

TO DO OR NOT TO DO THE DENTAL EXTRACTION IN CANCER PATIENTS, WHO RECEIVE BONE TARGETED AGENTS (BTAS): A SYSTEMATIC REVIEW, BSG, MASCC, PART 1

E. Vardas ${ }^{l}$, O. Nicolatou-Galitis ${ }^{l}$, E. Papadopoulou ${ }^{l}$, M. Kouri ${ }^{l}$, D.

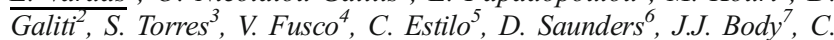
Migliorati $^{8}$, R.V. Lalla $^{9}$

${ }^{I}$ National and Kapodistrian University of Athens- Dental School, Clinic of Hospital Dentistry, Athens, Greece 
${ }^{2}$ National and Kapodistrian University of Athens- Dental School, Department of Oral Diagnosis \& Radiology, Athens, Greece

${ }^{3}$ Clementino Fraga Filho University Hospital- Universidade Federal do Rio de Janeiro, Departmento of Oral Pathology and Diagnosis, Rio de Janeiro, Brazil

${ }^{4}$ Azienda Ospedaliera di Alessandria, Oncology Unit, Alessandria, Italy ${ }^{5}$ Memorial Sloan Kettering Cancer Center, Department of Surgery, New York, USA

${ }^{6}$ Northern Ontario School of Medicine Northeast Cancer Centre- Health Sciences North, Department of Dental Oncology, Ontario, Canada

${ }^{7}$ Université Libre de Bruxelles- CHU Brugmann-Bruxelles- Belgium, Department of Medicine, Brussels, Belgium

${ }^{8}$ University of Florida College of Dentistry, Department of Oral and Maxillofacial Diagnostic Sciences, Florida, USA

${ }^{9}$ University of Connecticut- School of Dental Medicine- Farmington- CT-

USA, Department of Oral Medicine, Connecticut, USA

\section{Introduction}

Dental extraction is considered the main local risk factor (45\%-70\%) for the development of ONJ in cancer patients, who receive BTAs. However the actual risk of ONJ after a dental extraction is not clear.

Objectives

To determine the risk of ONJ development following dental extractions in cancer patients, who receive BTAs.

Methods

A systematic review of the literature between 2009-2017 was conducted by the BSG.

Results

6,249 titles were reviewed. Four articles ( 2 case series, 1 retrospective and one prospective study) were included in the review. 255 dental extractions were performed in 126 patients (mean age 61.7 years) receiving BTAs for prostate cancer (69), multiple myeloma (34), breast cancer (20), lung cancer (2) and non-hodgkin lymphoma (1). All patients received zoledronic acid; 3 patients also received pamidronate. The reason for dental extractions was available in 92 of 255 cases $(36,1 \%)$, either due to periodontal disease or dental caries. Zoledronic acid was discontinued in $64(50,8 \%)$ patients during 92 extractions (mean time 47.1 days). Antibiotics were administrated pre- and postoperatively in all patients. $156(61,2 \%)$ extractions were surgically performed, while $99(38.8 \%)$ were simple extractions. LLLT was applied in $30(11,8 \%)$ extraction sites. Healing time was available in $57(22,4 \%)$ extractions and ranged between 7 to 71 days. ONJ developed in 14 patients $(11,2 \%)$.

Conclusions

Although dental extractions are a risk factor for ONJ in cancer patients receiving BTA's, over $85 \%$ of extractions did not lead to ONJ. Therefore, extractions are not completely contraindicated in such patients, with adequate precautions.

\section{PS055}

PREFERENCES, BARRIERS AND FACILITATORS FOR PREOPERATIVE EXERCISE PARTICIPATION FOR ELDERLY TREATED FOR COLORECTAL CANCER AND THEIR SOCIAL NETWORK

C. Agasi-Idenburg-PT.- MSc ${ }^{l}, M$. Koning-van Zuilen-PT.- MSc. ${ }^{2}, M$. Stuiver-PT-PhD ${ }^{I}$, C. Punt-M.D-PhD, N. Aaronson- PhD, M. Westerman- $P h D^{5}$

${ }^{1}$ Antoni van Leeuwenhoek, Center for Quality of Life, Amsterdam, The Netherlands

${ }^{2}$ Utrecht University of Applied Sciences, Department Movement Studies, Utrecht, The Netherlands

${ }^{3}$ Academic Medical Centre Amsterdam, Department of Medical Oncology, Amsterdam, The Netherlands

${ }^{4}$ Netherlands Cancer Institute, Division of Psychosocial Research and Epidemiology, Amsterdam, The Netherlands
${ }^{5}$ Vu University, Department of Methodology and Statistics, Amsterdam, The Netherlands

\section{Introduction}

Over $85 \%$ of patients with colorectal cancer are older than 70 years when diagnosed. Surgery can have a strong impact on functional capacity, especially in frail elderly people. Prehabilitation programs have been proposed to improve physical capacity prior to surgery, which should improve functional outcomes. Uptake of such programs appears to be low in clinical practice. Reasons for this low uptake are largely unknown. Objectives

To generate insights into barriers, facilitators and preferences for physical activity based prehabilitation (PA-Prehab) as seen by frail elderly who are eligible for colorectal surgery, and by formal and informal caregivers.

\section{Methods}

This qualitative, exploratory study uses semi-structured interviews and a focus group. Elderly patients (EP), informal caregivers (IC) and formal caregivers (FC) were recruited through purposive sampling. All data were analyzed using the Thematic analysis approach.

Results

Initiating PA-Prehab may be difficult due to the emotional impact of the diagnosis (IC), physical discomfort (EP), and lack of time between diagnosis and surgery (FC). It is important that patients understand the potential benefits of PA-Prehab (EP), and are rewarded for their efforts (IC). PA-Rehab should be individualized (FC). Professionals prefer patients to exercise in groups with other patients, but patients prefer programs whereby they can exercise individually, near home, receiving professional advice only when needed; informal caregivers prefer that patients exercise individually but under direct professional supervision.

\section{Conclusions}

To successfully involve frail elderly in PA-Prehab programs several physical, personal and environmental factors should be considered. Preferences of FC and patients may not align.

\section{PS056}

\section{EXERCISE IN OLDER ADULTS WITH CANCER}

\author{
P. Cormie ${ }^{I}$ \\ ${ }^{T}$ Australian Catholic University, Institute for Health \& Ageing, \\ Melbourne, Australia
}

Older adults with cancer may experience serious chronic health and psychological sequelae including accelerated functional decline, fatigue, musculoskeletal symptoms, neuropathies, psychological distress, a higher risk of developing comorbid conditions and reduced quality of life. This presentation will summarise the evidence of the efficacy of exercise in counteracting the detrimental side effects of cancers and their treatment. The evidence emerging from epidemiological studies suggesting exercise confers benefit to cancer outcomes will also be presented. Practical strategies to incorporate exercise into clinical practice will also be discussed.

\section{PS057}

\section{THE IMPACT OF FALLS ON CANCER TREATMENT IN OLDER ADULTS WITH CANCER}

S. Sattar ${ }^{1}$, S. Alibhai ${ }^{2}$, S. Spoelstra ${ }^{3}$, M. Puts ${ }^{1}$

${ }^{T}$ University of Toronto, Lawrence S. Bloomberg Faculty of Nursing, Toronto, Canada

${ }^{2}$ University Health Network, Department of Medicine, Toronto, Canada

${ }^{3}$ Grand Valley State University, Kirkhof College of Nursing, Michigan, USA

\section{Introduction}

Falls are a major health issue among older adults. Cancer and its treatment can heighten their risks for falls and fall-related injuries. However, little is known 
about circumstances of falls, how falls are assessed and reported in oncology clinics, and how falls impact cancer treatment in older patients with cancer.

Objectives

The objective of this study is to address the mentioned gaps in geriatric oncology. Methods

This is a mixed-methods cross-sectional study that recruited communitydwelling older cancer patients at the Princess Margaret Hospital in Toronto who have experienced $\geq$ one fall within the past 12 months. Data collection included patient self-reported survey supplemented by chart review and oncologist interview.

\section{Results}

Ninety-fiveolder patients participated. The median age is 76 (range 6695); $63 \%$ are male; $68 \%$ have $\geq$ one functional limitation; $57 \%$ use a walking aid; over $59 \%$ experienced $>$ one fall. The most common diagnoses are prostate and breast cancers. Injurious fall rate is $48 \%$. The most common fall locations are staircase, sidewalk curb, and washroom. Four patients experienced impact on cancer treatment due to falls. Forty percent of participants did not report falls to their oncologists; of those who did, few were assessed. Over half the participants expressed high concerns about falling. Oncologists voiced that patients are not forthcoming in fall reporting.

\section{Conclusions}

Falls are uncommonly reported by older cancer patients, are rarely assessed by oncologists, and do not seem to affect cancer treatment. More work needs to be done to assess whether cancer treatments are associated with falls risk.

\section{PS058}

\section{MALNUTRITION AND OVERALL SURVIVAL IN OLDER PATIENTS WITH CANCER}

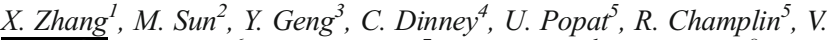

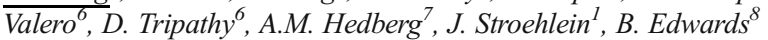

${ }^{1}$ University of Texas- MD Anderson Cancer Center, GastroenterologyHepatology and Nutrition, Houston, USA

${ }^{2}$ University of Texas- MD Anderson Cancer Center, Cancer Therapeutics, Houston, USA

${ }^{3}$ University of Texas- MD Anderson Cancer Center, General Internal Medicine, Houston, USA

${ }^{4}$ University of Texas- MD Anderson Cancer Center, Urology, Houston, USA

${ }^{5}$ University of Texas- MD Anderson Cancer Center, Stem Cell Transplantation, Houston, USA

${ }^{6}$ University of Texas- MD Anderson Cancer Center, Breast Medical Oncology, Houston, USA

${ }^{7}$ University of Texas- MD Anderson Cancer Center, Clinical Nutrition, Houston, USA

${ }^{8}$ Central Texas Veterans Administration, Geriatric Medicine, Temple, USA

\section{Introduction}

Malnutrition is a common condition in older adults with cancer

Objectives

To assess prevalence of malnutrition and its association with overall survival Methods

This is a single site, retrospective cohort study. Patients 65 years of age and older in cancer care underwent comprehensive geriatric assessments via referrals. Geriatric assessment included cognitive, functional, nutritional, physical, and comorbidity measurement. Malnutrition status was derived from the Mini nutritional assessment and clinical interviews. Patients received recommendations for nutritional supplementation, and when necessary, appetite stimulants. Patients were followed for 3 years. Analysis: Descriptive statistics, and univariate and multivariable survival analysis were used.

\section{Results}

We enrolled 456 patients with complete data that were included for final analysis. Patients had hematologic, gastrointestinal, urologic, breast, lung and gynecologic cancers. The mean (SD) age was 77.7 (6.9) years; males and females were equally represented. Forty-one percent presented malnutrition, 33\% died during the 3-year follow-up. In univariate Cox regression analysis, malnutrition was associated with all-cause mortality in all cancers $(\mathrm{HR}=1.46,95 \% \mathrm{CI}=1.06,2.01)$. In multivariate Cox regression analysis, malnutrition was associated with all-cause mortality $(\mathrm{HR}=2.38$, $95 \% \mathrm{CI}=1.14,4.97)$ in patients with solid tumors. This was not observed in hematologic malignancies.
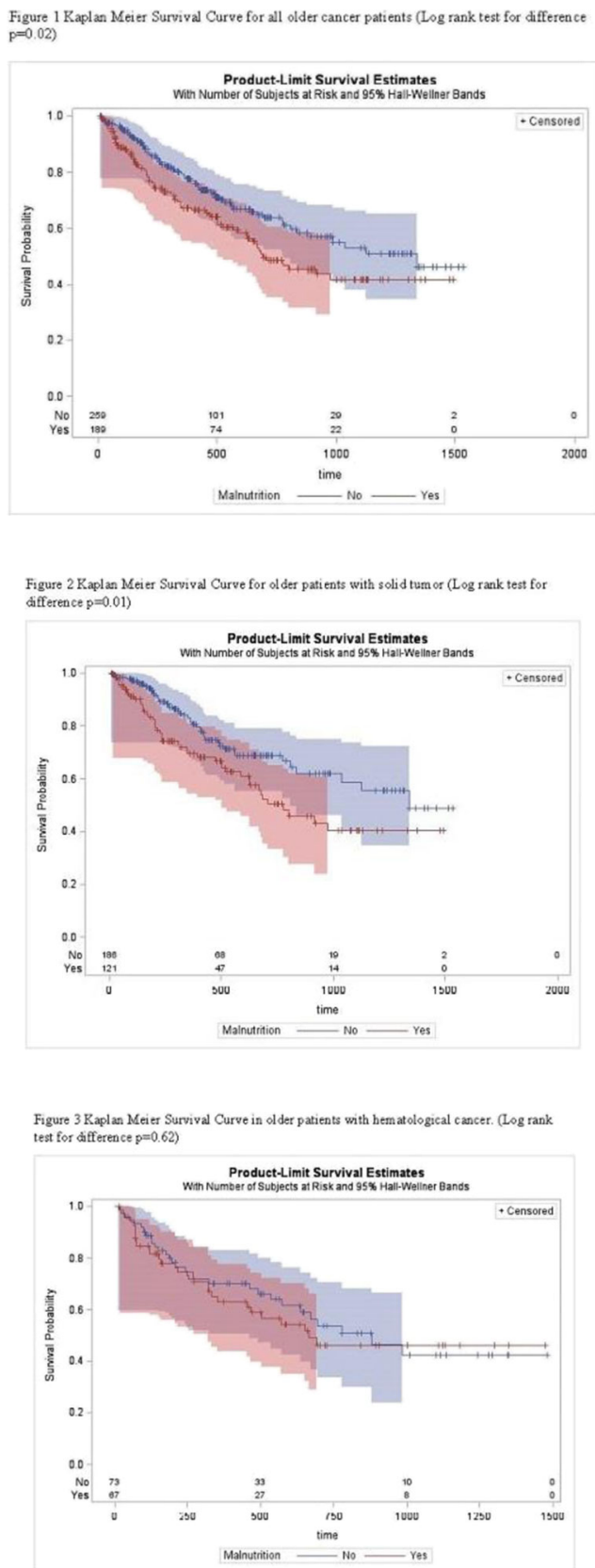

\section{Conclusions}

Malnutrition is commonly identified in older cancer patients. Despite nutritional recommendations for increased dietary intake, malnutrition at the time of initial visit remained a risk factor for mortality in older cancer patients, and an independent risk factor for mortality in patients with solid tumors. Prospective interventional studies are recommended. 


\section{PS059}

WRITING FOR PUBLICATION IN SUPPORTIVE CARE IN CANCER: KEY ISSUES AND LESSONS LEARNED

F. Ashbury ${ }^{1}$, P. Hesketh ${ }^{2}$, I. Olver ${ }^{3}$

${ }^{T}$ University of Toronto, Dalla Lana School of Public Health, Whitby, Canada

${ }^{2}$ Lahey Health Cancer Institute, Thoracic Oncology, Burlington, USA

${ }^{3}$ Sansom Institute for Health Research, Translational Cancer Research, Adelaide, Australia

The goal of this seminar is to facilitate participants' understanding of the requirements for preparing and submitting manuscripts for publication consideration to the journal, Supportive Care in Cancer ("SCC"). The presenters will describe key issues and lessons learned from three perspectives: editor, author, and peer-reviewer. The objectives of this session include: understanding SCC's submission requirements, expectations, and submission processes; positioning papers properly in the context of the journal; preparing the manuscript and elements of successful writing; reviewer approaches/considerations; and responding effectively to decisions and recommendations. Participants will benefit from the dialogue that emerges from the key issues and lessons learned to optimize success for publishing in SCC specifically, and the peer-reviewed literature in general.

\section{PS060}

CANCER PATIENTS' EXPERIENCES WITH AN EARLY PALLIATIVE CARE CONVERSATION: A QUALITATIVE STUDY OF AN INTERVENTION BASED ON THE SENSSTRUCTURE

\author{
M. Fliedner $^{1,2}$, S. Zambrano ${ }^{1}$, C. Lohrmann ${ }^{3}$, J. Schols ${ }^{2}$, R. Halfens ${ }^{2}$, S. \\ Eychmüller $^{1}$ \\ ${ }^{1}$ University Hospital Bern, University Center for Palliative Care, Bern, \\ Switzerland \\ ${ }^{2}$ Maastricht University, Department of Health Services Research- \\ CAPHRI, Maastricht, The Netherlands \\ ${ }^{3}$ Medical University of Graz, Department of Nursing Science, Graz, \\ Austria
}

\section{Introduction}

Person-centred palliative care interventions performed early in the disease trajectory of cancer patients, lessen patients' distress and increase adherence to their wishes. The SENS-structure, which focuses on Symptoms, End-oflife decisions, Network, and Support for the family, provides a pragmatic structure for interprofessional early palliative care conversations.

Patients' insights and experiences with these structured conversations as well as perceived benefits or potential negative effects remain unexplored.

\section{Objectives}

As part of a clinical trial testing the efficacy of SENS-structure, this qualitative study aimed at understanding patients' experiences with the intervention.

Methods

Interviews with conveniently selected patients with advanced cancer not amenable to curative treatment who received the intervention $(n=20)$ were performed. Verbatim transcriptions were analysed based on qualitative content analysis.

Results

Topics covered by SENS were relevant to patients' daily concerns: they felt they were taken seriously. Three main themes were identified: (1) Experiences with the SENS intervention which facilitated discussions about complex and not yet raised issues, putting patients into a contemplative mood; or talking about difficult topics e.g. advance care planning, which was perceived as relieving pressure and providing confidence about the future. (2) A feeling of empowerment which stimulated patients to take action, for example through anticipatory planning. Lastly, (3) impact on family communication, which encouraged discussion about potential wishes and concerns.

\section{Conclusions}

Patients benefited from conversations structured by SENS. They felt more self-confident and reassured by a supportive relationship with health professionals. Our data provides guidance on perceived relevant topics and the engagement of health professionals in structured early palliative care conversations.

\section{PS061 \\ EFFECT OF PALLIATIVE CARE ON QUALITY OF LIFE IN PATIENTS WITH LYMPHOMA}

\author{
Y. Gao ${ }^{l}$, C. Chen $^{2}$ \\ ${ }^{T}$ 1st affiliated hospital of Zhengzhou University, oncology, Zhengzhou, \\ China \\ ${ }^{2}$ Nursing College of Zhengzhou University, oncology, Zhengzhou, China
}

\section{Introduction}

Palliative care service (PCS) has been shown to be utilized less in patients with malignant lymphoma than in those with solid tumors. Lymphoma is progressive, can cause significant symptoms, and impacts on quality of life. Therefore PCS might have a role in the care of patients.

\section{Objectives}

To evaluate the effect of palliative care on the quality of life, symptom improvement, anxiety and depression in patients with lymphoma

\section{Methods}

104 patients with lymphoma were randomly assigned to control and intervention group. Patients of the control group were given routine oncology care, including symptom management, psychological support, health education. Patients of intervention group received PCS based on routine oncology care, which focused on symptom management, information support, share decision-making and humanistic care by palliative care team.

Data were collected at the first visit, 12 and 24 weeks after intervention. The clinical outcomes ware measured by the Functional Assessment Cancer Therapy-Lymphoma, Edmonton Symptom Assessment Scale, Hospital Anxiety and Depression Scale. The changes in groups were analyzed by generalized estimating equations and repeated measurement of variance analysis. Results

Baseline parameters were not significantly different. There were significant differences in quality of life and anxiety and depression of the two groups $(P<0.05)$. The scores of pain, fatigue, depression, anxiety and anxiety in the intervention group was lower compared with control group $(P<0.05)$. There was no difference in nausea and shortness of breath between the two groups. Conclusions

Early palliative care can effectively improve the quality of life of patients, reduce the patients' adverse symptoms and anxiety and depression.

\section{PS062}

DEVELOPMENT AND INITIAL VALIDATION OF A PROGNOSTIC NOMOGRAM FOR AMBULATORY PATIENTS WITH ADVANCED CANCER

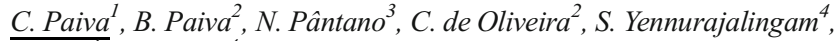 \\ D. Hui ${ }^{4}$, E. Bruera ${ }^{4}$ \\ ${ }^{1}$ Barretos Cancer Hospital, Department of Clinical Oncology, Barretos, \\ Brazil \\ ${ }^{2}$ Barretos Cancer Hospital, Palliative Care and Quality of Life Research \\ Group- Post-Graduate Program-Barretos Cancer Hospital- Barretos- \\ São Paulo-Brazil., Barretos, Brazil \\ ${ }^{3}$ Barretos Cancer Hospital, Researcher Support Center- Learning and \\ Research Institute- Barretos Cancer Hospital-Barretos- São Paulo-
} Brazil., Barretos, Brazil 
${ }^{4}$ The University of Texas M.D. Anderson Cancer Center-Houston- Texas, Department of Palliative Care and Rehabilitation Medicine, Houston, USA

\section{Introduction}

Accurate estimation of prognostic is a fundamental component in the management of patients with advanced cancer, helping oncologists define the continuity of anti-cancer palliative treatments and timely palliative care $(\mathrm{PC})$ referral.

\section{Objectives}

To develop and test a new prognostic tool in patients when they were first referred to $\mathrm{PC}$.

\section{Methods}

A total of 497 patients were analyzed (development set, $n=221$; validation set, $n=276$. Data from 35 potentially prognostic features were analyzed; the most accurate model was identified after multivariate Cox regressions. Parameters were built into a nomogram to estimate the probability of patient survival at 30,90, and 180 days. Calibration and discrimination properties of the Barretos Prognostic Nomogram (BPN) were evaluated in the validation phase of the study.

Results

The BPN was composed of gender, presence of distant metastasis, serum albumin concentration, white blood cell count and Karnofsky Performance Status (Figure 1). The C-index was 0.71. The values of the area under the curve (AUC) of the receiver operating characteristic (ROC) curve were $0.84,0.74$, and 0.74 at 30,90 and 180 days, respectively. There were good calibration results according to the Hosmer-Lemeshow test. According with the BPN score percentiles 25 (p25) and p75, the median survival times (95\% CI) were 313 (225-400) days (<p25), 129 (105-152) days (p-25-p75) and 37 (7-66) days $(>p 75)(p<0.001)$.

\section{Barretos Prognostic Nomogram (BPN)}
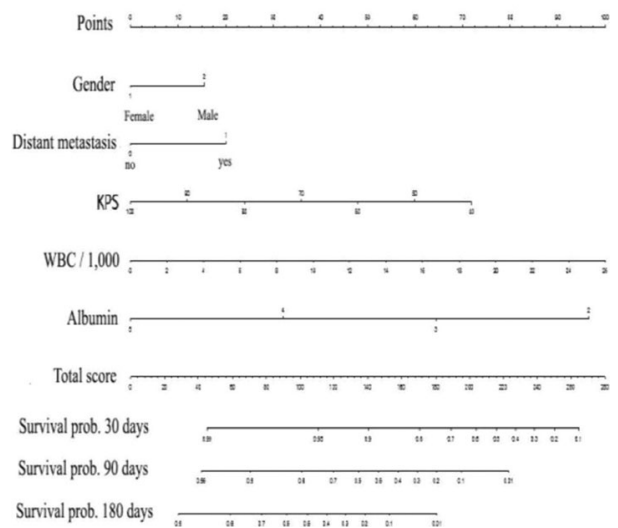

\section{Conclusions}

The BPN is a new prognostic tool with adequate calibration and discrimination properties. It is now available to assist oncologists in estimating the survival of adult patients with advanced solid tumors.

\section{PS063}

PRO: OPIOIDS ARE SAFE AND EFFICACIOUS FOR THE MANAGEMENT OF CANCER-RELATED DYSPNEA

\section{D. $\mathrm{Hui}^{\mathrm{I}}$ \\ ${ }^{T}$ University of Texas MD Anderson Cancer Center, Palliative Care, Houston, USA}

In this session, we shall discussed the pros and cons of using opioids for dyspnea relief. We will provide an updated review of the evidence supporting the use of opioids for dyspnea, focusing on randomized controlled trials and metaanalyses that have been completed in the past few years. We will the provided a balanced discussion of the potential risks related to opioid use, and touch on alternative strategies. We will conclude with practical recommendations for how opioids can be used to treat dyspnea in opioid naive and tolerant individuals.

\section{PS064}

ACUTE AND CHRONIC REDUCTION OF PHYSICAL ACTIVITY IN AGED LUNG CANCER PATIENTS

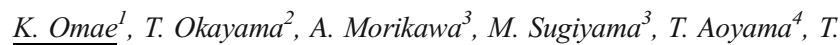
Naito

${ }^{1}$ Kyoto University, Department of Clinical Biostatistics, Kyoto, Japan

${ }^{2}$ Shizuoka Cancer Center, Division of Rehabilitation Medicine, Shizuoka, Japan

${ }^{3}$ Shizuoka Cancer Center, Division of Nursing, Shizuoka, Japan

${ }^{4}$ Shizuoka Cancer Center, Division of Nutrition, Shizuoka, Japan

${ }^{5}$ Shizuoka Cancer Center, Division of Thoracic Oncology, Shizuoka, Japan

\section{Introduction}

Reduction of physical activity brings about various health disadvantage for cancer patients. The activity is often measured in daily steps using a pedometer. However, it is unclear how to use the measurement for intervention, especially its timing.

Objectives

It is to explore whether time series analysis of step count can be useful tool to find an optimal timing of initiating supportive care.

\section{Methods}

This is the prospective observational study approved by the institutional review board (Trial registry No. UMIN000012845). Time series of step count data from 11 patients aged $\geq 70$ years with metastatic non-small-cell lung cancer were analyzed. Daily steps were measured with the pedometer (Lifecorder-GS, SUZUKEN, Japan). We used an autoregressive model to denoise the noisy step count data. By the model, the expected number of steps in each week was estimated by the actual step counts up to the previous weeks. We finally examined the trends of these estimated number of steps.

Results

We found that there were two clear patterns of decreases in the expected number of steps: acute and chronic reduction, observed as jump transitions and slope slip, respectively. In addition, such patterns were classified as reversible and irreversible reductions.

Conclusions

The expected number of steps estimated by the autoregressive model would be useful for diagnosing changes in physical activity. We need discrimination rule to detect the singularity point, namely irreversible days that expected physical activity is never improved without any intervention, and method of intervention in such situations.

\section{PS065}

\section{INTENSIVE PULMONARY REHABILITATION FOR LUNG} CANCER PATIENTS: IS IT FEASIBLE? IS IT EFFECTIVE?

\section{Shannon}

${ }^{T}$ University of Texas at MD Anderson Cancer Center, Pulmonary Medicine, Houston, USA

\section{Introduction:}

Information regarding the minimal duration of pulmonary rehabilitation (PR) that confers significant improvements in functional parameters is limited. This information is useful in the cancer setting where significant delays in definitive cancer therapies imposed by a course of PR is a major concern. 


\section{Objectives}

Determine the minimal duration of PR that confers significant improvements in functional parameters.

Methods

Nonsmall cell lung cancer (NSCLC) patients with Gold Stage IIIIV COPD (mean FEV1 $1.763 \mathrm{~L}+513 \mathrm{ml}$ ) and pretreatment poor performance status were referred to our PR program and prospectively studied. The outpatient, 3 week program consisted of aerobic/resistance training and educational sessions 3 days/week. Functional parameters, generated from pulmonary function testing, cardiopulmonary exercise testing (CPET), Borg scales, and $6 \mathrm{MWT}$ were assessed at baseline, weeks 2 and 3 of PR and at 12 weeks post PR

\section{Result}

Ninety-two patients $(63+8$ years, $74 \%$ males $)$ completed the program. Statistically significant improvements in values of VO2max $(12.2+1.6$ versus $19.3+2.1 \mathrm{ml} / \mathrm{kg} / \mathrm{min}, \mathrm{p}=.0001)$, peak exercise capacity ( $25 \mathrm{~W}$; $34 \%$ of baseline values, $\mathrm{p}=0.0074)$, and $6 \mathrm{MWD}(144 \mathrm{~m} ; 45.7 \%$ of initial values, $p=0.0002$ ) were seen at 2 weeks of PR. Two-week Borg scores of dyspnea and fatigue improved by 1.0 Borg unit, $\mathrm{p}<0.001$. Incremental improvements in all values were noted at week 3 . Values declined by week 12 but remained significantly higher than prePR baseline numbers. Conclusions

Significant gains in performance status may be seen as early as 2 weeks following PR . A short course of PR may benefit select cancer patients and improve cancer treatment options.

\section{PS066}

THE EFFECT OF SELF-EFFICACY-ENHANCING-BASED ACTIVE CYCLE OF BREATHING TECHNIQUE ON ELDER LUNG CANCER PATIENTS WITH LUNG RESECTION

\author{
J. Zhang ${ }^{\text {I }}, \mathrm{Y}_{\mathrm{Mei}}{ }^{2}$ \\ ${ }^{T}$ Sun Yat-senUniversity, School of Nursing, Guangzhou, China \\ ${ }^{2}$ Sun Yat-sen University, School of Nursing, Guangzhou, China
}

\section{Introduction}

Enhancing self-efficacy in the active cycle of breathing technique (ACBT) will be essential to realize the full benefit of pulmonary rehabilitation among elder lung cancer patients.

\section{Objectives}

To explore the effect of self-efficacy-enhancing-based ACBT on sputum clearance and cough strength, functional exercise capacity and postoperative pulmonary complication among elder lung cancer patients with lung resection. .

\section{Methods}

A quasi-experimental design was used. The control group $(n=35)$ received routine breathing exercise from September to December 2016; accordingly, the intervention group $(n=35)$ received the selfefficacy-enhancing-based ACBT in addition to routine perioperative care from January to April 2017. All the patients received 6 interventions: 3 interventions before operation, postoperative day 1 , day 2 and day 3, once a day. We compared the 24-hours wet sputum weight and postoperative pulmonary complications after surgery, the peak expiratory flow (PEF), 6-minute wallk test (6MWT), exercise self-efficacy from baseline until hospital discharge.

Results

The sputum weight of postoperative day 2 , and day 3 were significantly increased in intervention group compared with that in control group $(\mathrm{P}<0.05)$. The PEF(309.2 VS 225.6 L/min), 6MWT(465.4 VS 333.8 m)and exercise self-efficacy(77.8 VS 68.9)were also improved significantly, statistical differences were found $(\mathrm{P}<0.05)$. However, there is no significant difference on postoperative pulmonary complications between the two groups $(\mathrm{P}>0.05)$.

\section{Conclusions}

Conclusions: Self-efficacy-enhancing-based ACBT is a simple and effective short-term pulmonary rehabilitation method and should be advocated

\section{PS067}

CARBOXYMALTOSE IN THE TREATMENT OF CHEMOTHERAPY-INDUCED ANEMIA: A COST EFFECTIVINESS AND COST-MINIMIZATION ANALYSIS

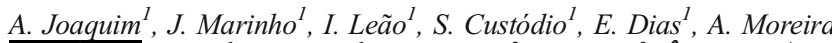

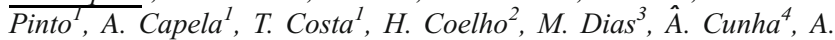
Macedo ${ }^{5}$

${ }^{1}$ Centro Hospitalar Vila Nova de Gaia Espinho, Medical Oncology Department, Vila Nova de Gaia, Portugal

${ }^{2}$ Centro Hospitalar Vila Nova de Gaia Espinho, Clinical Hematology Department, Vila Nova de Gaia, Portugal

${ }^{3}$ Centro Hospitalar Vila Nova de Gaia Espinho, Pneumology Department, Vila Nova de Gaia, Portugal

${ }^{4}$ Centro Hospitalar Vila Nova de Gaia Espinho, Imunohemotherapy Department, Vila Nova de Gaia, Portugal

${ }^{5}$ Keypoint, Keypoint, Lisbon, Portugal

\section{Introduction}

Chemotherapy-induced anemia (CIA) is common in cancer patients, adversely affects quality of life and impacts survival. Anemia has a multifactorial etiology, but iron deficiency (ID) is the dominant underlying mechanism.

\section{Objectives}

Study the effectiveness (hematopoietic response) and economic impact of Ferric Carboxymaltose (FCM) supplementation in CIA patients.

Methods

Prospective study between 2015-2016 with chemotherapy-treated patients for solid tumors, grade $\geq 2$ anemia and ID. Interventional group were patients treated with FCM (2015-2016). Control group (2013-2014) were patients that received blood transfusion only (no patient was treated with intravenous iron).

\section{Results}

Between 2015-2016, 99 patients were included and treated with FCM. The median age was $66(31-84)$ years, and 49 were male (Table 1$)$. Before study enrolment the majority of patients (81) presented relative ID. The mean baseline hemoglobin levels were $9.2(6.7-10.8 \mathrm{~g} / \mathrm{dL})$. Four weeks after FCM administration hemoglobin increased on average to 10.6 (7.8$14.2 \mathrm{~g} / \mathrm{dL})(\mathrm{p}<0.0001)$. A $16 \%$ reduction in the transfusional rate from control to the FCM group was observed (Table 2); relative risk 0.84 (95\% CI:0.74-0.94). Between the two periods, there was an absolute saving of 140 blood units.

The cost-effectiveness analysis showed a global advantage for the usage of FCM treatment in CIA, per patient $(-0.04 €)$ and chemotherapy cycle ($0.27 €$ ), relative to blood transfusion (table 3 ).

\begin{tabular}{|c|c|c|}
\hline & $\begin{array}{l}\text { Control group } \\
\text { (2013-2014) }\end{array}$ & $\begin{array}{l}\text { Ferric Carboxymaltose group } \\
(2015-2016)\end{array}$ \\
\hline Patients (n) & 1732 & 1811 \\
\hline ChTa cycles & 12322 & 13221 \\
\hline Number of transfusions & 194 & 189 \\
\hline RBC units & 657 & 517 \\
\hline Patients treated with $\mathrm{FCM}$ & 0 & 99 \\
\hline Total FCM vials & 0 & 319 \\
\hline Transfusion per patient (\%) & 11 & 10 \\
\hline Transfusional rate ${ }^{\mathrm{b}}(\%)$ & 5.3 & 3.9 \\
\hline
\end{tabular}

Number of RBC units per chemotherapy session. 


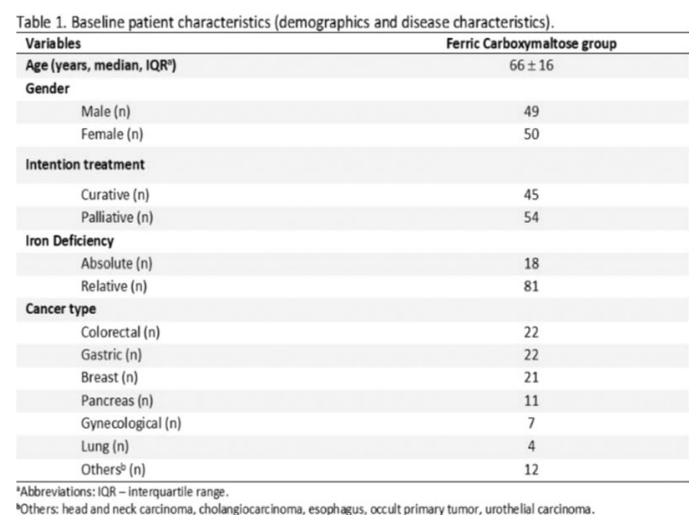

Table 3. Global itreatment costs (red blood cell transfusions + FCM treatment) and cost-effectiveness of FCM treatment (per patient and chemotherapy cycle.

\begin{tabular}{|c|c|c|}
\hline & Control group & Ferric Carboxymaltose group \\
\hline Transfusions ${ }^{2}$ & $126144,00 €$ & $99264,00 €$ \\
\hline Ferric Carboxymaltose' & . & $32562,40 €$ \\
\hline Total costs & $126144,00 €$ & $131826,40 \mathrm{E}$ \\
\hline Total cost per patient & $72.83 €$ & $72.79 €$ \\
\hline Total cost per ChT cycle & $10.24 €$ & $9.97 €$ \\
\hline Incremental cost per patient & . & $.0 .04 \varepsilon$ \\
\hline Incremental cost per ChT cycle & . & $-0.27 €$ \\
\hline
\end{tabular}

\section{Conclusions}

This study shows that FCM is an effective, cost-saving supporting treatment in patients with CIA and ID, improving anemia. Additionally, reduced the need for allogeneic transfusions saving blood units which are a crucial and limited resource.

\section{PS068}

REDUCING LEAD TIME AND ENHANCING QUALITY OF INFECTION MANAGEMENT IN A CANCER CENTER URGENT CARE CLINIC

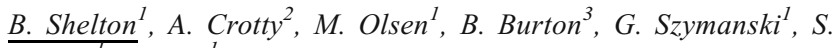
Krumm $^{I}, J$. Kane

${ }^{1}$ The Sidney Kimmel Comprehensive Cancer Center at Johns Hopkins, Oncology Nursing Administration, Baltimore, USA

${ }^{2}$ The Johns Hopkins School of Nursing, Graduate School-Clinical Nurse Specialist Track, Baltimore, USA

${ }^{3}$ The Sidney Kimmel Comprehensive Cancer Center at Johns Hopkins, Pharmacy, Baltimore, USA

\section{Introduction}

Febrile neutropenia and neutropenic sepsis are common complications in oncology patients, and associated with significant morbidity and mortality. Standardized protocols for sepsis management are proven to enhance patient outcomes.

\section{Objectives}

This Comprehensive Cancer Center's Urgent Care Clinic (OUCC) is a referral point for patients with potential infection. Project goals were to: 1) reduce time from clinic arrival to infection treatment, 2) enhance adherence to sepsis management recommendations, and 3) create consistency among clinicians. OUCC staff used lean principles and evidence-based literature to implement an algorithm aimed to enhance sepsis management

Methods

Practice changes included use of antimicrobial ordersets, streamlined radiology approval processes, and on-unit antimicrobial doses. Baseline data were analyzed for patients with possible infection treated in OUCC compared to an equivalent group of patients treated in the Emergency Department. Pre-and post-algorithm OUCC data were compared to determine efficacy of the algorithm to achieve the defined objectives.

\section{Results}

Chi-square tests were used to compare demographic variables between groups, collection of serum lactate levels, if antibiotics were administered within 60 minutes, and if fluids were adequate and timely. Increased number of OUCC patients received antibiotics within 60 minutes after algorithm implementation, $X^{2}(1, \mathrm{~N}=30)=7.5, p=.017$. Completion of timely lactate levels improved but did not reach statistical significance $\left(X^{2}(1, \mathrm{~N}=30)=3.33, p=.068\right)$.

\section{Conclusions}

Implementing a clinical algorithm resulted in consistent improvement in quality measures, however; administration of the recommended fluid volume offers an opportunity for continued practice improvement. Identified barriers to clinician protocol adherence has informed initiation of additional practice improvements.

\section{PS069}

CENTRAL VENOUS CATHETER INFECTIONS AMONG THE ADULT ONCOLOGY PATIENTS: A RETROSPECTIVE, SINGLE IRISH INSTITUTION EXPERIENCE

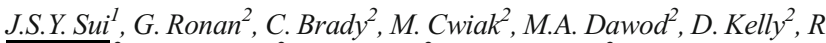 \\ $\overline{\text { Bambury }}^{2}$, D.G. Power ${ }^{2}$, S. O'Reilly ${ }^{2}$, D. O'Mahony ${ }^{2}$ \\ ${ }^{1}$ Mater Misericordiae University Hospital, Medical Oncology, Dublin 1, \\ Ireland \\ ${ }^{2}$ Cork University Hospital, Medical Oncology, Cork, Ireland
}

\section{Introduction}

Adult oncology patients(pts) often has central venous catheter(CVC) inserted to facilitate administration of chemotherapy and supportive medications. However, they are at higher risk of infection. This often leads to longer hospitalisation, delay in cytotoxic administration or potential catheter removal.

\section{Objectives}

We performed this retrospective study to evaluate the impact of CVC infections on oncology pts in our institute.

Methods

Pts who underwent CVC insertions between January 2012 and April 2017 in Cork University Hospital were included. Clinical characteristics of pts were obtained from hospital electronic database.

Results

Seven hundred and thirty-one pts were included, of which 544(74\%) were females with the median age of 57(range: 16-85). CVC insertion are common in $<66$ aged group, $n=548(75 \%)$ with mean length of catheter in-situ of 250 days. The most common cancers were breast(45\%), gastrointestinal(20\%), lymphoma(13\%) and gynae-oncology(9\%). 308 pts $(42 \%)$ were metastatic, $232(32 \%)$ were adjuvant and $75(10 \%)$ were treated neo-adjuvantly.

Cumulative incidence of CVC-related infection was $8 \%(n=57)$ with higher risk of infection in $<66$ years $(77 \%)$. Rates of infection were similar between port vs peripheral inserted central catheter(PICC): $50 \%$ vs $50 \%$. Positive blood cultured were identified in 72 cases. Types of micro-organisms cultured were gram positive $(72 \%)$, gram negatives $(21 \%)$ and fungal( $7 \%)$. The average length of hospitalisation was 14.5 days(range: $1-50)$. Four pts(1\%) died of CVC-related sepsis.

\section{Conclusions}

CVC used is associated with increased risk of infection in younger adults. Local guidelines are essential to identify pts suitable CVC insertion. CVC-related infection can increase burden of healthcare resources with prolonged hospitalisation and compromising cancer care. 


\section{PS070}

SAFETY OF TOPICAL VITAMIN K1 FOR EPIDERMAL GROWTH FACTOR INHIBITOR-INDUCED RASH

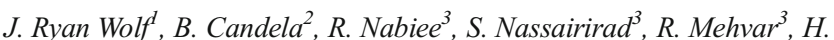 \\ Montezari', S.F. Wong ${ }^{3}$ \\ ${ }^{1}$ University of Rochester Medical Center, Dermatology, Rochester, USA \\ ${ }^{2}$ University of Rochester, School of Medicine \& Dentistry, Rochester, USA \\ ${ }^{3}$ Chapman University, School of Pharmacy, Irvine, USA
}

\section{Introduction}

Papulopustular rash occurs in $80 \%$ of patients receiving Epidermal Growth Factor Inhibitors (EGFRI) for cancer treatment. The rash often results in decreased quality of life and discontinuation of EFGRI therapy. Effective rash management is imminent to optimize therapeutic outcomes. Topical Vitamin K1, a phosphatase inhibitor, could reduce rash severity in the skin but must avoid systemic interference of EGFR inhibition in the tumor.

\section{Objectives}

This study evaluated the safety of a topical $0.1 \%$ Vitamin K1 product for potential use in patients with EGFRI-induced rash.

\section{Methods}

Topical application of $0.1 \%$ Vitamin K1 product resulted in Vitamin K1 blood concentrations ranging from 0 to $8.22 \mathrm{ng} / \mathrm{ml}$ in 10 healthy subjects. To determine if Vitamin K1 reversed cetuximab-induced EGFR inhibition, A549 lung cancer cells were treated in vitro with EGF (200ng), cetuximab (55mg and 160mg), and Vitamin K1 (2.5 to 100ng/ $\mathrm{ml}$ ) in $1 \% \mathrm{FBS} / \mathrm{RPMI}$ media. Phosphorylated-EGFR (p-EGFR) was detected by semi-quantitative ELISA. All statistical analyses (Univariate General Linear Model, ANOVA) were performed at significance level of 0.05 .

\section{Results}

EGF significantly increased p-EGFR (untreated vs. +EGF: mean difference [ $95 \% \mathrm{CI}=-0.3470[-0.0 .397,-0.297], \mathrm{p}<0.001)$. Cetuximab $(55 \mathrm{mg}$ or $160 \mathrm{mg})$ significantly decreased EGF-induced p-EGFR (+EGF vs. +cetuximab: mean difference $[95 \% \mathrm{CI}]=$ $0.140[0.090,0.190]$ and $0.261[0.031,0.401], \mathrm{p}$ 's $<0.001)$. None of the seven doses of Vitamin K1 increased p-EGFR in cetuximabtreated cells ( $\mathrm{p}$ 's $>0.670)$.

\section{Conclusions}

These data suggest that Vitamin K1 levels systemically absorbed by topical application would not reverse cetixumab-inhibition of EGFR. Our topical $0.1 \%$ topical Vitamin $\mathrm{K} 1$ product appears safe to use in patients receiving EGFRI-therapy.

\section{PS071}

\section{CUTANEOUS TOXICITY OF BRAF AND MEK INHIBITOR THERAPY IN CHILDREN}

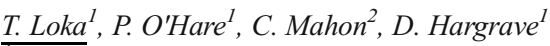

${ }^{T}$ Great Ormond Street Hospital NHS Trust, Department of Paediatric Oncology, London, United Kingdom

${ }^{2}$ Great Ormond Street Hospital NHS Trust, Department of Paediatric Dermatology, London, United Kingdom

\section{Introduction}

The mitogen-activated protein kinase pathway (MAPK) inhibitors include BRAF inhibitors(BRAFi), (dabrafenib) and MEK inhibitors (MEKi), (trametinib). They are emerging therapies in childhood cancer. Cutaneous adverse events (AE) are common and well-described in adult patients, but not in children.

Objectives

The purpose of this study was to document the spectrum and severity of cutaneous AE of BRAFi and MEKi in children and propose anticipatory management strategies.

\section{Methods}

We undertook an observational cohort study of all children treated with BRAFi and MEKi at Great Ormond Street Hospital for children. Thirtythree children and adolescents aged 1-20 (mean 8.4) years were treated with dabrafenib, $(n=15)$, trametinib $(n=17)$, or combination therapy (dabrafenib+trametinib, $n=3$ ) were studied. Median treatment duration was 51 (25-99), 42 (22-72) and 33 weeks respectively. Eleven (73\%) dabrafenib-treated children developed new melanocytic naevi (at 1-43 weeks), 9 (60\%) also developed persistent erythema nodosum-like (EN) eruptions (1-32 weeks). Four (26\%) developed diffuse alopecia, (9-40 weeks).

Results

Eleven trametinib-treated children (65\%) developed acneiform eruptions (1-17 weeks). Ten (57\%) developed paronychia (onset 4-25 weeks), of whom 8 (47\%) developed diffuse alopecia (4-17 weeks), and $8(47 \%)$ generalised xerosis (1-4 weeks). There were no cases of squamous malignancy or melanoma. No child ceased therapy because of cutaneous SE, however 2 children with severe acneiform rashes required dose reduction.

Conclusions

The cutaneous AE of MAPK pathway-targeted therapies in children differ from those seen in adults. In particular, new naevi and panniculitis in BRAFi-treated children and hair loss in both groups were common. Despite this, BRAFi and MEKI were well tolerated.

\section{PS072}

\section{LOW LEVEL LASER THERAPY IN THE TREATMENT OF CHEMOTHERAPY AND TARGET THERAPY INDUCED PALMAR-PLANTAR ERYTHRODYSESTHESIA}

\author{
S. Latifyan ${ }^{1}$, E. Chevalier ${ }^{1}$, D. Leanen ${ }^{1}$, M.F. Scharll ${ }^{1}$, M. \\ Vandenhoucke $^{I}$, M. Paesmans ${ }^{1}$, M. Yunakova ${ }^{1}$, M.T. Genot ${ }^{1}$ \\ ${ }^{I}$ Institut Jules Bordet, Laser therapy, Brussels, Belgium
}

\section{Introduction}

Palmar-plantar erythrodysesthesia (PPE) also called hand-foot syndrome, is a relatively frequent dermatologic toxicity associated with certain chemotherapeutic and target anti-cancer agents. PPE typically presents with dysesthesia and tingling in the hands and feet, which progress into ulceration and epidermal necrosis.

\section{Objectives}

We conducted a randomised prospective study of lowlevel laser therapy (LLLT) in 32 patients with a chemo or target therapy-induced PPE to evaluate the effect of LLLT.

\section{Methods}

We randomised 32 patients with PPE of grade I to III during active anticancer treatment for a one side hand and foot LLL treatment. Patients were their own controls. We used low level light diode for at least 2 weeks, 3 times a week for a total of 18 sessions.

\section{Results}

We treated a series of 27 females and 5 males, mean age of 58 years (range: 34-80). There were 30 solid and 2 hematologic malignancies, most of which breast cancer $(84 \%)$. Chemotherapy, target treatment and combination-induced PPE were of 62\%, 22\% and $16 \%$ respectively. Most of the patients underwent a minimum of 2 weeks of LLLT; mean number of sessions 28.5 (range: 3-160).

We observed that $75 \%$ of the patients reported a decreased pain and that the PPE grade decrease was $69 \%$.

\section{Conclusions}

There is a large body of evidence to support that LLLT, also called photobiomodulation, has an important role in the management of radiation and chemotherapy-related mucositis in cancer patients. Our study suggests that LLLT might be useful in the control of therapy-induced PPE. 


\section{PS073}

TOP 10 RESEARCH PRIORITIES FOR TEENAGE AND YOUNG ADULT CANCER IN THE UNITED KINGDOM: PARTNERSHIP BETWEEN YOUNG PEOPLE, CARERS AND PROFESSIONALS

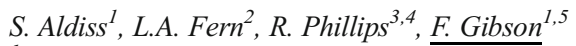

${ }^{1}$ University of Surrey, School of Health Sciences, Guildford, United Kingdom

${ }^{2}$ University College London Hospitals NHS Foundation Trust, Division of Oncology, London, United Kingdom

${ }^{3}$ University of York, Centre for Reviews and Dissemination, York, United Kingdom

${ }^{4}$ Leeds Teaching Hospitals NHS Trust, Department of Paediatric Haematology and Oncology, Leeds, United Kingdom

${ }^{5}$ Great Ormond Street Hospital for Children NHS Foundation Trust, Centre for Outcomes and Experience Research in Children's HealthIllness and Disability, London, United Kingdom

\section{Introduction}

The research agenda is frequently set by healthcare professionals and researchers. Young people with cancer, aged 13-24 years, have unique physical, psychological and social responses to their diagnoses, with distinct outcomes.

Objectives

We aimed to engage young people, carers and professionals in a systematic process to identify and prioritise research questions relating to cancer in young people.

\section{Methods}

We followed the James Lind Alliance process at all stages: created a multidisciplinary steering group of those involved in the care of this cancer population, including young people; gathered potential research questions from young people, carers and professionals via an online survey; checked submitted questions to ensure they were unanswered; undertook interim prioritisation via a second survey to identify the highest priority questions; held a final consensus meeting to reach agreement on the Top 10 research priorities.

\section{Results}

Eight hundred and fifty five potential questions were gathered from 292 respondents; refined into 208 unique questions. Seven were already answered and 16 were ongoing studies, therefore removed. One hundred and seventy four respondents completed the interim online survey prioritising 30 research questions. Prioritisation of the 30 questions was debated at a workshop attended by 25 young people, carers, and professionals from a broad range of roles. Consensus was reached on the Top 10 research priorities, see Table 1.

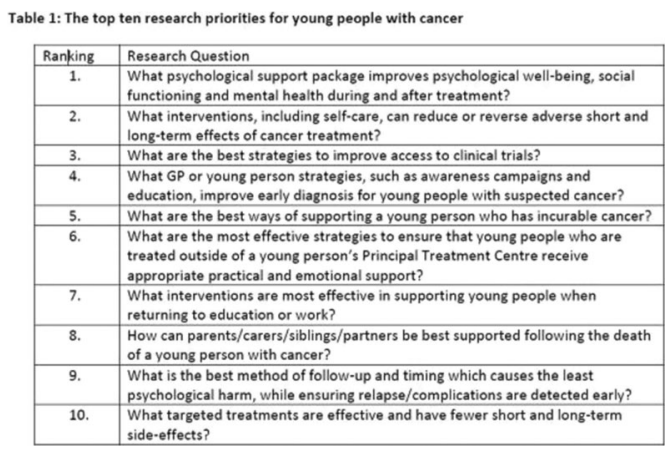

\section{Conclusions}

The Top 10 research priorities have been identified using a rigorous and person-centred approach involving stakeholders who are typically not involved in setting the research agenda. These will inform the funding of future research.
PS074

\section{SUPPORTING CAREGIVERS}

$\frac{\text { Y. Kim }}{{ }^{1} \text { Univer }}$

${ }^{T}$ University of Miami, Psychology, Coral Gables, USA

More family members will become caregivers of cancer patients and cancer survivors in coming decades, due to medical advances, early detection, and our aging population, all of which increase the number of cancer survivors. Studies have documented that cancer has an impact on the quality of life not only of the patients but also their family caregivers. Each person's quality of life is also influenced by other family members' resources and risk factors as well as the trajectory of the illness in the family. Social and health psychology provide a conceptually rich starting point for research on this set of phenomena. Several empirical studies testing the impact of cancer on the individual as well as the partner (family member) and experimental studies testing the pathways of dyadic interpersonal processes of cancer will be presented. Applications of relational approach in the Psycho-Oncology field and future directions will be discussed.

\section{PS075}

EXPLICIT PROGNOSTIC DISCLOSURE TO ASIAN WOMEN WITH BREAST CANCER: A RANDOMIZED SCRIPTED VIDEO-VIGNETTE STUDY (J-SUPPORT 1601)

M. Mori ${ }^{1}$, M. Fujimori co-primary author ${ }^{2}$, L. van Vliet ${ }^{3}$, T. Yamaguchi ${ }^{4}$, C. Shimizu, ${ }^{5}$, T. Kinoshita ${ }^{6}$, M. Kawahara ${ }^{7}$, H. Inoguchi ${ }^{8}$, Y. Matsuoka ${ }^{7}, J$.

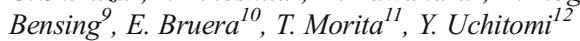

${ }^{I}$ Seirei Mikatahara General Hospital, Palliative Care Team, Hamamatsu, Japan

${ }^{2}$ National Cancer Center, Division of Cohort Consortium ResearchEpidemiology and Prevention Group-Center for Public Health Sciences- Division of Health Care Research-QOL Research GroupCenter for Public Health Sciences, Tokyo, Japan

${ }^{3}$ Nivel-Netherlands Institute for Health Services Research, Department of Communication, Utrecht, The Netherlands

${ }^{4}$ Tohoku University Graduate School of Medicine, Division of Biostatistics, Sendai, Japan

${ }^{5}$ National Cancer Center Hospital, Department of Breast and Medical Oncology, Tokyo, Japan

${ }^{6}$ National Cancer Center Hospital, Department of Breast Surgery, Tokyo, Japan

${ }^{7}$ National Cancer Center, Division of Health Care Research- QOL Research Group- Center for Public Health Sciences, Tokyo, Japan

${ }^{8}$ National Cancer Center Hospital, Department of Psychooncology, Tokyo, Japan

${ }^{9}$ Nivel - Netherlands Institute for Health Services Research-Utrecht University, Faculty of Social and Behavioral Science, Utrecht, The Netherlands

${ }^{10}$ The University of Texas MD Anderson Cancer Center, Department of Palliative Care and Rehabilitation Medicine-Division of Cancer Medicine, Houston, USA

${ }^{11}$ Seirei Mikatahara General Hospital, Palliative and Supportive Care Division, Hamamatsu, Japan

${ }^{12}$ National Cancer Center Hospital, Innovation Center for SupportivePalliative and Psychosocial Care- Division of Health Care ResearchQOL Research Group-Center for Public Health Sciences, Tokyo, Japan

\section{Introduction}

Non-disclosure of a bad news to advanced cancer patients remains a typical practice in Asia. Although the importance of advance care planning (ACP) with prognostic communication has increasingly been recognized worldwide, little is known if explicit prognostic disclosure positively affects Asian patients with advanced cancer. 


\section{Objectives}

To examine the effects of explicit prognostic communication at the time of cancer recurrence.

\section{Methods}

In this randomized, video-vignette study, women with breast cancer who had undergone curative surgery $(\mathrm{n}=105$, age: $53.8 \pm 8.2)$ viewed two videos of prognostic communication between a patient with recurrent breast cancer and her oncologist. Two videos differed only in the presence/absence of explicit prognostic disclosure. The primary outcome was participants' uncertainty $(0-10)$, and the secondary outcomes included State-Trait Anxiety Inventory (STAI)-State $(20-80)$, Patient Satisfaction Questionnaire $(0-$ $10)$, self-efficacy $(0-10)$, and willingness for ACP $(1-4)$.

\section{Results}

After viewing the video with explicit disclosure, compared with the video without it, participants exhibited significantly lower uncertainty (5.3 [SE, 0.2] vs. 5.7 $[0.2], \mathrm{p}=0.032)$ and higher satisfaction (5.6 [0.2] vs. 5.2 [0.2], $\mathrm{p}=0.010)$ without increasing anxiety (changes in STAI-State, 0.06 [0.5] vs. 0.6 [0.5], $\mathrm{p}=0.198$ ). No significant differences were observed in self-efficacy (5.2 [0.2] vs. 5.0 [0.2], $\mathrm{p}=0.277)$ and willingness for $\mathrm{ACP}(2.7[0.1]$ vs. 2.7 [0.1], $\mathrm{p}=0.240)$.

\begin{tabular}{|c|c|c|c|c|c|c|c|}
\hline \multirow[b]{2}{*}{ Effect } & \multicolumn{2}{|c|}{ Explicitness $(+)$} & \multicolumn{2}{|c|}{ Explicitness (-) } & \multirow[b]{2}{*}{ Difference } & \multirow[b]{2}{*}{$95 \% \mathrm{CI}$} & \multirow[b]{2}{*}{$p$} \\
\hline & Mean & SE & Mean & SE & & & \\
\hline Uncerainty ${ }^{2}$ & 5.3 & 0.2 & 5.7 & 0.2 & 0.4 & 0.04 .0 .8 & 0.032 \\
\hline Satisfaction $^{2}$ & 5.6 & 0.2 & 5.2 & 0.2 & -0.4 & $-0.7 .-0.1$ & 0.010 \\
\hline Anxiety ${ }^{b}$ & 0.06 & 0.5 & 0.6 & 0.5 & 0.6 & $-0.3,1.4$ & 0.198 \\
\hline Self-effícacy ${ }^{2}$ & 5.2 & 0.2 & 5.0 & 0.2 & -0.2 & -0.6 .0 .2 & 0.277 \\
\hline Willingness for $\mathrm{ACP}^{\mathrm{C}}$ & 2.7 & 0.1 & 2.7 & 0.1 & -0.1 & -0.2 .0 .1 & 0.240 \\
\hline \multicolumn{8}{|c|}{ Scores range from $0-10$ (low to high) } \\
\hline \multicolumn{8}{|c|}{ b: Differences in STAI-State scores $(20-80$. low to high $)$ before and after viewing the video } \\
\hline \multicolumn{8}{|c|}{ Score ranges from $1-4$ (low to high) } \\
\hline \multicolumn{8}{|c|}{ Abbreviations: SE, standard emor. CI. confidence interval: STAL. State-Trait Anxiety Inventory: ACP } \\
\hline
\end{tabular}

\section{Conclusions}

Explicit prognostic disclosure led to better outcomes than non-disclosure in Japanese women with breast cancer. When asked about prognosis by Asian cancer patients, clinicians may be encouraged to respect their wishes and explicitly discuss prognosis if deemed appropriate.

\section{PS076}

\section{UNDERSTANDING HOW COPING STRATEGIES AND QUALITY OF LIFE MAINTAIN HOPE IN PATIENTS DELIBERATING PHASE-I TRIAL PARTICIPATION}

D. van der Biessen ${ }^{1}$, P. van der Helm ${ }^{2}$, D. Klein ${ }^{1}$, S. van der Burg ${ }^{3}$, M. Ron $^{\prime}$, M. Lolkema, M. de Jonge ${ }^{l}$

${ }^{1}$ ErasmusMC Cancer Institute, Medical Oncology, Rotterdam, The Netherlands

${ }^{2}$ Leiden University of Applied Sciences, Applied Psychology, Leiden, The Netherlands

${ }^{3}$ Radboud University Medical Centre, IQ Healthcare, Nijmegen, The Netherlands

\section{Introduction}

Patients with advanced or incurable solid tumors, with a good performance status, can opt for studies that test novel-cancer drugs.

\section{Objectives}

This study aimed to understand how hope and motivation of patients considering phase-I trial participation are affected by psychological factors such as coping strategies and locus of control (LoC), and general well-being as measured by the quality of life (QoL).

\section{Methods}

An exploratory cross-sectional study was performed in patients with incurable cancer $(N=135)$ referred to our phase-I unit for the first time. Table1. Patients were potentially eligible for phase-I trial participation and participated in our study while deliberating trial participation. We used questionnaires on hope, motivation to participate, coping, LoC, and QoL. To investigate nature and magnitude of the relationships between the scales, a structural equation modeling (SEM) was fitted to the data.

\begin{tabular}{|c|c|}
\hline $\begin{array}{l}\text { Characteristic } \\
\text { Age, mean } \pm \text { standard deviation (range), y }\end{array}$ & \begin{tabular}{|l|} 
Value \\
$61.8 \pm 10.3(31-84)$
\end{tabular} \\
\hline \multicolumn{2}{|l|}{ Sex, No. $(\%)$} \\
\hline Male & $65(48.1)$ \\
\hline Female & $70(51.8)$ \\
\hline \multicolumn{2}{|l|}{ Marital status, No. $(\%)$} \\
\hline Married / living with partner & $112(82.9)$ \\
\hline Single / Separated / divorced / widowed & $23(17.0)$ \\
\hline \multicolumn{2}{|l|}{ Education level, №.(\%) } \\
\hline Primary education & $19(14.1)$ \\
\hline High school or college & $78(57.8)$ \\
\hline University & $34(25,1)$ \\
\hline Other or unknown & $4(3.0)$ \\
\hline Time since diagnose, (range), $y$ & 2.3 years $(0-16,8)$ \\
\hline \multicolumn{2}{|l|}{ Tumor Classification, №. (\%) } \\
\hline Breast & $9(6.6)$ \\
\hline Gastrointestinal & $51(37.7)$ \\
\hline Gynecological & $29(21.5)$ \\
\hline Lung & $7(5.1)$ \\
\hline All others & $39(28.8)$ \\
\hline \multicolumn{2}{|l|}{$\begin{array}{l}\text { WHO performance status at (not) signing informed } \\
\text { consent, No. (\%) }\end{array}$} \\
\hline 0 & $24(17.8)$ \\
\hline 1 & $106(78.5)$ \\
\hline 2 & $3(2.2)$ \\
\hline$\geq 3$ & $1(0.7)$ \\
\hline Unknown & $1(0.7)$ \\
\hline \multicolumn{2}{|l|}{ Phase I trials $\left(\mathrm{N}_{0}=18\right), \mathrm{N}_{0} .(\%)$} \\
\hline Single experimental agent $(\mathrm{no}=8)$ & $75(55.5)$ \\
\hline $\begin{array}{l}\text { Combination therapy, with } 1 \text { approved agent (no = } \\
\text { 10) }\end{array}$ & $59(43.7)$ \\
\hline None, & $1(0.7)$ \\
\hline \multicolumn{2}{|l|}{$\begin{array}{l}\text { Signed informed consent for phase I trial participation, } \\
\text { No. }(\%)\end{array}$} \\
\hline Yes & $125(92.6)$ \\
\hline No & $10(7.4)$ \\
\hline \multicolumn{2}{|l|}{ Start in phase I trial after consent $(\mathrm{N}=125)$, No. $(\%)$} \\
\hline Yes & $103(82.4)$ \\
\hline No & 22 (17.6) \\
\hline
\end{tabular}

Abbreviations: No., number; WHO, World Health Organization

\section{Results}

Hope significantly predicted the motivation to participate in phase-I trials. Predictors of hope were a combination of flexible and tenacious goal pursuit (both $p<0.01)$, internal $\mathrm{LoC}(p<0.01)$, and QoL $(p<0.01)$. The SEM showed an exact fit to the data, using a null hypothesis significance test: chi-square $(8)=9.30, p=0.32$. Figure $1 /$ Table2.

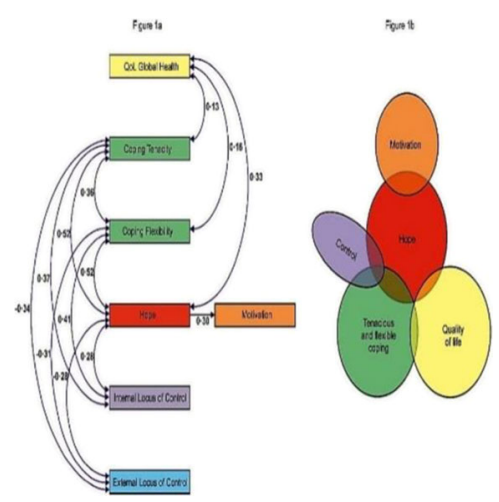


Table 2. Correlations in Patients Consenting to Phase-I Trial

\begin{tabular}{|c|c|c|c|c|c|c|c|}
\hline $\mathrm{n}=125$ & Motivation & n Hope & Flexibility & y Tenacity & $\begin{array}{l}\text { y LoC } \\
\text { intem }\end{array}$ & $\begin{array}{l}\text { LoC } \\
\text { extern }\end{array}$ & $\begin{array}{c}\text { Global } \\
\text { QoL }\end{array}$ \\
\hline Motivation & 1 & & & & & & \\
\hline Hope & $.38^{* *}$ & 1 & & & & & \\
\hline Flexibility & $.22^{*}$ & $.51^{\star \star}$ & 1 & & & & \\
\hline Tenacity & $.37^{\star \star \star}$ & $.54^{* *}$ & $.32^{\star \star *}$ & 1 & & & \\
\hline LoC intern & .15 & $.32^{* *}$ & $.45^{\text {t* }}$ & $.29^{ \pm *}$ & 1 & & \\
\hline LoC extern & $-.23^{k}$ & $-.23 *$ & $-.28^{* k}$ & $-.25^{*}$ & .12 & 1 & \\
\hline Global Qol & .09 & $.49^{* *}$ & $.24^{*}$ & $.33^{* *}$ & .08 &. .10 & 1 \\
\hline
\end{tabular}

\section{Conclusions}

Patients considering phase-I trial participation use a pact of tenacious and flexible coping, and control to stay hopeful. The psychological pact may promote an adaptation enabling them to adjust to difficult circumstances by unconsciously ignoring information, called dissonance reduction. This mechanism may impair their ability to provide a valid informed consent. We suggest including a systematic exploration of patients' social context and values before proposing a phase-I trial.

\section{PS077}

\section{IMMUNOTHERAPIES IN CANCER: PROMISE AND BURDEN}

\author{
R. Bartsch ${ }^{1}$ \\ ${ }^{T}$ Medical University of Vienna, Department of Medicine I- Clinical \\ Division of Oncology, Vienna, Austria
}

Toxicity of conventional chemotherapy such as nausea or neutropenia are still relevant today but oncologists have gained considerable expertise in the treatment and prevention of these side effects. Meanwhile, aplethora of targeted agents have become clinically available; while often considerably improving treatment options, management of specific side effects often posed a challenge to clinicians. The last years have seen another revolution in treatment strategies in many solid tumours with the introduction of immune-checkpoint modulators. These antibodies targeting CTLA-1, PD-1 or PD-L1 counteract tumour-associated inhibition of T-cell activation, thereby aiming to generate an anti-tumour response. Indeed, these drugs have proven highly active in several disease entities such as melanoma, lung cancer, urothelial or renal cell cancer; clinical trials in breast cancer are currently ongoing with early promising results observed in triple-negative disease.

By increasing T-cell activity, however, auto-immune reactions may result; this is a major issue especially with drugs targeting CTLA-4. While less pronounced with antibodies directed against PD-1 or PD-L1, side effects such as hypophysitis, thyroiditis, pneumonitis, adrenal gland insufficiency, hepatitis, guillain-barre syndrome or colitis may occur. As these toxicities are potentially life-threatening, early detection and treatment with corticosteroids is essential.

\section{PS078 \\ OFF-LABEL USE OF STATINS FOR THE PREVENTION OF RADIATION-INDUCED NORMAL TISSUE DAMAGE}

\author{
G. Fritz $^{1}$, V. Ziegler ${ }^{1}$, C. Henninger ${ }^{1}$ \\ ${ }^{T}$ Heinrich-Heine-Universitat, Toxicology, Düsseldorf, Germany
}

\section{Introduction}

Damage to normal tissue remains dose-limiting in radiotherapy. However, preventive pharmacological strategies are missing. Lipid-lowering drugs (i.e. statins) impact various genotoxic stress responses, making them promising drug candidates for an off-label use as radioprotectors.

Objectives

Here we investigated the usefulness of the HMG-CoA reductase inhibitor lovastatin for the prevention of both lung cell and lung tissue damage following fractionated irradiation in vitro and in vivo, respectively.

\section{Methods}

In our study we employed fractionated irradiation. Non-growing primary human lung fibroblasts, lung epithelial and lung endothelial cells were used as in vitro models. Moreover, fractionated irradiation of the mouse lung (BALB/c mice) was performed. We investigated the influence of lovastatin on IR-induced DNA damage formation and repair, DNA damage response (DDR), cell death and inflammatory responses.

\section{Results}

Fractionated irradiation triggered apoptotic cell death in endothelial cells in vitro, which was prevented by lovastatin. By contrast, lung fibroblasts did not undergo apoptosis upon irradiation. Lovastatin improved the repair of IR-induced DNA double-strand breaks (DSBs) in all lung cell types in vitro and, moreover, affected various components of the DDR machinery. In vivo, lovastatin counteracted IR-stimulated increase in breathing frequency, which is indicative of a beneficial effect of the statin on the development of radiation pneumonitis. Moreover, the level of residual DNA damage and apoptosis were reduced in the presence of the statin as investigated four weeks after irradiation.

\section{Conclusions}

Since statins are well tolerated in humans, their off-label use is suggested as a powerful pharmacological strategy to prevent radiotherapy-induced lung injury.

\section{PS079}

BAYESIAN ANALYTICS (BA) TO IDENTIFY RELATIONSHIPS BETWEEN CANCER TREATMENT REGIMENS, PATIENT COMORBIDITIES AND SPECIFIC TOXICITY RISK: TOXSCREEN.CARE DEMONSTRATION PROJECT

G. Ovsak Contributed Equally ${ }^{1}$, C. Cho Contributed Equally ${ }^{2}, L$. Saligan $^{3}$, A. Ross ${ }^{3}$, S. Sonis ${ }^{4}$

${ }^{1}$ Harvard Medical School, Harvard Medical School, Boston, USA

${ }^{2}$ Harvard School of Dental Medicine, Harvard School of Dental Medicine, Boston- MA, USA

${ }^{3}$ National Institutes of Health, NINR - Division of Intramural Research, Bethesda- MD, USA

${ }^{4}$ Primary Endpoint Solutions- Dana-Farber Cancer Institute- and Brigham and Women's Hospital, Primary Endpoint Solutions- DanaFarber Cancer Institute- and Brigham and Women's Hospital, BostonMA, USA

\section{Introduction}

Cancer regimen-related toxicity (CRRT) risk depends on the complex interaction of multiple variables. A tool capable of simultaneously analyzing large amounts of multifaceted medical data to define the collective impact of manifold factors on CRRT risks would be valuable in personalizing treatment choices.

\section{Objectives}

To demonstrate the utility of data-driven prediction of CRRT risk presented in an accessible, clinician-friendly, web-based interface.

\section{Methods}

De-identified data from cancer patients enrolled in National Institutes of Health (NIH) research protocols from 1999 (1,070,081 records) were retrieved through the NIH Biomedical Translational Research Information System and refined to solid tumors and select chemotherapeutic agents to yield data from 14,400 subjects. 9,209 subjects randomly selected as a training set were reviewed to identify tumor diagnosis, treatment regimen (RxR), co-morbidities (CM), and CRRT experience. For each RxR and toxicity pair, we used a BA scheme to calculate absolute and relative risk (AR/RR) of individual CRRT in patients which met a CM filter compared to the rest of the subjects. Validity was assessed using a holdout cohort $(\mathrm{n}=5,191)$. A query/output instrument was enabled (URL TOXScreen.care).

Results

AR/RR of CRRT were associated with pre-cancer RxR and CM. BA yielded a platform that informs consolidated multivariable risk assessment 
and allows for the addition of other modifiers (i.e. genomics, epigenomics, etc.). TOXscreen.care provides clinician access to assess patient CRRT risk. Conclusions

This demonstration project successfully established the potential utility of a BA approach as a means for clinicians to comprehensively assess CRRT risk factors.

\section{PS080}

\section{BUDESONIDE REDUCES NERATINIB-INDUCED} GASTROINTESTINAL INJURY AND DIARRHOEA IN RATS

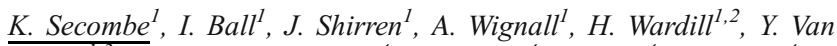
Sebille ${ }^{I, 3}, F$. Avogadri-Connors ${ }^{4}$, D. Martin ${ }^{4}$, E. Olek ${ }^{4}$, S. Moran ${ }^{4}, J$. Bowen $^{1}$

${ }^{1}$ The University of Adelaide, Adelaide Medical School, Adelaide, Australia

${ }^{2}$ South Australian Health and Medical Research Institute, Centre for Nutrition and Gastrointestinal Diseases, Adelaide, Australia

${ }^{3}$ University of South Australia, Division of Health Sciences, Adelaide, Australia

${ }^{4}$ Puma Biotechnology, Inc., Los Angeles, USA

\section{Introduction}

Neratinib is an irreversible pan-HER tyrosine kinase inhibitor recently approved by the FDA for extended adjuvant treatment of HER2+ breast cancer. Diarrhea is the most frequently observed adverse event with this agent. Our previously developed rat model for neratinib-induced diarrhea indicated that diarrhea pathogenesis includes anatomical disruption and inflammation in the ileum and colon.

Objectives

This study aimed to evaluate the corticosteroid budesonide as an intervention for reducing neratinib-induced diarrhea in our rat model.

Methods

Male Albino Wistar rats $(\mathrm{n}=48)$ were treated daily with $50 \mathrm{mg} / \mathrm{kg}$ neratinib and $1 \mathrm{mg} / \mathrm{kg}$ budesonide or vehicle control (5\% DMSO / 1\% carboxymethylcellulose). Budesonide and neratinib were administered by oral gavage 2 hours apart for 14 or 28 days. Diarrhea was scored using a 0-3 grading system. Gastrointestinal histopathological damage was assessed using an established injury score and inflammation was measured by multiplex cytokine/ chemokine ELISA. ErbB/pErbB expression was measured via Western blot. Results

Neratinib-induced diarrhea was decreased from $15.8 \pm 2.7$ days to $10.0 \pm 1$ days by budesonide $(\mathrm{p}=0.027)$. Budesonide reduced colonic injury scores $(\mathrm{p}=0.04)$ and increased ileal anti-inflammatory IL-4 expression $(\mathrm{p}=0.02)$, with a moderate negative correlation ( $\mathrm{rs}=-0.61)$ between days with moderate diarrhea and IL-4. Budesonide increased total and phosphorylated ErbB1 expression in the ileum and colon compared to neratinib only $(\mathrm{p}<0.05)$.

\section{Conclusions}

Budesonide successfully reduced the number of days with diarrhea, histopathological injury and mitigated inflammation. Protection appears to be via upregulation of anti-inflammatory cytokine production and increased ErbB1 expression. These findings warrant further investigation in clinical trials.

\section{PS081}

\section{COGNITIVE IMPAIRMENT IN AYA CANCER SURVIVORS}

A. Chan $^{1}$

${ }^{T}$ National University of Singapore, Pharmacy, Singapore, Singapore

As the cure rates for adolescent and young adult (AYA)cancers continue to improve and survivors live longer, survivorship issues in this group of patients are becoming increasingly relevant, and more in-depth research is needed. Among these undesirable toxicities, post-chemotherapy associated cognitive impairment remains inadequately studied in the AYA population. Cognitive toxicity may pose substantial challenges for AYA cancer survivors who wish to resume or maintain their usual life, including school, work and other social aspects, after treatment. In this talk, we will examine the studies that were conducted to evaluate the risk of chemotherapy-associated cognitive impairment in AYA cancer survivors. Furthermore, we will discuss the socioeconomic impact of cognitive impairment on long-term survivorship in AYA cancer survivors.

\section{PS082}

PREVALENCE OF PRESCRIPTION PSYCHOACTIVE MEDICATION USE IN ADOLESCENT SURVIVORS OF CHILDHOOD CANCER: A REPORT FROM THE CHILDHOOD CANCER SURVIVOR STUDY (CCSS)

Y.T. Cheung ${ }^{1}$, W. Liu' ${ }^{2}$, T.M. Brinkman ${ }^{3}$, D. Srivastava ${ }^{2}$, W.M.

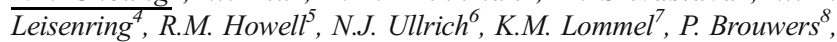
T.M. Gibson ${ }^{3}$, L.L. Robison ${ }^{3}$, G. Armstrong ${ }^{3}$, K.R. Krull ${ }^{3}$

${ }^{I}$ The Chinese University of Hong Kong, School of Pharmacy, Hong Kong, Hong Kong S.A.R.

${ }^{2}$ St. Jude Children's Research Hospital, Biostatistics, Memphis, USA

${ }^{3}$ St. Jude Children's Research Hospital, Epidemiology and Cancer Control, Memphis, USA

${ }^{4}$ Fred Hutchinson Cancer Research Center, Clinical Research Division, Seattle, USA

${ }^{5}$ The University of Texas MD Anderson Cancer Center, Radiation Physics, Houston, USA

${ }^{6}$ Boston Children's Hospital, Neuro-Oncology, Boston, USA

${ }^{7}$ University of Kentucky College of Medicine, Psychiatry, Lexington, USA

${ }^{8}$ National Institute of Mental Health NIH, U.S. Department of Health and Human Services, Washington DC, USA

\section{Introduction}

Long-term survivors of childhood cancer are at-risk for developing physical, emotional, and cognitive late effects which may be managed pharmacologically. The prevalence of psychoactive medication use in adolescent survivors is unknown.

\section{Objectives}

To compare the prevalence of psychoactive medication use in adolescent survivors, against adolescent siblings, and identify treatment, clinical and behavioral predictors of medication use.

Methods

Participants included 5665 adolescent $(\leq 18$ years old) survivors of childhood cancer from the CCSS and 921 sibling controls (Table 1). Parentreports of psychoactive medication use and behavioral functioning (Behavior Problems Inventory) were analyzed. Multivariable logistic regression was performed to compare odds ratios (OR) of risk factors for psychoactive medication use, adjusted for age, sex and race.

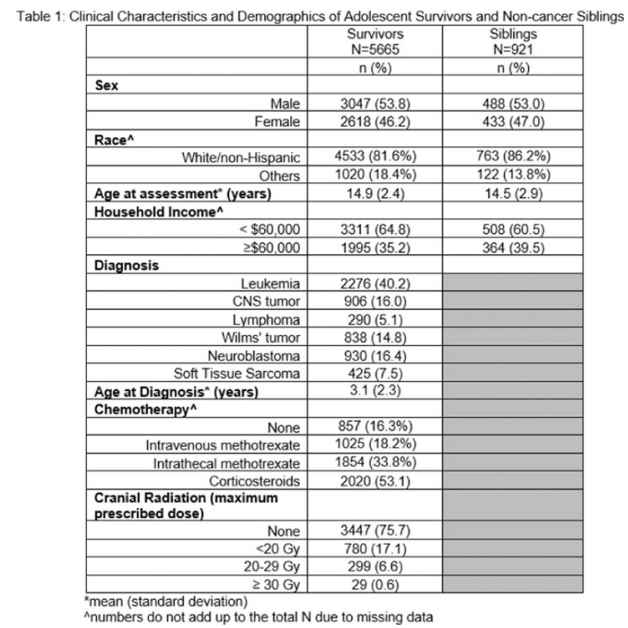




\section{Results}

Survivors were significantly more likely to report psychoactive medication use than non-cancer siblings ( $18 \%$ vs $6 \%, P<0.001$; Table 2$)$. Survivors of CNS tumors or those treated with higher doses of cranial radiation were more likely to use psychoactive medication (Table 3 ). Cancer-related pain was strongly associated with non-opioid (OR [95\% CI] 5.29 [2.52-11.1]) and opioid (OR 4.83 [2.71-8.63]) analgesic use. Use of stimulants, antidepressants and analgesics was more prevalent in survivors with decreased attention, antisocial behavior and emotional distress (Table 3 ), as well as those who received special education services (OR 2.39 [2.12-2.70]).
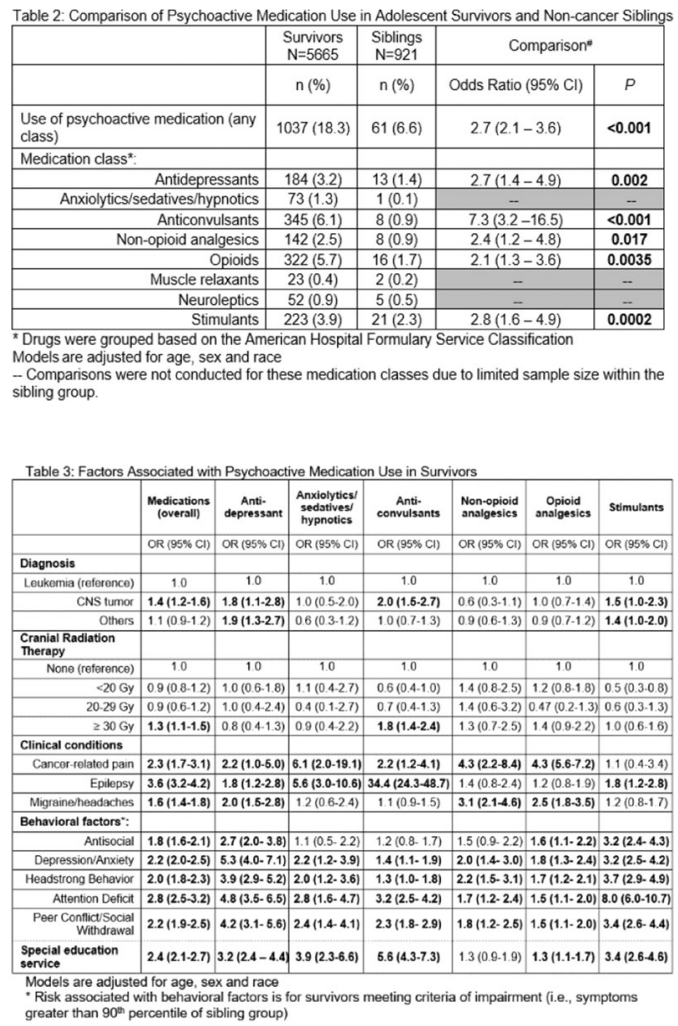

\section{Conclusions}

Use of psychoactive medication was more prevalent among adolescent survivors, and was associated with pain and more significant behavior problems. Future research should examine the impact of psychoactive medication use during adolescence on health-related quality of life and functional attainment during adulthood.

\section{PS083}

EFFECT OF FAMILY-ORIENTED REHABILITATION ON QUALITY OF LIFE IN CHILDREN WITH BRAIN TUMORS AND IN THEIR PARENTS: OBSERVATIONAL PROSPECTIVE STUDY

\footnotetext{
A. Rumyantsev ${ }^{1}$, G. Tseitlin ${ }^{2}$, A. Karelin ${ }^{3}$, M. Kokoreva ${ }^{4}$, I. Borodina ${ }^{5}, \underline{\text { T. }}$ Nikitina $^{6,7}$, A. Zinkovskaya ${ }^{8}$, N. Porfir'eva ${ }^{7}$, T. Ionova ${ }^{6,7}$

${ }^{T}$ Dmitry Rogachev National Medical Research Center of Pediatric Hematology- Oncology and Immunology, General Director, Moscow, Russia

${ }^{2}$ Dmitry Rogachev National Medical Research Center of Pediatric Hematology- Oncology and Immunology, Head of Psychosocial Rehabilitation Department, Moscow, Russia
}

${ }^{3}$ Dmitry Rogachev National Medical Research Center of Pediatric Hematology- Oncology and Immunology, Deputy General Director, Moscow, Russia

${ }^{4}$ Dmitry Rogachev National Medical Research Center of Pediatric Hematology- Oncology and Immunology, Psychosocial Rehabilitation Department, Moscow, Russia

${ }^{5}$ Dmitry Rogachev National Medical Research Center of Pediatric Hematology-Oncology and Immunology, Rehabilitation department for children with CNS tumors, Moscow, Russia

${ }^{6}$ University clinic- Saint-Petersburg State University, Quality of Life Monitoring Dapartment, Saint-Petersburg, Russia

${ }^{7}$ Multinational Center for Quality of Life Research, Oncology and Hematology Department, Saint-Petersburg, Russia

${ }^{8}$ Multinational Center for Quality of Life Research, Department of Biostatistics, Saint-Petersburg, Russia

\section{Introduction}

Family-oriented rehabilitation is promising to support cancer children and their families with the re-entry into 'normal' life.

Objectives

We aimed to evaluate quality of life (QoL) of children with brain tumors in remission and their parents during family-oriented rehabilitation.

\section{Methods}

Children of 6-17 y.o. and their parents underwent 4-weeks familyoriented rehabilitation after the end of cancer treatment. Children filled out child form of PedsQL TM questionnaire, parents - SF-36 and parent form of PedsQL TM before and after rehabilitation. Statistical analysis included t-test, Wilcoxon test, Generalised Estimation Equations (GEE) and $\chi^{2}$ test.

Results

In total 82 survivors ( $11.8 \pm 3.1$ y.o., $57 \%$ boys, median remission' duration -26 mos) and 82 parents ( $44.4 \pm 11.1$ y.o., $85 \%$ mothers) were enrolled. At baseline $78 \%$ children had significant QoL impairment $(\geq 25 \%$ decrease as compared to population norms), $22 \%$ - mild QoL impairment. After rehabilitation, according to child-reports children' QoL increased mainly in terms of emotional and psychosocial functioning $(\mathrm{p}<0.05)$; Integral QoL Index improved $(0.328$ vs $0.411, \mathrm{p}<0.001)$. According to parent-reports social, school and psychosocial functioning of children improved $(\mathrm{p}<0.05)$. In general, children' QoL improvement was revealed with adjustment for age, gender, duration of remission and baseline QoL (GEE, $\mathrm{p}<0.05$ ). After rehabilitation, the number of survivors with significant QoL impairment decreased, and with mild QoL impairment increased ( $78 \%$ vs $57 \%, 22 \%$ vs $43 \% ; \chi^{2}=7.1, \mathrm{p}<0.05$ ). Parents' QoL improved almost by all SF-36 scales; Integral QoL Index 0.393 vs 0.485 ( $\mathrm{p}<0.001)$. Conclusions

Family-oriented rehabilitation is effective in terms of QoL improvement both in surviving children with brain tumors and in their parents.

\section{PS084}

ADHERENCE TO THE WCRF/AICR RECOMMENDATIONS FOR CANCER PREVENTION IS ASSOCIATED WITH BETTER HEALTH-RELATED QUALITY OF LIFE AMONG COLORECTAL CANCER SURVIVORS

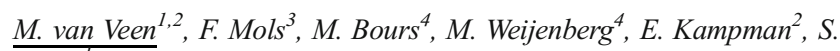
Beijer $^{I}$

${ }^{1}$ IKNL, Research, Utrecht, The Netherlands

${ }^{2}$ Wageningen University, Division of Human Nutrition, Wageningen, The Netherlands

${ }^{3}$ Tilburg University, CoRPS-Center of Research on Psychology in Somatic diseases- Department of Medical and Clinical Psychology, Tilburg, The Netherlands

${ }^{4}$ Maastricht University, Department of Epidemiology- School for Oncology and Developmental Biology GROW, Maastricht, The Netherlands 


\section{Introduction}

In 2007, the World Cancer Research Fund/American Institute for Cancer Research (WCRF/AICR) launched lifestyle guidelines for cancer prevention. Several studies show positive associations between adherence to non-cancer specific lifestyle guidelines and health related quality of life (HRQL) among mixed populations of cancer survivors. However, little is known about the association between adherence to the WCRF/AICR guidelines and HRQL in colorectal cancer (CRC) survivors.

Objectives

To investigate the association between adherence to WCRF/AICR recommendations and HRQL among CRC survivors.

Methods

In a cross-sectional PROFILES-registry study in 1,096 CRC survivors (mean time since diagnosis 7 years), WCRF/AICR adherence scores were calculated using questionnaires (Table 1) on dietary intake, physical activity (PA), and BMI. HRQL was assessed using the EORTC QLQ-C30. Associations between overall and disease-specific HRQL scores with the overall adherence scores and separately for BMI, PA and diet were investigated using linear regression analyses.

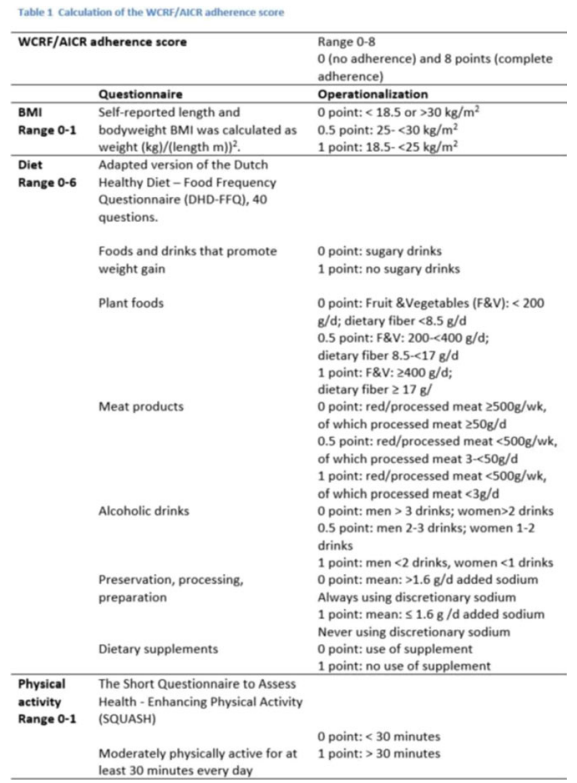

\section{Results}

Survivors in the highest tertile ( $>5.3$ points) of adherence scores had significantly higher mean physical and role functioning scores compared to survivors in the lowest tertile ( $<4.4$ points) ( 84.8 vs $78.3,86.5$ vs. 78.3 , respectively, $\mathrm{P}_{\text {trend }}<0.001$ for both). Separate analyses for BMI, diet and PA showed that only the continuous score for PA was significantly associated with physical ( $\beta$ 10.92; 95\% CI 8.59/13.25) and role functioning ( $\beta$ 11.00; 95\% CI 7.82/14.18).

\section{Conclusions}

Higher adherence to the WCRF/AICR guidelines was significantly associated with higher physical and role functioning among CRC survivors. PA seemed to be the main contributor. It is unknown whether the association reflects causality or reverse causation.

\section{PS085}

\section{DEVELOPING SUPPORTIVE CARE: EXPERIENCE FROM SERBIA}

\section{S. Bosnjak ${ }^{1}$}

${ }^{T}$ Institute for Oncology and Radiology of Serbia, Supportive Oncology, Belgrade, Serbia
Developing supportive care (SC) at the Institute for Oncology and Radiology of Serbia (IORS) has always been about envisioning that all patients and their families receive the support and care they need to maximize quality of live despite the disease and its treatment. Our mission has been to integrate supportive care interventions in all aspects of oncological care at the IORS. The role of MASCC in facilitating the development of supportive care in Serbia will be illustrated by using 2 examples: the prevention of chemotherapyinduced nausea and vomiting and cancer pain management. The Supportive Oncology Department (SOD) at the IORS operates as patient- and family-directed service, embedded in oncological care and provided from the time of diagnosis and throughout to the point when a disease-directed treatment is no longer possible or, in the case of a successful tumour response, to survivorship. The integration of supportive care and oncology is reflected in the clinical structure of the SOD (Supportive Oncology unit with 4 beds; mobile consultation team for inpatients; supportive care service for outpatients), activities in education (lectures in SC for oncologists, nurses, GPs / clinical rotation in SC / the development of local SC guidelines / publications) as well as in research activities / publications. Together with the Serbian Society for Medical Oncology, the SOD organized two regional education meetings on SC for Eastern Europe and the Balkan Region $(2016,2017)$ with the support from MASCC, ESO and ESGO and four former Presidents of MASCC. The 2017 meeting was also accredited by ESMO and involved 180 participants from 18 countries. The meeting was highly valued (1: poor to 4: excellent) for it's learning outcomes (3.76/4) as well as content: a) relevant to clinical work: $3.62 / 4$; b) well-balanced /evidence-based: $3.72 / 4$. The time provided for discussion/ questions was also highly appreciated (3.75/4). The SOD has been actively involved in efforts to develop supportive care in Serbia and include supportive and palliative care in the National Cancer Control Plan.

\section{PS086 \\ ASSESSMENT OF SYMPTOM BURDEN AMONG PATIENTS DIAGNOSED WITH GASTROINTESTINAL (GI) M A L I G N A N C I ES A D M I T T E D TO RE CEIVE CHEMOTHERAPY AT THE INSTITUTE FOR ONCOLOGY AND RADIOLOGY OF SERBIA (IORS)}

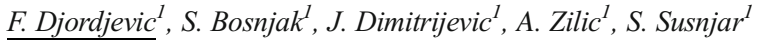 ${ }^{T}$ Institute of Oncology and Radiology of Serbia, Supportive and palliative care, Belgrade, Serbia}

\section{Introduction}

Routine symptom assessment represents the cornerstone of symptom management. The Edmonton Symptom Assessment System (ESAS) has been proposed as a screening tool in cancer patients. It consists of 11-point numerical rating scales (0-10) for self-report of nine common symptoms of cancer, with a 10th scale for a patient-specific symptom.

\section{Objectives}

The objective of this paper is to assess the symptom burden using an ESAS in patients with GI malignancies who were scheduled to receive first cycle of chemotherapy at the IORS.

\section{Methods}

ESAS was used to assess symptoms in patients with GI malignancies who were admitted to start chemotherapy at the IORS for the first time. Descriptive statistics methods were used to analyze collected data.

\section{Results}

ESAS records were collected from 30 patients (13 male and 17 female) with the median age of 59 years (range 25-80). All the patients had advanced malignant disease. Majority of patients $(70 \%)$ had performance status (PS) of 1. All nine ESAS symptoms were reported as mild (less 
than $4 / 10$ ) by majority of patients with mean scores of $2.46,1.71,1.80$, $1.59,2.03,2.02,1.04,2.19,2.06$ for pain, nausea, lack of appetite, shortness of breath, fatigue, depression, drowsiness, anxiety and well-being, respectively.

\section{Conclusions}

Although high symptom burden might be expected our analysis showed mild symptom in patients with metastatic disease. Our research supports use of ESAS as a useful, easy and not time-consuming screening tool for symptom burden. Further research will be conducted in order to examine the impact of ESAS in treatment decision-making process.

\section{PS087}

\section{IDENTIFYING GAPS IN IMMUNOTHERAPY EDUCATION BEYOND THE ONCOLOGY TEAM}

\author{
T. Perloff ${ }^{\text {, }}$, B. Guardado ${ }^{1}$, L. Lucas $^{\text {I }}$, P. Rattananont Ferris ${ }^{1}$ \\ ${ }^{T}$ The Association of Community Cancer Centers, Provider Education, \\ Washington, USA \\ Introduction
}

Clinical guidelines have rapidly included immunotherapy (IO) as standard of care across multiple tumor types. As a result, an increasing number of patients will be at risk for developing toxicities from these treatments which may not be reported until after discontinuation from oncological care.

\section{Objectives}

Our study goal was to assess gaps in IO education and care coordination among multidisciplinary, non-oncology providers.

Methods

A Needs Assessment Survey was electronically distributed to 156 non-oncology providers from December 2017 to January 2018. 150 non-oncology providers representing 12 unique disciplines completed the survey. The overall completion rate was $96 \%$. The survey assessed providers' level of comfort treating IO patients, communication between the primary cancer team, and the use of guidelines and educational tools for managing IO patients.

Results

$50 \%$ of providers do not feel comfortable generally treating an IO patient or recognizing and managing immune-related adverse events (irAEs). When asked to report comfort level in administering and prescribing appropriate and timely treatment to prevent IO adverse side effects, only $18 \%$ of providers reported a high comfort level. $15 \%$ of respondents were unaware their place of practice had guidelines for treating IO patients, but over $80 \%$ noted they would utilize guidelines.

\section{Conclusions}

This study demonstrates the need for IO provider education to extend beyond the oncology team. These findings pave the way for future research to tailor educational interventions for non-oncology providers. Addressing these problems in health care delivery could positively impact the survivorship of patients treated/being treated with IO.

\section{PS088}

AVAILABILITY OF AND ACCESS TO ANTIEMETICS NEEDED FOR THE PREVENTION OF CHEMOTHERAPY INDUCED NAUSEA AND VOMITING (CINV) AFTER HEC AND CARBOPLATIN IN SERBIA

\author{
A. Zilic ${ }^{1}$, S. Bosnjak ${ }^{1}$, I. Popovic ${ }^{2}$ \\ ${ }^{T}$ Institute for Oncology and Radiology of Serbia, Supportive oncology, \\ Belgrade, Serbia \\ ${ }^{2}$ Institute for Oncology and Radiology of Serbia, Pharmacy, Belgrade, \\ Serbia
}

\section{Introduction}

According to ESMO, management of cancer is predicited by the availability and access to anticancer treatment. Last approval, of the Republic Health Insurance Fund (RHIF) brought the access with the full reimbursement of several new anticancer drugs in Serbia. Antiemetics (AE) should also be accessible to accompany optimal anticancer therapy.

\section{Objectives}

To evaluate the availability, accessibility and affordability of $\mathrm{AE}$ recommended by the MASCC/ESMO guideline(www.mascc.org) for the prevention of CINV after HEC (non-AC and AC- based) and carboplatin.

\section{Methods}

Availability was evaluated by the formulary availability and marketing authorization in Serbia. Accessibility and affordability were assessed by RHIF coverage as fully reimbursed, reimbursed only for a specific indication or fully paid by the patient.

\section{Results}

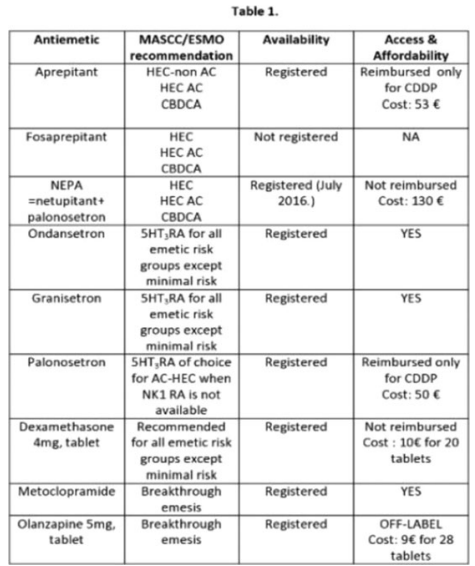

Ondansetron, granisetron and metoclopramide are available and accessible (Table 1). NEPA is now registered, however it's payed out off patients pocket and it's access depends on the personal affordability. Aprepitant and palonosetron are reimbursed only for cisplatin-based chemotherapy. For CINV, olanzapine is used as an off-label medication and therefore is not reimbursed by RHIF.

\section{Conclusions}

While RHIF reimbursement changes have improved the access to anticancer drugs in Serbia they didn't improve the access to AE. Optimal prevention of CINV in patients receiving AC-HEC and carboplatin is not possible in Serbia since aprepitant, NEPA and palonosetron are not reimbursed and their access brings out-of-pocket expenses for the patients. The major barrier for the use of olanzapine as an AE is the need for an offlabel use in this setting.

eP001

IMPACT OF FOSAPREPITANT IN THE PREVENTION OF NAUSEA AND EMESIS IN HEAD AND NECK CANCER PATIENTS UNDERGOING CISPLATIN-BASED CHEMORADIATION (CRT): A PROSPECTIVE COHORT STUDY

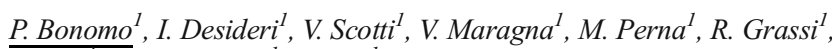

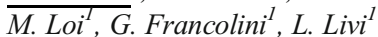 \\ ${ }^{1}$ University of Florence, Radiation Oncology, Florence, Italy
}

\section{Introduction}

Concurrent cisplatin-based CRT is standard treatment for head and neck squamous cell carcinoma (HNSCC). Intensity modulated radiotherapy (IMRT) is state of the art technique able to decrease late 
treatment morbidity. Preliminary evidence suggests that IMRT may worsen the intensity of chemotherapy-induced nausea and vomiting (CINV).

\section{Objectives}

The aim of our study is to report on the impact of fosaprepitant in the prevention of CINV in HNSCC.

\section{Methods}

Fosaprepitant was administered at a dose of $150 \mathrm{mg}$ over a 30 -minutes ev infusion as part of standard antiemetic prophylaxis. Acute toxicity was evaluated according to CTCAE v.4. In addition, incidence of CINV was estimated with the FLIE questionnaire. The timing of CINV assessment was as follows: twice during CRT (week 2 and 5) and one week after the end of treatment.

\section{Results}

Between 05/2017 and 11/2017, 22 patients were enrolled. Treatment and patients' characteristics are summarized in table 1. Mean dose intensity of cisplatin was $79.5 \%$. Observer-rated CINV rates are summarized in table 2. According to FLIE, the mean scores of patient-reported nausea and vomiting were 30.5 (range 21-49) and 30 (range 15-52) at week 2, 29.8 (range 15-52) and 29.2 (range 2645) at week 5, 27.4 (range 23-41) and 27.7 (range 23-41) after the end of CRT. In order to quantify the potential contribution of IMRT to CINV, dosimetric analysis of vomiting center structures (dorsal nucleus of vagus and area postrema) is ongoing.

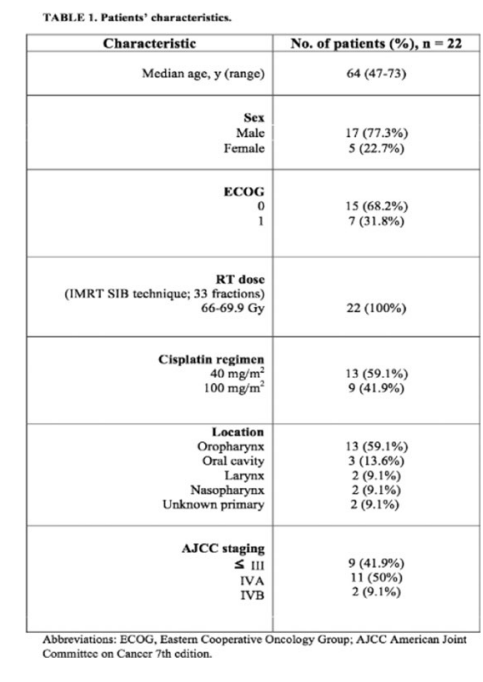

TABLE2. Aecute traturnent-related torichty yccording to CTCAE version 4.03.

\begin{tabular}{lcccc}
\hline \multirow{2}{*}{ Toxicity (CTCAE v 4.03) } & \multicolumn{4}{c}{ No. (\%) by toxicity grade } \\
\cline { 2 - 5 } Nausca & Grade 0 & Grade 1 & Grade 2 & Grade 3 \\
Vomiting & $8(36 \%)$ & $8(36 \%)$ & $6(28 \%)$ & 0 \\
& $16(73 \%)$ & $5(22.5 \%)$ & $1(4.5 \%)$ & 0
\end{tabular}

\section{Conclusions}

The use of Fosaprepitant in the prevention of CINV yielded a very low rate of moderate-severe nausea and vomiting

\section{eP002}

\section{ANTIEMETICS FOR PATIENTS WITH BREAST CANCER: AN EVALUATION OF IMPLEMENTING MASCC/ESMO RECOMMENDATIONS IN A SCOTTISH CANCER CENTRE}

${\text { K. } \text { Cowie }^{1}}^{1}$

${ }^{T}$ NHS Tayside, Pharmacy Department- Ninewells Hosptital, Dundee, United Kingdom

\section{Introduction}

Breast cancer patients receiving anthracycline and cyclophosphamide (AC) based chemotherapy are at high risk of chemotherapy induced nausea and vomiting (CINV); treatment delays and dose reductions reduce survival outcomes in this group so control of CINV is vital. MASCC/ESMO antiemetic guidelines recommend triplicate CINV prophylaxis for AC chemotherapy. At the time of this study, NK1 antagonists were not recommended in NHS Scotland outwith cisplatin-based regimens, in such circumstances MASCC/ESMO recommends the 5HT-3 antagonist palonosetron.

Objectives

This MSc project investigated effect of switching to breast cancer patients from ondansetron to palonosetron.
Historical Control

Dexamethasone 20mg IV

$+8 \mathrm{mg}$ BD $\times 3$ days PO

Ondansetron $16 \mathrm{mg}$ PO
Intervention Cohort

Dexamethasone $8 \mathrm{mg}$ IV $+8 \mathrm{mg}$ OD x 3 days PO

Palonosetron $0.25 \mathrm{mg}$ IV

\section{Methods}

The primary outcome was proportion of patients with complete response (CR) following first cycle i.e. no vomiting, no nausea, no rescue medication use. A historical cohort of patients receiving AC chemotherapy for breast cancer was compared to a matched group receiving the new CINV regimen.

Results

37 patients per cohort were evaluable. A higher CR was observed in the historical cohort, $43.2 \%$ vs. $24.3 \%$ though not statistically significant ( $\mathrm{p}=0.140,95 \% \mathrm{CI}-2.7$ to $40.5 \%)$.

\section{Conclusions}

The intervention was confounded by a coinciding reduction in dexamethasone and insufficiently powered due to educational project time constraints. However, CR rates were within the anticipated range and indicated there was insufficient evidence to support a 5-fold increase in cost associated with switching. Dexamethasone doses were restored.

NK1 antagonists were approved for use in 2017 warranting further evaluation.

\section{eP003}

INTRACELLULAR EMETIC SIGNALING EVOKED BY THE SUBSTANCE P NEUROKININ TYPE 1 RECEPTOR (NK1R) AGONIST GR73632 IN THE LEAST SHREW (CRYPTOTIS PARVA)

N. Darmani ${ }^{1}$, W. Zhong ${ }^{1}$

${ }^{T}$ COMP/WU HS, Basic Medical Sciences, Pomona, USA

\section{Introduction}

We recently published on the intracellular emetic signals involved following selective activation of serotonergic $5-\mathrm{HT}_{3}$ receptors (Zhong et al., 2014 PLOS One e104718). Despite the introduction of substance $\mathrm{P} \mathrm{NK}_{1} \mathrm{R}$ antagonists into the clinic for the prevention of chemotherapy-evoked vomiting in early 2000 s, intracellular signaling cascade(s) involved in $\mathrm{NK}_{1} \mathrm{R}$-mediated vomiting still remains unknown.

\section{Objectives}

To investigate potential brainstem signaling pathways following treatment of least shrews with the selective substance $\mathrm{P} \mathrm{NK}_{1} \mathrm{R}$ agonist GR73632.

\section{Methods}

Behavioral and Western blot techniques were employed in the least shrew model of vomiting.

\section{Results}

Besides GR73632 evoking robust vomiting over a 30-min observation period in the least shrew, an increase in substance P- 
immunoreactivity occurred in the shrew brainstem emetic nucleus DMNX at $15 \mathrm{~min}$ post GR73632 (5 mg/kg. i.p.)-injection, as well as time-dependent up-regulation of phosphorylation of several protein kinases in the brainstem tissue including: $\mathrm{Ca}^{2+} /$ calmodulin kinase $\mathrm{II} \alpha(\mathrm{CaMKII} \alpha)$, extracellular signal-regulated protein kinase $1 / 2$ $(\mathrm{ERK} 1 / 2)$ and protein kinase $\mathrm{C} \alpha / \beta(\mathrm{PKC} \alpha / \beta)$ as revealed by Western Blots. We further confirmed the dose-dependent anti-emetic effect of the $\mathrm{NK}_{1} \mathrm{R}$ antagonist netupitant (i.p.) against GR73632induced vomiting, as well as suppression the evoked emesis by antagonsts/inhibitors of L-type $\mathrm{Ca}^{2+}$ channel (LTCC) (nifedipine, s.c.), inositol trisphosphate receptor (IP3R) (2-APB, i.p.), ERK1/2 (U0126, i.p.), PKC (GF109203X, i.p.) and phosphatidylinositol 3kinase (PI3K) (LY294002, i.p.). $\mathrm{NK}_{1} \mathrm{R}$, LTCC, and $\mathrm{IP}_{3} \mathrm{R}$ were also required for GR73632-evoked CaMKII $\alpha$, ERK1/2 and $\mathrm{PKC} \alpha / \beta$ phosphorylation. In addition, ERK1/2 phosphorylation was sensitive to inhibitors of CaMKII, PKC and PI3K.

Conclusions

These findings indicate that LTCC/IP3R-dependent CaMKII/ $\mathrm{PKC} \alpha / \mathrm{PI} 3 \mathrm{~K}-\mathrm{ERK}$ signaling pathway is involved in NK1Rmediated vomiting.

\section{eP004}

GUIDELINES FOR THE PREVENTION OF CHEMOTHERAPY INDUCED NAUSEA AND VOMITING (CINV) DO NOT ADDRESS ECONOMIC VARIATION, AFFORDABILITY IN DIFFERENT POPULATIONS, AND DRUG AVAILABILITY

\author{
J.N. Galeas ${ }^{1}$, R.J. Gralla ${ }^{l}$, E. Azimi-Nekoo ${ }^{1}$ \\ ${ }^{T}$ Albert Einstein College of Medicine - Jacobi Medical Center, Medical \\ Oncology, Bronx, USA
}

\section{Introduction}

Several organizations have published effective guidelines for CINV. Evidence - based guidelines list the best known regimens for prevention of CINV in different emetic risk groups. While useful, this approach may not address economic realities of availability and affordability of antiemetics in most countries and for most patients.

Objectives

Establish a framework addressing affordability of CINV regimens in different economic settings to indicate guidance for improving antiemetic control.

\section{Methods}

We examined current guideline recommendations in 5 CINV risk categories (from high to minimal risk), and as a consensus group listed regimens for each setting as a Good regimen, a Better regimen, or Best regimen. The World Bank / WHO established four economic groups into which each nation is placed. Additionally, the WHO approached healthcare affordability based on income levels per country (especially minimum wage). Acquisition price and availability of recommended antiemetics in each country was determined.

Results

The greatest economic issues were seen in the two top emetic risk groups, where NK1-based regimens were often not affordable except in the highest income nation group (but not in all these countries). Widespread availability of generic agents has improved affordability. Two-agent regimens were affordable in most economic groups, as were olanzapine-based three agent regimens. Differences still existed among countries of the same economic group.

\section{Conclusions}

The Best antiemetic regimen should always be used where it is available and affordable. Where economic realities exist limiting Best regimen use, Good and Better regimens provide realistic CINV prevention for many nations. This model may be useful in other oncology settings.
eP005

AN OPEN MULTICENTER STUDY TO INVESTIGATE EFFICACY AND TOLERABILITY OF OLANZAPINE IN PATIENTS WITH ADVANCED CANCER SUFFERING FROM NAUSEA NOT INDUCED BY CHEMOTHERAPY OR IRRADIATION

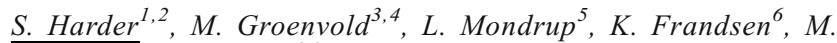
$\overline{\text { Neergaard }}$, J. Herrstedt ${ }^{8,9}$

${ }^{1}$ Odense University Hospital, Department of Oncology, Odense C, Denmark

${ }^{2}$ University of Southern Denmark, Institute of Clinical Research, Odense, Denmark

${ }^{3}$ Bispebjerg and Frederiksberg Hospitals, The Research UnitDepartment of Palliative Medicine, Copenhagen, Denmark

${ }^{4}$ University of Copenhagen, Department of Public Health, Copenhagen, Denmark

${ }^{5}$ Hospital of Southwest Jutland, The Palliative Care Team, Esbjerg, Denmark

${ }^{6}$ Bispebjerg and Frederiksberg Hospitals, Department of Palliative Medicine, Copenhagen, Denmark

${ }^{7}$ Aarhus University Hospital, The Palliative Care team, Aarhus, Denmark

${ }^{8}$ Zealand University Hospital, Department of Clinical Oncology, Roskilde, Denmark

${ }^{9}$ University of Copenhagen, Department of Clinical Medicine, Copenhagen, Denmark

\section{Introduction}

The anti-psychotic drug olanzapine is of interest because it is effective against chemotherapy-induced nausea and targets multiple receptors known to be involved in the emetic reflex arch. The drug has a half-life of 30 hours, which allows for a single daily administration.

\section{Objectives}

To investigate the anti-emetic effect and tolerability of olanzapine in patients with advanced cancer not receiving chemotherapy or irradiation.

\section{Methods}

Patients with advanced cancer (no curable treatment options) with at least 'moderate' nausea and/or one emetic episode within 24 hours were included if they had not received chemotherapy or irradiation within the previous 14 days and had no reversible causes of nausea/vomiting. The patients were administered $10 \mathrm{mg}$ olanzapine daily for five days (first dose subcutaneously and the following four orally). Nausea, vomiting and adverse effects were assessed for seven days.

\section{Results}

Twenty-one patients have been included. Eighteen patients experienced some degree of improvement (Table 1). Mean combined N/V score ( 0 100 ) at baseline was 68 . After 24 hours and seven days it was 20 and 21, respectively (Figure 1). We recorded no extrapyramidal symptoms, hypotension or seizures. Fatigue, dizziness and sedation were numerically (but not statistically significant) worse 24 hours after the first dose (Table 2). No adverse events seemed to be present at seven days.

\begin{tabular}{|c|c|c|c|c|c|}
\hline $\begin{array}{l}\text { Degree of } \\
\text { nausea }\end{array}$ & $\begin{array}{l}\text { At baseline: } \\
\text { None }\end{array}$ & Mild & Moderate & Severe & Total: \\
\hline $\begin{array}{l}\text { At 24-hours: } \\
\text { None }\end{array}$ & 0 & 0 & 5 & 4 & 9 \\
\hline Mild & 0 & 1 & 6 & 1 & 8 \\
\hline Moderate & 0 & 0 & 1 & 2 & 3 \\
\hline Severe & 0 & 0 & 0 & 1 & 1 \\
\hline Total: & 0 & 1 & 12 & 8 & 21 \\
\hline
\end{tabular}


Figure 1. Combined nausea/Nomiting (NN) scale (EORTC QLQ-C30) at baseline, at 24 . hours following injection of olanzapine and at seven days.

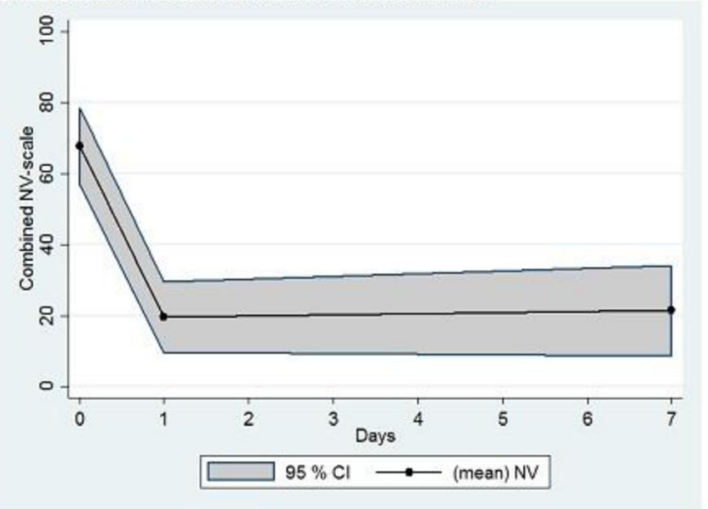

\begin{tabular}{|c|c|c|c|c|c|}
\hline Fatigue & $\begin{array}{l}\text { At baseline: } \\
0=\text { None }\end{array}$ & $1=$ Mild & 2=Moderate & $3=$ Severe & Total: \\
\hline $\begin{array}{l}\text { At 24-hours: } \\
0=\text { None }\end{array}$ & 0 & 1 & 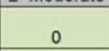 & 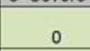 & 1 \\
\hline $1=$ Mild & 1 & 3 & 0 & 0 & 4 \\
\hline $2=$ Moderate & 1 & 2 & 7 & 2 & 12 \\
\hline $3=$ Severe & 0 & 0 & 1 & 3 & 4 \\
\hline Total: & 2 & 6 & 8 & 5 & 21 \\
\hline Dizziness & $\begin{array}{l}\text { At baseline: } \\
0=\text { None }\end{array}$ & $1=$ Mild & $2=$ Moderate & $3=$ Severe & Total: \\
\hline $\begin{array}{l}\text { At 24-hours: } \\
0=\text { None }\end{array}$ & 8 & 1 & 0 & 0 & 9 \\
\hline $1=$ Mild & 1 & 6 & 0 & 0 & 7 \\
\hline $2=$ Moderate & 2 & 3 & 0 & 0 & 5 \\
\hline $3=$ Severe & 0 & 0 & 0 & 0 & 0 \\
\hline Total: & 11 & 10 & 0 & 0 & 21 \\
\hline Sedation & $\begin{array}{l}\text { At baseline: } \\
0=\text { None }\end{array}$ & $1=$ Mild & $2=$ Moderate & $3=$ Severe & Total: \\
\hline $\begin{array}{l}\text { At 24-hours: } \\
0=\text { None }\end{array}$ & 10 & 1 & 0 & 0 & 11 \\
\hline $1=$ Mild & 3 & 3 & 2 & 0 & 8 \\
\hline $2=$ Moderate & 1 & 1 & 0 & 0 & 2 \\
\hline $3=$ Severe & 0 & 0 & 0 & 0 & 0 \\
\hline Total: & 14 & 5 & 2 & 0 & 21 \\
\hline
\end{tabular}

\section{Conclusions}

Olanzapine appears effective and tolerable as an anti-emetic in patients with advanced cancer. Recruitment continues and updated results will be presented. Future research should examine a lower dose $(5$ or $2.5 \mathrm{mg})$, preferably in a randomized controlled trial.

\section{eP006}

\section{WHAT OCCURS IN THE OTHER 20\% OF CANCER PATIENTS} WITH CHEMOTHERAPY-INDUCED NAUSEA AND VOMITING? A SINGLE INSTITUTION QUALITATIVE STUDY

\author{
D. Childs ${ }^{1}$, S. Looker ${ }^{2}$, J. Le-Rademacher ${ }^{3}$, J. Ridgeway ${ }^{4}$, C. Radecki \\ Breitkopf , A. Jatoi ${ }^{2}$ \\ ${ }^{1}$ Mayo Clinic, Department of Internal Medicine, Rochester, USA \\ ${ }^{2}$ Mayo Clinic, Department of Oncology, Rochester, USA \\ ${ }^{3}$ Mayo Clinic, Division of Biomedical Statistics and Department of \\ Health Sciences Research, Rochester, USA \\ ${ }^{4}$ Mayo Clinic, Center for the Science of Health Care Delivery, Rochester, USA \\ ${ }^{5}$ Mayo Clinic, Department of Health Sciences Research, Rochester, USA
}

\section{Introduction:}

Despite major advances in prophylaxis and management, $20 \%$ of patients who receive moderate to severe emetogenic chemotherapy continue to experience nausea and vomiting $(\mathrm{N} / \mathrm{V})$.

\section{Objectives}

In the current era of "highly effective antiemetic therapy," this qualitative study sought to fill a void in the published literature by reporting and understanding chemotherapy-induced $\mathrm{N} / \mathrm{V}$ in patients' own words.

\section{Methods}

Solid tumor patients with a history of poorly controlled N/V provided informed consent and then participated in a semi-structured interview, which was audio-recorded and transcribed. After data saturation, enrollment ceased; and inductive, qualitative analysis methods were employed. Results

The median age of these 20 patients was 56 years (range: 27, 83) with an equal gender split; half had gastrointestinal cancers. Two themes emerged. First, N/V remains a severe and multidimensional experience: "It's like shredding your muscles... It's doing it over and over again." This symptom complex has psychosocial implications: "Isolation is a big thing." Financial toxicity is also implicated: "I use [antiemetics] when I feel like it is absolutely necessary because it is so expensive I cannot afford it anyway." The second theme is under reporting; patients seemed to accept N/V and therefore underreport to providers: "God, if you're pumping poison in your system, you gotta expect some side effects."

\section{Conclusions}

These explicit data should motivate investigators to continue to design and conduct clinical trials to prevent/palliate this symptom complex and should remind healthcare providers of the importance of patient education about the availability of therapy for breakthrough symptomatology.

\section{eP007 \\ A STUDY OF THE EFFECTS OF OLANZAPINE AGAINST NAUSEA IN OUR DEPARTMENT}

\author{
H. Komatsu ${ }^{l}$, K. Ishida ${ }^{l}$, R. Kawagishi ${ }^{l}$, Y. Matsui $^{l}$, A. Sasaki $^{l}$ \\ ${ }^{T}$ Iwate Medical University, Surgery, Morioka-Iwate, Japan
}

\section{Introduction}

In cancer therapy, there are nausea and vomiting as side effects that patients feel strongly as distress. Currently the mechanism of emesis is also clarified, but it has not been controlled sufficiently yet. In NCCN guideline 2015, instead of aprepitant, an option to use olanzapine, a psychotropic drug with multiple receptors action, in combination with palonosetron and dexamethasone was presented.

\section{Objectives}

We examine backwardly the antiemetic effect of olanzapine in regimen using anthracycline drug in our department.

Methods

Among the 30 primary breast cancer patients who underwent preoperative or postoperative adjuvant therapy using anthracycline drugs from April to December, 2017, we decided to use olanzapine as an antiemetic 19 patients were used. For regimens using anthracycline drugs, FEC 100 therapy or TAC therapy was used.

\section{Results}

The median age was 46 years ( 30 to 61 ). There were 15 cases of FEC 100 therapy and 4 cases of TAC therapy. In all cases, improvement of Grade CTCAE v4.0 of nausea was improved, and 10 cases $(52.6 \%)$ were improved to asymptomatic. In all cases, olanzapine was started in the first or second course, and each regimen was progressed as planned.

\section{Conclusions}

In adjuvant chemotherapy, it is necessary to take into consideration the balance between maximum therapeutic intensity and minimal adverse reaction symptoms and try to prevent recurrence. Nausea and vomiting is a representative side effect, and control over it has an important role in the progress of drug therapy afterwards. Olanzapine, together with a marked antiemetic effect, could help smooth progress of cancer drug therapy. 


\section{eP008}

PILOT AND DEFINITIVE RANDOMISED DOUBLE-BLIND PLACEBO-CONTROLLED TRIALS EVALUATING AN ORAL CANNABINOID-RICH THC/CBD CANNABIS EXTRACT FOR SECONDARY PREVENTION OF CHEMOTHERAPYINDUCED NAUSEA AND VOMITING (CINV)

A. Mersiades ${ }^{1}$, A. Tognela ${ }^{2}$, P. Haber ${ }^{3}$, M. Stockler ${ }^{1,3,4}$, N. Lintzeris ${ }^{3,5}, J$. $\overline{\text { Simes }}^{\text {I, }}$, I. McGregor ${ }^{7}$, I. Olver ${ }^{8}$, D. Allsop ${ }^{3}$, C. Gedye ${ }^{9}$, A. Kirby ${ }^{I}$, R. Morton $^{1}$, K. Briscoe ${ }^{10}$, P. Fox ${ }^{11}$, S. Clarke ${ }^{12}$, M. Aghmesheh ${ }^{13}$, N. Wong ${ }^{1}$,

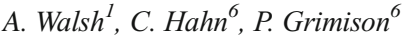

${ }^{I}$ NHMRC Clinical Trials Centre, University of Sydney, Sydney, Australia

${ }^{2}$ Macarthur Cancer Therapy Centre - South Western Sydney Local Health District, Dept of Medical Oncology, Sydney, Australia

${ }^{3}$ Sydney Medical School, University of Sydney, Sydney, Australia

${ }^{4}$ Concord Cancer Centre - Sydney Local Health District, Dept of Medical Oncology, Sydney, Australia

${ }^{5}$ Drug and Alcohol Services, South East Sydney Local Health District, Sydney, Australia

${ }^{6}$ Chris O'Brien Lifehouse, Dept of Medical Oncology, Sydney, Australia

${ }^{7}$ Lambert Initiative for Cannabinoid Therapeutics, University of Sydney, Sydney, Australia

${ }^{8}$ Sansom Institute for Health Research, University of South Australia, Adelaide, Australia

${ }^{9}$ Calvary Mater Hospital, Dept of Medical Oncology, Newcastle, Australia

${ }^{10}$ Mid-North Coast Cancer Institute - Coffs Harbour Hospital, Dept of Medical Oncology, Coffs Harbour, Australia

${ }^{11}$ Central West Cancer Care Centre - Orange Health Service, Dept of Medical Oncology, Orange, Australia

${ }^{12}$ Northern Cancer Institute, Dept of Medical Oncology, Sydney, Australia

${ }^{13}$ Illawarra Cancer Care Centre - Illawarra Shoalhaven Local Health District, Dept of Medical Oncology, Sydney, Australia

\section{Introduction}

$\mathrm{Up}$ to half of patients receiving moderate-to-highly emetogenic chemotherapy experience CINV despite optimal anti-emetic prophylaxis. Limited evidence suggests that tetrahydrocannabinolbased (THC) cannabinoid medications may reduce CINV, and addition of cannabidiol (CBD) may improve efficacy and tolerance.

Objectives

The aim of this multi-centre, randomised, placebo-controlled, phase II/III trial is to determine efficacy of addition of an oral cannabinoid-rich THC/CBD cannabis extract for control of CINV.

\section{Methods}

Target population is adult patients experiencing CINV during moderate and highly emetogenic chemotherapy regimens despite guideline-consistent prophylaxis, scheduled to receive at least 2 more consecutive cycles (A, B and, where applicable, C). Treatment consists of oral THC $2.5 \mathrm{mg} / \mathrm{CBD} 2.5 \mathrm{mg}$ (Tilray TNTC11M) capsules or placebo TDS days -1 to 5 , in addition to guideline-consistent anti-emetics, including rescue medications. In the pilot trial $(\mathrm{N}=80)$, subjects are randomised for cycle $\mathrm{A}$, with cross-over for cycle B, and preferred treatment for cycle $\mathrm{C}$. In the planned definitive trial $(\mathrm{N}=250)$, subjects are randomised to investigational product or placebo for cycles $\mathrm{A}, \mathrm{B}$ and $\mathrm{C}$ in a parallel design (Figure 1). The primary end-point is the proportion of patients gaining a complete response (no emesis and no use of rescue medications) $(0-120 \mathrm{~h})$, with additional efficacy endpoints of (i) complete response, (ii) no emesis, (iii) no significant nausea and (iv) no use of rescue medication during the a) acute, b) delayed, and c) overall phases of cycle A, B and C, (iv) adverse events, (v) quality of life, and (vi) cost-effectiveness.

Figure 1: Study schema

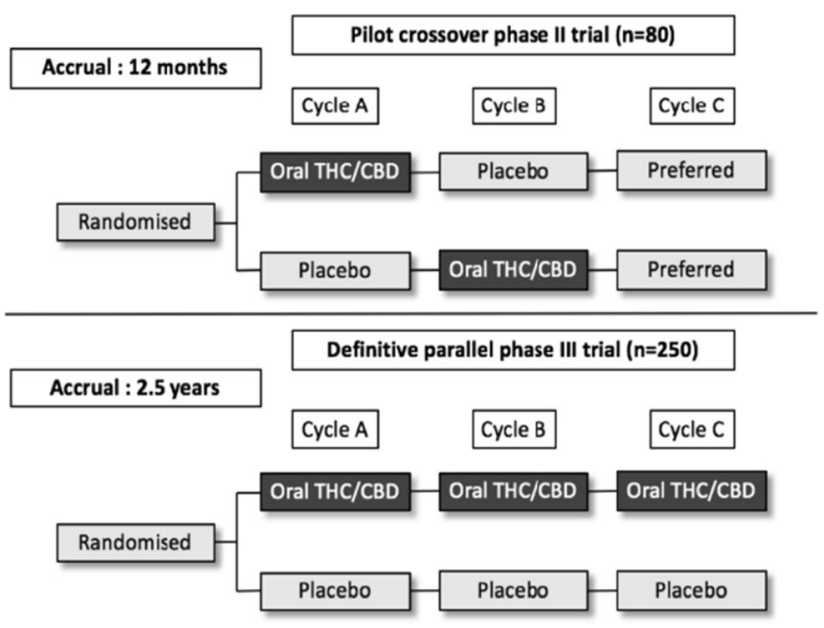

Results

Pre-results, as of 08/02/2018, 41 of 80 patients recruited.

ACTRN12616001036404

\section{Conclusions}

NA

\section{eP009}

WHAT ABOUT CAREGIVERS, PERCEPTION OF CHEMOTHERAPY INDUCED NAUSEA AND VOMITING IN CHILDREN? A PRELIMINARY REPORT

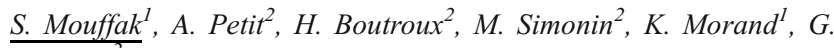
Leverger $^{2}$

${ }^{I}$ Armand Trousseau Children Hospital, Pharmacy, Paris, France

${ }^{2}$ Armand Trousseau Children Hospital, Pediatric Hematology and Oncology, Paris, France

Introduction:

The management of chemotherapy induced nausea and vomiting (CINV) has improved significantly over the last 15 years in adults, whereas in children, it is still complicated by several factors : Imprecise international recommendations until 2017, few antiemetic medicines indicated in pediatric population, or difficulties in nausea detection because of limited communication skills in young children.

\section{Objectives}

The purpose of this study was to assess caregivers' perception of chemotherapy induced nausea and vomiting in children receiving intravenous chemotherapy.

\section{Methods}

We conducted a prospective study recording daily the level of nausea experienced by children, up to 24 hours after the end of chemotherapy administration. At the same time, CINV perceptions were evaluated with physicians, nurses and nurse assistants. Patients' feeling and caregivers' perceptions were collected using a pictorial nausea rating scale adapted to children.

Results

40 patients' experiences and 114 caregiver's assessments were collected during two months. When children indicated they have no 
nausea $(25 / 40)$, caregivers assessment coincided. When patients described nausea $(14 / 40)$ or vomiting $(1 / 40)$, symptoms were underestimated by caregivers in $72 \%$ of cases, overestimated in $19 \%$ of cases and coincided with patient experience in $9 \%$ of cases. There were no significant differences between physicians, nurses and assistants answers $(\mathrm{p}=0.8)$.

Conclusions

This study shows that CINV in children is underestimated by caregivers, and thus undermanaged. The use of a pictorial nausea rating scale by caregivers should improve the detection of these symptoms. This type of scale should be used several times in a day in children receiving intravenous chemotherapy, as pain scale after surgery.

\section{eP010}

LOW DOSE OLANZAPINE IN CHEMOTHERAPY INDUCED NAUSEA AND VOMITING: THE IDEAL 8 PM ANTIEMETIC?

S. Mukhopadhyay ${ }^{1}$, P. Dutta ${ }^{2}$, B. Bhattacharya ${ }^{2}$, S. Banerjee ${ }^{2}, S$. Biswas $^{3}$, B. Samanta ${ }^{4}$

${ }^{1}$ Assistant professor, Pharmacology, Burdwan, India

${ }^{2}$ Burdwan Medical College, Radiotherapy, Burdwan, India

${ }^{3}$ Burdwan Medical College, Pharmacology, Burdwan, India

${ }^{4}$ Burdwan Medical College, Biochemistry, Burdwan, India

\section{Introduction}

Though olanzapine produces benefit in chemotherapy induced nausea and vomiting (CINV), sedation is often disproportionate and embarrassing. Therefore a lower dose needs exploration for CINV control, sedation and ideal time of dosing.

Objectives

To compare the efficacy, safety and sedation produced by two doses of olanzapine in control of CINV and also to find the preferred time of dosing.

Methods

A prospective, randomized, double blind and controlled study was conducted on the chemotherapy naïve, consenting cancer patients receiving moderately emetogenic chemotherapy and received Ondansetron and dexamethasone. Olanzapine $5 \mathrm{mg}$ or $10 \mathrm{mg}$ were added in the test and control groups respectively during day 1-3 either at daytime or evening. CINV was measured with MASCC antiemesis tool and sedation with a $100 \mathrm{~mm}$ VAS from day 1-5.

\section{Results}

Out of 69 enrolled patients, data of 52 patients were available for analysis. There was no statistically significant difference between the groups in control of CINV (Fig 1).

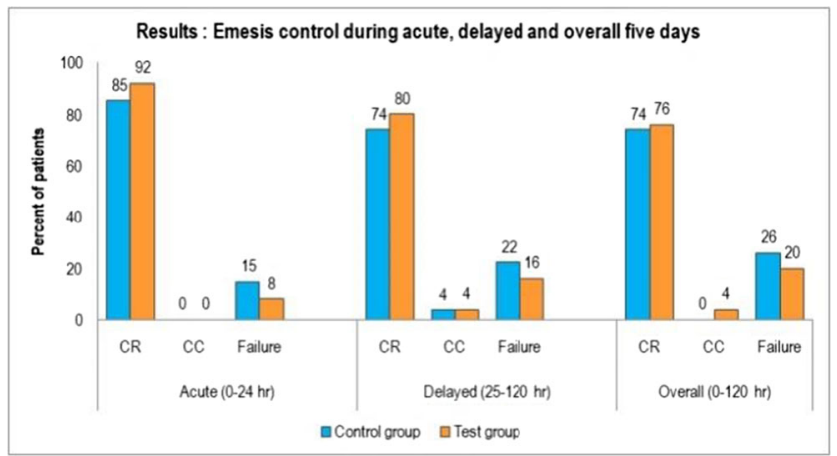

Delayed nausea was less in the control group $(1.22+0.43)$ compared to test $(2.4+1.25)$ but not statistically significant. Most of the patients had no nausea in both groups. (Fig 2)

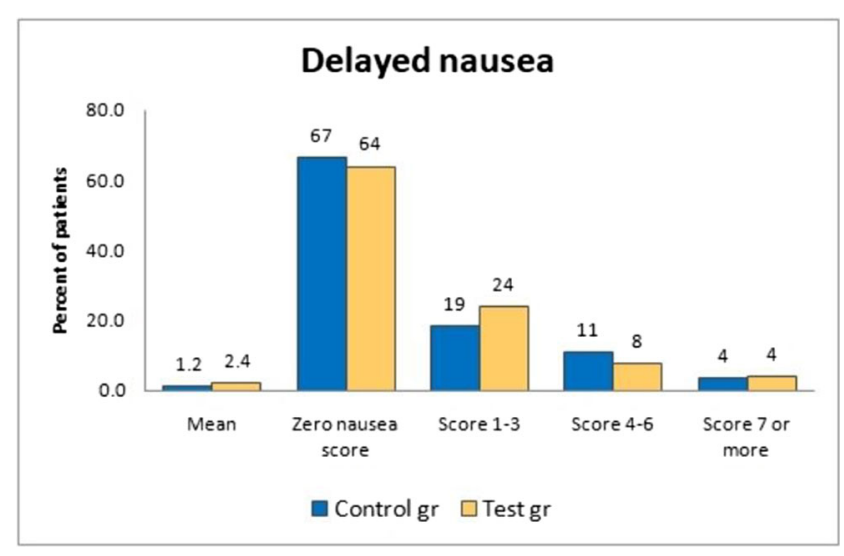

Sedation was significantly high in the control group during day 1-5. Less sedation was noted with daytime dose with $5 \mathrm{mg}$ olanzapine but not with $10 \mathrm{mg}$ dose. (Fig 3).

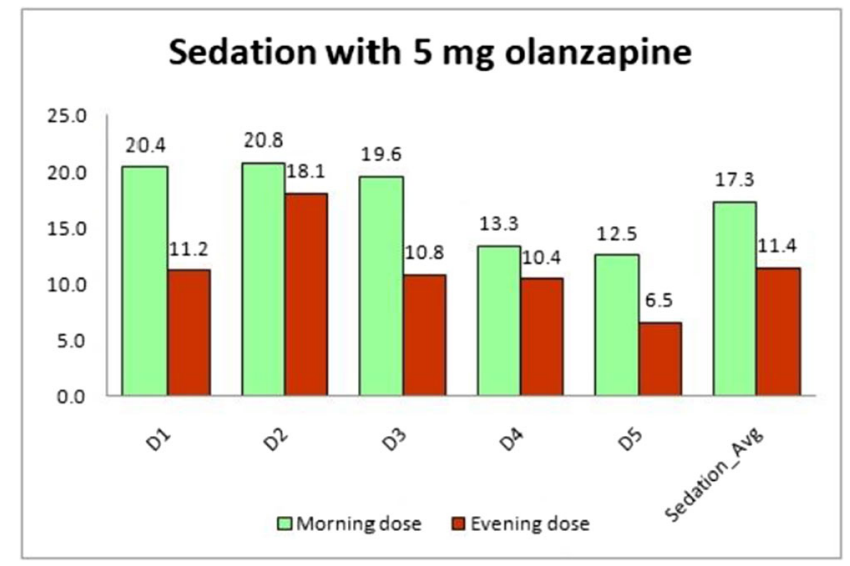

\section{Conclusions}

Olanzapine $5 \mathrm{mg}$ has similar efficacy with less sedation compared to $10 \mathrm{mg}$ dose in control of CINV. Evening dosing of $5 \mathrm{mg}$ olanzapine results minimal daytime sedation and has the potential of minimal interference of daytime activities.

eP011

A RANDOMIZED, DOUBLE-BLIND, PLACEBOCONTROLLED PHASE III STUDY EVALUATING OLANZAPINE 5MG COMBINED WITH STANDARD ANTIEMETIC THERAPY FOR THE PREVENTION OF CINV IN PATIENTS RECEIVING CISPLATIN-BASED CHEMOTHERAPY

K. Nakashima ${ }^{1}$, T. Yanai ${ }^{2}$, A. Masakazu $^{3}$, Y. Hirashima $^{3}$, H. Murakami $^{l}$, S. Hamauchi ${ }^{4}$, H. Ishikawa ${ }^{5}$, M. Sakurai ${ }^{5}$, T. Yamaguchi $^{6}$, S. Zenda ${ }^{7}$, Y.

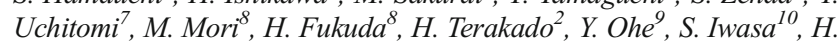
Hashimoto $^{2}$

${ }^{1}$ Shizuoka Cancer Center, Department of Thoracic Oncology, Shizuoka, Japan

${ }^{2}$ National Cancer Center Hospital, Department of Pharmacy, Tokyo, Japan

${ }^{3}$ Shizuoka Cancer Center, Department of Gynecologic Oncology, Shizuoka, Japan

${ }^{4}$ Shizuoka Cancer Center, Department of Gastrointestinal Medical Oncology, Shizuoka, Japan 
${ }^{5}$ Shizuoka Cancer Center, Department of Pharmacy, Shizuoka, Japan

${ }^{6}$ Tohoku University Graduate School of Medicine, Department of

Biostatistics, Sendai, Japan

${ }^{7}$ Japan Supportive- Palliative and Psychosocial Oncology Group, Japan Supportive- Palliative and Psychosocial Oncology Group, Tokyo, Japan

${ }^{8}$ National Cancer Center, Japan Clinical Oncology Group Data Center, Tokkyo, Japan

${ }^{9}$ National Cancer Center Hospital, Department of Thoracic Oncology, Tokyo, Japan

${ }^{10}$ National Cancer Center Hospital, Department of Gastrointestinal Medical Oncology, Tokyo, Japan

\section{Introduction:}

Olanzapine is effective for chemotherapy-induced nausea and vomiting (CINV). Although $10 \mathrm{mg}$ of olanzapine is widely used for CINV, patient sedation may be a concern. In Japan, three phase II studies revealed the efficacy and safety of $5 \mathrm{mg}$ of olanzapine combined with palonosetron (PALO), aprepitant (APR), and dexamethasone (DEX) for CINV induced by cisplatin-based chemotherapy. In these studies, complete response (CR: no vomiting, no rescue) rate in the delayed phase (24-120 h) was $83-95 \%$.

\section{Objectives}

The aim of this phase III study is to evaluate the efficacy of $5 \mathrm{mg}$ of olanzapine as compared with placebo, in combination with APR, PALO, and DEX, for the control of CINV induced by cisplatin-based chemotherapy.

Methods

Cisplatin-naïve patients receiving cisplatin-based $\left(\geq 50 \mathrm{mg} / \mathrm{m}^{2}\right)$ chemotherapy are enrolled. The two groups receive either $5 \mathrm{mg}$ of olanzapine or placebo daily on days 1 through 4 , in combination with APR, PALO, and DEX. We expected the CR rate of the placebo and olanzapine arms to be $65 \%$ and $75 \%$, respectively. According to this estimation, a total of 690 patients are required to achieve a significance level of $2.5 \%$ (one-sided) and $80 \%$ statistical power. The primary endpoint is $\mathrm{CR}$ rate in the delayed phase.

Results

This trial was registered in the UMIN Clinical Trials Registry as UMIN000024676.

\section{Conclusions}

This is the first phase III study to compare $5 \mathrm{mg}$ of olanzapine with placebo in combination with APR, PALO, and DEX for cisplatin-based chemotherapy. This trial will be completed by the second-quarter of this year.

\section{eP012}

EFFICACY OF COMBINATION OF APREPITANT, RAMOSETRON, AND DEXAMETHASONE FOR CHEMOTHERAPY-INDUCED NAUSEA AND VOMITING PREVENTION IN OVARIAN CANCER PATIENTS

\section{E.S. Paik ${ }^{l}$, M.K. Kim ${ }^{2}$, C.H. Choi ${ }^{l}$, D.S. Bae ${ }^{l}$}

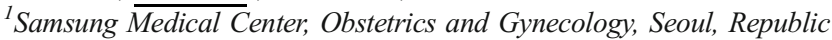
of Korea

${ }^{2}$ Samsung Changwon Hospital- Sungkyunkwan University School of Medicine, Department of Obstetrics and Gynecology, Changwon, Republic of Korea

\section{Introduction:}

Women with ovarian carcinoma that are treated with paclitaxel/ carboplatin are particularly susceptible to chemotherapy-induced nausea and vomiting (CINV).

\section{Objectives}

The current study evaluated the new combination (aprepitant/ramosetron/ dexamethasone, $20 \mathrm{mg}$ ) in ovarian cancer patients receiving multiple cycles of paclitaxel/carboplatin.

\section{Methods}

This is a prospective non-randomized single site study. Patients received the following regimen for the prevention of CINV-day $1,125 \mathrm{mg}$ aprepitant, $0.6 \mathrm{mg}$ ramosetron, and $20 \mathrm{mg}$ dexamethasone before chemotherapy; and days $2-3,80 \mathrm{mg}$ aprepitant each day. The primary end point was the proportion of patients with complete response (CR) during the $120 \mathrm{~h}$ following the first chemotherapy cycle. Toxicity assessments were conducted using the NCI-CTC investigator guide (version 3.0).

\section{Results}

Of the 89 patients enrolled, 85 patients were evaluable for efficacy and toxicity, and $68(80 \%)$ completed all 6 cycles. In cycle 1, the percentage of patients who achieved $\mathrm{CR}$ in the acute, delayed, and overall phases was $98.8 \%, 89.4 \%$, and $89.4 \%$, respectively. Of the 460 cycles, adverse events, drugrelated adverse events, and serious adverse events occurred in $179(38.9 \%), 35(7.6 \%)$, and 10 cycles $(2.2 \%)$, respectively. The most common adverse event was constipation $(12.4 \%)$ and headache $(11.1 \%)$. None of the patients discontinued the study because of adverse events.

\section{Conclusions}

The combination of aprepitant, ramosetron, and high-dose dexamethasone demonstrated efficacy for CINV prevention in ovarian cancer patients receiving paclitaxel and carboplatin.

eP013

THE GHRELIN AGONISTS, ANAMORELIN AND IPAMORELIN, ANTAGONIZE CISPLATIN-INDUCED ANOREXIA AND EMESIS IN THE FERRET

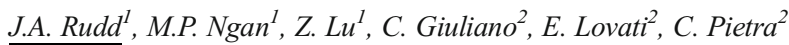
${ }^{T}$ Chinese University of Hong Kong, Emesis Research Group, Shatin, Hong Kong S.A.R.

${ }^{2}$ Helsinn Healthcare SA, Research and Development, Lugano, Switzerland

\section{Introduction}

Ghrelin has well-known appetite stimulating properties, gastric prokinetic activity, and short acting anti-emetic affects against cisplatin-induced emesis. However, clear anti-emetic effects are only seen following i.c.v. administration.

Objectives

To explore the potential of two potent synthetic ghrelin agonists, anamorelin and ipamorelin, to reduce cisplatin-induced acute and delayed emesis and associated anorexia.

\section{Methods}

Ferrets were administered anamorelin (1-3 mg/kg, i.p.), ipamorelin (1-3 $\mathrm{mg} / \mathrm{kg}$, i.p.), or saline $30 \mathrm{~s}$ before cisplatin $(5 \mathrm{mg} / \mathrm{kg}$, i.p. $)$, which indices acute and delayed emesis; treatment with saline or ghrelin agonists were repeated every $24 \mathrm{~h}$. In a separate experiment, anamorelin $(10 \mu \mathrm{g})$, or saline, were administered i.c.v. $30 \mathrm{~s}$ before cisplatin and repeated every $24 \mathrm{~h}$.

\section{Results}

Both compounds administered i.p. had no effect on cisplatin-induced emesis, but ipamorelin at $3 \mathrm{mg} / \mathrm{kg}$, i.p. antagonized the associated anorexia by 28 and $22 \%$ at 48 and $72 \mathrm{~h}$, respectively $(\mathrm{P}<0.05) ; 1 \mathrm{mg} / \mathrm{kg}$, i.p. also reduced anorexia by $20 \%$ at $72 \mathrm{~h}(\mathrm{P}<0.01)$. Anamorelin at $1 \mathrm{mg} / \mathrm{kg}$, i.p., reduced cisplatin-induced anorexia by $18 \%$ at $72 \mathrm{~h}(\mathrm{P}<0.05)$. Anamorelin, $10 \mu \mathrm{g}$, i.c.v., antagonized cisplatin-induced acute emesis by $60 \%(\mathrm{P}<0.05)$ and improved food and water intake by $22(\mathrm{P}<0.001)$ and $40 \%(\mathrm{P}<0.001)$, respectively; weight loss was also reduced by $27 \%$ $(\mathrm{P}<0.05)$ at $48 \mathrm{~h}$.

\section{Conclusions}

Brain penetrating ghrelin mimetics may have the potential to reduce weight loss and emesis induced by chemotherapeutic drugs. The effect on appetite may relate to an ability to reduce nausea, but this requires further investigation. 


\section{eP014}

EFFICACY AND QUALITY OF LIFE OF NEPA AS CINVPROPHYLAXIS IN HIGHLY OR MODERATELY EMETOGENIC CHEMOTHERAPY - INTERIM RESULTS OF A GERMAN PROSPECTIVE NON-INTERVENTIONAL STUDY

J. Schilling ${ }^{1}$,E. Wierick ${ }^{2}$, P. Wuelfing ${ }^{3}$, G. Oskay-Oezcelik ${ }^{4}$, M. Karthaus ${ }^{5}$ ${ }^{T}$ BNGO e.V., German Professional Association of Gynaeco-Oncology in Practices, Neuenhagen, Germany

${ }^{2}$ Practice for gynaecology, Practice, Weißkollm, Germany

${ }^{3}$ Mammazentrum Hamburg am Krankenhaus Jerusalem, Practice, Hamburg, Germany

${ }^{4}$ Praxisklinik/ Brustzentrum, Practice, Berlin, Germany

${ }^{5}$ Städtisches Klinikum Neuperlach, Städtisches Klinikum Neuperlach, Munich, Germany

\section{Introduction}

NEPA, a fixed dose combination of netupitant and palonosetron, is approved for the prevention of acute and delayed chemotherapy-induced nausea and vomiting in cancer patients receiving cisplatin-based highly emetogenic (HEC) or moderately emetogenic chemotherapy (MEC).

Objectives

Primary objective is the evaluation of quality of life (QoL) in adults receiving NEPA for CINV prevention in MEC or HEC. Secondary endpoints are efficacy and safety of NEPA.

\section{Methods}

This German prospective, non-interventional study evaluates NEPA in 2,500 cancer patients receiving single day or two day MEC or HEC. QoL is recorded by FLIE questionnaires. Complete response (CR, no vomiting, no rescue medication), additional medication, and safety are recorded in diaries over three consecutive chemotherapy cycles.

\section{Results}

In this interims analysis 1,997 patients have been included. In the MEC subgroup $53 \%$ and $21 \%$ received carboplatin- and oxaliplatin-based chemotherapy, respectively. In the HEC subgroup $87 \%$ received an anthracycline/cyclophosphamide-based regime.

MEC-patients $(n=401): 84 \%$ reported CR in cycle $1,85 \%$ in cycle 2 and $86 \%$ in cycle 3 . Efficacy, assessed by physicians on a 4-point scale, was rated very good/good for $90 \%, 93 \%$, and $94 \%$ in cycle 1,2 and 3, respectively $(n=630)$. HEC-patients $(\mathrm{n}=896): 81 \%$ reported CR in cycle $1,82 \%$ in cycle 2 and $83 \%$ in cycle 3 . Efficacy, assessed by physicians, was rated very good/good for $88 \%, 89 \%$, and $90 \%$ in cycle 1,2 and 3, respectively $(\mathrm{n}=1185)$.

NEPA was well tolerated with low-grade constipation being the most frequent treatment-related adverse event.

Conclusions

NEPA provided very effective CINV-prevention in this ongoing real life study.

\section{eP015}

THE PREVALENCE OF NAUSEA IN PATIENTS RECEIVING INTRAVENOUS CHEMOTHERAPY - A PROSPECTIVE, OBSERVATIONAL, REAL WORLD STUDY

\author{
T. Smit ${ }^{1}$, B. Rapoport ${ }^{2,3}$ \\ ${ }^{T}$ The Medical Oncology Centre of Rosebank, Pharmacy, Saxonwold, \\ South Africa \\ ${ }^{2}$ The Medical Oncology Centre of Rosebank, Oncology, Saxonwold, \\ South Africa \\ ${ }^{3}$ University of Pretoria, Department of Immunology- Faculty of Health \\ Sciences-, Pretoria, South Africa
}

\section{Introduction}

The development of effective anti-emetic treatments has contributed to the resolution of emesis in chemotherapy patients (pts). There is a growing concern that the emesis focus is primarily on vomiting. Nausea seems to be underestimated and its incidence and impact remains a major unmet medical need.

\section{Objectives}

The study focused primarily on nausea in patients undergoing highly-, moderately- or low emetogenic chemotherapy (HEC, MEC, LEC). The primary endpoint was no nausea during the initial 120-hours overall phase of cycle 1. Patients achieving a complete response (no vomiting) were also evaluated. Secondary endpoints included no nausea during day7 and day-10 of cycle 1 .

\section{Methods}

This prospective, observational single centre study enrolled 95 patients undergoing LEC (25 pts), MEC (24 pts) or HEC (46 pts) for malignancy. Prophylactic antiemetics were administered according to MASCC/ESMO 2016 guidelines. Patient diaries were used to collect data from day-1 to day5 , day- 7 and day-10 beginning with cycle-1 for up to 3 cycles.

\section{Results}

The incidence of nausea of the entire population was $59 \%$ compared to only $24 \%$ pts experiencing vomiting $\left(\mathrm{Chi}^{2}=23.5956 ; p>.000001\right)$. Significant variables predicted for nausea included gender, age and history of motion sickness. The level of emetogenicity did not correlate with the incidence of nausea $(\mathrm{LEC}=25 \%, \mathrm{MEC}=60 \%, \mathrm{HEC}=67 \%),\left(\mathrm{Chi}^{2}=5.1893 ; p>.07\right)$. Conclusions

Despite the usage of adequate antiemetic prophylaxis, chemotherapy induced nausea is under reported and remains a major unmet medical need. Further research should evaluate the association of nausea with patient risk factors.

\section{eP016}

EVALUATING THE ANTIEMETIC EFFECT OF APERPITANT ON PATIENTS UNDERGOING AUTOLOGOUS HEMATOPOIETIC STEM CELL TRANSPLANTATION (AHSCT) IN A TERTIARY TEACHING HOSPITAL

\author{
M. Tavakoli-Ardakani ${ }^{1}$, M. Mehdizadeh ${ }^{2}$, A. Ghadirifard $^{3}$ \\ ${ }^{T}$ School of pharmacy, clinical pharmacy, Tehran, Iran \\ ${ }^{2}$ Taleghani Hospital, Bone Marrow Transplant Center-, Tehran, Iran \\ ${ }^{3}$ School of pharmacy, Students' Research Committee- Shahid Beheshti \\ University of Medical Sciences, Tehran, Iran
}

\section{Introduction}

Hematopoietic Stem Cell Transplantation (HSCT) is one of the treatment modality for hematologic malignancies and is associated with significant nausea and vomiting.

\section{Objectives}

Aperpitant is a suitable medicine in acute and delayed emesis. We designed this study to evaluate the safety and efficacy of aprepitant in Autologous HSCT.

\section{Methods}

Eighty AHSCT patients were enrolled and randomly divided in equal two groups. There were not significant difference in age, gender, and type of malignancy between two groups. In Aprepitant group, patient received standard antiemetic regimen (administrating $8 \mathrm{mg}$ of Dexamethasone, IV injection and Granisetron, $1.5 \mathrm{mg}$, twice per day, IV injection) plus aprepitant (125 mg 60-90 minutes before administration of the first dose of moderately to highly emetogenic chemotherapy. On the next day or thereafter, $80 \mathrm{mg}$ in the morning until 3 days after the last drug of chemotherapy regimen). Nausea, vomiting, headache, anorexia, hiccup, diarrhea, fever, blood glucose, serum electrolytes, hepatic transaminase were evaluated during the study period.

\section{Results}

Mean scores obtained for nausea and vomiting in the acute phase for Aprepitant and control groups were not significant different. Mean scores obtained in the delayed phase for patients of Aprepitant and control groups was 10.300 and 17.750 respectively $(P$ value $=0.0001)$ and for vomiting in delayed phase it was 0.700 and 2.750 respectively $(\mathrm{P}$ value $=0.004)$. 


\section{Conclusions}

The results of this study suggest that the addition of aprepitant to granisetron and dexamethasone can improve the antiemetic effect especially in delayed emesis without increasing Adverse Drug Events (ADEs) in AHSCT patients.

eP017

ANTIEMETIC GUIDELINE COMPLIANCE OF NEUROKININ1 (NK1) RECEPTOR ANTAGONISTS (RA) IN THE HIGHLY EMETOGENIC CHEMOTHERAPY (HEC) SETTING

E.J. Roeland ${ }^{1}$, K. Ruddy ${ }^{2}$, T.W. LeBlanc ${ }^{3}$, G. Binder ${ }^{4}$, D.T. Amari ${ }^{5}$, A. Surinach $^{5}, Z . W^{5}, R$. Navari $^{6}$

${ }^{I}$ Massachusetts General Hospital, Oncology \& Palliative Care, BostonMA, USA

${ }^{2}$ Mayo Clinic, Department of Oncology, Rochester-MN, USA

${ }^{3}$ Duke Cancer Institute, Department of Medicine-Duke University School of Medicine, Durham- NC, USA

${ }^{4}$ Helsinn Therapeutics, HEOR \& Value-Based Medicine, Iselin- NJ, USA

${ }^{5}$ Genesis Research, Outcomes Research, Hoboken- NJ, USA

${ }^{6}$ University of Alabama Birmingham, School of Medicine, BirminghamAL, USA

\section{Introduction}

Chemotherapy-induced nausea and vomiting(CINV) risk substantially increases in subsequent cycles when not prevented upfront. Evidencebased antiemetic guidelines recommend a triplet prophylactic regimen $\left(\mathrm{NK}_{1} \mathrm{RA}+5 \mathrm{HT}_{3} \mathrm{RA}+\right.$ dexamethasone [DEX]) before receipt of HEC. Prior studies with limited data have demonstrated unsatisfactory adherence with antiemetic guidelines.

Objectives

This initiative evaluates the utilization of $\mathrm{NK}_{1}$-containing regimens for patients receiving HEC in the US.

Methods

Using the MarketScan Commercial and Medicare claims database from 2011-2016, antiemetic utilization was assessed in adult chemotherapy-naïve patients with $>7$ days between HEC cycles. Guideline adherence was defined as administration of $\mathrm{NK}_{1} \mathrm{RA}+5-\mathrm{HT}_{3} \mathrm{RA} \pm \mathrm{DEX}$ at chemotherapy initiation.

Results

A total of 111,497 patients were included; $35 \%$ received carboplatin, $24 \%$ anthracycline+doxorubicin (AC), and $18 \%$ cisplatin. Most (71\%) were female; $24 \%$ were $<50$ years, and $24 \%>65$ years. Guideline adherence was $45 \%$ (49,916 patients) and improved $\sim 1.7 \%$ points annually from 2011-2016; rates were $26 \%$ for carboplatin, $71 \%$ for AC, and $72 \%$ for cisplatin. Compliance declined with older age (Figure). Among patients receiving non-compliant prophylaxis of only a $5 \mathrm{HT}_{3} \mathrm{RA} \pm \mathrm{DEX}$ at initiation, over $20 \%$ of $\mathrm{AC}$ and cisplatin patients received an $\mathrm{NK}_{1} \mathrm{RA}$ in subsequent cycle(s); rates were consistently higher in females (Figure).

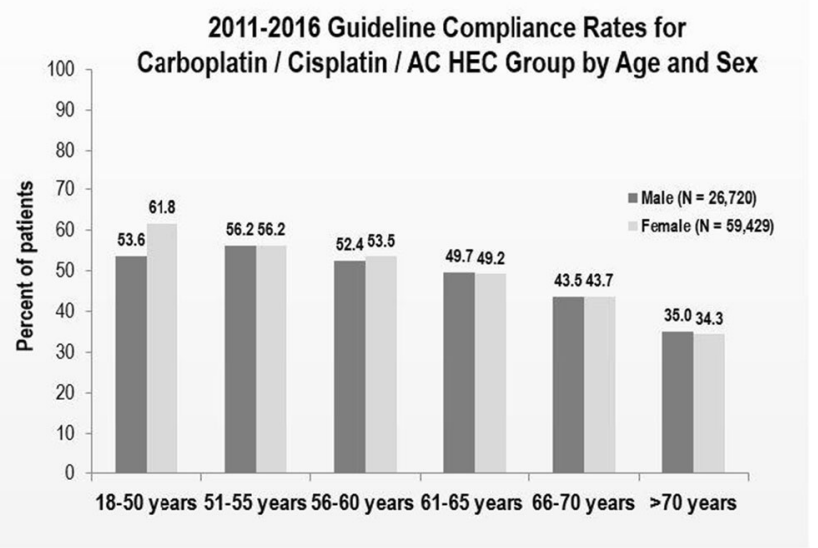

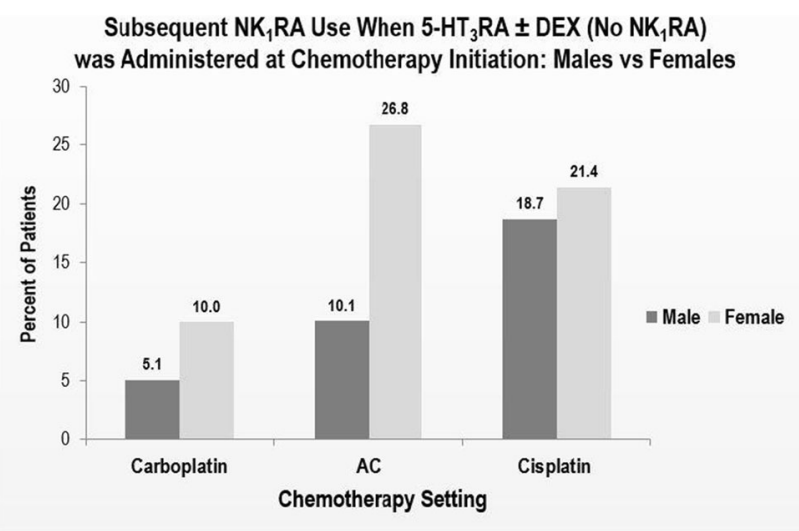

\section{Conclusions}

Substantial improvement in antiemetic guideline compliance is needed for patients receiving carboplatin, $\mathrm{AC}$, or cisplatin. Absence of upfront NK1RA use at chemotherapy initiation commonly results in subsequent use, especially among females, most likely due to breakthrough CINV. These findings emphasize the importance of clinicians/organizations taking deliberate action to improve compliance with evidence-based antiemetic guideline recommendations for upfront triplet prophylaxis in HEC.

\section{eP018}

EFFICACY OF INTRAVENOUS AND ORAL NEPA, A FIXED NK1/5-HT3 RECEPTOR ANTAGONIST COMBINATION, IN THE PREVENTION OF HIGHLY EMETOGENIC CHEMOTHERAPY (HEC)-INDUCED NAUSEA AND VOMITING (CINV)

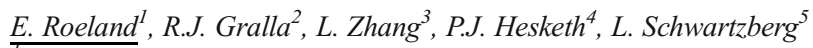
${ }^{T}$ Massachusetts General Hospital, Oncology \& Palliative Care, BostonMA, USA

${ }^{2}$ Albert Einstein College of Medicine, Jacobi Medical Center, Bronx- NY, USA

${ }^{3}$ Sun Yat-sen University Cancer Center, State Key Laboratory of Oncology in South China-Collaborative Innovation Center for Cancer Medicine, Guangzhou, China

${ }^{4}$ Lahey Hospital \& Medical Center, Lahey Health Cancer Institute, Burlington- MA, USA

${ }^{5}$ West Cancer Center, Hematology \& Oncology, Germantown- TN, USA

\section{Introduction}

The approval of oral NEPA, a novel fixed antiemetic combination comprised of the $\mathrm{NK}_{1} \mathrm{RA}$ netupitant $\left(300 \mathrm{mg}\right.$ ) and $5-\mathrm{HT}_{3} \mathrm{RA}$ palonosetron (PALO, $0.50 \mathrm{mg}$ ), was based on demonstrating superior prevention of CINV over PALO. An intravenous formulation of NEPA (fosnetupitant $235 \mathrm{mg} /$ PALO $0.25 \mathrm{mg}$ ) was developed to offer additional treatment options. In addition to showing pharmacokinetic bioequivalence, a clinical study showed comparable safety profiles for IV and oral NEPA.

\section{Objectives}

This post-hoc analysis presents the efficacy of IV and oral NEPA from pivotal studies in the non-AC HEC setting.

\section{Methods}

Data is compiled from 4 pivotal registration studies in adult chemotherapy-naïve patients with solid tumors undergoing predominantly cisplatinbased HEC. All studies had similar inclusion and exclusion criteria. In Study 1 , IV NEPA was administered as a single 30-minute infusion and in Studies 1-4 a single capsule of oral NEPA was given prior to HEC. All patients received dexamethasone on Days 1-4. Complete response (CR: no emesis/no rescue medication) rates are summarized for the overall phase $(0-120 \mathrm{~h})$ of the initial cycle of HEC for each study and pooled 
for oral NEPA. No formal statistical comparisons were done for this posthoc analysis.

\section{Results}

The overall CR rate was $76.8 \%$ for IV NEPA and $79.8 \%$ for oral NEPA for the pooled studies. These results compare favorably to aprepitant and rolapitant pivotal $\mathrm{HEC}$ studies where overall $\mathrm{CR}$ rates ranged from $62.7 \%-72.7 \%$

\begin{tabular}{|l|c|c|c|c|}
\hline Overall (0-120h) CR & \multicolumn{2}{|c|}{ IV NEPA } & \multicolumn{2}{c|}{ Oral NEPA } \\
\hline & N & $\%$ & N & $\%$ \\
\hline $\begin{array}{l}\text { Study 1 } \\
\text { (Schwartzberg et al) }\end{array}$ & 203 & 76.8 & 201 & 84.1 \\
\hline $\begin{array}{l}\text { Study 2 } \\
\text { (Hesketh et al) }\end{array}$ & & & 135 & 89.6 \\
\hline $\begin{array}{l}\text { Study 3 } \\
\text { (Gralla et al) }\end{array}$ & & & 75 & 84.0 \\
\hline $\begin{array}{l}\text { Study 4 } \\
\text { (Zhang et al) }\end{array}$ & & & 412 & 73.8 \\
\hline Pooled Studies 1-4 & & & 823 & 79.8 \\
\hline
\end{tabular}

\section{Conclusions}

Both IV and oral formulations of NEPA represent safe, effective and convenient single-dose prophylactic antiemetics targeting two distinct CINV pathways, offering comparable options for clinicians/patients in different settings.

\section{eP019}

A PHASE 3B SAFETY STUDY OF INTRAVENOUS (IV) NEPA FOR PREVENTION OF CHEMOTHERAPY-INDUCED NAUSEA AND VOMITING (CINV) IN BREAST CANCER (BC) PATIENTS RECEIVING ANTHRACYCLINE/ CYCLOPHOSPHAMIDE (AC)

L. Schwartzberg ${ }^{1}$, S. Sebastiani ${ }^{2}$

${ }^{T}$ West Cancer Center, Hematology \& Oncology, Germantown, USA

${ }^{2}$ Helsinn Healthcare SA, Medical Affairs, Lugano, Switzerland

\section{Introduction}

Approval of oral NEPA, the only fixed combination antiemetic comprised of the $\mathrm{NK}_{1}$ receptor antagonist (RA) netupitant (300mg) and $5-\mathrm{HT}_{3} \mathrm{RA}$ palonosetron (PALO, $0.50 \mathrm{mg}$ ) was based on demonstrating superior CINV prevention to PALO. The safety of oral NEPA was also well-established in the Phase $2 / 3$ clinical program. An intravenous formulation of NEPA (fosnetupitant $235 \mathrm{mg} / \mathrm{PALO}$ $0.25 \mathrm{mg}$ ) was developed to offer additional convenience for clinicians/patients. A bioequivalence approach was pursued for netupitant with clinical evaluation focused on showing similar safety for the IV and oral formulations. A prior pre-approval study showed comparable safety of IV and oral NEPA in patients receiving non-AC highly emetogenic chemotherapy.

Objectives

The present study evaluates the safety and tolerability of IV NEPA in BC patients receiving multiple cycles of AC.

Methods

This is a phase 3b, randomized, double-blind study (NCT03403712) in female chemotherapy-naïve $\mathrm{BC}$ patients receiving initial and repeated AC cycles (up to 4) at study centers in the US, Russia, Ukraine, and Georgia. A total of 400 patients stratified by region (US/non-US) and age $(<55 / \geq 55$ years) will be randomized $1: 1$ on day 1 of cycle 1 to receive either a single 30-minute infusion of IV NEPA or a single capsule of oral NEPA prior to AC. DEX will be administered in both groups on Day 1 of each cycle. The primary objective is to evaluate the safety of IV NEPA (relative to oral NEPA) based primarily on adverse events. No formal statistical comparisons are planned. Enrollment is expected to initiate in March 2018.
Results

Conclusions

eP020

ANTIEMETIC EFFICACY OF A PALONOSETRONDEXAMETHASONE SCHEDULE IN PEDIATRIC PATIENTS TREATED WITH MODERATELY OR HIGHLY EMETOGENIC CHEMOTHERAPY

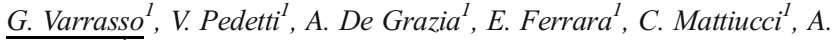 \\ Schiavetti ${ }^{I}$ \\ ${ }^{1}$ Policlinico Umberto I - Sapienza University of Rome, Pediatric Oncolgy \\ Unit, Roma, Italy
}

\section{Introduction}

Chemotherapy-induced nausea and vomiting (CINV) is the most frequent and distressing side effect of childhood cancer treatment.

\section{Objectives}

To evaluate the efficacy of palonosetron-dexamethasone association in the prevention of CINV in children receiving moderately (MEC) or highly emetogenic chemotherapy (HEC).

\section{Methods}

All consecutive patients with solid tumors were enrolled. The antiemetic schedule was as follows: palonosetron $5 \mathrm{mcg} / \mathrm{Kg}$ i.v. 30 minutes before chemotherapy on day 1 if chemotherapy duration was less than 3 days, every other day if chemotherapy lasted $\geq 3$ days. In the MEC courses dexamethasone at $0.1 \mathrm{mg} / \mathrm{kg}$ daily was associated to palonosetron until one day after chemotherapy. In the HEC courses dexamethasone was given at a dosage of $0.2 \mathrm{mg} / \mathrm{kg}$ daily during chemotherapy and at $0.1 \mathrm{mg} / \mathrm{kg}$ until 2 days after chemotherapy. Complete response (CR) was defined as no vomiting and no rescue therapy, complete control (CC) as $\mathrm{CR}$ and no more than mild nausea.

\section{Results}

29 patients (14 M, $15 \mathrm{~F}$ ), median age 7 yrs (range 1-18) were prospectively enrolled. A total of 160 courses, 105 MEC and 55 HEC were delivered. In the MEC courses a CR was obtained in $95.5 \%$ in the acute phase and in $100 \%$ in the delayed phase; CC was observed in $87 \%$ and $94.5 \%$ of acute and delayed phase, respectively. In the HEC courses CR and $\mathrm{CC}$ rates in acute and delayed phase were $89 \%, 98 \%, 83 \%, 98 \%$, respectively.

\section{Conclusions}

Our data show a very high antiemetic efficacy of palonosetrondexamethasone association in children receiving MEC or HEC.

\section{eP021}

INVOLVEMENT OF HISTAMINE H4 RECEPTOR IN THE DEVELOPMENT OF CHEMOTHERAPY-INDUCED ANOREXIA IN MICE

\section{K. Yamamoto $^{1}$, R. $_{\text {Okui }}{ }^{1}$, A. Yamatodani $^{2}$}

${ }^{T}$ Osaka University, Department of Medical Physics and EngineeringGraduate School of Allied Health Sciences- Faculty of Medicine, SuitaOsaka, Japan

${ }^{2}$ Osaka University, Professor Emeritus-, Suita- Osaka, Japan

\section{Introduction}

Cisplatin-based cancer chemotherapy often induces a biphasic pattern (acute and delayed phase) of anorexia, nausea and vomiting. Antiemetic agents proved to be effective to vomiting, but patients still experience anorexia and nausea, especially delayed anorexia. It is known that adaptive behavioral changes such as malaise and anorexia occur during the course of inflammation. Previous 
studies revealed that antagonism of the histamine $\mathrm{H} 4$ receptor has been shown to be anti-inflammatory.

Objectives

We investigated the involvement of $\mathrm{H} 4$ receptor in the development of chemotherapy-induced anorexia.

Methods

Mice were housed in individual food intake monitoring cages. On the day of the experiment, they received cisplatin $(7.5 \mathrm{mg} / \mathrm{kg})$ with or without pretreatment with granisetron $(0.5 \mathrm{mg} / \mathrm{kg})$, fosaprepitant $(30 \mathrm{mg} / \mathrm{kg})$, dexamethasone $(0.5 \mathrm{mg} / \mathrm{kg}$ ), and JNJ7777120 (H4 receptor antagonist $10 \mathrm{mg}$ / $\mathrm{kg}$ ), then their daily food intake was monitored for 3 days. Additionally, we examined the effect of JNJ7777120 on the cisplatin-induced expression of Tumor Necrosis Factor (TNF)- $\alpha$ mRNA in the hypothalamus.

\section{Results}

Cisplatin induced anorexia within 48 hours after administration of the drug, and lasted during observation periods. Antiemetic agents did not affect cisplatin-induced anorexia; however, JNJ7777120 completely abolished the anorexia. Cisplatin increased TNF- $\alpha$ mRNA expression in the hypothalamus and the time required to increase the expressions was comparable to the latency period of anorexia. In addition, pretreatment with JNJ7777120 completely inhibited the expression.

\section{Conclusions}

Results suggest the TNF- $\alpha$ mRNA expression in the hypothalamus via H4 receptor may contribute to the development of cisplatin-induced anorexia and $\mathrm{H} 4$ receptor antagonists have the potential to be candidates used as its treatment.

\section{eP022}

THE CINV CAUSED BY MULTIPLE-DAY CISPLATIN-BASED HIGHLY EMETOGENIC CHEMOTHERAPY CAN LAST A LONG PERIOD OF TIME IN PATIENTS WITH NSCLC

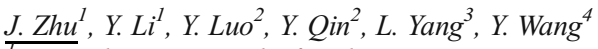 \\ ${ }^{T}$ West China Hospital of Sichuan University, Department of thoracic \\ cancer, Chengdu, China \\ ${ }^{2}$ West China School of Medicine- Sichuan University, West China School \\ of Medicine- Sichuan University, Chengdu, China \\ ${ }^{3}$ Cobaxer-biotech limited company, Cobaxer-biotech limited company, \\ Chengdu, China \\ ${ }^{4}$ Department of Oncology-AVIC 363 Hospital, Department of Oncology- \\ AVIC 363 Hospital, Chengdu, China
}

\section{Introduction:}

The 3-day cisplatin-based doublet chemotherapy is widely used in China for the patients with NSCLC regarded its lower renal toxicity, hydration is unnecessary. Aprepitant included antiemesis regimen had been proved to increase the prevention of CINV. But the real situation of CINV caused by the 3-day cisplatin-based chemotherapy is still unclear.

\section{Objectives}

To investigate the real situation of CINV in the entire cycle of the 3-day cisplatin-based chemotherapy.

Methods

96 chemotherapy-naive patients with NSCLC who received the first 3-day cisplatin-based doublet regimen (total dose of cisplatin: 75 $\mathrm{mg} / \mathrm{m}^{2}$ ) were enrolled in this study. Antiemetic regimen included granisetron and dexamethasone, aprepitant was recommended ( not covered by medical insurance). The observation period was from day 1 to day 21, CINV situation was recorded in the patient's diary according to MAT every day, while FLIE was used to evaluate the Qol.

\section{Results}

66 patients $(68.8 \%)$ were observed to experience the long lasting CINV beyond day 8 , which caused bad effect in some life functions. $37.5 \%$ patients who were administered aprepitant (Group A) still experienced the long lasting CINV exceed day 8. While the incidence rate of long lasting CINV was $81.3 \%$ in patients who were administered antiemetics excluding aprepitant (Group NA).

\begin{tabular}{|c|c|c|c|}
\hline Variable & Group $A(n=48)$ & Group NA ( $\mathrm{n}=48)$ & $P$-value \\
\hline Gender & & & 0.29 \\
\hline Male & 28 & 34 & \\
\hline Female & 20 & 14 & \\
\hline Age, years & & & 0.85 \\
\hline Meduan (range) & $62.5(30-78)$ & $60(39-79)$ & \\
\hline Stage & & & 0.22 \\
\hline 1 & 4 & 3 & \\
\hline II & 12 & 22 & \\
\hline $\mathrm{m}$ & 8 & 6 & \\
\hline $\mathrm{IV}$ & 24 & 17 & \\
\hline Pathological pattern & & & 0.29 \\
\hline Adenocarcinoma & 27 & 33 & \\
\hline Squamous carcinoma & 21 & 15 & \\
\hline Performance status (ECOG) & & & 0.67 \\
\hline Score 0 & 26 & 29 & \\
\hline Score 1 & 16 & 12 & \\
\hline Score 2 & 6 & 7 & \\
\hline Chemotherapy regmen & & & 1.00 \\
\hline Cicplatin/paclitaxel & 24 & 24 & \\
\hline Cisplatin/gemcitabine & 15 & 15 & \\
\hline Cisplatinvetoposide & 9 & 9 & \\
\hline
\end{tabular}

Time to complete remission of CINV

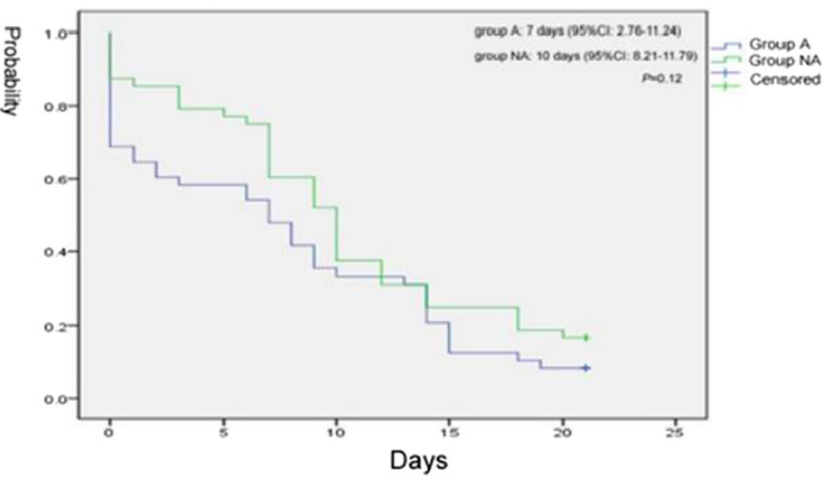

\section{Conclusions}

3-day cisplatin based chemotherapy can cause the long lasting CINV at an obvious ratio even with aprepitant. Patients' quality of life is affected by the long lasting CINV.

\section{eP023}

PROLONGING APREPITANT ADMINISTRATION INCREASES THE ANTIEMETIC EFFECT AGAINST MULTIPLE-DAY C I S P L A T I N - B A S E D H I G H LY EM E T O G E N I C CHEMOTHERAPY

J. Zhu ${ }^{1}, Y . L i^{1}, M . Y u^{1}$, B. $\mathrm{Liu}^{2}, P . \mathrm{Yu}^{2}, \mathrm{Q} . \mathrm{Li}^{3}, \mathrm{P} . \mathrm{Chen}^{4}, \mathrm{X} . \mathrm{Zeng}^{4}, \mathrm{~K} . \mathrm{Xie}^{5}$ ${ }^{T}$ West China Hospital of Sichuan University, Department of thoracic cancer, Chengdu, China

${ }^{2}$ Sichuan Cancer Hospital \& Institute, Sichuan Cancer Hospital \& Institute, Chengdu, China

${ }^{3}$ Department of Oncology- the 452nd hospital of People's Liberation Army, Department of Oncology- the 452nd hospital of People's Liberation Army, Chengdu, China

${ }^{4}$ Chengdu Cancer Hospital, Chengdu Cancer Hospital, Chengdu, China 'Sichuan Provincial Peoples' Hospital, Sichuan Provincial Peoples' Hospital, Chengdu, China 


\section{Introduction}

Even with the 3-day aprepitant, $37.5 \%$ patients received multplei-day cisplatin-based chemotherapy having long lasting CINV according to our prior investigation.

\section{Objectives}

This study was designed to prevent CINV better in the whole cycle by prolonging the dose of aprepitant.

\section{Methods}

The study enrolled chemotherapy-naive patients with lung cancer who were planed to receive 3-day cisplatin-based doublet chemotherapy from 5 major tumor centers in Chengdu. Patients agreeing using aprepitant were randomly recruited in PA group (6-day aprepitant ) or SA group (3-day aprepitant). Patients refused aprepitant entered $\mathrm{C}$ group. The investigation period lasted from the first day to the $21^{\text {st }}$ day of the first cycle. CINV was recorded in the patient's diary every day according to the MASCC antiemesis tool. The primary end points were the safety and the fully protected days (FPDs).

\section{Results}

A total of 150 patients were enrolled, 141 cases (PA: 47, SA: 45, C: 49) completed the study were analyzed. No study related death. Difference of side effects among the 3 groups was nonsignificant. The average FPDs of the PA group were $18.28 \pm 3.37$ days, significantly better than SA group (13.58 \pm 6.39 days, $P<0.001)$ and group $\mathrm{C}(10.94 \pm 6.71$ days, $P<0.001)$. Group SA was also advantage in completed response $(\mathrm{CR})$ rate of CINV, time to CR of CINV, and the life functions.

Table 1: Antiemetic treatment plan
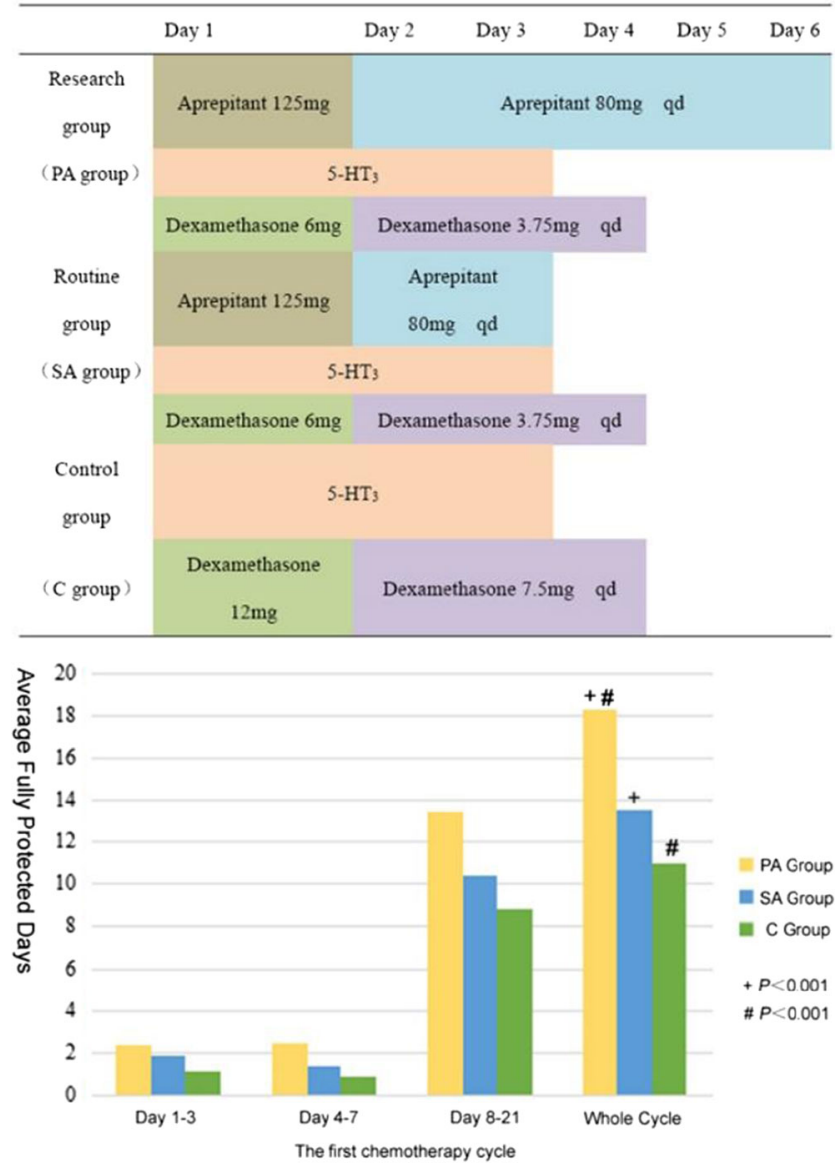

\section{Median time to $\mathrm{CR}$ of $\mathrm{CINV}$}

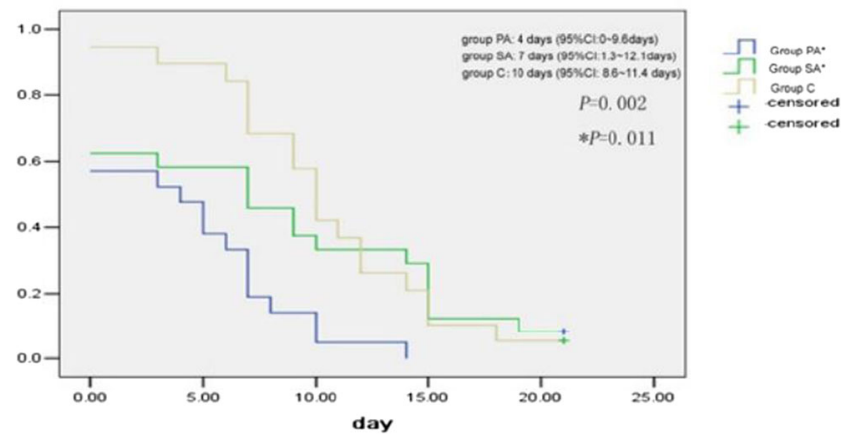

\section{Conclusions}

Prolonging aprepitant to 6 days shows a good safety and a better antiemetic effect among the whole cycle for patients receiving multiple-day cisplatin-based chemotherapy.

\section{eP024 \\ COMPUTATIONAL SIMULATION OF BONE TISSUE REMODELING PROCESS: A VIRTUAL TOOL FOR CLINICAL GUIDANCE}

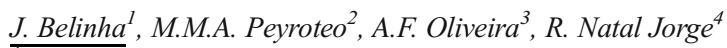

INEGI/ISEP - School of Engineering of the Polytechnic Institute of Porto, Department of Mechanical Engineering, Porto, Portugal

${ }^{2}$ INEGI - Institute of Science and Innovation in Mechanical and Industrial Engineering, Department of Design and Experimental Validation, Porto, Portugal

${ }^{3}$ Hospital Santo António - Hospital Center of Porto, Head of Orthopedics, Porto, Portugal

${ }^{4}$ Faculty of Engineering of the University of Porto, Mechanical Engineering Department, Porto, Portugal

\section{Introduction:}

Bone is a living tissue, capable to structurally react to external stimuli, such as mechanical loading. In this work, bone tissue adaptation to mechanical loading is predicted by means of a friendly computational tool using an advanced topologic optimization mathematical model for bone remodeling.

\section{Objectives}

The aim of this work was to deliver a mature computational tool capable to predict the bone tissue remodeling in several scenarios, such as healthy or cancerous bone structures requiring implants.

\section{Methods}

In order to obtain the computational discretization of the bone structure, discrete numerical methods are used, such as the Finite Element Method and meshless methods. Then, the remodeling algorithm seeks the minimization of the strain energy density field and considers a phenomenological law capable to predict the bone tissue's mechanical properties based on the bone apparent density.

\section{Results}

In this work, several clinical cases are studied, such as the remodeling simulation of a femur submitted to a hip replacement surgery, whose head was removed due to a cancer. The virtual results show that, using the proposed computational tool, it is possible to predict the trabecular distribution of bone structures, with or without femoral stems, as figure 1 shows. 


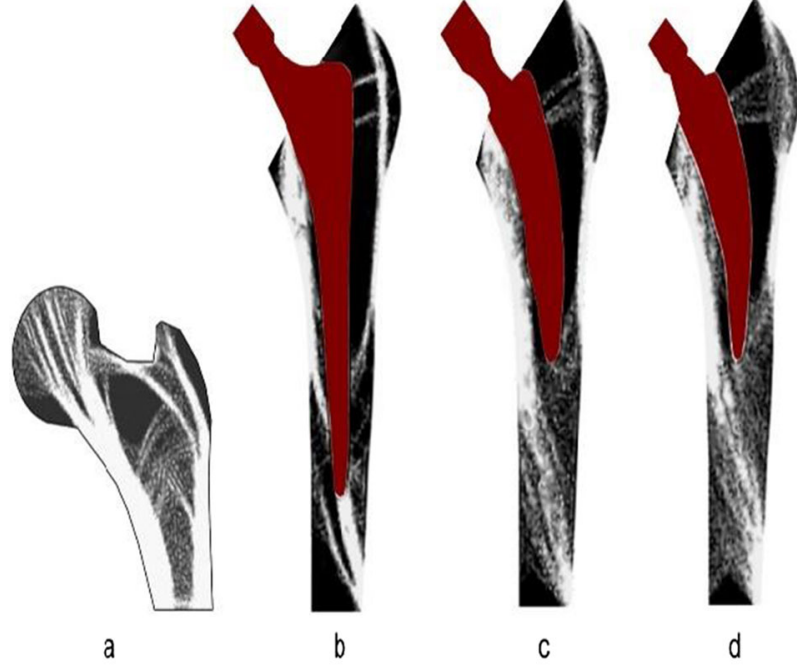

Figure 1 - (a) Expected remodeling of proximal femur. Expected remodeling due to the insertion of three distinct femoral stems (b) long, (c) medium and (d) short stem.

\section{Conclusions}

The numerical results obtained with the proposed computational tool do not perfectly match the reality. However, they are excitingly close, allowing the physician to select the most suited implant solution for each patient. Additionally, this mature computational tool was successfully used by trainee orthopedists and dentists, with no engineering background, showing its potential in the daily clinical practice.

eP025

A NOVEL CLINICAL SOFTWARE TO ASSESS BONE TISSUE FAILURE RISK USING THE FINITE ELEMENT METHOD COMBINED WITH A MEDICAL RADIOGRAPHY

\author{
J. Belinha ${ }^{I}$, G. Henriques ${ }^{2}$, A.F. Oliveira ${ }^{3}$, R. Natal Jorge ${ }^{4}$ \\ ${ }^{T} I N E G I / I S E P$ - School of Engineering of the Polytechnic Institute of \\ Porto, Department of Mechanical Engineering, Porto, Portugal \\ ${ }^{2}$ INEGI - Institute of Science and Innovation in Mechanical and \\ Industrial Engineering, Department of Design and Experimental \\ Validation, Porto, Portugal \\ ${ }^{3}$ Hospital Santo António - Hospital Center of Porto, Head of \\ Orthopedics, Porto, Portugal \\ ${ }^{4}$ Faculty of Engineering of the University of Porto, Mechanical \\ Engineering Department, Porto, Portugal
}

\section{Introduction}

Today, it is commonly accepted by the medical community that radiography is an efficient imaging technique to diagnose bone tumors. Although radiologists are capable to recognize such pathology, to them it is not clear if and how bone tumors influence bone tissue structural response.

\section{Objectives}

The main goal of this work is to provide a novel and user friendly clinical software capable to automatically read medical radiographies of femur bones and deliver its failure risk, regardless the pathology (bone tumor, osteoporosis, etc.).

Methods

First, in order to automatically recognize from the radiography the femur contour and domain, a new image segmentation technique was developed. The technique allows to build a computational model of the femur bone and simultaneously attribute mechanical properties to each point of the model (based on the grayscale of the radiography). Then, the virtual model is analyzed with the finite element method and the stress field is obtained, allowing to measure bone failure risk.

\section{Results}

The software was tested by trainee engineers and orthopedists using several clinical cases. The software was capable to consistently identify the femur bone domain and provide its failure risk, as shown in figure 1 . The results were in agreement with more complex computational mechanics analyses.
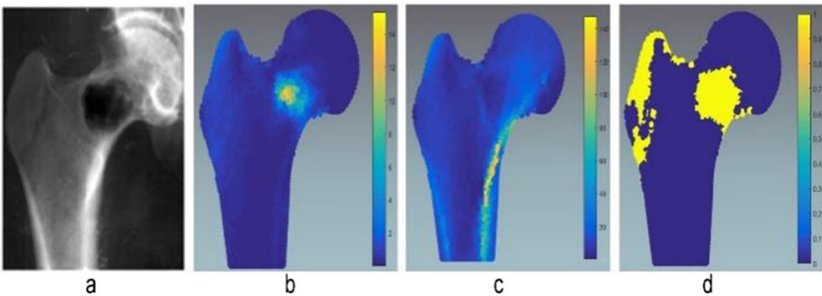

Figure 1 - (a) Detail of the initial radiography, in which it is possible to identify a bone tumor. ffter the automatic structural analysis, the software is capable to deliver: (b) the expected stress field, (c) the risk failure ratio and (d) the risk failure zones.

\section{Conclusions}

Since the developed software is an ongoing project, it allows recurring improvements and enhancements. The feedback provided by the testers allowed to improve significantly the software interface and usability. The robustness and consistency of the obtained results shows that the developed software has the potential to assist the clinical practice.

\section{eP026}

USE OF BONE PROTECTION IN PATIENTS WITH PRIMARY INTRACRANIAL TUMOURS ON LONG TERM CORTICOSTEROIDS

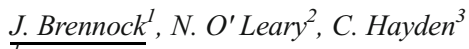

${ }^{T}$ Our Lady Of Lourdes Hosptial Drogheda Co. Louth, Palliative Medicine Department, Drogheda, Ireland

${ }^{2}$ Our Lady's Hospice- Harold's Cross- Dublin 6W, Palliative Medicine, Dublin, Ireland

${ }^{3}$ Our Lady's Hospice- Harold's Cross, Pharmacy, Dublin, Ireland

\section{Introduction}

Long term use of corticosteroids (CS) can be associated with significant morbidity, including development of glucocorticoid-induced osteoporosis and fractures. There are currently no specific standards pertaining to the use of bone protection in patients on long term CS in palliative care. As a significant proportion of these patients are on CS for prolonged periods, this area should be explored further.

Objectives

To ascertain current use of bone protection in a palliative cohort of patients with primary intracranial tumours on long term CS treatment

To identify patients in this cohort who would likely have benefitted from receiving bone protection

Methods

Standards used: American College of Rheumatology 2010 Recommendations for the Prevention and Treatment of GlucocorticoidInduced Osteoporosis. These recommend that patients on long term glucocorticoid treatment (dose $\geq 7.5 \mathrm{mg}$ prednisolone daily for $\geq$ three months) should be on bone protection therapy (bisphosphonate).

Methodology: Retrospective audit using chart review of patients with primary intracranial tumours on initial referral to Palliative Care Team (PCT). Results

32 eligible patients identified. $37.5 \%$ on steroids on admission, had been on steroids for $>$ three months on initial assessment and had $>$ three months to live. $12.5 \%$ had $>$ six months to live and were on steroids on first assessment, and $6.25 \%$ had been on $>3$ month course of steroids. 


\section{Conclusions}

$62 \%$ of patients initially assessed by PCT should have been considered for bone protection prior to referral. $45 \%$ of patients were not suitable for consideration for bone protection. 55\% which could have potentially been considered for bone protection by the PCT following initial assessment.

\section{eP027}

TREATMENT PROTOCOL OF DENTIGEROUS CYST IN THE MANDIBLE, IN PATIENT RECEIVING INTRAVENOUS BEVACIZUMAB DUE TO OVER-CANCER METASTASIS; A CASE REPORT

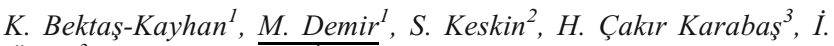
Özcan ${ }^{3}$, M. Soluk Tekkeşin ${ }^{4}$

${ }^{1}$ Istanbul Univercity- Faculty of Dentistry, Department of Oral and Maxillofacial Surgery, Istanbul, Turkey

${ }^{2}$ Memorial Hospital Oncology Centre, Department of Medical Oncology, Istanbul, Turkey

${ }^{3}$ Istanbul Univercity- Faculty of Dentistry, Department of Oral and Maxillofacial Radiology, Istanbul, Turkey

${ }^{4}$ Istanbul Univercity-Institute of Onkology, Department of Tumor Pathology, Istanbul, Turkey

\section{Introduction}

Various type of agents are used in cancer treatment including targeted therapy agent; bevacizumab. This has been used widely in metastatic cases with some side effects like GI problems, bleeding and MRONJ.

\section{Objectives}

Beside the complicative effects of cytotoxic CT on oral surgery, bevacizumab has a negative impact both on healing and the risk of developing MRONJ. Therefore, when oral surgery is necessary for these patients, multidisciplinary approach should be applied in cooperation with medical oncologist and dentist. The purpose of the treatment plan in this case; the multidisciplinary approach to oral surgery reduces the risk of complications that may arise as a result. Methods

Here we present a metastatic over cancer patient ( 52 years, female) who was referred to our department for dentigerous cyst-related wisdom tooth with bony retention. Bevacizumab was discontiniued after the consultation with the medical oncologist of the patient and marsupialisation at the cyst was performed in order to minimize the cyst and the risk of ONJ due to major surgery. For 3 mounths marsüpialisation didn't make a dramatic change in the dimension of the cyst. After radiological follow up enucleation and curettage was made.

\section{Results}

Oncologists, dentists and patients should have knowledge about the side effects of any kind of CT agent; it is important for the oncologist and the dentist decide on the most appropriate treatment procedure for the condition of the patient.

Conclusions

This case report shows that with multidisciplinary approach the complications can be avoided when oral surgery is needed in patients receiving antineoplastic agents.

\section{eP028}

EFFECT OF LEUKOCYTE-PLATELET RICH FIBRIN ON HEALING OF MEDICINE INDUCED OSTEONECROSIS OF JAW- PILOT STUDY

\author{
K. Dholam ${ }^{1}$ \\ ${ }^{T}$ Tata Memorial Centre, Dept of Dental and Prosthetic Surgery, Mumbai, \\ India
}

\section{Introduction}

Medicine-induced osteonecrosis of the jaw (MRONJ) is a relatively uncommon but potentially serious side effect of treatment with antiresorptive agents such as intravenous (IV) high potency bisphosphonates and denosumab, which are used to decrease the risk of skeletal-related events in patients with cancer and metastatic bone disease. Its incidence rate ranges from 1.3 to $1.9 \%$ depending on type of drug, mode of administration, frequency of drug, duration and other risk factors.

The management of MRONJ has been centered on efforts to eliminate or reduce the severity of symptoms, to slow or prevent the progression of disease, and to eradicate diseased bone. There is currently no gold standard for the treatment of MRONJ.

\section{Objectives}

To study the effect of Leukocyte-Platelet-rich fibrin $\left(\mathrm{L}-\mathrm{PRF}^{\mathrm{TM}}\right)$ on healing of Medicine-induced osteonecrosis of jaw (MRONJ) in a single study group.

\section{Methods}

Patients diagnosed with MRONJ and not responding to conservative medications for eight weeks were recruited. Pre-operative assessment was made of the site, stage, signs and symptoms. Minimal sequestrectomy and removal of necrotic, infected bone was carried out followed by placement of L-PRF ${ }^{\mathrm{TM}}$ and primary closure was achieved. To obtain L-PRF, patient's blood will be collected in a vacutainer and centrifuged. Response of each patient was recorded 1 month and 4 months postoperatively.

\section{Results}

Ongoing study and healing response of MRONJ lesions in our series of patient will be presented.

\section{Conclusions}

Ongoing study and healing response of MRONJ lesions in our series of patient will be presented.

\section{eP029}

THE USE OF ORAL LORATADINE IN THE MANAGEMENT OF G-CSF INDUCED BONE PAIN

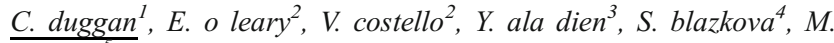
dowling

${ }^{1}$ oncology Department, Portiuncula Hospital, galway, Ireland

${ }^{2}$ Portiuncula hospital, oncology, galway, Ireland

${ }^{3}$ sligo general hospital, oncology, sligo, Ireland

${ }^{4}$ galway university hospital, oncology, galway, Ireland

${ }^{5}$ National university of Ireland, department of nursing studies, galway, Ireland

\section{Introduction}

The use of oral Loratadine in the management of G-CSF induced bone pain.

\section{Objectives}

Pilot study was to ascertain if bone pain induced by Granulocyte-Colony Stimulating Factors (G-CSFs) can be alleviated or eliminated by administration of the oral antihistamine Loratadine (Claritin $\left.{ }^{\circledR}\right)$.

\section{Methods}

The study was undertaken at a unit which treats mostly breast, colorectal and gynaecology patients. All eligible patients completed the Brief Pain Inventory (BPI) prior to the first cycle of chemotherapy and on Day 7 after the first cycle of chemotherapy. Oral Loratadine was commenced on day one of cycle 2 if the patient's pain was $\geq 3$ at its worst (Q3 on BPI). Each patient was instructed to take Loratadine $10 \mathrm{mg}$ for each remaining cycle of chemotherapy thereafter. Vitamin D and magnesium levels were also recorded at baseline.

\section{Results}

-The final sample of twelve patients, ten women and two men with a diagnosis of breast $(n=7)$, genitourinary cancer $(n=4)$, and colorectal $(n=1)$. Daily pain increased initially from pre-cycle one to post cycle 
one in all patients. Pain decreased towards the later cycles following commencement of Loratadine in cycle 2, with the exception of one patient. There was a statistically significant negative correlation noted between vitamin D levels and pain on pre-cycle $1(-0.67 ; \mathrm{p}=0.045)$. Similarly, there was a statistically significant negative correlation noted between magnesium levels and average pain on cycles $2(-0.642$; $\mathrm{p}=0.024)$ and $4(-0.822 ; \mathrm{p}=0.012)$.

Conclusions

Oral Loratadine is associated with pain reduction in cancer patients receiving G-CSFs.

\section{eP030}

MEDICATION-RELATED OSTEONECROSIS OF JAW (ONJ) IN CANCER PATIENTS RECEIVING BISPHOSPHONATES OR DENOSUMAB: TIME TRENDS AMONG 557 CASES OF A CANCER NETWORK (RETE ONCOLOGICA) DATABASE

\section{Fusco ${ }^{1}$, M. Cabras ${ }^{2}$, A. Gambino ${ }^{2}$, O. Bertetto ${ }^{3}$ \\ ${ }^{T}$ Azienda Ospedaliera di Alessandria, Oncology, Alessandria, Italy \\ ${ }^{2}$ Azienda Città della Salute di Torino, Dental School, Turin, Italy \\ ${ }^{3}$ Azienda Città della Salute di Torino, Rete Oncologica Piemonte Valle d'Aosta, Turin, Italy}

\section{Introduction}

As an almost unique experience, since 2005 a multidisciplinary study group collected data of cases of Osteonecrosis of Jaws (ONJ) in 221 cancer patients treated with Bisphosphonates (BP) observed in oncology and hematology centers of a regional network (Piemonte - Valle d'Aosta), with yearly incidence reduced from 60 (max on year 2006) to 21 cases (on 2008) (Fusco et al, ISRN Oncology 2013)

\section{ONJ TIME TREND (2004 - 2015)}

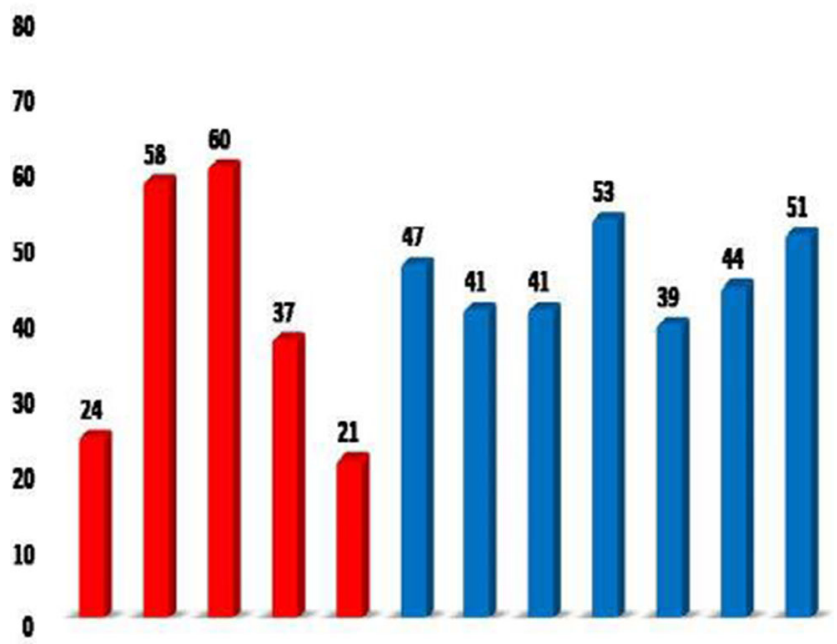

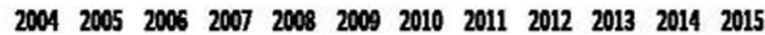

\footnotetext{
Objectives

Update of yearly incidence after 2008.

Methods

We asked for ONJ cases observed between 2009 and April 2016 from the same medical oncology, haematology, and oral care centers.

Results

We received data of further 336 cases in cancer patients: $66 \%$ were female and $34 \%$ male; primary neoplasm was : breast cancer
}

$150(45 \%)$, myeloma $70(21 \%)$, prostate cancer $70(21 \%)$, lung $18(5 \%)$, renal cell $10(3 \%)$, and other types of cancer or not specified $18(5 \%)$. The median number of cases per year was 44 (range 39-53) in years 2009-2015.

\section{Conclusions}

Preliminary data show an unexpected new increase of ONJ cases per year in spite of measures prescribed to reduce the ONJ risk (dental visit and oral care before antiresorptive treatment). Several possible reasons could be hypothesized : introduction of denosumab in bone metastatic patients; larger introduction of new agents (other than BP and denosumab) potentially inducing ONJ, and higher risk from antiseporptive plus antiangiogenic drugs; longer survival of some cancer patient subsets (ie, lung and renal cell cancer ); etc. The full collection of clinical data is warranted to explore these suggestions.

eP031

PROGRANULIN REGULATION OF AUTOPHAGY CONTRIBUTES TO ITS CHONDROPROTECTIVE EFFECT IN OSTEOARTHRITIS

F. Guo ${ }^{1}$, A. Hettinghouse ${ }^{2}$, W. Xie ${ }^{1}$, C. Liu $^{2}$

${ }^{T}$ Core Facility of Development Biology-Chongqing Medical UniversityChongqing 400016-China, Department of Cell Biology and GeneticsChongqing Medical University- Chongqing 400016-China, Chongqing, China

${ }^{2}$ New York University School of Medicine- New York- NY10003- USA, Departments of Orthopaedic Surgery- New York University School of Medicine- New York- NY10003- USA, New York, USA

\section{Introduction:}

Osteoarthritis (OA) is the most common degenerative joint disease,but the pathogenesis of $\mathrm{OA}$ is not fully understood.

Objectives

Whether PGRN regulates chondrocyte autophagy and if so, whether PGRN regulation of autophagy is important for PGRN's protective role in OA.

Methods

Mice DMM model;Primary PGRN-/- chondrocytes culture;Western blot;Q-PCR;FCM;IHC;Immunofluorescence.

Results

Autophagy-associated molecules are elevated in $O A$ chondrocytes. The mRNA levels of PGRN,ATG5 and ATG12 were significantly upregulated in OA chondrocytes compared to normal controls'.The protein levels of ATG5-ATG12,ATG5 and LC $3 \alpha / \beta$ were also clearly elevated in OA chondrocytes comparing with normal chondrocytes(Fig. 1).

PGRN is required for chondrocyte autophagy. Ramamycin-stimulated expressions of ATG5 and ATG12 have no significant difference in primary PGRN-/- chondrocytes. There is no significant difference in PGRN-/ - chondrocytes treated with or without IL-1 $\beta$ (Fig. 2). Taken together,PGRN is required for activation of autophagy in chondrocytes. Autophagy is important for PGRN's protective role in chondrocytes and OA. PGRN-mediated anabolism is largely lost in the presence of autophagy inhibitor BafA1 in human OA chondrocytes.BafA1 also inhibited PGRN's suppression on IL-1 $\beta$-induced catabolic metabolism in OA chondrocyte. We generated DMM OA model in 4 months-old mice,followed by intra-articular injection of PBS,PGRN $(6 \mu \mathrm{g})$ with or without BafA1(5ug) once a week.Recombinant PGRN clearly protected against $\mathrm{OA}$ progression in surgically induced OA models, and intraarticular injection of BafA1 significantly suppressed PGRN's protective effect(Fig. 3).It is demonstrated that autophagy regulated by PGRN contributes to its chondroprotective effect against OA.Mechanistic studies demonstrate that PGRN regulates autophagy through direct interaction with ATG5-ATG12(data not shown). 

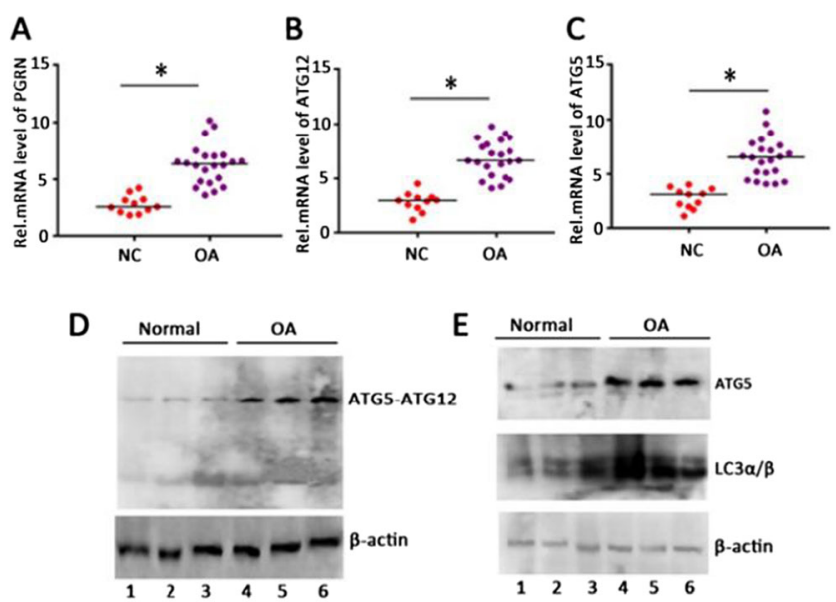

Fig.1. A-C, mRNA levels of PGRN, ATG5 and ATG12 in human normal and OA chondrocytess; D,E. Protein levels of ATG5-ATG12, ATGS and LC3 in human normal and OA chondrocytes.
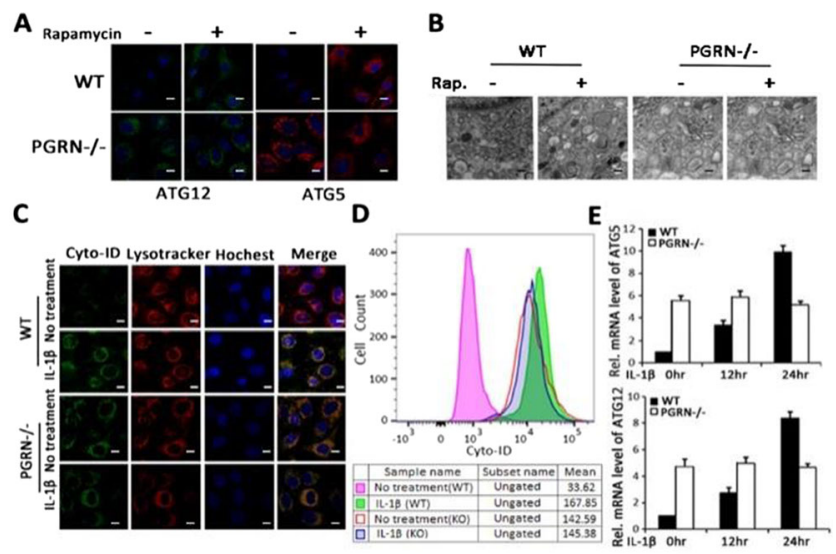

Fig.2. A. Immunoinfluence staining of ATGS and ATG12 in WT and PGRN KO primary murine chondrocytes with or without Rapamycin B. The autophagosome in WT and PGRN KO murine chondrocytes detected with TEM; $C$, D. The autophagy staining and flux in IL 1B. stimulated WT and PGRN KO murine chondrocytes detected with live image technique and FCM analysis. E. The mRNA levels of ATG5 and ATG12 in WT and PGRN KO human C2812 chondrocytes stimulated with IL 13

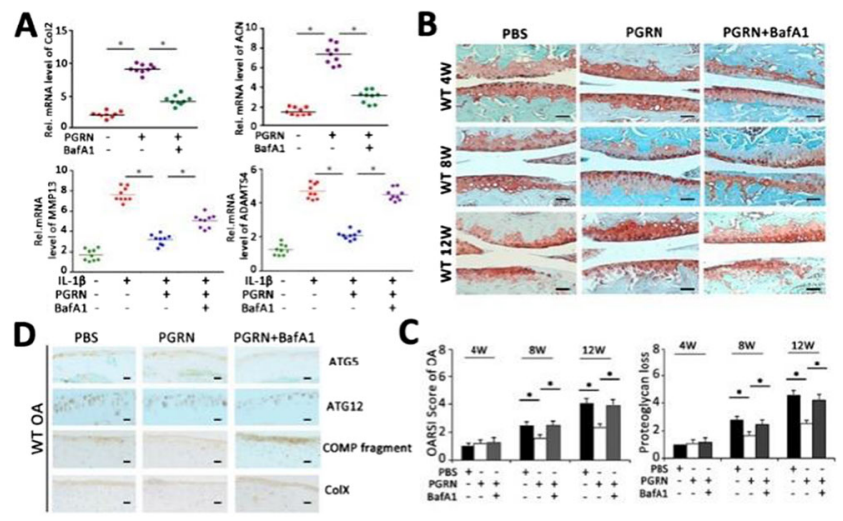

Fig.3. A.The mRNA levels of Col2, Aggrecan, MMP13 and ADAMTS4 in human OA chondrocytes, $n=9$; B. Safranin $O$ staining of knee joint; $n=8$. C. Osteoarthritis Research Society International (OARSI) score of $O A$ and loss of Aggrecan based on the result of Safranin $O$ staining; $D$.Immunohistochemistry of knee joint obtained from DMM mice treated with or without PGRN and BafA1.

\section{Conclusions}

PGRN-mediated regulation of chondrocyte autophagy is important for PGRN's chondroprotective role in OA.PGRN associated autophagy in chondrocyte homeostasis in particular.
eP032

PAIN, ANXIETY, AND DEPRESSION DURING BONE MARROW ASPIRATION AND BIOPSY

Y. Karacan ${ }^{1}$, B. Demircioglu ${ }^{2}, R . A l i^{2}$

${ }^{T}$ Uludag University Faculty of Medicine, Oncology Department, Bursa, Turkey

${ }^{2}$ Uludag University Faculty of Medicine, Hematologia Department, Bursa, Turkey

\section{Introduction}

Hematologic patients go through many processes until a diagnosis is made. The hardest and the most difficult one during this period is the diagnosis process. One of the most commonly used methods is bone marrow aspiration and biopsy (BMAB), which provides morphological, immunological, cytogenetical, and histopathological examination of the bone marrow.

Objectives

This study is performed with the aim of prospectively analyzing the factors that affect pain, anxiety, and depression in cases for whom bone marrow aspiration and biopsy are performed and the relationships among them.

Methods

This study consisted of 90 patients who consulted to Department of Hematology, Faculty of Medicine, Uludag University, and followedup for bone marrow aspiration and biopsy indication. The data was gathered using a personal information form, Hospital Anxiety Depression scale, and Wong-Baker face pain scale where generally face expressions exist.

\section{Results}

The average age of the patients who participated in the study was 50.90 years (16.59), and the pain level after the application was 1.98 (1.33). After the application, a positive relationship is determined between the pain level and anxiety $(r=-0.79 ; p=0.02)$, whereas this has a negative relationship with age $(\mathrm{r}=0.78 ; \mathrm{p}=0.03)$.

\section{Conclusions}

As a result, pain develops depending on bone marrow aspiration and biopsy; therefore, taking precautions aimed at alleviating pain and evaluation of pain before and after the application within the scope of total maintenance have been suggested.

\section{eP033}

CLINICAL INVESTIGATION OF CASES WITH MEDICATIONRELATED OSTEONECROSIS OF THE JAW RESULTING IN POOR OUTCOMES WITH CONSERVATIVE TREATMENT

\section{T. Konishi ${ }^{1}$, R. Kishi ${ }^{1}$, Y. Mizutani ${ }^{1}$ \\ ${ }^{T}$ national cancer center hospital east, dental, chiba prefecture, Japan}

\section{Introduction}

Treatment for medication-related osteonecrosis of the jaw (MRONJ) is often difficult once MRONJ has developed, resulting in significant deterioration in quality of life for patients due to the development of deleterious symptoms such as pain.

\section{Objectives}

Conservative treatment with long-term use of appropriate antibiotics can control infection and symptoms such as pain in many patients who have developed bone exposure. However, even when such appropriate treatment or procedure is provided, it occasionally fails to control the range of exposure associated with osteonecrosis of the jaw, resulting in an expanded bone exposure and an advanced clinical stage.

\section{Methods}

From among patients with malignant tumors who visited our department between 2010 and 2017, 100 patients diagnosed with MRONJ who received conservative treatment were included in this study. We 
retrospectively reviewed the patients suffering expanded bone exposure range during conservative treatment.

\section{Results}

Of 100 patients diagnosed with MRONJ in our hospital, 20 had renal dysfunction, and 47 discontinued antibiotics during clinical management. An expansion of bone exposure range during conservative treatment was observed in 11 patients. Of these 11 patients, 7 had renal dysfunction, 8 discontinued antibiotics during clinical management, and 5 discontinued but later resumed antibiotics due to exacerbation of symptoms.

Conclusions

As patients with renal dysfunction are at high risk of failure in the control of jaw bone exposure due to limited use of antibiotics, close monitoring (such as more frequent hospital visits) may be necessary when the temporary discontinuation of long-term administration of antibiotics is required.

\section{eP034}

A SYSTEMATIC LITERATURE REVIEW ON THE MA N A G E M E N T O F M E D I C A T I O N-R E L A T E D OSTEONECROSIS OF THE JAW AMONG PATIENTS WITH CANCER

M. Marcusssen ${ }^{1}$, O. Nicolatou-Galitis ${ }^{2}$, E. Vardas $^{2}$, E. Papadopoulou ${ }^{2}$, M. Kouri ${ }^{2}$, D. Galiti $^{2}$, N. Yarom ${ }^{3}$, S.K. Yom ${ }^{4}$, C. Ripamonti ${ }^{5}$, C. Van Poznak ${ }^{6}$, W. Tan $^{7}$, J.J. Body ${ }^{8}$, C. Migliorati ${ }^{9}$, R. lalla ${ }^{10}$

${ }^{1}$ Aalborg University Hospital, Clinical Cancer Research Center, Aalborg, Denmark

${ }^{2}$ National \& Kapodistrian University, Clinic of Hospital Dentistry, Athens, Greece

${ }^{3}$ Tel Aviv University, School of Dental Medicine-Oral Medicine Unit, Tel Aviv, Israel

${ }^{4}$ Memorial Sloan Kettering Cancer Center, New York, NY, USA

${ }^{5}$ Istituto Nazionale dei Tumori, Supportive Care Unit- Fondazione IRCCS, Milano, Italy

${ }^{6}$ University of Michigan, Department of Internal Medicine- Division of Hematology Oncology, Ann Arbor - Michigan, USA

${ }^{7}$ Mayo Clinic, Division of Hematology/Oncology-Department of Internal Medicine, Jacksonville- Florida, USA

${ }^{8}$ University Libre de Bruxelles, CHU Brugmann, Bruxelles, Belgium

${ }^{9}$ University of Florida, Department of Oral and Maxillofacial Diagnostic

Sciences, Gainesville- Florida, USA

${ }^{10}$ UConn School of Dental Medicine, Section of Oral Medicine, Farmington- CT, USA

\section{Introduction}

Medication-related osteonecrosis of the jaw (MRONJ) is a significant complication among cancer patients receiving Bone Targeting Agents (BTAs); however, debate is ongoing on the best management of MRONJ. Objectives

To perform a systematic review of the literature on the management of MRONJ in cancer patients.

Methods

We searched MEDLINE and Embase for English-language literature from January 2009 to December 2016 using a predefined search strategy combining medical subject headings with text words. We included papers that described the treatment of MRONJ-affected cancer patients.

\section{Results}

We identified 38 papers; 37 case series or reports and one randomized trial. Management typically comprised of medical/non-surgical treatment, or surgical treatment ranging from superficial curettage, debridement, and sequestrectomy to continuity resection and reconstruction. Additional treatment involved the use of drug holiday, hyperbaric oxygen, lowlevel laser therapy, platelet-rich plasma or the use of fluorescenceguided surgery. One randomized study reported that laser therapy did not improve outcome among 20 surgically treated patients. In another cohort of 30 cases, a correlation between histology-verified necrosis-free resection margins and complete healing was reported. Of 588 included patients, $414(70,4 \%)$ healed completely.

\section{Conclusions}

Due to the low level of evidence in the retrieved studies (lack of randomization and controls) and heterogeneous patient cohorts (various MRONJ stages and treatment modalities), no meta-analysis was feasible. Although some cases healed after conservative treatment, we observed a trend towards surgical resection of necrotic bone to achieve complete clinical healing at all MRONJ stages. Better quality studies are warranted, with standardized reporting of interventions and outcomes

\section{eP035}

OSTEONECROSIS OF THE JAW RELATED TO NONANTIRESORPTIVE MEDICATIONS/THERAPIES: A SYSTEMATIC REVIEW

O. Nicolatou-Galitis ${ }^{1}$, M. Kouri ${ }^{l}$, E. Papadopoulou ${ }^{1}$, E. Vardas $^{I}$, D. Galiti $^{1}$, J. Epstein ${ }^{2}$, S. Elad ${ }^{3}$, G. Campisi ${ }^{4}$, N. Tsoukalas ${ }^{5}$, K. BektasKayhan ${ }^{6}$, W. Tan ${ }^{7}$, J.J. Body ${ }^{8}$, C. Migliorati ${ }^{9}$, R. Lalla ${ }^{10}$

${ }^{1}$ University of Athens, Dental School-, Athens, Greece

${ }^{2}$ Cedars-Sinai Health System-CA and City of Hope National Medical Center-Duarte- CA, Samuel Oschin Comprehensive Cancer Institute, Los Angeles, USA

${ }^{3}$ University of Rochester Medical Center-Rochester, Eastman Institute for Oral Health-, New York, USA

${ }^{4}$ Sector of Oral Medicine "V. Margiotta" University of Palermo, Dept. Di.Chir.On.S-, Palermo, Italy

${ }^{5}$ Veterans Hospital NIMTS-, Oncology Department, Athens, Greece

${ }^{6}$ Department of Oral and Maxillofacial Surgery, Istanbul UniversityFaculty of Dentistry, Istanbul, Turkey

${ }^{7}$ Mayo Clinic Florida- Jacksonville, Hematology/oncology, Florida, USA

${ }^{8}$ Univ. Libre de Bruxelles, CHU Brugmann, Bruxelles, Belgium

${ }^{9}$ University of Florida College of Dentistry, Department of Oral and Maxillofacial Diagnostic Sciences, Florida, USA

${ }^{10}$ UConn, School of Dental Medicine, Farmington- CT, USA

\section{Introduction}

Osteonecrosis of the jaw (ONJ) related to medications without antiresorptive properties, such as antiangiogenics, tyrosine kinase inhibitors, mammalian target of rapamycin inhibitors, immune checkpoint inhibitors and conventional chemotherapy is increasingly reported.

\section{Objectives}

To review characteristics of ONJ in cancer patients receiving nonantiresorptive medications.

\section{Methods}

A systematic review of the literature between 2009-2017 was conducted by the BSG.

Results

Of 6,249 articles reviewed, 31 articles, describing 39 cases of ONJ related to non-antiresorptives were identified. No gender predilection was noted, median age was 64 years and ONJ stage 2 was most common, with a predilection for posterior mandible. Exposed bone, infectious manifestations and pain were common at diagnosis. In comparison to bone targeting agents (BTAs), radiology, histology and management were similar, with medication often discontinued. Delayed diagnosis (median 8 weeks) was noted.

Important differences included earlier time to ONJ onset (median 20 weeks), absence of trigger event $(41 \%)$, greater likelihood of healing and shorter healing time (median 8 weeks) as compared to BTA-related ONJ. Gastrointestinal cancers predominated, followed by renal cell carcinomas in contrast to breast, followed by prostate cancers in BTA-related ONJ, reflecting the different treatments. 


\section{Conclusions}

Data about non-antiresorptive-related $\mathrm{ONJ}$ is sparse and based mostly on case-reports. ONJ related to "non-antiresorptives" may have better prognosis compared to the BTA-related, suggested by greater likelihood of healing and shorter healing time. The delay to diagnosis highlights the need for more education. This is the first attempt to determine characteristics of the non-antiresorptive related ONJ and compare with the BTArelated ONJ.

\section{eP036}

CHANGES IN EPIDEMIOLOGICAL CHARACTERISTICS OF PATIENTS DIAGNOSED WITH MEDICATION RELATED OSTEONECROSIS OF THE JAW (MRONJ): THE SHEBA MEDICAL CENTER EXPERIENCE OF THE PAST FIFTEEN YEARS

S. Whitefield ${ }^{1,2}$, T. Sorel Lazarovici ${ }^{3}$, R. Yahalom $^{3}$, S. Friedlander -

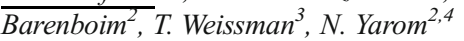

${ }^{1}$ Goldschleger School Of Dental Medicine- Tel-Aviv University- Israel, Department Of Oral Pathology And Oral Medicine, Tel Aviv, Israel

${ }^{2}$ Sheba Medical Center-Tel-Hashomer-Israel, Oral Medicine Unit, Tel Aviv, Israel

${ }^{3}$ Sheba Medical Center- Tel Hashomer, Department Of Oral And Maxillofacial Surgery, Tel Aviv, Israel

${ }^{4}$ Goldschleger School Of Dental Medicine- Tel-Aviv University- Israel, Department Of Dental Medicine, Tel Aviv, Israel

\section{Introduction}

MRONJ is affecting a dynamic population of patients, who received medication for osteoporosis and oncology treatment, the demographics are changing due to fluidity in treatment protocols. A change that the relevant health care givers need to be constantly up to date with.

Objectives

The aim of this study is to track the development of the epidemiological characteristics in the various patient populations diagnosed with MRONJ at the Sheba Medical Center.

Methods

The files of patients diagnosed with MRONJ from 2002-2016 were retrieved. MRONJ was diagnosed according to AAOMS diagnostic criteria. Data on demographics, medical background, type and duration of drug use and triggering events at presentation was collected.

Results

The study included 432 patients, 327 females (76\%) and 105 males (24\%). $63 \%$ of patients were in the $60-79$ year age bracket. There was a sharp increase in cases between 2008-2009, followed by a steady increase 2009-2012, and a slight decrease 2012-2016. A decrease in the proportion of multiple myeloma patients and an increase in proportion of patients with bone metastases of solid tumors, presenting with MRONJ has been observed in the past few years. In addition, an increase in proportion of cases in osteoporosis patients compared with oncology patients is evident.

Conclusions

As a result of changes in drug and treatment protocols and increased awareness of oncology care givers, including referral and consultation with specialists in Oral Medicine, there has been a change in the demographics and presentation of the disease.

\section{eP037 \\ DIFFICULTIES IN PROVIDING PALLIATIVE CARE IN RURAL INDIA (WEST BENGAL) - EXPERIENCE OF AN NGO}

M. Aditya $^{l}$

${ }^{T}$ Advocacy, Palliative Care, Purba Medinipur, India

\section{Introduction}

As in any developing countries state of West Bengal in India has a huge burden of cancer patients in advanced stage coming from rural area where awareness regarding the usefulness of palliative care in rather poor.

\section{Objectives}

Our goal is to give a pain free good quality of life in these advanced stage cancer patients. Objective of this study is to identify the main difficulties in achieving the above goal in a rural village setting in India.

\section{Methods}

Advanced cancer patients in need of palliative care in various villages in of rural India were selected for this study. Their symptoms and managements in that rural surroundings were evaluated by an NGO (under the guidance of a senior palliative care specialist) working in that area. An attempt was made to identify the main obstacles in getting proper palliative care in a rural setting.

\section{Results}

Pain, fatigue are the main symptoms effecting these patients. In most patients pain and other symptoms control were grossly inadequate due to lack of properly trained manpower in the rural India. However regular homecare visits by a group of social workers were of immense help in the last few months of life. NGO team was well guided by a palliative care specialist.

\section{Conclusions}

There is a wide gap of trained manpower in this filled in rural areas of India. Dedicated groups from rural area itself need encouragement and proper training, so that difficult symptoms can be managed locally along with necessary social and psychological support to these patients.

\section{eP038 \\ CANCER PAIN CONTROL BEFORE AND AFTER REFERRAL TO PALLIATIVE CARE CENTER IN KUWAIT}

\author{
A. Alansari ${ }^{l}$ \\ ${ }^{T}$ PCC, Palliative Medicine, Kuwait, Kuwait
}

\section{Introduction}

Still ineffective cancer pain management is a common problem especially among palliative care patients.

\section{Objectives}

Objectives: We aimed to assess the control of pain in cancer patients before and after referral to Palliative Care Center (PCC) in Kuwait.

\section{Methods}

Methods: Prospective cohort study was done on 35 adult patients with confirmed advanced cancer disease who were referred to PCC. Data was collected about pain severity and pain medications. Patients were assessed for pain on day of referral, day three, six and fourteen from referral to PCC.

Results

Results: Mean age was $58.7( \pm 14.79)$ years, 19 patients $(54.3 \%)$ were males. Mean Pain scores at time of referral to PCC 6.17( \pm 2.41$)$ while at Day 3 after referral was 3.94( $( \pm 1.76)$, Day 6 was 2.5( \pm 1.94$)$ and Day 14 was $1.7( \pm 0.8)$. Before referral to palliative care services, $42.9 \%(n=15)$ had severe pain and no patient was pain free while at Day 3 after referral, only $8.6 \%(n=3)$ had severe pain and $40 \%(n=14)$ had mild pain. By Day 6 , no patient had severe pain ( $p$ value $<0.001$ ). Using of adjuvant medications were statistically significant improved after referral to PCC. Before Referral, only $28.6 \%(n=10)$ of patients were on adjuvant medications but after referral, this percentage was increased to $71.4 \%(n=27)$ at Day 0 ( $\mathrm{p}$ value 0.020 ) and to $82.9 \%(\mathrm{n}=29)$ at Day 14 ( $\mathrm{p}$ value $<0.001$ ).

\section{Conclusions}

Conclusion: Referral to palliative care services is significantly improving control of pain in patients with advanced cancer. Routine and earlier referral to palliative care services is recommended. 
eP039

THE EFFECTIVENESS OF PALLIATIVE CARE SERVICE TO CONTROL CANCER PAIN

A. alsuhail ${ }^{1}$

${ }^{T}$ KING FA HAD MEDICAL CITY, Palliaitve care department, RIYADH,

Saudi Arabia

\section{Introduction}

Pain is one of most common symptoms in cancer patients. The pain in cancer patients is severe and progressive. The majority of the cancer patient's complaining of uncontrolled pain which makes their life miserable. Unfortunately, the pain in cancer patients is under-diagnosed and under treatment in the majority of cases.

Objectives

The aim of this study is to assess the effectiveness of palliative care service in control pain for cancer patients.

\section{Methods}

The palliative care nurse coordinator ( $\mathrm{PCNC}$ ) assesses pain severity based on numerical rate scale (NRS). PCNC assesses pain two times for each patient. The 1 st time before intervention and 2 nd time after one week of intervention or at discharge day if patients going home before seven days. The intervention is treating cancer pain management guidelines based on WHO ladder.

Results

The number of patients seen during the 12 months study period was 377 patients. The prevalence of cancer pain was $87 \%$, and the prevalence of moderate to severe pain was $56 \%$. The average pain score reduced from 5.29 (SD 2.84) at initial assessment to 1.42 (SD 1.5) after intervention (pvalue less the 0.001).

Conclusions

Majority of cancer patients has uncontrolled pain. The palliative care intervention is very effective to control cancer pain especially moderate to severe pain. Early referral to palliative care is highly recommended.

\section{eP040}

IS THERE A NEED TO RE-INTERPRET THE SCIENCE OF PROGNOSTICATION IN CANCER PAIN - RESULTS AND ANALYSIS OF AN OBSERVATIONAL TRIAL IN ADVANCED CANCER

R.D. Arora ${ }^{1}$

${ }^{T}$ All India Institute of Medical Sciences- New Delhi, Palliative Medicine, Ansari Nagar-New Delhi, India

\section{Introduction}

The Edmonton Classification System for Cancer Pain (ECS-CP) has been developed for the characterization of Pain. The number of days to achieve stable pain control has been used as a surrogate measure for the complexity of pain control. Complex pain syndromes usually require a longer time period to achieve stable pain control. The minimum number of follow up visits planned (3) were not completed which prompted a rethink on the idea of prognostication. An essential element of the science of prognostication is the component of survival which needs to be included in the ECS CP. Neutrophil Lymphocyte ratio (NLR) and Platelet Lymphocyte ratio (PLR) have been associated with poor prognosis in advanced cancer. Objectives

This paper puts forward an argument to combine these haematological indices as a marker of survival within the existent framework of ECS-CP. Methods

The recording of baseline pain was done at the initial visit by ECS-CP. Acute exacerbation of Pain (AEP) was recorded using the Alberta breakthrough pain assessment tool (ABPAT). A Patient self record of number of AEP in a day, opioid induced adverse effects and regularity in taking medication was maintained.

\section{Results}

A weak positive correlation was obtained between NLR, PLR and Pain intensity

Conclusions

A composite measure for prognostication of Pain which consists of the following components - pain assessment tools (ECS-CP, ABPAT), biochemical parameters (serum creatinine) and haematological parameters (NLR, PLR) needs to be developed.

\section{eP041}

CREATION OF A REGIONAL COOPERATIVE NETWORK IN FRANCE FOR INTRATHECAL ANALGESIA

A. Boden ${ }^{l}$, J. Pouymayou ${ }^{l}$, C. Combis ${ }^{2}$, M. Bourgouin-Ariba ${ }^{l}$, V. $\overline{\text { Mauries-Saffon }}{ }^{1}$, N. Caunes-Hilary ${ }^{1}$

${ }^{I}$ Institut Universitaire du Cancer Toulouse-Oncopole, Supportive Care, Toulouse, France

${ }^{2}$ Institut Universitaire du Cancer Toulouse-Oncopole, Pharmacy, Toulouse, France

\section{Introduction}

Intrathecal analgesia using implanted pump is an innovative and eficient treatment for intractable cancer pain. In France, only few referent centers like the Institut Universitaire du Cancer de Toulouse - Oncopole (IUCTO) may implement and refill pumps. Because of long and regular journeys for refilling necessity, a lot of patients do not benefit from this technique.

\section{Objectives}

The main objective is to create a regional cooperative network in french southwest: IUCT-O should remain the referent center for pump implemantation, but refilling procedure would be done in local hospitals in order to avoid patients long traveling.

Actually there is only two networks of this kind in France.

\section{Methods}

The IUCT-O pharmacy will be in charge of blend preparation (morphine, ropivacaine and ziconotide) and the supportive care department will train the local algologist / palliative care teams in intrathecal analgesia. All the refilling and programmation procedure will be delegated.

Results

The project has been under discussion with french health authorities for two years. Final autorization is currently under examination.

\section{Conclusions}

Refilling procedure is the main constraint standing in the way of intrathecal analgesia development. Creating this cooperative network will allow more patients to benefit from it, and also to get more comfort in their medical care.

\section{eP042}

THE RELATIONSHIP BETWEEN PAIN, ANALGESICS AND SURVIVAL IN PATIENTS WITH ADVANCED CANCER. A SECONDARY DATA ANALYSIS OF THE EUROPEAN PALLIATIVE CARE CANCER SYMPTOM STUDY

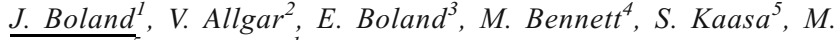 \\ $\overline{\text { Hjermstad }}^{3}$, M. Johnson ${ }^{1}$ \\ ${ }^{1}$ University of Hull, Hull York Medical School, Hull, United Kingdom \\ ${ }^{2}$ University of York, Hull York Medical School, York, United Kingdom \\ ${ }^{3}$ Hull and East Yorkshire Hospitals, Palliative medicine, Hull, United \\ Kingdom \\ ${ }^{4}$ University of Leeds, School of Medicine, Leeds, United Kingdom \\ ${ }^{5}$ Norwegian University of Science and Technology, Faculty of Medicine, \\ Trondheim, Norway
}




\section{Introduction}

Opioids are used to reduce cancer-related pain but an association between shorter survival and longer-term opioids has been observed. A better understanding of the relationship between pain, analgesics, cancer and survival is needed to inform clinical decision making.

Objectives

To investigate the relationship between pain, analgesics and survival within the European Palliative Care Cancer Symptom (EPCCS) prospective cohort study.

\section{Methods}

Secondary analysis of the EPCCS study. This included adults with advanced, incurable cancer receiving palliative care. A multilevel Weibull survival analysis model, adjusted for age, gender, Karnofsky Performance Status, cancer type and stage, was used to examine the association between pain, analgesics and time to death.

\section{Results}

The study included 1739 patients (average age 65.8 years (SD:12.4) range 21-97; men 50\%). Date of death or last follow-up was available for 1404 patients and the mean survival from baseline was 46.5 (1.5) weeks (95\% CI:43.6-49.3) and the median survival was 24 weeks, (IQR 8 to 69). Pain was reported by $76 \%$; $51 \%$ were taking non-opioid analgesics, $60 \%$ opioids and $24 \%$ co-analgesics. Opioids were associated with decreased survival $(\mathrm{p}<0.001)$, although causality cannot be determined. A sensitivity analysis of a subset with C-reactive protein $(C R P)(n=219)$ measures showed higher CRP measures were related to poorer survival $(\mathrm{p}=0.001)$; the relationship between survival and opioids remained.

Conclusions

Opioids were associated with reduced survival, which was maintained in the subgroup analysis adjusted for CRP. Prospective, powered studies are needed to confirm this finding to judge the benefits and risks of opioids in advanced progressive disease.

\section{eP043}

PREDICTORS AND TRAJECTORY OF PERFORMANCE STATUS IN PEOPLE WITH ADVANCED CANCER. A SECONDARY DATA ANALYSIS OF THE EUROPEAN PALLIATIVE CARE CANCER SYMPTOM STUDY

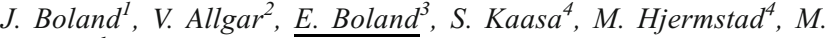 Johnson ${ }^{l}$ \\ ${ }^{1}$ University of Hull, Hull York Medical School, Hull, United Kingdom \\ ${ }^{2}$ University of York, Hull York Medical School, York, United Kingdom \\ ${ }^{3}$ Hull and East Yorkshire Hospitals, Palliative Medicine, Hull, United Kingdom \\ ${ }^{4}$ Norwegian University of Science and Technology, Faculty of Medicine, Trondheim, Norway}

\section{Introduction}

Performance status, a predictor of cancer survival and ability to maintain independent living, deteriorates in advanced disease. An understanding of predictors of change in performance status could help identification and management of those at risk of functional deterioration. Palliative care interventions may help support independent living and reduce health and social care costs. The relationship between symptoms, analgesics and performance status are poorly delineated.

Objectives

To explore whether quality of life, medication use and stage of disease predict the trajectory of Karnofsky Performance Status (KPS) in people with advanced cancer.

\section{Methods}

Secondary data analysis of the prospective, longitudinal European Palliative Care Cancer Symptom (EPCCS) study. This included adults with advanced, incurable cancer receiving palliative care, without severe cognitive impairment and who were not imminently dying. A multivariable regression model was built for KPS area under the curve per day.

\section{Results}

At baseline 1739 people were included and 1090 patients died. The mean daily KPS AUC was 41.1 (14.1). Opioids $(\mathrm{p}<0.001)$, co-analgesics $(\mathrm{p}=0.023)$, poorer physical functioning $(\mathrm{p}<0.001)$ and appetite loss $(\mathrm{p}=0.009)$ at baseline were explanatory factors for lower KPS AUC $\left(\mathrm{r}^{2}=34.8 \%\right)$. A subgroup analysis of participants with C-Reactive Protein $(C R P)$ data $(n=240)$, showed that only CRP $(p=0.040)$ and physical function $(\mathrm{p}<0.001)$ were associated with lower KPS AUC $\left(\mathrm{r}^{2}=34.0 \%\right)$.

\section{Conclusions}

Interventions targeting physical function, appetite and inflammation, such as those used for cachexia management, may help maintain KPS in people with advanced cancer. Further research assessing this association and the impact of managing systemic inflammation on clinical outcomes is needed.

\section{eP044}

AUDIT OF USE OF FENTANYL IN CONTINUOUS SUBCUTANEOUS INFUSION BY HOSPITAL SPECIALIST PALLIATIVE CARE TEAM

\author{
J. Brennock ${ }^{l}$, A. O'Gorman ${ }^{1}$ \\ ${ }^{T}$ Our Lady Of Lourdes Hosptial Drogheda Co. Louth, Palliative \\ Medicine Department, Drogheda, Ireland
}

\section{Introduction:}

Fentanyl is a strong mu opioid receptor agonist used by Specialist Palliative Care Team (SPCT) in continuous subcutaneous infusion (CSCI) for treatment of pain and dyspnoea in a specific patient cohort, and guidelines for its use have been developed locally to ensure safe and effective prescribing in practice.

Objectives

To measure current use of fentanyl in CSCI against set guidelines

Methods

Standards:

Locally produced and ratified - A Guideline for the Use of Fentanyl by Continuous Subcutaneous Infusion in a Syringe Pump by Specialist Palliative Care (SPC) Practitioners. These standards state that Fentanyl via syringe pump may be considered for use in patients who have: 1)An estimated glomerular filtration rate (eGFR) below $30 \mathrm{ml} / \mathrm{min}$, 2)Developed opioid toxicity 3)Previous history of opioid toxicity

\section{Results}

25 in-patients who were receiving SPCT input, 10 were on CSCI, with 8 of them (80\%) on Fentanyl. 2 (25\%) had eGFR <30, and $3(37.5 \%)$ had previously been opioid toxic, leaving 3 patients (37.5\%) who were on Fentanyl in CSCI which did not fit the criteria as set out in guidelines. On re-auditing post education: 25 in-patients who were receiving SPCT input, 9 were on CSCI, with 2 of them $(22.22 \%)$ on Fentanyl. 2 (100\%) had eGFR $<30$, which fulfilled criteria for use of Fentanyl in CSCI.

\section{Conclusions}

Interval objective improvement in use of Fentanyl in CSCI as per Guidelines following education session

\section{eP045}

A COMPARISON OF QUALITY OF LIFE, SEXUAL FUNCTION AND DEPRESSION BETWEEN WOMEN WITH GYNECOLOGICAL CANCERS AND RACE-MATCHED HEALTHY CONTROLS

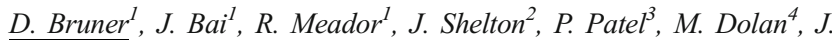

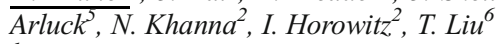

${ }^{1}$ Emory University, Nell Hodgson Woodruff School of Nursing, Atlanta, USA

${ }^{2}$ Emory University, School of Medicine, Atlanta, USA
} 
${ }^{3}$ Emory University, Radiation Oncology, Atlanta, USA

${ }^{4}$ Emory University, Obstetrics and Gynecology, Atlanta, USA

${ }^{5}$ Emory University, GYN OB: General, Atlanta, USA

${ }^{6}$ Emory University, Department of Radiation Oncology, Atlanta, USA

\section{Introduction:}

Treatments, especially radiotherapy (RT) for gynecologic cancers (GynCa), are associated with significant quality of life (QOL) decrements, however, few studies have compared patients to healthy controls and most reports under sample Blacks.

Objectives

Compare QOL, sexual function, and depression between women with GynCa and race-matched healthy controls.

Methods

Patients with GynCa were assessed at baseline (pre-RT) and 6 mos. postRT. Healthy controls who were post-menopausal were recruited at routine gynecology visits with one assessment timepoint. Both groups completed: Functional Assessment of Cancer Therapy-General (FACT-G), Female Sexual Function Index (FSFI), and Patient Health Questionnaire (PHQ-9). Spearman's correlations and Mann-Whitney U were used to examine associations between variables.

Results

Analysis includes 80 participants (27 GynCa and 53 controls) $(50 \%$ White, $50 \%$ Black). Groups were balanced on marital status. Patients compared to controls were younger ( 54 yrs. vs 63 yrs; $p=0.007$ ) and more met criteria for depression $(\mathrm{p}=0.02)$. Patients and controls both met criteria for sexual dysfunction at baseline. Patients had more sexual dysfunction preRT and lower QOL. 6-mos. post-RT patients showed improved sexual function compared with pre-RT, and similar results to controls. Racial comparisons demonstrated no differences between White and Black controls on any measure. White GynCa reported lower FSFI scores, less desire, and more pain than Black patients; Black and White's patients reported lower QOL than matched controls.

Conclusions

Women with cancer reported more depression and lower QOL than healthy controls at baseline and 6 mos. post-RT. Among GynCa, white women had more sexual dysfunction than Black women.

\section{eP046 \\ DEMOGRAPHICS OF OPIATE USE IN A REGIONAL ACADEMIC CANCER CENTER}

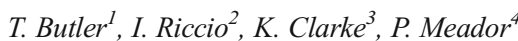

${ }^{T}$ Mitchell Cancer Institute, Medical Oncology, Mobile, USA

${ }^{2}$ Tulane University, Pre-medical, New Orleans, USA

${ }^{3}$ Howard University, Pre-Medical, Washington, USA

${ }^{4}$ Mitchell Cancer Institute, Administration, Mobile, USA

\section{Introduction:}

The national concern about opiate prescription safety and monitoring is coupled with the concern by oncologists to assure access of cancer patients to pain control. Identifying associated socioeconomic and other risk factors for patient's opiate use in the hematology-oncology population would allow insight into their care. A pilot quality assurance project allows exploration of characteristics of patients receiving opiate therapy to better understand the prescribing patterns in these patients.

Objectives

To perform a random review of demographics of 100 consecutive patients in a hematology-oncology clinic to identify important trends to predict the use of narcotic analgesics in this population

Methods

100 consecutive patient's charts were reviewed at university-affiliated cancer research institute as a quality assurance measure. Data gathered included age, gender, race, marital status, diagnosis, cancer stage, narcotics or anxiolytics prescribed, use of tobacco,alcohol, employment,
BMI and pain and fatigue scores, and these parameters analyzed for association with opiate use.

\section{Results}

$47 \%$ of the patients had narcotic use with a tendency to use of anxiolytics, antidepressants, positive tests for illicit drugs, to be previous or current smokers. In relation to pain, fatigue, income, and BMI, the models did not predict the use of narcotics, with $1.5 \%$ of narcotics use attributed to median household income, and the largest contributors fatigue $(13.3 \%)$, and pain $(8 \%)$.

\section{Conclusions}

Although there was a tendency to association of narcotic use among the patients sampled to use of anxiolytics, antidepressants, tobacco and illicit drugs, further analysis in larger populations would be warranted to better understand the patterns of use in these patients

\section{eP047}

INTRAVENOUS NALOXONE UTILIZATION AMONG HOSPITALIZED CANCER PATIENTS ON OPIOIDS AT A TERTIARY CANCER CENTER

S. Dalal ${ }^{1}$, L. Cheng ${ }^{2}$, A. DeJesus ${ }^{2}$, A. Rodriguez ${ }^{3}$, E. Bruera $^{1}$

${ }^{T}$ UT MD Anderson Cancer Center, Palliative Care- Rehabilitation and Integrative Medicine, Houston, USA

${ }^{2}$ UT MD Anderson Cancer Center, Department of Clinical Effectiveness, Houston, USA

${ }^{3} U T$ MD Anderson Cancer Center, Department of Lymphoma and Myeloma- VP Medical Affairs-, Houston, USA

\section{Introduction}

Intravenous naloxone is used to reverse respiratory depression and progressive sedation resulting from opioid overdose. Monitoring hospital's use of naloxone is an important metric to examine trends related to opioid safety.

\section{Objectives}

To quantitate naloxone use among hospitalized adult ( $>18$ years) cancer patients at a tertiary cancer center.

Methods

Inpatient admissions between January 01, 2017 and December 31, 2017 were retrospectively reviewed. Data collected included admission source (emergency center (EC), outpatient operation theater (OT), or others) and the administration of opioids and naloxone.

\section{Results}

We identified 15,861 unique cancer patients (solid tumors $77 \%$ and hematological malignancies 23\%) that accounted for 27,876 admissions, resulting in 225,997 inpatient-days. Total inpatient-days with opioid use was $150,775(67 \%)$ and inpatient-days with both opioids and naloxone use was 549 (445 unique patients). Incidence rate of naloxone administration per 1,000 inpatient opioid days was $3.64 \%$. Patients admitted via EC had higher frequency of naloxone administration $(1.75 \%, 234 /$ $13,388)$ as compared to OR $(1.41 \%, 95 / 6,726)$ and others $(1.30 \%, 101 /$ $7,762)(\mathrm{P}<0.05)$. Solid tumor inpatients had higher rates of naloxone use than hematological patients $(1.80 \%$ versus $0.93, \mathrm{P}<0.05)$.

\section{Conclusions}

We report on the incidence of naloxone use for opioid overdose among hospitalized cancer patients at a tertiary cancer center. We found patients admitted from the EC to have a higher frequency of naloxone administration as compared to admissions via the OR and others. This information is being used to drive improvements in patient safety.

\section{eP048}

ROLE OF PALLIATIVE RADIOTHERAPY IN BONE METASTASIS IN PATIENTS WITH CANCER IN GANJAVIAN HOSPITAL 
M. Dorchin ${ }^{1}$

${ }^{T}$ Ganjavian Hospital, Oncology, Dezfoul, Iran

\section{Introduction}

The management of painful bone metastases requires multidisciplinary care, with external beam radiation therapy (EBRT) providing relief that is effective and time efficient.

Objectives

Recent treatment-guideline publications for bone metastases greatly define the appropriate use of EBRT for this patient group, and they create a means by which treatment approaches may serve as quality measures of radiotherapy departments.

\section{Methods}

Between 1.6.2012 and 1.6.2013 a total of 48 patients, 33 patients with all Bone metastatic cancers esp. Breast and Prostate cancer was 11 males and 37 females with an average age of 38 years (minimum 27 and maximum 79 years). We reviewed the literature focusing on studies investigating the efficacy of hypo fractionated radiotherapy for bone metastases. We also addressed the problem of treating multiple skeletal lesions with irradiation.

\section{Results}

External beam irradiation that our patients treated in Ahwaz center and other radiotherapeutics centers achieves pain palliation in more than $75 \%$ of patients with bone metastases, even with EBRT down to a single-dose administration. The results of exclusive radiotherapy in the cord compression syndrome depend on a prompt diagnosis, patient presentation and the intrinsic radiosensitivity of tumor cells in three patients. Palsy can always be avoided in these patients.

\section{Conclusions}

In our study the efficacy of external beam irradiation in the palliation of bone metastasis-related symptoms is confirmed by this study, even with short treatments and single-dose administrations. This is important for both patient expectations and the necessity for improved resource allocation with reference to the territorial distribution and waiting lists of radiotherapy centers.

\section{eP049}

CANCER PAIN MANAGEMENT AND PAIN INTERFERENCE WITH DAILY FUNCTIONING AMONG CANCER PATIENTS IN GONDAR UNIVERSITY HOSPITAL

\section{E. Gebreyohannes ${ }^{1}$, H. Tegegn ${ }^{2}$}

${ }^{T}$ University of Gondar-College of Medicine and Health Sciences, Clinical Pharmacy, Gondar, Ethiopia

${ }^{2}$ University of Gondar, Clinical Pharmacy, Gondar, Ethiopia

\section{Introduction}

Cancer is an increasing public health burden for Ethiopia. Pain is among the most common symptoms in patients with cancer. Despite availability of several established guidelines for the management of cancer pain, many cancer patients frequently receive inadequate pain treatment and undertreatment is well documented.

Objectives

The aim of this study was to assess cancer pain prevalence, cancer pain interference, and adequacy of cancer pain treatment in the oncology ward of an Ethiopian teaching hospital.

Methods

This questionnaire-based study was conducted from February 15 to May 15, 2016, in the oncology ward of GUH, Gondar, North-West Ethiopia using Brief Pain Inventory-Short Form and Pain Management Index.

Results

Of 83 patients, total of $76(91.6 \%)$ cancer patients experienced pain with varying degree of severity, and 7 (8.4\%) patients experienced severe pain. Of the 76 cancer patients with pain, $68(89.2 \%)$ experienced pain interference with their daily activities. Fifty-four $(65 \%)$ patients were receiving inadequate cancer pain treatment with negative Pain Management Index

\section{Conclusions}

Based on the findings of our study, a significant percentage of patients with cancer experience pain of which majority of them were receiving inadequate cancer treatment despite experiencing pain interference with their daily activities. It is very crucial to anticipate and assess pain of the cancer patients as routine clinical practice to optimize analgesic therapy through identifying and intervening barriers of adequacy of pain management, thereby improving patient health outcome and quality of life.

\section{eP050}

TRANSDERMAL BUPRENORPHINE FOR CANCER PAIN MANAGEMENT: EXPERIENCE FROM A TERTIARY CARE CENTRE IN NORTHERN INDIA

\author{
V. Goel ${ }^{1}$, V. talwar ${ }^{1}$, N. patnaik ${ }^{2}$, S. raina ${ }^{1}$, P. dash ${ }^{1}$ \\ ${ }^{T}$ Rajiv Gandhi Cancer Institute and Reasearch Center, Medical \\ Oncology, delhi, India \\ ${ }^{2}$ action cancer hospital-paschim vihar, pathology, delhi, India
}

\section{Introduction}

Buprenorphine is partial agonist activity at the $\mu$-opioid receptor and competitive antagonist activity at the kappa opioid receptor. Transdermal therapeutic system buprenorphine should be of special value in cancer pain control. Such a buprenorphine formulation allows for a stepwise dose increase, similar to that reported for sustained-release morphine.

\section{Objectives}

To evaluate the effectiveness and tolerability of transdermal buprenorphine (TDB) treatment for cancer pain management.

\section{Methods}

In this prospective observational study, seventy-five patients with moderate to severe chronic cancer pain were included. Ages range was 18-70 years. For efficacy assessment Visual Analog Scale and the Wong-Baker FACES Pain Rating Scale were used and patients were also assessed for side effects profile of buprenorphine patch.

\section{Results}

There was significant improvement of visual analog scale and FACES pain scores from the baseline to the second day of application $(P<0.001)$. By the 15 th day, scores reached $1.25 \pm 0.393$ and $1.52 \pm$ 0.35 , respectively $(P<0.001)$. The sedation score increased on the second day to 2 in 7 patients and to 3 in 40 patients. By the seventh day, 50 patients had a sedation score of 1 . All patients returned to baseline by the 15th day. Itching was reported in 16 cases, and erythema occurred in 10 cases. No significant side effects were reported.

\section{Conclusions}

Transdermal buprenorphine was found to be an effective, safe, and welltolerated treatment for cancer-related chronic moderate to severe pain. In this study population, evaluation of vital signs and physical examination did not suggest any safety concerns while using transdermal buprenorphine.

\section{eP051}

VALIDATION OF THE SPANISH VERSION OF THE BREAKTHROUGH PAIN ASSESSMENT TOOL IN CANCER PATIENTS OF MEXICO

R. Gonzalez-Melendrez ${ }^{1}$, M.D.R. Guillén-Núñez ${ }^{2}$, A.P. Leonel ${ }^{3}$, W. Katherine $^{4}$, R.N. Reyna ${ }^{1}$, D.L.R.P. Victor ${ }^{I}$

${ }^{I}$ Instituto Nacional de Cancerologia, Pain clinic, Ciudad de Mexico, Mexico

${ }^{2}$ Instituto Nacional de Cancerología, Pain clinic, Ciudad de Mexico, Mexico 
${ }^{3}$ Universidad Autónoma de Baja California, Graduated, Mexicali- Baja California, Mexico

${ }^{4}$ Royal Surrey County Hospital- London., Supportive and Palliative Care, London, United Kingdom

\section{Introduction}

Currently there are no validated instruments in Spanish that allow the assessment of breakthrough pain in patients with cancer.

Objectives

To translate and validate the Breakthrough Pain Assessment Tool (BAT) in spanish (México)

\section{Methods}

A prospective, descriptive, longitudinal study was conducted in patients with cancer and pain treated in Mexico. Seventy patients participated, 140 questionnaires were analyzed, all patients were above 18 years-old. Through an interview, information was obtained related to their age, sex, oncological diagnosis, pain diagnosis and level of education. The scale was applied twice, with 4 days of difference between both measurements. To assess reliability, the Kappa test and the intra-class correlation coefficient were used. For consistency, Cronbach's alpha test was used.

\section{Results}

Reliability varied between 0.746 in the use of analgesic question, up to 1.00 in pain localization, 13 of the 14 items presented values above 0.8 , and 12 above 0.9 . On average, the consistency was 0.7

\section{Conclusions}

Discussion: We obtained the same alpha of cronbach (0.7) as the scale validated in English and Korean. Our re-test was at 4 days, the original at 24 hours. Possibly, the differences in some items vary, because they were applied in different clinical scenarios, our study is based on patients who attended to pain clinic, where regularly the analgesic treatment is adjusted.

Conclusion: The BAT Scale showed a high concordance and acceptable reliability in Mexican cancer patients.

\section{eP052 \\ ADVERSE DRUG INTERACTIONS OCCURRING IN PATIENTS ON ONCOLOGICAL SUPPORTIVE CARE}

\section{W. Jarostaw \\ ${ }^{T}$ University Hospital, Dept. of Pain Treatment and Palliative Care, KRAKÓW, Poland}

\section{Introduction}

In a patient undergoing supportive care in oncology and in palliative medicine, drug interactions may change the effectiveness of treatment and may increase the risk of side effects.

\section{Objectives}

The study analyzes the complications that occurred in 114 patients treated as part of adjuvant treatment and palliative medicine

Methods

A cause and effect relationship was assessed between pharmacological treatment and clinical picture of complications, looking for a combination of drugs that could interact with each other in pharmacokinetic and pharmacodynamic interactions

\section{Results}

Out of 114 cases of complications in 86 cases, associations of drugs that could be responsible for the emerging complications were identified. In 74 cases, it was pharmacokinetic interactions, while in 12 cases the interactions were pharmacodynamic. In this group of patients, the interactions concerned analgesics, spasmo- lytics, propulsive drugs, antifungals, as well as drugs from the group of benzodiazepines and neuroleptics. In most cases, interactions occurred at the cytochrome P450 level and were predictable.

\section{Conclusions}

The problem of drug interactions in palliative medicine and supportive care in oncology is gaining more and more practical significance, unfortunately it is neglected, which fundamentally changes the safety profile of the pharmacotherapy used

\section{eP053}

\section{DOES THE CHOICE OF A DRUG TO TREAT BREAKTHROUGH PAIN REQUIRE INDIVIDUALISATION?}

\section{J. Woron ${ }^{1}$}

${ }^{T}$ University Hospital, Dept. of Clinical Pharmacology Jagiellonian University College of Medicine Krakow, Krakow, Poland

\section{Introduction}

The choice of drugs used in breakthrough pain in a patient with cancer should be individualized. The fast and short-acting fentanyl preparations available on the pharmaceutical market today differ in pharmacokinetic parameters and tolerability.

Objectives

The study compares the frequency of side effects after using 4 different medicines containing fentanyl for the treatment of breakthrough pain.

\section{Methods}

94 reports were reviewed, which included a description of the side effects that occurred in patients who received the above-mentioned medicines containing fentanyl as an active ingredient. The causal relationship between the drugs used and the adverse effects described was analyzed

Results

In 42 cases, the side effects of Effentora have been described, the most common symptoms including burning and burning sensation at the place of application and were more pronounced when the patient was used with concomitant inflammatory or infectious lesions on the oral mucosa. In 30 cases, side effects occurred in patients taking PecFent and in most cases reported a feeling of nasal congestion and difficulty in breathing. In 15 cases, side effects have been described which have been associated with the use of Instanyl. In 7 cases, side effects occurred after the sublingual application of Vellofent and they had local intolerance in the form of oral mucosal irritation. In the largest number of $n=50$ cases, the PecFent and Effentora drugs were rotated into other fast and short-acting medicines containing fentanyl.

\section{Conclusions}

The choice of the drug used to treat breakthrough pain requires individualisation

\section{eP054}

\section{"AUDITING FINANCIAL PAIN OF CANCER PATIENTS"}

S. Joshi ${ }^{1}$, U. Joshi ${ }^{2}$

${ }^{T}$ Hospice india, Palliative medicine, Delhi, India

${ }^{2}$ Ascent capital, Finance, Delhi, India

Introduction

Audit measures practice against performance. Unlike research (which asks the question, "What is the right thing to do?"), 
clinical audit asks, "Are we doing the right thing in the right way (1)

Cancer treatment is one of the most costly treatments throughout the world. Financial pain is a growing concern and may at times lead to discontinuation of oncological treatment (2-11).

Objectives

Audit financial pain

Methods

METHODS AND RESULTS

A complete audit cycle, was done i.e.:

1. Setting an audit standard- at least $80 \%$ of patients should have a financial assessment completed, as part of their initial assessment on the existing institutional pain form.

2. Measuring current practice- pain assessment forms of cancer patients who attended pain clinic over past 6 months were analyzed for adequacy of financial pain assessment on the existing institutional proforma.

3. Results- $53 \%$ patients were found to be completely evaluated for financial assessment.

4. Planning and implementing change- The COST (Comprehensive Score for Financial Toxicity): A FACIT Measure of Financial Toxicity, was discussed and the five questions numbered 6 to 10 in the existing institutional pain form were replaced by the COST - FACIT (Version 1) questionnaire, and assess the results $(12,13)$.

5. Reassessing your practice

\section{Results}

Mentioned above

Conclusions

Audit exercise improved our assessment of financial pain.Results of this audit cycle can provide us a lead into the research for "coupling wealth management needs with oncological treatment".

\section{eP055}

COMPARATIVE STUDIES OF 1-DAY TRANSDERMAL FENTANYL AND 3-DAY TRANSDERMAL FENTANYL : EFFICACY OF OPIOID SWITCHING AND TOLERABILITY ASSOCIATED WITH UP-TITRATION

\section{T. Kanamori ${ }^{1}$ \\ ${ }^{T}$ Kyoto Min-Iren Chuo Hospital, Department of Pharmacy, Kyoto city, Japan}

\section{Introduction}

The pharmacological qualities are said to be "almost" equal between the worldwide standard 3-day transdermal fentanyl patches(3DP) and 1-day transdermal fentanyl patches(1DP), which are more popularly used in Japan. However, the actual clinical differences are unknown.

\section{Objectives}

To compare efficacy of opioid switching and tolerability associated with up-titration.

\section{Methods}

Retrospective study of the electronic medical record was conducted. Cancer patients who had achieved pain control with other opioids and switched appropriately to either 1DP(Fentos Tape $\left.{ }^{\circledR}\right)$ or 3DP(Durotep MT Patch $\AA$ ) was targeted. From these patients, a patient ratio of those who continued to have adequate pain control were compared. Adequate pain control was defined as "Rescue $\leq 2$ doses/day, and STAS(pain control) $\leq 1$, and side effects were tolerable" consecutively for 3 days. For those patients who did not have continued pain control, days required to re-achieve pain control were recorded.

For patients who had an up-titration due to pain aggravation, and continued usage was abandoned due to adverse events, a comparative study was made by dividing the concentration raise into 4 phases (Fig. 2).

Results

100 patients were treated with 1DP and 59 with 3DP (Table1).

\begin{tabular}{|c|c|c|}
\hline & $\begin{array}{l}\text { 1-day patches } \\
(n=100)\end{array}$ & $\begin{array}{c}\text { 3-day patches } \\
(n=59)\end{array}$ \\
\hline Age (years), mean (sd) & $73(11.5)$ & $70(10.3)$ \\
\hline Female & $46(46 \%)$ & $29(49 \%)$ \\
\hline Body mass index $\left(\mathrm{kg} / \mathrm{m}^{2}\right)$, mean $(\mathrm{sd})$ & $19.3(3.8)$ & $21.1(7.3)$ \\
\hline Serum albumin concentration $(\mathrm{g} / \mathrm{dL}$ ), mean $(\mathrm{sd})$ & $2.93(0.67)$ & $2.79(0.60)$ \\
\hline \multicolumn{3}{|l|}{ Use of opioids until start of transdermal fentanyl } \\
\hline Oral morphine & $6(6 \%)$ & $0(0 \%)$ \\
\hline Parenteral morphine & $8(8 \%)$ & $12(20 \%)$ \\
\hline Oral oxycodone & $69(69 \%)$ & $45(76 \%)$ \\
\hline Parenteral oxycodone & $2(2 \%)$ & $0(0 \%)$ \\
\hline Oral tramadol & $15(15 \%)$ & $1(2 \%)$ \\
\hline Oral dihydrocodeine & $0(0 \%)$ & $1(2 \%)$ \\
\hline Oral morphine equianalgesic dose (mg), median (range) & $30(5-240)$ & $30(7.5-150)$ \\
\hline \multicolumn{3}{|l|}{ Primary tumor } \\
\hline Gastrointestinal & $27(27 \%)$ & $21(36 \%)$ \\
\hline Urogenital & $24(24 \%)$ & $9(15 \%)$ \\
\hline Lung & $18(18 \%)$ & $9(15 \%)$ \\
\hline Pancreas & $10(10 \%)$ & $9(15 \%)$ \\
\hline Breast & $10(10 \%)$ & $4(7 \%)$ \\
\hline Liver & $7(7 \%)$ & $4(7 \%)$ \\
\hline Others & $4(4 \%)$ & $3(5 \%)$ \\
\hline
\end{tabular}

The 1DP group had a higher rate of continued pain control after switch (83.0\%vs61.0\%, OR:3.12, 95\%CI:1.49-6.53, p=0.0026). Furthermore, the 1DP group had more patients who re-achieved pain control within 10 days (Fig. 1).

\section{Fig. 1 Time needed for pain control re-achievement of 1DP and 3DP}

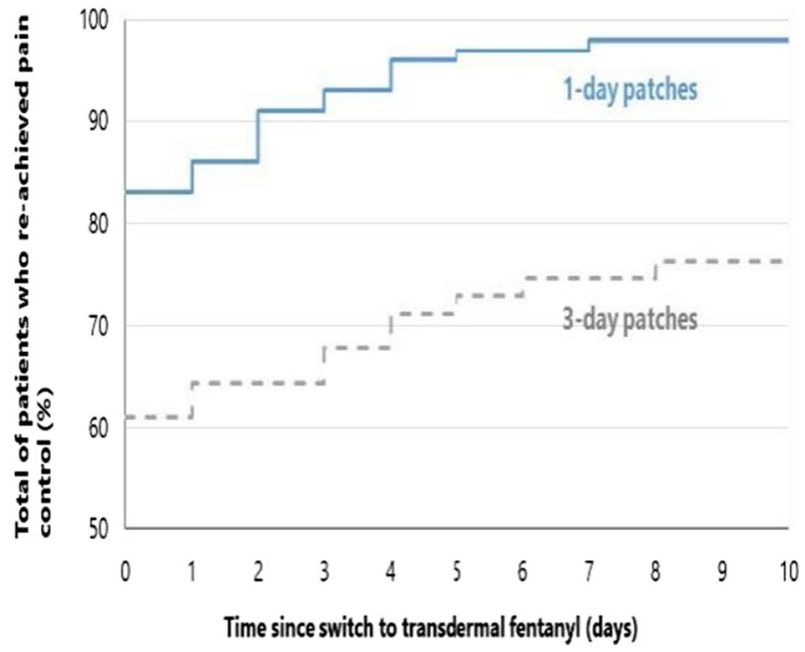


The 1DP group had higher rates of patients who underwent up-titration and continued usage was abandoned(18.2\%vs12.1\%, OR:1.62, 95\% CI:0.62-4.22, $\mathrm{p}=0.32$ ). The intolerance of phase1 especially stood out (Fig. 2).

Fig. 2 A comparative study of adverse events was made by dividing the concentration raise into 4 phases. At phase 1, transdermal fentanyl was increased from 12.5 to $25 \mu \mathrm{g} / \mathrm{h}$; phase 2,25 to $50 \mu \mathrm{g} / \mathrm{h}$; phase 3,50 to $75 \mu \mathrm{g} / \mathrm{h}$; phase 4 , above $75 \mathrm{\mu g} / \mathrm{h}$.

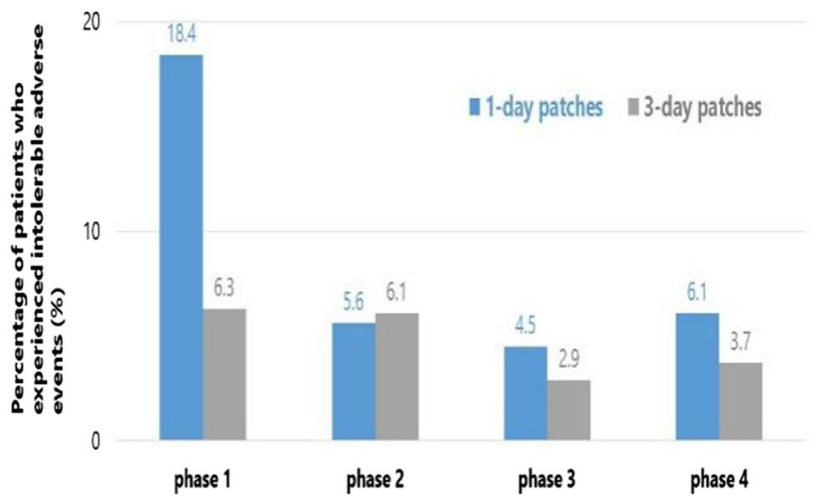

Conclusions

The 1DP switches more effectively than the 3DP, however, tends to have safety disadvantages after up-titration.

\section{eP056}

ANALGESIC AND PREVENTIVE EFFECTS OF FREE RADICAL SCAVENGERS IN CHEMOTHERAPY-INDUCED NEUROPATHIC PAIN IN RATS

H.K. Kim ${ }^{1}$, S.H. Hwang ${ }^{1}$, S. Abdi ${ }^{l}$

${ }^{T}$ The University of Texas MD Anderson Cancer Center, Pain Medicine, Houston, USA

\section{Introduction}

Advances in the treatment of cancer using various types of chemotherapy agents have led to improvement in the survival rate of cancer patients. Unfortunately, pain associated with the chemotherapy including taxanes, vinca alkaloids, and platinum complexes is a dose-limiting adverse effect which affects the quality of life of the survivals.

Objectives

The purpose of study was to determine the analgesic and preventive effects of Tempol, a free radical scavenger, on paclitaxel (PAC)-induced neuropathic pain in rats.

Methods

PAC-induced neuropathic pain was produced by interaperitoneal injections of PAC $(2 \mathrm{mg} / \mathrm{kg}$ on days $0,2,4,6)$ in adult male Sprague-Dawley rats. The pain behavioral tests as well as western blotting and live cell imaging of the L1-6 dorsal root ganglia (DRGs) were performed. Tempol was intraperitoneally injected or infused beginning on day 0,6 , or 20 .

\section{Results}

For the analgesic effect, the single injection and infusion of Tempol reduced pain behaviors on day 20. PAC increased the levels of inflammatory cytokines and mitochondrial superoxide anion in the
DRGs tissues and culture. However, Tempol decreased PACinduced cytokines and superoxide anion. For the prevention, the infusion of Tempol beginning on day 0 for 1 week did not affect the development of pain behavior. In contrast, the infusion of Tempol beginning on day 6 for 1 week completely prevented further development of pain behavior.

Conclusions

We conclude that Tempol alleviates and prevents chemotherapyinduced neuropathic pain in rats by reducing the levels of inflammatory cytokines and free radicals in the DRGs.

eP057

FREQUENCY, OUTCOMES AND PREDICTIVE FACTORS ASSOCIATED WITH OPIOID-INDUCED NEUROTOXICITY FOR PATIENTS WITH ADVANCED CANCER RECEIVING PALLIATIVE CARE

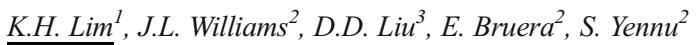

${ }^{T}$ Kangwon National University Hospital, Department of Internal Medicine, Chuncheon-si, Republic of Korea

${ }^{2}$ The University of Texas MD Anderson Cancer Center, Department of Palliative Care and Rehabilitation Medicine, Houston, USA

${ }^{3}$ The University of Texas MD Anderson Cancer Center, Department of Biostatistics, Houston, USA

\section{Introduction}

Opioid-induced neurotoxicity (OIN) is described as a spectrum of symptoms, from drowsiness to hallucinations, myoclonus, seizures, delirium and hyperalgesia.

\section{Objectives}

The aim of this study was to examine the frequency, outcomes, and predictive factors associated with OIN for in patients with advanced cancer receiving palliative care.

\section{Methods}

In this study, the eligibility criteria was: advanced cancer patients admitted to MD Anderson Cancer Center and seen by the palliative care specialist (PCS), $\geq 18$ years of age, and on opioid medication for $\geq 24 \mathrm{hrs}$. Out of 600 patients who were selected randomly from 3014consecutive consults from Jan 2014 to Dec 2014, 390 patients were eligible. Data including patients' demographics, ESAS, diagnosis and management of OIN, and overall survival (OS) were reviewed.

\section{Results}

348 patients were evaluable. $15 \%(\mathrm{n}=52)$ patients had OIN. Of these patients, $46 \%$ and $54 \%$ developed 1 and $\geq 2$ OIN symptoms respectively. The most common OIN symptom was delirium (44\%), followed by drowsiness (40\%), myoclonus (39\%) and hallucination (37\%). Opioid rotation/reduction and hydration were most common interventions for OIN. There was no significant difference in OS between patients with OIN and those without OIN ( $p=0.65)$. In multivariate analysis, high MEDD $(\mathrm{OR}=1.01, p=0.02)$, or high ESAS pain $(\mathrm{OR}=1.27, p=0.043)$, ESAS drowsiness $(\mathrm{OR}=1.28, p=0.007)$, and ESAS appetite $(\mathrm{OR}=1.29$, $p=0.014$ ) were significant associated with OIN.

\section{Conclusions}

OIN was reported in about $15 \%$ of the inpatients referred to PCS. Careful monitoring of OIN is needed for cancer patients reporting severe pain, severe drowsiness, poor appetite or receiving high MEDD.

\section{eP058}

CHEMOTHERAPY-INDUCED PERIPHERAL NEUROPATHY (CIPN) - A DESCRIPTIVE ANALYSIS IN A SPECIALIST CANCER CENTRE 
D. Magee ${ }^{I}$, S. Bachtold ${ }^{1}$, M. Brown ${ }^{I}$, J. Williams ${ }^{1}$, P. Farquhar-Smith ${ }^{1}$ ${ }^{T}$ Royal Marsden Hospital, Pain Management, London, United Kingdom

\section{Introduction}

Advances in cancer diagnosis and treatment have led to an increase in people living with cancer worldwide. Chemotherapy-Induced Peripheral Neuropathy (CIPN) is a significant cause of persistent pain and disability in this population. The incidence and severity of CIPN is often underreported. Identifying the prevalence and characteristics of CIPN may help identify key characteristics for targeted interventions.

\section{Objectives}

Identify the prevalence of CIPN in patients attending pain management clinics. Compare pain characteristics between patients with CIPN and those with non-CIPN pain.

\section{Methods}

Royal Marsden Pain Clinic attendees between January 2016 - November 2017 who consented and had a documented pain diagnosis were included. Data was captured using a bespoke tablet interface, complying with Information Governance requirements and Research Ethics Committee approval (16/LO/1989).

The following data were extracted and compared between the two groups:

- $\quad$ Brief Pain Inventory (BPI)

- Hospital Anxiety and Depression Scale (HADS)

- Patient Global Impression of Change Scale (PGIC)

- Douleur-Neuropathique-4 (DN4)

Results

A total of 601 patients were included, of which $113(18.8 \%)$ had a diagnosis of CIPN. Table 1 demonstrates the differences between the CIPN and non-CIPN population attending our pain management clinics.

Table 1

\begin{tabular}{|l|c|c|}
\cline { 2 - 3 } \multicolumn{1}{c|}{} & CIPN & non-CIPN \\
\hline$n$ & 113 & 488 \\
\hline $\begin{array}{l}\text { Neuropathic pain } \\
\text { (DN4 }>4)\end{array}$ & $85.8 \%$ & $50.7 \%$ \\
\hline $\begin{array}{l}\text { Pain Intensity } \\
\text { (BPI } \geq 7)\end{array}$ & $21.2 \%$ & $19.3 \%$ \\
\hline $\begin{array}{l}\text { Pain Interference } \\
\text { (BPI } \geq 7)\end{array}$ & $15.0 \%$ & $39.5 \%$ \\
\hline $\begin{array}{l}\text { Significant Anxiety } \\
\text { (HADS } \geq 7)\end{array}$ & $24.3 \%$ & $31.9 \%$ \\
\hline $\begin{array}{l}\text { Significant Derression } \\
\text { (HADS } \geq 7)\end{array}$ & $40.5 \%$ & $55.3 \%$ \\
\hline $\begin{array}{l}\text { Significant Improvement } \\
\text { (PGIC } \geq 5)\end{array}$ & $49.4 \%$ & $46.8 \%$ \\
\hline
\end{tabular}

\section{Conclusions}

In a specialist cancer centre, CIPN was seen in almost one-fifth of those attending pain management clinics. Previously reported data has suggested that neuropathic pain is of greater intensity and more difficult to treat. Here, reported pain intensities were similar between patients with CIPN and non-CIPN pain. $49.4 \%$ of patients with CIPN reported improvements (using PGIC) following engagement with the specialist pain service.

\section{eP059}

A COMPARATIVE EFFICACY OF EPIDURAL TRIAMCINOLONE AND BETAMETHASONE FOR
LUMBOSACRAL RADICULAR PAIN IN CANCER PATIENTS: A PROSPECTIVE RANDOMIZED STUDY

S. Mishra ${ }^{1}$, S. Bhatnagar ${ }^{1}$, S. thu-kar ${ }^{2}$

${ }^{T}$ IRCH-AIIMS- ANSARI NAGAR- NEW DELHI- INDIA, ONCOANAESTHESIA AND PALLIATIVE MEDICINE, New Delhi, India

${ }^{2}$ IRCH- AIIMS- Ansari NAGAR-NEW DELHI- INDIA, Radiology, New Delhi, India

\section{Introduction}

Epidural steroids have been in vogue for a long time for treatment of nonspecific low back pain as well as radicular pain of specific or undefined origin.

Objectives

- To compare the efficacy and side effects of Triamcinolone and Betamethasone in cancer patients with severe lumbosacral radicular pain.

- Opioid sparing effect of these agents.

- Assessment of improvement in Quality of life

Methods

After ethics committee approval; all cancer patients with lumber radicular pain were randomized in two groups: 1. Group 1- Epidural Betamethasone $12 \mathrm{mg}$

2. Group 2 -Epidural Triamcinolone $60 \mathrm{mg}$. Oral morphine was given as rescue analgesics in all patients. Patients pain score was assessed by VAS, satisfaction score by (0-10), Quality of life score (ECOG), rescue analgesic and side effects were also noted. All parameters were assessed immediately after the procedure, 1 week, 1 month and 3 months after the procedure.

Results

- There was significant improvement in back ache immediately after the procedure in both groups. At one week, 59\% of Group 1 and $73 \%$ of Group 2 showed improvement in lower back pain and lower limb pain $(\mathrm{p}=0.002)$. At one month $58 \%$ of Group 1 and $75 \%$ of Group 2 had improvement in leg or buttock pain. At three months, $54 \%$ of betamethasone and $71 \%$ of Triamcinolone patients showed improvement in lower back pain $(<0.001)$.

\section{Conclusions}

The epidural triamcinolone reduced lower back and radicular pain in more than half the patients. It can be good option for radicular pain in palliative care setting.

\section{eP060 \\ USG GUIDED SUPERIOR HYPOGASTRIC NEUROLYSIS IN PELVIC CANCER PAIN IN PALLIATIVE CARE}

S. Mishra ${ }^{1}$, S. bhatnagar ${ }^{2}$, S. thulkar ${ }^{3}$

${ }^{T}$ IRCH- AIIMS- NEW DELHI- INDIA, ONCO-ANAESTHESIA AND

PALLIATIVE MEDICINE, New Delhi, India

${ }^{2}$ IRCH-AIIMS- New Delhi INDIA, ONCO-ANAESTHESIA and PALLIATIVE MEDICINE, New Delhi, India

${ }^{3}$ IRCH- AIIMS- NEW DELHI- India, Radiology, new delhi, India

\section{Introduction}

Pelvic cancer pain is a chronic condition related to involvement of viscera, pelvic muscular structures or to the involvement of neural structures by tumor. The cancer pain can be effectively treated in $80 \%-90 \%$ of patients with systematic use of WHO analgesic ladder. Selective interventional analgesic therapy is needed in $10 \%-20 \%$ of patients with intractable cancer pain. 


\section{Objectives}

We have done the study to know the efficacy of Ultrasound-Guided Superior Hypogastric Plexus in pelvic cancer pain.

Methods

Study was conducted after Ethics Committee approval. Patients having severe pelvic pain not controlled with opioids were underwent anterior USG guided superior hypogastric neurolysis. Oral morphine was given as rescue analgesic. Various parameters recorded were pain score (VAS-Visual Analogue Scale), functional capacity, global satisfaction score \& adverse effects for three months.

Results

There was significant decrease in VAS score from baseline in all patients $(\mathrm{p}<0.05)$ throughout the study period. The daily morphine consumption was decreased significantly from the baseline, at 1 st week and at one month. There was improvement in functional capacity in patients from the baseline but it was statistically insignificant. The Global satisfaction score was better at 1 st week and at one month. Only one patient had transient weakness of both lower limbs and another had hypotension following the superior hypogastric block.

Conclusions

The anterior USG guided superior hypogastric plexus neurolysis technique is useful in relieving pelvic cancer pain.

eP061

EFFECTS OF TRANSCUTANEOUS ELECTRICAL NERVE STIMULATION ON PAIN AND PHYSICAL SYMPTOMS IN PATIENTS WITH CANCER: A PILOT RANDOMIZED CROSSOVER TRIAL

J. Nakano ${ }^{1}$, T. Fukushima ${ }^{2}$, S. Ishii ${ }^{2}$, K. Ishii ${ }^{3}$, A. Morishita $^{3}, H$. Kawachi $^{4}$, K. Hashizume ${ }^{5}$, K. Ueno ${ }^{5}$, E. Matsuura ${ }^{5}$, Y. Kusuba

${ }^{1}$ Nagasaki University, Department of Physical Therapy ScienceGraduate School of Biomedical Sciences, Nagasaki, Japan

${ }^{2}$ Nagasaki University Hospital, Department of rehabilitation, Nagasaki, Japan

${ }^{3}$ Nagasaki University Hospital, Palliative care team, Nagasaki, Japan

${ }^{4}$ Saga-Ken Medical Center KOSEIKAN, Department of rehabilitation, Saga, Japan

${ }^{5}$ Nagasaki University, Department of Nursing Science- Graduate School of Biomedical Sciences, Nagasaki, Japan

\section{Introduction}

Pain and other physical symptoms including nausea, constipation, and dyspnea are major clinical problems of patients with cancer. When these symptoms cannot be controlled by pharmacotherapy completely, transcutaneous electrical nerve stimulation (TENS) might be used for treatment. However, there are few studies on the use of TENS for physical symptoms of patients with cancer.

Objectives

This pilot study aimed to assess the effects of TENS on pain and physical symptoms in patients with cancer.

\section{Methods}

Patients with cancer (age, 70.1 \pm 7.7 years) were enrolled in this pilot study. Patients were randomly divided and administered with TENS and non-TENS for 1 week in a crossover manner separated by 3 days washout period. Electrical stimulation $(100 \mathrm{~Hz}, 20 \mathrm{~min}, 5$ days/week) was performed at four points: the center of the back for nausea and dyspnea mainly, back at the same dermatomal level as the origin of the pain, and both peroneal nerves for constipation. Pain level, number and quantity of opioid rescue dose, and number of evacuation were recorded. Physical symptoms were evaluated using EORTC QLQ-C15-PAL.
Table 1. Characteristics of cancer patienrts

\section{Variable}

Gender

$$
\begin{array}{r}
\text { Female, } n \\
\text { Male, } n
\end{array}
$$

Age, year

$70.1 \pm 7.7$

\section{Body mass index}

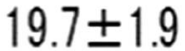

\section{Cancer}

$\begin{array}{rr}\text { Esophageal, } \mathrm{n} & 4(40 \%) \\ \text { Oral, } \mathrm{n} & 2(20 \%) \\ \text { Kidney, } \mathrm{n} & 2(20 \%) \\ \text { Intraperitoneal, } \mathrm{n} & 2(20 \%)\end{array}$

\section{Stage}

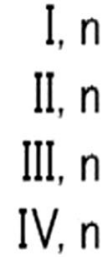

\section{Pain

Visceral, $n$
Neuropathic, $n$
Somatic, $n$

\section{Disease duration, day $\quad 377 \pm 415$}

n: number.

Results

Pain and nausea were reduced by TENS significantly though the number and quantity of opioid rescue dose were not changed. An improvement of the constipation by TENS was shown; however, the change did not reach significance. 
Table 2. Change from pre to post in pain and physical symptom

\begin{tabular}{rrrr}
\hline & TENS Group & non-TENS Group & $P$ value \\
\hline NRS of Pain & & & \\
Average in day & $-0.6 \pm 3.7$ & $0.0 \pm 2.5$ & n.s. \\
Maximum in day & $-2.1 \pm 2.0$ & $1.0 \pm 2.2$ & $\langle 0.01$ \\
& & & \\
EORTC QLQ-C15-PAL & & & \\
Global quarity of life & $4.9 \pm 20.9$ & $13.3 \pm 21.9$ & n.s. \\
Physical functioning & $6.7 \pm 11.7$ & $-2.7 \pm 11.0$ & n.s. \\
Emotinal functioning & $-4.2 \pm 27.0$ & $11.7 \pm 24.9$ & n.s. \\
Fatigue & $-2.0 \pm 28.3$ & $15.9 \pm 34.9$ & n.s. \\
Nausea and vomiting & $-20.0 \pm 34.0$ & $6.7 \pm 8.6$ & $<0.01$ \\
Pain & $-29.2 \pm 21.2$ & $6.6 \pm 8.6$ & $<0.01$ \\
Dyspnea & $-6.7 \pm 21.0$ & $-3.3 \pm 29.1$ & n.s. \\
Insomnia & $3.3 \pm 24.5$ & $0.0 \pm 27.2$ & n.s. \\
Loss of appetite & $-10.0 \pm 22.5$ & $13.3 \pm 28.1$ & n.s. \\
Constipation & $-20.2 \pm 17.2$ & $3.3 \pm 29.1$ & 0.08 \\
\hline
\end{tabular}

n. number. TENS transcutaneous electrical nerve stimulation. ns. not significant:

Table 3. Quantity of opioid rescue dose, evacuation, and performance status in TENS period

\begin{tabular}{|c|c|c|c|}
\hline & TENS Group & non-TENS Group & $P$ value \\
\hline Number of rescue dose, $n /$ day & $2.2 \pm 1.9$ & $2.6 \pm 2.2$ & n.s. \\
\hline Quantity of opioid in day*, $\mathrm{mg} /$ day & $52.7 \pm 94.9$ & $54.0 \pm 94.3$ & n.s. \\
\hline Number of evacuation, $n /$ day & $1.3 \pm 0.6$ & $1.2 \pm 0.5$ & n.s. \\
\hline Performance status & $2.8 \pm 0.4$ & $2.6 \pm 0.5$ & n.s. \\
\hline
\end{tabular}

\section{Conclusions}

This pilot study showed that TENS might improve pain, nausea, and constipation in patients with cancer. Further study is required to examine whether TENS is effective for patients with cancer as one of the palliative therapies.

\section{eP062}

STRATEGIES TO IMPROVE ROUTINE PAIN SCREENING IN AUSTRALIAN OUTPATIENT ONCOLOGY AND PALLIATIVE CARE CLINICS: PROCESS DATA FROM THE STOP CANCER PAIN TRIAL

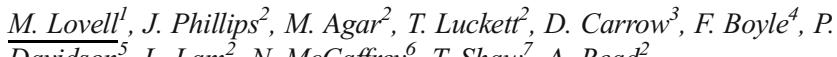
$\overline{\text { Davidson }}^{5}$, L. Lam ${ }^{2}$, N. McCaffrey ${ }^{6}$, T. Shaw ${ }^{7}$, A. Read ${ }^{2}$

${ }^{1}$ University of Sydney, Sydney Medical School, St Leonards, Australia
${ }^{2}$ University of Technology Sydney, IMPACCT - Improving PalliativeAged and Chronic Care through Clinical Research and Translation, Ultimo, Australia

${ }^{3}$ University of Technology Sydney, PaCCSC - Palliative Care Clincial Studies Collaborative, Ultimo, Australia

${ }^{4}$ Mater Hospital, The Patricia Ritchie Centre for Cancer Care and Research, North Sydney, Australia

${ }^{5}$ Johns Hopkins University, Department of Acute and Chronic Care, Baltimore, USA

${ }^{6}$ Deakin University, Deakin Health Economics- School of Health and Social Development, Burwood, Australia

${ }^{7}$ University of Sydney, WEDG - Workforce Education and Development Group, Camperdown, Australia

\section{Introduction}

Cancer Pain is a common and distressing symptom that is often undertreated. Australian Cancer Pain Management Guidelines have been developed, and a guideline implementation trial ('Stop Cancer PAIN') is currently underway at oncology and palliative care outpatient services. Clinical champions at each service are supported to facilitate pain screening and management recommendations.

Objectives

To identify strategies for increasing routine pain screening in outpatient oncology settings.

\section{Methods}

Data collected on the process of pain screening at two outpatient services were compared at three time points.

T1 - End of control phase

T2 - 2.5 months into intervention phase

$\mathrm{T} 3$ - End of intervention phase

\section{Results}

Service one had a $33 \%(1178 / 3521)$ pain screening rate of patient care episodes at T1. T2 saw a reduction in dedicated human resources to routine pain screening and a concomitant decrease in pain screening to $12 \%(430 / 3484)$. Facilitative strategies at this service were associated with an improvement in pain screening rates to $40 \%(315 / 787)$ by T3 and included:

- dedicated clinical staff to routine pain screening

- targeted focal clinics based on patient population

Service two had a screening rate of $17 \%(675 / 4010)$ at T1. At T2, the low screening rate of $17 \%$ (162/950) continued. Support for the importance of routine pain screening and modelling of the behaviour change by a senior clinician/champion, the screening rate improved to $42 \%(1135 / 2678)$ at T3.

\section{Conclusions}

While pain screening numbers remain sub-optimal, clinical champions were able to facilitate improvements by means of: authority to allocate adequate resources, modelling behaviour change and targeting relevant clinics. Successful strategies differed between services.

\section{eP063}

KNOWLEDGE AND ATTITUDE OF BACHELOR LEVEL NURSING STUDENTS REGARDING CANCER PAIN MANAGEMENT: A CROSS-SECTIONAL STUDY

\section{B. Sharma ${ }^{l}$}

${ }^{T}$ National Academy of Medical Sciences NAMS- Bir Hospital Nursing Campus, Nursing, Kathmandu, Nepal

\section{Introduction}

Pain is the worst human experience, if left undiagnosed and untreated, it may negatively affect the quality of life of an individual. For the successful pain assessment and management nurses require sufficient knowledge and appropriate attitudes regarding pain management. 


\section{Objectives}

To determine the knowledge and attitudes regarding cancer pain management among Bachelor nursing students studying in Government College in Nepal.

\section{Methods}

The descriptive, cross-sectional survey conducted with convenient sample of 47 students. Knowledge and Attitudes Survey Regarding Pain was used. Data were analyzed by descriptive statistics.

\section{Results}

The results showed, mean score of knowledge was 23 out of 37 (62.24\% out of $100 \%)$. Less than half of the students $(45 \%)$ recognized patients have pain even when vital signs are stable; respiratory depression rarely occurs while receiving stable opioid dosage; usual duration of 1-2 mg morphine IV is 4-5 hours; and recommended route of opioid is oral for patients with persistent cancer related pain. More than $80 \%$ students have agreed that patients should not be encouraged to endure pain; and there is no role of placebo for active pain management. About $100 \%$ of students agreed sedation assessment is recommended to prevent opioid-induced respiratory depression; and opioid doses should be adjusted in accordance with individual patient's response.

\section{Conclusions}

In general students have average knowledge and positive attitudes towards cancer pain management. Knowledge on pain pharmacology items was lower than that of non-pharmacology items. Study underlines need for review of curricula for in-depth and up-to-date pharmacological pain management and incorporates evidence-based research.

\section{eP064}

COMPARATIVE EFFICACY OF ULTRASOUND GUIDED CELIAC PLEXUS NEUROLYSIS VERSUS C ARM GUIDED TECHNIQUE FOR UPPER GASTROINTESTINAL MALIGNANCY PAIN

\section{S. Singh ${ }^{1}$, S.P. Singh Rana ${ }^{2}$ \\ ${ }^{T}$ Fortis Hospital Noida, Dept of pain \& Palliative medicine, Noida, India ${ }^{2}$ AIIMS Bhopal, Dept of Integrated Oncology, Bhopal, India}

\section{Introduction}

Patients with advanced gastrointestinal malignancies commonly present with pain in upper abdomen of varying severity. Neurolytic celiac plexus block not only alleviate severe pain of cancer but also improves quality of life and increases survival.

\section{Objectives}

Many techniques have been described for celiac plexus neurolysis, here we have compared efficacy of Ultrasound guided celiac plexus neurolysis versus $\mathrm{C}$ arm guided technique for upper gastrointestinal malignancy pain. Methods

20 patients in each group were enrolled into the study. Uncontrolled pain relief with more than $120 \mathrm{mg}$ morphine per day or side effects due to disease or treatment was considered as indication for celiac plexus neurolysis. The pain relief, analgesic consumption, breakthrough pain, changes of any medication, performance status of patients and side effects were recorded in each visit. Results

Pain score (NRS) decreased in both the groups but statistically significantly in ultrasound group from $1^{\text {st }}$ day onward (1.4 vs 1.9 at 1 week, 2.2 vs 2.6 at 1 month, and 1.8 vs 2.8 at 3 month. The Karnofsky score improved from the baseline of 60 to 90 subsequently in both groups. The linear quality of life scale showed an improvement from a scale of 2 to 4 in the follow up period. Only $11.4 \%$ patients required low dose oral morphine months after neurolysis in $\mathrm{C}$ arm group with none patient required morphine in ultrasound group.

\section{Conclusions}

Ultrasound guided techniques offers advantage of better pain relief, less postop discomfort, bed side technique, no radiation hazard, less complications and greater satisfaction for patients.

\section{eP065}

THE ROLE OF CERVICAL EPIDURAL ANALGESIA IN ADVANCED CANCER PAIN

J. Tachibana ${ }^{1}$, S. Hattori ${ }^{1}$, M. Yokota ${ }^{2}$

${ }^{T}$ Cancer Institute Hospital of JFCR, Department of Cancer Pain Management, Tokyo, Japan

${ }^{2}$ Cancer Institute Hospital of JFCR, Department of Anesthesiology, Tokyo, Japan

\section{Introduction}

Cancer pain in high dermatome areas such as jaw, neck, shoulder, and upper extremities can be refractory to systemic opioids. Cervical epidural analgesia may help these patient, although the procedure should be done by well experienced pain physician. This study shows the effectiveness of cervical epidural analgesia for these patients who is suffering with conservative pain treatments.

\section{Objectives}

The goal of this study was to assess the impact of cervical epidural analgesia on the quality of life, including pain scores, opioid dose and activity parameters, who has intolerable cancer pain in neck, shoulder, and upper extremities regardless of high dose opioids.

\section{Methods}

We review the chart from March, 2008 to September, 2017 and collected opioid dose, sleepiness, stress, performance status, and pain scores before and one week after starting cervical epidural analgesia from 37 patients. Parameter was obtained from MD Anderson Pain Inventory score sheet and clinical charts.

\section{Results}

37 patients were included in the study ( 5 head/mandible, 17 shoulder/ upper extremity, and 15 neck pain). One week after the induction of epidural analgesia, there was a significant reduction in NRS(numerical rating scale of $0-10$ ); from 8 to 3 , sleepiness scores; from 6 to 4 , and opioid dose; from $241.6 \mathrm{mg} /$ day (oral morphine equivalent dose) to $53 . \mathrm{mg} /$ day(epidurally given dose). No significant difference was noted in stress scores and performance status scores.

\section{Conclusions}

Our study shows promising efficacy of cervical epidural analgesia in patients with uncontrollable pain in neck and upper extremities. However, procedure should be considered under careful risk/benefit evaluation and pursued by well experienced pain specialists.

\section{eP066 \\ PAIN MANAGEMENT IN GERMAN CANCER PATIENTS - RESULTS OF A CROSS-SECTIONAL ONLINE SURVEY ON CANCER-RELATED BACKGROUND AND BREAKTHROUGH PAIN}

\section{Ueberall ${ }^{1}$ \\ ${ }^{T}$ Institute for Neurological Sciences, IFNAP, Nuernberg, Germany}

\section{Introduction}

Pain is a highly prevalent and one of the most feared complications of cancer. Data on the status quo of cancer pain management for Germany is rare.

\section{Objectives}

Assessment of pain management quality in German cancer patients under real-life conditions.

\section{Methods}

Nationwide German cross-sectional online survey of patients with cancer pain $(\mathrm{CP})$ between February $1^{\text {st }}$ to June $30^{\text {th }}$ 2017. Use of a standardized electronic cancer pain questionnaire to assess cancer-related background (BGCP) and breakthrough (BTCP) pain characteristics and treatment approaches. Use of predefined algorithms to assess medical undertreatment (UT) and malpractice (MP). 


\section{Results}

Participation of 5.576 patients with BGCP, $47.4 \%$ with BTCP. UT and/or MP (either isolated or in combination) occurred in $52.5 / 71.3 \%$ of BGCP/BTCP-patients. Overall, only $37.1 \%$ of patients presented formally with an adequate pain management. UT/MP had a significant impact on reported pain intensities and pain-related suffering. Overall wellbeing in adequately treated patients was reported as either "very good", "good", "satisfying" or "adequate" in $77.1 \%$, vs. $52.1 \%$ in patients with UT, $30.5 \%$ with MP, and $14.7 \%$ with a combination of UT and MP. The odds ratio for UT/MP-patients reporting their wellbeing as either "inadequate" or even "insufficient" vs. those treated adequately was 19.4 (95\%-CI: 16.2-23.3), those for MP-patients 7.7 (6.2-9.5), and for UT 3.1 (2.7-3.6; $\mathrm{p}$ for each comparison $<0.001$ ).

\section{Conclusions}

Adequate pain management of cancer pain patients in Germany is still the exception rather than the rule. UT/MP are frequent and affect 5/10 BGCPand 7/10 BTCP-patients with devastating consequences on affected individuals in comparison to those treated adequately.

\section{eP067}

PHYSICIANS SATISFACTION WITH BREAKTHROUGH CANCER PAIN TREATMENT FOR PATIENTS WITH BONE METASTASIS IN SPAIN CLINICAL PRACTICE

F. Villegas Estévez ${ }^{l}$, C. Ferrer Albiach ${ }^{I}$, M.D. López Alarcón ${ }^{2}$, M. de

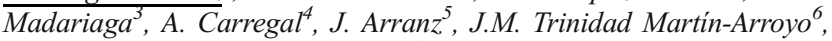
A.J. Jiménez López ${ }^{7}$, A. Sanz Yagüe ${ }^{7}$

${ }^{1}$ Hospital Provincial de Castellón, Pain Unit, Castellón, Spain

${ }^{2}$ Hospital General Universitario de Valencia, Pain Unit, Valencia, Spain

${ }^{3}$ Hospital Universitario Infanta Sofia, Pain Unit, Madrid, Spain

${ }^{4}$ Complejo Hospitalario Xeral Cíes, Pain Unit, Pontevedra, Spain

${ }^{5}$ Hospital Universitario Nuestra Señora de Candelaria, Pain Unit,

Tenerife, Spain

${ }^{6}$ Hospital Puerta del Mar, Pain Unit, Cádiz, Spain

${ }^{7}$ Kyowa Kirin Farmacéutica- S.L.U., Medical Department, Madrid, Spain

\section{Introduction}

An adequate breakthrough cancer pain (BTcP) management with analgesics is essential to improve the functionality of patients with bone metastases. Physicians' satisfaction with BTcP treatment might reflect an optimum BTcP control in patients with metastatic disease.

\section{Objectives}

To assess physicians' satisfaction with $\mathrm{BTcP}$ therapy prescribed for patients with bone metastasis in clinical practice in Spain.

Methods

Observational cross-sectional multicenter study. Pain specialists completed an electronic questionnaire aimed to gather clinical data on their BTcP patients and to evaluate their satisfaction with the treatment they have prescribed to them.

\section{Results}

A total of 79 physicians from radiation oncology (41.7\%), pain units $(27.8 \%)$ and palliative care units $(21.7 \%)$ participated in the survey and collected data from $386 \mathrm{BTcP}$ patients with bone metastatic disease. Most patients $(71.3 \%)$ were prescribed rapid-onset fentanyl preparations alone $(84.7 \%)$ or in combination with other analgesics $(15.3 \%)$, followed by rapid-release morphine $(9,3 \%)$ and NSAIDs $(5,4 \%)$. Most physicians $(71.8 \%)$ reported to be satisfied at some extent with prescribed BTcP therapy $(48.7 \%$ of them moderately satisfied, $37.2 \%$ slightly satisfied, and $14 \%$ extremely satisfied). In addition, mean reported satisfaction was 5 (being 1/extremely dissatisfied, 7/extremely satisfied). According to physicians, the most frequent factor contributing to reduce their satisfaction with treatment was organizational issues in each department $(25.9 \%)$ while only a $13.2 \%$ reported that the lack of efficacy of treatment was the main cause of dissatisfaction.

\section{Conclusions}

The results of our study indicate that, in general, pain specialists in Spain are satisfied with BTcP therapy for patients with bone metastasis.

\section{eP068}

\section{A SYSTEMATIC REVIEW OF POST-TREATMENT PAIN FOR BREAST CANCER}

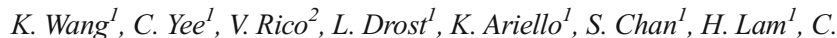
DeAngelis $^{2,3}$, E. Chow ${ }^{1}$

${ }^{1}$ Sunnybrook Odette Cancer Centre, Department of Radiation Oncology, Toronto, Canada

${ }^{2}$ Sunnybrook Odette Cancer Centre, Department of Pharmacy, Toronto, Canada

${ }^{3}$ University of Toronto, Leslie Dan Faculty of Pharmacy, Toronto, Canada

\section{Introduction}

Pain following treatment for breast cancer (BC) and persistent posttreatment pain has been linked to decreased quality of life $(\mathrm{QoL})$ in $\mathrm{BC}$ patients, but the incidence and severity of pain resulting from different treatments are not well-understood.

\section{Objectives}

To investigate the overall incidence and severity of post-treatment pain and to explore the efficacy of rescue treatments for pain and other toxicities in $\mathrm{BC}$ patients.

\section{Methods}

A systematic review of the literature was conducted using MEDLINE, Embase and Cochrane Central databases up to August 2017. Published studies addressing post-BC treatment pain, which reported on the incidence and/or severity of pain were eligible.

Results

Seventy-eight studies evaluated the severity of pain in BC patients due to different combinations of BC therapy. In a total of 39822 patients, $26.7 \%$ reported mild pain, $12.5 \%$ moderate pain and $5.4 \%$ reported severe pain. 22 studies evaluated the efficacy of various rescue versus standard or placebo treatments for the management of pain and related toxicities post-treatment. Of these studies, 10 reported a significant improvement in pain following rescue treatments such as exercise, physical therapy, analgesics and topical products.

\section{Conclusions}

Post-BC therapy pain is common in this population and rescue treatment efficacy could potentially be improved. More research on how specific $\mathrm{BC}$ treatments affect post-treatment pain may help our understanding in maintaining QoL in $\mathrm{BC}$ patients.

\section{eP069}

CHINESE FAMILY CAREGIVERS' PERCEPTION ON OPIOIDS AS ANALGESIC - INSIGHTS FROM CHICAPO SURVEY

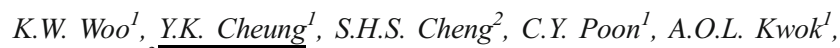
D.M.W. Tse

${ }^{1}$ Caritas Medical Centre, Palliative Care Unit, Kowloon, Hong Kong S.A.R.

${ }^{2}$ The Chinese University of Hong Kong, MBChB Programme, New Territories, Hong Kong S.A.R.

${ }^{3}$ Kowloon West Cluster- Hospital Authority, Princess Margaret Hospital, Hong Kong, Hong Kong S.A.R.

\section{Introduction}

Misconceptions about morphine and opioids as analgesic in cancer pain are thought to be common, however, prevalence was unknown. 


\section{Objectives}

To study the prevalence and correlates of misconceptions about opioids as analgesics among family caregivers of advanced cancer patients receiving palliative care.

\section{Methods}

This is a single centre cross sectional study. Family caregivers were invited to join the study by convenience sampling. Participating caregivers rated their agreement on a 20 -item self-developed questionnaire on opioids misconception using a Likert scale from 1 (very disagree) to 5 (very agree). Descriptive statistics and Chi-squared test were used to compare proportion between groups.

\section{Results}

Among 100 participants, (median age 56 years old), 60\% were female and $77 \%$ were main caregivers.

The 3 most commonly agreed/ strongly agreed items were:

- Morphine is reserved for severe pain. (77\%)

- Morphine is used in late stage but not early stage cancer patients. (61\%)

- Morphine has ceiling effect in pain control. (59\%)

The 3 most commonly disagreed /strongly disagreed items were:

- Taking morphine is shameful to tell the others. (88\%)

- Morphine should not be given to patients even with doctor's prescription. $(80 \%)$

- Morphine should not be used because of the historical pain from Opium Wars $(70 \%)$

In 14 out of 20 items, caregivers with misconceptions were significantly older. "Morphine prescription is a form of euthanasia" and "morphine can hasten death" were disagreed by $60 \%$ and $49 \%$ respondents.

Conclusions

Opioids misconception was common among family caregivers and required more attention and education.

\section{eP070}

\section{A PRECISION MEDICINE, MECHANISTIC APPROACH FOR CLINICAL TRANSLATION OF PHOTOBIOMODULATION THERAPY IN ONCOLOGY}

\author{
P. Arany ${ }^{I}$ \\ ${ }^{T}$ Universiity at Buffalo, Oral biology and Biomedical Engineering, \\ Buffalo- NY, USA
}

\section{Introduction}

The use of light as a therapeutic agent dates back to ancient civilizations. Modern medicine recognized its critical value in both destroying tumors (termed Photodynamic Therapy) as well as promoting therapeutic responses such as alleviation of pain and inflammation as well as promoting tissue healing (termed Photobiomodulation Therapy). More recent exploration has investigated the use of biophotonics treatments for both thermal and non-photothermal anti-tumor immunological responses. While many of these clinical applications are being actively being pursued in the clinic, there remain key questions about fundamental light-biological tissue interactions. Objectives

This work examined the molecular basis of the anti-inflammatory and wound healing promoting abilities of Photobiomodulation (PBM) therapy.

\section{Methods}

Using a combination of cell culture, tissue engineered scaffolds and animal (transgenic mice) models, we generated wound models and examined the effects of various parameters of PBM therapy including wavelengths and dose (Irradiance, fluence and time) on mitigating the inflammatory responses and promoting healing.

\section{Results}

We previously described the ability of near-infrared, low dose lasers to activate a latent TGF- $\beta 1$ complex. This work examined the role of PBMactivated TGF- $\beta 1$ in modulating the inflammatory responses and promoting lineage-restricted responses. We noted individual cell responses could customized by altering PBM treatment protocols for disease phasespecific therapeutic responses.

\section{Conclusions}

These observations together are enabling us to develop a molecular mechanism-based, precision medicine approach to optimizing clinical applications. Our current work is focused on human clinical translation studies for the safe and effective use of PBM therapies in oncology care including oral mucositis and post-radiation fibroses.

\section{eP071}

SUCROSOMIAL IRON: A NEW ALTERNATIVE FOR FIRST LINE ORAL SUPPLEMENTATION IN CANCER PATIENTS WITH IRON-DEFICIENCY ANEMIA

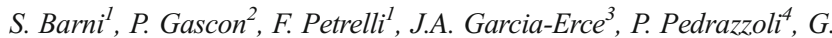

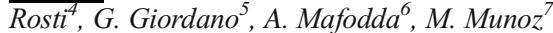

${ }^{1}$ ASST Bergamo Ovest, Oncology, Treviglio, Italy

${ }^{2}$ Hospital Clinic University of Barcelona, Division of Medical Oncology, Barcelona, Spain

${ }^{3}$ Blood and Tissue Bank of Navarra, Director, Pamplona, Spain

${ }^{4}$ Fondazione IRCCS Policlinico San Matteo, Medical Oncology, Pavia, Italy

${ }^{5}$ Regional Hospital 'A. Cardarelli', General Medicine and Hematology, Campobasso, Italy

${ }^{6}$ A.O. Ospedali riuniti B.M.M, Medical Oncology Unit, Reggio Calabria, Italy

${ }^{7}$ School of Medicine- University of Malaga, Perioperative Transfusion Medicine, Malaga, Spain

\section{Introduction}

Disorders of iron metabolism are commonly seen in onco-hematological clinical practice. Iron-deficiency (ID) anemia and cancer-associated anemia are usually treated with supportive therapies. Optimal management of these conditions are fundamental for patient management and quality of life.

Objectives

To summarize what it is considered optimal clinical practice for screening, diagnosis, treatment and monitoring of ID and anemia in cancer patients.

\section{Methods}

A position paper discussing a number of hot topics on anemia in cancer patients has been recently published to cover definitions, prevalence, consequences of anemia and ID. Moreover, based on evidence, recommendations on how to diagnose and monitor iron status and how to management ID with or without anemia were issued. In particular, the paper discusses also the role of newer oral iron formulations, such as Sucrosomial Iron, as well as their safety and cost issues.

\section{Results}

Anemia still represents a major complication of treatment in cancer patients (surgery, chemotherapy, radiotherapy, targeted therapies), aggravating physical impairment, and negatively affecting general outcome. Sucrosomial iron has been shown to have a higher absorption than ferrous sulfate, with excellent tolerability. Moreover, Sucrosomial iron seems to be a valid alternative to IV iron for treating anemia in several clinical settings, such as chronic kidney disease and cancer-related anemia, where inflammation and/or altered iron absorption are present.

\section{Conclusions}

Newer oral iron formulations, such as Sucrosomial iron, may represent a first line option for iron supplementation in case of ID or ID anemia, effectively increasing both hemoglobin levels and adherence to therapy.

eP072

THE PERCEPTIONS AND EXPERIENCES OF OSTEOPATHIC TREATMENT AMONG CANCER PATIENTS IN PALLIATIVE CARE: A QUALITATIVE STUDY 
M. Filbet $^{1}$, A. Steel ${ }^{2}$, T. Monsarrat ${ }^{1}$, C. Tricou ${ }^{l}$

${ }^{7}$ service de soins palliatifs Pavillon $1 K$ CHLS CHU de Lyon, Medecine palliative, Pierre-Benite, France

${ }^{2}$ Endeavour College of Natural Health, Australian Research Centre in Complementary and Integrative Medicine University of Technology, Sydney, Australia

\section{Introduction}

Osteopathy is practised in many countries throughout the world.In France, recently, this complementary medicine is acknowledged as a distinct health profession as well as the required training standards The majority of research on osteopathy focuses on lower back pain or cervicalgia and research which explores the effects of osteopathy on cancer-related symptoms is scarce.

Objectives

This research aimed to explore the perceptions and experiences of cancer patients receiving osteopathic treatment as a complementary therapy when it is used in addition to conventional treatment for cancer pain.

\section{Methods}

This qualitative study employed semi structured interviews of cancer patients in a palliative care in Lyon, France who received treatment from an osteopath alongside their conventional cancer treatment. We analyzed data using grounded theory and qualitative methods.

\section{Results}

We interviewed 16 patients. The themes identified through the analysis included a low awareness of osteopathy among the population and an $\mathrm{g}$ high level of misconceptions. The benefits of osteopathy were described as more than just the manual treatments with participants valuing osteopathy as a holistic, meditative and non-pharmaceutical approach. Participants also described the osteopathic treatments as assisting with a range of cancer-related health complaints such as pain, fatigue, and sleep problems. Offering osteopathic treatment at an accessible location at low or no cost were identified by participants as enablers to the continued use of osteopathy

Conclusions

The findings of this study provides preliminary data which suggests, when delivered alongside existing medical care, osteopathy may have health benefits for patients with complex conditions such as cancer.

\section{eP073}

HERB AND SUPPLEMENT USE BY PATIENTS WITH BREAST AND GYNECOLOGIC CANCER: A PROSPECTIVE STUDY ABOUT 114 CASES

H. langin ${ }^{1}$, E. tresch ${ }^{2}$, M.C. le deley ${ }^{2}$, G. marliot $^{3}$, I. sakji ${ }^{3}, M$. vanseymortier $^{4}$, C. abdeddaim ${ }^{5}, J$. bonneterre $^{6}$, E. lartigau $^{1}$, G. lefebvre ${ }^{7}$

${ }^{1}$ oscar lambret center, radiotherapy, lille, France

${ }^{2}$ oscar lambret center, biostatistics, lille, France

${ }^{3}$ oscar lambret center, pharmacy, lille, France

${ }^{4}$ oscar lambret center, clinical research, lille, France

5 oscar lambret center, oncology, lille, France

${ }^{6}$ oscar lambret center, senology, lille, France

${ }^{7}$ oscar lambret center, supportive care, lille, France

\section{Introduction}

Many patients with cancer commonly use complementary and alternative medicine (CAM). During their antineoplastic treatment they can use herbal medicine (HM). Potential drug interactions and adverse effects are possible. Quality and security of biological CAM are still unknown.

\section{Objectives}

The objective of this study was to identify the prevalence and awareness of CAM among breast (BR) and gynecologic (GYN) cancer patients undergoing chemotherapy, hormone therapy, or targeted therapy.

Methods

We conducted from June to September 2017 an observational prospective study. 114 patients were asked to complete a questionnaire regarding use of HM. Clinico-pathological characteristics were recorded. Antineoplastic treatment was also registered.

\section{Results}

The study participants included 62 BR locoregional, 31 BR advanced, 3 GYN localized, 15 GYN advanced. 59,3\%(95\%CI:49,7-68,4) used one or more CAM during treatment. Most popular CAM were respectively, homeopathy, HM, acupuncture. 60,2\% (95\% CI:50,5-69,2) knew HM, and 33 used it (29.2\% (95\% IC: $21.0-38.5)$ ). HM use was more prevalent in localized cancer $(\mathrm{p}=0,005) .8$ patients started it during treatment. 4 thought $\mathrm{HM}$ had synergistic effect on treatment. No patients thought HM was more efficient than anti-tumor treatment. Main reasons for CAM use were to improve health condition (22) or reduce treatment's adverse effects (20). Half of them believed HM is harmless, 33\% (95\% CI: 18-51.8) had informed their oncologist; 31 would appreciate information on herbs.

\section{Conclusions}

Our findings contribute to show that prevalence of CAM use in BR and GYN cancers is high. Oncologists could initiate discussions with PTS about HM to understand them and to improve supportive care

\section{eP074}

\section{SELENIUM FOR ADVERSE EFFECTS OF CANCER TREATMENTS}

P. Renner ${ }^{I}$, G. Dennert ${ }^{2}$, A. Kalisch ${ }^{3}$, M.A. Horneber ${ }^{3}$

${ }^{1}$ Institute of Cardiology, Department of Internal Medicine - Paracelsus Medical University - Klinikum Nürnberg, Nürnberg, Germany

${ }^{2}$ Social Medicine and Public Health with Focus on Gender and Diversity, Department of Applied Sciences - University of Applied Sciences Dortmund, Dortmund, Germany

${ }^{3}$ Institute of Oncology and Hematology, Department of Internal Medicine - Paracelsus Medical University - Klinikum Nürnberg, Nürnberg, Germany

\section{Introduction}

Selenium has been claimed to have beneficial effects for patients undergoing cancer treatment.

\section{Objectives}

To assess the efficacy of selenium to prevent or treat the side effects or toxicities of chemotherapy, radiotherapy or surgery.

Methods

We searched the Cochrane Central Register of Controlled Trials (CENTRAL), MEDLINE, EMBASE, the WHO International Clinical Trials Registry Platform (ICRTP), the German online register clinical trials, OpenGrey, and the NCI Clinical Trials register (last search: 21 October 2017). We included randomized controlled trials with adult patients undergoing cancer therapy using selenium for supportive care. Two authors independently screened abstracts, applied inclusion criteria, and extracted data. We used Cochrane's risk of bias assessment and GRADE.

\section{Results}

We included a total of nine studies with 457 cancer patients. All studies applied selenium orally in highly different doses. Seven studies reported benefits for selenium: one for cisplatin-induced renal toxicity, one for oral mucositis after high-dose chemotherapy, two for radiotherapy-induced oral or intestinal mucositis, one for protection of salivary glands during I-131 radiation, one for recurrences of erysipelas after surgery for breast cancer, and one for postoperative lymphedema after neck-dissection. All studies had methodological limitations, no study was judged as being at low risk of bias, and pooling of results was not possible due to heterogeneity.

\section{Conclusions}

The currently available evidence from randomized controlled trials is insufficient to give selenium a definite role in supportive cancer care. The results concerning effects on cisplatin-induced nephrotoxicity, oral mucositis during high-dose chemotherapy, and salivary protection during I-131 radiation could serve as a means of prioritizing research. 


\section{eP075}

COMPLEMENTARY THERAPIES FOR THE PREVENTION AND MANAGEMENT OF CHEMOTHERAPY-INDUCED PERIPHERAL NEUROPATHY

\section{A. Kalisch ${ }^{1}$, M. Rostock ${ }^{2}$, A. Stapf ${ }^{1}$, D. Steinmann ${ }^{3}$, M.A. Horneber ${ }^{1}$}

${ }^{T}$ Institute of Oncology and Hematology, Department of Internal Medicine

- Paracelsus Medical University - Klinikum Nürnberg, Nürnberg, Germany

${ }^{2}$ Hubertus Wald Tumor Center, University Cancer Center Hamburg,

Hamburg, Germany

${ }^{3}$ Department of Radiotherapy, Medical School Hannover, Hannover, Germany

\section{Introduction}

Chemotherapy induced peripheral neuropathy (CIPN), is a severe problem in oncology leading to dose reduction, treatment delay or discontinuation. Complementary therapies (CT) are supportive measures used as adjuncts to mainstream cancer care to control symptoms and enhance well-being.

Objectives

To update the available evidence about the efficacy and safety of CT for the prevention and management of CIPN.

Methods

We searched the Cochrane Central Register of Controlled Trials (CENTRAL), MEDLINE, and EMBASE. We included randomized trials with adults using CT for CIPN. Abstracts were screened, inclusion criteria applied, data extracted, and risk of bias assessed. Evidence was determined by a pre-defined grading system. The grades indicated a high, moderate, or low net benefit, no demonstrated effect or suggested harm.

\section{Results}

Overall, 25 CT were identified. Depending on whether the CT was used for prevention or management and what kind of chemotherapeutic agent was applied, the picture was different: Glutathione and omega-3-fatty acids were categorized to grade B for the prevention of platinum-induced neuropathies, whereas for the prevention of taxane-induced neuropathies only omega-3-fatty acids were categorized to grade B and glutathione was suggested to potentially cause harm. Low-level laser therapy and neurofeedback were categorized to grade B for the management of both, platinum- and taxane-induced neuropathies. For details see Table 1 and 2.

\begin{tabular}{|l|l|l|l|l|}
\hline \multicolumn{7}{|l|}{ Table 1: Complementary therapies for the prevention of CIPN } \\
\hline Grade & Oxaliplatin & Cisplatin & $\begin{array}{l}\text { Platinum/Taxane } \\
\text { combination }\end{array}$ & Taxanes \\
\hline $\begin{array}{l}\text { High } \\
\text { benefit }\end{array}$ & - & - & - & - \\
\hline $\begin{array}{l}\text { Moderate } \\
\text { benefit }\end{array}$ & $\begin{array}{l}\text { Glutathion } \\
\text { Omega--3atty acids }\end{array}$ & Glutathion & - & Omega-3-fatty acids \\
\hline $\begin{array}{l}\text { Low } \\
\text { benefit }\end{array}$ & $\begin{array}{l}\text { Acetylystetine } \\
\text { Exercise (multimodal) } \\
\text { Glutamine/glutamate } \\
\text { Menthol (topical) } \\
\text { Red sage }\end{array}$ & $\begin{array}{l}\text { Exercise (multimodal) } \\
\text { Menthol (topical) }\end{array}$ & $\begin{array}{l}\text { Exercise (multimodal) } \\
\text { Hypothermia }\end{array}$ & $\begin{array}{l}\text { Exercise (multimodal) } \\
\text { Pressure gloves } \\
\text { Hypothermia } \\
\text { Menthol (topical) }\end{array}$ \\
\hline No effect & $\begin{array}{l}\text { Alpha-lipoic acid } \\
\text { Calcium/Magnesium } \\
\text { Vitamin B complex } \\
\text { Vitamin E }\end{array}$ & $\begin{array}{l}\text { Neurotrophic peptide } \\
\text { (Org2766) } \\
\text { Vitamin E }\end{array}$ & Vitamin E & $\begin{array}{l}\text { Carnitine } \\
\text { Glutamine/glutamate } \\
\text { Vitamin B complex } \\
\text { Vitamin E E }\end{array}$ \\
\hline $\begin{array}{l}\text { Suggested } \\
\text { harm }\end{array}$ & $\begin{array}{l}\text { Goshajinkigan } \\
\text { Acupuncture } \\
\text { Amifostin } \\
\text { Carnitine }\end{array}$ \\
\hline
\end{tabular}

\begin{tabular}{|c|c|c|c|c|}
\hline Grade & \begin{tabular}{|l|} 
Oxaliplatin \\
\end{tabular} & \begin{tabular}{|l|} 
Cisplatin \\
\end{tabular} & $\begin{array}{l}\text { Platinum/Taxane } \\
\text { combination }\end{array}$ & Taxanes \\
\hline $\begin{array}{l}\text { High } \\
\text { benefit }\end{array}$ & - & - & - & none \\
\hline $\begin{array}{l}\text { Moderate } \\
\text { benefit }\end{array}$ & $\begin{array}{l}\text { Low-level laser therapy } \\
\text { Neurofeedback }\end{array}$ & $\begin{array}{l}\text { Low-level laser } \\
\text { therapy } \\
\text { Neurofeedback }\end{array}$ & $\begin{array}{l}\text { Low-level laser therapy } \\
\text { Neurofeedback }\end{array}$ & $\begin{array}{l}\text { Low-level laser therapy } \\
\text { Neurofeedback }\end{array}$ \\
\hline $\begin{array}{l}\text { Low } \\
\text { benefit }\end{array}$ & $\begin{array}{l}\text { Alpha-lipoic acid } \\
\text { Exercise (multimodal) } \\
\text { Menthol (topical) }\end{array}$ & $\begin{array}{l}\text { Alpha-lipoic acid } \\
\text { Exercise (multimodal) } \\
\text { Menthol (topical) }\end{array}$ & \begin{tabular}{|l|} 
All-trans-retinoic acid \\
Exercise (multimodal)
\end{tabular} & $\begin{array}{l}\text { Alpha-lipoic acid } \\
\text { Carnitine } \\
\text { Exercise (multimodal) } \\
\text { Menthol (topical) }\end{array}$ \\
\hline No effect & \begin{tabular}{|l|} 
Acupuncture \\
Alpha-Lipoic acid \\
Scrambler Therapy \\
(CALMARE \\
Transcutaneous electrical \\
nerve stimulation
\end{tabular} & $\begin{array}{l}\text { Acupuncture } \\
\text { Scrambler Therapy } \\
\text { (CALMARE }{ }^{\circ} \text { ) }\end{array}$ & \begin{tabular}{|l} 
Acupuncture \\
Scrambler Therapy \\
(CALMARE ${ }^{\circ}$ )
\end{tabular} & $\begin{array}{l}\text { Acupuncture } \\
\text { Scrambler Therapy } \\
\text { (CALMARE }{ }^{\circ} \text { ) }\end{array}$ \\
\hline $\begin{array}{l}\text { Suggested } \\
\text { harm }\end{array}$ & Goshajinkigan & Cannabinoids & - & Cannabinoids \\
\hline
\end{tabular}

\section{Conclusions}

Complementary therapies may be helpful when conventional treatment does not bring satisfactory relief or causes undesirable side effects in the prevention or management of CIPN. However, depending on the chemotherapeutic agent, CT could also cause harm

eP076

THE EFFECT OF USE COMPLEMENTARY AND ALTERNATIVE MEDICATION COMBINED CONVENTIONAL MEDICINE IN CHRONIC PAIN PATIENTS

S.P. Hsu ${ }^{l}$

${ }^{T}$ Shin Kong wu ho-su memorial hospital, Nursing department, Taipei, Taiwan R.O.C.

\section{Introduction}

Chronic pain is defined by NANDA as an "unpleasant sensory and emotional experience arising from actual or potential tissue damage or described in terms of such damage; At present, interest in growing in non-pharmacological interventions to control pain. Complementary and alternative therapies have enjoyed increasingly widespread use in recent years. Because of this trend, we were eager to obtain a better grasp on the actual number of people in our hospital's pain clinic who have used these modalities. In an effort to explore the use of complementary alternative medicine (CAM) by patients seen in a chronic pain clinic.

\section{Objectives}

Study purposes were to:(1) The Situation of chronic pain patients using CAM combined conventional medicine.(2) The outcome of chronic pain patients using CAM combined conventional medicine.

Methods

This study used a cross-sectional, descriptive correlational research design. A sample of 130 chronic pain patients using CAM combined conventional medicine, To structured questionnaires for data collection from outpatient department in Taipei, Taiwan.

Results

(1) There are significant differences of the pain control worked well of chronic pain patients using CAM.

(2) There are significant differences of the pain control worked well of chronic pain patients using the status of medicine.

(3) The pain control worked well of chronic pain patients using CAM combined conventional medicine.Conclusions

Non-pharmacological interventions to pain management is became important. Nurses have the skills, experience, responsibility and professionalism to assume the major role in the care of chronic pain sufferers.

eP077

THE EFFECTS OF MUSCLE RELAXATION THERAPY IN REDUCING HEAD AND NECK CANCER POSTOPERATIVE INDUCED FATIGUE AND SLEEP QUALITY -A RANDOMIZED CONTROLLED TRIAL

\section{T.W. Huang ${ }^{\text {I }}$, J.H. Lai $^{2}$}

${ }^{T}$ Taipei Medical University, School of Nursing-College of Nursing, Taipei, Taiwan R.O.C.

${ }^{2}$ Erlin Christian Hospital, Department of internal Medicine-, Chunghua, Taiwan R.O.C.

\section{Introduction}

There are 6,000 newly diagnosed head and neck cancers in Taiwan yearly. Objectives

This study examined the effectiveness of muscle relaxation intervention on fatigue and sleep quality of head and neck cancer patients. 


\section{Methods}

After informed consent, patients were randomized into two groups and outcome adjudicators were blinded. The two groups underwent pretesting before surgery (T0). The experimental group underwent Hendrickson muscle relaxation training for 15 minutes every night from the Day 3 of transfer from intensive care unit and the control group received regular care. The primary endpoint was the fatigue (Brief Fatigue Inventory) and sleep quality (Pittsburgh Sleep Quality Index- Taiwanese version), while secondary end points was the severity of depression level (VAS range from 0 to 10). All participants undertook the posttest before discharge (T1).

Results

A total of 67 patients were included and analyzed. 7 patients were dropped (6 patients developed serious conditions, 1 contracted a sexually disease); ultimately, completed with 30 patients in each group. General Estimated Equation analysis revealed that sleep quality $(\beta=0.38 ; p=$ $0.03)$ and fatigue $(\beta=0.09 ; \mathrm{p}=0.03)$ of the experimental group exhibited greater improvements than those of the control group. The depression level of the experimental group was significantly lower than of the control group on Day $5(0.33 \pm 1.83$ vs. $4.33 \pm 10.06 ; p=0.04)$.

Conclusions

The use of muscle relaxation intervention prove to be of greater benefit in decreasing fatigue and promote sleep quality. Hence, clinical workers could use as a reference for patient care.

\section{eP078}

EFFECT OF AROMATHERAPY MASSAGE ON CHEMOTHERAPY-INDUCED PERIPHERAL NEUROPATHIC PAIN AND FATIGUE IN PATIENTS RECEIVING OXALIPLATIN

N. Izgu ${ }^{1}$, L. Ozdemir ${ }^{1}$, F. Bugdayci Basal ${ }^{2}$

${ }^{T}$ Hacettepe University, Nursing Faculty, Ankara, Turkey

${ }^{2}$ Ataturk Chest Diseases and Surgery Training and Research Hospital, Medical Oncology Department, Ankara, Turkey

\section{Introduction}

Patients receiving oxaliplatin may experience peripheral neuropathic pain and fatigue. Aromatherapy massage, a non-pharmacological method, may help to control these symptoms.

Objectives

The aim of this open label parallel group quasi-randomized controlled pilot study was to investigate the effect of aromatherapy massage on chemotherapy-induced peripheral neuropathic pain and fatigue in patients receiving oxaliplatin.

Methods

Stratified randomization was used to allocate 46 patients to two groups: intervention $(\mathrm{n}=22)$ and control $(\mathrm{n}=24)$. Between week one and week six, patients in the intervention group (IG) received aromatherapy massage three times a week. There was no intervention in weeks seven and eight. The control group (CG) received routine care. Neuropathic pain was identified using the Douleur Neuropathique 4 Questions; severity of painful paresthesia was assessed with the Numerical Rating Scale; fatigue severity was identified with Piper Fatigue Scale.

Results

At week six, the rate of neuropathic pain was significantly lower in the IG, when compared to CG. The severity of painful paresthesia based on Numerical Rating Scale in the IG was significantly lower than that in the CG at weeks two, four, and six. At week eight, fatigue severity in the IG was significantly lower when compared to $\mathrm{CG} .(\mathrm{p}<.05)$.

\section{Conclusions}

This pilot study suggests that aromatherapy massage may be useful to relieve neuropathic pain and fatigue. However, there is a need for further clinical trials to validate the results of this study.
eP079

THE EFFECTS OF THERAPEUTIC YOGA IN WOMEN WITH CANCER UNDERGOING RADIATION THERAPY

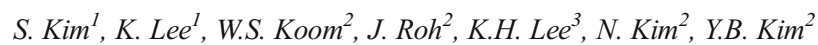

${ }^{T}$ Yonsei University College of Nursing, Department of Nursing, Seoul, Republic of Korea

${ }^{2}$ Yonsei University Health System, Yonsei Cancer Center, Seoul, Republic of Korea

${ }^{3}$ Korea Natural Therapy Yoga Association \& Cha University, Graduate School of Integrated Medicine, Sungnam, Republic of Korea

\section{Introduction}

Yoga is well known for its positive effects on improving functions of patients with cancer. Previous studies regarding yoga have focused on breast cancer patients, cancer survivors, and patients undergoing chemotherapy. However, a previous study reported that yoga was effective for improving functions in women undergoing radiotherapy. While this finding has been demonstrated in Western populations, there is a lack of evidence for patients undergoing radiation therapy in Korea.

\section{Objectives}

The purpose of this study was to examine the effects of yoga on anxiety, depression, quality of life, spiritual health, vital signs, and body composition in female cancer patients.

\section{Methods}

One-group, experimental, simple interrupted time-series design was applied with 27 women with cancer undergoing radiotherapy. Self-reported questionnaires, vital signs, and body composition were measured before and after intervention and after four weeks of follow-up. As an intervention, eight sessions of therapeutic yoga were provided over four weeks. Data were analyzed with descriptive statistics and repeated measures ANOVA.

\section{Results}

Yoga intervention was effective for improving partial quality of life, including physical and psychological well-being, in women with cancer undergoing radiotherapy. It helped decrease anxiety and depression, diastolic pressure, and body fat mass in both arms. However, yoga did not impact spiritual health, or body composition in areas other than the arms.

\section{Conclusions}

Based on these results, continuous therapeutic yoga intervention should be considered to improve physical and psychological function in patients undergoing radiation therapy. Moreover, continuous research should be concurrently organized to determine the exact dose and behavioral outcome indicators of the effects of yoga.

\section{eP080}

THE EFFECTS OF A NURSE-LED SUPPORTIVE CARE DELIVERY INCLUDING COMPLEMENTARY AND INTEGRATIVE MEDICINE (CIM) ON BREAST AND GYNECOLOGIC CANCER PATIENTS' QUALITY OF LIFE

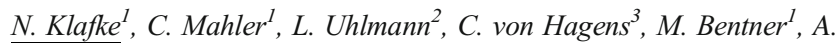
Schneeweiss $^{4}$, A. Mueller ${ }^{5}$, J. Szecsenyi ${ }^{6}$, S. Joos

${ }^{1}$ University Hospital Heidelberg, Department of General Practice and Health Services Research, Heidelberg, Germany

${ }^{2}$ University of Heidelberg, Institute of Medical Biometry and Informatics, Heidelberg, Germany

${ }^{3}$ University Women's Hospital Heidelberg, Department of Gynaecological Endocrinology and Reproductive Medicine- Head of Naturopathy and Integrative Medicine, Heidelberg, Germany

${ }^{4}$ National Center for Tumor Diseases- University Hospital Heidelberg, Head of Division Gynaecologic Oncology, Heidelberg, Germany

${ }^{5}$ Community Hospital Karlsruhe, Director of the Women's Clinic, Karlsruhe, Germany
} 
${ }^{6}$ University Hospital Heidelberg, Head of Department of General Practice and Health Services Research, Heidelberg, Germany

${ }^{7}$ University Hospital Tuebingen, Head of Institute of General Practice and Interprofessional Care, Heidelberg, Germany

\section{Introduction}

The interest in Complementary and Integrative Medicines (CIMs) is widespread in cancer patients. Therefore many cancer centers offer integrated approaches with CIM to their patients but the impact of those approaches on Quality of Life (QoL) is unclear.

Objectives

The aim of the CONGO (Complementary Nursing in Gynecologic Oncology) trial was to investigate the effects of a complex supportive nurse-led intervention using CIM on QoL.

Methods

From 7/2014 to 2/2016, we enrolled 251 patients diagnosed with breast and gynecologic cancer in a randomized trial of CIM supportive care integrated with routine care $(n=126)$ vs. routine care alone $(n=125)$ at two South German oncology centers (NCT, SKK). Patients completed the EORTC-QLQ-C30 weekly during their chemotherapy (T1: before, T2: mid-term, T3: end of chemotherapy maximally after 24 weeks), and at 6 month follow-up (T4). Mixed linear models were applied to assess the effects of this supportive care intervention.

\section{Results}

Compared to routine care, CIM supportive care improved patients' QoL at T4 (estimate $6.643[1.65 ; 11.64](p=0.010)$, but not at T3 (estimate $1.04[-4.89 ; 2.809], p=0.596)$. Similarly at T4, further group effects could be found for fatigue (effect estimate: $-7.04[-13.2 ;-.840],(p=0.0266)$, and emotional functioning (effect estimate: 8.196 [2.328; 14.07], $p=0.0065$ ) in favor of the intervention group.

\section{Conclusions}

In the long-term, breast and gynecologic cancer patients' health outcomes were improved by the integrated intervention. Further research is warranted to explain the mechanisms of this delayed effect, which may presumably has developed due to improvement of patients' self-efficacy and patient competence.

\section{eP081}

PRESCRIBING MEDICINAL CANNABIS IN AUSTRALIA FOR CANCER PATIENTS: A NEW ERA IN MEDICINE WITH ALL ITS COMPLEXITIES

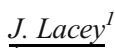

${ }^{T}$ Chris O'Brien Lifehouse Cancer Centre, Medical Oncology-Supportive Care and Integrative oncology, Sydney, Australia

\section{Introduction:}

Australia has recently introduced the limited prescribing of cannabis for symptom management in cancer patients with "refractory" symptoms. This presentation will look at the Australian model and the strategy used to develop a program to prescribe for selected patients, the limitations and strengths .

Objectives

To highlight the key strategies in developing prescribing medicinal cannabis for cancer related symptoms in Australia. Looking at clinical trials in the cancer population, the development of partnerships, the role of observational $/ \mathrm{n}=1$ studies in this setting.

Methods

Case studies, guidance document development, current clinical trials presented from the perspective of a palliative and supportive care specialist involved in studies, prescribing and policy development. Taking a look at prescribing skills required and strategies to improve evidence

Results

Australian prescribing of cannabis for symptom management in cancer patients is evolving rapidly. One proposed strategy is the $\mathrm{N}=1$ model whilst the gold standard remains the Randomised Control Trial. The skills of palliative care and supportive care training and experience in balancing benefit and side effect of medications to improve quality of life can be applied in this new field coupled with knowledge of the products available, their potential role as a therapeutic agent, potential adverse side effects and interactions will presented.

\section{Conclusions}

Medicinal Cannabis plays a growing role with ongoing exploration of $i$ it's potential in Supportive Cancer Care for the patient with refractory symptoms .

\section{eP082}

ENHANCED SUPPORTIVE CARE PROGRAM INCORPORATING INTEGRATIVE ONCOLOGY IN A COMPREHENSIVE CANCER SERVICE

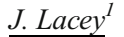

${ }^{T}$ Chris O'Brien Lifehouse Cancer Centre, Medical Oncology-Supportive Care and Integrative oncology, Sydney, Australia

\section{Introduction:}

Increasingly cancer services are incorporating integrative oncology services to enhance the holistic care of patients and meet patient expectations. Integrating evidence based complementary medicine (CM) into mainstream cancer care can provide viable options for relief of symptoms that may not be relieved by conventional treatments .In 2014 the Chris O'Brien Lifehouse comprehensive cancer centre opened with a mission to incorporate an integrative oncology (IO) service and develop supportive care from time of diagnosis.

\section{Objectives}

To present a model of holistic supportive care service development incorporating complementary therapies, exercise and supportive care holistic medical consultation to enhance the care of patients attending the comprehensive cancer service

Methods

Outlining the service development, strategies for service growth, use of PROMS and integration into mainstream cancer care. The IO service offers comprehensive medical specialist consultation, exercise physiologist and programs, individual therapies such as acupuncture, massage, reflexology and group classes in yoga, meditation and qi gong. Patients are referred via medical specialist or self-refer

\section{Results}

Between January 2015 and April 2017, 1,506 new patients attended the IO service. The majority were female $(66 \%)$ and between $51-70$ years (51\%). Occasions of service per month ranged from 450-700 in the past year. Over $60 \%$ of patients attended more than once. PROMs, MDT and mulitdisciplinary research have enhanced integration.

\section{Conclusions}

The IO service at the Chris O'Brien Lifehouse is currently the most comprehensive on offer in Australia. Lifehouse is proving to be a robust program model for future supportive care cancer services.

\section{eP083 \\ PATIENT EXPERIENCES AND VALUES OF ACUPRESSURE UTILIZED AFTER COLORECTAL CANCER SURGERY IN HOSPITAL}

\author{
Y.H. LIU ${ }^{\text {I }}$, C.W.H. Chan ${ }^{2}$ \\ ${ }^{T}$ the Chinese University of Hong Kong, the Nethersole school of nursing, \\ Hong Kong, China \\ ${ }^{2}$ the Chinese University of Hong Kong, the Nethersole school of nursing, \\ Hong Kong, Hong Kong S.A.R.
}




\section{Introduction:}

Postoperative gastrointestinal ileus is common among patients undergoing colorectal surgery. Acupressure is a promising intervention to improve postoperative gastrointestinal function; however, patients' experiences and values of acupressure have not been explored.

\section{Objectives}

To evaluate patients' experiences and values of acupressure after an acupressure intervention using acupen.

\section{Methods}

An exploratory qualitative method was used and face-to-face semistructured individual interviews of 11 patients undergone colorectal surgery and 10 family members were conducted to explore their experiences and values of acupressure. Transcripts of audio data were analyzed by a thematic content analysis approach.

Results

Themes were identified including general attitudes toward the acupressure experiences, areas for improvement, motivation to continue acupressure, values for acupressure and its hospital application. The majority of patients and family members recognized the procedure of acupressure and held positive or neutral attitude toward acupen. Aspects for improvement involve longer acupressure duration, a combination of multiple acupoints and using larger side of acupen. All patients thought acupressure helped them in certain ways like making them comfortable, facilitating their recovery from surgery or feeling good in general. No harms of acupressure were reported. Furthermore, the majority of patients were willing to continue acupressure after discharge. All patients supported the application of acupressure in hospital.

\section{Conclusions}

This study provides an insight into the experiences and values of patients undergoing acupressure after colorectal cancer surgery in hospital. The results can provide evidence for the clinical applicability of acupressure and give inspiration of evaluating participants' experiences and values in order to tailor intervention in clinical settings.

\section{eP084}

EFFICACY OF TRADITIONAL JAPANESE MEDICINE (KAMPO) IN SUPPORTIVE CARE IN CANCER: A METAANALYSIS OF RANDOMIZED CONTROLLED TRIALS

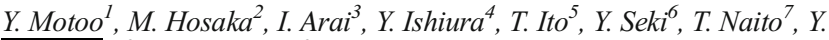
Masuzawa $a^{2}$, T. Nakayama ${ }^{2}$

${ }^{1}$ Kanazawa Medical University, Department of Medical Oncology, Ishikawa, Japan

${ }^{2}$ Kyoto University, Department of Health Informatics, Kyoto, Japan

${ }^{3}$ Nihon Pharmaceutical University, Department of Kampo Medicine, Saitama, Japan

${ }^{4}$ Kansai Medical University Medical Center, Department of RespiratoryOncology and Allergy Medicine, Osaka, Japan

${ }^{5}$ Showa University Northern Yokohama Hospital, Department of Internal Medicine, Yokohama, Japan

${ }^{6}$ Uonuma Institute of Community Medicine- Niigata University Medical and Dental Hospital, Department of Hematology, Niigata, Japan

${ }^{7}$ Shizuoka Cancer Center, Division of Thoracic Oncology, Shizuoka, Japan

\section{Introduction}

The EBM Committee of the Japan Society for Oriental Medicine makes available on its Evidence Reports of Kampo Treatment (EKAT) website a compilation of structured abstracts of randomized controlled trials (RCTs) on Kampo medicines.

\section{Objectives}

Our aim was to evaluate the efficacy of Kampo formulations in supportive care in cancer by meta-analyzing RCTs selected from the EKAT website.

\section{Methods}

We established the "Advanced EKAT", including risk of bias (7 items), safety, source of funding, and conflicts of interest, in addition to items in the original EKAT. Our meta-analysis protocol was registered on PROSPERO (CRD42017068380).

\section{Results}

In all, 48 RCTs in supportive care in cancer were reported before December 31,2016 . In the 4 studies about postoperative motility in patients with colorectal cancers or hepatocellular carcinoma, the number of days to first flatus was significantly reduced (mean difference [MD]: $-0.74 ; 95 \% \mathrm{CI}:-0.85,-0.63 ; P<0.0001)$ and length of hospital stay (MD: $-1.34 ; 95 \%$ CI: $-1.52,-1.16 ; P<0.0001$ ) was significantly shorter in the daikenchuto group compared to the control (without daikenchuto). In the 5 studies about chemotherapyinduced peripheral neuropathy, the incidence of grade 3 or higher neuropathy was significantly lower (risk ratio: 0.36 ; $95 \%$ CI: 0.18 , $0.74 ; P=0.005)$ in the goshajinkigan group compared to the control (without goshajinkigan). The risk of bias was high for allocation concealment, blinding, and selective reporting.

\section{Conclusions}

Although our meta-analysis had methodological limitations, we can conclude that some Kampo medicines are effective in supportive care in cancer.

\section{eP085 \\ EFFECT OF INHALATION AROMATHERAPY ON PROCEDURAL PAIN AND ANXIETY AFTER NEEDLE INSERTION INTO AN IMPLANTABLE CENTRAL VENOUS PORT CATHETER}

\section{E. Mutluay Yayla ${ }^{1}$, L. Ozdemir ${ }^{2}$}

${ }^{T}$ Health Sciences Faculty, Internal Medicine Nursing, Düzce, Turkey

${ }^{2}$ Nursing Faculty, Internal Medicine Nursing, Ankara, Turkey

\section{Introduction}

Needle insertion into an implantable central venous port cathetermay lead to procedural pain and anxiety in cancer patients.

\section{Objectives}

The aim of this a quasi-randomized controlled study was to investigate the effect of inhaler aromatherapy on procedural pain and anxiety during needle insertion into an implantable venous port catheter.

\section{Methods}

Sample of the study consisted of a total of 123 cancer patients, randomly selected to lavender group, eucalyptus group and control group. The stratified randomization was used in this study. Patients in lavender group were applied aromatherapy inhalation of cotton ball soaked in 3 drop lavender essential oils during 3 minutes before needle insertion into an implantable venous port catheter. Aromatherapy inhalation of cotton ball soaked in 3 drop eucalyptus essential oils was applied to eucalyptus group during 3 minutes before the needle insertion. No intervention was performed to the control group patients before needle insertion. The level of pain was measured with "Görsel Analog Skala" (VAS). The level of anxiety was evaluated by "State Anxiety Inventory" (STAI-I).

\section{Results}

The average VAS scoresof the lavender group were significantly lower than those of the control group $(\mathrm{P}<.05)$. There were no significant differences in the average VAS scores betweenthe eucalyptus group and the control group $(\mathrm{P}>.05)$. The average STAI-I scores of the lavender group, the eucalyptus group, and thecontrol group were not significantly different $(\mathrm{P}>.05)$.

\section{Conclusions}

Lavender essential oil applied by inhalation decreased pain level. Aromatherapy applied with lavender essential oil may be recommended to decrease pain level during needle insertion into an implantable venous port catheter. 
eP086

THE EFFECT OF LIGHT ON QUALITY OF SLEEP AND LIFE IN BREAST CANCER PATIENTS

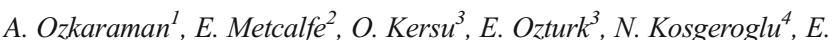
Colak', A. Ozen $^{2}$, G. Balcr Alparslan ${ }^{4}$

${ }^{1}$ Eskisehir health high school, Nursing of Internal Medicine Disease, Eskisehir, Turkey

${ }^{2}$ Eskisehir Osmangazi University, Faculty of Medicine, Eskisehir, Turkey

${ }^{3}$ Eskisehir Osmangazi University, Hospital, Eskisehir, Turkey

${ }^{4}$ Eskisehir Osmangazi University, Faculty of Health Sciences, Eskisehir, Turkey

\section{Introduction}

Sleep disorders cause many problems in oncology patients, which should be assessed and cured.

\section{Objectives}

This study aimed to evaluate the effect of bright white light on sleep and quality of life in breast cancer patients who received radiotherapy.

Methods

The sample of this randomized controlled study consisted of patients who had lower sleep quality. Patients who met the sampling selection criteria were randomly assigned to intervention and control groups. The intervention group was exposed to 10.000 lux bright white light for 30 minutes a day in a week while the control group patients were rested. Sleep and quality of life of each group of patients were assessed twice as before the radiotherapy treatment and on the eighth day of radiotherapy.

Results

Although the PSQI mean score of each group was greater than five points according to the first assessment performed before radiotherapy, the PSQI mean score decreased in both groups in the second assessment on the eighth day of radiotherapy. PSQI mean score was lower in the intervention group compared with the control group in the second measurement [(PSQI; Intervention $=5.0 \pm 2.32$, Control $=6.50 \pm 3.26),(\mathrm{U}=85.000$, $\mathrm{p}=0.260)]$. The mean scores of the functional subscale of intervention and control groups were determined to be greater in the second assessment compared with the first assessment. The mean score of the symptom subscale decreased in both groups in the second assessment while no difference was found for the mean score of global well-being.

\section{Conclusions}

It was determined that bright white light does not affect sleep and quality of life.

\section{eP087}

EFFICACY OF SAAM ACUPUNCTURE TREATMENT ON IMPROVEMENT OF IMMUNE CELL NUMBERS IN CANCER PATIENTS

\author{
H.M. ryoo ${ }^{1}$, D.J. Kim ${ }^{2}$ \\ ${ }^{7}$ Daegu Catholic University Medical Center, Hemato-Oncology, Daegu, \\ Republic of Korea \\ ${ }^{2}$ Daegu Haany Oriental Medical Hospital, Internal Medicine, Daegu, \\ Republic of Korea
}

\section{Introduction}

Acupuncture is currently used for the improvement of immunity in humans, but little has been reported on the efficacy of acupuncture in cancer patients. We assessed the effect of Korean Saam acupuncture on the improvement of immune cell numbers

Objectives

To collect preliminary data on the effects of Saam acupuncture with regard to the immunity in cancer patients.

Methods

Ten cancer patients were analyzed for improvements in immunity. Acupuncture was applied at the 5 acupuncture points, Jingqu (LU 8),
Zutonggu (BL 66), Yanggu (SI 5), Yangchi (TE 4), and Zhongwan (CV 12) for 2 weeks with 4 sessions. We assessed the effect of Korean Saam acupuncture on the immune system in cancer patients by measuring particular blood cell subsets, including CD3+, CD4+, CD8+, CD19+, and CD56+ cells, as well as total white blood cell count, absolute neutrophil count, and fatigue score. The measurement was performed before and after acupuncture and at a 2-week follow-up.

\section{Results}

There was a statistically significant increase in the number of $\mathrm{CD} 3+(\mathrm{P}=$ $0.023)$ and $\mathrm{CD} 8+$ cells $(\mathrm{P}<0.001)$ and T-cell subsets, as well as a decrease in the fatigue severity scale (FSS) score $(\mathrm{P}=0.001)$ after Saam acupuncture using the 5 acupoints.

\section{Conclusions}

Acupuncture may improve the immune system by increasing the counts of a few immune cells and relieve fatigue in cancer patients by decreasing FSS scores. Although this was a non-controlled study, it constitutes preliminary research investigating the potential effects of Saam acupuncture in increasing the counts of several immune cells in cancer patients.

\section{eP088 \\ KAMPO MEDICINE APPROACH FOR WESTERN MEDICINE RESISTANT EDEMA AND ASCITES OF CANCER PATIENTS}

D. Tanada

${ }^{T}$ Hyogo College of Medicine, Anesthesiology \& Pain Medicine, Nishinomiya, Japan

\section{Introduction}

For control of edema and ascites of cancer patients, Western medicine such as diuretics is often used, but in many cases it is difficult to control.

\section{Objectives}

In our Palliative Care Team, we use a Kampo medicine with water handling effect against western medicine resistant edema and ascites. This time we verified these effects.

\section{Methods}

Between December 2015 and November 2017, cases with our team intervening cases using Kampo medicine with water - handling effect against western medicine resistant edema and ascites were examined retrospectively.

Results

During that period, 77 cases were used Kampo medicine: $15.4 \%$ of the total intervention (501 cases). Breakdown and effectiveness rates was 36 cases of Goreisan: $44.4 \%$ (16/36 ), 26 cases of Saireito : 50\% (13/26), 7 cases of Eppikajutsuto:14.3\% (1/7), 6 cases of Saireito + Eppikajutsuto : $100 \%(6 / 6), 2$ cases of Goreisan + Eppikajutsuto:50\% (1/2) .

\section{Conclusions}

In this time Kanpo medicine cases were insufficient effect in Western medicine, but good symptom relief was obtained by Kanpo medicine use (an effective rate of $48 \%$ as a whole). In particular, the combined use of Saireito and Eppikajutsuto was effective (effective rate was $100 \%)$. As a factor of that, Saireito is a drug that combines antiinflammatory action and water-handling action. Furthermore, since Eppikajutsuto has an anti-inflammatory effect against exudative inflammation, it is considered that a favorable combination use effect was obtained.

\section{eP089 \\ VISCUM ALBUM ULTRADILUIDO IN THE TREATMENT OF MYELOMA IN DOG - CASE REPORT}

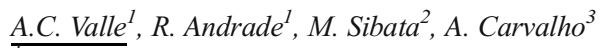

${ }^{T}$ UCB, Genomic Science and Biotechnology, Brasilia, Brazil

${ }^{2}$ Injectcenter-Brazil, Clinic, Ribeirao Preto, Brazil

${ }^{3}$ UNIP, Natural Medicine, Sao Paulo, Brazil 


\section{Introduction}

Myeloma is an uncommon malignancy in dogs. It corresponds to $0.3 \%$ of all malignancies, in this species, with few descriptions of the literature. Conventional treatment is not effective. However, complementary therapies become the best option for this care.

Objectives

Objective of this work is to report the treatment of canine myeloma through Viscum album ultradiluido (UVA).

Methods

Was attended at the veterinary office a dog, 13-year-old, feme, non-defined breed, with a history of discomfort in the lumbar region (04/17). After the $\mathrm{x}$ ray, a mass was seen on the L-4 vertebra. Magnetic resonance imaging showed a mass between L-3 and L-4 with $3 \mathrm{~cm}$ in diameter. a local biopsy $(05 / 17)$ was performed, which resulted in plasmacytic cells. The immunohystochemistry expressed cells were: CD79a and MUM1 and the non-expressed AE1AE3; CD3 and Lisozym, the phenotypic and morphological profile show myeloma. The patient was treated by complementary medicine (07/17) through daily applications of viscum ultradilution, subcutaneous, in potencies D3, D6, D9, D1, D30 daily.

Results

According to the literature, myeloma treatment is difficult and its survival is low through conventional therapy. Instead, the present case demonstrated an excellent result with complementary therapy, after 7 months of treatment with UVA, the tumor disappears completely, being confirmed by magnetic resonance imaging.

\section{Conclusions}

It is correct to say that the treatment was effective in its purpose, increasing the survival of the patient, improving their quality of life as well as providing complete cure. However, new studies and evaluation models are of fundamental importance for a better evaluation of similar case.

\section{eP090}

PERCEIVED BARRIERS OF EXERCISE PARTICIPATION IN PATIENT WITH HEMATOLOGICAL CANCER DURING HEMATOPOIETIC STEM CELL TRANSPLANTATION

\author{
M.S. Yu ${ }^{1}$, S.H. Hwang ${ }^{1}$, J.Y. Jeon ${ }^{1}$ \\ ${ }^{T}$ Exercise Medicine and Rehabilitation Lab, Department of Sport \\ Industry Studies- Yonsei University, Seoul, Republic of Korea
}

\section{Introduction}

Hematopoietic Stem Cell Transplantation (HSCT) is used primarily for severe hematological cancer worldwide. Many studies have verified the effects of inpatient exercise intervention during HSCT. Due to the unique treatment pattern that includes long-term high-dose intensity chemotherapy and the isolated ward life during HSCT, the perceived barriers for participating in exercise intervention needs to be ascertained.

Objectives

The objective of this study is to gain in-depth understandings about the perceived barriers of patients participating in the inpatient exercise intervention during HSCT.

Methods

This study used the phenomenological method to ascertain the meaning of participant's experiences. Six patients participated in semi-structured interviews, which were conducted face-to-face at Severance Hospital, Seoul. Interviews were constantly compared after being transcribed and coded. Data obtained was reviewed and organized into conceptual categories.

Results

The findings showed three main categories of barriers including eleven types of corresponding themes: (1) treatment-specific barriers (difficulties with nausea and vomiting, sore throat, eating disorder, lethargy), (2) personal attributes (anxiety from isolation, weakened willpower, disbelief, fear of deteriorating health, negative self-perceptions), (3) social environment (negative story from other patients, no encouragement from oncologist). These findings provide information to identify perceived barriers of HSCT patients.

\section{Conclusions}

The perceived barriers negatively influenced the HSCT patients' willingness to participate in the exercise intervention. These findings can provide in-depth information to develop inpatient exercise program that encourage the participation of patients.

\section{eP091}

INFLUENCE OF SUPPORTIVE INTERVENTION ON CAREGIVING BURDEN AMONG CANCER CAREGIVERS IN TWO SELECTED PRIMARY HEALTH CENTRES IN ODUKPANI, CROSS RIVER STATE, NIGERIA

\section{P.A. Akpan-Idiok ${ }^{l}$ \\ ${ }^{T}$ University of Calabar, Nursing Science, Calabar, Nigeria}

\section{Introduction:}

Cancer is a life limiting chronic disease that has become a major source of morbidity and mortality in developing countries with estimated annual incidence of 500,000 new cases in Nigeria. The burden of caring for this large population of patients by formal and informal caregivers in Nigeria has been under-studied.

\section{Objectives}

This study investigated the influence of information and communication intervention on caregiving burden among advanced cancer family caregivers in two selected primary Health centres in Odukpani, Nigeria.

\section{Methods}

A mixed method pre-test/post-test quasi-experimental research design was adopted for the study with 260 eligible purposively selected family caregivers. A well validated researcher developed questionnaire and an adopted standardized questionnaire and Focused Group Discussions question guide were used for data collection. Descriptive and inferential statistics were used in analyses and hypotheses testing at $<0.05$ levels of significance.

\section{Results}

Prior to intervention $78(60 \%)$ but after intervention $21(16.2 \%)$ of the respondents reported severe burden with many respondents (64(49.2\%) reporting a no burden experiences. Gender, age and relationship to the cared for significantly $(\mathrm{p}<0.05)$ determined caregivers' burden experiences after intervention. The findings indicate that information and communication $(X=25.92)$ were sensitive to mild and moderate burden reductions. There was significant $(p<0.05)$ difference in the mean levels of burden experienced by the respondents as a result of the intervention package administered to them.

\section{Conclusions}

The intervention package administered to the family caregivers of advanced cancer patients in the two selected study settings significantly reduced their burden levels experiences.

\section{eP092}

THE INTERDISCIPLINARY "HEALING THE HEALER GROUP": A QUALITATIVE STUDY OF THE EFFECTS OF A PILOT EDUCATIONAL INTERVENTION FOR HEALTHCARE TEAMS

G. Anandarajah ${ }^{1}, K$. Conner ${ }^{2}$

${ }^{T}$ Alpert Medical School of Brown University, Office of Medical Education, Providence- RI, USA

${ }^{2}$ Lawrence General Hospital, Palliative Care, Lawrence- MA, USA

Introduction:

Healthcare workers, including those working with severely ill cancer patients, are at risk for compassion fatigue and burnout. Few workplace interventions are described and evaluated in the literature.

\section{Objectives}

To evaluate effects of a pilot educational program designed to foster professional formation and decrease burnout in interdisciplinary healthcare teams. 


\section{Methods}

Educational approach - We developed the "Healing the Healer Group" program for interdisciplinary hospice providers, drawing from medical education, chaplaincy, social work and nursing literature. Key design elements: learning through reflection on practice; focus on complex patient/family interactions; healing through story telling; learning from peers; safety; and support. The 1-hour small group format includes: relaxation exercises; discussion of a spontaneous "case" by a participant; group reflection; and sharing of best practice approaches to challenges. Evaluation - A multi-method qualitative approach included analysis of session field notes, written feedback after 6 months, and semi-structured individual participant interviews after 1 year. Qualitative analysis used the immersion/crystallization method. IRB approval obtained.

\section{Results}

All hospice team members have participated. Field note analysis revealed several themes in cases: watching suffering, boundaries, emotional attachments, feeling alone, control, and dealing with chaos. Interviews revealed 4 major themes: personal validation; improved team cohesiveness; professional growth (learning) and personal growth (healing - emotional and spiritual). Format strengths: small group size, safety, spontaneous cases, and skillful facilitation. Challenge: protected time. All participants recommend continuing the program.

\section{Results: Common Themes in Healing the Healer Group Discussions (Field Notes)}

\begin{tabular}{|c|c|}
\hline IDG Group (nurses, social workers, chaplains) & Hospice Aids (HHA) Group \\
\hline $\begin{array}{l}\text { Watching suffering, when you know you can do } \\
\text { something, but not allowed to help (eg. } \\
\text { morphine, falls) }\end{array}$ & $\begin{array}{l}\text { Watching suffering (especially when family } \\
\text { cannot "see" eg. do not medicate) }\end{array}$ \\
\hline $\begin{array}{l}\text { Boundaries (mostly related to doing what is } \\
\text { needed vs. what is wanted) }\end{array}$ & $\begin{array}{l}\text { Boundaries-common theme (mostly related to } \\
\text { emotional attachments) }\end{array}$ \\
\hline $\begin{array}{l}\text { Understanding patient/family better (eg. } \\
\text { culture, complex dynamics, fears) }\end{array}$ & $\begin{array}{l}\text { Emotional attachment to patient and family } \\
\text { (becoming part of the family; but not really) }\end{array}$ \\
\hline $\begin{array}{l}\text { Letting go of control } \\
\text { Letting go of the relationship (occasional) }\end{array}$ & Letting go of the relationship (common) \\
\hline "a good death" - for who? & $\begin{array}{l}\text { What to do when patient relies on you for too } \\
\text { much support (wants you to stay, but you } \\
\text { cannot) }\end{array}$ \\
\hline Feeeling "àlone" (éspécially RNs); Seéking hèlp & $\begin{array}{l}\text { Caught in the middle - Balancing what the RN } \\
\text { wants, what the family wants and what the } \\
\text { patient wants }\end{array}$ \\
\hline \multicolumn{2}{|l|}{ Dealing with anger, unfairly directed at you } \\
\hline \multicolumn{2}{|l|}{$\begin{array}{l}\text { Dealing with chaotic situations - establishing } \\
\text { order while maintaining support and respect. }\end{array}$} \\
\hline $\begin{array}{l}\text { Strong dedication to job and care of patients/ } \\
\text { families }\end{array}$ & Strong dedication to the job and care of patients \\
\hline
\end{tabular}

Table: Effects of Healing the Healer Group on Group Members

\begin{tabular}{|c|c|}
\hline Themes & Subthemes \\
\hline Personal Validation & $\begin{array}{l}\text { - Feeling valued/appreciated (job satisfaction) } \\
\circ \text { By the team } \\
0 \text { By the organization }\end{array}$ \\
\hline Team Cohesiveness & $\begin{array}{l}\text { - Learning about others roles } \\
\text { - Increased compassion for teammates } \\
\text { - More likely to reach out for help }\end{array}$ \\
\hline $\begin{array}{l}\text { Professional Growth } \\
\text { (Learning ) } \\
\text { (learning both clinical skills } \\
\text { and "wisdom") }\end{array}$ & $\begin{array}{l}\text { - Learning different perspectives on their work \& how to handle challenges } \\
\text { - Reflection on own clinical performance } \\
\circ \text { Feedback from interdisciplinary colleagues } \\
\text { - Learning Confidence - Professional validation } \\
\text { - Learning from colleagues - tips and approaches } \\
\text { - Learning in a different way } \\
\text { Learning to find balance between professional boundaries and } \\
\text { compassionate care }\end{array}$ \\
\hline $\begin{array}{l}\text { Personal Growth } \\
\text { (Healing) }\end{array}$ & $\begin{array}{l}\text { - Emotional Healing } \\
\circ \text { Decreased isolation } \\
\circ \text { Decreases stress (a weight lifted; "emptying out") } \\
\circ \text { Improved resilience/job satisfaction } \\
\text { - Spiritual Healing } \\
\circ \text { Being deeply heard } \\
\circ \text { Addressing questions of meaning } \\
\text { - Closure/"Letting go" } \\
\circ \text { Increases ability to be present/centered }\end{array}$ \\
\hline
\end{tabular}

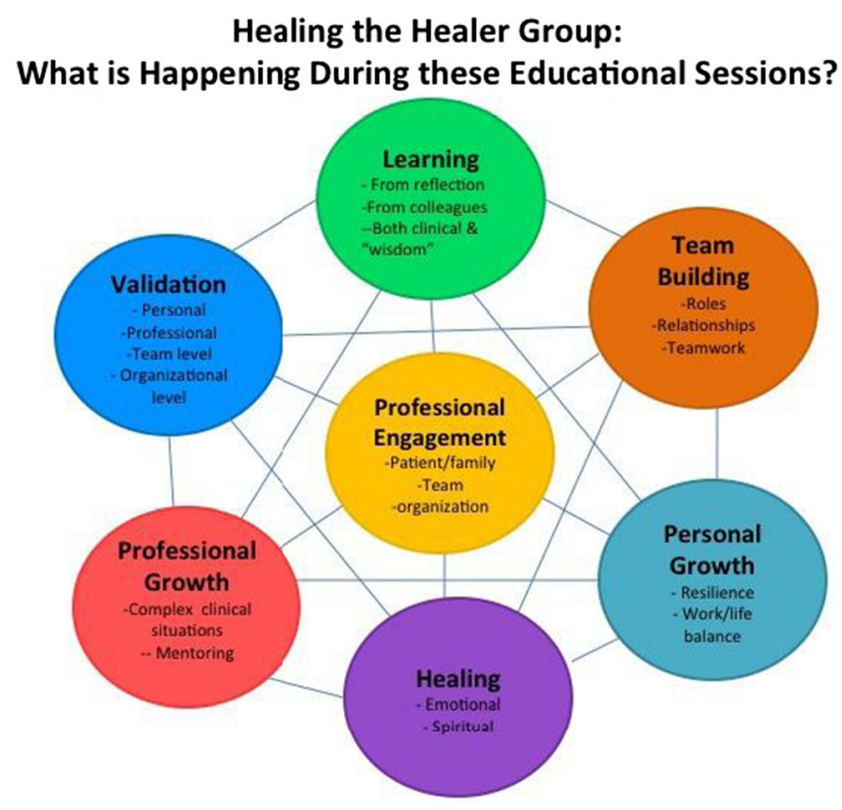

\section{Conclusions}

This pilot Healing the Healer Group appears to have the potential fill a significant need in the professional lives of hospice workers. Further study is needed.

\section{eP093}

\section{MODELS OF ACADEMIC PALLIATIVE MEDICINE IN INDIA}

\section{R.D. Arora ${ }^{1}$}

${ }^{T}$ All India Institute of Medical Sciences- New Delhi, Palliative Medicine, Ansari Nagar-New Delhi, India

\section{Introduction}

The primary hospital based models of service delivery include the consultative model, the integrated model and a model that combines the two.

Objectives

This paper attempts to put into focus the two existent academic models of Palliative Medicine in India which are offering a three year postgraduate training course in this subject. It attempts to compare and contrast the two, with an emphasis on their conceptualization, components and means to achieving the goal of palliation.

\section{Methods}

The paper is a distillation of the author's experiences at these two places. Results

There are two important models of Academic palliative medicine in the country, one bieng a consultative model based out of Tata Memorial Centre, Mumbai and the other an integrated inpatient model nested within the Department of Oncoanaesthesiology at the All India Institute of Medical Sciences, New Delhi. The first model demonstrates a deeply ingrained emphasis on the continuity of care which has lead to the creation of a qualified workforce capable of taking this service to the patients doorstep. The integrated model is the first of its kind in the country to house a dedicated six bedded inpatient unit which offers an opportunity to converge the science of Palliation with interventional pain management. It is one of the first units to offer training in intensive care to postgraduates in the subject. 


\section{Conclusions}

These models of Palliative Medicine which have been closely developed in concurrence with the religious, sociopolitical and cultural fibre of the community offer interesting examples of delivering palliation to the masses.

\section{eP094}

RESEARCH ON THE RELATIONSHIP OF COGNITION OF SYMPTOM DISTRESS AND SENSE OF COHERENCE IN CANCER CHEMOTHERAPY

K. Asaba ${ }^{1}$, A. Okawa ${ }^{2}$

${ }^{T}$ Nagoya University Graduate School of Medicine, Doctor's Course Nursing, Nagoya, Japan

${ }^{2}$ Yokkaichi Nursing and Medeical Care University, Faculty, 1200 Minoo Yokkaichi Mie, Japan

\section{Introduction}

The symptom is subjective, and it is important that I understand how a patient recognizes the symptom. However, the personal characteristic to influence the recognition of the symptom of the patient individual does not become clear enough.

\section{Objectives}

This study investigated the relationship between the symptom distress, quality of life(QOL), and sense of coherence in non-small cell lung cancer patients adjuvant chemotherapy.

Methods

The questionnaire covered demographics, self-perceived severity of symptom distress(Symptom Distress Scale), QOL(SF-8), Sense of Coherence(SOC). Descriptive statistics and correlation coefficients were calculated, and hierarchical multiple regression analysis was performed using QOL as a dependent variable.

\section{Results}

aaa

Conclusions

aaa

\section{eP095}

THE MOST FREQUENT TOPICS OF INTEREST FOR SUPPORTIVE CARE (SC) EDUCATION IN THE EASTERN EUROPE AND THE BALKAN REGION (EEBR)

N. Bakic ${ }^{l}$, A. Zilic ${ }^{1}$, S. Bosnjak ${ }^{l}$, J. Dimitrijevic ${ }^{1}$

${ }^{T}$ Institute for Oncology and radiology of Serbia, Intensive Care Unit/Supportive oncology, Belgrade, Serbia

\section{Introduction:}

The Second Regional Educational Meeting on SC in Cancer Patients for EEBR was held in Belgrade, Serbia (December 1-2, 2017). Topics covered were related to cancer-induced symptoms and treatment-related toxicities.

\section{Objectives}

To analyze the most preferred topics which would be selected for the agenda of the third regional educational meeting on SC for EEBR (2019).

\section{Methods}

Participants and faculty were invited to participate in the raffle and name and rank 3 SC topics they would like to see included in the next regional EEBR symposium. MASCC membership and ESMO booklets were offered for 3 prizes in the raffle. Descriptive statistic methods were used to analyze and identify the most frequent topics of interest.

\section{Results}

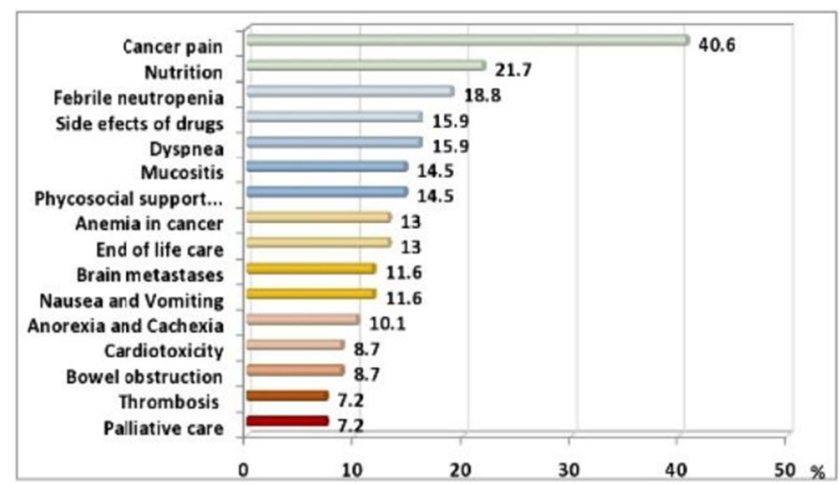

Out of 159 participants from the 18 countries, 140 (88\%) were physicians; $10(6.3 \%)$ were pharmacists and $9(5.7 \%)$ nurses. Evaluation forms were filed out by 75 (47\%) participants: 69/75 (92\%) were valid. Thirty two different SC topics of interest were identified: 10/32 are presented on Figure 1. Five most wanted topics were: cancer pain $(40.6 \%)$, nutrition $(21.7 \%)$, febrile neutropenia $(18.8 \%)$, dyspnea $(15.9 \%)$ and side- effects of cancer treatment $(15.9 \%)$. The $1^{\text {st }}$ and $2^{\text {nd }}$ educational SC EEBR meeting provided a good coverage of the most wanted topics. Cardiotoxicity, end-of life care and psychosocial support have never been covered.

\section{Conclusions}

Ten most interesting topics for SC education in EEBR region were identified. Three topics of interest were observed that have never been covered and will be considered for the next meeting agenda.

\section{eP096}

SYMPTOM NAVIC PROGRAM: TRAINING NURSES TO FACILITATE CANCER PATIENTS' SELF-MANAGEMENT OF SYMPTOMS WITHIN A STRUCTURED POGRAM

M. Bana ${ }^{1,2}$, S. Peters ${ }^{3}$, T. Manser ${ }^{4,5}$, S. Kropf-Staub ${ }^{6}$, E. Näf $f^{7}$, S. Zürcher $^{8}$, M. Eicher ${ }^{2,3}$

${ }^{1}$ School of Health Sciences HEdS-FR, HES-SO University of Applied Sciencies and Arts Western Switzerland-Fribourg, Winterthur, Switzerland

${ }^{2}$ Institut universitaire de formation et de recherche en soins IUFRS, University of Lausanne Unil, Lausanne, Switzerland

${ }^{3}$ Centre hospitalier universitaire vaudois CHUV, Departement of Oncology, Lausanne, Switzerland

${ }^{4}$ School of Applied Psychology FHNW, University of Applied Sciences and Arts Northwestern Switzerland, Olten, Switzerland

${ }^{5}$ Institute for Patient Safety, University Hospital Bonn, Bonn, Germany

${ }^{6}$ Hospital Group Lindenhof, Departement of Oncology, Berne, Switzerland

${ }^{7}$ Solothurner Spitäler AG, Departement of Practice Development in Nursing, Olten und Solothurn, Switzerland

${ }^{8}$ Hospital Group Lindenhof, Departement of Nursing Development, Berne, Switzerland

\section{Introduction}

We are currently pilot testing the Symptom Navi@ Program (SNCP) in a cluster-randomized design complemented with qualitative methods. The program includes symptom-specific information leaflets (Symptom Navi@ Flyers [SN@Flyers]), provided in semi-structured, nurse led consultations to facilitate cancer patients' self-management of symptoms. We designed a training for nurses using the Capability Opportunity Motivation - Behaviour model and a training manual validated with health care experts. 


\section{Objectives}

To present a preliminary evaluation of nurses' perspective of the training for implementing the SN@P in cancer outpatient services.

\section{Methods}

We used a questionnaire based on the training manual to evaluate nurses' preparedness for applying the SN@P. The questionnaire included five self-report scales (ranging from $1=$ "not at all" to $7=$ "greatest possible"), and two open-ended narrative questions. Potential impact on SNCP's acceptability within practice routines was measured by the 9-item work-related sense of coherence (WorkSoC), a 7-point validated Likert-scale. Nurses completed the questionnaires after each training course.

\section{Results}

To date, ten nurses from two oncology outpatient centres rated their training courses as supportive and appropriate (Median $=6$ for both courses, IQR 0.5 and 2 respectively). Narrative questions showed that they appreciated the detailed SNOFlyers' introductions, and reflections on how to apply semi-structured consultations into practice. Global Work-SoC Median score was 6 (IQR 2), with highest ranking on sub-dimension meaningfulness (Median $=6$, IQR 1).

Conclusions

The SN@P training seems to be meaningful for and well accepted by nurses. We will evaluate nurses' perspective of training at two additional centres.

\section{eP097}

THE ONCOLOGIC HOSPITAL MEDICINE FELLOWSHIP PROGRAM: MAKING THE CASE

N. Brito-Dellan ${ }^{1}$

${ }^{T}$ UT MD Anderson Cancer Center, General Internal Medicine, Houston, USA

\section{Introduction:}

There is an increased demand for expert oncological care in and out of the hospital, as a result of various factors including a growing aging population with a greater incidence of cancer, a shortage of oncologists and a decrease in house-staff working hours. These conditions have created the need for a physician with expertise in the management of the cancer patient in the hospital. Defined as the physician who focuses on the comprehensive care of hospitalized cancer patients, an oncology hospitalist provides value to the patients, their outpatient providers and the health care system as a whole.

Objectives

To train and educate the next generation of physicians in the management of complex medical problems of the cancer patient in the hospital setting.

Methods

The Oncologic Hospital Medicine Fellowship Program at MD Anderson Cancer Center, the first and only of its kind, provides advanced training in the management of complex medical problems of the hospitalized cancer patient. The main goal of the program is to facilitate expertise in the diagnosis, treatment, and multidisciplinary management of the hospitalized cancer patient, as well as to advance scholarship in the growing sub-discipline of oncology hospital medicine.

Results

The fellowship program is in its first year of implementation. The fellow is being recognized as an expert in oncologic hospital medicine.

\section{Conclusions}

Oncology Hospitalists focus on the comprehensive care of hospitalized cancer patients, they provide value, and the creation of a fellowship program aims at training the future generation of experts in the emerging discipline of oncologic hospital medicine.

\section{eP098}

DOCTORAL: THE FREE APP FOR TUTORING IN SUPPORTIVE ORAL CARE

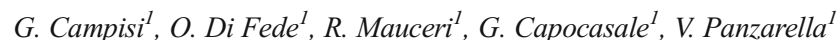
${ }^{T}$ Sector of Oral Medicine "V. Margiotta", Dept. Surgical- Oncological and Oral Sciences- University of Palermo, Palermo, Italy

\section{Introduction:}

Cancer patients are susceptible to a range of oral complications, including: mucositis, oro-dental infections, hyposalivation/ xerostomia, oral GVHD (Graft Versus Host Disease) and ONJ (OsteoNecrosis of the Jaw) drug-related. Therefore, it is essential that easily tools are made available, for being used also by nondental professionals, to perform appropriate clinical assessments and to manage patients' oral needs.

\section{Objectives}

To introduce the version 2.0 of DoctOral free app, created for tutoring medical/dental professionals (and students) in the clinical recognition of oral lesions and dental treatment planning in patients at risk of $\mathrm{ONJ}$ drugrelated.

\section{Methods}

The principal functions of the app are the guided paths based on the recognition of initial lesions in the oral cavity, differentiating them by Morphology/Chroma or Etiology. Each pathway is selected by multiple-choice questions, leading to one or more diagnostic hypotheses. The user will be able to take clinical photos of their own cases throughout the entire pathway for comparison with the final hypothesis/es (Fig. 1). At the end of the tree, if necessary, it is possible to request via e-mail a service support. The second function allows to consult some guided pathways decisional algorithm regarding the dental treatment of patients at risk of drug-related osteonecrosis of the jaw (in pre/post-therapy), according to the Recommendations of Italian Scientific Societies (SIPMO and SICMF) (Fig. 2-3).

Results

Up to date, DoctOral has been used by 1,295 (IOS) and 3,488 (ANDROID) users.

\section{Conclusions}

DoctOral could be a useful and simple tool for management of cancer patient oral health.

\section{eP099}

KNOWLEDGE OF HOSPICE AND ATTITUDE TOWARDS HOSPICE ADMISSION IN PATIENTS WITH ADVANCED CANCER IN PALLIATIVE MEDICINE SETTING IN THE INDIAN SOCIO-CULTURAL CONTEXT

G.G. Chinchalker ${ }^{1}$, J. Deodhar ${ }^{1}$, N. Salins ${ }^{1}$, M. Muckaden

${ }^{T}$ Tata Memorial Centre, Palliative Medicine, Mumbai, India

\section{Introduction}

Despite potential benefits of hospice enrollment, hospice facilities are underutilized among advanced cancer patients. No study has been done which reflects knowledge of and attitude of advanced cancer patients towards hospice.

\section{Objectives}

To understand knowledge of hospice, attitude towards hospice admission and factors associated with knowledge and attitude among advanced cancer patients receiving palliative care.

\section{Methods}

A cross sectional survey was conducted on 100 advanced cancer patients visiting Dept of Palliative Medicine, Tata Memorial Hospital, Mumbai, India, using convenience sampling \& a specially prepared content validated questionnaire, with questions for knowledge and attitude (response from strongly agree to strongly disagree). Analysis was done for 
descriptive statistics. Chi square test was applied to examine associations between knowledge, attitude and different factors.

\section{Results}

Participants included 54\% females, $61 \%$ aged $<60$ years, $98 \%$ with family support. 33\% participants had knowledge of hospice. Urban/suburban residence $(\mathrm{p}=0.04)$ and higher level of education $(\mathrm{p}=0.001)$ were significant factors associated with knowledge of hospice. Of these, $15(45 \%)$ and $9(27.2 \%)$ believed care at hospice is better than at home and hospital, respectively. Analysis of attitude revealed that $23 \%$ considered admission at hospice meant losing hope, $22 \%$ thought they would not be able to go back home, knowledge of hospice $(p<0.001)$ being a significant affecting factor. $19 \%$ with low finances $(\mathrm{p}=0.009)$ would prefer hospice stay.

\section{Conclusions}

Awareness of hospice is limited among palliative care patients. Culturally appropriate educational measures need to be implemented to bring about a positive change in understanding of hospice among patients $\&$ general public.

\section{eP100}

\section{QUIET ISSUE OF CANCER: SEXUALITY}

\author{
N. Nural ${ }^{1}$, Y. Clracı Yasar ${ }^{1}$, S. Öztürk ${ }^{l}$ \\ ${ }^{I}$ institue of health sciences, internal medicine nursing, trabzon, Turkey
}

\section{Introduction:}

Cancer process is biologically, psychologically and emotionally damaging to the patient's well-being and adversely affecting the quality of life of the patient. Sexuality, which is a normal course of life, is also greatly affected in the cancer process, but usually both patients and health professionals are reluctant to talk about it. Whereas, purpose of sexual health is not only about reproductive medical care and counseling but also strengthen personality, personal relationships and life. The aim of nursing care is a holistic patient approach. Nurses should be able to assess sexuality as part of holistic care and provide support for patients. Because of this, nursing education should also focus on the problem of sexuality of the patients.

Objectives

Methods

Results

Conclusions

\section{eP101}

HOLISTIC APPROACH TO SUPPORTIVE CARE: BULGARIAN EXPERIENCE

\section{Despotova-Toleva ${ }^{1}$, N. Yordanov ${ }^{2}$}

${ }^{T}$ Medical University, Medical Facullty, Plovdiv, Bulgaria

${ }^{2}$ Complex oncology center, Palliative care, Vraza, Bulgaria

\section{Introduction}

The best approach to supportive and palliative care for patient who suffers from incurable disease is the holistic approach and the natural place to provide such care is at home by patient's family, respectively by his/her general practitioner. There are no approved standards, no accredited program and no recognized specialty in palliative medicine in Bulgaria.

Objectives

1.To contribute adoption of a National Standard for better palliative care. 2. To justify the importance of general practitioners and the opportunities of general practice for providing better support and care for patients and their families.

\section{Methods}

Education and training for General Practitioners and other medical and non-medical specialists in Bulgaria in supportive, long term and palliative care.

\section{Results}

Since 2003 significant efforts are devoted to train GPs in palliative and long term care and special training programs for three educational levels were developed and offered in Medical University - Plovdiv (for undergraduate medical students, for postgraduate physicians and for $\mathrm{PhD}$ students. As a result, textbooks, monographs and manuals as well as patient oriented materials were published. Multidisciplinary seminars and workshops were organized with over 100 medical and nonmedical participants. National congresses with international participation and International conferences in the field are organized every year. Special journal with international editorial board was established and issued.

\section{Conclusions}

Combined efforts of general practitioners and other medical and nonmedical specialists are needed to provide high quality supportive and long term care using the holistic approach to achieve better care, and quality of life of the patients.

\section{eP102}

\section{ART2CARE PROGRAM: A POWERFUL TOOL FOR BUILDING RESILIENCE IN PAEDIATRIC CANCER CARE}

\author{
M. Elewah ${ }^{1}$ \\ ${ }^{T}$ Hermel Hospital, Pediatric Cancer, Cairo, Egypt
}

\section{Introduction}

When a child is diagnosed with a life threatening disease, the family is devastated. Child and family life changes from normal to abnormal life full of challenges inside the clinics, wards and hospitals corridors. In Egypt, pediatric cancer patients suffer from psychopathology and psychosocial support, where psychosocial support plays a major role in their treatment path or later as survivors. Long-term survivors of pediatric cancer demonstrate resilience in quality of life... 10-20\% score more than fourfold greater risk in PTSD compared to siblings Kazak et al (p.2002), and Stuber (2010). Art therapy in the medical setting proved to be a valuable resource for building resilience and a powerful psychosocial tool in rehabilitation of pediatric cancer patients and their families. (Council, 2012: Nainis, 2008).

\section{Objectives}

The theory and practice of art therapy for trauma powerfully informs art therapy with pediatric cancer patients in Art2Care program. The presentation will: (1) Describe the nature of traumatic experience through neuroscience; (2) Present case examples illustrating how art therapy helps patients preserve self-esteem, build resilience, and process trauma; (3) Explore the role of community support in creating better life quality for survivors (Boss, 2003).

\section{Methods}

Weekly workshops have been carried out since its inception in April 2014.

Results

ART2CARE program has served 12,335 patients, 2,983 families, with the help of 4,902 volunteers, using 41,380 kgs of art supplies, participating in 32 exhibitions, and 22 conferences, 18,005 Facebook friends and 1,804 followers on Instagram.

\section{Conclusions}

ART2CARE has proven to be a powerful tool for building resilience in pediatric cancer care.

\section{eP103}

CANCER AWARENESS IN IBADAN, SOUTHWEST NIGERIA: A POPULATION-BASED CROSS-SECTIONAL STUDY 
O. Fagbule ${ }^{1,2}$, E. Idiga $^{2}$, A. Owosho ${ }^{3}$, F. Lawal $^{4}$

${ }^{T}$ Cephas Health Research Initiative CHRI, Head and Neck Cancer, Ibadan, Nigeria

${ }^{2}$ University College Hospital UCH, Periodontology and Community Dentistry, Ibadan, Nigeria

${ }^{3}$ University of New England, College of Dental Medicine, Maine, USA

${ }^{4}$ University of Ibadan and University College Hospital UCH, Periodontology and Community Dentistry, Ibadan, Nigeria

\section{Introduction}

Cancer is a leading cause of death globally and the incidence is on the increase. Prognosis for advanced cases has remained poor even with advanced management protocol, making prevention and early presentation very important. A baseline information about the level of awareness becomes crucial for planning preventive health-promotion programs.

\section{Objectives}

To assess cancer awareness and associated factors among adults in Ibadan, Nigeria.

\section{Methods}

An interviewer-administered questionnaire-based cross-sectional survey was conducted among participants $(n=389)$ of eight dental-outreach programs across Ibadan, with $97 \%$ response rate. Information on sociodemography, cancer awareness and cancer-types were sought. Data were analyzed with SPSS version 17.

Results

Mean age was $36.4( \pm 15.8)$ years, $63.2 \%$ were females, and $39.1 \%$ had no formal education. Dependents, unskilled and skilled workers were $35.1 \%$, $61.5 \%$ and $3.4 \%$ respectively. The majority $(92.2 \%)$ have heard of cancer; breast cancer (BC) $(80.8 \%)$, cervical cancer (CC) (54.3\%), oral cancer (OC) $(39.7 \%)$, prostate cancer (PC) $(37.1 \%)$, and "cancer of blood" (CB) $(39.7 \%)$. Almost $40 \%$ either "believed" or "not sure" that cancer is caused by spiritual attack.

Females were more aware of cancer $(\mathrm{OR}=2.0 ; \mathrm{CI}=1.0-4.0 ; \mathrm{P}=0.041), \mathrm{BC}$ $(\mathrm{OR}=2.8 ; \mathrm{CI}=1.5-5.2 ; \mathrm{P}=0.001), \mathrm{CC}(\mathrm{OR}=2.9 ; \mathrm{CI}=1.8-4.7 ; \mathrm{P}<0.001)$. Unskilled workers were least aware of $\mathrm{BC}(\mathrm{OR}=3.0 ; \mathrm{CI}=1.0$ $8.3 ; \mathrm{P}=0.040)$, and $\mathrm{PC}(\mathrm{OR}=3.4 ; \mathrm{CI}=1.4-8.2 ; \mathrm{P}=0.007)$. Tertiary-level of education was a significant predictor of being aware of $\mathrm{CC}$ $(\mathrm{OR}=3.1 ; \mathrm{CI}=1.4-6.6 ; \mathrm{P}=0.004), \mathrm{PC}(\mathrm{OR}=2.4 ; \mathrm{CI}=1.1-5.1 ; \mathrm{P}=0.021), \mathrm{CB}$ $(\mathrm{OR}=2.9 ; \mathrm{CI}=1.3-6.1 ; \mathrm{P}=0.006)$ and rejecting spiritual attack as cause of cancer $(\mathrm{OR}=3.1 ; \mathrm{CI}=1.5-6.6 ; \mathrm{P}=0.003)$.

Conclusions

About $84 \%$ were aware of cancer as a disease, and almost $40 \%$ thought it could be caused by spiritual attack. Females were more aware and awareness was highest for $\mathrm{BC}$ and lowest for PC.

\section{eP104 \\ NURSING EXPERIENCE IN A BREAST CANCER PATIENT WITH CHEMOTHERAPY-INDUCED ANXIETY}

C. Guan Zhen ${ }^{l}$

${ }^{T}$ Chang Gung University, nursing, New Taipei City, Taiwan R.O.C.

\section{Introduction}

During chemotherapy, side effects may compromise the quality of life of the patient. If the condition becomes serious, chemotherapy-induced side effects could impede the advancement of the therapeutic regimen, cause uncertainties of treatment and prognosis, and thus lead to anxiety.

The patients developed concerns, anxiety and emotional distress or even frustration regarding her treatment and prognosis. She was becoming worried about her previous efforts could be in vain, and nothing to her was controllable.

Objectives

By sharing such experience, it is expected to perform a comprehensive nursing assessment, make patient-centered nursing plans, and provide individualized and consecutive nursing measures to improve self- protection, learn about basic knowledge of nursing care for side effects caused by chemotherapy, and thus develop adequate responses to situations resulted from treatment.

\section{Methods}

Through proper diet, personal hygiene and home-based self care instructions, the patient improved her self-protection skills, improved her white blood cell levels, and was thus able to resume the scheduled chemotherapy.

\section{Results}

Through sharing such experiences, similar findings were made in studies conducted by Hsu (2011) and $\mathrm{Wu}$ (2011), with the priority in patients with leukopenia being infection prevention; i.e. proper hand-washing and self-care skills are both helpful in reducing the possibility of infection.

\section{Conclusions}

As a result, if nursing staffs have evaluated the understanding of chemotherapy-induced side effects among candidate patients at early stage of the treatment, and give the patients proper self-care instructions accordingly, the quality of life of the patients during treatment will be greatly improved.

\section{eP105 \\ NURSING EXPERIENCE IN A BREAST CANCER PATIENT WITH CHEMOTHERAPY-INDUCED ANXIETY}

C. Guan Zhen ${ }^{1}$

${ }^{T}$ 0277282647, Far Eastern Memorial Hospital, New Taipei City, Taiwan R.O.C.

\section{Introduction}

During chemotherapy, side effects may compromise the quality of life of the patient. If the condition becomes serious, chemotherapy-induced side effects could impede the advancement of the therapeutic regimen, cause uncertainties of treatment and prognosis, and thus lead to anxiety. The patients developed concerns, anxiety and emotional distress or even frustration regarding her treatment and prognosis. She was becoming worried about her previous efforts could be in vain, and nothing to her was controllable.

\section{Objectives}

By sharing such experience, it is expected to perform a comprehensive nursing assessment, make patient-centered nursing plans, and provide individualized and consecutive nursing measures to improve self-protection, learn about basic knowledge of nursing care for side effects caused by chemotherapy, and thus develop adequate responses to situations resulted from treatment.

\section{Methods}

Through proper diet, personal hygiene and home-based self care instructions, the patient improved her self-protection skills, improved her white blood cell levels, and was thus able to resume the scheduled chemotherapy.

Results

Through sharing such experiences, similar findings were made in studies conducted by Hsu (2011) and $\mathrm{Wu}$ (2011), with the priority in patients with leukopenia being infection prevention; i.e. proper hand-washing and self-care skills are both helpful in reducing the possibility of infection.

\section{Conclusions}

As a result, if nursing staffs have evaluated the understanding of chemotherapy-induced side effects among candidate patients at early stage of the treatment, and give the patients proper self-care instructions accordingly, the quality of life of the patients during treatment will be greatly improved.

eP106

PATIENTS, RELATIVES AND HEALTH CARE PROFESSIONALS VIEWS REGARDING THE INFLUENCE 
ON REFERRALS OF THE TERMINOLOGY 'PALLIATIVE CARE CLINIC' IN A TEACHING HOSPITAL

\author{
N. Holtom ${ }^{1}$, S. Hlaing ${ }^{2}$ \\ ${ }^{T}$ Palliative Medicine Consultant, Norfolk + Norwich University Hospital \\ Foundation Trust, Norwich, United Kingdom \\ ${ }^{2}$ Palliative Care Specialty Doctor, Norfolk and Norwich University NHS \\ Foundation Trust, Norwich, United Kingdom
}

\section{Introduction}

Concern that patients, who could potentially benefit from symptom management and supportive care, had declined referral to the palliative care clinic because of negative connotations associated with its name prompted this study.

\section{Objectives}

To ascertain whether the terminology 'Palliative care Clinic' had an impact on willingness to refer and patient's willingness to accept referral as well as views regarding clinic rebranding

\section{Methods}

Consultants and nurses caring for adult patients with life limiting illnesses completed a semi-structured questionairre using case studies. Patients and relatives attending the palliative care clinic completed an interview questionairre following consultation.

\section{Results}

A majority of health professionals believed the terminology "Palliative Care Clinic" would deter them from referring certain patients, especially those with non-malignant disease, early in their illness and cancer survivors. Similarly $>80 \%$ of patients and relatives felt the terminology had negative connotations and did not represent their experience of the support they had gained from the clinic. Patients did not always understand why they had been referred which caused anxiety and distress. They were overwhelmingly in favour of rebranding the clinic and felt this would be less ambiguous and cause less distress

\section{Conclusions}

As the traditional roles associated with Palliative Care expand to meet the changing needs in the acute sector, it is important that services are acceptable to all patients that would potentially benefit. As a result of this survey the Palliative Care clinic has been rebranded 'The Symptom Management and Supportive Care Clinic' which professionals, patients and relatives supported and we felt best describes our role.

\section{eP107 \\ PATIENTS WITH MALIGNANT PANCREATIC MASSES DIAGNOSED AT A CANCER CENTER}

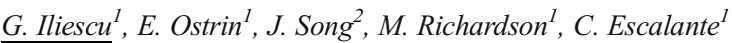 \\ ${ }^{T}$ The University of Texas- MD Anderson Cancer Center, General Internal \\ Medicine, Houston- TX, USA \\ ${ }^{2}$ The University of Texas- MD Anderson Cancer Center, Biostatistics, \\ Houston- TX, USA
}

\section{Introduction}

Introduction: Patients diagnosed with cancer frequently receive fragmented care as they move through presentation, diagnosis and then treatment and often experience delays. We developed a diagnostic clinic at a comprehensive cancer center to address this concern. We describe our findings.

Objectives

Objective: Our objectives are to describe patients (demographic, histology, stage, treatment received) with malignant pancreatic masses presenting to our clinic.

\section{Methods}

Methods: The study was approved by our institution's IRB. Data from our institution's Tumor Registry were extracted for patients with malignant pancreatic masses evaluated in our diagnostic clinic from 1/1/2011 through $8 / 31 / 2017$. This included both demographic and disease information. Descriptive statistics were utilized to summarize demographic and clinical characteristics of the cohort.

Results

Results: 100 patients with malignant pancreatic masses that were evaluated with a mean age of 67 years and male $52 \%$. The majority were white $(75 \%)$. The most common histology was adenocarcinoma $(73 \%)$. Only $9 \%$ were neuroendocrine tumors. $51 \%$ had distant disease by SEER staging. $77 \%$ of patients either received cancer treatment or had treatment recommendations outlined. The most common treatments received at our institution during the first 4 months after diagnosis were chemotherapy $(48 \%) ; 8 \%$ received surgery while $49 \%$ received no cancer treatment.

\section{Conclusions}

Conclusions: Patients with undiagnosed malignant pancreatic masses most frequently have metastatic disease. Of those receiving treatment, chemotherapy was most administered likely due to advanced stage while nearly half elected to receive no cancer treatment. The importance of symptom control and focus on quality of life should be considered during the diagnosis of this malignancy.

\section{eP108 \\ SUSPICION OF CANCER CLINIC IN A TERTIARY CENTER: INITIAL EXPERIENCE AND TREATMENT RECEIVED}

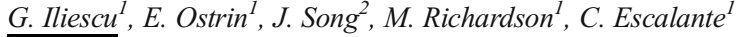

${ }^{T}$ The University of Texas- MD Anderson Cancer Center, General Internal Medicine- Unit 1465, Houston- TX, USA

${ }^{2}$ The University of Texas- MD Anderson Cancer Center, Biostatistics, Houston- TX, USA

\section{Introduction}

Introduction: Complexity of diagnostic tools and therapeutic options have increased exponentially over the last decade. Available data on the role of internists in diagnosis and oncology referral is scarce. We present our initial experience.

\section{Objectives}

Objectives: To describe patients (histology, stage, treatment modality) with malignancy presenting to clinic.

\section{Methods}

Methods: We analyzed medical records of patients evaluated in clinic between 1/1/2011 and 8/31/2017. Demographic and clinical characteristics were recorded. Clinical outcomes considered included: non-malignant or type of malignancy, stage at diagnosis and treatment vs palliative care referral.

\section{Results}

Results: Among 2135 patients, 898 (42\%) were diagnosed with cancer. Hematological malignancies represented 137 (15.2\%) lymphoma 117 (13\%) being most common. Lung 281 (31.3\%), gastrointestinal 208 (23.2\%) and cancer of unknown primary $50(5.6 \%)$ were the most common solid tumors. Most frequent histological diagnosis was adenocarcinoma 299 (33.3\%). Majority of patients for which AJCC staging was available (505) had advanced cancer (stage III and IV) $352(69 \%)$. The most frequent treatments were chemotherapy 387 (43.1\%), radiation 161 (17.9\%), surgery $119(13.3 \%)$ and immunotherapy 103 (11.5\%), while $341(38 \%)$ received no treatment.

\section{Conclusions}

Conclusions: A significant number of patients referred to a diagnostic clinic within a large tertiary cancer center are diagnosed with cancer. Experienced internists provided cancer diagnosis and transition to cancer treatment or palliative care. Age appropriate screening and increased suspicion in the primary care community with early referral to a center of excellence in cancer care may translate in improved survival and decreased percentage of patients that move to palliation. Further study is needed. 


\section{eP109}

PATIENTS WITH MALIGNANT LUNG LESIONS DIAGNOSED IN A CANCER CENTER

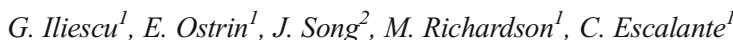

${ }^{T}$ The University of Texas- MD Anderson Cancer Center, General Internal Medicine- Unit 1465, Houston- TX, USA

${ }^{2}$ The University of Texas- MD Anderson Cancer Center, Biostatistics, Houston- TX, USA

\section{Introduction}

Introduction: Lung cancer is the most common non-skin cancer and the leading cause of death from malignancy in the United States. Low-dose $\mathrm{CT}$ screening in high-risk population showed reduction of the risk of death. False-positive results prompt costly or invasive testing that can result in complications. Limited data are available regarding diagnosis of abnormal lung lesions in a specialized internal medicine clinic.

Objectives

Objectives: To describe patients (demographics, histology, stage, treatment) with malignant lung lesions seen in our clinic.

Methods

Methods: We reviewed medical records of patients evaluated in Suspicion of Cancer Clinic between 1/1/2011 and 8/31/2017.

Results

Results: Of 2135 patients seen in clinic, 278 were diagnosed with lung cancer $(13.0 \%)$. Median age at diagnostic was 66 . Gender was nearly evenly distributed, (50.7\% female). The most common histological subtypes were adenocarcinoma 133 (47.8\%), squamous cell carcinoma 45 (16.2\%). Out of 199 cancers with AJCC staging, 138 (69\%) were diagnosed as stage III and IV.

Of 115 (41.4\%) patients commencing chemotherapy; 104 (37.4\%) received radiation therapy, $44(15.8 \%)$ patients qualified for surgical resection, $8(2.9 \%)$ have been treated with immune checkpoint blockade agents, and $83(29.9 \%)$ patients did not receive therapy.

Conclusions

Conclusions: Of all lung nodules, $13 \%$ were malignant. While this is higher than the $4 \%$ of lung nodules diagnosed as cancer in a CT-based lung cancer screening trial, our patient population represents a heterogeneous group. Patients with abnormal lung lesions should be referred to an institution with expertise in diagnosis and access to a multidisciplinary team skilled in the evaluation, diagnosis, and treatment.

\section{eP110}

OCCUPATIONAL EXPOSURE TO ANTINEOPLASTIC AGENTS: EVALUATION OF ONCOLOGY NURSES AWARENESS OF POTENTIAL RISK FOLLOWING HANDLING AND ADMINISTERING CHEMOTHERAPY IN A GREEK ONCOLOGY UNIT.

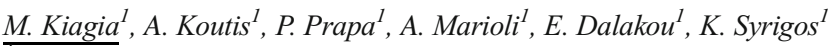
${ }^{T}$ Sotiria General Hospital, Oncology Unit GPP, Athens, Greece

\section{Introduction}

The exposure of the nursing staff to chemotherapy drugs is a critical issue that worries hospital staff.

Objectives

The aim of the study was to evaluate the concerns of nursing staff of Sotiria Hospital regarding the potential exposure to chemotherapy drugs, the knowledge and use of Personal Protective Equipment and their desire for additional training on this issue.

Methods

A quantitative research method with a closed-ended questionnaire was used. The sample of the study consisted of 98 nurses (Assistant Nurse and Registered Nurse ), with the basic requirement to work in chemotherapy drugs administration departments of the hospital.

\section{Results}

The majority of Sotiria Hospital nurses are aware of the possible risk of handling chemotherapy drugs. They reported their basic studies, seminars and internet as the main sources of information regarding this topic. The highest percentage of respondents are aware of ways to prevent the toxicity of chemotherapy drugs, and indeed take the appropriate precautions when transferring, administering and disposing of chemotherapy drugs. It was found that those with a University degree $[\mathrm{RN}](59.8 \%)$ desired more information (seminars, workshops, conferences) about the protection measures to be taken when administering chemotherapy drugs $(\mathrm{p}=0.027)$. In addition, those who had already attended seminars related to the topic, displayed higher nursing experience $(\mathrm{p}=$ 0.027).

\section{Conclusions}

The majority of nurses that participated in the study are aware of ways to protect them from the toxicity of chemotherapeutic drugs and taking precautionary measures when administering them, but additional education would be welcomed.

\section{eP111}

BREAST CANCER SCREENING IN INDIA - BARRIERS AND PERSPECTIVES

C. Kohli ${ }^{1}$, N. Dahiya ${ }^{2}$, S. Garg $^{2}$

${ }^{T}$ Vardhman Mahavir Medical College, Department of Community Medicine-, New Delhi, India

${ }^{2}$ Maulana Azad Medical College, Department of Community Medicine, New Delhi, India

\section{Introduction}

Breast cancer is the most common female cancer worldwide including urban India, where advanced stages at diagnosis, and rising incidence and mortality rates, making it essential to understand the perspectives of community about breast cancer. Despite many screening methods available for breast cancer, it is still not widely practiced.

\section{Objectives}

To determine the barriers and perspectives of general population about breast cancer screening in Delhi, India.

Methods

A cross sectional study was conducted among 220 females aged 18 years and more who gave written informed consent to participate in the study. Study participants were interviewed using a pretested semi -structured questionnaire. Data was collected to assess the barriers to practice breast cancer screening like Breast Self Examination. Data was analysed in SPSS 17 software. Ethical clearance was taken from Institutional Ethics Committee.

\section{Results}

Mean age (+SD) of study subjects was $30.12(+23.9)$ years. Most of the study subjects were graduates (43.2\%) and students (47.3\%). When inquired about their perception about Breast Self Examination (BSE), 118 $(57.6 \%)$ were aware that it should be done monthly. Correct procedure of doing BSE was known to only 92 (44.9\%) subjects. 106 (52.5\%) subjects were practising BSE. Among reasons of not practising BSE, no awareness about the need of BSE (24.9\%), perception of not being at risk of breast cancer $(31.8 \%)$ and no knowledge about the procedure $(29.5 \%)$ were most common.

\section{Conclusions}

Innovative Behavior Change Communication (BCC) interventions should be taken to make breast cancer screening acceptable and available to community.

\section{eP112}

SHORTENING AND ADAPTATION OF THE GERMAN BARRIERS QUESTIONNAIRE II - A QUESTIONNAIRE TO 
MEASURE PATIENT-RELATED BARRIERS TOWARDS THE MANAGEMENT OF CANCER PAIN

\section{A. Koller ${ }^{1}$, P. Jahn ${ }^{2}$}

${ }^{T}$ University of Vienna, Faculty of Social Sciences, Vienna, Austria

${ }^{2}$ Martin-Luther-University-Medical Faculty, Institute for Health and

Nursing Science, Halle-Wittenberg- Germany, Germany

\section{Introduction}

The Barriers Questionnaire (BQII, 27 items) is one of the most frequently used instruments to measure patient-related barriers towards cancer pain.

Objectives

The aim of this study was to shorten the German BQII (BQII-G) in order to decrease patient and clinician burden.

Methods

In this four-stepped mixed methods study, (1) baseline data of a cluster randomized trial with 207 adult oncology patients with cancer pain (10/ 2008-12/2009) were used for factor analysis and descriptive values. (2) With the content validity index, expert ratings of 4 nurses and 2 patients with experience in using the BQII-G were analyzed. (3) In a consensus process between the first and the last author results of (1) and (2) were synthesized. Decisions were guided by the overall aims to reduce patient burden and to keep the original structure of the BQII-G. In each dimension, at least one question was maintained. If results were ambiguous, expert ratings were preferred. (4) The baseline data of the cluster randomized trial were used for first psychometric testing of the short version (BQII-G12).

\section{Results}

Cronbach's a was 0.92 for the BQII-G. Steps 1 to 3 resulted in the BQIIG12 (12 items). The BQII-G12 explains $84.3 \%(r=0.92)$ of the variance of the BQII-G. Cronbach's alpha of the BQII-G12 was with 0.833 appropriate.

\section{Conclusions}

The BQII-G12 shows excellent psychometric properties in the preliminary testing. It therefore will support clinical practice and research by substantially reducing patient burden and resources needed to measure patient-related barriers to cancer pain management.

\section{eP113}

EVALUATION OF A NURSE-LED SELF-MANAGEMENT SUPPORT INTERVENTION FOR PATIENTS WITH CANCERRELATED PAIN: PRACTICABILITY OF A CLUSTER RANDOMIZED STEPPED WEDGE TRIAL (EVANTIPAIN)

\section{A. Koller ${ }^{\text {, H. Mayer }}{ }^{1}$ \\ ${ }^{T}$ University of Vienna, Faculty of Social Sciences, Vienna, Austria}

\section{Introduction:}

Stepped wedge cluster randomized trials (SW-CRTs) are considered useful to evaluate the implementation of complex interventions.

Objectives

In this presentation, experiences with the EvANtiPain study are discussed regarding the practicability of an SW-CRT in acute care.

\section{Methods}

ANtiPain, a cancer-pain self-management support intervention, was implemented on 17 wards (clusters) in 3 hospitals in Vienna following the SW-CRT plan (Figure 1). ANtiPain is based on (1) information, (2) skill building, and (3) coaching and was applied by designated staff nurses. Adult patients with cancer-related pain were recruited from 01/17-05/18. Patients treated before (control condition) and after implementation (intervention condition) were compared regarding pain-interference with daily activities (primary outcome), and other outcomes (e.g., pain intensity).

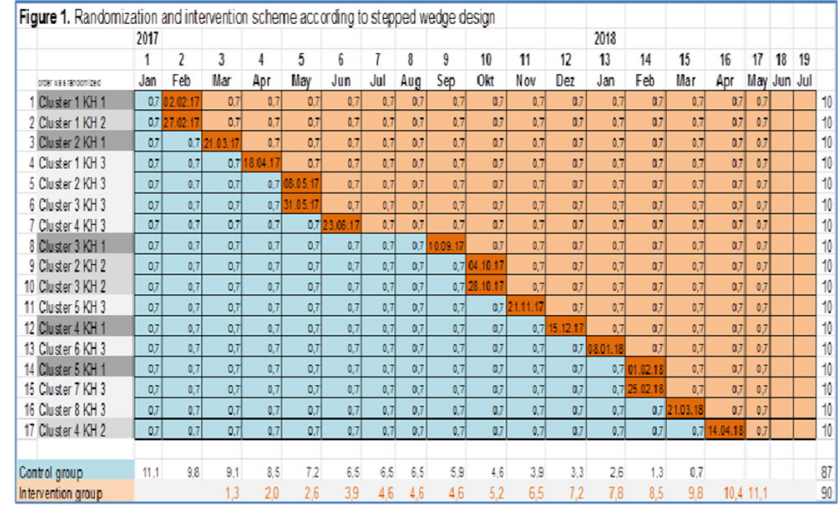

Results

Questions arising from the relatively inflexible nature of the SWCRT design are discussed. (1) Randomization steps should be evenly distributed over the study period (EvANtiPain: 24 days). Due to unexpected restructuring, two clusters lacked cancer patients. It is unclear how the exclusion of two clusters that leads to uneven distances between randomization steps will affect the analysis. In our study the recruitment rate lagged expectations $(57 \%$ of the expected rate). Because extensions (e.g., prolonging steps) are not recommended for SW-CRTs, slow recruitment was probably inadequately addressed. (2) Time trend analysis and within cluster comparisons are advantages of SW-CRT. However, it is unclear how these are affected by unevenly distributed recruitment within clusters like in EvANtiPain.

\section{Conclusions}

More discourse is needed to discuss adaptations of SW-CRT designs to increase their usability in dynamic clinical settings.

\section{eP114}

LOST IN TRANSLATION: EXPLORING THE NATURE OF INFORMATION AND SUPPORT ACCESSED BY NONENGLISH SPEAKERS TO AN AUSTRALIAN CANCER HELPLINE

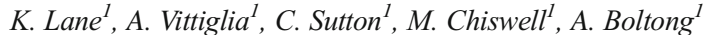 \\ ${ }^{1}$ Cancer Council Victoria, Cancer Information and Support Service,} Melbourne, Australia

Introduction:

In Australia, research shows non-English speakers (NES) with cancer have poorer experiences than those where English is a first language. NES also report limited access to culturally appropriate information and support in their preferred language. Cancer Council Victoria receives thousands of calls each year via their 131120 telephone line, with a small proportion from NES, utilising the Government's interpreting service. Although calls are routinely reviewed for quality purposes, this hasn't occurred for NES.

Objectives

To assess:

1) Accuracy of interpretation

2) Conversational barriers or facilitators

3) Whether NES calls utilised the same therapeutic model as Englishspeaking calls

\section{Methods}

Interpreter calls in 2016 were transcribed with consecutive pairs of dialogue between nurse and caller/nurse and interpreter analysed for content and accuracy. Transcripts also assessed quality issues and impacts on therapeutic communication using an established proforma. 


\section{Results}

16 calls were transcribed, representing four languages. Analysis showed information relayed from caller to nurse was accurate $65 \%$ of the time, while information from the nurse to the caller was only $73 \%$ accurate. Quality issues indicated non-compliance with best practice principles when working with interpreters by both interpreter and nurse. This created barriers that affected the nurse's ability to follow usual call process, impacting communication via the expected therapeutic model. Emotional concerns were rarely discussed.

Conclusions

Provision of qualified interpreters with knowledge of medical terminology and complexities is paramount for culturally appropriate care to NES. This project will inform development of targeted training mechanisms for Cancer Council staff to be implemented across similar services to increase NES access to culturally appropriate support.

\section{eP115}

ANALYSIS OF TELEPHONE COUNSELING FOR SYMPTOM MANAGEMENT IN GASTROINTESTINAL CANCER PATIENTS TREATED WITH CHEMOTHERAPY IN OUTPATIENT SETTING

\section{S.H. Lee ${ }^{1}$, Y.H. Kim ${ }^{1}$, M.H. Ryu ${ }^{2}$, J.H. Kim ${ }^{3}$}

${ }^{T}$ Asan Medical Center, Department of Nursing, Seoul, Republic of Korea

${ }^{2}$ Asan Medical Center, Department of Oncology, Seoul, Republic of Korea

${ }^{3}$ University of Ulsan, Department of Nursing, Seoul, Republic of Korea

\section{Introduction:}

Since an increasing number of cancer patients are receiving chemotherapy in outpatient setting and they could experience adverse events occurring at home, it is needed to offer appropriate symptom management information and supportive nursing intervention. This study investigated the use of our institution's telephone counseling program which is developed for managing symptoms effectively.

\section{Objectives}

The purpose of this study is analyzing the contents of telephone counseling about symptom management of gastrointestinal cancer patients receiving chemotherapy in outpatient setting.

Methods

After a specialist nurse provided chemotherapy education and information booklets to patients, the nurse received the calls and consulted the doctor if necessary. Raw data extracted from 1,022 telephone counseling calls requested by 660 patients from December 2015 to December 2016 were analyzed by using the pre-established telephone program.

\section{Results}

The most common cancers were $\operatorname{stomach}(36.8 \%)$, biliary $\operatorname{tract}(25 \%)$, and pancreas(23.2\%). The most frequent chemotherapy schedules that had been administered were Oxaliplatin plus Capecitabine(20.6\%) and Gemcitabine plus Cisplatin(13.4\%). Among the reported 33 symptoms, pain $(24.1 \%)$ was the most common, and diarrhea $(9.1 \%)$, nausea and vomiting $(8.0 \%)$, anorex$\mathrm{ia}(6.3 \%)$ and fever $(6.0 \%)$ were followed. And as a result, only $34.6 \%$ of the total calls resolved the problem by receiving telephone education and explanation from a specialist nurse, and the others were required attendance in the outpatient clinic $(58.7 \%)$ and emergency department $(6.7 \%)$.

\section{Conclusions}

Pain is the most common symptom of the patients with gastrointestinal cancer treated with chemotherapy at the outpatient setting. Therefore, it is important to develop education programs for patients and their families to provide appropriate intervention and symptom management.

\section{eP116}

AN ASSESSMENT OF SEXUAL INFORMATIONAL NEEDS OF BREAST CANCER PATIENTS IN RADIATION ONCOLOGY

\author{
L.H. Lim $^{1}$ \\ ${ }^{T}$ National Cancer Centre Singapore, Division of Radiation Oncology, \\ Singapore, Singapore
}

\section{Introduction:}

Research regarding the sexual impact of breast cancer treatment on Asian women is both sensitive and scarce.

\section{Objectives}

This study aims to assess and evaluate the sexual health needs and concerns of breast cancer radiotherapy patients. It is hoped that awareness will be increased and an appropriate intervention can be developed to address the needs of future breast cancer patients.

\section{Methods}

110 consecutive unselected breast cancer patients were recruited prospectively. Questionnaires were administered once for patient undergoing radiotherapy to the breast. This study employed an anonymous questionnaire; any breast radiotherapy patient who can read English can voluntarily receive and complete the survey. The questionnaire consisted of items addressing demographics, potential informational needs, and educational preferences.

Results

Patients' interest to address sexual concerns decreases with age $(\mathrm{p}=0.024)$. Coherently, sexual concerns of patients are reported to decrease with age $(\mathrm{p}=0.015)$ where $70 \%$ of all respondents below age 50 [age 20-29 (60\%); 30-39 (56.3\%); 40-49(55.1\%)] have stated to have sexual concerns regarding their treatment effects on their sexual health. Patients who underwent breast conservation surgery $(42.2 \%)$ and reconstruction surgery $(83.3 \%)$ were more likely to have concerns on sexual health versus patients who underwent mastectomy $(36.7 \%)(\mathrm{p}=0.032)$.
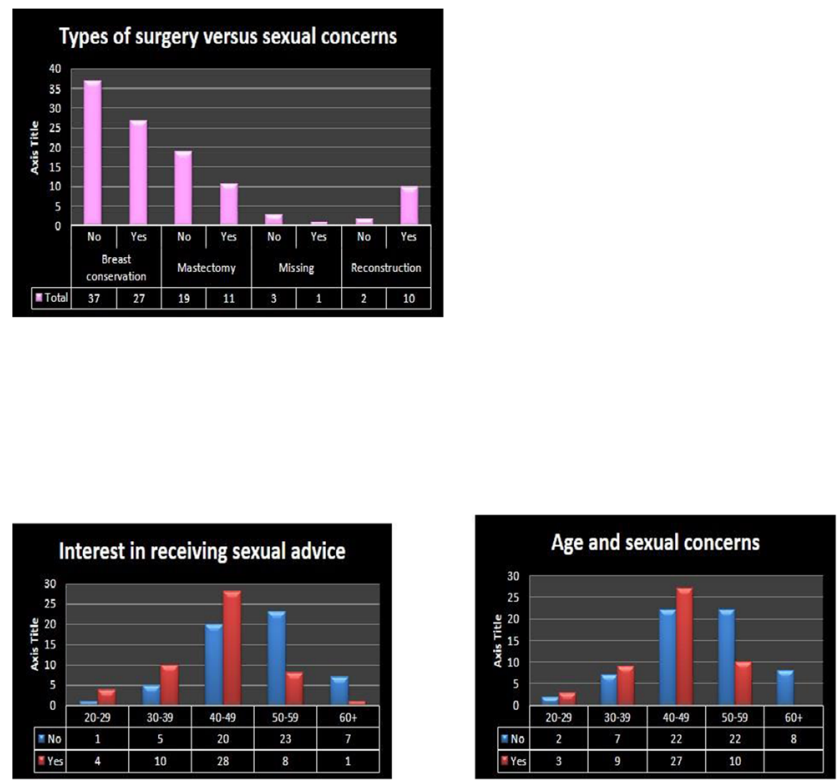

$74.2 \%$ of patients with sexual concern regardless of age would initiate conversation with their healthcare providers $(\mathrm{p}<0.001)$.

\section{Conclusions}

The results showed a staggering interest of female patients wanting information on this area which would not only boost their confidence and body image but also address concerns of the effect of breast radiotherapy on sexual health during their treatment. 
eP117

COMPETENCE BASED UNDERGRADUATE EDUCATION IN PALLIATIVE MEDICINE.

D. McMahon ${ }^{1}$, D. Walsh ${ }^{2}$

${ }^{7}$ Our Lady's Hospice \& Care Services, Academic Department of Palliative Medicine, Dublin, Ireland

${ }^{2}$ Levine Cancer Institute- Carolinas HealthCare System, Department of Supportive Oncology, Charlotte, USA

\section{Introduction}

In their first year after qualification, doctors care for patients who are dying or have palliative care (PC) needs. All medical students should receive core teaching on PC. Medical students value PC education but complete education feeling underprepared to deal with PC issues. To be effective, PC training should be competence based. In Ireland, the Health Service Executive (HSE) produced a competence framework (the Framework) in 2014 to provide for core competences in PC. The Framework was designed to inform academic curricula and professional development programmes.

Objectives

To revise and update the undergraduate palliative medicine curriculum (The Curriculum) at an Irish University, to comply with best international practice and the Framework.

\section{Methods}

A systematised literature search to identify international competence based curricula in undergraduate palliative medicine. A gap analysis determined where the Curriculum met or failed to meet: -

(a) the Framework,

(b) best international practice.

Using gap analysis, curriculum revision was performed using a consensus approach.

\section{Results}

Four relevant undergraduate medical curricula in PC were identified (UK, Canada, Australia and the European Association for Palliative Care). Each identified required knowledge, skills and attributes to satisfy core learning outcomes

Conclusions

Undergraduate medical education has historically involved student exposure to PC. It should now be competence based. A competence based PC curriculum is therefore a prerequisite. Future development is required to integrate PC principles throughout the medical curriculum and to introduce competence based assessments. Appropriate resources will be required.

\section{eP118}

\section{A NATIONAL SURVEY OF KOREAN MEDICAL STUDENTS FOR END OF LIFE CARE EDUCATION}

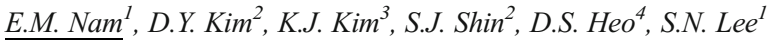 \\ ${ }^{T}$ Ewha Womans University College of Medicine, Department of Internal \\ Medicine, Seoul, Republic of Korea \\ ${ }^{2}$ Dongguk University Ilsan Hospital, Department of Internal Medicine, \\ Goyang, Republic of Korea \\ ${ }^{3}$ Dongguk University School of Medicine, Department of Medical \\ Education, Goyang, Republic of Korea \\ ${ }^{4}$ Seoul National University College of Medicine, Department of Internal \\ Medicine, Seoul, Republic of Korea
}

\section{Introduction:}

End of life (EoL) care is an important issue and adequate education and training in medical education is a key factor in care and management of terminally ill patients.

\section{Objectives}

We aims to explore the current knowledge and experience on EoL care at the undergraduate level in South Korea, and to measure attitudes of medical students and identify factors affecting the attitude toward EoL care.

\section{Methods}

An anonymous survey was designed involving fourth-year medical students of all 41 Korean medical schools. The topics of classroom and bedside teaching, including feedback experience during clinical clerkship related to EoL care were measured. Also, seven items for self-rated attitudes were identified towards EoL related education and practice.

\section{Results}

With a response rate of $49.2 \%$, the most acknowledged topics among students were bad news delivery in classroom teaching (83.9\%) and clinical experiences (43.9\%). Less than $20 \%$ received education or training related to communication such as advance care planning or discussion of advance directives for EoL care. Few participants (21.2\%) replied that they were ready for EoL care practice and most of the students insisted that they should have adequate knowledge and preparedness for clinical competency towards EoL care. More than one bed side experience affected positively all items of self-perceived attitudes.

\section{Conclusions}

The education for EoL care for medical students in Korea is exceptionally inadequate despite growing remarkable demand. This result let us know an unmet need for EoL care practice and medical schools have to develop the suitable EoL care curriculum on the routine medical education thoroughly.

\section{eP119}

EVALUATING THE EFFECTIVENESS OF KETAMINE PLUS ATROPIN AS ANAESTHESIA FOR INTRATHECAL CHEMOTHERAPY AND BONE MARROW ASPIRATION AT HOSPITAL, VIET NAM

H. Nguyen Thi Kim ${ }^{1}$

${ }^{T}$ Doctor, Hue Pediatric Center, Hue, Vietnam

\section{Introduction:}

Ketamine is a phencyclidine and cyclohexamine derivative. It is unique among the sedative analgesics in producing a dissociative state between the thalamus and the limbic system characterized by four features: sedation, analgesia, amnaesia and catalepsy. Ketamine and atropine have increasingly been used in recent years as an effective form of deep sedation/ anaesthesia in children in developed countries, but not in developing countries like Vietnam.

\section{Objectives}

This pioneer trial aimed to evaluate the effectiveness of using ketamine plus atropine as anaesthetic agents for paediatric oncology procedures.

\section{Methods}

A descriptive and prospective study on 80 children with acute leukemia was carried out from January 2015 to December 2017. Datas were analysed by Medcalc software.

\section{Results}

The total number of procedures was 250 . Bone marrow aspiration was performed 102 times and intrathecal chemotherapy given 148 times. All procedures were successfully completed. The dose of ketamine and atropine used $1.75 \pm 0.35 \mathrm{mg} / \mathrm{kg}$ and $0.100 \pm$ $0.042 \mathrm{mg}$ respectively. The time taken for anaesthesia to wear off was short: $8.5 \pm 7.6$ minutes. Only $0.4 \%$ experienced apnoea; $1.7 \%$ convulsion; $1.5 \%$ nystagmus, and hyperactivity; $1.8 \%$ excess salivation, and dreaming; $6.5 \%$ vomiting; none of the patients had laryngospasm or transient rash.

\section{Conclusions}

$2 \mathrm{mg} / \mathrm{kg}$ intravenous ketamine and $0.1 \mathrm{mg}$ atropine were found to be effective and suitable dose in children requiring deep sedation for painful procedures and produce only minimal side effects. We established a 
protocol with the above doses and continue to apply this in order to reduce pain, trauma, and complications and to practice safely.

\section{eP120}

PERIPHERAL NEUROPATHY AND FALLING RISK IN 65 YEARS AND OLDER PATIENTS RECEIVING CHEMOTHERAPY: A PROSPECTIVE STUDY

\author{
A. Ozkaraman ${ }^{1}$, N. Orlu' ${ }^{2}$, O. Usta Yesilbalkan ${ }^{3}$, A. Karadakovan ${ }^{3}$ \\ ${ }^{T}$ Eskişehir Osmangazi University. Faculty of Health Sciences, \\ Department of Nursing, Eskisehir, Turkey \\ ${ }^{2}$ Eskişehir Osmangazi University Hospital, Daily medical unit, Eskişehir, \\ Turkey \\ ${ }^{3}$ Ege University Faculty of Nursing, Internal Diseases Nursing, İzmir, \\ Turkey
}

\section{Introduction:}

In geriatric patients with cancer, both falling and falling-related problems are common repercussions of cancer treatment, along with the physiological decline that accompanies ageing

\section{Objectives}

Chemotherapy-related peripheral neuropathy is known to increase the risk of falls in the elderly. This study aims to assess the developmental state of peripheral neuropathy and the risk of falls in 65 years and older recipients of chemotherapy.

\section{Methods}

The study population comprised patients over 65 years who were first treated with chemotherapy between February 1, 2016, and October 31, 2017, in a a hospital in Eskișehir, Turkey. We collected data every month for 6 months using the Individual Identification Form, the National Cancer Institute Common Sense of Toxicity Criteria Sensory and Motor Neuropathy Scale and the Hendrich-II Falling Risks Scale. We evaluated the comparison of measurements at different time points with a normal distribution, analysis of variance at repeated measures and normal non-dispersive score variables by the Friedman test.

Results

The number of neuropathic patients increased in the sixth month than the first month in the 6-month follow-up period. In addition, it was determined that the Hendrich-II Falling Risks Scale score increased in the fifth and sixth months than the first and second months.

\section{Conclusions}

This study revealed that the degree of neuropathy and the risk of falling increased in the sixth month than the first month for individuals over 65 years who received chemotherapy. Hence, no statistically significant correlation existed between age and neuropathy grade and age and risk of falls in this study.

\section{eP121}

EFFECT OF CONSULTATION ON CLINICAL BREAST EXAMINATION AND MAMMOGRAPHY IN IRANIAN WOMEN

\section{P. Parsa ${ }^{1}$, A. Mirmohammadirad ${ }^{2}$, G. Roshanaiee ${ }^{3}$ \\ ${ }^{T}$ Chronic Disease Home care Research Center of Hamadan University of Medical Sciences, Mother and Child Health, Hamadan, Iran \\ ${ }^{2}$ Chronic Disease Home Care Research Center of Hamadan University of Medical Sciences, Mother and Child Health, Hamadan, Iran \\ ${ }^{3}$ Hamadan University of Medicial Sciences, Statistics, Hamadan, Iran}

\section{Introduction:}

Early diagnosis of breast cancer is directly related to success in treatment.

\section{Objectives}

The aim of this study was to determine the effect of consultation on clinical breast examination (CBE) and mammography among women in Hamadan, Iran.

\section{Methods}

This research was a clinical trial study. Eligible women aged $>40$ years attending to Hamadan health care centers in 2015 were randomly assigned to experimental and control groups ( $n=75$ in each group). The experimental group received 4 weekly sessions of breast cancer screening consulting based on health belief model. Knowledge on breast cancer, HBM constructs, and practices were compared between two groups before, one and three months after intervention.

\section{Results}

Before the intervention, no significant differences were observed in knowledge, HBM constructs and practice between intervention and control group. While one and three months post intervention significant differences were detected between two groups on HBM constructs (except susceptibility and severity) and knowledge ( $p<0.05)$.

\section{Conclusions}

The results show that consultation is a efficient method to promote CBE and mammography and breast cancer screening in Iranian women

\section{eP122}

\section{DO YOUR PATIENTS UNDERSTAND? ASSESS AND IMPROVE} HEALTH LITERACY AT YOUR CANCER PROGRAM

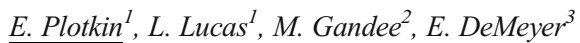 \\ ${ }^{T}$ Association of Community Cancer Centers, Provider Education, \\ Rockville, USA \\ ${ }^{2}$ Association of Community Cancer Centers, Development, Rockville, \\ USA \\ ${ }^{3}$ Beyond Oncology, Consulting, Dallas, USA
}

\section{Introduction:}

Although many cancer programs have made significant headway in providing patient-friendly materials, health literacy related to cancer diagnosis, treatment, and post-treatment follow-up requires ongoing attention. For patients with cancer, poor health literacy may result in facing increased risk of medical errors, chronic disease recurrence that leads to higher hospitalization rates, and suboptimal health outcomes.

\section{Objectives}

ACCC seeks to help cancer programs across the country to improve survivorship programming through the application of the health literacy principles.

\section{Methods}

ACCC constructed an expert advisory committee to inform the work, including several patient advocacy partner organizations. After an environmental scan was completed of relevant quality metrics, an online tool was formed to score programs in 6 domains: Health Literacy Program; Staff Training; Health Information; Navigation; Technology; Quality Measurement and Improvement

Results

To help cancer programs evaluate their current health literacy efforts and prioritize steps for improvement, a health literacy gap assessment tool was developed. This tool is intended to help cancer programs advance their delivery of patient-centered care by integrating key attributes of a health literate healthcare program. By completing the assessment tool, cancer programs can identify educational needs and pinpoint areas where targeted education could improve patient care.

\section{Conclusions}

No cancer program taking the assessment received a perfect score $(\mathrm{n}=219)$, indicating that there is work to be done across the board to adhere to national standards for a health literate organization and enhance patient centered care. Using this report, cancer programs can customize 
goals for improvement, considering resource level, infrastructure, administrative/leadership support and patient population.

\section{eP123}

CHALLENGES AND ACHIEVEMENTS ENCOUNTERED WHILE OFFERING COMMUNITY PALLIATIVE CARE IN SEMI-ARID AREA. IS VOLUNTEERING WORK WORTHY WHILE FULL TIME WORKING?

\section{S.M. Rithara ${ }^{1}$ \\ ${ }^{T}$ Kenya Medical Training College, NURSING, Nairobi, Kenya}

\section{Introduction:}

Community-based health care occurs when community members take on the responsibility of initiating and sustaining their own health care.

\section{Objectives}

To highlights the needs of palliative care in remote semi-arid area where health facilities are limited.

Methods

Experience with 58 trained community health volunteers for seven years in volunteering work with Ongata Ngong Palliative Community Care.

Results

There are several challenges and achievement while offering community supportive care. $65 \%$ appreciate the $\mathrm{HCBC}$ while $35 \%$ prefers being in hospital. $72 \%$ willing to learn while $38 \%$ declined. $86 \%$ hard to reach, $55 \%$ do not understand cancer either heard about it, 58\% prefers herbal treatment for cancer. $89 \%$ preventive measures, $62 \%$ few health facilities, $68 \%$ no roads, $40 \%$ of distance on foot, $25 \%$ vehicle, $35 \%$ truck/foot. $52 \%$ burnout, $48 \%$ no resource. STRENGTHS; Palliative Care trained volunteer, Holistic approach, ownership of the activities, willingness to support each other and good referral system, CHALLENGES; Language barrier, Under reporting of pain and other distressing symptoms, evaluation and monitoring, documentation and Culture constrain.

Conclusions

There is a lot of work needed to be done in the communities. Need to store data appropriately, community health volunteers should be guided on how to report the activities they carry out in the community and their specific catchment areas for proper follow up. Motivating trained palliative/oncology health educators to ensure supervision takes place and community commitment.

\section{eP124}

BENEFITS OF NATIONAL HOSPITAL INSURANCE FUND IN CARE OF PATIENTS WITH KAPOSIS SARCOMA IN POOR RESOURCE CONSTRAINED SETTING IN WESTERN KENYA

C. saina ${ }^{1}$

${ }^{T}$ college of Health Sciences, pharmacy, eldoret, Kenya

\section{Introduction:}

Cancer is athird leading cause of death in kenya,22000-41000 new cases occur each year,28000 kenyans die as aresult of cancer each year.

Kaposis sarcoma is the most common AIDS associated malignancy in western kenya and its a significant cause of morbidity and mortality.clinical features of k.s were investigated i a tertiary refferal centre in kenya between 1997-2017 with the ratio of 3:7 male to female,symptoms seen at presentation,only peripheral lymphadenopathy was found to be significantly associated with HIV compared to those with KS but non HIV Objectives

Identifying means of lessening the burden and ensuring that all patients cancer patients receive at an affordable cost

Methods

All patients attending Chulaimbo county refferal hospital were sensitized and recruited in April 2017 and were well educated on the benefits of acquiring NHIF scheme, Pharmacist incharge of running the clinic together with NHIF team and a peer educator from the community took the lead in ensuring that patients understood the benefits of having the cover and the need of having it.

\section{Results}

Health insurance status at diagnosis was recorded for $70 \%$ patients $23 \%$ had no NHIF, those who had NHIF compared to those who did not,the risk of treatment abandonment relative to event free survival was significantly $7 \%$

\section{Conclusions}

Patients with NHIF at diagnosis had significantly lower chance of abandoning treatment and a higher chance of KS remission.KS outcome could be improved by interventions that prevent treatment abandonment and specifically by increasing access to NHIF medical scheme.

\section{eP125}

EFFECTIVENESS OF PEDIATRIC RISK OF MORTALITY SCORE (PRISM III) IN INITIAL EVALUATION AND FOLLOW-UP OF CRITICALLY ILL CANCER PATIENTS AT PEDIATRIC-ONCOLOGY ICU IN SOUTH EGYPT

\author{
H. Sayed ${ }^{l}$, A. Ali ${ }^{l}$, M. Elzembely ${ }^{l}$

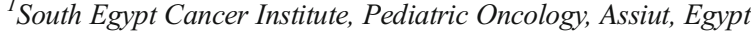

\section{Introduction:}

Pediatric risk of mortality score (PRISM III-12) is a physiology based predictor for risk of mortality

\section{Objectives}

We conducted this study to explore the ability of 1 st PRISM III-12 to predict the risk of mortality in critically ill cancer patients and the ability of serial PRISM III measured every 72 hours to follow up the patients' clinical condition during POICU stay.

\section{Methods}

We conducted prospective study from $1 \backslash 1 \backslash 2014$ to $1 \backslash 1 \backslash 2015$ in pediatric oncology intensive care unit (POICU) at South Egypt Cancer Institute, Egypt. The study population consisted of children with cancer admitted to POICU during the study period. Patients' demographics, underlying diagnosis, 1st PRISM III-12 score, reason for admission, stage of treatment were recorded at diagnosis, Serial PRISM III every 72 hours and types of therapeutic intervention were also recorded

\section{Results}

123 (78 males) children were included. Median age was 5 years (1-15 years).Death rate was $20 \% .1$ stPRISM III-12 mean was 19 (0-61). The mean 1st PRISM III-12 for survivors was significantly higher compared to non-survivors ( 15 vs. 37 respectively; P<0.001). $1^{\text {ST }}$ PRISM III-12 mean was significantly correlated to the reasons for admission and organ failures' number $(\mathrm{P}<0.001$ and $\mathrm{P}<0.001) .1^{\mathrm{ST}} \mathrm{PRISM} \mathrm{III-12}$ correlated weakly positive with the length of stay $(\mathrm{r}=0.2 ; \mathrm{P}=0.024)$. ROC curve for 1stPRISM III-12 was 0.913 (CI 95\% 0.85-0.98; P<0.001). Decline in serial PRISM III was significantly correlated with favorable (survivor) outcome $(\mathrm{P}<0.001)$.

\section{Conclusions}

PRISM III-12 can be used effectively in predicting the risk of mortality and following the clinical condition of patients during POICU stay.

eP126

HEMATOLOGIC MALIGNANCIES SURVIVORSHIP EDUCATIONAL INTERVENTION：AN UPDATE IN HEMATOLOGIC MALIGNANCIES FOR NURSES AND ADVANCED PRACTICE PROVIDERS (APP)

W. $\operatorname{Tan}^{1}$

${ }^{T}$ Mayo Clinic, Hematology oncology, Jacksonville, USA 


\section{Introduction:}

Educational interventions with the primary goal of providing education at the level of the learner have been often challenging. Most educational courses are geared towards multiple levels of learners.

Objectives

An educational intervention was planned and implemented with the primary aim to fill the gap of knowledge and improve confidence in APP and nurses .

Methods

An live educational intervention was planned and implemented with core topics in hematologic malignancy management April 18, 2017 Jacksonville, Florida. A post course survey was done.

\section{Results}

18 individuals attended the sessions: they represented 7 institutions including 4 hospitals, 1 Nursing school and 2 pharmaceutical companies. 4 learned more than 4 new topics, 6 learned 3, 5 learned 2 and 3 learned 1 new topic. The level of the talk was appropriate for their level of education and experience 16/18 and 2/18 felt it was above the level of their understanding. A second post survey to see if the course met the educational goals revealed ( 8 individuals volunteered for this): $8 / 8$ felt the course met its educational objective, $8 / 8$ felt that they acquired new knowledge, 2/8 gained abilities as a result of participating in the course, $2 / 8$ learned skills that they plan to implement in practice, $3 / 8$ felt that they obtained strategies that will improve patient care and influence medical outcome.

\section{Conclusions}

A learner focused planned educational intervention for nurses and APP in hematologic malignancy met its educational goal. This led to learner satisfaction and knowledge gain that they plan to implement/improve patient care.

\section{eP127}

SURVIVORSHIP EDUCATIONAL INTERVENTION THROUGH AN INTER-PROFESSIONAL TEAM FOR NEW ADVANCED PRACTICE PROVIDERS IN HEMATOLOGY/ONCOLOGY

W. $\operatorname{Tan}^{1}$

${ }^{T}$ Mayo Clinic, Hematology oncology, Jacksonville, USA

\section{Introduction:}

There is a significant gap of knowledge and confidence in new Advanced practice providers (APP) who are new to hematology/oncology.

Objectives

Develop a curriculum and implement a live educational intervention for new APP in Hematology/Oncology in Florida, USA.

\section{Methods}

An educational course known as the Rapid integration course was organized, with topics including basic physical examination/diagnostics, hematology/oncology pharmacology, pathology, radiation oncology, hem/onc procedures, hematology/oncology emergencies, giving bad news, billing/financial issues and clinical trials were discussed in a one day course. The speakers represented an inter-professional team that address these issues ( 8 speakers ARNP 3, PA 1, pathologist 1 , radiation oncologist 1 hematologist oncologist 2). A post course survey was conducted.

\section{Results}

70 healthcare professionals registered for the meeting, 52 actually attended the meeting. (25 ARNP,6 PA, 17 RN, 3 LPN and 1 other. 19/ 52 individuals completed the survey voluntarily. There was significant increase in knowledge 14/19 (73.68\%), significant change in competence $12 / 19(63.16 \%)$, felt that the course will change their practice performance $12 / 19(63.12 \%)$, plan to implement what they learned $16 / 18$ ( $88.8 \%$ and the course had an impact on how they will treat their future patients $15 / 18(83.3 \%)$.

\section{Conclusions}

A one day crash course in hematology/oncology that covered basic clinical topics improved knowledge, help improve perception of competence and help practitioners plan to implement what they learned which can potentially lead to a change the way they care for patients.

\section{eP128 \\ EDUCATION IN SUPPORTIVE CARE AT UNITED METHODIST CHURCH NURSING SCHOOL KISSY THE EASTERN PART OF FREETOWN SIERRA LEONE}

\author{
J. Bundor Tarawally ${ }^{I}$ \\ ${ }^{T}$ African organization and research and training in cancer South Africa \\ union in cancer control Switzerland. The max foundation USA/American \\ Society of clinical oncology USA/and partnership against concern \\ Canada
}

Sierra Leone situated in West Africa. It lies between Guinea and Liberia. The country has a population of about Seven Million people. The country is divided into four regions, they are as follows-Western Area with Freetown being the Capital City, Southern Province with Bo being the headquarter, Northern province with Makeni being the headquarter and Eastern province with Kenema being the headquarter.

The country is divided into twelve district. Kissy is situated in the Eastern part of Freetown.

The population of Kissy is about four hundred and fifty thousand people. There are five public hospitals and small health centers. United Methodist Church Hospital is located at the heart of Kissy. Education on supportive care is very important in all hospitals and health centers so that health care workers can apply it when necessary. Supportive care are given to improve the quality of life of patients who have serious or life threatening disease. The goal of supportive care is prevention, treats as early as possible the symptoms of the disease, side effects caused by treatment of a disease, psychological, social and spiritual problems related to a disease or its treatment also called comfort care, palliative care and symptom management. Description of how education on supportive care is innovative Education on supportive care is innovative because the presentation is done alongside with an illustration of international posters. The poster will help the students/participants to understand better the importance of supportive care for patients with life threatening disease. Furthermore the program is always interactive. Participants are also advised to replicate the knowledge gained during the session.

\section{OBJECTIVE OF THE STUDY}

1. To raise public education on supportive care.

2. To help the participants understand the importance of supportive care to patients with life threatening disease.

\section{METHODOLOGY}

This study was based on interviewing forty health care workers comprises of the following people nurses, caregiver, social workers, community health officers, chaplain and volunteers from the three institutions and community.

United Methodist church Nursing School, Kissy Nicole Terrace Health Center kissy, Kissy Health Center and Kissy Mess-Mess

1. Nurses -3

2. Social workers -2

3. Care givers -2

4. Community health officer -3

Nicole Terrace Health Center

1. Nurses -3

2. Social workers -2

3. Care givers -2

4. Community health officer -3

Kissy Health Center

1. Nurses -3

2. Social workers-2 
3. Care givers -2

4. Community health officer -3

Kissy Mess-Mess

Volunteers -5

Chaplain -5

During my interview with the different categories of people in the different health institutions and community, I discussed with them supportive care its importance and the impact it creates in the life of a patient with life threatening disease. It was one to one interview and information received was record.

According to my evaluation, I observed that, non of them have knowledge about supportive care and the impact it creates in the life of patients with life threatening disease.

\section{Result/findings of my research}

The findings of my research are as follows. - All the people in the different institutions and community have no knowledge about supportive care. Conclusion/statement of the statement of the problem

Since supportive care help to improve the quality of life of patients who have serious life threatening disease, Education on the issue/empowering the following people involved in the provide supportive care namelyNurses, care givers/volunteers, chaplain and social workers will help to prolong the lifes of patients with life threatening diseases.

\section{eP129}

HEALTH CARE PROFESSIONAL'S PRACTICE IN CANCER PREVENTION: SMOKING CESSATION COUNSELING AS AN EXAMPLE

\section{H.W. Tsai ${ }^{1}$, Y.H. Lai ${ }^{2}$, H.L. Wu ${ }^{3}$, K.C. Hsueh ${ }^{4}$}

${ }^{T}$ Hsin Sheng Junior College of Medical Care and management, nursing, Longtan Township, Taiwan R.O.C.

${ }^{2}$ School of Nursing-College of Medicine- National Taiwan University, nursing, Taipei, Taiwan R.O.C.

${ }^{3}$ Taiwan Adventist Hospital, Department of Thoracic Medicine-, Taipei, Taiwan R.O.C.

${ }^{4}$ Kaohsiung Veterans General Hospital, Departments of Family Medicine, Kaohsiung, Taiwan R.O.C.

\section{Introduction}

Smoking cessation is one of the most important issues in cancer prevention. In Taiwan, a 3-level (basic, intermediate, advanced) 48-hour smoking cessation educators (SCE) training programs has been conducted nationally by government. It is unclear what the current status of SCE trainees' practice of SC counseling before they received the comprehensive SCE training.

Objectives

(1) the implementation of SC counseling in SCE trainees, and (2) identify factors associated with SCE trainees' applying of counseling in SC.

Methods

This study is part of a major project. A cross-sectional survey was conducted among SCE trainee $(\mathrm{N}=250)$. The survey includes levels of SC counseling and attitudes toward SC practice. Data were analyzed by descriptive statistics and Pearson's correlation.

\section{Results}

The mostly applied SC counseling activities were as follow: "Provide resources and assistance", "Ask tobacco users if they are willing to quit", "Identify reasons for quitting". However, requiring more knowledge and skills, such as "set a quit date", "refer to web resources", "use cessation materials that are appropriate by age, culture, language, education, and pregnancy status" are less implemented. The correlation results shows that positive correlation between SC counseling and perceived responsibility $(\mathrm{r}=.39, \mathrm{p}<.01)$ and self-efficacy in $\mathrm{SC}(\mathrm{r}=.23, \mathrm{p}<.01)$.

Conclusions

The results suggested that there will be important to apply SC education to increase the health care professional to apply SC counseling.
Enhancing health care personnel's perceived responsibility and selfefficacy might also increase their willingness to apply SC counseling.

\section{eP130}

EMPOWERING ONCOLOGY NURSES IN PROVIDING NUTRITIONAL INFORMATION TO CANCER SURVIVORS

\author{
M. van Veen ${ }^{1,2}$, E. Kampman ${ }^{2}$, B. Kolen ${ }^{3}$, S. Beijer ${ }^{1}$ \\ ${ }^{T}$ IKNL, Research, Utrecht, The Netherlands \\ ${ }^{2}$ Wageningen University, Division of Human Nutrition, Wageningen, \\ The Netherlands \\ ${ }^{3}$ Elisabeth Tweesteden Ziekenhuis, Oncology, Tilburg, The Netherlands
}

\section{Introduction}

Previous research showed that more than half of cancer survivors have information needs on nutrition and cancer. Although many reported to have received nutritional information, nearly half still felt they lacked the appropriate information. Oncology nurses are the key figures in providing information about nutrition and cancer to cancer survivors.

Objectives

To assess nutritional knowledge of oncology nurses and to provide oncology nurses with tools to provide cancer survivors with appropriate nutritional information.

\section{Methods}

Oncology nurses were asked how they perceived their nutritional knowledge. Based on these results, we developed a nutritional advisory tool for nurses and investigated whether this tool supports nurses with the information provision to cancer survivors. For this evaluation, oncology nurses filled out a questionnaire before and four months after introduction of the tool.

\section{Results}

Most oncology nurses (95\%) feel that informing cancer survivors on nutrition and cancer should be part of their job, however, only $65 \%$ of these nurses perceived themselves as having sufficient knowledge on nutrition and cancer. After introduction of the advisory tool, more oncology nurses felt confident to give nutritional advice (before: $65 \%$ vs. after: $85 \%, \mathrm{p}<0.05)$. More survivors were informed about how to obtain a healthy lifestyle (before: $33 \%$ vs. after: $52 \%$, p<0.05). Instead of providing every survivor with a standard advice, the advice given was more tailored to the survivors' needs.

\section{Conclusions}

The nutritional advisory tool is a useful tool in helping oncology nurses to feel more confident with providing nutritional information to cancer survivors.

\section{eP131}

TUMOUR LYSIS SYNDROME MANAGEMENT IN PAEDIATRIC ONCOLOGY PATIENTS IN A SINGLE INSTITUTION. RIGOROUS SUPPORTIVE CARE IMPROVES COST EFFECTIVENESS AND DECREASES INVESTIGATIVE BURDEN IN CHILDHOOD CANCER

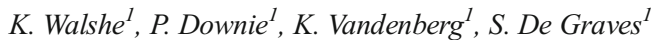 \\ ${ }^{T}$ Monash Children's, Children's Cancer Centre, Clayton, Australia
}

\section{Introduction:}

Tumour lysis syndrome and hyperuricaemia are known complications of acute leukaemia and non-Hodgkin lymphoma. TLS can be classified into clinical or laboratory TLS, with published guidelines for prevention and management of patients. Reported incidence in adult cancer patients is as high as $40 \%$ whereas the incidence in paediatrics is $<5 \%$ (Cairo and Bishop 2004)

\section{Objectives}

This study was to determine the incidence of clinical and laboratory TLS in a single institution, and estimate health care costs associated with 
management. The aim was to determine if costs could be reduced by developing a predictive model based on the published guidelines. Additionally, we wanted to evaluate if the risk stratification model was correctly identifying the highest risk patients and instituting timely administration of recommended supportive care.

Methods

103 acute lymphoblastic leukaemia and NHL patients were retrospectively reviewed. Patients were treated with hyper-hydration and either allopurinol or rasburicase. Serum electrolytes, urate, calcium, phosphate, and LDH were measured at baseline, and at 6 hourly intervals for a minimum of 48 hours. Records were examined to determine if there was a change in clinical management based on these results.

Results

Laboratory TLS occurred in 20\%. Clinical TLS occurred in 1 patient. Electrolytes, urate, Ca PO4 were measured every 6 hours. In 96\%, there was no change in parameters to indicate clinical concern.

Conclusions

New institutional procedures have been written based on these findings. Estimated cost savings are over 50,000 AUD per annum, and reduction in the unnecessary burden of repeated blood tests on children newly diagnosed with haematological cancers.

eP132

A QUALITATIVE TEXT-ANALYSIS OF PERCEPTIONS ON PALLIATIVE CARE: SURVEY OF STUDENT IN ONLINE COURSE FOR UNDERSTANDING CANCER IN JAPAN

K. WATANABE ${ }^{1}$, T. Tashiro ${ }^{2}$, S. Matsumoto ${ }^{3}$, M. Matoba $^{4}$

${ }^{T}$ Teikyo University School of Medicine, Department of Internal Medicine, Tokyo, Japan

${ }^{2}$ The Open University of Japan, Living and Welfare, Chiba, Japan

${ }^{3}$ NTT Medical Center Tokyo, Quality Indicator Section, Tokyo, Japan

${ }^{4}$ Japanese Red Cross Medical Center, Department of Palliative Care,

Tokyo, Japan

\section{Introduction}

Cancer has been one of the main causes of death in developed countries, and palliative care should be integrated from early phase of trajectory both to patients and their families. People are becoming interested in learning about cancer.

Objectives

To verify the effectiveness of education materials that are easy to understand for citizens.

Methods

A course "Understanding Cancer in Japan," which provides web-based education was launched aspart of lifelong health literacy education at the Open University of Japan since 2016. Students attending this interactive program learn about various aspects on cancer mechanism, prevention, screening, diagnosis, treatment, palliative care, cancer policies and welfare programs for 4 months. An anonymous self-administered questionnaire survey was conducted in the lecture about palliative care, including evaluation of the course. We analyzed each record using SPSS Text Analytics for Surveys.

Results

1,521 students of the all over Japan answered the questionnaire. 1,457 $(95.7 \%)$ students replied about a lecture as beneficial. The key words were divided with a linguistic method into 30 categories: "palliative care", "patients", "medical care", "treatment", "care", "oneself", etc. Five clusters were formed in the cluster analysis: Thoughts on introduction, social issues, quality of life, intervention, and perception.

Conclusions

Students were able to understand basic knowledge about significance of palliative care and we could grasp their image of the understanding with linguistic approach. Online course can be effective method for prevailing knowledge on palliative care and empowering attendees for advanced decision making. The feedback from students shows a utility as a educational program for citizens.
eP133

THE PROJECT OF MAKING CLINICAL RESEARCH POLICY OF SUPPORTIVE AND PALLIATIVE CARE IN JAPAN

S. Zenda ${ }^{1}$, T. Morita ${ }^{2}$, A. Inoue ${ }^{3}$, T. Yamaguchi ${ }^{4}$, Y. Uchitomi ${ }^{5}$

${ }^{T}$ national cancer center hospital east, Radiation Oncology, chiba prefecture, Japan

${ }^{2}$ Seirei Mikatahara Haspital, Palliative and Supportive care, Shizuoka, Japan

${ }^{3}$ Tohoku University Graduate School of Medicine, Palliative Medicine, Miyagi, Japan

${ }^{4}$ Tohoku University, Clinical Trial Data Management, Miyagi, Japan

${ }^{5}$ National Cancer Center Hospital, Innovation Center for SupportivePalliative and Psychosocial Care, Tokyo, Japan

\section{Introduction}

In Japan there are serious problems in the system of clinical research especially in supportive and palliative care. The main reason is that there is no stipulated guideline. The methodology of clinical research in supportive and palliative care is different from that in medical oncology field in terms of the style of multidisciplinary approaches, study design, and endpoints. To provide enough information for researchers, it is necessary to make rule guideline of both general remarks and detailed exposition.Here we conduct the project of making guideline and rule books of supportive and palliative care research field.

\section{Objectives}

Our guideline and rule books of supportive and palliative care research field are made for clinical researchers.

\section{Methods}

Supportive and Palliative care research guideline (General remarks): Considering the minor difference between each specific field, this guideline shows the basic concept of supportive and palliative care research. The contents in detail is as follows; definition of supportive care and palliative care, characteristics of study, subject of study, study design, endpoints and outcome measure, death with 30 days in palliative care research, Enforce system and management of the study.

\section{Results}

A proto version of Supportive and Palliative care research guideline (General remarks) were made on November 2017, and final version will be published after receiving public comments.

\section{Conclusions}

Our goal is that the quality of research in these fields will be raised to that in medical oncology field.

\section{eP134}

EXPERIENCE OF CANCER-RELATED FATIGUE AFTER COMPLETION OF CHILDHOOD CANCER TREATMENT: A LITERATURE REVIEW

Q.M. Alqahtani ${ }^{1}$

${ }^{1}$ King Saud University - College of Applied Medical Sciences, Rehabilitation- Physical Therapy, Riyadh, Saudi Arabia

\section{Introduction}

Fatigue is the most frequent debilitating symptom that may persist for years in survivors of childhood cancer. After completion of cancer treatment, children reported fatigue as a barrier for engaging in physical activity and an obstacle to successful school reentry.

\section{Objectives}

This review aimed to explore the literature regarding the experience of cancer-related fatigue in survivors of childhood cancer who aged $\leq 18$ years at the time of participation in the research. 


\section{Methods}

Using the Joanna Briggs Institute guideline, an electronic search of the AMED, CINAHL, Cochrane, EMBASE, MEDLINE, and PsycINFO and reference lists was conducted. Data were extracted and assessed for quality.

\section{Results}

Four studies were included for review with merely one study involved children younger than 13 years who survived brain tumours. After completion of cancer treatment, fatigue was perceived as a considerable problem that limited daily functioning and had an adverse impact on the ability to exercise. Moreover, most of children and adolescents managed fatigue by rest most of the times, and exercise was the single best treatment for fatigue recommended by adolescents. Furthermore, to the knowledge of the researcher, there is no study investigating parents' perspective of how their children perceive and manage fatigue after completion of cancer treatment.

\section{Conclusions}

Although fatigue is a distressing symptom reported by children who survived cancer, there has been limited research on fatigue experience among paediatric cancer survivors. Further research is needed to know how young children experience fatigue after completion of cancer treatment, and to explore parents' impact on fatigue management of their children.

\section{eP135}

HEART RATE VARIABILITY IN CANCER-RELATED FATIGUE

B. Brady ${ }^{1,2}$, M. Barrett ${ }^{1}$, M.J. Kennedy ${ }^{3,4}$, S. Cuffe $e^{3}$, R.T. Murphy ${ }^{3}$, D. Walsh $^{1,2,4}$

${ }^{1}$ Our Lady's Hospice \& Care Services, Academic Department of Palliative Medicine, Dublin, Ireland

${ }^{2}$ University College Dublin, School of Medicine, Dublin, Ireland

${ }^{3}$ St. James's Hospital, Department of Medical Oncology, Dublin, Ireland

${ }^{4}$ Trinity College Dublin, School of Medicine, Dublin, Ireland

\section{Introduction}

Cancer-related fatigue (CRF) pathophysiology is unclear. Cardiovascular autonomic nervous system (CVANS) dysfunction may contribute. CVANS can be measured by heart rate variability (HRV) and postural hypotension.

Objectives

Investigate CRF and CVANS function in solid tumours.

Methods

Treatment-naïve outpatients recruited from medical oncology. Fatigue measured subjectively (brief fatigue inventory [BFI]) and objectively (hand grip strength, timed up and go [TUG] and sit to stand [STS]). $\mathrm{BFI} \geq 3$ indicated fatigue.

HRV was recorded for five minutes each of spontaneous and paced breathing (BioPac Systems MP36 ${ }^{\mathbf{T M}}$ ). Analysis was performed using ARTiiFACT ${ }^{\mathbf{M}}$. HRV reported as root mean square of successive differences (RMSSD) and standard deviation of the NN (R-R) intervals $(\mathrm{SDNN})$. Lower SDNN represents loss of dynamic response. Postural hypotension defined as systolic blood pressure (BP) drop of $\geq 20 \mathrm{mmHg}$ or diastolic BP drop of $\geq 10 \mathrm{mmHg}$ on standing.

Results

10 participants $(6 \mathrm{~F})$

Age: Median 54 (range 40-73)

Cancer types: Breast 6, Oesophageal 4

Loco-regional disease: 3

BFI: Median 1.25 (range 0-5.9)

$\mathrm{BFI} \geq 3: 3 / 10(30 \%)$

\begin{tabular}{|l|r|r|}
\hline & Fatigued & Non-Fatigued \\
& Median (range) & Median (range) \\
$\mathrm{N}=3$ & $\mathrm{~N}=7$ \\
\hline Grip strength (kg force) & $23(18-33)$ & $35(14-48)$ \\
\hline TUG (s) & $8(7-8)$ & $7(6-9)$ \\
\hline STS (number in 30s) & $13(9-16)$ & $13(11-18)$ \\
\hline RMSSD, spontaneous & $16(6-25)$ & $18(11-79)$ \\
\hline RMSSD, paced & $18(8-71)$ & $23(17-86)$ \\
\hline SDNN, spontaneous & $22(18-27)$ & $30(17-124)$ \\
\hline SDNN, paced & $30(17-96)$ & $45(28-121)$ \\
\hline
\end{tabular}

Conclusions

1. Significant proportion of participants fatigued pre-treatment

2. Lower SDNN in fatigued participants indicates CVANS dysfunction

3. Orthostatic hypotension rarely present

\section{eP136}

CARDIAC MUSCLE FUNCTION IN CANCER-RELATED FATIGUE

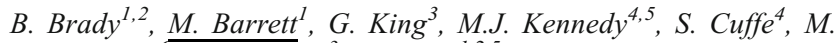
Cunningham , R.T. Murphy ${ }^{3}$,D. Walsh ${ }^{1,2,5}$

${ }^{1}$ Our Lady's Hospice \& Care Services, Academic Department of Palliative Medicine, Dublin, Ireland

${ }^{2}$ University College Dublin, School of Medicine, Dublin, Ireland

${ }^{3}$ St. James's Hospital, Department of Cardiology, Dublin, Ireland

${ }^{4}$ St. James's Hospital, Department of Medical Oncology, Dublin, Ireland

${ }^{5}$ Trinity College Dublin, School of Medicine, Dublin, Ireland

${ }^{6}$ St. Luke's Radiation Oncology Network, Radiation Oncology, Dublin, Ireland

\section{Introduction:}

Cancer-related fatigue (CRF) is a common, high-impact symptom but pathophysiology is unclear. One possible mechanism dysfunction of cardiac muscle. Cancer-related changes to cardiac muscle may precede any treatment-related cardiotoxicity. Cardiac biomarkers can be abnormal, even with normal cardiac imaging. Myocardial strain is a sensitive method of measuring left ventricular (LV) function.

\section{Objectives}

To investigate $\mathrm{CRF}$ and cardiac function in people with solid tumour malignancies.

\section{Methods}

Treatment naïve participants were recruited from medical oncology outpatient clinics. Fatigue was measured subjectively (brief fatigue inventory $[\mathrm{BFI}]$ ) and objectively (grip strength, timed-upand-go [TUG] and sit-to-stand [STS]). BFI $\geq 3$ was indicative of fatigue.

A 2D transthoracic echocardiogram assessed cardiac function (systolic: ejection fraction $[\mathrm{EF}]$; diastolic: isovolumic relaxation time [IVRT, LV relaxation] and mitral valve E/A ratio [E/A, LV filling velocities]. Left ventricular (LV) myocardial strain was analysed using EchoPAC software (GE Healthcare ${ }^{\mathrm{TM}}$ ). A cardiac biomarker, NT-pro brain natriuretic peptide (NT-BNP), was measured on a subset of participants who were scheduled for chemotherapy.

Results

- 45 participants $(30 \mathrm{~F})$

- $\quad$ Age: Median 57 (range 38-82)

- Cancer type: Breast 28, Oesophageal 16, Prostate 1

- Loco-regional disease: 26

- $\quad$ BFI: Median 1.7 (range 0-7.7)

- $\quad \mathrm{BFI} \geq 3: 14 / 45(31 \%)$ 


\section{Conclusions}

1. High proportion of participants fatigued pre-treatment.

2. Indicators of diastolic dysfunction present i.e. reduced E/A ratio in fatigued participants; prolonged IVRT in both groups

3. No difference in systolic function or myocardial strain between groups.

\section{eP137}

SYMPTOM CLUSTER AND QUALITY OF LIFE TRAJECTORIES IN BREAST CANCER PATIENTS BEFORE AND 1-YEAR AFTER STARTING CHEMOTHERAPY

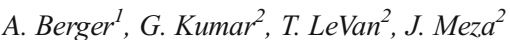 \\ ${ }^{T}$ University of Nebraska Medical Center, College of Nursing, Omaha, \\ USA \\ ${ }^{2}$ University of Nebraska Medical Center, College of Public Health, \\ Omaha, USA
}

\section{Introduction}

Breast cancer patients experience multiple concurrent symptoms before and after chemotherapy (CTX). Physical and psychological symptoms may persist after treatment and reduce quality of life (QOL) of survivors. Objectives

To determine differences in prevalence and severity of symptoms at three times within 1-year of starting CTX; and to identify symptom cluster (SC) trajectories over these times in breast cancer patients.

Methods

Symptoms were identified in breast cancer patients $(\mathrm{N}=219)$ before the start of CTX (baseline), 30 days after the last CTX, and 1-year after the first CTX treatment. Hospital Anxiety and Depression Scale (HADS) and Symptom Experience Scale measured symptoms. MOS-SF-36v2 questionnaire measured QOL. Exploratory factor analysis (EFA) was performed to identify symptom clusters at each time and clusters were compared over time.

\section{Results}

The prevalence and severity of symptoms gradually decreased over time $(<0.05$ for all symptoms). Fatigue (range $94.5 \%$ to $87.2 \%)$ was one of the most prevalent symptoms while depression (range $10.9 \%$ to $9.8 \%$ ) was the least prevalent. Results showed there was an improvement over time in both the physical and mental domain score of QOL ( $p<0.01)$. Two major SCs were identified at each time point. When compared, the number and type of symptoms in each cluster differed over time.

\section{Conclusions}

Findings suggest that symptoms in breast cancer patients improved over time; however, did not return to baseline 1-year after beginning CTX. Despite experiencing these symptoms, women reported QOL similar to population norms 1-year after the first CTX treatment.

\section{eP138}

\section{EVIDENCE-BASED CANCER-RELATED FATIGUE}

\author{
M. Chang ${ }^{1}$ \\ ${ }^{T}$ Shin Kong Wu Ho-Su Memorial Hospital, Nursing, Taipei, Taiwan \\ R.O.C.
}

\section{Introduction}

Cancer has been viewed as a chronic disease. Cancer-Related Fatigue is most common symptom in cancer patient and occurs both tumors and treatment.

\section{Objectives}

The aim of this paper is to summarize relevant systematic review study on intervention to reduce cancer-related fatigue in cancer patient adult.

Methods

Articles of systematic review or meta-analysis about Cancer-Related Fatigue published in five years and full text, were searched using Cochrane and PubMed databases.

\section{Results}

Yoga for improving health-related quality of life, mental health and cancer-related symptoms in women diagnosed with breast cancer.

Aerobic exercise can be regarded as beneficial for individuals with cancer-related fatigue during and post-cancer therapy, specifically those with solid tumors. There was a lack of evidence on the clinical effectiveness of massage for symptom relief in people with cancer.

Educational interventions may have a small effect on reducing fatigue intensity, fatigue's interference with daily life, and general fatigue, and could have a moderate effect on reducing fatigue distress.

\section{Conclusions}

The evidences were support yoga or aerobic exercise and psychological interventions could significantly reduced cancer-related fatigue, but massage or educational could not. CRF treatment effectiveness was associated with cancer stage, baseline treatment status, experimental treatment format, experimental treatment delivery mode, psychological mode, type of control condition. The medical staffs, patients and their families are encouraged to attach importance to CRF, and the care quality of cancer patients are also improved.

\section{eP139}

COMPARISON OF THE EFFECTS OF MENTAL AND PHYSIOLOGICAL INTERVENTION MEASUREMENT FOR CANCER SURVIVORS

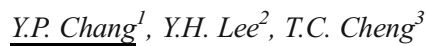

${ }^{T}$ DA-Yeh University, Department of Nursing, Changhua, Taiwan R.O.C.

${ }^{2}$ The National Health Research Institutes NGRI, Department of Administration, Miaoli County, Taiwan R.O.C.

${ }^{3}$ Chi Mei Medical Center-Liouying, Vice Superintendent Office, Tainan, Taiwan R.O.C.

\section{Introduction}

Cancer-related fatigue (CRF) is the most concerning symptom of cancer survivors, widely affecting patients' physical, emotional, and cognitive functions, with prevalence rates of up to $75-99 \%$. Stress management, cognitive behavior modification, relaxation, meditation, and mindfulness can help improve the patient's selfmanagement ability to relieve the symptoms of fatigue, anxiety, and depression, thereby improving their social relationships, sleep quality and QOL.

\section{Objectives}

The purpose of this study was to investigate the physical and psychological effects of stress management and Qigong for cancer survivors by evaluating their effectiveness at reducing physical and psychological stress and the effects of CRF.

\section{Methods}

Participants were randomly assigned to the stress management group (SMG) or the Qigong group (QG) according to the screening criteria. Improvements in CRF, QOL, and heart rate variability (HRV) were evaluated before and after 12 weeks intervention also at a three-month followup.

\section{Results}

Thirty-four participants completed SMG (n=17) or QG ( $\mathrm{n}=17)$ intervention. The results of the SM and Qigong intervention measures showed that SM effectively reduced CRF after 12 weeks of intervention and up to 3 months later. The significant effect of Qigong was only found at the 3month follow-up. The comparison of the levels of effectiveness of the SMG and QG interventions showed a significant difference in $\mathrm{LF} / \mathrm{HF}$ only after three months of follow-up.

\section{Conclusions}

Comparison of the effects of the SMG and QG showed an average growth rate of the SMG was larger than that of the QG, and the growing range was synchronous with a consistent growth trend. 
eP140

THE ASSOCIATION BETWEEN RESILIENCE AND FATIGUE IN COLORECTAL CANCER PATIENTS

Y.J. Chou ${ }^{l}$, K. Hsuan-Ju ${ }^{l}$, S. Shiow-Ching ${ }^{1}$

${ }^{T}$ National Taiwan University, Department of Nursing, Taipei, Taiwan R.O.C.

\section{Introduction}

Fatigue is the common symptom in colorectal cancer patients. Resilience is the ability to recover from illness, and it might be an important factor to cope with disease and fatigue in colorectal cancer patients.

Objectives

To explore the relationship between resilience and fatigue in colorectal cancer patients.

Methods

A cross-sectional study was conducted in surgery clinics at 2 medical centers in Taipei. Colorectal cancer patients who were diagnosed stage I to $\amalg$ within 5 years were recruited. A structured questionnaire was used to assess patients' demographic and clinical characteristics, fatigue characteristics, resilience, and depression. Analysis of Variance and Pearson's correlation were used to explore the relationship between fatigue, symptom distress, resilience, and depression.

\section{Results}

Among 97 patients, the mean age was $62.6(\mathrm{SD}=11.7)$. In overall, patients had mild level of fatigue $(\mathrm{M}=7.1, \mathrm{SD}=14.3)$, moderate resilience $(\mathrm{M}=137.6, \mathrm{SD}=23.4)$, and $23.7 \%$ of them had depressed mood (the score of CES-D $\geqq 10$ ). About $17.5 \%, 41.2 \%$, and $41.2 \%$ patients had low, moderate, and high resilience, respectively. Resilience was significant negatively associated with total fatigue $(\mathrm{r}=-.206, \mathrm{p}=.043)$, fatigue intensity $(\mathrm{r}=-.221, \mathrm{p}=.030)$, fatigue duration $(\mathrm{r}=-.201, \mathrm{p}=.048)$, average fatigue $(\mathrm{r}=-.208, \mathrm{p}=.041)$, and depression $(\mathrm{r}=-.462, \mathrm{p}<.001)$. Patients with low resilience had significantly higher level of average fatigue $(\mathrm{F}=3.819$, $\mathrm{p}=.025)$ and fatigue intensity $(\mathrm{F}=4.174, \mathrm{p}=.018)$ than patients with moderate resilience.

Conclusions

Colorectal cancer patients with higher level of resilience had the lower level of fatigue and depression. Therefore, resilience might be an important factor to assist colorectal cancer patients in managing and adapting disease and fatigue situations effectively.

\section{eP141}

AN INTEGRATED EXPERIENTIAL TRAINING PROGRAMME WITH COACHING TO REDUCE FATIGUE AND IMPROVE QUALITY OF LIFE AMONG CHILDREN WITH CANCER: A RANDOMISED CONTROLLED TRIAL

O.K. Chung ${ }^{I}$, H.C.W. Li ${ }^{1}$

${ }^{T}$ The University of Hong Kong, School of Nursing, Hong Kong, Hong Kong S.A.R.

\section{Introduction}

Cancer-related fatigue is a commonly reported symptom in children with cancer and negatively affects their quality of life. Despite strong evidence that regular physical activity reduces cancer-related fatigue, most children with cancer experience a decline in physical activity levels after diagnosis.

Objectives

To examine the effectiveness of an integrated experiential training programme with coaching in promoting physical activity, reducing fatigue, and enhancing self-efficacy and quality of life among Hong Kong Chinese children with cancer.

Methods

A randomised controlled trial was conducted in a public acute care hospital in Hong Kong. Participants were 70 Chinese children with cancer aged 9-18 years. Subjects in the experimental group received an integrated experiential training programme with coaching in promoting physical activity for six months. Subjects in the control group received usual medical care plus placebo intervention. The outcome measures were fatigue, physical activity levels, self-efficacy and quality of life assessed at baseline, and 6 and 9 months after starting the intervention.

Results

Between October 3, 2015, and October 5, 2016, we randomly assigned 70 eligible children with cancer to an experimental group $(n=37)$ or a control group $(n=33)$. The experimental group reported statistically significantly lower levels of cancer-related fatigue, and higher levels of physical activity, self-efficacy and quality of life than the control group at 9 months.

\section{Conclusions}

The integrated programme is effective in promoting physical activity, decreasing cancer-related fatigue, and enhancing self-efficacy and quality of life among Hong Kong Chinese children with cancer.

\section{eP142 \\ CHEMOTHERAPY-INDUCED-FATIGUE INDUCES A SPECIFIC GENE EXPRESSION PROFILE IN PERIPHERAL BLOOD MONONUCLEAR CELLS OF BREAST CANCER PATIENTS RECEIVING ADJUVANT OR NEOADJUVANT CHEMOTHERAPY}

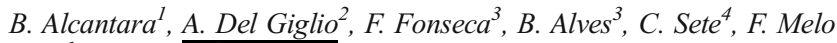
Cruz', D. Cubero

${ }^{I} F M A B C$, Medical Oncology, Santo Andre, Brazil

${ }^{2}$ FACULDADE DE MEDICINA DA FUNDACAO ABC, ONCOLOGIA E HEMATOLOGIA, SANTO ANDRE, Brazil

${ }^{3}$ FMABC, Clinical Pathology, Santo Andre, Brazil

${ }^{4}$ FMABC, Clinical Oncology, Santo Andre, Brazil

\section{Introduction}

Chemotherapy-Induced-Fatigue (CIF) is a frequent symptom that impairs patient functioning during chemotherapy for neoplastic disease.

\section{Objectives}

To evaluated if CIF can induce a specific gene expression profile in peripheral blood mononuclear cells (PBMNC) of patients with locoregional breast cancer (LRBC) receiving chemotherapy.

\section{Methods}

Initially, we sequenced the total transcriptome of the PBMNC of 3 patients who developed CIF collected before and after their initial chemotherapy first cycle. We employed the Ion Torrent ${ }^{\mathrm{TM}}$ System (Life Technologies) using the Ion AmpliSeq ${ }^{\mathrm{TM}}$ Transcriptome Human Gene Expression Kit (ThermoFisher Scientific). We sequenced a total of 12.345 transcripts from which we selected 26 out of 137 that had significant differential expression before and after chemotherapy. We then validated these results by RT-PCR in a different group of 28 patients with LRBC who developed CIF after their first cycle of chemotherapy and in 6 patients who also received chemotherapy but did not develop CIF (controls). We assessed CIF by the BFI and Chalder Questionnaires.

\section{Results}

We noted a significant increase in expression of DUSP18 and RHOBTB1 and a decreased expression of NCAN and RAET1G in patients who developed CIF after Chemotherapy. Control patients only had a significant decreased expression of NCAN.

\section{Conclusions}

CIF may induce specific changes in gene expression in PBMC of LRBC patients. Some of these changes such as decreased NCAN expression may reflect direct effects of chemotherapy as its also shared by controls. Furthermore, CIF may involve gene deregulation of Skeletal muscular (RHOBT1, DUSP18) and immune (RAETG1) systems. 


\section{eP143}

ENDOCRINE ABNORMALITIES AND THEIR ASSOCIATION WITH FATIGUE IN ADVANCED CANCER PATIENTS

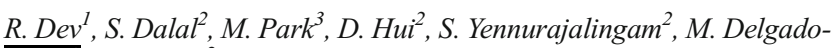
Guay $^{4}$, E. Bruera ${ }^{2}$

${ }^{1}$ University of Texas MD Anderson Cancer Center, Supportive Care and Palliative Medicine, Houston, USA

${ }^{2}$ University of Texas MD Anderson Cancer Center, Symptom Control \& Palliative Medicine, Houston, USA

${ }^{3}$ University of Texas MD Anderson Cancer Center, Department of

Biostatistics, Houston, USA

${ }^{4}$ Unviersity of Texas MD Anderson Cancer Center, Symptom Control \& Palliative Medicine, Houston, USA

\section{Introduction}

Fatigue in cancer patients is common and distressing symptom. Objectives

We investigated the association of endocrine abnormalities with fatigue in advanced cancer patients seen in a supportive care clinic.

Methods

A retrospective review of 234 consecutive cancer patients who had completed a laboratory workup for cancer fatigue collected the following: patient demographic data, cancer diagnosis, ECOG functional status, serum levels of total, free, and bio-available testosterone, $25(\mathrm{OH})$ vitamin $\mathrm{D}$, thyroid stimulating hormone (TSH), and body mass index (BMI). Symptoms were measured by the Edmonton Symptom Assessment Scale. We used descriptive statistics and Wilcoxon rank-sum test to examine the difference in fatigue between patients with normal and abnormal endocrinopathies.

\section{Results}

Patients were predominantly male $(66 \%)$ and white $(67 \%)$, with a median age of 62 (range, 22-91), a mean of BMI 25.8 (range, 15 54). Gastrointestinal (27\%) and lung (20\%) malignancies were predominant. 98 patients $(42 \%)$ had vitamin D deficiency $(25(\mathrm{OH})$ vitamin $\mathrm{D}<20 \mathrm{ng} / \mathrm{dL})$ and 64 patients $(28 \%)$ were insufficient $(20$ $\mathrm{ng} / \mathrm{dL} \leq 25(\mathrm{OH})$ vitamin $\mathrm{D}<30 \mathrm{ng} / \mathrm{dL})$. Low vitamin $\mathrm{D}$ levels were significantly more likely in non-Caucasian patients, $\mathrm{p}<0.001 .43$ patients $(18 \%)$ were noted to have thyroid dysfunction (TSH $>4.2$ $\mathrm{mcu} / \mathrm{L})$. In 143 male patients, 119 patients $(83 \%)$ were hypogonadic (bioavailable testosterone $(<61 \mathrm{ng} / \mathrm{dL})$. Hypogonadism was significantly associated with increased fatigue intensity, (mean 5.3 vs 4.3 , $\mathrm{p}=0.03$ ); however, no association was noted in patients with vitamin D deficiency or thyroid dysfunction.

\section{Conclusions}

Hypogonadism in male patients, but not low vitamin D levels or thyroid dysfunction, was significantly associated with fatigue in advanced cancer patients.

\section{eP144}

CANCER RELATED FATIGUE (CRF): PERCEPTION OF EFFORT OR TASK FAILURE?

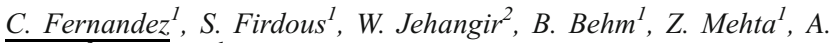 \\ Berger $^{3}$, M. Davis ${ }^{1}$ \\ ${ }^{1}$ Geisinger Medical Center, Palliative Medicine, Danville, USA \\ ${ }^{2}$ University of Vermont Medical Center, Hematology and Oncology, \\ Burlington, USA \\ ${ }^{3}$ Geisinger Medical Center, Research Support, Danville, USA
}

\section{Introduction}

Patient's rating of perceived effort (RPE) has been used to assess central fatigue. CRF is believed to be of central origin. The increased RPE with a motor task, such as the Finger Tapping Test (FTT), can easily be measured in the clinical setting.

\section{Objectives}

To correlate the FTT, RPE and the brief fatigue inventory (BFI) rated fatigue severity in cancer patients.

\section{Methods}

Subjective fatigue was assessed in adult patients with cancer by the BFI. Participants performed a modified FTT with the index finger of the dominant hand: 15 seconds $\times 2,30$ seconds $\mathrm{x} 2$ and 60 seconds $\times 2$ with 1 minute of rest between each time trial. RPE at the end of task was measured by the Borg 10 scale. Exclusions were brain metastasis, history of brain radiation, Parkinson's disease, Huntington's Chorea, multiple sclerosis, delirium and depression. Spearman's correlation coefficients were used to describe the relationships between BFI, FTT and Borg 10 scale.

Results

Thirty patients participated. Mean age was 56.2. 16 were females $(53.3 \%)$. The BFI mean score was 4.1, median 4.4. Tapping rate did not correlate with fatigue severity. Interestingly, the RPE to accomplish the FTT correlated with mean BFI: $\rho$ 0.513, p-value 0.0038 and median BFI: $\rho$ 0.541, p-value 0.0020 .

Conclusions

Cancer patients with fatigue require greater effort to complete a motor task which is a more sensitive indicator of CRF using FTT than task failure. A sense of effort is largely related to central corollary discharge to the supplementary cortex and hence central fatigue.

\section{eP145}

A LONGITUdinAL ASSESSMENT TO EVALUATE THE IMPACT OF HIGHER BODY MASS INDEX ON CANCERRELATED FATIGUE IN BREAST CANCER PATIENTS RECEIVING CHEMOTHERAPY

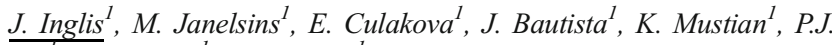 \\ Lin $^{I}$, I. Kleckner ${ }^{I}$, L. Peppone ${ }^{I}$ \\ ${ }^{1}$ University of Rochester School of Medicine and Dentistry, Surgery, \\ Rochester, USA
}

\section{Introduction}

Introduction: Obese breast cancer patients suffer increased all-cause mortality, inflammation, and decreased quality of life. Inflammation is associated with cancer-related fatigue (CRF).

\section{Objectives}

Objectives: To evaluate the impact of obesity on CRF in breast cancer patients through a secondary analysis of a prospective study.

\section{Methods}

Methods: Female breast cancer patients (aged 53 \pm 10.61 ) completed the multidimensional fatigue symptom inventory (MFSI) and the symptom inventory (SI) to measure CRF symptoms at baseline pre-chemotherapy (T1), post-chemotherapy (T2) and six months post-chemotherapy (T3). Subjects were categorized based on body mass index (BMI) at T1: Obese (OB: $\geq 30.0 \mathrm{~kg} / \mathrm{m}^{2} ; \mathrm{n}=294$ ), overweight (OV: $25.0-29.9 \mathrm{~kg} / \mathrm{m}^{2} ; \mathrm{n}=146$ ) and normal weight (NW: $\left.18.5-24.9 \mathrm{~kg} / \mathrm{m}^{2} ; \mathrm{n}=125\right)$. Multivariate regression models were used to evaluate obesity level on fatigue controlling for age, race, radiation history, hormonal therapy, cancer stage, exercise level and KPS score.

\section{Results}

Results: At T1, the obese had significantly higher CRF symptoms than normal weight subjects for both the MFSI Total $(\mathrm{OB}=11.4 \mathrm{vs} \mathrm{NW}=8.1$; $\mathrm{p}=0.03)$ and $\mathrm{SI}(\mathrm{OB}=3.5 \mathrm{vs} \mathrm{NW}=2.9 ; \mathrm{p}=0.02)$. Significantly higher SI fatigue scores persisted at $\mathrm{T} 2$ for the obese $(\mathrm{OB}=4.4 \mathrm{vs} \mathrm{NW}=3.8$; $\mathrm{p}=0.02)$ with a trend towards significance for the overweight $(\mathrm{OV}=4.4$ vs $\mathrm{NW}=3.8 ; \mathrm{p}=0.08$ ). Over the course of the study, obese subjects maintained higher MFSI scores for General, Mental, and Emotional subscales $(\mathrm{p}<0.05)$. 


\section{Conclusions}

Conclusion: Obese breast cancer patients may suffer increased fatigue. Weight loss interventions should be considered to address fatigue in obese breast cancer patients. Funding: NCI UGCA189961, R25 CA102618

\section{eP146}

\section{THYROTROPIN-RELEASING HORMONE BASED} THERAPEUTICS FOR CANCER-RELATED FATIGUE

\section{J. Kamath \\ ${ }^{T}$ University of Connecticut Health Center, Psychiatry, Farmington, USA}

\section{Introduction}

Thyrotropin-releasing hormone (TRH) is involved in the control of behavioral, metabolic, and immunological homeostasis, far beyond its role as a regulator of the thyroid axis. TRH agonism is known to exert arousing and analeptic actions in instances of behavioral depression.

\section{Objectives}

In a pilot, randomized, placebo controlled, crossover study, we established the efficacy and safety of intravenously administered TRH as a treatment for cancer-related fatigue (CF). Efforts are currently underway to develop a sublingual formulation of TRH as a treatment of CF.

Methods

Patients with cancer experiencing clinically significant fatigue were given TRH or placebo over a 4 week period. The primary outcome measure was the visual analog scale for energy (VAS-E) assessed at 3, 7 and 24 hour post TRH and saline (placebo) intravenous administration. The secondary outcome measures included the profile of mood states (POMS), a sixminute walking test, quality of life assessment, and selected serum markers of inflammation.

\section{Results}

A total of 8 patients completed the study. No unexpected side effects were seen. Clinically significant improvement in fatigue level was seen with the VAS-E and the fatigue and vigor subscales of the POMS ( $<<0.05)$. Increase in energy level was associated with functional and quality of life improvements, and decrease in serum C-reactive protein levels.

Conclusions

$\mathrm{TRH}$ administration was efficacious in the treatment of $\mathrm{CF}$ with a positive impact on quality of life. Current efforts to develop a sublingual formulation of TRH as a treatment of $\mathrm{CF}$ will be delineated including the ongoing pharmacokinetic study of the sublingual TRH formulation

\section{eP147}

PREDICTING EVENING CANCER-RELATED FATIGUE SEVERITY IN ONCOLOGY PATIENTS ONE WEEK FOLLOWING CHEMOTHERAPY

\author{
K. Kober ${ }^{I}$, X. Hu ${ }^{I}$, B. Cooper ${ }^{I}$, S. Paul ${ }^{1}$, C. Miaskowski ${ }^{l}$ \\ ${ }^{T}$ School of Nursing- University of California San Francisco, \\ Physiological Nursing, San Francisco, USA
}

\section{Introduction}

Effective symptom management is a critical component of cancer treatment. Computational tools that predict the course and severity of these symptoms can assist oncology clinicians to personalize the patient's treatment regimen and provide more timely interventions. Cancer-related fatigue (CRF) is the most common symptom associated with cancer and its treatments. CRF has a negative impact on the patients' ability to tolerate treatments and on their quality of life. One of the limitations to effective treatment of CRF is the availability of a valid and reliable model to predict the severity of CRF.

Objectives

To generate a model using demographic and clinical characteristics and CRF levels collected just prior to CTX administration (T1) to predict evening CRF severity 1 week after chemotherapy (CTX) administration (T2)

\section{Methods}

Support vector regression was used to predict the severity of the evening CRF at T2 in 689 patients ( $n=518$ training, $n=171$ testing). Model fit was assessed with 10-times 10 -fold cross-validation root-mean-square error (RMSE).

Results

Our model achieved high accuracy (mean reported CRF score $=5.7$, mean predicted $\mathrm{CRF}$ score $=5.6$ ) and low prediction error $(\mathrm{RSME} / \mathrm{mean}=0.269)$. Predictors with the highest importance were: morning and evening fatigue at $\mathrm{T} 1$, attentional function, sleep disturbance, and performance status. Predictors with the lowest importance were: living alone, caregiver to adult, level of education, cycle length, and number of metastatic sites.

\section{Conclusions}

This type of predictive model can be used to identify high risk patients, educate patients about their symptom experience, and improve the timing of pre-emptive and personalized symptom management interventions.

\section{eP148}

A PHASE III DOUBLE-BLIND PLACEBO-CONTROLLED RANDOMIZED STUDY OF DEXAMPHETAMINE SULFATE FOR FATIGUE IN PATIENTS SUFFERING FROM PRIMARY BRAIN TUMORS

\author{
F. Laigle-Donadey ${ }^{1}$, F. Ducray ${ }^{2}$, M. Boone ${ }^{3}, H$. Diallo ${ }^{4}$, C. Ramirez ${ }^{5}$, O.

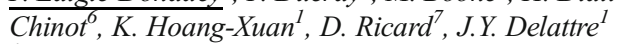 \\ ${ }^{I}$ AP-HP-Hôpital Pitié-Salpêtrière, Service de Neurologie Mazarin, \\ Paris, France \\ ${ }^{2}$ Hôpital Neurologique Pierre Wertheimer- CHU de Lyon, Service de \\ neuro-oncologie, Bron, France \\ ${ }^{3}$ CHU d'Amiens, Service d'oncologie médicale, Amiens, France \\ ${ }^{4}$ AP-HP-Hôpital Pitié-Salpêtrière, Unité de Recherche Clinique, Paris, \\ France \\ ${ }^{5}$ CHRU de Lille- hopital Roger Salengro, Service de Neurochirurgie, \\ Lille, France \\ ${ }^{6}$ CHU de la Timone, Service de Neuro-oncologie, Marseille, France \\ ${ }^{7}$ HIA Percy, Service de Neurologie, Clamart, France
}

\section{Introduction}

Many primary brain tumor (PBT) patients complain of severe fatigue, which contributes to decreased quality of life (QOL).

\section{Objectives}

We hypothesized that dexamphetamine sulfate, a psychostimulating drug, could result in reduced fatigue in PBT patients.

\section{Methods}

A phase III, multi-institutional, placebo-controlled randomized trial assessed the efficacy and tolerance of dexamphetamine administered up to $30 \mathrm{mg}$ /day during 3 months in PBT patients with non progressive disease. Patients should complain of severe fatigue, defined by a Multidimensional Fatigue Inventory (MFI) score $\geq 60$, and should not suffer from depression. Evaluation included treatment effect on fatigue (primary outcome). We also studied as secondary outcomes anxiety and depression (Hospital Anxiety and Depression Scale), QOL (EORTC QLQ C30 and BM20), cognitive functions (memory, speech, executive functions and apathy), as well as tolerance.

\section{Results}

From April 2013 to November 2016, 46 patients were enrolled in the study, with $41(89 \%)$ retained for analysis. Tolerance was generally good. There were no statistically significant differences at 3 months between the dexamphetamine and placebo subgroups in the MFI 20 score, as well as in any secondary outcomes (anxiety, depression, QOL and cognitive functions). 


\section{Conclusions}

Dexamphetamine up to $30 \mathrm{mg} /$ day, is well tolerated in non progressive PBT patients suffering from severe fatigue, but does not provide a significant benefit on fatigue, anxiety, depression, cognition, and QOL.

\section{eP149}

AN ORAL BOTANICAL EXTRACT REDUCES FATIGUE IN A RADIATION-INDUCED FATIGUE MOUSE MODEL

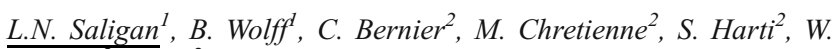
Pralong', J. Liu ${ }^{2}$

${ }^{1}$ National Institute of Nursing Research- National Institutes of Health, Symptoms Biology Unit, Bethesda- Maryland, USA

${ }^{2}$ Legacy Healthcare, Research and Development, Epalinges, Switzerland

\section{Introduction}

Cancer related fatigue (CRF) is a particularly prevalent debilitating condition with multifactorial etiology that can persist even during survivorship. No gold-standard treatment is approved for CRF due to insufficient understanding of underlying mechanisms.

Sustained inflammation is believed to play a key role in CRF.

We previously observed significant correlations between changes in the expressions of eight mitochondria-related genes (e.g., BCL2) with worsening of fatigue symptoms in radiation-treated men. We also observed that Cellium, a safe botanical extract, inhibited inflammation and normalized BCL2 levels in human alopecia models.

Objectives

To investigate the anti-fatigue effects of oral Cellium in a radiationinduced fatigue mouse model.

Methods

Two successive placebo-controlled experiments $(\mathrm{N}=40, \mathrm{n}=20$ Cellium, n=20 placebo) were conducted. Oral Cellium/placebo were provided to all mice before, during and following irradiation. Voluntary wheel running activity measuring distance and speed, served as the surrogate objective measure of fatigue-like behavior in these mice.

Results

Average cumulative post-irradiation running distance in the Cellium-group was up to $47 \%$ greater than the Placebo-group. The Cellium-group returned to initial running distance 5-8 days earlier than the Placebo-group with no between-group differences in bodyweight. Average speed improved post-irradiation in the Cellium-group by up to $77 \%$ compared to the placebo-group.

Conclusions

Oral Cellium had anti-fatigue effects in irradiation -induced fatigue mice, possibly related to its anti-inflammatory and BCL2 effects. Further investigations on the anti-fatigue effects of Cellium on cancer patients receiving treatment and cancer survivors are warranted.

\section{eP150}

\section{CORRELATION BETWEEN HANDGRIP STRENGTH AND} ONCOLOGICAL FATIGUE IN OLDER ADULTS CANCER SURVIVORS

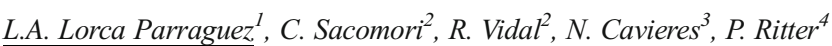
${ }^{T}$ Hospital del salvador, Medicina Física y Rehabilitacion, Santiago, Chile ${ }^{2}$ Universidad Bernardo O'Higgins, Escuela de Kinesiologia, Santiago, Chile

${ }^{3}$ Universidad Bernardo OHiggins, Escuela de Kinesiologia, Santiago, Chile

${ }^{4}$ Hospital del Salvador, Unidad del dolor y cuidados paliativos, Santiago, Chile
}

\section{Introduction}

Introduction: The functional limitations of aging are important predictors of mortality, morbidity and disability. Consequently, the manual grip force is used as an indicator of functional physical capacity. However, for those with cancer, fatigue has been described as a major concern during treatment, in advanced stages of the disease and after curative treatment.

\section{Objectives}

The aim of this study was to analyze the relationship between manual grip strength and oncological fatigue in elderly cancer survivors

\section{Methods}

Method: Consists of a cross-sectional observational study. Forty-three adult elderly cancer survivors who attended the polyclinics of the Hospital del Salvador participated. To evaluate fatigue, the Brief Fatigue Inventory was used and to evaluate the handgrip strength the digital hand-held dynamometer model Baseline ${ }^{\circledR}$ Hidraulic were used

\section{Results}

Results: Negative correlation was identified between the hand grip strength with the fatigue score (rho $=-, 416$ ) and with the Minimental score (rho =, 319).

\section{Conclusions}

Conclusion: There is a negative correlation between hand grip strength and oncological fatigue in older Adults Cancer Survivors in suggesting the need for kinesic treatment by means of exercises to improve these functions and and quality ol life in elderly cancer survivors.

\section{eP151}

FATIGUE AMONG WOMEN WITH BREAST CANCER: LONGITUDINAL RELATIONSHIP TO COGNITIVE PERFORMANCE, CHEMOTHERAPY AND CLINICAL FACTORS

\author{
D. Lyon ${ }^{1}$, R. Cohen ${ }^{2}, J$. Gullett $^{3}$, R. Fieo ${ }^{3}$, D. Lynch Kelly ${ }^{1}$, A. \\ Startkweather ${ }^{4}$, C. Jackson-Cook ${ }^{5}$ \\ ${ }^{1}$ University of Florida, College of Nursing, Gainesville- FL, USA \\ ${ }^{2}$ University of Florida, McNight Brain Institute, Gainesville- FL, USA \\ ${ }^{3}$ University of Florida, Department of Psychology, Gainesville- FL, USA \\ ${ }^{4}$ University of Connecticut, Nursing, Storrs, USA \\ ${ }^{5}$ VIrginia Commonwealth University, Cytogenetics, Richmond, USA
}

\section{Introduction}

Women with breast cancer may experience persistent cognitive deficits and fatigue after treatment. However, the interrelationships of fatigue and cognitive dysfunction is not well-understood, particularly across the active treatment regimen and into survivorship.

\section{Objectives}

The severity and impact of fatigue was examined longitudinally among women with early-stage breast cancer. Changes in fatigue across treatment phases and the relationship of fatigue severity and impact to on cognitive performance were examined.

\section{Methods}

Our group completed a 2-year prospective study of 75 women with early-stage breast cancer using an validated questionnaires and an objective measure of cognitive performance (CNSVS). Fatigue and performance-based cognitive testing was assessed at five time points beginning prior to chemotherapy and finishing 24 months after initial chemotherapy. Temporal changes in fatigue were examined as was the relationship between fatigue and cognitive performance at each time point

\section{Results}

Severity of reported fatigue and the extent to which it interfered with daily living varied over time. Fatigue severity and functional impact was moderate at baseline, increased significantly during chemotherapy, and returned to near baseline levels at two-years. At each time point, fatigue severity and impact were significantly associated with processing speed 
and complex attention performance. Neoadjuvant chemotherapy was associated with greater fatigue and poorer cognitive function.

Conclusions

The strong association between subjective fatigue and cognitive performance suggests that they are likely functionally related. That cognitive deficits were evident at baseline, whereas fatigue was more chemotherapy-dependent, suggests that fatigue and cognitive performance may share common bases, but likely also differ in underlying mechanisms.

\section{eP152}

CHEMOTHERAPY INDUCED FATIGUE AND SYMPTOM SEVERITY IN THE INDIAN CANCER PATIENTS: AN OBSERVATIONAL STUDY

\section{P. Prinja ${ }^{l}$, S. Mukhopadhyay ${ }^{2}$}

${ }^{T}$ Venkateshwar Hospital- New Delhi, Palliative Care, Delhi, India

${ }^{2}$ Burdwan Medical College, Pharmacology, Burdwan, India

\section{Introduction}

Significant numbers of chemotherapy patients develop fatigue during their course of cancer treatment. India, though have a huge burden of cancer, prevalence of fatigue in the Indian patients was not done earlier. There is also need for finding the rural and urban distribution of fatigue in India. Severity of cancer related symptoms and its correlation of fatigue also needs evaluation. Objectives

To find out the prevalence of Chemotherapy induced fatigue, cancer related symptom in Indian Cancer patients and to find out any correlation of fatigue with severity of symptoms detected by Edmonton Symptom Assessment Scale (ESAS).

\section{Methods}

A cross sectional study is being conducted over a period of three months on the urban based patients of North India who received at least three cycles of chemotherapy. Fatigue severity was noted using FACIT F score. Severity of cancer symptoms were noted by using ESAS. Correlation coefficients were analyzed to assess correlation of fatigue with ESAS.

\section{Results}

Data of 10 patients were available till the time of analysis. Fatigue was noted in $40 \%$ patients amongst whom $75 \%$ had severe pain, $50 \%$ reported loss of appetite and 50\% severe loss of wellbeing. Higher FACIT score (indicating less fatigue) were negatively correlated pain, appetite, tiredness and loss of wellbeing with correlation coefficient ( $\mathrm{r}$ ) values of $-0.83,-0.82$,0.82 and -0.87 respectively) as well as Shortness of breath (r, -0.71$)$.

\section{Conclusions}

Fatigue is prevalent in urban Indian cancer patients following chemotherapy. It is correlated with several cancer related symptoms. Further data will be available after completion of the study.

\section{eP153}

CORTICOMUSCULAR COHERENCE IN PRE-TREATMENT CANCER-RELATED FATIGUE VS CHRONIC FATIGUE SYNDROME

\author{
C.M. O'Higgins ${ }^{1,2,3}$, S. Dukic ${ }^{1}$, A. Rice ${ }^{3}$, B. O'Connor ${ }^{2}$, S. Cuffe ${ }^{4}$, E. \\ Hanrahan', E. Tiernan ${ }^{6}$, D. Walsh ${ }^{1,2}$, R.B. Reilly ${ }^{1,3,7}$ \\ ${ }^{1}$ Trinity College Dublin, School of Medicine, Dublin, Ireland \\ ${ }^{2}$ Our Lady's Hospice \& Care Services, Academic Department of \\ Palliative Medicine, Dublin, Ireland \\ ${ }^{3}$ Trinity College Dublin, Trinity Center for BioEngineering, Dublin, \\ Ireland \\ ${ }^{4}$ St James' Hospital, Department of Oncology, Dublin, Ireland \\ ${ }^{5}$ St. Vincent's University Hospital, Department of Oncology, Dublin, Ireland \\ ${ }^{6}$ St. Vincent's University Hospital, Department of Palliative Care, Dublin, \\ Ireland \\ ${ }^{7}$ Trinity College Dublin, School of Engineering, Dublin, Ireland
}

\section{Introduction}

Cancer-Related Fatigue (CRF) and Chronic Fatigue Syndrome (CFS) are common, yet their pathophysiology is poorly understood. Aetiology may be central or peripheral and originate anywhere from the brain to muscle fibre. Studies have shown a dissociation between brain and muscle signals during voluntary muscle fatigue. This suggests weakened corticomuscular coherence. Objectives

To objectively evaluate the effect of muscle fatigue on corticomuscular coherence by determining EEG-EMG coherence during a fatiguing motor task. We hypothesize that corticomuscular coherence in $\beta$ eta-band frequency would decrease significantly with fatigue in CRF and CFS compared to healthy controls (HC).

\section{Methods}

12 newly diagnosed, pre-treatment, non-small cell lung cancer CRF, 12 CFS and $12 \mathrm{HC}$ were enrolled. Participants completed a (subjective) Brief Fatigue Inventory (BFI) and performed a sustained isometric forearm contraction (Flexor Carpi Ulnaris (FCU) \& Flexor Carpi Radialis (FCR) muscles) at 30\% maximal level (MVC) until failure. Endurance Time (ET) was noted. Task evaluated in two stages; mild \& severe fatigue. EEG-EMG $\beta$-band frequency (associated with voluntary motor activity) coherence and power spectrum in each stage was computed.

Results

CRF and CFS had weaker MVC and earlier perceived exhaustion than HC (Table 1). EMG power (but not EEG) increased while coherence at lower ßeta-band $(15-25 \mathrm{~Hz})$ significantly decreased in severe fatigue compared to mild fatigue (Figure 1)

\begin{tabular}{|cccccc|}
\hline COHORT & GENDER & $\begin{array}{c}\text { AGE } \\
\text { (years) }\end{array}$ & BFI & ET(S) & MVC(N) \\
\hline $\begin{array}{c}\text { Healthy } \\
\text { Controls }\end{array}$ & $\begin{array}{c}\text { Female: } 5 \\
\text { Male: } 7\end{array}$ & $50 \pm 13$ & $2.3 \pm 2$ & $512 \pm 417$ & $351 \pm 152$ \\
\hline $\begin{array}{c}\text { Cancer- } \\
\text { Related } \\
\text { Fatigue }\end{array}$ & $\begin{array}{c}\text { Female: } 6 \\
\text { Male: } 6\end{array}$ & $65 \pm 11$ & $3.2 \pm 2$ & $201 \pm 165$ & $305 \pm 127$ \\
\hline $\begin{array}{l}\text { Chronic } \\
\text { Fatigue } \\
\text { Syndrome }\end{array}$ & $\begin{array}{c}\text { Female: } 8 \\
\text { Male: } 4\end{array}$ & $49 \pm 11$ & $6.5 \pm 1^{* *}$ & $291 \pm 165$ & $237 \pm 65^{*}$ \\
\hline
\end{tabular}

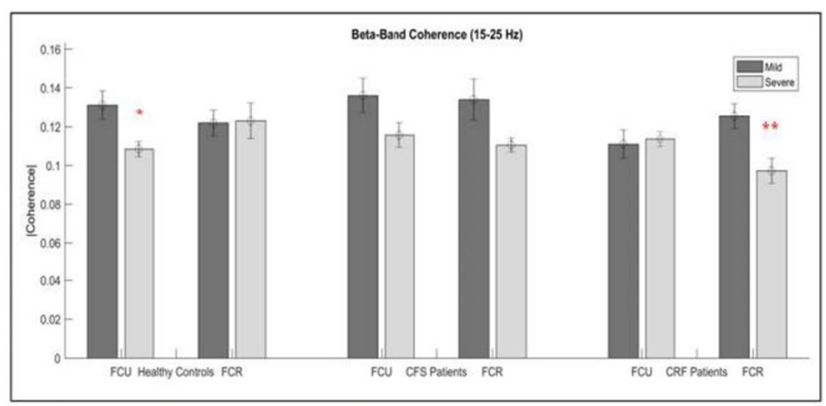

Figure 1: Lower Beta-band conerence changes over contralateral motor cortex for both muscles at each fatigue stage in all three cohorts. ( $\left.{ }^{\mathrm{P}} \mathrm{P}<0.05,{ }^{\mathrm{x} \times} \mathrm{P}<0.0001\right)$

\section{Conclusions}

Both CRF and CFS was associated with weakened corticomuscular coherence in a sustained submaximal motor activity at lower $\beta$ eta-band frequencies. This suggests central neural mechanisms contribute to both $\mathrm{CRF}$ and CFS with associated performance impairment. Interventions to improve coherence may reduce fatigue.

\section{eP154}

AMERICAN GINSENG IN REDUCING FATIGUE IN PATIENTS TREATED FOR HEAD AND NECK CANCER: A RANDOMIZED, DOUBLE-BLIND, PLACEBO CONTROLLED, MONO-CENTRE PHASE II STUDY 


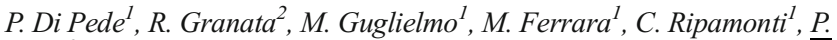
Bossi $^{2}$

${ }^{T}$ Fondazione IRCCS Istituto Nazionale dei Tumori, Supportive Care in Cancer-Department Oncology-Hematology, Milano, Italy

${ }^{2}$ Fondazione IRCCS Istituto Nazionale dei Tumori, Head and Neck Medical Oncology Department, Milano, Italy

\section{Introduction}

There are few data about fatigue treatment in Head and Neck cancer ( $\mathrm{HNC}$ ) patients (pts) during follow up (FU)

Objectives

To evaluate the efficacy of American Ginseng on fatigue reduction in $\mathrm{HNC}$ patients in FU

Methods

Consecutive eligible pts coming for planned follow-up visits were randomized 1:1 to receive American ginseng $1000 \mathrm{mg}$ /day or placebo for 8 weeks. Inclusion criteria were: curative intent treatment for primary $\mathrm{HNC}$ ended since more than 1 year, no evidence of disease; Brief Fatigue Inventory (BFI) score $\geq 4$, lack of overt clinical evidence of cognitive failure, no diagnosis of psychiatric disorder. Reduction in BFI score at the end of treatment was the primary endpoint. We conducted analysis according to intention to treat (ITT) and per protocol (PP)

Results

We enrolled 32 patients with the following characteristics: 22 males and 10 females, median age 55 years, mainly oropharyngeal cancer pts (15), of whom 12 with HPV positive cancer; median time since treatment end was 2.4 years. No significant differences in tumor site, HPV and gender were reported between treatment and placebo pts.

No major side effect was reported, except by 2 pts in treatment arm who interrupted treatment due to gastric intolerance. Mean difference in BFI score (after and before treatment) was 1.8 in ginseng group and 2.1 in placebo group ( $\mathrm{p}=\mathrm{ns}$ ), according to $\mathrm{PP}$ analysis, not different from ITT results $(\mathrm{p}=\mathrm{ns})$ Conclusions

Ginseng was quite tolerated, but not active in reducing fatigue in FU patients after treatment for $\mathrm{HNC}$

\section{eP155}

WAS LEVEL OF FEAR OF RECURRENCE ASSOCIATED WITH LEVEL OF FATIGUE IN COLORECTAL CANCER PATIENTS?

\section{S.C. Shun ${ }^{1}$, Y.J. Chou ${ }^{1}$, H.J. Kuo ${ }^{1}$}

${ }^{T}$ National Taiwan University, School of Nursing, Taipei City, Taiwan R.O.C.

\section{Introduction}

Fatigue is the common symptom in colorectal cancer patients and psychological distress has been shown as one of the most related factors. However, the relationship between fear of recurrence and fatigue has not been studies in colorectal cancer patients.

Objectives

To explore the relationship between fear of recurrence and fatigue in colorectal cancer patients.

Methods

A cross-sectional study was conducted in surgery clinics at 2 medical centers in Taiwan. Colorectal cancer patients diagnosed within 5 years were recruited. Fear of Cancer Recurrence Inventory-Short Form (FCRsf) and fatigue symptom Inventory were used to assess patients' fear of recurrence and fatigue characteristics. Generalized estimating equation was used to examine their relationships after controlling other confounding factors (symptom distress and depression).

\section{Results}

Among 97 patients with mild fatigue, 54.6\% ( $\mathrm{n}=54)$ had been identified as clinical levels of fear of recurrence (the score of FCR-sf $>=13$ ). No significant differences of levels of FCR and fatigue in patients with different treatment status (active treatment vs. survivors) and cancer stage. FCR was significant positively associated with average fatigue $(\mathrm{r}=.25, \mathrm{p}=.013)$ and patients with higher level of fear of recurrence $(>=13)$ had significant higher than level of average fatigue than patients with lower level than 13.

\section{Conclusions}

Colorectal cancer patients with higher level of fear of recurrence had the higher level of fatigue in average. Therefore, decreasing the level of fear of recurrence might be an important factor to help colorectal cancer patients in managing fatigue effectively. However, their causal effect needs to identify in the further study.

\section{eP156}

FATIGUE DURING AND AFTER NEOADJUVANT CHEMOTHERAPY AMONG WOMEN WITH BREAST CANCER: ASSOCIATION WITH CANCER-RELATED PHYSICAL SYMPTOMS, SLEEP QUALITY AND EMOTIONAL DISTRESS

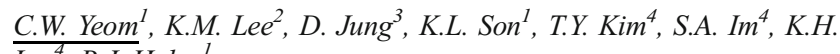
$\mathrm{Lee}^{4}$, B.J. Hahm ${ }^{1}$

${ }^{1}$ Seoul National University Hospital, Department of Neuropsychiatry, Seoul, Republic of Korea

${ }^{2}$ Seoul National University Hospital, Public Health Medical Service, Seoul, Republic of Korea

${ }^{3}$ Ulsan National Institute of Science and Technology, Department of Human Factors Engineering, Ulsan, Republic of Korea

${ }^{4}$ Seoul National University Hospital, Department of Internal Medicine, Seoul, Republic of Korea

\section{Introduction}

Fatigue is one of the most prevalent side effects of chemotherapy and could be influenced by several factors, such as physical symptoms and psychiatric symptoms. These influences on fatigue could alter during and after chemotherapy. Limited data existed on fatigue in neoadjuvant chemotherapy.

\section{Objectives}

This study evaluates the trajectory of fatigue and its association with cancerrelated physical symptoms, sleep quality, emotional distress among women with breast cancer during and after neoadjuvant chemotherapy.

\section{Methods}

In this prospective study, 111 participants reported severity of fatigue using the Fatigue Severity Scale (FSS), at baseline, at the end of chemotherapy, and at 8 months after completion of chemotherapy. Overall cancer-related physical symptoms, sleep quality, emotional distress were assessed serially using the M. D. Anderson Symptom Inventory (MDASI) except items of fatigue, insomnia, distressed and sad, the Pittsburgh Sleep Quality Index (PSQI) and the Hospital Anxiety and Depression Scale (HADS).

\section{Results}

Fatigue severity increased during chemotherapy and decreased after chemotherapy. On multivariate linear regression analyses, FSS during chemotherapy was significantly associated with MDASI $(\beta=0.38, \mathrm{p}<$ $0.001)$ during chemotherapy, adjusted age and FSS at baseline. FSS after chemotherapy was significantly associated with $\operatorname{HADS}(\beta=0.24, \mathrm{p}=$ $0.012)$, PSQI ( $\beta=0.23, p=0.005)$, MDASI $(\beta=0.23, p=0.009)$ after chemotherapy, adjusted age and FSS at baseline.

\section{Conclusions}

These findings imply that, in women with breast cancer, fatigue during chemotherapy is primarily affected by cancer-related physical symptoms, but fatigue after chemotherapy is further affected by emotional distress and poor sleep quality.

\section{eP157}

IN DEPTH UNDERSTANDING ON VALUES AND ISSUES RELATED TO HEALTH INSURANCE AMONG BREAST CANCER SURVIVORS IN LOW AND MIDDLE INCOME SETTING WITH UNIVERSAL HEALTH COVERAGE 
N.T. Bhoo Pathy ${ }^{1}$, Y.C. Kong ${ }^{2}$, L.P. Wong ${ }^{3}$, C.W. $\mathrm{Ng}^{3}$, P. Yehgambaram ${ }^{4}$, N.A. Taib ${ }^{5}$, A.F. Abdul Azizi $i^{6}$, W.Z. Wan Ishak ${ }^{7}$, C.H. Yip ${ }^{8}$, N. Bhoo Pathy ${ }^{3}$

${ }^{1}$ University Malaya, Social and Preventive Medicine, Kuala Lumpur, Malaysia

${ }^{2}$ National Clinical Research Centre, Clinical Epidemiology Unit, Kuala Lumpur, Malaysia

${ }^{3}$ University of Malaya, Department of Social and Preventive Medicine, Kuala Lumpur, Malaysia

${ }^{4}$ Kuala Lumpur Hospital, Department of Radiotherapy and Oncology, Kuala Lumpur, Malaysia

${ }^{5}$ University of Malaya, Department of Surgery, Kuala Lumpur, Malaysia

${ }^{6}$ Pantai Hospital Kuala Lumpur, Cancer Institute, Kuala Lumpur, Malaysia

${ }^{7}$ University Malaya Medical Centre, Department of Oncology, Kuala Lumpur, Malaysia

${ }^{8}$ Subang Jaya Medical Centre, Radiology and Oncology, Selangor, Malaysia

\section{Introduction}

Despite Malaysia's stellar achievement of Universal Health Coverage in delivery of primary care services, it is still struggling in provision of cancer care. Health insurance therefore plays an important role in alleviating financial toxicity following cancer.

Objectives

We investigated the motivators and barriers to health insurance ownership, value of health insurance, and issues in navigating health insurance among Malaysian breast cancer survivors.

Methods

In this qualitative inquiry, 9 focus group discussions (FGDs) were conducted with breast cancer survivors diagnosed within 12-24 months, from Hospital Kuala Lumpur (tertiary government hospital), University Malaya Medical Center (public academic hospital) and Pantai Hospital Kuala Lumpur (private hospital). All FGDs were audio recorded and transcribed verbatim. Data were examined using thematic content analysis.

Results

The major themes for motivators in obtaining private health insurance were "a back-up plan" and "expedited treatment" while themes for barriers were "high premium rates", "low awareness" on health insurance, "presence of comorbidities", "competing financial priorities", and "belief in fate and destiny". In terms of value of health insurance, participants mooted that "insurance saved my life" and "provided a sense of security". Nonetheless, issues faced in navigating health insurance included "slow claim processes due to red tape", "complicated policies and hidden clauses", "pay first, claim-later" and "rapid exhaustion of insurance limits".

Conclusions

While health insurance plays an important role in reducing the financial burden faced by breast cancer survivors, this study highlights the need to increase health insurance literacy, improve insurance regulation and revise existing medical coverage plans in low-and middle-income settings.

\section{eP158}

FINANCIAL DISTRESS AMONG ADVANCED CANCER PATIENTS ASSESSED IN A SUPPORTIVE CARE OUTPATIENT CENTER

M. Delgado-Guay ${ }^{I}$, M.G. De la Cruz ${ }^{1}$, J.L. Williams ${ }^{I}$, E. Bruera ${ }^{l}$

${ }^{T}$ The University of Texas MD Anderson Cancer Center, Palliative Care and Rehabilitation, Houston, USA

\section{Introduction}

Advanced cancer patients(AdCa) experience physical, psychological, existential/spiritual distress and financial distress(FD) associated with the disease or treatment. Regular assessments of these issues in the outpatient-setting are limited.

\section{Objectives}

To determine the frequency, intensity and correlates of self-reported FD (Distress/Suffering experienced secondary to financial issues) among these AdCa.

\section{Methods}

We reviewed 289 consults of AdCa evaluated by our Supportive Care Outpatient-Center between October-2012 and January-2013. We used Edmonton Symptom Assessment Scale adding two items following the same scale (0 to 10$)$ to evaluate: Spiritual Pain and FD, (ESAS-FS).

\section{Results}

Mean age(range): 60.1 years(22-92). 53\% were male. $189(65 \%)$ were White, 45(15\%) African American, and 34(12\%) were Hispanic. Type of cancer: head and neck $62(21 \%)$, gastrointestinal $57(20 \%)$, breast $40(14 \%)$, lung 32(11\%), urological 32(11\%), and others $45(16 \%)$.

187/289(65\%) AdCa had FD: mean(95\% Confidence-Interval) 4(3.5-4.4). AdCa with FD had worse Pain [mean(95\%CI) 5.12(4.7, 5.6) vs. 4.3(3.6, $4.9)](\mathrm{p}=0.028)$, depression $[3.8(3.3,4.2)$ vs. $1.5(1.1,2.1), \mathrm{p}<0.0001]$, anxiety [4.1(3.6, 4.6 vs. $1.6(1.1,2.1), \mathrm{p}<0.0001]$, Well-Being $[5.2(4.9,5.6)$ vs. 4.3 (3.7, 4.9), $\mathrm{p}=0.006]$, and Spiritual Pain[2.4(2.0, 2.9) vs. 0.5(0, 1.0), $\mathrm{p}<0.0001]$. FD correlated (Spearman) with Pain $\mathrm{r}=0.25, \mathrm{p}<0.0001$; Sleep $\mathrm{r}=0.29, \mathrm{p}<0.0001$; Depression $\mathrm{r}=0.41, \mathrm{p}<0.0001$; Anxiety $\mathrm{r}=0.45$, $\mathrm{p}<0.0001$; Drowsiness $\mathrm{r}=0.26, \mathrm{p}<0.0001$, Well-being $\mathrm{r}=0.25, \mathrm{p}<0.0001$; and Spiritual Pain $r=0.44, p<0.0001$. Logistic regression multivariate analysis showed association with Spiritual Pain[OR (95\% Wald CI) 1.575(1.2841.932), $\mathrm{p}<0.0001]$ and Anxiety[1.261 (1.083-1.488), $\mathrm{p}<0.0001]$.

\section{Conclusions}

FD was reported in more than $65 \%$ of AdCa. It correlates with physical and psychological, and spiritual distress. Using ESAS-FS allows identifying AdCa with FD evaluated in a Supportive Care Outpatient Clinic. More research is needed.

\section{eP159}

HEALTHCARE SYSTEMS AND FINANCIAL DISTRESS INEQUALITIES AMONG PATIENTS WITH ADVANCED CANCER

\author{
C. Barberet ${ }^{1}$, M. Filbet ${ }^{2}$, S. Sanchez ${ }^{3}$, M. Delgado $^{4}$, E. Bruera $^{5}$
}

${ }^{T}$ Centre Hospitalo-Universitaire de Grenoble, Department of Supportive and Palliative Care, Grenoble-, France

${ }^{2}$ service de soins palliaitss pavillon $1 K$ CHLS CHU de Lyon, Palliative Medecine, Pierre Benite LYON, France

${ }^{3}$ Hôpitaux Champagne Sud, Department of Medical Information Evaluation and Performance, Troyes, France

${ }^{4}$ The University of Texas MD Anderson Cancer Center, Department of Palliative-Rehabilitation- and Integrative Medicine-, Houston Texas, France

${ }^{5}$ University of Texas MD Anderson Cancer Center, Department of Palliative-Rehabilitation- and Integrative Medicine, Houston- TX, USA

\section{Introduction}

Financial distress (FD) is a common cause of suffering in patients with advanced cancer. There is limited data to compare the effects of FD of advanced cancer patient in two different health systems (US and France) Objectives

To compare the frequency and intensity of Financial Distress (FD) and its associations with symptom distress and quality of life (QOL) in patients with advanced cancer in France and US.

\section{Methods}

We conducted a secondary analysis of two cross-sectional studies with 292 patients enrolled in the USA (149 patients) and in France (143 patients). Selfrated FD (subjective experience of distress attributed to financial problems) numeric rating scale $(0=$ best, $10=$ worst $)$ and validated questionnaires assessed symptoms [Edmonton Symptom Assessment System (ESAS)], psychosocialdistress [Hospital Anxiety Depression Scale (HADS)], and QOL (FACT-G).

\section{Results}

The average age was 59 (10SD),144 (49\%) patients were female.In France FD was reported in $74(52 \%)$ vs. $129(88 \%)$ in the US $(\mathrm{p}<0.001)$. Severe FD $(\geq 4 / 10)$ was reported in 100 US patients $(68 \%)$ vs. $48(34 \%)$ in France $(\mathrm{p}<0.001)$. QOL was better in the USA than in France (respectively 69 versus $63 ; \mathrm{p}=0.003$ ). French patients had more anxiety ( 8 versus $6 ; p=0.008)$ and depression $(7$ versus $6 ; p=0.004)$. FD 
was associated with the country USA, single status $(0.907 ; \mathrm{p}=0.023)$ and the presence of metastasis $(1.538 ; \mathrm{p}=0.036)$.

\section{Conclusions}

Even lower than US patients with advanced cancer, the FD is hight in French patients despite a free cancer care access. Further research focusing on indirect costs is needed.

\section{eP160}

THE IMPACT OF DYSGEUSIA ON TREATMENT EXPOSURE OF ADJUVANT S-1 THERAPY AFTER CURATIVE GASTRECTOMY

\section{H. Ishikawa ${ }^{\text {, T. Kawakami }}{ }^{2}$, R. Negami ${ }^{3}$, E. Bando $^{4}$}

${ }^{1}$ Shizuoka Cancer Center Hospital, Department of Pharmacy, Sunto-

Nagaizumi-, Japan

${ }^{2}$ Shizuoka Cancer Center Hospital, Division of Gastrointestinal Oncology, Sunto-Nagaizumi-, Japan

${ }^{3}$ Shizuoka Cancer Center Hospital, Department of Nursing, SuntoNagaizumi-, Japan

${ }^{4}$ Shizuoka Cancer Center Hospital, Division of Gastric Surgery, SuntoNagaizumi-, Japan

\section{Introduction}

Patients (pts) who are treated with chemotherapy sometimes complain taste alteration and medical staffs often regard them as an adverse event that is inevitable. There are several reports of dysgeusia worsening quality of life, the impact of dysgeusia on treatment course is rarely reported.

Objectives

The aim of this study is to investigate the impact of dysgeusia on treatment outcome.

Methods

The data of pts who were treated with S-1 as adjuvant therapy after curative gastrectomy at Shizuoka Cancer Center from January 2007 to September 2013 were retrospectively analyzed. Inclusion criteria were as follows; ECOG performance status (PS) 0 or 1 ; pStage II/III under Japanese Classification of Gastric Cancer $14^{\text {th }}$ edition; and R0 resection. S-1 was administered for 4 weeks followed by a 2 -weeks rest.

\section{Results}

Two hundred and fourteen pts were satisfied inclusion criteria. Pts' background was median age, 66 years (range 48-87); PS 0/1, 150/64 pts; pStage II/III, 92/122 pts; total gastrectomy/distal gastrectomy, 91/123 pts. The incidence rate of dysgeusia was $28.0 \%$ (Grade 1/2: $21.5 \%$ / $6.5 \%$ ). The median time to emergence of dysgeusia (range) was 56 (2$210)$ days. Treatment completion rate in dysgeusia group and in nondysgeusia group was $65.0 \%$ and $75.3 \%$, respectively( $\mathrm{P}=0.173)$. Two pts discontinued adjuvant S-1 therapy by dysgeusia.

Conclusions

The completion rate of planned adjuvant S-1 therapy tends to be lower in dysgeusia group than non-dysgeusia group

\section{eP161}

FINANCIAL ADVOCACY UNCOVERED: AN EXPLORATION OF ROLES AND IMPLEMENTATION IN CANCER CARE

\section{P. Rattananont Ferris ${ }^{1}$, L. Lucas $^{1}$, T. Perloff ${ }^{l}$ \\ ${ }^{T}$ The Association of Community Cancer Centers, Provider Education, ROCKVILLE, USA}

\section{Introduction:}

The total cost of cancer care has made financial toxicity prevalent for patients treated in the US. While financial advocates have progressively become a vital member of the multidisciplinary team (MDT), few studies have examined their role in mitigating financial toxicity across disciplines.

\section{Objectives}

The goal of this study was to explore responsibilities of financial advocates and identify challenges related to the implementation of financial advocacy services.

\section{Methods}

An electronic survey was completed by 287 financial advocates in the Association of Community Cancer Centers (ACCC) network from October 2017 to November 2017. The survey assessed advocates' training, self-reported knowledge gaps related to insurance and patient assistance programs, and integration of financial distress screening into practice. Demographic information was collected to determine correlations with socioeconomic factors.

\section{Results}

Over $75 \%$ of financial advocates enroll patients into assistance programs, yet over $70 \%$ reported difficulty navigating them. $84 \%$ did not receive formal training on financial navigation, but $65 \%$ noted formal training would benefit them in their role. When asked about integration of financial distress screening into practice, $70 \%$ stated that they do not regularly screen patients despite $79 \%$ stating it is a critical issue to address. $89 \%$ of financial advocates expressed a need for information on the basics of costrelated health literacy along with tools to educate patients.

\section{Conclusions}

This study identifies the need for tailored education among financial advocates based on roles and educational background. Proactively addressing these issues in health care delivery may result in a better overall treatment plan for cancer patients and their caregivers.

\section{eP162}

ATTITUDES AND OPINIONS OF ELDERLY CANCER PATIENTS AND FAMILY CAREGIVERS ON END-OF-LIFE CARE DISCUSSION

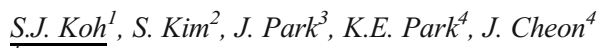

${ }^{T}$ Ulsan University Hospital- Ulsan University College of Medicine, Hematology and Oncology, Ulsan, Republic of Korea

${ }^{2}$ Changwon National University, Nursiing, Changwon, Republic of Korea

${ }^{3}$ Bobath Memorial Hospital, Oncology, Seongnam, Republic of Korea

${ }^{4}$ Ulsan University Hospital, Hematology and Oncology, Ulsan, Republic of Korea

\section{Introduction}

End-of-life (EOL) care decisions have become an urgent issue in Korea in response to recent legislation called the Life-Sustaining Treatment Decision Act of 2016.

\section{Objectives}

The present study attempted to explore attitudes and opinions on EOL discussion among elderly cancer patients and their family caregivers since communication regarding EOL care has been argued to be a major premise leading to the best decision making.

\section{Methods}

The attitudes and opinions of elderly cancer patients and their family caregivers were solicited through focus group interviews. The final sample consisted of 12 patients and 16 family members.

Results

Guided by content analysis, 5 themes were revealed. The identified themes were individual approach for delivering bad news and stakeholders involved in the discussion, contradictory attitudes toward advance care planning, mutual understanding, hope for the EOL care discussion process, and resistance to discussion of hospice-palliative care.

\section{Conclusions}

Study findings suggested that an approach focusing on the individualized needs of patients and family members is required in EOL care discussion for elderly cancer patients. 


\section{eP163}

DO PREOPERATIVE PSYCHO-SOCIAL FACTORS EFFECT ON POSTOPERATIVE COMPLICATIONS IN ELDERLY CANCER PATIENTS

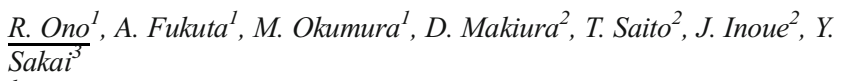

${ }^{1}$ Kobe University Graduate School of Health Sciences, Department of Community Health Sciences, Kobe, Japan

${ }^{2}$ Kobe University Hospital, Division of Rehabilitation, Kobe, Japan

${ }^{3}$ Kobe University Graduate School of Medicine, Division of Rehabilitation Medicine, Kobe, Japan

\section{Introduction}

With aging society, elderly cancer patients who have impaired psychosocial factors are increasing.

Objectives

The aim of this study is to examine the effect of preoperative psycho-social factors on the postoperative complications in elderly cancer patients.

Methods

Total 141 cancer patients who underwent radical surgery at a university hospital (mean age; 71.6years, female; $\mathrm{n}=26$ ) were participated in this cohort study. Psychological factor was assessed by depression, which defined as the geriatric depression scale $\geq 5$. Social factors were assessed by social frailty (SF) and social isolation (SI). Participants who met $\geq 2$ criteria (going out less frequently, rarely visiting friends, feeling unhelpful to friends or family, living alone, and not talking with someone every day) were defined as SF. Less than 12 points of the Japanese version Lubben Social Network Scale were defined as SI. The postoperative complications defined as the Clavien-Dindo grade $\geq 2$ within 30 days after surgery were collected by reviewing a medical record. Multiple logistic regression analyses were performed with depression, SF, and SI as each main explanatory variable, the presence of postoperative complications as outcome variable, and age, sex, smoking, drinking, grip strength, BMI, and clinical stage as confounding variables.

Results

The incidence rate of postoperative complication was $25.5 \%$. The prevalence of preoperative depression, SF and SI were $39.7 \%, 53.2 \%$ and $27.7 \%$, respectively. After adjusting for confounding factors, only SI affected the postoperative complications (odds ratio and 95\% CI; 3.0 and $1.1-8.2$ ).

Conclusions

To reduce the postoperative complications, it is important to assess and care for social factor, especially isolation.

\section{eP164 \\ SYMPTOM ASSESSMENT OF OLDEST OLD CANCER PATIENTS}

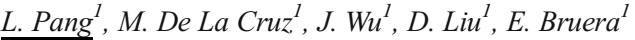 \\ ${ }^{T}$ UT MD Anderson Cancer Center, General Internal Medicine, Houston, USA}

\section{Introduction:}

The older population is a growing segment of the United States. The oldest old, described as those aged 85 and older, experienced the fastest growth. This population is also undergoing active cancer treatment. There are limited studies evaluating the symptoms of the oldest old cancer patient population.

Objectives

Our study aimed to evaluate the symptom severity among the general adult (GA), older adult (OA) and oldest old (OO) outpatient cancer patients on initial consult and followup.

Methods

Retrospective review of 441 patients composed of 200 patients in the 18-64 age group (GA), 200 in the 65-84 age group (OA) and 41 in those over 85 years old (OO). Demographic and clinical characteristics were collected and the differences between the groups at initial consult and first follow-up using Chi-Squared, Kruskal-Wallis and Wilcoxon signed-rank tests.

\section{Results}

Dyspnea $(\mathrm{p}=0.0225)$, financial distress $(\mathrm{p}=<0.0001)$, and spiritual pain $(p=0.0026)$ were significantly associated with age on initial consult. The OO reported more dyspnea than GA but less than OA, less financial distress than GA but more than OA. The OO reported lowest spiritual pain amongst all three groups. There was a trend among $\mathrm{OO}$ of less pain $(\mathrm{p}=0.0930)$ and nausea (0.0828). Among the OO, anxiety $(\mathrm{p}=0.0284)$ and sleep $(\mathrm{p}=0.0486)$ significantly improved on follow-up visit.

\section{Conclusions}

Our study shows that the oldest old cancer patients have different symptom severities compared to the general adult and older adult population. More research is needed to address the needs of this growing cancer population and focus on symptoms that can improve with palliative care intervention.

\section{eP165}

OLDER CANCER PATIENTS' INTERACTION WITH THE COMPUTER TO COMPLETE ELECTRONIC PATIENTREPORTED OUTCOME (EPRO) IS ASSOCIATED WITH FRAILTY AND OVERALL SURVIVAL.

\author{
A. Shahrokni ${ }^{I}$, S. Sarraf ${ }^{\text {, B. Borc-Grodzicki }}{ }^{1}$ \\ ${ }^{T}$ Memorial Sloan Kettering Cancer Center, Medicine, New York, USA
}

\section{Introduction}

The use of ePROs to further assess patients' symptoms has increased over time. Objectives

We aimed at assessing the relationship between older cancer patients' interction with a computer to complete $\mathrm{PRO}$ with frailty and overall survival. Methods

Since 2015, all older cancer patients who presented for preoperative evaluation completed an ePRO called electronic Rapid Fitness Assessment (eRFA) by using computers. The total eRFA score ranges from 0 to 13 with one point for each of these impairments; multiple comorbid conditions, poor Karnofsky Performance Scale (KPS), dependent for basic and instrumental activities of daily living (bADL iADL), fall in the past year, slow Timed Up and Go test, Limited Social Activity, depression, high distress, cognitive impairment, polypharmacy, weight loss \& poor social support. We collected data on whether patients were "independent" or "dependent" for using computers to complete eRFA.

\section{Results}

In total, 2463 older cancer patients completed eRFA. Those independent were younger ( 77.3 vs. 80.6 ), more likely to be male ( $56 \%$ vs. $46 \%$ ), married ( $62 \%$ vs. $48 \%$ ), be college graduate or have advanced degree $(63 \%$ vs. $35 \%)$ than dependents.Figure 1 shows the prevalence of the eRFA impairments among independents vs. dependents. Dependent patients were at higher risk for overall mortality after adjusting for age, gender, operation time and American Society of AnesthesiologistsPerformance Status classification ( $\mathrm{HR}=1.57,95 \%$ CI (1.22-2.0) (figure2).

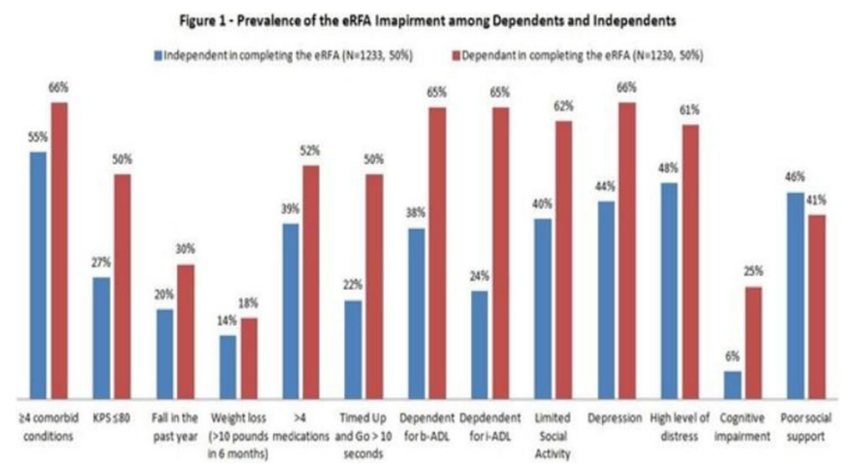


Figure 2- Overall Survival of Independent Patients vs. Dependents.

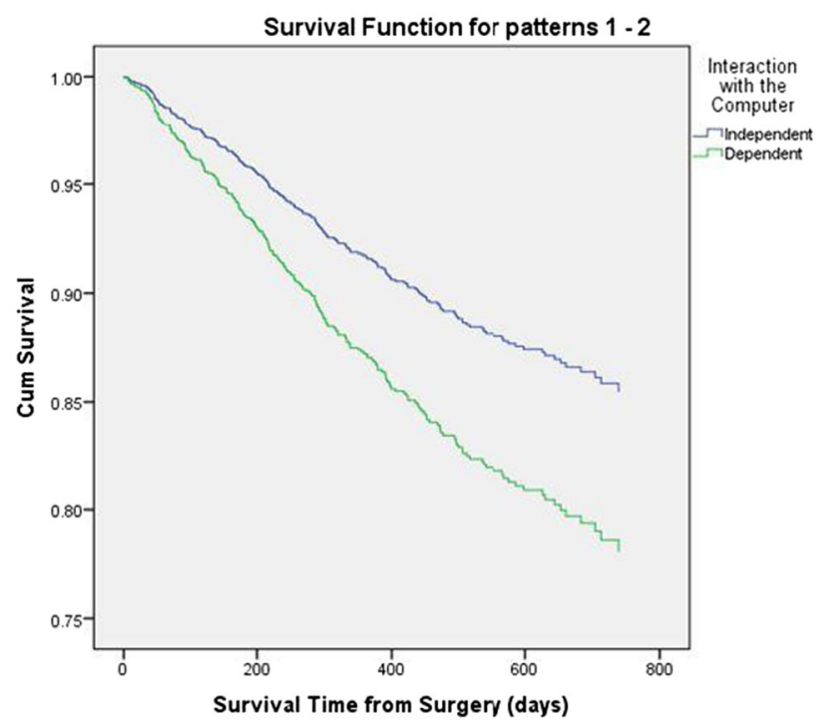

Conclusions

Older cancer patients' interaction with the computers to complete ePRO has a correlation with the frailty status. Future studies should assess whether the characteristics of this interaction can serve as a frailty screening method.

\section{eP166 \\ FROM HEALTHY TO VULNERABLE: OLDER MEN'S EXPERIENCES WITH PROSTATE CANCER AND CANCER CARE}

$\frac{B \text {. van } E e^{I}}{\text { Smits }}$, M. Hagedoorn ${ }^{2}$, A. Honkoop ${ }^{3}$, A. Kamper ${ }^{4}$, J. Slaets ${ }^{5}$, C. Smits

${ }^{1}$ Windesheim University of Applied Sciences, Research Group Innovating with Older Adults, Zwolle, The Netherlands

${ }^{2}$ University Medical Center Groningen, Health Sciences/Health Psychology, Groningen, The Netherlands

${ }^{3}$ Isala Hospital, Oncology Center, Zwolle, The Netherlands

${ }^{4}$ Isala Hospital, Geriatrics, Zwolle, The Netherlands

${ }^{5}$ Leyden Academy on Vitality and Ageing, Geriatrics, Leiden, The Netherlands

\section{Introduction}

Increasing numbers of older men have prostate cancer. However, the medical and psychosocial care takes younger patients as a starting point, and therefore poorly matches the needs and wishes of older patients with cancer. Care improvement is hindered by a lack of knowledge about older patients' experiences.

\section{Objectives}

This qualitative study aims to gain insight into the experiences of older men with prostate cancer.

Methods

Semi-structured interviews were conducted with 22 older (70+) men with prostate cancer. The interview transcripts were coded, and analyzed with Affinity Diagramming.

\section{Results}

Four themes emerged from the data: "Impact of prostate cancer", "dealing with prostate cancer and treatment", "involvement of and with others", and "experiences with professional care and the care trajectory". The experiences of these men with having prostate cancer and the care received varied widely. Several respondents coped reasonably well, were active and vital, and even offered support to others. However, prostate cancer and its treatment caused disturbing side-effects. For some men it was unclear if problems like incontinence, sexual dysfunction and fatigue were related to prostate cancer, comorbid diseases or old age. The information provided by professionals was not always sufficient to diminish their insecurity. Several men were reluctant to talk about their problems with other people, apart from their spouse.

\section{Conclusions}

Prostate cancer is a potentially limiting factor in older patients' lives. Some patients with complicated medical states and a lack of social support needed additional care, while others were less affected. More personalized information exchange is necessary.

eP167

SCREENING AND RISK FACTORS FOR MALNUTRITION IN OLDER PATIENTS WITH CANCER

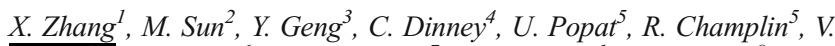

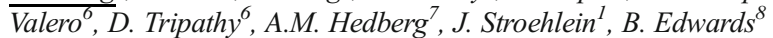

${ }^{1}$ University of Texas- MD Anderson Cancer Center, GastroenterologyHepatology \& Nutrition, Houston, USA

${ }^{2}$ University of Texas-MD Anderson Cancer Center, Cancer Therapeutics, Houston, USA

${ }^{3}$ University of Texas- MD Anderson Cancer Center, General Internal Medicine, Houston, USA

${ }^{4}$ University of Texas- MD Anderson Cancer Center, Urology, Houston, USA

${ }^{5}$ University of Texas- MD Anderson Cancer Center, Stem Cell Transplantation, Houston, USA

${ }^{6}$ University of Texas- MD Anderson Cancer Center, Breast Medical Oncology, Houston, USA

${ }^{7}$ University of Texas- MD Anderson Cancer Center, Clinical Nutrition, Houston, USA

${ }^{8}$ Central Texas Veterans Administration, Geriatric Medicine, Temple, USA

\section{Introduction}

Malnutrition is a common condition in older adults with cancer.

\section{Objectives}

To assess malnutrition in older cancer patients using three tools: the Mini Nutritional Assessment (MNA), weight loss, and BMI. To assess for risk factors for malnutrition

\section{Methods}

Cross sectional study. Patients 65 years of age and older in cancer care underwent a comprehensive geriatric assessment via referrals. Malnutrition was derived from the comprehensive screening tools and clinical interview. Analysis: Descriptive statistics, Sensitivity, Specificity, and Cohen's kappa statistic ( $\mathrm{K}$ ) for agreement, Cox proportional hazards.

\section{Results}

In total, 456 patients were included for final analysis. The mean age was 77.7 years. Males and females were equally represented. In 456 patients, $42 \%$ were clinically diagnosed as malnutrition. The MNA was performed in 354 patients, with $228(65 \%)$ categorized as malnourished or at risk of malnutrition. Weight loss $>3 \mathrm{~kg}$ was seen in $184(51 \%)$ of 359 patients and $\mathrm{BMI}<20 \mathrm{~kg} / \mathrm{m}^{2}$ was found in $30(7 \%)$ of 456 patients. The kappa statistic $(\mathrm{K})$ for MNA, weight loss and BMI index were 0.67, 0.45 and 0.08 respectively. The sensitivity for MNA, weight loss and BMI compared with the clinical diagnosis were $0.77,0.68$, and 0.80 , while the specificity were $0.96,0.78$, and 0.61 respectively. Risk Factors for malnutrition (univariate analysis): frailty, weight loss, slow gait speed, cognitive impairment, depression, ECOG, and KPS.

\section{Conclusions}

The MNA had the best validity in prediction of malnutrition. Cognitive impairment, major depression, frailty, weight loss, slow gait speed and performance measures were associated with malnutrition. 


\section{eP168}

EVALUATION OF CHEMOTHERAPY-INDUCED ORAL MUCOSITIS IN CHILDREN'S CANCER HOSPITAL

S. ABDELSAMIE ${ }^{1}$, D. Abdelmegeed ${ }^{2}$

${ }^{T}$ Children's cancer Hospital-Egypt-57357, Pharmaceutical services, Cairo, Egypt

${ }^{2}$ Children's Cancer Hospital-Egypt-57357, Pharmaceutical Services \& Sciences, Cairo, Egypt

\section{Introduction}

Oral mucositis $(\mathrm{OM})$ is common side effect experienced during chemotherapy course, impacting patient quality of life.

Objectives

To identify incidence of chemotherapy-induced oral mucositis (OM) in pediatric oncology hospital, patient populations at highest risk, and evaluate patient education concerning OM and adherence to mouth care.

\section{Methods}

105 patients/patient caregivers were interviewed to report any experience on OM since chemotherapy started, and to mention mouth care instructions they know. Patients' medical records (MR) were reviewed for related documentation.

\section{Results}

$83(79 \%)$ patients developed mucositis, with total 163 events $(\approx 2$ events per patient). Incidence was (85\%) and (79\%) within females and males, respectively; $92 \%, 81 \%, 72 \%$ within age ranges $(0.2-6),(7-12),(13-19)$ years; and 100\%, 87\%, 78\%, 75\%, 72\% in Acute Myeloid Leukemia, Non Hodgkin Lymphoma, Osteosarcoma, Rhabdomyosarcoma, Acute Lymphoid Leukemia, respectively; with $100 \%$ OM within relapsed patients, $77 \%$ during first line therapy. Incidence per patient was 3.67,1.8, $1.53,1.41,1.25,1.17,1,1$ for pre/post-operative chemotherapy, re-induction, first line induction, consolidation, second line therapy, continuation, intensification and re-intensification, respectively. OM severity was described in $70 \%$ of events, with grades $1,2,3,4$ contributed to $21 \%, 32 \%$, $12 \%, 5 \%$, respectively. $89 \%$ of patients used mouthcare agents; nonadherence was due to unacceptable product taste or unawareness. $36 \%$ discrepancy between patient answers and documentation was due to either inappropriate documentation (35\%) or unawareness (9\%). Event grades documented was $44 \%$ of 163 events.

\section{Conclusions}

$79 \%$ susceptibility to chemotherapy-induced OM was observed. Patient gender, age, diagnosis, protocol stage and disease stage were contributing factors to OM susceptibility. Event documentation and patient education were challenges.

\section{eP169}

EFFECTIVENESS OF LLLT IN THE PREVENTION AND TREATMENT OF CHEMOTHERAPY-INDUCED ORAL MUCOSITIS IN CHILDREN

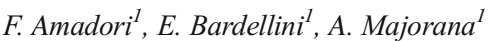 \\ ${ }^{T}$ University of Brescia, Dental Clinic, Brescia, Italy
}

\section{Introduction}

Chemotherapy still remains the mainstay of treatment for children with haematological malignancies. Oral mucositis $(\mathrm{OM})$ is a very painful common side effect of chemotherapy and affects from $40 \%$ to $80 \%$ of patients undergoing chemotherapy, affecting quality of life.

Objectives

The aim of this study is to verify the efficacy of LLLT both in preventing and in reducing the severity and pain of chemotherapy-induced OM in childhood.

Methods

Children between 3 and 18 years of age receiving chemotherapy for the first time for haematological malignancies or HSCT were enrolled. The study was organized into the prophylactic phase and the therapeutic phase. Laser or sham therapy started on the day before the beginning of chemotherapy and continued daily for 5 consecutive days; if the clinicians detected the onset of OM, laser or sham was performed daily for 5 consecutive days. Laser device features were $830 \mathrm{~nm}$ wavelength, power 150 $\mathrm{mW}$, energy density $4.5 \mathrm{~J} / \mathrm{cm}$.

Results

72 patients were enrolled. In 12 days, 24 patients developed OM. In the prophylactic phase, the difference in the presence of OM between the two groups resulted statistically significative just at $\mathrm{T} 1$ (day 4) $\left(\mathrm{c}^{2}=5.36\right.$, $\mathrm{p}=0.02$ ). In the therapeutic phase, the difference of OM grade and of VAS resulted statistically significative at the end of laser/sham OM treatment.

\section{Conclusions}

The main results of this research are, for the prophylactic phase, the delay in the time of onset of OM and, for the therapeutic aspect, the reduction in OM grade and pain, both in the groups treated by laser.

\section{eP170 \\ IMPACT OF LOW-LEVEL LASER THERAPY ON QUALITY OF LIFE IN HEAD AND NECK CANCER PATIENTS SUBMITTED TO CONCURRENT CHEMORADIATION}

H.S. Antunes ${ }^{1}$ D. Herchenhorn ${ }^{2}$ I.A. Small ${ }^{3}$ C.M.M Araújo ${ }^{4}$ C.M.P

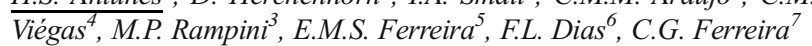

${ }^{1}$ Instituto Nacional de Câncer-INCA, Clinical Research Division, Rio de Janeiro, Brazil

${ }^{2}$ Grupo de Oncologia D'Or, Head and Neck Oncology Group, Rio de Janeiro, Brazil

${ }^{3}$ INCA, Clinical Research Division, Rio de Janeiro, Brazil

${ }^{4}$ INCA, Radiation Oncology Division, Rio de Janeiro, Brazil

${ }^{5}$ Private Practice, Dentistry, Rio de Janeiro, Brazil

${ }^{6}$ INCA, Head and Neck Surgery Division, Rio de Janeiro, Brazil

${ }^{7} D^{\prime} \mathrm{Or}$ Institute for Research and Education, Research and Education, Rio de Janeiro, Brazil

\section{Introduction:}

Oralmucositis $(\mathrm{OM})$ is a severe toxicity in head and neck squamous cell carcinomas (HNSCC) patients (pts) treated with chemoradiation (CRT) leading impact on quality of life.

\section{Objectives}

This study was designed to assess the efficacy of LLLT in reducing the incidence and/or severity of $\mathrm{OM}$ and evaluate the quality of life.

\section{Methods}

From June 2007 to December 2010, 94 HNSCC patients entered a prospective, randomized, double-blind, placebo-controlled phase III trial. Chemoradiotherapy consisted of conventional radiotherapy plus concurrent cisplatin every 3 weeks. A diode InGaAlP $(660 \mathrm{~nm}-100 \mathrm{~mW}-1 \mathrm{~J}-4 \mathrm{~J} /$ $\mathrm{cm}^{2}$ ) was used. OM evaluation was performed by WHO and OMAS scales every day from monday to friday and quality of life $(\mathrm{QoL})$ was assessed on the 1st, 20th and 39th RT fraction by EORTC questionnaires QLQ-C30 and QLQ-H \& N35.

\section{Results}

In the LLLT arm the incidence of OM Grade $3 / 4$ was only $6.4 \%$ versus $48 \%$ in the placebo arm; HR of 0.13 (IC 95\% $0.04-0.41$, p $<0.001$ ). On 39th RT fraction the results were significantly differents in QLQ-C30 in Physical functioning $(\mathrm{p}=0.011)$, Emotional functioning $(\mathrm{p}=0.038)$, Fatigue $(\mathrm{p}=0.009)$, Pain $(\mathrm{p}=0.027)$, Dyspnoea $(\mathrm{p}=0.027)$, and in QLQ-H\&N35, Pain $(p=0.004)$, Swallowing $(p=0.000)$, Trouble with social eating $(p=0.048)$, Opening mouth $(p=0.001)$, Dry mouth $(p=0.008)$, Sticky saliva $(\mathrm{p}=0.005)$. All in all the laser group had a better results than pacebo group.

\section{Conclusions}

Our results indicated that the laser group presented a better response to quality of life questionnaires than the placebo group due to the preventive LLLT to be an effective tool in reducing the incidence of grade 3/4 OM. 
eP171

CONSISTENCY AND MECHANISTIC IMPLICATIONS OF GENOMIC RISK PREDICTION STUDIES FOR CANCER REGIMEN-RELATED MUCOSAL INJURY: A SYSTEMATIC REVIEW

P. Bachour $^{1,2}$, S. Sonis ${ }^{1,2,3}$

${ }^{T}$ Harvard School of Dental Medicine, Oral Medicine- Infection- and Immunity, Boston, USA

${ }^{2}$ The Dana-Farber Cancer Institute and Brigham and Women's Hospital,

Oral Medicine, Boston, USA

${ }^{3}$ Primary Endpoint Solutions, n/a, Watertown, USA

\section{Introduction:}

While genomics hold promise in risk identification for regimen-based toxicities, inconsistencies in endpoints, methods, and design undermines their utility and meaningfulness.

Objectives

To assess concordance of genes or gene clusters in predicting oral or GI mucositis risk between candidate gene (CGS) and GWAS studies and to validate their functional relevance.

\section{Methods}

A PubMed literature search identified clinical studies published before August 2017 investigating associations of genetic variants in patients developing mucositis/enteritis. Search terms included select combinations of: mucositis, enteritis, toxicity, radiation, chemotherapy, gene, genetics, genomics, SNPs, GWAS, head and neck cancer, cancer.

Results

After filtering 1,383 papers using established exclusion criteria, 57 studies were identified. Three additional papers noted by reference review were added. Sample size, treatment, phenotype, toxicity scale and criteria, cancer, sample origin, studied and associated/significant genetic variants, and methods were recorded. 56 candidate studies (CGS) and 4 GWAS/Bayesian Network (BN) studies were identified. GeneAnalytics was used to characterize genes identified in GWAS/ BN and CGS studies to identify pathways, biological processes, and molecular functions. Exact concordance between CGS and GWAS/BN was demonstrated for ERK and PTK6 signaling and TGF- $\beta$ pathway. Overlap was observed for TGF- $\beta$ signaling, Wnt signaling, inflammatory response pathway, response to organic substance, and ATPase binding/activity. GWAS/BN studies independently identified an additional 14 pathways, 19 biological processes, and 19 ontological functions. Four studies included independent validation cohorts. Conclusions

CGS and GWAS have contributed to the identification of genomic mucositis risk prediction. Limited concordance between studies suggests that machine-learning algorithms, especially when study size populations are limited, may be desirable.

\section{eP172}

GUT-BRAIN AXIS DYSREGULATION IN CHEMOTHERAPY: COMPARING GUT TOXICITY, MICROBIAL SHIFTS AND NEUROIMMUNE IMPLICATIONS IN RATS WITH CHEMOTHERAPY-INDUCED INTESTINAL MUCOSITIS AND NSAID-INDUCED ENTEROPATHY.

\footnotetext{
J. Bajic ${ }^{l}$, C. Selway ${ }^{2}$, L. Bobrovskaya ${ }^{3}$, K. Lymn $^{4}$, G. Howarth $^{1,5,6}$, M. Hutchinson ${ }^{1,7}$

${ }^{1}$ The University of Adelaide, Physiology Department, Adelaide, Australia

${ }^{2}$ The University of Adelaide, Genetics and Evolution, Adelaide, Australia

${ }^{3}$ The University of South Australia, School of Pharmacy and Medical Sciences, Adelaide, Australia

${ }^{4}$ The University of Adelaide, School of Animal and Veterinary Sciences, Roseworthy, Australia

${ }^{5}$ The University of Adelaide, School of Animal and Veterinary Sciences, Adelaide, Australia
}

${ }^{6}$ Women's and Children's Hospital, Gastroenterology Department, North Adelaide, Australia

${ }^{7}$ The University of Adelaide, Australian Research Council Centre of Excellence for Nanoscale Biophotonics, Adelaide, Australia

\section{Introduction:}

The gut and the brain are primary targets for chemotherapy-induced damage resulting in gut toxicity and cognitive impairment.

\section{Objectives}

We compared aspects of gut-brain axis dysregulation in rats exposed to a high and low inflammatory response in the small intestine, utilising 5-Fluorouracil (5-FU; high dose) and Indomethacin (INDO; low dose), assessing gut toxicity, microbiota shifts and regional neuroimmune changes.

\section{Methods}

Female DA rats ( $\mathrm{n}=8$ /group) were randomly assigned to vehicle, 5 -FU $(150 \mathrm{mg} / \mathrm{kg})$ or INDO $(25 \mathrm{mg} / \mathrm{kg})$ with single IP injection and culled 72 hours later. Brain and spinal cord regions (X6) were isolated for western blot analysis using the glial markers GFAP (astrocytes) and IBA1 (microglia). Cecal microbial changes were detected by DNA extraction and 16S amplification sequencing. Myeloperoxidase (MPO) assay assessed acute intestinal inflammation.

\section{Results}

Bodyweight reduced $(\mathrm{p}=0.0001)$ and jejunal and ileal MPO expression significantly increased $(\mathrm{p}=0.0006$ and $\mathrm{p}=0.0014$, respectively) in rats treated with 5-FU compared to controls. Thoracic GFAP expression significantly decreased in 5 -FU treated rats $(\mathrm{p}=0.03)$ compared to controls. The cecal contents of 5-FU rats displayed less phylogenetic diversity than VEH and INDO groups and an increase in Bacteriodes with the addition of the bateria Phascolartobacterium compared to VEH and INDO.

\section{Conclusions}

Gut-brain axis dysregulation occurs following rats exposed to 5-FU. A single high dose of 5-FU induces changes from the gut to the brain, involving the microbiota and neuroimmune system. These central and peripheral changes highlight the need to focus future studies on the gut-brain axis side-effects occurring simultaneously in the chemotherapy setting.

\section{eP173}

ORAL MUCOSITIS IN STEM CELL TRANSPLANT PATIENTS: EPIDEMIOLOGY AND ECONOMICS

\author{
K. Berger ${ }^{I}$, T. Staudenmaier ${ }^{I}$, H. Ostermann ${ }^{I}$ \\ ${ }^{T}$ University Hospital of Munich, Oncology / Haematology Med III, \\ München, Germany
}

\section{Introduction:}

Oral mucositis $(\mathrm{OM})$ is a severe side effect of conditioning regimens in stem cell transplantation (HSCT). Clinical and economic data on OM in HSCT are limited.

\section{Objectives}

To determine incidence, risk factors and costs of OM and compliance regarding mouth washes.

\section{Methods}

Prospective, non-interventional single-center observational study. Inpatient allogenic and autologous HSCT patients $\geq 18$ years. OM assessment: WHO Oral Toxicity Scale. Compliance regarding mouth rinses was captured with a pre-tested patient questionnaire.

\section{Results}

45 HSCT patients (25 allogenic, 20 autologous) were consecutively enrolled. Twenty-six (58\%) patients developed OM (10 Grade I, 4 Grade II, 8 Grade III, 4 Grade IV). A history of oral lesions ( $48 \%$ vs $70 \%, \mathrm{p}=0.142)$ and age $\geq$ $65(31 \%$ vs $69 \%, \mathrm{p}=0.021)$ were associated with a lower OM incidence, history of smoking ( 1.77 vs $2.69, \mathrm{p}=0.036$ ), age $\geq 65$ ( 1.50 vs 2.36 ; $\mathrm{p}=0.179)$ and regular alcohol consumption ( 1.67 vs $2.40, \mathrm{p}=0.180)$ with a lower OM grade. Patients with unrelated donors ( 2.63 vs $1.29, \mathrm{p}=0.014)$ were associated with higher grades, females ( $80 \%$ vs $47 \%, R R=1.71, p=0.035)$ or 
previous $\mathrm{OM}(77 \%$ vs $50 \%, \mathrm{RR}=2.46, \mathrm{p}=0.101)$ with a higher incidence. OM-patients were less compliant with mouth rinses ( $35 \%$ vs $68 \%$, $\mathrm{p}=0.027)$. Costs of anti-infective and analgesic drugs in autologous HSCT OM patients vs non OM patients were Euro 802 ( $\mathrm{p}=0.044$ ) higher.

Conclusions

This study in a tertiary German teaching hospital shows a high burden of $\mathrm{OM}$ in HSCT. Awareness on OM and compliance regarding mouth washes is poor. The identification of risk factors offers the opportunity to identify patients at risk

\section{eP174}

VAGINAL MUCOSITIS IN PATIENTS RECEIVING CHEMORADIATION FOR GYNAECOLOGICAL CANCER: A FREQUENT BUT NEGLECTED SIDE EFFECT OF CANCER AND CANCER THERAPIES

S. Censabella ${ }^{I}$, S. Claes ${ }^{1}$, L. Van Bever ${ }^{I}$, S. Thielens ${ }^{2}$, P. Pannekoeke ${ }^{I}$, E. $\overline{\text { Van De Werf }}^{2}$, P. Bulens ${ }^{1}$

${ }^{1}$ Jessa Hospital, Medical Oncology, Hasselt, Belgium

${ }^{2}$ Limburg Oncology Centre, Radiotherapy, Hasselt, Belgium

\section{Introduction:}

Mucositis, the inflammation of mucous membranes, is a frequent and serious complication of cancer therapy. While the oropharyngeal cavity (oral mucositis) is the most frequent and well-documented mucositis site, mucositis affecting the vaginal canal has largely been neglected in oncology. Objectives

To document the incidence and extent of vaginal mucositis (VM) in women with gynaecological cancer.

Methods

Women scheduled for pelvic radiotherapy for gynaecological cancer were to complete numerical rating scales $(0-10)$ to assess the frequency and intensity of the main VM-symptoms before and at the end of external radiotherapy (i.e., after $25 * 1.8$ Gray). Patients also received concurrent chemotherapy.

Results

Of the 19 patients recruited so far (see Table 1 for patients' characteristics), $74 \%$ reported experiencing any degree of at least one VM-symptom. As can be seen on Figure 1, a non-negligible proportion of patients already experienced VM-symptoms before the start of radiotherapy. As might be expected, the frequency and severity of VM-symptoms increased as chemoradiation progressed.

Yet an examination of the distribution of scores for each VM-symptom revealed that the severity of VM might be higher than suggested by the mean scores (Figure 2).

Table 1. Patients characteristics

\begin{tabular}{|c|c|c|c|}
\hline \multirow[t]{2}{*}{ Mean Age (years, SD) } & $59(14)$ & Menopausalstatus & $N(\%)$ \\
\hline & & Pre-menopausal & $8(42.1)$ \\
\hline \multirow[t]{2}{*}{ Body Mass Index (M-SD) } & $29.5(8.1)$ & Post-menopausal & $6(31.6)$ \\
\hline & & Unknown & $5(26.3)$ \\
\hline TumourLocation & $N(\%)$ & & \\
\hline Cervix & $10(52.6)$ & Comorbidity & $N(\%)$ \\
\hline \multirow[t]{2}{*}{ Endometrium } & $9(47.4)$ & None & $9(47.4)$ \\
\hline & & Diabetes Mellitus & $2(10.5)$ \\
\hline Smoking Status & $N(\%)$ & Fibromyalgia & $1(5.3)$ \\
\hline Never smoked & $11(57.9)$ & $\begin{array}{l}\text { Rheumatic } \\
\text { disease }\end{array}$ & $3(15.8)$ \\
\hline Former smoker & $3(15.8)$ & Tuberculosis & $1(5.3)$ \\
\hline Current Smoker & $3(15.8)$ & Heart disease & $1(5.3)$ \\
\hline \multirow[t]{2}{*}{ Unknown } & $2(10.5)$ & Skin disease & $3(15.8)$ \\
\hline & & Unknown & $2(10.5)$ \\
\hline
\end{tabular}

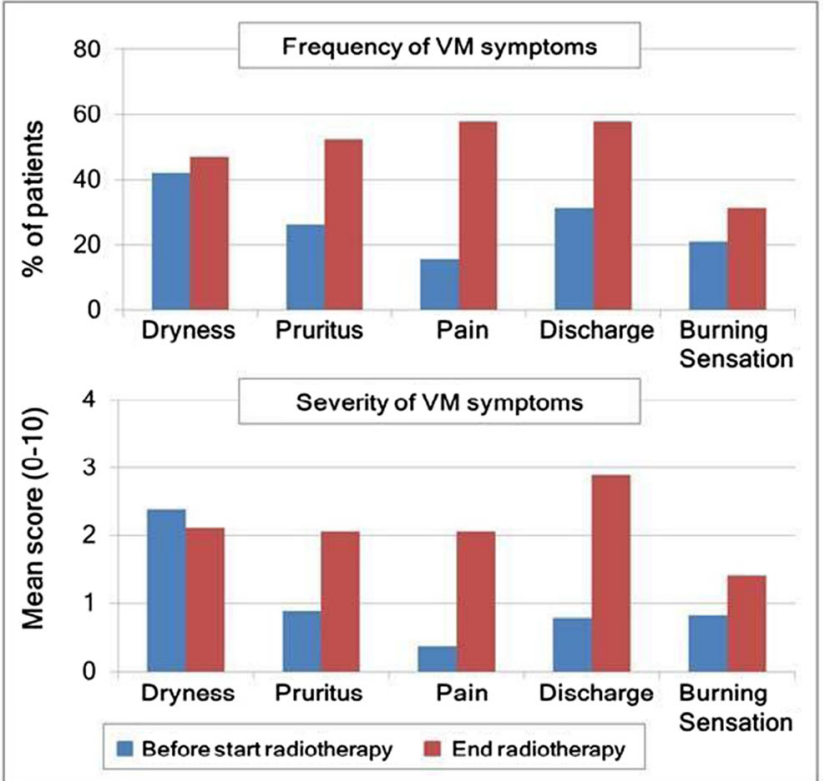

Figure 1. Frequency and severity of vaginal mucositis (VM): Percentage of patients reporting any degree of vaginal mucositis (numerical rating scale $>0$ ) and mean ratings per symptom before and after external radiotherapy (i.e., after $25^{\star} 1.8$ Gray)

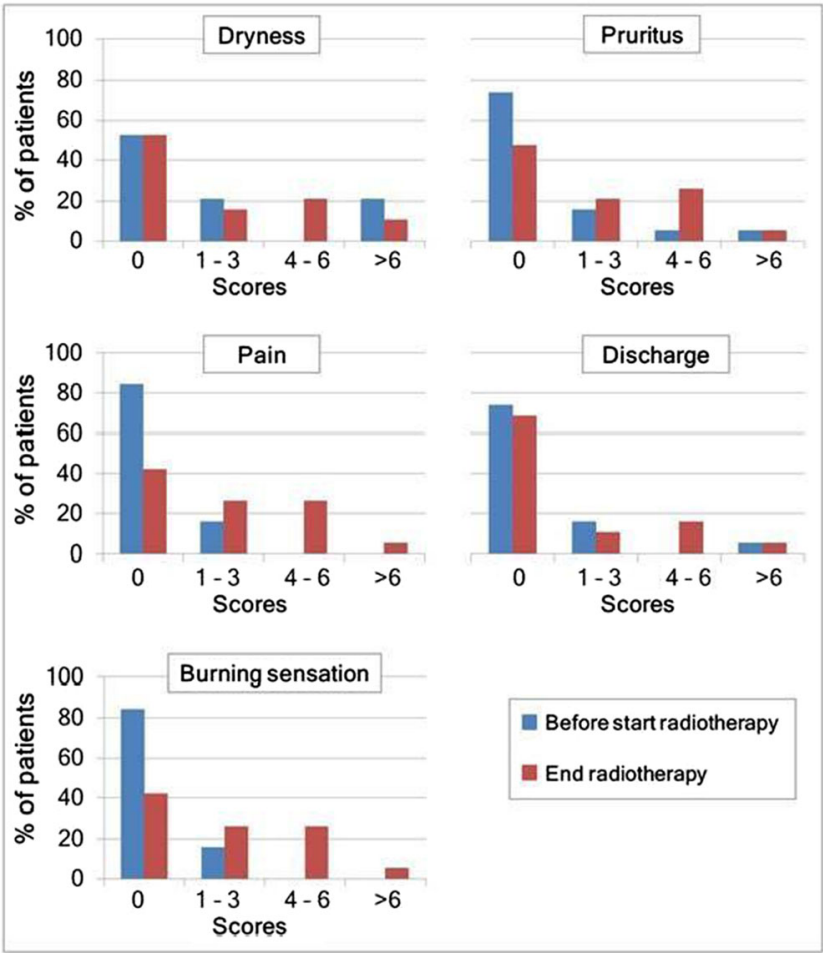

Figure 2. Distribution of the scores of the numerical rating scales for each VM symptom before and after external radiotherapy (25*1.8 Gray)

Conclusions

Our preliminary descriptive data indicate that VM is a rather frequent side effect in gynaecological cancer patients that aggravates during pelvic chemoradiation up to a moderate severity level and might therefore affect patients' quality of life. 
Although from a small sample, these prospectively collected data highlight the need for attention to vaginal mucositis in research but also in clinical practice.

\section{eP175}

INCIDENCE OF OTHER TOXICITIES IN PATIENTS WITH SEVERE GASTROINTESTINAL TOXICITY AND RELATIONSHIP TO COMORBIDITIES FOLLOWING 5FLUOROURACIL (5-FU) TREATMENT

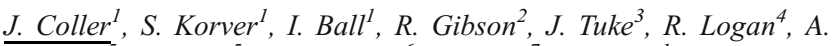

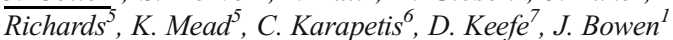

${ }^{1}$ The University of Adelaide, Adelaide Medical School, Adelaide, Australia

${ }^{2}$ The University of South Australia, Division of Health Sciences, Adeladie, Australia

${ }^{3}$ The University of Adelaide, School of Mathematical Sciences, Adelaide, Australia

${ }^{4}$ The University of Adelaide, Adelaide Dental School, Adelaide, Australia

${ }^{5}$ Flinders Medical Centre, Oncology, Bedford Park, Australia

${ }^{6}$ Flinders University, School of Medicine, Bedford Park, Australia

${ }^{7}$ Royal Adelaide Hospital, Medical Oncology, Adelaide, Australia

\section{Introduction:}

Severe gastrointestinal (GI) toxicity following 5-FU-based treatment is highly prevalent and negatively affects therapy, whilst other toxicities frequently reported can also negatively impact on quality of life. We have previously identified genetic markers in TLR2 and TNF that predict risk of severe GI toxicity. However, we have not yet examined links in the incidence of other toxicities or comorbidities.

Objectives

To investigate relationship between the incidence of other toxicities and comorbidities with severe GI toxicity.

\section{Methods}

One hundred and six participants who previously received 5-FU-based treatment for mixed cancers were recruited. GI toxicity, neuropathy, pain, skin toxicity and cardiotoxicity data (toxicity: symptoms Grade $\geq 3$ NCI's CTCAE v4, or requiring treatment cessation or reduction), demographics, comorbidities (BSA, smoking status, alcohol use, type 2 diabetes, cardiovascular disease, arthritis, asthma, GORD, thyroid activity) and treatment parameters were mined from clinical records. Chi-squared tests and logistic regression analyses determined any relationships.

\section{Results}

The incidences of neuropathy, pain, skin toxicity or cardiotoxicity were not different between patients who experienced severe versus not severe GI toxicity $(\mathrm{P}>0.05)$ : 34.7 versus $27.7 \%, 17.4$ versus $16.8 \%, 21.7$ versus $20.5 \%$, and 8.7 versus $16.8 \%$, respectively. In addition, there were no relationships between any comorbidities and the incidence of severe GI toxicity $(\mathrm{P}>0.05)$. Conclusions

Despite the potential for the occurrence of multiple toxicities in patients with severe GI toxicity and influence of comorbidities following 5-FU treatment, in our cohort we did not observe significant relationships between the occurrence of other toxicities or the comorbidities examined.

\section{eP176}

\section{EFFECT OF VITAMINS ON THE SEVERITY OF MUCOSITIS}

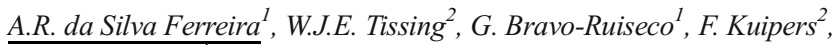
H.J.M. Harmsen ${ }^{I}$

${ }^{1}$ University Medical Center Groningen- University of Groningen, Department of Medical Microbiology, Groningen, The Netherlands

${ }^{2}$ University Medical Center Groningen- University of Groningen, Department of Pediatrics- Beatrix Children's Hospital, Groningen, The Netherlands

\section{Introduction:}

Chemotherapy-induced mucositis is a severe side-effect of anticancer treatment. This inflammatory disorder increases oxidative stress in the gut leading to alterations in the composition of the gut microbiota, including a decrease in anaerobic bacteria. Redox active compounds, such as vitamins, were shown to reduce inflammation in the gut and to enhance the growth of anaerobic bacteria.

\section{Objectives}

Determine the effect of vitamins in the development of mucositis and in the composition of the gut microbiota, using the methotrexate (MTX)induced mucositis rat model.

\section{Methods}

MTX-induced mucositis rats received daily oral gavage with vitamins, alone or in conjugation, and MTX $(45 \mathrm{mg} / \mathrm{Kg})$ or saline solution (control) was administrated via intravenous injection. The severity of mucositis was determined by measuring the food intake, body weight and plasma citrulline. The gut microbiota composition was characterized by $16 \mathrm{~S}$ rRNA sequencing. Bacterial strains isolated from MTX-induced mucositis rats were treated with different concentrations of vitamins to determinate their effect on bacterial growth.

\section{Results}

Vitamin supplementation in rats attenuated the severity of mucositis reflected by changes in body weight, food intake and plasma citrulline. The effect of vitamins on mucositis was dose dependent. The anaerobes Blautia coccoides and Roseburia intestinalis isolated from rats, significantly grew better under oxygen stress, when vitamins were supplemented.

\section{Conclusions}

Vitamins enhance the growth of anaerobic bacteria in the gut.This effect can be used to ameliorate mucositis in the MTX-induced mucositis rat model.

\section{eP177}

\section{CORRELATION OF SALIVARY AND PLASMA BUSULFAN CONCENTRATIONS: IMPLICATIONS FOR THERAPEUTIC MONITORING AND ORAL CRYOTHERAPY PROTOCOLS}

\author{
F.D.P. Eduardo ${ }^{1}$, L.M. Bezinelli, ${ }^{1}$, M.H. Ferreira ${ }^{2}$, D.L.C. Carvalho ${ }^{2}$, \\ F.C.P. Rosin ${ }^{I}$, C.E.D.S. Ferreira ${ }^{1}$, N. Hamerschlak ${ }^{1}$, L. Correa ${ }^{2}$ \\ ${ }^{1}$ Hospital Israelita Albert Einstein, Hematology, Sao Paulo, Brazil \\ ${ }^{2}$ School of Denstitry- University of São Paulo, General Pathology \\ Department, Sao Paulo, Brazil
}

\section{Introduction:}

Individual dose adjustment of intravenous busulfan during conditioning of hematopoietic-cell-transplantation(HCT) reduces its toxicity and improves transplantation outcomes. Therapeutic bulsulfan monitoring requires multiple blood samplings, which can cause patient discomfort. This agent is found in the saliva during the conditioning, but its salivary pharmacokinetics are unknown.

\section{Objectives}

The first aim of this study was to compare salivary and plasma busulfan concentrations during HCT-conditioning while analyzing the viability for future salivary busulfan therapeutic monitoring. The second aim was to propose an oral cryotherapy protocol based on the busulfan concentration curve in order to reduce busulfan's toxicity in the oral cavity.

\section{Methods}

We collected nine sets of blood and saliva samples from 20 patients undergoing allogeneic HCT. Salivary and plasma busulfan concentrations were measured using a previously standardized and validated ultra-high performance liquid chromatography method. 


\section{Results}

We found a significantly high correlation between plasma and salivary busulfan concentrations ( $r=0.92$, confidence-interval-0.89-0.93, $\mathrm{p}<0.001$ ). The drug peak in both fluids was $180 \mathrm{~min}$, which then decreased to half the peak concentration at $300 \mathrm{~min}$. Based on these results, oral mucositis occurrence can be derived from plasma and salivary busulfan concentrations. For an efficient oral cryotherapy protocol, the procedure should begin at least $30 \mathrm{~min}$ after the busulfan infusion and continue for at least $360 \mathrm{~min}$.

Conclusions

In conclusion, saliva can provide a good alternative matrix for busulfan therapeutic monitoring. The agreement between salivary and plasma concentration curves encourages adoption of oral cryotherapy for minimizing busulfan's toxic effects in the oral cavity.

We thank for Sao Paulo Research Foundation FAPESP (Process\#2016/ 03650-4), and AmigoH for financial support.

\section{eP178}

RISK FACTORS FOR DIGESTIVE TRACT MUCOSITIS IN MYELOABLATIVE AND NON-MYELOABLATIVE/REDUCEDINTENSITY CONDITIONINGS WITH BUSULFAN PRIOR TO HEMATOPOIETIC CELL TRANSPLANTATION

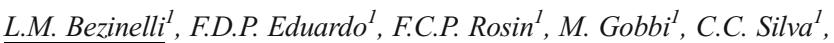
N. Hamerschlak ${ }^{l}$, L. Correa ${ }^{2}$

${ }^{1}$ Hospital Israelita Albert Einstein, Hematology, Sao Paulo, Brazil

${ }^{2}$ School of Denstitry- University of São Paulo, General Pathology Department, Sao Paulo, Brazil

\section{Introduction:}

Busulfan is an alkylating agent used as a major component of chemotherapy conditioning in hematopoietic cell transplantation (HCT). At myeloablative doses, busulfan is highly toxic, leading to risks of mortality due to conditioning. Non-myeloablative (NMA) or reduced-intensity conditioning (RIC) using busulfan have shown impaired toxicity. However, the effect of NMA/RIC in the digestive tract is controversial and poorly described.

Objectives

This study aimed to analyze whether myeloablative dose of busulfan is a risk factor for mucositis in the oral cavity $(\mathrm{OM})$, oropharynx/esophagus, and gastrointestinal tract.

\section{Methods}

We retrospectively retrieved clinical data of HCT patients $(n=100)$ who underwent MAC or NMA/RIC with busulfan. Frequency and time duration of mucositis in the oral cavity and oropharynx/esophagus, diarrhea, and prescription of total parenteral nutrition (TPN) and opioids were also collected.

\section{Results}

OM severity $(\mathrm{p}=0.009)$ and time duration of mucositis in oropharynx/esophagus $(\mathrm{p}=0.022)$ were frequently higher in MAC than NMA/RIC. A myeloablative dose of busulfan was a risk factor for $\mathrm{OM}$ grade $\geq 2(\mathrm{OR}=4.8, \mathrm{p}=0.002)$ and for mucositis in oropharynx/esophagus $\geq 5$ days $(\mathrm{OR}=2.64, \mathrm{p}=0.035)$. Overall survival (OS) in MAC was significantly higher than that in NMA/RIC $(p=0.017)$. No variables related to mucositis interfere significantly in OS.

Conclusions

In conclusion, myelossupression in busulfan-based regimens are predisposed to a high risk for severe $\mathrm{OM}$ and to prolonged mucositis in the oropharynx/esophagus. Oral care protocols must be emphasized in $\mathrm{HCT}$ patients undergoing MAC regimens with busulfan.

We thank for São Paulo Research Foundation - FAPESP (Process \# 2016/03650-4), and AmigoH for financial support.

eP179

THE PREVENTIVE ROLE OF INTRAVENOUS L-ALANYL LGLUTAMINE IN REDUCING INCIDENCE OF ORAL MUCOSITIS IN HEAD AND NECK CANCER PATIENTS RECEIVING RADIOTHERAPY WITH OR WITHOUT CHEMOTHERAPY.

A. Elfeky ${ }^{I}$, N.M. Sabry ${ }^{I}$, A.O. Barakat ${ }^{2}$

${ }^{T}$ Tanta University hospital, clinical oncology, Tanta, Egypt

${ }^{2}$ Head and Professor clinical oncology Department, Tanta University Hospitals

\section{Introduction}

Mucositis is a dose-limiting toxicity of cancer treatment with direct effects on survival

\section{Objectives}

This prospective phase 2 study to assess role of L-alanyl LGlutamine in reducing rate of oral mucositis for squamous head and neck cancer patients receiving radiotherapy with or without concurrent chemotherapy

\section{Methods}

From September 2014 to Septemper 2016, 100 H\&N cancer patients were treated at the Clinical Oncology Department, Tanta University Hospitals. Patients were randomized in 1:1 ratio into Group A treated by radiotherapy or concurrent chemoradiotherapy and Group B to receive same treatment plus intravenous Glutamine, that was infused daily at dose of $0.3-0.4 \mathrm{~g} / \mathrm{kg}$ diluted in NS and rate of $0.1 \mathrm{~g} / \mathrm{Kg} / \mathrm{hr}$. Radiotherapy dose was $65-$ 70 Gy using Linac 6MV photon beam. For concurrent chemotherapy, Cisplatin $(40 \mathrm{mg} / \mathrm{m} 2)$ was administered weekly

\section{Results}

Mucositis was assessed by WHO grading system. A significantly higher incidence of mucositis was reported in $45 \%$ of Group A patients compared with patients in group $\mathrm{B} 10 \% \mathrm{P}<0.001)$. Group B patients had significantly longer period free from mucositis compared to group A with median time (12 weeks vs 8 weeks) $\mathrm{P}<0.001$. A significant lower rate of radiotherapy interruption was reported in group B compared to group A (50\% vs $15 \%)$ $\mathrm{P}<0.001$. More Patients needed hospitalization in group A $(20 \%)$ vs $(5 \%)$ in group $\mathrm{B} P=0.059$. No adverse effects were observed related to glutamine

\section{Conclusions}

Intravenous L-alanyl L-Glutamine may be an effective measure to lower incidence or prevention of oral mucositis in head and neck cancer patients treated by radiotherapy or combined chemo-radiation

\section{eP180}

MATRIX METALLOPROTEINASE-1 (MMP-1) LEVELS AS A PREDICTIVE MARKER OF ORAL MUCOSITIS SEVERITY AMONG HEAD AND NECK CARCINOMA PATIENTS UNDERGOING RADIOTHERAPY

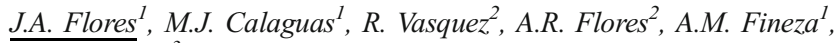
J.P. Dipasupil $l^{3}$ 
${ }^{1}$ Jose R. Reyes Memorial Medical Center, Radiotherapy, Manila, Philippines

${ }^{2}$ University of Santo Tomas, Medical Technology, Manila, Philippines

${ }^{3}$ Minute Healthcare, Lifestyle Medicine, Quezon City, Philippines

\section{Introduction}

Concurrent chemoradiotherapy is an established modality in the management of head and neck cancer. Although employed to improve quality of life, its association with several side effects remains a clinical threat. Matrix metalloproteinase-1 (MMP-1) degrades most extracellular matrix proteins including collagen. Found to increase in the occurrence of cancer, further rise ensues when exposed to radiotherapy and chemotherapy.

Objectives

To assess the adverse effects of radiotherapy in relation to the significant change in the level of MMP-1 on selected head and neck carcinoma patients.

\section{Methods}

This is an observational longitudinal approach on 20 head and neck cancer patients from Jose R. Reyes Memorial Medical Center. MMP-1 levels in the plasma samples of Day 0 and Day 10 was determined by ELISA method. Oral mucositis (OM), a RT-induced toxicity, was evaluated using the Radiation Therapy Oncology Group (RTOG) grading system. Correlation between MMP-1 level and complete blood count (CBC) of carcinoma patients was also analyzed.

Results

MMP-1 levels showed an increasing trend from Day 0 to Day 10 . However, p-value (0.148) presented a statistically insignificant correlation between MMP-1 level and radiotherapy. The RTOG measured $\mathrm{OM}$ of patients undergoing radiotherapy exhibited significant correlation with MMP-1 levels (p-value: 0.026). Parallel to $\mathrm{OM}$, certain hematological parameters of the $\mathrm{CBC}$ such as platelets and leukocytes also showed a significant relationship with levels of MMP-1 (p value of 0.0099 and 0.0397 , respectively).

Conclusions

The study results showed the potential role of MMP-1 as a predicitve marker to assess severity of oral mucositis among head and neck cancer patients undergoing radiotherapy.

\section{eP181}

MULTICENTER RANDOMIZED DOUBLE-BLIND CONTROLLED TRIAL TO EVALUATE THE EFFICACY OF LASER THERAPY FOR TREATMENT OF SEVERE ORAL MUCOSITIS INDUCED BY CHEMOTHERAPY IN CHILDREN. LAMPO RCT

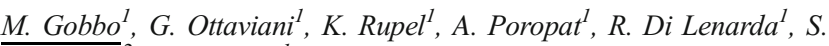 \\ $\overline{\text { Zacchigna }}^{2}$, M. Biasotto ${ }^{l}$ \\ ${ }^{1}$ University of Trieste, Dental Science - Oral Medicine and Pathology, \\ Trieste, Italy \\ ${ }^{2}$ International Centre for Genetic Engineering and Biotechnology, \\ Molecular Medicine Laboratory, Trieste, Italy
}

\section{Introduction}

Class IV diode laser photobiomodulation (PBM) has proven efficacy in the treatment of severe oral mucositis $(\mathrm{OM})$ in paediatric oncologic patients compared with placebo.

\section{Objectives}

Primary objective was reduction of OM grade (WHO scale) seven days after PBM. Secondary objectives were reduction of pain following a 0 -to-10 numeric pain rating (NRS) scale and need for analgesics.

\section{Methods}

A total of 101 eligible paediatrics suffering from a WHO grade $>2$ chemotherapy-induced OM were enrolled in 8 Italian hospitals. Patients were randomized into either PBM group or sham treatment group. PBM or sham treatment were performed for four consecutive days (days $+1-+4$ ). On days $+4,+7$ and $+11 \mathrm{OM}$ grade, pain and need for analgesics were evaluated by an operator blinded to treatment.

\begin{tabular}{|c|c|c|}
\hline & $\begin{array}{l}\text { Laser therapy } \\
\text { n-51 }\end{array}$ & $\begin{array}{l}\text { Sham therapy } \\
n=50\end{array}$ \\
\hline Age, median (QR) & $11.9(7.0-14.7)$ & TI.7(8.0-14.8) \\
\hline Male sex, number (\%) & $24(47.1 \%)$ & $30(60.0 \%)$ \\
\hline $\begin{array}{l}\text { Grade of } 0 \mathrm{M} \text {, number (\%) } \\
\text { grade } 3 \\
\text { grade } 4\end{array}$ & $\begin{array}{l}30(58.8 \%) \\
21(41.2 \%)\end{array}$ & $\begin{array}{l}27(54.0 \%) \\
23(46.0 \%)\end{array}$ \\
\hline $\begin{array}{l}\text { Type of oncologic disease, number (\%) } \\
\text { acute lymphatic leukemia (ALL) } \\
\text { transplantation } \\
\text { lymphoma } \\
\text { acute myeloid leukemia (AML) } \\
\text { other solid tumours }\end{array}$ & $\begin{array}{c}20(39.2 \%) \\
13(25.5 \%) \\
14(27.5 \%) \\
2(3.9 \%) \\
2(3.9 \%)\end{array}$ & $\begin{array}{l}21(42.0 \%) \\
13(26.0 \%) \\
9(18.0 \%) \\
2(4.0 \%) \\
5(10.0 \%)\end{array}$ \\
\hline Total body irradiation, number (\%) & $5(9.8 \%)$ & $8(16.0 \%)$ \\
\hline Neutrophil count, median (IOR) & $40.0(0.0-201.0)$ & $67.5(0.0-300.0)$ \\
\hline $\begin{array}{l}\text { Buccal swab, number (\%) } \\
\text { carried out } \\
\text { positive for fungi } \\
\text { positive for viruses }\end{array}$ & $\begin{array}{c}21(43.8 \%) \\
1 \\
2\end{array}$ & $\begin{array}{c}24(49.0 \%) \\
1 \\
1\end{array}$ \\
\hline $\begin{array}{l}\text { Presence of mouth or throst symptoms, } \\
\text { number }(\%)\end{array}$ & $51(100 \%)$ & $50(100 \%)$ \\
\hline $\begin{array}{l}\text { Relevance of mouth or throat symptoms, } \\
\text { number }(\%) \\
\text { none } \\
\text { moderate } \\
\text { severe } \\
\text { very severe }\end{array}$ & $\begin{array}{c}0 \\
5(9.8 \%) \\
20(39.2 \%) \\
26(51.0 \%) \\
\end{array}$ & $\begin{array}{c}0 \\
5(10.0 \%) \\
15(30.0 \%) \\
30(60.0 \%) \\
\end{array}$ \\
\hline Pain score, median (IQR) & $8.0(7.0-8.5)$ & $8.0(7.0-9.0)$ \\
\hline Presence of pain (pain score $>4$ ), aumber (\%) & $48(94.1 \%)$ & $45(90.0 \%)$ \\
\hline $\begin{array}{l}\text { Duration of pain in days before enrollment, } \\
\text { median (IQR) }\end{array}$ & $3.0(2.0-4.0)$ & $3.0(2.0-4.0)$ \\
\hline $\begin{array}{l}\text { Ongoing treatments } \\
\text { topical mucosal protectants } \\
\text { topical analgesics } \\
\text { systemic antimycotics } \\
\text { systemic antibiotics } \\
\text { systemic antivirals } \\
\text { oral analgesies } \\
\text { parenteral analgesics } \\
\text { parenteral nutrition }\end{array}$ & $\begin{array}{l}30(58.8 \%) \\
11(21.6 \%) \\
33(64.7 \%) \\
39(76.5 \%) \\
31(60.8 \%) \\
12(23.5 \%) \\
33(64.7 \%) \\
26(51.0 \%)\end{array}$ & $\begin{array}{c}34(68.0 \%) \\
9(18.0 \%) \\
38(76.0 \%) \\
37(74.0 \%) \\
26(52.0 \%) \\
11(22.0 \%) \\
38(76.0 \%) \\
28(56.0 \%)\end{array}$ \\
\hline
\end{tabular}

\section{Results}

51 patients were randomized into $\mathrm{PBM}$ group and 50 in sham group according to WHO grade. $93.7 \%$ of PBM patients and $72 \%$ of sham patients had a $\mathrm{WHO}<3$ on Day+7 $(\mathrm{p}=0.01)$.

Significant $(\mathrm{p}<0.006)$ decreasing of NRS was registered between day 0 (NRS: 8[7-9] in both groups) and day 7 (NRS: 1[0-3] in PBM group and $2.5[1-5]$ in sham group).

A reduced use of analgesics was reported in LT group (49\%) if compared to sham group (62\%) although not statistically significant. Neutrophil count was not significantly different between groups over time (NS). 
No significant adverse events attributable to treatment were recorded.

\begin{tabular}{|c|c|c|c|}
\hline \multicolumn{4}{|c|}{ Table 2. Study results } \\
\hline & $\begin{array}{l}\text { Laser therapy } \\
\left(\mathrm{n}=51^{*}\right)\end{array}$ & $\begin{array}{c}\text { Sham therapy } \\
(\mathrm{n}=50)\end{array}$ & $\mathbf{P}$ \\
\hline $\begin{array}{l}\text { OM grade at day }+7 \text {, number (\%) (main } \\
\text { study outcome) } \\
\text { - Grade } 4 \\
\text { - Grade } 3 \\
\text { - Grade }<3\end{array}$ & $\begin{array}{c}1(2.0 \%) \\
2(4.1 \%) \\
46(93.9 \%)\end{array}$ & $\begin{array}{c}8(16.0 \%) \\
6(12.0 \%) \\
36(72.0 \%)\end{array}$ & 0.01 \\
\hline $\begin{array}{l}\text { OM grade at day }+4, \text { number }(\%) \\
- \text { Grade } 4 \\
\text { - Grade } 3 \\
- \text { Grade }<3\end{array}$ & $\begin{array}{c}7(13.7 \%) \\
16(31.4 \%) \\
28(54.9 \%)\end{array}$ & $\begin{array}{l}12(24.0 \%) \\
19(38.0 \%) \\
19(38.0 \%)\end{array}$ & 0.19 \\
\hline $\begin{array}{l}\text { OM grade at day }+11, \text { number }(\%) \\
- \text { Grade } 4 \\
\text { - Grade } 3 \\
- \text { Grade }<3\end{array}$ & $\begin{array}{c}0(0.0 \%) \\
1(2.1 \%) \\
47(97.9 \%)\end{array}$ & $\begin{array}{c}5(10.0 \%) \\
5(10.0 \%) \\
40(80.0 \%)\end{array}$ & 0.02 \\
\hline $\begin{array}{l}\text { Self-reported pain score at day }+4 \text {, } \\
\text { median (IQR) }\end{array}$ & $4(2-6)$ & $5(3-7)$ & 0.07 \\
\hline $\begin{array}{l}\text { Self-reported pain seore at day +7, } \\
\text { median (IQR) }\end{array}$ & $1(0-3)$ & $2,5(1-5)$ & 0.006 \\
\hline $\begin{array}{l}\text { Self-reported pain score at day }+11 \text {, } \\
\text { median (IQR) }\end{array}$ & $0(0-1)$ & $1(0-3)$ & 0.01 \\
\hline $\begin{array}{l}\text { Absence of self-reported pain (score <5) } \\
\text { at day }+4 \text {, number }(\%)\end{array}$ & $23(45.1 \%)$ & $31(62.0 \%)$ & 0.09 \\
\hline $\begin{array}{l}\text { Absence of self-rcported pain (score <5) } \\
\text { at day }+7 \text {, number }(\%)\end{array}$ & $6(12.2 \%)$ & $15(30.0 \%)$ & 0.03 \\
\hline $\begin{array}{l}\text { Absence of self-reported pain (score }<5 \text { ) } \\
\text { at day }+11, \text { number }(\%)\end{array}$ & $1(2.1 \%)$ & $5(10.0 \%)$ & 0.21 \\
\hline $\begin{array}{l}\text { Analgesic use at day }+7, \text { number }(\%) \\
\text { - Parenteral } \\
\text { - Oral } \\
\text { - Topical } \\
\text { - Association } \\
\text { - No use }\end{array}$ & $\begin{array}{c}15(30.5 \%) \\
5(10.2 \%) \\
0 \\
4(8.2 \%) \\
25(51.0 \%)\end{array}$ & $\begin{array}{c}18(36.0 \%) \\
8(16.0 \%) \\
0 \\
5(10.0 \%) \\
19(38.0 \%)\end{array}$ & 0.60 \\
\hline $\begin{array}{l}\text { Neutrophil count ad day }+4 \text {, median } \\
\text { (IQR) }\end{array}$ & $100.0(0.0-800.0)$ & $104.0(0.0-580.0)$ & 0.98 \\
\hline $\begin{array}{l}\text { Neutrophil count ad day }+7 \text {, median } \\
\text { (IQR) }\end{array}$ & $770.0(100 .(0-1938.0)$ & $917.0(50.0-2100.0)$ & 0.79 \\
\hline $\begin{array}{l}\text { Neutrophil count ad day }+11 \text {, median } \\
\text { (IQR) }\end{array}$ & $1456.0(503.0 .4158 .0)$ & $1380.0(275.0-2875.0)$ & 0.32 \\
\hline Adverse events & & & \\
\hline
\end{tabular}

\section{Conclusions}

Conclusions: PBM is safe and effective and should be introduced as standard therapy for paediatrics patients affected by $\mathrm{OM}$ as it accelerates recovery from $\mathrm{OM}$ and reduces pain.

\section{eP182}

LOW-LEVEL LASER THERAPY AND MAGNETIC-INFRARED IRRADIATION FOR TREATMENT OF CHEMOTHERAPYINDUCED ORAL MUCOSITIS IN CHILDREN

\section{Gusev ${ }^{l}$, D. Prityko ${ }^{I}$ \\ ${ }^{T}$ St.Luka's Clinical Research Center for Children, Oncology, Moscow, Russia}

\section{Introduction}

Beneficial effects of low-level laser therapy (LLLT) for treatment of chemotherapy-induced oral mucositis (CIOM) have been demonstrated, although a number of studies in pediatric practice is limited.

Objectives

To appraise the efficacy of LLLT combined with magnetic-infrared irradiation (MIIR) for treatment of CIOM ulcers in children.

Methods

27 children aged from 3 to 6 years with malignant solid tumors who had developed CIOM grade 2 were randomized in two groups according to the treatment mode used: in the first $(\mathrm{n}=14)$ - LLLT+MIIR, in the second $(n=13)$ - topical anti-infective agents. The LLLT was applied using impulse irradiation $\lambda 890 \mathrm{~nm}, 4 \mathrm{~W}, 50 \mathrm{~Hz}$, spot size $-4 \mathrm{~cm}^{2}$, irradiation time/point $120 \mathrm{~s}$, energy density $1 \mathrm{~min} 0,006 \mathrm{~J} / \mathrm{cm}^{2}$, infrared radiation $40 \mathrm{~mW}$. The patient data was prospectively collected and then analyzed.

\section{Results}

In group 1, in all patients the marked reduction of CIOM symptoms and decrease of ulcers in size were noted on the day 1-2; the complete ulcers healing was achieved by the day 5-6 (mean 5,5). In contrast, in group 2 the recovery was delayed while the symptoms were subsiding after day 45 until day 9-11 (mean - 9.6). The estimated duration of treatment completed was then considerably reduced in group 1 compared to group 2 with a standardized mean difference $4.1, p=0,000$.

\section{Conclusions}

In our experience, LLLT + MIIR is very efficacious treatment for children with CIOM grade 2 thus producing greater healing of ulcers then conventional topical anti-infective agents.

\section{eP183}

ANALGESIC EFFICACY OF INDOMETHACIN ORAL SPRAY FOR OROPHARYNGEAL MUCOSITIS-INDUCED PAIN: RANDOMIZED, PLACEBO-CONTROLLED, DOUBLE-BLIND TRIAL

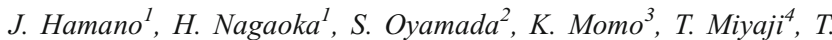

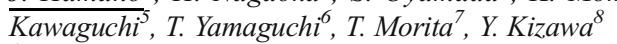

${ }^{1}$ University of Tsukuba, Faculty of Medicine, Tsukuba, Japan

${ }^{2}$ Japanese Organisation for Research and Treatment of Cancer, Department of Biostatistics, Tokyo, Japan

${ }^{3}$ Teikyo Heisei University, Department of Pharmaceutical Sciences, Tokyo, Japan

${ }^{4}$ University of Tokyo, Department of Clinical Trial Data ManagementGraduate School of Medicine, Tokyo, Japan

${ }^{5}$ Tokyo University of Pharmacy and Life Sciences, Department of Practical Pharmacy-School of Pharmacy, Tokyo, Japan

${ }^{6}$ Tohoku University Graduate School of Medicine, Division of Biostatistics, Sendai, Japan

${ }^{7}$ Seirei Mikatahara General Hospice, Department of Palliative and Supportive Care- Palliative Care Team, Hamamatsu, Japan

${ }^{8}$ Kobe University School of Medicine, Department of Palliative Medicine, Kobe, Japan

\section{Introduction}

Oropharyngeal mucositis-induced pain during anticancer therapy substantially reduces patient quality of life, sometimes necessitating dose reductions or treatment interruptions. No high-quality studies evaluating the analgesic efficacy of indomethacin (IM) oral spray (OS) for oropharyngeal mucositis-induced pain exist, although anti-inflammatory agents are recommended.

\section{Objectives}

We conducted a randomized, placebo-controlled, double-blind trial to evaluate the analgesic efficacy and safety of IM-OS for oropharyngeal mucositis-induced pain during anticancer therapy.

\section{Methods}

We enrolled adult cancer patients with oropharyngeal mucositis-induced pain during anticancer therapy who have a score of $\geq 4$ on the Brief Pain Inventory (BPI) item 5 at University of Tsukuba Hospital in Japan. Patients were randomly assigned in a 1:1 ratio to receive IM-OS or placebo-OS. $0.8 \mathrm{mg}$ of IM-OS or placebo was administrated 30 minutes before each meal and when patients had severe pain. Maximum usage was up to $20 \mathrm{ml}$ in 24 hours. The primary endpoint was the intergroup difference in the change of BPI-item 6 score at 30 minutes from baseline. Major secondary endpoints were questionnaire on meal and adverse events.

\section{Results}

From July 2015 through December 2016, 60 patients were enrolled and received IM-OS $(n=33)$ or placebo-OS $(n=27)$. The two groups had significant differences in the change of BPI-item 6 score at 30 minutes afterwards from baseline $(-1.3$ [95\% CI= $1.94-0.57] ; \mathrm{p}<0.001)$, which persist for 180 minutes. There were intergroup differences in scores for liquid drinkability $(p=0.027)$ and ease of conversation $(p=0.017)$. No IM-related adverse events occurred.

Conclusions

IM-OS reduces oropharyngeal mucositis-induced pain during anticancer therapy and could ease eating. 


\section{eP184}

DEVELOPMENT OF INTERPROFESSIONAL EVIDENCE BASED STANDARD OF CARE FOR PREVENTION AND TREATMENT OF MUCOSITIS, BOTH INPATIENT AND OUTPATIENT, ADULT AND PEDIATRIC

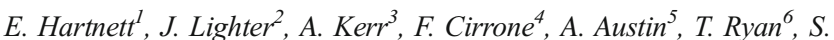
Gardner $^{\prime}$, D. Castillo ${ }^{8}$, J. Pontieri ${ }^{9}$, N. Sanfilippo ${ }^{10}$, A. Moursi ${ }^{11}$, F. De Los Reyes $^{12}$, L. Granowetter ${ }^{13}$, A.M. Araw ${ }^{14}$, A. Dikman ${ }^{15}$, P. Corby ${ }^{10}$, A. Fox $^{16}$, P. Hughes ${ }^{17}$, K. Laurent ${ }^{18}$, B. Guerrin ${ }^{19}$

${ }^{1}$ NYU Rory Meyers College of Nursing, Oral Health, New York, USA

${ }^{2}$ NYU Langone Health, Hospital Epidemiologist, New York, USA

${ }^{3}$ NYU College of Dentistry, Oral and Maxillofacial Pathology, New York, $U S A$

${ }^{4}$ NYU Langone Health, Adult Pharmacy, New York, USA

${ }^{5}$ NYU Langone Health, Information Systems, NewYork, USA

${ }^{6}$ NYU Langone Health, Perlmutter Cancer Cancer, New York, USA

${ }^{7}$ NYU Langone Health, Stephen D. Hassenfeld Childrens Center for Cancer \& Blood Disorders, New YorkUs, USA

${ }^{8}$ NYU Langone Health, Adult Nursing, New York, USA

${ }^{9}$ NYU Langone Health, Pediatric Nursing, New York, USA

${ }^{10}$ NYU Langone Health, Radiation Oncology, New York, USA

${ }^{11}$ NYU College of Dentsitry, Pediatric Dentistry, New York, USA

${ }^{12}$ NYU Langone Health, Pediatric Pharmacy, New York, USA

${ }^{13}$ NYU Langone Health, Pediatric Palliative Care. Stephen D. Hassenfeld Center for Children with Cancer and Blood Disorders, New York, USA

${ }^{14}$ NYU Langone Health, Adult Palliative Care, New York, USA

${ }^{15}$ NYU Langone Health, Gastroenterology, New York, USA

${ }^{16}$ NYU Langone Health, Adult Oncology Nursing Perlmutter Cancer Center, New York, USA

${ }^{17}$ NYU Langone Health, Adult Nursing Education, New York, USA

${ }^{18}$ NYU Langone Health, Pediatric Nursing Education, New York, USA

${ }^{19}$ NYU Langone Health, Pediatric Nursing Stephen D. Hassenfeld Center for Children with Cancer and Blood Disorders, New York, USA

\section{Introduction}

Mucosal damage secondary to cancer therapy occurs in $30 \%$ of patients receiving standard chemotherapy and $80 \%$ of patients receiving high dose chemotherapy. Mucositis is painful, interferes with nutrition, hydration, and often causes delay or reduction in chemotherapy. $20 \%$ of CLABSIs at NYU Langone Health (NYULH) in 2015 were secondary to mucosal translocation

Objectives

The goal of the NYULH Interprofessional Mucositis Workgroup is to decrease the incidence of mucositis in adult and pediatric oncology patients.

\section{Methods}

An interprofessional team of inpatient and outpatient, adult and pediatric medical providers, dentists, nurse practitioners, nurses, pharmacists, and IT collaborated to develop a standardized NYULH mucositis guideline for prevention and treatment.

\section{Results}

An evidenced-based standardized guideline for mucositis prevention and treatment across adult and pediatric inpatient and outpatient was developed.

\section{Conclusions}

This project suggests that interprofessional collaboration is an effective strategy for development and implementation of a standardized guideline for both pediatric and adult inpatients and outpatients.

\section{eP185}

REPRODUCTIVE CAPACITY OF ORAL EPITHELIAL CELLS AFTER EXPOSURE TO C. GLABRATA, C. KEFYR AND P. GINGIVALIS
T. Haverman ${ }^{1}$, F. Rozema ${ }^{1}$, J. de Soet ${ }^{2}$, A. Laheij ${ }^{1}$

${ }^{T}$ ACTA, Oral Medicine, Amsterdam, The Netherlands

${ }^{2}$ ACTA, Preventive Dentistry, Amsterdam, The Netherlands

\section{Introduction}

Both bacteria and fungi play a role in oral mucositis after chemo- and/or radiotherapy. In a previous study we showed that Porphyromonas gingivalis, Candida glabrata and Candida kefyr strongly inhibited wound closure in an in vitro scratch assay model. The question that remains to be elucidated is if this is caused by reduced cell migration or by reduced ability of cells to grow.

\section{Objectives}

Our aim was to study the reproductive capacity of oral epithelial cells when challenged with $P$. gingivalis, C. glabrata and C. kefyr.

Methods

A clonogenic assay in vitro was used. Oral epithelial cells (CA 922) were cultured and seeded in 6-well plates. Micro-organisms were added to the wells in different concentrations; mono-infections or mixed together. After one day the bacteria were removed and after seven days the epithelial cells were coloured with crystalviolet. Finally the colonies of oral epithelial cells that formed were counted using a stereomicroscope. The Kruskal-Wallis test was used to determine any differences in reproductive capacity of the epithelial cells under different bacterial conditions.

\section{Results}

No statistical difference was observed in the ability of a single cell to grow into a colony between control and epithelial cells exposed to different concentrations of C. glabrata $(\mathrm{p}=0.214), C$. kefyr $(\mathrm{p}=0.925)$ or P. gingivalis $(\mathrm{p}=0.762)$. This also applies to the mixed infections.

\section{Conclusions}

C. glabrata, C. kefyr or $P$. gingivalis did not influence the capacity of oral epithelial cells for continued proliferation.

\section{eP186}

ORAL MUCOSITIS RELATED TO ANTICANCER THERAPY: COMPARISON OF CYTOTOXIC AGENTS AND NEW DRUGS

\section{T. Kataoka}

${ }^{T}$ Kobe Minimally Invasive Cancer Center, Dental Oncology, Kobe, Japan

\section{Introduction}

Immune checkpoint inhibitors provide substantial benefits in advanced cancers, but their impact on the oral mucosa has not been compared with that of older cytotoxic agents.

\section{Objectives}

We retrospectively compared oral mucositis induced by cytotoxic agents and new agents (molecular targeted drugs or immune checkpoint inhibitors).

\section{Methods}

Clinical records for 1,103 regimens from January 2014 to December 2017 were analyzed. These included 424 regimens with molecular targeted drugs and 14 with immune checkpoint inhibitors.

\section{Results}

Oral mucositis caused by cytotoxic agents did not differ by proven period of onset, site, or mechanism. Characteristic lesions among some patients taking molecular targeted drugs were oral mucositis such as aphtha with EGFR tyrosine kinase inhibitors (TKIs); gingival bleeding with VEGF TKIs; and osteonecrosis with anti-RANKL antibody. Although the toxicities of immune checkpoint inhibitors are attributed to immune-reactivity against normal tissue, the mechanisms of their oral toxicity are still unclear. In this study, an 82-year-old male with oral malignant melanoma treated with nivolumab experienced severe oral mucositis, xerostomia and hyperglycemia. Severe xerostomia caused by hyperglycemia can increase the sensitivity of oral mucosa, leaving it susceptible to oral mucositis following damage by mechanical 
stimulation by the teeth, dentures, and food. Moreover, we recognized that his oral mucositis was similar to the radiation recall phenomenon, as he had been treated with radiation therapy 3 months before in the same area.

\section{Conclusions}

Clinical side effects of immune checkpoint inhibitors in the oral mucosa have been insufficiently investigated. Since these may involve the immune response, long-term follow-up translational research is warranted.

\section{eP187}

THE BILE ACID AGONIST TGR5 INDUCES INTESTINAL GROWTH AND AMELIORATES INTESTINAL MUCOSITIS IN MICE

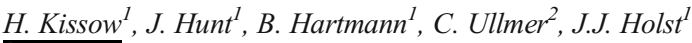

${ }^{T}$ University of Copenhagen, Department of Biomedical Sciences, Copenhagen, Denmark

${ }^{2}$ Roche Innovation Center, Roche Pharma Research and Early Development, Basel, Switzerland

\section{Introduction}

Stimulation of the bile acid receptor Takeda-G-protein-receptor-5 (TGR5) results in the secretion of glucagon-like peptide 1 (GLP-1) and glucagonlike peptide 2 (GLP-2). GLP-1 and GLP-2 analog treatments have previously been shown to have intestinotrophic effects and to ameliorate mucositis in rodents

\section{Objectives}

In this study we aim to investigate the intestinotrophic and protective effect of TGR5 activation

\section{Methods}

Female C57BL/6NJ mice were treated with the selective TGR5 agonist RO5527239 (30 mg/kg) or vehicle twice daily for ten days. Small intestinal weight was recorded and gut hormone analysis performed. In a subsequent study mice had a single injection with 5-fluorouracil (5-FU) and were treated with the TGR5 agonist $(30 \mathrm{mg} / \mathrm{kg})$ or vehicle twice daily until sacrifice $72 \mathrm{~h}$ after 5 FU. Body weight (BW) and intestinal parameters were explored; including intestinal weight, morphometry, histological analysis of mucositis severity and activity of myeloperoxidase

\section{Results}

Agonist treatment resulted in an increase in relative small intestinal weight $(p<0.0001)$. The concentration of intact GLP-1 and GLP-2 was increased in colonic tissue $(\mathrm{p}<0.01)$. Agonist treatment of 5-FU mice resulted in an increase in villus height $(\mathrm{p}<0.05)$ (Fig. 1 A) and crypth depth, a decrease in histological severity score (Fig. 1 B), a decrease in body weight loss $48 \mathrm{~h}$ after 5 -FU and a decrease in myeloperoxidase activity
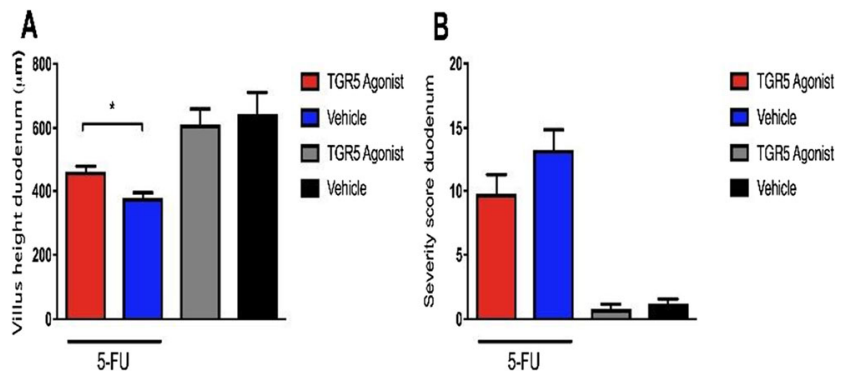

Conclusions

The study shows a TGR5 stimulated growth response in the small intestine associated with an increase in intact GLP-1 and GLP-2 in the colonic tissue. Furthermore, selective TGR5 activation is able to ameliorate 5-FU mucositis in mice.
eP188

SALIVARY A-AMYLASE AS A MARKER FOR SALIVARY GLAND TOXICITY IN HEAD AND NECK CANCER PATIENTS RECEIVING RADIOTHERAPY/ CHEMORADIOTHERAPY

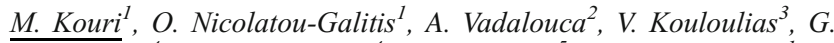
Maropoulos $^{4}$, P. Kanellopoulos ${ }^{4}$, E. Kyrodimos ${ }^{5}$, E. Papadopoulou ${ }^{1}$, E.

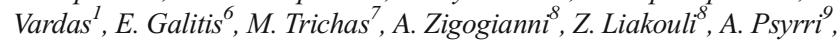
I. Kouvaris ${ }^{8}$

${ }^{1}$ National and Kapodistrian University of Athens, Clinic of Hospital Dentistry-School of Dentistry, Athens, Greece

"Athens Medical Center", Pain Clinic, Athens, Greece

${ }^{3}$ National and Kapodistrian University of Athens, 2 nd Radiology Department- Radiotherapy Unit- Medical School, Athens, Greece

4 "Laiko" General Hospital of Athens, Department of Biochemistry, Athens, Greece

${ }^{5}$ National and Kapodistrian University of Athens, ENT Clinic "Hippokrateio" Hospital-Medical School, Athens, Greece

${ }^{6}$ National and Kapodistrian University of Athens, Clinic of Orofacial Pain- School of Dentistry, Athens, Greece

7 "Iaso" Hospital, Radiotherapy Department, Athens, Greece

${ }^{8}$ National and Kapodistrian University of Athens, 1st Radiology Department- Radiotherapy Unit- Medical School- "Aretaieion" University Hospital, Athens, Greece

${ }^{9}$ National and Kapodistrian University of Athens, Oncology Department- "Attikon" University Hospital- Medical School, Athens, Greece

\section{Introduction}

Painful oral mucositis (OM), salivary gland dysfunction and xerostomia are significant radiation-toxicity related complications in head and neck cancer (HNC) patients. Salivary A-amylase can be a good salivary gland function indicator.

\section{Objectives}

To assess the A-amylase changes in HNC radiotherapy/ chemoradiotherapy.

\section{Methods}

Thirty-fourpatients (mean age 65.12 years, $79.4 \%$ males) were included in the study; 16 had oral/oropharyngeal, 7 laryngeal, 4 parotid and 7 other carcinomas. Whole saliva was collected and A-amylase was assessed before radiotherapy $(t 0)$, between $2^{\text {nd }}-4^{\text {th }}$ weeks (t1) and $5^{\text {th }}-7^{\text {th }}$ weeks (t2), using the 2-Chloro-NPG3Substrate-Photometric method. OM was recorded using the EORTC/RTOG grading, pain and xerostomia were assessed using a Verbal-Analogue-Scale (VAS).

\section{Results}

Twenty-five patients received chemoradiotherapy. Twenty-one received postoperative radiotherapy. Mean total dose was $63.18 \mathrm{~Gy}$. Thirty patients were assessed at t2: Amylase was reduced in 25 $(83.3 \%)$ patients from $165.09 \mathrm{U} / \mathrm{ml}$ to $49.48 \mathrm{U} / \mathrm{ml} ; 23(76.7 \%), 16$ $(53.3 \%)$, and $19(63.3 \%)$ patients had moderate-severe OM (RTOG:III-IV), pain and xerostomia (VAS:8-10) accordingly. Amylase was increased in 5 patients from $241.17 \mathrm{U} / \mathrm{ml}$ to $389.91 \mathrm{U} / \mathrm{ml}$; none of them had severe OM, pain or xerostomia. There was found a statistically significant reduction of the amylase levels $(\mathrm{p}<0.05)$; associated with more intense OM $(\mathrm{p}<0.05)$ and xerostomia $(\mathrm{p}<0.05)$ but not with pain $(\mathrm{p}>0.05)$. Eight $\mathrm{pa}-$ tients were assessed at t1: Amylase was reduced in 5 patients from $154.08 \mathrm{U} / \mathrm{ml}$ to $29.09 \mathrm{U} / \mathrm{ml}$ and was increased in 3 patients from $12.62 \mathrm{U} / \mathrm{ml}$ to $267.45 \mathrm{U} / \mathrm{ml}$.

\section{Conclusions}

The reduction of $\alpha$-amylase in most assessments (30/38) was considered related to salivary gland dysfunction and radiation-toxicity. A-amylase may serve as a marker for the radiation-induced salivary gland toxicity. 


\section{eP189}

A CASE STUDY OF THE USE OF AN ORAL CARE GUIDE IN CARING FOR HEAD AND NECK CANCER PATIENTS SUFFERING FROM ORAL MUCOSITIS

Y.W. $\operatorname{Lin}^{1}$

${ }^{T}$ head nurse, oncology ward, New Taipei City, Taiwan R.O.C.

\section{Introduction:}

Studies have shown that $80 \%$ to $90 \%$ of the patients with head and neck cancer undergoing radiation therapy may develop oral mucositis. If they also receive high-dose chemotherapy, oral mucositis occurs in approximately $20 \%$ to $40 \%$ of the cases. Studies have shown that a complete guide to oral care or maintenance of oral hygiene can prevent oral mucositis as well as reduce oral mucosal changes during the treatment .

Objectives

This oral care guide was used as an intervention measure in order to find out whether the severity of oral mucositis could be alleviated or not.

Methods

The guide to oral care in this study includes assessment of the oral mucosa, tooth-brushing tools and methods, use of dental floss and moisturizing by mouthwash

Results

The application of an oral care guide requires that the patient cooperate by implementing each step, so that oral mucositis may be prevented or alleviated. Though he still developed oral mucositis, which was as severe as Grade 3, there was no continual bleeding, nor did it turn into a whole area of fused ulcer. Until the end of the treatment, his oral mucositis remained of the same grade, without deterioration.

Conclusions

Though oral care is a common and very simple measure of intervention, its existence is significant. In the future, it is hoped that this oral care guide can be used clinically to benefit patients, so that the effectiveness of clinical work may be maximized and the quality of care may be enhanced.

\section{eP190}

NO COMMON GENE EXPRESSION ALTERATIONS BASED ON MUCOSITIS SEVERITY IN THREE INDEPENDENT CANCER COHORTS OF ACUTE MYELOID LEUKAEMIA, MULTIPLE MYELOMA AND TONSIL SQUAMOUS CELL CANCER

M. Marcusssen ${ }^{1}$, J.S. Bødker ${ }^{1}$, M. Sønderkar ${ }^{I}$, V. Charles ${ }^{1}$, I.

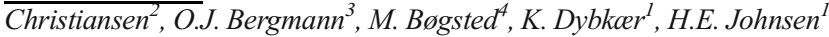
${ }^{I}$ Aalborg University Hospital, Clinical Cancer Research Center, Aalborg, Denmark

${ }^{2}$ Aalborg University Hospital, Department of Hematology, Aalborg, Denmark

${ }^{3}$ Aarhus University, School of Dentistry- Faculty of Health Sciences, Aarhus, Denmark

${ }^{4}$ Aalborg University, Department of Clinical Medicine, Aalborg, Denmark

\section{Introduction:}

Mucositis is a frequent clinical complication to chemotherapy among patients with acute myeloid leukemia (AML); however, the molecular mechanisms are not fully understod.

Objectives

First, we present molecular data from a pilot study using global gene expression (GGE) analysis on human mucosa biopsies before, during and after induction treatment for AML. Second, we compare mucosal GGE profile of AML patients with two previously published cohorts (multiple myeloma (MM), and tonsil squamous cell carcinoma (TSCC)) to identify common mucositis and disease related GGE profiles.

\section{Methods}

Six included de novo AML patients delivered three consecutive buccal biopsies for GGE analysis: before ADE (Cytarabine/Daunorubicin/ Etoposide) induction therapy (day0), day 3 of treatment (day3) and before initiation of second induction therapy (day27). Buccal biopsies from nine healthy, age and gender matched individuals served as controls (CON). The mucositis assessment was correlated to gene expression. Similar data from MM and TSCC cohorts were imported.

Results

Before treatment, RNU6-996P, a non-coding small nuclear RNA, was significant upregulated in AML mucosa compared to CON. In response to chemotherapy, genes involved in DNA damage and repair, and cell communication were altered: $P O L H$, HIST1H1, HISTIH2BM, and NOTCH1. A principal component analysis combining all three independent cancer cohorts, AML, MM and TSCC did not identify common GGE patterns based on clinical mucositis severity.

\section{Conclusions}

DNA damage and repair genes dominated the therapy-induced mucosal molecular changes of patients with AML, regardless of mucositis severity. The mucosal GGE profiles from three independent cancer cohorts did not identify common GGE alterations based on the severity of clinical mucositis.

\section{eP191}

CONDITIONING THERAPY-INDUCED ORAL MUCOSITISASSOCIATED SINGLE NUCLEOTIDE POLYMORPHISMS (SNPS) IDENTIFIED BY EXOME SEQUENCING ANALYSIS: A PILOT STUDY

J.L. Mougeot ${ }^{1}$, B. Hasséus ${ }^{2}$, K. Garming-Legert ${ }^{3}$, N. Rozario $^{4}$, A.

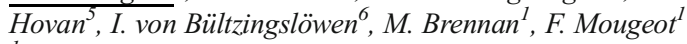

${ }^{1}$ Carolinas HealthCare System, Oral Medicine, Charlotte, USA

${ }^{2}$ Institute of Odontology-University of Gothenburg, Oral Medicine and Pathology, Gothenburg, Sweden

${ }^{3}$ Karolinska Institute, Dental Medicine, Stockholm, Sweden

${ }^{4}$ Carolinas HealthCare System, IAS CORE Analytics, Charlotte, USA

${ }^{5}$ University of British Columbia, Radiation Oncology, Vancouver, Canada

${ }^{6}$ University of Gothenburg, Department of Oral Microbiology and Immunology, Gothenburg, Sweden

\section{Introduction}

Oral mucositis $(\mathrm{OM})$ is a common dose-limiting side effect of conditioning therapy used in cancer or immunodeficiency treatment of hematopoietic stem cell transplant (HSCT) patients. There is a need to identify SNPs predictive of risk and severity of OM.

\section{Objectives}

Using exome-sequencing, our objective was to identify candidate SNPs associated with OM in HSCT patients.

Methods

Saliva DNA was extracted from HSCT patients $(n=63)$ with WHO-OM scores 2-4 (Group-2-4; $n=24$ ), or 0-1 (Group-0-1; n=39). Illumina HiSeq2000-paired-end platform was used for exome-sequencing. SNP calling was determined with GATK pipeline. Using a simplified logit model, a list of $309 \mathrm{SNPs} / 209$ genes was obtained (uncorrected p-value $<0.01)$. Assuming a "toxic gain in function", homozygous variant alleles overrepresented by more than $20 \%$ in Group-2-4 (possibly representing true positives independent from diagnosis) were determined. Proportions differences were evaluated by two-tailed 2 -sample z-test.

\section{Results}

Ten genes with SNP(s) were identified, including LAMC1 and ABCA4. LAMC1, essential for basal cell adhesion, had 8 SNPs that encompass $28 \mathrm{kbp}$. All SNPs combined were either heterozygous or homozygous for reference or variant genotype. Ten patients, eight in Group-2-4 and one with WHO-OM score 1, had variant homozygous genotype in LAMC1. 
Also, an overrepresented intronic homozygous SNP variant (100\% penetrance) was found in ABCA4, a gene adjacent to ARHGAP29 gene. In a previous cleft lip/palate association study, a different intronic SNP in ABCA4 was found within a long distance regulatory element of ARHGAP29. ARHGAP29 is critical for craniofacial development and involved in oral epithelial cell adhesion.

Conclusions

Exome-sequencing has potential to uncover SNPs relevant to OM.

\section{eP192}

SMOKELESS TOBACCO IS ASSOCIATED WITH MORE SEVERE BUT LESS PAINFUL MUCOSITIS

\author{
S. Mukhopadhyay ${ }^{1}$, A. Ghosh ${ }^{2}$, P. Dutta ${ }^{3}$, S. Biswas ${ }^{4}$ \\ ${ }^{T}$ Assistant Professor, Pharmacology, Burdwan, India \\ ${ }^{2}$ Burdwan Medical College, MBBS student, Burdwan, India \\ ${ }^{3}$ Burdwan Medical College Hospital, Radiotherapy, Burdwan, India \\ ${ }^{4}$ Burdwan Medical College, Pharmacology, Burdwan, India
}

\section{Introduction}

Head and neck cancer patients are likely to develop oral mucositis during their course of treatment with Radiation therapy or chemoradiation. Smoking is considered as a risk factor for the development of oral mucositis. However, the association of smokeless tobacco use and oral mucositis $(\mathrm{OM})$ was hardly ever explored.

Objectives

1. To find out any association between use of smokeless tobacco with development of $\mathrm{OM}$, in cancer patients undergoing radiation or chemotherapy.

2. To compare the development and severity of OM in such patients with smokers or non-tobacco users.

Methods

The head and neck cancer patients who developed OM during their course of radiation or chemotherapy were included in this observational study. The development, onset, severity were recorded and pain symptom in 0-10 NRS were noted among all patients. All data were compared among the subgroups of smokeless tobacco user, smokers and others.

Results

Significantly more (p, 0.033) number of grade 3 and grade 4 mucositis were noted among the smokeless tobacco users (Fig 1) and they had faster onset of mucositis (p, 0.002) compared to others (Table 1). However, severity of pain was less in this subgroup of patients though did not reach statistical significance.

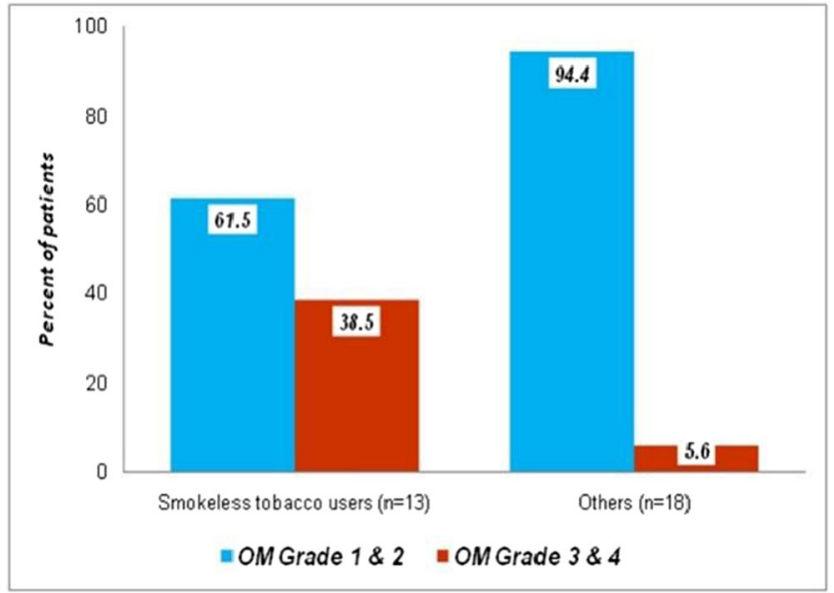

Figure 1: Severity of mucositis and smokeless tobacco

\begin{tabular}{|c|c|c|c|c|c|}
\hline & $\begin{array}{c}\text { Smokeless } \\
\text { tobacco only } \\
\text { (SL) }\end{array}$ & $\begin{array}{c}\text { Smoker+ } \\
\text { smokeless } \\
\text { (SS) }\end{array}$ & $\begin{array}{c}\text { Only } \\
\text { Smokers } \\
\text { (S) }\end{array}$ & $\begin{array}{c}\text { None } \\
\text { (N) }\end{array}$ & $p$ \\
\hline $\begin{array}{c}\text { Day of onset after start } \\
\text { of RT (mean) }\end{array}$ & $5.5 \pm 1.29$ & $5.8 \pm 1.6$ & $7.9 \pm 1.26$ & $8.7 \pm 2.2$ & 0.002 \\
\hline $\begin{array}{c}\text { Severity of Pain (mean } \\
\text { NRS) }\end{array}$ & $7 \pm 0.81$ & $9 \pm 0.86$ & $9 \pm 2.58$ & $9 \pm 1.7$ & 0.24 \\
\hline
\end{tabular}

Table 1. Onset time and severity of pain of Oral mucositis among four subgroups

\section{Conclusions}

The present study suggests that smokeless tobacco is associated with faster onset and higher grades of mucositis compared to smokers or tobacco nonusers but associated with less severe pain. However, future longitudinal study in larger population is recommended before fetching a definite conclusion.

\section{eP193}

EVALUATION OF THE EFFECTIVENESS OF A NEW MEDICAL DEVICE CONTAINING VERBASCOSIDE, POLYVINYLPYRROLIDONE, AND HYALURONIC ACID IN THE TREATMENT OF MUCOSITIS IN CANCER PATIENTS.

O. Nigro ${ }^{1}$, A. Giaquinto ${ }^{2}$, O. Ciotti ${ }^{2}$, A. Tuzi $^{2}$

${ }^{T}$ ASST-Sette Laghi- Ospedale di Circolo di Varese, Medical Oncology, varese, Italy

${ }^{2}$, Medical Oncology, Varese, Italy

\section{Introduction:}

Mucosal injury is the consequence of biologic events coupled with the influence of the oral environment and microbiome. Oral mucositis is one of the most common toxicities of chemoradiation therapies. Incidence and severity varies by chemoradiation regimens, radiation fields, and tumor site. The influence of mucositis on quality of life is greater among patients than the medical literature would suggest. Lack of experience in evaluating treatment toxicities is a daily problem in cancer patients.

\section{Objectives}

The aim of this study was to assess the efficacy of Verbascoside's (Mucosyte $\AA$ ) oral solution on mucositis in cancer patients.

\section{Methods}

Twenty-three patients with different grade mucositis treated with Mucosyte $®$ (3 rinses/day) were evaluated from October 2017 to January 2018. Patients were monitored every week until complete remission.

\section{Results}

Of 23 patients enrolled, 14 had G2 oral mucositis at baseline, 7 had G3 and 2 had G4. Median age was 63.3 years. Six patients were treated with carboplatin/paclitaxel for Non Small Cell Lung Cancer, seven with Paclitaxel for Breast Cancer, three with FOLFOX for Intestinal Adenocarcinoma, five with Cisplatin/Cetuximab for Squamous-cell Neck Cancer, two with Cisplatin/Cetuximab plus radiotherapy for Squamous-cell Neck Cancer. Mucosyte ${ }^{\circledR}$ treatment was able to rapidly shorten G3/4 oral mucositis to G1/2. All patients reached complete remission in a median time of 14.4 days (10-21 days).

\section{Conclusions}

The present study confirms that oral solution Mucosyte ${ }^{\circledR}$ is effective in the treatment of mucositis. Moreover, we recommend the use of Mucosyte ${ }^{\circledR}$ previously and during chemoradiation therapies.

\section{eP194}

LASER LIGHT EFFECT ON DORSAL ROOT GANGLIA NEURONS: A PRE-CLINICAL STUDY.

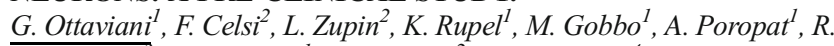

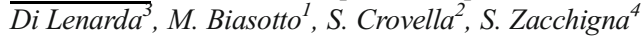


${ }^{1}$ University of Trieste, Dental Science - Oral Medicine and Pathology, Trieste, Italy

${ }^{2}$ I.R.C.C.S. materno infantile Burlo Garofolo, Immunology Lab., Trieste, Italy

${ }^{3}$ University of Trieste, Department of Medical- Surgical and Health Sciences, Trieste, Italy

${ }^{4}$ International Centre for Genetic Engineering and Biotechnology ICGEB, Cardiovascular Biology, Trieste, Italy

\section{Introduction:}

In oncological patients, affected by oral mucositis (OM), pain worsens the quality of life, often requiring high doses of analgesic drugs, and could lead to the halting of the therapies, with significant negative impact on their cancer prognosis. Supportive care and pain management due to $\mathrm{OM}$ has significant economic impacts: LT represents an excellent resource as supportive therapy with direct implication for improving cancer patient care.

Objectives

This study aimed at exploring the analgesic activities of LT in an in vitro model of sensory neurons, analysing cellular parameters possibly correlated to nociceptive pathways.

\section{Methods}

Primary culture of murine sensory neurons, derived from dorsal root ganglia (DRG; Figure 1), were employed in the experiments. The cells were irradiated with two LT protocols: $\lambda 800$ and $970 \mathrm{~nm}, 0.1 \mathrm{~W} / \mathrm{cm}^{2}, 6 \mathrm{~J} /$ $\mathrm{cm}^{2}, 5 \mathrm{~Hz}$. Immediately after irradiation, cells were analysed for production of ATP, radical oxygen species (ROS) and superoxide anion.

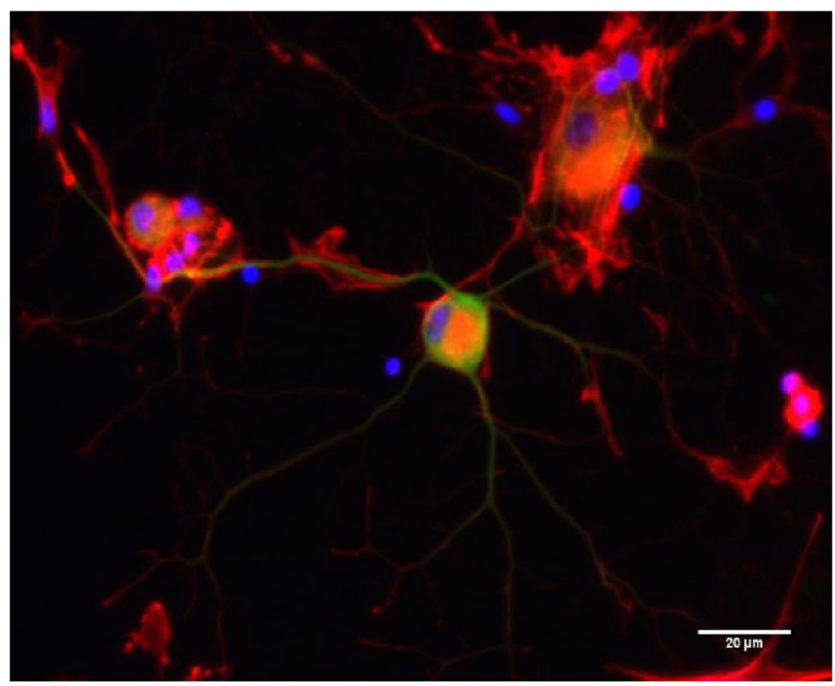

Results

Both the LT protocols were able to decrease the production of ATP and to increment ROS concentration, meanwhile only LT at $800 \mathrm{~nm}$ wavelength increased the level of superoxide anion (Figure 2).

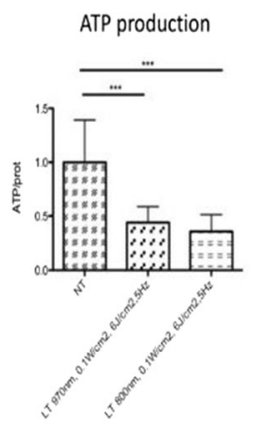

ROS production

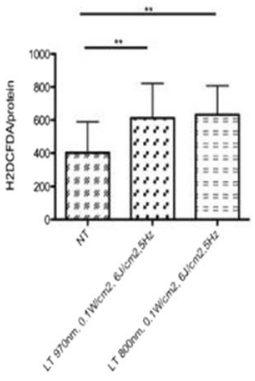

Superoxide anion production

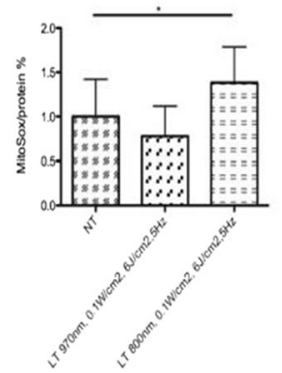

\section{Conclusions}

Our results highlight a direct effect of LT on DRG neurons, specifically LT appears to inhibit cellular metabolism, lowering ATP production and promoting ROS generation, thus suggesting a stress response to the nociceptive stimulus. Finally, the differential outcome on superoxide anion could be due to the specific target of $\lambda 800 \mathrm{~nm}$ on cytochrome $C$ oxidase enzyme. These findings suggest a direct and immediate metabolic effect of laser light on DRG neurons.

\section{eP195}

LASER THERAPY AS SUPPORTIVE TREATMENT FOR ORAL MUCOSITIS IS A SAFETY PROCEDURE IN ONCOLOGICAL PATIENTS

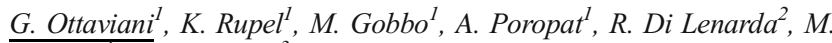 \\ Biasotto $^{1}$, S. Zacchigna ${ }^{3}$ \\ ${ }^{1}$ University of Trieste, Dental Science - Oral Medicine and Pathology, \\ Trieste, Italy \\ ${ }^{2}$ University of Trieste, Department of Medical- Surgical and Health \\ Sciences, Trieste, Italy \\ ${ }^{3}$ International Centre for Genetic Engineering and Biotechnology \\ ICGEB, Cardiovascular Biology, Trieste, Italy
}

\section{Introduction}

Laser therapy is emerging as a promising supportive treatment for oral mucositis induced by oncological therapies. However, its mechanisms of action and, more importantly, its safety in cancer patients, have never carefully investigated so far.

\section{Objectives}

The main purpose of the presented study is to analyze the behavior of cancer cells in vitro and in vivo after laser exposure.

\section{Methods}

Cell metabolism was assessed in vitro on mouse B16F10 melanoma cells, on human skin fibroblasts, commercial human umbilical vein endothelial cells and primary bone marrow-dendritic cells by ATPlite Luminescence Assay at 24 and 48 hours after different laser protocols exposure.

Both xenograft melanoma and ortotopic oral carcinogenesis mouse models were used to analyze tumor growth and invasiveness through a histological (hematoxylin and eosin stain) and immunohistochemistry characterization (CD4, CD8, DCIR2 and Melan-A). The analysis of the tumor infiltration was performed using FACS technology.

\section{Results}

In vitro cell metabolism and proliferation increased after laser treatment. Interestingly, in vivo laser therapy reduced tumor growth and invasiveness, indicating a beneficial influence on tumor microenvironment. Laser-treated tumors were delimited and infiltrated by immune cells, in particular by lymphocytes and dendritic cells. A paralleled effect was the enhanced secretion of type I interferons. In contrast, the number of pro-angiogenic macrophages was reduced in response to photobiomodulation, with consequent normalization of the tumor vasculature.

\section{Conclusions}

Our results encourage to perform laser therapy safely also in potentially dysplastic or neoplastic areas in oncological patients, affected by mucosal or cutaneous lesions.

\section{eP196}

EVALUATION THE EFFICACY OF FOMUKAL FOR TREATMENT OF ORAL MUCOSITIS INDUCED BY RADIATION THERAPY IN HEAD AND NECK CANCER PATIENTS

\section{Kiprian ${ }^{1}$, W. Rolski ${ }^{1}$, W. Michalski ${ }^{1}$}

${ }^{I}$ Maria Sklodowska Institute - Oncology Center, Department of Head and Neck Cancer, Warsaw, Poland 


\section{Introduction}

Oral mucositis $(\mathrm{OM})$ is the common and severe side effect of radiation therapy (RT). It can cause pain as well as lead to xerostomia, dysphagia and impaired quality of life. The currently available treatment schedules have limited effectiveness in reducing duration and severity of OM.

Objectives

We aimed to evaluate the safety and tolerability of neutral supersaturated calcium phosphate aqueous mouthwash solution (Fomukal) for RTinduced OM. Anti-inflammatory activity and analgesic effect have been attributed to calcium ions. Phosphate ions are supposed to protect mucosa from irritation caused by significant $\mathrm{pH}$ changes in the oral cavity.

Methods

Forty consecutive patients with head and neck cancer who received RT were included to pilot study. Treatment comprised mouth washing with Fomukal aside from standard OM treatment. The severity of OM, pain intensity, xerostomia and dysphagia were assessed at baseline, at weekly intervals during treatment and after 4 weeks follow-up and compared to the similar group with standard treatment.

\section{Results}

We noted the statistically significant reduction in mean severity of xerostomia, $\mathrm{OM}$, and dysphagia as well as the strong opioid consumption and frequency of prolonged hospitalization in Fomukal group. Similar differences between the observed groups were noted after four weeks follow-up.

\section{Conclusions}

Our preliminary findings demonstrated the safety and potential benefits of Fomukal implementation for radiation-induced OM treatment schedules. Furthermore studies, to evaluate the role of Fomukal in preventing and reducing mucosal side effects of $\mathrm{RT}$ in the randomized clinical setting, are warranted.

\section{eP197}

RELATING CHEMOTHERAPY-INDUCED GASTROINTESTINAL TOXICITY RISK TO PRE-TREATMENT MICROBIOME PROFILE: A PILOT STUDY

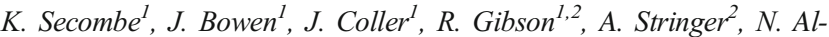 \\ Dasooqi $^{\text {I,2, }}$ B. Mayo $^{2}$, H. Wardill ${ }^{1,3}$ \\ ${ }^{1}$ University of Adelaide, Adelaide Medical School, Adelaide, Australia \\ ${ }^{2}$ University of South Australia, Division of Health Sciences, Adelaide, \\ Australia \\ ${ }^{3}$ South Australian Health and Medical Research Institute, Centre for \\ Nutrition and Gastrointestinal Diseases, Adelaide, Australia
}

\section{Introduction}

There is currently an urgent and unmet need to determine an individual's risk of developing gastrointestinal toxicity prior to chemotherapy. The microbiome has received attention for its role in regulating an individual's risk of gastrointestinal toxicity due to its ability to modulate the host's immune system and metabolise some chemotherapy agents. As such, pretreatment microbial profiling presents as a novel risk stratification method.

Objectives

To characterize pre-treatment microbial profiles in patients undergoing chemotherapy for cancer.

Methods

Patients with a variety of solid tumors including breast and colorectal were recruited from the Royal Adelaide Hospital. Stool samples were collected from patients before their chemotherapy treatment began (T0) and at day 5 (T5). Clinical case notes were used to determine clinical toxicity score and diarrhea severity. Microbiome composition was initially assessed using polymerase chain reaction, and will be validated by $16 \mathrm{~S}$ pyrosequencing techniques at the Australian Genome Research Facility. Results

Eight patients undergoing a folinic acid, fluorouracil and oxaliplatin (FOLFOX) chemotherapy regimen were recruited, with an average age of 62.65 years. Four of these patients went onto suffer from gastrointestinal toxicity symptoms such as diarrhea. Preliminary data shows that pre-treatment levels of E. coli and Bifidobacterium levels are increased in patients that went on to develop diarrhea, compared to patients with no symptoms. This will be validated with $16 \mathrm{~S}$ sequencing.

\section{Conclusions}

This pilot study is an important step in determining whether the gut microbiome can be used as a diagnostic marker in determining risk of severe chemotherapy-induced gastrointestinal toxicity.

\section{eP198}

PREDICTORS OF ORAL MUCOSITIS AND ITS IMPACT IN TRANSPLANTED PEDIATRIC PATIENTS PREVENTIVELY TREATED WITH PHOTOBIOMODULATION

W. Miranda-Silva ${ }^{1}$, E. Rodrigues Fregnani ${ }^{l}$, C. Arrais Rodrigues-Silva ${ }^{2}$, V. Rocha ${ }^{2}$, Y. Augusta Novis ${ }^{2}$, A. Gomes ${ }^{2}$, A.B. Mafra ${ }^{2}$, F. Paiva Fonseca ${ }^{3}$ ${ }^{1}$ IEP - Instituto de Ensino e Pesquisa/Hospital Sírio Libanês, Departament of Oral Medicine - Hospital Sírio Libanês, São Paulo, Brazil

${ }^{2}$ IEP - Instituto de Ensino e Pesquisa/Hospital Sírio Libanês, Bone Marrow Transplant Unit - Hospital Sírio Libanês, São Paulo, Brazil

${ }^{3}$ School of Dentistry- Universidade Federal de Minas Gerais, Department of Oral Surgery and Pathology- School of DentistryUniversidade Federal de Minas Gerais - Brazil, São Paulo, Brazil

\section{Introduction}

Oral mucositis $(\mathrm{OM})$ is the most relevant oral toxicity observed during antineoplastic therapy. It is observed in over $80 \%$ of patients submitted to HSCT, manifesting early after the allogeneic HSCT and commonly evolving to complete healing with no scar formation in approximately 7 to 10 days.

\section{Objectives}

To investigate the clinical features and outcomes associated to oral mucositis $(\mathrm{OM})$ in pediatric patients submitted to allogeneic hematopoietic stem cell transplantation (alloHSCT) preventively treated with professional dental care and photobiomodulation (PBM).

\section{Methods}

Medical data and OM presentation of all pediatrics who received alloHSCT between 2013 and 2016 at the Hospital Sírio-Libanês (São Paulo/Brazil) were retrospectively retrieved. The presence of OM was assessed and graded by two oral medicine specialists following the WHO guidelines and correlated with clinical parameters.

\section{Results}

Forty-nine consecutive patients were included. OM was diagnosed in 36 patients $(73.5 \%)$, being classified as aggressive in $36.1 \%$. Acute lymphoblastic leukaemia (ALL) as primary diagnosis and the use of myeloablative regimen were associated with OM development, but only the latter achieved significance in the logistic regression model. Primary diagnosis and the use of TBI were associated with aggressive OM. Neither the presence, nor the severity of OM affected the overall survival, whereas only the use of myeloablative regimen and a high body mass index proved to be determinants of lower OM-free survival.

\section{Conclusions}

In transplanted infants prophylactic treated with PBM and dental care, mucositis incidence and severity are decreased, it does not determine a lower overall survival rate, and it is associated with the conditioning regimen used.

\section{eP199}

IN VITRO HUMAN RECONSTRUCTED ORAL MUCOSA EXPOSED TO 5-FLUOROURACIL CAN MIMIC BLISTERING EVENTS RESEMBLING THE INITIATION OF ORAL MUCOSITIS

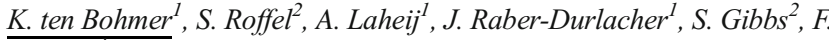 \\ Rozema \\ ${ }^{I}$ ACTA, oral medicin, Amsterdam, The Netherlands \\ ${ }^{2}$ ACTA, oral microbiology, Amsterdam, The Netherlands
}




\section{Introduction}

Oral mucositis is a painful inflammation of the oral mucosal tissues resulting as a serious side effect of cancer chemo- and or radio-therapy. There is an urgent need to develop physiologically relevant translational models allowing for assessing toxicities of cytotoxic therapy and studying the pathophysiology of oral mucositis.

\section{Objectives}

The aim of this study was to develop a human oral mucositis model and to establish a proof of concept testing of systemic toxicity caused by conventional chemotherapeutics.

Methods

Human reconstructed oral mucosa (ROM; differentiated epithelium on fibroblast populated collagen hydrogel) was constructed from TERT immortalized cell lines. Air-lifted ROM were exposed to 5-fluorouracil (10${ }^{2} \mathrm{M}$ and $10^{-5} \mathrm{M}$ ) via the culture medium for 24 and 48 hours. Hereafter, macroscopic photographs, tissue histology and metabolic activity were assessed.

\section{Results}

Multiple small in vitro blisters (separation of epithelium from fibroblasthydrogel) where observed covering ROM surface area when exposed to 5-fluorouracil. $10^{-5} \mathrm{M}$ 5-fluorouracil resulted in epithelial blisters occurring after $24 \mathrm{hrs}$ whereas $10^{-2} \mathrm{M}$ fluorouracil was cytotoxic resulting in a dead epithelial layer loosely attached to the hydrogel. The metabolic activity of ROM was significantly lower after exposure to 5-fluorouracil $\left(10^{-5} \mathrm{M}: \mathrm{p}=0,015 ; 10^{-2} \mathrm{M}: \mathrm{p}=0,035\right)$ compared to the vehicle after 24 hours and after exposure to $10^{-2} \mathrm{M}(\mathrm{p}=0,0001)$, but not $10^{-5} \mathrm{M}(\mathrm{p}=0,072)$ after 48 hours.

Conclusions

It was possible to mimic blistering events resembling the initiation of oral mucositis in vitro using organotypic human ROM exposed to a commonly used chemotherapeutic. This model provides a unique opportunity to investigate systemic toxicity without the use of animals.

\section{eP200 \\ A METHOTREXATE-INDUCED IN VITRO ORGANOID MUCOSITIS MODEL}

S. van $\operatorname{der} A a^{1,2,3}$, M. Peters ${ }^{2}$, P. Schulze ${ }^{2}, J$. Garssen ${ }^{2,4}$, L. Harthoorn ${ }^{2}$,

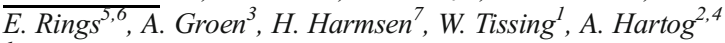

${ }^{1}$ UMC Groningen, Department of Pediatric Oncology, Groningen, The Netherlands

${ }^{2}$ Danone Nutricia Rsearch, Nutricia Advanced Medical Nutrition, Utrecht, The Netherlands

${ }^{3}$ UMC Groningen, Department of Pediatric Gastroenterology and hepatology, Groningen, The Netherlands

${ }^{4}$ Utrecht Institute for Pharmaceautical Sciences, Department of Pharmacology, Utrecht, The Netherlands

${ }^{5}$ Erasmus Medical Center Rotterdam, Department of Pediatrics, Rotterdam, The Netherlands

${ }^{6}$ Leiden University Medical Center, Department of Pediatrics, Leiden, The Netherlands

${ }^{7}$ UMC Groningen, Department of Medical Microbiology, Groningen, The Netherlands

\section{Introduction}

Chemotherapy-induced intestinal mucositis is frequently a doselimiting and costly side effect for which there is no treatment available. Here, we investigate whether intestinal organoids, a threedimensional in vitro tissue model incorporating many of the physical relevant features of the in vivo tissue, could be used to develop an in vitro mucositis model in which both mucositis development and recovery can be studied.

Objectives

To develop an in vitro model of methotrexate (MTX)-induced mucositis in small intestinal organoids.

\section{Methods}

Mice derived intestinal organoids were treated with different concentrations of MTX for 24-96 hours after which the metabolic activity was measured. Recovery of the metabolic activity was studied 24-96 hours after removal of the MTX. Citrulline and other amino acids concentrations were determined in the media. The inflammatory status of the MTXtreated organoids was studied by analyzing the cytokine and chemokine levels.

\section{Results}

Treatment with $100 \mathrm{ng} / \mathrm{ml}$ MTX for 96 hours decreased the metabolic activity of the organoids to $50 \%$ while ensuring recovery. The citrulline levels in the media were significantly reduced from $78 \mu \mathrm{M}$ to $52 \mu \mathrm{M}$ whereas the production of several chemokines significantly increased.

\section{Conclusions}

Intestinal organoids can be used for studying the initial response of the intestine to MTX treatment. Moreover, the described model might be suitable for screening interventions aiming to improve the course of intestinal mucositis, thereby potentially decreasing the number of animal experiments.

\section{eP201 \\ EARLY SALIVARY CHANGES IN MULTIPLE MYELOMA PATIENTS UNDERGOING AUTOLOGOUS HSCT}

S. van Leeuwen ${ }^{1}$, G. Proctor ${ }^{2}$, C. Potting ${ }^{3}$, S. ten Hoopen ${ }^{1}$, L. van Groningen $^{3}$, E. Bronkhorst ${ }^{1}$, N. Blijlevens ${ }^{3}$, M.C. Huysmans ${ }^{1}$

${ }^{1}$ Radboudumc, Dentistry, nijmegen, The Netherlands

${ }^{2}$ King's College London Dental Institute, Division of Mucosal \& Salivary Biology, London, United Kingdom

${ }^{3}$ Radboudumc, Hematology, Nijmegen, The Netherlands

\section{Introduction}

Saliva and especially salivary proteins play a major role in protecting the oral mucosa. Alterations in salivary proteins may affect oral mucosal health and may influence the severity of oral mucositis $(\mathrm{OM})$.

\section{Objectives}

One explorative observational study in 2 parts was performed to examine early salivary changes in relation to oral mucositis in multiple myeloma patients treated with high-dose melphalan and autologous hematopoietic stem cell transplantation (HSCT). Furthermore, due to protocol changes at the hospital, the effect of cryotherapy was evaluated.

\section{Methods}

Unstimulated (UWS) and stimulated (SWS) whole mouth saliva was collected and OM was scored with the OMNI instrument at days $-3,0,4,7,11$ and 15 after HSCT. Salivary flow rate, total protein (BCA assay), mucin 5B, albumin (western blot), total IgA, lactoferrin and myeloperoxidase levels (ELISA) were determined. Blood levels of polymorphonuclear leukocytes (PMNs) were recorded from medical charts.

\section{Results}

Trends of decreasing UWS and SWS flow rates and total IgA levels were observed. At days 7 and 11, increases in lactoferrin and albumin levels were found in UWS and SWS. Furthermore, a positive correlation was found between OMNI scores and albumin and lactoferrin levels in SWS. The fluctuating trend in salivary MPO levels reflected the changes in blood PMNs. In the second part of the study cryotherapy significantly lowered peak OMNI scores.

\section{Conclusions}

Compositional changes in saliva reflecting inflammation were found in the first days after HSCT and the use of cryotherapy in the second part was associated with a decreased OM severity.

Paper is under review 


\section{eP202}

HARNESSING TRANSGENIC ZEBRAFISH AS A NOVEL PLATFORM FOR THE STUDY OF CHEMOTHERAPY- AND TARGETED THERAPY- INDUCED GASTROINTESTINAL TOXICITY

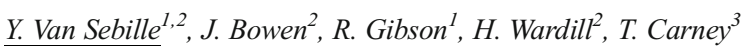

${ }^{T}$ University of South Australia, Health Sciences, Adelaide, Australia

${ }^{2}$ The University of Adelaide, Physiology, Adelaide, Australia

${ }^{3}$ Nanyang Technological University, Institute of Molecular and Cell Biology, Singapore, Singapore

\section{Introduction}

Gastrointestinal toxicity arising from cancer treatment remains a key reason for treatment discontinuation, significantly compromising remission. There are drawbacks to the currently used in vitro and rodent models, and a lack of translatability from in vitro to in vivo work. A screeningamenable alternative in vivo model such as zebrafish would, therefore, find immediate application.

Objectives

This study aimed to develop a transgenic reporter line of zebrafish to investigate its utility as an alternative vertebrate model to bridge the gap between simple in vitro cellular studies and complex in vivo models for understanding gastrointestinal toxicity.

Methods

Larvae zebrafish had genes up-regulated following gastrointestinal toxicity tagged with enhanced green fluorescent protein (eGFP). Transgenic larvae zebrafish were administered afatinib or SN38 in water, and assessed for eGFP induction. Adult zebrafish were administered afatinib via oral gavage, and SN38 via intraperitoneal injection. Fish were killed after 24 hours, had gastrointestinal tracts removed and assessed for histopathological damage, goblet cell changes and apoptosis.

Results

Treatment with either compound did not induce eGFP in the gastrointestinal tract of larvae. SN38 caused histopathological damage to adult zebrafish. The lack of eGFP induction is likely due to technical applications of the poorly soluble cancer drugs. Chemotherapy agents with high solubility and permeability would be more amenable to these models.

Conclusions

Further progress in this area would be greatly facilitated by the generation of robust and reproducible genetic models of zebrafish intestinal toxicity that mimic the known pathobiological pathways in rodents and humans, and can be readily induced in a short time-frame.

eP203

THE EFFECT OF A NOVEL ORAL CARE PROTOCOL IN DECREASING THE EXPRESSION OF CYTOKINES IN HEAD AND NECK CANCER PATIENTS RECEIVING CHEMORADIATION

R. Vasconcelos ${ }^{1}, J$. Moya $^{2}$, M. Gabinskiy ${ }^{2}, K$.Nightingale ${ }^{3}$, E. Queiroz ${ }^{4}$,

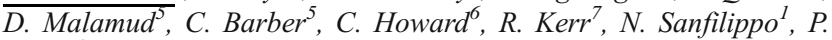
Corby $^{I}$

${ }^{1}$ New York Langone Health, Radiation Oncology Department, New York, USA

${ }^{2}$ New York Langone Health, Office of Science and Research, New York, USA

${ }^{3}$ New York Langone Health, Office of Science \& Research, New York, USA

${ }^{4}$ New York University College of Dentistry, Department of Population Health, New York, USA

${ }^{5}$ New York University College of Dentistry, Basic Science, New York, USA

${ }^{6}$ New York University College of Dentistry, Periodontology and Implant Dentistry, New York, USA

${ }^{7}$ New York University College of Dentistry, Department of Oral and Maxillofacial Pathology-Radiology \& Medicine, New York, USA

\section{Introduction}

Oral mucositis $(\mathrm{OM})$ is one of the most debilitating adverse effects in patients undergoing cancer treatment. Physiologically, chemotherapy (CT) and radiotherapy (RT) evoke a profound inflammatory response, resulting in mucosal injury, which can result in an increased susceptibility to infection.

\section{Objectives}

The objective of this pilot study was to asses the effects of a novel oral care protocol on OM severity and to evaluate salivary cytokines in head and neck cancer patients undergoing RT or CT/RT at the NYU Langone Laura and Isaac Perlmutter Cancer Center.

\section{Methods}

A total of ten participants were included in this study, and randomized to an Intervention Group (IG), or Control Group (CG). Subjects assigned to the CG received a standard of care oral hygiene on a bi-weekly basis. Subjects assigned to the IG received the Oral Mucosal Deterging and Dental Prophylaxis (OMDP) protocol weekly, which consisted of a periodontal surface debridement and cleansing and deterging of the oral mucosa surfaces.

\section{Results}

Salivary inflammatory biomarkers, noted in levels of IFN-gamma, IL10, IL12p70, IL13, TNF $\alpha$ and IL-6 had a significant increase in the CG and reduced or stayed the same under the IG. Although not statistically significant, a tendency of pain decrease was observed in the IG and difficulty in swallowing was statistically significant when both groups were compared $(\mathrm{p}=0,016)$.

\section{Conclusions}

These results suggest that overall inflammation was consistently higher as compared to baseline in the CG, providing encouragement for the effectiveness of the oral care protocol as a coadjutant treatment for this population.

\section{eP204 \\ PROPHYLACTIC PROBIOTICS FOR CANCER THERAPY- INDUCED DIARRHEA: A META-ANALYSIS}

H. Wardill ${ }^{1}$, Y. Van Sebille ${ }^{2}$, M. Ciorba ${ }^{3}$, J. Bowen $^{4}$

${ }^{T}$ South Australian Health and Medical Research Institute, Adelaide Medical School- The University of Adelaide, Adelaide, Australia

${ }^{2}$ The University of South Australia, Division of Health Sciences, Adelaide, Australia

${ }^{3}$ Washington University, School of Medicine-Division of Gastroenterology, Saint Louis, USA

${ }^{4}$ The University of Adelaide, Adelaide Medical School, Adelaide, Australia

\section{Introduction}

Strong preclinical data support prophylactic probiotics as an effective preventative strategy for diarrhea secondary to anti-cancer therapies. To determine the composite evidence that this approach translates to the clinic, we performed a meta-analysis of randomized controlled trials (RCTs) of prophylactic probiotics for the prevention of cancer therapy-induced diarrhea.

\section{Objectives}

To determine clinical efficacy of prophylactic probiotics for the prevention of cancer therapy-induced diarrhea via a meta-analysis.

\section{Methods}

A three-step search strategy was used to identify relevant studies (June 2000-June 2017) investigating probiotic intervention for diarrhea secondary to any cancer therapy. An initial search of PubMed/MEDLINE for articles published in the past 12 months to identify keywords and indexed terms, which were then used to conduct a thorough review of databases: PubMed, Embase, CINAHL and CENTRAL. A final review of reference lists was then conducted to verify strict inclusion criteria. Publication bias was assessed using sensitive analysis in RevMan 5.3 (The Cochrane Collaboration, Oxford, UK).

Results

Seven trials with a total of 1091 patients were included in this meta-analysis. Compared with placebo, prophylactic probiotics did not prevent or reduce the 
overall incidence of diarrhea or severe CTCAE Grade $\geq 3$ diarrhea $(\mathrm{RR}=0.81,95 \%, \mathrm{CI}: 0.60-1.09, \mathrm{Z}=1.41, \mathrm{P}=0.16 ; \mathrm{RR}=0.54,95 \% \mathrm{CI}: 0.25$ $1.16, \mathrm{Z}=1.58, \mathrm{P}=0.11$ ), nor did it influence the use of rescue medication ( $\mathrm{RR}=0.93,95 \% \mathrm{CI}: 0.53-1.65, \mathrm{Z}=0.24, \mathrm{P}=0.81$ ).

\section{Conclusions}

Current evidence does not support widespread implementation of probiotics for diarrhea secondary to cytotoxic therapy and the tyrosine kinase inhibitor, dacomitinib. Research efforts should be diverted to pair specific forms of gastrointestinal toxicity and their unique microbial phenotype to develop the ideal microbial protectant.

\section{eP205}

MMP INHIBITION, WITH AZD3342, SUPPRESSES TUMOUR GROWTH AND DELAYS SEVERE DIARRHOEA CAUSED BY METHOTREXATE

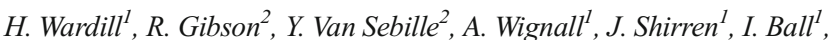
N. Williams ${ }^{1}$, K. Wanner ${ }^{I}, J$. Bowen ${ }^{I}$

${ }^{1}$ The University of Adelaide, Adelaide Medical School, Adelaide, Australia

${ }^{2}$ The University of South Australia, Division of Health Sciences, Adelaide, Australia

\section{Introduction}

The common cytotoxic mechanisms that underpin chemoefficacy and toxicity have hampered efforts to deliver effective supportive care interventions. This is particularly true for gastrointestinal toxicity, with numerous interventions failing due to unwanted inhibition of the tumoridical properties of chemotherapy. Matrix metalloproteinases (MMPs) have been implicated in both tumour growth and gastrointestinal toxicity, and as such MMP inhibitors (MMPIs) present as a novel therapeutic avenue to simultaneously enhance treatment efficacy and reduce toxicity.

\section{Objectives}

Determine the efficacy of MMP-9 and MMP-12 inhibition on tumour growth and gastrointestinal toxicity in a rat model.

Methods

Female tumour-bearing Dark Agouti rats $(\mathrm{N}=84)$ were divided into 4 groups: vehicle control; methotrexate (MTX) only ( 2 doses at $2 \mathrm{mg} / \mathrm{kg}$ intramuscularly); MMPI AZD3342 alone (20 mg/kg twice daily via oral gavage for 3 days) and MTX+AZD3342. Tumours were measured daily (for 5 days) using digital callipers. Gastrointestinal toxicity was assessed using validated clinical markers (diarrhoea/weight loss), histopathological analysis and functional intestinal barrier assessment.

Results

AZD3342alone supressed tumour growth ( $\mathrm{p}=0.003$ vs vehicle) but did not enhance the efficacy of MTX. AZD3342 delayed the onset of severe diarrhoea by 1 day (vs MTX) but was unable to improve the overall severity of diarrhoea. No changes were detected in tissue morphology or intestinal barrier function.

\section{Conclusions}

This study showed partial efficacy of AZD3342 in reducing tumour growth and delaying the onset of severe diarrhoea caused by MTX. We suggest further studies be undertaken around scheduling of administration of AZD3342 as well as investigation using different cytotoxic therapies that strongly activate MMP signalling.

\section{eP206}

EXPLORING THE ROLE OF "GLYCERINE PLUS HONEY" IN DELAYING CHEMORADIATION INDUCED ORAL MUCOSITIS IN HEAD AND NECK CANCERS

\author{
B. Yadav ${ }^{l}$ \\ ${ }^{T} B$ and $C$ Teaching Hospital, Department of Oncology, Birtamod, Nepal
}

\section{Introduction}

Oral mucositis is the most common and irritating side effect of chemoradiation in the treatment of HNSCC.

\section{Objectives}

To assess the efficacy of adding "Glycerine plus Honey" to standard management protocol,in terms of time to delay in oral mucositis $\geq$ grade 2 .

Methods

Hundred patients of oral cavity and oropharangeal cancers, planned for CRT (Dose:60-66Gy/30-33fractions/6 weeks) were randomized 1:1 to receive either home-made remedy made of "Glycerine plus Honey" added to the standard management protocol to prevent mucositis versus standard treatment alone.CTCAE v4 was used for assessing oral mucositis scores weekly.Chi square test was used to compare mucositis scores, weight loss,opoid use,ryles tube feeding and unplanned treatment breaks in each cohort.Independent T-test was used to compare means to assess the effect of treatment in delaying mucositis $\geq$ grade 2 .

Results

Significantly higher number of patients developed grade $\geq 2$ mucositis in control arm $[\mathrm{n}=43(86 \%)]$ compared to study arm $[\mathrm{n}=30(60 \%)]$ $(\mathrm{p}=0.003)$.CTCAE scores favored Glycerine plus honey at week 4 , and on last day of radiotherapy. Whereas,time to first occurrence of oral mucositis grade $\geq 2$ was $23.17( \pm 1.01)$ days for study arm [radiation dose $31.67 \mathrm{~Gy}( \pm$ $1.44)$ ], it was $20.65( \pm 0.8)$ days for control arm [radiation dose 28.14Gy $( \pm$ $1.16, \mathrm{p}=0.05)$.Study patients had lesser weight loss $(2.76 \mathrm{Kg})$ than control subjects $(3.9 \mathrm{Kg})$ with $\mathrm{p}=0.008$. There were significantly higher number of patients in control arm who required opoid analgesia,ryles tube insertion and had unplanned treatment breaks,compared to study arm.

Conclusions

Glycerine plus honey demonstrated superiority in delaying oral mucositis and the combination is safe and well tolerable.

eP207

DISTINGUISHING EPITHELIAL GROWTH FACTOR RECEPTOR TYROSINE KINASE INHIBITOR INDUCED O R A L M U C O S I T I S / T OMAT I T I S FROM PSEUDOMEMBRANOUS CANDIDIASIS: CASE SERIES

T. Yurikusa ${ }^{l}$, K. Furukawal

${ }^{T}$ shizuoka cancer centar, dentistry and oral surgery, Shizuoka, Japan

\section{Introduction}

Oral mucositis and stomatitis are relatively frequent with some targeted therapies, namely epithelial growth factor receptor tyrosine kinase inhibitor (EGFR-TKI).

\section{Objectives}

The purpose of this report is to show the importance of careful consideration in the administration of antifungal medications through two case studies of EGFR-TKI-induced oral mucositis/stomatitis.

\section{Methods}

Case 1: A 64-year-old woman with breast cancer was treated with trastuzumab and lapatinib. Within 3 weeks after start of treatment, the patient presented oral pain, diarrhea, and fever. The patient was hospitalized because of dysphagia due to Gr3 oral mucositis/stomatitis. There were multiple white lesions in the oral cavity that appeared to be pseudomembranous candidasis. Miconazole was prescribed by the attending physician, but the white lesion did not improve even after one week.

Case 2: A 88-year-old woman with EGFR mutation positive NSCLC was treated with osimertinib. In the 6th week after the start of osimertinib, the patient complained of oral pain and lip swelling. There were multiple white lesions in the oral cavity that appeared to be pseudomembranous candidasis. Miconazole was prescribed but the white lesions did not improve.

Results

The azole-based antifungal agents are metabolized by cytochrome P450 in the liver and may present drug interactions. Therefore, adverse drug 
reactions may be prolonged when administered concurrently with other drugs metabolized by the same mechanism. When using some EGFRTKIs, we should pay careful attention to the administration of antifungal medications.

\section{Conclusions}

EGFR-TKI-induced oral mucositis/stomatitis must be distinguished from pseudomembranous candidiasis.

\section{eP208}

AVAILABILITY AND ACCESS TO MEDICATIONS AND INTERVENTIONS RECOMMENDED BY THE MASCC/ESMO GUIDELINES IN THE COUNTRIES OF FORMER YUGOSLAVIA

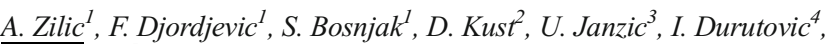 \\ M. Risteski ${ }^{5}$ \\ ${ }^{1}$ Institute for Oncology and Radiology of Serbia, Supportive oncology, \\ Belgrade, Serbia \\ ${ }^{2}$ University Hospital Center Sestre Milosrdnice, Department of Oncology \\ and Nuclear Medicine, Zagreb, Croatia \\ ${ }^{3}$ University Clinic Golnik, Department of Medical Oncology, Golnik, \\ Slovenia \\ ${ }^{4}$ Clinic for Oncology and Radiotherapy, Department of Medical \\ Oncology, Podgorica, Montenegro \\ ${ }^{5}$ PHI University Clinic of Radiotherapy and Oncology, Department of \\ radiotherapy and oncology, Skopje, FYR Macedonia
}

\section{Introduction}

Mucositis, as an oral and gastrointestinal mucosal damage, remains a highly significant toxicity of anticancer treatment. Guideline consistent prevention and treatment of mucositis depends on the availability and accessibility of medications and interventions.

\section{Objectives}

To evaluate availability and accessibility of medications/interventions recommended by the MASCC/ESMO mucositis guideline 2015. in the countries of former Yugoslavia: Serbia(SRB), Montenegro(MNE), Croatia(HRV), Macedonia(MKD),Bosnia and Herzegovina(BIH) and Slovenia(SVN).

Methods

Availability of medications was evaluated by the formulary availability and marketing authorization(registered or not). Accessibility was assessed by the coverage of medication/intervention by the National Health Insurance Fund (NHIF): as fully reimbursed or payed outof- pocket. Off-label use of medications is not reimbursed by the NHIF.

\section{Results}

Table 1.

\begin{tabular}{|c|c|c|c|c|c|c|c|}
\hline \multirow{7}{*}{$\begin{array}{l}\text { Medications and } \\
\text { interventions }\end{array}$} & & SRB & MNE & HRV & MKD & $\mathrm{BIH}$ & SVN \\
\hline & Amifostine & & & & & & \\
\hline & Octreotide & & & & & & \\
\hline & \begin{tabular}{|l|}
$\begin{array}{l}\text { Sucralfat } \\
\text { enemas }\end{array}$ \\
\end{tabular} & & & & & & \\
\hline & Sulfasalazine & & & & & & \\
\hline & Probiotic & & & & & & \\
\hline & $\begin{array}{l}\text { Hyperbaric } \\
\text { oxygen }\end{array}$ & & & & & & \\
\hline
\end{tabular}

Fully reimbursed Off- label
Not registered

Out of pocket
Not routine
Table 2.

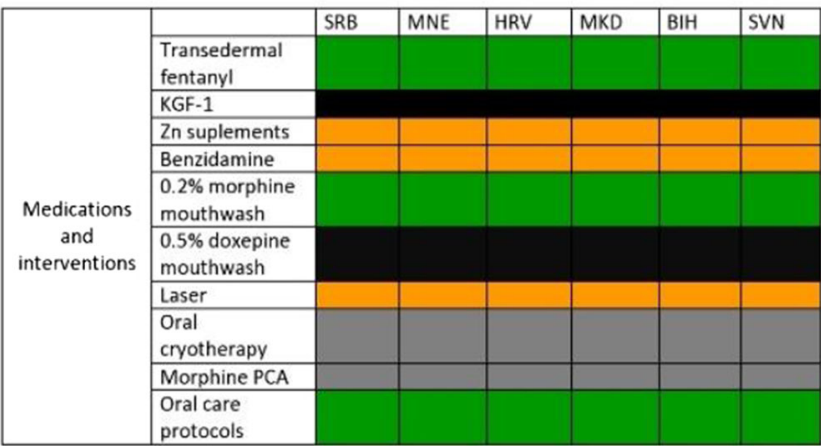

Fully reimbursed

Off- label

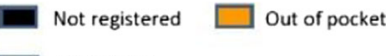

Gastrointestinal mucosistis:2/5 guideline-recommended medications are not available throughout the region, $3 / 5$ are not accessible due to their offlabel use $(2 / 5)$ or because of the lack of reimbursment (1/5) (Table 1.). Of special interest is the unaccess to octreotide needed for the treatment of potenially life threatening diarrhea.Oral mucositis:3/4 interventions can't be implemented, since oral cryotherapy and PCA morphine are not routinely used in the hospital setting and laser therapy is not reimbursed (Table 2.). 4/6 medications are not available. Opiods are widely available and accessible for transdermal use and as an oral solution.

\section{Conclusions}

There is no significant disparity in the availability and access to medications/interventions between countries of former Yugoslavia. Barriers in the availability and access to interventions and medications recommended by the guidelines preclude physicians in providing optimal care for patients with mucositis after cancer treatment in the region of former Yugoslavia.

\section{eP209}

PAIN DESCRIPTORS OF DOCETAXEL-ASSOCIATED ACUTE PAIN SYNDROME IN BREAST CANCER PATIENTS

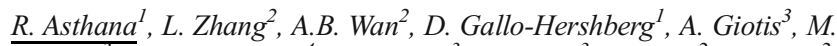
Pasetka $^{3}$, J. Van Draanen ${ }^{4}$, S. Goodall ${ }^{3}$, P.L. Diaz ${ }^{3}$, L. Drost $^{2}$, E. Chow ${ }^{2}$, C. De Angelis ${ }^{3}$

${ }^{1}$ University of Toronto, Pharmaceutical Sciences, Toronto, Canada

${ }^{2}$ Sunnybrook Odette Cancer Center, Radiation Oncology, Toronto, Canada

${ }^{3}$ Sunnybrook Odette Cancer Center, Pharmacy, Toronto, Canada

${ }^{4}$ University of British Columbia, Sociology, Vancouver, Canada

\section{Introduction}

Taxane Acute Pain Syndrome (TAPS) is defined as severe arthralgia and myalgia due to taxane chemotherapy that occurs 2-3 days after infusion, and lasts approximately 4 days.

\section{Objectives}

To assess pain descriptors used by patients during their experience of TAPS.

Methods

A clinical prospective cohort study was conducted on chemotherapy-naïve breast cancer patients who completed diaries on 3 consecutive docetaxel treatment cycles on days 1-7, 14, 21 (acute phase). Questionnaires to assess pain severity, descriptors of pain, and the interference in activities due to pain were adapted from the Brief Pain Inventory and the McGill Pain 
Questionnaire. Telephone questionnaire follow-up was completed at 1, 3, 6,9 and 12 months following treatment (delayed phase).

Results

The most commonly used descriptor for acute and chronic pain was "aching" (90-96\%). However, in the delayed phase of the study, "burning" (32-50\%), "radiating" (39-48\%), and "sharp" (40-69\%) were used more frequently. In both acute and chronic pain phases, most patients experienced moderate/severe pain regardless of the location. Pain in cycle 1 was predictive of pain in subsequent cycles $(p<0.0001)$. Pain in cycle 3 was predictive of chronic pain $(\mathrm{p}<0.002)$.

Conclusions

The subjective experience of TAPS varies from patient to patient. The descriptors used by patients may be reflective of the underlying mechanisms of TAPS. Based on the descriptors used by patients, we suspect that chemotherapy-induced pain (ChIP) from taxanes starts off as an acute inflammatory pain, which over time develops into neuropathic pain in a subgroup of patients.

eP210

NEUROIMMUNE CONSEQUENCES IN THE BRAIN AND SPINAL CORD OF MICE WITH ULCERATIVE COLITIS AND COLITIS-ASSOCIATED COLORECTAL CANCER

J. Bajic ${ }^{1}$, S. Mashtoub ${ }^{1,2,3}$, R. Safaeian ${ }^{4}$, L. Bobrovskaya ${ }^{5}, \underline{\text { G. }}$ Howarth $^{1,4,6}$, M. Hutchinson ${ }^{1,7}$

${ }^{T}$ The University of Adelaide, Physiology Department, Adelaide, Australia

${ }^{2}$ Women's and Children's Hospital, Department of Gastroenterology,

North Adelaide, Australia

${ }^{3}$ University of Western Australia, School of Medicine, Western Australia, Australia

${ }^{4}$ The University of Adelaide, School of Animal and Veterinary Sciences, Roseworthy, Australia

${ }^{5}$ The University of South Australia, School of Pharmacy and Medical Sciences, Adelaide, Australia

${ }^{6}$ Women's and Children's Hospital, Gastroenterology Department, North Adelaide, Australia

${ }^{7}$ The University of Adelaide, Australian Research Council Centre of Excellence for Nanoscale Biophotonics, Adelaide, Australia

\section{Introduction}

The risk of developing colorectal cancer (CRC) is higher in patients with ulcerative colitis (UC) and the pathogenesis of central comorbidities associated with both disorders is under investigated.

Objectives

We examined regional neuroimmunological consequences resulting from chronic gut inflammation in UC and colitis-associated CRC and hypothesized that glial dysregulation could contribute to central comorbidities associated with both disorders.

Methods

Female C57BL/6 mice ( $\mathrm{n}=8$ /group) were randomly allocated into three groups; saline, UC and CRC. UC was induced via ad libitum ingestion of $2 \% \mathrm{w} / \mathrm{v}$ dextran sulfate/sodium (DSS) in the drinking water every third week for seven weeks. Colitis-associated CRC was induced via single azoxymethane injection (day 0 ) followed by DSS/water cycles. UC group was euthanized at day 49 and the CRC group at day 62. CNS regions were isolated for western blot analysis using glial markers GFAP (astrocytes) and IBA1 (microglia).

Results

Colonic ulceration and tumour development was confirmed in UC and CRC groups (Mashtoub et al. J Gastro Hep (2016) 31:125). Hippocampal astrocytic expression was significantly elevated in both groups (UC; $\mathrm{p}=0.028$ and $\mathrm{CRC} ; \mathrm{p}=0.002$ ) and microglial expression increased in $\mathrm{CRC}$ rats $(\mathrm{p}<0.05)$ compared to vehicle controls. In the lumbar spinal region, GFAP expression also increased in CRC rats $(\mathrm{p}<0.05)$ compared to vehicle controls.

\section{Conclusions}

Glial activation contributes to neurodegenerative and pain disorders. Hippocampal glial activation may contribute to central comorbidities in $\mathrm{UC}$ and CRC whilst spinal changes may enhance pain signalling pathways. Future studies investigating glial morphological changes and proinflammatory influences will shed light on the interplay between mucosal injury and central comorbidities.

\section{eP211}

CANNABIS-RELATED COGNITIVE IMPAIRMENT: PROSPECTIVE EVALUATION OF POSSIBLE INFLUENCES ON CANCER PATIENTS DURING CHEMOTHERAPY TREATMENT

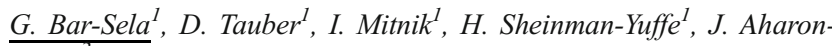 \\ Peretz $^{2}$ \\ ${ }^{1}$ Rambam health care campus, Oncology, Haifa, Israel \\ ${ }^{2}$ Rambam health care campus, Neuro-Cognitive Unit, Haifa, Israel
}

\section{Introduction}

In cancer patients, the use of medical cannabis has increased significantly during recent years. There is evidence that cannabis consumption may affect cognitive performance; however, this potential effect has not been investigated prospectively in cancer patients to date.

\section{Objectives}

We aimed to evaluate the effect of cannabis consumption on cognitive abilities as well as on symptom relief in cancer patients during chemotherapy treatment.

\section{Methods}

A prospective study was carried out on a group of 17 patients with cannabis treatment (case) paired to 17 patients without cannabis treatment (control). Participants completed self-reported questionnaires (The Hospital Anxiety and Depression Scale, Brief Fatigue Inventory, European Organization of Research and Treatment of Cancer core questions on the Quality of Life Questionnaire) and underwent the following neurocognitive tests: Montreal Cognitive Assessment (MoCA), Digit Symbol Substitution subtest (WAIS III) and Digital-Finger Tapping Test. The evaluation was conducted prior to the initiation of cannabis consumption and three months later during the period of cannabis use.

Results

Improvement in executive functioning was demonstrated in the case group. In aspects of symptoms, improvement in fatigue, appetite and sleep disorder was demonstrated after cannabis consumption. Patients consuming cannabis did not differ from the control group in cognitive functioning over three months of use. No significant cognitive decline was observed in either group over time.

\section{Conclusions}

These preliminary findings suggest that the short-term use of cannabis during chemotherapy treatment improved disease-related symptoms and did not affect cognitive skills in cancer patients.

\section{eP212}

\section{LOW DOSE METHADONE FOR CHEMOTHERAPY-INDUCED PERIPHERAL NEUROPATHY (CIPN): TWO CASE REPORTS}

\author{
E. Brawner ${ }^{1}$, J. Tsukanov ${ }^{I}$, E. Del Fabbro ${ }^{1}$ \\ ${ }^{7}$ Virginia Commonwealth University, Palliative Medicine, Richmond \\ Virginia, USA
}

\section{Introduction}

Chemotherapy-induced peripheral neuropathy (CIPN) manifests as pain, weakness, and numbness, usually in hands or feet. First line therapies may not be tolerated due to their side effect profile and patient factors i.e. hepatic/ renal function. Methadone, a mu-agonist, an NMDA receptor 
antagonist, and reuptake inhibitor of norepinephrine and serotonin, is safe in renal dysfunction, and an undervalued treatment option.

\section{Objectives}

Identify the potential of low dose methadone for management of CIPN, highlighting its use in renal dysfunction.

\section{Methods}

A) 59 yom with Multiple Myeloma, CKD, and severe CIPN leading to cessation of chemotherapy, decreased activity and insomnia. He obtained no relief from topical medications and did not tolerate duloxetine (mood disturbance). Gabapentin improved pain and sleep, but patient developed myoclonus. Patient was started on methadone $2.5 \mathrm{mg}$ BID and gabapentin was weaned.

B) 71 yof with small cell lung cancer stopped chemotherapy secondary to severe CIPN. She did not tolerate gabapentin, did not respond to amitriptyline, oxycodone, pregabalin and duloxetine. Pt was started on methadone $5 \mathrm{mg} \mathrm{BID}$ and pregabalin was weaned.

Results

A) Pain score decreased from 8 to 3 within two weeks, accompanied by improved function, quality of life and resolution of myoclonus.

B) Pain score decreased from 10 to 0 with improvement in function and quality of life within 1 week.

Conclusions

Low dose methadone merits consideration as treatment for CIPN in patients who have failed first line therapy or have renal dysfunction.

\section{eP213}

THE LIVED EXPERIENCE OF CHEMO BRAIN IN EARLY STAGE BREAST CANCER IN WOMEN 50 AND UNDER

P. Gibbons ${ }^{1}$

${ }^{T}$ MSKCC, Vascular Access, New York, USA

\section{Introduction}

Cognitive deficits (chemo brain) have been shown to affect those who have undergone treatment with chemotherapy. Women in this study who were treated for early stage breast cancer experienced chemo brain and described it as an inability to concentrate, remember details, and complete familiar tasks as quickly as they had done prior to their cancer diagnosis and treatment. They complained of short term memory loss, difficulty concentrating, struggling to make simple decisions, difficulty finding the right words, trouble multi-tasking and feelings of mental dullness. Learning about the experience of chemo brain may ultimately improve the lives of women who experience it.

Objectives

The purpose of this phenomenological study was to explore the lived experience of the chemo brain after undergoing chemotherapy for breast cancer.

\section{Methods}

van Manen's descriptive phenomenological methodology served to explore an everyday life understanding of the lived experience of chemo brain in 6 women age 50 and under with early stage breast cancer.

Results

The recurrent themes identified in this study were (1) Struggles with day to day life (2) Emotions related to diagnosis and treatment, (3) Fatigue (4) Coping and support, and (5) Feeling grateful. Findings of this study confirm the existence of cognitive changes during and after chemotherapy as well as describe the effects on daily life including fatigue, emotions, and the importance of support.

Conclusions

By understanding specific areas of cognitive dysfunction affected by chemotherapy, like memory, concentration, and task completion, findings of this study provide valuable information to cancer patients, as well as healthcare providers, including nurses.

\section{eP214}

HAND OR FOOT COVERING DOES NOT AFFECT EFFICACY OF CRYOTHERAPY FOR PREVENTION OF OBJECTIVE AND SUBJECTIVE SYMPTOMS OF CHEMOTHERAPY-INDUCED NEUROPATHY; DATA FROM THE SELF-CONTROLLED TRIAL

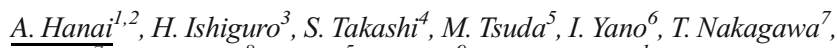
S. Imai', Y. Hamabe ${ }^{8}$, M. Toi ${ }^{5}$, H. Arai ${ }^{9}$, T. Tsuboyama ${ }^{1}$

${ }^{1}$ Kyoto University Graduate School of Medicine, Rehabilitation, Kyoto, Japan ${ }^{2}$ National Cancer Center, Center for Public Health Sciences, Tokyo, Japan

${ }^{3}$ International University of Health and Welfare- School of Medicine, Clinical Oncology, Nasushiobara, Japan

${ }^{4}$ Tokyo University of Science, Information and Computer Technology, Tokyo, Japan

${ }^{5}$ Kyoto University Graduate School of Medicine, Breast Surgery, Kyoto, Japan ${ }^{6}$ Kobe University, Pharmacokinetics and Pharmaceutics, Kobe, Japan

${ }^{7}$ Kyoto University Hospital, Clinical Pharmacology and Therapeutics, Kyoto, Japan

${ }^{8}$ Kyoto University Hospital, Nursing, Kyoto, Japan

${ }^{9}$ National Center for Geriatrics and Gerontology, Center for Gerontology and Social Science, Obu, Japan

\section{Introduction}

Chemotherapy-induced peripheral neuropathy (CIPN) is a frequent and disabling side effect of cancer treatment. In a self-controlled trial, we evaluated preventive effects of cryotherapy and concluded it was useful for preventing both the objective signs and subjective symptoms of CIPN. The patients used coverings to alleviate the cold discomforts, and there was no dropout due to cold intolerance. However, coverings might interfere with the skin temperature falls and the effects of cryotherapy.

\section{Objectives}

To evaluate the impact of coverings on the effects of cryotherapy.

Methods

Breast cancer patients who were planned to undergo weekly paclitaxel $\left(80 \mathrm{mg} / \mathrm{m}^{2}\right.$ for 1 hour) for an at least cumulative dose of $960 \mathrm{mg} / \mathrm{m}^{2}$. Each patient wore frozen gloves and socks on the dominant hand and foot, and the non-dominant side acted as the untreated control. The nonwoven fabric covers were applied when the patients complained of cold pain. The symptoms of CIPN (any grade) were assessed by Patient Neuropathy Questionnaire (subjective CIPN), tactile sensitivity (objective CIPN) or performance speed. The patients were divided into covering or noncovering group then CIPN symptoms were compared.

Results

In the subgroup analysis with the hazard ratio $(\mathrm{N}=40)$, cryotherapy with covering was also sufficient to prevent CIPN (Fig. 1).

\section{Fig 1. Subgroup Analysis}

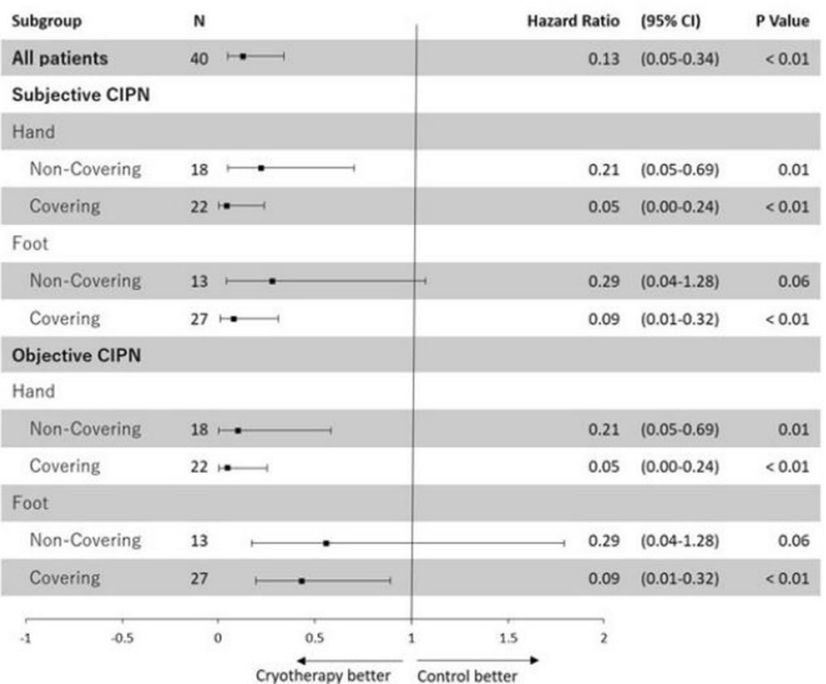


The scores of the performance speed also tend to be better in the both covering and non-covering group than the control group (Fig. 2).

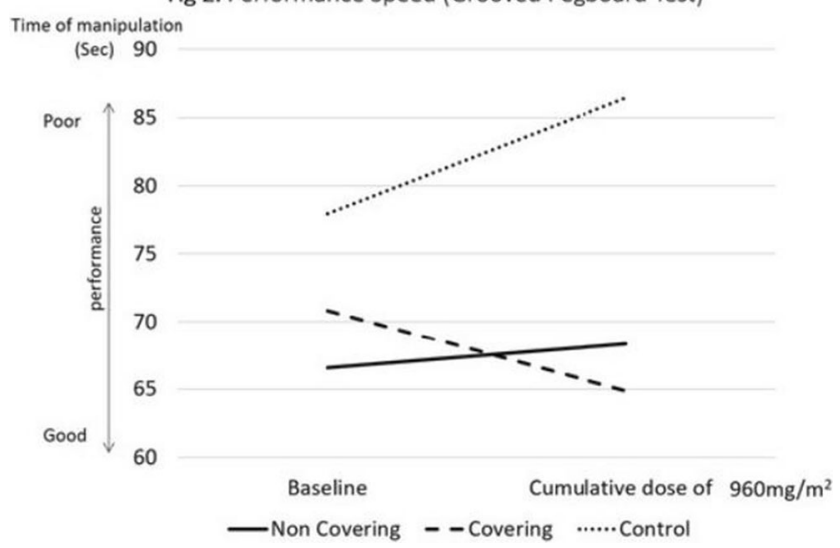

\section{Conclusions}

Coverings did not reduce the effectiveness of cryotherapy. It may suggest that cryotherapy with more comfortable skin temperature will be as effective as standard cryotherapy.

\section{eP215}

COGNITIVE FUNCTION AFTER RADIOTHERAPY FOR PRIMARY CNS TUMORS

D. Ivanoval

${ }^{T}$ Tokuda Hospital Sofia, Radiation Oncology, Sofia, Bulgaria

\section{Introduction}

The effect of radiotherapy on the long-term cognitive performance of patients treated for intracranial neoplasms is a major concern to clinicians and patients, particularly as long-term survival or cure is a possibility.

\section{Objectives}

To assess the neurocognitive effects of cranial radiotherapy on these patients, we analyzed cognitive performance data collected in a prospective clinical trial.

\section{Methods}

A total of 37 patients treated with radiotherapy for benign or malignant primary CNS tumors were included. The patients were evaluated with an extensive battery of psychometric tests (MMSE, BVRT,CERAD, MoCa, IST, TMT A and B) to quantify changes in verbal learning, memory, and executive function at baseline (before RT) and at approximately 3-month intervals for as long as 3 years after completing RT. To allow patients to serve as their own controls, cognitive performance was evaluated as change in scores over time. Data regarding cognitive function, fatigue, quality of life, and standard patient reported outcomes was collected to characterize the daily impact of treatment.

\section{Results}

Baseline test scores were below average compared with age-specific norms. At the second evaluation, the groups' mean test scores were higher than their initial performances on all psychometric measures, although the improvement was not statistically significant. Neurologic worsening due to disease recurrences was associated with worsening of the cognitive results.

Conclusions

Cognitive function was stable after RT in these patients evaluated prospectively during 3 years of follow-up. Slight improvements in some cognitive areas are consistent with practice effects attributable to increased familiarity with test procedures and content.
eP216

RELIABILITY AND VALIDITY OF THE KOREAN VERSION OF THERAPY INDUCED NEUROPATHY ASSESSMENT SCALE (TINAS) FOR COLORECTAL CANCER PATIENTS

\author{
H.J.jung ${ }^{1}$, K. Kim ${ }^{2}$, S. Chu ${ }^{2}$ \\ ${ }^{1}$ Severance hospital, nursing, Seoul, Republic of Korea \\ ${ }^{2}$ Yonsei university, nursing, Seoul, Republic of Korea
}

\section{Introduction}

With the increasing incidence of colorectal cancer in Korea, chemotherapy-induced peripheral neuropathy is an irreversible side effect that is closely related with patients' quality of life.

\section{Objectives}

The Therapy-Induced Neuropathy Assessment Scale (TINAS) was designed to measure the severity and course of neuropathy across various cancer treatments. The aim of this study was to evaluate the reliability and validity of the Korean version of the TINAS.

\section{Methods}

The original TINAS was translated into Korean through the process of forward and back translation. Subsequently, self-questionnaire surveys were conducted. A total of 146 patients with colorectal cancer was recruited in a university-affiliated hospital in Korea. For internal consistency reliability, Cronbach's alpha coefficient score was used; for external validity, confirmatory factor analysis (CFA) was conducted. Correlations between the TINAS and the Functional Assessment of Cancer TherapyColorectal (FACT-C) scale and MD Anderson Symptom Inventory (MDASI) were used to test the criterion validity of the TINAS.

\section{Results}

The Korean version of the TINAS showed reliable internal consistency with a Cronbach's alpha coefficient score for the total scale of .89. Factor loadings of the 13 items on the two sub $\neg$ scales ranged from .60 to .77 . The two subscales model was validated using CFA (RMSEA<.05, CFI $>$.9). The TINAS score was negatively associated with the quality of life of patients measured using the FACT-C scale $(\mathrm{r}=-.310, \mathrm{p}<.001)$, and positively associated with the MDASI score $(r=.682, p<.001)$.

\section{Conclusions}

The Korean version of the TINAS scale showed adequate reliability and validity in this study.

\section{eP217}

MANAGEMENT CHALLENGES IN A PATIENT WITH AGITATED DELIRIUM AND BENZODIAZEPINE WITHDRAWAL

S. $\mathrm{Kim}^{1}$, A. Haider ${ }^{2}$

${ }^{T} M D$ Anderson Cancer Center, Department of Palliative- Rehabilitation and Integrative Medicine, Houston, USA

${ }^{2}$ MD Anderson Cancer Center, Palliative-Rehabilitation and Integrative Medicine, Houston, USA

\section{Introduction}

Delirium remains underdiagnosed in terminally ill patients, which may result in inadequate treatment. The treatment of delirium in patients with a history of benzodiazepine abuse has not been well studied. We report a case of terminal delirium in an advanced cancer patient with a history of alprazolam abuse who responded to a regimen of chlorpromazine and lorazepam.

\section{Objectives}

Identify and treat Delirium in setting of benzodiazapine withdrawal.

\section{Methods}

N/A

Results

A 48-year-old man with a history of recurrent metastatic adenoid cystic carcinoma of the head and neck presented with confusion and recurrent 
falls. During the patient's hospitalization, he developed worsening delirium. Haloperidol was initiated intravenously as needed; however, even after receiving multiple doses, the patient's symptoms were not controlled. It was then discovered that the patient had a long history (years) of alprazolam abuse, although at an unknown dose as patient's delirium limited further evaluation. We then combined scheduled lorazepam and haloperidol, as we suspected benzodiazepine withdrawal may have been contributing to the patient's delirium. Addition of lorazepam appeared to improve control of agitation; however, both the nursing staff and family at the bedside reported worsening agitation after haloperidol administration. Haloperidol was exchanged for chlorpromazine. We continued to titrate chlorpromazine and lorazepam which provided good control of patient's psychomotor agitation.

\section{Conclusions}

This case illustrates the importance of determining the optimal drug combination in managing delirium, especially in patients with a history of benzodiazepine abuse. Neuroleptics remain the preferred class of drug but use of concurrent benzodiazepine in this patient population must be further investigated.

\section{eP218}

DELIRIUM IN ELDERLY PATIENTS UNDERGOING CHEMOTHERAPY FOR SOLID CANCERS AND LYMPHOMA

\author{
Y. Kirkova $^{1,2}$, Y.J. Hwang ${ }^{3}$ \\ ${ }^{T}$ Cleveland Medical Center, Medicine/ Geriatrics and Palliative Care, \\ Cleveland-OH, USA \\ ${ }^{2}$ Medstar Georgetown University Hospital, Medicine/Palliative Care, \\ Washington DC, USA \\ ${ }^{3}$ Case Western Reserve University School of Medicine, Medicine, \\ Cleveland- $\mathrm{OH}, \mathrm{USA}$
}

\section{Introduction}

Delirium in geriatric cancer patients is poorly understood.

Objectives

To determine the incidence and factors associated with delirium in elderly patients undergoing chemotherapy for solid cancers.

Methods

A retrospective chart review of consecutive patients $\geq 65$ years old was performed to identify 20 individuals who were admitted in 2015 with "delirium", "encephalopathy", "confusion", or "altered mental status" within 30 days of chemotherapy or chemoradiation for solid cancers and lymphomas. Descriptive and matched pair statistics compared demographics, chemotherapy, medications, comorbidities, and laboratory data between the identified patients and their controls matched by age, gender, and cancer diagnosis.

\section{Results}

We reviewed 123 patients hospitalized in an academic comprehensive cancer center. Median (range) age was $71(65$ to 89$)$. $60 \%$ were women. Most common cancers (\%) were: GI (22), Lymphoma (20), GU (18), Lung (15) and Breast (12). The most common admissions were: chemotherapy $(22 \%)$ and infection $(21 \%)$. Delirium incidence was $7.3 \%(\mathrm{~N}=$ 9/123). Five were men. Compared to matched controls, patients with delirium had a higher BUN, more acute kidney injury (AKI) and hyponatremia. There were trends in worse ECOG PS, lower Hct, Hgb, elevated ALT, AST, and more CAD, CKD stage III, depression, diabetes, and UTI.

Conclusions

Low delirium incidence in cancer patients $\geq 65$ years old within 30 days of chemotherapy was most likely due to delirium underreporting and excellent patient selection prior to chemotherapy. The most common admission reasons were infections and chemotherapy initiation. Elevated BUN, $\mathrm{AKI}$ and hyponatremia were significantly associated with delirium incidence, trends in worse ECOG, anemia, transaminitis and more comorbidities were observed.
eP219

GENE EXPRESSION AND PATHWAY ANALYSIS OF CHRONIC PACLITAXEL-INDUCED PERIPHERAL NEUROPATHY IN BREAST CANCER SURVIVORS

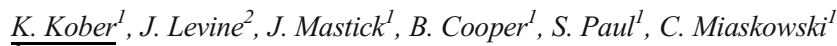
${ }^{T}$ School of Nursing- University of California San Francisco, Physiological Nursing, San Francisco, USA

${ }^{2}$ School of Medicine-University of California San Francisco, Department of Medicine, San Francisco, USA

\section{Introduction}

The prevalence of chemotherapy-induced peripheral neuropathy (CIPN) in cancer survivors is as high as $60 \%$. CIPN is the most debilitating symptom reported oncology patients and can lead to treatment discontinuation. No interventions are available to treat CIPN. A critical barrier to the development of efficacious interventions is the lack of understanding of the mechanisms underlying CIPN. In this pilot study, we evaluated the molecular characteristics associated with paclitaxel-induced peripheral neuropathy (PIPN).

Objectives

Compare differential gene expression (GE) between breast cancer (BC) survivors with and without PIPN.

\section{Methods}

GE in peripheral blood was assayed using RNA-seq. Differentially expressed (DE) genes and pathways were identified between survivors who received paclitaxel and $\operatorname{did}(n=25)$ and did not $(n=25)$ develop PIPN. Results

BC survivors with PIPN were significantly older; more likely to be unemployed; reported lower alcohol use; had a higher BMI, and poorer functional status; and had a higher number of lower extremity sites with loss of light touch, cold, and pain sensations, and higher vibration thresholds. No between group differences were found in the total dose of paclitaxel received or in the percentage of patients who had a dose reduction or delay due to PIPN. We found 140 DE genes and 21 DE pathways (e.g., signaling (PI3K/AKT, mTOR), oxidative stress (p53 signaling), and mitochondrial impairment (Mitophagy - animals)).

\section{Conclusions}

Chronic PIPN is associated with GE changes in peripheral blood. Our findings support previously hypothesized mechanisms of PIPN and suggest additional genes for validation and potential therapeutic targets.

\section{eP220}

COGNITIVE ASSESSMENT OF BRAZILIAN AND DANISH PATIENTS WITH METASTATIC CANCER: A PSYCHOMETRIC ANALYSIS OF INSTRUMENTS.

G.P. Kurita $^{1}$, J. Matuoka ${ }^{2}$, M. Nordly ${ }^{3}$, B. dos Santos ${ }^{4}$, M. Sandvad ${ }^{5}, J$. Højsted $^{6}$, L. Lundorff ${ }^{7}$, P. Sjøgren ${ }^{8}$, C.A. de Mattos-Pimenta ${ }^{4}$

${ }^{1}$ Rigshospitalet Copenhagen University Hospital, Palliative Research Group- Department of Oncology and Multidisciplinary Pain Centre, Copenhagen, Denmark

${ }^{2}$ University of Sao Paulo, University Hospital and School of Nursing, Sao Paulo, Brazil

${ }^{3}$ Copenhagen University Hospital, Palliative Research GroupDepartment of Oncology, Copenhagen, Denmark

${ }^{4}$ University of Sao Paulo, School of Nursing, Sao Paulo, Brazil

${ }^{5}$ Rigshospitalet Copenhagen University Hospital, Palliative Research Group-Department of Oncology, Copenhagen, Denmark

${ }^{6}$ Rigshospitalet Copenhagen University Hospital, Multidisciplinary Pain Centre, Copenhagen, Denmark

${ }^{7}$ Uddevalla Hospital, Department of Palliative Care, Uddevalla, Sweden ${ }^{8}$ Palliative Research Group- Department of Oncology- Rigshospitalet Copenhagen University Hospital, Department of Clinical MedicineFaculty of Health and Medical Sciences- University of Copenhagen, Copenhagen, Denmark 


\section{Introduction}

Cognitive dysfunction is subjective and sparsely assessed in patients with cancer.

\section{Objectives}

To analyze the performance of Brazilian and Danish patients with metastatic cancer on four neuropsychological tests and the cut-offs for cognitive dysfunction.

\section{Methods}

Convenience sample with 258 adult patients and 204 healthy controls (BR: 178 patients, 128 controls; DK: 80 patients, 76 controls), schooling $\geq 6$ years (Jul 2010-Nov 2015). Continuous Reaction Time (CRT), Finger Tapping Test (FTT), Digit Span Test (DST), and Trail Making Test-part B (TMTB) were analyzed regarding criterion validity, predictive value, sensitivity and specificity. Other variables: age, schooling, Karnofsky Performance Scale (KPS), pain, sleep, anxiety, depression, income, sensation of rest, and Mini Mental State Examination (MMSE).

\section{Results}

Significant weak correlations between patients' cognitive performance and age, schooling, KPS, pain, anxiety, depression, income, and MMSE. Predictive value analyses showed in the Brazilian group higher income associated with better performance on CRT $(P<0.018)$ and worse depression slightly associated with worse performance on DST backward $(P=0.029)$, while Danish group presented worse anxiety associated with worse performance on DST backward $(P=0.007)$ and higher age associated with worse performance on TMTB $(P=0.021)$. The tests were at most poor to differentiate patients and controls (ROC curves $\leq 0.69)$ and at most fair to predict MMSE score $\leq 26$ (ROC curves $\leq 0.74$ ).

Conclusions

Patients' cognitive performance was weakly related to sociodemographic and clinical variables. Income, age, depression and anxiety had different effects when comparing the nationalities. Further studies are necessary to confirm/understand these differences and to determine the tests cut-offs.

\section{eP221}

PERSISTENT CHEMOTHERAPY-INDUCED NEUROPATHY AMONG WOMEN WITH BREAST CANCER RECEIVING NEOADJUVANT CHEMOTHERAPY: ASSOCIATION WITH SLEEP QUALITY, DEPRESSION AND QUALITY OF LIFE

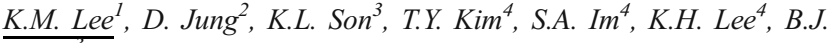 \\ $\mathrm{Hahm}^{3}$
}

${ }^{1}$ Seoul National University Hospital, Public Health and Medical Service, Seoul, Republic of Korea

${ }^{2}$ National Institute of Science and Technology, Department of Human Factors Engineering, Ulsan, Republic of Korea

${ }^{3}$ Seoul National University Hospital, Department of Neuropsychiatry, Seoul, Republic of Korea

${ }^{4}$ Seoul National University Hospital, Department of Internal Medicine, Seoul, Republic of Korea

\section{Introduction}

Chemotherapy-induced peripheral neuropathy (CIPN) is a common adverse reaction of chemotherapy, especially with taxanes. CIPN has been reported to have negative effects on quality of life and functional capacity, but the effects of persistent CIPN are not well understood.

Objectives

This study evaluates the impacts of CIPN and persistent CIPN on sleep quality, anxiety, depression and quality of life among women with breast cancer receiving neoadjuvant chemotherapy with docetaxel.

\section{Methods}

In this prospective study, participants reported neuropathic symptoms of tingling/numbness at baseline, at the end of chemotherapy, and at 8 months after completion of chemotherapy. Persistent CIPN was defined as CIPN occurring in the last chemotherapy cycle and also persisting over 8 months after completion of chemotherapy. Sleep quality, anxiety, depression and quality of life were assessed serially using the Pittsburgh Sleep Quality Index (PSQI), the Hospital Anxiety and Depression Scale (HADS) and the Functional Assessment of Cancer Therapy-Breast (FACT-B).

Results

Among 146 participants, $67(45.9 \%)$ experienced CIPN and 26 $(17.8 \%)$ reported persistent CIPN. On multivariate linear regression analyses, CIPN was significantly associated with PSQI ( $\beta=0.28, \mathrm{p}$ $<0.001)$ at the end of chemotherapy, adjusted for baseline PSQI. Persistent CIPN was significantly associated with depression subscale of HADS $(\beta=0.38, p=0.001)$ and FACT-B $(\beta=0.28, p=0.006)$ at 8 months after completion of chemotherapy, adjusted for each baseline variables.

\section{Conclusions}

A substantial proportion of women with breast cancer receiving neoadjuvant chemotherapy reported persistent CIPN with a significant negative impact on mood and quality of life.

\section{eP222}

\section{LONG TERM IMPROVEMENTS IN CHEMOTHERAPY INDUCED PERIPHERAL NEUROPATHY- OBJECTIVE IMPROVEMENT OR PATIENT ADAPTATION?}

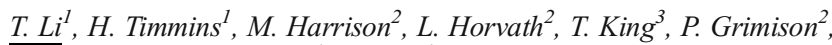 \\ D. Goldstein ${ }^{4}$, M. Kiernan ${ }^{1}$, S. Park ${ }^{1}$ \\ ${ }^{1}$ University of Sydney, ForeFront Clinic, Camperdown, Australia \\ ${ }^{2}$ Chris O'Brien Lifehouse, Oncology, Camperdown, Australia \\ ${ }^{3}$ Royal Prince Alfred Hospital, Haematology, Camperdown, Australia \\ ${ }^{4}$ Prince of Wales Hospital, Oncology, Randwick, Australia
}

\section{Introduction}

Chemotherapy-induced peripheral neuropathy is a disabling side effect that persists and affects cancer survivors' quality of life. Patient reported outcomes (PROs) are increasingly recognised as important in evaluating the impact of neuropathy. However, questions remain on whether patient reported improvements in the long-term reflect objective improvements or patient adaptation.

\section{Objectives}

To investigate whether sensory symptom improvement over time as described by a validated PRO questionnaire (FACT-GOG NTX) was reflected in objective tasks assessing sensory function of the upper (grating orientation task (GOT)) and lower limbs (2-point discrimination task (2PD)).

\section{Methods}

Forty-two patients (10 males; $54.4 \pm 11.9$ years) were assessed prospectively prior to neurotoxic chemotherapy (taxanes, platinums, or bortezomib), and at 3-months and 9-months post completion.

\section{Results}

At 3 -months post completion (mean $=3.0 \pm 0.8$ months), patients reported significantly more numbness and tingling in the hands (difference $=0.71$, $\mathrm{p}<0.01$ ) and feet (difference $=1.62, \mathrm{p}<0.01$ ) than at baseline. These results were reflected in objective tasks, demonstrated by significant decline in GOT (difference $=0.39, \mathrm{p}=0.01)$ and 2PD $($ difference $=2.35, \mathrm{p}<0.01)$ performance.

At 9-months post completion (mean=9.5 \pm 2.9 months) patients reported significantly less numbness and tingling in the hands (difference $=0.40$, $\mathrm{p}=0.02$ ) and feet (difference $=0.43, \mathrm{p}<0.01$ ) than at 3-months follow-up (Figure 1.). However objective tasks demonstrated no significant improvements $(\mathrm{GOT}$ (difference $=0.08, \mathrm{p}=0.20), 2 \mathrm{PD}$ (difference $=0.23$, $\mathrm{p}=0.29)$. 


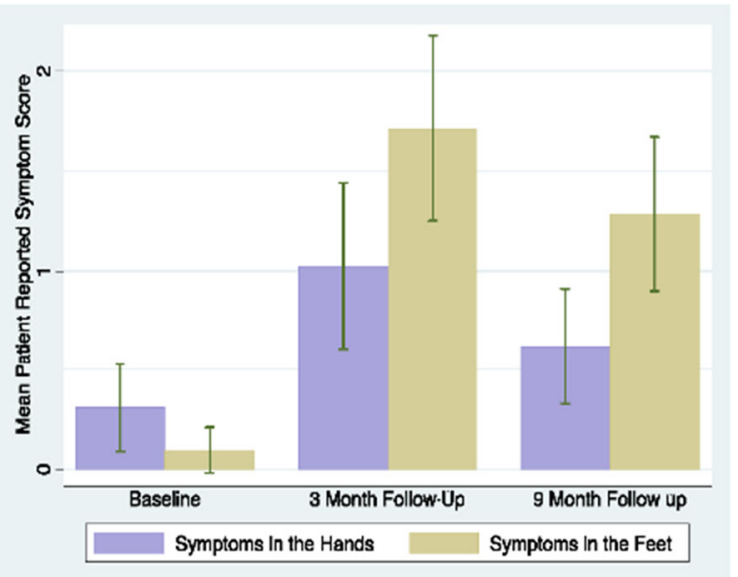

Figurel. Mean score (= standard error) of neuropathy symptoms (0-4) on the FACT-GOG NTX at 3 timcponts.

\section{Conclusions}

This study determined that patient reported improvements result from symptom adaptation rather than objective physiological improvements. Consequently, PROs should be used alongside objective measures to both capture patient experience and to quantify neurological deficits.

eP223

BGP-15 PREVENTS GASTROINTESTINAL SIDE-EFFECTS INDUCED BY OXALIPLATIN, BUT EXACERBATES SIDEEFFECTS OF 5-FU TREATMENT

R.M. McQuade ${ }^{1,2}$, V. Stojanovska ${ }^{2,3}$, A.C. Petersen ${ }^{4,5}$, R. Abalo $^{6}$, J.C. Bornstein', E. Rybalka ${ }^{2,4,5}$, K. Nurgali ${ }^{2}$

${ }^{1}$ Florey Institute for Neuroscience and Mental Health, Digestive Physiology and Nutrition Laboratory, Melbourne, Australia

${ }^{2}$ Victoria University, Centre for Chronic Disease, Melbourne, Australia

${ }^{3}$ Hudson Institute of Medical Research, The Ritchie Centre, Melbourne, Australia

${ }^{4}$ Victoria University, Institute of Sport- Exercise \& Active Living ISEAL, Melbourne, Australia

${ }^{5}$ Western Health, Australian Institute of Musculoskeletal Science AIMSS, Melbourne, Australia

${ }^{6}$ Universidad Rey Juan Carlos, Área de Farmacología y Nutrición y Unidad Asociada al Instituto de Química Médica IQM y al Instituto de Investigación en Ciencias de la Alimentación CIAL del Consejo Superior de Investigaciones Cientificas CSIC, Madrid, Spain

${ }^{7}$ Melbourne University, Department of Physiology, Melbourne, Australia

\section{Introduction}

Gastrointestinal (GI) side-effects of chemotherapy are a significant clinical hurdle resulting in dose reductions, delays and cessation of treatment, presenting a constant challenge in efficient and tolerable management of cancer. Previous studies have demonstrated that enteric neuropathy is a key contributor to the pathophysiology of chemotherapy-induced GI dysfunction.

Objectives

BGP-15 is a novel cytoprotective compound inhibiting poly(ADP-ribose) polymerase (PARP). PARP inhibitors have been reported to prevent chemotherapy-induced sensory peripheral neuropathy and are in clinical trials for various cancers. In this study we investigated the potential of BGP-15 to alleviate oxaliplatin (OXL) and 5-fluorouracil (5-FU)-induced enteric neuropathy and GI dysfunction.

Methods

Balb/c mice received either OXL (3mg/kg) or $5-\mathrm{FU}(23 \mathrm{mg} / \mathrm{kg})$ with and without BGP-15 $(15 \mathrm{mg} / \mathrm{kg})$ tri-weekly for 14 days. GI transit was analysed via in vivo $\mathrm{x}$-ray imaging prior to and following treatment.
After treatment colons were collected for assessment of ex vivo colonic motility, neuronal mitochondrial superoxide and cytochrome $c$ levels and immunohistochemical analysis of myenteric neurons.

Results

BGP-15 co-treatment ameliorated oxidative stress, increased enteric neuronal survival and alleviated GI dysfunction induced by OXL, but had no beneficial effect when administered in conjunction with 5-FU. In fact, BGP-15 exacerbated already present colonic inflammation and worsened GI dysfunction in 5-FU-treated mice, triggering mitochondrial superoxide production and membrane depolarisation in myenteric neurons.

\section{Conclusions}

This is the first study to investigate the impact of PARP inhibition on chemotherapy-induced neuropathy and gut function. The results of this work may have a profound impact on the clinical application of PARP inhibitors, particularly in cases where colonic inflammation is evident.

eP224

A CROSS-SECTIONAL EXAMINATION OF EXTRAPYRAMIDAL SIDE-EFFECTS (EPSE) IN A SPECIALIST PALLIATIVE MEDICINE INPATIENT UNIT

H. O'Brien ${ }^{1}$, F. Kiely ${ }^{2}$, A. Barry ${ }^{2}$

${ }^{T}$ Marymount University Hospital and Hospice- Curraheen- Cork, Palliative Medicine, Cork, Ireland

${ }^{2}$ Marymount University Hospital and Hospice-Cork- Ireland, Palliative Medicine, Cork, Ireland

\section{Introduction}

Extrapyramidal side-effects (EPSE) are serious potentially reversible side-effects of anti-psychotic and other medications that can cause distress for patients. A core principle of palliative care involves optimising quality of life. If side-effects of medications are burdensome it is imperative that we address this issue.

\section{Objectives}

The aim of the study was to determine and describe the burden of extrapyramidal side-effects (EPSE) in a specialist inpatient unit

Methods

Consenting patients who met inclusion criteria were assessed for EPSE with two validated screening tests, the Modified Simpson-Angus Scale (MSAS) and Barnes Akathesia Rating Scale (BARS). Additional demographic data was collected including medications associated with EPSE, previous history of EPSE and known risk factors that may predispose a patient to EPSE.

\section{Results}

$16(43 \%)$ inpatients met inclusion criteria. $11(68 \%)$ patients were taking regular medications associated with EPSE. Of those 4 (25\%) were taking $>/=2$ medications associated with EPSE. The MSAS revealed $8(50 \%)$ scored $<3,7(44 \%)$ scored 3-5 and $1(6 \%)$ scored 6-11. Seven patients had at least 1 "not-ratable score". In BARS (sitting+/-standing) $15(94 \%)$ scored $0 / 5,1(6 \%)$ scored $1 / 5$. Two $(12.5 \%)$ participants could stand for two minutes to complete the BARS.

\section{Conclusions}

$50 \%$ screened positive for EPSE. The complete BARS was unsuitable for most participants. The MSAS while allowing a notrateable score may underestimate EPSE. The frailty of an inpatient population impacts on applicability of screening tools and may therefore underestimate the burden of the problem in this population. Development of a population-specific screening tool warrants further investigation.

\section{eP225}

MORNING CHRONOTYPE IS A PROTECTIVE FACTOR AGAINST CHEMOTHERAPY-INDUCED PERIPHERAL NEUROPATHY IN WOMEN WITH BREAST CANCER 


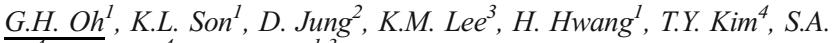
$\mathrm{Im}^{4}$, K.H. Lee, B.J. Hahm ${ }^{1,3}$

${ }^{I}$ Seoul National University Hospital, Department of Neuropsychiatry, Seoul, Republic of Korea

${ }^{2}$ Ulsan National Institute of Science and Technology, Department of Human Factors Engineering, Ulsan, Republic of Korea

${ }^{3}$ Seoul National University College of Medicine, Department of Psychiatry and Behavioral Sciences, Seoul, Republic of Korea

${ }^{4}$ Seoul National University Hospital, Department of Internal Medicine, seoul, Republic of Korea

\section{Introduction}

Breast cancer is one of the most frequently diagnosed cancers among women, and even in cases of early stage breast cancer which are operable, adjuvant chemotherapy is almost inevitable. Adjuvant chemotherapy often causes peripheral neuropathy, which have an adverse impact on quality of life. However, psychiatric factors associated with development of chemotherapy-induced peripheral neuropathy (CIPN) are still undetermined.

\section{Objectives}

The purpose of this study was to investigate whether chronotype was associated with the incidence of CIPN.

\section{Methods}

A total of 128 premenopausal women with early-stage breast cancer awaiting adjuvant chemotherapy after surgery without peripheral neuropathy were included. The presence of CIPN was defined as a response of 3 or higher on a peripheral neuropathy subscale in the M. D. Anderson Symptom Inventory. Candidate psychiatric factors associated with CIHF were assessed before chemotherapy, using the Composite Scale of Morningness, a measure of chronotype, the Pittsburgh Sleep Quality Index, and the Hospital Anxiety and Depression Scale.

\section{Results}

CIPN occurred in $41.4 \%$ of participants. The morning type was inversely associated with CIPN (reference: intermediate type, odds ratio [OR], 0.32 ; $95 \%$ confidence interval $[\mathrm{CI}], 0.11-0.81 ; \mathrm{p}=0.021$ ) in the univariate model, and the association remained significant (OR, 0.28; CI, 0.09$0.79 ; \mathrm{p}=0.022$ ) after adjusting for chemotherapy regimen, sleep quality, and anxiety.

\section{Conclusions}

The morning chronotype is a protective factor against the development of CIPN in patient with breast cancer. Chronotypes should be assessed and considered in the prediction and management of CIPN.

\section{eP226}

COGNITIVE PERFORMANCE AT DIAGNOSIS AND BEYOND DISEASE PROGRESSION IN NEWLY DIAGNOSED GLIOBLASTOMA PATIENTS

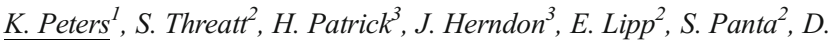

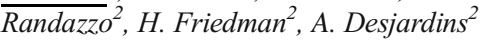 \\ ${ }^{1}$ Duke University Medical Center, Medicine, Durham, USA \\ ${ }^{2}$ Duke University, Neurosurgery, Durham, USA \\ ${ }^{3}$ Duke University, Biostatistics, Durham, USA
}

\section{Introduction}

All patients with central nervous system malignancies can develop impairments in cognition. This includes patients with glioblastoma (GBM), the most common and most lethal glial tumor in adults.

Objectives

Cognitive impairment can come from the underlying tumor, treatments (surgery, radiation, and chemotherapy) and tumor progression. Measures of cognition are commonly integrated into clinical trials, but after progression are rarely obtained or followed. We evaluated cognition after tumor progression in newly diagnosed GBM patients participating in a study of bevacizumab (BV) with concurrent chemoradiation, followed by
$\mathrm{BV}$ and temozolomide with the continuation of BV following progression.

\section{Methods}

In this prospective single-center trial, cognition was measured using a computerized battery of cognitive tests at time of study enrollment (before chemoradiation), after chemoradiation, and every 6 mos until study discontinuation or demise.

\section{Results}

The trial accrued 68 newly diagnosed GBM patients with mean age 55.4 yrs and $53 \% \mathrm{KPS} \geq 90 \%$. Subjects who continued study participation 6 mos following completion of chemoradiation were divided into two groups: subjects with $(n=16)$ and without $(n=33)$ tumor progression 6 mos after chemoradiation completion. Cognition for modalities including verbal and visual memory, reaction time, and psychomotor speed degraded in the tumor progression group in comparison to the group without progression. Previous work has shown that perceived subjective cognition did not decline over time in these groups.

\section{Conclusions}

Objective cognition, in contrast to subjective cognitive performance, declines after tumor progression in newly diagnosed GBM. Continued attention should be paid to monitoring cognitive performance in clinical trials at and beyond progression.

\section{eP227 \\ RANDOMIZED CONTROLLED TRIAL OF CRYOTHERAPY TO PREVENT PACLITAXEL-INDUCED PERIPHERAL NEUROPATHY (RU221511I); AN ACCRU TRIAL}

$\frac{\text { K. Ruddy }}{\text { I }}$, J. Le-Rademacher ${ }^{2}$, M. Lacouture ${ }^{3}$, M. Wilkinson ${ }^{4}$, A.

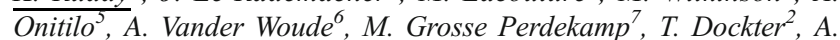
Tan $^{2}$, A. Beutler ${ }^{1}$, C. Loprinzi $i^{1}$

${ }^{1}$ Mayo Clinic, Department of Oncology, Rochester, USA

${ }^{2}$ Mayo Clinic, Department of Biostatistics, Rochester, USA

${ }^{3}$ Memorial Sloan Kettering Cancer Center, Department of Dermatology, New York, USA

${ }^{4}$ Inova, Hematology Oncology, Fairfax, USA

${ }^{5}$ Marshfield Clinic, Hematology/Oncology, Weston Center, USA

${ }^{6}$ Cancer and Hematology Centers of Western Michigan, Hematology/ Oncology, Grand Rapids, USA

${ }^{7}$ Carle Cancer Center, Hematology/Oncology, Urbana, USA

\section{Introduction}

Cooling the extremities during chemotherapy has been hypothesized to reduce neurotoxicity.

Objectives

To assess if cryotherapy prevents chemotherapy-induced peripheral neuropathy.

Methods

A randomized pilot trial compared cryotherapy to standard care (control) in patients initiating paclitaxel weekly for 12 weeks. On cryotherapy, the hands and feet were cooled between 15 minutes before and 15 minutes after each paclitaxel dose. The EORTC QLQ-CIPN20 was completed at baseline and weekly through paclitaxel. The area under the curve (AUC) for CIPN20 scores was calculated using the trapezoidal rule after adjusting for baseline score and compared between arms, using the Wilcoxon rank-sum test. Cross-study comparisons were performed using data from 2 prior similarly-conducted trials.

Results

Of 46 patients accrued, 4 withdrew or were ineligible, leaving 21 cryotherapy and 21 control patients. Cryotherapy was reasonably welltolerated (with 16 cryotherapy and 17 control patients completing study treatment), but EORTC QLQ-CIPN20 data did not differ between the arms in any of the neuropathy outcomes. However, patients in the control arm of the current trial experienced substantially less neuropathy than did placebo recipients in two similar trials that assessed minocycline and 
pregabalin in this setting. When the cryotherapy arm of the current trial was compared to the combined control arms from all three trials, it appeared that there was less neuropathy in the cryotherapy arm than the combined control arms (Wilcoxon Rank Sum $\mathrm{p}=0.004$ ).

Conclusions

While there was no difference in CIPN20 scores between the 2 study arms in the current trial, further investigation is needed.

\section{eP228}

\section{PERCEIVED COGNITIVE FUNCTION: ITS IMPACT ON} WORK IN BREAST CANCER SURVIVORS

\author{
D. Von $\mathrm{Ah}^{1}$, A. Crouch \\ ${ }^{T}$ Indiana University School of Nursing, Community and Health Systems, \\ Indianapolis, USA
}

\section{Introduction}

Breast cancer survivors (BCS) who represent approximately 3.2 million survivors' world-wide, often report ongoing symptoms, including perceived cognitive impairment. However, the long-term effects of perceived cognitive function on work-related outcomes in BCS is not well known. Objectives

The purpose of this study was to examine the relationship between perceived cognitive function including perceived cognitive impairment (PCI) and perceived cognitive ability (PCA) and work ability, work performance, work productivity, and intent to leave employment BCS

Methods

Employed BCS completed one-time questionnaires assessing PCI and PCA and work-related outcomes including, work ability, work performance, work productivity and intent to turnover. Descriptive statistics and separate regression models were used to analyze the data.

Results

Participants were 68 employed female BCS who were on average $52(\mathrm{SD}=8.6)$ years old and $5(\mathrm{SD}=3.8)$ years post-treatment with majority working full time. PCI was associated with poorer work ability $(B=-0.658)$, work performance (time- $B=0.647$; physical- $B=-$ 0.414 , and mental $-B=0.689)$ and work productivity $(B=0.731)$, but not intent to leave work. PCA was related to higher levels of work ability $(B=0.472)$, work performance (time- $B=-0.462$ and mental- $B=-0.453)$ and work productivity $(\beta=-0.494)$, but not physical demands or intent to turnover.

Conclusions

Employed BCS with negative perceptions of cognitive function reported poorer work outcomes with the exception of the intent to leave employment. In contrast, positive views of one's cognitive abilities were related to improved ratings of work outcomes again with the exception of intent to leave employment. Healthcare providers need to assess and address perceived cognitive functioning.to promote work-related outcomes in BCS

\section{eP229}

PREVALENCE AND RISK FACTORS OF CHRONIC NEUROPATHY IN 100 PATIENTS TREATED WITH OXALIPLATIN-BASED REGIMENS

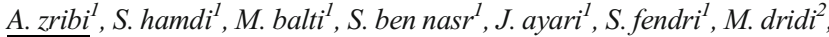 \\ A. haddaoui ${ }^{1}$, M.A. yousfi ${ }^{2}$ \\ ${ }^{1}$ military hospital tunis, medical oncology, tunisia, Tunisia \\ ${ }^{2}$ military hospital tunis, Internal pharmacy departement, tunisia, Tunisia
}

\section{Introduction:}

oxaliplatin is a chemotherapeutic agent largely used to treat a number of digestive cancers, its major side effect is chronic neuropathy

\section{Objectives}

The aim of our study was mainly to evaluate the prevalence of oxaliplatininduced chronic neuropathy and its risk factors in our population, and also to describe the consequences of this toxicity on the pursuit of the cures.

\section{Methods}

In our retrospective study, we included a total of 100 patients treated for colorectal cancer with oxaliplatin-based regimens: FOLFOX $\left(85 \mathrm{mg} / \mathrm{m}^{2}\right)$, FOLOFORINOX $\left(85 \mathrm{mg} / \mathrm{m}^{2}\right)$, GEMOX $\left(100 / \mathrm{m}^{2}\right)$ and XELOX $\left(100 / \mathrm{m}^{2}\right)$. The evaluation of the severity of neurotoxicity was based on OMS neurotoxicity scale and it was done before starting treatment and after each course

Results

$64 \%$ of our patients manifested neuropathy. $35 \%$ had manifested grade I neurotoxicity ,23\% had grade II and $6 \%$ had grade III . There were a close correlation between alcohol consumption, a high number of oxaliplatin cures and the incidence of chronic neuropathy, while no correlation was found between sex, age, high BMI, diabetes and neuropathy incidence . In $64.29 \%$ of cases, neuropathy required a dose adjustment . In $10.71 \%$ of cases, patients have had to delay their treatment. The discontinuation of oxaliplatin was decided in $11 \%$ of cases.

\section{Conclusions}

Oxaliplatin regimens can induce chronic neuropathy leading sometimes to delayed or even stop treatment. Alcohol consumption and a high number of cures of oxaliplatin (superior to six) are a risk factors that can predispose to this kind of neurotoxicity.

\section{eP230}

EXPANDING THE NEUTROPENIC FEVER ALGORITHMS FROM DICHOTOMIC (INPATIENT VS. OUTPATIENT) TO TRIPLE-SCENARIO MODEL (IN- VS. OUT- VS. HOME HOSPITALIZATION) FOR ADULT CANCER PATIENTS

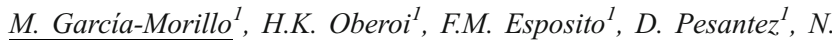

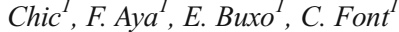

${ }^{1}$ Hospital Clinic de Barcelona, Medical Oncology, Barcelona, Spain

\section{Introduction}

Febrile neutropenia (FN) is a potentially life-threatening complication with a wide spectrum of clinical presentation and outcomes. Low risk patients with FN may be candidates for full outpatient management (FOM) with oral antibiotics. Home Hospitalization $(\mathrm{HH})$ with parenteral wide-spectrum antimicrobial therapy may be an alternative to conventional hospitalization $(\mathrm{CH})$ in this setting although scarce clinical evidence is currently available.

Objectives

To assess the rate, clinical characteristics and outcomes of consecutive patients with FN managed early discharged with $\mathrm{HH}$.

\section{Methods}

We reviewed the clinical characteristics and outcomes of consecutive episodes of FN in patients with non-hematological solid tumors managed according to a local triple-scenario approach protocol (FOM vs. $\mathrm{CH}$ vs. $\mathrm{HH}$ ) based on clinical and social variables (January 2012-June 2017).

Results

$491 \mathrm{FN}$ episodes in 436 patients were analyzed whose main baseline characteristics and FN-related outcomes are summarized in Table 1:

\begin{tabular}{llll}
\hline & FOM & CH & HH \\
& N=70 (10\%) & N=341 (79\%) & N=80 (11\%) \\
Mean age(years) & 56 & 62 & 58 \\
Male & $18(26 \%)$ & $179(53 \%)$ & $32(40 \%)$ \\
Cancer type & & & \\
Breast & $35(50 \%)$ & $85(25 \%)$ & $33(41 \%)$ \\
Lung & $8(11 \%)$ & $111(33 \%)$ & $24(30 \%)$
\end{tabular}




\begin{tabular}{llll} 
Colorectal & $5(7 \%)$ & $24(7 \%)$ & $1(1 \%)$ \\
$\begin{array}{l}\text { Neoadjuvant-Adjuvant } \\
\quad \text { chemotherapy }\end{array}$ & $10(15 \%)-23$ & $49(15 \%)-77$ & $15(19 \%)-25$ \\
$\begin{array}{l}\text { Comorbidities } \\
\text { COPD-Heart condition }\end{array}$ & $5(7 \%)-3(4 \%)$ & $44(13 \%)-32$ & $7(9 \%)-10$ \\
$\begin{array}{l}\text { Blood pressure } \\
\quad \text { < } 90 \text { mmHg }\end{array}$ & $7(10 \%)$ & $77(23 \%)$ & $12(15 \%)$ \\
$\begin{array}{l}\text { FN-RELATED } \\
\quad \text { OUTCOMES: }\end{array}$ & & & \\
$\begin{array}{l}\text { Hospital readmission } \\
\text { ICU admission }\end{array}$ & $5(7 \%)$ & $23(7 \%)$ & $5(6 \%)$ \\
Death & $4(6 \%)$ & $47(14 \%)$ & 0 \\
\hline
\end{tabular}

Conclusions

The HH approach was a safe alternative to FOM and $\mathrm{CH}$ for the management of FN. Further studies are needed to evaluate the potential benefits of $\mathrm{HH}$ in this setting.

\section{eP231}

MUCOSITIS AND INFECTION REDUCTION WITH PROBIOTICS IN CHILDREN WITH CANCER: A RANDOMISEDCONTROLLED FEASIBILITY STUDY PROTOCOL

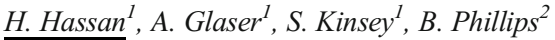 \\ ${ }^{T}$ University of Leeds, Leeds Institute of Cancer and Pathology, Leeds, \\ United Kingdom \\ ${ }^{2}$ University of York, Centre for Reviews and Dissemination, York, United \\ Kingdom
}

\section{Introduction}

Studies have investigated the use of probiotics in people with cancer. A recent systematic review and meta-analysis identified a paucity of randomised-controlled trials (RCT) investigating the use probiotics to reduce or prevent mucositis and infection in children with cancer.

Objectives

To evaluate the feasibility of undertaking an RCT to investigate the efficacy of probiotics for reducing or preventing mucositis and infection in children with cancers.

Methods

To undertake a randomised-controlled feasibility study with 20-40 participants aged 1 year or older, receiving chemotherapies likely to cause mucositis. Patients will be randomised to receive the probiotic VSL\#3 or placebo on day 1-14 of their chemotherapy. Patients, carers and healthcare professionals will be masked as to the allocation. Symptoms will be documented daily in a patient diary.

\section{Results}

Outcomes investigated will include analysis of the compliance of participants taking the probiotic on a daily basis and completion of patient diaries. Departmental referral and recruitment rates, numbers lost to follow up and evaluation of the participant/parent experience using telephone interviews will also be explored. Secondary outcomes investigated will include analysis of the incidence and severity of mucositis and infection, through the use of patient recorded diaries and review of clinical records.

\section{Conclusions}

Findings of the completed study will be used to assess if it is feasible to undertake a RCT to investigate whether probiotics can reduce the incidence and severity of mucositis and infection in children with cancer.

\section{eP232}

POSSIBLE VIRAL INVOLVEMENT IN DNA MOFIDYING OF PATIENTS WITH ACUTE LEUKEMIA

M. LEBEDENCO-BOTEZ ${ }^{1}$

${ }^{T}$ Coltea Clinical Hospital, Hematology, Bucharest, Romania

\section{Introduction}

I have noticed over the years as acute leukemia appear and the relapse of the disease occurs mainly in spring and autumn, so I want to study ,,possible viral involvement in DNA mofidying of patients with acute leukemia".

\section{Objectives}

For that, I want to research the possibility of involving certain viruses. Most leukemias are discovered by an infectious process

\section{Methods}

I believe that a screening test would be useful regarding common infection (for exemple: Influenza virus, coronavirus, parainfluenza, respiratory syncytial virus, rhinovirus, adenovirus, herpes simplex virus, HTLV). We make serological screening for usual infections, but we don't make screening for the viruses described above. A molecular analysis it would help us more. The number of samples available is 25 and the number anticipated in one year is 50

\section{Results}

To prove that a screening test would be useful regarding common infection.

Conclusions

Prove that a molecular analysis is useful regarding possible viral involvement in DNA mofidying of patients with acute leukemia.

\section{eP233}

POINT-OF-CARE SELF-TEST OF WHITE BLOOD CELL COUNT AND DIFFERENTIATION IN A POPULATION OF DANISH CANCER PATIENTS: A STUDY OF FEASIBILITY AND MEASUREMENT PERFORMANCE

T. Mattsson ${ }^{1}$, C.L. Lindhart ${ }^{2}$, J. Schöley ${ }^{3}$, L. Friis-Hansen ${ }^{4}$, J. Herrstedt ${ }^{5}$ ${ }^{T}$ Odense University Hospital, Oncology, Odense C, Denmark

${ }^{2}$ University College Absalon- Denmark, Nursing, Naestved, Denmark

${ }^{3}$ Clinical institute- University of Southern Denmark, University of Southern Denmark, Odense, Denmark

${ }^{4}$ University of Copenhagen-Denmark, Department of Clinical Biochemistry- North Zealand Hospital-Hilleroed-Denmark, Hilleroed, Denmark

${ }^{5}$ University of Copenhagen- Denmark, Department of OncologyUniversity Hospital Zealand- Roskilde Naestved-Denmark, RoskildeDenmark, Denmark

\section{Introduction}

Cancer patients in risk of neutropenia are generally monitored closely. This is done with planned and acute hospital laboratory measurements of white blood cell count and differentiation (WBC Diff). No prior studies have evaluated the use of point-of-care (POCT) devices to self-test WBC Diff in this population.

Objectives

The purpose of this study is to evaluate measurement properties and feasibility of cancer patients self-test using a POCT (HemoCue WBC DIFF®).

\section{Methods}

A feasibility and measurement study conducted in a University Hospital Oncology Department, comparing standard measurement of cancer patient's WBC count and neutrophil count with POCT measurements. The study included 82 patients; 60 ambulatory patients, 22 acute patients with suspected febrile neutropenia.

Results

A total of 106 POCT measurements were conducted. $46 \%$ of the patients were +70 years. Ninety percent of measurements were successful, and more than $90 \%$ of patients reported, they were both willing and able to use the POCT device to measure WBC DIFF at home. Bland-Altman analysis of differences between pairs of measures showed a small, but significant systematic disagreement between methods. POCT -0.37 [95\% CI $-0.63,-0.13]$ for leucocytes and $-0.38[-0.64,-0.13]$ for neutrophils. 
Weighted Deming regression analysis showed minimal, yet significant proportional bias between methods. WBC 0.92 [0.86. 0.97], neutrophils $0.86[0.79,0.91]$, with POCT increasingly underestimating compared to the standard method, the higher the count.

\section{Conclusions}

The instrument has sufficient measurement precision for self-monitoring and patient risk stratification of cancer patients in anticancer treatment. Patients were able and willing to conduct measurements.

\section{eP234}

USE OF GRANULOCYTE-COLONY STIMULATING FACTOR LENOGRASTIM - A NATIONAL SURVEY IN THE AMBULATORY CARE ENVIRONMENT

\section{R. Musch ${ }^{1}$, M. Groschek}

${ }^{T}$ Krebsheilkunde Lichtenberg, Hematology/Oncology, Berlin, Germany

${ }^{2}$ Hämatologie-Onkologie Stolberg, Hämatologie-Onkologie Stolberg, Stolberg, Germany

\section{Introduction}

Neutropenia is a common condition among patients with cancer and can be caused by the cancer itself or by certain types of chemotherapy drugs. In the field of prophylaxis of chemotherapy-induced febrile neutropenia $\mathrm{G}-\mathrm{CSF}$ overuse is as relevant as underuse.

\section{Objectives}

We performed a survey in oncological centers in Germany with focus on patient characteristics, lenograstim-dosing and -application to evaluate impact on chemotherapy.

\section{Method}

89 patients in 11 oncological centers participated in this project. The online-survey covered general information about the patient population, cancer disease, chemotherapy regimen and lenograstim treatment. The survey was performed starting from July 2016 until March 2017.

\section{Results}

Median age of patients was 67 years and $42 \%$ were female. Diseases were dominantly solid tumors. Decision-making between G-CSF and peg-GCSF was mainly based on effectivity, followed by suitable dosing and safety.

$90 \%$ of the G-CSF treated patients possessed individual risk factors. In more than $85 \%$ of chemotherapy cycles five or less days of lenograstim application were regarded as sufficient. $88 \%$ of chemotherapy cycles could be performed in the planned dose and schedule of treatment plan.

The majority of patients injected lenograstim themselves. Remarkably patient consultations regarding adherence to therapy and risk of neutropenia were conducted by medical assistants/nurses.

Conclusions

Most of the cancer patients treated with lenograstim possessed risk factors or comorbidities. In the majority of treatment cycles five or less applications of lenograstim were regarded as sufficient. Hints for overuse were not observed. This points toward an effective, cost-efficient and justified lenograstim treatment.

\section{eP235}

PROCALCITONIN AS AN EARLY SEPSIS BIOMARKER: CORRELATION OF PROCALCITONIN VALUES AND POSITIVE BLOOD CULTURES IN CANCER PATIENTS

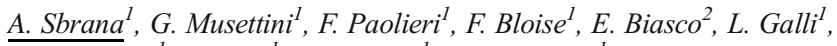
I.M. Brunetti ${ }^{1}$, S. Ricci ${ }^{1}$, A. Falcone ${ }^{l}$, A. Antonuzzol

${ }^{I}$ Azienda Ospedaliero-Universitaria Pisana, Oncologia Medica, Pisa, Italy

${ }^{2}$ Azienda USL Toscana Nord Ovest, DH Oncologico Portoferraio, Livorno, Italy

\section{Introduction}

Sepsis is a frequent complication in cancer patients, among whom it causes almost $20 \%$ of deaths. Early sepsis biomarkers could be useful to fasten diagnosis in order to offer patients the best appropriate treatments in the shortest time.

\section{Objectives}

In our study we wanted to confirm if procalcitonin (PCT) could be considered an early sepsis biomarker in cancer patients.

\section{Methods}

We analyzed patients that accessed our dedicated outpatient supportive care service with a clinical suspicion of sepsis from 2010 to 2016 . We considered only patients who were evaluated with both PCT and blood cultures (BCs). We analyzed the concordance between PCT positive levels ( $\geq 0.5 \mathrm{ng} / \mathrm{mL}$, according to our laboratory standard values) and a positive BC. We finally performed a ROC study to see which PCT cut-off was the best predictor of a positive $\mathrm{BC}$.

Results

90 patients were evaluable. 29 patients had a positive PCT: among these, $25(86.21 \%)$ had a positive BC and $4(13.79 \%)$ a negative one; 61 patients had a negative PCT: among these, $45(73.77 \%)$ had a negative BC and 16 $(26.23 \%)$ a positive one. The ROC analysis showed that the best cut-off for PCT was $0.88 \mathrm{ng} / \mathrm{mL}$. This value was related to a sensitivity of $74.1 \%$ (CI95\% 55-86.9), a specificity of $85.2 \%$ (CI95\% 73-92.5\%), a predictive positive value of $71.4 \%$, and a predictive negative value of $86.8 \%$.

\section{ROC Curve/ 0,01 / AUC $=0,807$}

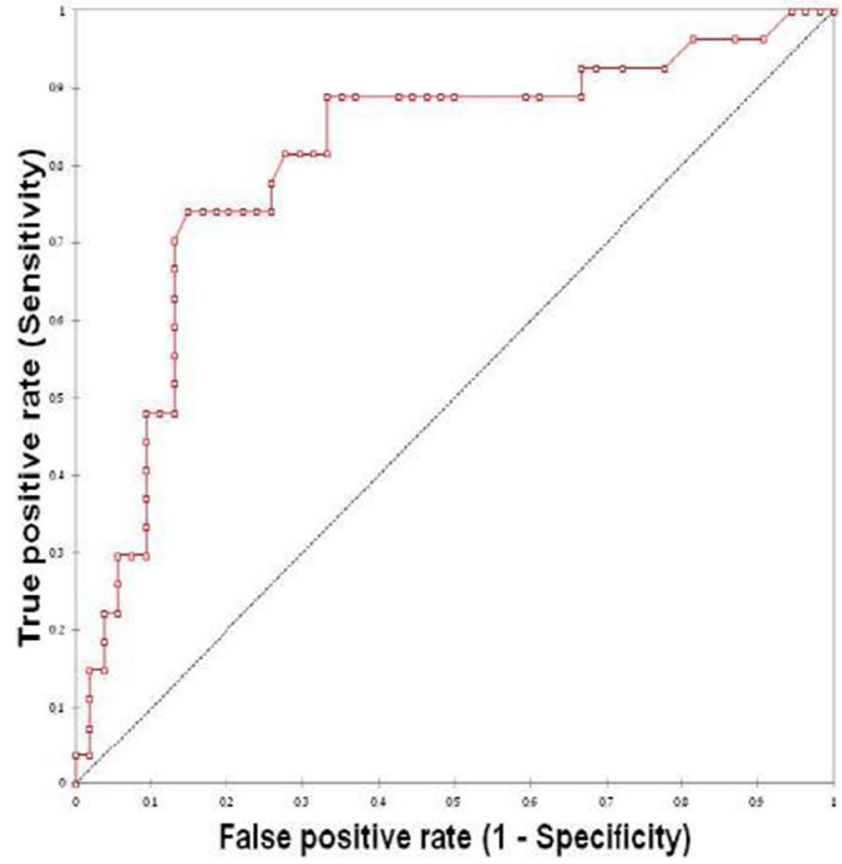

Figure 1-ROC curve for PCT

\section{Conclusions}

Our study confirmed the role of PCT as an early sepsis biomarker in cancer patients and its use should be implemented in everyday clinical practice.

\section{eP236}

INCIDENCE AND AETIOLOGY OF SEPSIS IN CANCER PATIENTS: THE EXPERIENCE OF A TERTIARY REFERRAL CANCER CENTER

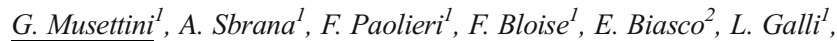
I.M. Brunetti ${ }^{I}$, S. Ricci ${ }^{l}$, A. Falcone ${ }^{l}$, A. Antonuzzo ${ }^{I}$ 
${ }^{1}$ Azienda Ospedaliero-Universitaria Pisana, Oncologia Medica, Pisa, Italy

${ }^{2}$ Azienda USL Toscana Nord-Ovest, DH Oncologico Portoferraio, Livorno, Italy

\section{Introduction:}

Sepsis is a frequent complication in cancer patients, among which it causes almost $20 \%$ of deaths. Aetiology of sepsis has been changing in the last years with a general shift from Gram-negative to Gram-positiverelated bacteremias and this has made empirical treatment of sepsis more challenging.

Objectives

In our study we evaluated the incidence and the aetiology of sepsis by analyzing blood cultures (BCs) performed in cancer patients with a clinical suspicion of sepsis that had been treated in our cancer center (both inand outpatients).

\section{Method}

We evaluated BCs performed from January 2011 to December 2016. In all patients BCs were taken from a peripheral vein and, when present, from a central-vein catheter.

Results

267 patients accessed our cancer center with a clinical suspicion of sepsis. 347 blood cultures were performed (since some patients underwent serial evaluations). $126 \mathrm{BCs}(36.31 \%)$ resulted positive. In 51 (40.5\%) a Gramnegative bacterium (mainly, E. coli) was isolated, in 41 (32.5\%) a Grampositive (mainly, Staphylococci spp), in $33(26.2 \%)$ multiple germs (i.e. Gram-negative and/or positive bacteria and/or fungi), and in $4(3.2 \%)$ fungi (mainly, Candida spp).

\section{Conclusions}

Though a relative increase of Gram-positive bacteria-related sepsis, in our reality Gram-negative bacteria-related septic complications still remain the most common ones. This implies a necessity of a broad-spectrum antimicrobial empirical therapy in all cancer patients with a clinical suspicion of sepsis, whereas an antimicotical empirical therapy might be avoided at the beginning.

\section{eP237}

RESISTANCES TO ANTIMICROBIAL AGENTS IN CANCER PATIENTS WITH SEPTIC COMPLICATIONS: AN EPIDEMIOLOGICAL STUDY OF AN ITALIAN CANCER CENTER

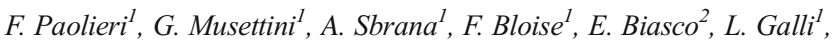
I.M. Brunetti ${ }^{l}$, S. Ricci ${ }^{l}$, A. Falcone ${ }^{l}$, A. Antonuzzo ${ }^{1}$

${ }^{1}$ Azienda Ospedaliero-Universitaria Pisana, Polo Oncologico, Pisa, Italy ${ }^{2}$ Azienda USL Toscana Nord-Ovest, DH Oncologico Portoferraio, Livorno, Italy

\section{Introduction}

Sepsis initial treatment is mainly empirical, since the availability of an antibiogram may require some days. The empirical therapy has to take into consideration the most commonly involved germs and their sensitivity to antimicrobial agents.

\section{Objectives}

In our study we evaluated the antibiotic sensitivity of germs isolated from basal blood cultures (BCs) performed in cancer patients with sepsis treated in our cancer center (both in- and outpatients).

Methods

We evaluated basal BCs from peripheral vein and/or central vein catheter performed from January 2011 to December 2016. Sensitivity to Penicillins (P), Cephalosporins (C), and Fluoroquinolones (F) was evaluated; in Gram-positive bacteria Glycopeptides $(\mathrm{G})$ were tested as well. Results

267 patients accessed our cancer center with a clinical suspicion of sepsis. 112 basal blood cultures were positive: Gram-positive bacteria were isolated in $42(37.5 \%)$, Gram-negative in $41(36.61 \%)$, fungi in 3 $(2.68 \%)$, and multiple germs were found in $26(23.21 \%)$. Among Gram-positive bacteria, $64.29 \%$ was resistant to $\mathrm{P}$, whereas $59.52 \%$ was sensitive to $\mathrm{F}$ and $85.71 \%$ to $\mathrm{G}$. Among Gram-negative bacteria, $51.22 \%$ was resistant to $P$, while $70.73 \%$ was sensitive to $C$ and $60.98 \%$ to $F$. In polymicrobial sepsis, we found out $61.54 \%$ of the cases resistant to $\mathrm{F}$ and $46.15 \%$ to $\mathrm{P}$.

\section{Conclusions}

In our reality therapy for sepsis from Gram-positive bacteria should include at least a Fluoroquinolone or a Glycopeptide, whereas in sepsis from Gram-negative bacteria an initial monotherapy with Cephalosporin might be adequate. In polymicrobial sepsis a combination of at least two antimicrobial agents should always be considered.

\section{eP238}

STUDY OF JAPANESE PATIENT RISK FACTORS FOR FEBRILE NEUTROPENIA IN NON-HODGKIN B-CELL LYMPHOMA RECEIVING R-CHOP: STOP FN2 SINGLE CENTER ANALYSIS IN JAPAN

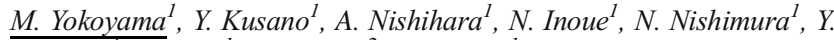

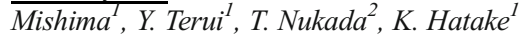

${ }^{1}$ Cancer Institute Hospital, Hematology Oncology, Tokyo, Japan

${ }^{2}$ Kyowa Hakko Kirin Co. Ltd., Medical Affairs, Tokyo, Japan

\section{Introduction:}

Cyclophosphamide, doxorubicin, vincristine, prednisolone, and rituximab (R-CHOP) is standard of care for patients (pts) with non-Hodgkin B-cell lymphomas (B-NHLs). We previously reported that the incidence of febrile neutropenia (FN) in B-NHL pts receiving R-CHOP without prophylactic granulocyte colony stimulating factor (GCSF) was $9.1 \%$ in cycle 1 and $12.3 \%$ throughout all cycles.

\section{Objectives}

To confirm the incidence of FN in Japanese pts treated with R-CHOP using prophylactic peg-GCSF.

\section{Methods}

From Jan 2015 to Jun 2017, in this single-center, retrospective, observational study, 239 patients with B-NHL who completed an R-CHOP at least three cycles were analyzed.

Results

The incidence of FN was $10.5 \%$ (25 of 239) in cycle 1 and $13.0 \%$ (31 of $239)$ throughout all cycles, with $80.6 \%$ (25/31) developing FN during in the first cycle. Risk factors for FN among pts with B-NHL treated with RCHOP were 65 years or older $(\mathrm{p}=0.0205)$, albumin $<35 \mathrm{~g} / \mathrm{L}(\mathrm{p}=$ $0.0093)$, and lack of prophylactic daily GCSF ( $<0.0001)$ and pegGCSF $(p<0.0001)$ in cycle 1 , and albumin $<35 \mathrm{~g} / \mathrm{L}(\mathrm{p}=0.0589)$ and hemoglobin $<12 \mathrm{~g} / \mathrm{dL}(\mathrm{p}=0.0133)$ throughout all cycles.

\section{Conclusions}

At $10.5 \%$ in cycle 1 and $13.0 \%$ throughout all cycles, the incidence of FN was almost the same previously we reported. We reconfirmed that the rate of FN in pts treated with R-CHOP was less than $20 \%$ in this clinical practice. To reduce the occurrence of $\mathrm{FN}$, primary prophylactic GCSF for pts with high risk factors of FN is recommended. (Clinical trial number: UMIN000029534)

\section{eP239}

E-HEALTH SUPPORT PROMOTES DIETARY IMPROVEMENTS IN WOMEN PREVIOUSLY TREATED FOR CANCER: RESULTS FROM THE AUSTRALIAN WOMEN'S WELLNESS AFTER CANCER PROGRAM

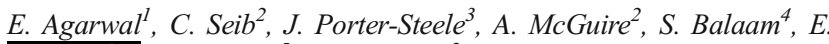
Harbeck, A. McCarthy ${ }^{5}$, D. Anderson ${ }^{2}$

${ }^{1}$ Bond University, Master of Nutrition \& Dietetic Practice Program, Gold Coast, Australia
} 
${ }^{2}$ Griffith University, Menzies Health Institute Queensland and School of Nursing and Midwifery, Gold Coast, Australia

${ }^{3}$ The Wesley Hospital, Choices Program, Auchenflower, Australia

${ }^{4}$ Queensland University of Technology, Australia Institute of Health and Biomedical Innovation and School of Nursing, Kelvin Grove, Australia

${ }^{5}$ University of Auckland, School of Nursing, Auckland, New Zealand

\section{Introduction}

Survivors of breast, gynaecological and blood cancer are at an increased risk of chronic disease, particularly if dietary choices are poor.

Objectives

This paper examines the efficacy of an e-health lifestyle program on compliance with World Cancer Research Fund (WCRF) and American Institute for Cancer Research (AICR) dietary guidelines in women after cancer.

\section{Methods}

A total of 351 Australian women participated in a multisite 12-week structured lifestyle intervention, the Women's Wellness after Cancer Program (WWACP). Participants were randomised into either an intervention group or a control group and they completed Food Frequency Questionnaires at three time-points (baseline, 12 weeks, and 24 weeks). Linear Mixed Models (LMM) and Generalized Estimating Equations (GEE) were used to examine changes in dietary patterns both withinand between- groups over the study period.

Results

Overall, groups were comparable at baseline with no significant differences noted for age, education, employment and income, or dietary choices $(p>0.05)$. Over the study period however, changes in dietary patterns were noted with a reduction in daily consumption of energydense foods and red meat across the cohort $(\mathrm{p}<0.05)$. LMM also showed that among the intervention group, there was a significant increase in total daily serves of fruit and vegetables and a significant decrease in waist-tohip ratio over time $(\mathrm{p}<0.05)$.

Conclusions

Conclusion of active treatment provides an unmissable window of opportunity for education and support for positive dietary changes targeting potential chronic disease risk factors.

\section{eP240}

NUTRITIONAL RISK IN ALLOGENEIC STEM CELL TRANSPLANTATION: RATIONALE FOR A TAILORED NUTRITIONAL PATHWAY

\author{
T. Aoyama ${ }^{\text {I }}$, O. Imataki ${ }^{2}$, H. Arai $^{3}$, T. Ikeda ${ }^{4}$ \\ ${ }^{T}$ Shizuoka Cancer Center, Nutrition, Shizuoka, Japan \\ ${ }^{2}$ Kagawa University Hospital- Kagawa-Japan, Hematology \& Stem Cell \\ Transplantation, Kagawa, Japan \\ ${ }^{3}$ University of Shizuoka-Shizuoka- Japan, Laboratory of Clinical \\ Nutrition and Management- Graduate School of Integrated \\ Pharmaceutical and Nutritional Sciences, Shizuokashi, Japan \\ ${ }^{4}$ Shizuoka Cancer Center-Shizuoka- Japan, Hematology \& Stem Cell \\ Transplantation, Shimonagakubo, Japan
}

\section{Introduction}

Hematopoietic stem cell transplantation carries nutrition-related risks. Therefore, nutritional therapy needs to be initiated before transplantation even takes place.

Objectives

We assessed nutritional risk among patients who underwent allogeneic stem cell transplantation.

\section{Methods}

We assessed nutrient supply (calorie supply and protein supply) by chart review. Assessments were made from the pretreatment phase of transplantation to after the end of parenteral nutrition in 51 patients who underwent allogeneic stem cell transplantation at Shizuoka Cancer
Center between 2007 and 2012. We compared nutrition-related adverse events and parameters between two groups: those in whom \% loss of body weight was $\geq 7.5$ and those in whom $\%$ loss of body weight was $<7.5$.

\section{Results}

A correlation was observed between changes in weight and skeletal muscle mass $(\mathrm{r}=0.89 ; \mathrm{P}<0.0001)$. A weak correlation was observed between $\%$ loss of body weight and nutrient supply of calories $(\mathrm{r}=0.517 ; \mathrm{P}=$ $0.0001)$. There were significant differences between the $\%$ loss of body weight $\geq 7.5$ group and the $\%$ loss of body weight $<7.5$ group in the following variables: $\%$ loss of body weight, nutrient supply from calories and protein; orally ingested nutrient supply from calories and protein; start day of oral intake; and acute graft-versus-host disease. Orally ingested calories were negatively correlated with nutrition-related adverse events in both groups.

\section{Conclusions}

Early and customized nutritional intervention may be optimal for all patients who undergo allogeneic stem cell transplantation to ameliorate body weight loss associated with nutrition-related adverse events.

\section{eP241}

TOTAL PARENTERAL NUTRITION IN MIDDLE EASTERN CANCER PATIENTS AT END OF LIFE : AN UNJUSTIFIED ABUSE

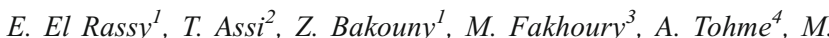

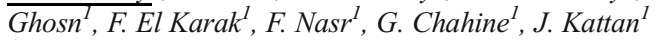

${ }^{I}$ Saint-Joseph University, Hematology-Oncology, Beirut, Lebanon

${ }^{2}$ Saint-Joseph University, Hematology-Oncology, paris, France

${ }^{3}$ Saint-Joseph University, Pharmacy, Beirut, Lebanon

${ }^{4}$ Saint-Joseph University, Internal medicine, Beirut, Lebanon

\section{Introduction}

The use of total parenteral nutrition (TPN) in terminally ill cancer patients is considered an aggressive approach with limited benefits.

\section{Objectives}

We examined the practice of TPN in end of life cancer patients and evaluated the patient and tumor characteristics that justify this practice. To our knowledge, this is the first study describing TPN in Middle Eastern cancer patients.

\section{Methods}

We conducted this retrospective observational study at Hotel-Dieu de France University Hospital, Lebanon. Eligible cases included cancer patients that died between the $1^{\text {st }}$ of January and the $31^{\text {st }}$ of December 2014. Patients and tumor characteristics and the management plan were retrieved from the hospital records.

\section{Results}

Among 129 patients enrolled, 39\% had received TPN among which $28 \%$ during the last 6 weeks. The mean duration of TPN was 33 days and 37 days between the end of TPN administration and death. TPN administration correlated negatively to hyperlipidemia $(\mathrm{OR}=$ $0.33 ; 95 \% \mathrm{CI}[0.12-0.87])$ and at least three cardiovascular risk factors $(\mathrm{OR}=0.28)$. However, it correlated positively to gastrointestinal tumors $(\mathrm{OR}=3.9)$ and imaging studies during the last month of life $(\mathrm{OR}=3.4)$. In multivariate analysis, only hyperlipidemia was found to be a significant determinant $(p=0.010$; ORa $=0.29)$. No correlation was found to hospitalization during the last two weeks of life or ICU admission.

\section{Conclusions}

The high percentage of TPN administration underlines the difficulty of adopting an optimal palliative approach in Middle Eastern cancer patients. This is applicable in our populations who seem to lack psychosocial maturity to accept a patient-centered supportive care approach. 


\section{eP242}

THE INVOLVEMENT OF NUTRITIONAL INTERVENTION FOR THE GASTROINTESTINAL PRE-CACHEXIA CANCER PATIENT USING SHORT TERM ORAL AMINO ACID SUPPLEMENTATION

M.Baba ${ }^{1}$, N. Kakita ${ }^{2}$, M. Nakai ${ }^{3}$

${ }^{T}$ Auru Visiting-Nursing Station, Dietitian, Kaizuka, Japan

${ }^{2}$ Kaizuka City Hospital, gastroenterology and hepatology, Kaizuka, Japan

${ }^{3}$ Auru Visiting-Nursing Station, none, Kaizuka, Japan

\section{Introduction}

Cancer-associated cachexia is characterized by loss of body weight and skeletal muscle. Sarcopenia is the major component of malnutrition and is a frequent complication that affects clinical outcomes. To date, appropriate earlier palliative care improves QOL of patient, however no effective medical intervention completely reverses cachexia yet to be determined. We will clarify the involvement of anti-catabolic treatment in precachexia stage for nutritional and prognostic factor.

\section{Objectives}

A total of 40 patients with gastrointestinal cancer patient in pre-cachexic period ( $65 \%$ male) were enrolled in the present study.

\section{Methods}

Patients are randomly assigned to two groups with (group A) or without (group B) ingestion of $3 \mathrm{~g}$ leucine-rich essential amino acid mixture twice a day for 3 months, and all patients had been performed a moderate physical therapy. Blood examinations and functional fitness parameters were collected at baseline, before and after 3-month intervention. Measurement of psoas and abdominal muscle mass on Computed Tomography images at L3 vertebra determined the degree of sarcopenia. Results

Clinical backgrounds of two groups were similar. However, walking speed, grip and controlling nutritional status score including albumin, total cholesterol and the number of lymphocyte were significantly maintained in group A after the 3-month intervention. And the reduce of SMI were significantly suppressed in group A, whereas prognosis was significantly poorer in group B.

\section{Conclusions}

We demonstrate that a suppression of skeletal muscle reducing using amino acid supplementation might be improved not only ADL but prognosis of gastrointestinal cancer patient in pre-cachexic period, suggesting its feasibility as a target of earlier therapeutic intervention.

\section{eP243}

CANCER ORGANISATION WEBSITES: DIETARY \& NUTRITIONAL ADVICE FOR SYMPTOM MANAGEMENT

\author{
M. Barrett ${ }^{1}$, P. Ui Dhuibhirr ${ }^{1}$, C. Njoroge ${ }^{2}$, D. Walsh ${ }^{1}$ \\ ${ }^{T}$ Our Lady's Hospice \& Care Services, Academic Dept of Palliative \\ Medicine, Dublin, Ireland \\ ${ }^{2}$ Dublin City University, School of Nursing, Dublin, Ireland
}

\section{Introduction}

Nutrition-impact symptoms are common at all cancer stages and increase the risk of malnutrition. Despite this, cancer patients are not routinely referred to a dietitian. Those with cancer may therefore seek information from alternative sources. National Cancer Organisations (NCO) offer both dietary and nutritional advice but the utility of this advice has not been evaluated. Dietary advice refers to how and when to prepare and serve food. Nutritional advice provides instructions about macro and micronutrient intake.

\section{Objectives}

To examine accessibility and relevance of advice for symptom control provided on $\mathrm{NCO}$ websites

\section{Methods}

We identified and searched English-language NCO websites for symptom management advice

\section{Results}

- Nine NCO websites were searched.

- Nutritional advice for anorexia, sore mouth and dysphagia often recommended high-protein and high-calorie intake. Examples of food and recipes were provided, but no website defined the diets (i.e grams per day).

- No micronutrient advice provided, except to increase sodium and potassium for diarrhoea.

- Little or no nutritional/diet advice for symptoms of early satiety and smell abnormalities.

- Dietary advice for anorexia and nausea \& vomiting (small frequent meals), constipation (increase fluids) and dysphagia (soft food) was consistent, but other advice was not.

- Healthcare professional input universally advised for specific guidance.

\section{Conclusions}

High-calorie, high-protein diets recommended for some symptoms but advice was non-specific.

Dietary advice practical for common symptoms. Other symptoms not addressed.

All websites recommended referral to dietitian or health care professional. Website navigation was challenging and negatively impacted information access.

\section{eP244}

EFFICACY OF GINGER (ZINGIBER OFFICINALE) IN AMELIORATING CHEMOTHERAPY-INDUCED NAUSEA AND VOMITING AND CHEMOTHERAPY-RELATED OUTCOMES: A SYSTEMATIC LITERATURE REVIEW UPDATE AND META-ANALYSIS

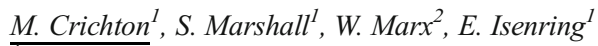 \\ ${ }^{T}$ Bond University, Faculty of Health Sciences and Medicine, Gold Coast, \\ Australia \\ ${ }^{2}$ Deakin University, Food \& Mood Centre- IMPACT Strategic Research \\ Centre-School of Medicine, Geelong, Australia
}

\section{Introduction}

Ginger has long been used in traditional medicine for alleviating nausea and vomiting; however, its use as an adjuvant therapy in patients undergoing chemotherapy is under-researched.

\section{Objectives}

A systematic literature review and meta-analysis was undertaken to evaluate the efficacy of ginger supplementation in the prevention and management of chemotherapy-induced nausea and vomiting.

\section{Methods}

Five electronic databases were searched from database inception to October 2017. Intervention studies which administered ginger supplementation and a control (placebo or anti-emetic) to adults receiving chemotherapy were included, critically appraised using the Cochrane Risk of Bias tool, and pooled using meta-analysis.

\section{Results}

Seventeen papers were included. Ginger supplementation of any dose or duration had no significant effect on nausea incidence or severity. Ginger administration for $>3$-days significantly reduced overall vomiting incidence (OR:0.58 [95\%CI:0.38-0.90] P=0.01; $\mathrm{n}=5$ studies; $\mathrm{I}^{2}=74 \%$ ) and delayed-phase vomiting incidence (OR:0.44 [95\%CI:0.25-0.78] $\mathrm{P}=0.005 ; \mathrm{n}=3$ studies; $\mathrm{n}=239$ participants $; \mathrm{I}^{2}=83 \%$ ). Sensitivity analysis did not explain the substantial heterogeneity in the pooled outcomes.

\section{Conclusions}

Ginger supplementation for $>3$-days may improve chemotherapyinduced vomiting incidence; however, existing research remains 
inconsistent. Further research using strong designs, adequate sample sizes and standardized ginger products is warranted prior to routine clinical prescription.

\section{eP245}

SUPPLEMENTAL PROPHYLACTIC INTERVENTION FOR CHEMOTHERAPY-INDUCED NAUSEA AND EMESIS (SPICE) TRIAL: PROTOCOL FOR A MULTI-CENTRE DOUBLE-BLIND PLACEBO-CONTROLLED RANDOMIZED TRIAL

M. Crichton ${ }^{1}$, W. Marx ${ }^{2}$, M. Alexandra ${ }^{3}$, S. Marshall ${ }^{4}$, A. Molassiotis ${ }^{5}, K$.

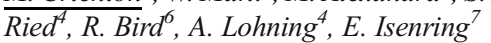

${ }^{1}$ Bond University, Faculty of Health Sciences and Medicine, Miami, Australia

${ }^{2}$ Deakin University, Food \& Mood Centre- IMPACT Strategic Research Centre- School of Medicine, Geelong, Australia

${ }^{3}$ University of Auckland, School of Nursing, Auckland, New Zealand

${ }^{4}$ Bond University, Faculty of Health Sciences \& Medicine, Gold Coast, Australia

${ }^{5}$ Hong Kong Polytechnic University, Hong Kong Polytechnic University, Hung Hom, Hong Kong S.A.R.

${ }^{6}$ Princess Alexandra Hospital, Division of Cancer Services, Brisbane, Australia

${ }^{7}$ Bond University, Faculty of Health Sciences and Medicine, Gold Coast, Australia

\section{Introduction}

There is significant recent interest in the role of ginger root (Zingiber officinale) as an adjuvant therapy for chemotherapy-induced nausea and vomiting.

Objectives

This study protocol aims to assess the efficacy (reduced incidence and severity of chemotherapy-induced nausea and vomiting, enhanced quality of life), safety, cost-effectiveness, and impact on gut microbiota of a standardized adjuvant ginger root supplement in adults undergoing single-day moderate-to-highly emetogenic chemotherapy.

Methods

Multi-site, double-blind, placebo-controlled randomized trial with two parallel arms, 1:1 allocation and target sample size of $\mathrm{N}=300$. The intervention comprises four capsules of ginger root consumed at specified times across the day (totaling $60 \mathrm{mg}$ of active gingerols/day), for five consecutive days commencing on the day of chemotherapy for Cycles 1 to 3 . The primary outcome is chemotherapy-induced nausea-related quality of life measured by the Functional Living Index - Emesis - 5 Day Recall (FLIE-5DR). Secondary outcomes include nutrition status; anticipatory, acute and delayed nausea and vomiting; fatigue; depression and anxiety; global quality of life; health service use and costs. Changes to the microbiome will be examined using 16S RNA analysis. Adverse events and adherence will also be assessed.

\section{Results}

The SPICE trial commenced recruitment in October 2017 and is anticipated to continue until October 2019. To date, 25 participants have been recruited and the study appears feasible.

Conclusions

The research gaps addressed by the SPICE Trial may guide future recommendations for specific ginger dosing regimens for use as an adjuvant for chemotherapy induced nausea and vomiting prevention and management.

\section{eP246}

INVESTIGATING MALNUTRITION AMONG CANCER PATIENTS RECEIVING CHEMOTHERAPY IN A TERTIARY CARE HOSPITAL OF PAKISTAN

\author{
A. Fatima ${ }^{I}$ \\ ${ }^{T}$ Shaukat Khanum Memorial Hospital and Research Centre, Oncology, \\ Lahore, Pakistan
}

\section{Introduction}

Cancer patients often suffer from Malnutrition which raises the risk of infections. Being immunocompromised, there is a marked reduction on quality of life (QoL) and health outcome. Malnutrition also enhances the incidence of postoperative complications such as delayed wound healing, wound dehiscence, morbidities and mortalities

\section{Objectives}

To investigate malnutrition among cancer patients and to assess the nutritional status of patients receiving chemotherapy.

\section{Methods}

The study was conducted in Sir Ganga Ram Hospital, Lahore. Simple screening tool (Short screening sheet) for malnutrition was used. Nutritional assessment of 80 patients receiving chemotherapy was done by assessing BMI, mid upper arm circumference MUAC, triceps skinfold thickness TST, serum albumin, Total lymphocytes count. Nitrogen Balance and intake of macronutrients were also analysed.

\section{Results}

According to full nutritional assessment, 42 patients (52.5\%) out of 80 were found malnourished. Short screening sheet identified 51 patients as malnourished who were receiving chemotherapy. The SSM had a specificity of 0.88 and sensitivity of 0.72 . $62 \%$ of the patients exhibited negative nitrogen balance.

\section{Conclusions}

Nutrition is the most neglected area of clinical care. Early nutritional support and counselling is essential in order to improve patients Quality of Life (QoL). Mass media should be involved so that adequate attention can be given to nutritional issues arising in diagnostic and therapeutic procedures

\section{eP247}

PROGNOSTIC IMPORTANCE OF SARCOPENIA AND INFLAMMATORY STATEMENTS IN STAGE III NON SMALL CELL LUNG CARCINOMA

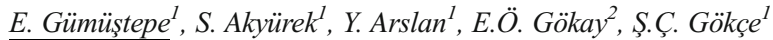 \\ ${ }^{T}$ Ankara University Medical School of Medicine, Radiation Oncology, \\ Ankara, Turkey \\ ${ }^{2}$ Ankara University Medical School of Medicine, Biostatistics, Ankara, \\ Turkey
}

\section{Introduction}

Sarcopenia is characterized by progressive loss of skeletal muscle mass, muscle strength and physical performance. Systemic inflammation is thought to contribute to sarcopenia.

\section{Objectives}

In this study, we aimed to investigate the prognostic significance of sarcopenia and inflammatory markers in stage III non small cell lung cancer (NSCLC).

\section{Methods}

Eighty-three patients with stage III NSCLC undergoing definitive chemoradiotherapy(KRT) in our clinic between April 2014 and August 2017 were evaluated. The cross-sectional area of muscle at the level of the third lumbar vertebra (L3) was measured using radiotherapy planning CT images. Systemic inflammatory markers investigated included serum lactate dehydrogenase (LDH), neutrophil/ lymphocyte ratio(NLR), Creactive protein(CRP), and albumin(alb).

\section{Results}

Sarcopenic patients was $52(62.6 \%)$. The mean age was higher in sarcopenic patients(68.3 \pm 9.7 vs $63.7 \pm 9.4, p=0.037)$. In sarcopenic patients, overall survival(OS) was 17.2 months, while non-sarcopenic patients were 19.58 months $(\mathrm{p}=0.310)$. Effects on OS were examined, it was found statistically significant that the presence of sarcopenia $(\mathrm{p}=$ $0.016)$, age $(p=0,001)$, low alb level $(p=0,009)$, NLR is higher than 4 ( $p$ $=0,003)$. Multivarible Cox regression analyzes showed independent prognostic significance in survival with RT dose $(\mathrm{HR}(95 \% \mathrm{CI})=1,2 ; \mathrm{p}$ 
$=0.001)$, low alb level $(\mathrm{HR}(95 \% \mathrm{CI})=2.42 ; \mathrm{p}=0.007)$ and age $(\mathrm{HR}(95 \% \mathrm{CI})=1,03 ; \mathrm{p}=0,044)$.

\section{Conclusions}

In our study, the presence of sarcopenia in patients with stage III NSCLC, as well as the low alb level and NLR of inflammatory markers was found to be a significant prognostic factor for OS.

\section{eP248}

PREVALENCE OF MALNUTRITION IN CANCER PATIENTS IN HOSPITAL SETTING - A CROSS-SECTIONAL ANALYSIS

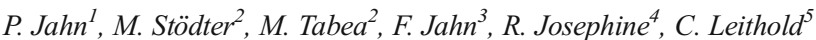 \\ ${ }^{T}$ Universitätsklinikum Halle Saale, Nursing Research Unit, Halle Saale, Germany \\ ${ }^{2}$ Martin-Luther-University Halle-Wittenberg, Institute for Nutritional \\ Science, Halle Saale, Germany \\ ${ }^{3}$ Universitätsklinikum Halle Saale, Department of Internal Medicine IV- \\ Hematology/Oncology, Halle Saale, Germany \\ ${ }^{4}$ SRH Wald-Klinikum Gera GmbH, Nutrition Therapy, Gera, Germany \\ ${ }^{5}$ Martin-Luther-University Halle-Wittenberg, Department of Internal \\ Medicine IV-Hematology/Oncology, Halle Saale, Germany
}

\section{Introduction}

Malnutrition is a frequent side effect in malign diseases, which affects patients' outcome and results in reduced overall survival. However, malnutrition in cancer patients is often underdiagnosed. Pirlich et al. (2006) executed the last prevalence study within a German hospital setting, thus the current situation remains unclear.

Objectives

The aim of this study was to assess the prevalence of malnutrition in a German University Hospital with special focus on the high-risk group of cancer patients.

Methods

This cross-sectional retrospective analysis from clinical recorded data comprises a collective of 455 patients at the University Hospital Halle. Clinical recorded data was validated with direct patient contact. The analysis included nutritional deficiency by Nutritional Risk Screening 2002 (NRS-2002) as well as patient-related data, laboratory parameter, primary diagnosis and diet-related secondary diagnoses.

\section{Results}

With a prevalence of $20.2 \%$, about every fifth inpatient had a nutritional risk, scored by NRS-2002. Patients with malignant diseases were malnourished in $38.2 \%$ compared with $13.0 \%$ in patients with nonmalignant diseases. Risk factors for malnutrition were the emergence of an oncological disease, diseases of the circulatory and digestive system, older age, BMI $<20.5 \mathrm{~kg} / \mathrm{m}^{2}$ and all four pre-screening parameters.

Conclusions

The prevalence of malnutrition in cancer patients remains high and has not changed in the last 10 years. Irrespective of a visible cachexia, malnutrition is often overseen. Therefore, the awareness of malnutrition needs to be increased due to implementation of training, screening and nutritional interventions as an essential part of supportive therapy.

\section{eP249}

DYSPHAGIA IN CANCER PATIENTS: COMMON, DANGEROUS, AND UNDER-DIAGNOSED

C. Kenny ${ }^{1,2}$, J. Regan ${ }^{3}$, D. Walsh ${ }^{1,2,4}$

${ }^{T}$ Our Lady's Hospice \& Care Services, Academic Department of Palliative Medicine, Dublin, Ireland

${ }^{2}$ Trinity College Dublin, School of Medicine, Dublin, Ireland

${ }^{3}$ Trinity College Dublin, Clinical Speech and Language Studies, Dublin, Ireland

${ }^{4}$ University College Dublin, School of Medicine \& Medical Science, Dublin, Ireland

\section{Introduction}

Dysphagia occurs in solid malignancies, even in tumours outside the head and neck or upper gastrointestinal tract. It is associated with aspiration pneumonia, increased healthcare costs, malnutrition, poorer prognosis, and worse quality of life. Dysphagia occurs frequently at end-of-life, but its natural history in cancer is unknown. Existing prevalence rates are not based on appropriately validated tools.

\section{Objectives}

This study examined dysphagia prevalence across the disease trajectory. It described dysphagia characteristics and associations with other disease features.

Methods

After a pilot study, 385 participants with solid malignancies outside the head and neck or upper gastrointestinal tract were recruited from two acute hospitals and one hospice using consecutive sampling. Individuals were screened for dysphagia. Those found to be symptomatic were evaluated qualitatively and with structured, validated tools (e.g. Mann Assessment of Swallowing Ability).

\section{Results}

$21 \%(n=80 / 385)$ noted dysphagia since cancer diagnosis. 89\% $(n=71 / 80)$ of them were undiagnosed. $91 \%(n=73 / 80)$ still had dysphagia. Of these, $70 \%(\mathrm{n}=52 / 74)$ required diet modification or safe swallow strategies. $30 \%$ $(n=22 / 74)$ needed intervention to prevent aspiration. Univariate regression showed dysphagia was associated with: palliative care service ( $\mathrm{p}=0.004)$; worse ECOG-PS ( $\mathrm{p}<0.001)$; lower quality of life $(\mathrm{p}=0.002)$. It was not associated with disease extent $(\mathrm{p}=0.673)$, which suggested presence throughout the course. Qualitative data revealed physiological insights not obtained during structured evaluation.

\section{Conclusions}

Dysphagia was common and clinically important, but pervasively underdiagnosed. Swallow screening must be routine and repeated in cancer patients. Existing tools need modification to accurately identify swallow difficulties. This is the first study to comprehensively describe dysphagia in this setting.

\section{eP250}

ASSESSMENT OF THE INITIATION TIMING OF DECREASE IN SKELETAL MUSCLE MASS IN PATIENTS WITH BREAST CANCER: A RETROSPECTIVE ANALYSIS

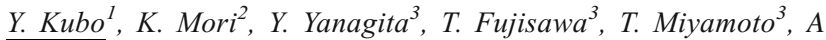 \\ $\overline{\text { Morishita }}^{3}$, T. Kazama ${ }^{1}$, S. Koizuka \\ ${ }^{I}$ Gunma prefectural cancer center, Palliative Medicine, Ota, Japan \\ ${ }^{2}$ Shizuoka Cancer Center, Clinical Research Center, Shizuoka, Japan \\ ${ }^{3}$ Gunma prefectural cancer center, Breast, Ota, Japan
}

\section{Introduction}

Decreased skeletal muscle mass is a poor prognostic factor in patients with cancer. Therefore, determining the precise initiation timing of this decline is imperative to prevent muscle mass decrease.

\section{Objectives}

This study aims to retrospectively analyze the initiation timing of muscle mass decrease in patients with breast cancer.

\section{Methods}

Among 2105 female patients with breast cancer who underwent breast cancer surgery from 2007 to 2017 , we retrospectively analyzed patients under the age of 65 years who died after metastasis. We assessed the change in the skeletal muscle index (SMI) by the squared height of the skeletal muscle in Computed Tomography images at the time of diagnosis, metastasis and within 1 year of death. We considered $P=0.016$ as statistically significant.

\section{Results}

The median age of the 21 enrolled patients at surgery was 43 (range: 30 $62)$ years. The median time from surgery date to metastasis was $23(2-60)$ months and to death was $42(12-124)$ months. The median (range) of 
SMI $\left(\mathrm{cm}^{2} / \mathrm{m}^{2}\right)$ at the time of diagnosis, metastasis, and within 1 year of death was $38.2(27.8-51.4), 34.6(25.7-52.1)$, and $31.0(21.0-42.3) \mathrm{cm}^{2} /$ $\mathrm{m}^{2}$, respectively. We observed no significant decrease in SMI at diagnosis and metastasis $(P=0.24)$, but it decreased significantly from the time of metastasis to before death $(P=0.010)$.

\section{Conclusions}

This study demonstrated that the skeletal muscle mass of patients with breast cancer decreases after metastasis. We believe that the findings of this study could help improve the prognosis of patients with breast cancer.

\section{eP251 \\ CLINICAL APPLICATION OF THE 2011 CANCER CACHEXIA CONSENSUS}

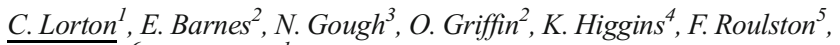
G. Stewart ${ }^{6}$, T.D. Walsh ${ }^{I}$

${ }^{I}$ Our Lady's Hospice \& Care Services, Academic Department of Palliative Medicine, Dublin, Ireland

${ }^{2}$ St Vincent's University Hospital, Department of Nutrition and Dietetics, Dublin, Ireland

${ }^{3}$ Mater Private Midwestern Radiation Oncology Centre, Department of Dietetics, Limerick, Ireland

${ }^{4}$ Tallaght Hospital, Department of Clinical Nutrition and Dietetics, Dublin, Ireland

${ }^{5}$ St Luke's Radiation Oncology Network, Department of Clinical Nutrition and Dietetics, Dublin, Ireland

${ }^{6}$ St Vincent's Private Hospital, Department of Dietetics, Dublin, Ireland

\section{Introduction}

The 2011 cancer cachexia consensus ${ }^{1}$ proposed stages (precachexia, cachexia and refractory) and assessment domains, including assessment of muscle mass. Objective measures of muscle mass are not routine in clinical practice. Assessment and classification by expert professionals may be feasible, even without this information.

\section{Objectives}

To assess if oncology dietitians could apply 2011 consensus $^{1}$ assessment domains and classification using standard clinical information.

\section{Methods}

This was a prospective multi-site observational study. Data from consecutive adults with cancer were collected during routine assessment by oncology dietitians in 5 hospitals.

Dietitians assessed weight, weight loss, food intake, nutrition symptoms, systemic inflammation, functional ability and disease status, and classified cachexia stage accordingly.

Results

200 patients were included (male 119; female 81), median age 65 years (19-93).

$12 \%$ were underweight, $40 \%$ overweight or obese. Two-thirds had lost $\geq 5 \%$ body weight. $65 \%$ had dietary intake $<75 \%$ of normal. Most had multiple nutrition symptoms or barriers (median $=2$; range 0-5). 69\% were ECOG 0-1. 49\% had metastatic disease. $91 \%$ were on active treatment.

Cachexia classification was applied in $88 \%$ of patients: $17 \%$ non-cachectic, $20 \%$ pre-cachectic, $52 \%$ cachectic and $11 \%$ refractory. Most common reasons unable to classify: no C-reactive protein, complex clinical situation, acute illness.

\section{Conclusions}

Experienced oncology dietitians were able to use clinical information to apply the cachexia classification for most patients. Almost two-thirds were considered cachectic. Future studies should validate clinical assessment against outcomes and objective muscle mass measures. Expert clinical assessment based on 2011 consensus domains could be cost-effective to screen for cachexia.

1. Fearon, Strasser. Lancet Oncol 2011
eP252

PRACTICE ANALYSIS AND MEDICO-ECONOMIC EVALUATION REGARDING THE PRESCRIPTIONS OF ARTIFICIAL NUTRITION IN THE MEDICAL ONCOLOGY SETTING OF BREST INSTITUTE OF ONCOLOGY AND HEMATOLOGY IN 2015. (ENROBE).

\author{
L. Monceau-Baroux ${ }^{1}$, J. Delarue ${ }^{2}$ \\ ${ }^{T}$ Centre Hospitalier Régional Universitaire, Medical Oncology, Brest, \\ France \\ ${ }^{2}$ Centre Hospitalier Régional Universitaire, Nutritional Sciences, Brest, \\ France
}

\section{Introduction:}

International evidence-based guidelines regarding the prescription of enteral $(\mathrm{EN})$ and parenteral nutrition $(\mathrm{PN})$ in cancer patients are now widely available. However, real-life practice does not always follow those guidelines. Nutritional management being part of supportive care in oncology, investigating the clinical and economic consequences of those real-life prescriptions was mandatory.

\section{Objectives}

The primary objective of this study was to evaluate if initiations of artificial nutrition (AN) made to adult patients with solid tumours in the oncological setting of our French University Hospital during the year 2015 complied with the grade A national and international guidelines available at that time (ASPEN, ESPEN, SFNEP).

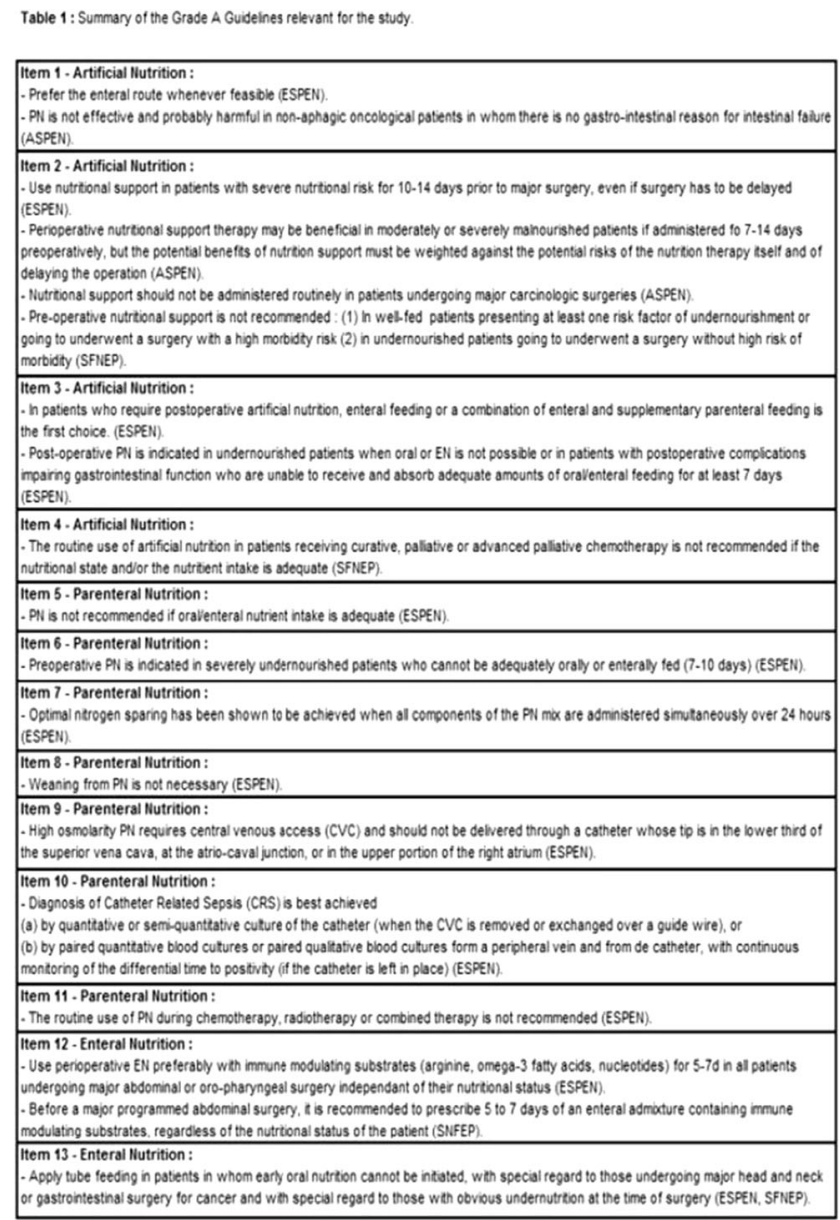

Secondary objectives were to study the clinical profile of the patients, evolution of their nutritional status under nutritional support, OS from the prescription time, complications rates and costs. 


\section{Methods}

Every computerized prescription of EN and PN made during the year 2015 was screened. Patients were included if it was their first exposure to AN. Both their digital and paper medical records were studied. They were separately analysed in 2 cohorts (EN / PN).

Results

109 patients were included (EN: 39, PN: 63, both: 7).

Table 2 : Characteristics of the patients

\begin{tabular}{|l|c|c|}
\cline { 2 - 3 } \multicolumn{1}{c|}{} & $\begin{array}{c}\text { Parenteral } \\
\text { Nutrition (n=70) }\end{array}$ & $\begin{array}{c}\text { Enteral } \\
\text { Nutrition (n=46) }\end{array}$ \\
\hline Gender & $43(61 \%)$ & $34(74 \%)$ \\
Male & $27(39 \%)$ & $12(26 \%)$ \\
\hline Female & $64[27 ; 85]$ & $64[44 ; 83]$ \\
\hline Median Age (years, range) & $28,5[11 ; 43]^{\mathrm{a}}$ & $29[17 ; 38]^{\mathrm{b}}$ \\
\hline Median albumin level (g/l, range) & $21,2[14,6 ; 36,7]^{\circ}$ & $20,7[11,1 ; 33,6]$ \\
\hline Median BMI (kg/m ${ }^{2}$, range) & $79,4[55,4 ; 102,1]^{\mathrm{d}}$ & $79,2[62,9 ; 98,4]^{\mathbf{6}}$ \\
\hline Median NRI (\%, range) & & \\
\hline Nutritional state & $8(11 \%)$ & $21(46 \%)$ \\
Missing data & $5(7 \%)$ & $3(7 \%)$ \\
Well fed & $15(21 \%)$ & $8(17 \%)$ \\
Moderately undernourished & $42(60 \%)$ & $14(30 \%)$ \\
Severely undernourished & & \\
\hline Tumor Type & $25(36 \%)$ & $2(4 \%)$ \\
Intrathoracic & $18(26 \%)$ & $2(4 \%)$ \\
Digestive & $16(23 \%)$ & $40(87 \%)$ \\
Upper digestive and respiratory tract & $3(4 \%)$ & $1(2 \%)$ \\
Urinary & $3(4 \%)$ & 0 \\
Gynaecologic & $2(3 \%)$ & $1(2 \%)$ \\
Neurologic & $2(3 \%)$ & 0 \\
Breast & $1(1 \%)$ & 0 \\
Endocrine glands & & \\
\hline Phase of the disease & $20(29 \%)$ & $42(91 \%)$ \\
Curative & $31(44 \%)$ & $4(9 \%)$ \\
Palliative & $16(23 \%)$ & 0 \\
Advanced Palliative & $3(4 \%)$ & 0 \\
Terminal & & \\
\hline
\end{tabular}

Missing data : ${ }^{3} 8$ patients ${ }^{\circ} 21$ patients ${ }^{\circ} 4$ patients ${ }^{\circ} 18$ patients ${ }^{\circ} 26$ patients

Prescriptions had and adequacy rate to the guidelines $\geq 82 \%$, except in the setting of perioperative nutrition. Evolution of the nutritional status, survival data, complications rates and estimation of the costs will be available in the poster.

Conclusions

This real-life study gave us a look at the current practice inside our institution. It will allow us to question and improve the nutritional management of our patients.

\section{eP253}

A FEASIBILITY STUDY OF EARLY MULTIMODAL INTERVENTION FOR ELDERLY PATIENTS WITH ADVANCED PANCREATIC AND NON-SMALL-CELL LUNG CANCER: THE NEXTAC-ONE STUDY

T. Naito ${ }^{1}$, S. Mitsunaga ${ }^{2}$, S. Miura ${ }^{3}$, N. Tatematsu ${ }^{4}$, T. Inano ${ }^{5}$, T. Mouri ${ }^{6}$, $\overline{\text { T. Tsuji }}$, T. Higashiguchi ${ }^{8}$, A. Inui ${ }^{9}$, T. Okayama ${ }^{10}$, T. Yamaguchi ${ }^{11}$, A.

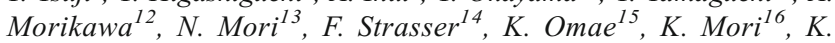
Takayama ${ }^{6}$

${ }^{1}$ Shizuoka Cancer Center, Division of Thoracic Oncology, Shizuoka, Japan

${ }^{2}$ National Cancer Center Hospital East, Department of Hepatobilliary \& pancreatic Oncology, Chiba, Japan

${ }^{3}$ Niigata Cancer Center Hospital, Department of Internal Medicine, Niigata, Japan

${ }^{4}$ National Cancer Center Hospital East, Division of Rehabilitation Medicine, Chiba, Japan
${ }^{5}$ Shizuoka Cancer Center, Division of Nutrition, Shizuoka, Japan

${ }^{6}$ Kyoto Prefectural University of Medicine, Department of Pulmonary Medicine, Kyoto, Japan

${ }^{7}$ Keio University School of Medicine, Department of Rehabilitation Medicine, Tokyo, Japan

${ }^{8}$ Fujita Health University School of Medicine, Department of Surgery and Palliative Medicine, Aichi, Japan

${ }^{9}$ Kagoshima University Graduate School of Medical and Dental Sciences, Department of Psychosomatic Internal Medicine, Kagoshima, Japan

${ }^{10}$ Shizuoka Cancer Center, Division of Rehabilitation Medicine, Shizuoka, Japan

${ }^{11}$ Kyushu University Hospital, DIvision of Nutrition, Kyushu, Japan

${ }^{12}$ Shizuoka Cancer Center, Division of Nusing, Shizuoka, Japan

${ }^{13}$ Aichi Medical University, Palliative Care Center, Aichi, Japan

${ }^{14}$ Cantonal Hospital, Oncological Palliative Medicine- Clinic Oncology and Hematology-Department of Internal Medicine, St. Gallen, Switzerland

${ }^{15}$ Kyoto University, Department of Clinical Biostatistics, Kyoto, Japan

${ }^{16}$ Shizuoka Cancer Center, Division of Clinical Research Center, Shizuoka, Japan

\section{Introduction}

Combination of exercise and nutritional intervention might improve functional-prognosis of cachectic cancer patients. However, high attrition and poor compliance to the intervention limits its efficacy.

\section{Objectives}

To test feasibility of an early induction of new multimodal-intervention specific for elderly cancer patients who are at high risk of cancer cachexia. Methods

This is the multi-center prospective single-arm study. We recruited 30 out of 46 screened patients aged $\geq 70$ years with advanced pancreatic or nonsmall-cell lung cancer scheduled to receive first-line chemotherapy. Eight-week educational intervention comprises of six sessions. Exercise-intervention combines daily low-intensity resistance-training and physical-activity promotive counseling using accelerometer. Nutritional-education includes self-management of nutritional impact symptoms. Supplements rich for branched-chain amino-acids was provided. We assessed attendance, compliance, and efficacy.

\section{Results}

Median age was 75 (range, 70-84) years. Cachexia was diagnosed in $40 \%$ at baseline. Twenty-nine patients attended $\geq 4$ of 6 sessions (96.7\%). One patient dropped out from deterioration of health status. Median proportion of compliant days for supplement consumption and exercise performance were 99 and $91 \%$, respectively. Body or skeletalmuscle mass index, 6-minute-walk-distance, and hand-grip strength were preserved. Time spent in moderate-to-vigorous physical-activity increased (mean change, $2.3 \mathrm{~min} /$ day) and 6-time sit-to-stand time shortened $(-0.5$ seconds) significantly $(\mathrm{p}<0.05)$.

\section{Conclusions}

Early induction of our multimodal intervention showed an excellent compliance and was potentially effective in preservation of physical function for elderly cancer patients receiving concurrent chemotherapy. We are now conducting a randomized phase two study of this strategy to measure its impact on functional-prognosis. (Grant-in-Aid from Japan Agency for Medical Research and Development, Trial-registration No.UMIN000023207)

eP254

HOW TO DISTINGUISH STARVATION FROM REFRACTORY CACHEXIA IN TERMINAL CANCER PATIENTS AND HOW TO PROVIDE NUTRITIONAL INTERVENTION?

\section{N. Nakajima $^{1}$}

${ }^{T}$ University of the Ryukyus, Community-based Medicine and Primary Care, Nishihara, Japan 


\section{Introduction}

Cachexia appears in terminal cancer patients and becomes gradually refractory cachexia. On the other hand, some patients with cachexia have starvation.

Objectives

This study aimed to identify terminal cancer patients under the condition of starvation among refractory cachexia, and to evaluate the effectiveness of nutritional intervention for these patients.

Methods

This was a prospective study. Study subjects were terminal cancer patients with severe malnutrition who admitted to the palliative care unit for last 6 years. We distinguished starvation from refractory cachexia using PPS, PPI, albumin(Alb) and transthyretin(TTR), and evaluated the changes of these indices and survival periods after nutritional intervention.

Results

Out of 223 cases considered to be refractory cachexia on admission, 26 patients were considered to be starveling. They have gotten significant improvement in several indices after nutritional intervention (Alb 2.1+/$0.2 \rightarrow 3.0+/-0.3, \mathrm{p}=0.01$, TTR $8.3+/-1.3 \rightarrow 21.6+/-5.3, \mathrm{p}=0.01$, PPS $24+/-$ $5 \rightarrow 61+/-8, \mathrm{p}=0.01)$. When we could maintain the improved PPS and Alb, TTR, we continued nutritional intervention (Effective group; $n=17$ ).If 'deterioration of PPS, Alb, TTR' occurred, we judged the transition to 'refractory cachexia' and reconsidered the nutritional intervention (Noneffective group; $n=9$ ).PPS was significantly higher in Effective group 2 weeks later $(\mathrm{p}=0.01)$.TTR and Alb were significantly higher in Effective group 3 weeks later $(p=0.003,0.04)$. Survival periods was significantly longer in Effective group (51+/-15 days vs $23+/ 6$ days, $\mathrm{p}=0.003)$.

Conclusions

In order to identify starvation from refractory cachexia, PPS and TTR were important. For the judgement of nutritional indication, PPS was most rapid and important.

\section{eP255}

THE EFFECTS OF ARTIFICIAL HYDRATION AND NUTRITION THERAPY FOR TERMINALLY ILL CANCER PATIENTS BASED ON THE JAPANESE CLINICAL GUIDELINE

\section{N. Nakajima ${ }^{1}$}

${ }^{T}$ University of the Ryukyus, Community-based Medicine and Primary Care, Nishihara, Japan

\section{Introduction}

Recently, there has been a growing interest in the use of artificial hydration and nutrition therapy (AHNT) for terminally ill cancer patients. The guideline for this purpose was published by Japanese Society for Palliative Medicine in 2007 and revised in 2013. In this revised guideline, nutrition-related problems were addressed in addition to hydration-related symptoms.

Objectives

The purpose of this study was to conduct AHNT based on this revised GL, and evaluate the effects on the alleviation of various symptoms and quality of life (QOL).

Methods

Of the 323 terminally ill cancer patients who admitted for palliative care during the last 2 years, 81 patients who were judged "inappropriate hydration and/or nutrition therapy" were prospectively analyzed. We performed guideline-based AHNT with standard pharmacologic therapy. The effects on the alleviation of hydration-related symptoms, appropriateness of nutritional therapy, QOL patient satisfaction and the feeling of benefit of AHNT were examined using a numeric rating scale, criteria of cancer cachexia, EORTC QLQ-C30, satisfaction scale and benefit scale to compare values measured before and one week after AHNT.

Results

Hydration-related symptoms (nausea;20, abdominal pain/distention;18, peripheral edema;32, and dyspnea; 16 cases) were significantly improved after performing guideline-based AHNT ( $\mathrm{p}=0.002, \mathrm{p}=0.005, \mathrm{p}<0.0001$, and $\mathrm{p}=0.020$, respectively). General QOL scores, global satisfaction and feeling of benefit were also significantly improved $(p<0.0001, p=0.0001$, and $\mathrm{p}=0.001$, respectively).

\section{Conclusions}

The provision of appropriate guideline-based AHNT could contribute to alleviating symptoms associated with hydration and nutrition-related problems, improving QOL, patients' satisfaction and feeling of benefit in terminally ill cancer patients.

eP256

UNDERSTANDING NUTRITIONAL PROBLEMS OF METASTATIC BREAST CANCER PATIENTS: OPPORTUNITIES FOR SUPPORTIVE CARE THROUGH EHEALTH

D.L. Oostra ${ }^{1}$, S.K. Mama ${ }^{2}$, S. Bluethmann ${ }^{1}$, J. Wiskemann ${ }^{1}$, K.H. $\overline{\text { Schmitz }}^{I}$, R.M. . Winkels ${ }^{I}$

${ }^{I}$ Penn State Cancer Institute-College of Medicine, Department of Public Health Sciences, Hershey PA, USA

${ }^{2}$ Penn State University, Department of Kinesiology, University Park PA, USA

\section{Introduction}

As a result of improved treatments, metastatic breast cancer patients are now living longer. However, they face potential side-effects of prolonged cancer treatment. Nutrition can play a vital role in managing sequelae; eHealth tools are emerging as promising delivery options for nutrition interventions.

\section{Objectives}

We qualitatively assessed nutritional problems of metastatic breast cancer patients and explored how to address those problems with eHealth tools.

\section{Methods}

Semi-structured phone or in-person interviews were conducted with 21 women participating in an ongoing study called Nurse AMIE, a tablet-based supportive care platform. Interviews were audiotaped, transcribed verbatim and analyzed to identify common themes.

\section{Results}

Most women stated that they currently or previously experienced nutritional problems due to loss of taste or appetite, and fatigue. Participants learned to cope with symptoms over time, but expressed that these issues were rarely addressed during routine clinical care. Participants expressed interest in learning about nutrition in general and specifically during treatment (e.g., foods to eat versus avoid). Specifically sugar and soy consumption were mentioned by many, as women were not sure to avoid them or not. Participants expressed interest in learning nutritional strategies to reduce fatigue and on how to cook/eat healthy while feeling fatigued.

Conclusions

Findings indicate the importance of providing metastatic breast cancer patients with sufficient information about nutrition, as patients report nutritional problems and uncertainty regarding nutritional strategies. Data from the study are a first step towards designing supportive care interventions that could fill the gap of not addressing nutritional concerns during routine clinical care.

\section{eP257}

IMPACT OF SARCOPENIA AND PHASE ANGLE ON SURVIVAL OF PALLIATIVE BREAST CANCER PATIENTS

D. PEREZ ${ }^{1}$, S. Allende ${ }^{1}$

${ }^{T}$ Instituto Nacional de Cancerologia, Palliative care, Ciudad de México, Mexico 


\section{Introduction}

The prognosis of patients with metastatic breast cancer is heterogeneous and can range from several months to many years. Sarcopenia has been evaluated as a separate condition in cancer patients. Palliative care plays a bigger role focusing mainly in the management and prevention of symptoms and side effects related to the cancer or its treatment.

Objectives

Was to evaluate the impact of sarcopenia and the phase angle (PA) in the survival of patients with breast cancer in palliative care.

Methods

We performed a prospective study, assessing the impact of sarcopenia, PA, on overall survival of palliative breast cancer patients treated at the National Cancer Institute in Mexico, between January 2014 and December 2016. Diagnosis of sarcopenia was based on definitions of skeletal muscle mass index (SMI) from the European Working Group on Sarcopenia. The InBody 720 was used to measure BIA. IBM SPSSStatistics v.21 was used.

\section{Results}

Sixty-six advanced breast cancer patients were included. The median age was 52 years. Fourteen percent of patients had sarcopenia $(n=9)$. The median PA value was $4.5^{\circ}$. The median body mass index (BMI) was 25.5. Association between patients with and without sarcopenia and PA was observed $(\mathrm{p}<0.0001)$. According to diagnosis of sarcopenia, overall survival was significantly different, showing a median survival of 171 , 138, and 14 days in patients with no sarcopenia, moderate and severe sarcopenia, respectively $(\mathrm{p}=0.01)$

\section{Conclusions}

We demonstrated that patients with sarcopenia have a shorter survival and that a low phase angle measurement is an independent variable that can be associated to detect sarcopenia.

\section{eP258}

CHEMOTHERAPY IMPACT ON WEIGHT LOSS AND GASTROINTESTINAL SYMPTOMS IN CANCER PATIENTS: A PILOT STUDY FROM CANCER CENTRE IN NORTHERN INDIA

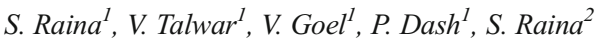

${ }^{1}$ Rajiv Gandhi Cancer Institute and Research centre, Medical Oncology, Delhi, India

${ }^{2}$ ESI Hospital PGIMSR-, Internal Medicine, Delhi, India

\section{Introduction}

Malnutrition is an important aspect of cancer management due to factors related to the disease and treatment.This is especially important for patients receiving chemotherapy. Malnutrition is an independent predictor of mortality and poor response outcome in cancer patients. The reported incidence of different grades of malnutrition in cancer patients is $40-80 \%$. Objectives

Our study aimed to evaluate the relationship of tumor type by location and presence of gastrointestinal symptoms with weight of patients receiving chemotherapy

Methods

After screening 440 patients 140 patients who had locally advanced unresectable and/or metastatic cancers receiving IV chemotherapy were included in our study. A detailed analysis of the patients' records was done to identify symptoms that factor in causing or indicating patients with malnutrition based on weight measurement.

Results

The distribution of tumors was Gall Bladder 25\%, Ovary $18.57 \%$, esophagus \& Gastroesophageal junction $15.71 \%$, Stomach $9.2 \%$ and Pancreas $10 \%$ and others $21.42 \%$. In the study patients $42.5 \%$ experienced consistent weight loss. The prevalence of weight loss was highest in Gall bladder cancer $(64.2 \%)$ and lowest in ovarian cancers (18.4\%). Common relevant symptoms experienced included nausea (58.2\%), anorexia
(45.4\%), constipation (32.5\%), abdominal pain (22.6\%), vomiting (8.6 $\%)$, and diarrhea $(4.6 \%)$.

It was observed that a higher percentage of patients with significant weight loss $(>5 \%)$ experienced nausea $(61.2 \%)$, anorexia $(49.5 \%)$, vomiting ( $12.4 \%)$, diarrhea $(6.8 \%)$.

\section{Conclusions}

We therefore conclude that these GI symptoms may serve as early markers of malnutrition and should be included in routine evaluation of cancer patient receiving chemotherapy to institute measures to manage malnutrition.

\section{eP259}

LINGUISTIC AND CONTENT VALIDATION OF THE PATIENTGENERATED SUBJECTIVE GLOBAL ASSESSMENT (PG-SGA) TRANSLATED AND CULTURALLY ADAPTED TO THE ITALIAN SETTING

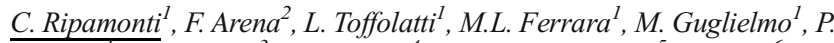
Di Pede ${ }^{I}$, F.D. Ottery ${ }^{3}$, A. Laviano ${ }^{4}$, H. Jager-Wittenaar ${ }^{5}$, P. Bossi ${ }^{6}$

${ }^{1}$ Fondazione IRCCS Istituto Nazionale dei Tumori, Supportive Care in Cancer-Department Oncology-Hematology, Milano, Italy

${ }^{2}$ University of Brescia- ASST Spedali Civili di Brescia-Brescia- Italy, Chair of Hematology and Unit of Blood Disease and Stem Cell Transplantation- Department of Clinical And Experimental Sciences, Brescia, Italy

${ }^{3}$ Hanze University of Applied Sciences- The Netherlands, Research Group Healthy Ageing- Allied Healthcare and Nursing-, Groningen, The Netherlands

${ }^{4}$ Sapienza University- Rome - Italy, Clinical Medicine, Roma, Italy

${ }^{5}$ University of Groningen- University Medical Center Groningen, Department of Maxillofacial Surgery, Groningen, The Netherlands

${ }^{6}$ Fondazione IRCCS Istituto Nazionale dei Tumori, Head \& Neck Complex Unit- Department Oncology-Hematology, Milano, Italy

\section{Introduction}

The Patient-Generated Subjective Global Assessment (PG-SGA) is an instrument to assess malnutrition and risk factors. The Boxes Weight, Intake, Symptoms and Activities/Function are to be completed by the patient, and the Worksheets by the professional. We translated and culturally adapted the original PG-SGA for the Italian setting, according to ISPOR Principles. Objectives

We aimed to test linguistic validity, i.e. perceived comprehensibility and difficulty, and content validity (relevance) of the Italian version of the PGSGA in patients and a multidisciplinary sample of healthcare professionals. Methods

Linguistic validity of the Italian PG-SGA was assessed in 120 Italian cancer patients, and linguistic and content validity were assessed in 81 Italian professionals (41 dietitians, 20 physicians, 18 medical students, 1 physiotherapist, 1 physiotherapy student). Comprehensibility, difficulty and content validity were operationalized by calculating item and scale indices. Scale indices $0.80-0.90$ were considered acceptable, and indices $\geq 0.90$ were considered excellent.

\section{Results}

Patients perceived comprehensibility and difficulty of the Boxes as excellent (S-CI=0.98, S-DI=0.96). Professionals perceived comprehensibility of the Worksheets as excellent ( $\mathrm{S}-\mathrm{CI}=0.92)$, difficulty as acceptable (S$\mathrm{DI}=0.85$ ), and content validity of the full PG-SGA as excellent (S$\mathrm{CVI}=0.92$ ). Dietitians gave higher scores on comprehensibility, difficulty and content validity of Worksheet 4 (physical exam) than the other professions. In Worksheet 4, the temples, clavicles, shoulders, interosseous muscles, scapula, and orbital fat pads were considered most difficult to evaluate.

\section{Conclusions}

Translation and cultural adaptation of the PG-SGA resulted in a Italian version that maintained its original purpose and meaning, and can be completed adequately by patients and professionals 
eP260

DYNAMIC OF ALBUMIN LEVEL DURING FIRST WEEK OF PARENTERAL NUTRITION AMONG THE PATIENTS WITH ADVANCED CANCER HAVE A PROGNOSTIC AND PREDICTIVE VALUE

\author{
A. Skripekova ${ }^{1}$, K. Krizanova ${ }^{1}$, L. Dzurillova $^{1}$ \\ ${ }^{T}$ National Cancer Institute Bratislava, Palliative Medicine Department, \\ Bratislava, Slovak Republic
}

\section{Introduction}

Parenteral nutrition (PN) of patients with advanced cancer is a controversial issue

Objectives

PN can be administered to patients with advanced cancer who are in anabolic state, which is characterized by increasing the level of albumin. Methods

We performed an analysis of 57 patients who were given parenteral nutrition. At the beginingwe defined a treatment goal. We obtained level of albumin before PN, during the first week of PN and on day 30, when we registered if the therapeutic goal was reached. We categorized the patients into two groups. The first group achieving the treatment goal was labeled as Th1. The second group who did not fulfill the treatment goal was labeled Th2. We performed an analysis to confirm, that the dynamic level of albumin during the first week can differ an anabolic state and will have a predictive value. We used a regression analysis linear model to correlate between figures in the group, which is expressed as p-value.

Results

28 patients had the treatment goal fulfilled (Th1) and 29 patients did not fulfill the treatment goal (Th2). When it comes to BMI, GPS, cholinesterase levels and levels of albumin, there were no differences between Th1 and Th2. Statisticaly significant dfference was in terms of dynamics of albumin levels during first week. Median survival in the Th1 group was 90 days and median survival in the Th2 group was 26.5 days. $(\mathrm{p}=0.002)$.

\section{Conclusions}

We can use the outcome of PN during first week to differentiate the patients whose catabolism is in the refractory stage.

\section{eP261 \\ ENTERAL SUPPLEMENTATION IN UNRESECTABLE PANCREATIC CARCINOMA-STYDY OF ONE CENTER}

M. Vaslamatzis $^{1}$, T. Bampouri ${ }^{2}$, N. Alevizopoulos ${ }^{1}$, M. Pavlakis $^{1}$, E.

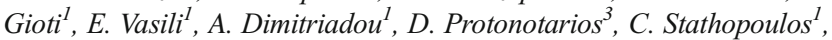
T. Tegos ${ }^{1}$

${ }^{T}$ Evangelismos General Hospital, Oncology, Athens, Greece

${ }^{2}$ Santorini Hospital, Public Health, Santorini, Greece

${ }^{3}$ North Medica, Enteral Nutrition, Athens, Greece

\section{Introduction}

Weight loss in patients(pts) with unresectable pancreatic cancer(UPC), as side effects of chemotherapy(CTP) or/and maldigestion due to pancreatic duct obstruction, is usual. Many other side effects(SE) from CTP such as neuropathy from oxaliplatin or/and hematological/gastrointestinal toxicities lead to delay of programmed CTP and reduction of treatment intensification.

Objectives

THE AIM of this study is to describe the clinical relevance of protein supplementation (Pentasure HP®) in pts with UPC receiving aggressive CTP(FOLFIRINOX)

Methods

Forty-two, 27men(m),15women(w)pts, median body weight(MBW) $63(39-88) \mathrm{kg}, \mathrm{m} / \mathrm{w}$ 64(39-88)/60,5(54-78), ECOG1(0-3), median weight loss $4(0-12) \mathrm{kg} / \mathrm{last}$ trimester with UPC (18 body, 13 head,11 tail) admitted consecutively in the Department of Clinical Oncology, Evangelismos General Hospital, Athens, Hellas between 11/2014-10/2017. Median serum albumin(MSA) was 3.5(2.5-4.6)gr/dl, m/w 3,5(3.6-4.1)/3.4(3-4.3)gr/ dl. All pts received 6 cycles of FOLFIRINOX,q15d with G-CSF primary prophylaxis, Pentasure HP® 60gr/d p.o.,and were reevaluated.

\section{Results}

CR,PR,SD and PD were documented in 5(12\%), 11(26\%), 15(36\%) and $11(26 \%)$ pts respectively. After CTP: MBW for all pts was $62,5(37-88) \mathrm{kg}$, $\mathrm{m} / \mathrm{w}$ 64(37-88)/60(54-79)kg, MSA 3,9(2,6-4,7), m/w 3.9(2.6-4.7)/4(2.74.4)gr/dl. The MSA in the subgroups of CR's,PR's,SD's,PD's respectively were: $3,6(3.1-4.3), 3.5(3-4.1), 3.6(2.6-4), 3.1(2.5-4.1) \mathrm{gr} / \mathrm{dl}$ before treatment and 4(3.8-4.7), 4(3.5-4.7), 3.7(3-4.2), 3,4(2.7-4)gr/dl, after CTP. Toxicity grade(gr) III was observed in $9 / 27(33 \%) \mathrm{m}, 5 / 15(33 \%) \mathrm{w}$, including hematologic $13(33 \%)$ and gastrointestinal 11(26\%) SE, in 58/ $252(23 \%)$ cycles. Neuropathy gr II was demonstrated in $7 / 42(17 \%)$ pts. The median delay of CTP was 4(0-7)days. No toxic death was observed.

\section{Conclusions}

Pts with UPC, in our study, treated with FOLFIRINOX/G-CSF and receiving Pentasure HP®: 1)did not reduce MBW, MSA, and received scheduled CTP with no major delays, 2)experienced less toxicity than expected, 3)had RR 38\% and SD $26 \%$.

\section{eP262}

SUBJECTIVE AND OBJECTIVE TASTE AND SMELL ABNORMALITIES: A POOLED ANALYSIS

A. Black ${ }^{1,2}$, P. Ui Dhuibhir ${ }^{3,4}$, M. Barrett ${ }^{3,5}$, D. McMahon ${ }^{2,3}$, D. Walsh ${ }^{2,3}$

${ }^{I}$ Dublin Institute of Technology, School of Biological Sciences, Dublin, Ireland

${ }^{2}$ Trinity College Dublin, School of Medicine, Dublin, Ireland

${ }^{3}$ Our Lady's Hospice \& Care Services, Academic Department of Palliative Medicine-Education and Research Centre, Dublin, Ireland

${ }^{4}$ University College Dublin, School of Nursing-Midwifery \& Health Systems, Dublin, Ireland

${ }^{5}$ Trinity College Dublin, School of Nursing and Midwifery, Dublin, Ireland

\section{Introduction}

Taste and smell abnormalities (TSA) occur in all cancer stages and affect nutritional intake and quality of life $(\mathrm{QoL})$. Previous research is limited by small sample sizes, varied assessment methods, and cancer treatment focus. TSA in treatment-naïve and advanced cancer groups are under studied and needs further investigation.

\section{Objectives}

Combine three study databases with identical methodologies to investigate TSA in treatment-naïve and advanced cancer. Determine associations between TSA and malnutrition risk, nutritional impact symptoms (NIS) and QoL.

Methods

Study datasets were pooled and analysed (Table 1)

\begin{tabular}{|lccc|}
\hline Disease stage & $\begin{array}{l}\text { Subjective TSA } \\
\text { Assessment }\end{array}$ & $\begin{array}{c}\text { Objective TSA } \\
\text { Assessment }\end{array}$ & abPG-SGA* \\
\hline Treatment-naïve (Study A) & 40 & - & 40 \\
Treatment-naïve (Study B) & 30 & 30 & 30 \\
Advanced Cancer (Study C) & 30 & 30 & 30 \\
Total & 100 & 60 & 100 \\
\hline
\end{tabular}

* Abridged Patient Generated Subjective Global Assessment

\section{TABLE 1: STUDY COHORTS AND ASSESSMENT TYPE}

\section{Results}

Combined dataset had 100 patients ( 66 females). All had subjective assessment and abPG-SGA. Sixty had objective TSA tests. Mean age: 62 . Median ECOG: 1 (range 0-3). 
TSA prevalence was $76 \%$ but depends on assessment type. The most common abnormalities were persistent bad taste $(52 \%)$ and stronger or weaker perception of taste (48\%) and smell (30\%). Sweet acuity was most commonly stronger (24\%) and salt mostly weaker (14\%). TSA severity correlated with lower QoL $(\mathrm{r}=.451, \mathrm{p}<0.01)$. Over half $(64 \%)$ were at malnutrition risk. TSA were significantly associated with NIS (dry mouth, early satiety, fatigue, lack of appetite), malnutrition risk and reduced intake.

\section{Conclusions}

TSA prevalence was high, but varied by assessment method. Over half of participants had persistent bad taste. Those with TSA were more likely to have NIS, be at malnutrition risk and eat less. Pooled analysis yielded significant associations between TSA and NIS, malnutrition risk and reduced intake, which were not observed in individual studies.

\section{eP263}

\section{VITAMIN D DEFICIENCY IN HONG KONG ADVANCED CANCER PATIENTS RECEIVING PALLIATIVE CARE}

\section{K.W. Woo ${ }^{1}, \underline{\text { C.Y.Poon }}^{1}$, A.O.L. Kwok ${ }^{1}$, D.K.H. $\mathrm{Ng}^{1}$, D.M.W. Tse ${ }^{2}$}

${ }^{I}$ Caritas Medical Centre, Palliative Care Unit, Kowloon, Hong Kong S.A.R.

${ }^{2}$ Kowloon West Cluster- Hospital Authority, Princess Margaret Hospital, Hong Kong, Hong Kong S.A.R.

\section{Introduction}

Vitamin D deficiency has been implicated in cancer symptom burden and mortality, however, the prevalence of vitamin D deficiency in Chinese advanced cancer patients receiving palliative care is unknown.

\section{Objectives}

To assess the prevalence of vitamin D deficiency, to identify its correlated factors as well as to analyse its impact on survival among advanced cancer patients receiving palliative care.

\section{Methods}

This was a retrospective cross-sectional single centre study. Baseline patient demographic data, functional status using Palliative Performance Scale, physical and psychological symptom distress using Edmonton Symptom Assessment System (ESAS) as well as Hospital Anxiety Depression Scale (HADS-Chinese Cantonese version) were conducted in recruitment interview. Vitamin D deficiency was defined as level less than $50 \mathrm{nmol} / \mathrm{L}$. Pearson and Spearman's rank correlation coefficient ( $r$ and $\rho$ respectively) were used to identify the correlation of continuous and ordinal data. Kaplan-Meier curve was used to determine the difference in survival.

\section{Results}

A total of 100 patients were recruited, $69 \%$ of patients suffered from vitamin D deficiency. Vitamin D level was positively correlated with age $(r=0.249, p=0.014)$ and PPS $(\rho=0.265, r=0.008)$, negatively correlated with HADS - depression subscale and ESAS - loss of appetite subscale. $(\rho=-0.200$ and $-0.220, p=0.046$ and 0.029 respectively). Patients with moderate or severe vitamin D deficiency had significant poorer survival that those with adequate vitamin D deficiency level at 180 day.

\begin{tabular}{|l|c|l|}
\hline & Number & $\begin{array}{l}\text { p-value of Log-Rank test using } \\
\text { Adequate as comparison }\end{array}$ \\
\hline Adequate $50 \mathrm{nmol} / \mathrm{L}$ or more & 31 & \\
\hline Mild deficiency $30-49 \mathrm{nmol} / \mathrm{L}$ & 34 & 0.052 \\
\hline Moderate deficiency $12.5-29 \mathrm{nmol} / \mathrm{L}$ & 29 & 0.020 \\
\hline Severe less than $12.5 \mathrm{nmol} / \mathrm{L}$ & 6 & $<0.001$ \\
\hline
\end{tabular}

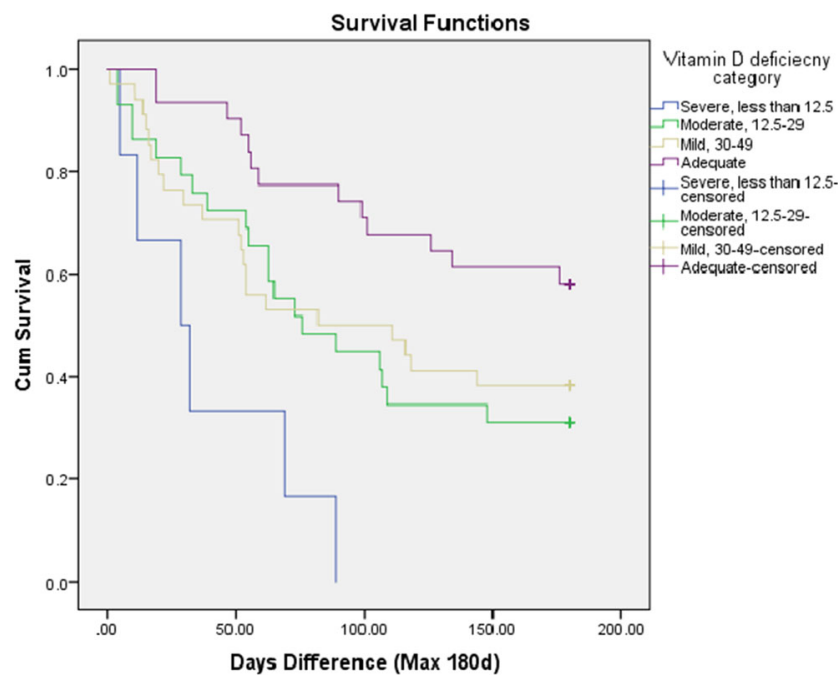

\section{Conclusions}

Vitamin D deficiency is common and is associated with lower HADSdepression score, anorexia and mortality in advanced cancer patients.

\section{eP264}

CORRELATION BETWEEN SKELETAL MUSCLE LOSS AND CLINICAL OUTCOMES AFTER CHEMOTHERAPY IN PATIENTS WITH ADVANCED LUNG CANCER

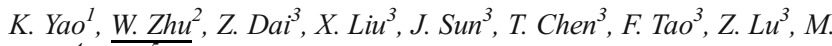
$\mathrm{Cong}^{4}, \mathrm{~J} \cdot \mathrm{Li}^{3}$

${ }^{I}$ National Cancer Center/Cancer Hospital- Chinese Academy of Medical Sciences and Peking Union Medical College, Department of Nutrition, Beijing, China

${ }^{2}$ National Cancer Center/Cancer Hospital-Chinese Academy of Medical Sciences, Department of Integrative Oncology, Beijing, China

${ }^{3}$ Cancer Hospital of Huan Xing Chao Yang District Beijing, Department of Integrative Oncology, Beijing, China

${ }^{4}$ National Cancer Center/Cancer Hospital-Chinese Academy of Medical Sciences and Peking Union Medical College, Department of Integrative Oncology, Beijing, China

${ }^{5}$ National Cancer Center/Cancer Hospital- Chinese Academy of Medical Sciences and Peking Union Medical College, Department of Medical Oncology, Beijing, China

\section{Introduction}

As a common comorbidity in patients with malignant disease, weight loss, especially skeletal muscle loss, was reported to relate to compromised prognosis after chemotherapy.

\section{Objectives}

The study aims to identify the correlation between the reduction of skeletal muscle mass and clinical outcomes after chemotherapy in patients with advanced lung cancer.

\section{Methods}

35 patients with advanced lung cancer treated with platinum-based doublet chemotherapy were enrolled. Skeletal muscle mass was measured with bioelectrical impedence analysis at baseline and after 2-cycle $(\mathrm{C} 2)$ chemotherapy, and the patients were subdivided into the decrease $(\mathrm{N}=15$, skeletal muscle loss $>2 \%)$ and control $(\mathrm{N}=20$, skeletal muscle loss $\leq 2 \%)$ groups. Nutritional status was evaluated with Patient-Generated Subjective Global Assessment (PG-SGA) method before and after C2 chemotherapy. Overall response rate (ORR) and adverse event rate after $\mathrm{C} 2$ chemotherapy were compared between groups.

\section{Results}

After chemotherapy, patients in the decrease group demonstrated reduced hemoglobin level $(128.93 \pm 14.14$ vs. $112.53 \pm 10.47 \mathrm{~g} / \mathrm{L})$ whereas the 
control group had improved nutritional status, as manifested by decreased PG-SGA score $(5.55 \pm 4.01$ vs. $3.25 \pm 2.84, \mathrm{P}<0.05)$ and elevated prealbumin level $(23.62 \pm 5.53 \mathrm{~g} / \mathrm{L}$ vs. $27.45 \pm 5.26 \mathrm{~g} / \mathrm{L}, \mathrm{P}<0.05)$. Compared with the control group, the decrease group displayed significantly lower ORR $(13.3 \%$ vs. $35 \%, \mathrm{P}<0.05)$ yet higher rate of grade I anemia $(66 \%$ vs. $35 \%, \mathrm{P}<0.05)$ and grade II-III gastrointestinal reaction ( $66 \%$ vs. $35 \%, \mathrm{P}<0.05)$.

\section{Conclusions}

Skeletal muscle loss in patients with advanced lung cancer is associated with worse response and increased toxicity, suggesting its potential as a predictor of clinical outcomes after chemotherapy.

\section{eP265}

ORAL COMPLICATIONS OF CANCER TREATMENT IN BREAST NEOPLASM : A RETROSPECTIVE OBSERVATIONAL STUDY IN AN HOSPITAL SETTING

\author{
J. Gonçalves ${ }^{I}, \underline{\text { R. Amaral Mendes }}{ }^{2}$ \\ ${ }^{1}$ Private practice, None, Porto, Portugal \\ ${ }^{2}$ Case Western Reserve University, Oral and Maxillofacial Medicine and \\ Diagnostic Sciences, Cleveland-OH, USA
}

\section{Introduction:}

Anti-cancer therapy and overall managing strategies regarding breast cancer have evolved greatly, significantly improving the prognosis.Nonetheless, the supportive care and management of oral complications is still often disregarded as a concern during the management of breast cancer, often failing to adopt holistic approaches that may increase the patients' quality of life during and after treatment.

\section{Objectives}

The objective of this study was to analyse and further characterise the main oral manifestations of cancer treatment associated with patients with breast neoplasm and ascertain if these patients have knowledge of these types of complications and if they are receiving treatment for them.

\section{Methods}

A retrospective observational study was carried out. Records from 32 patients undergoing chemotherapy were retrieved from the Hospital Santos Silva (CHVNG/E) files and reviewed. Patients were subsequently observed and a questionnaire applied. Tumour type, stage, therapeutic regimens, and oral manifestations were obtained and a statistical analysis performed using SPSS 21.0

\section{Results}

A sample of 32 patients was obtained. Invasive Carcinoma were the most common breast neoplasm form with an incidence of $56.25 \%(n=18)$. Stage IIA was the most common with an incidence of $25 \%(n=8)$. FEC , 46.9\% ( $n=15)$, was the most employed therapeutic chemotherapy regimens. Dysgeusia was the most common oral complication observed with an incidence of $81.3 \%(n=26)$

\section{Conclusions}

The awareness for oral complications during breast neoplasm between patients is very low and undervalued by them. More studies are needed so that the quality of life for the oncological patient can be significantly improved.

\section{eP266}

TREATMENT OF ORAL CHRONIC GVHD WITH DEXAMETHASONE AND TACROLIMUS: A RETROSPECTIVE STUDY

G.A. Ramos ${ }^{1}$, C.B. Lobo ${ }^{1}$, T.D.B.Silva ${ }^{1}$, M.C.R. Moreira ${ }^{2}$, H.S. Antunes ${ }^{3}$ ${ }^{1}$ INCA, Clinical Research Division, Rio de Janeiro, Brazil

${ }^{2}$ INCA, Bone Marrow Transplantation Center, Rio de Janeiro, Brazil

${ }^{3}$ Instituto Nacional de Câncer-INCA, Clinical Research Division, Rio de Janeiro, Brazil

\section{Introduction}

Chronic graft-versus-host disease (cGVHD) is the main immunological complication of allogeneic hematopoietic stem cell transplantation (HSCT), and may be affected multiple organs.

\section{Objectives}

The aim of this study was to evaluate the treatment of oral cGVHD with topical dexamethasone, topical tacrolimus and immunosuppressive medication.

\section{Methods}

This is a retrospective study that evaluated 30 patients who had undergone allogeneic HSCT, with oral cGVHD, from September 2012 to February 2017. The patients were evaluated and treated by three investigators by NIH scores, from diagnosis until cicatrization. The patients were treated with combined topical dexamethasone solution $0.1 \mathrm{mg} /$ $\mathrm{mL}(0.01 \%)$ and topical tacrolimus ointment $0.1 \%$, combined topical dexamethasone, topical tacrolimus and immunosuppressive medication and topical dexamethasone only.

\section{Results}

The results showed that the frequency of oral cGVHD was lichenoid changes $(63.4 \%)$ erythema $(20.3 \%)$ and ulcers $(16.3 \%)$. The median time of cicatrization of lichenoid changes, erythema, and ulceration using dexamethasone associated with the immunosuppressive medication was of 105,42 and 52.5 days respectively $(p=0.017)$. When we associated dexamethasone, tacrolimus and immunosuppressive medication, the median time of cicatrization of lichenoid changes, erythema and ulceration was of 98,35 and 73.5 days ( $p=0.044)$. When dexamethasone was used only, the median time of cicatrization of lichenoid changes, erythema and ulceration was $182,140,21$ days respectively $(\mathrm{p}=0.022)$.

\section{Conclusions}

We can conclude that we had a better response in patients who were treated with association of dexamethasone, tacrolimus and immunosuppressive medication and erythema has a better response them compared with lichenoid changes and ulceration.

\section{eP267}

\section{DENTAL IMPLANT SUCCESS IN IRRADIATED JAWS: A RETROSPECTIVE STUDY}

\section{K. Bektas-Kayhan ${ }^{1}$, E. Sancakli ${ }^{2}$, A. Balik', E. Baca ${ }^{2}$, T. Celakil ${ }^{2}$ \\ ${ }^{T}$ Istanbul University Faculty of Dentistry, Oral and Maxillofacial Surgery, Istanbul, Turkey \\ ${ }^{2}$ Istanbul University Faculty of Dentistry, Prosthodontics, Istanbul, Turkey}

\section{Introduction}

Head and neck cancer patients usually needs serious oro/dental constructive support after the cancer therapy. Although radiation -especially in curative doses- has a negative influence on osseointegration, dental implants are trustful partners in dental rehabilitation of these patients for many years.

\section{Objectives}

Our aim here to present the success of dental implants even in high radiation doses ( $>50 \mathrm{~Gy}$ ) of head and neck cancer patients.

\section{Methods}

The data of HNCPs who were referred to oral and maxillofacial surgery clinic from 2011-2017 were reviewed for inclusion criteria's: 1. Receiving total dose of radiotherapy $>50 \mathrm{~Gy}, 2$. Performed dental implant surgery by the author, 3 . Prosthetic rehabilitation was completed at least 6 months ago. The criteria were fulfilled by 53 patients ( 19 female, 34 male) with the mean age of 54.03. The success and complications of dental implants were evaluated.

\section{Results}

Mean follow up time was 32 months. 176 implants were inserted, and long-term success rate was calculated as $94,9 \%$. In total 9 implants were lost. Only 2 cases of osteoradionecrosis were evident with successful recovery in 4-10 months. 


\section{Conclusions}

1. The osseointegrated implant supported dental rehabilitation should be considered as reliable partners in dental rehabilitation of HNCPs. Under the special precautions, they are successful even in high doses of radiation.

2. Today, oral and maxillofacial surgeon and prosthodontists are inseparable part of oncology team with a positive impact to head and neck cancer patients' quality of life.

\section{eP268}

GRA F T-VE R S U S-H O S T D ISEASE (GVHD) IN HEMATOPOIETIC STEM CELL TRANSPLANT (HCST) PATIENTS, INTERIM ANALYSIS OF THE ORASTEM STUDY

N.M.A. Blijlevens ${ }^{1}$, I. von Bültzingslöwen ${ }^{2}$, A. Hovan $^{3}$, B. Hasséus ${ }^{2}$, M.C. Huysmans $^{1}$, K. Garming Legert ${ }^{4}$, R.G.A. Koppelmans ${ }^{5}$, A.M.G.A. Laheij $^{5}$, S. van Leeuwen ${ }^{1}$, A. Gruza ${ }^{3}$, N.L. Rozario ${ }^{6}$, F.R. Rozema ${ }^{5}$, J.E. Johansson', M.T. Brennan ${ }^{8}$

${ }^{1}$ Radboud University Medical Center, Department of Dentistry, Nijmegen, The Netherlands

${ }^{2}$ Sahlgrenska Academy, Not applicable, Gothenburg, Sweden

${ }^{3}$ BC Cancer Agency, Not applicable, Vancouver, Canada

${ }^{4}$ Karolinska Institute, Not applicable, Huddinge, Sweden

${ }^{5}$ Academic Center for Dentistry Amsterdam ACTA, Not applicable, Amsterdam, The Netherlands

${ }^{6}$ Carolinas Healthcare System, Center for Outcomes Research and Evaluation CORE, Charlotte, USA

${ }^{7}$ Sahlgrenska University Hospital, Department of Hematology and Coagulation, Gothenburg, Sweden

${ }^{8}$ Carolinas Healthcare System, Department of Oral Medicine, Charlotte, USA

\section{Introduction}

GVHD has a major impact on morbidity and mortality in allogeneic HSCT recipients.

Objectives

To evaluate prospectively the prevalence of GVHD and determine the relationship with objective and subjective oral findings.

Methods

Patients were prospectively evaluated at 100-day, 6, and 12 months following HSCT as part of Orastem study. Prevalence of GVHD was documented by the treating physician, and presence of symptoms and objective manifestations related to oral GVHD were assessed. Fishers Exact, ttest and Wilcoxon Rank-Sum tests were used to analyze the variables wherever deemed appropriate.

\section{Results}

This interim analysis included 96 patients (median age (interquartile range $=55.5(39.5-62)$ years, $54(56 \%)$ males). Medical diagnoses included: acute myelogenous leukemia $=36(38 \%)$, acute lymphoblastic leuke$\mathrm{mia}=13(14 \%)$, myelodysplastic syndrome $=12(13 \%)$, lymphoma $=9$ $(9 \%)$ and myelofibrosis=7 (7\%). Forty-nine $(51 \%)$ patients had GVHD during any follow-up visit. Type of conditioning regimen was not associated with GVHD. In the GVHD group, $30(61 \%)$ had oral GVHD with symptoms of lichenoid lesions $=24(49 \%)$, dry mouth $=11(22 \%)$, erythematous lesions $=6(12 \%)$ and ulcerations $=5(10 \%)$. The total mucosal lesion size was larger in the GVHD vs no-GVHD group $(\mathrm{p}=0.009)$. GVHD patients had more oral pain (max grade $=2)$ with functional limitations (CTC scale: 0-4) compared to the no-GVHD group (max grade=1) $(\mathrm{p}=0.006)$.

\section{Conclusions}

Prospectively documented oral GVHD is a common finding with allogeneic HSCT patients with increased rates of pain and functional limitations. The rate of GVHD in this interim analysis of Orastem indicates that future robust multivariate analysis will be possible.
eP269

THE IMPACTS OF DYSPHAGIA AND ITS ASSESSMENT ON HEAD AND NECK CANCER PATIENTS: A SYSTEMATIC REVIEW

Y.J. Chen $^{1}$

${ }^{T}$ Da-Yeh University, Department of Nursing, Changhua, Taiwan R.O.C.

\section{Introduction}

Dysphagia is the most common symptom in head and neck cancer patients due to multiple active cancer treatment. Although dysphagia could be examined by intrusive scope, the examination is not routinely and limited to understand how patients perceive dysphagia and its impact.

\section{Objectives}

The purposes of the study were: (1) to understand the impacts of dysphagia on HNC patients; (2) to identify the available dysphagia questionnaires for $\mathrm{HNC}$ patients.

\section{Methods}

Five electronic databases were systematically searched. Studies investigating dysphagia in head and neck cancer patients and published between 1995 and 2016 were included for aim 1. Psychometric studies were including if they measured dysphagia in head and neck cancer patients but excluding the medical examination or treatment for aim 2 .

\section{Results}

Twenty-six original studies were included; most of them were crosssectional and longitudinal quantitative studies, and mere studies were intervention studies. Most dysphagia research reported the association with emotional function, social eating, physical function and global QOL. There were 4 swallowing difficulties specific questionnaires, and 8 questionnaires containing swallowing items for $\mathrm{HNC}$ patients.

\section{Conclusions}

Many factors contribute to swallowing related QoL, so routine and long term swallowing assessment should be provided. The study suggested adopting the validated swallowing-specific questionnaires that give more information about swallowing. Patients should be coached and advised to continue oral intake and implementation of swallow rehabilitation by a multidiscipline team.

\section{eP270}

THERAPEUTIC POSSIBILITIES FOR PATIENTS SUFFERING FROM XEROSTOMIA AND HYPOSALIVATION INDUCED BY RADIOTHERAPY AND CHEMOTHERAPY

E. Ferreira $^{1}$, H.S. Antunes ${ }^{2}$, C. Andrade ${ }^{3}$, S. Santos ${ }^{4}$

${ }^{T}$ private, not aplicable, Rio de Janeiro, Brazil

${ }^{2}$ Instituto Nacional de Câncer, Coordination of clinical research, Rio de Janeiro, Brazil

${ }^{3}$ Faculdade Israelita de Ciência da Saúde Albert Einstein, Ensino, São Paulo, Brazil

${ }^{4}$ Faculdade Israelita de Ciência da Saúde Albert Einstein, Ensino, Rio de Janeiro, Brazil

\section{Introduction:}

One of the most common side effects of radiotherapy and chemotherapy treatment for head and neck cancer is the xerostomia and hyposalivation.

\section{Objectives}

Describe the main therapeutic possibilities forpatients suffering from xerostomia and hyposalivation induced by radiotherapy and chemotherapy.

\section{Methods}

The search strategy was done through articles indexed in the electronic databases National Library of Medicine (PUBMED) and Scientific Electronic Library Online (SCIELO), published between $01 / 2000$ and $05 / 2017$, using the following descriptors: salivary gland hypofunction, xerostomia, radiotherapy, treatment, dry 
mouth, with the crossings: [salivary gland hypofunction] or [radiotherapy], [dry mouth] or [radiotherapy], [xerostomia] or [radiotherapy], [salivary gland hypofunction] or [therapy], [xerostomia] or [therapy], [dry mouth] or [therapy]. Only articles in English involving treatment for hyposalivation and xerostomia in patients subjected to head and neck radiotherapy, alone or associated with chemotherapy, have been selected.

\section{Results}

Fifty articles have been used approaching the following therapeutics: radioprotectors, mitigators, low-power laser, transcutaneous electrical stimulation, bethanechol, cevimeline, masticatory stimulants, oral lubricants, surgical transfer of salivary gland, acupuncture, hyperbaric oxygen and gene therapy. The review identified scientific evidence in using muscarinic agonist stimulation, oral mucosa lubricants, surgical transfer of submandibular gland and acupuncture. Gene therapy also seems to be promising.

\section{Conclusions}

There is evidence that xerostomia and hyposalivation cause significant impact on quality of life, wherein the degree of injury is directly proportional to gland tissue exposure to the radiotherapy. Among therapies with scientific evidence, the muscarinic agonists and oral lubricants are the most clinically used ones, although symptom relief is temporary and short-lived.

\section{eP271}

IMPORTANCE OF ADHERENCE TO DENTAL TREATMENT IN THE IMPROVEMENT OF ORAL HEALTH AFTER HEAD AND NECK RADIOTHERAPY: CASE REPORT

E. Ferreira $^{1}$, H.S. Antunes ${ }^{2}$

${ }^{1}$ private, not aplicable, Rio de Janeiro, Brazil

${ }^{2}$ Instituto Nacional de Câncer, Coordination of Clinical Research, Rio de Janeiro, Brazil

\section{Introduction}

Radiotherapy (RT) is one of the treatment options for head and neck cancer patients and may generate oral toxicities that interfere in the quality of life.

\section{Objectives}

To show the importance of adherence to dental treatment, after radiotherapy and its impact on oral health.

\section{Methods}

Describing two clinical cases of patients with oropharyngeal tumors treated with linear accelerator, with dose of 70 Gy, 2 Gy per day, 5 days a week during 6 weeks. Case 1: Male, 59 years old, end of the RT in 2008. After RT, he presented xerostomia and kept semestral follow-up, daily mouthwash with sodium fluoride at $0.05 \%$ and gel at $2 \%$ at night, control of oral hygiene and diet. Case 2: Female 73 years old, end of radiotherapy in 2010. She attended the dental office 3 years after the end of RT with caries in all teeth, severe xerostomia and precarious oral hygiene. Endodontic, prosthetic and restorative treatments were performed. She was oriented to use silicone trays with sodium fluoride at $2 \%$ gel at night, oral lubricant, fluoride toothpaste and oral hygiene after every meal.

\section{Results}

Case 1: Ten years after RT, he keeps the same oral status of the beginning of the treatment.

Case 2: For 8 years, she keeps annual follow-up, but she does not follow the dental orientations correctly, always being necessary to perform new dental interventions.

\section{Conclusions}

Close maintenance of oral care, non-cariogenic diet and dental follow-up, influence positively both in quality life and in oral health of these patients.

\section{eP272}

TOBACCO USE, AWARENESS AND CESSATION AMONG MALAYALI TRIBES, YELAGIRI HILLS, TAMIL NADU, INDIA

D.L. Francis ${ }^{1}$

${ }^{T}$ Tamil Nadu Dr MGR Medical University, Public Health, Chennai, India

\section{Introduction}

Despite remarkable world-wide progress in the field of diagnostic, curative and preventive medicine, still there are large populations of people living in isolation in natural and unpolluted surroundings far away from civilisation, maintaining their traditional values, customs, beliefs and myths. India has the second largest tribal population of the world next to the African countries. About half of the world's autochthonous people live in India, thus making India home to many tribes which have an interesting and varied history of origins, customs and social practices.

\section{Objectives}

The present study was conducted to assess the tobacco use, awarness and its effect on health among Malayali tribes, Yelagiri Hills, Tamil nadu, India.

\section{Methods}

Data was collected from a cross-sectional survey, using a Survey Proforma, clinical examinationand a pre-tested questionnaire which included Demographic data, tobacco habits. An intra-oral examination was carried out by a single examiner to assess the Oral Health Status using WHO Oral Health Surveys - Basic Methods Proforma (1997)

\section{Results}

Among 660 study population, 381(57.7\%) had no formal education, $75 \%$ had the habit of alcohol consumption. Of those who had the habit of smoking, $26 \%$ smoked beedi, $10.9 \%$ smoked cigarette, $65 \%$ chewed raw tobacco, $18 \%$ chewed Hans and Prevalence of oral mucosal lesions in the study population was high.

\section{Conclusions}

From the results of this study it may be concluded that the Malayali tribes were characterized by a lack of awareness about oral health, deep rooted dental beliefs, high prevalence of tobacco use and limited access to health services.

\section{eP273}

MEDICATION-RELATED OSTEONECROSIS OF JAW (ONJ) IN CANCER PATIENTS: COMPARISON OF STAGE ACCORDING TO AMERICAN (AAOMS) AND ITALIAN (SIPMO-SICMF) STAGING SYSTEMS IN 58 PATIENTS

\author{
V. Fusco $^{1}$, A. Fasciolo ${ }^{2}$, L. Benzi ${ }^{3}$, A. Gambino ${ }^{4}$, M. $_{\text {Cabras }}{ }^{4}$, I. De

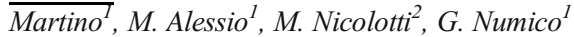 \\ ${ }^{1}$ Azienda Ospedaliera di Alessandria, Oncology, Alessandria, Italy \\ ${ }^{2}$ Azienda Ospedaliera di Alessandria, Maxillofacial Surgery, \\ Alessandria, Italy \\ ${ }^{3}$ Azienda Ospedaliera di Alessandria, Radiology, Alessandria, Italy \\ ${ }^{4}$ Azienda Ospedaliera Città della Salute di Torino, Dental School, Turin, \\ Italy
}

\section{Introduction}

Controversies exist among the ONJ definition (and staging system) of the American Association Oral Maxillofacial Surgery (AAOMS) task force, based on clinical features (mainly bone exposure), and the Italian proposal, supported by the Italian Societies of Oral Medicine (SIPMO) and Maxillofacial Surgery (SICMF), implying adoption of imaging tools (mainly Computed Tomography, CT) together with clinical features (Bedogni et al, Oral Disease 2012 ). 


\begin{tabular}{|c|c|c|c|c|}
\hline SPanc-skaf & & & & \\
\hline $\begin{array}{c}\text { stape } \\
\text { Monesstage } \\
\downarrow\end{array}$ & I & - & = & Totalcases \\
\hline 0 & 8 & 15 & 0 & $\mathbf{2 3}$ \\
\hline I & 6 & 4 & 0 & 10 \\
\hline . & 3 & D & 0 & $\boldsymbol{Z Z}$ \\
\hline - & a & 0 & 3 & 3 \\
\hline Iotal cases & 17 & 38 & 3 & $\mathbf{s}$ \\
\hline
\end{tabular}

Objectives

To compare the stage of ONJ cases at the first observation time in patients receiving antiresorptive therapy (bisphosphonates, denosumab) for malignant diseases, according to two different staging systems.

Methods

We reviewed cancer and myeloma patients with signs of ONJ after antiresorptives.

\section{Results}

We collected data about 58 patients. The AAOMS stage was $0 / \mathrm{I} / \mathrm{II} / \mathrm{III}$ respectively in 23/10/22/3 cases. The SIPMO-SICMF stage was I (alveolar bone)/ II (extralveolar bone) /III (complicated) respectively in 17/38/ 3 cases. The 23 AAOMS stage 0 cases (signs/symptoms without bone exposure) were reclassified as stage I(8) or II(15) respectively, according to the Italian system.

\section{Conclusions}

There is no unique ONJ definition and staging system, even if the AAOMS one is mostly reported. In our hands, in ONJ cases in cancer patients the AAOMS definition and staging system are insufficient, exposing patients to delayed diagnosis (and potentially inadequate treatment). It would be advisable to establish the diagnosis not only on the basis of the clinical data but also on the findings of the CT scan, since the latter technique offers greater information about extent and severity of the disease.

\section{eP274}

PERIOPERATIVE ORAL MANAGEMENT HAS A POSITIVE EFFECT IN MAINTAINING SERUM ALBUMIN AND LYMPHOCYTE COUNT THROUGH CANCER SURGERY

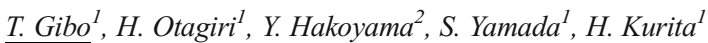

${ }^{T}$ Shinshu University School of Medicine, Department of Dentistry and Oral Surgery, Matsumoto, Japan

${ }^{2}$ Aizawa Hospital, Oral and Maxillofacial Surgery, Matsumoto, Japan

\section{Introduction:}

Perioperative oral management is expected to reduce systemic/local infection and some other adverse events caused by oral/dental bacteria. It is also expected to maintain and improve oral functions, which relates to good patient's recovery and quality of life. Perioperative management may produce good patient's immunonutritional condition.

\section{Objectives}

The purpose of this retrospective study is to assess whether perioperative oral management had a positive effect in maintaining nutritional and immune condition through cancer surgery.

Methods

181 patients who underwent cancer surgery for digestive or urinary organ in 2016 were available in the study. Of these, 63 patients underwent perioperative oral management (POM group) and other 118 patients did not (control group). Prognostic nutritional index (PNI, calculated from serum albumin and lymphocyte count; Ono et al, 1984) was assessed through the cancer surgery and was compared between the groups.

\section{Results}

PNI of the POM group tended to reveal less decline and better recovery after cancer surgery than that of the control group. If patients who resume ingestion within three days after the surgery were compared, the difference was statistically significant (repeated measure analysis, least-squares method, $\mathrm{p}<0.05$ ). Conclusions

The results of this study suggested the possibility that perioperative oral management might have a positive effect on maintaining serum albumin level and lymphocyte count through cancer surgery.

\section{eP275}

SURVIVAL ANALYSIS OF PATIENTS WITH METASTATIC BONE DISEASE ADMINISTERED ANTI-RESORPTIVE AGENTS

\section{T. Higuchi ${ }^{1}$, Y. Soga ${ }^{1}$, A. Sasaki ${ }^{2}$}

${ }^{T}$ Okayama University Hospital, Division of Hospital Dentistry, Okayama, Japan

${ }^{2}$ Okayama University Graduate School of Medicine- Dentistry and Pharmaceutical Sciences, Department of Oral and Maxillofacial Surgery and Biopathology, Okayama, Japan

\section{Introduction}

Anti-resorptive agents are administered during the overall management of patients with metastatic bone disease to delay and prevent skeletal-related events and side effects of anti-resorptive agent-related osteonecrosis of the jaw (ARONJ). Dental infection a risk factor associated with ARONJ. Thus, during survival, performing dental treatment and oral care is imperative in patients irrespective of ARONJ.

\section{Objectives}

This retrospective study aimed to evaluate overall survival rates of patients with metastatic bone disease in our hospital.

\section{Methods}

At our hospital, 270 patients were administered zoledronic acid $(n=110)$ or denosumab $(n=160)$ for metastatic bone disease from April 2012 to March 2015. The Kaplan-Meier analysis and log-rank test were used to estimate survival rates of all patients, including those administered zoledronic acid, or denosumab and those with a primary tumor.

Results

In total, 137 patients died. While the overall 1-year survival rate was $53 \%$, 3 -year survival rate was $19 \%$. The overall survival rate of the denosumab group was higher than that of the zoledronic acid group, and KaplanMeier survival estimates of both groups were significantly different $(P<$ 0.01 ). The overall survival rate of the breast cancer group was higher than that of the group of other primary tumors, and Kaplan-Meier survival estimates of both groups were significantly different $(P<0.01)$.

\section{Conclusions}

This study revealed that denosumab treated breast cancer patients showed longer survival than those with some other type of cancer. Overall, dental treatment and oral care is essential to ensure high survival rates among patients with metastatic bone disease.

\section{eP276}

PREVALENCE OF ORAL COMPLICATIONS DURING SYSTEMIC CANCER THERAPY

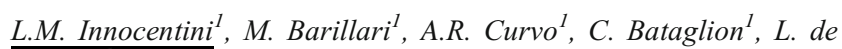
Macedo

${ }^{1}$ University of São Paulo- Ribeirão Preto Clinical Hospital- Ribeirão Preto School of Medicine, Department of Head and Neck SurgeryDentistry and Stomatology Division, Ribeirão Preto, Brazil 


\section{Introduction}

Oral side effects related to cancer therapies and dental disease are associated with a legion of signs and complaints in head and neck region. However, there is poor information about patients complains associated to oral health during systemic cancer therapy.

Objectives

To identify the need of oral care by active search of oral health problems during systemic cancer therapy.

Methods

Patients receiving systemic cancer therapy were evaluated from August 2016 to October 2017 in an Oncology Centre. All patients were interviewed twice a week and asked about their oral health complaints during the last month, and an experient dentist evaluated teeth and oral mucosa.

Results

Three hundred and twelve patients were included in this study, $5.3 \%$ with mucositis (95\% mild, 5\% severe), 5.3\% reported burning mouth, $13.1 \%$ reported dysgeusia, $14.4 \%$ reported xerostomia, $2.5 \%$ presented fungal infection and $0.3 \%$ viral infection detected by oral examination. About dental health, in upper jaw $36.3 \%$ were partially edentulous, $41.9 \%$ edentulous, in lower jaw $55.9 \%$ were partially edentulous, $27.8 \%$ edentulous, and oral hygiene is considered satisfactory in $65.9 \%$ and unsatisfactory in $34.1 \%$ with $44.7 \%$ of patients reporting need of dental treatment.

\section{Conclusions}

Clearly the standard time to prepare a treatments plan for patients with cancer is before they have undergone therapy. Although it is not possible all time because usually there is no time to think about oral health when cancer is discovered. The active search of oral health problems during systemic therapy should help to avoid interruptions in cancer therapy course.

eP277

USE OF A MULTI-PRONGED STRATEGY TO RAISE THE IMPLEMENTATION RATE OF ORAL CARE AMONG HEAD AND NECK CANCER PATIENTS

Y.W. Lin $^{1}$

${ }^{T}$ head nurse, oncology ward, New Taipei City, Taiwan R.O.C.

\section{Introduction}

This study proposed that the implementation of appropriate oral care education in a clinical care setting can not only promote oral comfort and reduce the incidence of lesions, but also prevent the occurrence of treatment-related side effects and, thus, improve the therapeutic effect of treatments.

Objectives

It was hoped that this oral care program would help increase the awareness of individualized health education among head and neck cancer patients, get them to attach more importance to oral care.

Methods

1.Morning meeting: In-service education and an oral care implementation process were promoted to fellow colleagues.

2.An oral care educational video was filmed. The film covered issues such as the importance of oral hygiene, assessment methods, cleaning procedures, tool preparation, cleaning steps, and mouth washing procedures. A QR code was created for the video, enabling patients and their family members to access it simply by scanning the code.

\section{Results}

Through the use of the health education leaflets and the QR code to promote access to the health education video and information on actual procedures, patients and their family members were able to understand the health information better, resulting in an increase in the oral care implementation rate of the patients to $85 \%$.

Conclusions

The nursing issues most commonly encountered by head and neck cancer patients are those related to the oral mucosa, and proper oral care or oral hygiene can help prevent the occurrence of issues such as oral mucositis, minimize oral mucosal changes during the treatment period, and increase the level of comfort felt by patients.

\section{eP278}

MANAGEMENT OF ORAL POTENTIALLY MALIGNANT DISORDERS AND ORAL CANCER IN NEPALESE DENTAL PATIENTS

A. Mainali ${ }^{l}$

${ }^{T}$ Nepal medical college, Oral Medicine and Radiology, Kathmandu, Nepal

\section{Introduction}

Oral potentially malignant disorders (OPMDs) describe oral lesions and conditions associated with a risk of malignant transformation. Erosive lichen planus is the commonest OPMD which has higher chances of turning into malignancy. Early detection and treatment remains the most important approach for reducing the risk of malignant transformation associated with OPMDs

\section{Objectives}

To determine the management of OPMDs and oral cancer among a group of Nepalese dental patients.

\section{Methods}

A questionnaire-based study was carried out following mailing of covering letter and self-administered questionnaire comprising 10 items to dental colleges in Kathmandu Nepal based on convenient sampling. The questionnaire was sent to all the specialists directly involved in the care of such patients.

\section{Results}

Out of the 5 dental colleges, we received responses from all colleges with 60 completely filled questionnaires. Among 60 respondents, 11 were oral medicine specialists and the rest were dental surgeons. $67.7 \%$ of the dentists gave topical steroid initially for erosive lichen planus and the rest prescribed only analgesics and anesthetics. There was lack of awareness regarding early treatment of erosive lichen planus. only $25 \%$ of the dentists said they had knowledge regarding Supportive care for cancer patients undergoing radiotherapy and management of complications like radiation mucositis.

\section{Conclusions}

OPMDs among dental patients are relatively common and awareness about oral cancer among Nepalese dental patients is low. Necessary steps need to be taken to improve dentists knowledge about treatment of OMPDS and oral cancer. Continuing dental education is required for improving attitudes toward early diagnosis, supportive care and treatment.

\section{eP279}

ORAL DISEASES AND SURVIVAL AFTER ALLOGENEIC HSCT

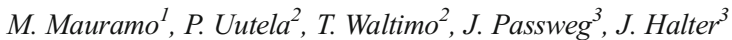 \\ ${ }^{T}$ Institute of Dentistry, Department of Oral and Maxillofacial diseases, \\ University of Helsinki, Finland \\ ${ }^{2}$ UZB University Centre for Dental Medicin, Department for Preventive \\ Dentistry and Oral Microbiology, Basel, Switzerland \\ ${ }^{3}$ University Hospital, Department of Hematology, Basel, Switzerland
}

\section{Introduction}

Hematopoietic stem cell transplantation (HSCT) recipients suffer from acute and long-term side effects in the oral cavity.

\section{Objectives}

The purpose of this study was to evaluate oral health and survival of allogeneic HSCT recipients up to 24 months post-HSCT. 
Methods

269 adult allogeneic HSCT recipients (m/f: 127/142; mean age: 50.6 years), treated in the Department of Haematology, University Hospital Basel, Switzerland, between 2008-2016 were included in the study. Dental examination, including panoramic radiography, DMFT index and measurement of stimulated saliva flow rate (SSFR) was conducted just prior and 6,12 and 24 months post-HSCT. The associations of oral health parameters and six months survival were examined.

Results

Forty patients (14.8\%) died within six months post-HSCT. Hyposalivation (SSFR $\leq 0.7 \mathrm{ml} / \mathrm{min}$ ) pre-HSCT was more common among deceased patients, compared with HSCT recipients who survived more than six months $(47.5 \%$ vs. $31.4 \%, \mathrm{P}<0.05)$. Caries incidence was also higher among deceased patients, compared with HSCT recipients who survived more than six months (mean $2.0 \pm 3.0$ vs. $0.88 \pm 2.0 ; \mathrm{P}<0.05$ ). There were no differences in DMFT or radiological attachment lost (RAL $>3 \mathrm{~mm}$ ) between deceased or survived HSCT recipients. Hyposalivation pre-HSCT was associated with increased DMFT score $(\mathrm{P}<0.05)$ and smaller number of teeth $(\mathrm{P}=<0.05) 24$ months post-HSCT, when HSCT recipients with hyposalivation were compared with HSCT recipients with normal SSFR.

Conclusions

Hyposalivation and caries are associated with increased decease risk postHSCT. Additionally, hyposalivation predisposes to dental diseases postHSCT. Thus, special care and regular dental examinations, including SSFR measurements, pre- and post-HSCT are recommended.

\section{eP280}

PATIENTS WITH DIGESTIVE SYSTEM CANCER WERE ASSOCIATED WITH LESS TEETH AND LESS DENTURE WEARER

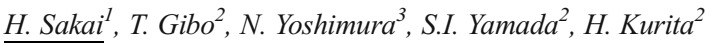

${ }^{T}$ Nagano Municipal Hospital, Dentistry Oral Surgery, Naganotommitake1333-1, Japan

${ }^{2}$ Shinshu University School of Medicine, Dentistry and Oral Surgery, Matumoto, Japan

${ }_{3}^{3}$ Shinshu Ueda Medical Center, Dentistry and Oral Surgery, Ueda, Japan

\section{Introduction}

The patients who suffered with digestive system cancer have often been assumed to have the greatest more dental treatment needs.

Objectives

The aim of this study is to survey prevalence of dental disease in the patients who underwent treatment of digestive system cancer.

Methods

This study was includingincluded 235 patients. The patients were aged 60 to 79 years-old who underwent treatment of digestive system cancer in our hospital. Dental condition was assessed and compared with the results of the Japanese National Survey of Dental Diseases in 2011 (60 to 79 years-old, $\mathrm{n}=1,619)$ for control.

\section{Results}

The patients group had significantly less decayed and filling teeth, while more missing teeth than the result of national survey (D-index 0.55 vs 0.98 , student t-test, $\mathrm{p}<0.05$; F-index 8.32 vs 10.10, $\mathrm{p}<0.05$; and M-index 10.6 vs 9.04, $\mathrm{p}=0.08$ ). Multi-tooth loss (more than eight teeth) was more frequently observed in the patient group than the results of national survey (ratio of more than 20 teeth remaining were $548.1 \%$ vs $37.3 \%$ in patients and $63.4 \%$ in standard, chi square test, $(\mathrm{p}<0.01)$. However, tThe ratio of denture wearer was lower in the patients group were $(34.8 \%$ vs $52.9 \%$, in patients and $50.2 \%$ in standard $(\mathrm{p}<0.01)$. Their oral disease status and treatment needs were higher compared to standard indicating a poor oral hygiene level.

\section{Conclusions}

The result of this study suggested that the patients with digestive system cancers more frequently associated with multi-tooth loss and less denture wearer.
eP281

SPIRONOLACTONE AMELIORATES RADIATION-INDUCED LATE FIBROSIS IN SALIVARY GLANDS IN A RAT MODEL

C. Elsurer ${ }^{1}$, Z.E. Celik' ${ }^{2}$, G. Yavas $^{3}$, C. Yavas $^{3}$

${ }^{1}$ Selcuk University, Department of Ear- Nose and Throat, Konya, Turkey

${ }^{2}$ Selcuk University, Department of Pathology, Konya, Turkey

${ }^{3}$ Selcuk University, Radiation Oncology, Konya, Turkey

\section{Introduction}

Management of head and neck cancer includes surgical resection, chemotherapy and radiotherapy (RT). While RT significantly improves survival, exposure to ionizing radiation usually results in unavoidable coirradiation of the normal tissues surrounding tumor. Salivary glands, that have a high impact on quality of life, are among these normal tissues affected by ionizing radiation.

\section{Objectives}

We hypothesized that pre-radiation treatment with a mineralocorticoid receptor (MR) antagonist, spironolactone (S), may have a radioprotective effect on salivary glands.

\section{Methods}

Thestudy included 30 adult rats divided into four groups. G1 $(n=8)$ was defined as control group and the rats in this group were sham irradiated. G2 ( $n=6)$ was defined as the 15 Gy RT group. G3 $(n=7)$ and G4 $(n=9)$ were defined as S-only and the RT-plus-S groups, respectively. Rats were sacrificed at the sixth hour and the twenty-first and hundredth days after RT. Salivary gland samples were taken for microscopic examination.

\section{Results}

Periductal-perivascular inflammation scores at the twenty-first and hundredth days tended to be higher in G2 than in G4, but the difference was not statistically significant. Periductal-perivascular fibrosis scores were significantly different between the groups at the hundredth day $(\mathrm{P}=0.010)$. Pairwise comparisons revealed that periductal-perivascular fibrosis scores were different between $\mathrm{G} 1$ and $\mathrm{G} 2(\mathrm{P}=0.014)$, between G2 and G3 (P=0.014), and between G2 and G4 (P=0.034)

\section{Conclusions}

MR blockade with spironolactone, when initiated before RT, ameliorates late radiation-induced fibrosis in submandibular glands. Whether reduced salivary gland fibrosis induced by spironolactone translates into decreased salivary gland dysfunction remains to be elucidated.

\section{eP282}

\section{POSSIBLE PEDIATRIC NEPHROTOXICITY OF CISPLATIN}

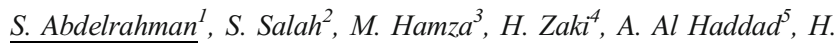
Moawad $^{4}$

${ }^{1}$ Children's cancer Hospital-Egypt 57357, Pharmaceutical Services \& Sciences, Cairo, Egypt

${ }^{2}$ Children's Cancer Hospital Egypt, Clinical Research, Cairo, Egypt

${ }^{3}$ Children's cancer Hospital-Egypt 57357, Clinical Resaerch, Cairo, Egypt

${ }^{4}$ Faculty of Pharmacy-Cairo University, Department of Pharmacology and Toxicology, Cairo, Egypt

${ }^{5}$ Children's cancer Hospital-Egypt 57357, Department of Pediatric Oncology \& Stem Cell Transplant, Cairo, Egypt

\section{Introduction}

Cisplatin is known for nephrotoxicity. Risk increases with increased cumulative dose, bolus dosage and associated nephrotoxic drugs.

Objectives

To identify incidence of Cisplatin nephrotoxicity within paediatric cancer patients, and associated risk factors. To identify most sensitive test(s) for early toxicity detection and possibility of using early therapy cycle(s) exposure as predictor of late nephrotoxicity. 


\section{Methods}

51 patients receiving Cisplatin-based chemotherapy were recruited, monitored and scored for nephrotoxic events throughout chemotherapy course (observational prospective), then correlated to patients' demographics, Cisplatin dosage, associated nephrotoxic drugs and disease risk, using logistic regression. Cramér's V was used to test strength of association between late nephrotoxicity relative to earlier cycles' toxicity. Also between different nephrotoxicity events relative to overall patient exposure. Results

$6(12 \%)$ patients developed grades 1,2 renal events. 14(27\%) developed grade 3 and $31(61 \%)$ developed grade 4 . Female gender, rapid Cisplatin dose rate and nephrotoxic drugs were significant predictors of higher toxicity grades upon univariate logistic regression (ODDs ratios $<1$, 95\% CI). Upon multivariate logistic regression, aminoglycoside therapy was significant predictor of higher nephrotoxicity score (ODDs ratio: $0.496,95 \% \mathrm{CI}$ ). Cramér's V between chemotherapy cycles: $1,2,4$ toxicity events \& late events were $>0.4$. Cramér's V between renal events' severity \& overall patient exposure were $>0.4$ for Sodium, Potassium, Phosphorus, Magnesium, creatinine ( $\mathrm{P}-$ values $<0.05)$.

Conclusions

$61 \%$ of children developed severe nephrotoxicity. Serum Potassium, Sodium and Phosphorus were most sensitive toxicity detectors. Nephrotoxicity during chemotherapy was indicator of late nephrotoxicity. Combination of female gender, higher Cisplatin dose rate, and nephrotoxic drugs increased nephrotoxicity severity. Aminoglycosides with/without other factors, increased nephrotoxicity risk.

\section{eP283}

WHAT PROGRESS IN BILATERAL WILM'S TUMOR MANAGEMENT IN ALGERIA?

\section{N. Bouterfas ${ }^{1}$, A. Mohandoussaid ${ }^{1}$, K.N. Benhalla ${ }^{1}$ \\ ${ }^{T}$ CHU Beni Messous, Pediatrics, Alger, Algeria}

\section{Introduction}

Nephroblastoma is the most common renal neoplasm in children and counts for $90 \%$, but bilateral disease is rare.

Objectives

to report on Algerian experience with BWT in children over a period of twenty years by describing patients characteristics and treatment modalities. Methods

We reviewed a series of 27 children aged less than 15 diagnosed with BWT between 1992 and 2015. Clinical and epidemiological data were collected from registry files. Investigations included abdominal US and/ or TDM, chest X-ray was systematically performed.Diagnosis was confirmed by histological studies of resected specimen. Pretreatment evaluation consisted on hematological and biochemical studies. Patients were classified and treated according to the SIOP protocol regimens with a follow up time 2 to 10 years.

Results

27 children with a BWT were registered between the 1992th and the 2015 th, among whom 23 with synchronous and 4 others with metachronous. The median age was of 30 months with a sex-ratio of 0.7. Two sisters presented with Perlman syndrome, another patient with a sexual ambiguity. Preoperative treatment consisted of chemotherapy with a median duration of 11 weeks, ( 8 to $26 \mathrm{ws}$ ). Doxorubicine was administered in half of the cases. 25 patients underwent surgery; a radical unilateral nephrectomy was realized in $37 \%$ and a conservative management was performed $63 \%$. No bilateral nephrectomy was made. Overall survival was $81.5 \%$. Four patients died from progressive disease, one death was owed to a cardio-toxicity.

Conclusions

Management of bilateral Wilms tumor in children constitutes a real challenge that to get complete remission, with renal function preservation as long as possible.

\section{eP284}

DEVELOPMENT AND USABILITY TESTING OF 'HELP': AN INFORMATION INTERVENTION FOR PARENTS OF CHILDREN WITH NEWLY DIAGNOSED ACUTE LYMPHOBLASTIC LEUKAEMIA

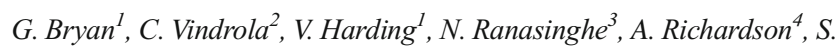

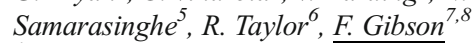

${ }^{1}$ UCL Great Ormond Street Institute of Child Health, Louis Dundas Centre for Children's Palliative Care, London, United Kingdom

${ }^{2}$ University College London, Department of Applied Health Research, London, United Kingdom

${ }^{3}$ Parent Representative, Parent Representative, London, United Kingdom ${ }^{4}$ University of Southampton, Health Sciences, Southampton, United Kingdom ${ }^{5}$ Great Ormond Street Hospital NHS Foundation Trust, Oncology and Haematology, London, United Kingdom

${ }^{6}$ University College London Hospitals NHS Foundation Trust, Cancer Clinical Trials Unit, London, United Kingdom

${ }^{7}$ University of Surrey, School of Health Sciences, London, United Kingdom ${ }^{8}$ Great Ormond Street Hospital NHS Foundation Trust, Centre for Outcomes and Experience Research in Children's Health- Illness and Disability ORCHID, London, United Kingdom

\section{Introduction}

Parents of children newly diagnosed with Acute Lymphoblastic Leukaemia (ALL) experience a high care burden. In our previous research parents described receiving minimal support to facilitate the acquisition of new knowledge. We developed an online intervention named HELP (Harmonising Education about Leukaemia for Parents) to facilitate easy access to information about leukaemia.

\section{Objectives}

To develop and usability test an information intervention for parents of children with ALL.

\section{Methods}

HELP incorporates text, links to resources and videos filmed with health professionals (HCPs) and parents. It was developed using a stepwise approach. An initial prototype of HELP was used to inform meetings with HCPs and parents. Feasibility testing was conducted with a diverse group of HCPs and two parent representatives. Parents of children with ALL ( $n=7)$ were recruited to undertake usability testing: incorporating thinking aloud while navigating HELP and the System Usability Scale questionnaire. HELP underwent two periods of refinement.

\section{Results}

Initial development of HELP was slow, engaging and recruiting HCPs was challenging. Filming videos with parents and HCPs, proved worthwhile as parents responded favourably to videos during usability testing. Data reports on user performance and parent satisfaction were collected. These were positive about content, less regarding style. Overall, parents stated that they would have found the resource useful.

\section{Conclusions}

The HELP website has been developed after extensive engagement with parents and input from HCPs. It has now undergone usability testing. We now have a resource that we are confident will meet the information needs of parents of children with ALL.

\section{eP285}

REVISED ANTI-EMETICS GUIDELINES AND THE IMPACT ON NUTRITIONAL STATUS DURING INDUCTION CHEMOTHERAPY IN CHILDREN WITH HIGH RISK NEUROBLASTOMA

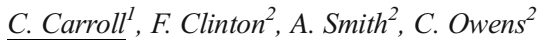 \\ ${ }^{T}$ Our Lady's Children's Hospital Crumlin, Clinical Nutrition and \\ Dietetics, Dublin, Ireland \\ ${ }^{2}$ Our Lady's Children's Hospital Crumlin, National Centre for Paediatric \\ Haematology \& Oncology, Dublin, Ireland
}




\section{Introduction}

High-Risk Neuroblastoma (HR NBL) treatment advocates intensive induction chemotherapy. The profoundly emetogenic agents used can result in compromised nutritional status. Our institution introduced new antiemetic guidelines in 2010 incorporating regular dexamethasone use, in addition to ondansetron, for all highly emetogenic protocols.

Objectives

To establish the impact of revised guidelines on emetic control, nutritional status and hospital admission frequency for febrile neutropenia (FNP).

Methods

A retrospective review of paediatric patients diagnosed with HR NBL between 2004 and 2017 and treated with rapid COJEC induction chemotherapy as per HR-SIOPEN NBL trial. The cohort was divided into two based on anti-emetics received.

Results

63 children were eligible for inclusion (median age 31 months, range 1-89 months). Group A had more emesis episodes than Group B (189 versus $116, P<0.0001)$ during induction. There was a significant difference in Weight-for-Age $\mathrm{Z}$ score change between the groups $(P=0.0027)$. Four children (13\%) in Group A lost $>10 \%$ body weight with; 0 in Group B. Nutrition support (NS) was utilised by 29 children (94\%) in Group A versus 22 children (69\%) in Group B. Group A had a median of 3 (range1-7) admissions for FNP versus a median of 2 (range 0-4) for Group B $(P=0.018)$.

Conclusions

The revised guidelines led to reduced emesis frequency for those in Group B. They required less NS, were better able to follow expected growth trajectories and had fewer FNP admissions. We propose that this may be due to better emesis control resulting in improved nutritional status and associated enhanced immune function.

\section{eP286}

DEVELOPMENT OF A FRAMEWORK TO ADDRESS FACTORS THAT INFLUENCE PARENTAL UNCERTAINTY AND HEALTH-RELATED QUALITY OF LIFE (HRQOL) IN CHILDREN WITH CANCER

I.J. Eche ${ }^{1}$, T. Aronowitz ${ }^{2}$

${ }^{T}$ Boston Children's Hospital, Hematology/Oncology, Boston, USA

${ }^{2} U M B$, College of Nursing and Health Sciences, Boston, USA

\section{Introduction}

Uncertainty is a prevalentpsychological distress in parents of children with cancer. The psychological trauma from the cancer diagnosis makes the parents prone to high levels of uncertainty which can affect their perception of their child's symptoms. Evidence indicates that parents of children with cancer report low child HRQOL scores when they have increased symptoms of depression, anxiety, and stress themselves, suggesting propensity for parental reporting bias.

\section{Objectives}

The CTE framework was constructed to identify and link the concepts $(\mathrm{C})$ that guides the study, the theory $(\mathrm{T})$ that is generated to test aspects of the model, and the empirical (E) measures that are used to operationalize the theoretical concepts.

Methods

A systematic literature review of the phenomenon of interest and framework was completed.

Results

The basic concepts identified are parental uncertainty, HRQOL, parental trait anxiety, parental depression and perceived social support. The proposed relations between the concepts are explained with linkages to their empirical indicators. The relation between parental uncertainty and child's HRQOL is hypothesized to be moderated by levels of parental trait anxiety and depression. It is also hypothesized that parental social support will mediate the relation between parental uncertainty and child's
HRQOL. The sociodemographic variables may also moderate the relation between parental uncertainty and child's HRQOL.

\section{Conclusions}

Despite recognition that uncertainty is a significant psychological stressor for parents of children with cancer, there is limited literature addressing how it impacts HRQOL in this population. The proposed framework will help to understand the relation between parental uncertainty and HRQOL in children with cancer.

\section{eP287}

FEBRILE NEUTROPENIA IN INDONESIAN PEDIATRIC CHILDHOOD CANCER

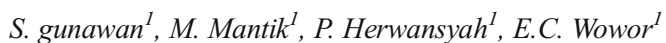

${ }^{T}$ Prof. Dr. dr. RD Kandou Hospital Manado/ Faculty of Medicine Sam Ratulangi University, of Child health, manado, Indonesia

\section{Introduction}

Offensive therapy of childhood cancers including systemic antineoplastic therapy has secondary effects on a variety of normal cells including hematopoietic elements of the bone marrow, often causing neutropenia. Neutropenia increases the risk for serious infection and is associated with remarkable morbidity and mortality. The approach to the treatment of the febrile neutropenia in pediatric patient has evolved substantially because high- and low-risk criteria have been defined and empirical antibiotics developed.

Objectives

To examine the characteristics of children with febrile neutropenia in several pediatric cancer center in Indonesia and their causing bacteria and antibiotics sensitivity results.

\section{Methods}

We reviewed literatures in PubMed and Google Search using keywords: febrile, neutropenia, children, cancer, and Indonesia. Literature was included when research was focused on the characteristics of children with febrile neutropenia or reported on febrile neutropenia-causing bacteria and antibiotics sensitivity results. Literature was excluded when full version of the paper was not accessible.

\section{Results}

There are 15 papers found but 4 papers were excluded. Papers came from six centers. The most common characteristics are male, age 5-6 years old, Absolute Neutrophil Count (ANC) $200-500 / \mathrm{mm}^{3}$, suffered from Acute Lymphoblastic Leukemia (ALL), and malnutrition.

The most common etiology of FN was gram negative bacteria (Escherichia coli and Pseudomonas aeruginosa). Antibiotics sensitivity to those bacteria were meropenem and amikacin.

\section{Conclusions}

FN are frequently occurred in ALL and malnourished children. Gram negative bacteria are the most common caused and sensitive to meropenem and amikacin.

\section{eP288}

IMPACT OF ENHANCED SUPPORTIVE CARE ON REDUCING THE MORTALITY RATES DURING TREATMENT OF PEDIATRIC ACUTE LEUKEMIA;A DEVELOPING COUNTRY EXPERIENCE

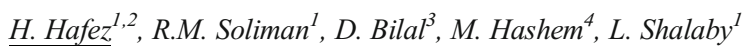

${ }^{T}$ National Cancer Institute - Cairo University, Pediatric Hematology/ Oncology, Cairo, Egypt

${ }^{2}$ Children Cancer Hospital Egypt CCHE 57357, pediatric oncology and BMT, Cairo, Egypt

${ }^{3}$ National Cancer Institute - Cairo University, Biostatistics, Cairo, Egypt ${ }^{4}$ Children Cancer Hospital Egypt CCHE 57357, Research Department, Cairo, Egypt
} 


\section{Introduction:}

Children with acute leukemia may experience high toxic mortality rates and treatment related mortality (TRM) which often occurs early in the induction phase

\section{Objectives}

The aim of the study was to assess the effectiveness of improving the supportive care measures in reducing the overall and induction deaths during therapy of pediatric acute leukemia

Methods

This is a retrospective study included patients with acute leukemia who presented to the National Cancer Institute, Cairo University between January 2011 and December 2013. Patients' data were collected and analyzed for the total and early death rates and proposed causes of death Results

\section{Fig.2:- Total and induction deaths during the three successive years}

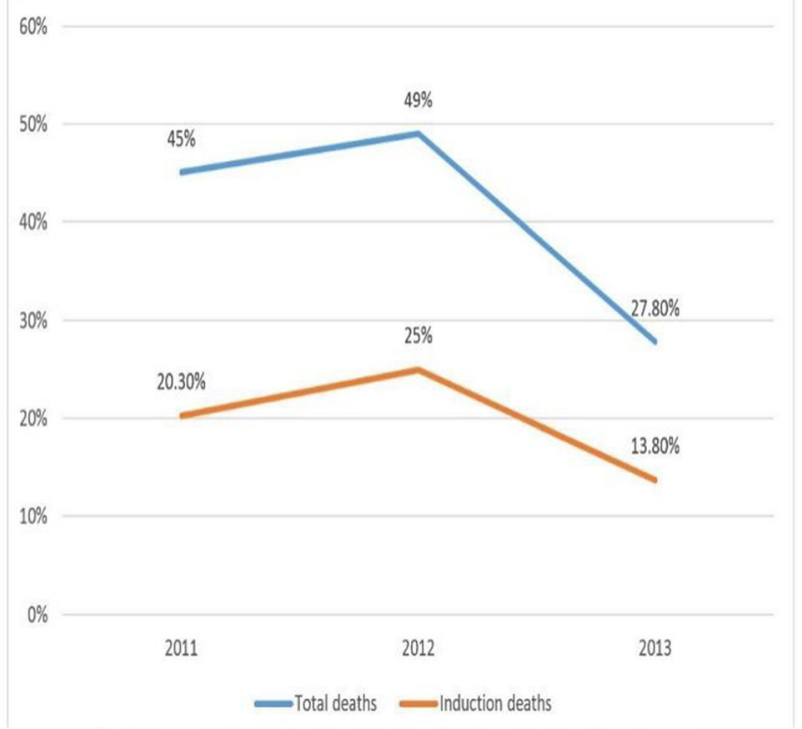

Fig.3:- Trend of proposed cause of early death throughout the three successive years.

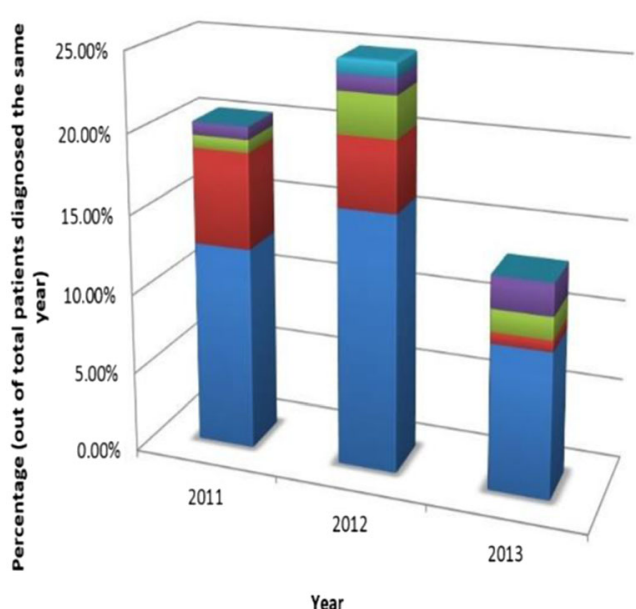

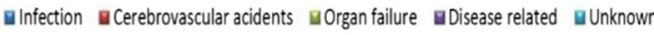

The study analyzed 370 patients, 253 with acute lymphoblastic leukemia (ALL), 100 with acute myeloid leukemia (AML) and 17 with mixed phenotypic. The total death rate was $40.5 \%(\mathrm{n}=150)$ and induction death rate was $19.2 \%(\mathrm{n}=71)$. Treatment related deaths were responsible for the majority (90\%) of the early deaths (EDs) and only $7 \%$ were disease related. Infections were the most common underlying cause of EDs (64.7\%) followed by cerebrovascular accidents (18.3\%). Using enhanced supportive care measures during 2013 had significantly reduced the overall and induction mortality rates $(27.8 \%$ and $13.8 \%$ respectively in 2013 versus $45 \%$ and $20.3 \%$ in 2011 and $49 \%$ and $25 \%$ in 2012)

\section{Conclusions}

treatment related deaths in pediatric acute leukemia remain a major challenge in developing countries and constitute an increasing fraction of all deaths. Accordingly, using well equipped centers with better supportive care guidelines is essential to improve the survival in this group of patients.

\section{eP289}

ESTIMATING MALNUTRITION AT DIAGNOSIS IN NEWLY DIAGNOSED PEDIATRIC ONCOLOGY PATIENTS

M. Hussaini ${ }^{1}$

${ }^{T}$ Aga Khan University Hospital, Oncology, Karachi, Pakistan

\section{Introduction:}

Nutritional depletion is a major problem among pediatric oncology patients in the developing world. This is associated with treatment related toxicity and mortality. Studies found that children with malignancies die due to the effects of undernourishment rather than the malignancy itself. Early identification, thorough assessment and management can help prevent complications

\section{Objectives}

The purpose of this study is to estimate the prevalence of malnutrition that is present at the time of diagnosis.

\section{Methods}

A sample size of 14 newly diagnosed patients within last three months (ie from June to August 2016) were taken to estimate the nutritional status at the time of diagnosis. Height, weight, BMI, height for age, weight for age and BMI for age percentiles were collected. Analysis was done using growth charts. Written consent was also taken.

\section{Results}

Overall analysis of the data showed that out of 14 patients, 8 children were found to have their height, weight and BMI percentiles below $25^{\text {th }}$ which indicates that they were already undernourished at the time of diagnosis. Furthermore, 5 out of these 8 children were found to have their percentiles of less than 10 which is a clinically significant finding.

\section{Conclusions}

The need for adequate nutritional assessment at the time of diagnosis is as significant as it is during and after the completion of treatment. Therefore, proper growth monitoring, timely reporting and management is required to prevent child from developing further complications. This study will to plan holistic strategies and integrate nutritional therapy as part of cancer therapy.

\section{eP290 \\ INCIDENCE OF FEBRILE NEUTROPENIA IN PEDIATRIC SOLID TUMOR AND LYMPHOMA PATIENTS TREATED WITH CHEMOTHERAPY}

A.Y. Jeon ${ }^{1}$, N. Han ${ }^{2}$, M.G. Kim ${ }^{3}$, Y. Cho ${ }^{1}$, E.J. Cho ${ }^{1}$, M.J. Kim ${ }^{1}$, A.J.

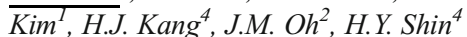

${ }^{I}$ Seoul National University Hospital, Department of Pharmacy, Seoul, Republic of Korea

${ }^{2}$ Seoul National University, College of Pharmacy and Research Institute of Pharmaceutical Sciences, Seoul, Republic of Korea 
${ }^{3}$ CHA University, Graduate School of Clinical Pharmacy, Pocheon, Republic of Korea

${ }^{4}$ Cancer Research Institute-Seoul National University College of Medicine, Department of Pediatrics, Seoul, Republic of Korea

\section{Introduction:}

Febrile neutropenia (FN) is a potentially life-threatening complication of cancer treatments, but information regarding the incidence of FN among different chemotherapy regimens for pediatric patients is scarce.

\section{Objectives}

We aimed to evaluate incidence of FN in pediatric solid tumor and lymphoma patients treated with chemotherapy and assess risk factors in each tumor type and regimen.

\section{Methods}

Pediatric solid tumor and lymphoma patients newly administered chemotherapy from 2011 to 2015 in Seoul National University Children's Hospital were included. Time-dependent cumulative incidence (CI) of FN with different tumor types and chemotherapy regimens was evaluated. Risk factors for FN were assessed.

\section{Results}

Of the 409 patients, central nervous system (CNS) tumor was the most common cancer $(27 \%)$. Incidence of $\mathrm{FN}$ were $45.5 \%$ in KSPNO regimen for low risk CNS germ cell tumor (GCT) $(\mathrm{CI}=0.520), 4.5 \%$ in $9952 \mathrm{~A}$ for low grade glioma $(\mathrm{CI}=0.048)$, $6.3 \%$ in 5961 modified-COP for non-Hodgkin's lymphoma (NHL) $(\mathrm{CI}=0.063), 80.0 \%$ in CarDEC for retinoblastoma $(\mathrm{CI}=0.906), 87.0 \%$ in $\mathrm{CCG} 7921 \mathrm{~A}$ for bone cancer $(\mathrm{CI}=0.870)$, and $78.8 \%$ in $\mathrm{CCG} 321 \mathrm{P} 2$ for neuroblastoma $(\mathrm{CI}=1.000)$. FN occurred mainly in the first cycle of chemotherapy in CNS tumors. Incidence of FN after treatment with cyclophosphamide/ dauorubicin/l-asparaginase/prednisolone/vincristine was significantly high compared to cyclophosphamide/prednisolone/vincristine $(H R=6.998, p=0.012)$ in NHL. In addition, low hemoglobin $(\leq 12$ $\mathrm{g} / \mathrm{dL})$ was the risk factor of $\mathrm{FN}$ in CNS tumor treated with KSPNO regimen for low risk CNS GCT $(O R=16.5, p=0.022)$.

\begin{tabular}{lc} 
Table 1. Patients characteristics & \\
\hline Demographic and clinical characteristics & All patients $(\mathrm{N}=409)$ \\
\hline Sex & $253(61.9)$ \\
Male $(\%)$ & $6.42 \pm 5.28(0.09-17.97)$ \\
Age, years & 120 \\
$0-1$ & 125 \\
$2-6$ & 67 \\
$7-11$ & 97 \\
$12-18$ & $0.888 \pm 0.457(0.227-2.082)$ \\
BSA (m) & \\
Baseline labs & $11.46 \pm 1.90$ \\
Hemoglobin $(\mathrm{g} / \mathrm{dL})$ & $337.79 \pm 137.88$ \\
Platelet $\left(\times 10^{3} / \mu \mathrm{l}\right)$ & $4487.65 \pm 5609.99$ \\
ANC $(/ \mu 1)$ & $9.9 \pm 5.90$ \\
BUN $(\mathrm{mg} / \mathrm{dL})$ & $0.45 \pm 0.35$ \\
Creatinine $(\mathrm{mg} / \mathrm{dL})$ & $3.99 \pm 0.57$ \\
Albumin $(\mathrm{g} / \mathrm{dL})$ & $35.54 \pm 26.37$ \\
AST $(\mathrm{IU} / \mathrm{L})$ & $21.91 \pm 20.18$ \\
ALT $(\mathrm{IU} / \mathrm{L})$ & $2.06 \pm 4.30$ \\
CRP $(\mathrm{mg} / \mathrm{dL})$ & $128.24 \pm 47.08$ \\
Schwartz $\mathrm{eGFR}$ & \\
\hline
\end{tabular}

$a_{\text {mean }} \pm \mathrm{SD}$ (range) $b_{\text {mean }} \pm \mathrm{SD}$

Abbreviations:

BSA, body surface area: ANC. absolute neutrophil count: BUN, blood urea nitrogen: AST, apartate transaminase: ALT, alanine aminotransferase: CRP, c-reactive protein: eGFR, estimated glomerular filtration rate
Figure 1. Time-dependent Cumulative Incidence of Febrile Neutropenia
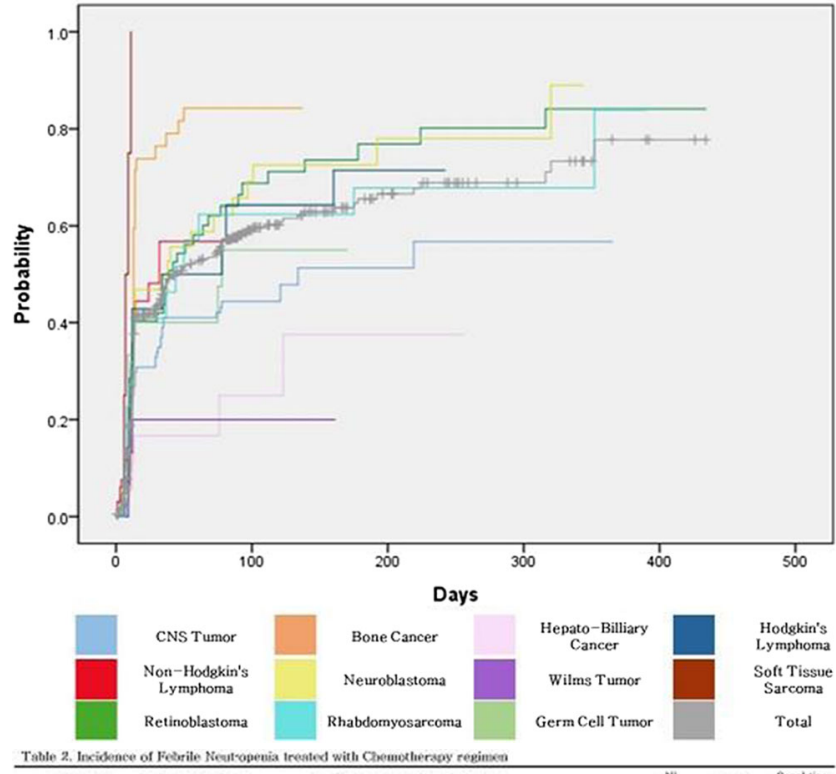

Hodgkin's
Lymphioma

Soft Tissue

Sarcoma

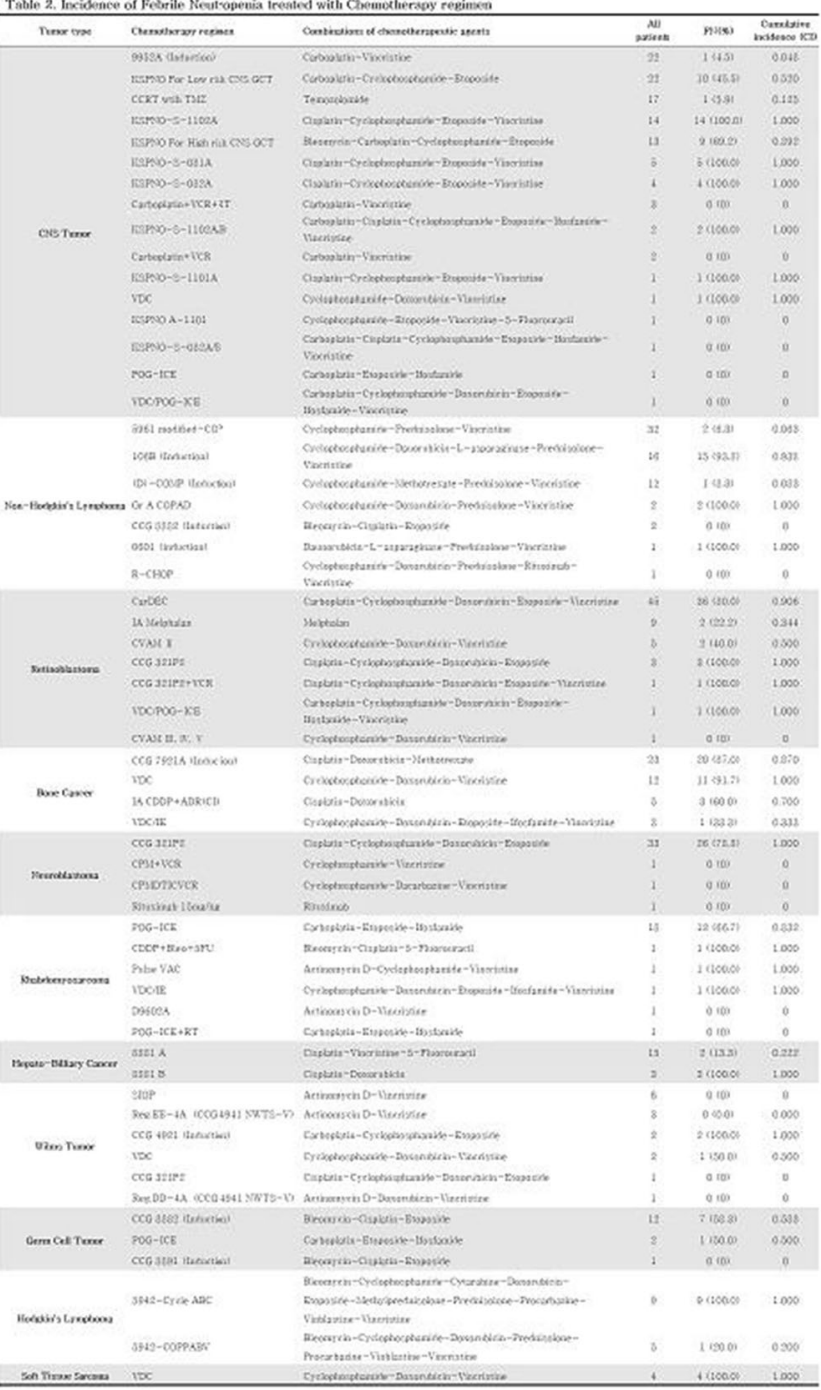

Conclusions

Incidence of FN in pediatric solid tumor and lymphoma patients is largely dependent on tumor type and chemotherapy regimens. 
eP291

NEWER TRENDS OF UMBILICAL CORD BLOOD STEM CELLS BANKING FOR MANAGEMENT OF PEDIATRIC HEMATOLOGIC MALIGNANCIES: AN UPDATE

C. Kohli ${ }^{1}$, J. Kishore ${ }^{I}$, N. Dahiya ${ }^{2}$

${ }^{7}$ Vardhman Mahavir Medical College, Department of Community Medicine, New Delhi, India

${ }^{2}$ Maulana Azad Medical College, Department of Community Medicine, New Delhi, India

\section{Introduction:}

Umbilical Cord Blood is a source of hematopoietic stem cells and is equivalent to those found in the bone marrow. These stems cells in cord blood are multi-potent in nature and have potential to treat hematologic malignancies like leukemia.

\section{Objectives}

The present study explored the recent trends about Umbilical Cord Blood Stem Cells Banking for future management of hematologic malignancies in India.

Methods

A study was conducted using mixed method approach incorporating both qualitative and quantitative research methods. A total of 345 pregnant females were selected randomly in a secondary care hospital in India. All pregnant women attending Antenatal Clinic constituted study population. As part of same study, data was collected to assess future role of Umbilical Cord Blood banking for hematologic malignancies in India after interviewing the major stakeholders. Ethical clearance was taken from Institutional Ethics Committee.

\section{Results}

Only $141(40.8 \%)$ females knew about the meaning of Umbilical Cord Blood stem cells. $102(29.5 \%)$ females perceived that Umbilical Cord Blood will help to treat hematologic malignancies in their future child. Stakeholders were of opinion that with high birth rate and a hub of medical tourism, Umbilical Cord Blood banking has a huge potential in India; though limited evidence in Indian settings about stem cell banking in hematologic malignancies and lack of understanding among masses remains major challenges.

Conclusions

Application of Umbilical Cord Blood for management of hematologic malignancies has great potential in India. There is a need to promote public private partnership and more research.

\section{eP292}

TREATMENT-RELATED MORTALITY IN PEDIATRIC ONCOLOGY: CATEGORIZATION, INCIDENCE AND ASSOCIATED RISK FACTORS

\author{
E.A. Loeffen ${ }^{1}$, R.R. Knops ${ }^{2}$, J. Boerhof ${ }^{1}$, L.C. Kremer ${ }^{2}$, W.J. Tissing ${ }^{1}$ \\ ${ }^{T}$ University of Groningen- Beatrix Children's Hospital- University \\ Medical Center Groningen- Groningen- the Netherlands, \\ Department of Pediatric Oncology/Hematology, Groningen, \\ The Netherlands \\ ${ }^{2}$ Emma Children's Hospital- Academic Medical Center- Amsterdam- \\ The Netherlands, Department of Pediatric Oncology, Amsterdam, \\ The Netherlands
}

\section{Introduction}

Although survival rates of children with cancer have increased substantially due to the introduction of intensive treatment protocols, these treatments themselves also lead to morbidity and mortality.

\section{Objectives}

We aimed to 1) obtain an overview of the various causes of death among children with cancer, and 2) identify factors contributing to treatmentrelated mortality (TRM).

Methods

Eligible participants were children diagnosed with cancer between 2003 and 2015 in the university hospitals of Groningen or Amsterdam (NL), and who had died between 2003 and 07/2017. Data on various demographic and disease/treatment factors were collected by use of a purpose-designed data collection form. Categorization of TRM or not-TRM was according to the classification of Alexander (2015), causes were further specified according to both this classification and the ICD-10.

\section{Results}

402 deaths were included in the analysis. Of these, 84 (20.9\%) were classified as TRM (causes see table 1), 317 (78.9\%) were classified as not-TRM, and for $1(0.2 \%)$ no classification was possible. In univariate logistic regression, several factors were significantly associated with increased occurrence of TRM; diagnosis of hematological malignancy, absence of relapse, allogeneic HSCT, and expected five year survival of $>40 \%$ (vs. $<40 \%$ ). In multivariate analysis, diagnosis of hematological malignancy and absence of relapse remained significant.

\section{Conclusions}

We found that 1 in 5 deaths in children with cancer were treatment-related. In multivariate testing, diagnosis of hematological malignancy was associated with occurrence of TRM. The same was true for absence of relapse, which we think is due to the higher rate of tumor-related deaths in this group.

\section{eP293}

DEVELOPMENT OF CHILDHOOD CANCER SUPPORTIVE CARE INDICATORS; EVALUATION OF FEBRILE NEUTROPENIA CARE IN THE NORTH OF THE NETHERLANDS

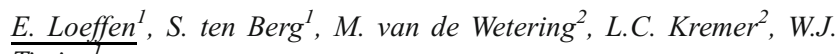
Tissing ${ }^{I}$

${ }^{1}$ University of Groningen-Beatrix Children's Hospital- University Medical Center Groningen- Groningen- the Netherlands, Department of Pediatric Oncology/Hematology, Groningen, The Netherlands

${ }_{2}^{2}$ Emma Children's Hospital-Academic Medical Center-Amsterdam- the Netherlands, Department of Pediatric Oncology, Amsterdam, The Netherlands

\section{Introduction}

Febrile neutropenia (FN) is a severe and potentially lifethreatening side effect of chemotherapy. Measuring care provided to children with cancer suffering from FN, would be very valuable in identifying ways to improve care. This measurement can be done with indicators.

\section{Objectives}

We aimed to 1) develop three types of indicators (structure, process, outcome) derived from existing evidence-based FN guidelines, and 2) do a pilot implementation of the developed indicators.

Methods

For a detailed flow diagram of the developmental process, see figure 1. The developed indicators were tested in 3 hospitals (1 university hospital, 2 shared care hospitals) in the North of the 
Netherlands, using retrospectively collected individual patient data. We included all children diagnosed with cancer between 0101-2014 and 31-12-2015.

\section{Results}

7 indicators were developed, see table 1. In the pilot, $119 \mathrm{FN}$ episodes in 59 patients were included. Notable findings were 1) $100 \%$ of episodes without focus $(n=108)$ were treated with guideline-consistent antibiotics (antipseudomonal beta-lactam), 2) in $12.5 \%$ of episodes with persistent fever $(n=14)$ guidelineconsistent additional testing (aspergillus diagnostics) was performed too late (no registered reason), 3) of all $\mathrm{FN}$ episodes $(\mathrm{n}=119), 18.5 \%$ resulted in ICU admittance and $1.7 \%$ resulted in death.

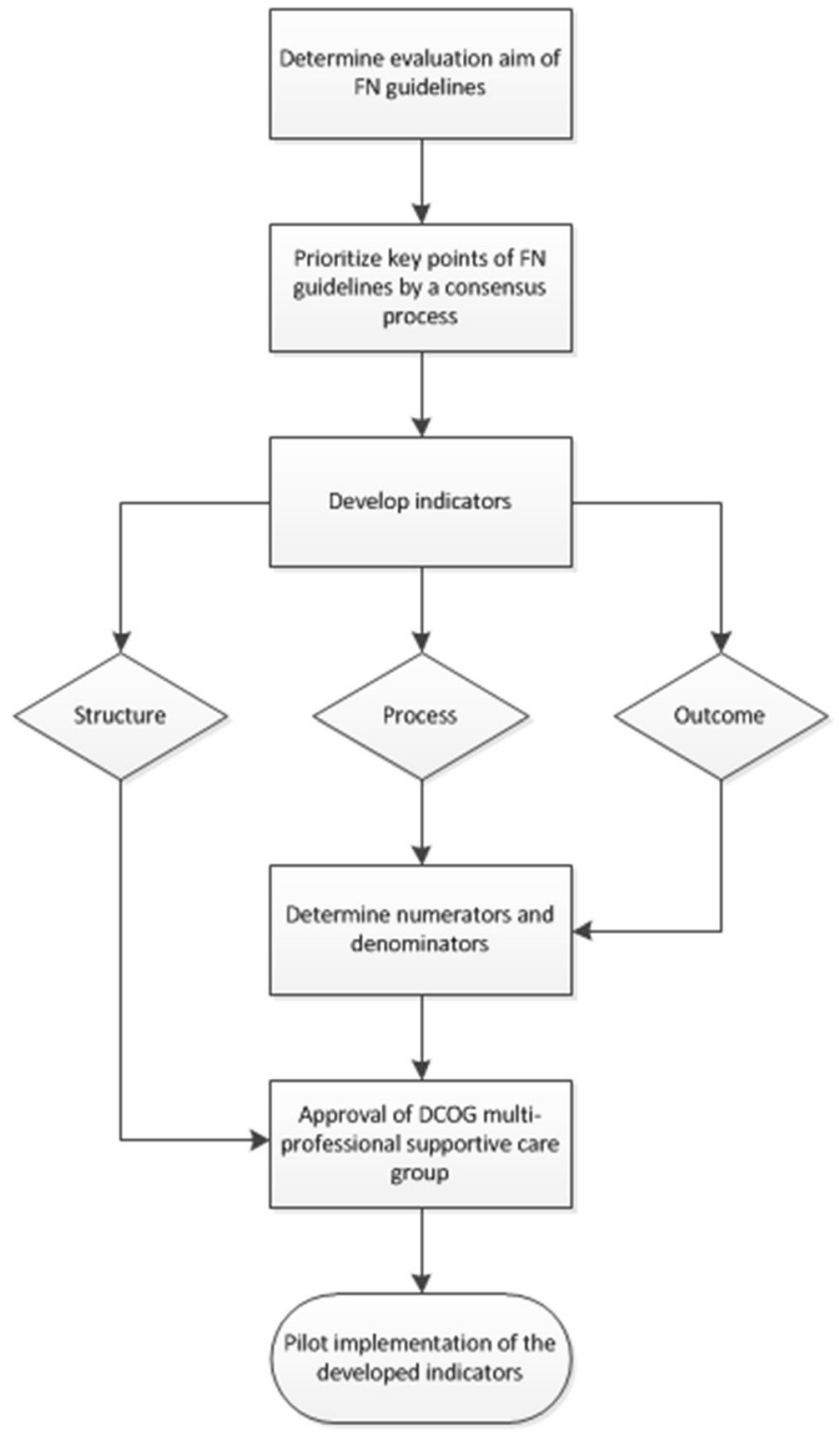

\begin{tabular}{|c|c|}
\hline & Structure indicators \\
\hline \multirow[t]{5}{*}{ Indicator 1} & $\begin{array}{l}\text { Having a general recommendation on the antimicrobial policy of FN in } \\
\text { children with cancer. }\end{array}$ \\
\hline & 1. No recommendation \\
\hline & 2. Verbal agreement \\
\hline & 3. Written recommendation in own document system \\
\hline & 4. According to the DCOG guideline \\
\hline \multirow[t]{4}{*}{ Indicator 2} & Is the recommendation according to the FN guideline? \\
\hline & 1. Yes \\
\hline & 2. No \\
\hline & Process indicators \\
\hline Indicator 3 & $\begin{array}{l}\text { Percentage of febrile neutropenia episodes without microbial focus, which } \\
\text { are treated with ceftazidim }\end{array}$ \\
\hline Numerator & $\begin{array}{l}\text { The number of FN episodes without microbial focus, for which patients } \\
\text { received ceftazidim according to the FN guideline }\end{array}$ \\
\hline Denominator & All episodes of FN without microbial focus \\
\hline Indicator 4 & $\begin{array}{l}\text { Percentage of febrile episodes in neutropenia with persistent fever without } \\
\text { focus ( }>96 \text { hours), in which an HRCT or BAL was performed }\end{array}$ \\
\hline Numerator & $\begin{array}{l}\text { The number of persistent FN episodes without microbial focus, in which an } \\
\text { HRCT/BAL was performed }\end{array}$ \\
\hline \multirow[t]{2}{*}{ Denominator } & All persistent FN episodes without microbial focus \\
\hline & Outcome indicators \\
\hline Indicator 5 & $\begin{array}{l}\text { The percentage of clinical FN episodes in children with cancer, in which a } \\
\text { patient is admitted to the ICU }\end{array}$ \\
\hline Numerator & $\begin{array}{l}\text { The number of clinical FN episodes in children with cancer, in which a } \\
\text { patient is admitted to the ICU }\end{array}$ \\
\hline Denominator & All clinical FN episodes \\
\hline Indicator 6 & Percentage of children with cancer who die during a clinical FN episode \\
\hline Numerator & The number of children with cancer who die during a clinical FN episode \\
\hline Denominator & $\begin{array}{l}\text { The total number of children with cancer diagnosed between 01-01-2014 and } \\
31-12-2015\end{array}$ \\
\hline Indicator 7 & The percentage of clinical FN episodes of which patients have died \\
\hline Numerator & The number of clinical FN episodes of which patients have died \\
\hline Denominat & $\mathrm{T}$ \\
\hline
\end{tabular}

Table 1 - developed indicators

\section{Conclusions}

To our knowledge this is the first project in which indicators for supportive care in childhood cancer were developed. The developed indicators have shown to be competent, as provided care was effectively evaluated. The indicator set will be implemented throughout the Netherlands to evaluate care for children with cancer suffering from FN, and to potentially identify ways to improve care.

eP294

DEVELOPMENT OF A CLINICAL PRACTICE GUIDELINE FOR ASSESSMENT, PREVENTION AND TREATMENT OF PAIN IN CHILDREN WITH CANCER

E. Loeffen ${ }^{1}$, R.L. Mulder ${ }^{2}$, A. Font-Gonzalez ${ }^{2}$, L.L. Dupuis ${ }^{3}$, M.D. van de

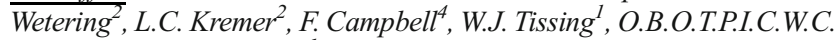
Guideline Working Group ${ }^{I}$

${ }^{1}$ University of Groningen- Beatrix Children's Hospital- University Medical Center Groningen- Groningen- the Netherlands, Department of Pediatric Oncology/Hematology, Groningen, The Netherlands

${ }^{2}$ Emma Children's Hospital-Academic Medical Center-Amsterdam- the Netherlands, Department of Pediatric Oncology, Amsterdam, The Netherlands

${ }^{3}$ The Hospital for Sick Children- Toronto- Canada, Department of Pharmacy and Research Institute, Toronto, Canada

${ }^{4}$ The Hospital for Sick Children- Toronto-Canada, Department of Anesthesia and Pain Medicine, Toronto, Canada 


\section{Introduction:}

High-quality evidence-based guidelines for supportive care in children with cancer are needed. In an earlier phase, clinicians prioritized pain in children with cancer as an area in which they seek guidance.

\section{Objectives}

To develop a clinical practice guideline (CPG) for pain in children with cancer with recommendations on 1) assessment of pain;2) pharmacological prevention and treatment of a) tumor-related pain, b) treatmentrelated pain, and c) procedure-related pain; and 3) physical and psychological prevention and treatment of a) tumor- and treatment-related pain, and b) procedure-related pain.

\section{Methods}

The international and inter-professional guideline panel comprises a core group and six working groups and includes 44 individuals. Previously 25 clinical questions were prioritized to be answered through systematic literature reviews. After performing the literature appraisal and independent dual data extraction of the included studies (see figure 1), the project group met in Amsterdam (35/44 members attending) to complete and discuss the evidence-to-decision frameworks and formulate draft recommendations.

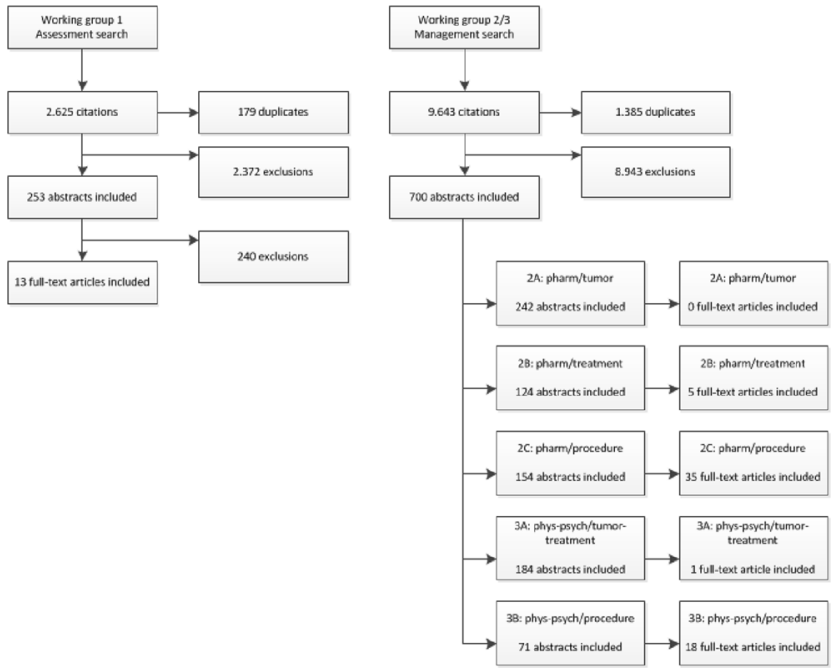

Figure 1. Flowchart of the selection process of the systematic review. In all steps, dual independent appraisal/extraction was performed, discrepancies were resolved by consensus.

\section{Results}

Various draft recommendations were formulated for all working groups. These recommendations are currently being discussed with the members not present at the guideline meeting and various patient- and parent-representatives. Hereafter the recommendations will be finalized.

\section{Conclusions}

We have developed a CPG regarding pain in children with cancer. Currently the recommendations are being finalized, these will be presented at the 2018 MASCC meeting. In the next phase we will work on implementation of the CPG.

\section{eP295}

DEALING WITH CANCER-RELATED BEREAVED PAEDIATRIC PATIENTS: LINKAGE BETWEEN RESEARCH AND PRACTICE

M. Mukelabai ${ }^{1}$

${ }^{T}$ ACCHAP MGT.CENTRE, Anaesthesia \& Pain Unit, Mufulira, Zambia

\section{Introduction}

Children can experience death at any age from be infancy to adulthood, during which time, each child is developing physically, emotionally, intellectually, spiritually, and socially.

\section{Objectives}

The main aim of the present study is to review some perspectives in dealing with cancer-related bereaved paediatric clients.

\section{Methods}

This in depth literature review explores theoretical perspectives in dealing with children arising from cancer-related parental demise.

Results

Although research into adult bereavement begun more than a century ago, it is only in recent years, in the West, that there has been a growing professional and public awareness of the needs of bereaved children.

We now know, for instance, that when the death of o parent is antcipated, children's bereavement may be affected by a number of factors, including the duration of the previous illness of the deceased parent, advance knowledge of the impending death as well as the degree of the child's awareness of the parent's death.

The first intra-psychic theory of grief was proposed by Freud (1917) and has stood as a precedent for scientific research into grief today. Lindemann's classic study of survivors of the Coconut Grove fire in Boston (1944) described adult grief as having both somatic and psychological effects, where distress, preoccupation with the deceased, guilty, anger, and loss of usual living were experienced.

\section{Conclusions}

Bereavement theories developed from research into bereaved adults would also appear relevant for bereaved children.

\section{eP296}

UNDERWEIGHT, NON-REMISSIONAL STATUS OF MARROW AND PROFOUND NEUTROPENIA- THE PREDISPOSING FACTORS FOR THE DEVELOPMENT OF RECURRENT FEVER IN CHILDREN WITH ACUTE LYMPHOBLASTIC LEUKEMIA

J. ramamoorthy $^{1}$, V. Radhakrishnan ${ }^{2}$, V. Vijayakumar ${ }^{3}$, T.G. Sagar ${ }^{4}$

${ }^{T}$ Cancer Institute- Adyar, Pediatric Oncology Unit- Department of Medical Oncology-, Chennai, India

${ }^{2}$ Cancer institute- Adyar-Chennai, Pediatric Oncology UnitDepartment of Medical Oncology, Chennai, India

${ }^{3}$ Cancer Institute, Department of Medical Microbiology, Chennai, India ${ }^{4}$ Cancer Institute- Adyar-Chennai, Department of Medical Oncology, Chennai, India

\section{Introduction}

Antibiotics are recommended to be continued till count recovery (CR) anticipating the development of recurrent fever (RF). The factors that predict recurrence of fever following defervescence (DS) during febrile neutropenia episode (FNE) have not been studied.

\section{Objectives}

The objective was to estimate the incidence and risk factors for RF in children with high risk FNE during intensive phases of acute lymphoblastic leukemia (ALL) therapy.

Methods

The study included 113 patients diagnosed with ALL. Total of 256 FNE in these patients between January 2016 and June 2017 were analyzed retrospectively. RF was defined as febrile episode developing 24 hours after and within 7 days of afebrile period. CR was defined as Absolute Neutrophil Count (ANC) $>500 / \mathrm{mm}^{3}$ or absolute monocyte count $>100$ / $\mathrm{mm}^{3}$ in peripheral blood.

\section{Results}

RF developed in 38/256 FNE (14.85\%). Evidence of documented infection (DI) was observed in 26/38 RF episodes and absence of CR was observed in 35/38. Risk factors associated with development of RF were: non-remissional status of marrow $(\mathrm{NRSM})(\mathrm{P}=0.007)$, profound neutropenia $(\mathrm{PN})(\mathrm{P}=0.002)$, DI $(\mathrm{P}=0.002)$ and underweight $(\mathrm{P}=0.037)$. Multivariate analysis revealed association between RF and NRSM $(\mathrm{P}=0.002), \mathrm{PN}(\mathrm{P}=0.001)$ and underweight $(\mathrm{p}=0.031)$. DS within 72 hours of FN but without $\mathrm{CR}$ was observed in $82 / 256$ episodes. $31 / 82$ 
(37.8\%) episodes developed RF \& was associated with DI ( $\mathrm{P}=0.02)$, underweight $(\mathrm{P}=0.01)$ and $\mathrm{PN}(\mathrm{P}=0.002)$ on univariate analysis; with underweight $(\mathrm{P}=0.006)$ and $\mathrm{PN}(\mathrm{P}=0.003)$ on multivariate analysis Conclusions

Underweight, PN and NRSM predispose the development of RF. These patients require require prolonged administration of antibiotics irrespective of DS and CR.

\section{eP297}

PEDIATRIC CANCER CARE: QUALITATIVE REVIEW OF CAREGIVERS KNOWLEDGE, ATTITUDE AND PRACTICES

\section{H. Saeed ${ }^{1}$, S. Javed Khan ${ }^{1}$, S. Anjum ${ }^{1}$, R. Muhammad Wali ${ }^{1}$}

${ }^{T}$ Shaukat Khanum Cancer Hospital and Research Center, Pediatric Oncology, lahore, Pakistan

\section{Introduction}

Knowledge, attitude and practices (KAP) of pediatric cancer patients' caregivers effects outcome. KAP differs by culture and ethnicity so local knowledge of KAP is essential. This is particularly important in developing countries where late presentation and abandonment lead to worse outcomes. Information about local KAP can help design interventions to target these issues and support families.

\section{Objectives}

Evaluate KAP of caregivers of pediatric oncology patients in a developing country.

Methods

A unique, semi-structured questionnaire was designed according to local culture. Sampling was opportunistic. Interviews were conducted in Urdu language at a tertiary-care cancer hospital February-August 2016). SPSS version 19 was used for analysis.

Results

Characteristic of the 61 caregivers interviewed are given in table 1 .

Caregiver perception of cancer and its causes is summarized in table 1 and figure 1 . They stated a variety of causes ranging from blaming illness in other children to pregnancy related issues. $13 \%$ accepted it as will of god. A quarter of patients consider cancer to be contagious and half feared for the rest of their family. Only $57 \%$ of caregivers were comfortable disclosing that their child had cancer.

Majority of caregivers $(68.9 \%, \mathrm{n}=42)$ were using spiritual healers either prior to or during treatment. Other forms of alternative medicine (CAM) were rare.

\begin{tabular}{|l|l|l|}
\hline \multicolumn{2}{|c|}{ Pable 1: Caregiver Characteristics (n=61) } & \multicolumn{1}{|l|}{ Number (n) } \\
\hline & & \\
\hline Relationship to patient: & 29.5 & 18 \\
\hline - Mother & 39.3 & 24 \\
\hline - Father & 8 & 5 \\
\hline - grandparent & 23 & 14 \\
\hline - Aunt/uncle/cousin & 60.7 & 37 \\
\hline Gender: Male & & \\
\hline Language: & 47.5 & 29 \\
\hline - Pashto & 21.3 & 13 \\
\hline - Urdu & 19.7 & 12 \\
\hline - Punjabi & 11.4 & 7 \\
\hline - others & & \\
\hline Education: & 31.1 & 19 \\
\hline - Illiterate & 27.9 & 17 \\
\hline - Primary school & 13.1 & 8 \\
\hline - 10years of school & 27.9 & 17 \\
\hline - College & & \\
\hline Profession: & 6.6 & 4 \\
\hline - Unemployed & 36.1 & 22 \\
\hline - Daily wages & 49.7 & 12 \\
\hline - Salaried Job & 37.7 & 23 \\
\hline - Self employed & 65.6 & \\
\hline Job loss due to illness & & \\
\hline & & \\
\hline
\end{tabular}

\begin{tabular}{|l|r|r|}
\hline \multicolumn{3}{|l|}{ Table 2. Caregiver Perception of Causes of Cancer } \\
\hline & Number (n) & Percentage(\%) \\
\hline Don't know & 27 & 44.3 \\
\hline Fate/Destiny/God given & 8 & 13 \\
\hline Specific Cause (given below): & 26 & 42.6 \\
\hline - Diet/toxins in food & 6 & \\
\hline - Worsening of infection & 4 & \\
\hline - Smoking/beetle nut & 4 & \\
\hline - Unhygienic conditions & 3 & \\
\hline - Hereditary & 3 & \\
\hline - Issues during pregnancy & 3 & \\
\hline - Trauma & 2 & \\
\hline - Illness/trauma in sibling & 2 & \\
\hline - Geographic predisposition & 1 & \\
\hline - Bomb blasts & 1 & \\
\hline - Discharging wound & 1 & \\
\hline - Punishment from God & 1 & \\
\hline - Blood borne & 1 & \\
\hline - Technology & 1 & \\
\hline
\end{tabular}

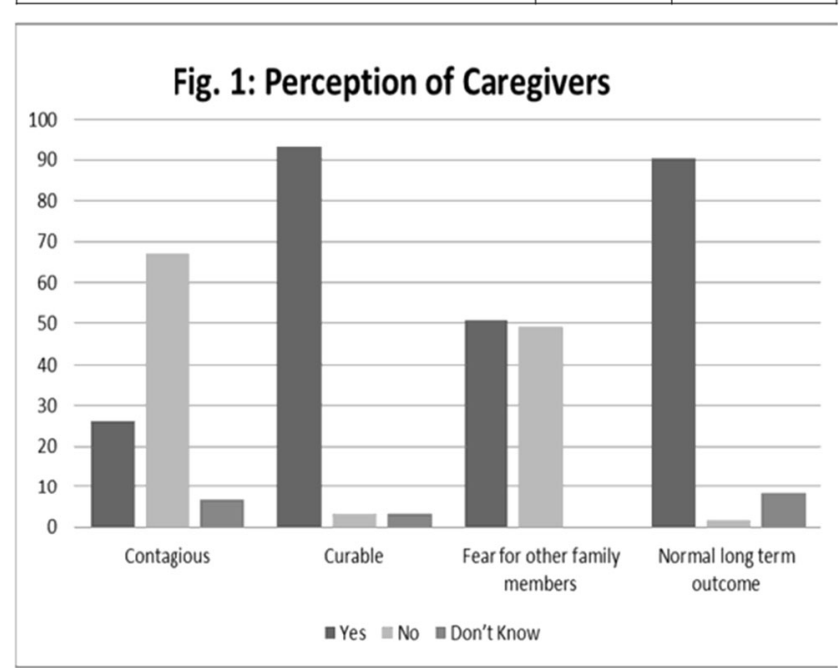

\section{Conclusions}

Caregivers have significant stressors (language and financial) that need to be addressed. Misconceptions about causes of cancer need to be clarified to reduce caregiver self- blame. Spiritual healing is a common form of CAM used that should be explored further.

\section{eP298}

THE MEDIATION EFFECT OF BONE HEALTH SELFEFFICACY ON THE RELATIONSHIP BETWEEN SYMPTOM DISTRESS AND EXERCISE INVOLVEMENT IN ADOLESCENTS WITH CANCER

\section{W.W. Wu ${ }^{l}$, F.H. Chao ${ }^{l}$}

${ }^{T}$ National Taiwan University, School of Nursing-College of Medicine, Taipei, Taiwan R.O.C.

\section{Introduction}

Symptom distress is usually experienced by adolescents who are receiving cancer treatment, and would affect the willingness of exercise involvement. Exercise involvement may enhance their quality of life. Enhancing bone health self-efficacy may mediate the negative impact of symptom distress on exercise involvement. 


\section{Objectives}

To describe the degree of bone health self-efficacy, symptom distress, and exercise involvement among adolescents with cancer, and to determine whether self-efficacy mediates the relationship between symptom distress and exercise involvement.

Methods

A cross-sectional study of 97 participants who were undergoing cancer treatment was conducted. Hierarchical multiple regression was performed to examine the mediation relationship.

Results

Bone health self-efficacy in calcium intake and in physical activity, symptom distress, and exercise involvement were correlated $(\mathrm{p}<.05$ and .01$)$. Health self-efficacy in physical activity partially mediated the adverse effect of symptom distress on exercise involvement for adolescents undergoing cancer treatment, accounting for $15.43 \%$ of the total variation with the value of $0.2019 \mathrm{R}^{2}$.

\section{Conclusions}

Health self-efficacy in physical activity partially mediated the adverse effect of symptom distress on exercise involvement for adolescents undergoing cancer treatment. Developing appropriate health education program that is feasible to pediatric hematology/oncology ward settings to enhance bone health self-efficacy in physical activity for adolescents undergoing cancer treatment is important.

\section{eP299}

THE MEDIATION EFFECT OF BONE HEALTH SELFEFFICACY ON THE RELATIONSHIP BETWEEN SYMPTOM DISTRESS AND EXERCISE INVOLVEMENT IN ADOLESCENT CANCER SURVIVORS

W.W. $W u^{l}$

${ }^{T}$ National Taiwan University, School of Nursing-College of Medicine, Taipei, Taiwan R.O.C.

\section{Introduction}

Adolescence is a key period for bone mass accumulation. Both cancer and cancer treatment may result in deficiencies in bone mineral density, which leads to greater risk of fractures and mortality later in life. Appropriate exercise is one of ways to remain/ enhance bone mass for adolescents. Enhancing bone health selfefficacy may be important to reduce the negative impact of symptom distress on exercise involvement.

\section{Objectives}

The objective of this study was to determine whether bone health selfefficacy mediated the relationship between symptom distress and exercise involvement.

\section{Methods}

113 participants was recruited. The hierarchical multiple regression was performed to examine the mediation relationship.

Results

Bone health self-efficacy in in physical activity, symptom distress, and exercise involvement were correlated $(\mathrm{p}<.05$ and .01$)$. Bone health self-efficacy in physical activity partially mediated the adverse effect of symptom distress on exercise involvement for adolescent cancer survivors, accounting for $14.12 \%$ of the total variation with the value of $0.3033 \mathrm{R}^{2}$

\section{Conclusions}

Bone health self-efficacy in physical activity partially mediated the adverse effect of symptom distress on exercise involvement for adolescent cancer survivors. In-service education that enhances nurses' awareness of the importance of self-efficacy in improving adolescents' exercise involvement is recommended. Developing bone health promotion education program that is easily be used in ambulatory setting in enhancing bone health self-efficacy in physical activity is important for adolescent cancer survivors.

\section{eP300}

MODIFIED METHOD FOR EXCISION OF ABDOMINAL NEUROBLASTOMA

L. Yanan ${ }^{I}$, H. Yang ${ }^{l}$

${ }^{T}$ The First Affiliated Hosptial of Zhengzhou University, pediatric surgery, Zhengzhou, China

\section{Introduction}

Neuroblastoma(NB) is one of the common extracranial solid tumors of children, It is not easy to excise the tumor which envelope the abdominal large vessels.

\section{Objectives}

The aim of this study is to discuss a technique for excision of neuroblastoma encasing abdominal vessels.

\section{Methods}

To explore the excision technique of abdominal neuroblastoma with trapped major vessels, retrospective review was performed in 123 cases that had undergone attempted surgical excision of abdominal neuroblastoma over the past ten years. Seventy-seven were male and the other 64 were female. The mean age was $21 \pm 17.5$ months ( 1 month to 10 years). Sixty-four cases were arrived from adrenal, 59 cases of which were from retroperitoneal.Forty five cases of those were surrounding major vessels, among which 39 cases were completely resected under tunica adventitia vasorum, 4 cases underwent palliative excision, and 2 cases received chemotherapy after surgical biopsy

\section{Results}

The complete tumor resection rate reached 95\% (117/123) in macrostate, with no perioperative death. Four cases with intraoperative vascular rupture repair had no postoperative recurrent bleeding or thrombogenesis.Bilateral renal failure after abdominal aorta repair in 1 case,and died 2 months later.In a male patient, the left kidney got atrophied and calcificated, though the tumor was resected from renal artery without vessel repair intraoperatively. adhesive intestinal obstruction in 2 cases, chyloperitoneum in 2 cases.All of them were cured with intravenous nutrition.

\section{Conclusions}

It is feasible to separate and excise the abdominal neuroblastoma surrounding large vessels under the tunica adventitia vasorum, which can significantly improve the resection rate of neuroblastoma and survival rate.

\section{eP301}

\section{TREATMENT OF STAGE 4S NEUROBLASTOMA}

H. Yang ${ }^{l}$, L. Yanan ${ }^{1}$

${ }^{I}$ The First Affiliated Hosptial of Zhengzhou University, pediatric surgery, zhengzhou, China

\section{Introduction}

Neuroblastoma(NB) is one of the common extracranial solid tumors of children, the prognosis $4 \mathrm{~S} \mathrm{NB}$ is good.

\section{Objectives}

We discuss the diagnosis and treatment of stage $4 \mathrm{~s}$ neuroblastoma.

\section{Methods}

A retrospective study was performed on 8 patients who were diagnosed with 4s NB, from Jan. 2013 to Dec. 2017,6 of whom were male and 2 were female. The age of patients range from 3 days to 7 months years old. The primary site was left adrenal (in 3 cases), right adrenal (in 3 cases), and para vertebral (in 2 cases). Distant disease was found in liver (in 6 cases), skin (in 2 cases), and marrow (in 1 case) respectively. The primary tumor was resected in five cases, three of which received chemotherapy post-operation. Three cases undergone "wait and see" strategy after aspiration biopsy. Results

After 3 months to 3 years, all patients were alive. The pathology result showed NB, but no MYCN was found amplified. One case was found 
morrow metastasis from primary site and skin nodule were resected. Bone marrow turned negative after 7 cycles chemotherapy. In one patient, tumor compressed the spinal, and the right leg was weak. As time went on, the tumor decreased and compression symptom got better without surgery. The primary and metastasis site of the other two observed cases were diminished slowly.

Conclusions

The prognosis 4S NB is good, Each child needs individual treatment because of the variation of disease. Active treatment may contribute to good outcome, especially for those life-threatening and spinal compression cases.

\section{eP302}

UROTHELIAL BLADDER TUMOUR IN CHILDHOOD: A REPORT OF ONE CASE AND A REVIEW

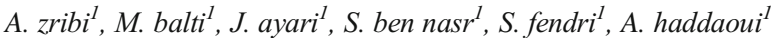 \\ ${ }^{T}$ military hospital, medical oncology, tunisia, Tunisia
}

\section{Introduction}

Urothelial bladder tumour in childhood is extremely rare, and almost all the reported cases have been low-grade tumours with a favourable outcome

\section{Objectives}

we report our case and a review of literatture

Methods

We reviewed the databases of PubMed for reports in English, searched using the keywords 'bladder', 'transitional cell carcinoma' and 'children'. We also describe a new case

\section{Results}

A 14-year-old boy, presented in july 2017 with recurrent attacks of painless haematuria. Hi has a family medical history of B-thalassemia. Typical risk factors of urothelial carcinoma, including no history of chemotherapy, radiation, or chemical exposure. He and his family denied history of tobacco usage and there was no family history of genitourinary malignancy. Physical examination revealed a normal abdominal and genitourinary examination.US showed a $12 * 11 \mathrm{~mm}$ vesical parietal tissue formation. The biopsy concluded at urothelial carcinoma GII pTa with no stromal invasion. Body CT scan was normal. The tumour was resected (tumorectomy).The histopathology confirmed the carcinoma. no adjuvant treament was needed. The child is currently asymptomatic and follow-up cystoscopy is scheduled.

\section{Conclusions}

Urothelial carcinoma, though rare in the pediatric population, must be considered in the differential diagnosis in pediatric population presenting with gross hematuria. Ultrasound is indicated, followed by cystoscopy and transurethral resection for diagnosis and therapeutic care. Appropriate treatment and followup remain as yet poorly defined.

\section{eP303}

LOST IN TRANSLATION: BUILDING INTERPRETER CONFIDENCE AND SKILL IN FACILITATING PALLIATIVE CARE CONVERSATIONS

\section{J. Abrahm ${ }^{1}$, J. Goldhirsch ${ }^{2}$}

${ }^{T}$ Dana-Farber Cancer Institute, Psychosocial Oncology and Palliative Care, Boston, USA

${ }^{2}$ Brigham and Women's Hospital, Social Work, Boston, USA

\section{Introduction}

Diversity of language and culture can create cultural barriers that result in health care disparities. In the US, for example, while over 300 languages are spoken, the English language and Western health care culture often bumps up against the languages and cultures of patients and their families.
The need for effective navigation of language and cultural barriers is no more urgent than in the field of palliative care, health care that emphasizes effective communication and attention to the medical, psychosocial and spiritual needs of patients and families coping with serious illness and even end-of-life. Palliative care clinicians can overcome linguistic and cultural barriers by integrating interpreters into their interdisciplinary teams. For successful integration and teamwork, however, both parties must be trained.

FIGURE I: CONFIDENCE SCORES
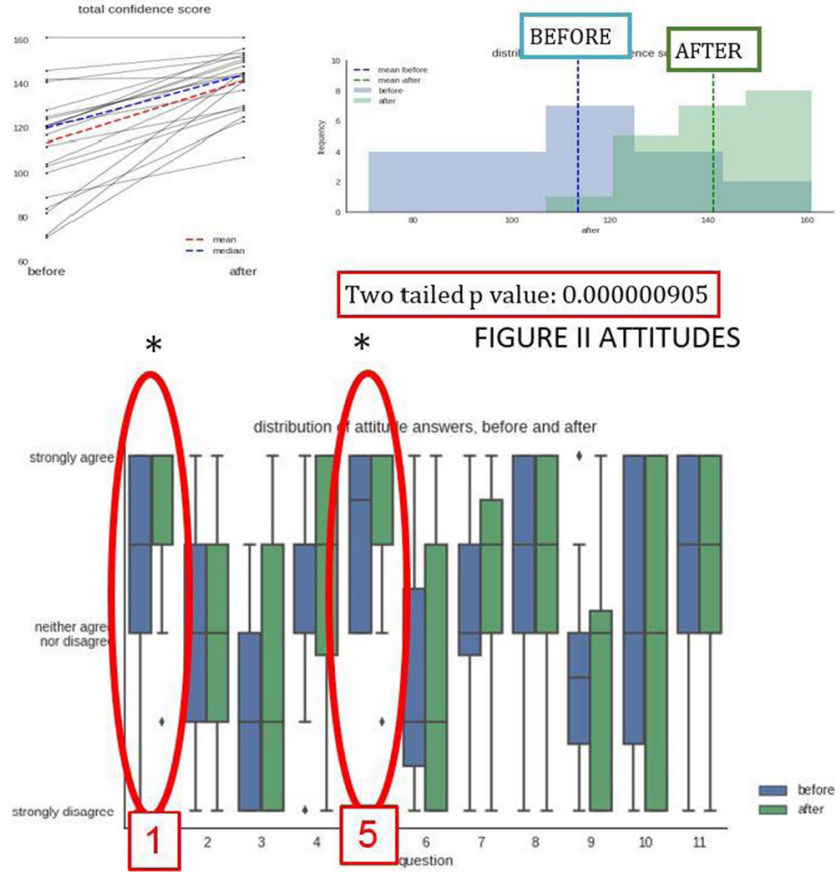

Objectives

Improve interpreter confidence in and attitudes towards participating in palliative care family meetings, including both the "pre-meeting" before the encounter, and meetings that included difficult issues facing patients and families, such as breaking bad news and code status discussions.

\section{Methods}

We held 6 monthly sessions, with a defined curriculum and readings, each facilitated by both an experienced palliative care social worker and physician. Pre- and post-course attitude and confidence evaluations of participants were completed.

Results

Figure I shows the highly significant improvement in confidence in the 30 interpreter participants; Figure II shows the significant changes in interpreter attitudes in the 11 domains assessed.

\section{Conclusions}

Well-structured dialogues between palliative care clinicians and interpreters, including didactics, discussions, and multidisciplinary roleplaying, caused highly significant improvements in confidence and attitudes among interpreters of palliative care conversations.

\section{eP304}

\section{ALOE VERA:A MULTIFACETED AYURVEDIC HERB USE IN} PALLIATIVE CARE OF ORAL CANCER PATIENTS

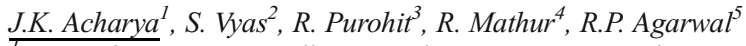

${ }^{T}$ Senior demonstrator, Palliative and supportive care, Bikaner, India

${ }^{2}$ Medical officer, Ayurved department, Bikaner, India

${ }^{3}$ Assistant professor, Medical education, Bikaner, India

${ }^{4}$ Senior professor, Medical education, Bikaner, India

${ }^{5}$ Senior professor and principal, Medicine medical education, Bikaner, India 


\section{Introduction}

In recent years, more head and neck cancer patients have been treated with radiotherapy and chemotherapy .Radiation-induced mucositis ,oral fibrosis, burning mouth syndrome are common and dose limiting toxicity of radiotherapy as well as chemotherapy among patients with head and neck cancers . A number of new agents applied locally or systemically to prevent or treat radiationinduced oral complications have been investigated, but there is no widely accepted prophylactic or effective treatment for them

Objectives

To review composition, actions, and clinical applications of ALOE VERA herb in palliative care of oral cancer patients .To establish its effectiveness as an invaluable adjunct in the treatment of oral cancer patients in palliative care.

Methods

A total 80 patients were randomized in double blind study to either $\mathrm{AV}$ (40patients)or placebo (40patients), at a dose $5 \mathrm{mg}$ three times a day. A visual Analog Scale and subjective symptoms were used for rating pain , oral fibrosis ,intra oral dryness, eating ability, oral discomfort .The patients were evaluated after 4 and 8 weeks.

Results

Data analysis study groups that had been given aloe vera ,by anova showed that there was a statistically significant difference $(\mathrm{p}<0.05)$ in pain ,eating ability , and oral discomfort as graded by visual analogue scale.

Conclusions

Within the limitations of this study aloe vera is effective as a natural healer and anti oxidant agent for preservation of oral mucous membrane and reduces oral complications. As a potential choice, Aloe vera gel for palliative patients undergoing head and neck cancer treatment prevent oral complications.

\section{eP305}

SPIRITUALITY IN PRIMARY PALLIATIVE CARE: A LONGITUDINAL STUDY OF FACTORS IMPACTING PHYSICIANS' SPIRITUAL CARE PROVISION

\section{G. Anandarajah ${ }^{1}$, L. Mennillo ${ }^{I}$, J. Roseman ${ }^{2}$ \\ ${ }^{T}$ Alpert Medical School of Brown University, Family Medicine, Providence- RI, USA \\ ${ }^{2}$ Nova Southeastern University, College of Osteopathic Medicine, Fort Lauderdale- FL, USA}

\section{Introduction}

Spiritual care (SC) is central to palliative care, however there remains a mismatch between patients' desire to have their spiritual needs addressed and physicians' SC provision. The shortage of specialty-trained palliative physicians, necessitates that all physicians provide primary palliative care, including SC. Although several quantitative studies explore physicians' barriers to SC, few qualitative studies exist and few explore facilitators. No longitudinal studies exist.

Objectives

To gain an in-depth understanding of factors influencing physicians' SC provision over time.

Methods

A 10-year longitudinal, individual interview study. In study year-1, we interviewed all residents in a USA primary care residency (full studygroup) regarding SC beliefs, experiences and skills. The longitudinal study-group (PGY1 subgroup) was interviewed again in study year-3 and year-11. Interviews were audio-recorded and transcribed. Three researchers analyzed transcripts using a grounded theory approach. IRB approved.

\section{Results}

We analyzed 59 interviews from 34 physicians. Physicians had diverse personal spiritual beliefs. Seven themes regarding SC facilitators emerged in both groups (response rate 89\%): patients' needs; practice setting; beliefs regarding role of physician; personal spiritual beliefs; life experiences (professional, personal); SC training; self-care and reflection. Longitudinal interviews (response rate 97\%) revealed thematic evolution and interactions over 10-years: patients' needs and belief in whole-person care remained primary motivators; cross-cultural SC communication training diminished impact of physicians' personal spiritual beliefs; life experiences enhanced SC skills; work environment helped or hindered SC provision; and spiritual self-care/reflection fostered patient-centered, compassionate SC.

\begin{tabular}{|c|c|}
\hline Themes & Examples of Quotations \\
\hline \multirow{2}{*}{$\begin{array}{l}\text { Patients' } \\
\text { Needs }\end{array}$} & "It's sinportant to our practice of medicine because it's important to maxy of our patients" \\
\hline & 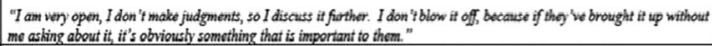 \\
\hline \multirow[t]{2}{*}{$\begin{array}{l}\text { Practice } \\
\text { Setting }\end{array}$} & 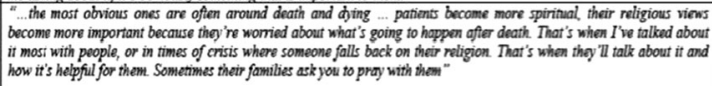 \\
\hline & $\begin{array}{l}\text { "they come to me with anviety, depression and stress. Those are all continons for which my avareness of the social } \\
\text { supports and their belief neworks are important." }\end{array}$ \\
\hline \multirow{2}{*}{$\begin{array}{l}\text { Beliets } \\
\text { Regarding } \\
\text { Role of } \\
\text { Physician }\end{array}$} & $\begin{array}{l}\text { "I guess my beliefs about a physician, at least a family medicine physicien, is very much the idea of - whole person health } \\
\text { and in that sense, I d do see religion and spirinaliny as components of that, important components of that." }\end{array}$ \\
\hline & $\begin{array}{l}\text { "Tes, Idefrnitely think it's part of my role. It's sach an important part of someone's perronality and their poyche, Ithink it } \\
\text { does make a difference in their healing and it's important to get a feel for that" }\end{array}$ \\
\hline \multirow{2}{*}{$\begin{array}{l}\text { Personal } \\
\text { Spiritual } \\
\text { Beliets }\end{array}$} & 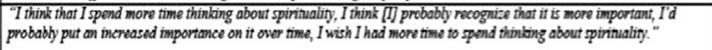 \\
\hline & 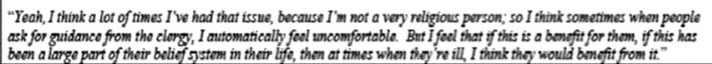 \\
\hline $\begin{array}{l}\text { lite } \\
\text { Experiences }\end{array}$ & 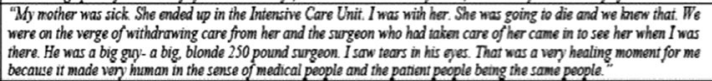 \\
\hline \multirow[t]{2}{*}{$\begin{array}{l}\text { Spiritual Caré } \\
\text { Training }\end{array}$} & 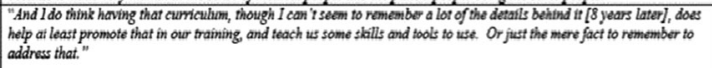 \\
\hline & $\begin{array}{l}\text { "Ifelt thke it was ok to think of it as par of my role, and that it wasn't sonething I hod to lenve out of my relationships with } \\
\text { patiens." }\end{array}$ \\
\hline \multirow[t]{2}{*}{$\begin{array}{l}\text { Self-Core and } \\
\text { Reflection }\end{array}$} & 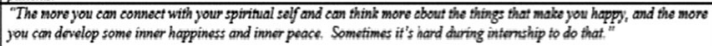 \\
\hline & $\begin{array}{l}\text { "I thmt that would be the dideal for providers to really spend nime taling care of themsebes so that they can really be } \\
\text { present for their patienss," }\end{array}$ \\
\hline
\end{tabular}

Conclusions

Findings suggest approaches to facilitate SC provision to seriously ill patients by non-palliative care specialists and areas for further research.

\section{eP306 \\ EXERCISE INTERVENTIONS FOR PALLIATIVE CANCER PATIENTS: PRELIMINARY RESULTS OF A SYSTEMATIC LITERATURE REVIEW}

G. Anandarajah ${ }^{1}$, H. Mennillo ${ }^{2}$

${ }^{T}$ Alpert Medical School of Brown University, Office of Medical Education, Providence- RI, USA

${ }^{2}$ Alpert Medical School of Brown University, Student, Providence- RI, USA

\section{Introduction}

Exercise interventions have been demonstrated to improve physical and emotional health for many conditions. However, the impact of exercise on cancer patients and especially on palliative care patients has only recently been gaining increased attention.

Objectives

To evaluate current research on effects of exercise on palliative cancer patients' well-being (physical, emotional) and quality of life (QOL).

\section{Methods}

This systematic literature review represents the first step of a comprehensive review utilizing multiple databases. We searched Medline/PubMed (January 2008 to January 2018) using MeSH terms "palliative cancer", "palliative carcinoma", "advanced cancer," and "palliative care" in combination with "exercise" and "exercise therapy." One author screened titles and excluded articles that were clearly not relevant, were not primary studies, or were non-English. Next, two authors independently screened abstracts to further exclude articles concerning non-palliative patients. Finally, both authors will review full articles. Given the paucity 
and heterogeneity of relevant studies, a quantitative meta-analysis is not possible. Therefore, authors are currently preparing a narrative synthesis. Results

Data analysis is ongoing. Preliminary analysis suggests that exercise interventions are feasible in palliative cancer patients and appear to improve QOL with some improvement in physical and mental functioning and mixed findings regarding fatigue. However, exercise interventions can also trigger emotional distress in palliative patients who may perceive inability to participate as a failure. Final analysis will be completed prior to conference presentation. Conclusions

Studies on this topic are sparse. Next steps: search for additional studies in other databases and in reference sections of included articles. Systematic review findings will guide future research.

\section{eP307}

\section{IS RESPITE CARE A REALITY IN ONCOLOGY?}

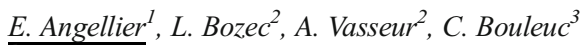

${ }^{T}$ Institut Curie, DISSPO 35 rue dailly, Saint Cloud, France

${ }^{2}$ Institut Curie, DOM 35 rue dailly, Saint Cloud, France

${ }^{3}$ Institut Curie, DISSPO 25 rue d'ULM, Paris, France

\section{Introduction}

Respite is necessary for families with severe cancer patients. Do we use it properly?

Objectives

To investigate patients outcome after admission for respite care.

Methods

Retrospective analysis of 18 months requests.

\section{Results}

24 patients, 5 men and 19 women were concerned. Severe symptoms or risk were motives of admission for 4 , and family respite or major anxiety for others. 2 requests were refused; for 4 caregivers and patients were informed that it could be transformed in definitive stay.

Median age: 70,5 years (46 to 89 ).

Median delay between request and admission: 3,5 days (1 to 33).

Median length of stay: 21 days (1 to 62), 19 if we exclude patients who were discharged, 21 for these latter ones (10 to 24).

4 patients went back home:

-1 is alive, 8 months after request

- 1 was hospitalized after 3 days at home : family could not cope anymore and expressed a need for hospitalization until death. She died 3 months after request

- 2 went back home and died 3 and 5 months after request

Other patients died during their stay in palliative care unit.

\section{Conclusions}

It remains difficult to assess time of End-Stage by vulnerable cancer patients who can quickly worsen. Maybe is the word respite not appropriate? We face with deliver bad news in the best timing as possible, including psychological timing of patients, caregivers, and families. Patients'information level has to be transmitted to palliative care units, and help them to turn the stay as a definitive one, especially because we can't hospitalize them elsewhere.

\section{eP308 \\ PROCESS CHALLENGES IN PALLIATIVE CARE FOR CANCER PATIENTS: A QUALITATIVE STUDY}

M. Ansari ${ }^{1}$, M. rassouli corresponder author ${ }^{1}$, M.E. akbari ${ }^{2}$, A. $\overline{\text { abbaszadeh }}^{1}, \overline{\text { A. akbarisari }}^{3}$

${ }^{I}$ Shahid beheshty university, school of nursing and midwifery, Tehran, Iran

${ }^{2}$ Shahid beheshty university, Cancer Research Centre, Tehran, Iran

${ }^{3}$ tehran university medical science, Department of Health Management

and Economics-School of Public Health, tehran, Iran

\section{Introduction}

Quality assessment is a critical component of determining the value of medical services, including palliative care.

\section{Objectives}

This study was conducted to explore stakeholder's perceptions of palliative care process challenges for cancer patients in Iran.

Methods

This qualitative study was carried out with 22 semi structured interviews from February 2016 to August 2017 in Hospitals of Tehran, Iran. Participants were selected through purposive sampling and included cancer patients and their family caregivers as well as healthcare providers, and health policy-makers. The interviews were analyzed using qualitative directed content analysis based on Donabedian model. In order to assess the accuracy and validity of the study, Lincoln and Guba's four criteria were used.

\section{Results}

from the analysis of the interviews, the codes were categorized into main category, namely process, and three identified subcategories were "Lack of attracting stakeholders participation ", "standardized care"and"applying educational and research approaches".

\section{Conclusions}

the result of this study showed that since palliative care in Iran is a young discipline, the process of providing services requires the attention of the health system to the; Standard models for providing palliative care services, training human resources in generalist and specialist palliative care groups, design of palliative medicine curricula and raising general culture of cancer and patient and caregivers empowerment.

\section{eP309}

IS THERE A NEED TO INCLUDE A REFERRAL FOR CHEMOTHERAPY AND MANAGEMENT OF RECURRENT HYPERCALCEMIA WITHIN THE PURVIEW OF PALLIATIVE MEDICINE?

R.D. Arora $^{1}$

${ }^{T}$ All India Institute of Medical Sciences- New Delhi, Palliative Medicine, Ansari Nagar- New Delhi, India

\section{Introduction}

In the era of Immunotherapy when the trajectory of illness is bieng redefined, there exists an urgent need to make palliative medicine more relevant to the advanced cancer patient.

\section{Objectives}

This paper tries to reinterpret the role of palliative medicine by way of a providing a synopsis of the management of two advanced cancer patients. Methods

A 43 year old female with type 2 diabetes mellitus, a known case of metastatic gall bladder cancer with malignant biliary stricture who had undergone PTBD with stenting presented with an episode of cholangiitis. She was prescribed intravenous antibiotics, subcutaneous Insulin and other supportive treatment, following which she was started on Palliative chemotherapy. A 31 year old female, a known case of adenocarcinoma colon presented with symptoms of Malignant bowel obstruction for which she was started on pharmacological management. She developed an episode of witnessed generalised tonic clonic seizure preceded by an episode of flash of lights with blurring of vision on day 8 of treatment. MRI examination and a neurology referral was suggestive of a diagnosis of PRES. Symptomatic management of hypertension was initiated.

\section{Results}

Management of these medical issues was important in improving the patients quality of life.

\section{Conclusions}

Initiation of chemotherapy and medical management of sepsis, electrolye imbalances and lower respiratory tract infections are important issues in 
the advanced cancer patient. Bringing these issues directly under the ambit of Palliative Medicine is an important step towards redefining the role of this nascent subspeciality.

\section{eP310}

WHAT DOES " EARLY" IN EARLY PALLIATIVE MEDICINE REALLY IMPLY?

R.D. Arora $^{1}$

${ }^{T}$ All India Institute of Medical Sciences- New Delhi, Palliative Medicine, Ansari Nagar- New Delhi, India

\section{Introduction}

Early palliative medicine has not been well defined. Multiple studies have failed to prove the unequivocal beneficial effects of this premise.

Objectives

To enlist the scope and approaches which define early palliative medicine. Methods

This paper is a distillation of the personal views of the author on the subject. Results

As we go through multiple definitions of palliative medicine which have existed since the time that this speciality came into existence and move towards the present we realize that there has been an attempt to limit its scope to a niche subgroup of advanced cancer patients. Boundaries between supportive oncology and palliative medicine are often blurred. The timing at which the patient is introduced to these services (with supportive oncology bieng the earlier of the two) and the differences in the primary providers (supportive oncology is usually considered the domain of medical oncologists) constitute the thin line of demarcation between the two. Conclusions

Introduction of the palliative medicine expert to the patients family at an earlier stage in the illness, his active involvement during the daily multispeciality oncology rounds, expanding his role to include management of medical issues such as side effects of chemotherapy( nausea, peripheral neuropathy, diarrhoea) may make palliative medicine more accessible to the patient and his family. It might go a long way in making the involvement of this expert acceptable at the later stage in the disease trajectory and negate the apprehension associated with such a referral.

\section{eP311}

THE CURRENT STATUS OF THE PALLIATIVE CARE CONSULTATION IN THE ONCOLOGICAL MANAGEMENT IN LEBANON

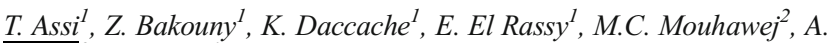
$\overline{\text { Tohme }}^{2}$, J. Kattan ${ }^{l}$

${ }^{1}$ Saint-Joseph University, Hematology-Oncology, Beirut, Lebanon

${ }^{2}$ Saint-Joseph University, Palliative care, Beirut, Lebanon

\section{Introduction}

Early palliative care consultation (PCC) has been associated with better oncological outcomes than late consults. Many factors contribute to the delay in PCC in conservative societies which may alter the efficacy outcomes and quality of life of cancer patients.

Objectives

We evaluated the role and the timing of PCC in Lebanese cancer patients and examined the factors that justify this practice in order to improve the quality of cancer care in undermined regions.

Methods

This is an observational study at Hotel-Dieu de France University Hospital, Lebanon that included all cancer patients that had a PCC between the 1st of January 2014 and 31st of June 2016. Patients and tumour characteristics as well as details concerning the PCC were retrieved from the hospital records.

\section{Results}

Among our study population including 210 patients, the median survival post-diagnosis and post-PCC were 18.7 and 0.6 months respectively. The delay in PCC correlated to the lack of family support $(\mathrm{HR}=1.77$, $95 \% \mathrm{CI}=1.16-2.68)$, ignorance of diagnosis $(\mathrm{HR}=0.64,95 \% \mathrm{CI}=0.45$ $0.91)$, availability of $\mathrm{NGO}$ support $(\mathrm{HR}=2.41,95 \% \mathrm{CI}=1.54-3.76)$. Among patients who were followed-up at home, those who had NGO support had longer follow-up outside the hospital $(\mathrm{OR}=0.54,95 \% \mathrm{CI}=$ $0.36-0.81)$ and were more likely to die at home instead of the hospital $(\mathrm{OR}=9.78,95 \% \mathrm{CI}=4.11-23.27)$.

\section{Conclusions}

The integration of PCC into the oncological armamentarium is still delayed until the late stages of cancer due to social and financial obstacles. Still, the delayed PCC seemed to benefit our patients.

\section{eP312}

EFFECTS OF STRONG CYTOCHROME P450 (CYP)3A4 AND CYP 2 D 6 INDUCERS AND INHIBITORS ON THE PHARMACOKINETIC (PK) PROFILE OF ANAMORELIN IN HEALTHY VOLUNTEERS

\author{
A. Bernareggi ${ }^{1}$, M.A. Matson ${ }^{2}$, E. Duus ${ }^{3}$ \\ ${ }^{T}$ Helsinn Healthcare SA, -, Lugano, Switzerland \\ ${ }^{2}$ Prism Clinical Research, St. Paul, Minnesota, USA \\ ${ }^{3}$ Helsinn Therapeutics US Inc., Iselin, New Jersey, USA
}

\section{Introduction}

The oral selective ghrelin receptor agonist anamorelin, which is currently in development, improved body weight, body composition parameters, and symptom burden in patients with advanced non-small cell lung cancer and anorexia/cachexia (ROMANA studies; Temel J. Lancet Oncol. 2016; Currow D. Ann Oncol. 2017). Anamorelin is metabolized by the liver, predominantly by CYP3A4 and marginally by CYP2D6. Strong CYP3A4/CYP2D6 inducers and inhibitors might alter its PK profile.

\section{Objectives}

The effects of the CYP3A4 inhibitor ketoconazole, the CYP3A4 inducer rifampin, and the CYP2D6 inhibitor paroxetine on the PK profile of anamorelin were evaluated in two phase I clinical trials in healthy volunteers.

Methods

Healthy volunteers received anamorelin orally, alone or in combination with oral ketoconazole, rifampin, or paroxetine (Table). Serial blood samples were collected in both studies to assess the PK parameters of anamorelin.

Results

Concomitant ketoconazole administration significantly increased anamorelin maximum plasma concentration $\left(\mathrm{C}_{\max }\right) 3.1$-fold and exposure $\left(\mathrm{AUC}_{0-\infty}\right)$ 3.2-fold (Table). Concomitant rifampin administration significantly decreased anamorelin $\mathrm{C}_{\max }$ 2.2-fold and $\mathrm{AUC}_{0-\infty} 3.1$-fold (Table). Concomitant paroxetine administration significantly increased anamorelin $\mathrm{C}_{\max }$ by $28 \%$, while anamorelin $\mathrm{AUC}_{0-\infty}$ was $15 \%$ lower post-paroxetine, but this unexpected decrease was not significant (Table).

\begin{tabular}{|c|c|c|c|}
\hline \multirow{3}{*}{$\begin{array}{l}\text { PK } \\
\text { parameter } \\
\text { (anamorelin) }\end{array}$} & \multicolumn{2}{|c|}{ CYP3A4 } & \multirow{2}{*}{$\begin{array}{c}\text { CYP2D6 } \\
\text { CYP2D6 inhibitor } \\
\text { (paroxet ine) }\end{array}$} \\
\hline & $\begin{array}{l}\text { CÝşA4 inhibitor } \\
\text { (kețoconazole) : }\end{array}$ & $\begin{array}{l}\text { CYPBA inducer } \\
\text { (rifampiñ) }\end{array}$ & \\
\hline & $\begin{array}{l}\text { Study } 1(\mathrm{~N}=12) \\
\text { (ANAM } 25 \text { mg alone or } \\
\text { with ketaconazole } 200 \mathrm{mg} \\
\text { every } 12 \text { hours for } 3 \text { doses) }\end{array}$ & $\begin{array}{r}\text { Study } \\
\text { (ANAM } 100 \mathrm{~m} \\
\text { rifampin } 600 \mathrm{mg} \\
\text { paroxetine } 20 \mathrm{~m}\end{array}$ & $\begin{array}{l}(\mathrm{N}=16) \\
\text { alone or with } \\
Q D \text { for } 7 \text { days or } \cdots \ldots \\
Q D \text { for } 11 \text { days) }\end{array}$ \\
\hline $\begin{array}{l}\text { Mean } \mathrm{C}_{\max } \\
\text { (SD), } \mathrm{ng} / \mathrm{mL}\end{array}$ & $\begin{array}{c}118(114) \text { vs } 368(120) \\
(p \leq 0.001)\end{array}$ & $\begin{array}{c}1006(340) \text { vs } 454(209) \\
\vdots \quad(p<0.001)\end{array}$ & $\begin{array}{c}796(353) \text { vs } 1017(514) ; \\
(p=0.003)\end{array}$ \\
\hline $\begin{array}{l}\text { Mean } t_{\max } \\
\text { (SD), hours }\end{array}$ & $\begin{array}{c}1.53(0.70) \text { vs } 0.64(0 ; 22) \\
(p \leq 0.01) \\
\vdots\end{array}$ & $\begin{array}{c}1.02(0.71) \text { vs } 0.86(0.50) \\
\vdots \quad(p=0.663)\end{array}$ & $\begin{array}{c}0.83(0.62) \text { ys } 0.72(0.43) \\
(p=0.400) \\
\vdots\end{array}$ \\
\hline $\begin{array}{l}\text { Mean AUC }{ }_{0-\text { D }} \\
\text { (SDD), } \\
\mathrm{ng}^{*} \mathrm{hr} / \mathrm{mL}\end{array}$ & 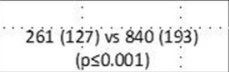 & $\begin{array}{c}2391 \text { (1019) vs 779.0 (435) } \\
\vdots \quad(p<0.001)\end{array}$ & $\begin{array}{l}2087(1271) \text { vs } 1780(1033) \\
(p=0.072)\end{array}$ \\
\hline
\end{tabular}




\section{Conclusions}

Concomitant administration of anamorelin should be approached with caution with strong CYP3A4 inhibitors and inducers because it may affect the $\mathrm{PK}$ profile of anamorelin. No restrictions regarding concomitant use of CYP2D6 inhibitors are needed.

\section{eP313}

THE PSYCHO SOCIAL AND ECONOMIC EFFECTS OF CARING FOR TERMINALLY ILL CANCER PATIENTS: THE CASE OF HOSPICE AFRICA UGANDA

N. Buyinza ${ }^{1}$

${ }^{T}$ Hospice A frica Uganda, Education, Kampala, Uganda

\section{Introduction}

Care giving at the end of life can be a challenging and confronting experience. The responsibilities of giving care to terminally ill cancer patients may include executing complex physical and mental tasks including financial planning, decision making, emotional support and coordination of care.

\section{Objectives}

To identify the psycho social and economic effects of caring for terminally ill cancer patients attending Hospice Africa Uganda

Methods

This was a mixed method study. Source of data was restricted to care givers of terminally ill cancer patients attending Hospice Africa Uganda. Sampling was simple random.

\section{Results}

This study showed that caretaking had statistically significant effects on the psychosocial wellbeing of caretakers as shown by the $p$ values $(<0.05)$. Particularly their relationship with $\operatorname{God}(\mathrm{p}<0.05)$, getting enough time to attend social gatherings $(\mathrm{p}<0.05)$ and caring for their children $(p<0.05)$. Fatigue and exhaustion as a result of giving prolonged care to the terminally ill $(\mathrm{p}<0.05)$ was as well reported.

Providing care to terminally ill cancer patients had minimal effect on the economic livelihood of care givers. It only had statistically significant influence on changes in income of caregivers $(\mathrm{p}<0.005)$, and the fact that caregivers could not find an alternative job alongside patient care $(\mathrm{p}<0.05)$

Conclusions

Care giving has a statistically significant effect on the psychosocial and economic well being of caregivers. We recommend that caregivers and family be given ongoing support so as to provide adequate care to cancer patients and their families and this should be initiated early in the disease trajectory.

\section{eP314}

WHAT IS PERSON-CENTRED CARE FROM A HAEMATOONCOLOGY PATIENT PERSPECTIVE IN THEIR LAST YEAR OF LIFE? A GROUNDED THEORY APPROACH

\author{
K. Campbell ${ }^{1}$ \\ ${ }^{T}$ Edinburgh Napier University, School of Health and Social Care, \\ Edinburgh, United Kingdom
}

\section{Introduction}

Haemato-oncology (HO) patients comprise $8.5 \%$ of the overall adult cancers within the United Kingdom (Cancer Research UK 2012). The literature suggests that in the last year of life transition to palliative care is late or non-existent for $\mathrm{HO}$ patients, potentially resulting in their care being compromised (Alt-Epping et al 2011); with many dying in hospital (Howell et al 2010 b). In contrast national guidance states that the HO patient should receive an early integration to palliative care services (National Institute for Health and Clinical Excellence 2003, 2004).
Various reasons have been cited as affecting the integration of palliative care services (Manitta et al 2010).

\section{Objectives}

To explore patient's perspective of patient centred care in last year of life. Methods

The data includes initial theoretical sampling and conducting 20 patient interviews. Constant comparison analysis supported an in-depth view of HO patient's perspective care in the last year of their lives.

\section{Results}

The sub categories identified include: Reversibility : Advances in treatment including emergency and routine admissions; Death and dying: leaving a legacy, affect on connections:Life transitions and loss; Independence vs dependency and Co-ordination of care.

\section{Conclusions}

This presentation will conclude that the above subcategories are interlinked and all need to be assessed giving an understanding of the whole patient, which will inform the development and implementation of future clinical pathways and interventions to ensure the quality of person centred care for the HO patient in the last year of their lives.

\section{eP315}

\section{SUPPORTING PATIENT SYMPTOM MANAGEMENT USING} EDMONTON SYMPTOM ASSESSMENT SCALE (ESAS)

\author{
$\underline{\text { S. Rauenzahn Cervantez }}{ }^{1}$, L. Tenner ${ }^{1}$, S. Schmidt ${ }^{2}, N$. Ali $^{3}$, S. Singh- \\ Carlson $^{4}$ \\ ${ }^{I}$ Mays Cancer Center UT Health San Antonio, Hematology Oncology, \\ San Antonio, USA \\ ${ }^{2}$ The University of Texas Health Science Center San Antonio, Department \\ of Epidemiology \& Biostatistics, San Antonio, USA \\ ${ }^{3}$ The University of Texas Health Science Center San Antonio, Clinical \\ Information Systems, San Antonio, USA \\ ${ }^{4}$ The University of Texas Health Science Center San Antonio, School of \\ Nursing, San Antonio, USA
}

\section{Introduction}

Research in supportive care demonstrates improvements in overall survival, quality of life, symptom management, and reductions in the cost of care. Despite the American Society of Clinical Oncology recommendation for early concurrent supportive care in patients with advanced cancer and high symptom burden, integrating supportive services is challenging.

\section{Objectives}

To implement an electronic medical record (EMR) provider alert of high symptom burden based on Edmonton Symptom Assessment Scale (ESAS) criteria.

To determine the impact an alert has on supportive service referrals.

\section{Methods}

ESAS scores were implemented in medical ambulatory oncology clinics to quantitatively assess symptom burden. An EMR alert was programmed for a total ESAS score $>30$ and any single response of $\geq 9$ to capture approximately $15 \%$ of our high symptom burden patient population. Providers could elect to accept the alert, placing an order for palliative consultation or decline prompts. Referral rates and symptom assessment scores were followed as metrics for EMR alert efficiency.

Results

Over 10 months, 9,710 patient visits used ESAS system resulting in 7,707 fully completed ESAS scores (79.4\% completion rate). There were 78 total consults to palliative-care, a referral rate of less than $1 \%$ of population, which was unchanged from prior to trigger implementation. Of the total completed ESAS forms, the trigger alerted 686 times $(8.9 \%$ of patient visits) with only 55 of those triggers leading to a supportive care referral $(8.0 \%)$. 


\begin{tabular}{|l|c|c|c|}
\hline & $\begin{array}{c}\text { All Visits } \\
(\mathbf{N}=9,710)\end{array}$ & $\begin{array}{c}\text { Entirely Completed } \\
\text { ESAS Visits } \\
(\mathbf{N}=7,707)\end{array}$ & $\begin{array}{c}\text { First Entirely } \\
\text { Completed ESAS Visit } \\
(\mathbf{N}=\mathbf{2 , 3 9 4 )}\end{array}$ \\
\hline Total Unique patients & 3,185 & 2,894 & 2,894 \\
\hline Total Follow-up visits & 6,525 & 4,813 & \\
\hline Palliative Care Consults, n (\%) & $78(0.80 \%)$ & $72(0.93 \%)$ & $28(0.97 \%)$ \\
\hline Hospice Consults, n (\%) & $55(0.58 \%)$ & $42(0.54 \%)$ & $6(0.2 \%)$ \\
\hline Trigger Fired, n (\%) & & $686(8.9 \%)$ & $282(9.7 \%)$ \\
\hline Palliative Care Consults & & 55 & 21 \\
\hline Hospice Consults & & 17 & 4 \\
\hline
\end{tabular}

\section{Conclusions}

This project highlights challenges of an EMR based alert system and need for continued efforts to improve supportive care referrals through provider education and tool implementation.

\section{eP316}

\section{A SAMPLE OF HOSPICE SATISFACTION OF HOSPICE WARD IN A MEDICAL CENTER}

\author{
C.T. Chang ${ }^{l}$ \\ ${ }^{T}$ SHIN KONG WU HO-SU MEMORIAL HOSPITAL, Department of \\ Nursing, Taipei, Taiwan R.O.C.
}

\section{Introduction}

In the end of life, "Good Death" is the expectation of patients and their families and is also an important indicator of tranquility care and care.

\section{Objectives}

The goal is to understand how Hospice Ward affects the patient's and family's satisfaction with Hospice and its associated impactors.

\section{Methods}

The testing period is from November 1, 2015 to October 31, 2017. A total of 130 patients who have been hospitalized for 5 days and who have died in the hospital, Relevant medical staff participating in the service of deceased patients, used the" Good Death Service Self-satisfaction Questionnaire" which included "Hospice Good Death Scale "and" A audit Scale for Death "to evaluate and analyze the related data and to explore the process of helping patients and their relatives in hospice ward. Results

The results showed that 36 patients $(27.6 \%)$ who did not know the deaths, the ratings of end-of-life service self-satisfaction rated $71.9 \%$. Ninetyfour patients $(72.3 \%)$ who were aware of the deaths rated $90.1 \%$ for self-satisfaction for end-of-life services. Nine patients $(6.9 \%)$ who did not reach their life goals given an average score of $66.1 \%$. The average score of 121 patients $(93.0 \%)$ who reached their life goal was $84.3 \%$.

Conclusions

This study found that patients whose life goals were reached, the higher the average rate of end-of-service satisfaction; if the symptom control scored of $80 \%$ or more, their end-of-life service evaluation would berelatively high; the understanding for end-stage patients of sickness condition may contribute to a good hospice result.

\section{eP317 \\ "YOU'RE NEVER LONELY!": A CASE REPORT WHICH WAS PERFORMED BY THE INTERECTION THEORY OF IDA JEAN ORLANDO}

Y. Clract Yasar ${ }^{1}$, N. Nural ${ }^{1}$, B.C. Demirbag $\breve{g}^{2}$

${ }^{T}$ institue of health sciences, internal medicine nursing, TRABZON, Turkey

${ }^{2}$ institue of health sciences, public health nursing, trabzon, Turkey

\section{Introduction}

While the cancer patient tries to suffer from physical problems, he also has negative feelings such as loneliness, suffering, hopelessness and anxiety about death. Empathizing with patients, understanding, evaluating and interpretting their feelings, and managing individual care process are requirements for professional nursing.

\section{Objectives}

Our case report was planned with the aim of identifying the nursing interventions that will help the patient's need of help observed by the nurse and disseminating the use of the purposeful nursing process via interaction model of Ida Jean Orlando from nursing theorists in the care of a hospitalized patient with a diagnosis of malignant neoplasm of the stomach.

\section{Methods}

Face-to-face interview performed during patient care were recorded in September 2017 with a 60-year-old female patient who was diagnosed with gastric malignant neoplasm at the Surgery Clinic of Trabzon Kanuni Training and Research Hospital.The nursing care plan was applied to the patient in accordance with the intended nursing process described in Orlando's theory and feedback was received.

\section{Results}

In interviews with the patient, it was observed through the patient's verbal and behavioral responses that the patient was in need of support because of advanced disease, physical problems, malnutrition and lack of care.

\section{Conclusions}

The patient's needs were solved with the purposeful nursing process and positive feedback was obtained from the patient.

\section{eP318}

HOPE AND LIFE ENGAGEMENT OF PATIENTS WITH CANCER AT THE ADVANCED STAGE

\author{
Y. Clracı Yasar ${ }^{I}$, N. Nural ${ }^{1}$, S. gülhan ${ }^{I}$ \\ ${ }^{T}$ institue of health sciences, internal medicine nursing, TRABZON, Turkey
}

\section{Introduction}

Cancer is a disease that causes both physical and mental problems. These problems adversely affect the patient's life expectancies and engagements.

\section{Objectives}

The purpose of this study is to evaluate the implications of hope and life engagement in advanced cancer patients and to examine the factors that affect them.

\section{Methods}

This study performed as a cross-sectional and descriptive study on 74 cancer patients with advanced stage who hospitalized in Kanuni Training and Research Hospital.Interview performed with emotional support by holding patient's hands and lasted about 1 hour.In the questionnaire the sociodemographic and emotional status of the patients were evaluated.As scale; the "integrative hope scale" and "life engagement scale" that were validated in Turkish validity were used.Parametric tests "Anova and t test" were used for the analysis of sociodemographic data and variables that could affect hope and life engagement. The significance level was accepted as 0.05 .

\section{Results}

The mean hope scale score of patients was $104,5946 \pm 6,80233$ and the mean life engegament scale score was $23,91 \pm 4,92340$. The variables which may affect mean hope score were age, fear of death, diagnosis, place of residence, support status, having children and gender.The variables which may affect mean life engagement score were place of residence, educational status, having children, level of pain, diagnosis, support status, agony.

\section{Conclusions}

Although the patients were advanced cancer patients, the level of hope and life engagement was found to be high.The environment they live in, their educational status, agony they experience, diagnosis, fear of death, support status and gender are influential in their desire to stay alive. 
eP319

EFFECT IVE ACHIEVEMENT OF PALLIAT IVE RADIOTHERAPY: A PROSPECTIVE STUDY

A. Clément-Zhao ${ }^{I}$, M. Luu ${ }^{2}$, J.E. Bibault ${ }^{I}$, C. Daveau ${ }^{I}$, S. Kreps ${ }^{I}, H$.

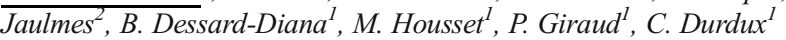

${ }^{1}$ Hôpital Européen Georges Pompidou, Radiotherapy, Paris, France

${ }^{2}$ Hôpital Européen Georges Pompidou, Palliative care unit, Paris, France

\section{Introduction}

Introduction The main goal of palliative radiotherapy is to reduce patient's discomfort. But sometimes patients didn't receive any benefit from this treatment because of rapid worsening of their general condition.

Objectives

Objectives This prospective monocentric study assessed the effective achievement of palliative radiotherapy.

\section{Methods}

Methods From 1st December 2015 to 29th February 2016, all consecutive patients treated for palliative radiotherapy in our hospital were included. The primary endpoint was the effective achievement of palliative radiotherapy in accordance to initial prescription (total dose, overall treatment time and fractionation). The secondary endpoints were the number of treatment breaks (temporary or definitive), the clinical benefit (complete, partial, absent or indeterminate) and the number of deaths.

\section{Results}

Results Fifty-nine patients were included in the study and 64 treatments were analysed ( 3 patients treated twice, one patient three times). Anatomic treatment sites were: bone $(70.3 \%$ : spine $31.3 \%$, pelvis $26.6 \%$, other $12.5 \%)$ and brain (21.9\%). Treatment goals were: pain control only (43.8\%), decompression only (21.9\%), pain control and decompression $(32.8 \%)$, haemostatic aim (1.6\%). Palliative treatment was achieved in 57 cases $(89 \%)$. Temporary stop of radiotherapy was necessary in 6 cases (9.4\%, 3 for medical reason, 3 for logistic reason). The main reason of interruption was worsening of performance status ( 7 cases). Palliative objective (complete or partial responses) was obtained in 44 cases $(68.8 \%)$. Conclusions

Conclusion Palliative radiotherapy was completed as originally planned in 51 cases $(79.9 \%$ ) with a clinical benefit for 44 cases $(68.8 \%)$. Radiation therapy must not be neglected as palliative treatment at the end-of-life.

\section{eP320}

TREATING ANOREXIA IN PEOPLE WITH ADVANCED CANCER. A RANDOMISED, DOUBLE BLIND, CONTROLLED TRIAL OF MEGESTROL ACETATE, DEXAMETHASONE OR PLACEBO

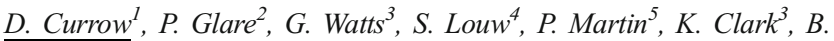
Fazekas $^{\circ}$, M. Agar ${ }^{1}$

${ }^{1}$ University of Technology Sydney, Faculty of Health, Sydney, Australia

${ }^{2}$ Sydney University, Pain Management Research Institute, Sydney, Australia ${ }^{3}$ Calvary Mater Newcastle, Palliative Care, Sydney, Australia

${ }^{4}$ McCloud Consulting Group, Biostatistics \& Data Management, Sydney, Australia

${ }^{5}$ Deakin University, Clinical Communication and End-of-Life Care, Melbourne, Australia

${ }^{6}$ Flinders University, College of Medicine and Public Health, Adelaide, Australia

\section{Introduction}

Anorexia is a significant symptom in advanced cancer. Current therapeutic options are limited.

Objectives

This multi-site, double blind, parallel arm, fixed dose phase III study compared megestrol acetate $480 \mathrm{mg}$ /day, dexamethasone $4 \mathrm{mg}$ /day and placebo for their net short-term effect on appetite and quality of life (QoL) in people with advanced cancer.

\section{Methods}

Inpatients or outpatients seeing a palliative care team with anorexia for $\geq 2$ weeks with a score $\leq 4$ on a $0-10$ numeric rating scale (NRS; $0=$ no appetite, $10=$ best possible appetite) were recruited. Participants were randomised to receive megestrol $480 \mathrm{mg}$, dexamethasone $4 \mathrm{mg}$ or placebo daily for up to 4 weeks. Primary response assessment occurred at day 7 , and responders were defined as having more than a $25 \%$ improvement in NRS compared to baseline.

\section{Results}

There were 190 people randomised (megestrol acetate $n=61$; dexamethasone $n=67$, placebo $n=62$ ). At week 1 (primary endpoint), $79 \cdot 3 \%$ of participants in the megestrol group, $65.5 \%$ in the dexamethasone group and $58.5 \%$ in the placebo group $(\mathrm{p}=0.067)$ were responders. No differences in weight, performance status or quality of life were reported. Treatment emergent adverse events occurred in the majority of participants $(90.4 \%)$, and included altered mood and insomnia. Hyperglycemia was more frequent in people on dexamethasone.

\section{Conclusions}

Although there was little difference between treatment groups for the primary or secondary effectiveness endpoints, there was a consistent trend in secondary end-points favouring megestrol acetate than dexamethasone or placebo. Sub-group analyses indicate megestrol acetate may be more effective in maintaining body weight for subjects whose appetite responded.

eP321

DIFFERENCES BETWEEN EARLY AND LATE INVOLVEMENT OF PALLIATIVE HOME CARE IN ONCOLOGY CARE: A FOCUS GROUP STUDY WITH PALLIATIVE HOME CARE TEAMS

\section{N. Dhollander ${ }^{1}$, L. Deliens ${ }^{2}$, S. Van Belle ${ }^{3}$, A. De Vleminck ${ }^{2}$, K. Pardon ${ }^{2}$ ${ }^{T}$ End-of-Life Care Research Group, Ghent University, Gent, Belgium \\ ${ }^{2}$ End-of-Life Care Research Group, Ghent University \& Vrije Universiteit Brussel VUB, Brussel, Belgium \\ ${ }^{3}$ Ghent University Hospital, Department of Medical Oncology, Ghent, Belgium}

\section{Introduction}

To date, no randomised controlled trials on the integration of specialised palliative home care (PHC) into oncology care have been identified. Information on whether existing models of integrated care are applicable to the home care system and how working procedures and skills of the palliative care teams might require adaptation is missing.

\section{Objectives}

To gain insight into differences between early and late involvement and the effect on existing working procedures and skills as perceived by $\mathrm{PHC}$ teams. Methods

Six focus groups were held with PHC teams in Flanders, Belgium. The discussions were transcribed verbatim and analysed using the method of constant comparative analysis.

Results

Differences were found concerning 1) reasons for initiation, 2) follow-up of the care, 3) focus on possibilities versus problems, 4) opportunity to provide holistic care, 5) empowerment of patients, and 6) empowerment of professional caregivers. A shift from a medical approach to a more holistic approach is the most noticeable. Being involved earlier also results in a more structured follow-up and in empowering the patient to be part of the decision-making process. Early involvement creates the need for transmural collaboration, which leads to the teams taking on more supporting and coordinating tasks.

\section{Conclusions}

Being involved earlier leads to different tasks and working procedures and to the need for transmural collaboration. Future research might focus 
on the development of an intervention model for the early integration of PHC into oncology care. To develop this model, components of existing models might need to be adapted or extended.

\section{eP322}

BARRIERS FOR THE EARLY INTEGRATION OF PALLIATIVE HOME CARE INTO THE TRAJECTORY OF ADVANCED CANCER PATIENTS: A FOCUS GROUP STUDY WITH PALLIATIVE HOME CARE TEAMS.

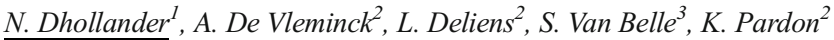
${ }^{T}$ End-of-Life Care Research Group, Ghent University, Gent, Belgium

${ }^{2}$ End-of-Life Care Research Group, Ghent University \& Vrije Universiteit Brussel VUB, Brussel, Belgium

${ }^{3}$ Ghent University Hospital, Department of Medical Oncology, Ghent, Belgium

\section{Introduction}

Even though the interest in the integration of palliative home care (PHC) in oncology care increases, $\mathrm{PHC}$ is still often involved late in the disease trajectory. Recently, some studies have been conducted exploring the barriers for early integration of PC in the hospital setting. Because PHC is organized differently compared with $\mathrm{PC}$ in a hospital setting, existing barriers for the early integration of $\mathrm{PHC}$ need further exploration.

Objectives

To identify barriers for the early integration of PHC into oncology care, as perceived by PHC teams.

Methods

Six focus groups were held with PHC teams in Flanders, Belgium. The focus group discussions were transcribed verbatim and analyzed using constant comparative analysis.

\section{Results}

Our findings confirm many barriers found in previous studies, such as the lack of available financial resources to ensure the provision of optimal integrated PC and the existing societal perception of PC as terminal care. A lack of knowledge of oncologists about the content and role of PC is also confirmed. Furthermore, our study revealed that professional caregivers working in the home context are missing important information on oncology care to provide optimal PC. Another additional barrier specific for this transmural model of integration is the discontinuity of care, as a result of a lack of communicational structure and a lack of central coordination.

\section{Conclusions}

The results of our study contribute to a better understanding of the factors hindering the provision of PHC concurrent with oncology care. For the home context, the transmural discontinuity of care seems to be an important additional barrier.

\section{eP323}

PALLIATIVE CARE, EARLY \& SYSTEMATIC (PACES) PROJECT: IMPACT ON PATIENT \& HEALTH SYSTEMS OUTCOMES

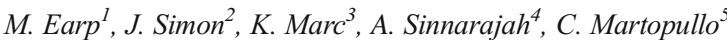 \\ ${ }^{1}$ University of Calgary, W21C, Calgary, Canada \\ ${ }^{2}$ University of Calgary, Oncology-Community Health Services, Calgary, \\ Canada \\ ${ }^{3}$ University of Calgary, Oncology, Calgary, Canada \\ ${ }^{4}$ University of Calgary, Oncology-Community Health Sciences, Calgary, Canada \\ ${ }^{5}$ Alberta Health Services, Tom Baker Cancer Centre- Psychosocial \\ Oncology, Calgary, Canada
}

\section{Introduction}

Strong evidence exists that early palliative care (PC) improves patient outcomes, and national guidelines, including Choosing Wisely Canada, recommend it.

\section{Objectives}

This knowledge translation project will study methods to adapt and implement evidence-based practices for delivering early and systematic PC at the health system level for patients with metastatic colorectal cancer (mCRC).

\section{Methods}

We have identified four gaps in providing early PC in mCRC in Alberta. We will close these gaps across the health care system by: 1) routinely identifying patients for early PC, 2) increasing the quality of patientprovider communication of care preferences by implementing the "Serious Illness Conversation Guide", 3) ensuring Temel's "five elements" of PC are consistently addressed, 4) improving access to family physicians and PC homecare services. The primary outcome is the number of patients receiving PC $\geq 3$ months before death. Secondary outcomes are patient and caregiver-focused (e.g., quality of life), systemfocused (e.g. number of days spent in hospital), and health-care professional focused (survey and focus groups of experiences).

\section{Results}

This study is in progress. Challenges have included securing project funding, developing an implementation plan that will work across the province (given resource diversity and needs by region) and engaging stakeholders across the province, avoiding change fatigue, and sustaining engagement to effect change.

\section{Conclusions}

Lessons learned are: Identify dedicated local clinical champions, get buyin from overall health system leaders, including each clinical area (e.g. Cancer Control Alberta, Community Care). Project success requires proper: funding for project personnel, process mapping and qualityimprovement methodology, and culture/change management expertise.

\section{eP324}

PALLIATIVE RADIOTHERAPY UTILISATION WITHIN A PALLIATIVE CARE UNIT1 1ANN PALLIAT MED 2017;6(SUPPL 2):S140-S146. DOI: 10.21037/APM.2017.09.07

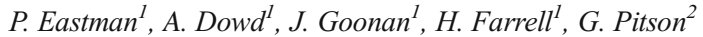 \\ ${ }^{T}$ Barwon Health, Department of Palliative Care, North Geelong, \\ Australia \\ ${ }^{2}$ Barwon Health, Department of Radiation Oncology, Geelong, Australia
}

\section{Introduction}

Palliative radiotherapy (RT) has demonstrated efficacy for symptom management in advanced malignancy however concerns exist about appropriateness in deteriorating/dying patients. There are limited data available investigating the use of RT for inpatient palliative care patients.

\section{Objectives}

Given the lack of available evidence, vulnerability of this cohort and the potential for both benefits and harms we evaluated palliative RT utilisation amongst patients admitted to our regional Australian Palliative Care Unit (PCU).

\section{Methods}

A retrospective-cohort study was undertaken incorporating all PCU inpatients between 2011 and 2016 who underwent RT. A range of clinicodemographic, radiotherapy-specific and outcome variables were collected. Opioid consumption was recorded as a surrogate for RT benefit. Comparisons in consumption were made between baseline and each of weeks one, two and three post RT.

Results

Fifty-four episodes of RT were analysed. Pain management was the commonest reason for RT treatment, with single fractions utilised in $68 \%$ of cases with this indication. Using the proportion of patients able to reduce their opioid consumption following RT as a response marker, response rates were between $32-41 \%$. The commonest discharge outcome was death with $20 \%$ and $43 \%$ of patients dying within 14 and 30 days of radiotherapy respectively. Median survival was 36 days. 


\section{Conclusions}

A small proportion of all PCU patients received RT with almost half dying during their admission. Response rates were lower than have been previously reported however this likely reflects, at least in part, issues using opioid consumption as response marker. Ongoing research is required to optimise the stratification of PCU inpatients to RT.

\section{eP325 \\ PROGNOSTIC FACTORS IN ADVANCED DISEASE - HOW TO SUPPORT DECISION-MAKING}

F. Ferreira ${ }^{l}$, J. Pereira ${ }^{l}$, L. Fernandes ${ }^{I}$, A.J. Pissarra ${ }^{l}$, L. Matos ${ }^{l}, J$.

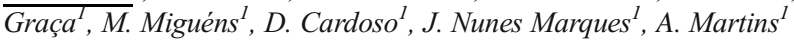

${ }^{1}$ Hospital São Francisco Xavier - Centro Hospitalar de Lisboa Ocidental, Medical Oncology, Lisboa, Portugal

\section{Introduction}

In Oncology is essential to recognise the end-of-life period, but there is lack of validated prognostic scales to help the management of the patient. Clinical prediction of survival (CPS), performance status (PS), some signs/symptoms and analytic factors showed prognostic value. There are two validated scores: Palliative Prognostic Score (PPP) and Palliative Prognostic Index (PPI).

\section{Objectives}

Identification of prognostic factors in end-stage disease (ESD) for predicting the survival and indication to initiate specific oncologic treatment (SOT).

Methods

Retrospective observational study including the patients admitted in a Portuguese Oncology department for one year, evaluation of patient/ disease characteristics and aferition of prognostic factos.

Results

From the 565 patients included, $37.5 \%$ had metastatic or locally advanced/irresectable disease. The main primary tumours were colon, prostate and pancreas. $38 \%$ died in the 6 months after the first appointment, 9 of them being submitted to chemotherapy in this period. More than $90 \%$ of the patients who didn't initiate SOT for clinician decision (38\%) died in the first year.

The prognostic value of various factors was evaluated: age, PS, number of comorbidities and previous medication, number of metastatic sites, symptomatic burden, analytic alterations and CPS. PPP and PPI were also calculated. Conclusions

PS and CPS showed to be the more relevant prognostic factors. $84 \%$ of the patients with $P S \geq 2$ died in the first year and only 6 of them initiated chemotherapy. The search for ways of help decision making in ESD is essential, as the failure to do it can have important clinical, economic and moral implications.

\section{eP326}

INTEGRATED PALLIATIVE CARE IN LUNG CANCER AT OUTPATIENT CLINIC. CLINICAL AND ADMINISTRATIVE OUTCOMES

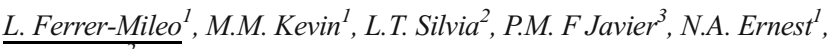 G.B. Jesús \\ ${ }^{1}$ Institut Català d'Oncologia, Medical oncology, Hospitalet Llobregat, Spain \\ ${ }^{2}$ Institut Català d'Oncologia, Palliative Care Department, Hospitalet Llobregat, Spain \\ ${ }^{3}$ Institut Català d'Oncologia, Clinical Research Unit, Hospitalet Llobregat, Spain}

\section{Introduction}

Lung cancer (LC) is one of the worldwide most common tumors, presenting a substantial symptom burden. Several studies describe that integrated early palliative intervention with standard oncological treatments improves symptom control, quality of life and life-expectancy.

\section{Objectives}

To compare clinical-administrative characteristics between early palliative care intervention (EPCI) and standard referral (SR) in patients diagnosed with LC at the Lung Cancer Clinic.

\section{Methods}

In this observational retrospective study, data were extracted from electronic records.. Eligible criteria to early intervention: $<3$ months LC diagnosis with SCLC stage III-IV, NSCLC and poor pain control with strong opioids, emotional distress, substance abuse, family frailty, ethical dilemma or poor performance status (Barthel $\leq 60$ ).

\section{Results}

From June to November 2016, 80 patients were selected. 34 patients were EPCI and 46 were SR. No differences in age, gender, pathology were observed. Median time from first oncology visit to palliative care (PC) visit was 29.45days (d) and $604 d(p<0.05)$, respectively. Higher burden of symptoms and emotional distress was objective in EPCI group. Although these patients needed more frequent follow-up visits ( $21 \mathrm{vs} 28 \mathrm{~d}$, respectively, $\mathrm{p}<0.05)$, there were no differences in emergency room visits or hospital admissions. PC improved symptoms control, except for asthenia and dyspnea, and reduced anxiety and sadness in both groups. Overall survival was $245 \mathrm{~d}$ vs $827 \mathrm{~d}(\mathrm{p}<0.05)$, respectively. However, lifeexpectancy after first visit in PC was longer (15d) for EPCI than SR, with no statistical differences.

\section{Conclusions}

Integrated palliative care in a LC Clinic might improve symptom management and emotional suffering in patients with advanced lung cancer.

\section{eP327 \\ FOUR-MONTH FEASIBILITY STUDY OF ELECTRONIC PATIENT-REPORTED SYMPTOMS DATA CAPTURE AND EXTRACTION: UNIVERSITY OF ROCHESTER NATIONAL CANCER INSTITUTE COMMUNITY ONCOLOGY RESEARCH PROGRAM}

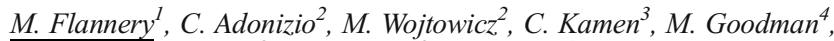
S. Obrecht ${ }^{4}$, C. Seiler ${ }^{2}$, A. Kleckner ${ }^{3}$, C. Pandya ${ }^{5}$, S. Mohile ${ }^{4}$

${ }^{1}$ University of Rochester, School of Nursing, Rochester, USA

${ }^{2}$ Geisinger Cancer Institute, Cancer Institute, Danville, USA

${ }^{3}$ University of Rochester, Surgery, Rochester, USA

${ }^{4}$ University of Rochester, Cancer Center, Rochester, USA

${ }^{5}$ University of Rochester, Population Health Management, Rochester, USA

\section{Introduction}

In oncology, research has demonstrated that routine electronic collection of patient-reported symptoms improves symptom relief, quality of life, and survival. However, widespread electronic collection of patientreported outcomes (PROs) is minimal due to difficulties with implementation and extraction of data to inform clinical care.

\section{Objectives}

1) to establish the feasibility of electronic capture of symptoms during routine clinical operations, 2) to extract relevant patient outcomes from the electronic medical record (EMR) and 3) identify challenges.

\section{Methods}

At Site1, symptom collection via EMRs was in place and, at Site2, symptom collection was initiated. A minimum data set for symptoms and outcome measures was established. For Aim2, a data dictionary and program code was developed for EMR extraction of demographics, tumor and treatment characteristics and health care utilization measures.

\section{Results}

Over four months, at Site1, 154/579 (27\%) of symptom surveys were completed and at Site2, 114/646 (18\%) of symptom surveys were completed. For Aim2, extraction was $100 \%$ successful for $21 / 30(70 \%)$ 
variables at both sites (e.g., demographic, treatment data, utilization variables), partially successful for some variables (e.g., staging, referrals) and unsuccessful for 2 variables (e.g., pathology). The largest barriers included challenges to changing standard practice (by patients, providers and staff) and to establishing a new integrated workflow.

Conclusions

Symptom survey completion rate was low at both sites, demonstrating that significant resources are required to implement routine symptom collection with the EMR and overcome barriers. Extracting data from the EMR was highly successful.

\section{eP328}

A SINGLE INSTITUTIONAL EXPERIENCE ON WHOLE BRAIN RADIOTHERAPY AS PRIMARY TREATMENT FOR BRAIN METASTASIS: A RETROSPECTIVE ANALYSIS OF PATIENT DEMOGRAPHICS, PROGNOSTIC INDICES AND TREATMENT-RELATED OUTCOMES

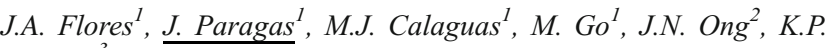 \\ Ferraris $^{3}$ \\ ${ }^{1}$ Jose R. Reyes Memorial Medical Center, Radiotherapy, Manila, \\ Philippines \\ ${ }^{2}$ Jose R. Reyes Memorial Medical Center, Neurology, Manila, Philippines \\ ${ }^{3}$ Jose R. Reyes Memorial Medical Center, Neurosurgery, Manila, \\ Philippines
}

\section{Introduction}

Brain metastasis represents an increasingly critical problem among cancer survivors, and in low-resource centers, most are still treated with whole brain radiotherapy (WBRT).

\section{Objectives}

The purpose of this study is to provide an awareness regarding patient demographics, prognostic model indexes and treatment-related outcomes among patients treated with WBRT in a government hospital in a lowmiddle income country (LMIC) like the Philippines

\section{Methods}

Records of 86 patients who were treated with WBRT were retrospectively reviewed from June 2012 to October 2017

\section{Results}

Most patients received a total dose of 30 Gy (10 fractions). Breast represented the most common primary site $(40 \%)$ in which majority (73\%) have 1-3 brain metastases, located in the cerebral cortices $(85 \%)$. Headache $(39 \%)$ is the primary symptom of patients to undergo WBRT. Most were classified as RTOG RPA Class II. The median survival (MS) time among patients who completed the WBRT was 93 days (range 3-240). Among the different factors, only GPA showed statistical significance with the MS (p-value: 0.02). MS was positively correlated with GPA scores but negatively correlated with the RPA scores. Breast was noted to have the best MS (73.9 days); unknown primary had the poorest MS (34.6). The total dose of 30 Gy WBRT had the best MS (93) compared to the higher 40 Gy with MS of 64 days.

Conclusions

WBRT has been a primary management for brain metastasis in LMIC like the Philippines. Different factors affect the median survival of these patients. Goals of treatment focus more on palliation and improvement of quality of life.

\section{eP329 \\ COMFORT CARE FOR A HEPATOCELLULAR CARCINOMA PATIENT IN HOSPICE WARD}

M. Gamea ${ }^{I}$

${ }^{T}$ Gharbia Cancer Society, Medical Oncology, Tanta, Egypt

\section{Introduction}

Cancer is the major cause of illness and mortality in Egypt . It is noteworthy that the psychosocial and spiritual needs of patients with terminal cancer patients often are neglected by families and healthcare providers in Egypt Objectives

The purpose of this study was to apply the Comfort Theory to explore the experience on comfort care of a patient with terminal HCC near the end of life in hospice ward

\section{Methods}

Data were collected and evaluated based on Kolcaba's theory of comfort. It directs nurses to assess physical, psycho-spiritual, sociocultural and environmental comfort needs of the patient; design holistic interventions to meet those needs; and measure the effectiveness of interventions to enhance the patient's comfort

Results

Four contexts of a terminal hepatocellular carcinoma patient experience are as follows: (a) Physical: abdominal distention resulted from ascites deterioration. (b) Psycho-spiritual: Fear of death anxiety resulted from the uncertainty of life-threatening. (c) Sociocultural: Altered family process resulted from family worry and conceals the patient's condition. (d) Environmental: Un-homelike setting related to the limitation of hospital hardware

\section{Conclusions}

The caring experience of this study provide important information to health professionals while caring terminal cancer patients based on their comfort needs. Health professionals could design an individualised care plan, establish a trusting relationship, control symptoms, provide complementary medicine, reconnect family relationship and create a homelike atmosphere to meet patients' needs. The study will help health professionals to perceive psychosocial and spiritual needs and properly provide support for terminal cancer patients

\section{eP330}

\section{PREDICTION OF SURVIVAL WITH THE CHANGING PATTERN OF VITAL SIGN IN TERMINALLY ILL CANCER PATIENTS}

S.I. Go ${ }^{I}$, J.H. Kang $^{2}$

${ }^{T}$ Gyeongsang National University Changwon Hospital- Gyeongsang National University School of Medicine, Internal Medicine, Changwonsi, Republic of Korea

${ }^{2}$ Gyeongsang National University Changwon Hospital- Gyeongsang National University School of Medicine, Internal Medicine, Jinju-si, Republic of Korea

\section{Introduction}

Prediction of impending death is of great concern to terminally ill cancer patients. Several prediction tools developed previously are rather complex to use by health care providers.

\section{Objectives}

To assess the clinical efficacy of the changing pattern of vital sign to predict survival in this population.

\section{Methods}

We retrospectively assessed all patients who died of advanced cancer from 2009 to 2013 and who were not treated with chemotherapy during the last 6 weeks of life. Patient's vital signs during the last 8 days of life were reviewed and analyzed for its association with death.

\section{Results}

A total of 1,357 patients were included. Mean systolic (SBP) and diastolic blood pressure (DBP) abruptly decreased during the last day of life. Mean respiratory rate $(\mathrm{RR})$ apparently increased since 2 days before death. Mean heart rate (HR) consistently increased and there was small difference of mean body temperature (BT) during the last 8 days of life. Based on the difference amounts of each vital sign, we defined the "vital sign risk" to die within 3 days according to the following risks: SBP decrease $\geq 10 \mathrm{mmHg}$, DBP decrease $\geq 10 \mathrm{mmHg}$, HR increase $\geq 10 / \mathrm{min}$, RR increase $\geq 5 / \mathrm{min}$, and 
BT increase $\geq 0.5^{\circ} \mathrm{C}$. The matched odds ratios for death within 3 days were $6.5,11.1,5.9,14.8$, and 3.4 in each vital sign risk compared with their counterparts. The positive predictive values for death within 3 days were $90.2 \%, 92.7 \%, 89.9 \%, 94.2 \%$, and $80.4 \%$, respectively.

\section{Conclusions}

The changing pattern of vital sign is useful to predict survival in terminally ill cancer patients.

\section{eP331 \\ PALLIATIVE CARE IN EGYPT: THE EXPERIENCE OF THE GHARBIAH CANCER SOCIETY}

M. Hablas $^{1}$

${ }^{T}$ Gharbia Cancer Society, Palliative Care, Tanta, Egypt

\section{Introduction}

The need for palliative care in middle and low resources countries, including Egypt, is emerging .TheGharbiahCancerSociety(GCS) is a nonprofit, nongovernmental hospital, located in Tanta, the Capital of the Gharbiah governorate in the mid-NileDelta. The Society provides acute care to patients with cancer including surgery,chemo-and radiotherapy. Review of 9year-data of Gharbiah population-based cancer registry from 1999to2007 revealed 3480 cancer cases/year, with AgeStandardizedRate(ASR) of 161.7/100,000 for males\&120.8/100.000 for females.

Objectives

About $70 \%$ of cases present in advanced stages(III\&IV) with liver cancer the most frequent cancer in male and breast cancer as the most frequent cancer in females. TheGCS started a comprehensive palliative care services in April2011 with 10-bed inpatient unit and 6days/week outpatient clinic. All palliative-care equipment were provided by public donations. Methods

Through collaboration with NationalCancerInstitute,Bethesda,Maryland and the SanDiegoHospice and the InstituteforPalliativeMedicine and MiddleEastCancerConsortium, a fellowship training program was developed for a medical-oncologist in palliative-medicine and End-of-LifeCare training course for nurses.

\section{Results}

The program succeeded in convincing local health authorities to increase the recommended opioids dose and to allow more physicians to prescribe opioids for cancer pain. In a period of 24 months, symptom management and palliative care were provided to 195 patients with advanced malignancies. The opioids consumption was increased by 30 folds.

\section{Conclusions}

The Major challenges for the program were inadequate public and healthprofessionals awareness of PalliativeCare services and lack of vehicles and finances to cover home visits. The initial results of the program warrant allocating more resources for coverage of a large number of trainees and instituting a home visits program.

\section{eP332}

CONCURRENT USE OF OPIOIDS AND BENZODIAZEPINES OR NON-BENZODIAZEPINES SEDATIVE HYPNOTICS AMONG CANCER PATIENTS REFERRED TO OUTPATIENT SUPPORTIVE CARE CENTER

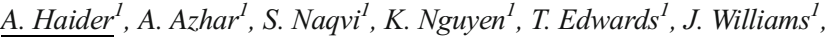 \\ P. Minjeong ${ }^{I}$, D. Liu' ${ }^{1}$, E. Bruera ${ }^{1}$ \\ ${ }^{I}$ The University of Texas M. D. Anderson Cancer Center, Palliative- \\ Rehabilitation and Integrative Medicine, Houston, USA
}

\section{Introduction}

Benzodiazepines (BZD) and non-benzodiazepines sedative hypnotics $(\mathrm{S} / \mathrm{H})$ have the potential for sedation and respiratory depression when prescribed concurrently with opioids.

\section{Objectives}

To evaluate the frequency and trend of concurrent use of opioids with BZD or S/H among patients referred to outpatient supportive care center. To explore the demographic and clinical predictors of concurrent use.

\section{Methods}

This is a retrospective review of 2000 randomly selected patients seen as first consultation between January 1, 2009 to December 31, 2016. Eligible patients had a diagnosis of cancer, 18 years or older and on opioids. We compared 300 randomly selected patients with concurrent use of opioids and BZD or S/H with 300 randomly selected patients on opioid only. We also collected data at first follow-up visit among eligible patients. Descriptive statistics and Wilcoxon rank sum test were utilized. Results

96 out of 221 (43\%) patients were on concurrent BZD or S/H during 2011 Vs 67 out of $217(31 \%)$ in $2016(\mathrm{P}=.028)$. Out of 600 patients reviewed for secondary analysis; Median Morphine Equivalent Daily Dose (MEDD) was statistically higher among patients with concurrent use of opioid with BZD or S/H $(75 \mathrm{mg} /$ day IQR $[40,145]$ versus opioid only group $(60 \mathrm{mg} /$ day IQR $[30,150]$; $\mathrm{P}=0.009)$ upon referral. At first follow up, patients among concurrent use group; half 142 (47\%) stopped BZD or $\mathrm{S} / \mathrm{H}$.

\section{Conclusions}

Concurrent use of opioids and BZD or $\mathrm{S} / \mathrm{H}$ has declined in recent years. Concurrent use group received a higher MEDD. After one supportive care consult; half of the patients successfully discontinued $\mathrm{BZD}$ or $\mathrm{S} / \mathrm{H}$

\section{eP333}

ASSESSMENT OF END OF LIFE PRACTICES IN A LARGE ONCOLOGIC INTENSIVE CARE UNIT

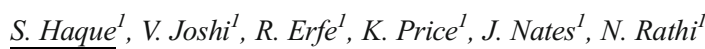

${ }^{T} U T M D$ Anderson Cancer Center, Department of Critical Care, Houston, USA

\section{Introduction}

Annually, the cost of treating intensive care unit (ICU) patients approaches $\$ 180,000,000,000$ in America. Timely end of life (EOL) decision-making is crucial to minimize patient suffering and limit costs. Objectives

This study examines when EOL decisions are made and which health care providers are making these decisions.

\section{Methods}

All admissions to our Medical ICU (MICU) over an 18 month period were retrospectively reviewed. The number of admissions admitted to the ICU with a do-not-resuscitate (DNR) or comfort care (CC) status, the number made DNR or CC during their ICU stay, and the number whose code status changed during the first 24 and 48 hours of ICU admission were determined. Time to change and involved provider were also noted.

\section{Results}

Among 2231 admissions to the MICU during the study period, 13 patients came with a DNR status and 813 were made DNR during their ICU stay. Of these, 439 cases changed code status within 24 hours and 541 within 48 hours of admission. The critical care team was responsible for $55.5 \%$ of the DNR status changes. No patients came to the ICU with a CC status. A total of 395 were made comfort care during their ICU stay; 39 cases within 24 hours and 110 within 48 hours of ICU admission. The critical care team was responsible for transition to $\mathrm{CC}$ in $90.1 \%$.

\section{Conclusions}

Changes in code status are frequently made within a day of ICU admission, suggesting that EOL decision making earlier in the hospital stay may decrease patient suffering and unnecessary healthcare costs. 


\section{eP334}

FACTORS AFFECTING LESBIAN, GAY, BISEXUAL, TRANSGENDER AND QUESTIONING PATIENTS' EXPERIENCES IN CANCER-RELATED PALLIATIVE CARE: A SCOPING REIVEW OF THE LITERATURE

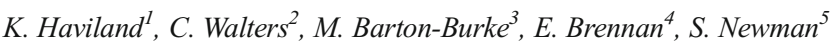
${ }^{T}$ Memorial Sloan Kettering Cancer Center, Supportive Care, New York, USA

${ }^{2}$ Memorial Sloan Kettering Cancer Center, Director- Patient \& Caregiver Engagement, New York, USA

${ }^{3}$ Memorial Sloan Kettering Cancer Center, Director of Nursing Research, New York, USA

${ }^{4}$ Medical University of South Carolina, Library, Charleston, USA

${ }^{5}$ Medical University of South Carolina, Nursing, Charleston, USA

\section{Introduction}

Discrimination, refusal of care, bias and derogatory statements towards lesbian, gay, bisexual, transgender and questioning (LGBTQ) persons by healthcare practitioners (HCP's) not identified at LGBTQ friendly creates fear of accessing healthcare. Higher rates of obesity, substance abuse, and incidences of anal, breast, cervical, colorectal, endometrial, lung and prostate cancer have been documented. Current healthcare curriculums do not prepare HCPs to provide sensitive palliative care to this population.

Objectives

To explore what is known from the existing literature about the barriers to providing cancer-related palliative care for the LGBTQ patient.

Methods

The Social Ecological Method was used to guide an analysis of existing literature in a five-stage framework using the following databases without restrictions: PubMed, Scopus, PsychINFO and Cochrane. Primary levels of interest included intrapersonal, interpersonal, institutional/organizational, historical/society/cultural and global experiences.

\section{Results}

A total of 1,372 citations were identified, 894 remained after duplicates were removed. Twenty-three met inclusion criteria, 2 additional manuscripts were identified by hand-searching and after review, 17 were chosen. Barriers to palliative cancer-care included discrimination, criminalization, persecution, fear, distress, social isolation, fear of aging, disenfranchised grief, bereavement and tacit acknowledgement. Limited knowledge, poor preparation of legal aspects of advanced care planning and end-of-life were identified as under-provided.

\section{Conclusions}

As a result of these barriers, current palliative cancer-care for LGBTQ patients is deficient; perpetuating marginalization and healthcare inequities. A gap in the literature exists exploring two-way perceptions between practitioners and LGBTQ patients. It is essential to collect clinical data to better understand the needs for LGBTQ palliative cancer care.

\section{eP335}

DEVELOPEMNT AND AUDIT OF PATHWAY TO GUIDE END OF LIFE CARE PROVISION FOR STEP DOWN PATIENTS FROM CRITICAL CARE COMPLEX (CCC) TO WARD BASED CARE

\author{
N. Holtom ${ }^{1}$, D. Shrimpton ${ }^{2}$, S. Hlaing ${ }^{2}$, S. Hutchinson ${ }^{3}$, T. Leary $^{4}$ \\ ${ }^{T}$ Palliative Medicine Consultant, Norfolk and Norwich University NHS \\ Foundation Trust, Norwich, United Kingdom \\ ${ }^{2}$ Norfolk and Norwich University NHS Foundation Trust, Palliative \\ Medicine, Norwich, United Kingdom \\ ${ }^{3}$ Norfolk and Norwich University NHS Trust, Critical Care Complex, \\ Norwich, United Kingdom \\ ${ }^{4}$ Norfolk and Norwich University NHS Foundation Trust, Critical Care \\ Complex, Norwich, United Kingdom
}

\section{Introduction}

Concern that patients being moved from the critical care complex (CCC) to ward based care were not always having their holistic palliative care needs assessed prompted the development of a pathway to guide management Objectives

To ensure that this patient group had their holistic palliative care needs assessed and effectively managed by a Palliative Medicine Physician prior to ward transfer and ongoing care and support was formally transferred to the palliative care consultants.

\section{Methods}

Reflective review of a case study, where seizures were poorly controlled in a patient with anoxic brain injury who was transferred to ward based care without a timely referral to palliative care, prompted the development of this pathway in conjunction with CCC colleagues. Following implementation of the pathway all end of life patients transferred from $\mathrm{CCC}$ to ward based care during 2017 were audited

Results

Establishment of 7 day palliative care service enabled a rapid response service to CCC to be developed. All end of life patients were assessed in $\mathrm{CCC}$ and formal handover made to palliative care physicians. Guidance was provided to ensure that all symptoms were anticipated proactively and that a subcutaneous syringe driver was started prior to withdrawal of treatment to ensure that patient's comfort was maintained. Relatives were introduced to the palliative care physician as a point of contact

Conclusions

Use of this pathway has demonstrated that the needs of this patient group are now proactively and effectively managed and that ongoing support for relatives and carers is always continued by the palliative care team.

\section{eP336}

THE END-OF-LIFE CARE EXPERIENCE OF A TERMINAL OVARIAN CANCER PATIENT WITH HOSPICE CARE

T.H. Hsu ${ }^{l}$

${ }^{1}$ Shin Kong Wu HO-SU Memorial Hospital, Nursing Department, Taipei, Taiwan R.O.C.

\section{Introduction}

An 48 years old woman was diagnosed with ovarian cancer in 2014 and received regular chemotherapy. Unfortunately, Liver and Colon metastasis were found in January 2017 with symptoms of pain, ascites, jaundice, edema and other symptoms. She divorced after giving birth to her daughter and her daughter was raised by her ex-husband. She silently cared for her daughter but never met her. She did not have family members during hospitalization and she felt very lonely and hopeless. The most worried about is her daughter, she wanted to apologize to her because she thought she did not being a good mother, which is the greatest regret in her life. Objectives

The purpose of this article was to present an end-of-life hospice care experience with a middle-aged woman who had a diagnosis of terminal ovarian cancer.

\section{Methods}

After establishing a good relationship with the patient and having many meetings with the medical team, she signed a DNR consent form and accepted hospice care . Assisted by doctors and nurses, social workers, psychologists and clinical Buddhist chaplain and other hospice-palliative multi-disciplinary interventions to help alleviate the physical discomfort and encourage her to express her wish to meet her and thank each other and apologize with the case Under the precious and beautiful memory, the case also shows that life has no regrets and died in peace in three weeks. Results

The patient finally passed away in the company of her daughter in a dignified, peaceful and painless manner.

\section{Conclusions}

A professional multi-disciplinary hospice team is essential. 


\section{eP337}

PATTERN AND PREDICTORS OF OUTPATIENT PALLIATIVE CARE REFERRAL AMONG THORACIC MEDICAL ONCOLOGISTS

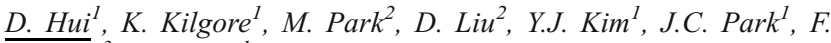
Fossella ${ }^{3}$, E. Bruera ${ }^{1}$

${ }^{1}$ University of Texas MD Anderson Cancer Center, Palliative CareRehabilitation and Integrative Medicine, Houston, USA

${ }^{2}$ University of Texas MD Anderson Cancer Center, Biostatistics, Houston, USA

${ }^{3}$ University of Texas MD Anderson Cancer Center, Thoracic Medical Oncology, Houston, USA

\section{Introduction}

There is significant variation in access to palliative care.

Objectives

We examined the pattern of outpatient palliative care referral among thoracic medical oncologists and identified oncologist characteristics associated with greater referral.

Methods

We retrieved data on all patients who died of advanced thoracic malignancies at our institution between January 1, 2007 and December 31, 2012. Using median as a cutoff, we defined two groups (high-referring and low-referring oncologists) based on their frequency of referral. We examined various oncologist and patient-related characteristics associated with outpatient referral.

\section{Results}

444 of 1642 decedents $(27 \%$ ) had an outpatient palliative care referral. The median proportion of referral among 26 thoracic oncologists was $30 \%$ (range $9 \%-45 \%$; median proportion of high-referring $37 \%$ vs. lowreferring $24 \%$ when divided into two groups at median). High-referring oncologists were significantly younger (age 45 vs. $56, \mathrm{P}=0.003$ ) than lowreferring oncologists; they were also significantly more likely to refer patients earlier (median interval between oncology consultation and palliative care consultation 90 days vs. 170 days, $\mathrm{P}=0.003$ ) and those without metastatic disease ( $7 \%$ vs. $2 \%, \mathrm{P}=0.004)$. In multivariable mixed-effect logistic regression, younger oncologists (odds ratio $[\mathrm{OR}]=0.97$ per year increase, 95\% CI $0.95-0.995 ; \mathrm{P}=0.02)$, younger patients $(\mathrm{OR}=0.98$ per year increase, 95\% CI 0.97-0.99; $\mathrm{P}=0.001)$ and non-metastatic disease status $(\mathrm{OR}=0.48,95 \%$ CI $0.29-0.78 ; \mathrm{P}=0.008)$ were significantly associated with outpatient palliative care referral.

\section{Conclusions}

The pattern of referral to outpatient palliative care varied widely among thoracic oncologists. Younger oncologists were not only referring a higher proportion of patients, but also earlier in the disease trajectory.

\section{eP338}

THE MINIMAL CLINICALLY IMPORTANT DIFFERENCE OF THE RICHMOND AGITATION-SEDATION SCALE IN CANCER PATIENTS WITH AGITATED DELIRIUM

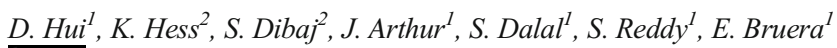
${ }^{T}$ University of Texas MD Anderson Cancer Center, Palliative CareRehabilitation and Integrative Medicine, Houston, USA

${ }^{2}$ University of Texas MD Anderson Cancer Center, Biostatistics, Houston, USA

\section{Introduction}

The Richmond Agitation-Sedation Scale (RASS) is commonly used to assess psychomotor activity; however, its minimal clinically important difference (MCID) has not been determined.

Objectives

The objective of this study is to identify the MCID for RASS using two anchor-based approaches.

\section{Methods}

This is a secondary analysis of a randomized controlled trial to compare the effect of lorazepam versus placebo as an adjuvant to haloperidol for persistent agitation in patients with delirium (Hui et al. JAMA 2017). The primary outcome was change in RASS (10-point numeric rating scale ranging from -5 [unarousable] to +4 [combative]) from baseline to 8 hours after treatment administration. The sensitivity-specificity and within-patient change methods were used to identify the MCID, with patient comfort after study intervention as perceived by caregivers and nurses being the anchors.

\section{Results}

90 patients were randomized and $58(64 \%)$ received the study medication for restless/agitation (mean baseline RASS 1.6). 23 (61\%) caregivers and $23(55 \%)$ of nurses perceived that the patient was more comfortable after treatment. Using the sensitivity-specificity method, the optimal RASS reduction was $\geq 4$-points according to both caregivers (sensitivity $61 \%$, specificity $80 \%$; area under the curve [AUC] 0.71 ) and nurses (sensitivity $73 \%$, specificity $84 \%$; AUC 0.78 ). The RASS cutoff based on withinpatient change method was similar ( -4.2 for caregivers; -4.0 for nurses).

\section{Conclusions}

For patients with persistent restlessness/agitation, a reduction of $\geq 4$ points in RASS was considered to be the MCID for both nurses and caregivers. These findings may have implications for sample size calculation and interpretation of treatment effect in future delirium trials.

\section{eP339 \\ QUALITY OF LIFE (QOL) IN SURVIVORS OF ADULT HAEMATOLOGICAL MALIGNANCY}

\author{
A. Immanuel $^{1}$, J. Hunt ${ }^{2}$, E. van Teijlingen ${ }^{2}, H$. McCarthy ${ }^{3}$ \\ ${ }^{T}$ Bournemouth University, Faculty of Health \& Social Sciences, \\ Christchurch, United Kingdom \\ ${ }^{2}$ Bournemouth University, Faculty of Health \& Social Sciences, \\ Bournemouth, United Kingdom \\ ${ }^{3}$ Royal Bournemouth Hospital, Haematology, Bournemouth, United \\ Kingdom
}

\section{Introduction}

The treatment for haematological malignancies is lengthy, complex and intense, leading to potentially debilitating symptoms and reduced health-related quality of life. Survivors can suffer long-term effects of both treatment and disease. The presentation is part of a mixed methods study and aims to deepen our understanding of the quality of lives of survivors of adult haematological malignancy and identify unmet needs. The study will contribute to the underresearched topic related to adult survivors of a haematological malignancy.

\section{Objectives}

The qualitative phase aims to explore in depth and improve understanding of the quantitative findings related to the quality of life among adults who have survived a hematological malignancy.

\section{Methods}

The participants $(\mathrm{n}=11)$ were adults who had completed treatment for a haematological malignancy and were between 1-5 years post treatment. A qualitative research process of inquiry was used to explore the quality of lives of survivors of haematological malignancy and identify unmet supportive care needs. In-depth face-to-face semi-structured interviews were conducted, based on the content and structure of the interviews envisaged to be grounded in the findings of the quantitative phase of the study.

\section{Results}

Four themes were identified, including physical wellbeing, psychological wellbeing, independence and unmet supportive care needs.

\section{Conclusions}

Survivors of haematological malignancies have unmet supportive care needs. Enhancing their physical, psychological wellbeing and addressing supportive care needs optimises their QoL. Knowledge and understanding of these factors may provide an insight into implementation measures to enhance the same. 
eP340

PALLIATIVE QUAD SHOT RADIOTHERAPY IN ADVANCED HEAD AND NECK CANCER (HNC) - AN ALTERNATIVE REGIMEN IN LOW RESOURCE COUNTRIES

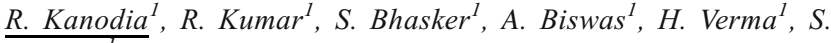 \\ Chander \\ ${ }^{1}$ All India Institute of Medical Sciences- New Delhi, RADIATION \\ ONCOLOGY, Ansari Nagar- New Delhi, India
}

\section{Introduction}

$\mathrm{HNC}$ is a major cancer burden in developing countries, especially in Southeast Asia, with nearly two-third presenting in advanced stages having dismal prognosis. We present a prospective study on palliative QUAD SHOT radiotherapy (RT) in advanced HNC patients.

Objectives

To evaluate symptom relief and tumor response in advanced HNC patients treated with QUAD-SHOT Radiotherapy.

Methods

Among 36 patients, stage IV-A and IV-B comprised 16 and 20 patients respectively. Palliative QUAD SHOT RT delivers 14 Gy in 4 fractions twice daily 6 hours apart on 2 consecutive days. The regimen was designed till 3 cycles given every 4 weekly if tumor did not progress.

\section{Results}

Median age of the group was 58 yrs with ECOG PS 2. Most common sites were oral cavity $(66.6 \%)$, oropharynx $(19.5 \%)$ and larynx (13.9\%). Mean duration of symptoms was 7.5 months with Pain (100\%), dysphagia (83.3\%) and neck swelling (75\%) being major symptoms. 23 patients $(63.9 \%)$ received $\geq 2$ cycles of QUAD SHOT RT of which 14 patients (38.9\%) completed three cycles. At 1 month post first cycle of QUAD SHOT RT, 23 patients had partial response while one patient each had stable and progressive disease. None had $\geq$ grade 2 dermatitis and mucositis. Mean PFS who received $\geq 2$ cycles of QUAD SHOT RT was 5.5 months.

Conclusions

Palliative QUAD SHOT RT is good alternative option in advanced HNC patients. It produces quick symptomatic relief in short duration of treatment with minimal side effects, thus can be employed effectively in areas with high patient burden in limited resource countries.

\section{eP341}

\section{DELIVERING OUTCOMES FROM A JOINT SUPPORTIVE} CARE AND ACUTE ONCOLOGY SERVICE

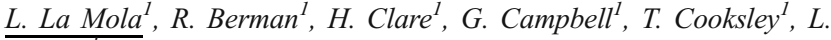 \\ Hacker \\ ${ }^{1}$ The Christie NHS Foundation Trust, Supportive Care, Manchester, \\ United Kingdom
}

\section{Introduction}

Enhanced supportive care (ESC) has transformed existing palliative care services in cancer centres.

Joint working with oncologists, rebranding of palliative care and provision of a rapid access clinic delivered jointly by palliative care and acute oncology have all proved beneficial in preventing crises leading to admission, ultimately improving patient satisfaction whilst delivering cost savings.

Objectives

Initially our aims were to remove barriers to palliative care, provide patients with the right information to allow them to make the most appropriate decisions about their treatment and enhance communication between primary and secondary care.

Methods

We rebranded "palliative care" to "supportive care".

Supportive care services were embedded within 3 oncology disease groups to enhance practice of all team members and provide specialist support for patients developing problems at a much earlier stage.
Audit and collection of patient and oncologist feedback was conducted throughout.

Phase 2 widened the scope of the service. We developed a "rapid access clinic" in collaboration with acute oncology to better address the problems faced by any cancer patient or cancer survivor without delay.

\section{Results}

Phase 1 demonstrated almost 600 avoided admissions over a three year period, reducing emergency admissions by more than $20 \%$.

A 6 month pilot of Phase 2 demonstrated 49 prevented admissions, 59 patients discharged early from hospital, and 101 patients with enhanced symptom control.

NHS England adopted this initiative and phase 1 is currently being rolled out as a CQUIN in 23 cancer centres nationwide.

\section{Conclusions}

Enhanced supportive care can positively impact on emergency admissions and patient outcomes

\section{eP342}

THE ROLE OF THE SPIRITUAL CARE AS AN ESSENTIAL ASPECT OF PALLIATIVE CARE

N. Lalic $^{1}$

${ }^{T}$ Institue for Pulmonary Diseases of Vojvodina, Department for pulmonary oncology, Novi Sad, Serbia

\section{Introduction}

The spiritual dimension of patients at the end of life manifests itself through questions such as "Why me?", "What happens when I die ?", "Is there meaning in life?" and is often ignored by clinicians fearful of making things worse. It is necessary to provide a holistic approach to help promote a collaborative process in attending to the various physical, psychological, social, and spiritual needs of patients at the end of their lives.

Objectives

The aim of study was to check does the oncological patient need the help of a spiritual person in the treatment? Does the therapeutic team of palliative care require the help of a spiritual person?

\section{Methods}

200 patients with lung carcinoma who started treatment in 2016 were divided according to the gender, school schedule, types of religion and to the stage of the disease.

Results

From 20 questions "After you become ill, do you consider that a priest can take part in the treatment for oncological patients?" Men responded positively with $38.9 \%$ and women with $71.6 \%$, ( $<<0.001)$. When asked if "Do you think that a priest can participate in the treatment of oncological patients?" Patients with a stage of the disease to IIIA (<IIIA) responded positively with $24.4 \%$, stage IIIB and IV (IIIB +) of the disease with $71.1 \%(\mathrm{P}<0.001)$.

\section{Conclusions}

The palliative care staff must recognize a patients spiritual and existential concerns, especially for patients in the advanced phase of the disease and they should be supported by the spiritual care specialist on the team.

\section{eP343 \\ CONCERNS ABOUT USING PALLIATION SEDATION THERAPY AND LEVELS OF GRIEF OF FAMILY MEMBERS OF TERMINAL AND CRITICAL PATIENTS}

C.C. Lin

${ }^{T}$ The University of Hong Kong, School of Nursing, Pok Fu Lam, Hong Kong S.A.R.

\section{Introduction}

Using palliative sedation therapy (PST) in the intensive care unit is a common and routine medical procedure. But it may cause profound family members' distress, even affect their levels of grief. 


\section{Objectives}

To identify the concerns of family members and to delve into the influence on family member's grief, as exerted by the use of PST on terminally and critically ill patients.

\section{Methods}

62 family members of terminally and critically ill patients were assessed, comprising family members of 31 patients who received PST and of 31 who did not received PST in Northern Taiwan. Statistical analyses included descriptive statistics, $t$ tests, one-way analysis of variance, and multiple regression.

\section{Results}

Whether used PST or not, the levels of family members' grief did not significantly influence. The average duration of using PST until to death was 11.43 days. Most of these patients continually and routinely used PST. The family member's highest concerns were "feeling the patient still had something more to do" $(4.40 \pm 0.56)$, followed by "wish there had been a chance for the entire family to discuss" $(4.33 \pm 0.61)$, and "distressed that they could not communicate with the patient" $(4.20 \pm 0.61)$. When the patients were older, the family members experienced less grief. The family members who were the spouses or parents of the patients and were incomparably close to the patients experienced more grief.

Conclusions

Use of PST is not related to family members' grief levels; however, PST may cause profound family members' distress. Understating their experiences with PST is of value to provide intensive emotional support.

\section{eP344}

\section{GUIDELINES ON SYMPTOM CONTROL AT END OF LIFE CARE IN THE ACUTE HOSPITAL}

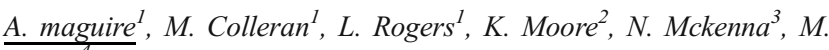 \\ Kerins \\ ${ }^{1}$ Naas General Hospital, Palliative Care, Naas, Ireland \\ ${ }^{2}$ Naas General Hospital, Medicine, Naas, Ireland \\ ${ }^{3}$ Naas General Hospital, Pharmacy, Naas, Ireland \\ ${ }^{4}$ Naas General Hospital, Accident and Emergency, Naas, Ireland
}

\section{Introduction}

Many patients die in acute hospitals under the care of clinical teams and may or may not require specialist palliative care input. Current Medical Council recommendations in Ireland advise that doctors should treat patients who are dying with dignity, optimize comfort and it recognizes family's communication needs. These guidelines were developed as part of an audit of symptom control of patients at the end of life in an acute hospital.

\section{Objectives}

To create a guideline to facilitate symptom management and care planning for patients who are imminently dying and to empower nonpalliative care medical practitioners in their clinical decision making.

Methods

A collaborative, interdisciplinary, interdepartmental process was involved in developing the guidelines.

\section{Results}

The guidelines were developed based on current evidence, palliative care practice, and feedback from the consultative process. They were presented to the doctors at Induction. Hard copies were printed, laminated and made available on the wards.

The guidelines include medication management, clinical practice and communication issues. They consist of prompts to aid individualized, patient-centered, clinical decision making including addressing reversible causes for deterioration if appropriate and consideration of the ceiling of care. Options for anticipatory symptom control medications and the patient's environmental setting were incorporated. Specialist palliative care advice and involvement can be sought at any stage of management.

\section{Conclusions}

The guidelines were developed to aid timely, effective, patient focused care for patients who are imminently dying and to support non-palliative medical practitioners in end of life care.

\section{eP345 \\ FILIPINO PATIENTS' AWARENESS OF THEIR PROGNOSIS AND PARTICIPATION IN DECISION MAKING AND TREATMENT PLANNING}

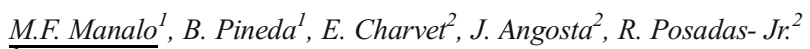

${ }^{T}$ The Medical City, Internal Medicine, Pasig, Philippines

${ }^{2}$ The Medical City, Cancer Center, Pasig, Philippines

\section{Introduction}

The trend in recent years has been one of greater patient participation in illness management decisions. Patients or their surrogates should be involved in decisions if they wish to do so, and their preferences and values need to be better taken into account.

\section{Objectives}

To determine advanced cancer patients' awareness and preferences for prognostic information, their preferences for treatments and role in decision making in the Philippines.

Methods

Two hundred patients with advanced cancer at the Cancer Center, The Medical City are recruited to the study. The previously validated survey instrument (APPROACH) based on FACIT has questions on awareness of and preferences for prognostic information and role in decision making and treatment preferences.

\section{Results}

Reasons why patients prefer disclosure over non-disclosure include ability to resolve unfinished business, right to know the truth, fostering cooperation between patient and physician, and relief from unnecessary treatments. There are fewer patients who prefer not to know and their reasons include: disclosure can cause them psychological and emotional distress, and it may be meaningless and could not give help to them. Some patients believe that what they don't know cannot hurt them. Thus, they prefer not to be informed of the gravity of their sickness. Filipinos, specifically tend to leave things up to fate or destiny, thinking that everything happens for a reason; and these includes their illness and anticipated death.

\section{Conclusions}

A greater awareness and understanding of cultural and family structure factors influencing end-of-life decision-making will assist the hospital staff in providing optimal supportive oncology and palliative care.

\section{eP346}

IMPACT OF EARLY PALLIATIVE CARE IN HOSPITALIZATION AND EMERGENCY ROOM VISITS AMONG BREAST CANCER PATIENTS TREATED AT INSTITUTO NACIONAL DE CANCEROLOGIA MEXICO, CITY.

L. Mendoza-Galindo ${ }^{I}$, C. Arce-Salinas ${ }^{1}$, R. Ramirez-Morales ${ }^{2}, S$. Allende-Pérez ${ }^{3}$, E. Monreal-Carrillo ${ }^{3}$, C. Arzate-Mireles ${ }^{3}$, D. PérezCamargo $^{4}$, E. Verastegui-Avilez ${ }^{3}$, D. Flores-Díaz ${ }^{1}, J$. Matus-Santus ${ }^{I}, L$. López-Rojas ${ }^{2}$

${ }^{1}$ Nacional Cancer Institute - Mexico City INCan, Medical Oncology, Mexico City, Mexico

${ }^{2}$ Nacional Cancer Institute - Mexico City INCan, Clinical Research, Mexico City, Mexico

${ }^{3}$ Nacional Cancer Institute - Mexico City INCan, Palliative Care, Mexico City, Mexico

${ }^{4}$ Nacional Cancer Institute - Mexico City INCan, Nutrition, Mexico City, Mexico 


\section{Introduction}

The earlier palliative care interventions are associated with greater reductions in average hospitalization days and less visits to emergency room (ER).

\section{Objectives}

To evaluate the reduction in hospitalization length and ER consultations with early palliative care intervention in comparison with patients treated with oncological recommendations

\section{Methods}

Newly diagnosed or relapsed metastatic breast cancer were included, patients were randomized to standard care given by the attending physician (control arm) or intervention by palliative team, which included psychological, nutritional and symptom support (experimental arm). Patients were stratified according to ER/PR, and HER2 status (positive vs negative). Local ethical committee approved protocol. We registered the number of ER consultations, hospitalizations (yes/no) number of hospitalization days, main cause of attention.

\section{Results}

53 patients were included, $33(62 \%)$ were assigned to experimental arm and $20(38 \%)$ to control arm, there was a trend of reduction in ER visits among experimental arm in comparison with control arm 39 vs $50 \mathrm{p}=0.074$. there was no difference in number of hospitalizations ( $48 \%$ vs $51 \%)$ neither days of hospitalization stay ( 78 vs 90 days) among groups $p=0.808$. However, according with the main cause of hospitalization there was a trend that patients in control arm the reason was uncontrolled symptoms $30 \%$ vs $12 \%$ and more patients in experimental arm were hospitalized due to disease progression $33.3 \%$ vs $25 \%$ with not statistical difference.

Conclusions

There was a trend that early palliative care intervention is associated with a reduction in ER consultations and hospitalizations due to uncontrolled symptoms, however, sample size should be increased.

\section{eP347}

\section{SCREENING PALLIATIVE PATIENTS WITH THE DISTRESS THERMOMETER: A FEASIBILITY STUDY}

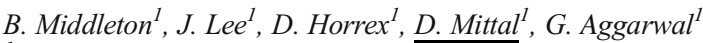 \\ ${ }^{I}$ Concord Centre for Palliative Care, Concord Repatriation General \\ Hospital, Concord, Australia
}

\section{Introduction}

Distress is poorly recognised and suboptimally managed at the end of life. Timely detection is crucial for relieving distress. The Distress Thermometer is a validated measure of self-reported distress in cancer patients but its use has not been validated in a palliative care inpatient population. At the Concord Centre for Palliative Care, standard care includes daily screening with a Symptom Assessment Scale and Palliative Care Problem Severity Score. It does not include an overall self-rated distress measure.

Objectives

To determine the feasibility and acceptability of implementing the Distress Thermometer in addition to standard care in an inpatient palliative care unit.

Methods

We administered the Distress Thermometer to 30 consecutive consenting patients. Acceptability, time to complete and reasons for distress were collected. We compared the detection rate with standard care and reviewed referrals to Psychology and Social Work.

Results

The mean time taken to complete the Distress Thermometer was 2.8 minutes. The screen was positive in $76 \%$ of patients. Fifty seven percent of patients found it easy or very easy to complete. In $10 \%$ of patients, distress was reported which had not been detected by standard screening. Twenty percent of patients had psychological or social distress detected by the Distress Thermometer where standard assessment had not triggered a referral for Psychology or Social Work review.

\section{Conclusions}

The Distress Thermometer is feasible and acceptable to use in clinical practice in a palliative care inpatient unit. Its use identified additional distress not detected with standard care and provided opportunities to improve management of palliative patients with distress.

\section{eP348}

THE PATIENT AND FAMILY MEETING PROGRAM: A PILOT OF EARLIER DISCUSSIONS IN A GENITOURINARY ONCOLOGIC CLINIC

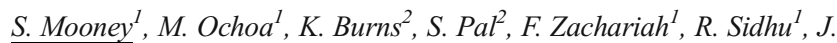
Prudhomme-Hunter $^{l}$, N. Garcia ${ }^{l}$, S. Buga ${ }^{l}$, W. Dale ${ }^{l}$, M. Loscalzo ${ }^{l}$

${ }^{1}$ City of Hope National Medical Center, Department of Supportive Care Medicine, Duarte, USA

${ }^{2}$ City of Hope National Medical Center, Department of Genitourinary Oncology, Duarte, USA

\section{Introduction}

Evidence shows family meetings in the ICU result in less aggressive care for critically ill patients. Less evidence exists for the benefit of earlier, values-based discussions in outpatient settings.

\section{Objectives}

We sought to increase the incidence of interdisciplinary family meetings in the genitourinary oncologic $(\mathrm{GU})$ clinic at City of Hope National Medical Center (COH). Objectives include efficient management of physician time and involving supportive care and hospice disciplines.

\section{Methods}

At $\mathrm{COH}$ we utilize an interdisciplinary approach to conduct family meetings. This involves a social work-led semi-structured format including orientation, pre-meeting to align messages, eliciting the patient's understanding of their condition, and efficient delivery of medical information. Afterwards, the physician is excused and remaining team members help the family reconcile medical information with values and goals. In this pilot, the Patient and Family Meeting Program (FMP) was applied in the GU clinic to patients with a prognosis of less than two years. Outcomes tracked included physician time in meetings, hospice and supportive care referrals, and place of death.

\section{THE PATIENT AND FAMILY MEETING PROGRAM}

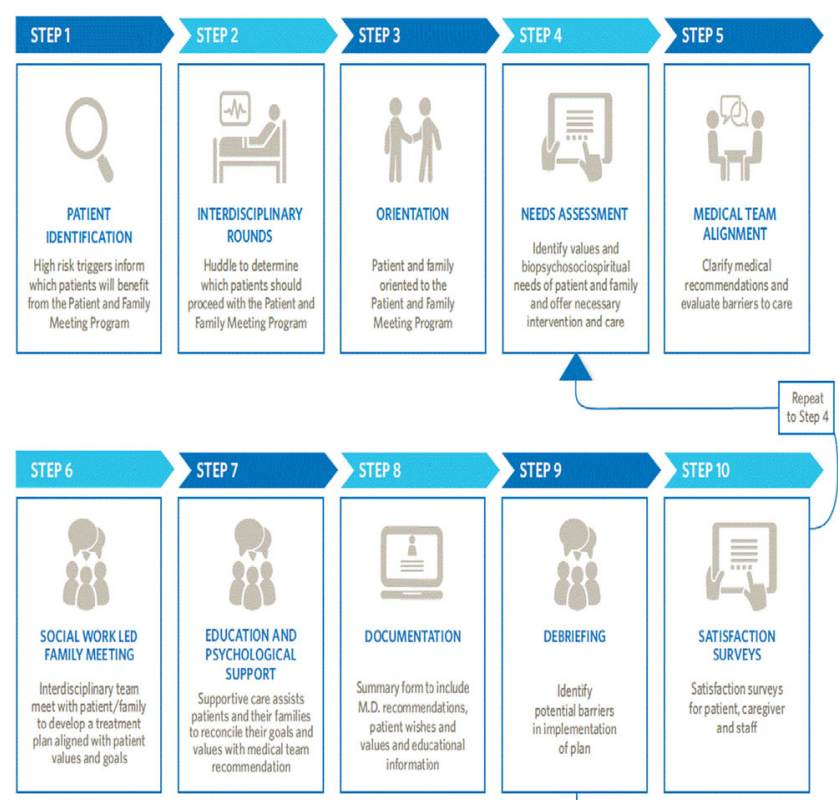




\section{Results}

Between 10/16/17 and 1/29/18, 26 orientations and 16 family meetings were held. 15 patients were referred to hospice, 13 patients admitted to hospice, and 4 died in home hospice. Average physician time in meetings was 17 minutes. 5 referrals were made to Supportive Medicine.

\section{Conclusions}

The FMP in the GU clinic has shown beneficial outcomes, including efficiency of physician time and involvement of supportive care and hospice. Work is needed to compare this intervention to standard of care and to expand to other specialties.

\section{eP349}

\section{EXPLORATORY STUDY OF FAMILY FUNCTIONING AND} QUALITY OF LIFE AMONG FAMILY CAREGIVERS OF TERMINAL CANCER PATIENTS IN JAPAN

\author{
M. Morishita-Kawahara $^{1,2}$, T. Kawahara ${ }^{3}$, K. Kamibeppu ${ }^{2}$ \\ ${ }^{T}$ National Cancer Center, Division of Health Care Research-Center for \\ Public Health Sciences, Tokyo, Japan \\ ${ }^{2}$ The University of Tokyo, Department of Family Nursing-Graduate School \\ of Health Sciences and Nursing-Faculty of Medicine, Tokyo, Japan \\ ${ }^{3}$ The University of Tokyo Hospital, Biostatistics Division- Clinical \\ Research Support Center, Tokyo, Japan
}

\section{Introduction:}

Family functioning of terminal cancer patients is unsettled because roles of family member can change while taking care of patients. Many studies have shown that low confliction is related to better QOL of family caregivers. Family caregivers of terminal cancer patients experience many troubles and confrontations, therefore confliction among family members cannot be avoided.

Objectives

To investigate family types of family caregivers of terminal cancer patients and to examine the relationship between family functioning and QOL.

Methods

We conducted a cross-sectional study. We included family caregivers of terminal cancer patients in general wards who were 20 years old or more. We measured family functioning and QOL using Family Relationship Index (FRI) and Caregiver Quality of Life Index-Cancer (CQOLC), respectively. We clustered them referring to FRI and compared QOL between clusters.

\section{Results}

We included 51 patients and their family caregivers. Their average FRI score was 8.5. They were clustered into 3 groups: supportive group (high cohesiveness, low expressiveness, low conflict), communicative group (high cohesiveness, high expressiveness, medium conflict), and conflictive group (low cohesiveness, low expressiveness, high conflict). Their average CQOLC score was 77.2, 74.9, 65.0, respectively. Post-hoc analysis showed that CQOLC score of supportive and communicative groups was significantly higher than that of conflictive group.

\section{Conclusions}

Their family functioning was not good because they need to make decisions in many scenes about the end of life care and therefore they often face conflicts. However, we found that confliction may not be negatively related to QOL if their expression is enough.

\section{eP350}

END OF LIFE CARE EXPERIENCE AT THE PEADIATIC ONCOLOGY UNIT AT THE UGANDA CANCER INSTITUTE: WHAT ROLE CAN ONCOLOGY NURSES PLAY?

\section{Mulyowa \\ ${ }^{T}$ Uganda Cancer Institute, Oncology, Kampala, Uganda}

\section{Introduction:}

Although the majority of childhood cancers are curable, this is not yet true for low resource countries. In Uganda, cancer care is only done at the Uganda Cancer Institute.
Between Jan $1^{\text {st }}$ and Dec $31^{\text {st }}$, there were 4,321 new cancer cases registered and about $\mathbf{8 \%}$ were among children. The mortality rate is $\mathbf{7 0 \%}$ annually, that is every $\mathbf{3}$ in $\mathbf{5}$ children diagnosed with cancer will not survive past one year after cancer diagnosis. Here, we review the factors contributing to poor outcomes and potential solutions.

\section{Objectives}

1. To determine the role of an oncology Nurse at the end of life care of children with cancer.

\section{Methods}

Since this is an experience, $\mathrm{i}$ will just describe and explain what we do.

\section{Results}

Majority of the children present with advanced disease at cancer diagnosis. The chemotherapy is prescribed for palliative intent more frequently and high rates of complications are observed. Family involvement in decisions regarding the role of palliative chemotherapy at end of life is low.

Nurses play an integral role, identifying symptoms, providing care coordination, because they are with the patients most of the time, day and night. Nurses also play a role in assuring clear communication with patients' and their caretakers.

\section{Conclusions}

Palliative Care Training for all the clinicians and other healthcare providers is essential, also educational initiatives for patients and their families regarding the type of cancer the child/patient has, treatment modalities should be emphasized.

\section{eP351 \\ MODELS OF PALLIATIVE CARE FROM HOSPITAL TO HOME IN SOUTHERN THAILAND}

K. Nilmanat ${ }^{1}$, N. Niyomthai ${ }^{1}$, M. Udchumpisai ${ }^{2}$, P. Podjamanpong ${ }^{3}$

${ }^{7}$ Prince of Songkla University, Department of Medical nursing- Faculty of Nursing, Hat Yai, Thailand

${ }^{2}$ Srisavarindhira Thai Red Cross Institute of Nursing, Department of adult and elderly nursing, Bangkok, Thailand

${ }^{3}$ Bangkok Puket Hospital, Nursing division, Puket, Thailand

\section{Introduction:}

Palliative care in Thailand has had a progressive development. The development of palliative care activism in Thailand is spotty and had limited support. Objectives

The purposes of this qualitative research were to describe the models of palliative care from hospital to home in Southern Thailand.

\section{Methods}

Three regional or tertiary level hospitals which were the palliative care contracting unit for secondary and primary care settings were selected. 92 participants included persons with terminal illness and their family caregivers, directors of selected hospitals, lord mayor, other health care providers involved in palliative care. In-depth interviews, focus group discussions, and observation were data collection methods while content analysis was applied. Results

The continuity of palliative care from hospital to home in selected hospital reflected fragmented care. The care process included identifying target group, conducting discharge planning, coordinating with the continuity of care center for transfer, and visiting patients at home. There were 3 models of palliative care continuity from hospital to home; hospital based palliative care consultation, integrated community based palliative care and volunteer support palliative care. Several barriers to promote the care continuity were identified. Conclusions

Findings from this study can be used to develop the seamless palliative care by promoting sustainable palliative care policy, supporting professional development on palliative care in both basic level and specialist level, establishing a minimum list Palliative-Specific Pharmaceuticals policy and essential palliative care medical equipment, strengthening care network in community, and promoting and enhancing volunteer work in palliative care. 


\section{eP352}

\section{AMBULATION STATUS AFTER EMERGENCY RADIOTHERAPY FOR METASTATIC SPINAL CORD COMPRESSION}

W. Onchan ${ }^{1}$, N. Pattamapaspong ${ }^{2}$, C. Madla ${ }^{2}$, P. Klunklin ${ }^{1}$, S. Chakrabhandu $^{l}$, E. Tharavichitkul ${ }^{1}$, B. Jia-Mahasap ${ }^{l}$, P. Sripan ${ }^{l}, I$. Chitapanarux ${ }^{I}$

${ }^{1}$ Faculty of Medicine- Chiang Mai University, Radiation Oncology, Chiangmai, Thailand

${ }^{2}$ Faculty of Medicine- Chiang Mai University, Radiology, Chiangmai, Thailand

\section{Introduction:}

Metastatic spinal cord compression (MSCC) occurs in 5-10\% of cancer patients and associated with poor quality of life. Radiotherapy (RT) is an effective treatment for palliation of symptoms from MSCC.

Objectives

To evaluate post-treatment ambulation status after palliative RT for MSCC.

Methods

Thirty-seven MSCC patients who were received emergency palliative RT within 24 hours after magnetic resonance imaging (MRI) between November 2015 and November 2016 were reviewed. Motor function and ambulation status were evaluated before and 2 months after RT.

Results

There were 18 men and 19 women, with a median age of 57 years. The primary tumor was predominantly lung cancer $(38 \%)$ followed by breast cancer (22\%). Radiotherapy outcomes were categorized into 3 groups according to ambulation status before and after RT (Not improved: non-ambulation before and after RT, Stable: ambulation before and after RT, Improved: nonambulation before and ambulation after RT). Improvement of ambulation status occurred in $46 \%$, stable in $19 \%$ and not improved in $35 \%$ of patients. Pre-radiotherapy motor power grade and short interval time (1-7 days) developing motor deficit before RT seem to be the prognostic factors for RT outcomes in univariate analysis with $\mathrm{p}$-value $<0.001$ and 0.019 , respectively. Table: Potential prognostic factors on ambulation status

\begin{tabular}{|c|c|c|c|c|}
\hline Characteristic & $\begin{array}{l}\text { Not improved } \\
\text { (N=13) }\end{array}$ & $\begin{array}{l}\text { Stable } \\
(\mathrm{N}=7)\end{array}$ & $\begin{array}{l}\text { Improved } \\
(\mathrm{N}=17)\end{array}$ & P-value \\
\hline Gender & & & & $>0.999^{\circ}$ \\
\hline Male & $6(33 \%)$ & $4(22 \%)$ & $8(45 \%)$ & \\
\hline Feande & $7(37 \%)$ & $3(16 \%)$ & $9(47 \%)$ & \\
\hline Median (IQR) of age (year) & $57(50-63)$ & $56(49-64)$ & $59(46-65)$ & $0.846^{b}$ \\
\hline Type of primary tumor & & & & $0.530^{2}$ \\
\hline Lung cancer & $5(36 \%)$ & $2(14 \%)$ & $7(50 \%)$ & \\
\hline Breast cancer & $2(25 \%)$ & $1(12 \%)$ & $5(63 \%)$ & \\
\hline Head and neck cancer & $1(33 \%)$ & $2(67 \%)$ & $0(0 \%)$ & \\
\hline Hematologic cancer & $2(29 \%)$ & $2(29 \%)$ & $3(43 \%)$ & \\
\hline Other & $3(60 \%)$ & $0(0 \%)$ & $2(40 \%)$ & \\
\hline Visceral metastases at the time of RT & & & & $0.475^{3}$ \\
\hline No & $6(32 \%)$ & $2(11 \%)$ & $10(57 \%)$ & \\
\hline Yes & $f(3 / \%)$ & $S(26 \%)$ & $f(3 / \%)$ & \\
\hline No. of involved vertebrae & & & & $0.515^{3}$ \\
\hline $1-2$ & $5(45 \%)$ & $1(9 \%)$ & $5(45 \%)$ & \\
\hline 23 & $8(31 \%)$ & $6(23 \%)$ & $12(46 \%)$ & \\
\hline RT regimens, Gy & & & & $0.586^{2}$ \\
\hline $5 \times 4$ & $5(26 \%)$ & $4(21 \%)$ & $10(53 \%)$ & \\
\hline $2 \times 6.5$ & $7(47 \%)$ & $2(13 \%)$ & $6(40 \%)$ & \\
\hline $1 \times 8$ & $1(33 \%)$ & $1(33 \%)$ & $1(33 \%)$ & \\
\hline Motor power grade before RT & & & & $<0.001^{2}$ \\
\hline $\mathbf{0}$ & $7(78 \%)$ & $0(0 \%)$ & $2(22 \%)$ & \\
\hline 1-3 & $6(29 \%)$ & $0(0 \%)$ & $15(71 \%)$ & \\
\hline $4-5$ & $0(0 \%)$ & $7(100 \%)$ & $0(0 \%)$ & \\
\hline $\begin{array}{l}\text { Motor power grade and time } \\
\text { developing motor deficits before RT }\end{array}$ & & & & $0.019^{\prime}$ \\
\hline Grade $0,>7$ days & $2(100 \%)$ & $0(\%)$ & $0(0 \%)$ & \\
\hline Grade $0,1-7$ days & $5(71 \%)$ & $0(0 \%)$ & $2(29 \%)$ & \\
\hline Grade $>0,>7$ days & $1(14 \%)$ & $0(0 \%)$ & $6(86 \%)$ & \\
\hline Grade $>0,1-7$ days & $5(24 \%)$ & $7(33 \%)$ & $9(43 \%)$ & \\
\hline
\end{tabular}

\section{Conclusions}

Emergency radiotherapy maintained and recovered ambulation status in MSCC patients with good motor power grade before radiotherapy.

eP353

ROLE OF MEGESTROL ACETATE VERSUS DEXAMETHASONE FOR IMPROVEMENT IN APPETITE IN PATIENTS WITH CANCER ASSOCIATED ANOREXIA CACHEXIA: A RANDOMIZED CONTROLLED PILOT TRIAL

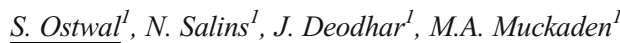

${ }^{T}$ Tata Memorial Hospital, Palliative Medicne, Mumbai, India

\section{Introduction:}

Anorexia is a frequent and distressing symptom in patients with advanced cancer and other life-limiting illnesses. Prolonged periods of anorexia, can lead to both serious physical and psychological distress to patients and their families, resulting in poorer quality of life.

\section{Objectives}

To compare clinical benefits between Dexamethasone and Megestrol acetate (MA) on Appetite, lean body weight and quality of life in an Indian setting.

Methods

A prospective, randomized controlled pilot study conducted in outpatient clinic of Palliative Medicine from September to December 2015. Registered adult advanced cancer patients meeting the inclusion criteria and exclusion criteria were assessed for anorexia, measured by ESAS scale, lean body weight (by Hume's Formula) and QOL (EORTC-QOL PAL15). Patients received either Dexamethasone (4mg) or MA (160mg in divided doses) for 21 days. Follow up done at days 7, 21 and 35. CTRI No: REF/2015/10/009871

\section{Results}

Total 40 patients were enrolled in the study. Patients in both groups showed statistically significant improvement in appetite, lean body weight and quality of life at weeks 3 and 5, compared to baseline. However, study failed to show any significant difference for primary (appetite improvement) and secondary (lean body weight and quality of life) objectives between two groups, at weeks 3 and 5. Dexamethasone was found to be associated with more side effects.

\section{Conclusions}

Comparison between Megestrol acetate and dexamethasone failed to show any statistical significant difference in primary and secondary objectives. However, Improvement in fatigue and other symptom scores were seen to be statistically significant in MA arm

\section{eP354}

IMPACT OF PALLIATIVE CARE ON REDUCTION AGGRESSIVE END OF LIFE CARE MEASURES AMONG BRAZILIAN PATIENTS WITH ADVANCED CANCERS

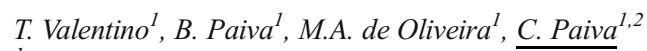

${ }^{1}$ Barretos Cancer Hospital-Barretos-São Paulo-Brazil, Research Group on Palliative Care and Health-Related Quality of Life GPQual, Barretos, Brazil

${ }^{2}$ Barretos Cancer Hospital, Department of Clinical Oncology, Barretos, Brazil

\section{Introduction}

Relevant indicators on the quality of care for patients with advanced cancer (ACPs) are aggressive care at the end of life (EOL).

\section{Objectives}

To evaluate the impact of palliative care (PC) consultations on aggressive EOL care measures among patients with ACPs. 


\section{Methods}

Data from ACPs treated at a Brazilian Cancer Hospital who died between 2010 and 2014 were retrieved. ACPs were categorized as early (>3 m before death) and late ( $<3 \mathrm{~m}$ before death). Quality of EOL care indicators were extracted from medical charts, including emergency room visits, hospitalization, ICU admissions, and systemic antineoplastic therapy use within the last 30 days of life. A modified composite score for aggressiveness of care (mCSAC) was used (range: 0-5; the higher the score, the more aggressive the care).

Results

Patients not consulted in PC were more likely to receive aggressive care compared to consulted patients (mCSAC $\geq 1: 87.4 \%$ vs. $52.8 \%, \mathrm{p}<0.001$ ). mCSAC were not different between early and late PC. However, patients later referred to $\mathrm{PC}$ were more likely to have $\geq 2$ emergency room visits ( $9.8 \%$ vs. $4.6 \%, p=0.004), \geq 2$ hospitalizations ( $14.3 \%$ vs. $9.9 \%$; $\mathrm{p}=0.054)$, systemic anticancer use $(14.8 \%$ vs. $7.7 \%, \mathrm{p}<0.001)$ and less likely to die at home $(6.3 \%$ vs. $12.0 \%, \mathrm{p}=0.002)$ compared with early PC. Conclusions

ACPs consulted by the PC team receive less aggressive care at the EOL compared with patients never consulted. Early PC seems to add further benefits.

\section{eP355}

IS IT POSSIBLE TO BE HAPPY AFTER CANCER? FACTORS INFLUENCING THE PERCEPTION OF HAPPINESS OF PATIENTS UNDERGOING PALLIATIVE CARE ONLY

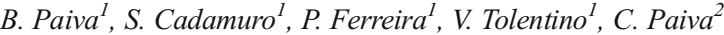 \\ ${ }^{1}$ Barretos Cancer Hospital-Barretos- SP-Brazil, Research Group on Palliative Care and Health-Related Quality of Life GPQual, Barretos, Brazil \\ ${ }^{2}$ Barretos Cancer Hospital, Department of Clinical Oncology, Barretos, Brazil}

\section{Introduction}

Identifying factors associated with greater happiness perception among cancer patients, particularly those undergoing palliative care (PC), may target efforts to promote measures that increase the chance of making them happier, even living in a disease setting.

Objectives

To identify the impact of cancer on the individual perception of happiness and its associated conditions.

\section{Methods}

A cross-sectional study investigating advanced cancer patients (ACP) undergoing PC only. Patients answered the Pemberton Happiness Index (PHI) and questions addressing issues potentially associated with their happiness. PHI scores ranged from 0 to 10 ; the higher the score, the higher the happiness. PHI $>7$ was the cut-off for happiness. Variables with $\mathrm{p}$-values $<0.2$ in univariate analyzes were included in a logistic regression model. Values of $\mathrm{p}<0.05$ were considered significant.

\section{Results}

110 patients were included. Mean age $(\mathrm{SD})=51.4$ (13.3) years. Mean PHI score was $7.2(\mathrm{SD}=1.7) ; 60(59.4 \%)$ patients were classified as "happy". $35(35 / 44,79.5 \%)$ and $5(5 / 44,11.3 \%)$ patients said that improving their health and having more money was what they still lacked to be happy, respectively. Satisfaction with financial aspects $(\mathrm{OR}=4.95$, $\mathrm{p}<0.001)$, perception of importance of religiosity/spirituality $(\mathrm{R} / \mathrm{E})$ $(\mathrm{OR}=4.94, \mathrm{p}<0.001)$, frequent contact with nature $(\mathrm{OR}=2.59, \mathrm{p}=0.019)$ and more leisure activities $(\mathrm{OR}=4.96, \mathrm{p}=0.034)$ were variables associated with happiness in the multivariate analysis.

Conclusions

Even facing a disease with a very poor prognosis, approximately $60 \%$ of patients can be considered happy. Some factors are associated with happiness among patients receiving PC only and should be comprehensively addressed by PC health professionals.
eP356

RELATIONSHIP BETWEEN EASTERN COOPERATIVE ONCOLOGY GROUP PERFORMANCE STATUS (ECOG-PS) AND ACTIGRAPHY-DERIVED ACTIVITY PARAMETERS IN A HETEROGENEOUS GROUP OF ADVANCED CANCER PATIENTS

S.D. Patel ${ }^{1,2}$, A. Davies ${ }^{1}$, D.J. Dijk ${ }^{2}$

${ }^{T}$ Royal Surrey County Hospital/St Luke's Cancer Centre, Supportive and Palliative Care, Guildford, United Kingdom

${ }^{2}$ University of Surrey, Faculty of Health and Medical Sciences, Guildford, United Kingdom

\section{Introduction}

Discrepancies between physician-assessed and patient-assessed performance status, have been associated with an increased risk of mortality. Quantification of activity through actigraphy may be a better, objective method for prognostication. The actigraphy-derived dichotomy index $(\mathrm{I}<\mathrm{O})$ has been reported to be of prognostic value in the metastatic colorectal cancer population. However, the association between the $\mathrm{I}<\mathrm{O}$ and ECOG-PS has not been fully explored.

\section{Objectives}

To assess the relationship between physician-assessed ECOG-PS scores and the $\mathrm{I}<\mathrm{O}$ and other actigraphy-derived activity parameters such as daytime activity and mean 24-hour activity.

Methods

50 adult outpatients with advanced cancer and an estimated prognosis of less than a year were recruited as part of a feasibility study. Patients and the palliative care physician independently assessed the patient's ECOGPS both at baseline and after 7 days. Participants were instructed to wear an Actiwatch Spectrum Plus ${ }^{\circledR}$ for seven consecutive 24-hour periods on their non-dominant arm, and to concurrently complete a sleep diary.

\section{Results}

On Day 8, there was moderate agreement between the palliative care physician and individual patient's assessment of their ECOG-PS, with a Kendall's correlation of $0.70(p<0.001)$. A moderate negative correlation was observed between physician-assessed ECOG-PS and the dichotomy index $(\mathrm{I}<\mathrm{O})(\mathrm{r}=-0.55 ; \mathrm{p}=0.0003)$. There was no correlation between physician-assessed ECOG-PS and mean daytime activity $(\mathrm{r}=-0.29$; $\mathrm{p}=0.073)$ or mean 24-hour activity level $(\mathrm{r}=-0.2 ; \mathrm{p}=0.218)$.

\section{Conclusions}

Physician-assessed ECOG-PS and patient-assessed ECOG-PS scores are moderately correlated. A poor performance status is significantly associated with a measure of day-night difference in activity, but not with absolute activity measures.

\section{eP357}

PREVALENCE AND SURVIVAL OUTCOMES IN PALLIATIVE CARE PATIENTS WITH DEEP VEIN THROMBOSIS: RESULTS OF THE NATIONAL CANCER INSTITUTE IN MEXICO

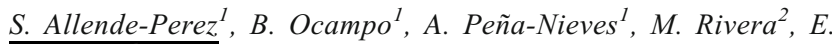 \\ Verástegui $^{I}$ \\ ${ }^{1}$ Instituto Nacional de Cancerología, Palliative Care Service, Mexico \\ city, Mexico \\ ${ }^{2}$ Instituto Nacional de Ciencias Médicas y Nutrición Salvador Zubiran, \\ Hematology and oncology, Mexico City, Mexico
}

\section{Introduction}

Patients with cancer are at high risk to develop deep vein thrombosis (DVT). Cancer-associated thrombosis represents a major cause of morbidity and mortality in these patients, accounting for the second leading cause of cancer-related death, nonetheless, DVT is frequently underdiagnosed. Objectives

Establish the prevalence of DVT and overall survival in palliative care (PC) patients during a 5-year period. 


\section{Methods}

A retrospective analysis was performed in palliative care patients of the National Cancer Institute in Mexico from 2012 to 2016. Clinical and demographic characteristics, were obtained from the Medical Records. Descriptive statistics were used. Overall survival was calculated using Kaplan-Meier estimates, comparing groups with log-rank test, using STATA v.12.

Results

We identified 2,301 patients. The median age was 55 years, $71.5 \%$ $(\mathrm{n}=1,646)$ were female. The most frequent cancer diagnoses were: breast (22.1\%, $\mathrm{n}=509)$, stomach $(16.3 \%, \mathrm{n}=375)$, cervical $(16 \%, \mathrm{n}=369)$, and colorectal $(13.9 \%, \mathrm{n}=320)$. In all the cohort, the prevalence of DVT was $12.3 \%$, mostly females $(9.7 \%)$. DVT was most frequently diagnosed in breast $(3.2 \%)$, cervical $(3.1 \%)$, and colorectal cancers (1.4\%). Differences in survival were observed according to the underlying diagnosis $(\mathrm{p}=0.006$ ), but no differences were observed when comparing groups with and without DVT $(\mathrm{p}=0.688)$.

\section{Conclusions}

Prevalence of DVT was low in our cohort. No differences in survival were observed when comparing groups with DVT and without DVT. We hypothesize that our results are a consequence of the timely diagnosis and management offered by our multidisciplinary group in the Department of PC to our cancer patients during the first 24-hour period after diagnosing DVT.

\section{eP358}

\section{THE UNIQUE SUPPORT PATH FOR METASTATIC BREAST CANCER PATIENTS}

\section{E. Plotkin ${ }^{1}$, M. Gandee ${ }^{2}$, L. Lucas $^{1}$}

${ }^{T}$ Association of Community Cancer Centers, Provider Education, Rockville, USA

${ }^{2}$ Association of Community Cancer Centers, Development, Rockville, USA

\section{Introduction}

An estimated 150,000 to 250,000 women in the U.S. are currently living with metastatic breast cancer. While breast cancer is a high-profile disease, receiving significant research funding and prevention initiatives, patients with metastatic breast cancer face unique challenges. Public messaging about a "cure" is so pervasive that people diagnosed with metastatic breast cancer can be stigmatized by the perception that they've failed to take care of themselves.

\section{Objectives}

Expand the current breast cancer conversation to address gaps between early and metastatic disease and improve the treatment and management of metastatic breast cancer in the community setting.

Address the communication challenges that cancer care teams face such as: Patientperception that their cancer is curable, patient level of engagement during conversations with providers, providers who may "minimize" the severity of the disease, and lack of awareness of available resources.

\section{Methods}

An environmental scan was completed. An expert advisory panel was formed. 5 different community cancer centers completed in-depth focus groups on their care path.

\section{Results}

A process improvement workbook was created with a map of care from metastatic diagnosis through end of life. Relevant resources were attributed to five stages: Diagnosis, Current Treatment, Monitoring Treatment, Treatment Failure, Hospice. Six principles were identified and assigned: Empower the patient, reframe the conversation, reduce patient isolation, offer logistical support, connect patients with support in the community, and collaborate in the interest of patients.

Conclusions

This patient population should be given specialized care to address their unique diagnosis and improve communications with their care team.

\section{eP359}

SPIRITUAL NEEDS AND SPIRITUAL WELL-BEING OF THE TERMINALLY ILL PATIENTS, THAILAND

\section{P. pokpalagon ${ }^{I}$, S. chaiviboontham ${ }^{l}, N$. phinitkhajorndech ${ }^{1}$ \\ ${ }^{T}$ Ramathibodi School of Nursing, Faculty of Medicine Ramathibodi Hospital-Mahidol University, Bangkok, Thailand}

\section{Introduction}

Spiritual care is an essential domain of palliative care, which focuses on the needs of the whole person and their family.

\section{Objectives}

The study aims to descriptively analyze and compare the spiritual needs and spiritual well-being among Thais with terminally ill who were receiving care from four different settings (community/home-based care, faith-based HIV organization, faith-based cancer organization, and hospice care units) and compare the spiritual well-being by the frequency of spiritual practice.

\section{Methods}

One hundred and seventy Thais with terminally ill receiving treatment in one of four selected settings were recruited to complete a demographic questionnaire, a spiritual need form, and a spiritual well-being questionnaire.

\section{Results}

The results revealed that the top three spiritual needs were: 1) to forgive someone; 2) to ask for forgiveness; 3) to receive advice or assistance from people around them. The terminally ill patients receiving care at faith-based cancer organization and community/home-based care had a significantly higher mean score of spiritual needs than those in the other settings. Subjects receiving care at faith-based cancer organization had a significantly higher mean score than those receiving care in the other settings. Subjects having more frequency of spiritual practice (making merit, mental development, wisdom development) had a significantly higher mean score of spiritual well-being than subjects having less frequency of spiritual practice or not practice.

Table 1 comoarison of spiritual needs and soiritual well-being within four palliative care settings in $=170$ )

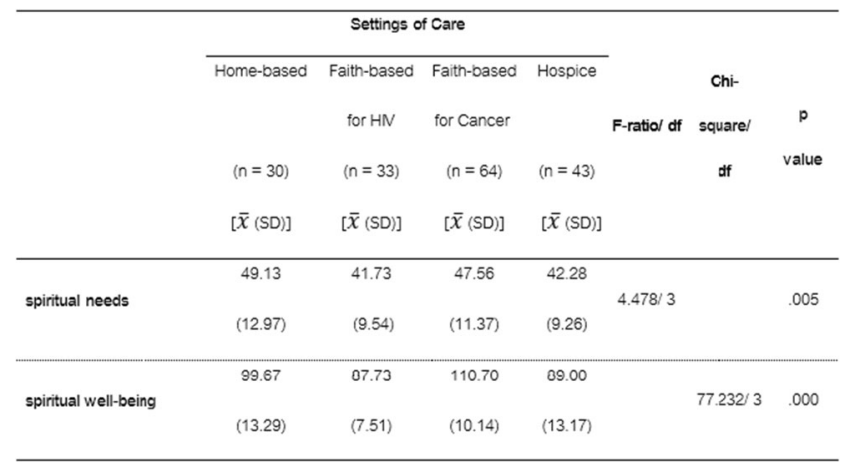

Table 2 Mean Scores of spiritual well-being by the frequency of spiritual practice $(n=170)$

\begin{tabular}{|c|c|c|c|c|}
\hline Frequency of spiritual practice & Number & $\begin{array}{c}\text { Mean score of } \\
\text { spiritual well-being }\end{array}$ & SD & Chi-square \\
\hline Frequency of making merit & & & & $38.085^{* * *}$ \\
\hline Usually & 70 & 106.97 & 12.29 & \\
\hline Sometimes & 91 & 93.08 & 14.03 & \\
\hline Rarely or never & 7 & 87.00 & 10.50 & \\
\hline Frequency of mental development & & & & $9.841^{*}$ \\
\hline Usually & 68 & 102.46 & 14.36 & \\
\hline Sometimes & 77 & 97.26 & 14.96 & \\
\hline Rarely or never & 23 & 91.78 & 14.12 & \\
\hline Frequency of wisdom development & & & & $20.504^{* * *}$ \\
\hline Usually & 41 & 107.24 & 13.21 & \\
\hline Sometimes & 74 & 97.89 & 15.00 & \\
\hline Rarely or never & 52 & 93.19 & 13.31 & \\
\hline
\end{tabular}




\section{Conclusions}

Spiritual practices have been shown to improve spiritual well-being so that incorporating spiritual practices as appropriate.

\section{eP360 \\ APPLYING IPOS QUESTIONNAIRE IN PALIATIVE CARE INPATIENT UNIT - NEW CHALLENGES FOR A ROMANIAN CENTER}

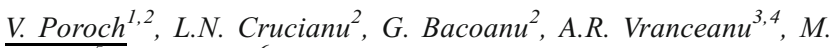
Boanca ${ }^{3}$, R. Postolica ${ }^{6}$

${ }^{1}$ University of Medicine and Pharmacy "Grigore T.Popa" of IasiRomania, Surgery- Palliative Care Department, Iasi, Romania

${ }^{2}$ Regional Institute of Oncology-Iasi-Romania, Palliative Care Department, Iasi, Romania

${ }^{3}$ Regional Institute of Oncology - Iasi- Romania, Medical OncologyPalliative Care Department, Iasi, Romania

${ }^{4}$ University of Medicine and Pharmacy "Grigore T.Popa" of IasiRomania, Palliative Care Department, Iasi, Romania

${ }^{5}$ University of Medicine and Pharmacy "Grigore T.Popa" of IasiRomania, Family Medicine- Palliative Care Department, Iasi, Romania ${ }^{6}$ Regional Institute of Oncology- Iasi-Romania, Medical Oncology, Iasi, Romania

\section{Introduction}

Integrated Palliative care Outcome Scale (IPOS) has recently been introduced in Romania in the practice of palliative care.

Objectives

To highlight the experience of applying IPOS questionnaire and the results obtained in Palliative Care Department within Regional Institute of Oncology Iasi.

Methods

Study conducted between February and July 2017 on 62 patients, test - retest type (at the time of admission and after 10 days) to which the IPOS was applied.

\section{Results}

There were statistically significant differences between the time of admission and re-evaluation after 10 days of dyspnea, fatigue, nausea and vomiting, loss of appetite, constipation, drowsiness, oral cavity problems, mobility, concerns about the illness, perception of family concerns and self-esteem, emotional state, communication with the family and the level of information received. Also, there were significant differences between the self-care capacity after 10 days of delivering palliative care compared to admission (being higher after the delivered care), between the need for approaching to spirituality/ religion after 10 days of palliative care compared to admission (the need for spiritual support for 10 days of palliative care being higher), significant differences between the intensity of pain perceived after 10 days of palliative care compared to the one seen at admission (less 10 days later).

\section{Conclusions}

IPOS has been viewed by patients and professionals as a more streamlined assessment method, capturing their most important concerns, both in terms of symptoms and the need for information, practical activities, anxiety, depression or mood disorders, family concern, reconciliation with oneself, well-being in general.

\section{eP361}

PALLIATIVE CARE EDUCATIONAL NEEDS FOR CANCER PATIENTS IN IRAN: A SWOT ANALYSIS

M. Rassouli ${ }^{1}$, M. Ansari ${ }^{1}$, M.E. akbari ${ }^{2}$, A. abbaszadeh ${ }^{1}$, A. akbari sari ${ }^{3}$ ${ }^{7}$ Shahid Beheshti University of Medical Sciences, Department of Nursing-School of Nursing and Midwifery, Tehran, Iran

${ }^{2}$ Shahid Beheshti University of Medical Sciences, Cancer Research Centre, Tehran, Iran
${ }^{3}$ Tehran University of Medical Sciences, Department of Health Management and Economics- School of Public Health, Tehran, Iran

\section{Introduction:}

By acceptance of palliative care as a part of health system of each country and due to increasing prevalence of cancer, special focus on stakeholder's educational needs is of vital importance so that palliative care services are improved and the quality of life of patients is enhanced.

\section{Objectives}

This study was conducted to explore the educational needs of stakeholders of palliative care for cancer patients in Iran.

Methods

This qualitative study with 20 semi-structured interviews was conducted from August 2016 to February 2017 in Shohadaye Tajrish and Emam Khomeini Hospitals of Tehran. Participants were selected through purposive sampling and included cancer patients and their family caregivers as well as healthcare providers, experts and policy-makers. The data were analyzed through Conventional Content Analysis of Landman and Graneheim using MAXQDA10 software. Statements of each main category of the study were summarized in SWOT categorizes.

\section{Results}

A total of 546 codes were extracted from the analysis of the interviews and four main categories and four subcategories were identified. The four main identified categories included: "academic education planning", "workforce education", "public awareness", and "patient and caregiver empowerment" that contained four subcategories as follows: "strengths", "weaknesses", "opportunities" and "threats" (SWOT).

\section{Conclusions}

Meeting the educational needs of the stakeholders of palliative care requires policy-makers to identify the factors leading to strategies that are based on the use of opportunities, the removal of weaknesses, and coping with the threats to which the organization is faced.

\section{eP362}

ADDRESSING URGENT SYMPTOMS TO IMPROVE PATIENT OUTCOMES IN COMMUNITY SPECIALIST PALLAITIVE CARE THROUGH USE OF AN EMERGENCY MEDICATION KIT

K. Reed ${ }^{l}$

${ }^{T}$ Calvary Health Care Bruce, Specialist Palliative Care, Canberra, Australia

\section{Introduction}

Community Specialist Palliative Care Services (CSPCS) aims to provide relief of suffering for those with a life limiting illness whilst meeting end of life goals of care, in the home. With an increasing number of patients wanting end of life care at home with high acuity, appropriate and timely emergent symptoms management is essential.

Evidence shows a commonality in emergent symptoms for which emergency medications can be used meet patients needs.

Objectives

Increase ability of nursing staff to give appropriate symptom management from time of admission to CSPCS to reduce suffering and meet patient and carer goals of care whilst reducing cost burdens of healthcare such as after hours medical visits, emergency department presentations etc.

\section{Methods}

An uncontrolled prospective pilot intervention research trial took place over 13 weeks recruiting 99 newly referred CSPCS patients and their carers in the ACT, giving them access to a parenteral Emergency Medication Kit (EMK) from time of admission.

An Audit at 2years post adoption in standard practice, provided further comparison. 
Results

\begin{tabular}{lll}
\hline Unwanted Outcomes Averted by use of EMK & Trial & Audit \\
After Hours PCMO Review & $94 \%$ & $98 \%$ \\
Hospital Admission & $92 \%$ & $98 \%$ \\
Hospice Admission & $94 \%$ & $100 \%$ \\
Deaths & & \\
PPoD Achieved & $56 \%$ & $91 \%$ \\
Home Deaths & $43 \%$ & $69 \%$ \\
Home Deaths with EMK Use & $77 \%$ & $80 \%$ \\
\hline
\end{tabular}

Conclusions

An EMK provides cost-effective, emergency symptom management for CSPCS patients to meet goals of care, reduce suffering and reduces burden on the healthcare system.

A third arm of research is now underway into inclusion of oral formulations to improve the EMK and patients' satisfaction and outcomes.

\section{eP363}

ELECTROCHEMOTHERAPY FOR THE PALLIATIVE TREATMENT OF CUTANEOUS METASTASIS AND MALIGNANT WOUNDS AMONG FILIPINO PATIENTS

\author{
C.M. Reyes-Habito ${ }^{I}$, E. Cutillar ${ }^{1}$, M. Manalo-Igot ${ }^{1}$, C. Ngelangel $^{I}$ \\ ${ }^{T}$ Asian Hospital and Medical Center, Asian Cancer Institute, Muntinlupa, \\ Philippines
}

\section{Introduction}

Cutaneous metastasis and malignant wounds decrease the quality of life of cancer patients. Electrochemotherapy is a localized treatment which enhances the uptake of chemotherapy through the application of electric pulses.

Objectives

This observation study was done to evaluate the efficacy, safety and clinical outcome of a four-electrode electrochemotherapy device (IQ Wave ${ }^{\circledR}$ ) for the treatment of cutaneous metastasis among Filipino patients

\section{Methods}

13 cutaneous metastasis patients were treated with 1-5 electrochemotherapy sessions at least 4 weeks apart from February to November 2017. Bleomycin or Cisplatin was given intratumorally, intravenously or both prior to application of electric pulses, based on the ESOPE protocol. Concomitant chemotherapy was given in 9 patients while 4 had none. Lesions were measured using a ruler and patients were evaluated using the RECIST criteria every 4 weeks.

Results

There were 11 BRCA patients, 1 head and neck SCCA patient, and 1 sarcoma patient. Small BRCA cutaneous metastasis lesions objectively resolved in 2/13 patients after two sessions. Large fungating tumors of head and neck SCCA and sarcoma and two large ulcerating BRCA tumors were palliatively controlled, with decrease of tumor exudate, bleeding and pain after at least 1 session. Disease progression, however occurred within 4 weeks post electrochemotherapy in $8 / 13$ patients. Adverse events were: post inflammatory hyperpigmentation (13), itchiness (7), post-procedure pain (3), skin infection (2), skin ulceration (1), nausea and vomiting (1), rash (1).

Conclusions

Electrochemotherapy can be used for the palliative control of cutaneous metastasis/ malignant wounds, with minimal side effects.

eP364

SYMPTOM MANAGEMENT IN PALLIATIVE CARE: EVIDENCE-BASED NURSING EDUCATION
E. Rieder ${ }^{1}$, S. Häusermann ${ }^{2}$

${ }^{T}$ Institute of Nursing, Institut of Nursing, Winterthur, Switzerland

${ }^{2} Z H A W$, Insitute of Nursing, Winterthur, Switzerland

\section{Introduction}

The module "Palliative Care" is integrated in the Bachelor program of Nursing at the Zurich University of Applied Sciences.

\section{Objectives}

The aim of the module is to provide the students with competencies to care for palliative patients in primary health care.

\section{Methods}

This learning arrangement is based on the definition of evidence-based nursing / health care; applying the best available research results (evidence) along with reflected clinical expertise, patient preferences and the resources (staff, material, time) available when making decisions about health care.

\section{Results}

Key aspects include the bio-psycho-social and spiritual assessment as well as building the capacity for evidence-based nursing interventions for symptom management; i.e. problems with nutrition, dyspnoea, pain, constipation and anxiety.

The students present a poster on the management of palliative symptoms as part of their examination. Some of these posters will be partly visualised

\section{Conclusions}

Changes in the Swiss educational infrastructure and health care, as well as the socio-demographic development demand the best available evidence of registered nurses.

Patient care must be based on the latest evidence-based practice. Student nurses should be introduced to the principles of evidence-based nursing as part of pre-registration education and apply this knowledge in the practice.

\section{eP365}

IS CLINICAL SUPPORT SUPERVISION IMPORTANT FOR NEWLY QUALIFYING PALLIATIVE CARE NURSES? CLINICAL PRACTICE EXPREINCE

S.M. Rithara ${ }^{1}$

${ }^{T}$ Kenya Medical Training College, NURSING, Nairobi, Kenya

\section{Introduction}

Palliative nursing training is one of the Kenya Medical Training College higher Diploma course. Qualifying palliative care nurses require clinical supervision to boost their work morale, encourage and share new ideas among the trainers nurses, mentors

\section{Objectives}

To find out the importance of supportive supervision in already qualified palliative nurses

\section{Methods}

A survey done between September 2013 to September 2017 on 73 qualified palliative care students and their mentors from KMTC program.

\section{Results}

clinical support supervision is importance and very challenging exercises. Improves patient's care through sharing new ideas and encouraging each other. 66\% trained PC nurses practicing, 34\% different department while $16 \%$ become mentors. $53 \%$ lack of managers support, $38 \%$ no Out patient clinics, $62 \%$ basic medicine available, clinical performance improved $70 \%$ campared first qualified class, $68 \%$ county nurses aware of training at KMTC. $72 \%$ lack of palliative books and references materials. $65 \%$ lack of supportive care locally and internationally. $32 \%$ in oncology/ palliative clinics. $64 \%$ need oncology incoperated with palliative program. $80 \%$ impoved palliative care while integrated with oncology while $10 \%$ not referred. $42 \%$ role conflict in oncology/palliative, $78 \%$ supportive improve their care, $22 \%$ no need. $48 \%$ prefers workshop while $52 \%$ one on one. $79 \%$ require manuals $21 \%$ notes and handouts. 


\section{Conclusions}

There is need for more interaction between newly qualified palliative nurses and seniors in the field. To improve palliative care services nurses need attend seminars/conferences and symposiums to learn new knowledge and skills. Programs set aside for supportive supervision to enhance the knowledge. Research on the benefit of integrated Oncology/palliative care clinic

\section{eP366}

THE INVOLVEMENT OF CANCER PATIENTS IN THE FOUR STAGES OF DECISION-MAKING IN CONTINUOUS SEDATION UNTIL DEATH. A QUALITATIVE STUDY

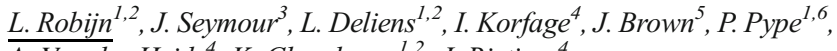
A. Van der Heide ${ }^{4}, K$. Chambaere ${ }^{1,2}$, J. Rietjens ${ }^{4}$

${ }^{1}$ Vrije Universiteit Brussel, Family Medicine and Chronic Care, Brussels, Belgium

${ }^{2}$ Ghent University, Internal Medicine, Ghent, Belgium

${ }^{3}$ University of Sheffield, School of Nursing and Midwifery, Sheffield, United Kingdom

${ }^{4}$ Erasmus University Medical Centre Erasmus MC, Department of Public Health, Rotterdam, The Netherlands

${ }^{5}$ De Montfort University, School of Nursing and Midwifery-Centre for the Promotion of Excellence in Palliative Care-, Leicester, United Kingdom

${ }^{6}$ Ghent University, Department of family Medicine and Primary Healthcare, Ghent, Belgium

\section{Introduction}

Involving patients in decision-making is considered to be particularly appropriate towards the end of life. Professional guidelines emphasize that the decision to initiate continuous sedation should be made in accordance with the wishes of the dying person and be preceded by their consent.

\section{Objectives}

To describe the decision-making process preceding continuous sedation until death with particular attention to the involvement of the person who is dying. Methods

Qualitative interviews with 26 physicians, 30 nurses and 24 relatives caring for 24 patients with cancer who received continuous sedation until death in Belgium, UK, and the Netherlands.

\section{Results}

We distinguished four stages of decision-making: initiation, information exchange, deliberation and the decision to start continuous sedation until death. There was wide variation in the role the patient had in the decisionmaking process. At one end of the spectrum (mostly in UK), the physician discussed the possible use of sedation with the patient, but took the decision themselves. At the other end (mostly in BE and NL), the patient initiated the conversation and the physician's role was largely limited to evaluating if and when the medical criteria were met.

Conclusions

Decision-making about continuous sedation until death goes through four stages and the involvement of the patient in the decision-making varies. Acknowledging the potential sensitivity of raising the issue of end-of-life sedation, we recommend building into clinical practice regular opportunities to discuss the goals and preferences of the person who is dying for their future medical treatment and care.

\section{eP367}

OUTCOMES OF HYPOFRACTIONATED RADIOTHERAPY IN ELDERLY PATIENTS WITH ADVANCED BLADDER CANCER

W. Rolski ${ }^{1}$, D. Kiprian ${ }^{1}$

${ }^{T}$ Maria Sklodowska Institute - Oncology Center, Department of Head and Neck Cancer, Warsaw, Poland

\section{Introduction}

Advanced bladder cancer may cause uncontrolled symptoms like pain or bleeding which often lead to reduced quality of life. Many patients are not suitable for radical treatment, therefore radiation therapy is commonly used in the palliative setting.

\section{Objectives}

The aim was to evaluate the efficacy of radiotherapy for improvement of local symptoms from bladder cancer in patients over the age of 75 considered unsuitable for curative treatment or systemic therapy through the advanced stage and/or comorbidities.

\section{Methods}

Between 2010 and 2017, 37 eligible patients with biopsy-proven bladder cancer received hypofractionated RT. Patients received 20 Gy in 5 daily fractions $(n=23)$ or single 6-7 Gy fraction $(n=14)$ according to KPS. We reviewed patient's demographics, performance status, relief of symptoms and toxicity.

\section{Results}

The median age was 79 years (range 75-87) and median KPS was 60 (range 40-70). The main indication was hematuria $(n=24)$, uncontrolled pain $(n=11)$ or urinary urgency $(n=2)$. Evaluation of palliative effect related to radiotherapy was conducted 3-4 weeks after treatment. In the absence of single dose effect (or symptom relapse) subsequent fraction was administered ( $\mathrm{n}=10$, range 3 weeks- 11 months). The rates of clinically relevant improvement in hematuria, reduction of pain and urinary urgency were $87 \%(21 / 24), 72 \%(8 / 11$, concomitantly with analgesic regimen modification) and 50\% (1/2) respectively. The median duration of response was 16 weeks (range 3 weeks -11 months). The acute or late toxicity was insignificant.

\section{Conclusions}

Hypofractionated radiotherapy manages successful and long-term palliation. Presented results suggest that even single fraction treatment also appears effective.

\section{eP368}

DEVELOPMENT OF MINI-COMPREHENSIVE GERIATRIC ASSESSMENT FOR THE ELDERLY CANCER PATIENTS BASED ON RELATIONSHIPS BETWEEN COMPREHENSIVE GERIATRIC ASSESSMENT ITEMS AND PERFORMANCE STATUS

H.M. ryoo ${ }^{1}$, K.U. Park ${ }^{2}$

${ }^{T}$ Daegu Catholic University Medical Center, Hemato-Oncology, Daegu, Republic of Korea

${ }^{2}$ Keimyung University Dongsan Hospital, Internal Medicine, Daegu, Republic of Korea

\section{Introduction}

The comprehensive geriatric assessment (CGA) is a multidimensional method used by oncologists to detect and evaluate multiple age-related problems and to plan and coordinate proper interventions. Because its main drawback is the time required, efforts have been made to evaluate screening instruments suitable for preliminarily assessing elderly patients. Objectives

The main aim of this study was to establish mini-comprehensive geriatric assessment for the elderly cancer patients (MCGA-C) based on the relationships between CGA items and performance status. We developed MCGA-C consisted of a total of 14 questions which were extracted from the 5 domains of CGA.

Methods

Patients aged $\geq 65$ years with cancer $(n=237)$ were evaluated by ECOGPS and 5 domains of CGA: Instrumental Activities Daily Living (IADL), the Mini Nutritional Assessment (MNA), Hospital Anxiety and Depression Scale (HADS), Charlson's Comorbid Index (CCI), MiniMental State Exam (MMSE). Functional Assessment Cancer TherapyGeneral (FACT-G) was used a reference test. 


\section{Results}

We tested the validity of using the receiver operating characteristics (ROC) curve. The value of the area below the ROC was $0.824(95 \%$ CI: $0.764-0.874)$ with a statistically significant degree of accuracy $(p<.0001)$. Through ROC curve analysis, Youden Index value of 55 points was determined as the poor health reference point. The discrimination ability of CGA and MCGA-C appeared to be quite similar.

Conclusions

On the basis of our data, MCGA-C is highly predictive of impaired functional status and can thus be considered a useful preliminary means of assessing older patients with cancer before undertaking a full CGA.

\section{eP369}

COST-EFFECTIVENESS ANALYSIS OF MANAGING CANCER AND LIVING MEANINGFULLY PSYCHOSOCIAL INTERVENTION FOR PATIENTS WITH ADVANCED CANCER

\section{U.Saha ${ }^{1}$, A. Ruco ${ }^{2}$, G. Rodin ${ }^{3}$, S. Hales ${ }^{3}$, C. Lo $^{3}$}

${ }^{T}$ Princess Margaret Cancer Center and University of Toronto, Regional Cancer Program and IHPME, Toronto, Canada

${ }^{2}$ University of Toronto and St.Michael's Hospital, Institute of Health Policy-Management and Evaluation, Toronto, Canada

${ }^{3}$ Princess Margaret Cancer Center, Supportive Care, Toronto, Canada

\section{Introduction}

Psychosocial interventions address the mental distress patients may experience during or after their cancer treatment. Managing Cancer and Living Meaningfully (CALM) is a brief, psychotherapy intervention aimed at facilitating psychological adjustment in individuals diagnosed with advanced cancer.

Objectives

The intervention is clinically effective but, the cost-effectiveness of this intervention remains to be explored.

\section{Methods}

A cost-effectiveness analysis from the perspective of the funder was conducted using a decision tree model. Model inputs were derived from RCT trial data and costs were estimated using data holdings at the Princess Margaret Cancer Centre (PM). Change in depression severity tiers as measured by PHQ-9 questionnaire at 6 months was the primary outcome. The incremental costeffectiveness ratio (ICER) was calculated and one-way and probabilistic sensitivity analyses were conducted to assess the robustness of the findings.

\section{Results}

A total of 54 participants in the intervention arm and 59 in the control arm were included in our study. The ICER for the base case scenario was $\$ 1,439.15 /$ tier. This ranged from $\$ 798 /$ tier to $\$ 4,949 /$ tier when sensitivity analyses were conducted. Monte Carlo probability distribution histogram showed that the ICER is less than $\$ 1,300 /$ tier in $50 \%$ of simulations and less than $\$ 5,000$ / tier in $97.5 \%$ of model runs.

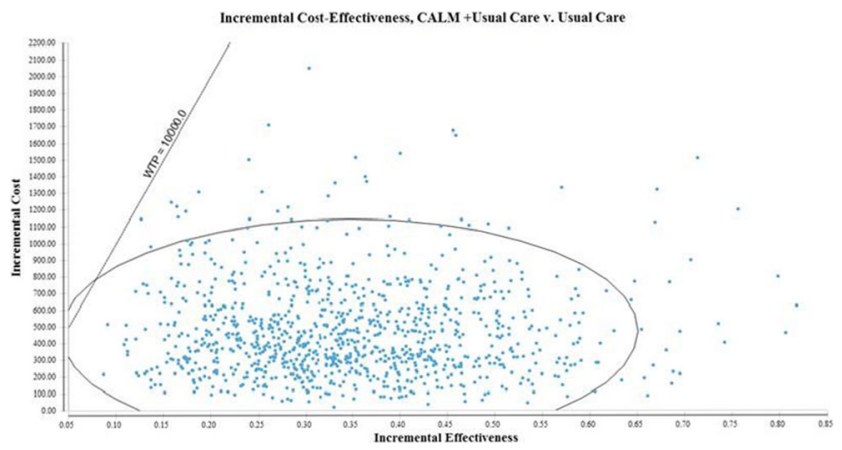

Table 2. Model inputs for the base case scenario

\begin{tabular}{|c|c|c|c|c|}
\hline $\begin{array}{l}\text { Change in PHQ-9 tier (primary } \\
\text { outcome) at } 6 \text { months }\end{array}$ & $\begin{array}{l}\text { Value/ } \\
\text { Proportion }\end{array}$ & $\begin{array}{l}\text { Payoff gained } \\
\text { per case }\end{array}$ & $\begin{array}{l}\text { Total } \\
\text { effect }\end{array}$ & $\begin{array}{l}\text { Average } \\
\text { Cost }\end{array}$ \\
\hline \multicolumn{5}{|c|}{ Intervention arm (CALM + Usual Care) } \\
\hline Better by 3 tiers & $11 \%$ & 3 & 0.333 & \\
\hline Better by 2 tiers & $35 \%$ & 2 & 0.704 & \\
\hline Better by 1 tier & $17 \%$ & 1 & 0.167 & \\
\hline No change & $19 \%$ & 0 & 0 & \\
\hline Worse by 1 tier & $2 \%$ & -1 & -0.019 & \\
\hline Death & $17 \%$ & 0 & 0 & \\
\hline Total & & & 1.18 & $\$ 474.92$ \\
\hline \multicolumn{5}{|l|}{ Control arm (Usual Care alone) } \\
\hline Better by 3 tiers & 12 & 3 & 0.356 & \\
\hline Better by 2 tiers & 19 & 2 & 0.373 & \\
\hline Better by 1 tier & 19 & 1 & 0.186 & \\
\hline No change & $34 \%$ & 0 & 0 & \\
\hline Worse by 1 tier & 8 & -1 & -0.085 & \\
\hline Death & 8 & 0 & 0 & \\
\hline Total & & & 0.85 & So \\
\hline
\end{tabular}

Figure 2. Tornado graph for the one-way sensitivity analyses.

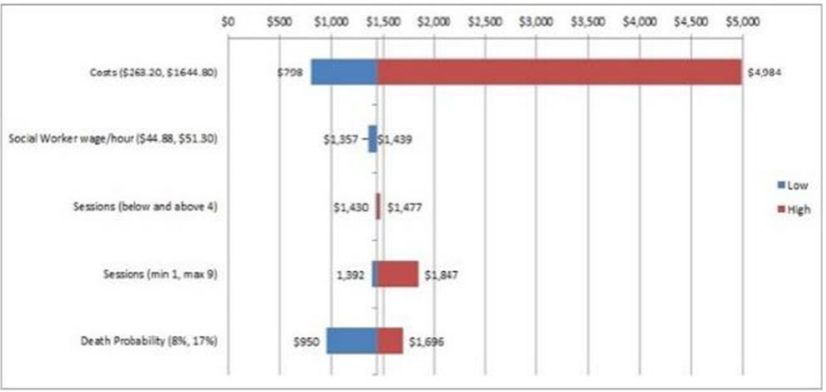

Conclusions

Cost-effectiveness analysis for the CALM intervention resulted in an ICER of $\$ 1,439.15$ with $97.5 \%$ of model runs falling below the $\$ 5,000 /$ tier willingness to pay threshold.Results suggest that the CALM intervention may be delivered in a cost-effective manner to reduce depression by 1 tier in PHQ-9 compared to usual care alone in a large urban healthcare centre.

\section{eP370}

CLINICAL PRACTICE TOOLS FOR MULTIDIMENSIONAL UNMET HEALTHCARE NEEDS IN ADVANCED CANCER: A SYSTEMATIC LITERATURE REVIEW

E.B. Schmidt ${ }^{1}$, F. Strasser $^{1}$

${ }^{1}$ Cantonal Hospital St. Gallen, Oncological Palliative Medicine- Clinic Medical Oncology and Hematology-Dept. Internal Medicine, St. Gallen, Switzerland

\section{Introduction:}

Unmet needs of advanced cancer patients may indicate quality cancer care. By tracking unmet needs in clinical practice, palliative interventions can be catered to align with patient priorities, also by supporting healthcare providers' (HCP) decision making about tailored intervention delivery, namely when encountering complex needs. Current need tools, however, focus on outcomes less routine care.

\section{Objectives}

To identify everyday clinical practice tools for recognizing and addressing unmet healthcare needs for advanced cancer patients. 
Methods

PRISMA methodology was followed exploring the databases PubMed and CINAHL in January 2018 applying three search strings (using both exploded heading terms and free text): 1) patient need;2) validated instrument; and 3) clinical practice. Cancer was intentionally not included to discover tools used for other diseases. After eliminating duplicates, 1428 article titles/abstracts were reviewed by two researchers until consens for orginial work excluding survivor or caregiver populations, lacking patient needs assessment in title or abstract; focused only on quality-of-life, satisfaction or single symptom. Hand searches were conducted in retrieved papers.

Results

43 articles ( 38 search, 5 handsearch) reporting needs tools were included. However, none covered all criteria demanded for needs monitoring in routine clinical practice: a) concrete real-time intervention oriented, b) designed to assess patient-perceived unmet need, and c) inclusive of multiple dimensions of palliative interventions (physical and psychological symptoms, illness/prognosis understanding, professional support networks, decisional processes, end-of-life preparation, spirituality, family support) and supportive care.

Conclusions

A new, validated tool is needed to track unmet healthcare needs of advanced cancer patients in routine care to guide tailored interventions

\section{eP371}

DIURNAL VARIATION PATTERN OF ANTIPSYCHOTIC DRUG, BENZODIAZEPINE DOSING IN TERMINAL CANCER PATIENTS

Y. Seol ${ }^{1}$

${ }^{T}$ Pusan National University Hospital, 8, Busan, Republic of Korea

\section{Introduction}

Delirium is highly prevalent in those with serious or advanced medical illnesses. It is associated with many adverse consequences, including significant patient, family, and health care provider distress.

Anecdotal observation suggests that cancer patients in palliative care units receive higher doses ofpsychoactive medications during evening shifts than day and night shifts.

\section{Objectives}

To determine the dosing patterns and total doses of psychoactive medications according to nursing shift in a cohort of cancer patients in a palliative care unit.

Methods

The sample consisted of 256 patients admitted to the palliative care unit. Data on time, dosage, and route of administration of the drugs were collected.

Results

A total of 194 patients qualified for the drug model for first generation antipsychotics (haloperidol, quetiapine) and 62 patients for benzodiazepine (lorazepam). The mean age was 63 years. Doses of both benzodiazepin and first generation antipsychotics were higher during the evening shifts (4 PM to midnight) than during the day or night shifts. Compared with women, men received higher doses of both benzodiazepin and first generation antipsychotics.

\section{Conclusions}

In this observational sample of terminal cancer patients, data indicated a positive association between dose levels of benzodiazepin and first generation antipsychotics during the evening nursing shifts relative to other shifts. Further investigation is needed to determine potential causes and to evaluate the impact on outcomes of sleep deprivation and delirium

\section{eP372}

AUDIT OF HOME PARENTERAL NUTRITION IN PALLIATIVE CARE PATIENTS

\author{
C. Shaw ${ }^{1}$, L. Eldridge ${ }^{1}$, J. Droney ${ }^{2}$, A. Halley ${ }^{2}$ \\ ${ }^{T}$ The Royal Marsden NHS Foundation Trust, Nutrition and Dietetics, \\ London, United Kingdom \\ ${ }^{2}$ The Royal Marsden NHS Foundation Trust, Symptom Control and \\ Palliative Care Team, London, United Kingdom
}

\section{Introduction}

The provision of parenteral nutrition in palliative care is controversial in the United Kingdom. Home Parenteral Nutrition (HPN) is not recommended for patients with incurable disease and a short life-expectancy. HPN may be considered for patients with malignant obstruction, a prognosis of more than 3 months and a WHO performance status of less than 2.

\section{Objectives}

To determine whether the provision of home parenteral nutrition for palliative patients at a tertiary cancer centre in the UK was in accordance with European guidance (Bozzetti et al, 2009).

\section{Methods}

A retrospective audit of patient records was undertaken at a tertiary cancer treatment centre.

\section{Results}

Between June 2012 and May 2017, 34 patients were considered for home parenteral nutrition with 22 patients being discharged on HPN. The average age was 56.5 years with range $31-84$ years with the majority of patients either having gastrointestinal $(45 \%)$ or gynaecological $(40 \%)$ cancers. PN was used appropriately in patients who were unable to eat $(100 \%)$ and in median survival time of longer than $2-3$ months (median survival 121 days). However, the standard was not met in terms of documentation of prognosis with $27 \%$ recorded and performance status with $45 \%$ having this documented in the electronic patient records.

\section{Conclusions}

The provision of home parenteral nutrition is complex, requires specialist management and appropriate patient selection. Recommendations have been made following the audit regarding patient selection, documentation of prognosis and performance status and improvements to methods of communication with the patient and specialist services.

\section{eP373}

SYMPTOM PREVALENCE AND FUNCTIONAL STATUS AMONG PATIENTS WITH ADVANCED CANCERS OF THE HEAD AND NECK

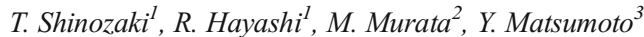

${ }^{T}$ National Cancer Center Hospital East, Department of Head and Neck Surgery, Kashiwa, Japan

${ }^{2}$ National Cancer Center Hospital East, Department of Nursing, Kashiwa, Japan

${ }^{3}$ National Cancer Center Hospital East, Department of Palliative Medicine, Kashiwa, Japan

\section{Introduction}

Little is known about quality of life and functional status of patients with terminally ill head and neck cancers.

\section{Objectives}

To clarify the symptom and functional status with terminal head and neck cancer.

\section{Methods}

We conducted a prospective, observational study to examine quality of life and functional status in terminally ill head and neck cancer patients. EORTC QLQ C15-PAL was used to assess QOL. QOL score and Clinician reported outcome were assessed every week. 


\section{Results}

Of the 29 patients meeting inclusion criteria, 27 were observed until death. There was no significant difference in the quality of life score between baseline and Week 3. Seventeen patients $(63.0 \%)$ could speak and nine patients $(33.3 \%)$ could have oral intake upon study entry. Twenty-two patients $(81.5 \%)$ received enteral nutrition. Eight patients fed through a percutaneous gastrostomy(PEG) tube. Thirteen patients $(48.1 \%)$ required dressing changes for fungating tumors. No patient had hemorrhagic shock. Patients with oral cancer tend to receive a benefit with PEG.

Conclusions

We observed how patients with HNC live during the last month of life in an acute hospital setting. Our data suggests that route of nutrition intake may play a role in $\mathrm{QOL}$

\section{eP374}

AN EXPLORATION OF THE VIEWS OF HEALTHCARE STAFF TOWARDS RAPID UNANTICIPATED DISCHARGE FOR END OF LIFE CARE IN AN ACUTE HOSPITAL IN SINGAPORE

S. Shivananda ${ }^{1}$, G. Pang $^{2}$

${ }^{T}$ National Cancer Centre, Palliative Medicine, SINGAPORE, Singapore

${ }^{2}$ National Cancer Centre, Dept of Palliative Medicine, Singapore, Singapore

\section{Introduction}

Terminal Discharge (TD) is a process of discharging a terminally ill patient home as per the wishes of the patient/family members to fulfill the wish of a home death. Little is known about how staff implementing this discharge process feel about the practice in Singapore

Objectives

The aim of this study was to explore the experience of doctors and nurses in dealing with the TD and hereby determine the barriers and facilitators involved during the process

Methods

Face-to-face semi-structured interviews were conducted in English with eight doctors and nurses (paired sample) separately who were involved in implementing the TD in an acute hospital in Singapore. All interviews were audio taped,transcribed verbatim and analysed using qualitative framework analysis

\section{Results}

16 encounters were analysed and 5 major categories and several sub themes factors that would affect the participants were identified:

(1) Logistic complexity of organising a TD (2) Emotionally charged /stressful nature of the discharge process (3) Lack of knowledge and practical experience (4) Pre-empt TD (5) Coping of the staff/Perception of TD Conclusions

$\mathrm{TD}$ is a process that requires several tasks to be completed within a short period of time to be able to discharge a terminally ill patient to fulfill the wish for a home death. Staffs were emotionally charged during this process and the knowledge and practical experience of the staff played a crucial part in the speedy implementation of TD

\section{eP375}

TERMINAL ILLNESS \& END OF LIFE CARE: THE IMPACT OF THE BIOPSYCHOSOCIAL- SPIRITUAL MODEL IN INDIAN PATIENTS

\section{S.P. Singh Rana ${ }^{1}$, S. Singh ${ }^{2}$, A. Bundiwal ${ }^{2}$ \\ ${ }^{T} M D-F I P P-D P M-F I A P M$, Dept of Anaesthesia-Pain \& Palliative med- icine, Bhopal, India \\ ${ }^{2}$ Fortis Healthcare, Dept of Integrated Oncology, Noida, India}

\section{Introduction}

Patients with cancer or life limiting illness entering in last phase of illness need expertise and multidisciplinary care. A combination of a rapidly changing clinical situation and huge psychosocial and spiritual demands present a big challenge that can only be met with competence, commitment and human compassion. Recognizing the terminal illness and last few hours of life along with compassionate Biopsychosocial care helps to keep alive the hope and improves quality of life of these patients.

\section{Objectives}

The aim was to detect the depression among patients and relatives, functional restoration after providing care, hope survival and pain relief.

\section{Methods}

We applied the Biopsychosocial- spiritual model of care in the treatment of 30 cancer patients at the last few days of terminal illness.

Results

Will be discussed in the conference.

\section{Conclusions}

A more comprehensive model of managing end of life care issues is needed that is mechanism-based and multimodal, using combination therapies including multidisciplinary treatment where appropriate, tailored to the needs of an individual, with the aim to optimize pain relief with minimization of adverse effects.

\section{eP376}

IDENTIFYING THE COMMUNICATION, INFORMATION AND SUPPORT NEEDS OF STAKEHOLDERS IN PALLIATIVE AND END-OF-LIFE CARE USING A QUALITATIVE ANALYSIS OF FREE-TEXT DATA.

\author{
S. Sivell ${ }^{1}$, J. Baillie ${ }^{2}$, J. van Godwin ${ }^{3}$, A. Byrne ${ }^{1}$, A. Nelson ${ }^{1}$ \\ ${ }^{T}$ Cardiff University, Marie Curie Palliative Care Research Centre- \\ Division of Population Medicine- School of Medicine, Cardiff, United \\ Kingdom \\ ${ }^{2}$ Cardiff University, School of Healthcare Sciences, Cardiff, United \\ Kingdom \\ ${ }^{3}$ Cardiff University, DECIPHer Centre-Social Sciences, Cardiff, United \\ Kingdom
}

\section{Introduction}

Palliative and end-of-life care research is under-recognised; stakeholders are likely to have a range of unmet communication, information and support needs. The James Lind Alliance (JLA) Priority Setting Partnership (PeolcPSP) identified the pertinent 'treatment uncertainties' for palliative and end-of-life care, including cancer and focusing on interventional based research. However, respondents submitted comments and questions which did not always meet the criteria of the JLA methodology.

Objectives

To undertake a supplementary analysis of free text data generated by the JLA PeolcPSP national survey (UK), focusing on communication, information and support needs.

\section{Methods}

A supplementary thematic qualitative analysis of the JLA PeolcPSP freetext data was undertaken in the final dataset of 1403 responses. The original coding framework was reviewed and refined until agreement was reached, concentrating on communication and decision-making.

\section{Results}

Four overarching themes were identified:

1) Availability, accessibility and improving information: focusing on support and guidance, as well as improving and finding information.

2) Effectiveness of communication between stakeholders: improve communication, openness and transparency between stakeholders; improving the communication of diagnosis and prognosis; exploring decisionmaking responsibilities.

3) Eliciting preferences and values: treatment decisions, Advance Care Planning and preferred place of care.

4) Spiritual and psychosocial needs: providing emotional and psychological supportfor patients, carers and professionals. 


\section{Conclusions}

Qualitatively analysing free text data identified a wide range of communication and decision-making concerns, as well as psychosocial support, which may not always fit into interventional methodology. Both quantitative and qualitative methodologies are needed to address the needs of all stakeholders in palliative and end-of-life care.

\section{eP377}

PILOT INVESTIGATION OF A SPA BATH FOR MANAGING PATIENT PAIN, ANXIETY AND WELL-BEING IN AN ACUTE PALLIATIVE CARE SETTING

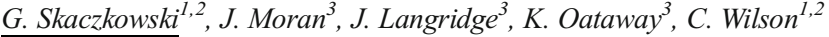 \\ ${ }^{T}$ La Trobe University, School of Psychology \& Public Health, Bundoora, \\ Australia \\ ${ }^{2}$ Austin Health, Olivia Newton-John Cancer Wellness \& Research \\ Centre, Heidelberg, Australia \\ ${ }^{3}$ Austin Health, Palliative Care Unit, Heidelberg, Australia
}

\section{Introduction}

The goal of palliative care is to reduce the symptom burden and improve quality of life for patients at the end-of-life. Complementary therapies provide an avenue for addressing patient concerns where pharmacological measures are inappropriate for medical reasons or do not align with patients' personal, cultural or health beliefs. One such intervention that has received relatively little attention in a palliative care context is the use of water-based relaxation.

\section{Objectives}

This pilot study examined the impact of a simple spa bath for reducing symptoms and improving well-being within a palliative care hospital setting. Methods

52 patients completed (or were assisted in completing) pre- and post-bath measures of pain, anxiety and well-being.

\section{Results}

The provision of a spa bath decreased patients' self-reported pain and anxiety, and improved self-reported well-being (all $p$ 's $<.001$ ).

Conclusions

These results suggest that the simple provision of a spa bath can improve patients' quality of life. This intervention can be conducted within the normal course of nursing duties and requires minimal additional training. These preliminary results justify further investigation of these effects.

\section{eP378}

ACUTE CARE MEDICAL INTERVENTIONS IN THE PALLIATIVE CARE UNIT VERSUS INPATIENT HOSPICE

M. Vidal

${ }^{T} M D$ Anderson Cancer Center, Palliative Care, Houston, USA

\section{Introduction:}

In a survey in the United States, only $23 \%$ reported having dedicated palliative care beds. Barriers that are often cited include poor reimbursement for services, limited institutional support and resources. Patients admitted to an Acute Palliative care unit (APCU) benefit from the multidisciplinary approach from an interdisciplinary team. There could be occasionally misconceptions about the differences between inpatient hospice (IH) and an APCU. Even though the psychosocial component of palliative care is extremely important a significant amount of medical interventions also are necessary to provide optimal care during this time Objectives

Identify the differences in medical interventions between APCU and IH. Methods

A retrospective chart reviewed was performed in 100 consecutives patients admitted to the APCU and 112 patients admitted to $\mathrm{IH}$ prior to
October 2013. From this data we identified the acute medical interventions ordered by the palliative Care specialist during the first 5 days of admission.

\section{Results}

A total of 100 patients from the APCU and 112 patients from IP were reviewed. In the APCU $100 \%$ of patients had iv fluids vs $7 \%$ in $\mathrm{IH}$. Antibiotics were given in $52 \%$ of APCU patients vs $2 \%$ in the IH. Steroids were given in $48 \%$ of APCU patients vs $30 \%$ in inpatient hospices.

\section{Conclusions}

The APCU in comparison to inpatient hospices had higher rate of acute medical interventions with the most common been iv fluids, antibiotics, laboratory and radiologic diagnostic tests. This represent the importance of Acute Palliative Care Units in advanced cancer patients with complicated situations to achieve comfort when transitioning to EOL

\section{eP379}

EXPERIENCE-BASED DESIGN：USING QUALITY IMPROVEMENT STRATEGIES TO IDENTIFY FAMILY AND STAFF EXPERIENCES IN CONVERSATIONS AT END-OF-LIFE

\author{
J. Wong $^{1}$, B. Hollister ${ }^{1}$, E. McRobert ${ }^{2}$ \\ ${ }^{1}$ Sunnybook Health Sciences Centre, Veterans Centre, Toronto, Canada \\ ${ }^{2}$ University of Toronto, Factor-Inwentash Faculty of Social Work, \\ Toronto, Canada
}

\section{Introduction}

In this facility, "palliative" and "long-term" care increasingly overlap due to aging of current and potential residents. Increased turnover has increased the need for quality end-of-life care. Effective communication impacts the quality of care at end-of-life, but "communication" may hold different meaning for different stakeholders. The need to fully understand stakeholder perspectives is essential when designing quality improvement projects at end-of-life.

\section{Objectives}

The aim of this project was to use stakeholder experiences to guide education priorities related to end-of-life communication practices.

Methods

1. Key informant interviews were conducted with "most involved persons" (MIPs) who had experienced the death of a loved one in the target patient care areas.

2. Staff surveys were used to collect qualitative information regarding education needs with respect to conversations at endof-life.

3. Modified emotional mapping techniques from experience-based design (EBD) were used to translate MIP and staff experiences into opportunities for improvement.

Results

The use of MIP and staff informants allowed for the identification of perceived and mis-/un-perceived education needs. Learning objectives were designed based on alignment between MIP and staff themes. Converging themes from these groups were: 1) care planning and disease management, 2) anticipatory grief, and 3) the impact of power and roles on discussions at end of life.

\section{Conclusions}

EBD allows for identification of key areas for quality improvement, and leverages direct experiences of patients/families and staff to maximize frontline engagement. This project demonstrates the how the application of qualitative strategies in quality improvement can be a motivator for change. 
eP380

PILOT RANDOMIZED PHASE II TRIAL OF THE ENHANCING QUALITY OF LIFE IN PATIENTS (EQUIP) INTERVENTION FOR PATIENTS WITH ADVANCED LUNG CANCER

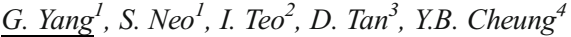 \\ ${ }^{T}$ National Cancer Centre Singapore, Palliative Medicine, Singapore, \\ Singapore \\ ${ }^{2}$ Duke-NUS Medical School, Lien Centre for Palliative Care, Singapore, \\ Singapore \\ ${ }^{3}$ National Cancer Centre Singapore, Medical Oncology, Singapore, \\ Singapore \\ ${ }^{4}$ Duke-NUS Medical School, Centre for Quantitative Medicine, \\ Singapore, Singapore
}

\section{Introduction}

New models of care that are effective and feasible for widespread implementation need to be developed for the delivery of early supportive and palliative care for advanced cancer patients.

Objectives

The main objective was to pilot the Enhancing Quality of life in Patients (EQUIP) intervention for patients with advanced lung cancer, to determine the feasibility and acceptability of the EQUIP intervention, data completion rate of patient-reported outcome measures in the context of the EQUIP trial and a tentative estimate of efficacy of the EQUIP intervention on quality of life and mood.

Methods

This was a pilot randomized phase II trial. Patients randomized to the control group received usual care, which comprised standard oncology care as well as referral for palliative care services if deemed appropriate by the primary oncologist. In addition to usual care, patients randomized to the intervention group individually received the EQUIP intervention which comprised four face-to-face educational sessions with a nurse.

Results

A total of 69 patients were recruited. In the intervention group, 30 of 35 patients $(85.7 \%)$ completed all four EQUIP sessions. Acceptability of the EQUIP sessions was high: All patients were satisfied with the topics shared and felt they were useful. However, there was no significant difference between intervention and control groups in quality of life at 12 weeks after baseline.

Conclusions

This pilot study showed that nurse-directed face-to-face educational sessions were feasible and acceptable to advanced lung cancer patients. However, there was no indication of benefit of the EQUIP intervention on quality of life and mood.

\section{eP381}

OUTCOMES OF EMBEDDED PALLIATIVE CARE OUTPATIENTS CONSULTS ON TIMING OF PALLIATIVE CARE ACCESS, SYMPTOMS, AND END-OF-LIFE QUALITY INDICATORS AMONG ADVANCED NON-SMALL CELL LUNG CANCER PATIENTS

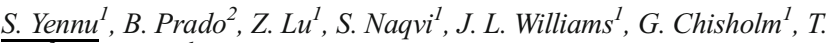 \\ Lim $^{3}$, E. Bruera \\ ${ }^{1}$ University of Texas MD Anderson Cancer Center, Palliative- \\ Rehabilitation \& Integrative Medicine, Houston, USA \\ ${ }^{2}$ Hospital Israelita Albert Einstein, Department of Oncology and \\ Hematology, Sao Paulo, Brazil \\ ${ }^{3}$ Veterans Health Service Medical Center, Department of Internal \\ Medicine-Division of Hematology and Oncology, Seoul, Republic of Korea
}

\section{Introduction}

There are no studies comparing the outcomes of embedded (EPC) consults as compared to independent outpatient palliative care consults (PC).

\section{Objectives}

To determine the timing of palliative care access, symptoms and EOL outcomes of advanced non-small cell lung cancer patients referred to EPC.

\section{Methods}

We retrospectively reviewed a random sample of EPC (Aug, 2012 - Jun, 2013) and PC consults (Jan 2009 -Jul 2012) at MD Anderson Cancer Center. Baseline features, symptoms (ESAS), EOL quality outcomes (intensive care unit deaths and admissions, emergency center visits, hospitalizations and length of stay, cancer treatments, hospice discussions and referrals, advanced care planning (ACP) discussions, and completion of advanced directives), time from referral to first consult, ACP date to death, and overall survival from consult to death were retrieved.

Results

340 were evaluable $(\mathrm{EPC}=147)$. At baseline, mean ECOG $(2.2$ vs $1.9, \mathrm{p}<.001)$, and median pain (6 vs $5, \mathrm{p}=.038$ ) were higher in EPC. Time from referral to first consult was shorter (median 0 vs 7 days, $\mathrm{p}<.001)$ and dyspnea was better in EPC at follow-up $(-1$ vs $0, \mathrm{p}=.039)$. A higher proportion in EPC (77\% vs $58 \%, \mathrm{p}<.001)$ had ACP discussions and these occurred earlier (median 4 vs 1 month before death, $\mathrm{p}<.001)$. No other significant differences in symptoms or EOL outcomes were found.

\section{Conclusions}

EPC consults at referral had earlier access and worse pain and performance status. EPC was not associated with significant improvement in symptoms or EOL outcomes except for better dyspnea control, and more frequent as well as earlier ACP discussions.

\section{eP382 \\ ABOUT SOME MENTAL DISORDERS IN CANCER PALLIATIVE CARE}

B. Zaydiner ${ }^{1}$, S. Savina ${ }^{2}$, V. Fedunenko ${ }^{3}$

${ }^{T}$ Regional Cancer Hospital, Out-Patient Care, Rostov-on-Don, Russia

${ }^{2}$ Medical Centre "Hyppocrates", Outpatient Care, Rostov-on-Don, Russia

${ }^{3}$ State Medical University, Surgery \#3, Rostov-on-Don, Russia

\section{Introduction}

As the mental disorders in cancer patients are underrecognized, such situation burdens patients' state

\section{Objectives}

To assess management options for some of these disorders in bedside settings

\section{Methods}

Observational survey was undertaken. The patients were evaluated at home; neuropsychiatric manifestations were detected with clinical examination \& routine tests (usually MMSE and CAM).

Results

1042 patients (men 489 , women 553 of an average age $66.7 \pm 5.8$ ) were consulted for variety of malignancies. Mental disorders were identified in 437 of 1042 (41.9\% of total cohort), nosologic prevalence were: depressions \& other mood disturbances 153 patients (35.0\%), cognitive impairment 92 (21.1\%), delirium (hyperactive, hypoactive, mixed) 186 patients $(42.6 \%)$, other disorders $6(1.4 \%)$. The most difficult cases for differentiating were the ones when delirium was superimposed on dementia (38 patients). The delirium's fluctuating consciousness permitted to set it apart from dementia. Identification of the new-onset delirium necessitated immediate intervention which contributed to disorder reversal or at least improvement.

\section{Conclusions}

The risk factors for delirium were a MMSE score $<20$, visual \& hearing deficit, dehydration. Being mentally aware is a preference reiterated by cancer patients 


\section{eP383}

MEANING CENTERED PSYCHOTHERAPY GROUP FOR CAREGIVERS OF PALLIATIVE CANCER

A. Zemaitis ${ }^{1}$, G. Bulotiene ${ }^{2}$

${ }^{T}$ Vilnius University, Faculty of Medicine, Vilnius, Lithuania

${ }^{2}$ National Cancer Institute, Department of Physical Medicine and

Rehabilitation, Vilnius, Lithuania

\section{Introduction}

Taking care of family members ill with advanced cancer causes a significant psychological problem. There is a lack of interventions addressing existential issues in caregivers. Meaning Centered Psychotherapy (MCP) is a psychotherapeutic intervention which includes new treatment approaches designed to enhance the spiritual well-being and meaning of life.

Objectives

The objective of our study is to describe the application of MCP to the caregivers of patients with advanced cancer in Lithuania.

Methods

A case study of 2 participants from the MCP-C group will be presented. The participants' mental state was measured with The Functional Assessment of Chronic Illness Therapy - Spiritual Well-Being scale (FACIT-Sp) and Distress Thermometer (DT) questionnaires.

Results

We observed the growth of the historical source of meaning in Member 1, including an increased understanding of her and her family origins. The acceptance of her father's disease led to a significant growth of the attitudinal source of meaning as well.

Member 2 had a significant increase in the creative source of meaning. Member 2 also changed the attitude towards her ill husband's persistent care. The anguish she felt became the meaning of life.

Both members emphasized the importance of group support.

Members' spiritual well-being increased during MCP: at the beginning FACIT-Sp avg. score 23; in the end avg. score 38 . DT average values decreased from 6.8 to 2.5 .

Conclusions

This case study suggests MCP to be a promising method to increase the spiritual well-being and to reduce existential distress in the caregivers of cancer patients.

\section{eP384}

SURVEY ON RESILIENCE AND ITS INFLUENCING FACTORS AMONG SPOUSES OF ADVANCED CANCER PATIENTS IN MAINLAND CHINA

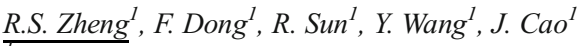 \\ ${ }^{T}$ Tianjin Medical University Cancer Institute and Hospital, Department \\ of Hepatobiliary Cancer, Tianjin, China
}

\section{Introduction}

Resilience is related to psychological adjustment to cope with stressful encounter. Emphasis on physical and mental health of caregivers has been a hot topic in clinics and research area. Spouses as the most important caregivers take responsibility of care, and offer support to cancer patients. However, little is known about advanced cancer patients' spouses' resilience.

Objectives

To describe resilience among Chinese spouses of cancer patients who are in end-of-life stage and to explore its influencing factors.

\section{Methods}

210 participants were recruited from January 2017 to February 2017 in a city, China. The questionnaires, including Connor-Davidson Resilience Scale-10, Zarit Caregiver Burden Interview, Trait Coping Style Questionnaire and self-designed demographic questionnaire, were delivered to potential participants. The data were analysed.

\section{Results}

192 participants completed the questionnaire. The overall resilience score for paricipants was $57.12 \pm 13.56$ which indicated that these participants were in middle level of resilience. There was a strong relationship between resilience and positive coping style $(\mathrm{r}=0.412, \mathrm{p}=0.001)$, negative coping style $(\mathrm{r}=0 .-314$, $\mathrm{p}=0.000)$, and caregiving burden $(\mathrm{r}=0 .-302, \mathrm{p}=0.010)$. The spouses who had greater resilience levels were male, with higher education and income, medical insurance and with assistant-caregiver. Five factors were significantly independent predictors explaining $48 \%$ of the variance of resilience, including income, assistant-caregiver, caregiving burden, positive and negative coping style.

\section{Conclusions}

The resilience of advanced cancer patients' spouses was moderate, and it was associated with income, assistant caregiver, caregiving burden and coping style. Nursing staff are advised to promote the resilience of advanced cancer patients' spouses, and nursing educators, researchers should develop appropriate programs aimed to improve their resilience.

\section{eP385 \\ ACHIEVING TIMELY SYMPTOM CONTROL FOR PATIENTS NEEDING OPIOID INFUSIONS: A QUALITY IMPROVEMENT PROJECT}

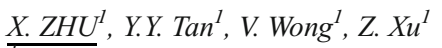

${ }^{T}$ National Cancer Centre Singapore, Division of Palliative Medicine, Singapore, Singapore

\section{Introduction}

In patients with poorly controlled pain/dyspnoea, continuous opioid infusions (COI) provide fast and effective symptom control. Singapore National Guidelines for Palliative Care recommend a turnaround time of $<1$ hour for urgent orders, but delays in initiation of COI may occur, impeding the provision of symptom relief for patients. Therefore, a quality improvement project was undertaken

\section{Objectives}

To achieve turnaround time of $<60$ minutes in patients prescribed COI for moderate to severe pain/dyspnoea in a medical ward within a tertiary hospital Methods

Average baseline data of the COI turnaround time is 101 minutes. Based on the data, a flowchart to delineate common causes of delay, brainstorming of possible interventions was done by the study team consisting of a doctor, palliative care nurses, ward nurses and a pharmacist. As this is a quality improvement project, Plan-Do-Study-Act (PDSA) cycles were used to test the effectiveness of each intervention. There was ongoing data collection of the abovementioned data fields.

Results

Baseline data was collected from April-May 17: 57.14\% of total 21 cases had moderate to severe symptoms, only $33.33 \%$ cases got COI set up within 60 mins. 4 interventions targeting nursing, pharmacy and medical workflows were tested since $25 / 07 / 17$. Till date, intervention involving workflow standardization and placement of flowcharts at medication carts as reminders for nurses displayed preliminary effectiveness in reducing COI turnaround time: $100 \%$.

\section{Conclusions}

Standardizing workflow and providing reminders for nurses via flowchart format at medication carts showed preliminary effectiveness in reducing COI turnaround time. Results will be updated after data collection completes in Jan 2018.

\section{eP386}

INTERVENTIONS TO MANAGE COMPASSION FATIGUE IN ONCOLOGY NURSES: A SYSTEMATIC PRISMA REVIEW

\author{
E. Akgün Çıtak ${ }^{l}$, S. Kav ${ }^{l}$ \\ ${ }^{T}$ Baskent University, Health Sciences Faculty Nursing Department, \\ Ankara, Turkey
}




\section{Introduction}

Oncology nurses are regularly exposed to high-stress situations that may lead to compassion fatigue. Types of interventions and evidence of its effectiveness on compassion fatigue hasn't been established.

Objectives

The aim of the review is to determine effectiveness of the interventions to manage compassion fatigue in oncology nurses.

\section{Methods}

Electronic databases (CINAHL®, PubMed and WOS) were used for searching the studies with the following key words "compassion fatigue", "interventions or strategies" and "oncology nursing". The methodology used for this systematic review was based on the Preferred Reporting Items for Systematic Reviews and Meta-analyses (PRISMA) guidelines. Identified publications were screened by using the following inclusion criteria; randomized controlled trials (RCTs), intervention studies and English language full text journals published in last 10 years. Descriptive studies, reviews, conference abstracts, letters to the editor were excluded. Results

The initial search identified 64 articles and 8 studies met eligibility criteria. Majority (5) of the studies from USA and rest of them were from Canada and Portugal. Non-randomized, pre-test and post-test design were used in intervention studies. Most of the studies were pilot and included small sample ranging from 10 to 45 participants. Effectiveness of the interventions were measured by The Professional Quality of Life Scale. Mindfulness, support group, knitting and education were applied over 4 to 6 weeks period. The interventions were effective in decreasing compassion fatigue level except one study that using mobile application. Conclusions

The findings of these small scale studies support that interventions can be effective in reducing nurses compassion fatigue.

\section{eP387 \\ POSTTRAUMATIC GROWTH AND DEATH ANXIETY IN CAREGIVERS OF CANCER PATIENTS}

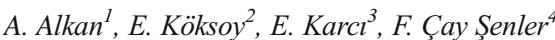

${ }^{T}$ Osmaniye Public Hospital, MEdical Oncology, OSmaniye, Turkey

${ }^{2}$ Kastamonu Public Hospital, Medical Oncology, Kastamonu, Turkey

${ }^{3}$ Bağcllar Education and Research Hospital, Medical Oncology, IStanbul, Turkey

${ }^{4}$ Ankara University School of Medicine, Medical Oncology, Ankara, Turkey

\section{Introduction}

Caregivers of cancer patients are exposed to numerous psychosocial problems, Post traumatic growth (PTG) is defined as a positive psychological change that occurs following a meaningfully challenging or traumatic life event.

\section{Objectives}

The purpose of the study is to define the predictors of PTG and death anxiety (DA) and evaluate the impact of DAN on PTG

Methods

The study is designed as a multicenter survey study. Caregivers of cancer patients were evaluated with structured questionnaires to assess the DAN and PTG and clinical parameters associated with them. The validated PTG scale and Templer death anxiety scale were used.

Results

231 participants were evaluated in 3 different cancer centers. $25.6 \%$ of the participants had at least one cancer screening after the diagnosis. Age more than 40 (73.0 vs $65.0, \mathrm{p}=0.024)$ and low monthly income ((72 vs 65 , $\mathrm{p}=0.034$ ) were associated with higher PTG scores. Female sex, low income, not working, having chronic disease, history of psychiatric illness were associated with higher DAN scores. In multivariate analysis, female sex was the only risk factor for high DAN scores. $(\mathrm{RR}=2.0,95 \% \mathrm{CI}, 1.0-3.9$, $\mathrm{p}=0.046$ ). There was a positive correlation between PTG and DAN scores $(r=0.13, p=0.037)$. Positive impacts were detected on philosophy of life (15.0 vs $13.0, p=0.035)$ and changes in relationship $(17.0$ vs $14.0, p=0.01)$

\section{Conclusions}

It's the first data about the association between DAN and PTG. We found a positive impact of death anxiety on positive psychological changes in caregivers of cancer patients. The caregivers who have risk factors should be cautiously evaluated.

\section{eP388}

THE IMPACT OF PSYCHOLOGICAL SUPPORT FOR ADULT PATIENTS IN THE CLINIC OF CHEMOTHERAPY OF THE MURATSAN HOSPITAL COMPLEX

\author{
Y. Asribabayan ${ }^{1}$, K. Alisa ${ }^{2}$, D. Zohrabyan ${ }^{3}$, L. Harutyunyan ${ }^{3}$, G. \\ Tamamyan $^{3}$ \\ ${ }^{I}$ Clinic of Chemotherapy of Muratsan Hospital Complex of Yerevan State \\ Medical University, Psychosocial Service, Yerevan, Armenia \\ ${ }^{2}$ Clinic of Chemotherapy of Muratsan Hospital Complex of YSMU, \\ Psychosocial Service, Yerevan, Armenia \\ ${ }^{3}$ Clinic of Chemotherapy of Muratsan Hospital Complex of Yerevan State \\ Medical University, Clinic of Chemotherapy, Yerevan, Armenia
}

\section{Introduction}

According to international cancer care standards, effective cancer care must include psychosocial services. In Armenia there is a huge stigma about psychosocial support. With the goal of providing high-standard quality care, Psychosocial Oncology Service was established at the Chemotherapy Clinic of Muratsan University hospital in 2016. Two clinical psychologists were involved in the service to work with adult and pediatric population. Due to the budget limitations, they have been working 2 days per week.

\section{Objectives}

Find out the main impact areas of psychological support for adult patients.

\section{Methods}

The analysis of clinical records was used to assess the main areas of impact of the psychological support among adult patients

\section{Results}

During one year, 58 (25.6\%) adult patients have received psychological support, $65,5 \%$ of them have been involved in regular psychological work. According to the clinical records, the main area of work was the revision of attitudes toward the diagnosis (1), self-presentation in the society in the context of changes in appearances (2) and reduction of anxiety levels (3). The work with families was focused on psycho-education: the acceptance of patients' negative emotions (1), the effective communication with patients (2), speaking out about the diagnosis (3).

\section{Conclusions}

Despite the resistance about psychological support, psychosocial service was successfully initiated. One of the main limitations for service development was the time restriction for specialists. Based on the relevance of the service it was changed to full time format from December 1, 2017.

\section{eP389}

\section{SYSTEMATIC REVIEW OF THE RELATIONSHIP BETWEEN} CHILDHOOD SEXUAL ABUSE AND CANCER OUTCOMES IN MEN

G. Cartagena $^{1}$, E.L. Kacel ${ }^{2}$, D.B. Pereira ${ }^{3}$

${ }^{T}$ University of Florida, Department of Clinical and Health Psychology, Gainesville- FL, USA

${ }^{2}$ University of Florida, Department of Clinical and Health Psychology, Gainesville, USA

${ }^{3}$ University of Florida, Department of Clinical and Healthy Psychology, Gainesville, USA

\section{Introduction}

A history of childhood sexual abuse (CSA) may negatively impact initiation, treatment, and survivorship of cancer. Many published studies on this topic have explored outcomes in women, as women comprise the 
majority of adults with CSA histories. To date, few published studies have explored how CSA may affect cancer outcomes among the approximately $17 \%$ of adult men with CSA histories.

\section{Objectives}

To (a) assess current literature surrounding CSA and cancer outcomes in men, and (b) posit a literature-based model of cancer outcomes in this population.

\section{Methods}

49 peer-reviewed, published articles concerning CSA and cancer were identified through a systematic search of online databases and content review. After further review, 13 articles were identified as relevant to men with CSA histories. These articles were organized thematically and conceptualized in a psychoneuroimmunological framework.

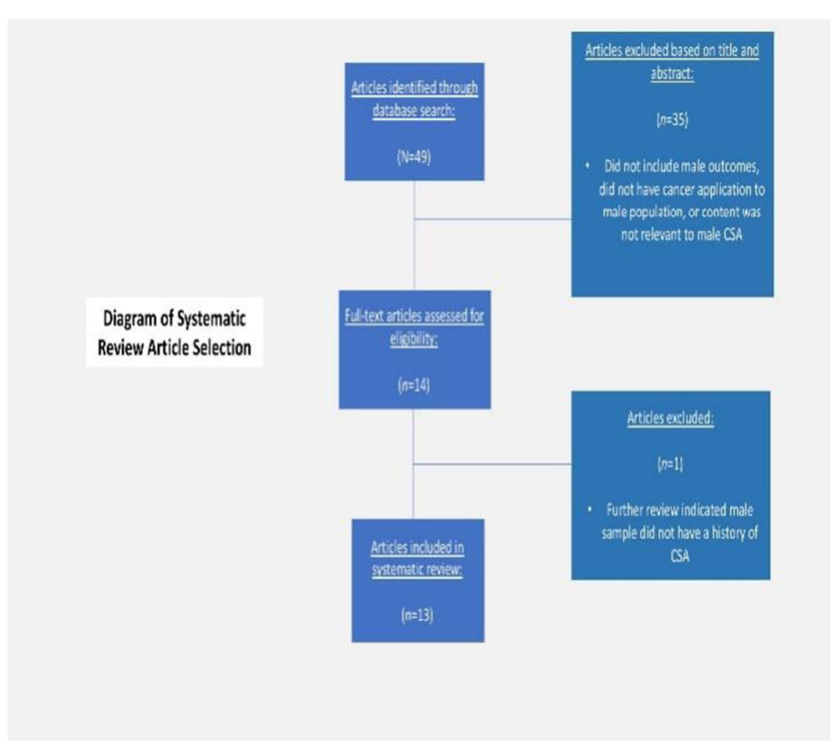

\section{Results}

Information from this review suggests a psychoneuroimmunological path toward cancer risk/outcome for men with CSA history. Psychological responses include fear/shame, depression/anxiety, low life-satisfaction, low abuse reporting, never-screening for cancer, decreased checkups, avoiding medical encounters, low likelihood of having health insurance, smoking, emotional eating, and retraumatization. Neuroendocrine/ immunological responses includeHPA Axis response and inflammatory immune responses. Cancer initiation outcomes include abnormal cell proliferation, angiogenesis, and lung cancer risk. Survivorship outcomes include poor health status, increased BMI, and risk of comorbid illness. Proposed Psychoneuroimmunological Pathway of Cancer Outcomes for Men With a History of CSA

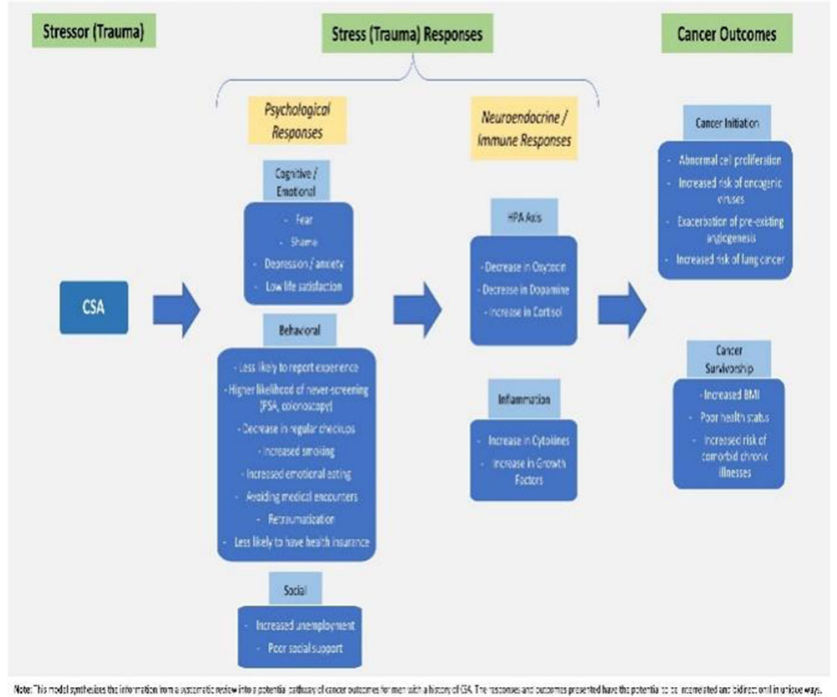

\section{Conclusions}

CSA may propagate psychoneuroimmunological responses that negatively impact cancer initiation/survivorship for men. As this systematic review highlights the paucity of research in this area, future studies might examine these potential relationships to address CSA trauma and improve cancer outcomes for men.

\section{eP390 \\ THE PREDICTORS OF ANXIETY AND DEPRESSION IN HEAD AND NECK CANCER PATIENTS}

Y.J. Chen $^{1}$

${ }^{T}$ Da-Yeh University, Department of Nursing, Changhua, Taiwan R.O.C.

\section{Introduction:}

Head and neck cancer (HNC) is one of the most common cancer in the worldwide, and the fourth rank of top ten common cancer of male in Taiwan. HNC patients suffer from physical and psychological dysfunction from cancer diagnosis. However, previous studies indicated that anxiety and depression significantly impacted on patients' mental health and quality of life.

Objectives

Therefore, the purposes of the study are to explore: (1) the severity of anxiety and depression; and (2) the predictors of anxiety and depression.

\section{Methods}

The study was a cross sectional study with consecutive sampling. Patients were assessed by structured questionnaires, including Hospital Anxiety and Depression Scale (HADS), Symptom Severity Scale (SSS), and background information after treatment. Data was analyzed by descriptive statistics and logistic regression.

\section{Results}

The results found that (1) in a total of 108 patients, the severity of psychological distress was mild at average, but around $32 \%$ of patients were identified anxiety and depression; (2) marital status, tumor stage, continued alcohol, tobacco, and betel nut used after treatment, and symptom severity were significantly predicted to anxiety. (3) gender, marital status, tumor stage, continued alcohol, tobacco, and betel nut used after treatment were significantly predicted to depression.

\section{Conclusions}

In conclusion, HNC patients suffered from anxiety and depression after cancer treatment. The screening and assessment of anxiety and depression should be earlier and routinely. Future studies may provide mental support or referral service to improve their mental health.

\section{eP391 \\ BEING DIAGNOSED WITH CANCER: THE EXPERIENCES OF PATIENTS WITH NON-HODGKIN'S LYMPHOMA}

\author{
D. Chircop ${ }^{1}$, J. Scerri ${ }^{2}$ \\ ${ }^{T}$ University of Malta, Department of Nursing, Msida, Malta \\ ${ }^{2}$ University of Malta, Department of Mental Health, Msida, Malta
}

\section{Introduction}

Patients with non-Hodgkin's lymphoma (NHL) are often diagnosed with cancer at an advanced stage. This is due to a lack of screening programmes for NHL and delays in help seeking as the symptoms experienced are fairly non-specific in nature. As a result, receiving an unexpected diagnosis of NHL may psychologically affect patients.

\section{Objectives}

To explore the lived experiences of patients on receiving a diagnosis of NHL.

\section{Methods}

A qualitative design was used. Six adult patients diagnosed with NHL were recruited and participated in two semi-structured interviews at 
different time points. Transcribed texts were analysed using interpretative phenomenological analysis.

\section{Results}

Three main themes emerged: 'Emotional reaction to the diagnosis', 'Struggling with a lack of understanding' and 'Searching through past experiences'. Most of the participants expressed shock, disbelief and fear at the news of being diagnosed with NHL. A sense of relief was expressed by one participant, who could now provide a label to the pain experienced.

Conclusions

This study highlights the responses of patients with NHL on receiving a diagnosis. These responses are influenced by their knowledge about the illness, type of symptoms experienced, their own personal circumstances and the manner in which the diagnosis was given. The lack of awareness and knowledge about NHL among patients and primary health care professionals presents an additional psychological challenge to this patient group when compared to patients with other types of cancers. The results highlight the importance of providing information in a patient-sensitive manner.

\section{eP392}

IS IT ALL IN THE LABEL? THE RELATIONSHIP BETWEEN USING A MEDICAL TERM VERSUS EUPHEMISM AND PSYCHOLOGICAL OUTCOMES AND HEALTH BEHAVIOURS AMONG INDIAN CANCER PATIENTS

M. Chittem ${ }^{1}$, T. Epton ${ }^{2}$, R. Tanikella ${ }^{1}$

${ }^{T}$ Indian Institute of Technology Hyderabad, Liberal Arts, Hyderabad, India

${ }^{2}$ University of Manchester, Manchester Centre for Health Psychology, Manchester, United Kingdom

\section{Introduction}

The term "cancer" has negative connotations; especially in India where cancer is a taboo. Instead, euphemisms (e.g., 'lump') are often used. Patients who use euphemisms could differ in psychological outcomes and health behaviours compared to those who use medical terms.

Objectives

This study investigates the relationship between patient use of medical terms versus euphemism and psychological outcomes and health behaviours in Indian cancer patients.

Methods

Patients $(N=350)$ were recruited from cancer hospitals in Hyderabad, India. They were asked "what words do you use to describe your illness?". Scores on the Brief illness perception questionnaire (BIPQ), Cancer stigma scale (CASS), Hospital anxiety/ depression scale (HADS), Brief COPE, Spontaneous self-affirmation scale (SSAS), and a health behaviour scale were recorded. Mann-Whitney Us were used to explore differences between euphemism users (i.e., used euphemism as their $1^{\text {st }}$ word; EU) and medical term users (MTU) groups.

Results

$51 \%$ were EUs. Compared to MTUs, EUs reported lower responsibility on the CASS; less self-distancing, emotional support, positive reframing, planning, acceptance, religion and blame on the COPE; and greater illness identity, less understanding of illness, and lower perceptions of personal control on the BIPQ. There were no differences between groups in anxiety and depression. EUs ate a healthy diet on fewer days than MTUs and were less likely to spontaneously self-affirm.

Conclusions

Using euphemisms is associated with negative illness perceptions, using less of the coping strategies and less spontaneous self-affirmation use. Future research needs to examine methods to overcome cultural taboos associated with cancer terminology in order to improve patient wellbeing.
eP393

SUPPORTIVE CARE NEEDS AND THEIR ASSOCIATIONS WITH PSYCHOLOGICAL WELL-BEING, ILLNESS BELIEFS AND QUALITY OF LIFE AMONG CANCER PATIENTS IN INDIA

M. Chittem ${ }^{1}$, C. Rowland ${ }^{2}$, S. Jakkula ${ }^{1}$, R. Tanikella ${ }^{1}$

${ }^{T}$ Indian Institute of Technology Hyderabad, Liberal Arts, Hyderabad, India

${ }^{2}$ University of Manchester, Centre for Health Psychology, Manchester, United Kingdom

\section{Introduction}

Residual unmet needs can impact negatively on patients' well-being and life experiences. Assessing patients' needs offers a direct measure of their support preferences and also helps identify gaps in service provision.

\section{Objectives}

To examine patients' supportive care needs and associations between these and psychological distress, symptom experience, illness perceptions and quality of life (QoL) among cancer patients in India.

\section{Methods}

Patients $(N=522)$ were recruited from cancer hospitals in Hyderabad, India and were interviewed using a structured, closed questions survey on one occasion. Using validated scales, patients were asked about their supportive care needs, anxiety and depression, symptoms, illness perceptions, and quality of life (QoL).

\section{Results}

Most participants had unmet needs $(80.3 \%)$ with only $1.9 \%$ reporting no needs and this did not differ by diagnosis or treatment status. Those with unmet needs were significantly younger and had fewer children than those with satisfied needs ( $p$ 's $<0.05$ ). Patients with unmet needs were significantly more anxious $(\mathrm{p}=.02)$ and depressed $(\mathrm{p}<.001)$ than those who were satisfied, they also had greater physical and psychological symptoms $(p ' s<.001)$ and a more negative perception of their illness $(\mathrm{p}<.001)$. Furthermore, overall QoL $(\mathrm{p}<.01)$ and $\mathrm{QoL}$ across all domains $(p ' s<.001)$ was significantly lower for patients with unmet needs. Logistic regression analysis is underway to explore these associations further.

Conclusions

Patients with unmet needs had higher levels of psychological distress, more physical and psychological symptoms, negative illness beliefs and lower QoL. These findings suggest that patients may benefit from psycho-oncology services. Further, future research can examine the impact of these psycho-oncological services on patients' well-being.

\section{eP394}

A RANDOMISED CONTROLLED TRIAL OF PSYCHOEDUCATIONAL INTERVENTIONS FOR REDUCING UNCERTAINTY AND ANXIETY, AND IMPROVING SEXUAL FUNCTIONING AMONG GYNAECOLOGICAL CANCER PATIENTS IN HONG KONG

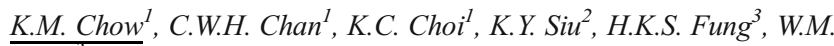
Shum $^{3}$

${ }^{1}$ The Chinese University of Hong Kong, The Nethersole School of Nursing, Hong Kong, Hong Kong S.A.R.

${ }^{2}$ Prince of Wales Hospital- Hospital Authority, Department of Obstetrics and Gynecology, Hong Kong, Hong Kong S.A.R.

${ }^{3}$ Queen Elizabeth Hospital- Hospital Authority, Department of Obstetrics and Gynecology, Hong Kong, Hong Kong S.A.R.

\section{Introduction}

The diagnosis of gynaecological cancer and the effects of related treatments have adverse effects to sexual functioning. Unpredictable disease outcomes and the complexity of cancer treatment also lead to uncertainty in gynaecological cancer patients. High levels of uncertainty are 
associated with high levels of anxiety, which may negatively impact on quality of life. Psycho-educational interventions for gynaecological cancer patients were found to have positive effects on sexual functioning and psychological outcomes. However, such interventions are still not commonly available.

\section{Objectives}

This study aims to implement a 4-session programme of psycho-educational interventions for gynaecological cancer patients in Hong Kong, and evaluate its effects on uncertainty, anxiety and sexual functioning.

\section{Methods}

This study is a randomised controlled trial with a mixed-method design. Participants were randomised into intervention or attention control groups. Chinese versions of the Mishel's Uncertainty in Illness Scale (C-MUIS), the anxiety subscale in the Hospital Anxiety and Depression Scale (HADS), and the Sexual Function-Vaginal Changes Questionnaire (SVQ) were used for outcome assessment.

\section{Results}

A total of 200 participants will be recruited and the study is in process. The results will be presented in the conference. It is expected that participants in the intervention group will demonstrate a significant reduction in uncertainty and anxiety, as well as a significant improvement in sexual functioning when compared with those in the attention control group. Conclusions

The evidence-based, theory-driven and cultural-adapted programme of psycho-educational interventions for Hong Kong Chinese gynaecological cancer patients is feasible and expected to be effective in minimising uncertainty and anxiety, and improving sexual functioning.

\section{eP395}

\section{HOW IS SEXUALITY IN TUNISIAN CANCER PATIENTS?}

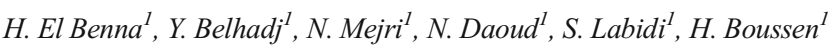
${ }^{T}$ Abderrahmen Mami Hospital Ariana- University Of Tunis El Manar, medical oncology, Ariana, Tunisia

\section{Introduction}

Sexual dysfunction is a common side effect of cancer therapy. It represents a quality of life issue rarely expressed by patients.

Objectives

We aim to evaluate sexual life change in Tunisian patients after cancer diagnosis.

\section{Methods}

We interviewed 66 adult cancer patients, regardless of location. Data about disease characteristics were collected from patients records. We elaborated a hetero-questionnaire addressing different items: libido, level of intimacy, excitement, body image, satisfaction and use of drugs to improve sexual life. Patients were asked to rate each item from 1 to 4 to compare their current status with their pre-cancer diagnosis status. All patients gave their informed consent.

Results

Meanage was 50 years, $53 \%$ of patients were females. Most of the patients were under active medical treatment (86\%), $42 \%$ were in the palliative setting. Eighty six percent of patients had regular sexual partner. Sexuality was considered as important as before cancer diagnosis in only $22 \%$ of patients. The intimacy level decreased after cancer diagnosis in $70 \%$ of patients. Sexual desire was unchanged in $38 \%$ patients. The mean frequency of sexual activity was 2.14 intercourses per month. Forty six per cent of patients experienced difficulties in reaching orgasm and $7 \%$ declared using medication before or during intercourse. Sixty per cent of patients reported that they were not informed about possible changes in their sexual life.

\section{Conclusions}

Cancer diagnosis is a disturbing event leading to changes in sexual life. Promoting more systematic information about sexuality is compulsory. Standardized assessment tools and intervention strategies to help patients are needed.
eP396

USE OF CAREGIVER REACTION ASSESSMENT SCALE IN ASSESSMENT OF CAREGIVER BURDEN AMONG A SAMPLE OF CAREGIVERS OF PATIENTS WITH BREAST CANCER

B. Fadipe $^{\text {I }}$, Y.O. Fadipe , A.T. Olagunju $^{3}$

${ }^{T}$ Lagos University Teaching Hospital- Lagos- Nigeria, Department of Psychiatry Consultation-Liaison, Festac Town, Nigeria

${ }^{2}$ Lagos University Teaching Hospital- Lagos- Nigeria, Department of Family medicine, Lagos, Nigeria

${ }^{3}$ Lagos University Teaching Hospital- Lagos- Nigeria, Department of Psychiatry Consultation-Liaison, Lagos, Nigeria

\section{Introduction}

Cancer caregiving puts enormous stress not only on patients but also their loved ones whose roles have been described as being of an acute and intensive nature. In Nigeria, there is paucity of studies that examine burden of caregiving in patients with Breast Cancer.

\section{Objectives}

This study set out to determine burden of care and associated factors among caregivers of patients with Breast cancer using the Caregiver Reaction Assessment Scale(CRA).

\section{Methods}

We carried out a cross-sectional study among Breast cancer patients using questionnaires administered to patients and their caregivers( $\mathrm{n}=127$ pairs $)$, attending the outpatient clinic of Lagos University Teaching Hospital. The CRA was used to assess caregiver burden, Oslo social support instrument assessed social support. The patient-caregiver dyad was interviewed using a sociodemographic and clinical history questionnaire to determine associated factors. Logistic regression analysis was used to identify factors associated with caregivers' burden.

Results

Almost half(47.5\%) of breast cancer patient caregivers reported high burden of caregiving. The highest burden was experienced in the domain of schedule $(44.9 \%)$ while the lowest burden was in the area of finance and social support(34.6\%). Following binary logistic regression, having a concurrent medical problem among caregivers and caregiver difficultly to get help from others in the care of their relatives were predictors of caregiver burden.

\section{Conclusions}

Caregiver burden remains a challenge experienced by family caregivers of patients with Breast cancer especially in a developing country where cancer services are scare and payment is largely out-of-pocket. Caregivers' own health status and social support were relevant factors in caregivers' experience of burden.

\section{eP397 \\ BREAKING AND MANAGING BAD NEWS TO CANCER PATIENT AND FAMILY: NURSING INTERVENTION}

M. Ferreira ${ }^{1}$, P. Alves ${ }^{2}$

${ }^{T}$ Instituto Português de Oncologia de Lisboa Francisco Gentil E.P.E., Oncologia Médica e Neurologia, Lisbon, Portugal

${ }^{2}$ Escola Superior de Enfermagem de Lisboa, Fundamentos de Enfermagem, Lisboa, Portugal

\section{Introduction}

The process of breaking and managing bad news in oncology is complex and susceptible of being source of discomfort for all participants. Because of its proximity to the cancer patient and the family when providing care, nurses are a key element in this process, being essential to develop competencies in this area.

\section{Objectives}

Develop skills in breaking and managing bad news to the cancer patient and family, as well as to promote the improvement of nursing care. 


\section{Methods}

This work was based on a project methodology. Initially the process of breaking and managing bad news was identified as a training need, not only personal but also of the nurses of the Internal Medicine Ward where I was working. Then was performed an internship for three months in places considered having good practices in this subject. Throughout the journey were applied questionnaires, constructed documents to support the practice, made reflection on action, provided health care to cancer patient/family and performed training sessions to nurses.

Results

Skills were acquired in the use of communication techniques, in the development of the relationship with cancer patient/family and in my emotional management, allowing an advanced nursing practice. The nurses who received skills training considered the contributions positive to their practice, enhancing the importance of knowing about guidelines for breaking and managing bad news.

\section{Conclusions}

It is possible to develop skills in this area based on professional experience and using this methodology, as long as nurses are aware of their training needs and are available to improve their practice.

\section{eP398}

\section{HOPE AND PATIENTS WITH ADVANCED CANCER}

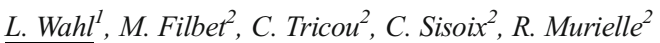

${ }^{T}$ Univ. Lyon- University Claude Bernard Lyon 1-, general practitioner department-, F-69008 Lyon, France

${ }^{2}$ Service de soins palliatifs pavillon $1 \mathrm{~K}$ CHLS CHU de Lyon, medecine palliative, Pierre-Benite, France

\section{Introduction}

Hope is a key issue in advanced cancer. When the cancer progresses, doctors find it difficult to reconcile their duty to inform patients while allowing them to maintain hope.

Objectives

Our aim was to explore the role and meaning of hope for patients with advanced cancer, in order to find clues to solve this dilemma.

\section{Methods}

This qualitative study used semi-structured interviews, and data analysis used grounded theory. Patients were recruited form two academic medical centers.

\section{Results}

We interviewed 18 patients. Despite the traumatic experience of cancer, hope remains a vital need. Patients construct hope in various ways. Sometimes patients express the need to escape reality. In these cases, they maintain hopes that might not be realistic, but that help them to keep on living. Other patients base their hopes on things for which there is no scientific explanation. Finally, it appears that uncertainty is part of the experience of cancer. Patients want their doctor to acknowledge that despite scientific knowledge, medicine cannot predict the future. In that sense, uncertainty is probably the key to accommodating medical information and hope in advanced cancer. It could be a way of sharing honest information with patients while, at the same time, giving them the opportunity to maintain hope.

Conclusions

Hope is necessary to live and has to be taken into account by healthcare professionals at all stages of cancer. Professionals need to understand the mechanisms of hope in order not to liken hope to denial.

\section{eP399}

BURNOUT POTTER BRIEF TEST AS SCREENING TOOL BEFORE AN INTRODUCTORY COURSE TO LAUGHTER YOGA FOR ONCOLOGY STAFF MEMBERS

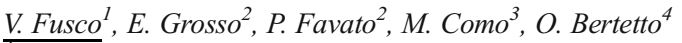 \\ ${ }^{T}$ Azienda Ospedaliera di Alessandria, Oncology, Alessandria, Italy
}

${ }^{2}$ Chiara Luce ONLUS, Psychology, Novi Ligure, Italy

${ }^{3}$ Azienda Ospedaliera di Alessandria, Nursing staff, Alessandria, Italy

${ }^{4}$ Azienda Ospedaliera Città della Salute di Torino, Rete Oncologica Piemonte Valle d'Aosta, Turin, Italy

\section{Introduction}

In order to prevent burnout in professionals (nurses, doctors, technicians, etc) dealing with cancer patients, an introductive one-day course of Laughter Yoga was offered to the staff of a regional cancer network. Laughter Yoga is a practice codified since 1995 by the Indian doctor Madan Kataria, based on psychological and physiological positive effects of voluntary laughter, supposed to be equivalent to those ones derived from spontaneous laughter. Exercises (clapping, eye contact, body movement, breathing exercises, acting and visualization techniques) have the final aim of breaking down inhibitions and of laughing without any humorous reason for consecutive minutes.

\section{Objectives}

Screening of burnout risk in self-selected cancer staff professionals.

\section{Methods}

The Potter brief test (Potter,1994), adapted in Italian language (Gitto et al, 2016), was proposed to and self-administered by attendees, in six one-day courses, immediately before the beginning of the Laughter Yoga introducing session. Twenty-five items (about exhaustion, depersonalization, etc) were self evaluated as 1 (seldom), 2 (sometimes), 3 (usually), 4 (often), 5 (always); the total score was so classified: 25-50, it is all OK; $51-75$, it is better to adopt preventive measures; 76-100, you are at risk of burnout; 101-125, please ask for help.

Results

The score in 148 attendees was : no burnout risk in $75 \%$; moderate risk in $22 \%$; potentially high risk in $3 \%$.

\section{Conclusions}

The Potter brief test is a reliable screening test before intervention to prevent burnout and it is going to be tested in order to evaluate efficacy of the Laughter Yoga as a preventive tool.

\section{eP400}

A VIDEOCONFERENCE GROUP INTERVENTION TO IMPROVE QUALITY OF LIFE OF CANCER CAREGIVERS: LESSONS LEARNED

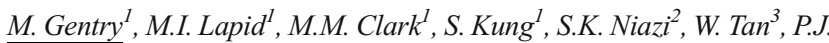
Atherton $^{4}$, J.A. Sloan ${ }^{4}$, K.J. Whitford ${ }^{5}$, J.M. Hubbard ${ }^{6}$, A.L. Cheville ${ }^{7}$, Y.I. Garces $^{8}$, T.A. Rummans ${ }^{I}$

${ }^{1}$ Mayo Clinic, Psychiatry and Psychology, Rochester, USA

${ }^{2}$ Mayo Clinic, Psychiatry and Psychology, Jacksonville, USA

${ }^{3}$ Mayo Clinic, Hematology Oncology, Jacksonville, USA

${ }^{4}$ Mayo Clinic, Health Sciences Research-Division of Bomedical Satistics and Informatics, Rochester, USA

${ }^{5}$ Mayo Clinic, Internal Medicine, Rochester, USA

${ }^{6}$ Mayo Clinic, Medical Oncology, Rochester, USA

${ }^{7}$ Mayo Clinic, Physical Medicine and Rehabilitation, Rochester, USA

${ }^{8}$ Mayo Clinic, Radiation Oncology, Rochester, USA

\section{Introduction}

Caregiving can negatively impact the health and well-being of the caregiver. Interventions for reducing distress and improving coping skills have been shown to be effective in improving quality of life (QOL) for caregivers. Telemedicine has the potential to help improve access to these interventions for caregivers.

\section{Objectives}

We explored the feasibility and acceptability of an interactive videoconference intervention to improve cancer caregiver QOL.

Methods

This study enrolled caregivers in a structured multidisciplinary intervention of 8 videoconference group sessions provided over 4 weeks. Groups 
were led by trained facilitators and included components of physical therapy, occupational therapy, psychosocial education, cognitivebehavioral intervention, supportive discussion, spiritual reflection, and mindfulness therapy. Feasibility was based on $25 \%$ recruitment, $80 \%$ session participation, and $80 \%$ questionnaire completion rates. The Linear Analog Self-Assessment (LASA) was used to assess QOL at baseline and post-intervention.

Results

20 caregivers were enrolled, who were mostly geriatric ( $80 \%$ were $>65$ years) female $(76.5 \%)$ married $(88.2 \%)$ to the patient, Caucasian $(100 \%)$ and highly educated (100\%). $60 \%$ attended $1-5$ sessions, $15 \%$ attended 68 sessions, 25\% attended no sessions. $6(30 \%)$ had pre- and post- intervention LASA scores. Overall QOL 6.2 vs 7.7; Mental well-being 6.8 vs 8.2; Physical well-being 6 vs 7.2; Emotional well-being 5.7 vs 7.2; Social activity 5.2 vs 6.2 ; Spiritual well-being 6.5 vs 7.2 ; Fatigue 6.2 vs 6.2 ; Social support 8 vs 7.3 .

\section{Conclusions}

The caregivers reported higher QOL in multiple domains after participating in intervention. Offering QOL interventions to caregivers through telehealth remains promising, however, more effective strategies are needed to overcome barriers to participation.

\section{eP401}

EVALUATION OF DISTRESS SCREENING WITH THE DISTRESS THERMOMETER IN CANCER INPATIENTS 4 YEARS AFTER IMPLEMENTATION

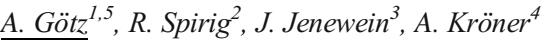

${ }^{T}$ Univerity Hospital Zürich, Oncology/ Radiooncology, Zürich, Switzerland

${ }^{2}$ Univerity Hospital Zürich, department of Nursing and Allied Health Care Professionals, Zürich, Switzerland

${ }^{3}$ Univerity Hospital Zürich, department of psychiatry and psychology, Zürich, Switzerland

${ }^{4}$ Univerity Hospital Zürich, Comprehensive Cancer Centre, Zürich, Switzerland

${ }^{5}$ Witten/Herdecke University, PhD Student, Witten, Germany

\section{Introduction}

When caring for cancer patients, finding an appropriate screening instrument to identify and assess increased psychosocial distress is crucial. In 2012, we implemented the Distress Thermometer (DT) at the Comprehensive Cancer Center at the University Hospital Zurich (USZ). Since then, the DT has been routinely used by nurses with all cancer inpatients.

Objectives

To identify adherence to the screening guideline and the documented benefits of the DT screening.

Methods

This retrospective descriptive study evaluated the distress screening since 2012. Data of all patients treated in the USZ were included. Every inpatient who was screened using the DT at least once during the treatment was labeled as screened. The data was collected from the electronic patient documentation system and analyzed descriptively.

Results

Since 2012, 11184 patients have been treated. In total, 15095 screenings were documented and $40.6 \%$ of the patients were screened. Male, married and emergency patients and patients with hematology diseases, brain tumors or head and neck cancer were screened more frequently $(\mathrm{p}<$ $0.001)$. More female, younger, single and emergency patients wished to be transferred to the psychooncology service $(\mathrm{p}<0.001)$. $7.9 \%$ of all patients were forwarded to the psychooncology service and $37.7 \%$ to a social worker. Patients with an increased distress level had a significantly reduced distress level in a repeated screening after a visit to a psychooncologist.

\section{Conclusions}

Certain patient groups were preferably screened. Despite increased distress levels, only a few patients were referred to psychooncology. Nurses seem not aware of the benefit of psychooncological visits.

eP402

DO PATIENTS TREATED WITH CHEMOTHERAPY OR CHECKPOINT INHIBITORS FOR ADVANCED NON-SMALL CELL LUNG CANCER (NSCLC) REGRET HAVING RECEIVED TREATMENT? A PROSPECTIVE EVALUATION IN 164 PATIENTS

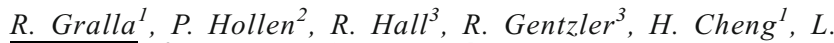
Carhuapoma $^{2}$, J. Crawford ${ }^{4}$, M. Lesser ${ }^{5}$

${ }^{1}$ Albert Einstein College of Medicine, Medical Oncology, New York- NY, USA

${ }^{2}$ University of Virginia, School of Nursing, Charlottesville- VA, USA

${ }^{3}$ University of Virginia, Medical Oncology, Charlottesville- VA, USA

${ }^{4}$ Duke University, Medical Oncology, Durham- NC, USA

${ }^{5}$ Feinstein Institute for Medical Research, Biostatistics, Manhasset- NY, USA

\section{Introduction}

Although chemotherapy for NSCLC is common, little is known regarding patients' views on the decision to receive treatment. In a recent systematic review of 59 studies about regret (Becerra Perez 2016), none focused on NSCLC.

\section{Objectives}

To determine a profile of the extent of regret, factors contributing to regret, and factors predicting risk of regret.

\section{Methods}

Patients receiving systemic treatment for advanced NSCLC were enrolled in a phase III, randomized trial and randomized to either usual care or enhanced care using the DecisionKEYS decision aid. All patients completed every 3-week PRO assessment using the electronic LCSS including the 3-Item Global Index (3-IGI: distress, activities, QL) and the Decision Regret Scale (“DRS," O'Connor 1999) at 3 months after starting treatment.

\section{Results}

164 patients entered; 160 received treatment; $43 \%$ women; $92 \%$ Stage IV; median PS $=1$; mean age $63.86 \%$ of patients alive at 3 months completed the DRS. Overall, $13 \%$ (95\% CI: $7.4 \%$ - 19.2\%) of patients expressed regret. Nearly all patients expressing regret at 3 months, had a $20 \%$ decline in the 3-IGI score at 6 weeks after starting treatment compared with baseline (15\% versus only $1 \%$ not having the $20 \% 3$-IGI decline, $\mathrm{p}=0.01)$. Results were similar in both randomization groups.

\section{Conclusions}

Regret is not common in patients receiving systemic therapy for advanced NSCLC. Patients ultimately having regret could be identified early using the 3-IGI of the LCSS. Early identification of risk for regret allows for interventions to address this issue.

Support: NIH/NCI R01 CA157409

\section{eP403}

IS EMOTION REGULATION ASSOCIATED WITH CANCERRELATED PSYCHOLOGICAL SYMPTOMS?

\author{
A.J. Guimond ${ }^{l}$, H. Ivers ${ }^{1}$, J. Savard ${ }^{1}$ \\ ${ }^{T}$ Université Laval, School of Psychology, Québec, Canada
}

\section{Introduction}

Breast cancer patients frequently report a combination of psychological symptoms including anxiety, depression, fear of cancer recurrence (FCR), insomnia, fatigue, pain, and cognitive impairments. In the general 
population, emotion regulation (ER) is considered a central mechanism underlying the development of psychological disorders. However, the relationships between ER and cancer-related psychological symptoms have received little attention.

\section{Objectives}

To examine the cross-sectional and prospective relationships between subjective (cognitive reappraisal, expressive suppression and experiential avoidance) and objective measures (high frequency heart rate variability [HF-HRV]) of ER and a set of psychological symptoms (anxiety, depression, FCR, insomnia, fatigue, pain, and cognitive impairments) among women receiving radiation therapy for breast cancer.

Methods

81 participants completed a battery of self-report scales before (T1) and after (T2) radiotherapy. HF-HRV at rest and HF-HRV reactivity during a worry-induction task were measured at $\mathrm{T} 1$.

\section{Results}

Canonical correlation analyses revealed that higher levels of experiential avoidance and expressive suppression were cross-sectionally associated with higher levels of all symptoms at $\mathrm{T} 1(\mathrm{R}=.73, p=.0002)$ and at $\mathrm{T} 2$ (except pain; $\mathrm{R}=.75, p<.0001$ ). Higher levels of suppression and reappraisal measured at T1, as well as a greater HF-HRV reactivity, were associated with reduced FCR and with increased cognitive impairments, depression, insomnia and fatigue between $\mathrm{T} 1$ and $\mathrm{T} 2(\mathrm{R}=.59, p=.03)$.

\section{Conclusions}

These results suggest that maladaptive ER strategies, assessed subjectively, may act as a transdiagnostic mechanism underlying several psychological cancer-related symptoms.

\section{eP404}

IS EMOTION REGULATION A COMMON MECHANISM UNDERLYING CLUSTERS OF PSYCHOLOGICAL SYMPTOMS IN BREAST CANCER PATIENTS?

\section{A.J. Guimond ${ }^{l}$, H. Ivers $^{l}$, J. Savard ${ }^{l}$ \\ ${ }^{T}$ Université Laval, School of Psychology, Québec, Canada}

\section{Introduction}

Breast cancer patients tend to experience numerous psychological symptoms that co-occur and form clusters. It has been proposed that a common psychological mechanism may underlie the membership of symptoms in a given cluster, but this hypothesis has never been investigated. Maladaptive emotion regulation (ER) is one possible common mechanism.

\section{Objectives}

This study examined the cross-sectional and prospective relationships between subjective (experiential avoidance, expressive suppression and cognitive reappraisal) and objective measures (high frequency heart rate variability [HF HRV]) of ER and clusters of psychological symptoms including anxiety, depression, fear of cancer recurrence, insomnia, fatigue, pain, and cognitive impairments among women receiving radiation therapy for breast cancer.

Methods

81 women completed a battery of self-report scales before (T1) and after (T2) radiotherapy. Resting HF-HRV and HF-HRV reactivity during a worry-induction task were measured at $\mathrm{T} 1$.

\section{Results}

Latent profile analyses identified between two and four clusters of patients with similar levels of symptoms across time points and between T1 and T2. Discriminant analyses showed that a higher level of avoidance at $\mathrm{T} 1$, and higher levels of avoidance and suppression at T2, predicted membership in symptom clusters that included more severe symptoms cross-sectionally (both $p$ s $<.001$ ). A higher level of avoidance and a lower level of reappraisal and HF-HRV reactivity at T1 predicted membership in a cluster of symptoms that were severe at $\mathrm{T} 1$ and that decreased slightly at T2 $(p=.02)$.

\section{Conclusions}

Maladaptive ER strategies, more particularly avoidance, are a possible psychological mechanism underlying clusters of cancer-related psychological symptoms.

\section{eP405 \\ PSYCHOSOCIAL DISTRESS AMONG CANCER PATIENTS: A SINGLE INSTITUTION EXPERIENCE AT THE STATE OF QATAR}

A. Hassan ${ }^{1}$, S.Eziada ${ }^{2}$, A. Bashir ${ }^{2}$, A. Gameil ${ }^{2}$, H. Elmalik ${ }^{2}$, A. Allam ${ }^{2}$

${ }^{T}$ Hamad General Hospital, Oncology, Doha, Qatar

${ }^{2}$ National Center for Cancer Care \& Research-Hamad Medical Corporation, Medical Oncology, Doha, Qatar

\section{Introduction}

The prevalence of psychosocial distress is up to $45 \%$ among cancer patients. It is crucial to identify and treat distress in order to improve quality of life.

Objectives

Aim of the study is to not only report on prevalence of distress among cancer patients, but to also analyze the effect of the disease stage using the Distress Thermometer.

\section{Methods}

We studied distress among 3 groups, each consisting of 100 patients: those initially diagnosed, patients undergoing treatment, and patients who were referred to the palliative team. Different variables such as time of screening, sex, age, nationality, and tumor type were analyzed.

\section{Results}

There was an overall incidence of $62 \%$ (level $\geq 4)$ and $17 \%$ (level $>7)$ patients having severe distress. Similarly, there was $75 \%$ of distress among patients who were referred to palliative care, whereas those who were at their initial phase $(56 \%)$ and undergoing treatment (54\%). In addition, women (69\%) had more distress than men (53\%). Expatriates had higher levels of distress, compared to Qataris $(51 \%$ versus $7 \% p=0.001)$. Breast $(69 \%)$ and lung (70\%) cancer patients had the most distress. Physical causes of distress were the most common (80\%), followed by emotional causes (69\%).

Conclusions

The prevalence of significant psychosocial distress among our patients was higher than that reported internationally. Distress screening should be considered as an essential component of initial assessment

\section{eP406 \\ FACTORS INFLUENCING PSYCHOSOCIAL ADJUSTMENT IN PATIENTS AFTER RECONSTRUCTIVE SURGERIES FOR ORAL CANCER}

\section{W.L. Hsiao ${ }^{1}$, T.J. Wang $^{2}$}

${ }^{T}$ National Taiwan University, Nursing, Taipei, Taiwan R.O.C

${ }^{2}$ School of Nursing- National Taipei University of Nursing and Health Science, Nursing, Taipei, Taiwan R.O.C.

\section{Introduction}

Appropriate psychosocial adjustment is crucial for patients with oral cancer to cope with multiple stressors of their disease and to balance their lives within the restrictions imposed by the consequences of cancer and its treatments. Knowledge on predictive factors of psychosocial adjustment in oral patients after reconstructive surgeries has been limited.

\section{Objectives}

The study aim was to explore the relationships among, facial disfigurement, depression, social support, and psychosocial adjustment in patients after reconstructive surgeries for oral cancer

Methods

A cross-sectional study with a predictive correlational design. A convenient sample of 77 oral cancer patients post reconstruction surgeries was 
recruited from surgery outpatient clinics of three general hospitals in Taiwan. Data were collected with the study questionnaires, including the Facial Disfigurement Scale, the Social Support Scale, the Center for Epidemiologic Studies Depression Scale, and the Psychosocial Adjustment to Illness Scale.

\section{Results}

The mean score on the Psychosocial Adjustment to illness Scale was 413.01 ( $\mathrm{SD}=32.32$ ), indicating that these participants were struggling in adjusting to their illness. $71.4 \%$ of the participants were maladjusted. Employment status, financial status, location of tumor, perceived facial disfigurement, depression, family social support, and provider social support explained $68 \%$ of the variance in psychosocial adjustment $(F(4,95)=16.4, p<0.001) .32 .5 \%$ of the participants were clinically depressed.

Conclusions

The level of psychosocial adjustment in oral cancer patients is suboptimal. Overall, the patients who had no job, with a lower income, perceived severer facial disfigurement, severely depressed, and with weak family social support reported poorer psychosocial adjustment to their illness.

\section{eP407}

QUALITY OF LIFE IN CHILDREN OF CANCER PATIENTS IN CHILD-CENTRED COUNSELLING: NORM COMPARISONS AND ASSOCIATED FACTORS

\section{Inhestern ${ }^{I}$, C. Bergelt ${ }^{1}$}

${ }^{T}$ University Medical Center Hamburg-Eppendorf, Department of Medical Psychology, Hamburg, Germany

\section{Introduction}

A parental cancer diagnosis can increase the burden not only for the patients but also for their minor children. Hence, child-centred support offers have been developed to support children in coping and to prevent long-term consequences.

\section{Objectives}

The aim of this study was to assess the quality of life $(\mathrm{QoL})$ in children of cancer patients using child-centred support, to identify factors associated with QoL in children and to compare QoL to normative values.

\section{Methods}

We used data on 89 children (0-18 years) of 57 families attending childcentred counselling. Children's QoL was assessed before the first counselling session using the Kidscreen-10-Index (parent version). We conducted descriptive statistics, norm comparisons and multiple linear regression analysis.

\section{Results}

In $67 \%$ of the families, the mother was ill. Main diagnoses were neoplasms of digestive organs (23\%) and breast cancer (21\%). $61 \%$ of the families had two or more children. Of the 89 children, $53 \%$ were male; mean age was 9 years $(\mathrm{SD}=4.4)$. Parents seeking for child-centred support reported significantly lower QoL in their children compared to age- and gender-adapted norm values. Lowest QoL was reported in children of parents with CNS tumours and lymphoid/hematopoietic neoplasms. Female gender in the child, lower family functioning and cancer diagnosis (CNS, lymphoid/hematopoietic, breast) were associated with lower QoL in children.

Conclusions

Children of cancer patients in child-centred counselling show lower QoL compared to norm values, indicating the need for child- and familycentred counselling to support the children of cancer patients. A familycentred approach improving family functioning may help to enhance the children's QoL.

\section{eP408}

HEALTH CARE PROFESSIONALS, VIEWS ON THE TREATMENT AND CARE OF METASTATIC CASTRATE RESISTANT PROSTATE CANCER: RESULTS OF A SURVEY

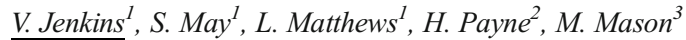

${ }^{T}$ University of Sussex, SHORE-C BSMS, Brighton, United Kingdom

${ }^{2}$ University College London, Oncology, london, United Kingdom

${ }^{3}$ Cardiff University, School of Medicine, Cardiff, United Kingdom

\section{Introduction}

Unfortunately, most advanced or metastatic prostate cancer will progress and become castrate resistant (CRPC). The management of metastatic (m)CRPC requires a multidisciplinary team (MDT) approach, with the patient receiving information from a number of different specialties and therefore important that MDT members provide patients with consistent information to guide treatment decisions.

\section{Objectives}

As part of an on-going study examining the EXperiences, TREatments and Quality Of Life (EXTREQOL) in men with mCRPC, we explored UK Health Care Professionals' views about this patient population.

\section{Methods}

A study specific survey (online and paper version) was available to members of the British Urology Group (BUG) and British Association of Urology Nurses (BAUN), between September 2016 and February 2017. It examined the current clinical practice and management of men with mCRPC.

\section{Results}

The response rate from nurses was lower than that of clinicians (28 nurses, 81 clinicians). The term mCRPC was rarely used with patients (20\%) but commonly with colleagues $(75 \%)$; the preferred phrase was "advanced prostate cancer" (59\%). During the diagnostic consultation clinicians indicated they almost always discussed current symptoms (92\%), PSA levels (88\%), treatment (89\%) and side effects (92\%), but topics such as prognosis (28\%), amelioration of side effects (56\%), and psychosocial well-being (11\%) were less regularly covered.

\section{Conclusions}

Delaying progression and palliation of symptoms are primary therapeutic aims of many treatments for mCRPC. Providing clear information on important topics such as prognosis and amelioration of side effects is vital in order to maximise a patient's quality of life.

\section{eP409}

SELF-EFFICACY AND RESILIENCE STATUS AMONG FAMILY CAREGIVERS OF CANCER PATIENTS IN TURKEY

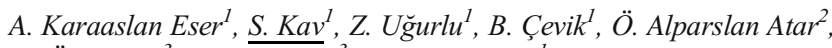 \\ H. Özençaktr ${ }^{3}$, A. Karabulut ${ }^{3}$, E. Akgün Çıtak ${ }^{l}$ \\ ${ }^{1}$ Baskent University, Faculty of Health Sciences Department of Nursing, \\ Ankara, Turkey \\ ${ }^{2}$ Baskent University Adana Hospital, Nursing Directorate, Adana, Turkey \\ ${ }^{3}$ Baskent University Ankara Hospital, Nursing Directorate, Ankara, Turkey
}

\section{Introduction}

Introduction: In diseases requiring long-term care such as cancer, the patients' problems affect both patients and family caregivers. Self-efficacy and psychological resilience can influence family members to cope with the problems they experience during the caregiving process.

\section{Objectives}

The aim of this study was to determine the status of psychological resilience and self-efficacy among family caregivers of cancer patients.

\section{Methods}

The study has been conducted at two ambulatory treatment centers of medical oncology department affiliated with a university hospital. Sample was included the 117 family caregivers of patients with cancer over 18 years and volunteer to participate. In data collection, sociodemographic data form, Turkish version of the Connor-Davidson Resilience Scale (CD-RISC) and Self-efficacy scale were used. 


\section{Results}

The mean age of the participants were $44.23 \pm 14.5$ (range: 19-23 years). Half of them were male (50.4\%), and university graduates $(54.7 \%)$. Mean duration of the caregiving was $24.12 \pm 28.56$ (range 2-180) months and $41.9 \%$ of them were spouse. One in third of the patients (35.9\%) diagnosed with gynecologic cancers and $26.5 \%$ of them were described as dependent to the caregivers. Self-efficacy scale mean was $87.81 \pm 13.43$ (range: 35-111) and 69.34 16.75 (range: 24-100) for CD-RISC. Male caregivers had higher self-efficacy scale scores and positive acceptance of change $(p<0.05)$. A positive significant correlations were found between self-efficacy scale and CD-RISC scores (r:0.26; $<<0.004)$.

Conclusions

Our results showed that caregivers reported moderate level of resilience and self-efficacy.

\section{eP410}

CANCER-RELATED DYSFUNCTIONAL BELIEFS ABOUT SLEEP MAY INFLUENCE INSOMNIA OF CANCER PATIENTS REGARDLESS OF DEPRESSION

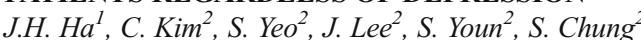

${ }^{1}$ Seoul Metropolitan Eunpyeong Hospital, Department of psychiatry, Seoul, Republic of Korea

${ }^{2}$ Asan Medical Center, Department of psychiatry, Seoul, Republic of Korea

\section{Introduction}

Depression is closely related to insomnia even in the absence of cancer, so in most of previous studies depressive symptoms are regarded as the factor influencing insomnia in cancer patients. Dysfunctional beliefs about sleep also may be related with insomnia and depression simultaneously.

Objectives

The aim of this study was to explore whether dysfunctional beliefs about sleep may influence insomnia regardless of depression.

Methods

All of 183 cancer patients who visited sleep clinic for cancer patients in Asan Medical Center (January November, 2017) were included in this study. At the first time of visit, patients were assessed with Insomnia Severity Index (ISI), Patient' Health Questionaaire-9 (PHQ-9), Fear of Progression (FoP), State and Trait Anxiety Inventory (STAI), and Cancerrelated Dysfunctional Beliefs about Sleep (C-DBS).

Results

Patients were $54.4 \pm 11.6$ years old, and $64 \%$ were female. Most common cancer of the subjects were breast (44\%), gastric (7.9\%), and pancreatic cancer (7.4\%). The cancer stages were as follows; I (27.4\%), II (27.9\%), III (19.6\%), and IV (25.3\%). The high C-DBS score was correlated with high ISI, PHQ-9, and FoP scores (all, $\mathrm{p}<0.01$ ) but not with age, cancer stage, and STAI score. Linear regression analysis revealed that severe insomnia was predicted by high C-DBS and PHQ-9 scores. Among patients who were not depressed ( $\mathrm{n}=71$, PHQ-9 $\leq 9)$, C-DBS was the only predicting factor for insomnia.

Conclusions

Dysfunctional beliefs about sleep were considered to be influenced by their depressive symptoms. However, we observed that those beliefs can influence insomnia symptoms of cancer patients regardless of their depression.

\section{eP411}

DETERMINATION OF LEVELS OF DEATH ANXIETIES AND DEATH DEPRESSION IN PATIENT RELATIVES WHO ARE CAREGIVERS OF ONCOLOGY PATIENTS

Z. Koç ${ }^{l}$, T. Kavalalı Erdoğan ${ }^{l}$, C. Atmaca Palazoğlu ${ }^{2}$, Z. Sağlam ${ }^{l}, S$. Masat $^{1}, \bar{C} \cdot \mathrm{K}_{\mathrm{Koc}} \mathrm{C}^{3}$
${ }^{I}$ Ondokuz Mayis University Faculty of Health Science, Nursing, Samsun, Turkey

${ }^{2}$ Gümüşhane University, Health Services Vocational School, Gümüşhane, Turkey

${ }^{3}$ Haccetepe University, Faculty of Medicine Student, Ankara, Turkey

\section{Introduction}

The attitudes and reactions developed by people towards death can vary from individual to individual.

\section{Objectives}

This study was conducted descriptively in order determine levels of death anxieties and depression related with death in patient relatives who are caregivers of oncology patients.

\section{Methods}

This study was conducted between 15.01.2015 and 22.04.2015. Seventy oncology patients and their relatives comprised the study group and 70 patients and relatives who were receiving due to a chronic disease other than cancer comprised the study group. Data was obtained by using a 30question questionnaire and Death Anxiety Scale and Death Depression Scale.

Results

Average Death Anxiety Scale score of the patient relatives in the study group was determined to be $9.0 \pm 2.6$ and average Death Anxiety Scale score of the patient relatives in the control group was determined to be $8.4 \pm 2.4$. No statistically significant difference has been determined in both study group and the control group $(\mathrm{p}=0.215)$. Total average Death Depression Scale score of patient relatives in the control group was determined to be 10.6 \pm 2.7 and total average Death Depression Scale score of patient relatives in the control group was determined to be $9.6 \pm 2.6$. A statistically significant difference has been determined in both study group and the control group ( $\mathrm{p}=0.043)$.

\section{Conclusions}

In this study, although patient relatives of oncology patients had more intensive feelings of death depression, sadness and loneliness compared to the control group, it was determined that no significant difference was present between both groups in terms of death anxiety.

\section{eP412}

IF DIAGNOSES MAKE DIFFERENCES IN LEVELS OF FEAR OF CANCER RECURRENCE AND CARE NEEDS: COMPARISON AMONG THREE TYPES OF CANCERS

\section{Y.H. Lai $^{1}$}

${ }^{T}$ National Taiwan University NTU, College of Medicine- NTU Cancer Center- NTU Supportive Cancer Care Research Team, Taipei, Taiwan R.O.C.

\section{Introduction}

Fear of cancer recurrence (FCR) is the most severe psychological distress in cancer patients. However, relatively limited research has explored the levels of FCR and care needs among different types of cancer populations.

\section{Objectives}

The purpose of this study is to explore three major types of cancer survivors (Early Stage Lung Cancer, GYN Cancer, Head \& Neck Cancer) of their experiences in FCR and care needs in a medical center in Northern Taiwan.

\section{Methods}

The validated Chinese version of Fear of Recurrence (FoR-Chinese) questionnaire and Supportive Care Needs Scale (SCNS) were used to assess patients. Cancer patients who were operable and have already completed their major anti-cancer treatments and with disease-free 
conditions were eligible. IRB approval and patients consents were obtained before data collection.

\section{Results}

A total of 210 subjects were recruited with 70 in each group. Patients with early stage lung cancer reported to have the highest concerns about the cancer recurrence and care needs. Compared to the other two types of cancer patients, GYN cancer patients reported to have relatively lower levels in both concerns.

\section{Conclusions}

Although most of these subjects were in early stage and operable, FCR and unmet care needs are still their concerns. Differences of cancer diagnosis might lead to different concerns. Individualized care based on a systematic assessment of FCR and care needs should be applied to improve cancer patients' life quality.

\section{eP413}

\author{
ANXIETY, DEPRESSION AND RELATED FACTORS IN \\ FEMALE CANCER PATIENTS IN ONCOLOGY OUTPATIENT \\ SETTINGS IN TAIWAN \\ Y.H. Lee I , C. Jui-Chun ${ }^{2}$, L.In-Fun ${ }^{3}$, S. Hui-Hsuan ${ }^{4}$ \\ ${ }^{T}$ National Taiwam University, School of Nursing, Taipei, Taiwan R.O.C. \\ ${ }^{2}$ Mackay Medicine College, Department of Nursing, Taipei, Taiwan \\ R.O.C. \\ ${ }^{3}$ Mackay Memorial Hospital, Department of Nursing, Taipei, Taiwan \\ R.O.C. \\ ${ }^{4}$ Mackey Memorial Hospital, Department of Internal Medicine, Taipei, \\ Taiwan R.O.C.
}

\section{Introduction}

In Taiwan, psychosocial care needs tend to be relatively neglected in outpatient settings.

\section{Objectives}

This study aims to (i) examine the prevalence and severity (levels) of anxiety and depression among female cancer patients in outpatient settings in Taiwan; and (ii) identify the factors related to anxiety and depression.

Methods

A cross-sectional study design was conducted to recruit the eligible patients from oncology outpatient setting in a teaching medical center in Northern Taiwan. Structured questionnaires were used, included (1) Hospital Anxiety and Depression Scale (HADS); (2) Social Support Scale (MOS-SSS); (3) Selfmanagement Ability Scale; and (4) Background Information form (BIF). Linear regression was conducted to identify the factors related to anxiety and depression.

\section{Results}

A total of 116 female cancer patients were recruited in this study. The major results showed that (i) $17.2 \%$ and $21.6 \%$ of patients had the risk of anxiety and depression, respectively (at risk cases indicated anxiety or depression scores $=8-21$ in this study). The severity (levels) of anxiety and depression were $4.2(\mathrm{SD}=4.1)$ and 4.1 ( $\mathrm{SD}=4.0)$, respectively; (ii) patients who had lower social support, lower self-management ability, worse physical function, and not having attending social support group reported higher levels of anxiety and total variance explained $34.7 \%$; patients who had lower social support, lower self-management ability, and having comorbidity reported higher levels of depression and total variance explained $44.3 \%$.

Conclusions

There were still about one-fifths of female patients at risk of anxiety and depression. Factors related anxiety and depression were identified in this study should be addressed for alleviating patients' distress.
eP414

\section{MEANING OF MOTHERHOOD TO YOUNG WOMEN WITH} BREAST CANCER

P. Alexandria Pinto de Magalhães ${ }^{1}$, E. Assunção Caetano de Loyola ${ }^{2}, T$. da Silva Vaz Paterra ${ }^{3}$, M. Lopes Borges ${ }^{4}$, A.P. Alonso Reis ${ }^{5}$, A. Ferreira Ouchi França ${ }^{6}, M$. Sanches Panobianco ${ }^{7}$

${ }^{I}$ National Service of Commercial Learning, Health and wellness, Catanduva, Brazil

${ }^{2}$ Prof of University of Alfenas, Department of Nursing, Alfenas, Brazil

${ }^{3}$ Master's Degree student of College of Nursing of Ribeirão Preto - USP, Postgraduate Nursing Program in Public Health, Ribeirão Preto, Brazil ${ }^{4}$ assistant professor of College of Nursing of Ribeirão Preto - USP, Department of Maternal and Child Nursing and Public Health, Ribeirão Preto, Brazil

${ }^{5}$ PhD student of College of Nursing of Ribeirão Preto - USP, Postgraduate Nursing Program in Public Health, Ribeirão Preto, Brazil ${ }^{6}$ PhD student of State University of Western Paraná, Center for education- letters and health, Foz do Iguaçu, Brazil

${ }^{7}$ Prof Dr of College of Nursing of Ribeirão Preto - USP, Department of Maternal and Child Nursing and Public Health, Ribeirão Preto, Brazil

\section{Introduction}

Introduction: Breast cancer compromises the building of the female existence in young women, mainly the motherhood.

\section{Objectives}

Aim: To comprise the meanings of the breast cancer experience to young women related to motherhood.

Methods

Method: A qualitative study developed in a Mastology Outpatient Clinic and a Mastectomized Rehabilitation Nucleus in Brazil. There were used Discourse of the Collective Subject (DCS) as the methodological approach, and Symbolic Interacionism as the theoretical approach. The data collection occurred between February, 2014 to January, 2015. Inclusion criteria: women between 18 to 40 years old, and with up to one year of the breast cancer diagnosis. Twelve women were interviewed using the guiding question: "Tell me about being a young woman with breast cancer in relation to motherhood". Thematic Content Analysis was applied to the DCS.

\section{Results}

Results: Four theoretical categories were established: "The motherhood has shown me a huge love. But the cancer shackes me at this age, because I have many dreams", "Impossibility of having children after treatment", "Importance of breastfeeding", and "My children are my strength to fight against breast cancer". To young women who had children, it meant "overcoming", "a light at the end of the tunnel" and "strength". To the others, it meant to give up the idea of being a mother to avoid suffering and frustrations. It meant fear of not breastfeeding or not to continue breastfeeding.

\section{Conclusions}

Conclusion: Despite of the difficulties related to motherhood, it was recognized as the source of the strength to believe in curing the disease.

\section{eP415}

DETERMINING THE ATTITUDES OF COPING WITH DISTRESS LEVELS OF ONCOLOGY PATIENT RELATIVES Z. Koç ${ }^{1}$, S. Masat ${ }^{1}$, Z. Sağlam ${ }^{1}$, T. Kavalalı Erdoğan ${ }^{1}, \dot{I}$.C. Koç ${ }^{2}$

${ }^{1}$ Ondokuz Mayis University Faculty of Health Science, Nursing, Samsun, Turkey

${ }^{2}$ Haccetepe University, Faculty of Medicine Student, Ankara, Turkey

\section{Introduction:}

The oncology patient relatives may experience a high level of distress during the diagnosis, treatment and prognosis processes of their patients. 
Objectives

This study was conducted as a descriptive study aiming to determine the attitudes of coping with distress levels of the oncology patients' relatives. Methods

The research was conducted between 11.02.2017 and 22.06.2017 with the participation of 211 relatives of the oncology patients who were under treatment in the chemotherapy unit of a hospital. The data were collected using a questionnaire aiming to determine the sociodemographic and clinical characteristics of the patients and their relatives, Coping Attitudes Assessment Scale and Distress Thermometer.

\section{Results}

This study revealed that, of the patients relatives, $64 \%$ were female and $36 \%$ were male, $72.5 \%$ were working, $79.1 \%$ had equal incomes to their expenses, $47.4 \%$ were the wives of the patients, $82 \%$ lived in the same house with the patient and $49.8 \%$ had been taking care of the patients for 3-6 months. In this study, $10.9 \%$ of the patients relatives were found to get the score of 4 and above, which is the cut-off score, on the distress thermometer. They were observed to obtain the highest score from the Active Coping sub-dimension and the lowest score from the Denial subdimension.

\section{Conclusions}

In this study, approximately one-tenth of the patient relatives had high levels of distress and they were determined to benefit from the approaches of using social support and suppressing other preoccupations in order to be able to cope with their distress.

\section{eP416}

DETERMINATION OF LEVELS OF DEPRESSION AND HOPELESSNESS OF RELATIVES OF PATIENTS RECEIVING ONCOLOGICAL TREATMENT

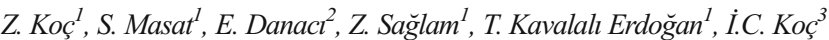

${ }^{1}$ Ondokuz Mayis University Faculty of Health Science, Nursing, Samsun, Turkey

${ }^{2}$ Bülent Ecevit University, Ahmet Erdoğan Health Services Vocational School, Zonguldak, Turkey

${ }^{3}$ Haccetepe University, Faculty of Medicine Student, Ankara, Turkey

\section{Introduction}

Cancer is an important health problem affecting both patients and their relatives in many dimensions including physical, social, economic and spiritual dimensions.

\section{Objectives}

This study was conducted in order to determine levels of depression and hopelessness of relatives of patients receiving oncological treatment.

Methods

The research was performed with participation of 151 relatives of oncology patients who received outpatient treatment in chemotherapy unit of a university hospital between 18.03.2015 and 25.06.2015. Data was obtained by using a 24-question questionnaire, Beck's Depression Inventory and Beck's Hopelessness Scale.

\section{Results}

In this study; it was determined that $43 \%$ of patients were women, $57 \%$ were men, $28.5 \%$ diagnosed with gastrointestinal cancer, $36.4 \%$ were at stage 3 , and $29.8 \%$ were diagnosed within last year. It was also determined that of the relatives of oncology patients; $67.5 \%$ were women, $32.5 \%$ were men, $49 \%$ were working, $69.5 \%$ had incomes equal to expenditures, $34.4 \%$ were patients' husband/wife, $31.1 \%$ were patients' son/daughter, $58.9 \%$ were living in same houses and $35.1 \%$ had taken care of the patient for more than a year. In this study, average Beck's Depression Inventory score was determined to be $16.2 \pm 10.4$ and average Beck's Hopelessness Scale score was determined to be $8.0 \pm 3.2$.

\section{Conclusions}

It was observed that status of hopelessness in patient relatives was moderate and $22.5 \%$ had moderate-level depression. It was also determined that depression and hopelessness scores of patient relatives differed in accordance with characteristics such as age, gender, marital status, educational status, occupation, degree of relationship, status of sharing the same house.

eP417

DEPRESSION AMONG BREAST CANCER PATIENTS AS COMPARED WITH THAT IN FEMALES WHO UNDERGO BREAST CANCER INSPECTION AND SCREENING

C. Minowa ${ }^{I}$

${ }^{T}$ Takasaki University of Health and Welfare, Department of Nursing, Takasaki, Japan

\section{Introduction}

The number of women with depression has been rapidly increasing worldwide. Consequently, it is thought that the prevalence of depression is also rapidly increasing in breast cancer patients. However, few studies have examined depression among breast cancer patients as compared with that in females who undergo breast cancer inspection and screening.

\section{Objectives}

The aim of this study was to assess the prevalence of depression in breast cancer patients as compared with that in females who undergo breast cancer inspection and screening.

\section{Methods}

Advertising posters were put on the wall of the waiting room at the clinic to recruit participants for the survey. A questionnaire comprising demographic characteristics and the Hospital Anxiety and Depression Scale (HADS) was used to collect data from females visiting a breast disease clinic.

Results

The participants were 193 women who visited a breast disease clinic, who were classified into the following three groups: breast cancer $(n=66)$, breast cancer screening $(n=102)$, breast inspection $(n=25)$. A HADS depression score of 8 or more was observed in 40 of the patients with breast cancer $(60.6 \%), 58$ of those who had undergone breast cancer screening (56.9\%), and 16 of those seeking breast inspection (64.0\%). There was no significant difference in the prevalence of depression among the 3 groups. Conclusions

These results suggest that screening for depression may be needed not only for breast cancer patients but also for patients who seek breast inspection and breast cancer screening.

\section{eP418}

THE EFFECTS OF EYE CONTACT WHEN DISCLOSING PROGNOSIS TO WOMEN WITH BREAST CANCER: A RANDOMIZED SCRIPTED VIDEO-VIGNETTE STUDY (JSUPPORT 1601)

M. Fujimori ${ }^{1}$, M. Mori co-primary author ${ }^{2}$, L. van Vliet ${ }^{3}$, T. Yamaguchi ${ }^{4}$,

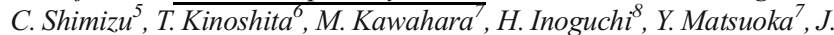
Bensing $^{9}$, E. Bruera $^{10}$, T. Morita ${ }^{11}$, Y. Uchitomi $^{12}$

${ }^{1}$ National Cancer Center, Division of Cohort Consortium ResearchEpidemiology and Prevention Group-Center for Public Health Sciences- Division of Health Care Research- QOL Research GroupCenter for Public Health Sciences, Tokyo, Japan

${ }^{2}$ Seirei Mikatahara General Hospital, Palliative Care Team, Hamamatsu, Japan

${ }^{3}$ Nivel - Netherlands Institute for Health Services Research, Department of Communication, Utrecht, The Netherlands

${ }^{4}$ Tohoku University Graduate School of Medicine, Division of Biostatistics, Sendai, Japan

${ }^{5}$ National Cancer Center Hospital, Department of Breast and Medical Oncology, Tokyo, Japan

${ }^{6}$ National Cancer Center Hospital, Department of Breast Surgery, Tokyo, Japan

${ }^{7}$ National Cancer Center, Division of Health Care Research- QOL Research Group-Center for Public Health Sciences, Tokyo, Japan 
${ }^{8}$ National Cancer Center Hospital, Department of Psychooncology, Tokyo, Japan

${ }^{9}$ Nivel - Netherlands Institute for Health Services Research- Utrecht University, Faculty of Social and Behavioral Science, Utrecht, The Netherlands

${ }^{10}$ The University of Texas MD Anderson Cancer Center, Department of Palliative Care and Rehabilitation Medicine- Division of Cancer Medicine, Houston, USA

${ }^{11}$ Seirei Mikatahara General Hospital, Palliative and Supportive Care Division, Hamamatsu, Japan

${ }^{12}$ National Cancer Center Hospital, Innovation Center for SupportivePalliative and Psychosocial Care-Division of Health Care ResearchQOL Research Group- Center for Public Health Sciences, Tokyo, Japan

\section{Introduction}

Oncologists' empathic communication has been shown to improve trust and reduce patient distress when a bad news is delivered. However, little is known if non-verbal empathy, such as eye contact, with explicit prognostic disclosure positively affects patients with advanced cancer.

Objectives

To assess the interaction effect of eye-contact with explicit prognostic communication at the time of cancer recurrence.

Methods

In this randomized, experimental $2 \times 2$ video-vignette study, women with breast cancer who had undergone curative surgery ( $\mathrm{n}=105$, age: $53.8 \pm 8.2)$ viewed four videos of prognostic communication between a patient with recurrent breast cancer and her oncologist. The videos differed only in the presence or absence of "eye contact" and "explicit prognostic disclosure". The main outcomes included participants' trust in the oncologist $(0-10)$, their six basic emotions $(0-$ 6 ), and their rating on the oncologist's compassion $(0-50)$.

\section{Results}

No interaction between eye contact and explicitness was found. After viewing the video with eye contact, compared with the video without it, participants exhibited significantly higher rating on trust (6.8 [SE, 0.2] vs. $3.5[0.2], \mathrm{p}<0.001$ ), lower negative emotions (mean of 5 negative emotions: anger/sadness/fear/disgust/surprise) (1.3 [0.2] vs. 2.4 [0.2], $\mathrm{p}<0.001$ ), and lower rating on the oncologist's compassion (reflecting higher oncologist's compassion) (18.5 [1.3] vs. 31.3 [1.3], p<0.001). No significant differences in the outcomes were observed between videos with and without explicit disclosure.

\section{Conclusions}

Eye contact is recommended for busy oncologists when disclosing prognosis, as it may convey empathy without taking extra time, and help oncologists build rapport with patients.

Table. Main effects of eye contact and explicit prognostic disclosure on outcomes

\begin{tabular}{lcccccccc}
\hline & \multicolumn{3}{c}{ Eye contact } & & \multicolumn{3}{c}{ Explicitness } \\
\cline { 2 - 3 } \cline { 7 - 8 } Effect & Difference & $95 \% \mathrm{CI}$ & $p$ & & Difference & $95 \% \mathrm{CI}$ & $p$ \\
\hline Trust $^{2}$ & -3.3 & $-3.7 .-3.0$ & $<0.001$ & & -0.2 & -0.5 .0 .2 & 0.372 \\
Physician compassion $^{\circ}$ & 12.8 & 10.6 .15 .1 & $<0.001$ & & -1.1 & -3.4 .1 .1 & 0.322 \\
Six basic emotions $^{c}$ & & & & & & & \\
$\quad$ Anger & 1.6 & 1.3 .1 .9 & $<0.001$ & & 0.2 & -0.1 .0 .5 & 0.275 \\
Sadness & 0.7 & 0.3 .1 .0 & $<0.001$ & & 0.2 & -0.1 .0 .5 & 0.130 \\
Fear & 0.7 & 0.5 .1 .0 & $<0.001$ & & 0.1 & -0.2 .0 .4 & 0.356 \\
Disgust & 1.5 & 1.2 .1 .8 & 0.075 & & 0.1 & -0.2 .0 .4 & 0.398 \\
Happiness & -0.2 & $-0.4,0.02$ & $<0.001$ & & -0.02 & -0.2 .0 .2 & 0.840 \\
Surprise & 0.7 & $0.4,1.1$ & $<0.001$ & & 0.05 & -0.3 .0 .4 & 0.754 \\
\hline
\end{tabular}

: : Scores range from $0-10$ (low to high)

$\checkmark$ : Score ranges from $0-50$ (high to low)

': Differences in scores $(0-6$, low to high) before and after viewing the video

Abbreviations: $\mathrm{CI}$, confidence interval.
eP419

ANTI-OESTROGENIC HORMONE THERAPY AND RISK FACTORS FOR DEPRESSIVE DISORDER IN WOMEN WITH BREAST CARCINOMA UNDER ADJUVANT TREATMENT: A SYSTEMATIC REVIEW OF THE LITERATURE

C. Oliveira ${ }^{1}, J$. Ramos ${ }^{1}$, M. Nascimento ${ }^{2}$, D. Martins-Branco ${ }^{3}$, E. Gouveia $^{3}$, L. Monteiro ${ }^{4}$

${ }^{1}$ Hospital Prof. Dr. Fernando Fonseca, Psychiatry, Amadora, Portugal ${ }^{2}$ Hospital Vila Franca de Xira, Psychiatry, Vila Franca de Xira, Portugal ${ }^{3}$ Instituto Português de Oncologia de Lisboa FG, Medical Oncology, Lisbon, Portugal

${ }^{4}$ Instituto Português de Oncologia de Lisboa FG, Psychiatry, Lisbon, Portugal

\section{Introduction}

Women with breast carcinoma (BC) have increased risk of depression. Anti-oestrogenic hormone therapy (HT) is often indicated as a possible cause.

Objectives

We aim to summarize evidence on this topic and to address factors associated with depression in BC survivors under HT.

\section{Methods}

Systematic review of literature using 3 electronic databases (Medline, Embase and PsycInfo) until December 31, 2016. We included randomized controlled trials (RCTs) and observational studies (ObS) of adult women with non-metastatic BC under adjuvant treatment.

Results

We identified 393 articles, 23 met the inclusion criteria: 5 RCTs and 18 ObS. $11 \mathrm{ObS}$ analysed HT as an independent factor for depression: 2 favoured the reduction of risk, 4 showed association with depression and 5 showed no association. 7 studies (4 RCTs and $3 \mathrm{ObS}$ ) reported no differences between HT drugs. 12 studies identified factors associated with depression during HT: i) 1 RCT demonstrated that physical exercise and hypocaloric diet may reduce the risk; ii) $11 \mathrm{ObS}$ identified the following risk factors: active smoking $(\mathrm{n}=1)$, psychosocial variables $(\mathrm{n}=$ $1)$, menopausal symptoms $(n=2)$, chemotherapy $(n=2)$ and expression of oestrogen receptors in the tumour $(n=1)$. The most frequently used scale was "Beck Depression Inventory".

\section{Conclusions}

The role of HT in the emergence of depression in $\mathrm{BC}$ survivors remains unclear. However, the possibility that HT induces depressive states in a vulnerable subgroup of patients should not be excluded. This review identified risk factors and valid tools for evaluating depression that should be considered in future prospective studies.

\section{eP420}

DYADIC RELATIONSHIPS AMONG SENSE OF MASTERY, CANCER-RELATED DISTRESS, AND CORTISOL IN PATIENTS AND CAREGIVERS IMPACTED BY ABDOMINAL AND PELVIC MALIGNANCIES

A. Padron-B.S. ${ }^{1}$, S. Wong- Ph.D. ${ }^{2}$, M.L. Armstrong- Ph.D. ${ }^{3}$, T. GeorgeM.D. ${ }^{4}$, D.B. Pereira- Ph.D. ${ }^{3}$

${ }^{1}$ University of Florida, Clinical \& Health Psychology, Gainesville- FL, USA

${ }^{2}$ Wake Forest University, School of Medicine, Winston-Salem, USA

${ }^{3}$ University of Florida, Clinical \& Health Psychology, Gainesville, USA

${ }^{4}$ University of Florida, Department of Medicine-Division of Hematology-Oncology, Gainesville, USA

\section{Introduction}

The Biobehavioral Model of Tumorigenesis posits that stress appraisals are associated with cancer outcomes via effects on mood, cortisol, and immunity. However, this model does not account for dyadic effects of 
stress on outcomes, which is a literature gap given the $4,000,000$ caregivers aiding adult cancer patients.

\section{Objectives}

To examine dyadic effects of stress appraisals on cancer-related distress and appraisals/distress on salivary cortisol in patients/caregivers affected by abdominopelvic cancers.

Methods

Participants were 16 patient-caregiver dyads affected by gynecologic or pancreatic cancers enrolled in mixed methods studies exploring quality of life using Photovoice. Self-mastery and cancer-related distress were assessed by the Sense of Mastery Scale and Impact of Events Scale, respectively. Cortisol was assessed in pancreatic cancer dyads via enzyme immunoassay. Actor-Partner Interdependence Model (APIM) analyses were used. Qualitative data were analyzed to elaborate upon APIM analyses.

\section{Results}

Contrary to hypotheses, greater caregiver self-mastery was associated with greater patient distress avoidance $[t(13.0)=-2.71, p=0.018]$. There were no significant partner/actor effects of self-mastery on cortisol. However, higher patient distress intrusion was marginally associated with more regulated caregiver diurnal cortisol $[t(4.19)=-2.32, p=0.085]$. Qualitative analyses revealed that female patients reported unmet psychosocial needs despite their male partner/caregivers use and belief in the effectiveness of problem-focused coping to manage dyadic distress.

Conclusions

Preliminary results suggest inverse effects of caregiver self-mastery on patient cancer-related distress avoidance. Qualitative findings highlight unmet psychosocial needs of female cancer patients.

\section{eP421}

COGNITIVE BEHAVIORAL EFFECTS ON TOTAL WAKE TIME IN WOMEN WITH GYNECOLOGIC MALIGNANCIES AND INSOMNIA

A. Padron ${ }^{1}$, G. Cartagena ${ }^{2}$, C.S. McCrae ${ }^{3}$, M.E. Robinson ${ }^{2}$, L. Trinastic ${ }^{4}$, R.A.Postupack ${ }^{5}$, D. Esperza-Duran ${ }^{6}$, E. Kacel ${ }^{2}$, G. Schultz ${ }^{7}, J$. Castagno $^{7}$

${ }^{1}$ University of Florida, Clinical and Health Psychology, Gainesville- FL, USA

${ }^{2}$ University of Florida, Clinical and Health Psychology, Gainesville, USA

${ }^{3}$ University of Missouri, Psychiatry, Missouri, USA

${ }^{4}$ PBS Health Care, Potomac Behavioral Solutions, Arlington, USA

${ }^{5}$ University of Florida, Clinical and Health Psychology, Middletown, USA

${ }^{6}$ Loma Linda University, Institute for Behavioral Health, Loma Linda, USA

${ }^{7}$ University of Florida, Obstetrics and Gynecology, Gainesville, USA

\section{Introduction}

Sleep disturbance is a common outcome associated with cancer diagnosis and treatment that contributes to depression, low quality of life, and morbidity. While there is evidence for the effectiveness of Cognitive Behavioral Therapy for insomnia (CBTi) in breast cancer survivors, there exists a lack of empirical evidence on its efficacy within gynecologic malignancies.

Objectives

To examine the effects of a CBT intervention for insomnia and pain (CBTip) subjective Total Wake Time (TWT) in women with confirmed gynecologic cancers.

\section{Methods}

Participants were 35 women with insomnia status/post-surgery for gynecologic cancer randomized to either a 6-week individual CBTip intervention $(N=18)$ or a 6 -week individual Psychoeducation program $(N=17)$. Subjective TWT was assessed via 14 days of sleep diaries at baseline (Wk0), post-intervention (Wk7), 2 month follow-up (Wk14), and one- year follow-up (Wk66). Mixed linear modeling (MLM) was conducted to examine longitudinal group differences on subjective TWT with planned contrasts examining group differences in TWT at Wk7, Wk14, and Wk66.

\section{Results}

Immediately post-intervention at $\mathrm{Wk} 7$, CBTip participants showed a nonsignificant trend toward lower TWT $(b=-28.15$, $p=0.055)$ compared to Psychoeducation participants. By Wk14, this trend was fully significant, such that CBTip participants demonstrated significantly lower TWT $(\mathrm{b}=-32.24, p=0.036)$ compared to Psychoeducation participants. However, there were no group differences in TWT at Wk66.

\section{Conclusions}

CBTip significantly reduced subjective TWT in women with gynecologic cancers and insomnia during the acute postsurgical treatment phase. Future research will focus on bidirectional effects of sleep and Hypothalamic-pituitary-adrenal (HPA) axis dysregulation.

\section{eP422}

DOCTOR, ARE YOU HEALTHY? A CROSS-SECTIONAL INVESTIGATION OF ONCOLOGIST BURNOUT, DEPRESSION, AND ANXIETY AND AN INVESTIGATION OF THEIR ASSOCIATED FACTORS

C. Paiva ${ }^{I}$, B. Martins ${ }^{2}$, B. Paiva ${ }^{2}$

${ }^{T}$ Barretos Cancer Hospital-Barretos- SP-Brazil, Department of Clinical Oncology, Barretos, Brazil

${ }^{2}$ Barretos Cancer Hospital-Barretos-SP-Brazil, Health-Related Quality of Life Research Group GPQual- Learning and Research Institute, Barretos, Brazil

\section{Introduction}

Doctors who work at cancer hospitals are at high risk of developing emotional distress.

\section{Objectives}

This study evaluated the prevalence of burnout, anxiety, and depression in a sample of oncologists of various specialties and sought to identify how much of this distress is explained by specific pre-established characteristics.

\section{Methods}

This cross-sectional study used online surveys. Burnout was measured using the Maslach Burnout Inventory (MBI), and anxiety and depression were measured using the Hospital Anxiety and Depression Scale (HADS). The variables associated with $\mathrm{p}$ values $<0.05$ in the univariate analyses were included in blocks of hierarchical binary logistic regression models to identify the predictors of burnout, depression, and anxiety.

\section{Results}

Of the 227 physicians (response rate $=70.5 \%), 132(58.1 \%)$ were identified as having burnout (high emotional exhaustion [EE] and depersonalization [DP]); furthermore, 28 (12.3\%) had depression (HADS-D $\geq 11$ ), and $44(19.4 \%)$ had anxiety (HADS-A $\geq 11)$. The block of perceptions related to the workplace explained $21.6 \%, 5.8 \%$, and $10.8 \%$ of the variances of burnout, depression, and anxiety, respectively. On the other hand, the outside-of-work characteristics block explained only $3.6 \%$, $10.5 \%$, and $3.6 \%$ of the variances of burnout, depression, and anxiety, respectively.

\section{Conclusions}

Work-related stressors are associated with burnout, but few are associated with anxiety and depression. Outside-of-work characteristics explained little of the distress reported by physicians. Strategies focused on perceptions of professional recognition and lower workloads that stimulate positive relationships between doctors and other health professionals are desirable in oncological context. 


\section{eP423}

EVALUATION OF BURNOUT, ANXIETY AND DEPRESSION SYMPTOMS IN NURSING PROFESSIONALS WORKING IN ONCOLOGY PALLIATIVE CARE

B. Paiva ${ }^{1}$, J. de Menezes ${ }^{2}$, T. Valentino ${ }^{3}$, C. Paiva ${ }^{4}$

${ }^{1}$ Barretos Cancer Hospital-Barretos- SP-Brazil, Research Group on Palliative Care and Health-Related Quality of Life GPQual, Barretos, Brazil

${ }^{2}$ Barretos Cancer Hospital-Barretos- SP-Brazil, Palliative Care Unit, Barretos, Brazil

${ }^{3}$ Barretos Cancer Hospital- Barretos- São Paulo-Brazil, Research Group on Palliative Care and Health-Related Quality of Life GPQual, Barretos, Brazil

${ }^{4}$ Barretos Cancer Hospital-Barretos- SP-Brazil, Clinical Oncology Department, Barretos, Brazil

\section{Introduction}

Palliative care (PC) patients often require increased emotional support, symptom improvement, and end-of-life support, requiring specific and comprehensive attention from nurses, which can lead to burnout, anxiety, and depression.

Objectives

To evaluate the levels of burnout, anxiety and depression among nursing professionals.

\section{Methods}

A cross-sectional study conducted at a PC Unit from reference oncology institution in Brazil. Registered nurses and nursing technicians with more than three months of experience in the PC unit were included. To assess burnout, we used the Maslach Burnout Inventory (MBI), that measures emotional exaustion (EE), depersonalization (PD), and professional achievement (PA). Burnout was defined as high EE combined with high DP. Symptoms of anxiety and depression were measured with the Hospital Anxiety and Depression Scale (HADS); HADS-A or HADS-D> 11 scores defined anxiety and depression, respectively.

\section{Results}

Of the 56 professionals, $30.1 \%(17 / 55), 17.8 \%(10 / 56)$ and $7.4 \%$ (4/54) were identified with burnout, anxiety and depression, respectively. Regarding burnout domains, 25\% (14/55) presented elevated levels of EE, $10.7 \%(6 / 55)$ high DP and $10.7 \%(6 / 55)$ low PA. Difficulties in relationship with the multiprofessional team $(\mathrm{p}=0.009)$ and overwork $(\mathrm{p}=0.011)$ were associated with burnout; dissatisfaction with institutional norms $(\mathrm{p}=0.029)$ were associated with anxiety.

Conclusions

The identified rates of burnout, anxiety and depression are lower than expected. Since teamwork is an essential component of palliative care, strategies to improve relationship between nurses and other member of PC team are warrant.

\section{eP424}

CANCER CARE EXPERIENCES AND UNMET NEEDS OF LESBIAN, GAY, AND BISEXUAL PEOPLE WITH CANCER: A SYSTEMATIC REVIEW OF QUALITATIVE EVIDENCE

K. Lisy ${ }^{1,2}$, M. Peters ${ }^{3}$, P. Schofield ${ }^{2,4,5}$, M. Jefford ${ }^{1,5}$

${ }^{1}$ Peter MacCallum Cancer Centre, Australian Cancer Survivorship Centre, Melbourne, Australia

${ }^{2}$ Peter MacCallum Cancer Centre, Department of Cancer Experiences Research, Melbourne, Australia

${ }^{3}$ University of South Australia, the Rosemary Bryant AO Research Centre- School of Nursing and Midwifery, Adelaide, Australia

${ }^{4}$ Swinburne University, Psychological Sciences, Melbourne, Australia

${ }^{5}$ The University of Melbourne, Sir Peter MacCallum Department of

Oncology, Parkville, Australia

\section{Introduction}

People who identify as sexually and/or gender diverse may face additional challenges in receiving equitable and appropriate cancer care leading to worse outcomes and avoidable harm. By synthesizing the cancer care experiences and unmet needs of this diverse and often overlooked population group, areas for improvement may be identified and changes implemented to support improved outcomes.

\section{Objectives}

To explore existing qualitative evidence regarding cancer care experiences and unmet needs of people who identify as sexually and/or gender diverse.

Methods

A qualitative systematic review and meta-synthesis of English language studies was undertaken. Following literature searching and study selection, methodological quality was examined and relevant qualitative data were extracted verbatim and synthesized using thematic analysis.

\section{Results}

Fifteen articles including lesbian, gay, and bisexual (LGB) people following a cancer diagnosis were included. No relevant studies published before 2002 were located. Studies including gender diverse people were not identified. Most participants were women with breast cancer or men with prostate cancer. Synthesis of 106 findings produced six core themes; sexual orientation disclosure, experiences and fear of homophobia, positive and negative healthcare professional behaviours, heterocentric systems and care, inadequacy of available support groups, and unmet needs for patient-centred care and LGB-specific information.

\section{Conclusions}

LGB people receiving care for cancer face challenges including anxiety, invisibility, isolation, and may receive inadequate care. Training and education of healthcare professionals is recommended to address challenges and practice gaps. Culturally appropriate care includes use of inclusive language, the provision of tailored information, involving partners in care and avoiding heterosexual assumptions.

\section{eP425}

\section{INFLUENCE OF ANEMIA ON COGNITIVE FUNCTIONS IN} CANCER PATIENTS

D. petranovic ${ }^{1}$, A. Petranovic ${ }^{2}$

${ }^{T}$ Clinical Hospital Rijeka, hematology, Rijeka, Croatia

${ }^{2}$ Faculty of humanities and social sciences, Psychology, Rijeka, Croatia

\section{Introduction}

Cancer patients are vulnerable to neurocognitive dysfunctions for a number of reasons.

\section{Objectives}

The aim was to analyse hypotesis that anemia influences cognition in therapy naive cancer patients and that cognitive functions could be improved by correction of anemia.

\section{Methods}

In Clinical Hospital Center of Rijeka, Croatia a total of 400 patients were categorised by prospective fourfold coupling in four groups (100 pts in each group) on the basis of two independent variables: cancer and anemia (1: anemic cancer pts, 2: with cancer but no anemia, 3: anemic patients without cancer, 4: healthy persons). Cognitive abilities were evaluated twice (before an after correction of anemia) by Complex Reactionmeter Drenovac (CRD) for perceptive abilities (detection, identification, visual orientation, spatial visualisation), memory (short term memory, maze learning, actualisation of memorized contents), thinking (operative thinking, problem solution, convergent thinking), psychomotor reactions ( simple and complex), dynamic features of CNS function (excitability, agility, stability, balance, endurance, reliability), attention (atention span, concentration, vigilance) and functional disturbances ( rigidity, agitation, perseverance, regression). 


\section{Results}

We found that hemoglobin $(\mathrm{Hb})$ level was in positive correlation with cognitive abilities, more then age, education and gender. Anemia and cancer had additive negative effect and cognitive abilities in cancer patients with anemia were the worst among all groups. Correction of anemia improved cognition but once anemic, cancer patients never achieved results of other groups.

Conclusions

We found that anemia has great impact on cognition. By correcting anemia, we could improve cognitive functions and so enhance quality of life in cancer patients.

eP426

CONDITIONS OF REFUSAL OF PSYCHO-ONCOLOGICAL SUPPORT SERVICES IN DISTRESSED CANCER PATIENTS. HOW CAN WE OPTIMIZE PSYCHO-ONCOLOGICAL SUPPORT SERVICES?

T. Pichler ${ }^{1}$, A. Dinkel ${ }^{2}$, K. Hermelink ${ }^{3}$, P. Heußner ${ }^{4}$, B. Marten-Mittag ${ }^{2}$, E. Telzerow ${ }^{4}$, P. Herschbach ${ }^{5}$

${ }^{1}$ Klinikum rechts der Isar - Technische Universität München, Comprehensive Cancer Center, Munich, Germany

${ }^{2}$ Klinikum rechts der Isar - Technische Universität München, Klinik und Poliklinik für Psychosomatische Medizin und Psychotherapie, Munich, Germany

${ }^{3}$ Klinikum der Universität München - Ludwig-Maximilians-Universität München, Klinik und Poliklinik für Frauenheilkunde und Geburtshilfe, Munich, Germany

${ }^{4}$ Klinikum der Universität München - Ludwig-MaximiliansUniversität München, Medizinische Klinik und Poliklinik III, Munich, Germany

${ }^{5}$ Klinikum rechts der Isar - Technische Universität München, Comprehensive Cancer Center- Roman Herzog Krebszentrum, Munich, Germany

\section{Introduction}

About one third of all cancer patients are so severely burdened by mental illness or pronounced subsyndromal disorders that they need professional psycho-oncological support. However, if such support is offered to these patients, about $60-80 \%$ refuse help.

Objectives

The aim of this study by the Comprehensive Cancer Center is to identify sociodemographic, medical and psychological conditions for the refusal of psycho-oncological help despite high psychological distress in order to be able to optimize the access to patients in need in the future.

Methods

In a multicenter study in Germany a total of $n=972$ inpatients with various cancer diagnoses were examined. Measuring instruments for psychological distress were the Distress Thermometer (Mehnert et al., 2006) and the FBK-R10 (Book et al., 2011). Further, we examined potential aspects regarding the refusal of psycho-oncological support services including sociodemographic (e.g. age), medical (e.g. tumor entity) and psychological conditions like depression (PHQ-4; Kroenke et al., 2009; Löwe et al., 2015), personality (BFI-10; Rammstedt \& John, 2007), social support, self-efficacy (ASKU; Beierlein et al., 2012), attitude towards psycho-oncological support and status of information.

Results

Data collection was accomplished in October 2017, results are expected by the end of February 2018 .

Conclusions

To be expected by March 2018 .
eP427

EXPLORING THE IMPACT OF PROVIDING MEN WITH INFORMATION ABOUT POTENTIAL PROSTATE CANCER TREATMENT OPTIONS PRIOR TO RECEIVING THEIR BIOPSY RESULTS

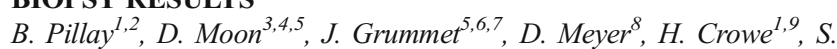

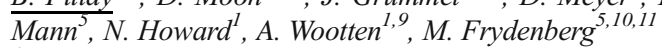

${ }^{1}$ Epworth Healthcare, Epworth Prostate Centre, Victoria, Australia

${ }^{2}$ Royal Melbourne Hospital, Department of Urology, Victoria, Australia

${ }^{3}$ Epworth Healthcare, Epworth Centre for Robotic Surgery, Victoria, Australia

${ }^{4}$ University of Melbourne, Surgery, Victoria, Australia

${ }^{5}$ Australian Urology Associates, Australian Urology Associates, Victoria, Australia

${ }^{6}$ Monash University, Department of Surgery, Victoria, Australia

${ }^{7}$ Alfred Health, Urology, Victoria, Australia

${ }^{8}$ Swinburne University, Department of Statistics Data Science and Epidemiology, Victoria, Australia

${ }^{9}$ Australian Prostate Centre, Australian Prostate Centre, Victoria, Australia

${ }^{10}$ Epworth Healthcare, Clinical Institute of Specialty Surgery, Victoria, Australia

${ }^{11}$ Monash Health, Department of Urology, Victoria, Australia

\section{Introduction}

A cancer diagnosis often causes anxiety, thus hampering patients' ability to make treatment decisions. There is little research assessing the impact of providing men with information about prostate cancer $(\mathrm{PCa})$ treatment options at time of referral for a prostate biopsy.

\section{Objectives}

Study objectives were to determine whether receiving an information booklet about PCa treatment options prior to biopsy was acceptable to patients, and if receiving this information influenced patients' levels of anxiety, depression and distress.

\section{Methods}

Patients undergoing prostate biopsy were randomised to receive either a standard biopsy instruction sheet (Control) or an information booklet about PCa treatment options in addition to the instruction sheet (Intervention). Ninety-eight patients completed validated Depression, Anxiety and Distress measures pre-biopsy and after receiving their biopsy results. Scores from baseline to follow-up were compared for both groups using mixed model repeated measures analysis.

\section{Results}

There were no significant differences between groups in terms of changes in psychological symptoms from baseline to follow-up (all $p>.05$ ). Control group patients receiving positive biopsy results reported an increase in distress $(p=.031)$ and depression scores $(p=.046)$ from pre- to post-biopsy. Intervention group patients receiving positive biopsy results showed no significant change in psychological symptoms. $87 \%$ of patients reported that the resource made it easier to understand subsequent explanation of treatment options.

\section{Conclusions}

Findings support the provision of written information regarding $\mathrm{PCa}$ treatment options at time of referral for a biopsy. Patients did not experience increased psychological distress as a result of the intervention, and preferred to be given this information prior to their biopsy.

eP428

PATH OF AWARENESS FOR THE CONSTRUCTION OF NEW PERSONAL MEANINGS. QUALITATIVE ANALYSIS OF THE PURPOSE IN LIFE (PIL) QUESTIONNAIRE IN AN ONCOLOGIC ITALIAN POPULATION

L. Buonaccorso ${ }^{1}$, G. Martucci ${ }^{2}$, G. Miccinesi ${ }^{3}$, A. Maruelli $^{4}$, C. Ripamonti $^{5}$ 
${ }^{1}$ Santa Maria Nuova Hospital - IRCCS Reggio Emilia, Psycho-Oncology, Reggio Emilia, Italy

${ }^{2}$ Italian League for the Fight Against Cancer LILT, Psycho-oncology, Reggio Emilia, Italy

${ }^{3}$ ISPO-Institute for the Study and Prevention of Cancer, Clinical Epidemiology, Firenze, Italy

${ }^{4}$ LILT and Center for Oncological Rehabilitation-CERION of Florence, Psychologic Unit, Firenze, Italy

${ }^{5}$ Fondazione IRCCS Istituto Nazionale dei Tumori, Supportive Care in Cancer, Milano, Italy

\section{Introduction}

The psychological intervention aimed to sustain patients in their personal search for meaning through a personal elaboration of the oncologic disease experience, may promote their physical, psychological and spiritual wellness during therapy.

\section{Objectives}

We investigated the meaning that oncological patients give to their illness, and the resources they put in practice to build new experiences of meaning and hope.

\section{Methods}

We conducted a thematic analysis of the qualitative parts B and C of the PIL questionnaire, which investigates the dimension of meaning, in 158 consecutive cancer patients on oncologic treatments, supportive care and/ or rehabilitation.

\section{Results}

We found the recurrence of a strong sense of positivity, present in the majority of the interviews. In particular, this positivity is showed by the use of words associated frequently to selfconsciousness and self-evaluation, desire of happiness and desire of contributing to the good of significant others and of taking care of your own life in order to give a positive contribution.

\section{Conclusions}

Conclusions. In our analysis it emerges that the presence of a purpose in life is linked to change, or in other words to the chance of understanding, through illness, what one might aspire to. The disease often seems to be the moment apt to rethink selfperception and needs

\section{eP429}

THE DEVELOPMENT OF DELTA: A DECISION AID TO HELP PARENTS CONSIDERING ENROLING THEIR CHILD IN A PAEDIATRIC ONCOLOGY CLINICAL TRIAL

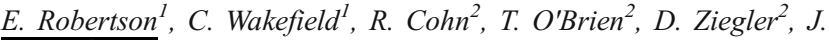
Fardell $^{I}$

${ }^{1}$ Sydney Children's Hospital, Behavioural Sciences Unit- Kids Cancer Centre, Randwick, Australia

${ }^{2}$ Sydney Children's Hospital, Kids Cancer Centre, Randwick, Australia

\section{Introduction}

Families often find the decision to enrol in a clinical trial difficult, and can experience significant decisional anxiety, uncertainty and therapeutic misconception. Delta is a world-first online decision-aid to support families deciding on participation in a paediatric oncology clinical trial. Delta provides clear information, alongside a values-clarification exercise (VCE) to support their decision-making process. Delta aims to improve decision satisfaction, the quality of informed consent, and improve clinical trial knowledge.

Objectives

This study details the Agile website development process of Delta, and user-testing results.

\section{Methods}

We developed Delta using the Agile process, with the support of a 16member steering committee. Agile relies on collaborations, flexibility, and multiple iterations. For user-testing, we conducted eye-tracking and think-aloud interviews with healthcare professionals $(n=6)$ and parents $(\mathrm{n}=7)$.

Results

Most participants $(n=11 / 13)$ reported the content was easy to read and flowed logically. Eye-tracking data showed participants read lines to completion, suggesting engagement with content. There were a few long fixations, indicative of cognitive processing. Participants reported minor difficulty navigating Delta $(n=7 / 13)$. Participants found the VCE useful and accurate in reflecting how they were leaning $(n=10 / 13)$, but felt it could be improved by providing a clearer introduction to the exercise $(n=6 / 13)$. Overall, participants reported high acceptability $(n=12 / 13)$ and good usability $(\mathrm{n}=8 / 13)$.

\section{Conclusions}

Delta acts as proof-of-concept for the use of Agile for development of an online decision-aid for health purposes. User-testing provided positive feedback, with requested changes now implemented. Agile has allowed for the development of a high-quality and high-value project completed within the constraints of cost, schedule and scope.

\section{eP430}

\section{DESIRE TO HASTEN DEATH IN ADVANCED CANCER PATIENTS EVALUATED AT A LATIN AMERICAN PALLIATIVE CARE SERVICE}

O. Rodríguez-Mayoral ${ }^{1}$, L. Ascencio-Huertas ${ }^{1}$, M. PauneroQuezadas $^{I}$, L.A. Rios-Ochoa ${ }^{1}$, E. Verastegui ${ }^{1}$, D.G. Marvin ${ }^{2}$, S. Allende-Pérez ${ }^{1}$

${ }^{I}$ Instituto Nacional de Cancerología, Palliative Care Unit, Mexico City, Mexico

${ }^{2}$ The University of MD Anderson Cancer Center, Department of Palliative Care-Rehabilitation and Integrative Medicine, HoustonTexas, USA

\section{Introduction}

Desire to Hasten Death (DHD) might be present in patients with advance cancer. Multiple distressing physical, psychosocial, symptoms can be related to it. There is limited literature in the characteristics of these patients in Latin America.

Objectives

To describe the prevalence and factors associated with advanced cancer patients' DHD evaluated palliative-care psychiatrist.

\section{Methods}

This is a retrospective chart reviewed performed at the Palliative Care Service of the Instituto Nacional de Cancerología in Mexico City from January to December 2015. We included patients referred for psychiatric assessment. DHD was defined as the presence of ideas of death and/or suicidal ideation and/or request for euthanasia and/or medically assisted suicide. Excluded patients were those with delirium, dementia, psychosis or uncontrolled physical symptoms.

\section{Results}

From 220 patients (seen in that period); 64 (29\%) patients were included for analysis. 59\% were women, the mean age was 49 years old $(\mathrm{SD} \pm 16)$. 41/64 (64\%) met criteria for major depressive disorder, 23/64 (36\%) for generalized anxiety disorder and/or panic disorder, and 7/64 (11\%) for the substance use disorder (Table 1). 28/64 (44\%) expressed some form of DHD (Table 2). 


\begin{tabular}{|c|c|c|c|c|c|c|c|c|}
\hline & & \multicolumn{2}{|c|}{ All } & \multicolumn{2}{|c|}{ With DHD } & \multicolumn{2}{|c|}{ Without DHD } & \multirow[b]{2}{*}{$\mathrm{p}$} \\
\hline & & $\mathrm{N}$ & $\%$ & $\mathrm{~N}$ & $\%$ & $\mathrm{n}$ & $\%$ & \\
\hline \multirow{3}{*}{$\begin{array}{l}\text { Age (mean - } \\
\text { Sex }\end{array}$} & & 48.72 & 15.696 & 49.96 & 10.854 & 47.75 & 18.718 & 0.01 \\
\hline & Female & 37 & 57.8 & 20 & 31.3 & 17 & 26.6 & \\
\hline & Male & 27 & 42.2 & 8 & 12.5 & 19 & 29.7 & 0.05 \\
\hline \multicolumn{9}{|c|}{ Marital status } \\
\hline & Single & 27 & 42.2 & 10 & 15.6 & 17 & 26.6 & \multirow{4}{*}{0.17} \\
\hline & Married/cohabiti & 25 & 39.1 & 14 & 21.9 & 11 & 17.2 & \\
\hline & Widowed & 7 & 10.9 & 1 & 1.6 & 6 & 9.4 & \\
\hline & Divorced & 5 & 7.8 & 3 & 4.7 & 2 & 3.1 & \\
\hline \multicolumn{9}{|l|}{ Religion } \\
\hline & Catholic & 59 & 92.2 & 26 & 40.6 & 33 & 51.6 & \multirow{3}{*}{0.66} \\
\hline & Christian & 4 & 6.3 & 2 & 3.1 & 2 & 3.1 & \\
\hline & None & 1 & 1.6 & 0 & 0.0 & 1 & 1.6 & \\
\hline \multicolumn{9}{|c|}{ Oncologic diagnosis } \\
\hline & Gastrointestinal & 9 & 14.1 & 5 & 7.8 & 4 & 6.3 & \multirow{11}{*}{0.36} \\
\hline & Hematology & 4 & 6.3 & 0 & 0.0 & 4 & 6.3 & \\
\hline & Liver and bile ouct & 5 & 7.8 & 3 & 4.7 & 2 & 3.1 & \\
\hline & Lung & 5 & 7.8 & 3 & 4.7 & , & 3.1 & \\
\hline & Skin and soft tissue & 7 & 10.9 & 2 & 3.1 & 5 & 7.8 & \\
\hline & Ginecology & 8 & 12.5 & 5 & 7.8 & 3 & 4.7 & \\
\hline & Urology & 9 & 14.1 & 4 & 6.3 & 5 & 7.8 & \\
\hline & Breast & 9 & 14.1 & 4 & 6.3 & 5 & 7.8 & \\
\hline & Head and Neck & 4 & 6.3 & 0 & 0.0 & 4 & 6.3 & \\
\hline & Melanoma & 3 & 4.7 & 1 & 1.6 & 2 & 3.1 & \\
\hline & Unknown & 1 & 1.6 & 1 & 1.6 & 0 & 0.0 & \\
\hline \multicolumn{9}{|l|}{ Karnovsky } \\
\hline & 40 & 3 & 4.7 & 1 & 1.6 & 2 & 3.1 & \multirow{6}{*}{0.76} \\
\hline & 50 & 9 & 14.1 & 4 & 6.3 & 5 & 7.8 & \\
\hline & 60 & 10 & 15.6 & 4 & 6.3 & 6 & 9.4 & \\
\hline & 70 & 22 & 34.4 & 12 & 18.8 & 10 & 15.6 & \\
\hline & 80 & 14 & 21.9 & 4 & 6.3 & 10 & 15.6 & \\
\hline & 90 & 6 & 9.4 & 3 & 4.7 & 3 & 4.7 & \\
\hline \multicolumn{9}{|l|}{ PPI } \\
\hline & A & 19 & 29.7 & 13 & 20.3 & 18 & 28.1 & \multirow{3}{*}{0.90} \\
\hline & B & 17 & 26.6 & 10 & 15.6 & 13 & 20.3 & \\
\hline & c & 1 & 1.6 & 5 & 7.8 & 5 & 7.8 & \\
\hline \multicolumn{9}{|c|}{ Major depressive disorder } \\
\hline & Yes & 41 & 64.1 & 26 & 40.6 & 15 & 23.4 & \multirow{2}{*}{0.0001} \\
\hline & No & 23 & 35.9 & 2 & 3.1 & 21 & 32.8 & \\
\hline \multicolumn{9}{|c|}{ Anxiety disorder } \\
\hline & Yes & 23 & 35.9 & 6 & 9.4 & 17 & 26.6 & \multirow{2}{*}{0.04} \\
\hline & No & 41 & 64.1 & 22 & 34.4 & 19 & 29.7 & \\
\hline \multicolumn{9}{|c|}{ Substance use disorders } \\
\hline & Yes & 7 & 10.9 & 3 & 4.7 & 4 & 6.3 & \multirow{2}{*}{0.96} \\
\hline & No & 57 & 89.1 & 25 & 39.1 & 32 & 50.0 & \\
\hline
\end{tabular}

\begin{tabular}{|c|c|c|c|c|c|c|c|c|}
\hline \multicolumn{9}{|c|}{ Table 2. Desire for Hasten Death classification } \\
\hline & \multicolumn{2}{|c|}{ Dead ideas } & \multicolumn{2}{|c|}{ Suicidal ideasjplan } & \multicolumn{2}{|c|}{$\begin{array}{l}\text { Euthanasia request / } \\
\text { Medical assisted suicide }\end{array}$} & \multicolumn{2}{|c|}{ Desire to Hasten Death } \\
\hline & $\mathrm{N}$ & $\%$ & $\mathrm{~N}$ & $\%$ & $\mathrm{~N}$ & $\%$ & $\mathrm{~N}$ & $\%$ \\
\hline Yes & 27 & 42.2 & 9 & 14.1 & 6 & 9.4 & 28 & 43.8 \\
\hline Noo & 37 & 57.8 & 55 & 85.9 & 58 & 99.6 & 36 & 56.3 \\
\hline
\end{tabular}

\section{Conclusions}

Desire to Hasten Death highly frequent in advanced cancer patients evaluated by psychiatric palliative care service. Multiple distressful psychiatric pathology, mainly the depression, is associated with this issue. Multiple interdisciplinary interventions are needed. More research is warranted.

\section{eP431}

PSYCHIATRIC DISORDERS AND CHEMICAL COPING AMONG ADVANCED CANCER PATIENTS EVALUATED BY PALLIATIVE CARE SERVICE AT A COMPREHENSIVE CANCER CENTER IN MEXICO

O. Rodríguez-Mayoral ${ }^{1}$, M. Delgado-Guay ${ }^{2}$, S. Allende-Perez ${ }^{1}$, E. Verastegui

${ }^{1}$ Instituto Nacional de Cancerología, Palliative Care Unit, Mexico City, Mexico

${ }^{2}$ The University of MD Anderson Cancer Center, Department of Palliative Care- Rehabilitation and Integrative Medicine, Houston- Texas, USA

\section{Introduction}

Patients while facing advanced illness might develop maladaptive coping strategies. Chemical coping can affect the way patients face their issues and cause devastating health issues if not detected/treated on time. Limited literature has been done in advanced cancer patients evaluated by Palliative Care services in the Latin America setting.

\section{Objectives}

To describe the sociodemographic, characteristics, and psychiatric comorbidities in cancer patients with diagnosed with chemical coping.

Methods

We include Cancer Patients evaluated by Palliative Care Team who were referred for psychiatry evaluation at the Instituto Nacional de Cancerología, Mexico City, from March 2016 to March 2017. Patients were interviewed by a Palliative Care psychiatrist of the team. Chemical coping was defined by: the use of a substance to cope with emotional distress and is characterized by the inappropriate and/or excessive use of a substance.

\section{Results}

We include 16 patients who met clinical criteria for Chemical Coping. 10/ $16(63 \%)$ were men, mean age of 48 years $(\mathrm{SD} \pm 14), 57 \%$ met criteria for major depressive disorder, $50 \%$ for some anxiety disorder, $63 \%$ for some personality disorder. The $86 \%$ had a history of substance use (tobacco $81 \%$, alcohol $56 \%$, benzodiazepines $31 \%$, cannabis, cocaine $19 \%$ and volatile solvents $6 \%$. The chemical coping was with opioids (68\%), benzodiazepines $(25 \%)$ and alcohol $(6 \%)$.

\begin{tabular}{|c|c|c|}
\hline & $\mathrm{n}$ & $\%$ \\
\hline Age (mean. DE) & 47.63 & \pm 14.4 \\
\hline Education in years (mean. DE) & 9.38 & \pm 4.8 \\
\hline \multicolumn{3}{|l|}{ Sex } \\
\hline Male & 10 & 62.5 \\
\hline Female & 6 & 37.5 \\
\hline \multicolumn{3}{|l|}{ Oncologic diagnosis } \\
\hline Gastrointestiral & 2 & 12.5 \\
\hline Hematology & 1 & 6.3 \\
\hline Sarcoma & 2 & 12.5 \\
\hline Lung & 2 & 12.5 \\
\hline Skin and soft issue & 1 & 6.3 \\
\hline Ginecology & 4 & 25 \\
\hline Urology & 4 & 25 \\
\hline \multicolumn{3}{|l|}{ Psychiatric diagnosis } \\
\hline Major depressive disorder & 9 & 56.3 \\
\hline Anxiety disorder & 8 & 50 \\
\hline \multicolumn{3}{|l|}{ Personality disorder } \\
\hline Borderline & 2 & 12.5 \\
\hline Histrionic & 2 & 12.5 \\
\hline Narcissist & 2 & 12.5 \\
\hline Dependent & 1 & 6.3 \\
\hline Obsessive & 1 & 6.3 \\
\hline Not otherwise specified & 2 & 12.5 \\
\hline \multicolumn{3}{|l|}{ History of substance abuse/dependence } \\
\hline Tobacco & 13 & 81.3 \\
\hline Alcohol & 9 & 56.3 \\
\hline Benzodiazepines & 5 & 31.3 \\
\hline Cannabis & 3 & 18.8 \\
\hline Cocaine & 3 & 18.8 \\
\hline Volatile Solvents & 1 & 6.3 \\
\hline ire of haste & 11 & 68.8 \\
\hline
\end{tabular}

\section{Conclusions}

Latino Advance Cancer patients can experience Chemical coping with opioids, benzodiazepines and alcohol. There is a high prevalence of multiple psychiatric comorbidities in this population. Personality disorders might be a factor contributing to the chemical coping. More research is needed. 


\section{eP432}

LIFE STRESSORS, UNHEALTHY LIFESTYLE BEHAVIOURS AND THE HEALTH OF WOMEN PREVIOUSLY TREATED FOR CANCER: RESULTS FROM THE AUSTRALIAN WOMEN'S WELLNESS AFTER CANCER PROGRAM

$\underline{C . S e i b}^{1}$, J. Porter-Steele ${ }^{1,2}$, A. McGuire ${ }^{I}$, S. Balaam $^{3}$, A. McCarthy ${ }^{4}$, D. $\overline{\text { Anderson }}^{1}$

${ }^{1}$ Griffith University, School of Nursing and Midwifery and Menzies Health Institute Queensland, Gold Coast, Australia

${ }^{2}$ Wesley Hospital, Choices Cancer Support Program, Brisbane, Australia

${ }^{3}$ Queensland University of Technology, Institute of Health and

Biomedical Innovation and School of Nursing, Brisbane, Australia

${ }^{4}$ University of Auckland, School of Nursing, Auckland, New Zealand

\section{Introduction:}

There is growing evidence to suggest that chronic life stress increase susceptibility to unhealthy lifestyle behaviours and decreased quality of life in women after cancer, though the extent and nature of correlations are difficult to determine because of differences across studies.

\section{Objectives}

This paper aims to: (1) examine the life stressors reported by women after cancer; (2) determine their relationship with stress appraisal and health and health behaviours, and; (3) test alternate models to determine which best described associations between life stress and several health indicators.

\section{Methods}

Data were drawn from 351 Australian women participating in a multisite lifestyle trial called the Women's Wellness After Cancer Program. A model building framework compared the accumulative risk vs. sensitive period stress exposure hypotheses and the best fitting model explored correlations between stress exposure, appraisal, and depressive symptoms.

\section{Results}

Many women reported life stressors other than their cancer diagnosis and treatment. Modelling suggested that women who reported the greatest adversity across the lifespan, also reported higher perceived stress ( $\mathrm{p}=$ 0.02 ) more unhealthy lifestyle behaviours (physical inactivity, increased body mass, and poor dietary indicators), and had a 2.38 higher odds of major depressive symptoms (95\% CI 1.80 - 2.95) compared with those without accumulated life stressors.

\section{Conclusions}

Findings showed that major depressive symptoms were increased in women with an accumulative risk of ife stress and highlights the need for supportive stress-management strategies for women previously treated cancer in an effort to possibly avert future health concerns.

\section{eP433}

\section{A PROTOCOL FOR THE LONGITUDINAL ASSESSMENT OF PATIENT-REPORTED OUTCOMES AT A LARGE COMPREHENSIVE CANCER CENTRE IN AUSTRALIA \\ G. Skaczkowski $^{1,2}$, C. Wilson ${ }^{1,2}$ \\ ${ }^{7}$ La Trobe University, School of Psychology \& Public Health, Bundoora, Australia \\ ${ }^{2}$ Austin Health, Olivia Newton-John Cancer Wellness \& Research Centre, Heidelberg, Australia}

\section{Introduction}

Patients with cancer may suffer a significant burden of distress at all points in their care experience. However, there are many challenges in tracking patient outcomes over the entire cancer pathway and in integrating these ongoing patient-reported outcome measures (PROMs) with standard hospital-based care

\section{Objectives}

We outline a protocol for the ongoing collection of PROMs within a comprehensive cancer centre in metropolitan Melbourne, Australia.

\section{Methods}

The protocol outlines an observational study examining all aspects of patients' physical, psychological and social well-being via a series of self-report surveys. A process of consultation with health care professionals and patient consumers is underway to inform survey content and to examine how PROMS may be integrated with patients' clinical data.

\section{Results}

Data collection is scheduled for 2018. Preliminary data on patient needs throughout the cancer pathway, and correlates of these, will be presented. The process of establishing this study within the hospital setting will be discussed, including any challenges encountered and recommendations for other centres.

\section{Conclusions}

This project will provide a more comprehensive picture of the aspects of care patients find most distressing, the needs of patients throughout the cancer pathway, the impact on clinical outcomes, and the individual-level factors that moderate and mediate these relationships. This information will inform the design of future interventions addressing patient wellbeing, and will facilitate better use of resources in supportive care management.

\section{eP434}

REFERRAL OFFER AND ACCEPTANCE FOLLOWING SUPPORTIVE CARE PROBLEM IDENTIFICATION IN AN AUSTRALIAN COMPREHENSIVE CANCER SERVICE

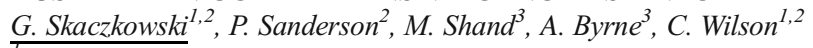

${ }^{T}$ La Trobe University, School of Psychology \& Public Health, Bundoora, Australia

${ }^{2}$ Austin Health, Olivia Newton-John Cancer Wellness \& Research Centre, Heidelberg, Australia

${ }^{3}$ North Eastern Melbourne Integrated Cancer Service, North Eastern Melbourne Integrated Cancer Service, Heidelberg, Australia

\section{Introduction}

The effectiveness of distress and problem identification for addressing patients' needs depends on appropriate subsequent triaging to supportive care services. Little is known about this process and the factors associated with referral offer and service uptake.

\section{Objectives}

This study aimed to identify the factors that predict utilisation of support services.

\section{Methods}

A retrospective review of patient records identified Distress Thermometer and Problem Checklist (DT/PC) completions from 1/1/13-30/6/14. Patients' demographic, disease and treatment details, distress levels, identified problems, referral offers and acceptance of supportive care services were recorded.

Results

729 patients completed the DT/PC, with half reporting a high level of distress (i.e. 4 or more). Most patients were offered referrals $(61 \%)$ and most referrals were accepted (71\%). Referrals were more likely to be offered to patients with higher distress $(p=.025)$ and more likely to be accepted by patients with lower distress $(p=.049)$. Referrals were more likely to be offered and accepted by patients with a higher number of problems $(p ' s<.001)$. The type of problem experienced was the main predictor of the type of referral offered (e.g. patients displaying physical problems were more likely to be offered physical referrals, than patients with emotional problems). 


\section{Conclusions}

Results indicate that within a large comprehensive cancer centre in Melbourne, Australia, triage to supportive care services via problem identification with the DT/PC accurately reflected patients' needs. Referrals were driven by the level and type of problems experienced by patients.

\section{eP435}

MORNING CHRONOTYPE IS A PROTECTIVE FACTOR AGAINST CHEMOTHERAPY-INDUCED HOT FLASHES IN PREMENOPAUSAL WOMEN WITH BREAST CANCER

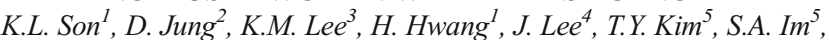
$\overline{\text { K.H. Lee }}^{5}$, B.J. Hahm ${ }^{1,3}$

${ }^{1}$ Seoul National University Hospital, Neuropsychiatry, Seoul, Republic of Korea

${ }^{2}$ Ulsan National Institute of Science and Technology, Human Factors Engineering, Ulsan, Republic of Korea

${ }^{3}$ Seoul National University College of Medicine, Psychiatry and Behavioral Sciences, seoul, Republic of Korea

${ }^{4}$ Armed Forces Medical Command, Health Management, Seongnam, Republic of Korea

${ }^{5}$ Seoul National University Hospital, Internal Medicine, Seoul, Republic of Korea

\section{Introduction}

Adjuvant chemotherapy in patients with breast cancer often causes hot flashes, impairing quality of life. However, the psychiatric factors associated with the development of chemotherapy-induced hot flashes (CIHFs) remain undetermined.

\section{Objectives}

The purpose of this study was to investigate whether chronotype was associated with the incidence of CIHFs.

Methods

A total of 119 premenopausal women with early-stage breast cancer awaiting adjuvant chemotherapy after surgery without hot-flashes were included. The presence of CIHF was defined as having moderate-to-high level of hot flashes symptom by the hot flashes subscale in the Menopause Rating Scale, at 6 months after the completion of chemotherapy. Chronotype (morning/intermediate/evening) was assessed with the Composite Scale of Morningness before adjuvant chemotherapy. To examine the association between chronotype and CIHF, we built logistic regression models, adjusting for age, body mass index, sleep quality, and radiation therapy.

\section{Results}

CIHF occurred in $50.4 \%$ of participants. The morning type was inversely associated with CIHF (reference: intermediate type, odds ratio [OR], $0.37 ; 95 \%$ confidence interval $[\mathrm{CI}], 0.16-0.94 ; \mathrm{p}=0.040$ ) in the univariate model, and the association remained significant (OR, 0.37; CI, 0.13$0.96 ; \mathrm{p}=0.045$ ) after adjusting for age, body mass index, sleep quality, and radiation therapy.

\section{Conclusions}

The morning chronotype is a protective factor against the development of $\mathrm{CIHF}$ in patient with breast cancer. Chronotypes should be assessed and considered in the prediction and management of CIHF.

\section{eP436}

PARTICULAR BIOLOGICAL MODEL OF THERAPEUTIC RESISTANCE IN THE ONCOLOGICAL PATIENT WITH DEPRESSION

I. Marinescu ${ }^{1}$, D. Marinescu ${ }^{1}$, M. Schenker ${ }^{2}$, D.G. Malaescu ${ }^{3}$, P.O. Stovicek $^{4}$

${ }^{T}$ University of Medicine and Pharmacy of Craiova, Department of Psychiatry, Craiova, Romania
${ }^{2}$ University of Medicine and Pharmacy of Craiova, Department of Oncology, Craiova, Romania

3 “Titu Maiorescu" University- Bucharest, Department of Morphopathology, Craiova, Romania

4 “Titu Maiorescu” University-Bucharest, Department of Pharmacology, Craiova, Romania

\section{Introduction}

Due to high depression rate in oncology (40-48\%), psychiatric therapeutic approach is an important target in oncology management.

\section{Objectives}

Oncological chemotherapy works frequently through neurotoxic mechanisms (platinum based agents). Also, radiotherapy treatment causes atrophy of hipocampus, thalamus and prefrontal cortex. Combining chemotherapy with radiotherapy increases cognitive disconnectivity by altering hippocampal-frontal circuits.

\section{Methods}

Alteration of hippocampal structure was demonstrated on animal model (rat) by highlighting neuronal necrosis and vacuolization of white matter. From clinical perspective, hippocampal neurotoxic syndrome associates mild cognitive impairment.

\section{Results}

Disconnectivity to the thalamic structure increases pain sensitivity and a loss of functional integrity with cerebral amygdala, resulting in impulsivity-aggressiveness behavioral disorders, up to suicidal behavior. Hippocampal and thalamic atrophy during chemotherapy or radiotherapy represent an indirect suicide risk marker of in oncological disease. Atrophy of the frontal cortex causes changes in working memory, loss of ideative flexibility and the appearance of obsessive-compulsive disorder symptoms. These mechanisms following neurotoxic effect of oncology-specific treatment can be amplified by dopamine-blocking psychotropic medication (D2 blocking antipsychotics or serotoninergic activators). This pharmacological mechanism increases the oxidative stress in the described cognitive structures and amplifies neuronal excitotoxicity through a high glutamate level, ultimately causing apoptosis.

Conclusions

The described mechanisms can be an evolutionary risk model in the oncology patient with depression, in which the specific oncology therapy, the psychotropic medication and the cognitive-behavioral psychotherapeutic interventions, can not benefit from an adequate therapeutic response (secondary therapeutic resistance). Neuroprotection and stimulation of cognitive circuits are required by alternative means (physical exercise, melotherapy, non-invasive methods for interhemispheric reconnection).

eP437

\section{DIFFERENTIATED MANAGEMENT OF DEPRESSIVE} DISORDERS IN ONCOLOGY PATHOLOGY

I. Marinescu ${ }^{1}$, D. Marinescu ${ }^{1}$, R.A. Schenker ${ }^{2}$, D.G. Malaescu ${ }^{3}$, P.O. Stovicek $^{4}$

${ }^{T}$ University of Medicine and Pharmacy of Craiova, Department of Psychiatry, Craiova, Romania

${ }^{2}$ University of Medicine and Pharmacy of Craiova, Doctoral School, Craiova, Romania

3 "Titu Maiorescu" University- Bucharest, Department of Morphopathology, Craiova, Romania

4 “Titu Maiorescu” University- Bucharest, Department of Pharmacology, Craiova, Romania

\section{Introduction}

Epidemiological data prove depression as the most frequent psychiatric disorder associated with oncological pathology.

\section{Objectives}

We believe that depression in oncology can be split in three clinical categories. 


\section{Methods}

The correct identification of these depression types and adopting differentiated strategies may improve the prognosis and the life quality of the oncology patient with depression.

\section{Results}

1. In the prodromal phase, which we interpret as a real paraneoplazic syndrome. It precedes the oncologic disease by 5 years, correlated with the multi-systemic neurobiologic depression model and with moderate symptomatology (Hamilton scale $<23$ ), insomia, tiredness, algic elements frequently interpreted as fibromyalgia. Antidepressants associated or not with antipsychotic medication determines negative oncologic evolution through 2 mechanisms: A. Incomplete terapeutic response, with persistent symptomatology associated with biologic markers (increase of reactive $\mathrm{C}$ protein levels, 6 and 8 interleukins). B. Consecutive advers events mechanisms induced by psychotropic medication intensifying inflamatory mechanisms and ARN markers modification mechanisms. Paraneoplazic depression requires multidisciplinary evaluation, associating depression markers with oncologic ones, for early diagnosys. 2. Associated with the disease, consecutive to oncologic diagnosis, under psychotraumatic and distress conditions, decreasing the patient's life quality and associating an avoidance behavior that delays treatment compliance. Evaluation and treatment require a psychoterapeutic team with psycho-oncologic competence. 3. Consecutive to neurotoxic side-effects of medication, with hippocampal atrophy, leading to secondary therapeutic rezistance, associated with the increase of 6 and 8 interleukins (progression factors of angiogenesis).

\section{Conclusions}

Immune and inflammatory markers regards depression as an independent risk factor leading to unfavorably evolution. Consequently the customized treatment must consider such symptoms.

\section{eP438}

THE PARENTING CONCERNS QUESTIONNAIRE: A VALIDATION STUDY WITH PORTUGUESE PARENTS WITH CANCER

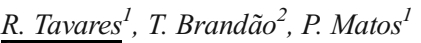

${ }^{T}$ Center for Psychology at University of Porto - Faculty of Psychology and Education Sciences- University of Porto- Portugal, Relational dynamics- processes of change and well-being, Porto, Portugal

${ }^{2}$ Centro de Investigação em Psicologia- Departamento de PsicologiaUniversidade Autónoma de Lisboa Luís de Camões- 1150-023 LisboaPortugal - Center for Psychology at University of Porto, Psychology, Porto, Portugal

\section{Introduction:}

When a parent receives the diagnosis of cancer, he/she starts to have specific concerns about the children. Although some studies start to focus on parenting concerns of parents with cancer, instruments that assess the worries of these parents are not common.

Objectives

This study assesses the psychometric properties of the Parenting Concerns Questionnaire (PCQ) in a sample of Portuguese parents with cancer.

\section{Methods}

The PCQ was completed by 290 adults with cancer who are parents of at least one minor children, using an online survey. Participants reported on sociodemographic and clinical data, parenting concerns, parental sense of competence, depression and anxiety symptoms, health-related quality of life, as well as behavioral of their children. Confirmatory factorial analysis (CFA) was used to examine factor structure of the PCQ. Pearson correlation analyses provided information regarding convergent and concurrent validity of the measure. Cronbach alpha was used to test reliability.

\section{Results}

CFA provided relevant information regarding the factor structure. The pattern of relationships with depression, anxiety symptoms, and healthrelated quality of life provided evidence of the convergent and concurrent validity of the PCQ.

\section{Conclusions}

Exploring parenting concerns provides additional relevant information about the experiences and psychological distress of parents with cancer. The PCQ can be an important tool for identifying parents with cancer who might benefit from additional support in parenting by psychological intervention.

\section{eP439}

\section{DEVELOPING A GROUP INTERVENTION PROGRAM FOR MOTHERS WITH BREAST CANCER: A PARTICIPATORY METHODOLOGY}

R. Tavares ${ }^{1}$, T. Brandão ${ }^{2}$, A.R. Oliveira ${ }^{3}$, P. Matos $^{1}$

${ }^{T}$ Center for Psychology at University of Porto - Faculty of Psychology and Education Sciences- University of PortoPortugal, Relational dynamics- processes of change and well-being, Porto, Portugal

${ }^{2}$ Centro de Investigação em Psicologia- Departamento de PsicologiaUniversidade Autónoma de Lisboa Luís de Camões- 1150-023 LisboaPortugal - Center for Psychology at University of Porto, Psychology, Lisbon, Portugal

${ }^{3}$ Center for Psychology at University of Porto, Relational dynamics- processes of change and well-being, Porto, Portugal

\section{Introduction}

When mothers confront Breast Cancer (BC) diagnosis, concerns regarding their parenting abilities and children's adaptation raise. However, the few existing group interventions do not promote emotional expression neither the sharing of experiences during the sessions.

\section{Objectives}

This study aimed to co-jointly discuss and build a group intervention program for mothers with $\mathrm{BC}$, by involving participants with $\mathrm{BC}$ through a participatory methodology.

\section{Methods}

Qualitative data was collected from three Focus Groups with a total of 18 women who had dependent children when received $\mathrm{BC}$ diagnosis. Participants were encouraged to talk about parenting issues/difficulties in the context of $\mathrm{BC}$, potential targets of intervention, as well as, potential benefits derived from their participation in the program. They were also invited to discuss on a preliminary version of a program. Transcripts were analyzed using content analysis, following the next steps: pre-analysis, encoding, categorization, and interpretation.

\section{Results}

Findings indicated that mothers need help on several issues regarding parenting. The Focus Group discussions provided information about the best way to deliver group interventions namely in terms of: issues or topics to target, the structure of sessions, frequency, localization, and timing of the group intervention. Some participants also suggested other types of support.

\section{Conclusions}

The use of a participatory methodology to develop intervention programs, by taking into account the specific parenting needs of mothers with BC, can increase the efficacy of these programs. Evidence-based interventions help health care professionals to better handle the specific needs of families and, consequently, contribute to decrease concerns and anxiety levels these mothers/families. 
eP440

\section{PSYCHOLOGICAL DISTRESS AND CONCERNS IN THAI PATIENTS WITH HEPATOCELLULAR CANCER}

S. Temtap ${ }^{I}$, K. Nilmanat ${ }^{2}$

${ }^{T}$ Prince of Songkla University, Nursing Division- Songkhlanagarind hospital, Songkhla, Thailand

${ }^{2}$ Prince of Songkla University, Adult and elderly nursing, Songkhla, Thailand

\section{Introduction}

Psychological distress is valued as the sixth vital sign and commonly reported by patients with cancer, particularly advanced cancer. However, little is known about the psychological distress and concerns among patients with hepatocellular cancer.

\section{Objectives}

This cross sectional descriptive study aimed to describe the prevalence of anxiety and depression in cancer patients with hepatocellular cancer.

Methods

Inclusion criteria were: individuals over the age of 20 who had a diagnosis of advanced or terminal hepatocellular cancer based on Barcelona Clinic Liver Cancer (BCLC) staging system, who were aware of their diagnosis, and who had sufficient physical and mental stability. Eligible patients were invited to complete the questionnaires including the demographic questionnaire and HADS questionnaire. Furthermore, clinical data were reviewed and collected from medical information record. Open-end questions to reflect concerns was used to gather sample's concerns. Mean, standard deviations, range, frequency and percentage were calculated for descriptive purposes. Content analysis was used.

\section{Results}

A total of 96 patients ranged in age from 32 to 83 years, mostly male $(82.29 \%)$. Majority $(76.04 \%)$ of sample were Buddhist. No patients had normal psychological state, 29 (30.2\%) broader line anxiety, 67 (69.8\%) with definite anxiety and these rates were seen in 32 (33.3\%), 64 (66.7\%) for depression, respectively. Psychological concerns were related to family life plan, distressful symptoms, social isolation, and the curable treatments.

\section{Conclusions}

Anxiety and depression are common psychological distress experienced by patients with advanced hepatocellular cancer. The findings highlight the needs for regularly psychological screening and intervention for patients with advanced hepatocellular cancer.

\section{eP441}

COPING STRATEGIES IN THAI PATIENTS WITH ADVANCED HEPATOCELLULAR CARCINOMA

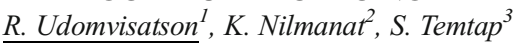

${ }^{T}$ Prince of Songkla University, Radiotherapuetic Intervention Department, Songkhla, Thailand

${ }^{2}$ Faculty of Nursing-Prince of Songkla University, Department of Adult and Elderly Nursing, Songkhla, Thailand

${ }^{3}$ Songkhlanagarind hospital- Prince of Songkhla University, Division of Nursing-, Hat Yai- Songkhla, Thailand

\section{Introduction}

Introduction: Hepatocellular carcinoma (HCC) is the most common of cancer mortality.Psychological distress among persons with cancer is common. Little is known about how patients with advanced hepatocellular carcinoma cope with cancer.

Objectives

This cross-sectional descriptive study aimed to describe coping strategies among patients with advanced or terminal stage hepatocellular carcinoma.

Methods

Data were collect from hospitalized patients at medical units in a tertiary care hospital in southern Thailand. The subjects consisted of 96 Thai patients with advanced or terminal stage hepatocellular carcinoma who were recruited from University hospitals in southern Thailand Research instruments consisted of 3 parts: (1) a demographic data form, (2) The Karnofsky Performance Status Scale, and (3) coping strategies questionnaire. Cronbach's alphas for coping strategies questionnaire were 0.84 respectively. Descriptive statistics was used.

Results

The sample employed both coping strategies, including problem-focused coping, emotional-focused coping. Three most common coping strategies used by subjects were accepting the treatments and adhere to professional advice, seek for support from health care providers when feeling unwell, and expressing emotional distress with their loved one.

\section{Conclusions}

The results of this study can be used for developing nursing program to promote effective coping strategies among patients with advanced or terminal stage hepatocellular carcinoma to help them live well at the end of life.

eP442

COGNITIVE BEHAVIORAL EFFECTS ON PERCEIVED STRESS MANAGEMENT SKILLS AND QUALITY OF LIFE IN WOMEN WITH GYNECOLOGIC MALIGNANCIES

C.M. Ulfig ${ }^{I}$, M. Marsiske ${ }^{l}$, M.H. Antoni ${ }^{2}$, E. Kacel $^{l}$, R. Postupack ${ }^{l}$, L. Trinastic $^{1}$, D. Esparza-Duran ${ }^{1}$, C.S. McCrae ${ }^{3}$, M.E. Robinson ${ }^{1}$, L.B.

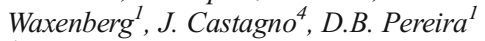

${ }^{1}$ University of Florida, Clinical and Health Psychology, Gainesville- FL, USA

${ }^{2}$ University of Miami, Department of Psychology, Miami- FL, USA

${ }^{3}$ University of Missouri, Department of Psychiatry, Columbia- MO, USA

${ }^{4}$ University of Florida, Obstetrics and Gynecology, Gainesville- FL, USA

\section{Introduction}

Diagnosis and treatment of gynecologic cancer is distressing, often resulting in multiple challenges and demands for patients. Several studies have displayed relationships among perceived stress management skills (PSMS), mood, and quality of life (QoL) in oncology patients; however, literature is lacking among those with gynecologic malignancies. Cognitive Behavioral Therapy, an empirically based psychological intervention may represent an integral component to improve patient outcomes.

\section{Objectives}

To examine the effects of a 6-week individual Cognitive Behavioral Therapy intervention for insomnia and pain (CBTi.p.) on PSMS and QoL in women with gynecologic cancer from baseline (T1) to postintervention (T2), 6-8 week follow-up (T3), and 1-year follow-up (T4).

\section{Methods}

Participants were 35 women $(M$ age $=59.11, S D=11.47)$ with confirmed gynecologic malignancies and clinically significant sleep disturbances. Participants were randomized to CBTi.p. $(N=18)$ or Psychoeducation only $(N=17)$. PSMS was assessed with the Measure of Current Status-Modified. Health related QoL was assessed with the Functional Assessment of Cancer TherapyGeneral (FACT-G), including the FACT-Emotional Well-Being Subscale.

\section{Results}

Mixed linear models were used to examine CBTi.p. effects on PSMS and QoL. Women randomized to CBTi.p. demonstrated significant increases in PSMS $(\mathrm{b}=.298, p=.009)$ and emotional well-being $(\mathrm{b}=.082, p=.028)$ from T1 to T3 compared to women randomized to the Psychoeducation condition. CBTi.p. associated improvements at $\mathrm{T} 3$ were maintained at $\mathrm{T} 4$. Conclusions

A 6-week individual CBT intervention improved PSMS and emotional well-being in women with gynecologic malignancies and clinical sleep disturbances throughout 1-year follow-up. Our future research will 
explore whether parallel improvements occur in inflammatory/ angiogenic cytokines and stress hormones.

\section{eP443}

CORRELATES AND PREDICTORS OF ANXIETY AND DEPRESSION AMONG CANCER PATIENTS IN ONE SELECTED REFERRAL HOSPITAL IN KIGALI -RWANDA M.G. Uwayezu ${ }^{l}$

${ }^{T}$ College of medicine and health sciences, Nursing, Kigali, Rwanda

\section{Introduction}

Introduction: Different studies have found that cancer patients face many challenges that may lead to anxiety and depression which increase mortality and morbidity.

\section{Objectives}

Objective: To explore the correlates and predictors of anxiety and depression disorders among cancer patients in one Selected Referral Hospital in Rwanda.

\section{Methods}

Methods: The descriptive Cross sectional design, quantitative approach was used. Sample size was 96 participants.A self completed questionnaire was used to collect social demographic data; perceived social support scale was used to evaluate the level of social support. Depression and anxiety were assessed by Hospital Anxiety and Depression Scale with a cut off more than 11 for depressive and anxiety symptomatic cases. Data was analyzed using the Statistical Package for the Social Sciences version 21.Chi square was used to examine the association between different variables and depression or anxiety. Logistic regression analysis was used to test the predicting factors.Ethical principles were observed throughout the study.

Results

Results: The prevalence of depression was (35.4\%) and anxiety $(26.1 \%)$.Anxiety was associated with cancer treatment ( $p$ $=0.019)$. Depression was correlated to the types of cancer $(\mathrm{p}=0.019)$, stage of cancer $(\mathrm{p}=0.022)$, employment status and anxiety $(\mathrm{p}=0.001)$. Being employed is the protector of depression while non-employment is the predictor of depression and having at least 3 cancer symptoms increase the likelihood to develop depression.

Conclusions

Conclusion: The study reported the prevalence and risks factors of anxiety and depression among the participants. Creation of psycho-oncology unit, routine screening and early treatment of anxiety and depression related to cancer are recommended.

Key words: Anxiety, Cancer, Correlation, Depression, Predictors.

\section{eP444}

THE PSYCHOSOCIAL ADJUSTMENT CHANGING TREND AND ITS RELATED FACTORS IN ORAL CANCER PATIENT AFTER RECONSTRUCTIVE SURGERY

W. Tsae-Jyy ${ }^{1}$, K. Pei-Ling ${ }^{2}$, L. Ming-Hsiou ${ }^{3}, C$.Wei-Wei ${ }^{4}$, L. ShuChiung, S. Shih-Chieh ${ }^{6}$, L. Yu-Ping ${ }^{7}$

${ }^{1}$ National Taipei University of Nursing and Health Sciences, School of Nursing, Taipei City, Taiwan R.O.C.

${ }^{2}$ University of Kang Ning, School of Nursing, Taipei City, Taiwan R.O.C.

${ }^{3}$ Tri-service General Hospital, Department of Nursing, Taipei, Taiwan R.O.C.

${ }^{4}$ Hualien Tzu chi hospital- Taiwan, Cardiothoracic surgery department, Hualien City, Taiwan R.O.C.

${ }^{5}$ Veterans General Hospital- Taipei, Nursing Department, Taipei, Taiwan R.O.C.

${ }^{6}$ Chang Gung Memorial Hosptial Linkou Branch, Department of Otolaryngology, Taoyuan City, Taiwan R.O.C.
${ }^{7}$ Oriental Institution of Technology, Nursing Department, New Taipei City, Taiwan R.O.C.

\section{Introduction}

Surgical resection is the primary treatments for oral cancer. After tumor resection, patient often have consequent facial deformities, dysphagia, and communication skill deficiencies, which may negatively affect their psychological adjustment.

\section{Objectives}

The purpose of this study is to explore the changing trend of postoperative psychosocial adjustment and its related factors in oral cancer patients.

\section{Methods}

This is a prospective longitudinal study. A convenient sample of 50 oral cancer patients admitted for reconstructive surgeries were recruited from two medical centers. Data were collected at preoperative (T1), one month (T2), three months (T3) and five months after discharge (T4). The instruments include the Facial disfigurement scale, the EORTC QLQ-H\&N35, the Psychosocial Adjustment to Illness Scale (PAIS) and the Social Support Scale.

Results

The average scores of psychosocial adjustment were $43.42(S D=18.59)$, $49.79(S D=22.46), 51.20(S D=21.44)$, and $48.93(S D=21.81)$ measured at T1, T2, T3, and T4, respectively. Result of GEE showed a significant time effect on psychosocial adjustment. Result of multivariate GEE showed that economic status, pain, speech, social contact and familial support were significant predictors of the changing trend in psychosocial adjustment.

\section{Conclusions}

In general, oral cancer patients reported poor postoperative psychosocial adjustment and it deteriorated gradually over time. Patients with poor financial status, lacking familial support, having pain, speech difficulties, and social contact difficulties were at the greatest risk for psychosocial maladjustment. Welfare referring, family social support strengthening, pain management, language therapy, and social contact may help patients to adapt their postoperative life.

\section{eP445}

"IT'S LIKE FALLING OFF A CLIFF": USING THE SERVICE USER VOICE TO CO-DESIGN AN END TO END PSYCHOLOGICAL SUPPORT PATHWAY FOR PEOPLE AFFECTED BY CANCER

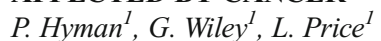

${ }^{T}$ Healthy London Partnership/National Health Service England, Transforming Cancer Services Team, London, United Kingdom

\section{Introduction}

Service users in London describe not having their psychological needs met at key points along the cancer pathway, with far more attention given to their physical health than their mental health.

\section{Objectives}

The aim of this project was to co-design a flexible, responsive, usercentred, psychological support pathway across the whole system to improve outcomes, experience and quality of life.

\section{Methods}

Consideration of key national drivers, the literature and a mapping of current service provision in London, including psycho-oncology services, was undertaken. Two stakeholder events were held involving service users, health professionals and decision makers to identify current gaps in service provision and how to address these. Additionally, focus groups and qualitative interviews with young adults affected by cancer were conducted.

Results

Thematic analysis from the focus group, interviews and stakeholder events identified key themes including: 
- how to prevent distress and promote adjustment

- the need for better provision of information on local resources and sources of support

- the gap in psychological support post-treatment

- the expert role of psycho-oncology teams which should be available across the whole pathway

- education and training needs of the wider workforce

- the wider impact on mental health of financial difficulties or fertility concerns.

Feedback on the draft pathway was received from service users, health professionals and decision makers and revised accordingly.

\section{Conclusions}

This project highlights the importance of listening to cancer patients in all aspects of service design. A psychological support pathway with clear recommendations for decision makers on implementation has been developed for London.

\section{eP446}

DEPRESSION SYMPTOMS AND VITAMIN D LEVEL IN A DVANCED CANCER PATIENTS: DID ALL MEASUREMENTS CORRELATE SIMILARLY?

K.W. Woo ${ }^{1}$, C.Y. Poon ${ }^{1}$, I.Y.Y. $\mathrm{Ng}^{2}$, V.W.C. Cho ${ }^{2}$, A.O.L. Kwok ${ }^{1}$, D.K.H. $\mathrm{Ng}^{l}$, D.M.W. Tse

${ }^{I}$ Caritas Medical Centre, Palliative Care Unit, Kowloon, Hong Kong S.A.R.

${ }^{2}$ Caritas Medical Centre, Department of Clinical Psychology, Kowloon, Hong Kong S.A.R.

${ }^{3}$ Kowloon West Cluster- Hospital Authority, Princess Margaret Hospital, Hong Kong, Hong Kong S.A.R.

\section{Introduction}

Correlation between vitamin D deficiency and different depression measures are not known despite vitamin D deficiency is associated with depression among advanced cancer patients.

Objectives

To assess the correlation between vitamin D deficiency and different depression measures, including Hospital Anxiety Depression Scale (HADS-Chinese Cantonese version), Geriatric Depression Scale (GDS) as well as Edmonton Symptom Assessment System (ESAS) - depression item.

\section{Methods}

This was a subgroup analysis of a retrospective cross-sectional single centre study, which was decided to assess the prevalence of vitamin D deficiency in advanced cancer patients. Participating patient's physical and psychological distress were assessed using ESAS and HADS. Patients who had GDS performed within 1 week of study enrolment would be recruited in this subgroup analysis. Spearman's rank correlation coefficient $(\rho)$ were used to identify the correlation of ordinal data.

Results

Among 100 participants of original study, 42 patients had GDS documented within 7 days when HADS was performed and they were enrolled into this subgroup analysis. Their mean vitamin D level was $46.3 \mathrm{nmol} / \mathrm{L}$ (range: $7-115$, standard deviation 25.6) Twenty-four $(57.7 \%)$ had vitamin D deficiency as defined as level less than $50 \mathrm{nmol} / \mathrm{L}$. Vitamin D level was negatively correlated with HADS-depression subscale $(\rho=-0.373, r=0.015)$ but not HADS-total score, GDS as well as ESAS-Depression subscale $(\rho=-0.303,-0.200$ and $-0.123, p=0.054,0.203$ and 0.437 respectively).

Conclusions

Vitamin D level was correlated with depression symptoms severity in terms of HADS but not GDS or ESAS-Depression. Further studies are needed to address which domains of depression are affected by vitamin $\mathrm{D}$ deficiency.

eP447

EFFICACY OF CRYPTOMPHALUS ASPERSA SECRETION CREAM IN THE TREATMENT AND PREVENTION OF RADIODERMATITIS IN CANCER PATIENTS: BETTER TO MOVE FORWARD AT A SNAIL'S PACE

S. BalajiSubramanian $^{1}, K$. Sathiyal, K. Balaji, ${ }^{1,2}, M$. Thirunavukarasu ${ }^{I}$

${ }^{1}$ Gleneagles Global hospitals, Radiation Oncology, Chennai, India

${ }^{2}$ VIT University, School of Advanced Scienes, Vellore, India

\section{Introduction}

Inspite of using advanced radiotherapy techniques, $85 \%$ of patients develop acute skin reaction which adversely affects patient outcomes. Preventive measures have remained elusive and were primarily focussed on skin hygiene and topical steroids.

\section{Objectives}

Purpose of the present case series report is to share the perceived clinical benefits of Cryptomphalus Aspersa secretion (SCA) based cream used prophylactically for Head \&Neck and Breast cancer patients undergoing radiotherapy.

\section{Methods}

Seventeen consecutive biopsy proven Head \& Neck and Breast cancer patients; 12 Females (70\%): Median Age 55 years (range 23-73) undergoing radiotherapy treatment were given SCA cream Radiocare $^{\mathrm{TM}}$ for application to the treatment field 1-2 times a day after each RT session. Primary sites were head neck cancers $(n=8)$ and Breast cancer $(n=9)$. Signs of Radiodermatitis were measured qualitatively with RTOG clinical scoring weekly twice. Table 1: Characteristics of 17 consecutive patients who had undergone radiotherapy with SCA-
Cream for skir. care.

\begin{tabular}{|c|c|}
\hline Clinical Parameter & Values \\
\hline $\begin{array}{l}\text { Gender } \\
\text { (Male: Female) } \\
\text { Age }\end{array}$ & $5: 12$ \\
\hline $\begin{array}{l}\text { Age } \\
\text { Median (range) (yrs) } \\
\text { Site of disease }\end{array}$ & $55(23-73)$ \\
\hline $\begin{array}{l}\text { Head \& Neck } \\
\text { Breast }\end{array}$ & $\begin{array}{l}9(53 \%) \\
8(47 \%)\end{array}$ \\
\hline $\begin{array}{l}\text { Breast } \\
\text { Whole breast RT : Chest wall RT } \\
\text { H\&N }\end{array}$ & $(3: 6)$ \\
\hline Post Operative RT : Radical RT & (3:5) \\
\hline $\begin{array}{l}\text { H\& N (HPE) }(n=8) \\
\text { Squamous cell carcinoma } \\
\text { Adenoid cystic carcinoma } \\
\text { Chondrosarcoma } \\
\text { Angiosarcoma }\end{array}$ & $\begin{array}{l}5 \\
1 \\
1 \\
1\end{array}$ \\
\hline $\begin{array}{l}\text { Dose of radiotherapy Breast cancer } \\
(\mathbf{G y})(\mathrm{n}=9) \\
50 \mathrm{~Gy} \\
50+12.5 \text { Gy boost to STV } \\
\text { Dose of radiotherapy Head \& Neck }\end{array}$ & $\begin{array}{l}6(67 \%) \\
3(33 \%)\end{array}$ \\
\hline $\begin{array}{l}\text { Tumors }(\mathrm{Gy})(\mathrm{n}=8) \\
<60 \mathrm{~Gy} \\
>60 \mathrm{~Gy} \\
\text { Concurrent chemotherapy(n) }\end{array}$ & $\begin{array}{l}4(50 \%) \\
4(50 \%) \\
4\end{array}$ \\
\hline
\end{tabular}

\section{Results}

SCA cream delayed the onset of grade 2 dermatitis (Usual onset ${ }^{1}: 25$ to 30 days was delayed by 5 days). Breast cancer patients (Post-BCS Vs MRM) showed similar rates of grade2 skin reaction. At completion acute grade 3 toxicity was higher in patients receiving concurrent chemo (0.005), bilateral neck RT(0.008) and a radical dose (0.005). Age (0.49), presence of flap (0.0), Use of bolus (0.0) did not influence the outcome. 


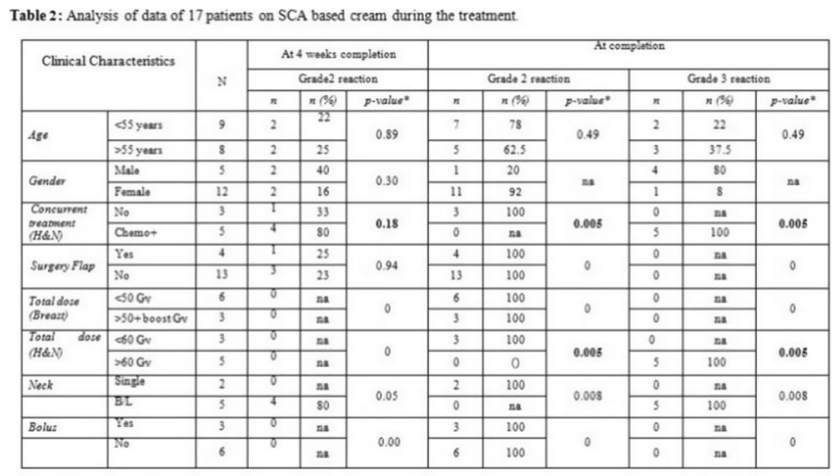

$-Z$ test for two population proportion

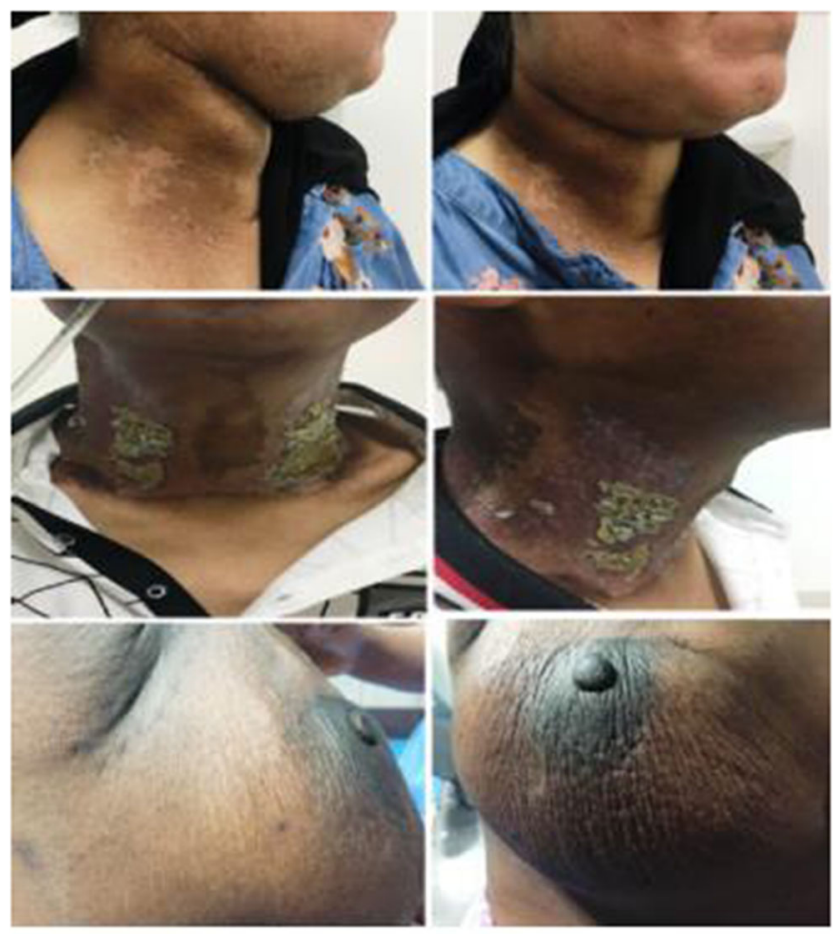

Figure 1: Skin reactions

\section{Conclusions}

SCA cream is effective for delaying acute skin reaction in adjuvant setting. Further randomized studies with a larger sample size and combination of more than one agent are required to improvise outcomes in radical radiotherapy and concurrent chemoradiation patients.

\section{eP448}

ACUTE MOIST DESQUAMATION IN BREAST CANCER PATIENTS TREATED WITH HYPOFRACTIONATED VS CONVENTIONAL RADIOTHERAPY: PRELIMINARY ANALYSIS

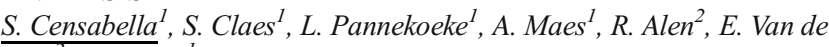
Werf', P. Bulens $^{1}$
${ }^{1}$ Jessa Hospital, Medical Oncology, Hasselt, Belgium

${ }^{2}$ Limburg Oncology Centre, Radiotherapy, Hasselt, Belgium

\section{Introduction}

Radiotherapy-induced moist desquamation (RIMD) is not only a painful side effect but also a cause of treatment interruption that might threaten treatment outcomes.

The risk of developing RIMD depends on multiple factors, among which the total irradiation dose and the dose per fraction. As small dose changes can produce large effect on healthy tissues, radiotherapy delivered over a shorter time with larger doses per fraction (i.e., hypofractionated regimen) might increase the risk of RIMD.

\section{Objectives}

To compare the incidence of RIMD in patients receiving hypofractionated radiotherapy (HF-RT) with that of historical controls having received conventional radiotherapy (CRT).

\section{Methods}

This retrospective study included breast cancer patients treated with HF-RT (16\#2.66 Gray [Gy] followed by a 5\#2.5-Gy boost) in 2014-2015. They were compared with a group of historical controls from a previous study treated with CRT $(25 \# 2$ Gy + 8\#2-Gy).

All patients were older than 50 and node-negative, had had lumpectomy but no prior adjuvant chemotherapy, and received identical skin care during radiotherapy.

The incidence of RIMD was recorded and compared through Chi-square tests.

\section{Results}

Patients in the HF-RT-group were significantly older and had larger mean breast size (as measured by tangential field separation) than controls (see Table 1), so that they were slightly at higher risk of developing RIMD.

However, the incidence of RIMD was statistically equivalent in the two groups (see Table 2).

Table 1. Patients' characteristics

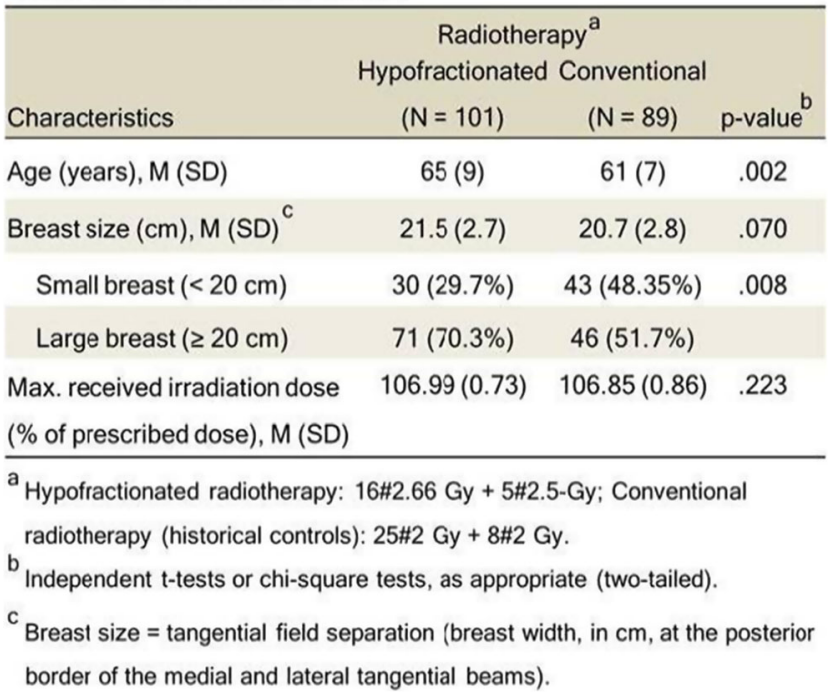


Table 2. Incidence of radiotherapy-induced moist desquamation (RIMD) per group and breast size

\begin{tabular}{|c|c|c|c|}
\hline \multirow{2}{*}{$\begin{array}{l}\text { N (\%) with } \\
\text { RIMD }\end{array}$} & \multicolumn{2}{|c|}{ Radiotherapy $^{a}$} & \multirow{2}{*}{$\begin{array}{l}\text { P-value } X^{2} \\
\text { (two-sided) }\end{array}$} \\
\hline & Hypofractionated & Conventional & \\
\hline Small breast $^{b}$ & $3 / 30(10 \%)$ & $2 / 43(4,7 \%)$ & .373 \\
\hline Medium breast & $8 / 60(13.3 \%)$ & $7 / 38(18.4 \%)$ & .496 \\
\hline Large breast & $2 / 11(18,2 \%)$ & $1 / 8(12,5 \%)$ & .737 \\
\hline Total & $13 / 101(12.9 \%)$ & $10 / 89(11.2 \%)$ & .825 \\
\hline \multicolumn{4}{|c|}{$\begin{array}{l}{ }^{a} \text { Hypofractionated radiotherapy: } 16 \# 2.66 \text { Gy }+5 \# 2.5-G y \text {; Conventional } \\
\text { radiotherapy (historical controls): } 25 \# 2 \text { Gy }+8 \# 2 \text { Gy. }\end{array}$} \\
\hline \multicolumn{4}{|c|}{$\begin{array}{l}{ }^{b} \text { Breast size }=\text { tangential field separation (width, in } \mathrm{cm} \text {, at the posterior } \\
\text { border of the medial and lateral tangential beams; }<20=\text { small breast }\end{array}$} \\
\hline
\end{tabular}

Conclusions

These preliminary data indicate that faster, hypofractionated radiotherapy does not lead to higher rates of moist desquamation than conventional radiotherapy.

eP449

SENSOR-CONTROLLED SCALP COOLING TO PREVENT CHEMOTHERAPY-INDUCED ALOPECIA IN BREAST CANCER PATIENTS IN THE CLINICAL ROUTINE: SAFETY AND EFFECTIVENESS IN THE NEOADJUVANT, ADJUVANT, OR PALLIATIVE SETTING

C.M.Kurbacher ${ }^{1}$, S. Herz' ${ }^{2}$, A.T. Kurbacher ${ }^{3}$, G. Kolberg ${ }^{3}$, N. Kettelhoit ${ }^{3}$, J.A. Kurbacher

${ }^{I}$ Gynecologic Center Bonn-Friedensplatz, Gynecologic Onncology, Bonn, Germany

${ }^{2}$ Gynecologic Center Bonn-Friedensplatz, Study office, Bonn, Germany

${ }^{3}$ Gynecologic Center Bonn-Friedensplatz, Gynecologic Oncology, Bonn, Germany

${ }^{4}$ Gynecologic Center Bonn-Friedensplatz, Gynecology II General Gynecologiy and Obstetrics, Bonn, Germany

\section{Introduction}

Among women, chemotherapy (Ctx)-induced alopecia is one of the most terrifying side effects of anti-cancer treatment.

Objectives

This retrospective analysis was initiated to obtain detailed information about the effectiveness and safety of sensor-controlled scalp cooling (SCSC) using the Paxman system (Paxman, Huddersfield, UK) to prevent CIA in patients (pts) with primary or recurrent/metastatic breast cancer (BC) exposed to neoadjuvant (NACT), adjuvant Ctx (ACT), or palliative Ctx (PCT).

Methods

109 pts were included: NACT, 47 (54.6\%); ACT, 40 (45.4\%); PCT 22; dose-dense (dd) Ctx, 38 (44.2\%); non-dd Ctx 48 (55.8\%); premenopausal, 48 (55.8\%); postmenopausal, 38 (44.2\%). The following Ctx regimens were used: $\mathrm{A}+\mathrm{T} / \mathrm{A} \rightarrow \mathrm{T}, 41$ (37.6\%), $\mathrm{T} \rightarrow \mathrm{A}, 23$ (26.7\%), T, 34 (31.2\%), non-A/non-T, $11(10.1 \%)$. Pts were subjected to SCSC during each Ctx cycle. CIA was quantified according to the Dean score (DS) determined 3 wks after the last Ctx cycle.

Results

Success rate was $64.0 \%$ (DS 0-2). Reasons for discontinuation was CIA in $21(24.4 \%) 1$ and side effects (local discomfort, pain, vomiting) in 9 pts
(8.35), which all resolved spontaneously. The relative risk (RR) to experience CIA was 1.18 (CI: 0.91-1.53) for post- vs premenopausal pts, 1.27 (CI: 0.99-1.64) for Ctx-naïve vs pretreated pts, 1.18 (CI: 0.89-1.56) for dd Ctx vs non-dd Ctx, 1.42 (CI: 1.03-1.80, $\mathrm{p}=0.0502$ ) for NACT/ACT vs PCT, and $1.42(1.11-1.85,0.0118)$ for A-based Ctx vs non A-based Ctx. Success rates for $\mathrm{A}+\mathrm{T} / \mathrm{A} \rightarrow \mathrm{T}, \mathrm{T} \rightarrow \mathrm{A}, \mathrm{T}$, and non-A/non- $\mathrm{T}$ were $48.8 \%$, $73.9 \%, 79.4 \%$, and $90.9 \%(\mathrm{p}=0.0149)$.

Conclusions

SCSC could safely and effectively prevent CIA in BC pts. All therapeutic subgroups benefited from SCSC.

\section{eP450}

ZINC DEFICIENCY ASSOCIATED WITH EPIDERMAL GROWTH FACTOR RECEPTOR- TYROSINE KINASE INHIBITOR USE

C.W. Lu ${ }^{1}$, W.H. Chung ${ }^{1}$, W.T. Chen ${ }^{1}$, C.C. Chi ${ }^{1}$, R. Hui ${ }^{1}$, C.F. Lu ${ }^{2}$

${ }^{T}$ Chang-Gung Memorial Hospital, Dermatology, Taipei, Taiwan R.O.C.

${ }^{2}$ Chung Shan Hospital, Dermatology-Research Center of Cutaneous Disorders-, Taipei, Taiwan R.O.C.

\section{Introduction}

Epidermal growth factor receptor - tyrosine kinase inhibitor (EGFR-TKI) plays an important role in non-small cell lung cancer treatment. Although those therapies are very effective, they may also cause severe cutaneous adverse effect. In our clinical observation, some cutaneous presentation and histopathological finding of those cutaneous side effects were similar to zinc deficiency and acrodermatitis enteropathica, a kind of skin disease related to zinc deficiency.

\section{Objectives}

To determine whether there is relationship between EGFR-TKI therapy and zinc deficiency.

\section{Methods}

We retrospectively collected the zinc level in 99 cancer patients under EFGR-TKI therapy with skin toxicity and 35 cancer patients under other anti-cancer therapy like immune check-point inhibitor or chemotherapy. The skin toxicity included papulopustular eruption, xerosis and dysgeusia. The normal range of zinc was between $70-120 \mathrm{ug} / \mathrm{dL}$. We compare these 2 groups using statistics. On the other hand, we treated patients with zinc gluconate and record the change of the EGFR-TKI related skin toxicity.

Results

In EGFR-TKI group, there are 88 patients (89\%) have zinc deficiency. In non- EGFR-TKI group, there are 11patients (31\%) under zinc deficiency status. The odds ratio is 3.7 , the $95 \%$ confidence interval is from 1.4 to 9.5. The $\mathrm{P}$ value of 2 tail Fisher's exact test is 0.008 .

\section{Conclusions}

We determine a significant relationship between zinc deficiency and EGFR-TKI therapy in this study. But More delicate study design to known whether the zinc level was correlated to the severity of EGFRTKI induced skin toxicity is ncessory.

\section{eP451}

THE EFFECTS OF A CREAM ON RADIATION-INDUCED DERMATITIS IN DIFFERENT CANCER PATIENTS. R.U. Nabi ${ }^{l}$, A.K. Sengupta ${ }^{1}$, S. Ahmed ${ }^{1}$, K.R. Mani ${ }^{1}$, S.M. Yusuff ${ }^{2}$

${ }^{T}$ United Hospital Limited, Oncology, Dhaka, Bangladesh

${ }^{2}$ Chittagong Medical College Hospital, Radiotherapy, Chittagong, Bangladesh

\section{Introduction}

Radiation dermatitis is one of the major limiting factors in radiotherapy which ranges from mild erythema and dry desquamation to more severe moist desquamation and ulceration which, usually causes significant pain. 


\section{Objectives}

Objective of this observational study was to assess ability of Ocoxin cream, a topical formulation manufactured by Catalysis S.L., Madrid, Spain to reduce the severity of radiation dermatitis in patients with different types of cancer.

\section{Methods}

The trial enrolled 18 cancer patients (mean age: 52 years; $78 \%$ women) who had the incidence of dermatitis during their course of RT. Breastcancer (50\%), head-and-neck (33\%) and cervical-cancer (22\%). RT with photon beam was suggested in all cases and concurrent-chemotherapy was administered in $9(50 \%)$ patients with head-and-neck and cervicalcancers. Patients used Ocoxin cream topically 3 times daily for 6 weeks. The severity of dermatitis and pain were assessed using Radiation Therapy Oncology Group (RTOG) skin-reaction-assessment-scale and McGill-Pain Questionnaire-Short form respectively.

Results

At baseline, the mean RTOG grade of radiation-dermatitis was 3.2 among all patients. After 3 weeks of Ocoxin-cream use the mean RTOG grade decreased to 1.7 , followed by a remarkable reduction to 0.33 after 6 weeks of treatment with Ocoxin-cream. Similarly assessment with McGill-Pain-Questionnaire-Short form described a significant reduction of pain from discomforting distressing pain (mean 2.7 at baseline) to almost no pain after the end of Ocoxin cream treatment. Moreover, no adverse effect of Ocoxin cream was reported during the study.

Conclusions

Based on these results, we conclude that Ocoxin cream could be beneficial to reduce the severity of radiation dermatitis.

\section{eP452}

THE PREVENTIVE EFFECT OF DHL-HISZNNA, AN A-LIPOIC ACID DERIVATIVE, FOR CHEMOTHERAPY-INDUCED ALOPECIA: A SINGLE-ARM PHASE 3 STUDY

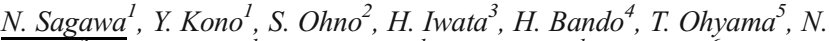

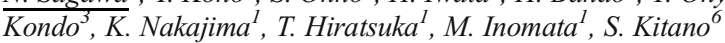

${ }^{I}$ Oita University Faculty of Medicine, Gastroenterological and Pediatric Surgery, Yufu, Japan

${ }^{2}$ The Cancer Institute Hospital- The Japanese Foundation for Cancer Research, Breast Oncology Center, Tokyo, Japan

${ }^{3}$ Aichi Cancer Center, Department of Breast Oncology, Nagoya, Japan

${ }^{4}$ Faculty of Medicine- University of Tsukuba, Department of Breast and Endocrine Surgery, Tsukuba, Japan

${ }^{5}$ Kurume University, Biostatistics Center, Kurume, Japan

${ }^{6}$ Oita University, Oita University, Oita, Japan

\section{Introduction}

Chemotherapy-induced alopecia (CIA) is one of the most distressing and unavoidable adverse events of chemotherapy in breast cancer patients. There is no drug to prevent CIA.

\section{Objectives}

The application of DHL-HisZnNa, an $\alpha$-lipoic acid derivative, had a preventive effect on CIA in rodent models. Therefore, we performed a multicenter clinical trial to assess whether the application of DHLHisZnNa reduces CIA in breast cancer patients.

Methods

The clinical trial was a multicenter, single-arm study on women planning to receive adjuvant chemotherapy. During chemotherapy, $4 \mathrm{ml}$ of a topical product containing DHL-HisZnNa was applied to the scalp four times a day. The primary end point was the proportion of participants with grade 2 alopecia ( $\geqq 50 \%$ hair loss, Common Terminology Criteria for Adverse Events version 4.0)

\section{Results}

101 participants were enroll. The mean age was 50 years; $34 \%$ of participants received 4 cycles of chemotherapy, and $66 \%$ received 8 cycles. The rate of grade 2 alopecia was $100 \%$, Grade 2 alopecia was occurred in all pts, but the recovered rate to less than G1 were 93\% and 100\% at three and six months after the completion of chemotherapy, respectively. All adverse events $(n=11)$ were grade 1 or grade 2 ,and these included scalp pain $(n=3)$, pruritus $(n=3)$, and rash $(n=5)$.

\section{Conclusions}

The preventive effect of CIA by the application of DHL-HisZnNa was not shown, but the promoting function of hair growth was suggested in this study. We have a new study plan to confirm the interesting function of this application.

\section{eP453}

CHILL GROUP MONITORS THE INCIDENCE OF CHEMOTHERAPY-INDUCED ALOPECIA WITH AND WITHOUT SCALP COOLING

C.J. van den Hurk' ${ }^{1}$, A. Young ${ }^{2}$, J.R. Nangia ${ }^{3}$, H.S. Rugo ${ }^{4}$, M.E.

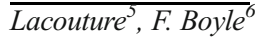

${ }^{I}$ Netherlands Comprehensive Cancer Organisation IKNL, Research, Utrecht, The Netherlands

${ }^{2}$ University hospital Coventry and Warwickshire NHS Trust, Warwick Clinical Trials Unit/Cancer Research Centre, Coventry, United Kingdom ${ }^{3}$ Baylor College of Medicine, Lester \& Sue Smith Breast Center, Houston, USA ${ }^{4}$ University of California San Francisco Helen Diller Family Comprehensive Cancer Center, Breast Oncology and Clinical Trials Education, San Francisco, USA

${ }^{5}$ Memorial Sloan Kettering Cancer Center, Dermatology ServiceDepartment of Medicine, New York, USA

${ }^{6}$ Mater Hospital/ Patricia Ritchie Centre for Cancer Care and Research, Oncology, Sydney, Australia

\section{Introduction}

Cancer related Hair loss, International Leadership and Linkage (CHILL) is an international collaboration focused on sharing (research) information about treatment-induced alopecia. One of the projects is the CHILL registry, a database to direct future work.

\section{Objectives}

The main objective for the registry is data gathering about the efficacy of various scalp cooling devices in clinical practice. Data will be used to inform patients and their health care providers about the added value of scalp cooling during their specific chemotherapy treatment regimen and identifying best scalp cooling practices.

\section{Methods}

Participating sites are in Australia, the Netherlands, the UK and USA. Patients complete an online questionnaire before each chemotherapy, with or without using scalp cooling. The registry is hosted by the Dutch Cancer Registry. For example treatment regimen, disease stage and age are recorded. The primary outcome is percentage of hair loss by the HairQoL scale. Data is transferred into the publically accessible decision aid on the CHILL website: www.cancerhairloss.org. Investigators can access regional data and compare results across sites.

Results

The incidence of alopecia by chemotherapy regimen and dose and regional use of scalp cooling will be presented.

\section{Conclusions}

The CHILL registry will inform patients and providers about the use of scalp cooling in clinical practice and will provide data to direct future research.

\section{eP454}

A NEW SOLUTION TO MANAGE THE TREATMENTINDUCED XEROSTOMIA IN HEAD AND NECK CANCER PATIENTS: A PRELIMINARY STUDY.

F. Amadori ${ }^{1}$, E. Bardellini ${ }^{1}$, A. Majorana ${ }^{1}$

${ }^{T}$ University of Brescia, Dental Clinic, Brescia, Italy 


\section{Introduction}

Despite the Literature purposes various methods for the prevention and treatment of radiation-induced xerostomia, this side effect has still a high prevalence among patients treated for head and neck cancer, affecting quality of life. Previous paper demonstrated the effectiveness of malic acid in the treatment of xerostomia induced by antidepressant and antihypertensive drugs

Objectives

The purpose of this preliminary study is to evaluate the effectiveness of a solution mainly composed by malic acid in patients with a reduced salivary flow rate after radiation for head and neck cancer

Methods

Twenty participants treated with radiotherapy to the head and neck and experiencing xerostomia, for at least six months, of grade 1 or 2 (RTOGEORTC scale) were invited to participate in the trial and divided in two different arms, the experimental one, treated with Salivacktiv â (malic acid), and the control one, treated with saline solution. Both arms followed the same administration protocol. Assessments at baseline, 1 month and 6 months were performed through the Xerostomia Questionnaire (XQ)

\section{Results}

The group treated with novel solution of malic acid showed a significant improvement $(\mathrm{p}<0.05)$ for XQ's values in the experimental arm at 1 and at 6 months

\section{Conclusions}

Malic acid could be useful to help patients with reduced salivary flow rate and xerostomia but with a minimal residual functionality of salivary glands (RTOG-EORTC grade 1 or 2), due to radiation therapy for head and neck cancer

\section{eP455}

DELIVERING IMMUNOTHERAPY IN REGIONAL AUSTRALIA: A REAL WORLD TOXICITY ANALYSIS

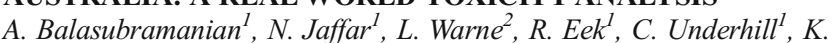
Clarke $^{\text {I, }}$ C. Steer ${ }^{1}$

${ }^{1}$ Border Cancer Hospital, Border Medical Oncology, Albury, Australia

${ }^{2}$ Border Cancer Hospital, Oncology, Albury, Australia

\section{Introduction}

Immuno-oncology has heralded the opportunity to gain excellent and sustained clinical outcomes for patients with advanced malignancies. Given its unique profile, monitoring and management of these toxicities provides a challenge for patients in regional settings.

\section{Objectives}

We performed a retrospective audit to analyze the incidence and severity of immune related adverse events at a single institution in regional Australia.

\section{Methods}

Medical records were retrieved for all patients who received immune checkpoint inhibitor therapy (Nivolumab, Pembrolizumab, or Ipilimumab) from the $1^{\text {st }}$ January 2016 to $5^{\text {th }}$ October 2017, at a single regional practice (Border Medical Oncology) in Albury, New South Wales. Toxicities were graded according to the Common Terminology Criteria for Adverse Events (CTCAE) classification.

\section{Results}

96 consecutive patients were treated over this period, with 2 excluded from analysis due to insufficient data. 53 patients $(56.4 \%)$ received Nivolumab, 40 received (42.6\%) Pembrolizumab and 1 received (1.1\%) Ipilimumab monotherapy. 4 patients $(4.3 \%)$ were treated with combination Ipilimumab/Nivolumab. Lung cancer and melanoma were represented in 72 patients ( $76.6 \%)$. 44 patients $(46.8 \%)$ were noted to have grade 1 4 immune related toxicities, $9(9.6 \%)$ of whom had grade 3 or 4 events (table 1). 3 out of the 4 patients who received combination therapy had grade 3 or 4 toxicity that precluded further therapy.
Table 1

\begin{tabular}{|l|l|l|l|}
\hline Toxicity & Grade $2(n=16,17.0 \%)$ & $G 3 / 4(n=9,9.6 \%)$ & Total $(n=44,46.8 \%)$ \\
\hline Pneumonitis & 6 & 4 & 12 \\
\hline Colitis & 5 & 2 & 14 \\
\hline Dermatitis & 3 & 0 & 12 \\
\hline Thyroid & 3 & 0 & 8 \\
\hline Hepatitis & 2 & 2 & 4 \\
\hline Arthralgias & 0 & 0 & 3 \\
\hline Diabetes & 0 & 1 & 1 \\
\hline Addison's & 1 & 0 & 1
\end{tabular}

\section{Conclusions}

This study describes the delivery of immunotherapy in a regional setting to have similar toxicity rates compared with existing literature from metropolitan centres. Our preliminary data suggests further work is needed to assist with careful patient selection when using combination immunotherapy.

\section{eP456}

A CHALLENGING CASE OF NEW-ONSET PAROXYSMAL ATRIAL FIBRILLATION WITH RAPID VENTRICULAR RESPONSE SECONDARY TO AUTOIMMUNE THYROIDITIS AFTER A SINGLE DOSE OF NIVOLUMAB-IPILIMUMAB N. Brito-Dellan

${ }^{T}$ UT MD Anderson Cancer Center, General Internal Medicine, Houston, USA

\section{Introduction}

Immune-checkpoint inhibitors are revolutionizing clinical immunotherapy by inducing tumor responses in various tumor types. Unfortunately, their application is also associated with a spectrum of immune-related adverse events (irAEs)

\section{Objectives}

To learn to promptly recognize and manage serious immunotherapyrelated thyroiditis

\section{Methods}

Direct patient care and literature review

\section{Results}

This is a 61 years-old gentleman with distal esophageal adenocarcinoma metastatic to the liver, who received his first cycle of nivolumabipilimumab 2 weeks prior. He was admitted to the hospital with progressive dysphagia, nausea, vomiting, and diarrhea, for which he underwent percutaneous gastrostomy tube placement. The following morning, the patient developed near syncope, lightheadedness, and diaphoresis. He was found to have an irregularly irregular tachyarrhythmia with heart rates exceeding 170, requiring emergent IV metoprolol, IV digoxin and IV diltiazem. Acute coronary syndrome and acute pulmonary embolism were ruled out. Thyroid function tests showed a very elevated free T4 at 7.77 , and a very elevated total T3 at 360 . Additional workup confirmed the diagnosis of immunotherapy-related thyroiditis. Anti-TSI antibodies were negative, anti-TPO antibodies were positive. The patient was successfully managed with beta-blockers for symptomatic and heart rate control. No corticosteroids were necessary. Following the hyperthyroid phase, the patient became euthyroid and then hypothyroid.

\section{Conclusions}

In immunotherapy-related thyroiditis, hyperthyroidism is produced by destruction of follicular thyroid cells with consequent liberation of thyroid hormones. In clinical trials with immune checkpoint inhibitors, the incidence of thyroiditis is as high as $10 \%$ with a single agent and $13 \%$ with 
combinations. Prompt recognition and assertive management are key to ensure best outcomes.

eP457

THROMBOTIC MICROANGIOPATHY IN A PATIENT WITH METASTATIC ESOPHAGEAL ADENOCARCINOMA TREATED WITH AN ICOS AGONIST ALONG WITH NIVOLUMAB

N. Brito-Dellan ${ }^{l}$

${ }^{T}$ UT MD Anderson Cancer Center, General Internal Medicine, Houston, USA

\section{Introduction}

JTX-2011 is an agonist monoclonal antibody that targets ICOS, inducible co-stimulator of T-cells. Thrombotic microangiopathy (TMA) induced by the use of an icos agonist in combination with nivolumab has not been described.

Objectives

To learn to promptly recognize and manage serious immunotherapyrelated adverse events

\section{Methods}

Direct patient care and literature review

Results

A 65-year-old female with metastatic esophageal adenocarcinoma, diagnosed in September 2016 most recently treated with immunotherapy, began noticing scleral icterus two weeks after a second dose of JTX2011-nivolumab. Three days later she became hypotensive and hypoxemic, requiring admission to the hospital where initial exam confirmed jaundice and hepatomegaly, and labs showed normocytic anemia (hemoglobin 5.8), thrombocytopenia (platelets 39), elevated LDH (> 7000), indirect hyperbilirubinemia. Blood smear showed anisopoikilocyosis, many spherocytes and schistocytes, teardrop forms, nucleated RBCs, burr cells, bands, metamyelocytes; no blasts and no hypo or hypersegmented neutrophils were seen. These findings were consistent with TMA. She was started on methylprednisolone 1 $\mathrm{mg} / \mathrm{kg}$ IV every 12 hours and best supportive care. Her hemolytic process was relentless, and she developed severe hypoxemic respiratory failure, unresponsive to mechanical ventilation, succumbing five days after admission.

\section{Conclusions}

TMA is a disorder characterized by microvascular occlusion due to platelet aggregation, thrombocytopenia and organ damage. Thrombocytopenia, schistocytosis, and elevated levels of LDH are enough to form a diagnosis in clinical practice. The majority of cases are idiopathic, but several etiologies have been recognized, including cytotoxic chemotherapies. TMA may also be caused by the underlying malignancy itself. Clinicians should be aware of TMA due to its potentially lethal evolution.

\section{eP458}

RECURRENT ORAL MUCOSITIS IN METASTATIC MELANOMA PATIENT TREATED WITH CHECKPOINT INHIBITOR, PEMBROLIZUMAB: CASE REPORT AND LITERATURE REVIEW

J. Dimitrijevic ${ }^{l}$, S. Bosnjak ${ }^{l}$, S. Matkovic ${ }^{2}$, N. Babovic ${ }^{2}$, S. Susnjar ${ }^{l}$

${ }^{T}$ Institute of Oncology and Radiology of Serbia, Oncology intensive care unit and supportive oncology mobile team, Belgrade, Serbia

${ }^{2}$ Institute of Oncology and Radiology of Serbia, Department for soft tissue sarcoma- melanoma and urologic malignancies, Belgrade, Serbia

\section{Introduction}

Pembrolizumab is up to date strategy against various types of cancer including melanoma. It has an unique type of adverse events (AE's) described as immune-related AE's (irAE's) connected with excessive immune system activation. Immune-related oral mucositis (irOM) with pembrolizumab is rarely described in literature.

Objectives

To report a case of a 57-year-old patient with metastatic melanoma treated with pembrolizumab who developed a recurrent irOM.

Methods

Assessment and treatment of irOM was performed according to the MASCC/ESMO guidelines for oral mucositis and ESMO guidelines for management of immunotherapy toxicity. IrAE's severity was assessed by CTCAE and WHO criteria. A retrospective literature search of published data was conducted.

Results

Patient developed grade 2 irOM after the first cycle and grade 4 irOM after the second cycle of pembrolizumab (Figure 1). OM was judged as an irAE's because of its recurrent nature and after other causes were excluded. Treatment included: parenteral highdose corticosteroids and dexamethasone oral rinses with local and systemic analgesia and parenteral nutrition. IrOM of grade 4 was rather unresponsive to therapy: after one month it was downgraded to grade 3 . Additional immunosuppressive agents are not accessible in Serbia for irAE's (off-label indication). Anti-PD1 antibody induced oral mucositis is reported, but rarely for pembrolizumab. Rapoport et al. 2017 described immunerelated oral manifestations in the form of lichenoid reactions, but not OM.
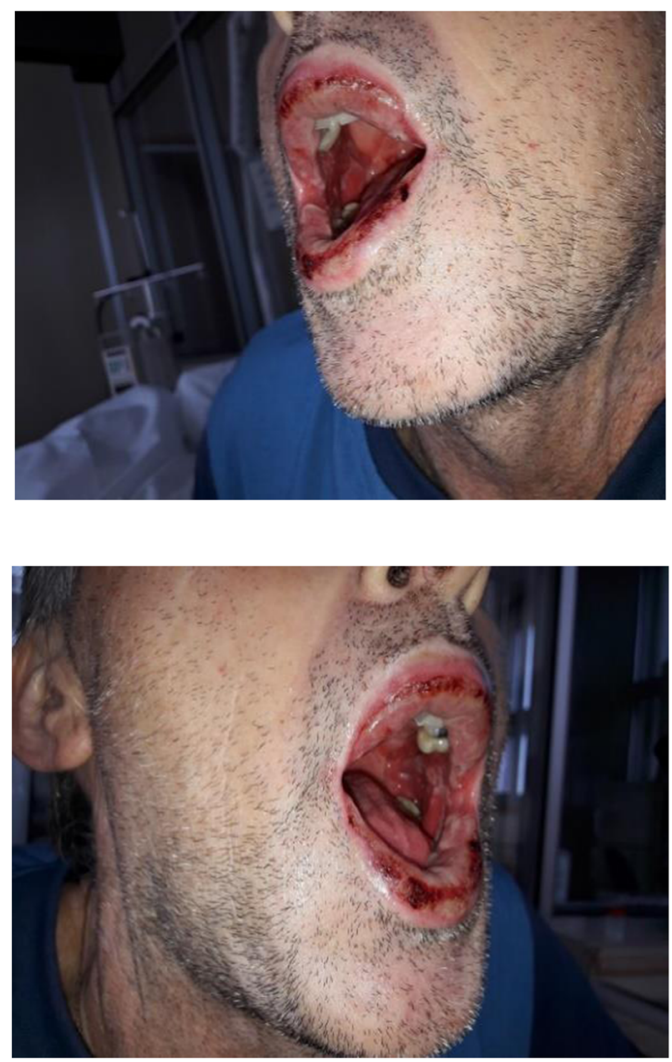


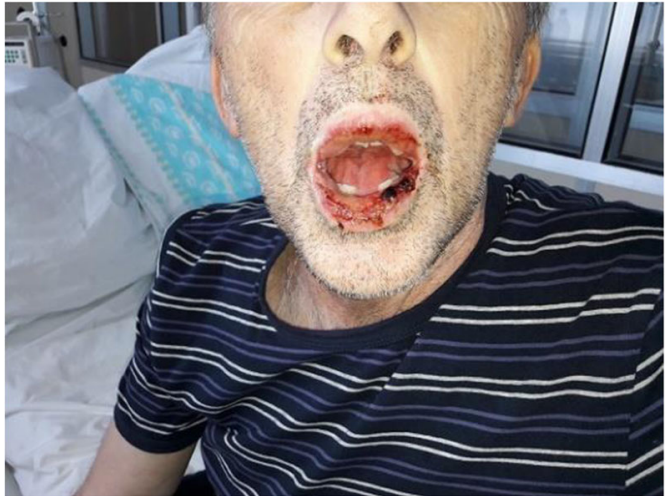

\section{Conclusions}

Immune-related oral mucositis is an uncommon irAE of pembrolizumab. The best approach to the management of irOM is yet to be defined.

eP459

INCIDENCE OF TREATMENT COMPLICATIONS DURING FCR TREATMENT IN PATIENTS WITH CHRONIC LYMPHOCITIC LEUKEMIA - EVERYDAY CLINICAL PRAXIS

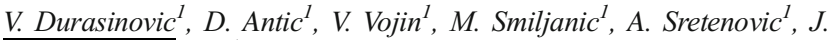
Bila $^{I}$, B. Mihaljevic ${ }^{1}$

${ }^{1}$ Clinic for hematology, Lymphoma department, Belgrade, Serbia

\section{Introduction:}

Imunnochemotherapy in chronic lymphocitic leukemia (CLL) can be complicated with toxicities.

Objectives

The aim was to asses incidence of treatment complications in pts with CLL treated with FCR protocol.

\section{Methods}

$70(74,5 \%)$ male and $24(25,5 \%)$ female, median age 63(39-79)years, from february 2014. till dec 2016. with diagnosed CLL treated with FCR were analysed.

\section{Results}

The most were in 2nd Rai CS- 47(50\%)pts, then 28(29,8\%)pts in 4 th, $10(10,6 \%)$ pts in $3 \mathrm{rd}$ and $9(9,6 \%)$ pts in 1 st. $62(66 \%)$ pts were previously treated and $32(34 \%)$ untreated. In 44(46,8\%) therapy was postponed. Reasons were: neutropenia 26(59\%), infection during neutropenia $12(27,3 \%)$, and infection alone $6(13,7 \%)$ and usually occured after 3rd cycle of therapy. Late onset of neutropenia (LON) occured in $15(15,8 \%)$ pts, and was frequent in older population $\geq 65$ y $21(55,3 \%)$ vs $9(16,1 \%)$ pts, $p<0,001$. G CSF was administered in $57,9 \%$ pts. CR/PR were achived in $86(89,5 \%)$ pts and $9(9,6 \%)$ pts had stable/progressive disease. $30(32 \%)$ pts had dose reduction. $3 / 30$ pts received $90 \%$ of dose, $7 / 30$ received $75-90 \%$, and $20 / 30$ pts received $<75 \%$ of dose. The main reason was reduced creatinine clirens-15, doctors decision 8 , neutropenia- 5, infection 1 and other $1 \mathrm{pt}$. Urgent hospitalisations $(\mathrm{UH})$ were needed in $22,1 \%$ of pts. Progression was more frequent in pts with incomplete therapy $8(23,4 \%)$ vs $1(1,8 \%)$ pts, $\mathrm{p}=0,001$. Prolonged neutropenia, LON, use of GCSF, UH were not statistically significantly different in groups with complete or incomplete therapy.

Conclusions

In about one third of pts receiving FCR we can expect complications that need treatment reduction. Treatment reduction can impact the outcome.
eP460

VALUE OF NON-SPECIFIC SIGNAL SYMPTOMS AS A PRODROME OF IMMUNE-RELATED ADVERSE EVENTS: CONSECUTIVE ANALYSIS OF 200 PATIENTS WITH NONSMALL CELL LUNG CANCER RECEIVING NIVOLUMAB R. Higashiyama ${ }^{l}$, H. Horinouchi ${ }^{l}$, K. Sekine ${ }^{l}$, Y. Matsumoto $^{l}$, S.

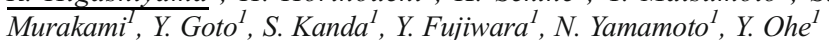

${ }^{I}$ Nantional Cancer Center Hospital, Department of Thoracic Oncology, Tokyo, Japan

\section{Introduction}

Immune-related adverse events (irAEs) are sometimes fatal problems, however detection at an early stage is difficult.

\section{Objectives}

We examined the significance of non-specific symptoms as a prodrome of irAEs in patients with non-small cell lung cancer (NSCLC).

\section{Methods}

We reviewed consecutive patients who received nivolumab for metastatic NSCLC between December 2015 and August 2017. Nivolumab was administered at a dose of $3 \mathrm{mg} / \mathrm{kg}$ every two weeks. We recorded the patient demographics, irAEs, and signal symptoms (SSs). Non-specific symptoms (fever, fatigue and headache) occurring 7 days or earlier before the onset of specific irAEs were considered as SSs. The associations between clinical information, including SSs before irAEs, were evaluated statistically using the Fisher exact test and logistic regression.

\section{Results}

A total of 200 patients received nivolumab. Among them, 129 (64\%) were male, the median age was 64 years (range, 34-83 years), $24(12 \%)$ had a PS of 2 or more, $147(73.5 \%)$ were smokers, and $46(23 \%)$ had squamous NSCLC. SSs occurred in 37 (18.5\%) of the 58 patients (29\%) who experienced irAEs. The SSs occurred a median of 19 days (range, 7154 days) before the subsequent irAEs. The SSs were positively associated with the irAEs $(\mathrm{P}<0.01$, Fisher exact test). A logistic regression analysis showed that the occurrence of the irAEs was significantly higher in male (odds ratio [OR]; 5.92, 95\% C.I.; 2.04-17.2) and patients who experienced SSs (OR; 30.90, 95\% C.I.;10.16-93.97).

\section{Conclusions}

SSs might play an important role in the early detection of irAEs in patients receiving immune-checkpoint blockade therapy.

\section{eP461}

\section{GREEN CARD TO IMMUNOTHERAPY M. Nazare ${ }^{l}$, A. Duarte ${ }^{l}$ ${ }^{T}$ Hospital da Luz, Pharmacy, Lisbon, Portugal}

\section{Introduction:}

Following 2 cases with severe immunological adverse reactions not identified in the context of urgency by non-cancer specialists, there was a need to develop a strategy to flag these patients and optimize the implementation of appropriate support measures.

\section{Objectives}

Definition of the structure of an identification card for all cancer patients undergoing immunotherapy.

\section{Methods}

The card, which is a little larger than an ATM card, is delivered to the patient at the doctor's office, filled with information regarding the prescribed drug, identification of the attending physician and emergency contact. Besides the recommendation to read the checklist for more frequent adverse events that require monitoring, the patient is advised to keep the card with him or her and to present it whenever he or she visits a doctor of another specialty or another institution. All health professionals have available on the card,information regarding mechanism of action of the therapy and associated toxicity, measures of action and means to contact the oncologist. 


\section{Results}

A identification card was designed for these patients, not only associated to a specific drug / laboratory but to a pharmacotherapeutic group, in order to ensure that the adverse reactions associated with this biological therapy are detected early when observed by other medical specialties, or in another health unit.

Conclusions

This initiative of the pharmaceutical service arises from the institutional need to create a method that allows, in urgent and / or emergency situations, health professionals to have rapid access to important safety information regarding the patient being treated with immunotherapy.

\section{eP462}

\section{CARDIOVASCULAR TOXICITY OF IMMUNE CHECKPOINT} INHIBITORS: ANALYZING EMERGING TRENDS WITHIN THE FAERS DATA

X. Pundole ${ }^{I}$, M. Sarangdhar ${ }^{2}$, M.E. Suarez-Almazor ${ }^{1}$

${ }^{T}$ The University of Texas MD Anderson Cancer Center, General Internal Medicine, Houston - TX, USA

${ }^{2}$ Cincinnati Children's Hospital Medical Center, Division of Biomedical Informatics - Cancer and Blood Diseases Institute, Cincinnati - OH, USA

\section{Introduction}

With increasing use ofimmune checkpoint inhibitors (CPI), the spectrum of immune related adverse events (AE) is growing rapidly giving rise to new toxicities. Objectives

To identify novel cardiovascular AEs with the use of CPI in the US Food and Drug Administration Adverse Events Reporting System (FAERS).

\section{Methods}

FAERS reports of cancer patients from 2011 to 2017 were mined using AERSMine (https://research.cchmc.org/aers/), an open-access web-based AE mining application, for signals of CPI-related toxicity. All Medical Dictionary for Regulatory Activities (MedDRA) coded cardiovascular AE reports were evaluated. Measures of disproportionality were calculated using well-established pharmacovigilance metrics, Relative Risks (RR) and safety signals (information component (IC)).

\section{Results}

We identified 30,939 unique patients with cancer and reports of CPIassociated toxicities. Statistically significant cardiovascular AEs included: (1) NIVOLUMAB: pericarditis ( $\mathrm{n}=39$; RR, $1.51 ; \mathrm{IC}, 0.59)$, pericardial effusion $(n=77 ; R R, 1.44 ;$ IC, 0.53$)$, cardiac failure $(n=127 ; R R, 1.62 ; \mathrm{IC}, 0.70)$ (2) PEMBROLIZUMAB: congestive cardiac failure $(n=18$; RR, 1.72; IC, 0.78) (3) IPILIMUMAB: ventricular tachycardia ( $\mathrm{n}=17$; RR, 2.22; IC, 1.15), atrial fibrillation ( $\mathrm{n}=99$; RR, 1.38; IC, 0.47) (4) DURVALUMAB: atrial fibrillation ( $n=10$; RR, 2.20; IC, 1.14), decreased ejection fraction $(n=3$; RR, 8.28; IC, 3.05). Increased rates of myocarditis, autoimmune myocarditis, and cardiac tamponade were observed with CPI therapy (odds ratio $>1, p<$ 0.005). Higher rates of cardiovascular AEs were observed in men and the elderly population ( $>65$ years) on CPIs $(\mathrm{p}<0.005)$.

Conclusions

Several CPI-related cardiovascular toxicity signals were detected. Although rare, these toxicities may become more recognizable with the expanding use of CPI

\section{eP463}

PNEUMONITIS FOLLOWING IMMUNE CHECKPOINT INHIBITION AMONG ELDERLY PATIENTS WITH ADVANCED NONSMALL CELL LUNG CANCER. DOES AGE MATTER?

\author{
V. Shannon ${ }^{l}$ \\ ${ }^{T}$ University of Texas at MD Anderson.Cancer Center, Pulmonary \\ Medicine, Houston, USA
}

\section{Introduction}

Immunosenescence may impact toxicity profiles of immune checkpoint inhibitors (ICIs). ICI safety profiles in the elderly are extrapolated from investigations involving young adults, as elderly patients were underrepresented in clinical trials. Pneumonitis represents one of the most severe and potentially lethal adverse events observed in ICI clinical trials. The influence of age in ICI-associated pneumonitis is unknown.

\section{Objectives}

Determine age influence on clinical presentations and outcomes of ICIrelated pneumonitis among patients with nonsmall cell lung cancer (NSCLC).

Methods

Patients with NSCLC and pneumonitis following anti-PD-1 therapies $(n=209)$ were retrospectively analyzed. Pneumonitis diagnosis was based on compatible histories and chest imaging findings, and the exclusion of competing diagnosis on bronchoscopically-obtained samples. Patients were grouped according to age ( $>65$ years; 50 - 64 years; $<50$ years).

\section{Results}

Patient stratification by age included 54\% $>65$ years; 31\%, 50-64 years and $14 \%<50$ years. Pneumonitis developed 2.1 months (mean) after anti-PD-1 therapy. Among patients aged $>65$ years, pneumonitis onset was earlier (1.8 months, versus 3.2 and 3.3 months for $50-64$ and $<50$ years, respectively, $\mathrm{p}<0.04$ ); more severe (grade 3 - 4 pneumonitis, $7.6 \%$ - age $>65 ; 2.9 \%$ - age 50 - 64;0\% - age $<50, \mathrm{p}<0.05)$; and required longer steroid tapering schedules (mean 7.1 weeks, versus $<5.2$ weeks for patients age $<65$ ). No pneumonitis-related deaths were reported.

\section{Conclusions}

Earlier onset and more severe pneumonitis was seen among persons aged $>65$. Immunosenescence and excess comorbidities may contribute to greater pneumonitis vulnerability in this age group. Further studies are needed.

\section{eP464}

PULMONARY SARCOIDOSIS FOLLOWING IMMUNE CHECKPOINT THERAPIES:A SINGLE CENTER EXPERIENCE

V. Shannon ${ }^{I}$

${ }^{T}$ University of Texas at MD Anderson Cancer Center, Pulmoanry Medicine, Houston, USA

\section{Introduction}

Pulmonary sarcoidosis as an immune-related adverse event following immune checkpoint inhibitors (ICIs) deserves critical attention because if often mimics underlying cancer progression, resulting in premature discontinuation of cancer therapies.

\section{Objectives}

Characterize the clinical presentation, treatment and outcome of ICIrelated sarcoidosis.

\section{Methods}

Medical records of 18 patients with biopsy-proven pulmonary sarcoidosis following anti-PD-1- and anti-CTLA-4-containing cancer regimens were extracted from the pulmonary ICI-database and retrospectively reviewed. Results

The study included 11 men and 7 women with a median age of 63 (range 34-78) who were treated with anti-CTLA-4 $(n=4)$ or anti-PD-1 $(n=2)$ monotherapies or anti-CTLA-4/PD-1 combinations $(n=12)$ for melanoma, renal or prostate carcinomas. Sarcoidosis developed de novo in 14 patients and following a 12-23 year period of inactive sarcoidosis in 4 . Findings included PET-positive intrathoracic lymphadenopathy $(n=12)$, diffuse lymphadenopathy above/below the diaphragm $(n=5)$, parenchymal lung disease $(n=3)$ and skin lesions $(n=5)$. Presenting symptoms included dry cough and dyspnea $(\mathrm{n}=11)$. The median time interval between ICI initiation and signs/symptoms was 109 days. Outcomes included 1). complete disease resolution following ICI withdrawal alone $(n=2$, mean time to resolution-2.6 months; 2$)$. complete disease resolution 
following drug discontinuation and systemic corticosteroids ( $\mathrm{n}=9$, mean time to resolution-3.7 months); 3). symptom improvement with stable but persistent lymphadenopathy despite ICI withdrawal and corticosteroids $(n=6)$. Rechallenge using alternative ICI therapies was initiated in 5 patients without evidence at 6 months of sarcoidosis recurrence. There were no sarcoidosis-related deaths.

Conclusions

Anti-CTLA-4-based therapies confer the highest rates of sarcoidosis. Rechallenge with ICIs may be safe in some patients. Long-term followup is needed.

\section{eP465}

CHARACTERISTICS AND INCIDENCE OF IMMUNERELATED TOXICITIES AMONG OLDER ADULTS ON IMMUNOTHERAPY-BASED PHASE I TRIALS

I. Subbiah ${ }^{1}$, L. Huang ${ }^{2}$, M.J. Schneck ${ }^{2}$, A. Lui ${ }^{2}, K . H^{2}{ }^{3}$, S. Rezai ${ }^{2}$, V. Subbiah', A. Naing ${ }^{2}$, D. Hong ${ }^{2}$

${ }^{1}$ UT MD Anderson Cancer Center, Palliative Care, Houston, USA

${ }^{2} U T$ MD Anderson Cancer Center, Investigational Cancer Therapeutics

A Phase I Program, Houston, USA

${ }^{3}$ UT MD Anderson Cancer Center, Biostatistics, Houston, USA

\section{Introduction}

Older adults age $65 y+$ represent a low proportion of participants in early phase trials with novel therapeutics, often over a concern about toxicities. Objectives

To that end, we investigated the participation and incidence of immunerelated adverse events (irAEs) among older adults on phase 1 immunotherapy trials.

Methods

We queried a prospectively maintained department database and gathered clinical characteristics and irAEs of 422 patients treated on immunotherapy-based phase I trials bw 04/2009-09/2015.

Results

Overall, 116 of 422 patients were aged $65 \mathrm{y}+(27 \%$, median $70 \mathrm{y}), 50$ were adolescent/young adults (AYA) aged 15-39y (12\%, median 30y), 256 aged 40-64y (61\%, median 56y). Primary cancers were GI $(n=108$, $26 \%)$, thoracic/head/neck $(\mathrm{n}=84,20 \%), \mathrm{GU}(\mathrm{n}=54,13 \%)$, and GYN $(\mathrm{n}=47,11 \%)$. Among the three age groups, the median PFS was comparable. Older adults had a higher incidence of both low grade 1/2 (p0.02) and high grade $3 / 4$ irAE (p0.14) vs mid-age or AYA.

When assessing the irAE rates of older adults to AYA and mid-age pts, the odds ratio of high grade irAEs was 1.81 (95\% CI 1.01, 3.24; p 0.05) and low grade irAEs was 1.85 (95\% CI 1.20, 2.85; p 0.0055). Most common low grade irAEs among all pts were fatigue $(n=76,18 \%)$, dermatitis $(\mathrm{n}=59,14 \%)$, fever $(\mathrm{n}=29,7 \%)$ and anorexia $(\mathrm{n}=28,7 \%)-$ older adults had a higher incidence of low grade fatigue ( $25 \%$ vs $15 \%$, p0.025).

Conclusions

Older adults remained underrepresented on immunotherapy-based phase I trials. Older adults did have a higher likelihood of experiencing an immune-related toxicity than the mid age and AYA pts.

\section{eP466}

SELECTION OF PATIENT REPORTED OUTCOMES QUESTIONS REFLECTING SYMPTOMS FOR PATIENTS WITH METASTATIC MELANOMA RECEIVING IMMUNOTHERAPY

L.K. Tolstrup $^{1}$, L. Bastholt ${ }^{1}$, K.B. Dieperink ${ }^{1}$, A.D. Zwisler ${ }^{2}$, H. Pappot ${ }^{3}$ ${ }^{T}$ Odense University Hospital, Department of Oncology, Odense C, Denmark

${ }^{2}$ REHPA - Knowledge Centre for Rehabilitation and Palliative Care, Department of Oncology, Nyborg, Denmark
${ }^{3}$ Copenhagen University Hospital- Rigshospitalet, Department of Oncology, Copenhagen, Denmark

\section{Introduction}

Toxicity-monitoring plays an important role in all cancer treatment, however, early recognition is vital for detecting and treating immune-related symptoms. Preparing a Patient Reported Outcomes tool and including melanoma patients receiving immunotherapy in the reporting of symptoms, may optimize toxicity-monitoring.

Objectives

The objective of this study was to identify the symptoms and their equivalent questions to include from the Patient-Reported Outcomes Common Terminology Criteria for Adverse Events (PRO-CTCAE) library for melanoma patients, receiving immunotherapy and, further, to evaluate if all relevant symptoms are covered by this tool.

\section{Methods}

To establish the relevant symptoms, three measures were taken. First, a literature review was carried out in three databases. Second, a chart audit was performed including medical records from melanoma patients receiving immunotherapy. Finally, the product information for the relevant immunotherapies was studied.

\section{Results}

Ten articles were included as a result of the literature review. As for the chart audit, a total of 37 patients (48 treatments with immunotherapy) were included. Overall, the reported symptoms from the literature review aligned with those identified in the chart audit. The examination of the product information supported the findings from review and chart audit, revealing only one additional symptom.

\section{Conclusions}

When preparing a Patient Reported Outcomes tool it is important that the preparatory work of selecting questions is done properly. By going through the literature, performing a chart audit, and examining the product information, the most important and relevant symptoms have been uncovered, facilitating the design of a PRO-questionnaire, based on PROCTCAE, that fits the patient population under investigation.

\section{eP467}

EXEMESTANE FACILITATES THE DEVELOPMENT OF EVEROLIMUS INDUCED MTOR INHIBITOR ASSOCIATED STOMATITIS (MIAS)

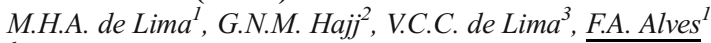

${ }^{1}$ AC Camargo Cancer Center, Stomatology, Sao Paulo, Brazil

${ }^{2}$ AC Camargo Cancer Center, International Research Center, Sao Paulo, Brazil

${ }^{3}$ AC Camargo Cancer Center, Medical Oncology Departament, Sao Paulo, Brazil

\section{Introduction}

Everolimus is an antineoplastic drug, classified as an inhibitor of the mammalian Target of Rapamycin (mTOR), and indicated for breast, kidney and neuroendocrine malignancies treatment. Side effects everolimusrelated are common, and mTOR inhibitor associated stomatitis (mIAS) is one of them. Clinically characterized as an ulcerated aphthous-like lesion, mIAS can cause serious complications to the general health condition of the patient, leading to dose reduction or suspension, mainly among breast cancer patients.

\section{Objectives}

Thus, we suggested that the association of everolimus and exemestane could contribute to increase the development of mIAS.

\section{Methods}

Clinical data from patients, who used everolimus, associated or not with exemestane, from March 2016 to September 2017 were collected and in vitro experiments with Dysplastic Oral Keratinocytes (DOK) treated with everolimus and/or exemestane (MTT assay and scratch wound 
healing assay) were performed. SPSS and GraphPad Prism were used for statistical analysis.

\section{Results}

One hundred and thirty patients were evaluated. The study population was predominantly female, the mean age was 60 years, and most were stage IV breast cancer. Almost $31 \%$ of the patients developed mIAS. Breast cancer patients (treated with exemestane and everolimus) had a 2.29-fold higher risk to develop mIAS when compared to patients with other tumors (treated with everolimus alone). In vitro experiments showed that the association of everolimus and exemestane increased cell death and inhibited cell migration of DOK when compared with DOK treated with everolimus only.

\section{Conclusions}

Clinical data and in vitro experiments suggest that the association of everolimus and exemestane may increase the risk of developing mIAS.

\section{eP468}

EXAMINATION OF LOPERAMIDE AND CROFELEMER IN A RAT MODEL OF NERATINIB-INDUCED DIARRHEA

J. Bowen ${ }^{l}$, K. Secombe ${ }^{1}$, I. Ball ${ }^{1}$, J. Shirren ${ }^{l}$, A. Wignall ${ }^{1}$, H. Wardill ${ }^{1}, Y$.

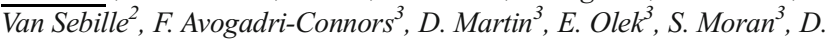
Keefe $^{I}$

${ }^{1}$ University of Adelaide, Adelaide Medical School, Adelaide, Australia

${ }^{2}$ University of South Australia, Health Sciences, Adelaide, Australia

${ }^{3}$ Puma Biotechnology-Inc, Los Angeles, California, USA

\section{Introduction}

Neratinib is an irreversible pan-HER tyrosine kinase inhibitor approved for extended adjuvant treatment of HER2+ breast cancer. Diarrhea is a frequent adverse event that is currently managed by loperamide. Crofelemer has recently been suggested as a novel approach to prevent HER2-inhibitor associated diarrhea.

\section{Objectives}

This study assessed loperamide and crofelemer for prevention of neratinib-induced diarrhea, and absorption of neratinib, in an established rat model.

\section{Methods}

Male Albino Wistar rats $(\mathrm{n}=64)$ were evenly divided into groups and received daily oral; 1) $50 \mathrm{mg} / \mathrm{kg}$ neratinib, 2) neratinib $+25 \mathrm{mg} / \mathrm{kg}$ crofelemer, 3) neratinib $+0.4 \mathrm{mg} / \mathrm{kg}$ loperamide, or 4) vehicle. Groups were killed at the end of each week for 4 weeks. Diarrhea was scored using a 0-3 grading system. For pharmacokinetic evaluation, blood was collected over $24 \mathrm{~h}$, with serum neratinib concentration determined by LC-MS.

Results

Neratinib caused diarrhea in all rats. Loperamide was associated with significantly increased severity of diarrhea and reduced weight gain compared to neratinib and crofelemer groups $(p<0.05)$. Crofelemer significantly reduced diarrhea in week one of treatment $(\mathrm{p}<0.05)$ but subsequently had no effect. Serum neratinib was increased in rats co-treated with loperamide (303 $\mathrm{ng} / \mathrm{ml})$ compared to neratinib alone $(198 \mathrm{ng} / \mathrm{ml})$. Crofelemer was associated with a decrease in $0-24 \mathrm{~h}$ AUC $(39890 \mathrm{ng} * \mathrm{~h} / \mathrm{ml})$ compared to neratinib alone $(60983 \mathrm{ng} * \mathrm{~h} / \mathrm{ml})$, but did not alter weekly concentrations.

Conclusions

In this model, the use of anti-diarrhea medications was associated with altered absorption of neratinib and modest impacts on diarrhea. Further investigation is required to determine the best mechanistic target for prevention of neratinib-induced diarrhea.
eP469

\section{COLESEVELAM REDUCES NUMBER OF DAYS WITH} NERATINIB-INDUCED DIARRHEA IN A RAT MODEL

J. Bowen ${ }^{1}$, K. Secombe ${ }^{I}$, I. Ball ${ }^{1}$, J. Shirren ${ }^{1}$, A. Wignall ${ }^{1}, H$. Wardill ${ }^{1}, Y$

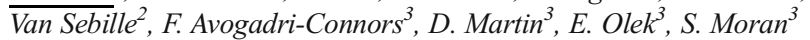

${ }^{1}$ University of Adelaide, Adelaide Medical School, Adelaide, Australia

${ }^{2}$ University of South Australia, Health Sciences, Adelaide, Australia

${ }^{3}$ Puma Biotechnology-Inc, Los Angeles, California, USA

\section{Introduction}

Neratinib is an irreversible pan-HER tyrosine kinase inhibitor recently approved by the FDA for extended adjuvant treatment of HER2+ breast cancer. Diarrhea is the most frequently observed adverse event with this agent, although the underlying pathogenesis is unclear. Our previously developed rat model for neratinib-induced diarrhea found that neratinib causes anatomical disruption in the ileum, however the role of bile acid malabsorption has yet to be investigated.

\section{Objectives}

This study aimed to evaluate whether the bile acid sequestrant, colesevelam, is an effective intervention for neratinib-induced diarrhea in our rat model.

\section{Methods}

Male Albino Wistar rats $(\mathrm{n}=48)$ were treated daily with $50 \mathrm{mg} / \mathrm{kg}$ neratinib and $300 \mathrm{mg} / \mathrm{kg}$ colesevelam or vehicle control. Colesevelam and neratinib were orally gavaged 4 hours apart for 14 or 28 days. Diarrhea was scored using a 0-3 grading system. Gastrointestinal histopathology was assessed using an established injury score and fecal bile acids were measured at the end of the study.

\section{Results}

Colesevelam reduced neratinib-induced diarrhea from $15.8 \pm 2.7$ to 10.0 \pm 2.1 days ( $\mathrm{p}=0.033$ ). Colesevelam was unable to prevent histopathology in the distal ileum at 28 days, although reduced injury in the proximal (3.12) and distal (1.37) colon compared to vehicle control (5.29 and 2.63, respectively). Concentration of fecal bile acids were increased in rats treated with colesevelam compared to vehicle control $(\mathrm{p}=0.0023)$ and neratinib alone $(\mathrm{p}=0.01)$, although neratinib itself was not associated with increased bile acids.

\section{Conclusions}

Colesevelam successfully decreased number of days with diarrhea and reduced histopathological injury in the colon. These findings warrant further investigation in clinical trials.

\section{eP470}

NASAL VESTIBULITIS: AN UNDER-RECOGNIZED AND UNDER-TREATED SIDE EFFECT OF CANCER TREATMENT

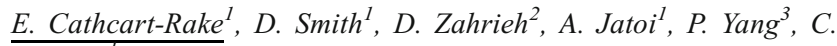
Loprinzi

${ }^{1}$ Mayo Clinic, Medical Oncology, Rochester-MN, USA

${ }^{2}$ Mayo Clinic, Health Sciences Research-Cancer Statistics, RochesterMN, USA

${ }^{3}$ Mayo Clinic, Epidemiology, Phoenix- AZ, USA

\section{Introduction}

Dryness, crusting, and bleeding of the nares due to inflammation, termed nasal vestibulitis, is infrequently reported as a side effect of cancer treatments.

Objectives

To describe the frequency of nasal vestibulitis symptoms, along with associated factors, among patients undergoing cancer-directed therapy.

\section{Methods}

Patients undergoing systemic antineoplastic therapy were interviewed regarding the presence of nasal symptoms. Fisher's exact tests were used to identify groups in which frequencies of nasal symptoms were significantly $(\mathrm{p}<0.05)$ higher than the comparator arm. To account for potential 
confounding factors, including demographic variables and concurrent therapies, logistic regression analyses were performed, and estimated proportions with their standard errors (SEs) and odds ratios were reported (ORs).

\section{Results}

Forty-one percent of the 100 surveyed patients had nasal symptoms, including dryness, pain, bleeding, and scabbing. Higher frequencies were reported among those who had received taxanes (71\%), and VEGFrelated therapies $(78 \%)$. For the patients who had received taxanes, after controlling for other factors, the odds of experiencing nasal symptoms was 4.86 times higher than for patients who did not receive taxanes $(90 \%$ CI: $2.01,11.76)$. For patients who received VEGF-related therapies, after controlling for other factors and exposure to taxanes, the odds of experiencing nasal symptoms was 7.38 (90\% CI:1.68, 32.51) times higher than for patients who did not.

Sixty-one percent of patients with symptoms reported them to their provider, but only $41 \%$ of chart notes contained documentation of such; $49 \%$ of patients reported treating their symptoms.

\section{Conclusions}

Nasal vestibulitis symptoms are common among oncology patients, but infrequently recorded or treated by providers.

\section{eP471}

ROSE GERANIUM IN SESAME OIL NASAL SPRAY: A POTENTIAL TREATMENT FOR NASAL VESTIBULITIS? E. Cathcart-Rake ${ }^{I}$, D. Smith ${ }^{1}$, D. Zahrieh ${ }^{2}$, C. Loprinzi ${ }^{1}$

${ }^{T}$ Mayo Clinic, Medical Oncology, Rochester- MN, USA

${ }^{2}$ Mayo Clinic, Health Sciences Research-Cancer Statistics, Rochester$M N, U S A$

\section{Introduction}

A rose geranium in sesame oil spray product has been anecdotally noted to appear to improve nasal vestibulitis symptoms.

Objectives

To evaluate whether patients with nasal vestibulitis associated with cancer-directed therapy experience symptomatic improvements from rose geranium in sesame oil nasal spray.

Methods

Patients with breast cancer prescribed rose geranium nasal spray were evaluated regarding whether this product improved symptoms. Patient medical information, as well as documentation of symptoms, was gleaned from medical charts. Questionnaires were sent to patients regarding their experiences.

\section{Results}

Of the 40 patients with breast cancer who were prescribed rose geranium nasal spray, $100 \%$ were receiving cancer-directed therapy: $66 \%$ were receiving chemotherapy, most frequently with taxanes; others received targeted therapy. Twenty-one patients returned surveys, 20 had used the spray product. Data are portrayed in the table.

\section{Conclusions}

Rose geranium in sesame oil nasal spray appears to improve patientreported nasal symptoms associated with cancer-directed therapy.

\section{eP472}

USEFULNESS OF INSTRUCTING AND SUPPORTING SIDE EFFECTS MANAGEMENT TO ADVANCED THYROID CANCER PATIENTS WHO ARE TAKING MOLECULAR TARGETED DRUG

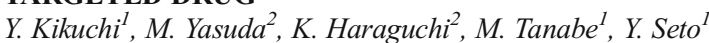

${ }^{T}$ The University of Tokyo Hospital, Breast and Endocrine Surgery, Tokyo, Japan
${ }^{2}$ The University of Tokyo Hospital, Nursing Department, Tokyo, Japan

\section{Introduction}

Lenvatinib, a molecular targeted drug for advanced thyroid cancer, has been reported to contribute significantly to prolonging PFS. On the other hand, this suggests the necessity of long-term side effect management.

Objectives

We examined effectiveness of nursing support for patients who are on long-term lenvatinib administration by checking diary of medication.

Methods

We give diary of medication and asked them to fill in blood pressure, appetite, changes in skin findings, or other symptoms. Then nurses check the diary when monthly outpatient visit.

\section{Results}

Case 1 . The patient was bothered by rising blood pressure, foot sole redness since he started taking medicine. He reported to be experiencing symptomatic exacerbation due to changes in daily activities such as work fatigue and drunk habits. After hearing that, we started to list those daily activities in medication diary. By doing this, he came to have a habit of looking back on his physical condition. Case 2. The patient suffered stomatitis, appetite loss and diarrhea by taking lenvatinib. A nurse instructed oral self- care, introduction of herbal medicine, her symptoms were relieved. By checking the diary, in addition to sharing information, the patient herself was able to check symptoms objectively.

\section{Conclusions}

The check of medication diary by a nurse not only can find patients side effects but also the patients can be recognized and evaluated side effects by patients Molecular target therapy is often administered for a long period of time. Supporting by multi-occupation such as nurse plays a big role to continue taking medicine while keeping QOL.

eP473

CARDIOTOXICITY OF IMMUNE CHECKPOINT INHIBITORS M. Morgado ${ }^{1,2,3,4}$, C. Lopes $^{1}$, S. Morgado ${ }^{4}$, I. Freire ${ }^{1,4}$, O. Fonseca ${ }^{1,4}$

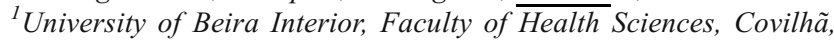
Portugal

${ }^{2}$ Polytechnic Institute of Guarda, School of Health Sciences, Guarda, Portugal

${ }^{3}$ University of Beira Interior, CICS-UBI - Health Sciences Research Centre, Covilhã, Portugal

${ }^{4}$ Hospital Center of Cova da Beira, Pharmaceutical Services, Covilhã, Portugal

\section{Introduction}

Immune checkpoint inhibitors (ICPI) are monoclonal antibodies which inactivate the inhibitor molecules of checkpoints, restoring the cytotoxic T-cells. They showed improved clinical outcomes in different types of cancer, but high-grade, immune-related adverse events may occur, namely myocarditis.

\section{Objectives}

To review the cardiotoxicity related to ICPI therapy.

Methods

Search of articles that correlate cardiotoxicity and immune checkpoint inhibitors therapy, published from 2016 to 2017 in PubMed's electronic database. Data analysis of FDA Adverse Events Reporting System (FAERS) was also accomplished.

Results

Ipilimumab had 11051 reported adverse events and of those $4.52 \%$ $(n=499)$ were cardiac disorders. Of the $4.52 \%, 99 \%(n=494)$ were serious cases and $40.1 \%(\mathrm{n}=200)$ resulted in death; myocarditis represented $10.4 \%$ cases $(n=52)$ of which $57.7 \%(n=30)$ ended in death. Nivolumab had 16213 reported adverse events and of those $5.99 \%(n=971)$ were cardiac disorders. Of the $5.99 \%, 99.5 \%(n=966)$ were serious cases and 
$34.5 \%(n=335)$ resulted in death; myocarditis represented $10.3 \%(n=100)$ cases of which $53 \%(n=53)$ ended in death.

\section{Conclusions}

Myocarditis is a rare but potentially fatal T-cell-driven drug reaction. Cardiac monitoring is not performed routinely in most immunotherapy treatments, being the true incidence of ICPI-induced myocarditis unknown. It is important to be aware of this cardiotoxic effect because immune-mediated myocarditis has an early onset, nonspecific symptomatology and a fulminant progress making it important to be monitored by ECG, troponin levels and other tests.

\section{eP474}

TARGETED THERAPY WITH NK-1R ANTAGONIST FOR ORAL MUCOSITIS AND OTHER TISSUE REACTION IN HAMSTER MODEL AND COMPARISON OF ORAL MUCOSITIS TREATMENT WITH LOW LEVEL LASER S. PS, M. Mohan ${ }^{2}$, A.A. Eid ${ }^{3}$

${ }^{T}$ Qassim University, Maxillofacial Surgery and Diagnostic Sciences, Buraidah, Saudi Arabia

${ }^{2}$ Qassim University, Prosthetic Dental Sciences, Buraidah, Saudi Arabia ${ }^{3}$ Qassim University, College of Dentistry, Buraidah, Saudi Arabia

\section{Introduction}

Studies done on NK-1R antagonist role, in tissue reactions namely, pruritis, mucositis are very few, those directed shows affirmative; and a causal association could convey foundation of targeted therapy for tissue reactions.

\section{Objectives}

1) To investigate whether the NK-1R antagonist has a therapeutic effect on mucositis, pruritis, and hair loss in the mouse model.

2) Comparison of effectiveness of NK-1R antagonist with low level laser in oral mucositisMethods

The hamsters were divided into four groups, control group with no treatment; Group A, receiving the 5-FU peritoneal injection only; Group B, receiving the 5-FU peritoneal injection and NK-1R antagonist; Group C, receiving the 5-FU peritoneal injection and low level laser. Hamsters were intraperitoneally administered $5-\mathrm{FU}$ at $80 \mathrm{mg} / \mathrm{kg}$ on days, 0,6 , and 9 . NK$1 \mathrm{R}$ antagonist, $1 \mathrm{mg} / \mathrm{kg}$, were administered intraperitoneally on Day 1,2 and 3rd. And low intensity red laser was administered on Day 2, 5 and 7 to the scratched cheek pouches

\section{Results}

Group A, showed, complete ulceration of the cheek pouch at the end of the 13th day, and very minimal small patches of alopecia $(2-5 \mathrm{~cm} 2)$, and biting, licking of hind paw. Group B, showed, Presence of erythema, and minimal evidence of erosion in the cheek pouch, with no scratching or no biting or licking the hind paw, and very good coat condition. Group C, showed, minimal presence of erythema in the cheek pouch, and very minimal small patches of alopecia and biting, licking or hind paw.

\section{Conclusions}

The targeted therapy with NK-1R antagonist in tissue reactions could reduce the symptoms.

\section{eP475}

A COMPARATIVE ANALYSIS OF PARTICIPATION AND CLINICAL BENEFIT AMONG OLDER ADULTS VERSUS MIDDLE AGE AND AYA PATIENTS ON PHASE I CLINICAL TRIALS WITH VEGF/VEGFR INHIBITORS

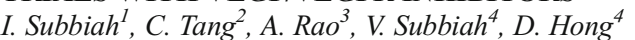

${ }^{T}$ UT MD Anderson Cancer Center, Palliative Care, Houston, USA

${ }^{2}$ UT MD Anderson Cancer Center, Radiation Oncology, Houston, USA

${ }^{3}$ UT MD Anderson Cancer Center, Bioinformatics, Houston, USA
${ }^{4} U T$ MD Anderson Cancer Center, Investigational Cancer Therapeutics A Phase I Program, Houston, USA

\section{Introduction}

Older adults aged 65 years and above remain underrepresented in cancer clinical trials.

Objectives

We hypothesized that older participation in early phase trials with VEGF/VEGFR (VEGF/R) inhibitors was lower than cancer prevalence in this group and lower than other age groups (middle age, adolescent/ young adults [AYA]).

Methods

We identified and separated patients treated on VEGF/R-inhibitor-based phase I trials from 12/1/2004-07/31/2013 into 3 age-based cohorts, AYA (15-39y), middle age (40-64y), older adults $(65 \mathrm{y}+)$. We analyzed clinical/ treatment characteristics and response outcomes, calculating the odds ratios (OR) of clinical benefit (defined as $\mathrm{SD} \geq 6$ months, $\mathrm{PR}, \mathrm{CR}$ ) for older adults and AYAs versus middle age participants.

\section{Results}

Of 1489 patients, 278 were older adults (18\%, median age 68.9y), 220 AYA (15\%, median age 32.6y), 991 middle age $(67 \%$, median age $53.8 \mathrm{y})$. Common malignancies included gastrointestinal $(n=438,29 \%)$, gyneco$\operatorname{logic}(n=234,16 \%)$, and thoracic/head/neck $(n=216,15 \%)$. Median time to treatment failure did not vary significantly between the 3 age-based cohorts ( $3 \mathrm{~m}$ in older adults, $3.5 \mathrm{~m}$ middle age, $3.3 \mathrm{~m}$ AYA). OR of achieving clinical benefit in older adults vs middle age (OR 1.10, p 0.19 [twotailed], p 0.09 [one-tailed]) and AYA vs middle age (OR 0.85, p 0.31 [proportions z-test, two tailed], p 0.15 [one-tailed]) showed no significant differences.

\section{Conclusions}

Older adults accounted for $<20 \%$ of participants on phase I clinical trials with VEGF/R inhibitors but those who participated were just as likely to achieve a clinical benefit as AYA and middle age patients. These findings merit further exploration into patient selection for early phase trials.

\section{eP476}

SAFETY AND EFFICACY PROFILE OF NERATINIB IN BREAST CANCER: POOLED REANALYSIS BASED ON RANDOMIZED CONTROLLED TRIALS

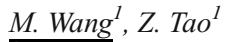

${ }^{T}$ West China Hospital of Sichuan University, state key laboratory, Chengdu, China

\section{Introduction}

Neratinib (HKI-272), a novel pan-HER tyrosine kinase inhibitor(TKI) against HER2-amplified breast cancers, is currently being tested in a number of trials for its clinical activity.

\section{Objectives}

To systematically evaluate the efficacy and safety of neratinib with available phase I, II, and III data, and elaborate on the safety profile of panHER TKIs.

\section{Methods}

A comprehensive search of PubMed, Medline, Embase was performed for all relevant clinical trials. Adverse events (AE) of any grades and grades $\geq 3$ were summarized for event rates. Odd ratios (ORs) were calculated for controlled trials. A random effect model was applied if heterogeneity was observed $\left(\mathrm{P}<0.10\right.$ or $\left.\mathrm{I}^{2}>50 \%\right)$. Progression free survival (PFS) was measured with hazard ratio (HR) extracted from KaplanMeier survival curves.

\section{Results}

4665 patients from 19 articles were included in the analyses. The most frequently occurred all-grade AEs of neratinib monotherapy was diarrhea $(88.0 \%)$, nausea $(42.4 \%)$ and fatigue $(26.3 \%)$. The frequency of grade $\geq 3$ diarrhea was significantly higher than control $(\mathrm{OR}=11.969$, 
95\% CI 6.441-22.244). The common adverse events of neratinibpaclitaxel-combined therapy included diarrhea(77.7\%), nausea $(57.2 \%)$, neutropenia $(31.5 \%)$, anemia $(31,5 \%)$ and stomatitis $(27.6 \%)$. For neratinib plus paclitaxel therapy, $29.4 \%$ of patients presented with peripheral sensory neuropathy and $50.7 \%$ with alopecia. The efficacy analysis on patients' PFS could not demonstrate the superiority of neratinib $240 \mathrm{mg}$ /day (HR=0.93, 95\% CI 0.57-1.50).

\section{Conclusions}

Neratinib based therapy lead to higher frequency of some adverse events with acceptable tolerability in patients with HER2+ advanced breast cancer. The efficacy results however are inconclusive since neither inferiority nor non-inferiority of treatment with neratinib could be demonstrated.

eP477

IMPACT OF USING COMMUNITY PALLIATIVE CARE VOLUNTEERS IN PROVIDING PALLIATIVE CARE: A MODEL USED IN KITOVU PALLIATIVE CARE PROGRAM, MASAKA, UGANDA

D.B. Abila

${ }^{T}$ Makarere University, College of Health Sciences, Kampala, Uganda

\section{Introduction}

Living and dying with incurable illness in poverty and pain is common in sub-Saharan Africa. In many countries, minimal or no resources have been dedicated to supportive or palliative care of chronic diseases like cancer. There exist huge shortages of health workers who can offer palliative care services.

\section{Objectives}

To evaluate the impact of using community palliative care volunteers in delivering palliative care services to patients suffering with cancer.

Methods

Reviewed the successful implementation of Kitovo Palliative Care program, Uganda which provides cancer supportive care. It's a freestanding mobile clinic system run by a church based organisation. It's formed by multi-disciplinary team of volunteers recruited through churches.

\section{Results}

The program was reliant on volunteers for case finding, ongoing social support, and end-of-life care. They built relationships and trust between patients and the community. This increased the willingness to use palliative care services. A sense of hope was instilled among the patients. Pain dominated the lives of many patients, most felt the volunteers were sent by God.

Nurses were grateful that people could now die in peace and dignity and that community attitudes to death and dying were changing positively. Community leaders described them as "community consultants". A local politician said that the community now identify patients and alert the volunteers.

\section{Conclusions}

Volunteer training and ongoing motivation were the observed challenges. Palliative care programs must be configured to respond to local needs and customs, be community based, and be integrated with local health and social care, with accessible referral pathways between and across services.

\section{eP478}

“CANCER TURNED MY LIFE UPSIDE DOWN." QUALITY OF LIFE IN ARAB CANCER SURVIVORS'

\section{Alananzeh ${ }^{1}$}

${ }^{T}$ Ingham Institute for Applied Medical Research, Centre for Applied

Nursing Research, Liverpool, Australia

\section{Introduction}

The cancer trajectory is associated with a negative impact on cancer survivors' quality of life (QOL). Little is known about the QOL among Arab cancer survivors.

\section{Objectives}

This study compared health-related quality of life in a sample of ArabAustralian (first generation immigrant) and Arab-Jordanian cancer survivors.

\section{Methods}

This mixed methods study was conducted between March 2016 and January 2017. Eligible participants ( $n=143$ ) were recruited from two public hospitals in Sydney, Australia and a specialist cancer center in Amman, Jordan. Participants aged over 18, diagnosed with any type or stage of cancer and up to 5 years post-diagnosis completed the Functional Assessment of Chronic Illness Therapy-Spiritual Well-Being (FACIT$\mathrm{Sp})$. Thirty semi-structured interviews were conducted with participants-18 in Australia and 12 in Jordan.

\section{Results}

Overall, both Arab Australians and their Jordanian counterparts reported low level of QOL. There is no statistically significant difference between the two groups in term of the overall score and sub-domains. Interview participants' (in both groups) revealed unique quality of life concerns across four domains of physical (lack of energy, meeting the family needs), psychological (sadness, nervousness), sex life (low satisfaction with their sex life) and functional well-being (not been able to enjoy their life, not been able to work inside or outside the house).

\section{Conclusions}

This paper provides insight that Arab cancer survivors experience a low level of QOL. Findings suggest a need to develop a suitable intervention to improve the Arab cancer survivors QOL.

\section{eP479}

\section{STUDY OF HEALTH-RELATED QUALITY OF LIFE IN CHILDHOOD CANCER SURVIVOR AT SOUTH EGYPT CANCER INSTITUTE}

A. Ali $i^{1}$

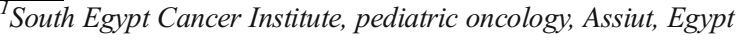

\section{Introduction}

Background: Thanks to therapeutic improvements in the past decades, survival rates in childhood cancer have increased to $75-80 \%$, resulting in a growing population of long-term survivors. However, cancer and cancer treatments have been associated with adverse late effects. Therefore, health and quality of life of survivors are a matter of increasing concern. Comprehensive data on the burden of late effects of childhood cancer and its risk factors, and data on quality of follow-up care in long-term survivors are scarce

\section{Objectives}

The present study was designed toassess the health related quality of life (HRQOL) and psychological distress in childhood cancer survivors and comparing them with age and sex matched controls.

\section{Methods}

Sixty childhood cancer survivors and Sixty matched apparently healthy control. have been subjected to thorough history taking include age, sex, diagnosis, duration of survival,type of treatment and complications, thorough clinical examination with special emphasis on disease related complications. All patients and controls were subjected to assessment of quality of life, using the Short Form - 12 Health Survey (SF-12). and assessment of psychological distress by the self reported Hospital Anxiety and Depression Scale (HADS) questionnaire.

\section{Results}

The results revealed significantly lower HRQOL scores in childhood cancer survivors compared to controls.

Also the study revealed significantly higher depression and anxiety scores in survivors compared to healthy controls especially in female and 
presence of disabling complications, while significant increase in anxiety scores in solid tumors and older age.

Conclusions

Childhood cancer survivors have significantly lower HRQOL and more psychological distress as anxiety and depression than healthy controls.

\section{eP480}

EFFECTS OF HEALTH BELIEFS MODEL BASED NURSING INTERVENTIONS PERFORMED AT HOME ON PREVENTION OF LYMPHEDEMA IN PATIENTS RECEIVING RADIOTHERAPY AFTER BREAST SURGERY

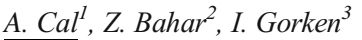

${ }^{T}$ Ondokuz Mayis University Faculty of Health Science, Public Health

Nursing, Samsun, Turkey

${ }^{2}$ Koc University Faculty of Nursing, Public Health Nursing, Istanbul, Turkey

${ }^{3}$ Dokuz Eylul University Faculty of Medicine, Radiation Oncology, Izmir, Turkey

\section{Introduction:}

The incidence of lymphedema occurring after breast surgery poses a high risk for public health.

\section{Objectives}

The study was conducted to determine home care needs and barriers to maintain behavior of lymphedema management in women developing lymphedema after breast surgery and to examine effects of health beliefs model based nursing interventions at home on lymphedema development.

\section{Methods}

The study had qualitative and experimental designs. In the qualitative design, data were collected from 14 women at face-to-face in-depth interviews based on Health Beliefs Model and content analysis of obtained data were made. In the experimental stage, data were collected from 72 women after breast surgery, of whom 37 were in the intervention group and 35 were in the control group. Data collection was performed at three home visits at three-months intervals by using Quick- Disabilities of the Arm, Shoulder and Hand Scale, EORTC QLQ-BR23, Strategies Used by Patients to Promote Health Scale and Arm Circumference Measurement Form.

\section{Results}

Barriers to lymphedema prevention and homecare needs after breast surgery were examined under the themes of lymphedema development, coping with lymphedema and effects of lymphedema on life. In the experimental stage of the study, upper extremity functions improved, sideeffects decreased and the quality of life was enhanced, frequency of lymphedema development and the cost averages was lower in the women offered nursing interventions at home compared to the control group.

\section{Conclusions}

Nursing interventions based on Health Belief Model by home visits performed at regular intervals is recommended for development of lymphedema prevention behavior after breast surgery.

\section{eP481}

CHEMOTHERAPY-INDUCED NAUSEA AND VOMITING (CINV) IN A SAMPLE OF WOMEN WITH GYNECOLOGICAL CANCER: A LONGITUDINAL STUDY

V.E. Di Mattei ${ }^{1,2}$, L. Carnelli ${ }^{2,3}$, P. Taranto ${ }^{2}$, M. Mazzetti $^{2}$, M. Bernardi $^{4}$, S. Piacenti ${ }^{I}$, P.M.V. Rancoita ${ }^{5}$, F. Negrini ${ }^{2}$, F. Carzaniga ${ }^{2}$, M. Petrone ${ }^{6}$, E. Rabaiotti ${ }^{6}$, M. Candiani ${ }^{6,7}$

${ }^{1}$ Vita-Salute San Raffaele University, Faculty of Psychology, Milan, Italy ${ }^{2}$ San Raffaele Hospital, Clinical and Health Psychology Unit Department of Clinical Neurosciences, Milan, Italy
${ }^{3}$ University of Milan-Bicocca, Department of Psychology, Milan, Italy

${ }^{4}$ University of Parma, Languages Department, Parma, Italy

${ }^{5}$ Vita-Salute San Raffaele University, University Centre of Statistics in the Biomedical Sciences CUSSB, Milan, Italy

${ }^{6}$ San Raffaele Hospital, Department of Obstetrics and Gynecology, Milan, Italy

${ }^{7}$ Vita-Salute San Raffaele University, Faculty of Medicine, Milan, Italy

\section{Introduction}

Despite advancements in the control of emesis, chemotherapy-induced nausea and vomiting (CINV) still represent major problems for patients undergoing chemotherapy and can deeply affect quality of life and everyday activities.

\section{Objectives}

This study aimed to measure the frequency and intensity of CINV and its variation over time, in order to gain a better understanding of the extent to which patients experience it.

\section{Methods}

One hundred and twenty-one patients treated for gynecological cancer at the San Raffaele Hospital completed the MASCC Antiemesis Tool (MAT) after their first chemotherapy infusion; 85 of these patients completed the questionnaire after the third infusion.

\section{Results}

The age range of the sample was $32-85$ years (mean=58.19; $\mathrm{SD}=13.82$ ). Thirty-five percent of the patients experienced acute nausea after the first infusion and $44 \%$ after the third infusion; $48 \%$ reported delayed nausea after the first infusion and 58\% after the third infusion. Only a small percentage of patients reported acute and delayed vomiting (5-18\%). The intensity of nausea was significantly different from 0 in all four cases $(\mathrm{p}<.001)$. A significant increase in the intensity of acute nausea $(p=.017)$ was found between the third infusion and the first.

\section{Conclusions}

The findings suggest that nausea represents an important problem that is still common among cancer patients undergoing chemotherapy; it is possible that an exhaustion of psychosocial resources and the accumulation of the effects of antineoplastic drugs contribute to the increased intensity of acute nausea over time. These should be further investigated in order to properly address these patient issues.

\section{eP482}

ORAL HEALTH-RELATED QUALITY OF LIFE IN CHILDREN AND ADOLESCENTS UNDERGOING ANTINEOPLASIC THERAPY: PILOT STUDY

\section{Carrillo ${ }^{I}$, M. Gianesella ${ }^{1}, J$. Rojz ${ }^{I}$}

${ }^{T}$ Instituto de Oncologia Pediátrica - GRAACC/UNIFESP, Dentistry, São Paulo, Brazil

\section{Introduction}

Children and adolescents with cancer may present with oral complications due to its treatment and/or disease that may compromise their quality of life.

Objectives

To assess the oral health related quality of life of children and adolescents undergoing treatment for cancer at a pediatric oncology center.

\section{Methods}

Ten patients undergoing treatment for cancer aged 8 to 14 years old were assessed between May and July 2015. Information collected was: demographic data, medical history, oncologic diagnoses and chief oral complaint. Measures of caries experience (DMFT/deft index) and periodontal disease (Community Periodontal Index) were calculated. Oral health related quality of life was assessed by the Portuguese validated version of the Child Perceptions Questionnaire in two groups of children: 8 to 10 years old and 11 to 14 years old. 


\section{Results}

Most of the patients were undergoing chemotherapy $(n=6)$, three were undergoing chemotherapy associated with head and neck radiotherapy, and one were undergoing head and neck radiotherapy. Xerostomia, dry lips, dysgeusia and orofacial pain were the most oral complaints among patients. The mean $\mathrm{dmft}$ was 4.2, DMFT was 0.9 and CPI was 2.7. Regarding quality of life, $50 \%$ of subjects aged 8-10 reported experiencing oral symptoms "Often" or "Everyday/Almost everyday" while 67\% of subjects aged 11-14 reported experiencing functional limitations "Often" or "Everyday/Almost everyday". There was a significant difference between child's and parents' perception on functional limitations on 8 to 10 years old group.

Conclusions

Oral complications due to cancer therapy may have a negative impact on quality of life of children and adolescents undergoing cancer therapy.

\section{eP483}

\section{EFFECT OF CHEMOTHERAPY ON SLEEP QUALITY IN CANCER PATIENTS}

F. Çay Șenler ${ }^{l}$, B.D. Işıkoğlu- ${ }^{1}$, A. Demirkazık ${ }^{l}$, G. Utkan ${ }^{1}$, Y. Ürün ${ }^{1}$, H. Akbulut ${ }^{l}$

${ }^{T}$ Ankara University Faculty of Medicine, Medical Oncology, Ankara, Turkey

\section{Introduction}

Insomnia is a sleep disorder characterized by difficulty falling in or staying asleep. Insomnia is a common problem in cancer patients and impairs quality of life. Numerous causes and risk factors for insomnia had been identified. Chemotherapy is among them. However, there is no evidence that chemotherapy has direct effects on sleep in the literature.

\section{Objectives}

The aim of this study was to evaluate the effect of chemotherapy on sleep quality in cancer patients.

Methods

Chemotherapy naive outpatients in Medical Oncology Department of Ankara University Faculty of Medicine were evaluated between March 2017 and November 2017. Turkish version of Pittsburgh Sleep Quality Index (PSQI) survey was used to assess sleep quality and disturbances before chemotherapy and 3 months after the start of chemotherapy. Turkish version of Hospital Anxiety and Depression (HAD) Scale was also used and medical data of patients were collected as possible factors associated with sleep disorder.

\section{Results}

One hundred patients were evaluated. Patient characteristics are listed in Table-1. A global sum of PSQI 5 or greater indicates a poor sleeper. Any significant effect of chemotherapy on pain, disturbing symptoms, anxiety, depression and sleep quality were not shown. Before chemotherapy 48 patients and after chemotherapy 50 patients were found poor sleeper.

\begin{tabular}{|c|c|}
\hline Variable & $\mathrm{n}$ (100 patients) \\
\hline $\begin{array}{l}\text { Gender } \\
\text { Fcmalc } \\
\text { Male }\end{array}$ & $\begin{array}{l}46 \\
54\end{array}$ \\
\hline $\begin{array}{l}\text { Age } \\
\text { Median (range) } \\
>65 \text { years }\end{array}$ & $\begin{array}{l}60(28-83) \\
37\end{array}$ \\
\hline $\begin{array}{l}\text { Cancer Type } \\
\text { Lung cancer } \\
\text { Breast cancer } \\
\text { Other }\end{array}$ & $\begin{array}{l}33 \\
22 \\
45\end{array}$ \\
\hline $\begin{array}{l}\text { Stage } \\
\text { Early stage } \\
\text { Advanced stage } \\
\text { Relapse disease }\end{array}$ & $\begin{array}{l}41 \\
39 \\
20\end{array}$ \\
\hline $\begin{array}{l}\text { Medical Causes of Insor } \\
\text { Drigs } \\
\text { Pain } \\
\text { Co-morbidity }\end{array}$ & $\begin{array}{l}35 \\
40 \\
52 \\
\end{array}$ \\
\hline $\begin{array}{l}\text { Psychiatric morbidity } \\
\text { Anxiery } \\
\text { median score (range) } \\
\text { Depression } \\
\text { median score (range) }\end{array}$ & $\begin{array}{l}10 \\
21 \\
5(0-19) \\
38 \\
5(0-21)\end{array}$ \\
\hline
\end{tabular}

\section{Conclusions}

In this study, no significant effect of chemotherapy on sleep quality has been demonstrated.

\section{eP484}

MINDFULNESS-BASED STRESS REDUCTION TO IMPROVE QUALITY OF LIFE AND MOOD IN CANCER PATIENTS: PRELIMINARY RESULTS

J. Mebis ${ }^{1}, S$. Censabella ${ }^{1}, N$. Cardinaels $^{1}$, S. Engels ${ }^{1}$, G. Orye ${ }^{2}, S$.

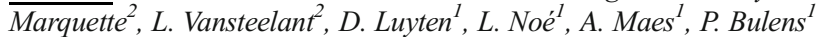

${ }^{1}$ Jessa Hospital, Medical Oncology, Hasselt, Belgium

${ }^{2}$ Jessa Hospital, Division of Gynaecology, Hasselt, Belgium

\section{Introduction}

Mindfulness-Based Stress Reduction (MBSR) is a group intervention based on meditation increasingly used to improve quality of life (QoL). In our Cancer Center, MBSR is offered since many years to any cancer patients who have undergone cancer therapy, yet its efficacy has not been evaluated to date.

\section{Objectives}

To assess the effect of our MBSR-program on QoL, mood, and mindfulness skills immediately and 3 months after the program.

\section{Methods}

Cancer patients who subscribed to our MBSR-program in 2016-2017 were recruited. The program is standardized and provided by a certified trainer during 8-week (one 3h-session/ week) to small groups (8-12 patients).

General and cancer-specific QoL, mood, and mindfulness skills were measured before the start, at the end, and 3 months after MBSR, using the brief World Health Organisation Quality of Life scale (WHOQOL-Bref), the Functional Assessment of Cancer Therapy - General (FACT-G), the Positive and Negative Affect Schedule (PANAS), and the Kentucky Inventory of Mindfulness Skills (KIMS), respectively.

Data were analysed by means of paired t-tests.

\section{Results}

Sixty-four cancer patients completed the study (see Table 1 for patients characteristics).

Table 1. Patients characteristics

\begin{tabular}{|c|c|c|c|}
\hline Gender & $N(\%)$ & Tumour Stage & $N(\%)$ \\
\hline Male & $6(9)$ & 0 & $3(5)$ \\
\hline \multirow{2}{*}{ Female } & $58(91)$ & 1 & $13(20)$ \\
\hline & & 2 & $29(45)$ \\
\hline \multirow[t]{2}{*}{$\begin{array}{l}\text { Mean age (years, } \\
\text { SD) }\end{array}$} & $53(11)$ & 3 & $9(14)$ \\
\hline & & 4 & $1(2)$ \\
\hline TumourLocation & $N(\%)$ & Unknown & $9(14)$ \\
\hline Brain & $1(2)$ & & \\
\hline Breast & $47(73)$ & Cancertherapy & $N(\%)$ \\
\hline Colorectal & $1(2)$ & Chemotherapy & $42(66)$ \\
\hline Gynaecologic & $6(9)$ & Radiotherapy & $46(72)$ \\
\hline Hematologic & $3(5)$ & $\begin{array}{l}\text { Endocrine } \\
\text { therapy }\end{array}$ & $40(63)$ \\
\hline Skin & $2(3)$ & Other & $13(20)$ \\
\hline Urogenital & $2(3)$ & & \\
\hline Head and Neck & $1(2)$ & $\begin{array}{l}\text { Mean time since } \\
\text { in }\end{array}$ & ecruitment \\
\hline \multirow[t]{3}{*}{ Soft tissue } & $1(2)$ & months) & $M(S D)$ \\
\hline & & Diagnose & $20(39)$ \\
\hline & & Latest therapy & $9(10)$ \\
\hline
\end{tabular}

* Primary surgery, chemo-, or radiotherapy (endocrine and targeted therapy were mostly ongoing at recruitment) 
Preliminary statistical analyses revealed significant improvement in quality of life, mood, and mindfulness skills at the end of MBSR (p's $<0.05$, see Table 2). These improvements remained mostly stable at 3 months follow-up.

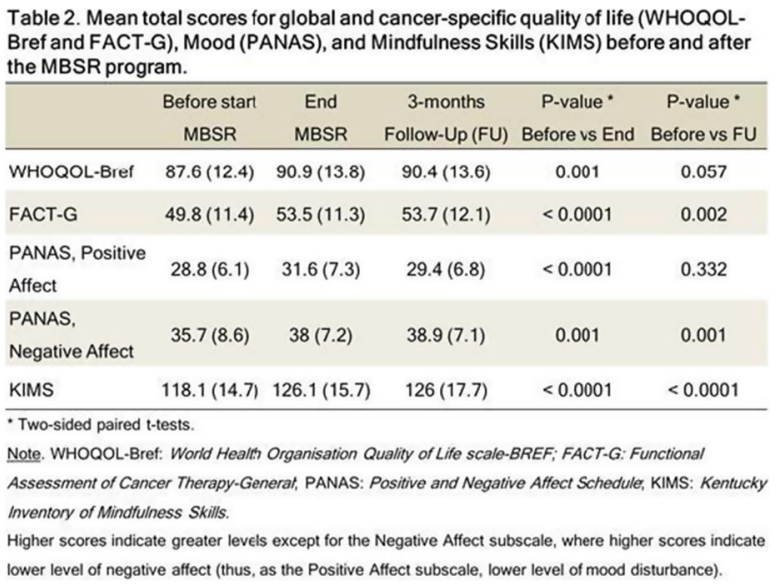

\section{Conclusions}

These preliminary results showed that our MBSR-program improved quality of life, mood, and mindfulness skills in cancer patients. These improvements remained stable at retesting 3 months later, suggesting, consistent with the literature, long-lasting beneficial effects.

\section{eP485}

THE FEAR OF DISEASE PROGRESSION, EMOTIONAL DISTRESS, AND QUALITY OF LIFE ASSOCIATED WITH PROSTATE CANCER PATIENTS

Y.P. Chang ${ }^{\text {, }}$ D.C. Lee ${ }^{2}$, T.C. Cheng ${ }^{3}$, Y.H. Lee ${ }^{4}$

${ }^{T}$ DA-Yeh University, Department of Nursing, Changhua, Taiwan R.O.C.

${ }^{2}$ DaYeh University, Department of Information Management, Changhua, Taiwan R.O.C.

${ }^{3}$ ChiMei Hospital- Liuying-, Department of Urology, Tainan, Taiwan R.O.C.

${ }^{4}$ The National Health Research Institutes, Department of Administration, Miaoli, Taiwan R.O.C.

\section{Introduction:}

Prostate cancer is a high incidence of cancer for men in 2015, with about 1.6 million new cases reported and a rate of increase of $66.1 \%$ during the past ten years. Cancer patients experience a great deal of psychological stress and physical discomfort resulting from treatments, which ultimately could affect the quality of life of patients and their families. Patients' perception regarding disease treatment and prognosis, as well as their own physical and mental indicators could offer medical care providers and patient themselves the bases for decision-making on future care and life quality.

Objectives

The purpose of this study is to assess the effects of different treatments on patients' fear of disease progression, emotional distress, and life quality. Methods

Three therapy modules of treatments were employed in this study on prostate cancer patients: hormonal treatment (HT), a combination of hormone treatment with either chemotherapy, radiotherapy, or surgery (Mix Treatment), and placebo treatment on the control group (CG). The study was designed as a cross-sectional study of 38 patients for the diagnosis and treatment of prostate cancer.

\section{Results}

By descriptive statistics, the control group scored better than the other two treated groups in emotional distress and quality of life, indicating that cancer treatment has a negative impact on patients' life or psychological status. These results also show that mixed treatment can indeed reduce cancer patients' fear of the disease progression.

\section{Conclusions}

For cancer patients, quality of life can be used as an important indicator for evaluating health and medical measures.

\section{eP486}

EVALUATION OF QUALITY OF LIFE AND COST OF CARE IN METASTATIC COLORECTAL CANCER PATIENTS RECEIVING AMBULATORY CHEMOTHERAPY

P. Chansriwong ${ }^{I}$, S. Sirilerttrakul ${ }^{I}$, M. Jirajarus ${ }^{1}, N$. Wannakansophon ${ }^{I}$, S. Vongterapak, S. Ckumdee ${ }^{1}$, P. Tangteerakoon ${ }^{1}$, E. Sirachainan ${ }^{1}$

${ }^{1}$ Ramathibodi Hospital- Mahidol University, Internal Medicine, Bangkok, Thailand

\section{Introduction}

Colorectal cancer is an important health problems in Thailand; chemotherapy remains the most suitable treatment for metastatic patients. The chemotherapy regimens are based on a 48 hours 5-fluorouracil (5-FU) infusion combined that need patients to be hospitalised, consequence to decrease quality of life ( QoL) and increase cost of treatment. Ambulatory chemotherapy helps patients to live normal lives by administer treatment at patients' home.

\section{Objectives}

Compares the QoL score and cost difference in treating ambulatory chemotherapy (AC) patients compared with inpatient treatment.

\section{Methods}

An observational cohort which enrolled 75 patients at the Ramathibodi hospital from Dec 2015 to Nov 2016. AC administered by the central venous access device (CVAD). Chemotherapy regimen as FOLFOX or FOLFIRI, 5-FU were in the elastomeric infusion pump and administered at the patients' home. Nurse coordinators followed up with the patients by phone. The Functional Assessment of Cancer Therapy-General and Colorectal (FACT-G and FACT-C) scale and cost of treatment questionnaire were collected at time of enrolment, 2 months and end of treatment. Results

75 patients are enrolled that 30 patients treated with $\mathrm{AC}$ and 45 patients treated with inpatient. 65 returned the questionnaire( response rate $86 \%$ ). Intention to treat analysis revealed significantly improved in social well being $(\mathrm{p}<0.001)$ in AC group (in Figure 1). This analysis demonstrated trend of QoL improvement for the FACT-G $(\mathrm{p}=0.059)$. The AC reduced cost about 483 US dollars per cycle of chemotherapy.

Table I. FACT. C subseales for patients at time of enrolment, 2 months and end of treatment

\begin{tabular}{|c|c|c|c|c|c|c|c|}
\hline & \multicolumn{3}{|c|}{$\begin{array}{l}\text { Ambulatory } \\
\text { Mean (SD) }\end{array}$} & \multicolumn{3}{|c|}{$\begin{array}{l}\text { Inpatient } \\
\text { Mean (SD) }\end{array}$} & \multirow[t]{2}{*}{ p value } \\
\hline & enrolment & 2 months & $\begin{array}{l}\text { end of } \\
\text { treatment }\end{array}$ & enrolment & 2 months & $\begin{array}{l}\text { end of } \\
\text { treatment }\end{array}$ & \\
\hline Enctons & $\begin{array}{l}18.44 \\
(4.35)\end{array}$ & $\begin{array}{l}19.28 \\
(5.37)\end{array}$ & $\begin{array}{l}19.77 \\
(2.86)\end{array}$ & $\begin{array}{l}18.03 \\
(4.01)\end{array}$ & $\begin{array}{l}18.69 \\
(4.58)\end{array}$ & $\begin{array}{l}19.61 \\
(3.73)\end{array}$ & 0.583 \\
\hline Functions & $\begin{array}{l}18.35 \\
(4.79)\end{array}$ & $\begin{array}{l}18.54 \\
(5.09)\end{array}$ & $\begin{array}{l}17.30 \\
(3.32)\end{array}$ & $\begin{array}{l}16.58 \\
(6.01)\end{array}$ & $\begin{array}{l}16.56 \\
(6.60)\end{array}$ & $\begin{array}{l}18.55 \\
(5.62)\end{array}$ & 0.175 \\
\hline$x^{2}=000$ & $\begin{array}{l}20.52 \\
(4.43)\end{array}$ & $\begin{array}{l}21.29 \\
(5.05)\end{array}$ & $\begin{array}{l}20.92 \\
(3.15)\end{array}$ & $\begin{array}{l}19.47 \\
(5.65)\end{array}$ & $\begin{array}{l}21.28 \\
(4.91)\end{array}$ & $\begin{array}{l}21.75 \\
(4.79)\end{array}$ & 0.332 \\
\hline 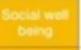 & $\begin{array}{l}22.03 \\
(3.40)\end{array}$ & $\begin{array}{l}21.24 \\
(3.84)\end{array}$ & $\begin{array}{l}20.38 \\
(2.87)\end{array}$ & $\begin{array}{l}18.51 \\
(4.18)\end{array}$ & $\begin{array}{l}17.66 \\
(3.99)\end{array}$ & $\begin{array}{l}18.30 \\
(3.62)\end{array}$ & $<0.001$ \\
\hline Fostio & $\begin{array}{c}79.05 \\
(12.88)\end{array}$ & $\begin{array}{c}76.53 \\
(16.34)\end{array}$ & $\begin{array}{l}78.38 \\
(8.07)\end{array}$ & $\begin{array}{c}72.58 \\
(15.12)\end{array}$ & $\begin{array}{l}74.18 \\
(16.47)\end{array}$ & $\begin{array}{c}78.23 \\
(14.90)\end{array}$ & 0.059 \\
\hline $\begin{array}{l}\text { Fostec } \\
\text { coseses }\end{array}$ & $\begin{array}{l}97.05 \\
(15.11)\end{array}$ & $\begin{array}{l}94.70 \\
(19.94)\end{array}$ & $\begin{array}{l}96.24 \\
(10.03)\end{array}$ & $\begin{array}{r}90.02 \\
(18.77)\end{array}$ & $\begin{array}{l}92.27 \\
(20.02)\end{array}$ & $\begin{array}{c}97.20 \\
(17.78)\end{array}$ & 0.087 \\
\hline 105 & & 0,0 & & & 1,496 & & \\
\hline
\end{tabular}




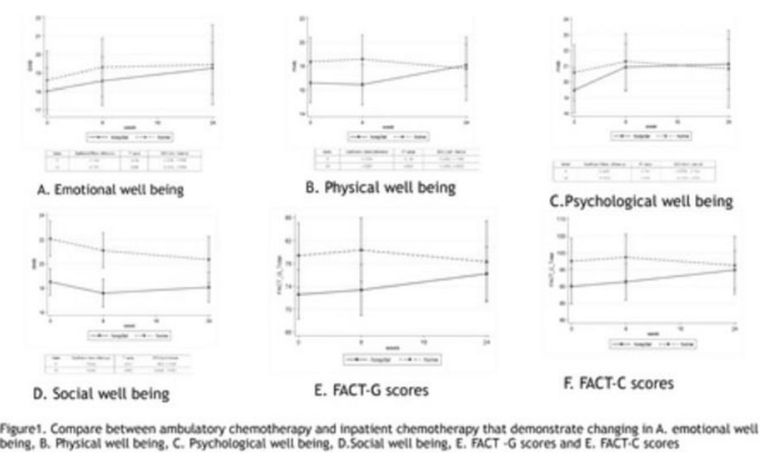

\section{Conclusions}

These data suggest that ambulatory chemotherapy improve QoL, especially in social well being and reduced cost of care in mCRC patients.

eP487

BODY IMAGE AND QUALITY OF LIFE AMONG COLORECTAL CANCER SURVIVORS: A CRITICAL REVIEW L.W. Cheung ${ }^{1}$, K.M. Chow ${ }^{1}$

${ }^{1}$ The Chinese University of Hong Kong, The Nethersole School of Nursing, Hong Kong, Hong Kong S.A.R.

\section{Introduction}

Colorectal cancer and its treatment have negative impacts on body image and quality of life. These impacts are becoming increasingly crucial as the survival rates have been rising.

Objectives

This review aims to identify, summarise and appraise literature investigating body image and quality of life among colorectal cancer survivors. Methods

A comprehensive search was conducted to identify relevant articles from MEDLINE, PsycINFO, CINAHL and British Nursing Index from 2007 to 2017.

\section{Results}

A total of 19 studies were included in this review. Colorectal cancer survivors with stoma reported having significantly lower body image which was persistent and worsen over time. Survivors who were welleducated, employed, married, had rectal cancer or pelvic dissection were found to have poorer body image. For the outcome of quality of life, colorectal cancer survivors reported having poorer quality of life than the general population, but improvement was observed with time. Similarly, survivors with stoma had poorer quality of life than those without it, yet some studies reported no significant difference. Gender, age, tumour stage and site were found to be determinants of quality of life, but the results were inconclusive. Regarding the association between body image and quality of life, body image disturbance was found to be correlated with poor emotional quality of life.

Conclusions

Colorectal cancer survivors, especially those with stoma, generally reported poorer body image and quality of life. Appropriate nursing interventions should be identified to address their needs. Future studies in various cultural context other than Western countries should be conducted.

\section{eP488}

\author{
QUALITY OF LIFE OF PATIENTS WITH A TRACHEOSTOMY \\ AFTER DISCHARGE

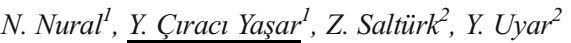

${ }^{1}$ Institue Of Health Sciences, Internal Medicine Nursing, Trabzon, Turkey ${ }^{2}$ Okmeydanı Training And Research Hospital, Clinic of ENT, Istanbul, Turkey

\section{Introduction}

Changes in airway management, nasal function, swallowing and taste, loss of voice, neck and shoulder mobility problems, and impaired social functioning have a considerable impact on the quality of life of patients with a permanent tracheostomy.

Objectives

The purpose of this study was to analyze QOL in patients with apermanent tracheostomy and the factors affecting their QOL

\section{Methods}

This is a cross-sectional and descriptive study. Fortyfive patients underwent total laryngectomy at Okmeydanı Training and Research Hospital Ear Nose and Throat Clinic in 2015 and 36 of those discharged with permanent tracheostomy participated in this study. Sociodemographic data werecollected along with data on QOL using EORTC QLQ C-30. The nonparametric Kruskall Wallis and Mann Whitney $U$ tests were used for the analysis of sociodemographic data and variables that could affect QOL. The significance level was accepted as 0.05 .

Results

The mean common wellbeing and quality of life score was $47.6 \pm 14.4$, which is below average. Regarding functional status, cognitive and emotional functions were affected the most. Regarding the symptom subscale, insomnia and fatigue were the factorsinfluencing decreasedquality of life the most. Moreover, age and social environment were major contributors of quality of life in the symptom subscale. With an increase of age and a decline in social environment,quality of life declines. Social environment also affects patients' wellbeing, as does continuing one's professional life. Conclusions

Our study revealed that the level of quality of life was low, especially in physical status, and that loneliness was a diminishing factor

\section{eP489}

PROSPECTIVE ASSESSMENT AND SIGNIFICANCE OF QUALITY OF LIFE IN WOMEN WITH ADVANCED CERVICAL CANCER IN A TERTIARY CARE INSTITUTE OF DELHI, INDIA

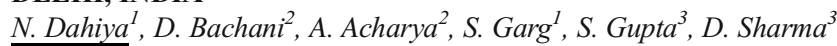
${ }^{T}$ Maulana Azad Medical College and LN Hospital, Community Medicine, Delhi, India

${ }^{2}$ Lady Hardinge Medical College, Community Medicine, Delhi, India

${ }^{3}$ All India Institute Of Medical College, Radiation Oncology, Delhi, India

\section{Introduction}

Cervical cancer is the most frequently diagnosed cancer among women in India.

\section{Objectives}

This study assessed the impact of chemo-radiation therapy on QOL of cervical cancer patients after 6 months of treatment.

\section{Methods}

This follow up study was undertaken at cancer center of AIIMS, New Delhi. Sixty eight women with advanced cervical cancer (stage $2 b$ to $4 b$ ) were included. Structured questionnaires on Quality of Life EORTC QLQ-C30 and EORTC QLQ-CX24) were used. QOL was assessed before initiation and after 6 months of treatment.

\section{Results}

The mean age of women at the time of detection was $53.03 \pm 11.14$ years. Unusual discharge from vagina, post-menopausal bleeding and pain abdomen were the most common presenting complaints. Squamous cell carcinoma was found to be the most common cell type (97\%). Six months survival was $90.3 \%$ The mean global health score of cervical cancer 
patients after six months of treatment was 59.5 , which was significantly higher than the pre - treatment score of 50.15 ( $\mathrm{p}=0.000054)$. Physical, cognitive and emotional functioning $(\mathrm{p}<0.05)$ improved significantly after treatment. Fatigue, pain, insomnia and appetite loss improved but episodes of diarrhea increased after treatment. Mean "symptoms score" using EORTC QLQ-CX24 post treatment was 12.55 which was significantly lower as compared to pre- treatment scores $25.66(\mathrm{p}=0.00001)$. Sexual enjoyment and sexual functioning decreased significantly after treatment.

Conclusions

QOL of newly diagnosed cervical cancer patients significantly improved following chemo-radiation therapy. To further improve QOL, interventions focusing on social and psychological support and physical rehabilitation may be needed.

\section{eP490}

UTILITY OF ACCEPTANCE AND COMMITMENT THERAPY FOR GIVING A BETTER QUALITY OF LIFE AMONG BREAST CANCER PATIENTS IN KOLKATA

A. Datta ${ }^{1}$, P. Chaudhuri ${ }^{2}$

${ }^{T}$ Netaji Subhas Chandra Bose Cancer Research Institute, Psychooncology, Kolkata, India

${ }^{2}$ Institute of Psychiatry, Psychiatry, Kolkata, India

\section{Introduction}

Acceptance and commitment therapy (ACT) is one of the newer forms (third wave) therapy which helps a cancer patient to increase acceptance level about their disease as well as their present situation and give better quality of life through developing acceptance ability.

Objectives

The present study had assessed effect of structured ACT intervention on depression acceptance level, meaning of life and quality of life among breast cancer patients who were undergoing chemotherapy.

Methods

Data was collected from 151 breast cancer patients those who were undergoing chemotherapy were willing to undergo psychological treatment, with no history of past psychiatric illness. Their baseline of depression, acceptance levels, meaning of life and quality of life were assessed using validated tools. The participants were randomly divided into two groups: experimental (ACT, 4 sessions over 2 months) and control group. Depression, acceptance level, meaning of life and quality of life were measured during post intervention on 2 months follow-up.

\section{Results}

The prevalence of depression in patients with cancer range from $31 \%$ to $71 \%$. Psychological variables were significantly associated with meaning of life and quality of life. At baseline, the factors that significantly more influenced psychological variables were sociodemographic factors rather than clinicopathological factors. Per and post intervention outcomes were analyzed and showed that changes in psychological flexibility predicted improvement in depression, acceptance level and quality of life.

\section{Conclusions}

ACT is an effective therapeutic option for treating depression and improves acceptance level that leads a better quality of life among breast cancer patients undergoing chemotherapy.

\section{eP491}

PSYCHOSOCIAL RESOURCES IN LONG-TERM BREAST, COLORECTAL AND PROSTATE CANCER SURVIVORS: PREVALENCE AND ASSOCIATIONS WITH HEALTHRELATED QUALITY OF LIFE

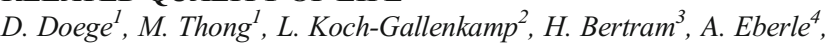
B. Holleczek, M. Waldeyer-Sauerland ${ }^{6}$, A. Waldmann ${ }^{6,7}$, S.R. Zeissig', H. Brenner ${ }^{2,9,10}$, V. Arndt ${ }^{1}$
${ }^{1}$ German Cancer Research Center DKFZ, Unit of Cancer SurvivorshipDivision of Clinical Epidemiology and Aging Research, Heidelberg, Germany

${ }^{2}$ German Cancer Research Center DKFZ, Division of Clinical Epidemiology and Aging Research, Heidelberg, Germany

${ }^{3}$ Cancer Registry of North Rhine-Westphalia, Cancer Registry of North Rhine-Westphalia, Münster, Germany

${ }^{4}$ Leibniz Institute for Prevention Research and Epidemiology, Bremen Cancer Registry, Bremen, Germany

${ }^{5}$ Ministry of Social Affairs- Health- Women and Family Affairs, Saarland Cancer Registry, Saarbrücken, Germany

${ }^{6}$ Ministry of Health and Consumer Protection, Hamburg Cancer Registry, Hamburg, Germany

${ }^{7}$ University Lübeck, Institute of Social Medicine and Epidemiology, Hamburg, Germany

${ }^{8}$ Cancer Registry of Rhineland-Palatinate, Cancer Registry of Rhineland-Palatinate, Mainz, Germany

${ }^{9}$ German Cancer Research Center DKFZ, German Cancer Consortium DKTK, Heidelberg, Germany

${ }^{10}$ German Cancer Research Center DKFZ, Division of Preventive Oncology, Heidelberg, Germany

\section{Introduction}

Many long-term cancer survivors still have to adjust to possible adverse consequences of the illness or treatment. Resources can play an important role in this adjustment process, but research on this topic is limited, especially for very long-term survivors.

Objectives

This study explores, which resources are most frequently indicated by different subgroups of cancer survivors, and what role resources play for functioning and health-related quality of life (HRQL) in cancer survivors with and without recurrence.

Methods

The sample of 6030 breast, colorectal and prostate cancer survivors (5-16 years post-diagnosis) was recruited in a German multi-regional population-based study. Personal resources were assessed by a 27 -item checklist; HRQL was assessed by the European Organization for Research and Treatment of Cancer Quality of Life Questionnaire - Core 30 (EORTC QLQ-C30). General linear models were used to analyze associations of resources with HRQL.

Results

Participants indicated on average 11.4 (SD 5.1) resources as helpful. Family, activities with others, and partnership were indicated most commonly overall, but frequencies varied according to age, sex, and tumor site. Physical activity, health, professional help, calmness, hope, optimism, and hobbies were most important in explaining HRQL variance. Cancer survivors with recurrence and many resources reported similar HRQL as survivors without recurrence and only few resources.

\section{Conclusions}

The study underlines the importance and situational variability of personal and social resources for cancer survivors' HRQL, even years postdiagnosis. Not only the availability, but also the individual perception and significance of resources should be considered in follow-up cancer care.

\section{eP492}

QUALITY OF SLEEP IN PATIENTS WITH CANCER; A CROSSSECTIONAL STUDY

${ }_{\text {B.Fadipe }}^{1}$, A.T. Olagunju ${ }^{2}$, M. Habeebu ${ }^{3}$, B. Adegboyega ${ }^{3}$, A.A. Ojeyinka

${ }^{1}$ Lagos University Teaching Hospital- Lagos- Nigeria, Psychiatry Consultation-Liason, Festac Town, Nigeria

${ }^{2}$ Lagos University Teaching Hospital- Lagos- Nigeria, Psychiatry Psycho-Oncology, Lagos, Nigeria 
${ }^{3}$ Lagos University Teaching Hospital-Lagos- Nigeria, Radiotherapy and Oncology, Lagos, Nigeria

${ }^{4}$ Lagos University Teaching Hospital- Lagos- Nigeria, Psychiatry, Lagos, Nigeria

\section{Introduction}

Sleep is essential to human health, serving restorative functions with enormous physical and emotional benefits. In cancer, illness symptoms, treatment side-effects and psychological or physical co-morbidity may prevent patients from enjoying qualitative sleep. However, till date, there has been no known study examining sleep quality among people with cancer from this environment.

Objectives

This study is set out to determine the quality of sleep and associated factors among patients with cancer in a Nigerian oncology clinic.

Methods

A total of 115 consenting adults with cancer were recruited into the study. Participants were administered a designed questionnaire to profile their clinico-demographic characteristics and Pittsburgh Sleep Quality Index (PSQI) to measure quality and patterns of sleep. Data was analyzed using SPSS-20.

\section{Results}

The mean age of participants was $47.33( \pm 12.86)$ years, majority of them were female $(86.1 \%)$ and had breast cancer $(67.8 \%)$ with slightly above half presenting with advanced stages. The average duration of sleep of was $6.35( \pm 1.85)$ hours. Eighty $(69.6 \%)$ participants reported poor sleep quality. Nineteen $(16.5 \%)$ reported having to use sleep medication three or more times a week. Participants who experienced pain in the month prior to the study $(\mathrm{X} 2=13.117, \mathrm{p}=<0.001)$,

Conclusions

Findings in this study suggest the experience of poor sleep quality in association with pain, use of sleep medication and advanced cancers. These should be considered in managing patients as a means to improve quality of life in patients with cancer.

\section{eP493}

\section{EXPERIENCES OF KNOWLEDGE MOBILIZATION FOR} DECISION MAKING AMONG AND TOWARDS ONCOLOGICAL PATIENTS AND THEIR NONPROFESSIONAL CAREGIVERS (BINOMIAL) IN MEXICO R. GARCIA

${ }^{T}$ CENTRO DE INVESTIGACIONES Y ESTUDIOS SUPERIORES EN ANTROPOLOGIA SOCIAL, ANTROPOLOGIA MEDICA, SAN CRISTOBAL DE LAS CASAS, MeXico

\section{Introduction}

The social aspects of the patient and their family environment are increasingly considered in strategies to overcome cancer without impairing their quality of life. However, little is known about their experiences in the processes and mechanisms of knowledge mobilization to perform their care work, particularly in Mexico

Objectives

Identify the knowledge needs of the binomials (oncology patients and their non-professional caregivers), where they obtain their knowledge and how do they mobilize it to carry out the work of care.

Methods

During 2016 and 2017, an exploratory study, mixed methods, was conducted in Mexico. Fourteen in-depth interviews with binomials, participant observations in two face-to-face and two online mutual help groups, 97 semi-structured interviews with medical staff from three hospitals and online interviews with academic experts on this field.

Results

There were few coincidences between the knowledge mobilization strategies reported as successful by the binomials and those described by the medical staff and academic experts. Among others, two very important aspects were identified: invisibility of non-professional caregivers by medical personnel and the importance and relevance of the use of online networks and internet regardless of socioeconomic characteristics

\section{Conclusions}

Current policies regarding care and accompaniment to non-professional caregivers of oncological patients do not incorporate neither in their design nor in their implementation the strategies and processes of knowledge mobilization reported as feasible and successful. It is needed to know the opinions and experiences of all actors involved if we want to improve their wellbeing.

\section{eP494}

\section{PROCESS DEVELOPMENT FOR ENHANCED RECOVERY IN} MEDICAL ONCOLOGY

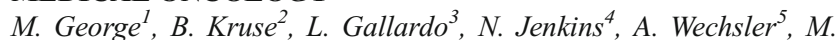

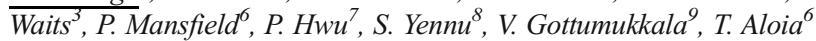

${ }^{1}$ The University of Texas MD Anderson Cancer Center, Internal Medicine, Houston, USA

${ }^{2}$ The University of Texas MD Anderson Cancer Center, Institute for Cancer Care Innovation, Houston, USA

${ }^{3}$ The University of Texas MD Anderson Cancer Center, Nursing Administration, Houston, USA

${ }^{4}$ The University of Texas MD Anderson Cancer Center, Nursing Education, Houston, USA

${ }^{5}$ The University of Texas MD Anderson Cancer Center, Emergency Medicine, Houston, USA

${ }^{6}$ The University of Texas MD Anderson Cancer Center, Surgical Oncology, Houston, USA

${ }^{7}$ The University of Texas MD Anderson Cancer Center, Cancer Medicine, Houston, USA

${ }^{8}$ The University of Texas MD Anderson Cancer Center, Palliative Care Medicine, Houston, USA

${ }^{9}$ The University of Texas MD Anderson Cancer Center, Anesthesiology and Perioperative Medicine, Houston, USA

\section{Introduction}

Complications related to cancer treatment or symptoms can delay a patient's return to intended oncologic therapy (RIOT). Multiple factors contribute towards patient hospitalizations, complications, and delays for RIOT. Enhanced recovery is a pathway utilized within surgery to improve patient outcomes.

\section{Objectives}

The purpose of this pilot project was to determine if implementation of an enhanced recovery pathway was feasible in the medical oncology setting for patients experiencing unintended hospitalizations.

\section{Methods}

An evaluation of current enhanced recovery programs in surgery showed use of multi-disciplinary approaches such as optimal opioid use, nonopioid alternatives, and functional mobility along with fluid and nutrition management. A multi-disciplinary stakeholder team was established. Process maps and order sets were created to help generate awareness of multimodal symptom management alternatives. Patients were engaged through daily checklists and education. A survey of opiate awareness was conducted to highlight areas for additional education.

\section{Results}

Key components to feasibility was multi-disciplinary teamwork and patient engagement. Others included system level and leadership support, and continued focus on the end goal of RIOT. The barriers to the process included engagement from all oncology clinical teams and additional opioid and adjuvant medication education for clinical staff.

\section{Conclusions}

Implementing an enhanced recovery pathway in medical oncology for patients experiencing unintended hospitalizations is potentially feasible. 
The impact of an enhanced recovery pathway in medical oncology on patients is yet to be determined. Reducing symptoms and enhancing recovery is patient-centric and a strong commitment to innovation and a multi-disciplinary team are drivers for success.

\section{eP495}

CAN PRO EVALUATION DETERMINE EARLY IF A PATIENT IS EXPERIENCING BENEFIT OR FUTILITY FROM TREATMENT: RESULTS OF A PROSPECTIVE TRIAL USING THE LCSS IN NSCLC

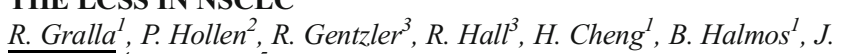

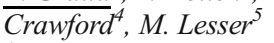

${ }^{1}$ Albert Einstein College of Medicine, Medical Oncology, New York- NY, USA

${ }^{2}$ University of Virginia, School of Nursing, Charlottesville- VA, USA

${ }^{3}$ University of Virginia, Medical Oncology, Charlottesville- VA, USA

${ }^{4}$ Duke University, Medical Oncology, Durham- NC, USA

${ }^{5}$ Feinstein Institute for Medical Research, Biostatistics, Manhasset- NY, USA

\section{Introduction}

Early determination of treatment effect in advanced NSCLC can prevent unnecessary exposure to toxic and costly therapy. Analysis in mesothelioma suggested that a $20 \%$ decline after 2 cycles of chemotherapy in the 3Item Global Index (3-IGI) of the LCSS PRO measure identified patients unlikely to benefit. The 3-IGI (distress, activities, QL) takes $<2$ minutes to perform.

Objectives

To determine if a $20 \% 3$-IGI decline predicts poor NSCLC outcomes.

Methods

164 patients with NSCLC receiving treatment were evaluated with the LCSS every 3 weeks. Patients were also randomized 1:1 so that physicians knew results of the LCSS immediately in half of the patients.

Results

Characteristics: Stage IV-92\%; 43\%-female; median PS=1; mean age 63; $46 \%$ minorities. The LCSS was completed after just 2 treatment cycles, and immediately prior to planning for the next cycle ( 6 weeks post-baseline). Patients with a $20 \%$ decline in the 3-IGI compared with baseline had a median survival of 7.6 months, contrasted to 15.8 months for those without this degree of 3 -IGI decline $(p=0.01)$. Even with PRO decline after 2 treatment cycles, patients in the $20 \%$ decline group received a median of 2.3 additional cycles of the same chemotherapy.

\section{Conclusions}

Assessing change from baseline of the 3-IGI of the LCSS identifies after just 2 cycles of treatment those patients who have poor response and survival outcomes if continued on the same therapy. This assessment is rapid, easy, and inexpensive. Acting on 3-IGI changes can result in better decisions concerning treatment, changing therapy, toxicity, and cost savings.

Support: NIH/NCI R01 CA157409

\section{eP496}

USE OF INDIVIDUAL PATIENT OUTCOME SCAE(IPOS) AS SCREENING TOOL TO IDENTIFY WHICH PATIENTS COULD BENEFIT FROM REFERRAL TO THE SYMPTOM MANAGEMENT AND SUPPORTIVE CARE CLINIC

N. Holtom ${ }^{l}$, C. Barry ${ }^{l}$

${ }^{T}$ Norfolk and Norwich University Hospital NHS Foundation Trust, Palliative Medicine, Norwich, United Kingdom

\section{Introduction}

Patient outcome measures are a way in which information regarding a patient's physical and psychological symptoms and practical issues relating to illness can be formally assessed. Timely referral to palliative care services is highlighted as best practice for patients with metastatic breast cancer.

\section{Objectives}

To assess whether IPOS could be used as an effective screening tool to identify which metastatic breast cancer patients would benefit from referral to The Symptom Management and Supportive Care Clinic .

\section{Methods}

Patients were identified by their oncologist at follow up clinic. Patients were asked to complete the IPOS after the consultation and to self rate their current problems on a scale of $0-4(0=$ not at all, $4=$ overwhelming $)$ Those patients who identified any domain as affecting them severely or overwhelmingly were considered suitable for assessment and offered referral by their specialist nurse.

Results

Data was collected for 23 patients during the pilot study. The mean IPOS score was 17.9 and top ranking issues were family anxiety, pain, weakness, inability to share feelings with family/friends and poor mobility. Although the mean IPOS score is significant it should not be used as the only factor triggering referral as patients scoring 3 or 4 in any domain may also benefit from assessment.

\section{Conclusions}

It was not easy to anticipate patients scoring highly and IPOS should therefore be used routinely for patients with metastatic breast cancer who are being treated with palliative intent. All IPOS are discussed in a metastatic breast cancer MDT attended by the palliative medicine consultant, oncologists and specialist nurses

\section{eP497}

\section{THE EXPLORATION FOR THE NEEDS OF ELDERLY} PROTECTIVE GEAR

C.Y. Hsu ${ }^{l}$, F.M. Lai $^{2}$

${ }^{T}$ Dayeh University, Department of Nursing, Changhua, Taiwan R.O.C.

${ }^{2}$ Dayeh University, Design and Materials for Medical Equipment and Devices, Changhua, Taiwan R.O.C.

\section{Introduction}

According the elderly with their age increasing, those people experienced their wrist muscle getting weakness, therefore, Da-Yeh University designed to develop the new wrist protectors and expected to be meet with the needs of elderly protective gear.

\section{Objectives}

The analysis of the needs with elderly protective gear will be established the future basis for the innovation of the wrist protectors.

\section{Methods}

In this study, a structured questionnaire is designed and developed as "A New Assistive Living Well Quality Questionnaire" to assess the suitability and importance of the questions through the expert validity. A group of 30 elderly persons who are willing to participate and with experiences used the wrist protectors are Group A, and the other 30 elderly are Group B who never used the wrist protectors, and analyzed the differences between the two groups.

Results

The design and development of new wrist protectors has been granted a patent certificate at the current stage (No: New M 461414) in Taiwan. This study assumes that the elderly's demand for protective gear is primarily based on convenience, including convenience of storage, no difficulty to use and the weight of protective gear whether with comfort; all of those factors will influence their experience the convenience and quality of life. This research is still processing as well.

\section{Conclusions}

The results of this research will provide the future medical product development and care team with an understanding of the needs of the elderly, and the provision of more suitable and comfortable protective gear to enhance the quality of life. 
eP498

\section{CANCER CACHEXIA STAGES: DO THEY PREDICT QUALITY OF LIFE?}

P. Kasvis $^{1,2}$, R. Kilgour $^{2,3}$, A. Vigano ${ }^{1,2}$

${ }^{T}$ McGill University Health Centre, Supportive and Palliative Care Division, Montreal, Canada

${ }^{2}$ McGill University Health Centre, McGill Nutrition and Performance Laboratory, Montreal, Canada

${ }^{3}$ Concordia University, Department of Exercise Science, Montreal, Canada

\section{Introduction}

Cancer cachexia is associated with decreased quality of life (QoL) in advanced cancer patients. It is unclear whether cancer cachexia stages (CCS) will predict differences in commonly used QoL measures.

Objectives

To determine the association between CCS and two commonly used measures of QoL: 1) "Wellbeing" on the Edmonton Symptom Assessment Scale and 2) the Distress Thermometer.

Methods

Five hundred and twelve cancer patients who took part in a Cancer Rehabilitation Program at the McGill University Health Centre (Montreal, Quebec) were classified into the following CCS stages: non-cachexia $(\mathrm{NC}=172)$, pre-cachexia $(\mathrm{PC}=115)$, cachexia $(\mathrm{C}=154)$, and refractory cachexia $(\mathrm{RC}=71)$. Mixed model ANOVA was used to determine differences in wellbeing and distress among the CCS stages. A score $\geq 4$ indicates a level of distress or lack of wellbeing requiring intervention.

\begin{tabular}{|c|c|c|}
\hline Criteria & & $\begin{array}{l}\text { Tools/Parameters and } \\
\text { their cut-off values }\end{array}$ \\
\hline $\begin{array}{l}\text { Abnormal } \\
\text { biochemistry }\end{array}$ & A & $\begin{aligned} \mathrm{CRP} & >10 \mathrm{mg} / \mathrm{L} \text { or } \\
\mathrm{WBC} & >11,000 / \mathrm{L} \text { or } \\
\mathrm{Alb} & <32 \mathrm{~g} / \mathrm{L} \text { or } \\
\mathrm{Hgb} & <120 \mathrm{~g} / \mathrm{L} \text { in men } \\
& <110 \mathrm{~g} / \mathrm{L} \text { in women }\end{aligned}$ \\
\hline $\begin{array}{l}\text { Decreased } \\
\text { food intake }\end{array}$ & B & aPG-SGA box 2 score $\geq 1$ \\
\hline $\begin{array}{l}\text { Moderate } \\
\text { weight loss }\end{array}$ & $\mathrm{C}$ & $\begin{array}{l}0-5 \% \text { in the past } 6 \\
\text { months }\end{array}$ \\
\hline $\begin{array}{l}\text { Significant } \\
\text { weight loss }\end{array}$ & D & $\begin{array}{l}>5 \% \text { in the past } 6 \\
\text { months }\end{array}$ \\
\hline Performance & G & $\begin{array}{l}\text { aPG-SGA box } 4 \text { score }>2 \\
(=\text { ECOG }>2)\end{array}$ \\
\hline
\end{tabular}

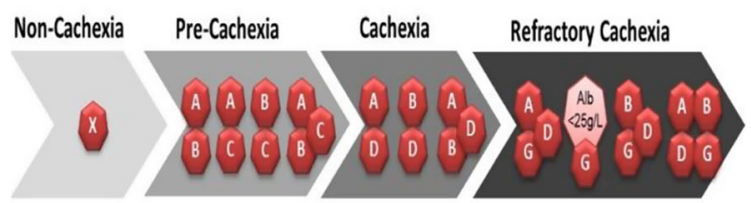

Alb: serum albumin: : aPG-SGA: abridged Patient-Generated Subjective Global Assessment; BMI: body mass index; CRP: C-reactive protein, ESAS: Edmonton Symptom Assessment System; Hgb. hemoglobin; WBC: white blood cells count.

\section{Results}

Average wellbeing in all CCS was $>4$. Only RC patients showed a significantly greater lack of wellbeing when compared to the other 3 groups $(\mathrm{p}<0.0001)$. Similarly, $\mathrm{RC}$ patients demonstrated significant increases in distress than the $\mathrm{NC}$ and $\mathrm{C}$ groups $(\mathrm{p}<0.0024)$.

\section{Relationship between distress and cachexia stage}

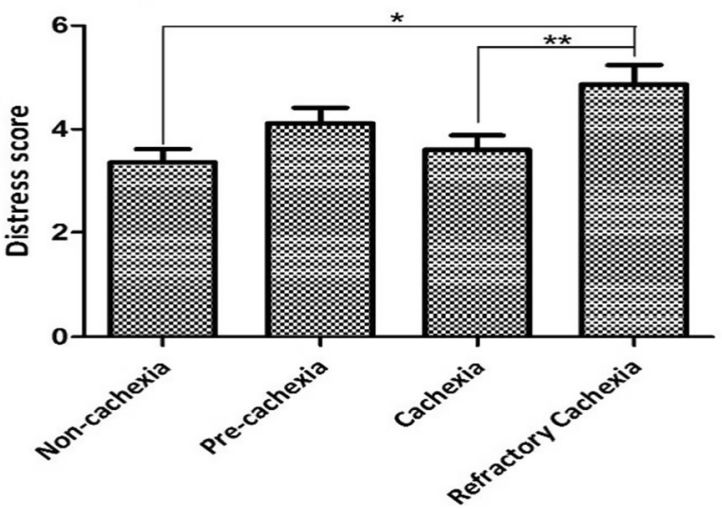

Relationship between wellbeing and cachexia stage

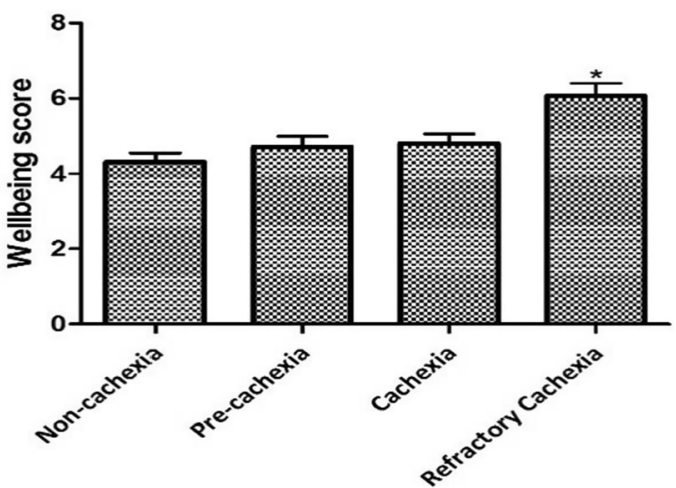

Conclusions

Patients in the most severe stage of cachexia (RC) exhibit significantly greater distress and less wellbeing than those in other cachexia stages. Thus, comfort care measures should be of primary importance in RC patients.

eP499

THE USE OF A STANDARDIZED CHINESE HERBS (CH) FORMULA IN PATIENTS WITH ADVANCED LUNG CANCER: A FEASIBILITY STUDY

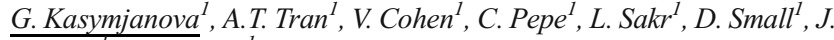
Agulnik', R.T. Jagoe ${ }^{I}$

${ }^{I}$ Peter Brojde Lung Cancer Centre- Segal Cancer Centre- Jewish General Hospital, Pulmonary Oncology, Montreal, Canada

\section{Introduction}

Increasing numbers of cancer patients are using $\mathrm{CH}$. A growing number of laboratory studies and clinical trials, suggest that $\mathrm{CH}$ may have a number of different beneficial effects for cancer patients. However, the heterogeneity of $\mathrm{CH}$ interventions used and the outcomes reported, still make it challenging to draw firm conclusions about the clinical usefulness of $\mathrm{CH}$.

Objectives

The primary objective: to establish if taking a specific standardized $\mathrm{CH}$ formula at the recommended dosage for six weeks, was 
acceptable for patients. The secondary objective: to determine if there were any indications of toxicity. Finally, quality of life were measured in each patient.

\section{Methods}

A single-arm, prospective study of a 6-week intervention with a $\mathrm{CH}$ formula in patients with stage 4 NSCLC.

Results

We found that advanced lung cancer patients in this study were interested in using the $\mathrm{CH}$ formula. Completion $(93 \%)$ and adherence $(98 \%)$ levels were very high and most patients perceived the $\mathrm{CH}$ treatment as easy to take and were willing to take the $\mathrm{CH}$ used in the study again if it was available. About half the patients reported adverse events (AEs), all of which were mild (Grade 1, 2 ) and only a small minority (8\%) were potentially related to $\mathrm{CH}$. No biochemical or hematological evidence of toxicity was observed. Overall, there was improvement in quality of life, specifically tiredness, sleepiness, well-being and the composite trialoutcome index.

\section{Conclusions}

This study provides preliminary evidence that short-term use of a carefully selected and prepared $\mathrm{CH}$ formula in patients with metastatic lung cancer is acceptable and safe.

eP500

EVALUATION OF LIFESTYLE CHANGES IN PATIENTS DIAGNOSED WITH LUNG CANCER

M. Kiagia ${ }^{I}$, E. Koutsikouri ${ }^{I}$, A. Marioli ${ }^{I}$, E. Dalakou ${ }^{I}$, G. Tourkantonis ${ }^{I}$, I. Karambelas ${ }^{1}$, K. Syrigos

${ }^{1}$ Sotiria General Hospital, Oncology Unit GPP, Athens, Greece

\section{Introduction}

Lung cancer remains the main cause for morbidity and mortality among all cancer types worldwide. Both the disease and its treatment modalities cause symptoms which undermine patients' quality of life affecting their physical, social and psychological wellbeing.

Objectives

Evaluation of the impact on QoL in patients with lung cancer and the effect of the disease symptoms on their lifestyle, as well as the investigation of the factors which affect their QoL.

Methods

The sample of the study consisted of 100 lung cancer patients attending a Greek oncology clinic. QLQ-C30 \& QoL-LC13 questionnaires were used along with another one to record the demographic characteristics. Data analysis was conducted with the use of the statistical software SPSS and the statistical significance was set at $<0.05$.

\section{Results}

The patients' QoL was found satisfactory.

Cognitive Function was at $65.93 \%$, Social Function at $67.50 \%$, Physical Function at $65.93 \%$ with the General Health at $60.83 \%$. Financial Difficulties were at $41.0 \%$. The most common symptoms were fatigue, constipation, insomnia, dyspnea and alopecia. Pain was found at $14.63 \%$ with those under medication for its management scoring less in the General Health and Physical Function variable $(p=0.032 \& 0.001$ respectively). Diarrhea was significantly higher among patients without children $(p=0.038)$ and insomnia among the working patients $(\mathrm{p}=0.043)$

\section{Conclusions}

Factors that affects the quality of life of patients with lung cancer have been found to be marital status, the existence of children, work and medication for pain management. Gender, age and educational level were not found to be factors influencing the quality of life.
eP501

COMPETENCE AS A MEDIATING FACTOR BETWEEN AUTONOMY SUPPORT AND COLORECTAL CANCER SURVIVORS' PHYSICAL ACTIVITY AND QUALITY OF LIFE K. Kim ${ }^{1}$, H.J. Jung ${ }^{1}$, S. Chu ${ }^{2}$

${ }^{T}$ Yonsei University, College of Nursing, Seodaemun-gu, Republic of Korea

${ }^{2}$ Yonsei University, College of Nursing, Seoul, Republic of Korea

\section{Introduction}

Physical activity is considered an important factor predicting the survival rate and improved health-related quality of life of cancer patients after treatment. However many cancer survivors are inactive. Selfdetermination theory has been used to initiate behavioral change and to explain persistence.

\section{Objectives}

This study aimed to investigate the mediating role of intrinsic needs (autonomy, competence, and relatedness) in the relationship between autonomy support and physical activity/quality of life.

\section{Methods}

A survey was conducted in a hospital in Seoul, Korea. Participants were 242 colorectal cancer survivors (CRC-S) who responded to a set of questionnaires that include the following: a socio-demographic questionnaire, the Health Care Climate Questionnaire, the Autonomous self-regulation scale, the Perceived Competence Scale, the Multidimensional Scale of Perceived Social Support, the Global Physical Activity Questionnaire and the EORTC QLQ-C30 (global).

\section{Results}

The results showed that autonomy $(\beta=0.110, \mathrm{P}<0.01)$, competence $(\beta=$ $0.062, \mathrm{P}<0.01)$ and relatedness $(\beta=0.278, \mathrm{P}<0.01)$ were strong factors influencing quality of life, but only competence $(\mathrm{b}=0.262, \mathrm{P}<0.01)$ influenced the physical activity of the participants. This study demonstrated that competence directly or indirectly affects the quality of life of CRC-S through physical activity.

\section{Conclusions}

Base on these findings, in order to maximize the quality of life of CRC-S, health care providers' autonomy support is necessary to promote patients' autonomy, competence, and relatedness. In addition, to increase physical activity, autonomy support that promotes competence is necessary.

\section{eP502}

QUALITY OF LIFE AND ORAL PAIN IN HEAD AND NECK CA N C E R PAT I E N T S R C E IVING RADIO/ CHEMORADIOTHERAPY

M. Kouri ${ }^{1}$, O. Nicolatou - Galitis ${ }^{1}$, A. Vadalouca ${ }^{2}$, V. Kouloulias ${ }^{3}$, G.

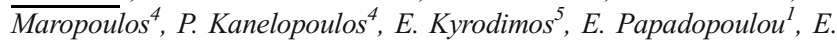

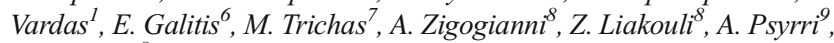
I. Kouvaris ${ }^{8}$

${ }^{I}$ National and Kapodistiran University of Athens, Clinic of Hospital Dentistry-School of Dentistry, Athens, Greece

2"Athens Medical Center", Pain Clinic, Athens, Greece

${ }^{3}$ National and Kapodistiran University of Athens, 2nd Radiology Department- Radiotherapy Unit-Medical School, Athens, Greece

4 "Laiko" General Hospital of Athens, Department of Biochemistry, Athens, Greece

${ }^{5}$ National and Kapodistiran University of Athens, ENT Clinic "Hippokrateio" Hospital-Medical School, Athens, Greece

${ }^{6}$ National and Kapodistiran University of Athens, Clinic of Orofacial Pain-School of Dentistry, Athens, Greece

7 "Iaso" Hospital, Radiotherapy Department, Athens, Greece

${ }^{8}$ National and Kapodistiran University of Athens, 1st Radiology Department- Radiotherapy Unit- Medical School- "Aretaieion" University Hospital, Athens, Greece 
${ }^{9}$ National and Kapodistiran University of Athens, Oncology Department"Attikon" University Hospital, Athens, Greece

\section{Introduction}

Oral pain (OP) is a significant complication, with adverse effect on Quality of Life (QoL), in Head and Neck cancer (HNC) patients receiving radio/chemoradiotherapy.

Objectives

To assess OP related changes on $\mathrm{QoL}$, in $\mathrm{HNC}$ patients receiving radio/ chemoradiotherapy.

Methods

Thirty patients were included in the study (mean age 64.3); 14(46.67\%) had oral/oropharyngeal cancer. QoL was assessed with the EORTC QoL Questionnaire Core-30 (EORTC QLQ-C30)V3.0 and the Head and Neck module (QLQ-H\&N35) before $(\mathrm{t} 0)$ and at 5-7 weeks ( $\mathrm{t} 1)$ of radiotherapy. Patients evaluated OP using a Verbal Analogue Scale $(0-10$, considered as 0:no symptoms, 1-4:mild, 5-7:moderate and 8-10:severe) at t1.

Results

Seven patients experienced severe OP, ten moderate, nine mild and four had no OP. Patients with moderate OP had significantly lower Functional Scales scores $(\mathrm{p}<0.02)$ in $\mathrm{t} 1$ compared to t0 assessment, while the reduction of Global QoL was not significant $(\mathrm{p}=0.160)$; Symptom Scales and H\&N35 scores were significantly increased in $\mathrm{t} 1$ compared to t0 assessment, except for Nausea/Vomiting, Insomnia, Financial problems, Diarrhoea, Speech and Teeth problems, Opening mouth, Coughing and Weight gain (statistically insignificant). In patients with severe OP, Global QoL, Physical, Role and Social Functions were significantly decreased in t1 compared to t0 assessment $(\mathrm{p}<0.006)$ while the reduction of Emotional and Cognitive Functions was insignificant; Symptom Scales and H\&N35 scores were significantly increased while Nausea/Vomiting, Insomnia, Constipation, Financial problems, Diarrhoea, Teeth problems, Coughing and Weight gain were not significant.

Conclusions

OP was related to most of the QoL score changes. Appropriate interventions can be directed towards managing OP and improving QoL of the patients.

\section{eP503}

\section{ASSESSING QUALITY OF LIFE FOR CHILDREN WITH BRAIN TUMORS USING THE PATIENT-REPORTED OUTCOMES MEASUREMENT INFORMATION SYSTEM (PROMIS) \\ J.S. Lai ${ }^{1}$, J. Beaumont ${ }^{2}$, S. Goldman ${ }^{3}$ \\ ${ }^{T}$ Northwestern University, Medical Social Sciences, Chicago, USA \\ ${ }^{2}$ Terasaki Research Institute, Terasaki Research Institute, Los Angeles, USA \\ ${ }^{3}$ Ann \& Robert H. Lurie Children's Hospital of Chicago, Hematology- Oncology \& Stem Cell Transplantation, Chicago, USA}

\section{Introduction}

Health-related quality of life (QOL) of childhood brain tumor (BT) patients and survivors has not been well-studied, partially due to the wide range of functional impact and treatment effects varying upon the tumor characteristics. PROMIS offers an opportunity to better understand QOL of BT by comparing to the US pediatric general population.

Objectives

This study evaluated QOL reported by BT using PROMIS - Anxiety, Depression, Fatigue, Peer Relationship, Mobility, and Upper Extremity Function.

Methods

Data from 227 BT children aged 8-17 (mean age $=12.8 \mathrm{yr}$; $54.2 \%$ boys; $79.1 \%$ white) were analyzed. Averaged years since diagnosis $=4.3$ with $18 \%<=1$ year. Patients completed PROMIS measures during clinical visits. Parents rated children's QOL using a single item (poor/fair/good/ very good/excellent).

\section{Results}

Overall, BT were less anxious (mean Tscore $=43$ ), depressed (mean Tscore $=45$ ) and fatigued (mean Tscore $=44$ ); had similar peer relationships (mean Tscore $=49.5$ ) and upper extremity function (mean Tscore $=48.4$ ); and slightly worse mobility (mean Tscore $=47.7$ ) compared to the US general population (mean Tscore $=50$ ). BT receiving chemotherapy (versus not) reported worse anxiety, depression, and upper extremity function; BT receiving radiation reported worse mobility \& peer relationships; years since last treatment was associated with anxiety, fatigue, upper extremity function and peer relationships. Parent-reported child's QOL was significantly associated with all QOL measures.

\section{Conclusions}

Overall, BT didn't demonstrate worse QOL when compared to their peers; yet wide ranges of scores were noted. Treatment types and time since last treatment was associated BT's QOL. Understanding influential factors to BT with inferior QOL is warranted to develop targeted interventions improving BT's QOL.

\section{eP504}

CORRELATIONS OF SYMPTOM DISTRESS,PHYSICAL FUNCTION,AND QUALITY OF LIFE IN THE PATIENTS WITH LUNG CANCER UNDERGOING INITIAL CHEMOTHERAPY

P.C. $\mathrm{Lai}^{1}$

${ }^{T}$ NO.95 Wenchang RD.- Shilin Dist.- Taipei 111- Taiwan. ROC., Nursing Department.Shin Kong Wu HO-SU Memorial Hospital. Taipei- Taiwan. ROC., Taipei, Taiwan R.O.C.

\section{Introduction}

Past studies indicate that some factors that may affect the quality of life(QOL) in the patients with lung cancer undergoing initial chemotherapy, confirm that the impact of lung cancer patients QOL factors gradually be taken seriously. Diagnosis to treatment of symptom distress and physical functions is an important factor affecting the quality of life.

Objectives

The aim of this study was to explore the symptom distress, physical function, and QOL in patients with lung cancer under Initial chemotherapy from pretreatment through eighth week of chemotherapy.

Methods

The symptoms distress, physical function, and QOL of 75 patients with lung cancer was assessed from pretreatment through the course of chemotherapy. The symptom distress, physical function, and QOL were evaluated using multivariate linear regression.

Results

A total of 75 patients completed a questionnaire to fill out. No significant difference in the QOL in different basic properties. Symptom distress, physical function and QOL presented significant positive correlation between the severity of symptoms, and QOL have a significant negative correlation between physical activity and QOL function presents significant negative correlation. The QOL has explanatory power factor, including symptom severity and functional physical activity, a total of $53.6 \%$ of all explain the variance.

\section{Conclusions}

Conclusions found symptom distress, physical activity function, QOL in the patients with lung cancer undergoing initial chemotherapy has significant explanatory power. Recommended that health care workers provide individual care of patients according to the severity of symptoms, reduce the severity of symptoms, improve physical activity and, in order to improve QOL. 
eP505

IMPROVEMENT OF QUALITY OF LIFE FOR ADVANCED C A N C E R P A T I E N T W I T H I N J ECT I O N O F COMPLEMENTARY PG2 - A DOUBLE BLIND, MULTICENTER, RANDOMIZED PHASE IV STUDY

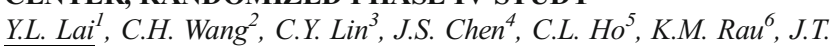
Tsai', C.S. Chang ${ }^{8}$, S.P. Yeh ${ }^{9}$

${ }^{1}$ Mackay Memorial Hospital, Hospice Palliative Care Center, Taipei City, Taiwan R.O.C.

${ }^{2}$ Chang Gung Memorial Hospital, Department of Internal Medicine, Keelung City, Taiwan R.O.C.

${ }^{3}$ Chi Mei Hospital, Division of Hematology and Oncology, Tainan,

Taiwan R.O.C.

${ }^{4}$ Chang Gung Memorial Hospital, Department of Internal Medicine, Taoyuan City, Taiwan R.O.C.

${ }^{5}$ Tri-Service General Hospital, Division of Hematology and Oncology, Taipei, Taiwan R.O.C.

${ }^{6}$ Chang Gung Memorial Hospital, Department of Internal Medicine, Kaohsiung City, Taiwan R.O.C.

${ }^{7}$ Taipei Medical University - Shung Ho Hospital, Division of Radiation Oncology, Taipei, Taiwan R.O.C.

${ }^{8}$ Changhua Christian Hospital, Division of Hematology and Oncology, Changhua County, Taiwan R.O.C.

${ }^{9}$ China Medical University Hospital, Division of Hematology and Oncology, Taichung, Taiwan R.O.C.

\section{Introduction}

Quality of Life (QoL) was proposed as a survival predictor in advanced cancer patients. PG2 injection was extracted and purified from Astragalus membranaceus. In Traditional Chinese medicine, Astragalus membranaceus is commonly used to treat the deficiency of qi (vital energy). We evaluated the efficacy of PG2 injection in improving QoL in advanced cancer patient with or without palliative radiotherapeutic or chemotherapeutic treatments in this study.

Objectives

The aim of this study was to evaluate the effect of PG2 injection on QoL in advanced progressed cancer patients under standard palliative care.

Methods

Patients with advanced cancer receiving standard palliative care were enrolled and randomized at a 1:1 ratio into two arms of PG2 injection treatment: $500 \mathrm{mg}$ dose $(500 \mathrm{mg}$ in $500 \mathrm{ml}$ saline, 3 times per week for 4 weeks) or $250 \mathrm{mg}$ dose $(250 \mathrm{mg}$ in $500 \mathrm{ml}$ saline, 3 times per week for 4 weeks) for two cycles. Symptoms and Quality of Life Assessments were analyzed by 11 questions (SS11) from European Organization for Research and Treatment of Cancer QoL Questionnaire (EORTC QLQ$\mathrm{C} 30$ ). Patients were divided into untreated, palliative chemotherapy treated, and palliative radiotherapy treated groups for analysis.

Results

Three hundred and ten patients were enrolled in this study. High and low doses of PG2 injection significantly improve patients' appetite loss $(\mathrm{p}<0.0001, \mathrm{p}=0.0004)$, fatigue $(\mathrm{p}<0.0001, \mathrm{p}<0.0001)$, insomnia $(\mathrm{p}=0.0002, \mathrm{p}<0.00001)$, and global QoL $(\mathrm{p}<0.0001, \mathrm{p}<0.0001)$ after two cycles of treatments. Patients with or without therapeutic treatments were both analyzed.

\section{Conclusions}

PG2 injection can significantly improve several indicators for QoL in advanced cancer patients without safety concern.

\section{eP506}

COGNITIVE COMPLAINTS IN CANCER SURVIVORS AND EXPECTATIONS FOR MANAGEMENT: RESULTS FROM A NATIONAL SURVEY

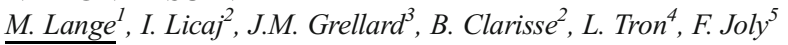

${ }^{1}$ Centre François Baclesse, INSERM ANTICIPE, Caen, France

${ }^{2}$ Centre François Baclesse, Clinical Research Unit, Caen, France

${ }^{3}$ Centre François Baclesse, Clinical Resaerch Unit, Caen, France

${ }^{4}$ INSERM, Anticipe, Caen, France

${ }^{5}$ Centre François Baclesse, Clinical Research Unit- INSERM Anticipe, Caen, France

\section{Introduction}

Cognitive complaints are common among cancer survivors. Few studies have assessed cognitive complaints using large sample and information on patients' expectations for management of cognitive disorders are scarce.

\section{Objectives}

We aimed to assess cognitive complaints using a large national survey. Methods

This online surveywas proposed by Seintinelles association to cancer survivors, except brain primary or metastasis cancer and without progressive psychiatric or neurological disease. Cognitive complaints were assessed by a list of questions. Patients were asked about their expectations for management of cognitive difficulties and knowledge about chemotherapy-associated cognitive problems.

\section{Results}

Among 1610 eligible participants (median age 52 [21-84]), $>85 \%$ were breast cancer survivors. Median post-cancer treatment time (excluding hormone therapy) was 2.83 years [0.8-33]. 273 (17\%) had a weekly consumption of psychotropic medications. 1379 participants $(86 \%)$ had cognitive complaints whose $1214(88 \%)$ related to cancer treatments. Cognitive difficulties started during chemotherapy (419 (35\%)), after chemotherapy (360 (30\%)) and during hormone therapy (186 (15\%)) and lasted a median of 2 years [0.08-32]. $618(40 \%)$ had pre-existing knowledge about chemotherapy-associated cognitive problems and this was significantly associated with their cognitive complaints $(\mathrm{p}=0.01)$. Among survivors with cognitive complaints related to cancer treatments, 909(75\%) would like to receive cognitive rehabilitation, 439(48\%) psychological support and 294(32\%) physical activity.

\section{Conclusions}

Using a national survey, we observed a large proportion of participants with cognitive complaints. For the majority of them, complaints were due to cancer treatments. Most participants would like to receive management and especially cognitive one. Cognitive program should be included in the portfolio of oncology supportive care.

\section{eP507}

COGNITIVE IMPAIRMENT IN BREAST CANCER PATIENTS BEFORE SURGERY?

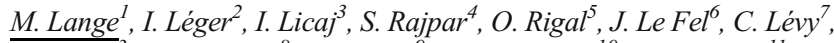

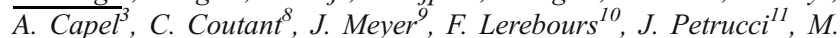
Campone $^{12}$, L. Vanlemmens ${ }^{13}$, A.L. Martin ${ }^{14}$, C. Mesleard ${ }^{14}$, J. Adnet ${ }^{15}$, F. André ${ }^{4}$, F. Joly ${ }^{3}$, S. Dauchy ${ }^{16}$

${ }^{1}$ Centre François Baclesse, INSERM ANTICIPE, Caen, France

${ }^{2}$ CHU Bicetre - Gustave Roussy, UF SSR des Maladies Neurovirales Supportive Care, Bicetre, France

${ }^{3}$ Centre François Baclesse, Clinical Research Unit, Caen, France

${ }^{4}$ Gustave Roussy, Medical Oncology, Villejuif, France

${ }^{5}$ Centre Henri Becquerel, Medical Oncology, Rouen, France

${ }^{6}$ Centre Henri Becquerel, Supportive Care, Rouen, France

${ }^{7}$ Centre François Baclesse, Medical Oncology, Caen, France

${ }^{8}$ Centre Georges François Leclerc, Medical Oncology, Dijon, France

${ }^{9}$ Centre Georges François Leclerc, Supportive Care, Dijon, France

${ }^{10}$ Institut Curie, Medical Oncology, Saint Cloud, France

${ }^{11}$ Institut Curie, Clinical Research Unit, Saint Cloud, France

${ }^{12}$ Institut de Cancérologie de l'Ouest, Medical Oncology, Nantes, France

${ }^{13}$ Centre Oscar Lambret, Medical Oncology, Lille, France

${ }^{14}$ UNICANCER, Research \& Development, Paris, France 
${ }^{15}$ Centre Georges François Leclerc, Clinical Research Unit, Dijon, France

${ }^{16}$ Gustave Roussy, Supportive Care, Villejuif, France

\section{Introduction}

Cognitive dysfunction is a common complaint among breast cancer patients after chemotherapy. Nevertheless, $20-30 \%$ of women have cognitive impairment after surgery and before any adjuvant treatment. These disorders may be explained by the impact of breast cancer surgery anesthesia. However, very few studies focused on cognitive functions before any treatment.

\section{Objectives}

In the French national cohort CANTO, a subgroup of patients had cognitive assessment before any treatment (surgery or neo-adjuvant treatment)

\section{Methods}

Breast cancer patients without metastasis were eligible. Episodic, working memory, executive functions, processing speed, attention, cognitive complaints, anxiety, depression and fatigue were assessed with neuropsychological tests and self-reported questionnaires, before any treatment. Cognitive impairment was defined according to ICCTF recommendations.

\section{Results}

Results concern 264 women ( $54 \pm 10.8$ years), with breast cancer diagnosis (stage I-II, 69\%) within 37 days in average (SD: 70.3). Only six patients $(2.3 \%)$ had a cognitive impairment in at least one cognitive domain (episodic memory and working memory). Twenty percent $(n=52)$ had significant cognitive complaints (PCI, FACT-Cog). Significant anxiety or depression symptoms were observed respectively in $46 \%$ and $13 \%$ of patients. They were related to cognitive complaints $(\mathrm{p}<0.001)$.

\section{Conclusions}

This study is the first which assessed cognition before any breast cancer treatment, using a large national cohort. Twenty percent had significant cognitive complaints, however very few patients had objective cognitive impairment, contrasting with the rate of cognitive impairment observed before adjuvant treatment and after surgery in breast cancer patients. These results suggest that surgical procedure itself, possibly by general anesthesia, could be involved in cognitive impairment in breast cancer patients.

\section{eP508}

RESILIENCE AND ITS PREDICTORS IN CAREGIVERS OF PATIENTS WITH ADVANCED ORAL CAVITY CANCER DURING SURVIVAL

L.Y. Lee ${ }^{\text {I }}$ S.C. Chen ${ }^{2}$

${ }^{T}$ China Medical University Bengain Hospital, Department of Nursing, Yulin, Taiwan R.O.C.

${ }^{2}$ Chang Hung University of Science and Technology, Department of Nursing, Taoyuan, Taiwan R.O.C.

\section{Introduction}

Anti-cancer treatments lead to varying levels of physical and psychological disturbance during treatment and prolong to survival period.

\section{Objectives}

The purpose of the study was to identify resilience and related factors in primary caregivers of patients with advanced oral cavity cancer during the survival period.

Methods

This study was a cross-sectional correlational design. Study was conducted to recruited subjects from RT outpatients department and cancer center in a medical center in northern Taiwan. Eligible subjects were assessed for the Instrument Activities of Daily Living Scale (IADL), and background information form. Primary caregivers were assessed the related variables using the Resilience Scale (RS), Caregiver Reaction Assessment (CRA),
Distress Thermometer (DT), Medical Outcomes Study Social Support Survey (MOS SS), and a background information form.

\section{Results}

Patients had moderate level of resilience, with the lowest resilience in "I usually take things in stride", "I feel that I can handle many things at a time", and "I am determined". Caregivers had higher level of self-esteem burden $(b=0.397, p<0.001)$, lower level of lack of family support burden $(b=0.378, p<0.001)$, and greater social support $(b=0.408, p<0.001)$ were associated with positive resilience.

\section{Conclusions}

Caregiving burden and social support were the important factors associated with resilience of caregivers of oral cavity cancer patients. Survivorship care plan are recommended to help cope with caregiving burden.

\section{eP509}

\section{FACTORS INFLUENCING ON TIME OF DELAY IN INITIAL REFERRAL, DIAGNOSIS AND TREATMENT IN CHILDREN WITH CANCER}

S. Mehranfar ${ }^{1}$

${ }^{T}$ Urmia University of Medical Sciences, Medical Genetic, Urmia, Iran

\section{Introduction}

Cancer has been one of the most critical health problems especially in developing countries. Since the diagnosis and treatment of this disease in its early stages can reduce the related mortality rates and complications and reduce the costs tangibly, it was attempted to investigate the factors affecting the patients' delay in referring to physicians in the beginning and subsequently propose some solutions to tackle this problem and reduce its resultant injuries.

\section{Objectives}

What are the type of training can we have an effective role in detecting early stages? Whar are factors are important for these goals?

\section{Methods}

80 parents of the children with different types of cancers were studied via both questionnaire and person-to-person interview after announcing their informed consent.

\section{Results}

The highest delay in patients' initial referral was due to the following factors: lack of attention or ignoring the first symptoms, delay in referring to the physician, low economic status and even lack of family support for the patient. In addition, visiting several doctors after the initial diagnosis, uncertainty about the first proposed method, and high cost of treatment can be mentioned as the main causes of delays in the start of treatment.

\section{Conclusions}

Education plays an important role in identifying the signs of cancer. In addition, proper relationship and cooperation between the health system and physicians as well as provision of adequate information to patients could lead to the long-term cooperation of these patients in continuing their treatment. It can be effective on strategic planning in the health system.

\section{eP510}

SATISFACTION AND PERCEPTION OF IMPLANTABLE PORTS (IP) IN CANCER PATIENTS AFTER THE END OF THERAPY: A SURVEY BASED ASSESSMENT

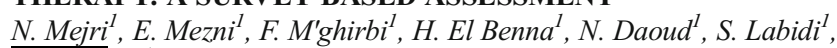
H. Boussen ${ }^{l}$

${ }^{1}$ Abderrahmen Mami Hospital Ariana- University Of Tunis El Manar, Medical oncology, Ariana, Tunisia

Introduction:

After successful termination of adjuvant therapy, IP removal can be discussed. 
Objectives

We aimed to investigate IP-specific satisfaction in patients treated in the adjuvant setting for a cancer and for whom IP is still in place during follow-up.

\section{Methods}

Eighty three patients having an IP, treated in the adjuvant setting, who finished therapy since at least 6-weeks were asked to answer a satisfaction questionnaire including items evaluating IP-specific aspects: Satisfaction, quality of life, body image and anxiety. Patients had to respond according to a scale of 5 levels: "not at all (1)" to "very mutch(5)".

\section{Results}

Patient's satisfaction was low $(1+2)$ with IP impact: on daily activity in $20 \%$, arm mobility in $17 \%$, driving $13 \%$, clothing $14 \%$, working $26 \%$ and during sleep in $34 \%$. Patient's satisfaction was high $(4+5)$ with cosmetic results in $61 \%$, absence of a foreign body feeling in $41 \%$. Sixty three percent of patients did not feel a need to remove it and $64 \%$ did not fear complications. IP did not interfere with social coping in $49 \%$, but $31 \%$ felt ashamed when someone sees the IP. The IP mediated a security feeling in $59 \%$, represented a reminder of the illness in $75 \%$. Eighty five percent of patients did not regret the IP implantation and $88 \%$ would recommend it to another patient. Among the $10 \%$ of patients who had IP removal $54 \%$ do not desire another implantation.

Conclusions

IP removal should be a shared patient-physician decision, based on several criteria: patient's satisfaction with his quality of life and IP specific anxiety.

\section{eP511}

\section{THE EFFECT OF INTRAVENOUS HIGH-DOSE VITAMIN C ON QUALITY OF LIFE OF CANCER PATIENTS N. Mikirova}

${ }^{T}$ Riordan Clinic, Bio-Communication Research Institute, Wichita, USA

\section{Introduction}

Ascorbic acid has been studied for decades for its potential role in preventing chronic diseases. It has also generated interest in using it against cancer. High-dose intravenous vitamin C (IVC) has been administered by physicians for many decades as a complementary and alternative therapy for cancer patients.

\section{Objectives}

Recently, evidence has been accumulating which indicates that IVC may improve the quality of life (QOL) of cancer patients, both in the presence and absence of adjuvant chemo-and radiotherapy. In our review we describe the recent studies examining the effects of IVC injections (7.5-100 grams) on cancer- and chemotherapy-related QOL.

\section{Methods}

We searched MEDLINE for human studies examining the safety and effectiveness of IVC use in cancer patients.

\section{Results}

The summary of the preclinical studies and clinical trials that were conducted on 25 patients with ovarian cancer (stage III and IV), 60 patients with advanced cancer, 39 patients with terminal cancer, 125 breast cancer patients (stage IIa-IIIb) and 14 pancreatic cancer patients demonstrated that IVC can reduce the severity of cancer symptoms and the side-effects of chemotherapy and radiotherapy, in particular of nausea, loss of appetite, fatigue, depression, sleep disorders, dizziness. Improvements in physical, cognitive, emotional, social functioning, and an improvement in overall health were also observed. IVC had effect on prolongation of the survival time of cancer patients.

Conclusions

IVC is safe and can improve the QOL of cancer patients. The additional studies to evaluate the usefulness of IVC for patients with cancer are warranted.
eP512

\section{DIFFERENCE IN HEALTH-RELATED QUALITY OF LIFE AND FATIGUE BETWEEN CANCER SURVIVORS AND HEALTHY SUBJECTS}

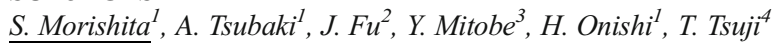

${ }^{T}$ Niigata University of Health and Welfare, Institute for Human

Movement and Medical Sciences, Niigata City, Japan

${ }^{2}$ University of Texas MD Anderson Cancer Center, Department of Palliative- Rehabilitation \& Integrative Medicine, Houston, USA

${ }^{3}$ Niigata University of Health and Welfare, Department of Nursing, Niigata, Japan

${ }^{4}$ Keio University School of Medicine, Department of Rehabilitation Medicine, Tokyo, Japan

\section{Introduction}

We compared quality of life (QOL) and fatigue in long-term cancer survivors and healthy subjects. We also investigated the difference in the relationship between QOL and fatigue in cancer survivors and healthy subjects.

\section{ObjectivesMethods}

Thirty-six cancer survivors ( 9 men) and 29 healthy subjects ( 6 men) were enrolled in this study. Participants were evaluated for health-related QOL and fatigue. Health-related QOL was assessed using the Medical Outcome Study 36-item Short-Form Health Survey. Fatigue was measured using the Brief Fatigue Inventory (BFI).

\section{Results}

Cancer survivors exhibited lower QOL scores in the physical functioning, physical role function, bodily pain, and general health domains $(\mathrm{p}<0.05)$. The usual fatigue subscale score was significantly higher in cancer survivors than in healthy subjects $(\mathrm{p}<0.05)$. Subscales of QOL significantly correlated with subscales of fatigue in both groups $(p<0.05)$. Additionally, these correlations were more prevalent in cancer survivors than in healthy subjects.

\section{Conclusions}

Our results show that QOL correlates with fatigue. Furthermore, QOL correlates with fatigue in cancer survivors more than it does in healthy subjects. Thus, relieving fatigue might contribute to higher QOL in cancer survivors. We believe that the findings of this study will be relevant in the context of planning rehabilitation for cancer survivors.

\section{eP513}

EXCELLENCE IN CANCER CARE: SUPPORTING THE PERSON WITH HEAD AND NECK LYMPHOEDEMA

J. Nixon $^{1,2}$, A. Pigott ${ }^{1}$, B. Cartmill ${ }^{3}$, J. Turner ${ }^{4}$

${ }^{T}$ Princess Alexandra Hospital, Occupational Therapy Department, Brisbane, Australia

${ }^{2}$ The University of Queensland, School of health and Rehabilitation Sciences, Brisbane, Australia

${ }^{3}$ Princess Alexandra Hospital, Speech Patholgy Department, Brisbane, Australia

${ }^{4}$ The University of Queensland, Faculty of Medicine, Brisbane, Australia

\section{Introduction}

Up to $75 \%$ of patients who experience head and neck cancer, have some form of head and neck lymphoedema (HNL).

\section{Objectives}

This study aims to examine the supports required to manage distress and quality of life in people with HNL, to help support direction of future care. Methods

This study used a mixed method explanatory design, with a prospective repeated measures component to examined distress, quality of life (QoL) and body image during a 22 week treatment program for people who had HNL. Subsequently, a qualitative approach was used to explore the 
patient experience of distress associated with HNL, and to help provide direction for future care.

\section{Results}

Distress associated with HNL was statistically different at the 3 time points during the treatment program (baseline, week six and week 22), with improvements in distress from baselineto week 6 $(p=0.015)$, and from baseline to week $22(p=0.007)$. No significant differences in overall QoL, body image or fear of cancer progression were reported. Thematic analysis of the patient reported experience revealed six major themes associated with the distress related to HNL, with a focus on the supportive care provided by treating clinicians.

\section{Conclusions}

This study found that distress related to HNL reduced in association with the delivery of a lymphoedema treatment program and support provided by a lymphoedema therapist. These findings have been used to inform clinical practice, with distress now routinely been monitored in the lymphoedema service.

\section{eP514}

DEVELOPING AN EFFECTIVE MODEL FOR PREVENTING CERVICAL CANCER AMONG THE POOR POPULATION OF CHIAPAS, MEXICO

H. Ochoal, R. Garcia ${ }^{2}$, E. Garcia ${ }^{3}$

${ }^{T}$ El Colegio De La Frontera Sur, HEALTH HEALTH, San Cristobal De Las Casas, Mexico

${ }^{2}$ El Colegio De La Frontera Sur, Health, San Cristobal De Las Casas, Mexico

${ }^{3}$ Universidad de Ciencias y Artes de Chiapas, Nutrición, Tuxtla Gutiérrez, Mexico

\section{Introduction}

Cervical cancer is a major public health problem in developing countries. In Mexico, Chiapas reported in 2013, a mortality rate of 17.2 per 100,000 women $\geq 25$ yrs

Involvement of community stakeholders in the prevention program was promoted as an effective strategy, especially among indigenous and poorest areas.

Objectives

To implement and evaluate a comprehensive cervical cancer prevention model to reduce the mortality from this disease among rural indigenous women of Chiapas, South Mexico, in order to introduce potential improvements in the state / national cervical cancer prevention program

Methods

We conducted a demonstration project with an accompanying health systems research to assess the feasibility and effectiveness of a cervical cancer prevention model. We did: health interview survey (1050 households), 19 FGD, in depth interviews, accompanying operations research and participatory workshops

\section{Results}

758 women had a Pap smear test $(77 \%)$ of those women interviewed (984), of which 613 undergone VIA (62.3\%) and a sub-sample of $248(25 \%)$ was selected for HPV DNA testing. $80 \%$ of participants in the follow-up activities for confirmation and treatment of the abnormalities detected of the women with a positive screening test result.

\section{Conclusions}

If the screening services are centered to the women needs and concerns, treating them with warmth and respect, and a highquality service is provided, a high participation of the women will be obtained, improving in consequence the impact on the cervical cancer mortality levels.
eP515

IMPACT OF SUPPORT GROUP INTERVENTION ON QUALITY OF LIFE OF PATIENTS WITH ADVANCED BREAST CANCER

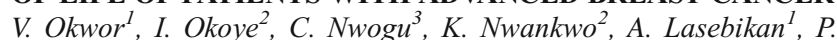
Okere $^{2}$, S. Ezeofor ${ }^{2}, N$. Iloanusi ${ }^{2}$, T. Onyeka ${ }^{4}, S$. Arua $^{5}, M$. Imhansoloeva ${ }^{6}, C_{\text {O Okwor }}^{7}, C$. Bright $^{8}, F$. Kanu $^{8}$

${ }^{1}$ University of Nigeria Teaching Hospital Enugu, Radiation Medicine, Enugu, Nigeria

${ }^{2}$ University of Nigeria Enugu Campus, Radiation Medicine, Enugu, Nigeria

${ }^{3}$ Lakeshore cancer center, Surgery, Lagos, Nigeria

${ }^{4}$ University of Nigeria Enugu Campus, Anaesthesiology, Enugu, Nigeria

${ }^{5}$ Natioanl Hospital Abuja, Radiotherapy, Abuja, Nigeria

${ }^{6}$ Lumenplus Consulting., Epidemiology unit, Ibadan, Nigeria

${ }^{7}$ University College Hospital, Chemical Pathology, Ibadan, Nigeria

${ }^{8}$ University of Nigeria Teaching Hospital Enugu, Radiotherapy, Enugu, Nigeria

\section{Introduction}

Health-related quality of life is an important end point in breast cancer care. Assessing quality of life in breast cancer patients could contribute to improved treatment outcome and could even serve as monitor along with medical parameters. The need for psychosocial support is well established among breast cancer patients with levels of psychological distress and depression, particularly after diagnosis and treatment. Cancer support helps patients to cope with cancer by adjusting their attitudes, knowledge, and expectation about the disease.

\section{Objectives}

1. To determine the impact of support group intervention on quality of life of patients with advanced breast cancer

Methods

A prospective cohort study involving breast cancer patients using a system of breast cancer support group that organises a two month period meeting for all breast patients in University of Nigeria Teaching Hospital. Educational information on breast cancer, nutritional needs, exercise and the need for regular follow up in breast cancer care were given to them as well as the means of overcoming the psychosocial burden in cancer. QOL assessment on selected patients with advanced breast cancer using WHO BREF was administered.

\section{Results}

At the end of the intervention,14 women $(12.4 \%)$ were censored due to death, while post assessment was carried out for only 99 women. There was a significant positive correlation between availability of social support to respondents (FS score) and the four quality of life domains $(\mathrm{p}<0.05)$. Also, quality of life significantly increased across all domains at post intervention $(\mathrm{p}<0.05)$.

\section{Conclusions}

Patients receiving support group intervention all had improved quality of life.

\section{eP516}

\section{ASSESSMENT OF QUALITY OF LIFE OF CANCER PATIENTS UNDERGOING CHEMOTHERAPY IN A SELECTED DISTRICT HOSPITAL IN RWANDA}

U. Pauline ${ }^{l}$, M. Mukeshimana

${ }^{T}$ Univeristy of Rwanda, Nursing, Kigali, Rwanda

\section{Introduction}

Quality of life is the most important patient outcome among patients living with cancer.Quality of life helps in measuring nursing and medical interventions 


\section{Objectives}

To assess the quality of life of cancer patients undergoing chemotherapy in a Selected District Hospital based on the level of functional scale, symptoms scale, and global health status/ QoL scale.

\section{Methods}

The study conducted at Butaro Hospital. A quantitative descriptive crosssectional survey was used. Data was collected from 5 April -5 May 2017. (EORTC) QoL Questionnaire-Core 30 vision 3 was used. The instrument was translated into Kinyarwanda language and was pretested. The target population was 90 . The sample size consisted of 73 patients . The data were analyzed using SPSS vision 20.

\section{Results}

The QoL core 30 showed that the global health status and quality of life was low with transformed score mean and standard deviation of 28.76(SD=20.03)which indicate poor quality of life while the functional scale the score was $51.55(\mathrm{SD}=19.12)$ indicated good quality of life. the symptoms scale, the score was high $61.13(\mathrm{SD}=17.10)$ which indicate the poor quality of life, this signifies high symptomatic.Pain 78.76( $\mathrm{SD}=35.050)$, fatigue $78.08(\mathrm{SD}=23.56)$ and financial difficulties 98.63( $\mathrm{SD}=11,70)$ were the major common symptoms

\section{Conclusions}

The quality of life and global health status scale scored low and symptoms scale scored high, indicated poor quality of life among our participants. Health care professionals must take into consideration the value of quality of life as well as cancer therapy and their side effects experienced by cancer patients undergoing chemotherapy in order to improve the quality of life of cancer patients.

\section{eP517}

\section{HEALTH-RELATED QUALITY OF LIFE OF BREAST CANCER PATIENTS, FIVE YEAR FOLLOW-UP}

E. Roine ${ }^{l}$, T. Saarto ${ }^{l}$, C. Blomqvist ${ }^{l}$, H. Sintonen ${ }^{2}$

${ }^{T}$ Helsinki University Hospital, Comprehensive Cancer Center, Helsinki, Finland

${ }^{2}$ University of Helsinki, Department of Public Health, Helsinki, Finland

\section{Introduction}

Adjuvant treatments of breast cancer (BC) lead to improved survival but are associated with adverse effects that disrupt patient well-being. Measuring health-related quality of life (HRQoL), gives information on the patients' own views of their subjective well-being and what dimensions are most profoundly affected.

\section{Objectives}

To measure HRQoL in BC patients and to compare their HRQoL to that of the general population.

\section{Methods}

$573 \mathrm{BC}$ patients aged 35-68 years (mean 53 years) recruited to a one-year exercise intervention study were after adjuvant treatments randomly allocated into an intervention or control group. The patients filled in the $15 \mathrm{D}$ generic HRQoL questionnaire at baseline and 6, 12, 18, 24 and 30 months and 3 and 5 years after the beginning of the intervention. The HRQoL of the patients was compared to that of age-standardized general female population.

Results

Compared to the general population, $\mathrm{BC}$ patients had statistically significantly worse mean HRQoL score at baseline. At five-year follow-up, the BC patients' HRQoL score had improved but was still statistically significantly worse than that of the general population. The most significant differences were on the dimensions of sleep, excretion, mental function, distress, vitality and sexual activity. The impairment in HRQoL was pronounced in younger ( $<53$ years) patients at both time points. Older patients ( $>52$ years) had better mean HRQoL score than younger patients at baseline and at five-year follow-up.

\section{Conclusions}

Five years after the start of treatment, BC patients still had worse mean HRQoL score than the general population and the difference was greatest in younger patients.
eP518

\section{CHARACTERIZING THE EFFECTS OF RADIATION DERMATITIS ON QUALITY OF LIFE}

A. Rzepecki ${ }^{1}$, M. Birnbaum ${ }^{2}$, N. Ohri ${ }^{3}$, J. Fox ${ }^{3}$, R. Kabarriti ${ }^{3}$, W. Bodner ${ }^{3}$, M. Keyur ${ }^{3}$, J. Daily ${ }^{4}$, S. Kalnicki ${ }^{3}$, B. McLellan ${ }^{2}$

${ }^{1}$ University of Michigan Medical School, Dermatology, Ann Arbor, USA ${ }^{2}$ Albert Einstein College of Medicine, Dermatology, New York City, USA ${ }^{3}$ Montefiore Medical Center, Radiation Oncology, New York City, USA

${ }^{4}$ Albert Einstein College of Medicine, Microbiology, New York City, USA

\section{Introduction}

Radiation dermatitis (RD) is a common adverse event of radiation therapy (RT). Patients may experience skin changes and pain. Little is known about how this radiation-induced skin toxicity can affect patients' quality of life (QoL).

\section{Objectives}

This study aimed to 1) assess the impact of RD on QoL in patients undergoing RT, focusing on 3 domains: emotions, symptoms, and functioning, and 2) evaluate the change in QoL during RT and its association with the severity of RD.

\section{Methods}

We are presenting preliminary results from a prospective trial during which cancer patients undergoing RT completed the Skindex-16, a QoL survey. Responses were recorded during initiation and upon completion of RT. Pre- and post-treatment Skindex-16 responses were compared using the sign test. Spearman rank correlation was used to assess associations between changes in Skindex scores and dermatitis grade.

Results

47 patients were included in the study. $58 \%$ developed grade 1 dermatitis, $32 \%$ developed grade 2 dermatitis, and $10 \%$ developed grade 3 dermatitis. Patients reported significantly higher Skindex scores for all 16 questions after completing RT $(\mathrm{p}<0.002)$. Dermatitis grade was significantly $(p<0.05)$ associated with change in Skindex score for 7 out of 16 elements. These associations were most common for questions assessing the functional domain ( $4 / 5$ elements) and less common for questions addressing symptomatic (1/4) and emotional (2/7) domains.

\begin{tabular}{|l|l|}
\hline $\begin{array}{c}\text { Factors significantly associated } \\
\text { with higher grade dermatitis }\end{array}$ & $\begin{array}{l}\text { Factors NOT significantly associated with } \\
\text { higher grade dermatitis }\end{array}$ \\
\hline Hurting (Q3) & Itching (Q1) \\
\hline $\begin{array}{l}\text { Persistence/recurrence of the skin } \\
\text { condition (Q5) }\end{array}$ & Burning or stinging (Q2) \\
\hline Worry about skin condition (Q6) & Skin irritation (Q4) \\
\hline Interactions with others (Q12) & Appearance of skin condition (Q7) \\
\hline Desire to be with people (Q13) & Frustration about skin condition (Q8) \\
\hline Show affection (Q14) & Embarrassment about skin condition (Q9) \\
\hline Work or do what you enjoy (Q16) & Annoyance about skin condition (Q10) \\
\hline & Feeling depressed about skin condition (Q11) \\
\hline & Daily activities (Q15) \\
\hline
\end{tabular}

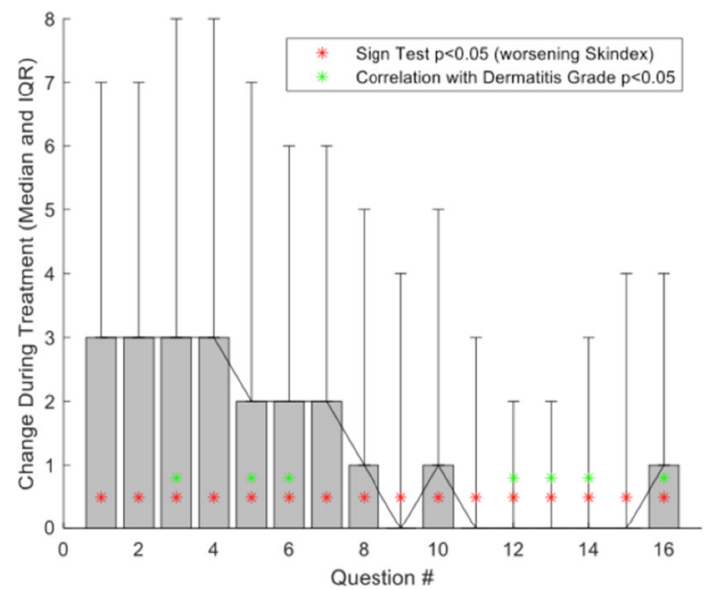




\section{Conclusions}

Severity of RD is significantly associated with impact on QoL, especially in the functional domain. These domains may not be captured in standard grading systems. Supportive interventions should focus on the functional domain in patients undergoing RT.

\section{eP519}

THE EFFECT OF INDIVIDUALIZED PATIENT EDUCATION AND EMOTIONAL SUPPORT ON THE QUALITY OF LIFE AND SELF-EFFICACY OF BREAST CANCER PATIENTS - A PILOT STUDY

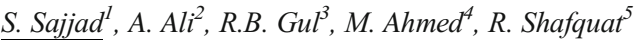

${ }^{T}$ The Aga Khan University, School of Nursing and Midwifery, Karachi, Pakistan

${ }^{2}$ King Abdul Aziz University, King Abdul Aziz University, Jeddah, Saudi Arabia

${ }^{3}$ Shifa Tameer-e-Millat University, Faculty of Nursing and Midwifery, Islamabad, Pakistan

${ }^{4}$ Oncology \& Radiotherapy Institute NORI, Nuclear Medicine Department, Islamabad, Pakistan

${ }^{5}$ The Aga Khan University, Community Health Sciences, Karachi, Pakistan

\section{Introduction}

Patient education has potential to improve the quality of life $(\mathrm{QoL})$ and self-efficacy (SE) of cancer patients.

Objectives

The study aims were (a) to determine the effect of individualized patient education on breast cancer patients' QoL and SE and (b) to identify the association between QoL and SE.

Methods

A quasi-experimental design was employed. The intervention and control groups consisted of 25 breast cancer women, each. The intervention comprised: written and verbal patient education, nurse's availability during chemotherapy session, and follow-up calls between patient and nurse to discuss patients' concerns. The control group received hospital's routine care. Functional Assessment of Cancer Therapy Breast (FACT-B) and self-efficacy tools were used to obtain QoL and SE data, at baseline (T1) and at the sixth week (T2) after completion of a chemotherapy. Inferential statistics were applied to identify the between-and-withingroup differences for QoL and SE and to determine the association between QoL and SE.

\section{Results}

A statistically significant improvement was observed in the overall QoL score of the intervention group, from $\mathrm{T} 1$ to $\mathrm{T} 2$ as compared to the control group. The intervention's effect size was moderate $(0.655)$. The SE scores remained unchanged over time in the intervention group and significantly decreased from T1 to T2 in the control group. Moreover, a positive association was found between $\mathrm{QoL}$ and $\mathrm{SE}$ at $\mathrm{T} 1$ and $\mathrm{T} 2$, in the intervention group.

\section{Conclusions}

Individualized patient education and support was found to be effective in improving the patients' QoL and SE. Larger, multi-center studies are recommended to establish the findings of this study.

eP520

AUDIT ON INCIDENTS AND KNOWLEDGE OF NURSES REGARDING CHEMOTHERAPY EXTRAVASATIONS AT DAY CARE ONCOLOGY, AT A TERTIARY CARE HOSPITAL IN KARACHI, PAKISTAN.

Z. Samoon ${ }^{l}$

${ }^{T}$ Aga Khan University Hospital, Oncology, karachi, Pakistan

\section{Introduction}

Extravasations of cytotoxic agents after its intravenous administration results in serious damage to the tissues leading to local injury and tissue necrosis. Worldwide incidence of extravasation ranges from $0.1 \%-6 \%$. Patient education, early identification and management can reduce morbidity associated with chemotherapy extravasation.

Objectives

The aim of this study is to determine the hospital based incidence of chemotherapy extravasation. We also aim to assess the knowledge regarding identification, management and patient education of nurses regarding extravasation.

Methods

This audit was conducted at day care oncology of Aga Khan University Hospital to assess the knowledge of nursing staff regarding identification and management of chemotherapy extravasations and teaching to the patients about signs and symptoms of extravasations. In addition we also recorded number of incidents of extravasation reported from 12th February till 15th June 2016.

\section{Results}

Total numbers of twelve nurses were audited; out of twelve, seven nurses $(58.33 \%)$ did not have adequate knowledge of early identification, management and components of teaching of patients regarding chemotherapy extravasation. Between 12th February till 15th June 2016, 5461 cytotoxic drugs were administered. Seven $(0.128 \%)$ incidents of extravasations were reported during this period. Four $(57 \%)$ were due to vinorelbine and two $(28 \%)$ were due to docetaxel.

\section{Conclusions}

Our hospital based incidence of chemotherapy extravasation is comparable to international data. This may be reduced further by improving knowledge of nurses regarding prevention, prompt identification, management and education of patients.

\section{eP521}

PATIENT REPORTED SYMPTOMS OF CANCER TREATMENT AND PATIENT SATISFACTION IN BNGO PRACTICES - A GERMAN SURVEY INCLUDING THE NCCN DISTRESS THERMOMETER

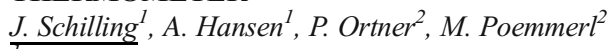

${ }^{T}$ BNGO e.V., German Professional Association of Gynaeco-Oncology in Practices, Neuenhagen, Germany

${ }^{2}$ Pomme-med GmbH, Pomme-med GmbH, Munich, Germany

\section{Introduction}

Gynaeco-oncologists associated in the BNGO conduct routine patient surveys on symptom burden and satisfaction with cancer treatment. The current survey includes the NCCN distress thermometer.

Objectives

To monitor the quality of life of patients with gynaecologic tumors and their satisfaction with their treatment in BNGO practices.

\section{Methods}

Since August 2017, 1116 patients have completed a printed questionnaire on the distress they are experiencing, tumor- and treatment-related symptoms, and their satisfaction with the practice and the physician. Chemotherapy patients also reported the frequency of side effects and rated the most distressing on a scale of 1 (least) to 10 (most).

\section{Results}

On the 11-point scale of the NCCN distress thermometer $(0=$ no distress, $10=$ extreme distress) $66 \%$ circled numbers between 0 and 5 (moderate) $99 \%$ of patients said the practice staff was very good or good and $100 \%$ rated their treating physician as very good or good. $34 \%$ of all patients perceived fatigue as the most distressing symptom, followed by problems sleeping (33\%). $69 \%$ of patients received chemotherapy. $83 \%$ of them reported alopecia, followed by fatigue ( $54 \%) .47 \%$ of chemotherapy patients considered bone pain and $44 \%$ alopecia an extremely distressing or 
the most distressing symptom. After the end of chemotherapy, $36 \%$ of patients still reported fatigue and $34 \%$ still experienced peripheral neuropathy.

\section{Conclusions}

Symptoms preventable by supportive care medications such as vomiting are rare. Peripheral neuropathy and fatigue often persist after the end of chemotherapy. Patients are well satisfied with their cancer treatment in BNGO practices.

\section{eP522}

HEALTH-RELATED QUALITY OF LIFE AMONG 8807 LONGTERM CANCER SURVIVORS WITH AND WITHOUT CARDIOVASCULAR DISEASE AT TIME OF CANCER DIAGNOSIS: RESULTS FROM THE POPULATION-BASED PROFILES REGISTRY

D. Schoormans ${ }^{1}$, F. Mols ${ }^{1}$, O. Husson ${ }^{2}$

${ }^{T}$ Tilburg University, Medical and Clinical Psychology, Tilburg, The Netherlands

${ }^{2}$ The Institute of Cancer Research, Department of Clinical Studies, London, United Kingdom

\section{Introduction}

Cancer and cardiovascular diseases (CVD) are common illnesses in the Western world. Cancer patients with a CVD present at their cancer diagnosis often have limited treatment options, since many cancer treatments are cardiotoxic.

\section{Objectives}

We will examine the relation between presence of CVD at cancer diagnosis and HRQoL and whether this relation is mediated by age, gender and cancer treatment.

\section{Methods}

Co-morbid CVD status at time of cancer diagnosis and cancer treatment information was extracted from the Netherlands Cancer Registry (NCR). HRQoL was examined by the EORTC QLQ-C30 within the PROFILES registry on average 9.4 years after cancer diagnosis.

\section{Results}

In total 8807 cancer survivors diagnosed with one of the following cancers; colorectal, prostate, ovarium, thyroid, endometrium, Hodgkin and non-Hodgkin lymphoma, chronic lymphocytic leukemia and multiple myeloma were included. One in five survivors $(n=1794,20.4 \%)$ had a co-morbid CVD at cancer diagnosis. Preliminary results show that cancer survivors with a co-morbid CVD at the time of cancer diagnosis report lower HRQoL-scores on overall QoL $(B=-5.07)$, physical $(B=-7.94)$, role $(\mathrm{B}=-6.93)$, cognitive $(\mathrm{B}=-2.49)$, emotional $(\mathrm{B}=-3.48)$, and social functioning $(\mathrm{B}=-3.75)$, while adjusting for age, gender, marital status, educational level and time since cancer diagnosis. For overall QoL, physical and role functioning small clinically relevant differences were found. Additional analyses exploring possible mediation effects for age, gender and cancer treatment will be presented at the conference.

\section{Conclusions}

Preliminary results show that cancer survivors who have a co-morbid CVD at the time of cancer diagnosis report significantly lower HRQoL scores.

\section{eP523}

\section{AUCTOX: A NEW METHOD TO EVALUATE THE SAFETY OF} ANTICANCER DRUGS

V. Launay-Vacher $^{1}$, C. Helissey ${ }^{2}$, B. Laguerre ${ }^{3}$, D. Spaeth ${ }^{4}$, T. Lloret ${ }^{5}, J$.

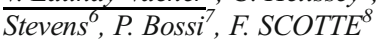

${ }^{1}$ Pitié-Salpêtrière University Hospital, Service ICAR, Paris, France

${ }^{2}$ Military Hospital Begin, Unité de Recherche Clinique, Saint-Mande, France
${ }^{3}$ Centre Eugène Marquis, Department of Medical Oncology, Rennes, France

${ }^{4}$ Centre d'Oncologie de Gentilly, Oncology, Nancy, France

${ }^{5}$ Direct Medica, oncology, Boulogne-Billancourt, France

${ }^{6}$ DirectMedica, Oncology, Boulogne-Billancourt, France

${ }^{7}$ Istituto Nazionale dei Tumori, Head and Neck Cancer Medical Oncology Unit, Milan, Italy

${ }^{8}$ Foch Hospital, Medical Oncology and Supportive Care, SURESNES, France

\section{Introduction}

The evaluation of anticancer drugs safety currently relies on the NCICTCAE classification. Most often in clinical trials, attention is focused on severe adverse events (AE) i.e. grade 3 or more. However, AE of lower grades can have a significant deleterious impact, especially when recurrent or permanent.

\section{Objectives}

We aimed at defining a new method to better describe the impact of treatment on quality of life (QoL).

Methods

Patients with metastatic prostate cancer treated with either docetaxel (DOC) or cabazitaxel (CABA) were recorded for toxicities (NCICTCAE). Their grades were plotted against time under treatment, to calculate the area under the curve of toxicities (AUCtox) that takes into account both the intensity and duration of toxicities, and the number of events. AUCtox was calculated for any toxicity reported at least once. The association with QoL, evaluated by EQ-5D-3L was determined.

\section{Results}

In total, 146 patients were evaluable [DOC 85 (58\%), CABA 61 (42\%)]. Median follow-up was 4.0 months. Patients underwent a median number of 6 cycles, with a median number of 15.0 evaluations (monitoring calls). At least one grade $\geq 3 \mathrm{AE}$ was reported by $45.2 \%$ of patients, most frequently fatigue (20.6\%) and pain (12.3\%). A negative impact on global health status, assessed by the VAS of EQ-5D-3L, was identified for 3 AEs based on grade alone vs. 8 when considering AUCtox.

\section{Conclusions}

Current evaluation of toxicity based on grade alone underestimates the impact of anticancer drugs on QoL. Our method, using AUCtox, can better detect this impact, allowing a more patient-oriented evaluation of drug safety.

\section{eP524}

\section{SUPPORTIVE BENEFITS OF A NUTRITIONAL SUPPLEMENT IN BREAST CANCER PATIENTS RECEIVING RADIATION THERAPY}

A.K. Sengupta ${ }^{1}$

${ }^{T}$ United Hospital Limited, Cancer Care Center, Dhaka, Bangladesh

\section{Introduction}

Several depression related symptoms like fatigue, loss of appetite, weight loss, nausea and emesis arise during and after radiation treatment, negatively affecting the quality of life and even morbidity. Oral Oncoxin Solution (OOS) contains green tea polyphenols such as epigallocatechin 3-gallate, vitamin B6 and vitamin C, three products whose anticancer and radiosensitizing action has been proven in several studies. OOS also contains cinnamic acid, a compound which may inhibit tumor growth, as well as glycyrrhizinic acid, whose action as anti-inflammatory and immunomodulator has been reported.

\section{Objectives}

General well being.

Quality of life.

Methods

Total 63 female breast cancer patients receiving radiotherapy as a part of their cancer treatment were enrolled in this study. 27 patients were 
assigned to receive $25 \mathrm{ml}$ Oncoxin Oral Solution (OOS) twice daily for 3 months. Another 36 patients did not receive OOS or any specific nutrition and they were managed conservatively (control group). Patient's general well-being \& quality of life (QOL) were assessed using Karnofsky's Performance Status (KPS) and Beck's Depression Inventory-II (BDI-II) forms respectively.

Results

In weeks 3-4 of radiotherapy, KPS significantly decreased by almost $50 \%$ (45 \pm 7.2$)$ in patients of control group; while KPS in patients of OOS group remained stable or even increased (87 \pm 8.53$)$. Moreover, according to BDI-II assessment, incidences of post RT-adverse effects were about $92 \%$ patients of control group compared to about $44 \%$ patients of OOS group.

\section{Conclusions}

This study indicates that OOS is effective as supportive care to improve general well being \& quality of life (QOL) in Breast cancer patients receiving radiation treatment.

\section{eP525}

FEASIBILITY STUDY OF AN INTER-PROFESSIONAL SUPPORTIVE “CARE-BOARD” IN LUNG CANCER

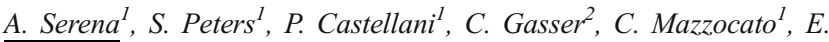
Manuela ${ }^{3}$

${ }^{1}$ Centre Hospitalier Universitaire Vaudois, Département d'oncologie, Lausanne, Switzerland

${ }^{2}$ Ligue suisse contre le cancer, Prévention- prise en charge et suivi, Bern, Switzerland

${ }^{3}$ Institut Universitaire de Formation et de recherche en SoinsUniversité de Lausanne, Département d'oncologie-CHUV, Lausanne, Switzerland

\section{Introduction}

Preliminary findings have shown that integrated inter-professional supportive care approaches improve patient's quality of life and might even impact overall survival. The University Hospital of Lausanne is the first Swiss comprehensive cancer center to develop and implement an inter-professional board called "Care-Board" (CB) during early treatment phase that is organized in complementary with the Tumor-Board. The aim of the $\mathrm{CB}$ is to facilitate a standardized inter-professional consensus process that results in the agreement on supportive care recommendations for lung cancer patients.

\section{Objectives}

To assess the feasibility of the CB model during a pilot phase.

Methods

The pilot phase (October-December 2017) included seven CB meetings. We analyzed participation rate and the related reasons for the absence, the meeting duration, the devoted time on supportive care for each patient and the most frequent discussed patient's supportive care needs.

\section{Results}

CB members were two clinical nurse specialists, two oncologists, a palliative care physician, a psycho-oncologist, a social worker, and a complementary medicine nurse. The average participation rate of the team members was $70 \%$. The most common reasons for absence were "holidays" (30\%), "clinical activities" (35\%), and "congress/continuing education" (11\%). The meeting duration was 50 (SD 8) minutes with an average time of 20 (SD 5) minutes per patient. Discussed patient's supportive care needs concerned mainly psychological/financial issues and integration of palliative care.

Conclusions

Feasibility results of this new inter-professional supportive CB showed promising result. Future directions may include adapting and testing this new approach for other cancer populations.
eP526

VALIDATION OF EDMONTON SYMPTOM ASSESSMENT SYSTEM (ESAS) IN ARABIC LANGUAGE, ESAS-A

O. Shamieh ${ }^{1}$, Z. Amin ${ }^{1}$, A. Al-Hawamdeh ${ }^{I}$, F. Abu Farsakh ${ }^{1}$, M. Abu

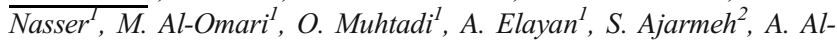
Tabba', K. Ammar ${ }^{3}$, D. Al-Rimawi ${ }^{3}$, D. Hui ${ }^{4}$

${ }^{1}$ King Hussein Cancer Center, Palliative Care, Amman, Jordan

${ }^{2}$ King Hussein Cancer Center, Nursing, Amman, Jordan

${ }^{3}$ King Hussein Cancer Center, Office of Scientific Affairs, Amman, Jordan

${ }^{4}$ MD Anderson Cancer Center, Supportive Care and Rehabilitation, Huston, USA

\section{Introduction}

Standardized symptom assessment is essential in palliative care practice and require the use of a validated tool.

\section{Objectives}

To evaluate the psychometric properties of the Arabic version of the Edmonton Symptom Assessment System, ESAS in advanced cancer patients in a tertiary cancer center in Jordan

\section{Methods}

A rigorous translation of ESAS from English to Arabic utilizing reverse translation and piloting method was used to develop the final Arabic version of ESAS, followed by a prospective crosssectional study to test for psychometric analysis including internal consistency reliability, responsiveness, convergent/discriminant validity and utility.

\section{Results}

240 patients with advanced cancer were included. 120 (50\%) were females, Chronbach's alpha for internal consistency was 0.844.For ESAS responsiveness, test - retest was done for all patients who scored 4 and above in the initial assessment and the tool was found to be reliable to detect improvement in all symptoms, after palliative intervention. Multitrait scaling analysis showed that all item-scale correlation coefficient met the set standards of Convergent Validity $>0.40(100 \%)$. Discriminant Validity is $87.8 \%$ [(90-11)/90]. We found strong correlation between anxiety and depression items in ESAS compared with stress and depression scores in Hospital Anxiety Depression Scale, HADS. However depression in ESAS was strongly correlated with stress scores and moderately correlated with depression scores in HADS.

\section{Conclusions}

The Arabic version of ESAS, ESAS - R was found to be a reliable, valid, responsive, and feasible tool for symptom assessment in Arabic speaking patients with advanced cancer .

\section{eP527}

SUPPORTIVE CARE NEEDS OF PATIENTS FOLLOWING TREATMENT FOR COLORECTAL CANCER: RESULTS FROM THE UK COLORECTAL WELLBEING (CREW) COHORT STUDY

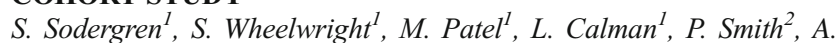

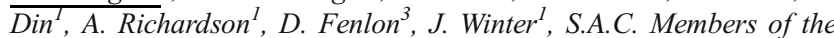
Study Advisory Committee ${ }^{I}$, C. Foster

${ }^{1}$ University of Southampton, Health Sciences, Southampton, United Kingdom

${ }^{2}$ University of Southampton, Social Statistics, Southampton, United Kingdom

${ }^{3}$ University of Swansea, Human and Health Sciences, Swansea, United Kingdom

\section{Introduction}

People living with and beyond colorectal cancer (CRC) might have longterm physical and psychosocial needs for help associated with altered 
bowel function and stoma placement. Given finite resources, their specific needs might not be recognised or supported.

\section{Objectives}

To investigate unmet needs of CRC patients at the end of treatment and whether these improve over time. To identify predictors of need following treatment and implications of unmet need for recovery of health and wellbeing.

Methods

As part of the UK ColoREctal Wellbeing (CREW) cohort study, 872 adults undergoing curative intent surgery for CRC were recruited from 29 UK centres. Questionnaires pre-surgery to 5 years post-surgery included assessments of health status, health-related quality of life (HRQoL), social support and self-efficacy. Supportive care needs (SCNs) were assessed at 15 and 24 months.

Results

At 15 months, 526 patients completed the SCNS and 502 patients at 24 months. Prevalence of unmet needs was relatively low (64\% at 15 months, $62 \%$ at 24 months reported no unmet needs). Psychological and physical unmet needs were the most common and did not improve over time. HRQoL at the beginning of treatment predicted unmet needs at the end of treatment. Unmet SCNs, specifically physical and health system and information needs, predicted poorer HRQoL at the end of treatment.

\section{Conclusions}

Unmet needs in CRC survivors persist over time and impact HRQoL. Evaluation of HRQoL at the start of treatment can help identify vulnerable patients. Assessment and management of unmet needs should be integrated into patient-tailored survivorship care.

eP528

DEVELOPMENT OF A HEALTH-RELATED QUALITY OF LIFE QUESTIONNAIRE SPECIFICALLY FOR PEOPLE WITH ANAL CANCER TREATED WITH CHEMORADIOTHERAPY; THE EORTC QLQ-ANL27

S. Sodergren ${ }^{1}, C$. Johnson ${ }^{2}, A$. Gilbert $^{3}$, K. Tomaszewski ${ }^{4}, W . C h u^{5}, H$.

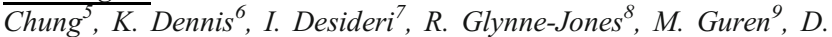
Kardamakis $^{10}$, K. Nugent ${ }^{2}$, H. Schmidt ${ }^{11}$, D. Sebag-Montefiore ${ }^{3}$, A.S. Darlington $^{1}$, V. Vasiliou ${ }^{12}$

${ }^{1}$ University of Southampton, Health Sciences, Southampton, United Kingdom

${ }^{2}$ University of Southampton, Cancer Sciences, Southampton, United Kingdom

${ }^{3}$ University of Leeds, Leeds Radiotherapy Research Group, Leeds, United Kingdom

${ }^{4}$ Ignatianum Academy, Health Outcomes Research Unit, Krakow, Poland ${ }^{5}$ University of Toronto, Department of Radiation Oncology, Toronto, Canada

${ }^{6}$ University of Ottawa, Division of Radiation Oncology, Ottawa, Canada

${ }^{7}$ University of Florence, Experimental and Clinical Biomedical Sciences,

Florence, Italy

${ }^{8}$ Mount Vernon Hospital, Centre for Cancer Treatment, Northwood, United Kingdom

${ }^{9}$ Oslo University Hospital, Oncology and K.G. Jebsen Colorectal Cancer Research Centre, Oslo, Norway

${ }^{10}$ University of Patras, Radiation Oncology, Patras, Greece

${ }^{11}$ Martin Luther University Halle-Wittenberg, Institute for Health and Nursing Science, Halle, Germany

${ }^{12}$ Bank of Cyprus Oncology Centre, Radiation Oncology, Nicosia, Cyprus

\section{Introduction}

Anal cancer is rare and the current standard of care is concurrent chemoradiotherapy (CRT). While treatment outcomes are promising, associated toxicities are common, potentially long lasting, and impact health-related quality of life (HRQoL). There is both a paucity of and need for research into patient reported outcomes.

\section{Objectives}

Our objective was to develop an anal cancer HRQoL measure using EORTC Quality of Life Group Guidelines.

\section{Methods}

We generated a list of HRQoL issues facing anal cancer patients treated with CRT by systematically reviewing the literature and conducting interviews with patients and health care professionals (HCPs). We then formulated questions using the EORTC Item Library. The provisional question list was pilot tested with patients from 11 centres across eight countries.

\section{Results}

From our literature review and interviews with 43 patients, we generated a list of $197 \mathrm{HRQoL}$ issues. A subsequent review of this list by $34 \mathrm{HCPs}$ and 10 patients resulted in the retention of 65 issues, which were used in the draft questionnaire tested by 100 patients. Our analyses led to the modification and removal of questions resulting in a 27-item questionnaire, the EORTC QLQ-ANL27 covering 4 areas of problem (bowel, pain, sexual, stoma).

\section{Conclusions}

The EORTC QLQ-ANL27 measures acute and chronic effects of anal cancer and CRT and is the first anal cancer-specific HRQoL instrument. The EORTC QLQ-ANL27 is suitable for use in clinical trials as well as clinical practice in promoting engagement and ongoing regular contact with HCPs thus facilitating the management of side effects and provision of psychological support where needed.

\section{eP529}

\section{METASTASIS DIRECTED MANAGEMENT OF RECURRENT BREAST CANCER ON SYMPTOM IMPROVEMENT AND} PERFORMANCE STATUS

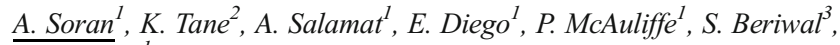
R. Johnson ${ }^{l}$

${ }^{I}$ MWH, Surgical oncology, Pittsburgh, USA

${ }^{2}$ Hyogo Cancer Center, Surgery, Hyogo, Japan

${ }^{3}$ MWH, Radiation Oncology, Pittsburgh, USA

\section{Introduction}

Metastatic site specific treatment in recurrent breast cancer (RBC) is indicated in patients (pts) with symptomatic disease.

\section{Objectives}

To investigate performance status and metastases specific symptom improvement in $\mathrm{RBC}$ pts who received intervention to distant site metastasis (DSM), and the complication rates of site specific interventions in this group of pts.

\section{Methods}

A prospectively-maintained cancer registry at a high-volume tertiary academic center was retrospectively reviewed. From 2006 to 2016, we identified pts who initially underwent standard of care treatment for their newly diagnosed with stage I-III primary BC and were subsequently diagnosed with distant recurrence during follow-up surveillance. All pts in this cohort received systemic therapy and additional interventions to DSM (included surgery (S), radiation therapy (RT), and radiofrequency ablation). Outcomes included post intervention morbidity, change of metastases specific symptoms (MSS) and performance status (PS), length of hospital stay (LOS) due to intervention, and progression free survival (PFS).

\section{Results}

Treatment and complication data were reported after 525 interventions. MSS and PS data were available in 266 and 106 pts respectively. Symptom improvement was seen in $68 \%$ (180/266) of cases. PS was improved in 19\% (20/106) of cases, and not changed in 68\% (72/106) of cases after intervention. Forty eight percent (135/279) of pts were 
admitted in the hospital after interventions and average LOS was $5 \pm 8.4$ days (1-87). PFS for intervention was $4 \pm 0.3$ months (95\%CI; 3-5).

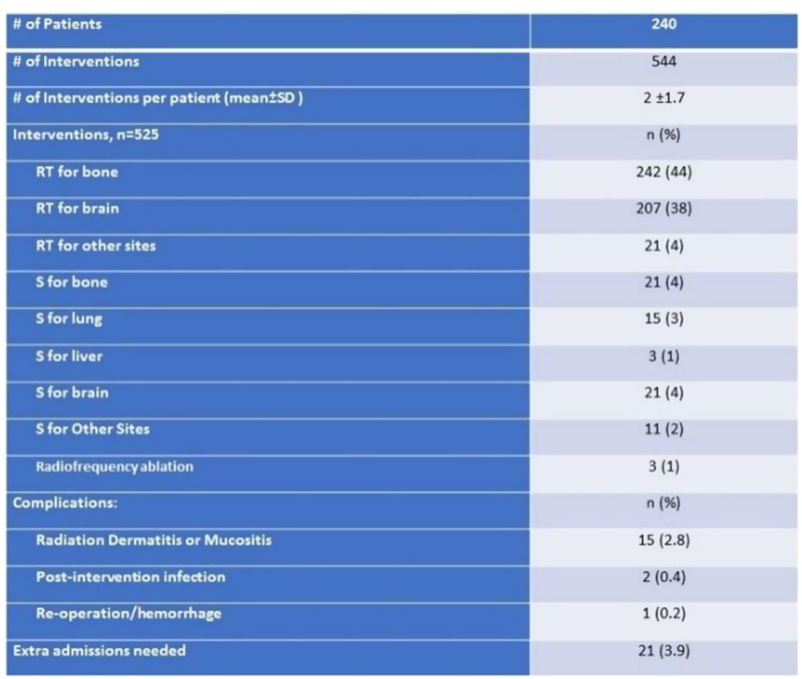

\section{Conclusions}

MSS was improved in 68\% and PS was improved in 19\% with DSM specific interventions. Intervention to DSM did not increase the rate of overall complications.

\section{eP530}

STUDY ON THE INFLUENCE OF RADIOTHERAPY AND ASSOCIATED CHEMOTHERAPY ON COGNITIVE PERFORMANCE IN PATIENTS WITH GLIOBLASTOMA

D.F.V. Pirscoveanu ${ }^{1}$, I. Marinescu ${ }^{2}$, R.A. Schenker ${ }^{3}$, M. Schenker ${ }^{4}$, D. G. Malaescu $^{5}$, P.O. Stovicek ${ }^{6}$

${ }^{1}$ University of Medicine and Pharmacy of Craiova, Department of Neurology, Craiova, Romania

${ }^{2}$ University of Medicine and Pharmacy of Craiova, Department of Psychiatry, Craiova, Romania

${ }^{3}$ University of Medicine and Pharmacy of Craiova, Doctoral School, Craiova, Romania

${ }^{4}$ University of Medicine and Pharmacy of Craiova, Department of Oncology, Craiova, Romania

5 “Titu Maiorescu" University-Bucharest, Department of Morphopathology, Craiova, Romania

6"Titu Maiorescu" University- Bucharest, Department of Pharmacology, Craiova, Romania

\section{Introduction}

The purpose of this study was to evaluate cognitive performance in patients diagnosed with glioblastoma during radiotherapy and associated chemotherapy and to monitor whether these therapies have significant influence on the cognitive status of these patients.

\section{Objectives}

In the study we included 17 patients diagnosed with glioblastoma and their cognitive performance was examined compared to a control group of 20 healthy subjects with a similar educational level.

\section{Methods}

Both patients and the control group were assessed cognitively using the Montreal Cognitive Assessment Scale (MoCA) and Addenbrooke's Cognitive Examination Revised (ACE-R) scales.

\section{Results}

Patient cognitive evaluation was performed in dinamics: baseline was done after surgery, but prior to radiotherapy initiation. The second evaluation was done after 6 weeks of chemotherapy-associated radiotherapy (temozolomide), and the third evaluation was performed after three temozolamide treatments. For the statistical study, the Student test was used ( $p<0.05$ statistically significant). After 6 weeks of radiotherapy simultaneous with temozolamide, we did not notice any statistically significant cognitive decline compared to the baseline and the control group. The final evaluation after the three temozolomide administrations also revealed a slight cognitive impairment but without statistical significance in the patient population. The most affected cognitive domains were attention and language, while the least damaged cognitive domain was orientation.

\section{Conclusions}

Our conclusion was that radiotherapy and cytostatic treatment had no statistically significant influence from the cognitive impairment point of view on patients with glioblastoma included in the study.

\section{eP531}

CORRELATION OF HEALTH-RELATED QUALITY OF LIFE WITH QUALITY OF SYMPTOM MANAGEMENT: BASED ON PERSONALIZED SYMPTOM GOALS IN OUTPATIENT PALLIATIVE CARE SETTING

K. Tagami ${ }^{1,2}$, T. Miura $^{2,3}$, T. Kawaguchi ${ }^{4}$, M. Yoshihisa ${ }^{2}$, Y. Watanabe-

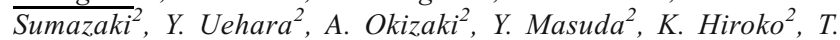

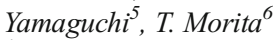

${ }^{I}$ Tohoku University School of Medicine, Palliative Medicine, Sendai, Japan

${ }^{2}$ National Cancer Center Hospital East, Palliative Medicine, Kashiwa, Japan

${ }^{3}$ National Cancer Center Hospital East, Biomarker DiscoveryExploratory Oncology Research \& Clinical Trial Center, Kashiwa, Japan ${ }^{4}$ Tokyo University of Pharmacy and Life Sciences, Practical Pharmacy, Hachioji, Japan

${ }^{5}$ Tohoku University Graduate School of Medicine, Biostatistics, Sendai, Japan

${ }^{6}$ Seirei Mikatahara General Hospital, Palliative and Supportive CarePalliative Care Team, Hamamatsu, Japan

\section{Introduction}

Personalized symptom goals (PSG) have been proposed as a good indicator of symptom management. The correlations of health-related quality of life (HRQOL) with quality of symptom management based on PSG, have not yet presented.

\section{Objectives}

The aim of this study is to clarify the achievement of PSG and the correlations of HRQOL with achievement of PSG in outpatient palliative care setting.

\section{Methods}

We performed a cross-sectional study among cancer patients who visited the palliative care outpatient clinic of our institute between April 2016 and September 2016. We investigated their Edmonton Symptom Assessment System-revised (ESAS-r) scores and PSG scores on ESAS$r$ physical symptoms. HRQOL scores were measured by Functional Assessment of Cancer Therapy - General. The relationship between HRQOL scores and the number of symptoms not achieving PSG (ESAS-r score > PPG score) was examined using the JonckheereTerpstra test. To compare HRQOL scores between patients achieving (ESAS-r score < PPG score) and not achieving PSG, the Student t test was performed.

Results

A total of 171 patients were enrolled (37.4\% female, median 68 years old). The prevalence of PSG achievement was as follows: pain (36.4\%), 
tiredness $(35.7 \%)$, drowsiness $(45 \%)$, nausea $(80 \%)$, lack of appetite $(58.6 \%)$, and dyspnea (61. 4\%). The number of symptoms not achieving PSG was associated with low HRQOL scores ( $<<0.01)$.Poor management to achieve PSG of pain, tiredness, lack of appetite and dyspnea had association with low HRQOL scores $(\mathrm{p}<0.05)$.

Conclusions

We first showed the correlations of HRQOL with quality of symptom management, based on PSG.

\section{eP532}

IMPACT OF MEDIUM-HIGH ACCUMULATED IRRADIATION DOSE IN NECK AND GRADE 3 DERMATITIS IN POORQUALITY SLEEP FOR PATIENTS WITH HEAD AND NECK SQUAMOUS CELL CARCINOMA

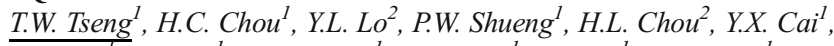

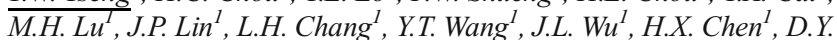

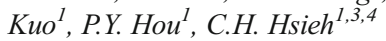

${ }^{1}$ Far Eastern Memorial Hospital, Division of Radiation OncologyDepartment of Radiology, New Taipei City, Taiwan R.O.C.

${ }^{2}$ Far Eastern Memorial Hospital, Department of Nursing, New Taipei City, Taiwan R.O.C.

${ }^{3}$ Faculty of Medicine- School of Medicine, National Yang-Ming University-, Taipei, Taiwan R.O.C.

${ }^{4}$ Institute of Traditional Medicine- School of Medicine, National YangMing University, Taipei, Taiwan R.O.C.

\section{Introduction}

The correlations of irradiation dose and dermatitis in sleep quality for head and neck squamous cell carcinoma (HNSCC) patients are not clear and worth to investigate.

Objectives

To estimate the relative factors to the sleep quality for patients with HNSCC receiving radiotherapy with or without chemotherapy.

\section{Methods}

From August 2015 to December 2016, a total of 20 patients with HNSCC who undergo radiotherapy with or without chemotherapy at Far Eastern Memorial Hospital are enrolled prospectively. Participants completed questionnaires of Pittsburgh Sleep Quality Index (PSQI). Data analysis used descriptive, correlational statistics and generalized estimation equation (GEE). Results

Of the 20 participants, the incidences of grade 3 for neck area received less than $46 \mathrm{~Gy}(\leq 46 \mathrm{~Gy})$ and medium-high irradiation dose that was larger than 46 Gy ( $>46$ Gy) were $1 \%$ and $13 \%$, respectively, $p<0.001$. Compared to the neck area $\leq 46 \mathrm{~Gy}$, the accumulated dose $>46 \mathrm{~Gy}$ increased the risk of severe dermatitis significantly $(p<0.001)$. Meanwhile, poor sleep quality was associated with neck area received dose $>46$ Gy $(p=0.039)$ and grade 3 dermatitis $(p=0.013)$, respectively. Conclusions

People with HNSCC underwent radiotherapy with or without chemotherapy that experienced poor-quality sleep are likely to show a positive correlation with neck accumulated irradiation dose $>46$ Gy and grade 3 dermatitis. The risk of severity of dermatitis in neck is associated with accumulated radiation dose. More HNSCC patient to be enrolled to confirm the impact of medium-high irradiation dose and grade 3 dermatitis in poor sleep quality is warranted.

\section{eP533}

\section{THE EFFECT OF HEALTH LITERACY ON SELF-EFFICACY AND QUALITY OF LIFE: CANCER PATIENTS}

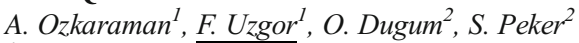

${ }^{1}$ Eskisehir Osmangazi University, Faculty of Health SciencesDepartment of Nursing, Eskisehir, Turkey
${ }^{2}$ Private Umit Hospital, Medical Oncology Clinic, Eskisehir, Turkey

\section{Introduction}

Cancer and symptoms related to the treatment of cancer decrease quality of life by affecting an individual's life physically, emotionally, socially and economically. Having health knowledge and a sense of self-efficacy can improve cancer management and improve quality of life.

\section{Objectives}

This research was conducted to evaluate the effect of health literacy in cancer patients on self-efficacy and quality of life.

\section{Methods}

This cross-sectional, descriptive study was conducted with cancer patients who were monitored and treated at the policlinics and clinics of Medical Oncology in a hospital in Eskisehir. The data were collected by means of "Individual Presentation Form", "Health Literacy Questionnaire-European Union (HLS-EU)", "Self-Efficacy for Managing Chronic Disease Scale (SMCD)" and "European Organization for Research and Treatment of Cancer Quality of Life Scale (EORTC QLQ-C30). Research data was analyzed using the IBM Statistical 21.0.

Results

While mean scores of 111 patients who participated in the study was $36,09 \pm 6.42$ for HLS-EU and $6.07 \pm 1.80$ for SMCD; EORTC global health state point was determined as $67.56 \pm 17.38$. Whilst a significant positive correlation was found between the HLS-EU and global health state scores (r: 0.199,p: 0.036) of the patients, a significant negative correlation was revealed between HLS-EU and symptom subscale scores $(\mathrm{r}:-0.334, \mathrm{p}<0,01)$. A positive correlation was found between SMCD score and respectively EORTC global health state $(\mathrm{r}: 0.411, \mathrm{p}<0,01)$ and functional subscale scores $(\mathrm{r}: 0.531, \mathrm{p}<0,01)$, while a negative correlation was found between SMCD and symptom subscale scores $(\mathrm{r}:-0.334, \mathrm{p}<0,01)$.

Conclusions

As health literacy level of cancer patients and chronic disease management self-efficacy score increases, general health status increases and disease symptoms decrease.

\section{eP534}

H E A L T H - R E L A T E D Q UA L I T Y OF L IFE : A N OBSERVATIONAL PROSPECTIVE STUDY IN POTENTIAL PHASE-I ONCOLOGY TRIAL PARTICIPANT

D. van der Biessen ${ }^{I}$,W. Oldenmenger ${ }^{1}$, P. van der Helm ${ }^{2}$, D. Klein ${ }^{1}$, E. Oomen-de Hoop ${ }^{I}$, R. Mathijssen ${ }^{1}$, M. Lolkema ${ }^{1}$, M. de Jonge ${ }^{l}$

${ }^{1}$ ErasmusMC Cancer Institute, Medical Oncology, Rotterdam, The Netherlands

${ }^{2}$ Leiden University of Applied Sciences, Applied Psychology, Leiden, The Netherlands

\section{Introduction}

Systematic assessment of patient-reported outcomes could help improve the delivery of adequate symptom-oriented care in patients with cancer during phase-I trial participation.

\section{Objectives}

This study aims to describe the variations in patient-reported outcomes like health-related quality of life (HRQoL) and symptoms scores in patients with advanced cancer at pre-consent, before and during phase-I trial participation.

Methods

In this observational prospective exploratory cohort study at the Erasmus MC Cancer Institute, patients completed the EORTC QLQ-C30 questionnaire at pre-consent $(n=124)$, baseline $(n=97)$, and first evaluation of a phase-I trial $(n=76)$ (SFigure 1). The Mann-Whitney U test was used to test the differences between eligible and ineligible patients. For eligibility, univariate logistic regression was performed with age, sex, marital state, 
performance status, Royal Marsden score, and the EORTC QLQ-C30, at pre-consent as independent factors.

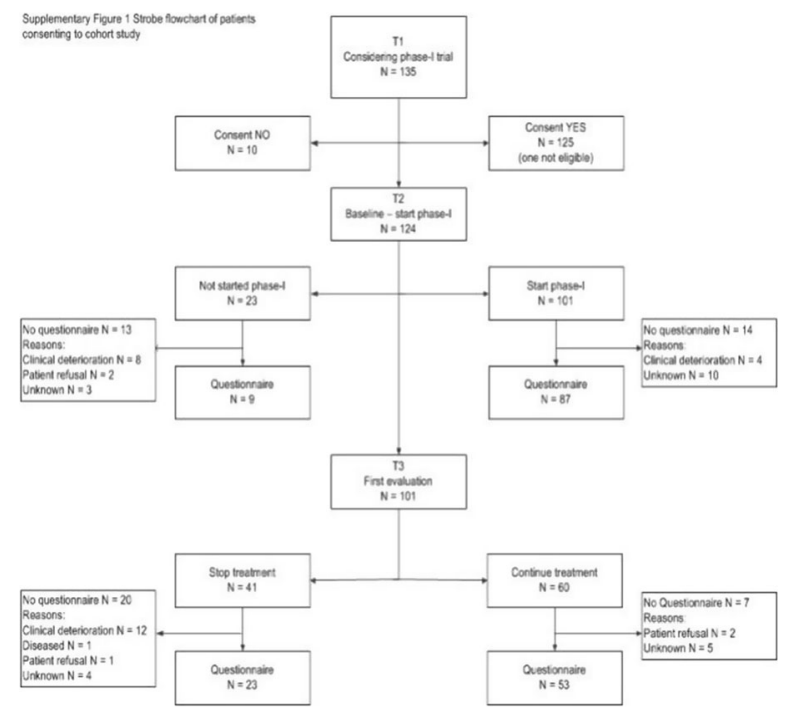

Results

Differences in HRQoL scores were found between eligible patients $(n=102)$ and ineligible patients. At pre-consent, eligibility was associated with a significant better global health $\mathrm{OR}=0946,95 \%$ CI [0.918, 0.975], $p=0.001$, better physical OR $=959,95 \%$ CI $[0.933,0.985], p=0.002$, role functioning $\mathrm{OR}=0.974,95 \%$ CI $[0.957,0.991], p=0.003$, and appetite loss OR 1.022, 95\% CI [1.007, 1.028], $p=0.004$ (Table 1). During the first two cycles on trial, role functioning and appetite loss seem to make a clinical difference.

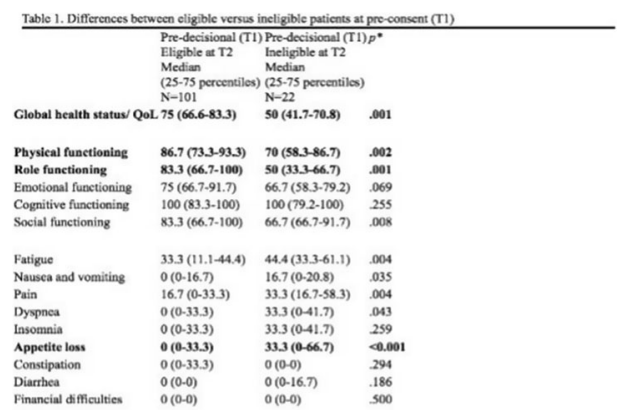

Abbreviations: QoL- Quality of Life, $p$-velues based on Mann-Whitney U test. ${ }^{-T h e}$ bold items are significant
at $\leq 0.0033$.

Conclusions

Patients who are ineligible for phase-I trial participation have lower HRQoL measures. For both potential phase-I trial candidates and participating patients HRQoL measures, as a tool to guide for symptom-orientated care to improve patients' outcomes and trial accrual, should be further explored.

eP535

\author{
ASSOCIATION OF COGNITIVE IMPAIRMENT AND QUALITY \\ OF LIFE IN BREAST CANCER SURVIVORS \\ D. Von $A h^{1}$, V. Champion ${ }^{1}$, P. Monahan ${ }^{2}$, T. Stump ${ }^{2}$, A. Crouch ${ }^{1}$, D. \\ Cella $^{3}$, F. Unverzagt ${ }^{4}$ \\ ${ }^{1}$ Indiana University School of Nursing, Community and Health Systems, \\ Indianapolis, USA \\ ${ }^{2}$ Indiana University School of Medicine, Department of Biostatistics, \\ Indianapolis, USA \\ ${ }^{3}$ Northwestern University, Department of Medical Social Sciences, \\ Chicago, USA \\ ${ }^{4}$ Indiana University School of Medicine, Department of Psychiatry, \\ Indianapolis, USA
}

\section{Introduction}

The diagnosis and treatment for breast cancer can disrupt quality of life. Many breast cancer survivors (BCS) also incur lingering symptoms, such as cognitive impairment, which has also been associated with diminished quality of life. However, the impact of cancer survivorship and cognitive impairment on quality of life is not well known.

\section{Objectives}

The purpose of this study was to examine whether cognitive impairment, breast cancer status (BCS versus healthy control [HC]), or both, are associated with quality of life, including psychological (depressive symptoms, overall well-being, post-traumatic stress and personal growth) and physical well-being (physical functioning and fatigue).

Methods

498 BCS who were 3 to 8 years post-chemotherapy treatment and 397 HC completed subjective memory and quality of life questionnaires and a one-time neuropsychological assessment, including tests of attention, memory, speed of processing, and verbal fluency. Cognitive impairment was defined as scoring 1.5 standard deviations below the mean of the control group on each test and overall composite. Separate linear regression models were used controlling for age, education, and income.

\section{Results}

BCS reported significantly more memory loss than $\mathrm{HC}(\mathrm{p}<0.000)$, with $21 \%$ of the BCS reporting clinically significant memory loss compared to HC. Cognitive performance did not differ significantly between BCS and HCs; however, a sub-sample of BCS $(7.2 \%)$ were clinically impaired compared to $\mathrm{HC}$. BCS with cognitive impairment had significantly greater post-traumatic stress and less personal growth. Perceived memory loss was significantly associated psychological and physical well-being.

\section{Conclusions}

Cognitive impairment although infrequent is significantly associated with psychological well-being among BCS.

eP536

PRIMARY SURGICAL TREATMENT OF LOCALLY ADVANCED BREAST CANCER

E.P. Weledji ${ }^{I}$

${ }^{T}$ University of Buea, Surgery, Buea, Cameroon

\section{Introduction}

Locally advanced breast cancer remains an important clinical problem, particularly in resource-poor settings. The prevalence is high in developing countries because of minimal breast awareness programmes, neglect, and the limited use of the multidisciplinary approach in management. 


\section{Objectives}

Neoadjuvant chemotherapy is the mainstay of management, but the question as to whether primary surgical care could be used in resource-poor settings is of certain value and interest.

\section{Methods}

A case of a 42-year-old African woman with a locally advanced carcinoma of the right breast and heavy nodal involvement only (T3, N2, M0) is presented. She underwent a simple mastectomy with en bloc lymphadenectomy to level II (axillary vein) followed by adjuvant radiotherapy to the mastectomy bed and systemic chemotherapy. There was no clinical evidence of local recurrence or distant metastases at 2 years' follow-up. Results

Optimum management of locally advanced breast cancer is multidisciplinary. Neoadjuvant chemotherapy is the mainstay of management. Primary surgical treatment may be acceptable in selected patients.

Conclusions

Primary surgical treatment may be acceptable in selected patients with locally advanced breast cancer. Further studies in the form of a clinical trial would ascertain the beneficial role of surgery in locally advanced breast cancer, especially in resource-poor settings.

eP537

LIVING WITH AND BEYOND CANCER: HOW WE ARE TRANSFORMING CANCER CARE SERVICES ACROSS LONDON

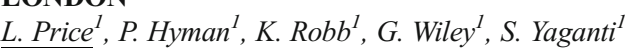

${ }^{T}$ Healthy London Partnership/National Health Service England,

Transforming Cancer Services Team, London, United Kingdom

\section{Introduction}

There are 9 million people in London. Half will experience cancer directly in their lifetime and many others will be affected. The Transforming Cancer Services Team (TCST) works to ensure Londoners have access to world class care before and after a cancer diagnosis.

Objectives

Living With and Beyond Cancer (LWBC) (part of TCST) supports cancer management from diagnosis through to post treatment/cancer as a longterm condition (LTC). We work in partnership providing guidance and support to decision makers and leading change within a complex, evolving landscape.

\section{Methods}

We support system change and promote the delivery of exceptional cancer care across London through:

- Co-design - work is developed with stakeholders (including users).

- Leading with innovation and expertise

- Developing comprehensive guidance documents with implementation plans

- Developing resources/ facilitating education

- Utilising cancer analytics innovatively

Results

- Quality of life improvement - Developing physical activity, lymphoedema, rehabilitation and psychological service guidance, improving primary care, with potential to reduce unnecessary health visits through increase in early detection, awareness and access to optimum care

- Recovery package and stratified follow up guidance - supporting decision makers to plan for and deliver cancer as a LTC strategically

- Primary Care Education -leading inter-professional learning enabling better support for cancer

- Psychological Care Leadership - Delivering an end-to-end pathway for cancer related psychological support
- Cancer Analytics: Instrumental in workbook publication on Cancer Prevalence in England: 21 year prevalence in partnership with Public Health England.

\section{Conclusions}

LWBC is working to transform cancer care across London. Work is shaping the national agenda. Learnings are applicable internationally.

\section{eP538}

MULTIDISCIPLINARY APPROACH FOR PATIENTS TREATED WITH HEAD AND NECK RADIOTHERAPY, NURSE, DENTIST AND NUTRITIONIST IN THE SAME PROTOCOL.

A.L. Yahoo ${ }^{1}, K$. braz ${ }^{1}$, Y. boaventura da silva ${ }^{2}$, R. hanriot ${ }^{3}$ ${ }^{T}$ Hospital Alemao Oswaldo Cruz, Oncologia, Sao Paulo, Brazil

${ }^{2}$ Hospital Alemao Oswaldo Cruz, Radioterapia, Sao Paulo, Brazil

${ }^{3}$ Hospital Alemao Hoswaldo Cruz, Radioterapia, Sao Paulo, Brazil

\section{Introduction}

Radiotherapy on Head \&Neck (HN) fields often leads to oral mucositis(OM), acute skin reactions, difficult feeding and important weight loss. These complications may further interrupted the proposed treatment.

Objectives

The purpose of this study was to assess the effects of an multidisciplinary protocol (MP) which involves oral care, nutritionist and nurse at all stages during the oncological treatment.

\section{Methods}

Between July 2016 to December 2017, 34 patients received IntensityModulated Radiation Therapy ( IMRT) on HN fields at Oswaldo Cruz Hospital Sao Paulo-Brasil. The total dose ranged from 35 to $70 \mathrm{~Gy}$, with mean single dose daily of 2,02Gys. All 34 patients were include in our MP and were prospectively evaluated for OM, radiodermits, weight loss. They received oral care instruction, and low laser therapy was applied an a specific protocol. They received instructions from nurse and nutritionist since the treatment begins. Symptom check-list including measures of pain, oral examination, dysphagia were perfomed weekly. We used WHO scale, RTOG scale and CTC for toxicity criteria.

Results

At 60 Gy 17,6\%,6 patients had any mucositis ,20 (58,8\% GII) and only 8 had GIII OM. Patients had skin events not significant. None of 34 need feeding tube, the nutricionist prescribed oral supplement and specific diet. None of 34 need treatment interruption.

Conclusions

Our study showed that a protocol with intensive multidisciplinary patient approach comprises a promising supportive therapy to reduce acute complications from $\mathrm{HN}$ cancer treatment. Randomized trails are warrented to better define its role on patient quality of life.

\section{eP539}

EXPLORING FACTORS INFLUENCING OF LIFESTYLE ON POSTOPERATIVE HEALTH RELATED LIFE QUALITY OF COLORECTAL CANCER PATIENTS

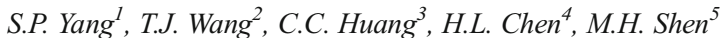

${ }^{T}$ Cathay General Hospital, Nurse Practitioner of General Surgery, Taipei, Taiwan R.O.C.

${ }^{2}$ National Taipei University of Nursing and Health Sciences, School of Nursing, Taipei, Taiwan R.O.C.

${ }^{3}$ Cathay General Hospital, General surgery, Taipei, Taiwan R.O.C.

${ }^{4}$ Cathay General Hospital, Nursing, Taipei, Taiwan R.O.C.

${ }^{5}$ Cathay General Hospital, Colorectal Surgery, Taipei, Taiwan R.O.C.

\section{Introduction}

Colorectal cancer is the third leading cause of cancer death and its incidence rate is continuously increasing. Tumor resection is primary 
treatment for colorectal cancer. Studies had shown lifestyle were related to the risk of death with colorectal cancer. However, only few studies have exploratored the impact of lifestyle on health related life quality of colorectal cancer patients. The study is to explore influences health related life quality of patients with colorectal cancer.

Objectives

The study showed workers's, level of education above college, good of enconomic, surgery of laparoscopic approach, albumin $>3.5 \mathrm{~g} / \mathrm{dl}$ and small amount of alcohol consumption had significantly's higher healthrelated quality of life. Physical Activity is positive correlation with health-related quality of life. Age and Charlson comorbidity index are negative correlation with health-related quality of life.

Methods

The study is a descriptive correlational design. A convenient sample of 106 colorectal cancer patients scheduled for cancer resection surgeries will be recruited from general surgery or rectal surgery clinics of a medical center in Taipei, in Taiwan.

Results

The participants' average score on health-related quality of life was $107.43(S D=13.95)$. The study showed the participants' health-related quality of life were satisfactory after operation. Results of linear regression showed that age, albumin and level of education were the significant predictive factors of health-related quality of life.

Conclusions

Results of the study inform us the important influencing factor of the patients' health-related quality of life as well as provide a foundation for developing interventions to promote health related quality of life.

\section{eP540}

INVESTIGATION OF THE ASSOCIATION BETWEEN PHYSICAL FUNCTIONS AND QUALITY OF LIFE LEVEL IN PATIENTS UNDERGOING AUTOLOGOUS HEMATOPOIETIC STEM CELL TRANSPLANTATION

\author{
V. Yildiz Kabak ${ }^{l}$, Y. Ekinci ${ }^{1}$, S. Atasavun Uysal ${ }^{1}$, T. Duger ${ }^{1}$ \\ ${ }^{T}$ Hacettepe University, Faculty of Health Sciences Department of \\ Physiotherapy and Rehabilitation, Ankara, Turkey
}

\section{Introduction}

The physical functions and quality of life (QOL) level are negatively affected in patients undergoing hematopoietic stem cell transplantation (HSCT).

\section{Objectives}

The aim of this study was to investigate the association between physical functions and QOL in adult patients who hospitalized for autologous HSCT.

Methods

This study was conducted at Hacettepe University Bone Marrow Transplantation Unit in Turkey. The participants(female: 17, male: 5) who planned to HSCT were included in this study. Their mean age was $47.36 \pm 13.03$ years and the mean duration after diagnosis was $17.68 \pm 6.26$ months. Participants had different cancer diagnosis; lymphoma( $(n=10)$, multiple myeloma $(n=9)$, and others $(n=3)$ and were assessed by physiotherapist before HSCT. The QOL was assessed with The European Organization of Research and Treatment of Cancer Quality of Life Core QuestionnaireC30(EORTC QLQ-C30). The physical function tests include handgrip and quadriceps femoris muscle strength, timed up and go-3 meter(TUG-3m), timed up and down stairs, 30 seconds sit to stand test, and 6 minute walk test.

Results

There was a moderate association between the functional score of the EORTC QLQ-C30 and TUG-3m ( $\mathrm{r}=-0.577, \mathrm{p}=0.045)$. The relationship between functional score and 30 second sit to stand test was in strong correlation $(\mathrm{r}=0.767, \mathrm{p}=0.004)$. There was no relationship in other parameters and QOL.

\section{Conclusions}

We found moderate to strong correlations between physical functions and QOL. Therefore, increasing patients' level of physical fuction at initial phase, positively contribute their QOL and their HSCT period. The exercise programs should be started as early as possible before HSCT to maintain physical function and to adapt patients to the exercises.

\section{eP541}

EXPLORING THE RELATIONSHIPS AMONG HEALTHY LIFE STYLE AND HEALTH RELATED QUALITY OF LIFE IN ENDOMETRIAL CANCER SURVIVORS

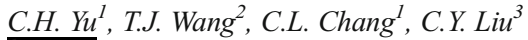

${ }^{T}$ Mack Memorial Hospital- Taiwan, Department of Obstetrics and Gynecology, taipei, Taiwan R.O.C.

${ }^{2}$ School of Nursing- National Taipei University of Nursing and Health Sciences - Taiwan, School of Nursing- National Taipei University of Nursing and Health Sciences - Taiwan, Taipei, Taiwan R.O.C.

${ }^{3}$ National Taipei University of Nursing and Health Sciences, Department of Language Speech Pathology and Audiology-and Department of Midwifery and Women Health Care, Taipei, Taiwan R.O.C.

\section{Introduction}

Endometrial cancer was the sixth most common cancer for women in Taiwan. Few studies have been conducted in Taiwan to investigate the relationships among health life style in endometrial cancer survivors.

\section{Objectives}

Explore the influences of healthy life style on health related quality of life in endometrial cancer survivors.

Methods

Participants were 134 women ( mean age $=54.31 \pm 9.63$ years) diagnosed with endometrial cancer and who had completed their treatment before data collection. Each woman completed structured questionnaires, including Fruit and Vegetable Intake questionnaire, Taiwanese version of the International Physical Activity Questionnaire, the Pittsburgh Sleep Quality Index questionnaire, and the Functional Assessment of cancer therapy-General Scale.

Results

The participants' average score of health-related quality of life was 88.14( $\mathrm{SD}=12.85)$. Results of ANOVA tests showed that the participants' quality of life scores were significantly different among different marital status $(F=4.95, p=0.008)$, and income groups $(F=3.20, p=0.026)$. The participants' life quality was significantly related to comorbidity $(\mathrm{r}=$ $0.294, p=0.01)$, sleep quality $(r=-0.400, p=0.001)$. Obesity, smoking, drinking, exercise and fruit and vegetable intake were not statistically significant related to health-related quality of life.

\section{Conclusions}

Although the healthy lifestyle were not significantly related to quality of life, the results showed that only small percentages of endometrial cancer surviors met the exercsie or diet recommendations of the American Cancer Society.

\section{eP542}

THE ANTIDIARRHEAL EFFICACY OF A PROPRIETARY AMINO ACID MIXTURE IN NEUROENDOCRINE TUMOR PATIENTS

Q. Yu ${ }^{1}$, A. Chauhan ${ }^{1}$, R. Miller ${ }^{2}$, L. Luque ${ }^{3}$, H. Weiss ${ }^{4}$, L. Anthony ${ }^{1}$

${ }^{T}$ University of Kentucky College of Medicine, Division of Medical Oncology Markey Cancer Center, Lexington, USA

${ }^{2}$ University of Kentucky College of Medicine, Dietician Consultant Markey Cancer Center, Lexington, USA 
${ }^{3}$ Entrinsic Health Solutions, Clinical Operations, Miami, USA

${ }^{4}$ University of Kentucky College of Medicine, Department of Biostatistics, Lexington, USA

\section{Introduction}

Over 100,000 gastroenteropancreatic neuroendocrine tumor (GEPNET) patients are currently living in the United States. One common quality-oflife limiting symptom in GEPNET patients is diarrhea, resulting from excessive serotonin production, secondary to post-operative short bowel syndrome, steatorrhea from somatostatin analogs, bile acid colitis or intestinal bacterial overgrowth. A novel amino acid based oral rehydration solution (Enterade $\left.{ }^{\circledR}\right)$ is currently under Phase II clinical trial for antidiarrheal effects in post bone marrow transplant patients (NCT02919670).

Objectives

We conducted a pilot study of Enterade ${ }^{\circledR}$ in neuroendocrine tumor (NET) patients with quality-of-life limiting diarrhea to evaluate its antidiarrheal efficacy.

Methods

Medical records of all the NET patients treated with Enterade ${ }^{\circledR}$ for symptomatic diarrhea were evaluated. Patients were treated at Markey Cancer Center from 05/2017 to 01/2018 (8 Oz bottle BID for 1 a week).

Results

Among the 23 patients at the time of abstract submission, 7 patients had small bowel NET, 5 had bronchial NETs, 2 had colorectal NETs, 3 had NETs of unknown primary site, 3 had gastric NETs, 2 had pancreatic NETs and 1 had high grade prostate NET. Fourteen had histories of prior bowel resection either for primary NET resection or debulking. Fifteen were on somatostatin analogs at the time of Enterade ${ }^{\prime}$ 's initiation. Seventeen reported subjective improvement in diarrheal symptoms. Twelve out of these seventeen patients reported over $50 \%$ reduction in diarrhea frequency.

\section{Conclusions}

73.9\% NET patients reported reduction in diarrhea frequency with Enteradeß. $52.173 \%$ reported more than $50 \%$ reduction. A prospective Phase II study of Enterade ${ }^{\circledR}$ in GEPNET patients with quality-of-life limiting diarrhea is planned.

\section{eP543}

THE EFFECT OF BREAST CANCER PATIENTS' PHYSICAL ACTIVITY LEVELS ON THEIR SYMPTOMS AND QUALITY OF LIFE

S. Dibekci ${ }^{l}$, F. Arikan ${ }^{2}$

${ }^{1}$ Akdeniz University, Hospital, Antalya, Turkey

${ }^{2}$ Akdeniz University, Faculty of Nursing, Antalya, Turkey

\section{Introduction}

Physical activity is important in increasing the degree of psychological and physical well being of cancer patients

Objectives

This study was planned to determine the effect of physical activity levels to symptoms and quality of life with breast cancer patients.

Methods

This descriptive study was conducted in the Chemotherapy Unit of Akdeniz University's Hospital from September 2015 to June 2016. In total, 128 individuals who met the inclusion criteria were included in the study. The study data were collected using a patient introduction form, the International Physical Activity Questionnaire, the EORTC-QLQ C30Quality of Life Scale and the EORTC QLQ- BR23 Quality of Life Scale.

\section{Results}

Of the patients, $53.9 \%$ were aged between 46 and 60 . On average, the patients were diagnosed with breast cancer $30.91 \pm 37$ months before the study, and $41.4 \%$ of them were in the second stage of the disease. This study found that $32.8 \%$ of the patients were active at a low level, while $35.94 \%$ were moderately active, and $31.2 \%$ were highly active. There was a negative correlation between the patients' International Physical Activity Questionnaire scores and scores on EORTC-QLQ C30for general symptoms, fatigue, pain, labored breathing, lack of sleep, loss of appetite $(\mathrm{p}<0.01)$. On the other hand, there was a positive correlation between their scores on functional health, occupational function, social function and general health $(\mathrm{p}<0.05)$

\section{Conclusions}

This study found that patients with high and moderate physical activity levels had fewer symptoms, and their functional status and general quality of life were much better

\section{eP544}

THE IMPACT OF PHYSICAL THERAPY PROGRAM IN FITNESS OF CHILDREN AND ADOLESCENTS WITH CANCER

E. Astorga ${ }^{1}$, D. Vargas $^{2}$

${ }^{T}$ Fundación Nuestros Hijos, Oncological Rehabilitation Center, Santiago, Chile

${ }^{2}$ Universidad Mayor, Physical Therapy, Santiago, Chile

\section{Introduction}

Been a cancer survival is not free of side effects, either due to treatment or due to the same disease, especially in the pediatric population. Among the main sequelae is the physical condition, directly affected by prolonged hospitalizations and complications of treatment. International evidence shows that there are improvements in the physical condition of subjects undergoing guided exercise programs, but at the national level there are no studies that talks about physical condition in pediatric patients with cancer. This research analyzed the impact of a physical therapy program on the fitness of this population.

Objectives

Evaluate the impact of a physical therapy program on fitness in children and adolescents with cancer in the admission and 3 month after exercise therapy program at Oncological Rehabilitation Center (CROFNH).

\section{Methods}

21 children and adolescents between 6 and 17 years who got into a physical therapy program at CROFNH between January 2016 to February 2017. Their physical condition was assessed through Timed Up and Go (TUG) and 6 Minute Walking Test (TM6M). Their performance was compared at the admission, at the end of physical therapy program and with reference values of healthy children.

\section{Results}

In the TUG test, the children spent a time of $6.67 \mathrm{~s}$. at the first time walk, and after the physical therapy program $4.69 \mathrm{~s}$. In the TM6M they initially walked an average distance of $489.50 \mathrm{~m}$. and after the physical therapy program $549.74 \mathrm{~m}$.

\section{Conclusions}

A 3-month physical therapy program significantly improves physical condition in children and adolescents with cancer.

\section{eP545}

EVALUATING THE CLINICAL BENEFITS OF EXERCISE REFERRAL FOR PEOPLE LIVING WITH CANCER: A CASE STUDY FROM ABERDEEN, SCOTLAND

J. Björkqvist

${ }^{T}$ University of Aberdeen, School of Medicine-Medical Sciences and Nutrition, Aberdeen, United Kingdom

\section{Introduction}

People living with cancer (PLWC) experience long-lasting adverse effects of their disease and treatment. Physical activity has been shown to be safe and clinically effective for PLWC. Despite this evidence physical activity 
is still not prescribed to PLWC as part of routine cancer care. Macmillan Cancer Support, Sport Aberdeen and NHS Grampian entered a partnership to deliver the Move More Aberdeen (MMA) exercise referral programme for PLWC.

\section{Objectives}

To evaluate the impact of a 12-week community-based physical activity programme for PLWC.

Methods

MMA delivery started in January 2015 with 12-week community-based physical activity groups of moderate-intensity and high-intensity. The cancer and physical activity standard evaluation framework (CaPASEF) was used to measure physical activity levels, fatigue and quality of life at baseline, 3, 6 and 12 months. A 6-minute walk test, sit-to-stand test and hand grip strength test were later introduced at baseline and 12 weeks to assess MMA's impact on cardiorespiratory fitness, strength and mobility. Results

MMA has generated 380 referrals to date. Referrals were highest for prostate $(25 \%)$, breast $(24 \%)$, bowel $(9 \%)$ and lung $(7 \%)$ cancer. MMA has demonstrated a long-term increase in physical activity levels $(\mathrm{P}<0.01)$, decrease in fatigue $(\mathrm{P}<0.05)$ and improvement in quality of life $(\mathrm{P}<0.05)$ for participants. Results from objective measurements will be available in May 2018.

Conclusions

MMA has successfully supported PLWC into a lifestyle of long-term, independent physical activity for health. The programme has improved access and adherence to physical activity for PLWC and demonstrates a feasible model for integrating physical activity into standard cancer care.

\section{eP546}

EFFECTS OF A SWALLOWING EXERCISE EDUCATION PROGRAM ON DYSPHAGIA-SPECIFIC HEALTH-RELATED QUALITY OF LIFE IN ORAL CAVITY CANCER PATIENTS POST-TREATMENT: A RANDOMIZED CONTROLLED TRIAL S.C. Chen ${ }^{l}$

${ }^{T}$ Chang Gung University of Science and Technology, Department of Nursing, Taoyuan, Taiwan R.O.C.

\section{Introduction}

Swallowing rehabilitation can be used to improve swallowing dysfunction and quality of life.

\section{Objectives}

The purpose of this study was to evaluate the effects of a swallowing exercise education program (SEEP) on swallowing ability, depression, and dysphagia-specific health-related quality of life (QOL) in oral cavity cancer (OCC) patients.

\section{Method}

This was a prospective, randomized controlled study with 2 groups, using a pre- and post-repeated measures design. A total of 76 participants were randomly assigned to an experimental group $(\mathrm{n}=38)$ and a control group $(\mathrm{n}=38)$. The experimental group participated in a SEEP, and the control group received normal care. Patients were assessed at 5 time points: baseline assessment (T0), and then 1, 2, 3, and 6-months (T1, T2, T3, and T4) after participating in the SEEP or receiving normal care. Results

Patients in the experimental group had significantly greater emotional dysphagia QOL compared to those in the control group. In the experimental group, post-education (T4) swallowing ability and depression were better than baseline (T0). For both group, functional dysphagia QOL and physical dysphagia QOL were greatest at $\mathrm{T} 0$, decreased from $\mathrm{T} 1$ and $\mathrm{T} 2$, and slightly decreased at $\mathrm{T} 3$ and $\mathrm{T} 4$. There were no differences between the groups and within groups with respect to dysphagiaspecific health-related QOL, global dysphagia QOL, functional dysphagia QOL, and physical dysphagia QOL.

\section{Conclusions}

Conclusions: The SEEP was effective in improving emotional dysphagia QOL during the initial 6 months after treatment of patients with OCC.

eP547

MULTI-STAKEHOLDER ATTITUDES REGARDING RISKBASED THRESHOLDS FOR INITIATING IMPAIRMENTDIRECTED INTERVENTIONS AMONG PATIENTS WITH BREAST CANCER

A. Cheville ${ }^{l}$, J. Basford ${ }^{l}$

${ }^{T}$ Mayo Clinic, Physical Medicine and Rehabilitation, Rochester, USA

\section{Introduction}

Physical impairments are prevalent following breast cancer (BC) treatment and associated with poor outcomes. Predictive models permit estimation of impairment risk, and can be the basis for directing screening and validated risk reduction measures.

\section{Objectives}

The objective of this study was to characterize diverse clinical stakeholders' risk thresholds for initiating $\mathrm{BC}$ impairment-directed education, screening, preventive activities, and specialist referrals.

\section{Methods}

A purposive sample of 40 breast cancer survivors, as well as 10 Breast Clinic physicians, 10 breast surgeons, 10 radiation oncologists, 10 medical oncologists, 10 Breast Clinic nurses, and 10 cancer rehabilitation therapists from academic and community settings were queried about their thresholds for initiating "More frequent and sensitive screening," "Education," "Instruction in preventive activities," and "Referral to a physical or occupational therapist." Participants indicated on numerical rating scales the risk level $(0-1.0)$ at which they would start activities for lymphedema, upper quadrant pain, functionally limiting chemotherapy-induced peripheral neuropathy, shoulder contracture, and fatigue.

\section{Results}

Across impairments, mean risk thresholds were consistently ordered as follows: education (.07-.12), prevention (.14-.25), screening (.25-.31), and therapist referral (.37-.45). BC Survivors had the lowest thresholds for initiating education; lymphedema .02 , pain .04 , neuropathy .02 , contracture .03 , and fatigue .03 , while therapists had the lowest thresholds for screening and referrals. Among physicians, risk thresholds varied widely across impairments, interventions, and disciplines, however breast surgeons had significantly higher thresholds for screening, prevention, and therapist referral, $\mathrm{p}<0.03$.

\section{Conclusions}

Risk thresholds for initiating BC impairment-directed activities are inconsistent across stakeholder groups. BC survivors, however, had the lowest thresholds for initiating impairment-directed education.

eP548

THREE YEAR FOLLOW-UP AFTER REHABILITATION - A RANDOMISED STUDY AMONG RADIATED PROSTATE CANCER PATIENTS

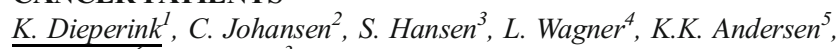
L.R. Minet ${ }^{6}$, O. Hansen ${ }^{3}$

${ }^{1}$ University Hospital Odense, Department of Oncology, Odense, Denmark

${ }^{2}$ Danish Cancer Society Research Center, Survivorship, Copenhagen, Denmark

${ }^{3}$ Odense University Hospital, Department of Oncology-Academy of Geratric Cancer research AgeCare, Odense, Denmark

${ }^{4}$ University of Southern Denmark, Unit of Nursing, Odense, Denmark

${ }^{5}$ Danish Cancer Society Research Center, Statistics-Bioinformatics and Registry, Copenhagen, Denmark 
${ }^{6}$ Odense University Hospital, Department of Rehabilitation, Odense, Denmark

\section{Introduction:}

Rehabilitation may help PCa patients treated with androgen deprivation therapy and radiotherapy.

Objectives

The purpose was a) to study the long-term effects of a multidisciplinary rehabilitation intervention for prostate cancer $(\mathrm{PCa})$ patients and $\mathrm{b}$ ) to study status of treatment-related adverse effects and quality of life (QoL) at different time points.

Methods

PCa patients $n=161$ were in 2010-2012 randomized to either a 4 hour program of two nurse lead psychosocial support sessions followed by two instructive sessions by a physical therapist $(n=79)$, or usual care $(n=82)$. The primary outcome, irritative urinary sum-score (EPIC-26) was significantly improved after six month. In this follow-up study all participants reported disease-specific QoL (EPIC-26) and general QoL (SF-12) three years after radiotherapy. PSA and the pelvic floor strength were examined. Survival status, descriptive analysis and linear regression analysis adjusting for baseline scores were conducted.

\section{Result}

Six patients had deceased, one from the intervention group and five from the control group. 143/155 (92\%) responded to the questionnaire. Three years after radiotherapy none of the EPIC domains were significantly different between groups. However, patients in the intervention group showed a trend for better irritative urinary sum score (0-100) with 86.1 SD 13.3 compared to 82.9 SD 15.0 in controls $P=.069$. Significantly more patients in the control group had moderate to severe bowel problems 10 (14.3\%) compared to the intervention group 2 (2.9\%) $P=.016$.

\section{Conclusions}

We did not find a long-term effect of multidisciplinary rehabilitation in irradiated PCa patients. However, the intervention may positively influence bowel QoL.

\section{eP549}

\section{SUPERVISED AND HOME-BASED EXERCISE IN PATIENTS NEWLY DIAGNOSED WITH MULTIPLE MYELOMA - A RANDOMIZED CONTROLLED FEASIBILITY STUDY R.F. Larsen ${ }^{1}$, M. Jarden ${ }^{2}$, L.R. Minet ${ }^{3}$, U.C. Frølund ${ }^{4}$, N. Abildgaard ${ }^{5}$ ${ }^{T}$ Zealand University Hospital, Department of Occupational Therapy and Physiotherapy, Roskilde, Denmark \\ ${ }^{2}$ Copenhagen University Hospital Rigshospitalet, Department of Hematology Finsen Center, Copenhagen, Denmark \\ ${ }^{3}$ Odense University Hospital, Department of Rehabilitation, Odense, Denmark \\ ${ }^{4}$ Zealand University Hospital, Department of Hematology, Roskilde, Denmark \\ ${ }^{5}$ Odense University Hospital, Department of Hematology, Odense, Denmark}

\section{Introduction}

Exercise is considered to be a feasible, safe and beneficial complementary treatment for patients with hematological cancer. Exercise interventions (EI) in primarily younger patients with multiple myeloma (MM) have only been conducted in three randomized controlled trials (RCT) and one single arm pilot study. Our ongoing RCT examines the effect of an individualized, early initiated EI in newly diagnosed patients with MM, irrespective of age, on muscle strength, physical function and activity.

Objectives

The aim of the present study is to report the interim analysis of feasibility and safety of the EI.

\section{Methods}

A two-center RCT with blinded outcome assessors. Baseline tests are carried out 3-4 days after starting anti-myeloma treatment, followed by randomization to control group or intervention group (IG). The intervention is a 10-week supervised and home-based exercise program comprising aerobic and strengthening exercises and physical activity. Feasibility outcome measures are eligibility, acceptance and drop-out rates. Further, adherence, tolerability and safety (adverse events (AE)) of the supervised exercise sessions (SES) are obtained.

\section{Results}

Of 49 patients screened, $80 \%$ were eligible for inclusion; $77 \%$ accepted participation. In IG, five patients dropped out (29\%); three prior to starting the EI. Adherence and tolerability was high; one patient missed one SES; two patients discontinued after one SES each due to non-serious AE (pain and dyspnea/dizziness). No serious AE (e.g. pathological fractures) were reported.

\section{Conclusions}

Early initiated EI in patients with MM, regardless of treatment regimen, is feasible, safe and tolerable. Early initiated exercise may be important in preventing physical decline during treatment for MM.

\section{eP550}

EFFECT OF LOW-INTENSITY EXERCISE THERAPY ON THE PHYSICAL FUNCTION, AND MENTAL AND PHYSICAL SYMPTOMS OF PATIENTS WITH HEMATOLOGIC MALIGNANCY UNDERGOING CHEMOTHERAPY

$\underline{\text { T. Fukushima }}^{1,2}$, J. Nakano $^{3}$, S. Ishii ${ }^{2}$, A. Natsuzako ${ }^{2}$, J. Sakamoto $^{3}$, M. Okita

${ }^{I}$ Nagasaki University Graduate School of Biomedical Sciences, Locomotive Rehabilitation Science, Nagasaki, Japan

${ }^{2}$ Nagasaki University Hospital, Rehabilitation, Nagasaki, Japan

${ }^{3}$ Nagasaki University Graduate School of Biomedical Sciences, Physical Therapy Science, Nagasaki, Japan

\section{Introduction}

Mid- to high-intensity exercise improves physical function and symptomatology in patients with hematologic malignancies. Low-intensity exercise therapy (LIET) is often adopted in cases where chemotherapeutic side effects preclude more intensive exercises. However, it remains unclear whether LIET is effective in patients with hematologic malignancies.

\section{Objectives}

To investigate the effects of LIET on the physical and mental symptoms and functions, in patients with hematologic malignancies undergoing chemotherapy.

\section{Methods}

Forty-four patients hospitalized for chemotherapy performed LIET. The exercise intensity of LIET was defined as achieving $<40 \%$ of the predicted maximum heart rate calculated using the Karvonen formula. LIET consisted of 20-40 minutes of walking and resistance training, per day. LIET frequency was adjusted according to the patient's condition. Muscular and physical function, activities of daily living (ADLs), psychological distress, and quality of life (QOL) were evaluated upon initiation of rehabilitation and at discharge. Participants were divided into high- and low-frequency groups according to their LIET frequency. Two-way repeated measures analysis of variance was used for statistical analysis.

\section{Results}

Muscle function was maintained, while physical function, ADLs, psychological distress, and QOL were significantly improved in the highfrequency group. In the low-frequency group, muscle function of the lower-limb was significantly reduced and no other improvement was observed.

Conclusions

LIET was effective in improving the physical functions and symptomatology in the high-frequency group, but not in the low-frequency group. 
Thus, LIET could be a potential treatment strategy for patients with hematologic malignancies undergoing chemotherapy who are unable to perform mid- or high-intensity exercise.

eP551

FUNCTIONAL AND POSTURAL STABILITY ASSESSMENT ON CANCER PATIENTS WHO UNDERWENT INPATIENT REHABILITATION UPON DISCHARGE AND ONE MONTH AFTER DISCHARGE

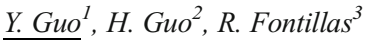

${ }^{T}$ University of Texas MD Anderson Cancer Center, PalliativeRehabilitation and Integrative Medicine, Houston, USA

${ }^{2}$ Xinqiao Hospital, Department of Digestive Disease, chongQing, China

${ }^{3}$ University of Texas MD Anderson Cancer Center, rehabilitation Services, Houston, USA

\section{Introduction}

Previous studies have shown that deconditioned cancer patient can improved their function through inpatient rehabilitation. Whether these patient can maintain their functional gain has not been assessed.

Objectives

To examine whether these patients can maintain their fall risk and postural stability.

\section{Methods}

This is a prospective pilot study. Deconditioned cancer patients who underwent inpatient rehabilitation or rehabilitation mobile team were recruited and assess at the time of discharge and four weeks after discharge. Outcome measures recorded at baseline and after four weeks included the Get Up and Go test; the timed 50-foot fast walk; fall risk test and postural stability test on Balance System SD® (Biodex, New York, USA).

Results

Twenty four patients assessed at baseline, only 9 patients (38\%) completed both assessments. Both functional tests (Get Up and Go test; the timed 50-foot fast walk test) were significantly improved from discharge to 4 weeks post-discharge. ( $P=0.011$ and $P=0.015$, respectively;). No statistical difference was found in the fall risk test and postural stability between discharge and 4 weeks post-discharge $(P=0.373$ and $P=0.106$, respectively)

Conclusions

Among the 9 patients who completed the study, their functional level continue to improve and fall risk and postural stability indexes were well maintained.

\section{eP552}

PHYSIATRIST AND ONCOLOGY PATIENT EXPECTATIONS REGARD ING FUNCTION AFTER INPATIENT REHABILITATION

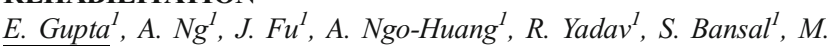

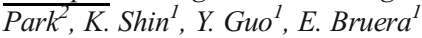

${ }^{1}$ The University of Texas MD Anderson Cancer Center, PalliativeRehabilitation and Integrative Medicine, Houston, USA

${ }^{2}$ The University of Texas MD Anderson Cancer Center, Biostatistics, Houston, USA

\section{Introduction}

Cancer rehabilitation plays a key role in the care of patients undergoing treatment as well as survivors. As the value of inpatient rehabilitation becomes more recognized by patients and oncologists, physiatrists are concerned regarding unrealistic expectations of rehabilitation goals

Objectives

The primary objective of this prospective study was to determine the difference between expectations of function after an inpatient rehabilitation stay between cancer patients and physiatrists, and how they align with actual functional status after rehabilitation.

\section{Methods}

Patients and physiatrists completed an admission, expected and discharge functional status using the Barthel Index and an ad hoc assessment gathering patient reported sources of expectations for rehabilitation. Quality of life and symptom management were measured by the Functional Assessment of Chronic Illness Therapy- General (FACT-G) and Edmonton Symptom Assessment System.

\section{Results}

Patients' expected median discharge functional score was higher by 2 and 5 points when compared to physiatrists' expected and actual discharge functional scores, respectively. $99 \%$ of patients felt family and friends motivated them to achieve their goals and $82 \%$ reported motivation due to obtaining further cancer treatment if stronger. Upon discharge, patients reported 6 point improvement in total symptom distress and 23 point improvement in FACT-G.

\section{Conclusions}

Patients significantly over-estimate goals for cancer rehabilitation when compared to physiatrists' goals and actual achieved function. These unmet expectations for functional recovery may impact their impression of future oncological care, and better communication should be explored. Inpatient rehabilitation significantly improves symptom distress and quality of life.

\section{eP553}

\section{TESTING EFFICACY OF A PULMONARY REHABILITATION PROGRAM FOR POST LUNG CANCER RESECTION SURGERY \\ W.L.Hsiao ${ }^{\text {, T.J. Wang }}$ \\ ${ }^{T}$ National Taiwan University, Nursing, Taipei, Taiwan R.O.C.}

\section{Introduction}

Pulmonary rehabilitation enhancing lung expansion and ventilation may help to improve oxygenation and reduce postoperative lung complications in patients with lung resection for removing lung cancer.

\section{Objectives}

The purpose of this study is to test the effects of an early rehabilitation intervention on oxygenation, postoperative complications, and recovery from lung cancer surgery.

\section{Methods}

An experimental design. Ninety patients for lung cancer surgeries was recruited from thoracic surgery units of a medical center. Patients were randomly assigned to the intervention or the control group. The intervention includes a 5-day preoperative home-based rehabilitation and a 5-day postoperative in-hospital rehabilitation. The main components of the rehabilitation were aerobic and strength exercises as well as breathing training by using an incentive spirometry.

\section{Results}

The GEE showed a significant group by time interaction effect on $\mathrm{SpO} 2 / \mathrm{FiO} 2$ ratio. As for the parameter estimates, from postoperative day 1 to day 4 , the $S / F$ ratio improvement in the intervention group was 74.49 (Wald $\mathrm{X}^{2}=46.42, \mathrm{p}<0.001$ ) more than in the control group. Result of Chi-square test showed that the number of postoperative lung complications in the intervention group $(\mathrm{n}=1)$ was significantly less $\left(\mathrm{X}^{2}=8.39, \mathrm{p}=0.004\right)$ than it in the control group. Result of the duration of chest tube drainage in the intervention group $(2.00 \pm 1.00$ days $)$ was significantly shorter $(t=-$ $2.32, \mathrm{p}=0.022)$ than it in the control group $(2.56 \pm 1.25$ days $)$.

\section{Conclusions}

Pulmonary rehabilitation designed to enhance lung expansion and ventilation may help to reduce postoperative lung complications and promote patients' recovery from lung cancer surgery. 
eP554

THE RELATIONSHIP BETWEEN THE ANXIETY TO USE IPSILATERAL LIMB AND IPSILATERAL SHOULDER ACTIVE RANGE OF MOTION AND INSTRUMENTAL DAILY LIVING ACTIVITIES AFTER BREAST CANCER SURGERY R. Kato ${ }^{1}$, H. Tajiri ${ }^{1}$, K. Tajiri ${ }^{1}$, A. Tanuma ${ }^{1}$

${ }^{T}$ Shizuoka cancer center hospital, rehabillitation, 1007 ShimonagakuboNagaizumi-, Japan

\section{Introduction}

The patients with breast cancer, who underwent surgery, has often feel anxiety in resuming instrumental daily living activities (IADL) early after surgery.

Objectives

To explore relationship between the anxiety to use ipsilateral limb and ipsilateral shoulder active range of motion (AROM) and IADL in patients with anxiety.

Methods

We retrospectively reviewed 27 breast cancer patients who underwent surgery with axillary lymph node dissection and perioperative occupational therapy in 2010. Patients were classified to anxiety group (AG, $n=14$ ) and non-anxiety group (NAG, $n=13$ ) according to the questionnaire about anxiety to use ipsilateral limb at first outpatient visit after discharge (11.0 \pm 2.9 days after surgery). We compared AROMs of shoulder and Frenchay Activities Index (FAI) score between two groups.

Results

AROMs and FAI before surgery were not statistically significant between two groups. Patients in AG had worse AROM in shoulder flexion (129 $\pm 28^{\circ}$ vs $\left.150 \pm 15^{\circ}, \mathrm{p}<0.05\right)$ and abduction $\left(124 \pm 43^{\circ}\right.$ vs $\left.155 \pm 21^{\circ}, \mathrm{p}<0.05\right)$ than those in NAG. However, both groups had similar FAI score $(26.5$ \pm 8.3 vs $25.0 \pm 6.9, \mathrm{p}=0.6$ ).

Conclusions

We found that patients in AG continued IADLs as same as those in NAG despite having restricted AROM of the shoulder. This might lead to developing anxiety to use limb in AG. Careful induction of occupational therapy with psychological support might reduce anxiety.

\section{eP555}

\section{NORDIC POLE WALKING FOR INDIVIDUALS WITH CANCER: A FEASIBILITY STUDY

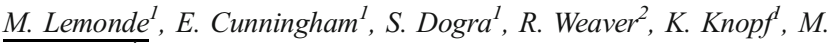 Nonoyama ${ }^{1}$ \\ ${ }^{1}$ University of Ontario Institute of Technology, Faculty of Health Sciences, Oshawa- Ontario, Canada \\ ${ }^{2}$ Rowan University, Faculty of Health \& Exercise Science, Glassboro, USA}

\section{Introduction}

Individuals with cancer experience high symptom burden that impacts their quality of life. An individualized, community-based Nordic pole walking (NPW) program may help.

\section{Objectives}

Primary objective: to assess the feasibility of a NPW program for individuals with non-small cell lung (NSCLC), prostate, colorectal, and endometrial cancer.

Secondary objective: to determine the effects of NPW on physical function and health-related quality of life (HRQoL).

Methods

Eight-week multi-centred randomized controlled feasibility study: NPW (supervised and independent NPW sessions) or control (usual daily routine). The Thabane four-criterion framework (2010) assessed feasibility (Process, Resources, Management, Scientific). Descriptive and non- parametric comparative statistics assessed effects of NPW over time and between groups.

Results

$\mathrm{N}=8$ were recruited, mean age 67 years. The study was feasible with recommended modifications: 1) recruit participants at hospital cancer centres; 2) individualize NPW and integrate behavioural change techniques; 3 ) use pedometers or accelerometers to measure physical activity levels; 4) organize NPW NSCLC programs alongside programs for other chronic respiratory diseases. Compared to baseline, the NPW group had a significant improvement in the 30-s chair stand test (median 10.5 to 14.3, $\mathrm{p}<0.05$ ) and decreased thigh circumference (median right thigh: 49.4 to $48.5 \mathrm{~cm}$; left thigh: 49.0 to $46.3 \mathrm{~cm}, \mathrm{p}<0.05$ ). There were no significant improvements between groups, though there was a trend toward improved physical activity and HRQoL.

Conclusions

Examining the effects of NPW on individuals diagnosed with cancer is feasible with modifications, and may improve physical function. Further research with larger sample sizes should be completed to more conclusively determine the impact of NPW.

\section{eP556}

HEART RATE AND BORG SCALE IS ASSOCIATED WITH EXERCISE-TOLERANCE TIME AFTER TRANSPLANTATION IN ALLOGENEIC HEMATOPOIETIC STEM CELL TRANSPLANTATION PATIENTS

S. Morishita ${ }^{1}$, T. Tanaka ${ }^{2}$, T. Wakasugi ${ }^{2}$, T. Harada ${ }^{2}$, K. Kaida ${ }^{3}, K$.

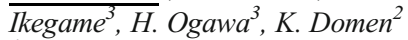

${ }^{1}$ Niigata University of Health and Welfare, Institute for Human Movement and Medical Sciences, Niigata City, Japan

${ }^{2}$ Hyogo College of Medicine, Department of Rehabilitation Medicine, Nishinomiya, Japan

${ }^{3}$ Hyogo College of Medicine, Division of Hematology-Department of Internal Medicine, Nishinomiya, Japan

\section{Introduction}

\section{Objectives}

We analyzed the results of the exercise-tolerance test, performed before and after transplantation, in patients who had undergone allogeneic hematopoietic stem cell transplantation (allo-HSCT). Furthermore, we investigated the relationship between Heart Rate (HR), changed HR, Borg scale, and exercise-tolerance time in allo-HSCT patients before and after transplantation.

\section{Methods}

Our study included 28 patients (19 men and 9 women) who had undergone allo-HSCT between May 2015 and March 2017. Patients performed the exercise-tolerance test on a bicycle for up to 2 weeks before and 3 weeks after transplantation. For participants who finished this test before and after transplantation, we investigated the following: exercisetolerance time, HR, changed HR between pre- and post-test, and the Borg scale.

\section{Results}

The exercise-tolerance time was significantly decreased after alloHSCT $(\mathrm{P}<0.05)$. HR, changed HR, and the Borg scale score were not significantly correlated with exercise-tolerance time at before transplantation. However, HR, changed HR, and Borg scale significantly correlated with exercise-tolerance time at after transplantation $(\mathrm{P}<0.05)$.

\section{Conclusions}

In allo-HSCT patients, HR, changed HR, and Borg scale may not influence exercise-tolerance before transplantation. However, after transplantation these physiological and psychological parameters influence the exercise-tolerance time in allo-HSCT patients. We should recognize the change in these characteristics between pre- and post-transplantation in allo-HSCT patients. 
eP557

PULMONARY FUNCTION AND HEALTH-RELATED QOL AFTER 1 YEAR IN SURGICALLY TREATED PATIENTS WITH MALIGNANT PLEURAL MESOTHELIOMA

S. Morishita ${ }^{1}$, T. Tanaka ${ }^{2}$, M. Hashimoto ${ }^{3}$, T. Nakamichi ${ }^{3}$, Y. Uchiyama ${ }^{4}$, S. Hasegawa ${ }^{3}, K$. Domen ${ }^{4}$

${ }^{1}$ Niigata University of Health and Welfare, Institute for Human Movement and Medical Sciences, Niigata City, Japan

${ }^{2}$ Hyogo College of Medicine Hospital, Department of Rehabilitation, Nishinomiya, Japan

${ }^{3}$ Hyogo College of Medicine, Department of Thoracic Surgery, Nishinomiya, Japan

${ }^{4}$ Hyogo College of Medicine, Department of Rehabilitation Medicine, Nishinomiya, Japan

\section{Introduction}

Pulmonary function and health-related quality of life (HRQOL) at one year of follow-up in surgically treated patients with malignant pleural mesothelioma (MPM) have not been evaluated in detail.

Objectives

The aim of this study was to investigate the change in pulmonary function and HRQOL from the preoperative period to the time of hospital discharge and at 1 year after surgery in MPM patients who underwent pleurectomy/decortication (P/D).

Methods

The study subjects were 16 male patients with MPM who underwent P/D. We investigated the change in pulmonary function and HRQOL from the preoperative period to the time of hospital discharge and at 1 year after surgery in MPM patients.

Results

FVC and FEV1 at the time of hospital discharge were significantly lower compared to preoperative values $(\mathrm{p}<0.05)$. They increased significantly at 1 year after surgery compared to values at the time of hospital discharge $(p<0.05)$. Scores in all eight domains of the SF-36 scale were significantly lower at the time of hospital discharge compared to 1 year after surgery $(p<0.05)$. Scores in physical functioning, body pain, general health, vitality, social functioning, and mental health in QOL were significantly higher at 1 year after surgery than at the time of hospital discharge $(\mathrm{p}<0.05)$

Conclusions

These results are relevant to physicians, nurses, and rehabilitation teams as they may provide insight into the development of customized rehabilitation strategies for patients with MPM who undergo P/D.

\section{eP558}

CANCER PATIENTS INTERESTS IN PARTICIPATION IN CANCER REHABILITATION: A POSTAL SURVEY

\section{E. Ohlsson-Nevo ${ }^{I}$, I. Alkebro $^{2}$, J. Ahlgren $^{3}$}

${ }^{T}$ Örebro university, School of Health and Medical Sciences, Örebro, Sweden

${ }^{2}$ Örebro University Hospital, Onkology Department, Örebro, Sweden

${ }^{3}$ Örebro Unversity Hospital, Onkology Department, Örebro, Sweden

\section{Introduction}

Rehabilitation for cancer patients aims at preventing and reducing the physical, mental, social and existential consequences of a cancer disease and its treatment. Cancer rehabilitation efforts should give the patient support according to their individual needs to with the purpose of to live as good a life as possible.

Objectives

The present study is part of a larger project, with the purpose to investigate rehabilitation needs, social stigma, health-related quality of life and the extent of given information, for people diagnosed and treated for cancer, in a region of Sweden.
The aim of this study is to describe rehabilitation cancer patients Methods

A total of 1179 cancer patients diagnosed with 28 different cancer diagnosis between 150801-160701, were identified for a postal survey with the purpose to investigate rehabilitation needs, social stigma, healthrelated quality of life and the extent of given information. The response rate was $60 \%$.

Results

The analysis is in progress and the results will be presented at the conference.

\section{Conclusions}

The results can give an indication of what cancer rehabilitation services need to be developed, and integrated in the care of cancer patients to reduce the cancer disease negative effect on a patient's quality of life.

\section{eP559}

DEVELOPMENT OF LOW-INTENSITY HOME-BASED RESISTANCE TRAINING COMBINED WITH PHYSICAL ACTIVITY COUNSELING FOR ELDERLY PATIENTS WITH ADVANCED CANCER: THE EXERCISE COMPONENT OF THE NEXTAC PROGRAM

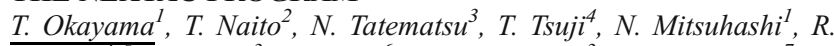

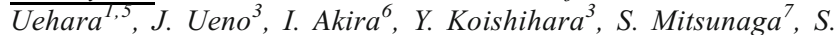

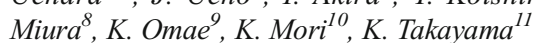

${ }^{1}$ Shizuoka Cancer Center, Division of Rehabilitation medicine, Shizuoka, Japan

${ }^{2}$ Shizuoka Cancer Center, Division of Thoracic oncology, Shizuoka, Japan

${ }^{3}$ National Cancer Center Hospital East, Department of Rehabilitation, Chiba, Japan

${ }^{4}$ Keio University School of Medicine, Department of Rehabilitation medicine, Tokyo, Japan

${ }^{5}$ Kanagawa Cancer Center, Division of Rehabilitation medicine, Kanagawa, Japan

${ }^{6}$ Niigata Cancer Center Hospital, Department of Rehabilitation, Niigata, Japan

${ }^{7}$ National Cancer Center Hospital East, Department of Hepatobiliary Pancreatic Oncology, Chiba, Japan

${ }^{8}$ Niigata Cancer Center Hospital, Department of Internal Medicine, Niigata, Japan

${ }^{9}$ Kyoto University, Department of Clinical Biostatistics, Kyoto, Japan

${ }^{10}$ Shizuoka Cancer Center, Clinical Research Center, Shizuoka, Japan

${ }^{11}$ Kyoto Prefectural University of Medicine, Department of Pulmonary Medicine, Kyoto, Japan

\section{Introduction}

The NEXTAC program was a multimodal intervention for elderly patients with advanced cancer to maintain their nutritional status and physical function during systemic chemotherapy.

\section{Objectives}

The aim of this presentation is to show algorithm of the exerciseprescription and its compliance.

\section{Methods}

Exercise-intervention combines daily low-intensity resistance training with physical-activity counseling using accelerometer. Three educational sessions were planned in 8-week study period. Exercise-prescription consists of 3 or 5 of following 5 exercises: sit-to-stand, calf-raise, knee-extension, knee-raise, and side-leg-raise with or without ankle-weight. Physiotherapist chose optimal prescription according to the modified Borg-scale. Patients performed each exercise 10-times repetition for 3 sets a day at home. A target step was calculated as adding 2000 steps on the average patient's steps with a maximum of 8000 steps. Instructors counseled patient to promote their indoor and outdoor activity. Compliance was assessed by diary. 


\section{Results}

Twenty-eight out of 30 patients attended all exercise sessions. Median proportion of days in which patients completed full of exercise program and performed full or modified exercise program was 41 and $91 \%$, respectively. Patients skipped or modified exercise program mainly due to feeling unmotivated or too ill to perform exercise. Patients kept or increased indoor or outdoor activity in 79 and $69 \%$ at the end of intervention, respectively. As a result, time spent in physical activity $(\geq 1.8$ METs $)$ increased in $69 \%$ of patients.

Conclusions

Our exercise program was feasible and promoted patient's voluntary participation in physical-activity for the elderly patients with advanced cancer.(Grant-in-Aid from Japan Agency for Medical Research and Development, Trial Registration No.UMIN000023207)

\section{eP560}

IMPACT OF A 11 WEEK EXERCISE PROGRAM IN HEAD AND NECK CANCER PATIENTS TREATED WITH RADICAL CHEMO-RADIOTHERAPY: A RANDOMIZED CONTROLLED TRIAL

S. Samuel ${ }^{\text {, A. }}$ Maiya ${ }^{2}$, D. Fernandes ${ }^{3}$

${ }^{T}$ Kasturba Medical College-Mangalore- Manipal Academy of Higher Education, Physiotherapy, Mangalore- Karnataka, India

${ }^{2}$ School Of Allied Health Sciences-Manipal Academy of Higher Education-Manipal, Physiotherapy, Manipal, India

${ }^{3}$ Kasturba Medical College- Manipal Academy of Higher Education-Manipal, Radiotherapy \& Oncology, ManipalKarnataka, India

\section{Introduction}

There is a dearth of literature regarding the effects of exercise training in Head and Neck Cancer patients receiving Chemo-radiotherapy. Increase in survival rates, owing to improvement in cancer care also authenticates the need for rehabilitation programs for this patient population.

Objectives

The primary objective of this study was to study the effectiveness of exercise based rehabilitation on Head and Neck Cancer patients receiving Chemo-radiotherapy on their functional capacity and Quality of Life while the secondary objective was to study the effect of exercise on fatigue and blood parameters Haemoglobin and platelets.

Methods

A Randomized controlled trial was conducted on 148 patients with Head and Neck Cancer undergoing Chemo-radiotherapy to evaluate the effectiveness of exercise on functional capacity, Quality of Life,Fatigue \& blood parameters .The control group received Physical Activity recommendations along with Standard care, while the exercise group received a structured exercise program for a period of 11 weeks ( 7 weeks in hospital during CRT and 4 weeks home based).

\section{Results}

There was a significant improvement in the functional capacity $(\mathrm{p}<.001)$, quality of life $(p<.001)$ and decrease in fatigue $(p<.001)$ in the exercise group while the blood parameters did not show a significant difference between the groups.

\section{Conclusions}

Our results show that a 11 week exercise training program in Head and Neck Cancer patients receiving Chemo-radiotherapy helps in maintaining and improving the functional capacity and Quality of Life and prevents deterioration of fatigue levels in the exercise group.
eP561

FACTORS INFLUENCING NECK AND SHOULDER FUNCTION AFTER ORAL ONCOLOGY TREATMENT: A FIVE-YEAR PROSPECTIVE COHORT STUDY IN 123 PATIENTS

C. Speksnijder ${ }^{l}$

${ }^{T}$ UMC Utrecht, Oral and Maxillofacial Surgery and Special Dental Care, Utrecht, The Netherlands

\section{Introduction}

Insight into late effects is critical for maximizing function of neck and shoulders and minimizing symptom burden in long-term survivors of oral cancer.

Objectives

The aim of this study was to identify factors influencing shoulder and/or neck function in patients treated for oral oncology up to five years after treatment.

\section{Methods}

Lateral flexion of the neck, ipsilateral forward flexion, and abduction of the shoulder were measured. Potential factors were entered into a linear mixed model analysis to create a multivariate model for describing the results.

\section{Results}

Neck and shoulder function were negatively influenced by higher age before intervention. Ipsilateral shoulder abduction and forward flexion were worse when there was a bigger neck dissection, and when a oral reconstruction was needed. Thereby, abduction was worse in women and anteflexion was worse when the tumor was more extended and related to the location of the oral tumor.

\section{Conclusions}

This study identified high risk patients for neck and/or shoulder impairments over a five year follow up.

\section{eP562}

IMPROVEMENT OF REFRACTORY LOWER EXTREMITY EDEMA WITH MULTILAYERED COMPRESSION BANDAGING: A CASE REPORT

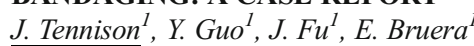

${ }^{T}$ The University of Texas MD Anderson Cancer Center, Department of Palliative- Rehabilitation- and Integrative Medicine, Houston, USA

\section{Introduction}

Lower extremity edema, which can be caused by several factors, is often poorly managed with commonly prescribed compression stockings and diuretics. Compression bandaging is the standard of care for lymphedema and venous edema, while the standard treatment for refractory peripheral edema is diuretics. Unless a rehabilitation specialist is already involved in a patient's care, referral to a lymphedema-certified therapist for compression bandaging to treat refractory edema is rare.

\section{Objectives}

This case demonstrates that compression bandaging can be successful in the management of refractory peripheral edema.

\section{Methods}

A lymphedema-certified therapist performed lower extremity multilayered compression bandaging and obtained serial limb circumference measurements. Regular weight measurements were also obtained.

\section{Results}

The patient's weight decreased from $94.5 \mathrm{~kg}$ on compression bandaging day 1 to $86.3 \mathrm{~kg}$ on day 7 . The circumferences of the affected limbs also decreased with compression bandaging (see Table 1).

Conclusions

This case demonstrates the utility of multilayered compression bandaging, typically used in the management of lymphedema, in the control of 
peripheral edema, refractory to diuretic therapy. More research is necessary to identify the most effective combination of physical therapy, compression therapy, and pharmacological treatment for patients with multiple comorbidities. Documenting serial limb circumference measurements and weight are essential outcome measurement tools with compression bandaging.

\section{eP563}

GROUP EXERCISE AS A STRATEGY IN IMPROVING ATTITUDE TOWARDS PHYSICAL ACTIVITY AMONG CANCER SURVIVORS: EXPERIENCES FROM A LOW RESOURCE SETTING

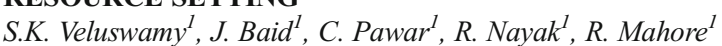

${ }^{T}$ MS RAMAIAH MEDICAL COLLEGE, Physiotherapy, Bangalore, India

\section{Introduction}

Exercise Interventions have been shown to positively influence complication of cancer therapies such as fatigue, anxiety, exercise intolerance, functional impairment and quality of life. Culturally, there is lack of positive attitude towards exercise in general population in India. The challenge to improve exercise and physical activity attitude among cancer survivors is far greater.

\section{Objectives}

Describe the experience of using group exercise as a means to improve attitude towards physical activity among cancer survivors in a low resource setting

Methods

Design: A retrospective mixed-methods analysis of department statistics on exercise participation among cancer survivors and patient experiences towards exercise.

Setting: Oncology in-patient services of a medical teaching hospital.

Program and Intervention: Internal audit of in-patient physiotherapy services for cancer survivors revealed negative attitude towards physical activity, resulting in poor participation in exercise program. Group exercise was tried as a strategy to improve attitude and participation in exercise program. Exercise classes were conducted five-days a week.

\section{Results}

A total of 139 survivors were offered Physiotherapy services between Sep-Nov 2017. Ca Breast (25.8\%), Cervix (17.2\%), and HNC (10.8\%) contributed to nearly $50 \%$ of survivors. All were offered exercise counselling of which $83(59.7 \%)$ participated in a median (IQR) of $3(2,8)$ sessions. There was a positive change in survivors' attitude towards exercise. Exercise leaders emerged who motivated other survivors to participate in group exercise sessions. This led to improvements in survivors exercise capacity and independence in ADL.

Conclusions

Group exercise can be a useful strategy to motivate cancer survivors to exercise and improve their physical activity levels.

\section{eP564}

\section{TRISMUS AND ITS INFLUENCING FACTORS IN ORAL} CANCER PATIENTS

K. Pei-Ling ${ }^{1}$, W. Tsae-Jyy ${ }^{2}$, L. Ming-Hsiou ${ }^{3}$, C. Wei Wei ${ }^{4}$, L. Shu-Chiung ${ }^{5}$

${ }^{1}$ University of Kang Ning, School of Nursing, Taipei City, Taiwan R.O.C.

${ }^{2}$ National Taipei University of Nursing and Health Science, School of Nursing, Taipei City, Taiwan R.O.C.

${ }^{3}$ Tri-service General Hospital, Department of Nursing, Taipei City, Taiwan R.O.C.

${ }^{4}$ Tzu Chi Hospital, Department of Cardiothoracic Surgery, Hualien, Taiwan R.O.C.

${ }^{5}$ Veteran General Hospital, Department of Nursing, Taipei, Taiwan R.O.C.

\section{Introduction}

Many oral cancer survivors suffer from trismus. This complication has major adverse impacts on individual quality of life. Knowledge regarding the risk factors for trismus is essential for developing intervention to prevent or effectively manage this devastating complication.

\section{Objectives}

The aim of the study was to explore the prevalence of trismus and its influencing factors in oral cancer patients.

\section{Methods}

A longitudinal descriptive correlation design was used. Sixty-five oral cancer patients were recruited from two medical centers in northern Taiwan. The maximum incisal opening was assessed before their surgery, discharge, and one month, three months, and six months post surgery.

\section{Results}

$70.2 \%$ and $78.9 \%$ of the study participants had trismus prior to and after receiving oral cancer excision surgery respectively. The mean maximum incisal opening distance was $31.0(\mathrm{SD}=9.2)$ prior to surgery, $27.6(9.8)$ and 27.0(10.5) one month and three months post surgery respectively. Advanced cancer stage and practicing oral exercise were important predictors of trismus. Only $56.1 \%(n=32)$ of the study participant did oral rehabilitation exercise after cancer excision surgery. 18 (31.6\%) study participants had not received any information regarding oral rehabilitation exercise.

\section{Conclusions}

Patients with advance stage of oral cancer showed higher risk for developing trismus. Medical professionals should pay attention to this high risk group and provide them adequate oral rehabilitation exercise. Some participants quick oral rehabilitation exercise due to pain $(n=9)$ and feeling not being effective $(n=7)$. Future studies should develop and test intervention for reducing discomfort during oral rehabilitation exercise and to enhance exercise adherence.

\section{eP565}

USING GUIDANCE TO IMPROVE LYMPHOEDEMA SERVICES FOR PEOPLE LIVING WITH AND BEYOND CANCER (LWBC): THE EXPERIENCE OF THE TRANSFORMING CANCER SERVICES TEAM (TCST) IN LONDON

K. Robb ${ }^{1}$, L. Price ${ }^{1}$

${ }^{T}$ Healthy London Partnership/National Health Service England, Transforming Cancer Services Team, London, United Kingdom

\section{Introduction}

Lymphoedema can occur when the lymphatic system is damaged by cancer or cancer treatment. It can significantly impact on individuals and the health care system. The National Health Service in London has prioritised lymphoedema care, but gaps in services and workforce remain. Objectives

The TCST developed guidance to assist decision-makers to improve lymphoedema services. Work focused on adults LWBC, with the view that additional services would also benefit.

\section{Methods}

Development involved:

- A multidisciplinary steering committee

- Collaboration with a wide range of stakeholders

- Mapping specialist lymphoedema services including challenges facing decision-makers

- $\quad$ Publication of a guidance document, service specification and business case.

Implementation involved:

- Influencing the geographical areas where workforce and service provision was most challenged 
- Key messaging around the economic argument for change

- Supporting the development of local action plans

Results

- The guidance has been widely disseminated

- A review of wound care and lymphoedema in North East London has led to plans for a new fully integrated service

- A new community of practice is supporting the workforce and driving service improvements

- TCST work has influenced and shaped national guidance by the voluntary sector.

Conclusions

TCST has influenced measurable improvement in lymphoedema care across London. This has occurred by identifying gaps in service delivery and outlining what good care looks like. Learning from this piece of work is being used across the UK, but is also applicable to an international audience.

\section{eP566}

DEVELOPMENT AND FEASIBILITY TESTING OF 'CHAT' AN ADAPTIVE ONLINE HOLISTIC NEEDS ASSESSMENT TOOL FOR PATIENTS WITH CANCER

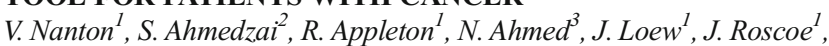
J. Dale

${ }^{1}$ University of Warwick, Warwick Medical School, Coventry, United Kingdom

${ }^{2}$ National Specialty Lead for Cancer Research, NIHR CRN Cancer Cluster, Leeds, United Kingdom

${ }^{3}$ University of Sheffield, Health Services Research Section-ScHARR, Sheffield, United Kingdom

\section{Introduction}

Patients with cancer have changing needs relating to physical, psychosocial, financial and other consequences of disease or treatments. Holistic needs assessment (HNA) is valuable for planning care and helping patient self-management. However current paper-based and fixed electronic HNA tools are rigid, cannot adapt to changing situations and consequently may not be completed.

Objectives

To develop and pilot an adaptive HNA system.

Methods

We have developed an online adaptive HNA called Composite Holistic Adaptive Tool (CHAT). This allows patients to determine which of several domains they want to complete, and also the depth of detail they want to enter for each domain. According to the HNA responses, patients can be alerted to online resources via embedded links. 'Red flag' symptoms can be alerted to healthcare professionals. We first developed CHAT for use in prostate cancer patients (CHAT-P). The completed package has now been used in a feasibility trial (ICARE-P) of patients attending their primary care practitioners and hospital specialists.

Results

41 men with prostate cancer were recruited from 14 general practices in the Birmingham, UK area -29 to the group who were offered CHAT-P and 12 to a control group with conventional care. Median age was 72 years (range 65-79). Responses have been positive and even men with limited or no experience of digital technology have been enthusiastic. Preliminary indications are that men are using the system to identify a range of concerns, with psychological and social issues being particularly prominent.

Conclusions

CHAT is an innovative HNA technology which is feasible in clinical practice.
eP567

\section{THE ACCURACY OF IMMINENT DEATH DIAGNOSIS IN} PALLIATIVE CARE SETTING

A. alsuhail ${ }^{1}$

${ }^{T}$ KING FAHAD MEDICAL CITY, Palliaitve care department, RIYADH, Saudi Arabia

\section{Introduction}

Prognostication or estimation of time of death is important for the patients and their family members as they need this information for preparation and planning their future and for the physicians as they desire to be accurate in their prognostication skills in order to plan and deliver better care.

Objectives

The study aims to evaluate the current situation regarding the accuracy of palliative care physicians' clinical predictions of time of death from initiation of imminent death protocol. The research hypothesis suggests that patients will die within 14 days' post imminent death diagnosis (IDD).

\section{Methods}

The imminent death protocol was developed for very sick patients who were expected to survive for less than two weeks. In order to initiate the imminent death protocol, the patient has to be assessed by two palliative care physicians who can diagnose a patient for imminent death. The time between imminent death diagnosis (IDD) and death will be documented.

Results

During study period, 48 patients died in PCU. The majority $28 / 48$ (58\%) died with imminent death diagnosis (IDD). In IDD group, 25/28(89.3) died within 14 days of diagnosis while $3 / 28(10.3 \%)$ died after 14 days. The median survival is 50 hours (CI 35-85).

\section{Conclusions}

Imminent death diagnosis (IDD) is a critical skill for Palliative care physicians to make a proper care plan. Our study showed that $90 \%$ of PCU patient died within 14 days' post imminent death diagnosis. The median survival was two days. We recommend further study to explore how IDD could improve care plan for dying patients.

\section{eP568}

RANDOMIZED TRIAL TO EVALUATE THE IMPACT IN OVERALL SURVIVAL OF EARLY PALLIATIVE CARE INTERVENTION AMONG METASTATIC BREAST CANCER PATIENTS. PRELIMINARY RESULTS.

C.H. Arce-Salinas ${ }^{1}$, E. Monreal ${ }^{2}$, L. Mendoza-Galindo ${ }^{1}$, S. AllendePerez $^{2}$, R. Ramirez-Morales ${ }^{3}$, C. Arzate-Mireles ${ }^{2}$, D. Perez-Camargo ${ }^{4}$, D. Flores-Diaz ${ }^{1}$, E. Verastegui-Aviles ${ }^{2}$, J.A. Matus-Santos ${ }^{\text {, }}$ L.A. LopezRojas $^{1}$

${ }^{I}$ Nacional Cancer Institute - Mexico City INCan, Breast Cancer, Mexico City, Mexico

${ }^{2}$ Nacional Cancer Institute - Mexico City INCan, Palliative Care, Mexico City, Mexico

${ }^{3}$ Nacional Cancer Institute - Mexico City INCan, Clinical Research, Mexico City, Mexico

${ }^{4}$ Nacional Cancer Institute - Mexico City INCan, Nutrition Deparment, Mexico City, Mexico

\section{Introduction}

Usually palliative care is giving at the end of life, however at our Institution, only $10 \%$ of patients received this intervention, besides that WHO guidelines recommended the early supportive care introduction. In lung cancer patients has been demonstrated 
benefit in overall survival when received in conjunction with oncological treatment supportive care. However, in breast cancer has not been demonstrated the benefit.

\section{Objectives}

To evaluate the impact in overall survival with early palliative care intervention, among metastatic breast cancer patients

Methods

Newly diagnosed or relapsed metastatic breast cancer were included, patients were randomized to standard care given by the attending physician (control arm) or intervention by palliative team, which included psychological, nutritional and symptom support (experimental arm). Patients were stratified according to ER/PR, and HER2 status (positive vs negative). Local ethical committee approved protocol.

Results

53 patients were included, $33(62 \%)$ were assigned to experimental arm and $20(38 \%)$ to control arm, patients in experimental arm have visceral disease in $72.7 \%$ (24), and $31.2 \%$ have more than 3 sites of metastatic disease in comparison with $75 \%(15)$ and $45 \%$ of patients in control arm respectively, $p=0.306$. At median follow-up of 30 months (2-36) there were no difference in overall survival among groups $36 \%$ and $47 \%$ respectively are still alive $\mathrm{p}=0.806$

\section{Conclusions}

This small trial have failed to demonstrated benefit in overall survival with early palliative care intervention. However, other benefits like quality of life and symptom control should be evaluated. Sample size should be increased to evaluate the benefit of the intervention

\section{eP569}

\section{COMPREHENSIVE CHARACTERIZATION OF PREMATURE CORONARY HEART DISEASE IN THE NHLBI LONG TERM TRANSPLANT SURVIVOR COHORT}

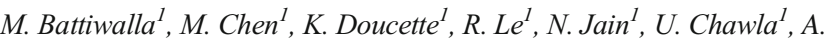
Biancotto $^{2}$, E. Koklanaris ${ }^{l}$, A. Remaley ${ }^{I}$, P. Anandi ${ }^{l}$, R. Childs $^{l}$, S. Ito ${ }^{l}$, P. Pophali $^{1}$, X. Tian ${ }^{1}$, J. Barrett ${ }^{I}$

${ }^{I}$ National Institutes of Health, National Heart Lung and Blood Institute, Bethesda, USA

${ }^{2}$ National Institutes of Health, National Institute of Allergy and Infectious Diseases, Bethesda, USA

\section{Introduction}

Premature coronary heart disease (CHD) complicates hematopoietic cell transplantation (HCT) survivor-ship. Allogeneic HCT has a 3 -fold higher incidence than autologous HCT. The long latency before event, poor understanding of the unique patho-biology and lack of validated screening techniques have been challenging.

Objectives

To understand the incidence and patho-biology of CHD in the NHLBI long term HCT survivor cohort study.

Methods

Comprehensive analysis included transplant and traditional cardiac risk variables; Framingham risk score calculation; cardiac $\mathrm{CT}$ scans (angiography plus calcium scoring) to unequivocally identify early CHD; NMR-based serum lipid profiling and proteomic (SOMAscan) for unbiased bio-marker discovery. Statistical methodology included multivariable modeling, ROC-curves and random forest methodology (and machine learning)

Results

At a median duration of 8 years post HCT, $\sim 43 \%$ of allogeneic HCT survivors had completely asymptomatic early CHD. Conventional
Framingham risk score was extremely insensitive, with $87 \%$ categorized as "low-risk". Chronic graft versus host disease was not associated with CHD. Surprisingly, abnormal triglyceride particles (but not HDL or LDL) identified by NMR lipid profiling were strongly associated with CHD. Troponin T (TNNT2), Troponin I (TNNI2),Interleukin-1 Receptor accessory protein (IL1RAP) and Ectonucleoside triphosphate diphosphohydrolase 5 (ENTPD5) were identified as biomarkers (out of $>1300$ analytes) associated with early CHD (76\% vs. $9 \%$, OR30.5, $\mathrm{P}=2 \times 10-9$ ).

\section{Conclusions}

Comprehensive characterization of our long term HCT survivor cohort has yielded valuable insights into premature CHD in this susceptible population.

\section{eP570}

PROGNOSTIC ROLE OF COMORBIDITY IN COLORECTAL CANCER PATIENTS: RESULTS FROM A POPULATIONBASED STUDY IN GERMANY

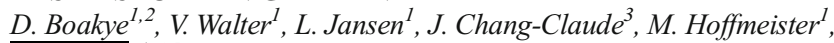
H. Brenner ${ }^{1,4,5}$

${ }^{I}$ German Cancer Research Center DKFZ, Clinical Epidemiology and Aging Research, Heidelberg, Germany

${ }^{2}$ Heidelberg University, Medical Faculty Heidelberg, Heidelberg, Germany

${ }^{3}$ German Cancer Research Center DKFZ, Unit of Genetic Epidemiology- Division of Cancer Epidemiology, Heidelberg, Germany

${ }^{4}$ German Cancer Research Center DKFZ, German Cancer Consortium DKTK, Heidelberg, Germany

${ }^{5}$ German Cancer Research Center DKFZ and National Center for Tumor Diseases NCT, Division of Preventive Oncology, Heidelberg, Germany

\section{Introduction}

There is limited evidence on the prognostic role of comorbidity among patients with colorectal cancer (CRC) and whether the prognostic impact varies by stage, site or age. Such evidence might provide better insight into the necessity of geriatric assessment in specific populations.

\section{Objectives}

We investigated the association of comorbidity and CRC prognosis in a population-based setting.

\section{Methods}

We extracted ICD-10 codes of comorbidities reported in the discharge records of 3950 CRC patients who were diagnosed in 2003-2012 and recruited into a population-based study on CRC in Germany. Overall comorbidity was then quantified with the Charlson comorbidity index (CCI). Hazard ratios (HR) and their 95\% confidence intervals (CI) from multivariable Cox regression were used to examine associations with 5-year overall, CRC-specific, disease-free and recurrence-free survival.

Results

Comorbidity was associated with decreased overall [CCI1 $(\mathrm{HR}=1.27 ; 95 \% \mathrm{CI}=1.08-1.49)$, CCI 2 ( $\mathrm{HR}=1.51 ; 95 \% \mathrm{CI}=1.27$ 1.79), $\mathrm{CCI} \geq 3(\mathrm{HR}=1.99 ; 95 \% \mathrm{CI}=1.66-2.38)]$, CRC-specific [CCI $1(\mathrm{HR}=1.16 ; 95 \% \mathrm{CI}=0.95-1.41)$, CCI $2(\mathrm{HR}=1.28 ; 95 \% \mathrm{CI}=1.02-$ 1.60), $\mathrm{CCI} \geq 3(\mathrm{HR}=1.46 ; 95 \% \mathrm{CI}=1.13-1.88)]$ and disease-free survival [CCI 1 (HR=1.18; 95\% CI=1.01-1.38), CCI 2 $(\mathrm{HR}=1.39 ; 95 \% \quad \mathrm{CI}=1.17-1.65), \mathrm{CCI} \geq 3 \quad(\mathrm{HR}=1.75,95 \%$ $\mathrm{CI}=1.47-2.10)]$. Comorbid CRC patients also had poor recurrence-free survival, but the association was significant only in patients with $\mathrm{CCI} \geq 3 \quad(\mathrm{HR}=1.32 ; 95 \% \mathrm{CI}=1.05-1.65)$. Particularly pronounced effects of comorbidity on prognosis were observed in rectal cancer, early stage and younger patients. 

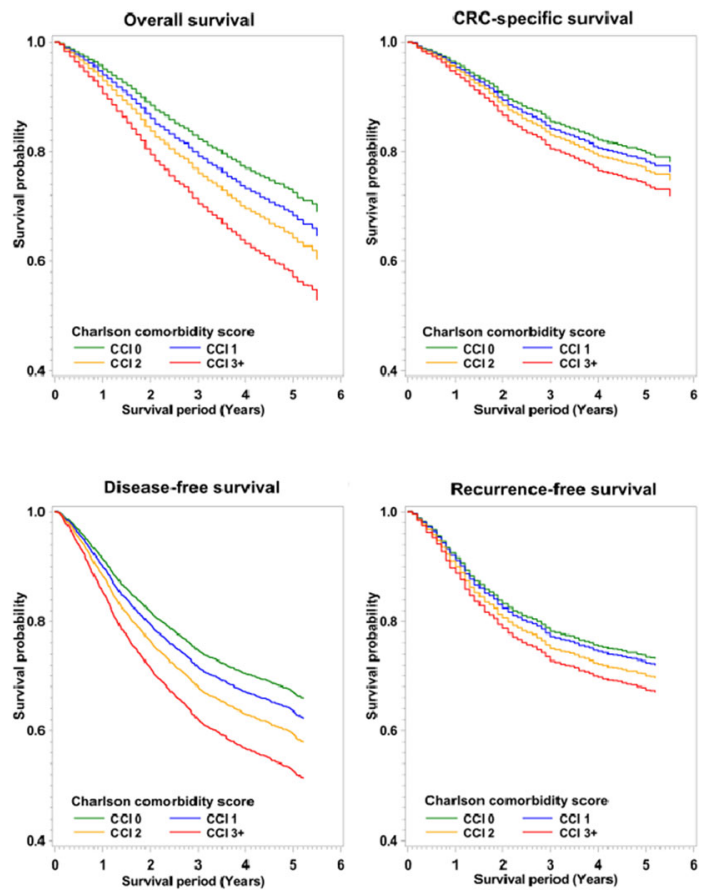

\section{Conclusions}

Comorbidity is strongly associated with CRC prognosis and its effect potentially differs by site, stage and age. The extent to which geriatric assessment might improve CRC care or prognosis should be evaluated by future studies.

\section{eP571}

\section{SYSTEMATIC REVIEW OF THE IMPLEMENTATION AND IMPACT OF HOLISTIC NEEDS ASSESSMENTS FOR PEOPLE AFFECTED BY CANCER, WITH AN EMPHASIS ON SURVIVORSHIP}

K. Campbell ${ }^{1}$, J. Young ${ }^{1}$, L. Johnston ${ }^{1}$

${ }^{T}$ Edinburgh Napier University, School of Health and Social Care, Edinburgh, United Kingdom

\section{Introduction}

International government and clinical policy recommends individuals affected by cancer should have their holistic needs assessed. Implementation of holistic needs assessments (HNA) is however heterogeneous.

Objectives

The objective of the systematic review is to answer "Does the way in which HNA is implemented, impact on the outcomes?"

Methods

MEDLINE, AMED, CINAHL, PsycINFO and the Psychological and Behavioural Sciences Collection were searched (2000 - 2017). Search strategy ensured inclusion of international HNA tools and synonyms for 'needs'. The Mixed Methods Appraisal Tool was applied to assess quality not exclude studies.

Results

24/ 851 met the inclusion criteria: randomised controlled trials $(n=4)$, local service evaluations $(n=9)$ and feasibility studies on the use of HNA $(n=11)$. Variability in assessment tool, purpose, approach and post assessment actions were found. Evidence on patient outcomes is diffuse and dominated by those of measurable interest to researchers. There is evidence on the way in which HNA is implemented impacts on both process and subsequent primary outcomes.

\section{Conclusions}

This review of global studies brings into sharp focus the gap in rigorous evidence on the impact of HNA and in our understanding of 'how' assessment can actually improve outcomes. It identifies that the critical, process and intermediate outcomes of HNA and care planning are under researched. There is a need to reconceptualise HNA as a means to an end and not an end in itself. This will help to identify key service priorities and relevant measures of success in meeting the needs and enhancing future self-management in this heterogeneous and complex population.

eP572

A LONGITUDINAL QUALITATIVE STUDY OF TRANSFORMATIONAL CHANGE: INDICATORS OF POSITIVE CHANGE IN CANCER AFTER CARE SERVICES.

K. Campbell ${ }^{1}$, L. Johnston ${ }^{1}$

${ }^{T}$ Edinburgh Napier University, School of Health and Social Care, Edinburgh, United Kingdom

\section{Introduction}

Edinburgh Napier University (ENU) have conducted a programme of educational research since 2011 on the transformational change of the Cancer Nurse Specialist (CNS) workforce. In addition, since 2014, ENU is carrying out an evaluation of 'The Transforming Care after Treatment' (TCAT) programme in Scotland: implementation of 25 projects, each with different local approaches to supporting people to live with and beyond cancer. A key evaluation question is "To what extent has the programme been successful at influencing attitudes, behaviours and priorities related to after care locally, regionally and nationally?

\section{Objectives}

To showcase our results from two programmes of research, highlighting indicators of positive change of CNS practice over the last seven years. Methods

A longitudinal mixed methods study of two key projects in transformational change. Data collected from focus group $(\mathrm{n}=6)$ discussions with CNS (2011); survey of TCAT wider stakeholders $(n=195)$ (2015 and 2016); local project focus group, at two time points $(n=5)$; stakeholders at two time points $(\mathrm{n}=10)$.

\section{Results}

Data prior to the TCAT programme showed that despite policy developments relating to cancer survivorship, challenges remained in terms of the interface between different professional roles and responsibilities. Pre and post project focus groups reveals integrated working and partnerships evolving due to building infrastructure to strengthen referral and signposting pathways.

\section{Conclusions}

By combining two programmes of research Edinburgh Napier University has been able to demonstrate significant transformational change over 5 years influencing attitudes, behaviours and priorities related to after care of people with cancer locally, regionally and nationally.

\section{eP573}

FEAR OF CANCER RECURRENCE IN CHINESE CANCER SURVIVORS: A CROSS-SECTIONAL STUDY

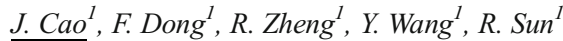

${ }^{T}$ Tianjin Medical University Cancer Institute and Hospital, Nursing Department, Tianjin, China

\section{Introduction}

Fear of cancer recurrence (FCR) is the most common concern and distressing experience for cancer survivors. High FCR levels could lead 
to distress, low quality of life, increased medical expenses and limited plans for future. However, little is known about Chinese cancer survivors' FCR, since China has the largest number of cancer survivors in the world. Objectives

The aim is to explore FCR of cancer survivors and to analyse its influence factors in the Chinese cultural context, and to provide valuable evidence for cancer survivors' FCR intervention development.

Methods

Two hundred cancer survivors, who had finished hospital-based therapies and were during follow-up period, were recruited from June 2017 to September 2017 in a city, China. The questionnaires, including demographic and disease characteristics, the Concerns about Recurrence Questionnaire(CARQ-4) and Mishel Uncertainty in Illness Scale for Adults(MUIS-A), were distributed to the potential participants.

Results

Ninety-two participants completed the questionnaire. Nearly $87.9 \%$ respondents reported clinical FCR (the scores of FCR $>12$ ). There was a strong relationship between FCR and uncertainty in illness $(r=0.314$, $p=0.002)$. The survivors who had significantly greater FCR had been diagnosed more than five years $(p=0.002)$, lower income $(p=0.005)$, no medical insurance $(p=0.049)$, undergone $\operatorname{surgery}(p=0.034)$ and unemployed $(p=0.032)$. By multiple linear stepwise regression analysis, four factors were proved to be significantly independent predictors, including uncertainty in illness, back to work, undergone surgery and medical insurance.

\section{Conclusions}

FCR was frequent among Chinese cancer survivors, and it has connections with uncertainty in illness, back to work, undergoing surgery and medical insurance. Further research is needed to develop appropriate interventions to decrease it.

\section{eP574}

\section{A MOBILE-BASED MULTI-SYMPTOM MANAGEMENT PROGRAM ON SYMPTOM BURDEN AND QUALITY OF LIFE OF BREAST CANCER SURVIVORS: A PILOT STUDY

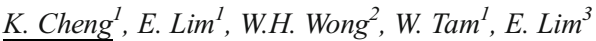 \\ ${ }^{T}$ National University of Singapore, Nursing, Singapore, Singapore \\ ${ }^{2}$ National University Hospital, Surgery, Singapore, Singapore \\ ${ }^{3}$ National Cancer Centre Singapore, Medical Oncology, Singapore, \\ Singapore}

\section{Introduction}

Breast cancer survivors (BCS) often experience side effects and symptoms post-treatment which can increase symptom burden and affect quality of life (QOL).

Objectives

This study aimed to develop a mobile-based symptom management program for BCS, and determine its feasibility and preliminary effects on symptom burden, psychological distress and QOL.

\section{Methods}

Female BCS aged 21 or above diagnosed with breast cancer between stage 0 -III and had completed treatment within five years were recruited. Phase one used individual qualitative interviews to explore the needs, perceptions and preferences for symptom management. Phase two was a pilot, randomized controlled trial to determine the preliminary intervention effects on symptom burden, psychological distress and QOL.

Results

Nine participants participated in phase one. The five categories which emerged were: experiencing diverse post-cancer treatment symptoms; dealing with post-cancer treatment symptoms; mismatched expectations between supply and demand of support/resources from hospital and healthcare professionals; demand for technology-based symptom management programms; and enhancing usage of technology-based symptom management programme. 17 subjects participated in phase two.
Significant within-group differences were found from baseline to postintervention in the intervention group for the Hospital Anxiety and Depression Scale anxiety sub-scale score and the Memorial Symptom Assessment Scale Global Distress Index score. The study implementation process was smooth and coordinated, and improvements were made to the main study based on information collected on study feasibility.

\section{Conclusions}

The findings suggest the potential of the intervention to contribute positively towards symptom management in BCS post-treatment.

\section{eP575}

IMPAIRMENT-DRIVEN PATTERNS OF HEALTH CARE UTILIZATION AMONG BREAST CANCER SURVIVORS OVER TWO DECADES FOLLOWING TREATMENT

A. Cheville ${ }^{1}$

${ }^{T}$ Mayo Clinic, Physical Medicine and Rehabilitation, Rochester, USA

\section{Introduction}

The impact of treatment-related impairments remains under-researched and its effect on healthcare (HC) utilization is unknown.

\section{Objectives}

To delineate the magnitude and patterns of $\mathrm{HC}$ utilization between breast cancer (BC) survivors with and without one of its most common, remediable and functionally limiting sequelae; upper extremity lymphedema. Methods

Design, Setting, Participants: We conducted a population-based, retrospective longitudinal cohort study of Olmsted County, MN residents who were newly diagnosed with $\mathrm{BC}$ in the 21 year period beginning on January 1, 1990 and concluding December 31, 2010. HC utilization was characterized using the Berenson-Eggers Type of Service (BETOS) categories. Overall $\mathrm{HC}$ utilization as well as its compartmentalization into the BETOS categories of: 1) Evaluation and management, 2) Procedures, 3) Imaging, 4) Tests, 5) Durable medical equipment, 6) Physical/ occupational therapy, 7) Other, and 8) Exceptions/Unclassified.

\section{Results}

The cohort included 1906 subjects of which 94\% (1800) had records meeting the inclusion criteria. Mean follow-up per survivor was 8.8 years (median, 8, range 1 - 23 years); 1239 were living and residing in Olmsted County at the study's conclusion. Analysis revealed that 1 ) survivors with BC-associated lymphedema used $>30 \%$ more services annually than their unaffected counterparts; 2) their increased utilization lessened but persisted for at least 10 years after diagnosis; and 3) this finding of increased utilization is robust, extending across all BETOS categories, and is further amplified as BMI increases.

\section{Conclusions}

BC-related lymphedema is associated with a significant, $>30 \%$, increase in $\mathrm{HC}$ utilization that extends for a prolonged period.

\section{eP576}

SEVERE FATIGUE AND LONG TERM QUALITY OF LIFE AMONG EPITHELIAL OVARIAN CANCER SURVIVORS: A GINECO CASE/CONTROL VIVROVAIRE STUDY

D. Ahmed-Lecheheb ${ }^{1}$,E. Kalbacher ${ }^{2}$, D. Berton-Rigaud ${ }^{3}, O$. Tredan ${ }^{4}, M$. Fabbro $^{5}$, A.M. Savoye ${ }^{6}$, J.E. Kurtz ${ }^{7}, J$. Alexandre ${ }^{8}$, P. Follana ${ }^{9}$, V. Delecroix $^{10}$, N. Dohollou $^{11}$, C. Roemer-Becuwe ${ }^{12}$, G. De Rauglaudre ${ }^{13}$ A. Lortholary ${ }^{14}$, K. Prulhiere ${ }^{15}$, N. Heutte ${ }^{1}$, J.M. Grellard ${ }^{1}$, C. Lhommé $^{16}$, A. Floquet ${ }^{17}$, F. Joly ${ }^{18}$

${ }^{1}$ Centre François Baclesse, Clinical Research Department, Caen, France ${ }^{2}$ University Hospitla of Besançon, Oncology Department, Besançon, France

${ }^{3}$ Institut de Cancerologie de l'Ouest, Oncology Department, Nantes, France 
${ }^{4}$ Centre Léon Berard, oncology Department, Lyon, France

${ }^{5}$ Institut de Cancérologie de Montpellier, Oncology Department, Montpellier, France

${ }^{6}$ Centre Jean Godinot, Oncology Department, Reims, France

${ }^{7}$ University Hospital of Strasbourg, Oncology Department, Strasbourg,

France

${ }^{8}$ Assistance Publique - Hôpitaux de Paris- Hôtel Dieu, Oncology

Department, Paris, France

${ }^{9}$ Centre Antoine Lacassagne, Oncology Department, Nice, France

${ }^{10}$ Clinique Mutualiste de l'Estuaire, oncology Department, Saint Nazaire,

France

${ }^{11}$ Polyclinique Bordeaux Nord Aquitaine, Oncology Department, Bordeaux, France

${ }^{12}$ Centre d'Oncologie de Gentilly, Oncology Department, Nancy, France

${ }^{13}$ Institut Sainte Catherine, oncology Department, Avignon, France

${ }^{14}$ Centre Catherine de Sienne, Oncology Department, Avignon, France

${ }^{15}$ Institut du Cancer Courlancy Reims, Oncology Deparment, Reims,

France

${ }^{16}$ Gustave Roussy, Oncology Department, Villejuif, France

${ }^{17}$ Institut Bergonie, Oncology Department, Bordeaux, France

${ }^{18}$ Centre François Baclesse, Oncology and Clinical Research

Departments, Caen, France

\section{Introduction}

Epithelial ovarian cancer (EOC) prognosis remains poor but $\geq 40 \%$ of patients can have long survival. EOC and its treatments are deleterious on long term Quality-of-Life (QoL): fatigue, physical, functional disorders and various symptoms. Few data exist on QoL among EOC Survivors (EOCS).

Objectives

We conducted a case-control study comparing severe fatigue and QoL between EOCS without relapse $\geq 3$ years after first-line chemotherapy and age-matched healthy women.

Methods

Controls from whole population and EOCS from 27 French centers completed validated self-questionnaires: Fatigue, QoL, neurotoxicity (FACT/F,FACT/G,FACT/O-TOI,FACT/Ntx), anxiety/depression (HADS), sleep disturbance (ISI), physical activity (IPAQ). Severe fatigue was defined by FACT-F score $<37$ and sensitive neuropathy using the 6 dedicated FACT/Ntx items.

Results

318 pairs were analyzed. Median age: 65 (20-86). FIGO stage: (I/II $=49 \%$, $\mathrm{III} / \mathrm{IV}=47 \%$ ). Years from chemotherapy end (mean $=6 \pm 3$ ). EOCS experienced more frequently severe fatigue $(26 \%$ vs $13 \%, p=0.0004)$ and poor quality-of-sleep (63\% vs 47\%, $p=0.0003$ ). Similarly, EOCS reported lower functional wellbeing score (17.5 vs 19.7, $p=0.0002)$, poorer QoL score related to EOC and treatment (FACT/O-TOI, 72 vs 77, $p=0.001$ ), and poorer neurotoxicity FACT/NTx score ( 35 vs $39, p<0.0001$ ). Especially, $27 \%$ of EOCS complained about sensitive neuropathy. Half of EOCS and controls reported anxiety ( $53 \%$ vs $47 \%, \mathrm{p}=0.11$ ); more EOCS noticed depression $(22 \%$ vs $13 \%, \mathrm{p}=0.01)$. Active physical activity was noted for $18 \%$ of both groups.

Conclusions

This case-control study highlighted that EOCS presented more severe fatigue, with depression, sleep disturbance, poorer long term QoL related to EOC and treatment, associated with neurotoxicity complains. Such late treatments-effects should be considered by clinicians to adapt EOCS management

eP577

COGNITIVE IMPAIRMENT IN OLDER BREAST CANCER SURVIVORS: A COMPREHENSIVE REVIEW OF THE LITERATURE

A. Crouch ${ }^{1}$, D. Von $A h^{2}$
${ }^{1}$ Indiana University School of Nursing, Clinical Nursing Science, Indianapolis, USA

${ }^{2}$ Indiana University School of Nursing, Department of Community \& Health Systems, Indianapolis, USA

\section{Introduction}

Breast cancer is the most common cancer in women. Aging is the greatest risk factor for the development of cancer. With improved screening and treatment, more women are surviving; however, breast cancer survivors (BCS) can be left facing disturbing symptoms including cognitive impairment $(\mathrm{CI})$. Little research has been conducted in older BCS experiencing CI.

\section{Objectives}

The objectives of this integrative review were to: (1) describe the incidence of CI in older BCS, (2) identify the cognitive domains impacted and measures used to assess CI, and (3) determine factors associated with $\mathrm{CI}$ in older BCS.

\section{Methods}

Databases searched included PubMed, CINAHL, PsycINFO, and MEDLINE. This review focused on studies that examined CI in female BCS who were 65 years of age or older. Eligible studies were published in peer-reviewed journals, written in English, and measured CI subjectively or objectively. We excluded studies that included women with a dementia diagnosis.

Results

Eight quantitative studies met inclusion criteria. Six used prospective designs and 2 were cross-sectional.Before treatment, incidence of $\mathrm{CI}$ ranged between $11 \%-41 \%$ using objective measures and ranged from $6 \%-64 \%$ when subjective measures were used. Four primary cognitive domains of impairment were identified, including memory, executive function, speed of processing, and language/verbal functioning. Factors associated with $\mathrm{CI}$ included older age, increased number of comorbidities, poorer physical functioning, frailty and poorer quality of life.

\section{Conclusions}

$\mathrm{CI}$ is a common and significant problem in older BCS. Healthcare providers need to assess and address CI to promote quality of life in older BCS.

eP578

UNDERSTANDING HEALTHCARE PROVIDERS' ROLE IN CANCER SURVIVORS' EXPERIENCES RETURNING TO WORK AFTER PRIMARY TREATMENT: A LONGITUDINAL QUALITATIVE STUDY

E. Drake $^{1,2}$, R. Urquhart ${ }^{1,3}$

${ }^{T}$ Nova Scotia Health Authority, Department of Surgery, Halifax, Canada

${ }^{2}$ Dalhousie University, Faculty of Health, Halifax, Canada

${ }^{3}$ Dalhousie University, Department of Surgery, Halifax, Canada

\section{Introduction}

The number of cancer survivors is growing due to increased cancer incidence and improved cancer survival through earlier detection and better treatments. With cancer survivorship comes practical and economic implications, with many survivors reporting difficulties reintegrating into the work environment following an absence due to treatment.

\section{Objectives}

To explore cancer survivors' perspectives on and experiences with their healthcare providers' roles in their return to work (RTW) following primary cancer treatment.

\section{Methods}

A prospective qualitative longitudinal study, using in-depth telephone interviews (three interviews/survivor), was used to provide a rich understanding of how 13 cancer survivors experienced RTW. Data were analyzed using thematic analysis. 


\section{Results}

Preliminary findings indicate that (1) healthcare providers play an important role in their RTW process, but that oftentimes the system lacks adequate psychosocial care. Thus, many survivors feel (2) they are left to advocate for the provision of these services themselves and, as a result, their needs are often unmet or sub optimally met. When available and accessed, (3) specialist psychosocial oncology providers/programs helped patients deal with their diagnosis and the challenges that present after surviving cancer, often times connecting them with others who understand what they are going through and helping them with the social, emotional and practical components of the RTW process.

Conclusions

Preliminary results suggest that access to specialist psychosocial oncology providers/programs within the healthcare delivery model enhances survivors' RTW after primary treatment.

eP579

INTEGRATING SELF-MANAGEMENT INTO DAILY LIFE FOLLOWING TREATMENT FOR HEAD AND NECK CANCER: SURVIVORS' EXPERIENCES

S. Dunne ${ }^{I}$, L. Sharp ${ }^{2}$, R. Gooberman-Hill ${ }^{3}$, D. Desmond ${ }^{4}$, O. Eleanor ${ }^{5}$,

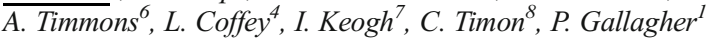

${ }^{1}$ Dublin City University, School of Nursing and Human Sciences, Dublin, Ireland

${ }^{2}$ Newcastle University, Institute of Health and Society, Newcastle, United Kingdom

${ }^{3}$ University of Bristol, School of Clinical Sciences, Bristol, United Kingdom

${ }^{4}$ Maynooth University, Department of Psychology, Maynooth, Ireland

${ }^{5}$ University College Cork, Dental School and Hospital, Cork, Ireland

${ }^{6}$ National Cancer Registry Ireland, National Cancer Registry Ireland, Cork, Ireland

${ }^{7}$ National University of Ireland Galway, Academic Department of Oto Rhino Laryngology, Galway, Ireland

${ }^{8}$ St. James's Hospital, Head and Neck Cancer Programme, Dublin, Ireland

\section{Introduction}

Self-management may help cancer survivors to better deal with challenges to their physical, functional, social, and psychological wellbeing presented by cancer and its treatment. Nonetheless, little is known about how people integrate cancer self-management practices into their daily lives.

Objectives

The aim of this study was to describe and characterise the processes through which head and neck cancer (HNC) survivors attempt to integrate self-management into their daily lives following primary treatment.

Methods

Using a purposeful critical case sampling method, $27 \mathrm{HNC}$ survivors were identified through four designated cancer centres in Ireland and participated in face-to-face semi-structured interviews. Interviews were audio-recorded, transcribed and analysed using thematic analysis.

Results

Six themes which describe HNC survivors' attempts to integrate selfmanagement into their daily lives following primary treatment were identified: grappling with having to self-manage, trying out self-management strategies, becoming an expert self-manager, struggling to integrate selfmanagement strategies into daily life, avoiding self-management and bracketing self-management.

Conclusions

This is the first study to describe HNC survivors' attempts to integrate self-management into their daily lives following primary treatment. The findings indicate that $\mathrm{HNC}$ survivors exhibit highly individualised approaches to self-management and abandon self-management strategies that fail to meet their specific needs. Survivors may also need skills training and structured support to assist their transition between inpatient care and having to self-manage after primary treatment, and/or ongoing support deal with persistent and recurring challenges such as eating difficulties and fear of recurrence.

\section{eP580}

HOLISTIC NEEDS ASSESSMENTS FOR TEENAGERS AND YOUNG ADULTS AFTER CANCER TREATMENT IN SCOTLAND IDENTIFIED A SIGNIFICANT BURDEN OF UNMET NEEDS

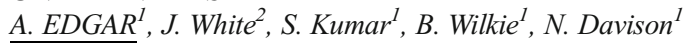

${ }^{T}$ Royal Hospital for Sick Children, Paediatric Oncology, Edinburgh, United Kingdom

${ }^{2}$ The Beatson West of Scotland Cancer Centre, Oncology, Glasgow, United Kingdom

\section{Introduction}

Psychosocial issues are common amongst Teenagers and young adults (TYA) after cancer treatment and TYA report feeling unsupported.

Objectives

Todetermine the burden of needs amongst TYA after cancer treatment and evaluate whether these needs are being met.

\section{Methods}

Scottish TYA (16-24 years) completing cancer treatment, between April 2016-April 2017, were identified. Treatment Summaries (TS) and Holistic Needs Assessment (HNA) were completed and distributed (TS only) to patients and health professionals. Qualitative analysis identified concerns.

\section{Results}

Fifty-one of 83 eligible TYA were recruited (response rate 61\%); 26 $(51 \%)$ males, median (range) age $22(16-25)$ years with overrepresentation of germ cell tumours (39\%), lymphomas (27\%), bone tumours (14\%). TS data was available for $51(100 \%)$ and raw HNA data for 26 patients (50\%). 45 TYA (88\%) reported concerns: 35 (69\%) reported 1-5 concerns; 2 (4\%) reported 6-10 concerns; 5 (10\%) reporting 11-15 concerns; 1 (2\%) reported 1 concern and $2(4 \%)$ reported $>20$ concerns: general appearance, physical fitness and emotional issues, were the most numerous. One third of TYA were referred for psychological support; two-thirds benefitted from third sector support programmes.

\section{Conclusions}

Almost $90 \%$ of TYA reported at least one concern after cancer treatment, with more than two-thirds of patients reporting five to 10 concerns, and $10 \%$ reporting up to 15 concerns. While support services, largely provided by third sector, are in place for many patients, further evaluation of the Health Needs Assessments, exploring relationships to diagnoses, and identification of gaps in services, are required to inform future developments.

\section{eP581}

INTRODUCTION OF TREATMENT SUMMARIES AND HOLISTIC NEEDS ASSESSMENTS FOR TEENAGERS AND YOUNG ADULTS AFTER CANCER TREATMENT IN SCOTLAND: A NATIONAL FEASIBILITY STUDY

A. EDGAR ${ }^{l}, J$. White ${ }^{2}$, S. Kumar ${ }^{1}$, B. Wilkie ${ }^{l}$

${ }^{T}$ Royal Hospital for Sick Children, Paediatric Oncology, Edinburgh, United Kingdom

${ }^{2}$ The Beatson West of Scotland Cancer Centre, Oncology, Glasgow, United Kingdom

\section{Introduction}

The Scottish Government recommends that all teenagers and young adults (TYA) treated for cancer, receive a Treatment Summary (TS) and 
Holistic Needs Assessment (HNA) to aid communication, education, self-management and identify areas of concern.

Objectives

i) To determine the feasibility of implementing TS and HNA for Scottish TYA after cancer treatment; and ii) to share TS with health professionals to improve communication and engagement.

Methods

Scottish TYA (16-24 years) completing cancer treatment, between April 2016 -April 2017, were identified at the weekly National TYA Multidisciplinary Team (TYA MDT) meeting. Treatment Summaries and HNA were completed and distributed to patients and health professionals.

Results

Of 83 eligible patients, 51 were recruited (response rate 61\%); $26(51 \%)$ males, median (range) age $22(16-25)$ years. Among non-recruits: 2 relocated, 1 relapsed, lead consultant deemed the process 'not relevant' $(n=6)$, or did not respond $(n=23)$. Cancer diagnoses: germ cell tumours (39\%), lymphomas $(27 \%)$ and bone tumours $(14 \%)$ over represented; carcinoma $(8 \%)$, melanoma $(2 \%)$ and CNS tumours $(2 \%)$ underrepresented.

\section{Conclusions}

Treatment Summaries and Holistic Needs Assessments were completed for almost two thirds of TYA identified after cancer treatment. Recruitment failure was due largely to consultant disengagement. Based on incidence data, current pathways do not allow identification of almost half of patients at the end of treatment. Completion of TS/HNA is labour intense and adequate time and training must be provided. By sharing Treatment Summaries we have improved communication and engagement with Primary Care and provided clear pathways for referral of patients to hospital when problems arise.

eP582

COST-EFFECTIVENESS ANALYSIS OF AN EXERCISE INTERVENTION TO TEST THE SAFETY AND EFFICACY OF PRESCRIBED EXERCISE IN PROSTATE CANCER SURVIVORS WITH BONE METASTASES

K. Edmunds ${ }^{1}$, P. Reeves ${ }^{1}$, D.A. Galvão ${ }^{2}$, R.U. Newton ${ }^{2}$

${ }^{T}$ Hunter Medical Research Institute, Health Research Economics, New Lambton Heights, Australia

${ }^{2}$ Edith Cowan University, Medical and Health Sciences, Joondalup, Australia

\section{Introduction}

The beneficial effects of prescribed exercise for prostate cancer survivors are widely known, yet few exercise intervention studies include survivors with bone metastases due to concerns of fragility fracture. These patients tend to have muscle atrophy and functional impairment from long term androgen deprivation resulting in considerable disease burden.

Objectives

This presentation reports on the first cost-effectiveness analysis (CEA) of a RCT of an exercise intervention for men with metastatic prostate cancer. Methods

The analysis was conducted from societal and healthcare perspectives. Mean costs were calculated for the intervention vs usual care. Outcome measures were the cost and incremental cost-effectiveness ratios for significant changes in body mass, physical activity and physical function. Quality adjusted life years (QALYs) were also calculated.

\section{Results}

The exercise program was both safe and effective. Significant mean differences were found between groups in a number of outcomes, however, significant differences between mean QALYs were not found. Compared with usual care, health sector costs were $\$ 352$ higher for the intervention. From the healthcare funder perspective, the incremental cost effectiveness ratio (ICER) for low intensity physical activity was $\$ 4.27$ (95\% UI \$3,
$\$ 11)$ per minute increase per week or $\$ 73.14(95 \%$ UI $\$ 45, \$ 190)$ per MET hour increase per week.

\section{Conclusions}

Prescribed exercise for prostate cancer survivors with metastatic bone disease is important in addressing the risk of fragility fractures and skeletal complications that cause significant morbidity and mortality and high treatment costs. More CEAs need to be conducted. For findings to be policy relevant, there are trial design implications.

\section{eP583}

\section{DO PEOPLE IMPROVE HEALTH BEHAVIOR AFTER THEIR PARTNER IS DIAGNOSED WITH CANCER? A DANISH COHORT STUDY}

N. Ezendam ${ }^{1}$, R. Karlsen ${ }^{2}$, J. Christensen ${ }^{3}$, T. Anne ${ }^{4}$, L. Van de Poll-

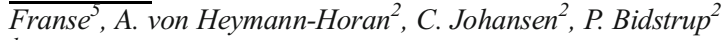

${ }^{I}$ Netherlands Comprehensive Cancer Organisation, Research, Utrecht, The Netherlands

${ }^{2}$ Danish Cancer Society Research Center, Survivorship, Denmark, Denmark

${ }^{3}$ Danish Cancer Society Research Center, Statistics- Bioinformatics and Registry, Denmark, Denmark

${ }^{4}$ Danish Cancer Society Research Center, Diet- Genes and Environment, Denmark, Denmark

${ }^{5}$ Netherlandsd Cancer Institute, Psychosocial Research and Epidemiology, Amsterdam, The Netherlands

\section{Introduction}

The cancer diagnosis might lead to behavioral change among partners of cancer patients.

\section{Objectives}

Assess if partners of cancer patients make more health behavior changes compared to persons whose partner remained cancer-free.

\section{Methods}

Nested in the Danish Diet, Cancer and Health study - a prospective cohort study - 672 couples where one person was diagnosed with cancer and 5534 couples who remained cancer-free were included. Questionnaires assessing diet, physical activity, smoking, alcohol and BMI were administered in 1993-1997 and 2000-2002. Logistic regression analyses were used to assess health behavior change among partners of cancer patients, and for the two subgroups of 1 . partners of patients who died after cancer and 2. partners of patients who remained alive after cancer.

\section{Results}

Compared to partners of persons who remained cancer-free: firstly, partners of cancer patients were more likely to decrease their alcohol intake, secondly, partners of persons who remained alive were less likely to decrease BMI, thirdly partners of persons who died after cancer were more likely to decrease alcohol intake, BMI and fruit intake, and to increase sugared beverages and fourthly, men tended to improve and women to worsen their physical activity following the cancer diagnosis of their partner.

\section{Conclusions}

Partners of cancer patients both improve and worsen certain health behaviors especially after the patient dies. Future research may assess effects of health behavioral change interventions for both cancer patients and their partners, as these interventions might be necessary to capitalize on the teachable moment of behavioral change.

\section{eP584}

LAUGHTER YOGA FOR CANCER PATIENTS, CANCER SURVIVORS AND CAREGIVERS: AN INITIAL EXPERIENCE

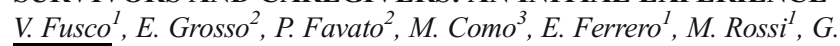
Numico 
${ }^{1}$ Azienda Ospedaliera di Alessandria, Oncology, Alessandria, Italy

${ }^{2}$ Chiara Luce ONLUS, Psychology, Novi Ligure, Italy

${ }^{3}$ Azienda Ospedaliera di Alessandria, Nursing staff, Alessandria, Italy

\section{Introduction}

Laughter Yoga is a practice codified since 1995 by the Indian doctor Madan Kataria, based on psychological and physiological positive effects of voluntary laughter, supposed to be equivalent to those ones derived from spontaneous laughter. Routine exercises include clapping, eye contact, body movement, breathing exercises, acting and visualization techniques, characterized by children-like playfulness. The final aim is to share consecutive minutes of breaking down inhibitions and laughing without any humorous reason

Objectives

Test Laughter Yoga as an aid to well-being, in cancer patients under active treatment and in cancer survivors, as well as in caregivers

Methods

Cancer patients (under adjuvant treatment or under therapy of wellcontrolled metastatic disease), cancer survivors (under regular follow-up check-up program), and their caregivers were invited to join Laughter Yoga bi-monthly meetings of an existing group (initially including nurses, doctors, hospital staff, charity voluntary team).

\section{Results}

Forty cancer patients and caregivers joined at least two meetings in six months. A satisfaction questionnaire revealed high positive evaluation in $90 \%$ and positive evaluation in $7.5 \%$. The median number of people attending the meetings increased from 14 to 28 per meeting, due to diffusion of invitation among cancer patients and their caregivers.

Conclusions

Preliminary data show high level of acceptance and satisfaction among cancer patients and survivors, and their caregivers. Strongest points of the experience are: well-being time sets shared by patients, caregivers and oncology staff ; easy process of acquiring knowledge of the techniques; emotional bonding.

\section{eP585}

CANCER SURVIVORSHIP NEEDS OF INDIGENOUS PEOPLE IN CANADA: A KNOWLEDGE TRANSLATION STRATEGY W. Gifford ${ }^{1}$, R. Thomas ${ }^{2}$ I.D. Graham ${ }^{3}$

${ }^{T}$ University of Ottawa, Faculty of Health Sciences School of Nursing, Ottawa, Canada

${ }^{2}$ University of Ottawa, Faculty of Health Sciences School of Rehabilitation, Ottawa, Canada

${ }^{3}$ Ottawa Hospital Research Institute- University of Ottawa, Centre for Practice-Changing Research-Department of Epidemiology \& Community Medicine-, Ottawa, Canada

\section{Introduction}

Cancer among Indigenous people in Canada is increasing faster than overall cancer rates.

Lack of culturally safe survivorship supports that incorporate traditional values and practices and recognize the historical impacts of colonization contributes to poor health outcomes and low 5year survival rates. Little has been done to address the unique survivorship needs of Indigenous peoples and few supports have considered the intersections of culture, history, and marginalization.

Objectives

To understand how healthcare approaches and practices can address the unique cancer survivorship needs of Indigenous people in Canada and improve healthcare delivery and outcomes.

Methods

As an early stage of a larger study, a research retreat was held with an Advisory Group $(n=13)$ of Indigenous Elders, traditional teachers, survivors, caregivers, providers, and non-Indigenous researchers to determine how survivorship supports can be developed and implemented. A video highlighting Indigenous people's experiences with cancer, developed from our previous photovoice study, was viewed and a focussed discussions of how messages from the video could be incorporated into survivorship practices. The meeting was audio-taped, transcribed and inductively analyzed.

Results

Five themes emerged as integral to Indigenous survivorship: healing supports (spirituality, nature, art, traditional medicines); cultural healthcare (holistic, patient-centered, community based); caregiver support; social isolation (art, leadership training, peer support); and system navigation. Art, community and culture were central to all themes. Based on results, a logic model is guiding implementation.

\section{Conclusions}

Collaboratively working with Indigenous people can facilitate understanding of their unique cancer survivorship needs to improve healthcare services, quality of life, and health outcomes.

\section{eP586}

WEIGHT LOSS AFTER BREAST CANCER AND INFLAMMATORY CYTOKINE RESPONSE IN OVERWEIGHT AND OBESE AFRICAN AMERICAN WOMEN

K. Griffith ${ }^{1}$, S. Zhu $^{2}$, A. Ryan ${ }^{3}$

${ }^{T}$ The George Washington University, School of Nursing, Washington, USA

${ }^{2}$ University of Maryland Baltimore, School of Nursing, Baltimore, USA

${ }^{3}$ University of Maryland Baltimore, School of Medicine, Baltimore, USA

\section{Introduction}

African American (AA) women are more likely to be overweight or obese at breast cancer (BC) diagnosis and following adjuvant treatment compared to whites. Increased adiposity in $\mathrm{BC}$ is associated with greater inflammation, cancer cell proliferation and cancer progression, and shorter survival.

\section{Objectives}

We investigated the influence of weight loss on inflammatory cytokines in overweight and obese AA women who had completed adjuvant treatment for breast cancer.

\section{Methods}

In this single-group longitudinal study participants $(n=22)$ received a tailored weight loss intervention. Cytokine measurements (IL-1b,IL-6, IL-8, TNF- $\alpha$ ) were evaluated at baseline (T1), after the 12-week intervention phase (T2), and post-maintenance at 24 weeks (T3). Bivariate linear regression was used to determine association between weight loss and change in cytokine levels. Participants hadamean (SD) age of 45.5 (6.9)(range 31-63), and body mass index (BMI) at baseline was $34.8(4.2) \mathrm{kg} / \mathrm{m}^{2}$ (range $\left.27.1-47.2 \mathrm{~kg} / \mathrm{m}^{2}\right)$.

\section{Results}

The majority of participants (59\%) had stage I or II BC, and had completed chemotherapy $(81 \%)$ and/or radiation $(77 \%)$. Overall mean weight change was 2.25 (2.5) kg from T1 to T2 (range +1.5 to $-9.1 \mathrm{~kg}$; $\mathrm{p}<.001$ ), which was associated with a reduction in IL-6 $(\mathrm{p}=.022)$ but not other cytokines.

\section{Conclusions}

Racially-disparate expression of IL-6 has been reported in AA BC patients, which is implicated in more aggressive BC. Our findings underscore the need for additional study of fat mass reduction influence on proinflammatory cytokine levels in order to evaluate influence on $\mathrm{BC}$ risk reduction in AA women. 
eP587

IMPACT OF SURVIVORSHIP CARE PLANS ON CANCER SURVIVORS' PATIENT-REPORTED OUTCOMES: A SYSTEMATIC REVIEW AND META-ANALYSIS

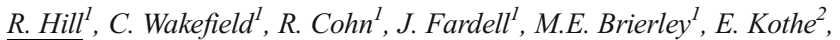
$\overline{K . H e t h e r i n g t o n}{ }^{I}$, R. Mercieca-Bebber ${ }^{3}$

${ }^{1}$ University of New South Wales, School of Women's and Children's Health- Faculty of Medicine, Kensington, Australia

${ }^{2}$ Deakin University, School of Psychology, Geelong, Australia

${ }^{3}$ The University of Sydney, NHMRC Clinical Trials Centre, Camperdown, Australia

\section{Introduction}

The Institute of Medicine (USA) recommends survivorship care plans (SCPs) as part of cancer survivorship care. However, several integrative and narrative reviews have found no difference in satisfaction with care/information, distress, or quality of life between cancer survivors who receive SCPs and those who do not (controls).

Objectives

To compare patient-reported outcomes (PROs) of cancer survivors who received SCPs versus controls.

\section{Methods}

We searched EMBASE, MEDLINE, PsycINFO, PubMed, CINAHL, Cochrane Databases and Google Scholar (inception-27 July 2017) and reviewed reference lists. Randomized studies comparing PROs of cancer survivors who received a SCP versus controls, reported in English, and reported data at comparable time points, were eligible for inclusion in the meta-analysis. Following data extraction by two authors, we used random effects models to pool standardized mean differences for each outcome, using R v3.4.0.

\section{Results}

Of 4461 identified articles, 10 were eligible, totalling 1582 participants. At 6-months post-intervention, there were no significant differences between the groups on physical functioning ( $g=-0.037,95 \%$ CI $[-0.17$, $0.09])$, satisfaction with information provision $(\mathrm{g}=0.131,95 \%$ CI $[-0.02$, $0.28]$ ) and self-efficacy $(\mathrm{g}=0.019,95 \% \mathrm{CI}[-0.22,0.26])$. There were also no significant differences between groups at 12-months post-intervention on anxiety $(\mathrm{g}=-0.008,95 \%$ CI $[-0.26,0.24])$, depression $(\mathrm{g}=0.030,95 \%$ CI $[-0.16,0.23])$, cancer-specific distress $(\mathrm{g}=-0.036,95 \%$ CI $[-0.17$, $0.09])$ or satisfaction with follow-up care $(\mathrm{g}=0.137,95 \%$ CI $[-0.14$, $0.42])$.

\section{Conclusions}

SCPs do not improve cancer survivors' PROs. Future research is needed to ascertain how SCP interventions can be improved and whether they impact any outcomes not studied in this review.

\section{eP588}

\section{ADOLESCENT AND YOUNG ADULT CENTRAL NERVOUS SYSTEM TUMOR SURVIVORS: DOCUMENTATION OF LATE EFFECTS RISKS AND SCREENING RECOMMENDATIONS IN BRITISH COLUMBIA, CANADA

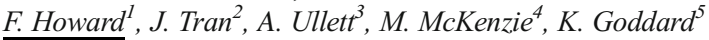 \\ ${ }^{T}$ University of British Columbia, School of Nursing, Vancouver, Canada \\ ${ }^{2}$ University of British Columbia, MD Undergraduate Program, Vancouver, Canada \\ ${ }^{3}$ BC Cancer Agency, Specialized Clinics- Patient Experience \& Interprofessional Practice, Vancouver, Canada \\ ${ }^{4}$ BC Cancer Agency, Medical Oncology, Vancouver, Canada \\ ${ }^{5}$ BC Cancer Agency, Radiation Oncology, Vancouver, Canada}

\section{Introduction}

Survivors of adolescent and young adult (AYA) central nervous system (CNS) neoplasms are at risk for late effects (LE) - treatment-related health problems occurring more than 5 years after therapy. Since, in Canada, AYA survivors are usually followed in the community, information must be conveyed to primary care providers to guide risk-based follow-up care. Objectives

To assess documentation of LE risks and screening recommendations (SR) in medical records of AYA CNS tumor survivors treated with radiation therapy.

Methods

The medical records of all patients diagnosed with a CNS neoplasm (benign or malignant) at ages 15-39 years, treated between 1985 and 2010 in the province of British Columbia, surviving $>5$ years and discharged to the community were assessed. Documentation of LE and SR were extracted, and analyzed descriptively.

\section{Results}

Among 132 survivors (52\% female), treated with radiation therapy (95\% partial brain, $10 \%$ craniospinal, $8 \%$ partial spine, and $4 \%$ whole brain) and chemotherapy (17\%), $19 \%$ of charts included no documentation of LE risks, $26 \%$ included only non-specific documentation, and 55\% had minimal documentation ( 1 or $2 \mathrm{LE}$ ). Documentation of at least one specific LE increased from $24 \%$ in $1980-1989$, to $54 \%$ in $1990-1999$, to $86 \%$ in $2000-2010$. Based on treatment information, all survivors were at high-risk for LE, such as radiation induced neoplasm, meningioma and cerebrovascular events. Yet, SR were documented in only $25 \%$ of charts.

\section{Conclusions}

The documentation of LE risks and screening recommendations has been limited, highlighting the need to improve written communication with primary care providers.

\section{eP589}

DEVELOPMENT AND VALIDATION OF THE SUPPORTIVE CANCER NEEDS-GASTRIC INTESTINAL CHINESE VERSION (SCN-GI-C) IN POST-OPERATIVE GASTRIC CANCER PATIENTS

M.P. Hsiao ${ }^{1}$, Y.H. Lee ${ }^{2}$, Y.H. Lai $^{3}$

${ }^{T}$ National Taiwan University Hospital NTUH, Department of Nursing, Taipei, Taiwan R.O.C.

${ }^{2}$ National Taiwan University NTU, School of Nursing, Taipei, Taiwan R.O.C.

${ }^{3}$ National Taiwan University NTU, School of Nursing- NTU Cancer Center \& NTU Supportive Cancer Care Research Trem, Taipei, Taiwan R.O.C.

\section{Introduction}

Gastric intestinal (GI) cancers are major problems worldwide. Patients with GI cancers usually experience lots of distress and unmet care needs related to GI distress. However, as our knowledge, there is no instrument to assess GI cancer specific care needs.

\section{Objectives}

(1) Develop the Supportive Cancer Needs- GI - Chinese version (SCNGI-C) and (2) Evaluate its psychometric properties in post- operative gastric cancer patients.

Methods

An instrument testing study was conducted and post-operative gastric cancer patients $(\mathrm{N}=133)$ were recruited in a medical center in Taiwan. The psychometrics of 17-item SCN-GI-C was evaluated by internal consistency reliability, content - and construct validity and discriminant validity. IRB approval was obtained before data collection.

Results

SCN-GI-C had satisfactory content validity and internal consistency reliability (Cronbach's $\alpha=0.85$ ). Four factors structure was identified to explain $66.5 \%$ of variance in SCN-GI-C. The SCN-GI-C can also differentiate patients with different cancer stages, surgical procedures and receiving reconstruction or not. Patients with more symptoms reported to have higher GI related care needs. 


\section{Conclusions}

SCN-GI-C a brief instrument with good psychometrical properties. Using this instrument to assess GI cancer patients' care needs are suggested clinically to enhance patients care.

eP590

CHALLENGES IN A TECHNOLOGY-BASED SUPPORT CARE INTERVENTION AMONG ASIAN AMERICAN BREAST CANCER SURVIVORS

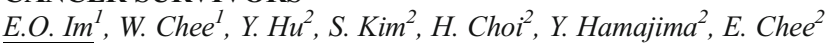

${ }^{T}$ Duke University, School of Nursing, Durham, USA

${ }^{2}$ Duke University, Nursing, Durham, USA

\section{Introduction}

In this era of computer and mobile technologies, technology-based interventions are widely adopted and used in support care for cancer patients across the globe. Furthermore, with increasingly diverse populations across the globe, cultural tailoring becomes prevalent in the technologybased interventions.

\section{Objectives}

The purpose of this presentation is to discuss the challenges that the research team encountered in a culturally tailored technology-based support care intervention among Asian American breast cancer survivors.

Methods

This was a randomized repeated measures pretest/posttest control group intervention study among 330 Asian American breast cancer survivors. The intervention group used the intervention and the American Cancer Society (ACS) website on breast cancer for 3 months, and the control group used only the ACS website for 3 months. To identify the challenges in using the intervention, the minutes of research team meetings and research diaries of research team members were analyzed using content analysis.

Results

First, technology literacy was a major challenge. Second, incorporating diversities within each ethnic group was another challenge. Third, Asian cultural heritage made the participants reluctant to discuss their personal experience and issues. Fourth, the qualifications of the interventionists needed to be changed at several times due to recruitment difficulties. Fifth, several technical incidents happened during the intervention process (e.g., delays in security certifications during regular updates). Finally, adherence and retention rates during major holidays and vacations were lower than usual.

\section{Conclusions}

Researchers need to consider possible challenges in using technologybased support care interventions among racial/ethnic minority cancer patients.

eP591

ASIAN AMERICAN BREAST CANCER SURVIVORSHIP: THE EFFICACY OF A TECHNOLOGY-BASED PROGRAM ON HEALTH OUTCOMES

W. Chee ${ }^{1}$, Y. Lee ${ }^{2}, X . \mathrm{Ji}^{3}$, E. Chee ${ }^{4}$, E.O. Im ${ }^{1}$

${ }^{T}$ Duke University, Nursing, Durham, USA

${ }^{2}$ Chung-Ang University, Nursing, Seoul, USA

${ }^{3}$ University of Delaware, Nursing, Newark, USA

${ }^{4}$ North Carolina State University, Biomedical Engineering, Raleigh, USA

\section{Introduction}

Marginalized groups tend to be more interested in technology-based interventions than those not marginalized. However, there exist few existing technology-based programs for marginalized groups, especially for Asian American breast cancer survivors.

\section{Objectives}

This study aimed to explore the preliminary efficacy of a technologybased program on survivorship experience of Asian American breast cancer patients.

\section{Methods}

This was a pilot study using a randomized repeated measures pretest/ posttest control group design. The participants included 94 Asian American breast cancer survivors. The instruments included the Perceived Isolation Scale, the Personal Resource Questionnaire, the Support Care Needs Survey-34 Short Form, and the Mishel Uncertainty in Illness Scale-Community. The data were analyzed using the repeated measures ANOVA.

\section{Results}

Significant changes were found in the isolation scores $(F=9.937, p<$ $0.01)$, the resource scores $(F=6.612, p<0.05)$, the support care need scores $(F=8.299, p<0.01)$, and the uncertainty scores $(F=8.722, p<$ 0.01 ) of the intervention group from the pre-test to the post-test. Compared with the control group, there were more decreases in the isolation scores $(M=-0.43, S D=0.79)$, more increases in the resource scores $(M=0.53, S D=0.59)$, and more decreases in the uncertainty scores $(M=$ $-0.49, S D=0.87$ ) among the intervention group.

\section{Conclusions}

The efficacy of the program needs to be further tested with a larger number of diverse groups of Asian American breast cancer survivors.

\section{eP592}

PRACTICAL ISSUES IN USING MULTIPLE LANGUAGES IN A SUPPORT CARE INTERVENTION FOR ASIAN AMERICAN BREAST CANCER SURVIVORS

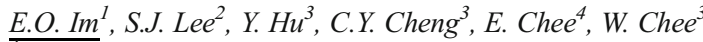

${ }^{T}$ Duke University, School of Nursing, Durham, USA

${ }^{2}$ Korea National Open University, Nursing, Seoul, USA

${ }^{3}$ Duke University, Nursing, Durham, USA

${ }^{4}$ North Carolina State University, Nursing, Durham, USA

\section{Introduction}

With an increasing number of racial/ethnic minorities in the U.S., the use of multiple languages became essential in support care interventions for cancer patients. The use of multiple languages promotes the understanding of educational materials related to support care and helps communicate effectively with patients regarding their health care needs.

\section{Objectives}

The purpose of this presentation is to identify practical issues in the use of multiple languages in a support care intervention for Asian American breast cancer survivors.

\section{Methods}

In a large-scale support care intervention study among Asian American breast cancer survivors, research team members wrote memos on issues in the use of multiple languages and plausible reasons for the issues. Then, the memos and written records were analyzed using a content analysis.

Results

Five themes/issues were identified. First of all, difficulties in recruiting and retaining bilingual research team members were prominent. Second, it was essential to maintain consistency in translation process and keep cultural and conceptual equivalence. Third, multiple IRB protocol modifications needed to be made to accommodate the use of multiple languages in all study materials. Fourth, existing translated versions or those in development needed to be identified in advance. Finally, special arrangements needed to be made for technological aspects related to electronic multiple-language versions.

\section{Conclusions}

Researchers need to consider and effectively manage the challenges in the use of multiple languages through various strategies. 
eP593

PERFORMANCE ON WORKING MEMORY TASKS AND PREFRONTAL CORTEX OXYGENATION IN CANCER SURVIVORS: A FUNCTIONAL NEAR-INFRARED SPECTROSCOPY FEASIBILITY STUDY

P. Jean-Pierre ${ }^{1}, H$. Bobo $^{2}$, A. Jean-Pierre ${ }^{3}$, H. Spahic ${ }^{4}$

${ }^{T}$ Florida State University, BSSM, Tallahassee, USA

${ }^{2}$ Florida State University College of Medicine, CoM, Tallahassee- Fl, USA

${ }^{3}$ Saint Mary's College, Biology, Notre Dame IN, USA

${ }^{4}$ University of Notre Dame, Biology, Notre Dame IN, USA

\section{Introduction}

Cancer and treatment-related neurocognitive dysfunction (CRND) is experienced by many patients and survivors.

Objectives

To use functional near-infrared spectroscopy (fNIRS) to assess CRND.

Methods

We used fNIRS to evaluate prefrontal cortex (PFC) hemodynamics/ neuronal activities during n-Back working-memory-tasks in 15 adult participants. We performed a general linear model to extract activation responses from each channel, and plotted $\beta$ values as a topological map for different tasks to determine significant activated regions. We completed linear mixed-effects models (LMEM) to represent the covariance structure associating common random effects (e.g., participants) to observations with similar levels as the grouping variable. Fixed effects were fNIRS channels location, age, sex, and cognitive tasks.

\section{Results}

The analysis showed no significant differences in age, sex, and task sublevels for the n-Back working memory test $(p>0.05)$. We found statistically significant group differences in channels $2,4,6$, and 16 for the $n-$ Back $(p<0.05)$. The results also showed bilateral activations in the right ventrolateral prefrontal cortex (R-VLPFC) for the older adults, and mild deactivations in the R-VLPFC for the younger adults. Increasing working memory load showed that DLPFC and VLPFC were negatively correlated. Overall, participants showed increased PFC activation with increasing working-memory load (0-3-Back). There was a slight right hemispheric dominance in younger participants for the lower n-Back condition, whereas older participants used both hemispheres.

Conclusions

The findings support the use of fNIRS to assess CRND. Decreased activation in cancer patients may reflects cognitive decline. The R-VLPFC might be useful as a biomarker of CRND-related hemodynamic changes.

\section{eP594}

SELF-MANAGEMENT NEEDS OF BREAST CANCER SURVIVORS AFTER TREATMENT: RESULTS FROM A FOCUS GROUP INTERVIEW

S.H. Kim ${ }^{1}$

${ }^{T}$ Inha University, Department of Nursing, Incheon, Republic of Korea

\section{Introduction}

Despite growing interest in the development of self-management (SM) intervention for cancer survivors, we have little insight into the kind of SM support needed.

Objectives

The purpose of this study was to explore the SM needs of breast cancer survivors who had completed treatment.

Methods

Using a qualitative study design, we interviewed a focus group of 20 breast cancer survivors who underwent cancer therapy (mean time since diagnosis, 3.4 years) at a university hospital in South Korea. Data were analyzed via conventional content analysis.

\section{Results}

The focus group interviews revealed 10 subthemes that we organized into five themes about posttreatment SM needs in breast cancer survivors: 1) symptom management needs, 2) emotional management needs, 3) information acquisition needs, 4) need for a relationship with healthcare providers, and 5) adaptation needs. Specifically, participants needed to acquire SM skills relieving symptoms (e.g., peripheral neuropathy, insomnia), dealing with emotional problems (e.g., fear of recurrence, anxiety, depression), and adapting to changes following from cancer treatment (e.g., body image, lifestyle, and role changes). In particular, participants expressed a substantial need for a constructive relationship with healthcare providers who, by respecting and communicating with them, would cause them to feel empowered. Participants placed a high value on building self-confidence for successful SM.

\section{Conclusions}

Our findings provide useful insights into identifying areas of need for SM support in breast cancer survivors. Themes and subthemes identified in this study can contribute to the development of breast cancer survivorship care plans, emphasizing posttreatment SM.

\section{eP595}

CO-MORBIDITIES AFTER CANCER AND THEIR MANAGEMENT. RESULTS OF A NATIONAL SURVEY OF BREAST CANCER SURVIVORS

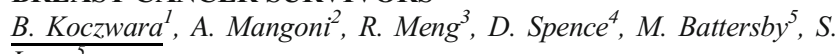
Lawn $^{3}$

${ }^{I}$ Flinders Centre for Innovation in Cancer, Medical Oncology, Adelaide, Australia

${ }^{2}$ Flinders University, Clinical Pharmacology, Adelaide, Australia

${ }^{3}$ Flinders University, Flinders Centre for Epidemiology and Biostatistics, Adelaide, Australia

${ }^{4}$ Breast Cancer Network Australia, Breast Cancer Network Australia, Melbourne, Australia

${ }^{5}$ Flinders University, Flinders Human Behaviour and Health Research Unit, Adelaide, Australia

\section{Introduction}

Breast cancer survivors experience significant burden from non-cancer chronic conditions but little is known about their impact and management.

\section{Objectives}

We conducted a survey of breast cancer survivors to examine the prevalence and impact of chronic diseases, care received, and its alignment with principles of chronic disease management.

\section{Methods}

A 22-item online survey was developed incorporating questions about presence of chronic conditions using the Charlson Comorbidity Index (CCI), functional status using Vulnerable Elders Survey (VES), and perceived quality of care for cancer and non-cancer conditions using the Patient Assessment of Care for Chronic Conditions Survey (PACIC). Members of Breast Cancer Network Australia (BCNA) were invited via email to complete the survey either online or through direct mail.

Results

The survey was sent to 2,198 members of BCNA and 177 responses were received $(8.1 \%)$. All respondents were women aged 32-88 (median 60 yrs). The majority were Caucasian $(171 ; 99 \%)$, married $(116,68 \%)$ and had private insurance $(137 ; 80 \%)$. Other health conditions were reported by $159(89 \%)$ with the most common conditions reported including chronic pain, fatigue, peripheral neuropathy and mental disorders. Mean PACIC scores were 2.2 (SD 1.0) for cancer and 2.4 (SD 1.0) for chronic disease consistent with poor concordance with Chronic Care Model. Presence of comorbidities was associated with perception of poor health and poor health care. 


\section{Conclusions}

Chronic conditions are common among breast cancer survivors, but their management is poorly aligned with chronic disease management principles. Strategies are needed to better assess and manage chronic conditions.

\section{eP596}

HAZARDS OF CANCER FROM EXPOSURE TO RADIOFREQUENCY ELECTROMAGNETIC FIELDS FROM MOBILE PHONE BASE STATIONS- A SYSTEMATIC REVIEW

\section{Kohli ${ }^{1}$, J. Kishore ${ }^{1}$, N. Dahiya ${ }^{2}$}

${ }^{T}$ Vardhman Mahavir Medical College, Department of Community Medicine, New Delhi, India

${ }^{2}$ Maulana Azad Medical College, Department of Community Medicine, New Delhi, India

\section{Introduction}

Government of India has decided to limit the radio-frequency electromagnetic fields (base station emissions) to one-tenth of the existing level; 0.92 Watt per square meter compared to the previous standard 9.2 Watt per square meter as a precautionary measure.

Objectives

To present a systematic review of the scientific literature concerning hazards of cancer from radio-frequency electromagneticradiation from mobile base stations that have been investigated till August 2017 in India and other countries.

Methods

A systematic search of Medline, EMBASE, ISI Web of Knowledge and the Cochrane Library literature was done till August 2017 to identify all relevant peer-reviewed scientific papers published before that date. Search key words included "cellular phone," "mobile," "phone," "cancer" and "base station." Human trials and epidemiological studies were included that have addressed hazard of cancer.

\section{Results}

A total of 124 studies were found but only 14 were found meeting inclusion criteria. 4 studies were randomized control trials and rest were epidemiologic studies. No study was done in Indian settings. No study could document any hazards of cancer from mobile towers.

Conclusions

All studies were unable to find a direct link between exposure to radiofrequency electromagnetic fields from mobile phone base stations and the hazard of cancer. There is no convincing scientific literature to prove a direct causal association of radio-frequency electromagnetic radiation emitted from mobile towers and cancer risk. No study has come out with duration of exposure necessary for the development of cancer attributed from radio-frequency electromagnetic.

eP597

EMPLOYMENT CHALLENGES FACED BY BREAST CANCER SURVIVORS: RESULTS FROM AN UPPER MIDDLE-INCOME ASIAN SETTING

$\underline{\text { Y.C. Kong }}^{1}$, N.T. Bhoo-Pathy ${ }^{2}$, L.P. Wong ${ }^{2}$, A.F. Aziz ${ }^{3}$, N.A. Taib ${ }^{4}$, P. Yehgambaram $^{5}$, M. Yusof ${ }^{3}$, S. Subramaniam ${ }^{1}$, C.H. Yip ${ }^{6}$, N. Bhoo-Pathy ${ }^{2}$ ${ }^{1}$ National Clinical Research Centre, Clinical Epidemiology Unit, Kuala Lumpur, Malaysia

${ }^{2}$ University of Malaya, Department of Social and Preventive Medicine, Kuala Lumpur, Malaysia

${ }^{3}$ Pantai Hospital Kuala Lumpur, Pantai Hospital Kuala Lumpur, Kuala Lumpur, Malaysia

${ }^{4}$ University of Malaya, Department of Surgery, Kuala Lumpur, Malaysia ${ }^{5}$ Kuala Lumpur Hospital, Department of Radiotherapy and Oncology, Kuala Lumpur, Malaysia
${ }^{6}$ Subang Jaya Medical Centre, Subang Jaya Medical Centre, Subang Jaya, Malaysia

\section{Introduction}

Cancer survivorship is an important component of the cancer control continuum. Yet, very little is known on how breast cancer affects employment among survivors, particularly in low- and middle-income settings.

\section{Objectives}

We aim to gain an in-depth understanding of the employment challenges faced by breast cancer survivors in an upper-middle income Asian setting.

\section{Methods}

Nine focus group discussions (FGDs) were conducted with multiethnic breast cancer survivors representing various socioeconomic backgrounds in Malaysia. Patients diagnosed 12-24 months prior to the study were recruited from a general public hospital, a public academic hospital and a private hospital. Data from the FGDs were examined using thematic content analysis.

\section{Results}

Major themes relating to impact of cancer diagnosis on employment were "decreased work ability", "job loss", "long absenteeism" and "hostile work environment". Coping strategies frequently mentioned to offset income loss from employment changes include "savings", "part-time work" or financial support from "family/friends" or "social security". In regards to returning to work, participants mentioned themes such as "need money", feeling "more happy" or having a "supportive work environment". Nonetheless, participants emphasized the "discrimination" they faced when seeking employment after active treatment. Specifically, participants' older age and their frequent need to take time-off from work for their cancer follow-ups were perceived as disadvantages when compared to younger, healthy applicants.

\section{Conclusions}

It is evident that a breast cancer diagnosis severely disrupts employment and return to work in middle income settings. Multisectoral interventions are urgently required to improve the employment status of our cancer survivors, including legislative reforms to prevent discrimination.

\section{eP598}

RELATIONSHIPS BETWEEN COMORBIDITY AND HEALTHRELATED QUALITY OF LIFE OUTCOMES AMONG NONHODGKIN LYMPHOMA SURVIVORS

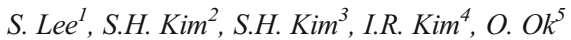

${ }^{T}$ Asan Medical Center, Oncology, Seoul, Republic of Korea

${ }^{2}$ Inha University, Department of Nursing, Incheon, Republic of Korea

${ }^{3}$ Asan Medical Center, Cancer Edu-Info Center, Seoul, Republic of Korea

${ }^{4}$ Samsung Medical Center, Cancer Education Center, Seoul, Republic of Korea

${ }^{5}$ Samsung Medical Center, Division of Hematology-OncologyDepartment of Medicine, Seoul, Republic of Korea

\section{Introduction}

Many non-Hodgkin lymphoma (NHL) survivors are increasing due to earlier diagnosis and more effective treatment. NHL survivors have comorbidities that influence their quality of life.

\section{Objectives}

The aim of this study was to assess the prevalence of comorbidities and the association between comorbidities and the health-related quality of life (HRQoL) among Korean NHL survivors.

\section{Methods}

A cross-sectional study was conducted among 570 NHL survivors who had been treated at any of 3 Korean hospitals from 1989 through 2010. HRQoL was assessed with the European Organization for Research and Treatment of Cancer (EORTC) Quality of Life Questionnaire Core 30 (QLQ-C30). Comorbidity was assessed with Charlson Comorbidity Index. 


\section{Results}

The proportion of participants without any comorbidity was $39.1 \%$. Subjects with comorbidity generally reported lower scores for most EORTC QLQ-C30 scales (except dyspnea, constipation) when compared to number of comorbidities. NHL survivors with GI disease showed clinically meaningful worse social functioning than those without GI disease. We observed clinical meaningful worse physical and role functioning in NHL survivors with musculoskeletal disease.

Conclusions

Comorbidities are common health problems in NHL survivors. Comorbidities have significantly negative influence on QOL. These findings suggested comprehensive care for NHL survivors.

eP599

EFFECTIVENESS OF MUSICAL TRAINING IN REDUCING DEPRESSIVE SYMPTOMS AND IMPROVING QUALITY OF LIFE IN CHILDHOOD BRAIN TUMOURS SURVIVORS: A RANDOMIZED CONTROLLED TRIAL

H.C.W. Lit ${ }^{l}$, O.K.J. Chung ${ }^{1}$

${ }^{T}$ The University of Hong Kong, School of Nursing, Hong Kong, Hong Kong S.A.R.

\section{Introduction}

Evidence suggests that survivors of paediatric brain tumours have more negative psychological sequelae than do survivors of other childhood cancers. Of all psychological sequelae, depression is the most commonly experienced by survivors of paediatric brain tumours. Increasing evidence suggests the use of musical training to improve psychosocial well-being in children with autism. Nevertheless, the effectiveness of musical training in enhancing psychological well-being and quality of life among childhood brain tumours survivors remains relatively unknown.

Objectives

To examine the effectiveness of musical training in reducing depressive symptoms, enhancing self-esteem and quality of life in survivors of paediatric brain tumours.

Methods

A pilot randomized controlled trial was conducted. Fifty-eight children surviving from brain tumours having medical follow-ups at the outpatient clinic of an acute-care hospital were invited to participate in the study and randomly allocated to the experimental $(n=30)$ and control groups $(n=28)$. Subjects in the experimental group received a weekly 45-minutes lesson on musical training for 52 weeks, whereas the control group received usual care. Subjects' depressive symptoms, level of self-esteem and quality of life were assessed at the time of recruitment, 6- and 12-month after starting the intervention.

\section{Results}

Subjects in the experimental group reported statistically significant fewer depressive symptoms, higher level of self-esteem and quality of life than children in the control group who received usual care at 6-and 12-month follow up.

\section{Conclusions}

The musical training was investigated to be effective in reducing the depressive symptoms, improving self-esteem and quality of life in childhood brain tumours survivors.

\section{eP600}

THE ASSOCIATION BETWEEN HYPOMAGNESEMIA AND SURVIVAL IN PATIENTS WITH OVARIAN CANCER

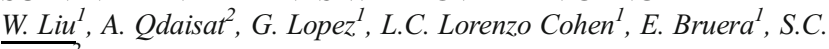
$\overline{\text { Yeung }^{2}}$

${ }^{I}$ MD Anderson Cancer Center, Palliative- Rehabilitation \& Integrative Medicine, Houston, USA
${ }^{2}$ MD Anderson Cancer Center, Department of Emergency Medicine, Houston, USA

Introduction

Hypomagnesemia is strongly associated with rapid disease progression and increased complications in patients with diabetes. Though it is a known side effect of several antineoplastic agents, its impact on cancer patient outcomes is not frequently studied.

\section{Objectives}

We aimed to examine magnesium abnormalities and survival in ovarian cancer patients who have received platinum-based chemotherapy.

\section{Methods}

We included ovarian cancer patients presenting to MD Anderson Cancer Center (MDACC):1) between 1/1/2004 and 12/31/2014;2) age 18 years or older; 3 ) first treatment (surgery or chemotherapy) at MDACC within 60 days of diagnosis; 4) chemotherapy containing cisplatin or carboplatin; and 5) interval between treatments $<3$ months. The final cohort consisted of 303 patients. Vital signs and laboratory tests were recorded for baseline (30 days before and on the first treatment day) and treatment course (after first treatment day to 30 days after the last treatment). The association between clinical parameters and survival were analyzed.

\section{Results}

Mean patient age was 62 years. Higher baseline heart rate $(\mathrm{OR}=1.03$, $\mathrm{P}<0.001)$ and the prevalence of hypomagnesemia during treatment course $(\mathrm{HR}=1.10, \mathrm{P}<0.001)$ were significantly associated with shorter survival independent of age, cancer stage, hyponatremia, hypokalemia, and chemotherapy cycles. Intravenous magnesium sulfate replacement $(\mathrm{g})$ ( $\mathrm{HR}=0.96, \mathrm{P}=0.002)$ was associated with longer survival.

\section{Conclusions}

Cardiovascular deconditioning prior to treatment and prevalence of hypomagnesemia are prognostic of survival for ovarian cancer patients receiving platinum-based chemotherapy and surgery. Optimizing cardiovascular condition should be an integral part of the overall treatment preparation. Future research is needed for strategies to detect and prevent hypomagnesemia in this patient population.

\section{eP601}

EFFECTS OF EXERCISE INTERVENTIONS ON ADULTS WITH LYMPHOMA: RESULTS FROM A SYSTEMATIC REVIEW

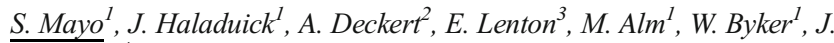
Kuruvilla ${ }^{4}$

${ }^{1}$ University of Toronto, Lawrence S. Bloomberg Faculty of Nursing, Toronto, Canada

${ }^{2}$ Princess Margaret Cancer Centre- University Health Network, Department of Supportive Care, Toronto, Canada

${ }^{3}$ University of Toronto, Gerstein Science Information Centre, Toronto, Canada

${ }^{4}$ Princess Margaret Cancer Centre- University Health Network, Department of Medical Oncology and Hematology, Toronto, Canada

\section{Introduction}

Lymphoma survivors represent a rapidly growing population requiring supportive care strategies to mitigate the late and long-term health consequences of treatment. While exercise has been associated with a range of positive outcomes in mixed cancer populations, an understanding of the effects of exercise in this specific disease and treatment context is necessary.

\section{Objectives}

To summarize evidence regarding the effects of exercise interventions on adults with lymphoma.

Methods

We searched seven bibliographic databases up to March 2017 for randomized and non-randomized trials of exercise interventions in adults 
with lymphoma. Two reviewers independently performed study selection, quality appraisal and data extraction. The review protocol is registered on PROSPERO (\#CRD42017079441).

\section{Results}

Of 4892 references screened, four randomized controlled trials were eligible for review. Interventions varied by exercise program type, frequency, intensity, and duration. Trials also differed in sample size (39 to 122 participants), length of follow-up (post-intervention to 6 months), and outcomes measured. All four studies tested the exercise intervention among patients during lymphoma treatment; two included patients offtreatment. Despite methodological limitations, each study reported good adherence and improvement in outcomes related to physical function, symptom severity and/or quality of life. A quantitative analysis currently in progress will provide insight into the consistency and magnitude of these effects.

\section{Conclusions}

Preliminary evidence from this review suggests that exercise interventions are feasible and may offer health benefits to adults with lymphoma. Few studies have tested the effect of exercise interventions on quality of life outcomes among lymphoma survivors who have completed treatment.

\section{eP602}

\section{ALTERATIONS IN GENE EXPRESSION INDUCED BY} OALIPLATIN BASED CHEMOTHERAPY ON PERIPHERAL LEUKOCYTES AS MARKERS OF SINUSOIDAL OBSTRUCTION SYNDROME OF THE LIVER

M. Morales ${ }^{1}$, A. Rodriguez Chamorro ${ }^{2}$, M. Fernandez del Castillo ${ }^{3}, R$. Gonzalez-Fernandez ${ }^{4}$, P. Martin-Vasallo ${ }^{4}$

${ }^{1}$ Hospital Universitario N S Candelaria, Medical Oncology, Santa Cruz. de Tenerife, Spain

${ }^{2}$ University of La Laguna, Medicine, La Laguna, Spain

${ }^{3}$ Hospital Universitario N S Candelaria, Radiology, Santa Cruz de Tenerife, Spain

${ }^{4}$ University of La Laguna, Biochemistry and Molecular Biology, La Laguna, Spain

\section{Introduction}

Colorectal cancer represents the third most frequently diagnosed malignancy in the world and a considerable number of patients will receive adjuvant oxaliplatin based chemotherapy.

Objectives

Oxaliplatin produces long term side effects, like peripheral neuropathy and liver injury as Sinusoidal Obstruction Syndrome (SOS). Since a considerable number of patients with colorectal cancer treated with adjuvant oxaliplatin based chemotherapy will be cured, we designed the following study to search the effects of oxaliplatin based chemotherapy on gene expression of peripheral leukocytes with the following aims: 1) to assess the effects of the therapy on gene expression; 2) to stablish correlations among gene expression variation and SOS, and 3) to search for molecular markers of long term toxicity.

Methods

Peripheral leukocytes from 27 patients with colon adenocarcinoma, scheduled to receive adjuvant or neoadjuvant chemotherapy, were isolated with Ficoll-Hypaque gradient, previously and after three FOLFOX- or CAPEOX-cycles (OX). We isolated mRNA from leukocytes from 27 patients in both situations, prior- and post- three cycles treatment and the expression of selected genes was measured by qRT-PCR.

Patients entering the study had normal liver functions test and signed informed contest. Clinical examination, laboratory tests and imaging studies were performed as indicated in clinical guide lines.

Results

OX chemotherapy varied AIF1, AGAP1, ROMO1, LMNA, SESN1, $T H B S 1$ and SERPINH1 genes expression in a manner that correlates with
SOS laboratory parameters (bilirubin, AST, ALT, GGT, AP LDH, Hb and platelets) and imaging studies

Conclusions

These genes could be used as early markers and evolution markers of SOS.

\section{eP603}

OMEGA-3 ( $\Omega 3$ ) SUPPLEMENTATION VERSUS OMEGA-6 $(\Omega 6)$ SUPPLEMENTATION FOR PAIN AMONG BREAST CANCER SURVIVORS: A URCC NCORP PHASE II FEASIBILITY RCT L. Peppone $^{1}$, J. Inglis ${ }^{1}$, K. Mustian ${ }^{1}$, K.P. Loh ${ }^{1}$, E. Culakova ${ }^{1}$, I.

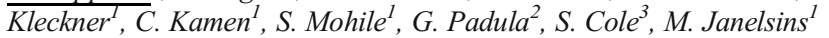

${ }^{1}$ University of Rochester School of Medicine, Surgery, Rochester, USA

${ }^{2}$ Summa Health, Medical Director, Akron, USA

${ }^{3}$ Dayton Clinical Oncology Program, Medicine, Dayton, USA

\section{Introduction}

Pain is common side effect for breast cancer survivors. The cause is often multifactorial and can have a detrimental effect on treatment adherence and quality of life.

Objectives

We conducted a secondary analysis of a multi-site, blinded phase II RCT examining the efficacy of $\omega 3$ polyunsaturated fats (fish oil) versus $\omega 6$ polyunsaturated fats (soybean oil) for improving pain

Methods

Breast cancer survivors 4-36 months post-adjuvant therapy were randomized into 3 arms: 1) High-dose $\omega 3$ ( $\omega 3 ; 6 g /$ day), 2) Low-dose $\omega 3 / \omega 6$ $(\omega 3 / \omega 6 ; \omega 3: 3 \mathrm{~g} /$ day and $\omega 6: 3 \mathrm{~g} /$ day) and 3) High-dose $\omega 6(\omega 6 ; 6 \mathrm{~g} /$ day) for 6 weeks. Pain was assessed via the Brief Pain Inventory Short Form at pre- and post-intervention. Serum was collected for inflammatory biomarker analysis. ANCOVAs, controlling for baseline pain and hormone therapy, were used to calculate mean pre- to post-intervention change.

Results

Mean baseline Worst Pain levels were similar between groups $(\omega 3=4.9, \omega 3 / \omega 6=5.6, \omega 6=4.7 ; p=0.43)$. The mean Worst Pain score decreased by 1.7 points for $\omega 6,0.8$ points for $\omega 3 / \omega 6$, and 0.8 points for $\omega 3$ ( $\omega 6$ vs $\omega 3$ p-value $=0.18$ ). The mean decrease in Current Pain score was more pronounced $(\omega 6=-1.8$ vs $\omega 3 / \omega 6=-0.8$ vs $\omega 3=-0.5 ; \omega 3$ vs $\omega 6 \mathrm{p}=0.05$ ) and indicated a dose-response. The $\omega 6$ group had a significant reduction in the inflammatory biomarker IL-6 compared to the $\omega 3$ group (IL-6 change: $\omega 3=+0.17$ vs $\omega 6=-0.30 ; p<0.01)$.

\section{Conclusions}

$\omega 6$ supplementation reduced pain levels compared to $\omega 3$ supplementation in breast cancer survivors. $\omega 6$ also significantly reduced IL- 6 levels compared to $\omega 3$, indicating a potential mechanism of action. Funding: NCI-R03CA175599/UG1CA189961. Nordic Naturals, Inc. supplied all study agents.

\section{eP604}

THE LIFE CYCLE OF PERSONAL SOCIAL NETWORKS OF WOMEN LIVING WITH BREAST CANCER IN THAILAND S. Purinthrapibal ${ }^{1}$, L. Batten ${ }^{2}, J$. Bodd $^{3}$, K. Nilmanat $^{4}$

${ }^{T}$ Prince of Songkla University, Nursing Administration, Songkhla, Thailand

${ }^{2}$ College of Health Massey University, Rsearch Centre for Maori Health and Development, Palmerston North, New Zealand

${ }^{3}$ Massey University, Collage of Health, Palmerston North, New Zealand ${ }^{4}$ Prince of Songkla University, Adult and elderly nursing, Songkhla, Thailand 


\section{Introduction}

Few support network studies of breast cancer women are exploring the qualitative dimensions of personal social networks. Many network studies are cross sectional rather than longitudinal. Consequently, a lack of knowledge regarded the complete PSNs of women with breast cancer was identified, in particular, how PSNs develop and change over time.

Objectives

To explore the characteristics of the personal social networks of women living with breast cancer in Thailand.

\section{Methods}

The network-relatedexperiences were gathered of 11 personal social networks from 11 women with breast cancer and their 30 network members. Longitudinal data collection during 11 months of fieldwork included a series of interviews, observation, and network mapping. Data were analysed thematically.

\section{Results}

The women's networks when living with breast cancer developed through five overlapping phases as a network life-cycle: i) network origination, ii) network expansion, iii) network adjustment, iv) contraction of the non-family components, and v) persistence of involvement of family and close members in each network. A diagnosis of cancer recurrence resulted in the original phases of network development recurring; however, development was quicker than with the primary diagnosis.

\section{Conclusions}

The personal social networks of women living with breast cancer were fluid with a phased life cycle and associated with specific features. Those features related to the disease, treatment burdens, and culturally specific social relationships resulting in a family-centered network. Therefore, nurses and other health professionals should consider women's personal social networks as one of key elements of the women's experiences of their illness journey.

\section{eP605}

\section{RETHINKING SURVIVORSHIP: COMMUNICATION IS THE} KEY

G. Rodriguez ${ }^{1}$, N. Dixit ${ }^{2}$

${ }^{T}$ University of California- San Francisco, Internal Medicine, San

Francisco, USA

${ }^{2}$ University of California- San Francisco, Division of Hematology/ Oncology, San Francisco, USA

\section{Introduction}

Improved treatment of breast and colon cancer has increased the number of cancer survivors. The Institute of Medicine in its 2005 report Cancer Patient to Cancer Survivor: Lost in Transition identified transition from active cancer treatment to long-term follow-up care as a phase where the role of different providers remains unclear. Most cancer survivors have additional comorbidities and would benefit from better care coordination between specialists and primary care providers (PCPs).

\section{Objectives}

1. To understand the knowledge and attitudes of PCPs of breast and colon cancer survivors in our safety net hospital and affiliated clinics.

2. To assess communication between PCPs, oncologists and patients.

Methods

The National Cancer Institute's Survey of Physician Attitudes Regarding the Care of Cancer Survivors was sent electronically to 220 PCPs in 12 primary care clinics in the San Francisco Health Network (SFHN) affiliated with Zuckerberg San Francisco General Hospital. The response rate was $50 \%$ with 110 responses while 81 providers completed each field.

Results

Only $19.05 \%$ of PCPs reported receiving a comprehensive summary of cancer treatment from oncologists, while $14.28 \%$ received an explicit follow-up care plan documenting recommendations for future care and surveillance. Almost half of PCPs reported having discussions with patients about which provider will follow-up cancer (47.56\%) vs. medical issues $(39.56 \%)$. (Refer to Table1).

\begin{tabular}{|c|c|c|}
\hline \begin{tabular}{|l|} 
Communication \\
\end{tabular} & Always/Almost Always/Often & Sometimes/Rarely/Never \\
\hline $\begin{array}{l}\text { Receive a comprehensive summary including cancer } \\
\text { treatment information from the patien's oncology specialist. }\end{array}$ & $19.05 \%$ & $80.95 \%$ \\
\hline \begin{tabular}{|l|} 
Provide a summary of the patient's pastnon-cancer medical \\
history for the patient's oncology specialist.
\end{tabular} & $35.72 \%$ & $64.38 \%$ \\
\hline $\begin{array}{l}\text { Receive information from the oncology specialist in a timely } \\
\text { manner. }\end{array}$ & $34.52 \%$ & $65.48 \%$ \\
\hline $\begin{array}{l}\text { Experience difficulties transfering patient care } \\
\text { responsibilities between you and the oncology specialist. }\end{array}$ & $24.80 \%$ & $75.20 \%$ \\
\hline $\begin{array}{l}\text { Receive from the patient's oncologist an explicit follow-up } \\
\text { care plan documenting recommendations for future care and } \\
\text { surveillance. }\end{array}$ & $14.28 \%$ & $85.72 \%$ \\
\hline $\begin{array}{l}\text { Communicate with patients' other physicians about. } \\
\text { responsibility of followup care for theircancer. }\end{array}$ & $26.83 \%$ & $75.17 \%$ \\
\hline $\begin{array}{l}\text { Have a specific discussion with the patient regarding } \\
\text { recommendation for future care and surveillance. }\end{array}$ & $27.38 \%$ & $76.62 \%$ \\
\hline $\begin{array}{l}\text { Discuss with your patient who will provide care for other } \\
\text { medical issues. }\end{array}$ & $39.56 \%$ & $60.44 \%$ \\
\hline $\begin{array}{l}\text { Discuss with the patient who will provide followup care for } \\
\text { cancer. }\end{array}$ & $47.56 \%$ & $52.44 \%$ \\
\hline
\end{tabular}

\section{Conclusions}

SFHN providers reported suboptimal communication between oncologists, PCPs and patients. Poor delineation of care was perceived as key factor for missed care of cancer survivors. Improving communication can significantly optimize cancer survivors' care.

\section{eP606}

CO-CREATING AND ADAPTING A SELF-MANAGEMENT PROGRAM FOR BREAST CANCER SURVIVORS INTEGRATED IN THE CLINICAL PATHWAY OF BREAST CENTERS IN SWITZERLAND (COSS PILOT STUDY).

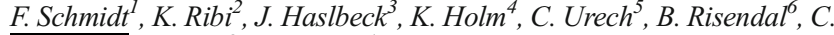
Foster', D. Fenlon ${ }^{8}$, M. Eicher ${ }^{I}$

${ }^{1}$ Institute of Higher Education and Research in Healthcare-IUFRS, Faculty of Biology and Medicine, Lausanne, Switzerland

${ }^{2}$ International Breast Cancer Study Group, not applicable, Bern, Switzerland

${ }^{3}$ Swiss Cancer League, Follow-up Care, Bern, Switzerland

${ }^{4}$ Patient Advocates for Cancer Research \& Treatment Association PACRT, not applicable, Geneva, Switzerland

${ }^{5}$ University Hospital of Basel, Women'sHealth ClinicGynecological Social medicine and Psychosomatics, Basel, Switzerland

${ }^{6}$ Community and Behavioral Health Colorado School of Public Health- University Colorado, Comprehensive Cancer Center, Colorado, USA

${ }^{7}$ University of Southampton, Faculty of Health Sciences, Southampton, United Kingdom

${ }^{8}$ Swansea University, not applicable, Swansea, United Kingdom

\section{Introduction}

The peer-led Cancer Thriving and Surviving Program (CTS) has positive effects on cancer survivor's skills, quality of life, and on clinical outcomes. Until now, the CTS has not been introduced in Swiss cancer care, nor adapted to the growing population of female breast cancer survivors.

\section{Objectives}

To adapt the CTS for Breast Cancer Survivors in Switzerland (CTS-BC$\mathrm{CH}$ ) and to determine its integration into the clinical pathway of Swiss breast centers.

Methods

The CTS was first translated into German, then three workshops were conducted with transdisciplinary working groups (breast cancer survivors, breast care nurses, psycho-oncologists, and researchers) using a co-creative and participatory approach. At 
these workshops, presentation of the CTS followed by discussions allowed identification of breast cancer specific topics. Final agreement of the CTS-BC-CH and its clinical integration was sought by consensus conference with workshop participants, additional breast cancer survivors, health care professionals and international experts.

Results

The 6-day CTS was extended to 7 days. Its contents were modified to better address the needs of breast cancer survivors, specifically content related to "My exercise", "Being a woman and having breast cancer" and "My (working) life". New interactive activities were created: e.g. "Take a stand" to help patients in making decisions. Transition from acute treatment to follow-up care was agreed as the optimal time point to introduce the program into the clinical pathway.

Conclusions

The CTS-BC-CH is now ready for pilot testing at two breast centers to evaluate the feasibility of its clinical introduction and its preliminary effectiveness on patient outcomes.

\section{eP607}

RECOVERY OF QUALITY OF LIFE FOLLOWING CURATIVE INTENT SURGERY FOR COLORECTAL CANCER: RESULTS FROM THE COLORECTAL WELLBEING (CREW) STUDY

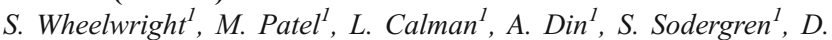

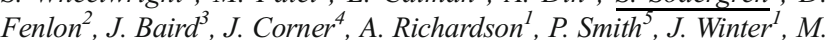
Study Advisory Committee ${ }^{1}$, C. Foster ${ }^{1}$

${ }^{1}$ University of Southampton, Health Sciences, Southampton, United Kingdom

${ }^{2}$ Swansea University, College of Human and Health Sciences, Swansea, United Kingdom

${ }^{3}$ University of Southampton, Medicine, Southampton, United Kingdom

${ }^{4}$ University of Nottingham, Research and Knowledge Exchange, Nottingham, United Kingdom

${ }^{5}$ University of Southampton, Social Sciences, Southampton, United Kingdom

\section{Introduction}

Whilst five year survival rates for colorectal cancer (CRC) in England are approaching $60 \%$, little is known about the recovery of quality of life (QOL) because previous studies worldwide have been cross-sectional or have not assessed QOL pre-surgery.

Objectives

To estimate the proportion of CRC survivors whose self-reported QOL returns to pre-surgery levels and to identify baseline predictors of five year QOL.

Methods

A representative cohort of 872 non metastatic CRC patients from 29 UK cancer centres received questionnaires at baseline (presurgery), 3, 9, 15, 24, 36, 48 and 60 months. QOL, self-efficacy, mental health, social support, affect, socio-demographics, clinical and treatment characteristics were assessed (primary outcome: Quality of Life in Adult Cancer Survivors Scale, QLACS). Recovery was defined as reaching $90 \%$ or more of the baseline QLACS generic summary score (QLACS-GSS). A generalised linear model was used to identify which baseline factors predicted QLACS-GSS at 60 months.

\section{Results}

The proportion of CRC survivors with recovered QOL peaked at 15 months (71.4\%)(Figure 1). At 60 months, over a third had not recovered their baseline QOL. Baseline health status, well-being, positive and negative affect, social support and presence of stoma predicted QLACS-GSS at 60 months.

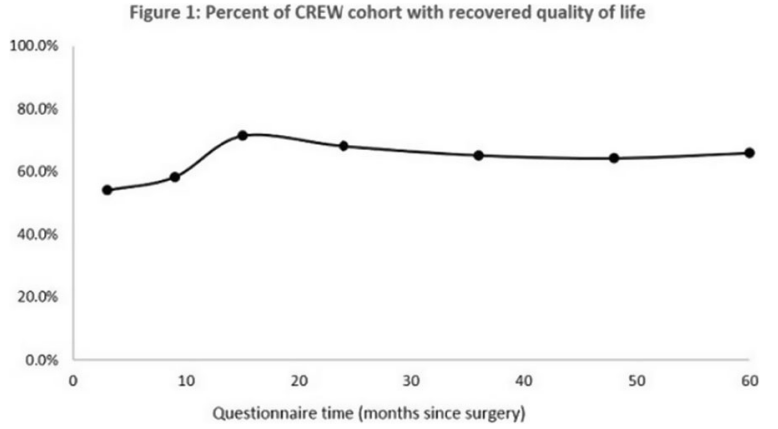

Conclusions

Around one third of CRC patients have not returned to pre-surgery levels of QOL five years after treatment. Baseline psychosocial factors, social support and stoma use, independent of treatment or disease characteristics, predict QOL at five years. Early psychosocial interventions, potentially pre-treatment, are needed to improve long term outcomes for CRC survivors.

\section{eP608}

THE ROLE OF COMORBID DIABETES ON THE SYMPTOMS OF BREAST CANCER SURVIVORS

S. Storey ${ }^{I}$, A. Cohee ${ }^{I}$, W.G. Gathirua-Mwangi ${ }^{l}$, V. Champion ${ }^{l}$

${ }^{T}$ Indiana University School of Nursing, Community Health Systems, Indianapolis, USA

\section{Introduction}

Introduction. Studies have noted women with diabetes have a $23 \%$ higher risk of developing breast cancer (BC) than those without. BC and diabetes share common symptoms contributing to decreased quality of life. Research in other cancers, has demonstrated those with diabetes have increased symptoms, however, a similar body of research as it relates to $\mathrm{BC}$ survivors (BCS) is lacking.

\section{Objectives}

Objectives. The purpose of this study was to determine if differences exist between BCS with/without diabetes on physical and attention function, sleep and fatigue.

\section{Methods}

Methods. This descriptive study used data from a larger quality of life study. BCS were stages I-IIIa, 3-8 years post chemotherapy, with no recurrence. Sociodemographic variables included age, income, education and marital status. Medical history included diabetes and BMI. Physical function, attention function, sleep and fatigue were measured. Independent $t$ tests compared the means of symptoms. Separate regression analyses were ran to assess the relationship of diabetes on each of the dependent variables.

\section{Results}

Results. 121 BCS had diabetes. BCS were primarily Caucasian, married and well educated. BCS with diabetes were older, had higher BMI, lower education and income. BCS with diabetes had poorer physical function $(p=.000)$ and attention function $(p=.017)$. BCS with diabetes did not report increased sleep disturbance $(p=.149)$ or greater fatigue $(p=.098)$.

\section{Conclusions}

Conclusion. This study indicates that BCS with diabetes experience poorer physical function and attention function. Baseline assessments of these symptoms at initial diagnosis, prior to initiation of and throughout treatment are important to assess to decrease symptom severity. 
eP609

PSYCHOLOGICAL IMPACTS, SYMPTOM AND SLEEP STATUS IN TAIWANESE HEAD AND NECK CANCER PATIENTS

Y.C. Su ${ }^{\text {I, Y.Y. Fang }}{ }^{\text {I, Y.H. }}$ Lai $^{2}$

${ }^{T}$ National Taiwan University, School of Nursing, Taipei, Taiwan R.O.C.

${ }^{2}$ National Taiwan University NTU- Taipei- Taiwan, School of Nursing. NTU Supportive Cancer Care Research Team \& NTU Cancer Center, Taipei, Taiwan R.O.C.

\section{Introduction}

Due to the complex treatment modalities, patients with head and neck cancer (HNC) patients might experience impacts on psychological, physical and sleep functions.

Objectives

(1) explore the current status of psychological impact of cancer, symptom severity and sleep quality, and (2) examine the relationship among psychological impact of cancer, symptom severity and sleep in Taiwanese HNC patients after major treatments.

Methods

This is a cross-sectional survey study to recruit HNC participants from outpatient clinics of a medical center in Northern Taiwan. The eligible subjects were those who were diagnosed with HNC and who have already completed their major treatments (surgery, chemotherapy and/ or radiation therapy) for at least 3 months. Patients were approached by trained senior oncology nurses. Patients' consent and IRB approval were obtained before data collection. We used Impact Event Scale (IES), Pittsburgh Sleep Quality Index, and Symptom Severity Scale (SSS) to assess patients' psychological impacts, symptoms, and sleep, respectively.

Results

A total of 120 subjects were recruited. The overall score of PSQI was 4.64 $(\mathrm{SD}=3.64)$ and IES was $8.46(\mathrm{SD}=10.23)$, respectively. The major symptom severity as their descending order of mean was: dry mouth, chewing difficulty, taste change, and shoulder tight. Generally, patients had higher levels of impacts of cancer reported to have higher sleep problems with correlation as 0.52 . Patients with fatigue and shoulder tight had higher sleep problems.

Conclusions

A systematic clinical assessments for HNC of their sleeping problems, impact of cancer and symptoms are suggested.

eP610

DRY MOUTH, PAIN AND SLEEP DISTURBANCE IN NASOPHARYNGEAL CARCINOMA (NPC) SURVIVORS IN TAIWAN

Y.T. Su ${ }^{1}$, Y.Y. Fang ${ }^{1}$, Y.H. Lai $^{2}$

${ }^{T}$ National Taiwan University NTU, School of Nursing, Taipei, Taiwan R.O.C.

${ }^{2}$ National Taiwan University NTU, School of Nursing. NTU Cancer Center \& NTU Supportive Cancer Care Research Team, Taipei, Taiwan R.O.C.

\section{Introduction}

Nasopharyngeal carcinoma (NPC) is a highly radiosensitive cancer with relatively good prognosis with 5-year survival rate as $67.1 \%$ to $81.6 \%$. However, due to the long-term side-effects of radiotherapy (RT), NPC survivors might suffer from those physical distress.

Objectives

This study aims to explore the current status of dry mouth, pain and sleep disturbance and their relationship in NPC survivors in Taiwan.

\section{Methods}

A cross-sectional study was conducted to recruit NPC patient who had completed concurrent chemo-radiotherapy (CCRT) or RT more than 3 months in a medical center in Northern Taiwan. The Xerostomia Questionnaire (XQ), Brief Pain Inventory (BPI), Medical Outcome Study-Sleep Scale (MOS-SS), and Background Information Form were used to assess patients' distress.

Results

There were 113 patients recruited. The results showed that patients' sleep hour per day was 7.0 hours $(S D=1.7)$ with major sleep problem as sleep adequacy, snoring, and sleep disturbance. The average total score on $\mathrm{XQ}$ was $43.6(\mathrm{SD}=16.8)$ with the highest problems as frequency of sipping liquids to aid in swallowing food and swallowing difficulty. More than one-fourth of patients $(26.5 \%)$ reported to have pain. Overall, patients with more severe dry mouth and pain intensity reported to have more sleep disturbance, with $r=0.54$ and $r=0.25$, respectively.

\section{Conclusions}

Dry mouth, pain and sleep disturbance are three major problems for NPC survivors. Clinically, careful assessment and related interventions should be applied to increase patients' care quality.

\section{eP611}

\section{RESEARCH OF RECOGNITION OF THE EXERCISE EFFECT AND EXERCISE SUPPORT NEEDS OF BREAST CANCER SURVIVORS \\ N. Tamai ${ }^{1}$, M. Kamizato ${ }^{2}$, Y. Kimura ${ }^{1}$, R. Nisida ${ }^{1}$, M. Nozaki $^{l}$ \\ ${ }^{T}$ Meio University, Department of Nursing, Okinawa, Japan \\ ${ }^{2}$ Okinawa Prefectural College of Nursing, Department of Nursing, Okinawa, Japan}

\section{Introduction}

Exercise is known to have positive effects to prevent recurrence and side effects in cancer patients. However, recognition of these effects and exercise support needs have not been clarified.

\section{Objectives}

The purpose of this study is to clarify recognition of the exercise effect and exercise support needs of breast cancer survivors.

Methods

A self-administered questionnaire on "recognition of the effect of exercise," and "need for exercise support" was carried out, and responses were analyzed.

\section{Results}

One hundred forty-one breast cancer survivors (age $60.4 \pm 12.6$ years) responded. Average period after diagnosis was $45.6 \pm 67.3$ months, $35.4 \%$ of patients were Stage I. $60.3 \%$ of patients showed symptoms, with "fatigue" by 38 patients $(44.7 \%)$ the most frequent. 59 patients $(41.9 \%)$ had reduced activity after diagnosis, and 11 patients $(7.8 \%)$ had increased activity. Highest recognition of exercise effect was "improvement of sleep" $(72.3 \%)$, followed by "improvement of physical functions" $(70.9 \%)$, and "prevention of falling" $(70.2 \%)$. Low recognition items were "alleviation of fatigue" (36.1\%), "alleviation of pain" (41.2\%), and "recurrence prevention" patients (46.1\%). 122 patients $(90.4 \%)$ need various exercise supports, such as "exercises with family" and "exercise facilities for cancer survivors."

\section{Conclusions}

The breast cancer survivors recognize that the exercise improves the lifestyle, but does not recognize that exercise relieves a symptom and prevents recurrence of cancer very much. Since $90 \%$ of breast cancer survivors need exercise support, it was suggested that transmission of right information about the exercise and the development of the exercise support program were necessary. 
eP612

"STILL A CANCER PATIENT" - ASSOCIATIONS OF CANCER IDENTITY WITH PATIENT-REPORTED OUTCOMES AND HEALTH CARE USE AMONG CANCER SURVIVORS FROM THE POPULATIOIN-BASED CAESAR STUDY

M. Thong I', E.M. Wolschon ${ }^{2}$, L. Koch-Gallenkamp ${ }^{3}$, A. Waldmann ${ }^{4}$, M. Waldeyer-Sauerland $^{4}, R$. Pritzkuleit ${ }^{5}, H$. Bertram ${ }^{6}, H$. Kajüter ${ }^{6}, A$. Eberle ${ }^{7}$, B. Holleczek ${ }^{8}$, S. Zeissig' ${ }^{9}$, H. Brenner ${ }^{3}$, V. Arndt ${ }^{1}$

${ }^{1}$ German Cancer Research Center DKFZ, Cancer Survivorship, Heidelberg, Germany

${ }^{2}$ University of Lübeck, Institute for Social Medicine and Epidemiology, Lübeck, Germany

${ }^{3}$ German Cancer Research Center DKFZ, Division of Clinical Epidemiology and Aging Research, Heidelberg, Germany

${ }^{4}$ Ministry of Health and Consumer Protection, Hamburg Cancer Registry, Hamburg, Germany

${ }^{5}$ Cancer Registry of Schleswig-Holstein, Cancer Registry of SchlewsigHolstein, Lübeck, Germany

${ }^{6}$ Cancer Registry of North Rhine-Westphalia, Cancer Registry of North Rhine-Westphalia, Münster, Germany

${ }^{7}$ Leibniz Institute for Prevention Research and Epidemiology - BIPS, Bremen Cancer Registry, Bremen, Germany

${ }^{8}$ Saarland Cancer Registry, Saarland Cancer Registry, Saarbrücken, Germany

${ }^{9}$ Cancer Registry of Rhineland-Palatinate, Cancer Registry of Rhineland-Palatinate, Mainz, Germany

\section{Introduction}

The concept of cancer identity is gaining attention as more individuals are now living with cancer as a chronic illness. Research is limited and results suggest that a self-identity as 'cancer patient' rather than 'cancer survivor' is associated with depression and lower health-related quality of life (HRQL).

\section{Objectives}

We aimed to identify factors associated with patient identity and investigate the associations between patient identity and treatment, health care use, psychosocial distress, and HRQL.

Methods

We used data from the population-based CAncEr Survivorship: A multiRegional (CAESAR) study. Breast, colorectal, and prostate cancer survivors diagnosed in 1994-2004 completed a postal survey on self-identity, health care use, psychological distress, and HRQL in 2009-2011. We calculated odds ratios and the $95 \%$ confidence interval of having a patient identity. Analyses were adjusted for age, sex, education and cancer stage, where appropriate.

Results

Of the 6057 respondents, colorectal cancer survivors $(25 \%)$ were least likely to consider themselves as patients and prostate cancer survivors $(36 \%)$, the most. Being male, of younger age, comorbidity, higher cancer stage, and disease recurrence were associated with patient identity. Treatment was associated with patient identity except among female colorectal cancer survivors. Having a patient identity was associated with higher health care use within the past 12 months. Survivors who still consider themselves as patients were more likely to be depressed and reported significantly lower HRQL.

Conclusions

A significant proportion of cancer survivors still consider themselves as patients 5-15 years post-diagnosis. Sensitivity to individuals' selfidentityshould be considered when exploring their cancer experience.

\section{eP613}

RESILIENCE AND POSITIVE PSYCHOLOGICAL CHANGES AFTER A CANCER DIAGNOSIS AND TREATMENT P. Tu ${ }^{l}$
${ }^{1}$ Chung Yuan Christian University, Psychology, Taipei City, Taiwan R.O.C.

\section{Introduction}

A growing body of literature has examined the positive psychological changes in people's lives following cancer diagnosis and treatment, and one of the representative viewpoints is the "Posttraumatic Growth Model". PTG is defined as a cognitive process accompanied with transformational psychological change that is experienced as a result of struggle from highly challenging life circumstances. Theoretically, resilience, defined a positive adaptation in the face of adversity, is as a construct distinct form but related to PTG. Recent vision of resilience-distress continuum included the possibility of personal growth beyond baseline functioning following a cancer diagnosis, but some studies assumed resilient outcomes may provide little need or opportunity for PTG.

\section{Objectives}

The relation between two concepts could have important implications for both preventive interventions as well as cancer psychosocial consultations. Therefore, this project adopted a cross-session design to investigate the relationship between psychological resilient and PTG after a cancer diagnosis and treatment.

\section{Methods}

A total of 201 Taiwanese breast cancer survivors completed the selfreported measures describing resilience, coping style, psychological growth and quality of life. The average age was 51.53 and time since diagnosis was 58 months.

\section{Results}

Hierarchical analysis showed that resilience significantly predicted higher psychological growth and health-related quality of life, and the effects were moderated by Positive-Affect coping. In addition, this study also found Negative-Affect coping had direct impact on lowering healthrelated quality of life, but no effect on psychological growth.
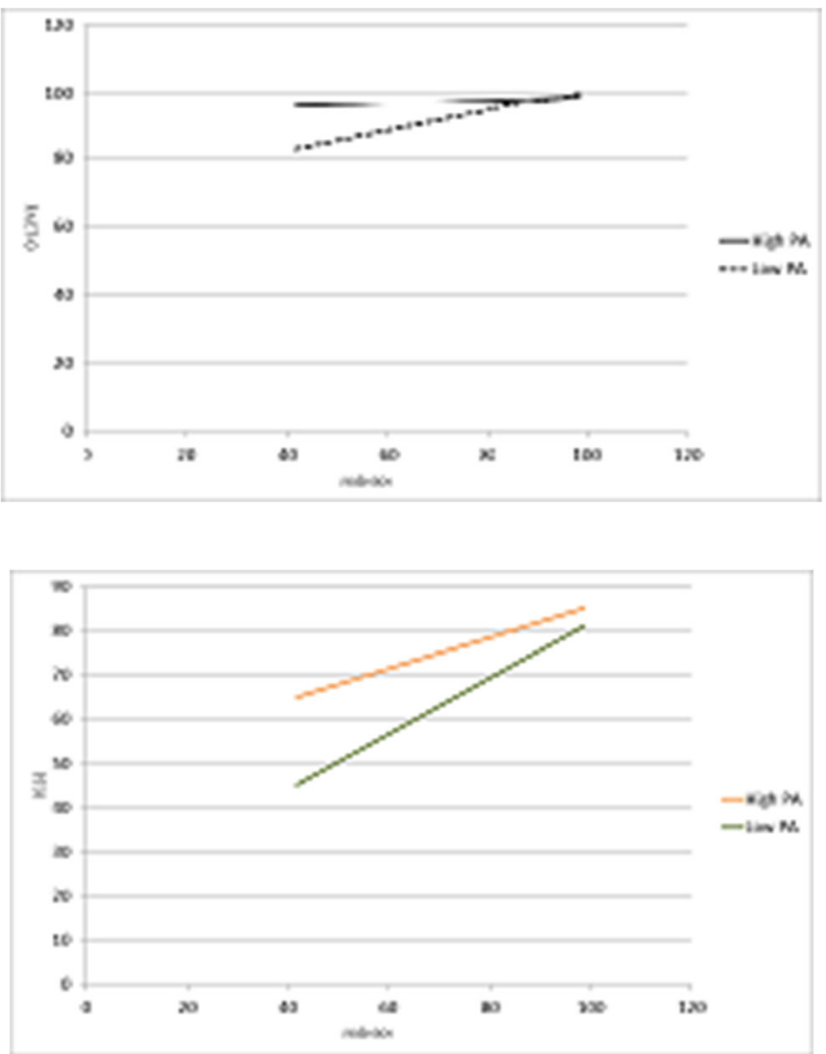


\section{Conclusions}

These findings emphasized that developing resilience and adaptive coping style help the breast cancer survivors to improve well-being and psychological growth.

eP614

A RETROSPECTIVE AUDIT, USING A HOLISTIC NEEDS QUESTIONNAIRE, TO IDENTIFY THE UNMET NEEDS OF UVEAL MELANOMA SURVIVORS THAT ARE UNDERGOING REGULAR LIVER SURVEILLANCE

J. Upton ${ }^{1}$

${ }^{T}$ The Clatterbridge Cancer Centre, Integrated Care, MERSEYSIDE, United Kingdom

\section{Introduction}

In the UK there are around 500 new cases of uveal melanoma diagnosed each year. Around $30 \%$ of these cases will die of distant metastases before 5 years after diagnosis and around $45 \%$ at 15 years after diagnosis. Unlike cutaneous melanoma, approximately $90 \%$ of distant metastases from UM occur in the liver. National guidance recommends life-long liver surveillance to enable early detection of metastases. Research studying anxiety for patients enrolled in cancer screening programmes is variable, however, all studies concur that anxiety is a common problem observed in patients undergoing surveillance for cancers. This presentation reports the findings of an audit that has studied the unmet needs of cancer survivors who are undergoing regular liver surveillance having previously been diagnosed with uveal melanoma.

Objectives

- Identify patterns between unmet needs and time points within the patient's surveillance journey

- Identify statistically significant trends in the unmet needs of the patients

Methods

Using the National Cancer Survivorship Initiative Holistic Needs Assessment questionnaire (2012), a retrospective audit of 119 uveal melanoma survivors undergoing regular liver surveillance was conducted.

Results

Unmet needs were identified in $43 \%$ of patients, with $52 \%$ of patients reporting emotional concerns. An average of $41 \%$ reported physical concerns throughout the trajectory. Baseline visits showed higher levels of unmet physical and emotional needs with the level of concern reported as higher.

\section{Conclusions}

In most of the categories concerns and unmet need improved with the elapse of time, however, some physical symptoms presented later and could be late effects of primary treatment or reflect an ageing population.

\section{eP615}

USING LONGITUDINAL INTERVIEWS TO EXPLORE CANCER SURVIVORS' EXPERIENCES RETURNING TO WORK AFTER PRIMARY TREATMENT: INSIGHT INTO THE METHOD

R. Urquhart ${ }^{1}$, E. Drake ${ }^{1}$

${ }^{T}$ Dalhousie University, Surgery, Halifax, Canada

\section{Introduction}

Despite rising long-term survival after a cancer diagnosis, survivors often face long-term effects of their cancer and its treatment. From a practical perspective, many report substantive challenges returning to work following cancer treatment.

\section{Objectives}

To understand the feasibility and value of using longitudinal interviews to explore cancer survivors' return to work (RTW) following primary cancer treatment.

\section{Methods}

We conducted a prospective, longitudinal study to explore cancer survivors' RTW after primary treatment and understand how their needs and experiences changed over the course of RTW. Specifically, we conducted three interviews per survivor at the following timepoints: end of primary treatment, 3 months post-primary treatment, and 9 months post-primary treatment. Data were analyzed using thematic analysis.

Results

Thirteen survivors enrolled in this study. Only one survivor did not complete the third interview, for a total of 38 (of a possible 39) interviews. Survivors' experiences with RTW changed over the course of the study. This included both internal (e.g., contemplations on the meaning of one's work) and external (e.g., organizational responses to illness) changes. Regarding the latter, while survivors frequently experienced supportive coworkers upon RTW, many perceived coworkers became less supportive of the person's long-term side effects (e.g., fatigue, pain) as time from treatment increased.

Conclusions

While infrequent in practice, longitudinal interviewing is feasible in the cancer survivor population. By providing an in-depth picture of survivors' evolving experiences and narratives, the method reveals important issues that would not be identified in a traditional "single snapshot" study design.

\section{eP616}

CHEMOTHERAPY-INDUCED CARDIOTOXICITY IN PATIENTS WITH COLORECTAL CANCER - SYSTEMATIC REVIEW

L. Weisser ${ }^{1}$, V. Arndt ${ }^{1}$, I. Finke ${ }^{1}$

${ }^{T}$ German Cancer Research Center DKFZ, Clinical Epidemiology and Aging Research, Heidelberg, Germany

\section{Introduction}

Progress in treatment with chemotherapy has led to an increased survival in patients with colorectal cancer (CRC). This is one reason, why potential negative consequences of chemotherapy need to be considered carefully during cancer therapy. One major negative consequence is the cardiotoxicity of certain chemotherapy-regimens which can lead to limitations of the chemotherapy-dosage, impaired quality of life or, at worst, to an earlier death.

\section{Objectives}

The aim is to systematically review the prevalence of cardiovascular events in colorectal cancer patients who underwent chemotherapy and identify possible risk groups.

\section{Methods}

A systematic review will be conducted using the databases PubMed and Web of Science. The protocol will be registered in the international prospective register of systematic reviews (PROSPERO). Full-texts will be screened by a second independent reviewer. The PRISMA flow diagram is used to document the selection-process at all stages.

\section{Results}

A total of 2,949 papers were retrieved from the databases. After title- and abstract-screening, a number of $n=95$ papers is eligible for full-textscreening. Preliminary results indicate that especially 5 -fluorouracil plays a major role in the cardiotoxicity of CRC patients. Final results will be presented in June 2018.

\section{Conclusions}

The results of this systematic review will help to assess cardiovascular risk in CRC patients due to certain types of chemotherapy-regimens. The 
identification of risk groups can contribute to more considerate cancer therapy planning and monitoring and thereby improve care of CRC patients.

eP617

\section{DESIGNING AND IMPLEMENTING A PRIMARY CARE-LED PATHWAY FOR STABLE PROSTATE CANCER PATIENTS} S. Yaganti ${ }^{I}$, L. Price ${ }^{I}$

${ }^{T}$ Healthy London Partnership/National Health Service England, Transforming Cancer Services Team, London, United Kingdom

\section{Introduction}

In the UK, Prostate Cancer remains the most common cancer in men. With improved treatments more men are surviving (Cancer Research UK 2015). The National Institute of Clinical Excellence (NICE) recommends patients stable two years after radical treatment or undergoing "watchful waiting" are offered follow-up outside of hospital (NICE Prostate Cancer: CG175 2014).

Objectives

The Transforming Cancer Services Team for London (TCST) developed a primary care stratified pathway that:

- Facilitated safe transfer of patients from secondary to primary care

- Improved service user experience including access to relevant services

- Improved information given to patients about their follow-up care and supported self-management.

- Provided training for health care professionals to ensure care addresses all medical and holistic needs.

\section{Methods}

Using evaluation data collected at the "proof of concept phase" the TCST worked in collaboration with service users, primary and secondary care clinicians to develop a suite of resources including a business case and a comprehensive service specification to deliver, implement and evaluate the pathway.

\section{Results}

527 patients were safely transferred to primary care in the "test site" of 61 practices and a further 420 practices have signed up to provide the service for their patients. The pathway, deliverable at scale, will release over 11,000 hospital appointments per year across London and has shaped national guidance.

\section{Conclusions}

The pathway demonstrates the feasibility of a primary care-led follow-up service that's cost effective, holistic, supports selfmanagement and integrates prostate cancer follow-up into long term condition management. Reducing the variation in service provision remains a challenge.

\section{eP618}

PSYCHOSOCIAL ADAPTATION OF ADULT CANCER SURVIVORS: A OUALITATIVE STUDY

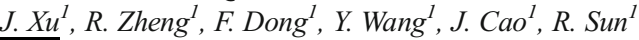

${ }^{T}$ Tianjin Medical University Cancer Institute and Hospital, Nursing Department, Tianjin, China

\section{Introduction}

Cancer survivors might experience clinically relevant psychosocial issues after completing treatment, though some of them successfully adjust during survivorship. Moreover, these psychosocial problems are considered to interfere in survivors' adoption of healthy behaviors, and jeopardize their quality of life. However, little evidence is available on cancer survivors' experience and perspectives with psychosocial adaptation.

\section{Objectives}

To explore how adult cancer survivors experience their psychosocial adaptation in Chinese cultural context.

\section{Methods}

Eighteen cancer survivors were recruited from a cancer center in mainland China, and they were then interviewed individually face-to-face with a semi-structured interview guide. Thematic analysis was used to analyse the data.

\section{Results}

Four main themes emerged from the data: engaging in daily life, interaction with others, seeking spiritual support and nurturing positive attitudes towards life. Though some ones experience significant burden from psychosocial issues, the most tried to adapt their new life and engaged themselves in educational achievement,work engagement and friendship.They sought assistant and support not only from family members but from colleagues, friends and moreover, from Buddhism and God, which were seen as strong spiritual support.However, some survivors expressed a feeling of strong burden as they worried that cancer may return and had no good psychosocial adaptation.

\section{Conclusions}

The deficit in psychosocial adaption of Chinese cancer survivors is evident but after a long time of struggle they had good psychosocial adaption. For those who had poor psychosocial issues, they are advised to enhance coping abilities. There is a urgent need to develop appropriate coping strategies and programs about promoting psychosocial adaptation.

eP619

THE RELATIONSHIP BETWEEN PREOPERATIVE COMPLETE BLOOD COUNT WITH PROGNOSTIC PARAMETERS AND SURVIVAL IN ENDOMETRIAL CANCER PATIENTS

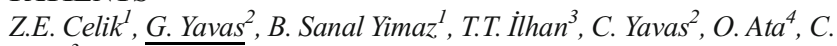
Celik $^{3}$

${ }^{1}$ Selcuk University, Pathology, Konya, Turkey

${ }^{2}$ Selcuk University, Radiation Oncology, Konya, Turkey

${ }^{3}$ Selcuk University, Department of Obstetrics and Gynecology-Division of Gynecologic Oncology, Konya, Turkey

${ }^{4}$ Selcuk University, Medical Oncology, Konya, Turkey

\section{Introduction}

Several inflammatory response biomarkers, including monocyte-tolymphocyte ratio (MLR) neutrophil-to-lymphocyte ratio (NLR), and platelet-to-lymphocyte ratio (PLR) have been reported to predict survival in various cancers.

\section{Objectives}

We evaluated the relationship between preoperative complete blood count with prognostic parameters and survival in endometrial cancer patients.

\section{Methods}

One hundred and forty four patients with the diagnosis of endometrial adenocarcinoma were included the present study. The relation of the complete blood count parameters including white blood cell count (WBC), platelet count (Plt), hematocrit (Hct), hemoglobin (Hb), NLR, PLR, MLR with prognostic parameters such as tumor size, tumor grade, FIGO stage, cervical involvement, positive lymph node number, extracapsular extension of lymph nodes, lympho-vascular invasion, myometrial invasion depth and disease free survival and overall survival investigated statistically.

\section{Results}

Patient with grade III tumors had higher WBC count than those with grade I tumors $(p=0,04)$. PLR and MLR was significantly lower in patients with lymphovascular invasion than those with no invasion ( $\mathrm{p}=0,018$ and $\mathrm{p}=0,028$ respectively). FIGO stage I-II patients had lower PLR than stage III-IV patients $(\mathrm{p}=0.03)$. No relation was determined between pretreatment complete blood count parameters and tumor size, 
cervical involvement, positive lymph node number, extracapsular extension of lymph nodes, myometrial invasion depth and disease free survival and overall survival $(\mathrm{p}>0.05)$.

\section{Conclusions}

Using of multiple pretreatment complete blood count parameters may provide information on outcome of the patients with endometrial cancer. Further studies with larger series and long term follow-up are needed to support the results of the present study.

\section{eP620}

\section{MAXIMUM OXYGEN CONSUMPTION IN CHILDHOOD CANCERS AFTER ACTIVE CANCER TREATMENT: A SYSTEMATIC REVIEW}

V. Yildiz Kabak ${ }^{1,2}$, E. Van Breda ${ }^{2,3}$, T. Duger ${ }^{1}$, J. Mohammed ${ }^{2,4}$, P. Calders $^{2}$

${ }^{1}$ Hacettepe University, Department of Physiotherapy and Rehabilitation, Ankara, Turkey

${ }^{2}$ Ghent University, Department of Physiotherapy and Rehabilitation, Ghent, Belgium

${ }^{3}$ University of Antwerp, Department of Physiotherapy-Research Group MOVANT, Antwerp, Belgium

${ }^{4}$ Bayero University Kano, Department of Physiotherapy, Kano, Nigeria

\section{Introduction}

Three-quarters of childhood cancer survivors (CCS) have post-treatment related problems, of which impairments in physical fitness is one of the most important. Presently, the impairments in physical fitness for CCS are not adequately described in the literature.

\section{Objectives}

The aim of this study was to describe the impairments in the level of physical fitness level by means of maximum oxygen consumption $\left(\mathrm{VO}_{2} \max \right)$ in CCS.

\section{Methods}

Following the PRISMA-guidelines, a systematic search was performed in PubMed, Web of Science and Embase using a combination of the following predefined keywords:"exercise capacity" OR "aerobic capacity" OR "fitness" OR "cardiorespiratory fitness" OR "cardiopulmonary fitness" OR "physical fitness" OR "exercise testing" OR "exercise tolerance" OR "exercise" OR "oxygen consumption" AND "leukemia" OR "childhood cancer" OR "childhood cancer survivor". Studies that met our inclusion criteria were reviewed on methodological quality and evidence synthesis using the Newcastle-Ottawa Scale.

\section{Results}

Totally 2644 articles were identified from the database search. After screening based on the eligibility (abstracts) and inclusion (full texts) criteria, 14 articles remained. The risk of bias scores was between low to high (2/8 to 6/8). Totally 635 CCS (age: $12.3-24.3$ years) were reported in the included studies.The majority (12/14) of the included studies revealed that $\mathrm{VO}_{2}$ max in $\mathrm{ml} / \mathrm{kg} / \mathrm{min}$, was significantly lower in CCS compared to the healthy controls or normative data $(32.48[22-41.4]$ vs. 41.36[28.5-49.58]).

\section{Conclusions}

We concluded from our systematic review that CCS has poor fitness even after active cancer treatment. Therefore, physical activity interventions that are aimed at improving to physical fitness should be emphasized in CCS.

\section{eP621}

FATIGUE AND PSYCHOLOGICAL DISTRESS IN PATIENTS WITH GASTROINTESTINAL STROMAL TUMORS (GIST) RECEIVING TARGETED THERAPY

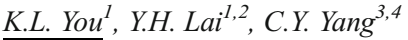

${ }^{I}$ National Taiwan University, School of Nursing, Taipei City, Taiwan R.O.C.

${ }^{2}$ National Taiwan University \& National Taiwan University Cancer Center, Supportive Cancer Care Research Team, Taipei, Taiwan R.O.C.

${ }^{3}$ National Taiwan University Hospital, Department of General Surgery, Taipei, Taiwan R.O.C.

${ }^{4}$ National Taiwan University, College of Medicine, Taipei, Taiwan R.O.C.

\section{Introduction}

Gastrointestinal Stromal Tumors (GIST) is one of the rare cancers with increasing survival time due to the effective targeted therapy. However, long-term or lifetime use of tyrosine kinase inhibitors (TKI) might cause physical and psychological distress.

\section{Objectives}

The aims of this study were to examine (1) the current status of fatigue, anxiety and depression; and (2) examine the relationship among fatigue, anxiety and depression in GIST patients undergoing TKI treatment.

\section{Methods}

This is a cross-sectional study to recruit GIST patients treated with targeted therapy (Glivec in this study) after surgery from surgical outpatient clinic in a medical center in northern Taiwan. Patients were assessed of their anxiety, depression and fatigue by Hospital Anxiety and Depression Scale (HADS), Fatigue Symptom Inventory (FSI) and background information.

Results

A total of 80 subjects were recruited. The results revealedthe mean scores of anxiety and depression were $3.4(\mathrm{SD}=3.8)$ and $4.0(\mathrm{SD}=3.7)$; and $3.8 \%$ and $6.3 \%$ of them were identified as anxiety and depression cases, respectively. The mean score of average fatigue intensity in the past week was $2.8(\mathrm{SD}=2.1)$ and fatigue interference was $2.1(\mathrm{SD}=2.5)$. Patients with higher fatigue is significantly related to higher anxiety and depression with $r=.65$ and $r=.47$, respectively.

Conclusions

Targeted therapy, in this study, Glivec, might lead to fatigue. Although a relatively longer survival time than other cancer patients, GIST patients still experienced mild to moderate psychological distress. Further interventions are suggested to help them to handle fatigue, anxiety and depression.

\section{eP622}

\section{EXPLORATION CULTURAL FACTORS THAT INFLUENCE MEDICAL DECISION MAKING OF CANCER TREATMENT IN JAVANESE PATIENTS}

A.K. Adha ${ }^{l}$, I. Luthfi Kholisa ${ }^{l}$, S. Setiyarini ${ }^{l}$

${ }^{T}$ Universitas Gadjah Mada, Study Programme of Nursing, Sleman, Indonesia

\section{Introduction}

In the case of cancer, shared decision making has been recommended by WHO internationally. It was important to resulting a good bio-psychosocio-spiritual outcome for the patients. However, Javanese society was known still retains cultural values which affect the patients in the decision-making process.

Objectives

this study aims to explored the cultural factors that influence it.

\section{Methods}

This research was a descriptive explorative research with qualitative approach. The sample in the study consisted of 8 patients, two families and a doctors obtained through purposive sampling. Data obtained from depth interviews to the patients, family and doctors, then the data was processed using content analysis.

\section{Results}

In this study, medical decision-making of cancer treatment in Javanese patients influenced by cultural factors such as 1) kinship principles (sedulur), this causes the extended family and the nearest ones such as 
neighbors, girlfriends, heads of constituent hamlets support patients to take a medical treatment, 2) the attitude of obedience and acceptance (nrimo ing pandum), which motivated the patients to heal, 3 ) the division of social class into the have people (priyayi) such as a doctor, a nurse and the have not people (wong cilik) which mean the patients itself, it led feeling of trust to the hospital services.

Conclusions

All of the cultural factors above influence the Javanese patients in the medical decision process, so the type of medical decision making was paternalistic.

\section{eP623}

CAN CUMULATIVE DISADVANTAGE EXPLAIN BREAST CANCER DISPARITIES IN BLACK WOMEN?

S. Bigatti ${ }^{1}$, T. Weathers ${ }^{1}$, K. Mintus ${ }^{2}$, B. $^{\prime}$ Herbert $^{3}$, A.M. Storniolo ${ }^{4}$, L. Robison ${ }^{T}$ H. Tanaka ${ }^{3}$

${ }^{1}$ Fairbanks School of Public Health at IUPUI, Social and Behavioral Sciences, Indianapolis, USA

${ }^{2}$ Indiana University Purdue University Indianapolis, Sociology, Indianapolis, USA

${ }^{3}$ Indiana University School of Medicine, Medical and Molecular Biology, Indianapolis, USA

${ }^{4}$ Indiana University School of Medicine, Hematology/Oncology, Indianapolis, USA

\section{Introduction}

Breast cancer mortality rates for all Black women are $39 \%$ higher than for white women, and tend to be more common and aggressive among Black women $<45$ years. The accumulated social, economic, and interpersonal stressors experienced over the life course, known as cumulative disadvantage (CD), may partially explain these disparities.

\section{Objectives}

To developed and test a comprehensive instrument of CD, the LIFE Tool, and validate it behaviorally and physiologically.

Methods

Recruited 15 Whiteand 15 Black women who had donated normal tissue to a tissue bank. Qualitative interviews, focused on exposure to lifetime stressors, were used to improve and add to items from existing instruments of life course stressors. Telomere length, a biomarker of cellular age and fitness, was obtained from the tissue bank and was the physiological validation tool. Respondents completed the final CD instrument and their telomere length was assessed using DNA extracted from peripheral blood leukocytes.

\section{Results}

Qualitative and quantitative assessments were correlated for childhood $(\mathrm{r}=0.74, p<0.001)$, lifetime ( $\mathrm{r}=0.62, p=0.002)$, and adulthood stressors $(\mathrm{r}=0.42, p=0.022)$. Telomere length was not correlated to $\mathrm{CD}$ score, and subjective assessments of the impact of stress on health were not different between groups. We found distinct patterns by race. Black women with high CD scores experienced substantial shortening of telomeres between two sample time points, white women with low/moderate CD scores gained length between samples.

Conclusions

Findings provide preliminary evidence of validity of this instrument and of $\mathrm{CD}$ as a potential contributor to cellular damage, a physiological precursors of breast cancer

\section{eP624}

A STUDY COMPAIRING NAILS IN PATIENTS RECEIVING TAXANE CHEMOTHERAPY - A STUDY OF NAIL POLISH VERSUS NO NAIL POLISH TO REDUCE NAIL DAMAGE (CONPART)

G. Blanchard ${ }^{l}$, R. Bilsborough ${ }^{l}$, D. Cook ${ }^{l}$, J. Lombard ${ }^{l}$, I. Nordman $^{l}, K$. Randall $^{1}$
${ }^{1}$ Calvary Mater Newcastle, Medical Oncology, Waratah, Australia

\section{Introduction}

Taxane-induced onycholysis has not been clearly linked with ultraviolet (UV) light exposure, however clinically it has been recommended that patients wear dark-coloured nail lacquer to prevent photo-induced onycholysis. In-vitro testing in our lab using human skin cells cultured in 96-well plates with plastic covers coated with various coloured lacquers and exposed to UV radiation showed that all coloured lacquers including neutral-beige equally protected the cells against UV damage.

\section{Objectives}

To test the hypothesis that nail lacquer protects from taxane-induced onycholysis and determine if there is an observable difference in nail toxicity.

Methods

To test the in vitro result in breast cancer patients receiving adjuvant Docetaxel a pilot study of 20 participants was proposed. Participants painted nails of one hand with neutral -beige lacquer. The participants other hand was used as a control.

For the study to be considered non- inferior a $>=30 \%$ reduction in nail changes will be identified.

\section{Results}

Interim results of $\mathrm{N}=5$ patients who have completed their full course of chemotherapy have shown a benefit in 1 patient with a $>30 \%$ observable difference in the hand using the nail lacquer. The study is ongoing.

\section{Conclusions}

Using a cell-based in vitro model, neutral-beige nail lacquer protected skin cells from UV-induced cell death. The benefit of nail lacquer in ameliorating taxane-induced onycholysis in the clinical setting is currently being explored in this pilot study at the Calvary Mater Newcastle. Currently, patients that have been assessed showed a $20 \%$ noninferiority from the use of nail lacquer.

\section{eP625}

INFLUENCE OF COMORBIDITY ON COLORECTAL CANCER TREATMENT PATTERN: RESULTS FROM A POPULATIONBASED STUDY IN GERMANY

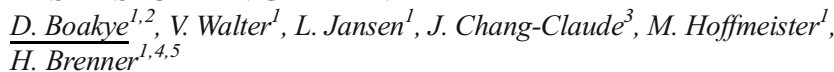

${ }^{1}$ German Cancer Research Center DKFZ, Clinical Epidemiology and Aging Research, Heidelberg, Germany

${ }^{2}$ Heidelberg University, Medical Faculty Heidelberg, Heidelberg, Germany

${ }^{3}$ German Cancer Research Center DKFZ, Unit of Genetic EpidemiologyDivision of Cancer Epidemiology, Heidelberg, Germany

${ }^{4}$ German Cancer Research Center DKFZ, German Cancer Consortium DKTK, Heidelberg, Germany

${ }^{5}$ German Cancer Research Center DKFZ and National Center for Tumor Diseases NCT, Division of Preventive Oncology, Heidelberg, Germany

\section{Introduction}

Colorectal cancer (CRC) is predominantly diagnosed at old age, when comorbidities are common and may influence treatment decisions.

\section{Objectives}

We investigated the extent to which comorbidity was associated with treatment patterns in a population-based cohort of CRC patients in Germany.

\section{Methods}

We used the ICD-10 codes to extract comorbidities from the discharge records of $5067 \mathrm{CRC}$ patients diagnosed in 2003-2015. Overall comorbidity was then quantified with the Charlson comorbidity index (CCI). Adjusted odds ratios (OR) and their 95\% confidence intervals (CI) from multivariable logistic regression were used to investigate associations 
with the type of CRC surgery, length of perioperative hospital stay and receipt of chemo/radiotherapy in the 3-year follow-up data $(N=3971)$.

Results

Patients with moderate-severe comorbidity $(\mathrm{CCI} \geq 2)$ had increased odds of open versus laparoscopic surgery $(\mathrm{OR}=1.47 ; 95 \%$ $\mathrm{CI}=1.12-1.93)$ and longer pre- $(\mathrm{OR}=1.68 ; 95 \% \mathrm{CI}=1.32-2.14)$ and post-surgical $(\mathrm{OR}=1.78 ; 95 \% \mathrm{CI}=1.51-2.10)$ hospital stays. In stage II-III colon cancer patients, comorbidity was associated with receiving adjuvant chemotherapy less often (CCI 1: $\mathrm{OR}=0.49 ; 95 \% \mathrm{CI}=0.29-0.83, \mathrm{CCI} \geq 2$ : $\mathrm{OR}=0.35 ; 95 \% \mathrm{CI}=0.22$ 0.58). In stage II-III rectal cancer patients, patients with comorbidity were also less likely to receive adjuvant chemotherapy (CCI 1: $\mathrm{OR}=0.59 ; 95 \% \mathrm{CI}=0.38-0.92, \mathrm{CCI} \geq 2$ : $\mathrm{OR}=0.66 ; 95 \%$ $\mathrm{CI}=0.43-1.02$ ) and neoadjuvant chemoradiotherapy (CCI 1: $\mathrm{OR}=0.68 ; 95 \% \mathrm{CI}=0.45-1.00, \mathrm{CCI} \geq 2: \mathrm{OR}=0.53 ; 95 \% \mathrm{CI}=0.36-$ 0.79 ).

\section{Conclusions}

Comorbidity is strongly associated with longer hospital stay and less receipt of cancer treatments in CRC patients. Future studies should evaluate to what extent improved prevention or management of comorbidities and geriatric syndromes can further enhance CRC care.

\section{eP626}

\section{STRUCTURED INTERDISCIPLINARY BEDSIDE ROUNDS IN} A DANISH ONCOLOGY WARD - PATIENTS' EXPERIENCES H. Bødtcher ${ }^{1}$, T. Bager ${ }^{1}$, S. Toftdal ${ }^{1}$, S. Ottesen ${ }^{1}$

${ }^{T}$ Zealand University Hospital, Department of Oncology and Palliative Care, Roskilde, Denmark

\section{Introduction}

Hospitalized cancer patients usually suffer from complex issues that requires interdisciplinary attention. Good communication between the multiple professionals and the patient is important to ensure a shared plan for care and treatment. Structured interdisciplinary bedside rounds (SIBR) has been associated with improved interdisciplinary collaboration, quality of communication, understanding of the plan and patient safety. In 2017 the concept of SIBR were implemented in our oncology ward. The first day after admission the patient complete a symptom assessment questionnaire and the outcome contributes to the agenda for the following SIBR. The patient relatives, physician, nurse, physiotherapist, occupational therapist, dietician, social worker, and clergyman participate in the SIBR. The patients' experience with SIBR is unknown.

Objectives

To investigate patients' and relatives' expectations and experiences with the SIBR in relation to their understanding of the plan, the follow-up, the communication, the level of involvement, the time spent and the participation of multiple professionals.

Methods

Patients and relatives who participate in SIBR are invited to three semistructured interviews: before SIBR, immediately after and the day of discharge. The interviewees are included consecutively until information richness. The transcription and analysis is conducted concurrently with the data collection.

\section{Results}

Preliminary data analysis suggest that the patients and their relatives are highly satisfied with the SIBR. Final results will be available in the beginning of 2018

\section{Conclusions}

Patients' experiences of SIBR will affect the future development and organization of the bedside rounds. Preliminary results suggest that the SIBR improves the information and involvement of the patients.
eP627

DEVELOPMENT OF INTERNATIONAL GUIDELINES AND EDUCATION RESOURCES FOR CANCER-ASSOCIATED THROMBOSIS (CAT)

T. Butler ${ }^{1}$, C. Escalante ${ }^{2}$, C. Rojas-Hernandez ${ }^{2}$, C. Font ${ }^{3}$, T. Oo $^{2}$

${ }^{T}$ Department of Veteran Affairs, Pharmacy, Nashville, USA

${ }^{2}$ University of Texas MD Anderson Cancer Center, Department of General Internal Medicine, Houston, USA

${ }^{3}$ Hospital Clinic de Barcelona, Medical Oncology Department, Barcelona, Spain

\section{Introduction}

Thrombosis risk is estimated to be as high as $18 \%$ in cancer patients and a source of morbidity and mortality. The hemostasis study group organized members to develop clinical practice guidelines for cancer-associated thrombosis (CAT). The 2017 MASCC/ISOO workshop hosted by the hemostasis/education study group revealed that further education materials might be indicated to improve patient understanding.

\section{Objectives}

To review the recent literature to determine recommendations on CAT with an initial focus on inpatient practice primary prevention. Of note, information will also be collected to develop patient friendly educational tools.

Methods

The co-chairs met with MASCC members periodically through teleconference to discuss project development. We adapted literature search methods similar to previous clinical practice guidelines published by MASCC/ISOO. The co-chairs conducted the initial literature search using MEDLINE and the Cochrane database and eliminated studies if the abstract revealed exclusion criteria. All other studies were pulled and divided to each participant. Grading and recommendations will follow previous guidelines by MASCC/ISOO.

Results

1806 studies were reviewed during the literature search. 1282 were eliminated based on the abstract. The following 524 studies have been assigned to each member and the full article will be reviewed for inclusion/exclusion criteria and grading, if applicable.

\section{Conclusions}

Each participant will review at least 48 studies with the goal of developing guidelines and educational tools for inpatient CAT prophylaxis. The target date is to present to the participants of the hemostasis study group in Vienna and to the executive leaders of MASCC/ISOO in 2018.

eP628

\section{INFUSION REACTIONS IN ONCOLOGY}

P. Cavacol, S. Ponte 2 , C. Cabral ${ }^{2}$, M.H. Miranda ${ }^{2}$, A.M. Mourão ${ }^{3}$

${ }^{1}$ Hospital de São Francisco Xavier - Centro Hospitalar de Lisboa Ocidental, Pharmacy, Lisboa, Portugal

${ }^{2}$ Hospital de São Francisco Xavier - Centro Hospitalar de Lisboa Ocidental, Oncology Day Hospital, Lisboa, Portugal

${ }^{3}$ Hospital de São Francisco Xavier - Centro Hospitalar de Lisboa Ocidental, Oncology Service, Lisboa, Portugal

\section{Introduction}

Virtually all chemotherapeutic agents have the potential to initiate infusion reactions, which can be defined as unexpected reactions that cannot be explained by known toxicity profile of the drug. These reactions range from mild flushing to anaphylaxis. Because of the frequency, potential severity and need for rapid intervention during infusion reactions, the presence of protocols is mandatory.

\section{Objectives}

To analyze the prevalence of infusion reactions and compliance to existing protocol in oncology patients. 
Methods

Analysis of prevalence of infusion reactions and compliance to existing protocol, through a descriptive, retrospective study, conducted between June and December of 2016, in oncology sector of a general central hospital. Data were collected by hospital records review.

\section{Results}

During the study period a total of 27 reactions were reported in 26 patients, the majority during second and subsequent administrations $(n=18)$. Cytotoxics with highest number of reactions were oxaliplatin $(n=10)$, paclitaxel $(n=4)$ and rituximab $(n=3)$. Patients experienced flushing $(n=15)$, cardiovascular symptoms, like tachycardia and changes in blood pressure $(n=13)$, tongue swelling $(n=8)$ and dysarthria $(n=7)$. Majority of reactions were handled using algorithms described in current ward protocol $(\mathrm{n}=25)$. Of the 26 patients, 5 suspended or had their chemotherapy substituted, in 2 a desensitization protocol was used, one completed full course of chemotherapy.

\section{Conclusions}

Infusion reactions to chemotherapy are unpredictable adverse events with potentially lethal consequences. Accurate grading of reactions is essential in determining how to proceed with treatment. When quickly addressed, these reactions are easily reversible and, if necessary, use of desensitization protocols can be considered in an individualized manner.

\section{eP629}

\section{MEDICATION REVIEW IN PATIENTS WITH PRESCRIPTION OF DRUGS SUBJECT TO ADDITIONAL MONITORING

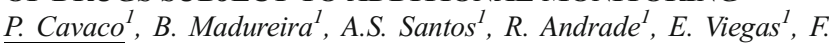 Falcão ${ }^{T}$ \\ ${ }^{1}$ Hospital de São Francisco Xavier - Centro Hospitalar de Lisboa Ocidental, Pharmacy, Lisboa, Portugal}

\section{Introduction}

Polymedication increases the risk of developing drug interactions, and this risk is higher as the number of drugs used increases. At the hospital, medication review is performed for patients receiving treatment with drugs subject to additional monitoring.

\section{Objectives}

Characterize the profile of drug interactions in oncological / haematological patients proposed for treatment with drugs subject to justification

\section{Methods}

Descriptive, observational, retrospective study conducted between January and December 2016 in a central general hospital. Oncological / haematological patients with drugs subject to justification were included. Information was collected through hospital records. Drug interactions were manually screened and classified using Lexi-interact data base risk rating.

\section{Results}

Total of 174 patients that had drugs subject to justification were included. We identified 57 drug interactions between drugs to treat comorbidities and proposed therapy $(32,7 \%$ of patients). Majority of patients were on 5 or more drugs. Interactions identified had the following risk classification: 48 risk C, 5 risk D and 4 risk $X$. Groups with highest number of interactions were cardiovascular system, CNS and drugs used to treat endocrine diseases. Everolimus and bortezomib had highest number of drug interactions/ number of requests. Management plan included monitoring, suggestions to change therapy and therapy modification (risk X nteractions).

\section{Conclusions}

Knowledge of potential drug interactions can lead to development of institutional strategies to minimize it and to prevent significant changes in therapy goal. Thus, it's important to identify criteria for selecting patients who can benefit most from this type of evaluation.
eP630

ORALLY-ADMINISTERED EMU OIL REDUCES DISEASE SEVERITY IN A MURINE MODEL OF COLITISASSOCIATED COLORECTAL CANCER

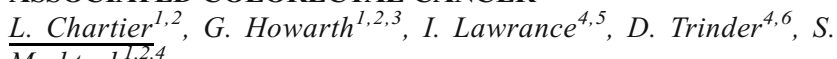
Mashtoub ${ }^{1,2,4}$

${ }^{1}$ The University of Adelaide, Adelaide Medical School, Adelaide, Australia

${ }^{2}$ Women's and Children's Hospital, Gastroenterology Department, North Adelaide, Australia

${ }^{3}$ The University of Adelaide, School of Veterinary and Animal Sciences, Roseworthy, Australia

${ }^{4}$ University of Western Australia, School of Medicine, Murdoch, Australia ${ }^{5}$ Saint John of God Hospital, Centre for Inflammatory Bowel Diseases, Nedlands, Australia

${ }^{6}$ Harry Perkins Institue of Medical Research, Medical School, Nedlands, Australia

\section{Introduction}

Ulcerative colitis is a chronic inflammatory bowel disorder. Colitis patients are at an increased risk of developing colitis-associated colorectal cancer (CA-CRC). Previously, we identified the protective and reparative properties of Emu Oil (EO) in models of gastrointestinal damage.

\section{Objectives}

To determine whether EO could reduce chronic colitis and prevent the onset of neoplasia in an azoxymethane (AOM)/dextran sulfate sodium (DSS) model of CA-CRC.

\section{Methods}

C57BL/6 mice were injected (i.p.) with AOM, followed by three DSS/water cycles (seven days DSS, 14 days water) ad libitum. Mice (n=9/group) were orally-administered water or EO $(80 \mu \mathrm{L}$ or $160 \mu \mathrm{L})$ thrice weekly for nine weeks. Bodyweight and disease activity index (DAI) were measured daily. Burrowing activity and colitis progression were analyzed throughout the trial. At sacrifice, tumor number and size were recorded.

\section{Results}

AOM/DSS induced significant bodyweight loss $(24 \%$; $<<0.05)$ compared to normal controls which was attenuated following EO-treatment (maximum bodyweight loss; $80 \mu \mathrm{L}: 7 \%$; $160 \mu \mathrm{L}: 11 \%$; p $<0.05$ ). EO reduced DAI of AOM/DSS animals (20\%; $\mathrm{p}<0.05)$. Burrowing activity was impaired by AOM/DSS on days 48 and $61(\mathrm{p}<0.05)$; an effect that was restored by EO on day $61(\mathrm{p}<0.05)$. Additionally, EO ameliorated colonoscopically-assessed colitis severity compared to AOM/DSS controls $(\mathrm{p}<0.05)$. EO-treatment $(80 \mu \mathrm{L})$ resulted in fewer small tumors in CA-CRC treated mice $(\mathrm{p}<0.05)$ compared to AOM/DSS controls. Finally, EO in normal mice did not elicit any demonstrable effect.

\section{Conclusions}

EO improved clinical indicators of colitis and reduced the number of small colonic tumors in CA-CRC mice, suggesting potential for EO application in colitis- and colon cancer-management.

\section{eP631}

\section{RAISING AWARENESS ON RARE VASCULAR VENOUS TUMORS}

B. CHESHMEDZHIEVA ${ }^{1}$, L. Despotova-Toleva ${ }^{2}$, V. Anastasov ${ }^{3}, Y$. Zahariev $^{3}$

${ }^{1}$ Medical University Plovdiv, Vascular surgery, PLOVDIV, Bulgaria

${ }^{2}$ Medical University, Medical Faculty, Plovdiv, Bulgaria

${ }^{3}$ Medical University, Vascular surgery, Plovdiv, Bulgaria

\section{Introduction}

Primary venous leiomyosarcoma of greater saphenous veinis very rare and unusual but aggressive tumor with a high degree of local recurrence and early haematogenic metastases. 


\section{Objectives}

To raise awareness to rare case of leiomyosarcoma firstly presented with deep venous thrombosis.

\section{Methods}

The authors present a rare clinical case of a 73 years old man with leiomyosarcoma of vena saphena magna. Literature review and analysis on these rare cases was also performed.

\section{Results}

We report 73 years old patient treated with Xarelto and Detralex for deep venous thrombosis of vena saphena magna, femoralis communis dextra and the distal part of vena iliaca externa dextra. Small palpable painful inguinal node. Followed by vascular surgeon for 6 months. Because of discomfort, pain and increasing size of the inguinal node formation he was admitted to internal diseases department. CT suspected for enlarged para aortal packets of lymph nodes. Inguinal nodes biopsy has revealed leiomyosarcoma from vascular origin. After surgical removal and venous recanalization the patient was sent to oncology committee. No chemo or radiotherapy was suggested. No pathological findings on PET 12 month after operation. Now the patient is good general condition and has good quality of life.

\section{Conclusions}

Reporting such cases is very important for raising awareness to very rare venous tumors. Combined efforts of different medical specialists - general practitioners, vascular surgeons, pathologists, radiologists etc. are needed for recognition and proper treatment of these serious life-threatening diseases and contribute to better prognosis and quality of life.

\section{eP632}

THE EFFECTS OF THORACIC EPIDURAL ANALGESIA DURING PERCUTANEOUS RADIOFREQUENCY ABLATION FOR HEPATOCELLULAR CARCINOMA

\section{E.J. Choi ${ }^{l}$}

${ }^{T}$ Pusan National University Yangsan Hospital, Department of Anesthesia and Pain Medicine, Yangsan, Republic of Korea

\section{Introduction}

Percutaneous radiofrequency ablation (RFA) is a useful and safe treatment for hepatocellular carcinoma (HCC). However, RFA performed under local anesthesia with intravenous sedation induces pain during or after the procedure.

Objectives

This study reviewed the efficacy of thoracic epidural anesthesia during percutaneous RFA for HCC.

\section{Methods}

Patients who underwent RFA for HCC in the past five years were divided into the epidural group and control group (local anesthesia with monitored anesthesia care (MAC)). We retrospectively reviewed opioid consumption during and after the procedure, changes in pain (numeric rating scale; NRS) after the procedure, presence of respiratory depression, changes of blood pressure during the procedure, length of hospital stay, postoperative nausea and vomiting (PONV) and voiding difficulty.

\section{Results}

Compared to the control group, thoracic epidural anesthesia led to a reduction of opioid consumption during and after the procedure. Further, thoracic epidural anesthesia also decreased severity of postprocedural pain and respiratory depression. Groups were not different with respect to blood pressure during the procedure, length of hospital stay. PONV and voiding difficulty were higher in the epidural group. Conclusions

Thoracic epidural anesthesia is an effective anesthetic and analgesic method during RFA for hepatic cancer.
eP633

\section{SPIRITUALITY AND RELIGIOSITY IN LATIN AMERICAN PALLIATIVE CARE HEALTH CARE PROFESSIONALS (LAHCP)}

M. Delgado-Guay ${ }^{I}$, M. Reyes Donoso ${ }^{2}$, M. Lopez Saca ${ }^{3}$, M. Riveros Rios $^{4}$, N. Colautti ${ }^{5}$, I. Espin Gonzalez ${ }^{6}$, G. Toledo Ahumada ${ }^{7}, T$. Pastrana Uruena ${ }^{8}$

${ }^{I}$ The University of Texas MD Anderson Cancer Center, PalliativeRehabilitation and Integrative Medicine, Houston, USA

${ }^{2}$ Grupos de Espiritualidad para vivir con Dignidad, Spiritual Care, Santiago, Chile

${ }^{3}$ Hospital Nacional Dr. Juan José Fernández. Zacamil., Cuidado Paliativo, San Salvador, El Salvador

${ }^{4}$ Universidad Nacional de Asunción-, Hospital de Clínicas- Cuidado Paliativo, Asuncion, Paraguay

${ }^{5}$ Hospices Casa de la Bondad, Psicologia y Cuidado Espiritual, Cordova, Argentina

${ }^{6}$ Instituto Oncológico Luis Razetti, Cuidado Paliativo, Caracas, Venezuela

${ }^{7}$ Instituto Religiosas Hijas de Santa Ana, Cuidado Espiritual, Santiago, Chile

${ }^{8}$ University Hospital RWTH Aachen, From the Spirituality Commission of the Asociacion Latino Americana de Cuidados Paliativos ALCP, Aachen, Germany

\section{Introduction}

Spirituality/religiosity are common with Latino cultural-values and daily-lives. These elements are essential in delivering Quality Palliative-Care. There is limited literature regarding LAHCP' spiritual/religious characteristics, or how these commitments shape their clinical engagement.

Objectives

To describe demographics, personal/professional role of spirituality/ religiosity(numeric-scale $0=$ the lowest, $10=$ the highest), spiritual-care/assessment provided and resources at their work areas.

\section{Methods}

From June/1 to December/31, 2017, a crossectional study using an anonymous and voluntary Online-Survey was provided to active member of ALCP.

Results

235/353 members from 20 Latin-America-Countries participated, Response-rate: $67 \%$. Median age $47(\mathrm{SD}+/-12), 75 \%$ were women. $80 \%$ were Catholic. $58 \%$ were physicians, $19 \%$ nurses, $12 \%$ psychologists, and other team-members $12 \%$. Median-time of working in Palliative-Care: 9 years(+/-7). LAHCP considered themselves spiritual(median: 8/10, range 0-10) and religious(5, 0-10). LAHCP considered Spirituality/Religiosity very important in their lives $(9 / 10,0-10$ and $6 / 10,0-10$, respectively) higher levels of Spirituality in psychology compared with physicians $(9 / 10 \pm 1$ vs $8 / 10 \pm 2, p=0.009$ ), and Religiosity in nurses. LAHCP reported that Spirituality/Religiosity were a source of strength and comfort $(9 / 10, \mathrm{SD}+/-2)$, helped them to cope with their problems, including illness $(8 / 10, \mathrm{SD}+/-3)$, and helped them to keep their quality of life in a stressful work environment(8/10, SD+/-4), highlysignificant in those belonging to a church-community $(\mathrm{p}=0.000)$, time working in Palliative-Care $(p=0.01)$, age $(p=0.03) .179$ / $235(76 \%)$ provided spiritual-assessment/care to their patients; mostly those highly spiritual $(\mathrm{p}<0.0001)$. The reasons spiritualassessment wasn't provided: lack of training $(18 \%)$, lack of time(11\%), worries about patients/caregivers' privacy(6\%)

\section{Conclusions}

Most of LAHCP considered themselves spiritual/religious. LAHCP assigned a highly important role to Spirituality/Religiosity in life. More spiritual care education is needed to fully integrate it in their daily practice. 


\section{eP634}

THE EFFECT OF COLLEAGUE SOLIDARITY AND OCCUPATIONAL COMMITMENT ON ONCOLOGY NURSES' TURNOVER INTENTION IN MAINLAND CHINA

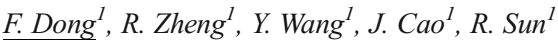

${ }^{T}$ Tianjin Medical University Cancer Institute and Hospital, Nursing

Department, Tianjin, China

\section{Introduction}

Colleague solidarity of nurses, recognized as support among nurses and the sharing of professional knowledge, methods and skills with each other, is a key indicator to evaluate the team work. High colleague solidarity level could lead to effective communication among nurses, improved performance and maintained stability of nursing team. Otherwise, occupational commitment is a good predictor to turnover intention. Little is known about Chinese oncology nurses' colleague solidarity and occupational commitment and the effects on turnover intention.

Objectives

To investigate the effect of nurses' colleague solidarity and occupational commitment on their turnover intention.

Methods

250 registered oncology nurses were recruited from June 2017 to July 2017. The questionnaires, including the Colleague Solidarity of Nurses Scale, the Occupational Commitment Questionnaire and Turnover Intention Questionnaire, were distributed to the potential participants.

\section{Results}

222 participants completed the questionnaire. The total scores of colleague solidarity, occupational commitment, and turnover intention were $91.29 \pm 9.29,87.56 \pm 14.25,9.29 \pm 4.00$. There was a significant negative correlation between colleague solidarity $(r=-0.269, p=0.000)$, occupational commitment $(r=-0.550, p=0.000)$ and turnover intention. Negative colleague solidarity, opportunity commitment and the whole occupational commitment entered the multiple regression equation and explained $36.1 \%$ of the variance of oncology nurses' turnover intention level.

\section{Conclusions}

Chinese oncology nurses have moderate level of colleague solidarity, opportunity commitment and low level of turnover intention, and there were negative connections among these variables. Nursing managers are advised to pay attention to nurses with negative opinions about nursing work, improve their colleague solidarity, and promote occupational commitment, which may in turn decrease turnover intention.

\section{eP635}

\section{N-DIHYDROGALACTOCHITOSAN AS A POTENT IMMUNE ACTIVATOR IN THE COURSE OF THE TREATMENT OF METASTATIC CANCER}

A. ElHussein ${ }^{1}$, M. Hamblin ${ }^{2}$

${ }^{T}$ The National institute of Laser Enhanced Science- Cairo universityEgypt, Laser Applications in photo-chemistry and photobiology, Cairo, Egypt

${ }^{2}$ Wellman Center for Photomedicine- Massachusetts General HospitalHarvard Univetrsity, Dermatology, Boston, USA

\section{Introduction}

Immunotherapy has become one of the fastest growing areas of cancer research. A promising in situautologous cancer vaccine (inCVAX) uses a novel immune activator, $N$-dihydrogalactochitosan (GC), that possesses the ability to stimulate dendritic cells (DC). inCVAX is a combination treatment procedure involving treatment of the tumor with a thermal nearinfrared laser to liberate whole cell tumor antigens, followed by injection of GC (a glucosamine polymer with galactose attached to the amino groups) into the treated tumor thereby inducing a systemic antitumor immune response. We studied the stimulatory action of GC on relatively immature DCs (DC2.4 cell line) in vitro.

\section{Objectives}

The objectives are to study the stimulating effect of GC on the professional antigen presenting cells, DCs in vitro and to visualize the binding as well as comparing such activation (if any) with lipopolysaccharide (LPS) as a positive control that activates DCs via binding to Toll-like receptors (TLR4).

\section{Methods}

The DCs' expression of MHC II, CD80, and CD11c and IL12 were tested after the introduction of GC (1gm).

\section{Results}

GC at $1 \mathrm{mg} / \mathrm{mL}$ was a potent stimulator for DC with limited toxicity, giving increased expression of major histocompatibility complex class 2, CD80, and CD11c. Confocal imaging also revealed qualitatively increased uptake of antigen by DCs after the introduction of GC. To visualize cellular uptake, GC was conjugated with FITC-fluorophore revealing its cellular internalization after 8 hours. In some cases GC was more effective than the toxic TLR4 agonist.

\section{Conclusions}

inCVAX is a promising therapeutic approach for the treatment of primary and metastatic cancer

\section{eP636}

INTRODUCTION OF A SPEECH PATHOLOGY ASSISTANT ROLE FOR SWALLOW SCREENING IN A HEAD AND NECK RADIOTHERAPY CLINIC

\section{J. Frowen ${ }^{1}$, R. Hughes ${ }^{1}$, N. Kiss ${ }^{2}$}

${ }^{T}$ Peter MacCallum Cancer Centre, Nutrition and Speech Pathology, Melbourne, Australia

${ }^{2}$ Deakin University, Institute for Physical Activity and Nutrition, Melbourne, Australia

\section{Introduction}

Best practice suggests that all moderate-high risk head and neck $(\mathrm{H} \& \mathrm{~N})$ cancer patients are seen by a speech pathologist for assessment and education when commencing definitive or post-operative radiotherapy. However this is not always feasible due to high patient numbers and limited staffing.

\section{Objectives}

To investigate the effectiveness of a speech pathology assistant (SP-A) role for swallowing screening and education for specific patients in a multidisciplinary H\&N treatment clinic.

\section{Methods}

A training module was established to up-skill the SP-A in the screening and education to be undertaken. A pre-test post-test design compared outcomes in two separate groups prior to and then following implementation of the SP-A role. Outcomes included: best practice compliance, speech pathology time spent with complex patients, swallowing-related admissions and patient satisfaction.

\section{Results}

Fifty-one patients were included pre-implementation and 35 post-implementation, with $16(46 \%)$ patients receiving SP-A screening/education post-implementation. The proportion of moderate-high risk patients seen by speech pathology pre-implementation was $63 \%$, compared to $94 \%$ seen by speech pathology or SP-A post-implementation $(\mathrm{p}=0.002)$. There was a (non-significant) increase in speech pathology time spent with complex patients post-implementation (total minutes per patient: mean $252, \mathrm{SD}=144$ vs $279, \mathrm{SD}=115$ ). There was no increase in swallowing-related admissions, and a (non-significant) increase in patient satisfaction post-implementation, indicating no compromise to patient safety or satisfaction. 


\section{Conclusions}

Implementing a speech pathology assistant role for screening patients within the H\&N treatment clinic allowed delivery of best practice and increased time for speech pathologists to spend with complex patients, without compromising patient safety or satisfaction.

\section{eP637}

PATIENT'S DECISIONAL CONTROL PREFERENCES IN PALLIATIVE CARE: SURVEY FROM AN INDIAN TERTIARY CANCER CENTRE

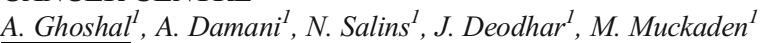

${ }^{T}$ Tata Memorial Centre, Palliative medicine, Mumbai, India

\section{Introduction}

The frequency of passive decisional control (patients prefers physician to make decisions) has been reported to be variable but generally larger among patients living in developing countries.

Objectives

This study aimed to determine the frequency of passive DCP among patients with advanced cancer in a tertiary cancer center, and identify its association with their socio-demographic and clinical characteristics.

\section{Methods}

150 patients referred to palliative care underwent assessment of decisional control preferences using validated tools including Control Preference Scale tool, Satisfaction with Decision Scale and understanding of illness questionnaire. Information regarding patient characteristics including age, gender, education, marital status, employment, Karnofsky Performance Scale, cancer stage and type, religion were also collected. Descriptive statistics and Logistic regression analysis were performed.

Results

Median age was 48 years, Karnofsky 90, and 55.3\% were men. Shared, Active (patient prefers to make decision by his/her own) and Passive DCP were $20.7 \%, 26.7 \%$ and $52.7 \%$ respectively $(\mathrm{n}=150) .51 .3$ were satisfied by the way the actual decisions were made. $70.7 \%$ felt that their cancer was curable. Passive DCP did not vary across regions. Multivariate analysis shows that the passive DCP was significantly associated in better KPS [expB 1.07 (1.01-1.15), $\mathrm{p}=0.03$ ].

Conclusions

There are significant differences in DCP with KPS. Patients report high level of satisfaction with their treatment decision, though they have poor understanding of their prognosis and understand their treatment to be of curative intent. Individualized understanding DCP and focus on illness understanding may be important for quality care and patient satisfaction outcomes.

\section{eP638}

KNOWLEDGE TRANSLATION PLAN GUIDES: A PRAGMATIC, CONCEPTUAL SYNTHESIS

I. Graham ${ }^{1}$, C. McCutcheon ${ }^{2}$, K. Mrklas ${ }^{3}$

${ }^{T}$ University of Ottawa, School of Epidemiology and Public Health, Ottawa, Canada

${ }^{2}$ Ottawa Hospital Research Institute, Clinical Epidemiology Program,

Ottawa, Canada

${ }^{3}$ University of Clagary, Public Health, Calgary, Canada

\section{Introduction}

Knowledge translation (KT) guides help users to devise KT strategies for their research and its use. While the number and diversity of these guides has grown, their nature and content remain poorly understood. This pragmatic review breaks down the similarities and differences between KT guides and assesses their quality.
Objectives

1. Present a comprehensive overview of the KT guidance readily accessible to researchers and knowledge users; and

2. Assist researchers and knowledge users with an analysis of the guides' key attributes, gaps, strengths and weaknesses, and alignment with evidence and best practice.

\section{Methods}

We developed a Google plain search strategy to mimic common user search techniques, in consultation with an academic librarian. Term clusters for knowledge translation (16) and guide (9) were joined with a Boolean operator (AND), then searched with/without quotations. English language guides providing advice on KT plan development (e.g., tangible steps, instructions, general advice) were included. Mixed methods were used to extract pre-defined variables and inductively capture key elements and themes.

\section{Results}

Few guides describe an underlying conceptual framework or evidence base, nor are they independently evaluated. Common guide characteristics include consideration of context (barriers, facilitators, user needs); research knowledge; audience; strategy types; and monitoring, evaluation, and resource requirements. Through an inductive construction of content categories we will demonstrate the most common KT advice offered, describe the degree to which guides are congruent with the latest KT evidence, and present a composite guide that synthesizes distinct elements of all included guides.

\section{Conclusions}

This review provides information upon which to select KT guides.

eP639

\section{ILLUSTRATION OF THE DISCREPANCY BETWEEN RESULTS FROM CLINICAL TRIALS WITH CANNABIS AND REPORTS FROM THE FIELD - SHOULD THE TRADITIONS OF CLINICAL RESEARCH BE MODIFIED? \\ J. Grunfeld \\ ${ }^{T}$ Asaf HaR $o f e h$ Medical Center- affiliated to the Tel Aviv University, Oncology, Tel Aviv, Israel}

\section{Introduction}

Recently, the medical use of cannabis has been gaining attention. Whereas claims of remarkable clinical benefit are being propagated throughout social media, the medical institution remains aloof, backing its stance with the lack of reliable data, and the failure to reproduce the claims in the few clinical trials conducted to date. Notably, firsthand clinician and patient experience supports the proposition that cannabis harbors significant potential to improve the management of hitherto poorly treated clinical challenges.

\section{Objectives}

To derive insights relevant to the formulation of strategies to exploit the posited therapeutic potential of cannabis.

\section{Methods}

Apposition of results from published clinical trials with cannabis with direct observations of patients using cannabis for medical purposes.

The data for analysis are drawn from a literature survey from the PubMed data base and from selected cases illustrating points of contention.

\section{Results}

Clinical trials assessing the medical use of cannabis are limited to a small number of indications compared to numerous conditions reportedly responding well to cannabis preparations in uncontrolled random observations. Remarkably, strict and consistent protocols have failed to achieve the desired results in certain indications where inconsistent loosely controlled modes of use apparently succeeded. 


\section{Conclusions}

Myriad sporadic observations of clinical benefits from cannabis have so far proven difficult to capture by means of traditional clinical research. One strategic, possibly essential, approach to tackle this failure may be to reformulate the rules underpinning clinical research in a manner better adapted to the study of clinical efficacy of complex preparations such as cannabis based products.

\section{eP640}

ONCOLOGY-HOSPITALIST SYSTEM CARING FOR PATIENTS WITH ADVANCED CANCER IN A TERTIARY CANCER CENTER IN KOREA

S.J. Heo ${ }^{1}$, H.D. Koh ${ }^{1}$, B.C. Ahn ${ }^{l}$, G. Lee ${ }^{1}$, J.B. Lee ${ }^{1}$, H.J. Choi ${ }^{1}$, J. Sohn ${ }^{l}$, $\overline{J . B . A h n}$

${ }^{I}$ Yonsei Cancer Center- Yonsei University College of medicine, Internal Medicine, Seoul, Republic of Korea

\section{Introduction}

In Korea, hospitalists system has been emerged since 2016 which helps to reduce working time of medical residents and improve their training environment after the government implemented the Special Law on Medical Residents, ensure safe hospital environment, and provide higher quality healthcare. Yonsei cancer center prelusorily introduced oncologyhospitalist system in March 2016 and became a model for caring patients with advanced cancer.

Objectives

This study compares outcomes for patients, resource utilization and patient satisfaction before and after oncology-hospitalist system.

\section{Methods}

We retrospectively analyzed 993 admissions from Mar 2015 to Jun 2016 and compared for two groups; A (before system) and B (after system). Baseline patient characteristics, admission/discharge type, length of stay, error rates of chemotherapy prescription, numbers of code blue, notice of discharge rates, 30-day readmission rates, and patient satisfaction were compared.

Results

Age, primary cancer type, Charlson comorbidity index score and admission/discharge type were not different but female sex increased in group B because of switching female room. Though more patients with stage IV $(80.8 \%$ vs $85.6 \%, P=0.043)$ or who needed supportive care (37.2\% vs $43.8 \%, P=0.034)$ were admitted in group $\mathrm{B}$, it consequently showed similar length of stay, number of code blue, and 30-day readmission rate. Error rates of chemotherapy prescription $(9.7 \%$ vs $4.8 \%$, $P=0.048)$, notice rates of discharge $(75.0 \%$ vs $86.6 \%, P=0.001)$ and patient satisfaction score (mean 9.59 vs $9.70, P=0.020$ ) were significant different.

\section{Conclusions}

Oncology-hospitalist system significantly improved efficiency of resource utilization and patient satisfaction. It would grow into comprehensive role for caring hospitalized patients and changing medical system.

\section{eP641}

REPORT ON THE APPLICATION OF ANESTHESIA CARE ON PROSTATE CANCER PATIENTS TREATED USING DA VINCI ROBOTIC SURGERY

C.Y. Hsu ${ }^{l}$, S.C. Hsu

${ }^{T}$ Dayeh University, Department of Nursing, Changhua, Taiwan R.O.C.

\section{Introduction}

The Da Vinci robotic arm can provide surgeons with high-resolution 3D stereoscopic vision, and this advanced robotic arm is capable of simulating the flexibility of human fingers. Furthermore, this arm can reach small and narrow spaces beyond the reach of human hands and perform fine surgical movements. In the field of urology, nearly all radical prostatectomies are performed using the Da Vinci robotic arm. Owing to the high average age of patients who undergo a prostatectomy, the chronic diseases that the patients suffer from may affect anesthetic care plans; even the head-low feet-high posture may pose an increased threat to anesthesia safety.

\section{Objectives}

A pre-anesthesia assessment needs to be carried out to reduce the risks of anesthesia, and enhance the safety and quality of anesthetic care. With the expectation, that patients will be able to enjoy the benefits of minimally invasive surgery with the aid of the Da Vinci robotic arm.

\section{Methods}

Deep interview with an anesthetic nurse who working in Taiwan of Changhua Christian Hospital, and the application of full anesthetic care to prostate cancer patients treated using Da Vinci robotic surgery was investigated in this work.

\section{Results}

This interviewing provides knowledge and clinical experience for the care of such patients.

\section{Conclusions}

The study is to help ensure that this surgical method and full anesthetic care will become more thorough in the future.

\section{eP642}

EVALUATION OF SIDE EFFECTS AND CHEMOTHERAPY EFFICACY IN PATIENTS WITH HEPATITIS C (HCV) OR B (HBV).

J. Huszno ${ }^{1}$, Z. Kolosza $a^{2}$

${ }^{T}$ Maria Sk? odowska Curie Memorial Cancer Centere and Institut of Oncology Gliwice, Clinical and Experimental Oncology Department, Gliwice, Poland

${ }^{2}$ MSC Memorial cancer centere and Institute of Oncology Gliwice Branch, Biostatistics Unit-, Gliwice, Poland

\section{Introduction}

Viral infections can lead to hepatic complications, such as: liver cirrhosis, liver failure or hepatocellular carcinoma and to non-hepatic complications, which may limit the use of cytostatics and increase the risk of chemotherapy complications.

\section{Objectives}

The aim of the study was to evaluate the influence of viral infections on the efficacy and safety of chemotherapy in breast cancer patients.

\section{Methods}

A retrospective analysis was conducted on the medical records of 219 breast cancer patients who were diagnosed and treated with chemotherapy at the MSC Memorial Cancer Center and Institute of Oncology, Gliwice Branch. All patients underwent blood tests for anti-HCV and HBsAg antibodies. Laboratory tests have confirmed viral infections in $2 \%$ of patients. The number of leukocytes, neutrophils, platelets and hemoglobin was assessed with respect to the CTCAE version 4.0.

Results

All patients received chemotherapy regiments with anthracycline and taxanes. In all cases, there was observed transaminases increase in grade 1-2. No one of patient had hepatological side effects in grade $>$ G3, which would require termination of chemotherapy. Hepatological side effects occurred in a similar percentage as in patients without HCV infection. $8 \%$ of patients required a dose reduction or delay of the next chemotherapy cycle. All patients completed the systemic treatment. 53\% of patients with a viral infection remain in regular follow up without relapse. There was no intensification of $\mathrm{HCV}$ infection under the influence of chemotherapy. 


\section{Conclusions}

Hepatological side effects occurred in a similar percentage as in patients without viral infection. Viral infection did not influence systemic treatment and infection intensification.

eP643

\section{DAILY WEATHER CONDITIONS AND ANTICIPATED DEATH} FROM CANCER

I.C. Hwang ${ }^{1}$, H.K. Ahn ${ }^{2}$

${ }^{T}$ Gachon University Gil Medical Center, Family Medicine, Incheon, Republic of Korea

${ }^{2}$ Gachon University Gil Medical Center, Medical Oncology, Incheon, Republic of Korea

\section{Introduction}

The effect of weather conditions on human health has been documented. However, the role of daily weather on anticipated death remains unclear. Objectives

To evaluate the relationship between daily weather conditions and death in terminally ill cancer patients.

\section{Methods}

We retrospectively searched a medical database of 935 consecutive terminally ill cancer patients who passed away in the palliative care unit from October 2009 to September 2013. We used Poisson regression to assess the relative risk (RR) of anticipated death based on various weather variables, using mean values calculated from the 10 days around the time of death.

Results

The final study population consisted of 745 patients with a mean age of $65.9 \pm 12.2$ years. The mean palliative prognostic index (PPI) score in the last week of life was $8.0 \pm 3.8$. After adjustment for age, sex, cancer type, and PPI score, RRs were 1.69 (95\% CI, 1.17-2.46) for low temperature, 2.57 (1.77-3.77) for high diurnal temperature variation, 2.35 (1.61-3.36) for low humidity, and 1.75 (1.22-2.53) for high levels of sunlight.

Conclusions

The results of our study suggest weather conditions may be a predictor of death in terminally ill cancer patients.

eP644

\section{A PROSPECTIVE STUDY OF PREOPERATIVE BLOOD ORDERING SCHEDULE,INDICATIONS AND ACTUAL USAGE OF BLOOD AND ITS COMPONENTS AMONG PATIENTS UNDERGOING CURATIVE SURGERIES FOR CANCER IN INDIA \\ M.B. Inamdar ${ }^{1}$, N. Hulikal ${ }^{1}$, S.K.V. Babu ${ }^{2}$ \\ ${ }^{T}$ SVIMS, surgical oncology, tirupati, India \\ ${ }^{2}$ SVIMS, Transfusion medicine, tirupati, India}

\section{Introduction}

MSBOS is designed for effective utilisation of blood bank resources. The figure is derived from locally accepted tariff of blood usage.Study was undertaken in order to achieve effective utilisation of resources and cost reduction in a resource limited developing country.

Objectives

To derive institute specific MSBOS for elective curative cancer surgeries. To study the preoperative ordering schedule of blood and its components, To study the indications for intraoperative and immediate (48 hours) postoperative blood transfusion among patients undergoing cancer, study the pattern of use, CT ratio, TI ratio and T\% for blood components.

Methods

Prospective study

Inclusion criteria- all malignancies between age 18 to 80 years undergoing curative surgery
Exclusion Criteria-All patients undergoing surgery for Brain turmors, spinal cord tumors, benign tumors, any coagulopathy or hematological disorder,any surgery without a curative intent eg palliative resections/ surgery.All patients who are not willing for blood transfusion.

Data tabulated in excel sheet 2016

Descriptive statistics like means and percentages were derived

Results

Total of 740 patients in one year of which 167 were males and 573 female patients. A total of 1370 packed red blood cells and 267 FFPs were reserved of which $403 \mathrm{PRBC}^{*}$ and 122 FFPs were used constituting $29.4 \%$ and $45 \%$ respectively of the reserved units. Almost $71 \%$ of prbc were over reserved and so were $55 \%$ of the FFPs.

\section{Conclusions}

The MSBOS for cross matching has a profound impact on patient care and helps in effective utilisation of resources and reduces the cost. We propose that MSBOS in all major institutes must be made standard of care.

eP645

EDOXABAN VERSUS DALTEPARIN FOR TREATMENT OF VENOUS THROMBOEMBOLISM (VTE) ASSOCIATED WITH CANCER: HOKUSAI VTE-CANCER RANDOMIZED TRIAL G. Raskob ${ }^{1}, N$. van $\mathrm{Es}^{2}$, P. Verhamme ${ }^{3}, M$. Carrier ${ }^{4}, M$. Di Nisio ${ }^{5}, D$.

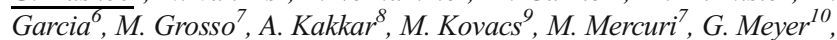
A. Segers ${ }^{11}$, M. Shi ${ }^{12}$, T.F. Wang $^{13}$, E. Yeo ${ }^{14}$, G. Zhang ${ }^{12}$, J. Zwicker ${ }^{15}$, J. Weitz $^{16}$, H. Buller ${ }^{2}$

${ }^{1}$ University of Oklahoma Health Sciences Center, College of Public Health, Oklahoma City, USA

${ }^{2}$ Academic Medical Center- University of Amsterdam, Department of Vascular Medicine, Amsterdam, The Netherlands

${ }^{3}$ University Hospitals Leuven, Department of Vascular Medicine and Haemostasis, Leuven, Belgium

${ }^{4}$ Ottawa Hospital Research Institute and University of Ottawa, Department of Medicine, Ottawa, Canada

${ }^{5}$ University "G. D'Annunzio" Chieti, Department of Medicine and Ageing Sciences, Chieti, Italy

${ }^{6}$ University of Washington, Department of Medicine- Division of Hematology, Seattle, USA

${ }^{7}$ Daiichi Sankyo Inc, Clinical Development, Basking Ridge, USA

${ }^{8}$ Thrombosis Research Institute, Thrombosis Research, London, United Kingdom

${ }^{9}$ London Health Sciences Center, Division of Hematology-Department of Medicine, London, Canada

${ }^{10}$ Hopital Européen Georges Pompidou- Universite Paris Descartes, Department of Respiratory Disease, Paris, France

${ }^{11}$ ltreas Academic Research Organization, Clinical Research, Amsterdam, The Netherlands

${ }^{12}$ Daiichi Sankyo Inc, Biostatistics, Basking Ridge, USA

${ }^{13}$ The Ohio State University Wexner Medical Center, Division of Hematology-Department of Internal Medicine, Columbus, USA

${ }^{14}$ University Health Network- University of Toronto, Division of Hematology-Department of Medicine, Toronto, Canada

${ }^{15}$ Beth Israel Deaconess Medical Center-Harvard Medical Schoo, Division of Hematology - Oncology, Boston, USA

${ }^{16}$ McMaster University, The Thrombosis and Atherosclerosis Research Institute, Hamilton, Canada

\section{Introduction}

Direct oral anticoagulants are effective for treatment of venous thromboembolism (VTE) but their role for cancer- associated VTE is uncertain. Objectives

We performed a randomized, open-label non-inferiority trial in 1050 cancer patients with acute VTE to evaluate edoxaban vs dalteparin. 
Methods

Patients received either low-molecular-weight heparin for 5 days followed by oral edoxaban $60 \mathrm{mg}$ once daily ( $30 \mathrm{mg}$ if creatinine clearance 30 to $50 \mathrm{ml} / \mathrm{min}$, body weight $<60 \mathrm{~kg}$, or strong P-gp inhibitor use), or subcutaneous dalteparin 200 units $/ \mathrm{kg}$ once daily for one month followed by 150 units $/ \mathrm{kg}$ thereafter. Treatment was given for up to 12 months. The primary outcome was the composite of the first recurrent VTE or major bleeding event during follow-up for 12 months. All suspected outcomes and severity of major bleeding were adjudicated by a committee without knowledge of treatment assignment.

\section{Results}

A total of 1046 patients were included in the modified-intention-to-treat analysis (522 edoxaban and 524 dalteparin). The primary outcome occurred in 67 patients $(12.8 \%)$ given edoxaban and in 71 patients $(13.5 \%)$ given dalteparin (hazard ratio $0.97 ; 95 \% \mathrm{CI}, 0.70$ to $1.36 ; \mathrm{P}=0.006$ for noninferiority). The risk difference (edoxaban minus dalteparin) for recurrent VTE was $-3.8 \%$ (95\% CI,-7.1 to -0.4), and for major bleeding was $3.1 \%$ (95\% CI, 0.5 to 5.7). Severe major bleeding occurred in 12 patients, $2.3 \%$, in each group. Survival free of recurrent VTE and major bleeding was $55.0 \%$ and $56.5 \%$ in the edoxaban and dalteparin groups, respectively.

Conclusions

Oral edoxaban is noninferior to subcutaneous dalteparin for treatment of cancer-associated VTE.

\section{eP646}

A GENETIC VARIANT RS13293512 IN THE PROMOTER OF LET-7 IS ASSOCIATED WITH AN INCREASED RISK OF BREAST CANCER IN CHINESE WOMEN

R. Sun ${ }^{l}$, J. Gong ${ }^{I}$, H. Zou ${ }^{I}$

${ }^{T}$ Yunnan University of Chinese Traditional Medicine, Yunnan University of Chinese Traditional Medicine, Kunming, China

\section{Introduction}

Breast cancer is the most common invasive cancer and the leading cause of cancer death among women worldwide the exact etiology is still unknown.the first association study demonstrated that single-nucleotide polymorphisms (SNPs) in the promoter region of miRNAs may contribute to the susceptibility to cancer. The lethal-7 (let-7)is a microRNAs.we want to evaluate the association of the SNPs with the breast cancer in Chinese women.

Objectives

Growing evidence has demonstrated that SNPs in the promoter of miRNA influence individuals' susceptibility to human diseases.

Methods

We examined two SNPs rs 10877887 and rs 13293512 in the promoters of let-7 family to determine if the two SNPs were related to the occurrence of breast cancer. Genotyping of the two SNPs was performed by polymerase chain reaction and restriction fragment length polymorphism analysis or TaqMan assay in 301 breast cancer patients and 310 age matched controls.

Results

We found a higher frequency of rs13293512 CC genotype and rs $13293512 \mathrm{C}$ allele among breast cancer patients. Stratification analysis showed that rs13293512 CC genotype was associated with an increased risk of breast cancer in patients with negative estrogen receptor, patients with negative progesterone receptor (adjusted OR $=1.92 ; 95 \%$ CI, 1.11$3.33 ; P=0.02$ ), patients with T1-2 stage cancer (adjusted $\mathrm{OR}=1.77 ; 95 \%$ CI, 1.07-2.93; $P=0.03$ ), and patients with N1-3 stage cancer (adjusted $\mathrm{OR}=1.89 ; 95 \% \mathrm{CI}, 1.13-3.17 ; P=0.015)$.

Conclusions

These findings suggest that rs 13293512 in the promoter of let-7a-1/let-7f$1 /$ let-7 d cluster may be a possible biomarker for the development of breast cancer in Chinese women.
eP647

ADVERSE OCULAR SIDE EFFECTS OF ORAL ANTICANCER DRUG S-1: A RETROSPECTIVE STUDY OF 103 CASES

H. Kashiwagi ${ }^{I}$, Y. Hirofumi ${ }^{2}$, F. Akira ${ }^{2}$, H. Satoshi ${ }^{2}$, M. Nozomu ${ }^{2}$, Y. Tomoya $^{2}$, T. Akiko ${ }^{2}$, Y. Kentaro ${ }^{2}$, T. Takahiro ${ }^{2}$, O. Yusuke ${ }^{2}$, K. Takeshi $^{2}$

${ }^{1}$ Shizuoka Cancer Center, Ophthalmology, Nagaizumi, Japan

${ }^{2}$ Shizuoka Cancer Center, Gastrointestinal Oncology, Nagaizumi, Japan

\section{Introduction}

Recognition of adverse side effects (epiphora) by anticancer drug s-1 remains insufficient.

\section{Objectives}

To report epiphora caused by lacrimal disorder associated with S-1 chemotherapy.

\section{Methods}

This study retrospectively assessed 103 patients ( 74 men and 29 women; aged 47-79 years) who were diagnosed as this side effects by an ophthalmologist at the Shizuoka Cancer Center between September 2009 and December 2014.

\section{Results}

Of the 103 patients, 93 were diagnosed with gastric cancer, and 10 with pancreatic cancer. Seventy-six patients with gastric cancer and the 10 with pancreatic cancer who received S-1 monotherapy were assigned to group $\mathrm{A}$ and $\mathrm{B}$, respectively; while 17 patients with gastric cancer who received $\mathrm{S}-1$ and Cisplatin co-therapy were assigned to group $\mathrm{C}$. The time to onset of epiphora was $11.55,7.20$, and 18.35 weeks in groups A, B, and C, respectively. All cases were classified as either mild (112 eyes) or advanced ( 92 eyes) based on probing and lacrimal irrigation. Lacrimal tube insertion with dacryoendscopic observation was performed in the advanced cases ( 85 eyes). Lacrimal canaliculus stenosis or obstruction was observed in $71.76 \%(122 / 170)$ of the cases; while the rate of complete lacrimal tube insertion was $89.41 \%(76 / 85)$ and the rate of epiphora improvement in the 76 eyes was $82.89 \%$ (63/ 76). Epiphora improvement in the mild cases was $94.64 \%(106 /$ 112). Corneal damages were observed in $16.8 \%(13 / 77), 30.0 \%$ $(3 / 10)$, and $5.5 \%(1 / 18)$ in group A, B, and C.

Conclusions

This study highlights the need for early detection of this condition and establishment of preventive methods.

\section{eP648}

EVALUATION OF THE USE OF RASBURICASE IN PROPHYLAXIS AND MANAGEMENT OF TUMOUR LYSIS SYNDROME AMONGST CANCER PATIENTS IN QATAR N. Kassem $^{1}$, S. El Azzazy ${ }^{1}$, H. El Omri ${ }^{2}$, M.A. Yassin ${ }^{2}$, H. McCarthy ${ }^{3}$, H. Ghazouani $^{4}$

${ }^{I}$ Department of Pharmacy, National Center for Cancer Care and Research, Hamad Medical Corporation, Doha, Qatar

${ }^{2}$ Department of Hematology and Bone Marrow Transplantation (BMT), National Center for Cancer Care and Research, Hamad Medical Corporation, Doha, Qatar

${ }^{3}$ School of Pharmacy, Queen's University, Belfast, UK

${ }^{4}$ Department bioinformatics, National Center for Cancer Care and Research, Hamad Medical Corporation, Doha, Qatar

\section{Introduction}

Tumour lysis syndrome (TLS) is an ontological emergency caused by the breakdown of malignant cells resulting in massive release of cellular components into the blood.

Rasburicase is a urate oxidase enzyme used for prophylaxis and treatment of hyperuricemia associated with TLS

\section{Objectives}

To evaluate the use of rasburicase in cancer patients in Qatar, characterize the impact of updating the institutional TLS guidelines on optimizing its 
use and to assess the efficacy of a single rasburicase dose in lowering uric acid levels.

\section{Methods}

It is a retrospective chart review cohort study in a tertiary care hospital providing oncologyand haematology services for adult patients in Qatar. The study included 103 patients divided into 2 groups based on the date of the TLS guidelines update; 48 patients before and 55 patients after the guidelines update.

\section{Results}

The rate of compliance to the institutional TLS guidelines after the guidelines update increased from $77 \%$ to $87.2 \% \quad(\mathrm{P}=0.17)$, from $45.8 \%$ to $78.2 \%(\mathrm{P}=0.001)$ and from $56 \%$ to $94.5 \%(\mathrm{P}<$ 0.001 ) in terms of proper indication, proper duration and G6PD screening, respectively. However, monitoring of uric acid and electrolytes was not improved. Rasburicase consumption and cost were significantly reduced by $42.5 \%$ after the guidelines update. Furthermore, the single dose rasburicase was efficacious in normalizing uric acid within 24 hours in $98.4 \%$ of patients.

Conclusions

Updating the institutional TLS guidelines had a significant impact on optimization of rasburicase use and compliance to guidelines in our institution. Moreover, the use of a single rasburicase dose demonstrated efficacy in lowering serum uric acid levels.

\section{eP649}

ASSESSMENT OF PRACTICE OF MANAGING CONSTIPATION IN PATIENTS ON ORAL MORPHINE AT INITIAL THREE SUBSEQUENT VISITS AT J.O.Y HOSPICE MBALE UGANDA IN 2014

W. KAWUBIRI

${ }^{T}$ J.O.Y HOSPICE MBALE, HEALTH, MBALE, Uganda

\section{Introduction}

Constipation is a common problem among persons receiving opiates for treatment of pain. It is often under assessed and under treated by both physicians and nurses, leaving many patients to grapple with this problem alone. (Susan 2004). A standard was put in place that all patients prescribed an opioid (ladder step2: Codeine or ladder step 3: morphine) must simultaneously be prescribed laxatives, whether previously constipated or not. (Dr. Merriman. A et.al (2012).

\section{Objectives}

The purpose of this audit was to assess the practice of managing constipation in patients on oral morphine at the initial three subsequent visits at J.O.Y Hospice Mbale district Uganda from $1^{\text {st }} /$ January/2014 to $31^{\text {st }}$ December/2014. .

\section{Methods}

It was a retrospective audit that included all patients prescribed oral morphine while excluding those files of patients on oral morphine missing dates on which they were seen, files having unclear handwriting and files for patients who didn't turn up for the initial three subsequent

Results

Of the 53 patients on oral morphine, who came at JOY Hospice, $86.8 \%$ were assessed for constipation and $13.2 \%$ were not assessed. $96.2 \%$ were prescribed a laxative only and a few $3.8 \%$ were not prescribed anything. Bisacodyl was the commonest drug prescribed to prevent constipation at $96 \%, 4 \%$ patients given Movicol powder. None was prescribed lactulose, Senna or given nutritional advice to prevent constipation.

\section{Conclusions}

It was recommended that all patients on oral morphine must be assessed for constipation at all visits, prescribed a laxative and nutritional advise given. continuous medical education at health centre was recommended.
eP650

\section{PROCALCITONIN AS A DIAGNOSTIC MARKER FOR BACTEREMIA IN TERMINAL CANCER PATIENTS}

J.E. Kim I', Y.S. Choi ${ }^{2}$, C.W. Lee ${ }^{2}$, S.J. Cho ${ }^{3}$

${ }^{1}$ Korea University Graduate School, Department of Medicine, Seoul, Republic of Korea

${ }^{2}$ Korea University Guro Hospital, Department of Family Medicine, Seoul, Republic of Korea

${ }^{3}$ Shamyook Medical Center, Department of Family medicine, Seoul, Republic of Korea

\section{Introduction}

An increasing number of patients with cancer have been dying of multiorgan failure caused by infection. Elevated serum procalcitonin (PCT) is found in bacteremia, and in patients with neoplasms or imminent death regardless of their infection status.

\section{Objectives}

We aimed to assess if serum PCT levels could be used to diagnose bacteremia in terminal cancer patients.

\section{Methods}

We analyzed 106 patients in whom PCT, C-reactive protein, and blood culture were concurrently requested in a hospice/palliative care center at a university hospital from January 2014 to December 2015. The factors affecting positive blood culture results were assessed via logistic regression analysis. Receiver operating characteristic (ROC) curve analysis was performed to evaluate the diagnostic usefulness of the serum PCT levels for bacteremia, and the PCT cut-off level for predicting positive blood cultures was determined.

\section{Results}

Patients were included 58 men and 48 women. The mean serum PCT level was $3.68 \pm 12.77 \mathrm{ng} / \mathrm{mL}$. The serum PCT levels exhibited significant correlation in the prediction of positive blood cultures in terminal cancer patients $(p=0.024)$ and after adjusting for confounding variables, serum PCT levels were significantly correlated with bacteremia $(p=0.021)$. Also, a ROC curve analysis was performed to investigate the usefulness of the serum PCT level in the prediction of positive blood cultures. The area under the curve of the serum PCT level was 0.643 and serum PCT level over $1.72 \mathrm{ng} / \mathrm{mL}$ can predict positive blood culture results (sensitivity $50.0 \%$, specificity $81.8 \%$ ).

\section{Conclusions}

Serum PCT level measurement is useful for predicting positive blood cultures in terminally ill cancer patients.

eP651

LYNCH SYNDROME RISK ASSESSMENT AMONG OVARY CANCER BASED ON SCREENING METHODS

\section{M.K. Kim}

${ }^{T}$ Samsung Changwon Hospital, Obstetrics and Gynecology, ChangwonSi, Republic of Korea

\section{Introduction}

BRCA mutation is the most common hereditary cancer syndrome about ovary . But mismatch repair genes related Lynch syndrome is also associated with it.There is not much study about risk evaluation about this among Korean population

\section{Objectives}

We investigate to know Lynch syndrome risk evaluation about ovary cancer in korea

\section{Methods}

We searched database to find ovary cancer patients who was counseled and tested about Lynch syndrome in Department of Obstetrics and gynecology, Samsung Changwon Hospital by single surgeon . Clinical information was extracted from the medical record including age, family and 
personal history of cancer, immunohistochemistry (IHC) of MLH1/MSH2, microsatellite instability test (MSI).

\section{Results}

Twenty six patients was found.mean age was 54(16 75) and fifty percent was serous type (13/26). There were 2 patients with Lynch syndrome related cancer family history (stomach and ovary) among their $1^{\text {st }}$ degree relatives. Only one patient has abnormal MLH1 IHC.There were three unstable MSI patients (BAT26, D2S123, D55346, and NR21).

\section{Conclusions}

We found three abnormal MSI patients among this population. Cost effective algorithm using multiple genetic testing to find Lynch syndrome associated ovary cancer patients should be developed through large population study.

\section{eP652}

\section{WRAPPED WITH LOVE- ART THERAPY IN PAEDIATRIC PALLIATIVE CARE}

J. King ${ }^{1}$

${ }^{T}$ Bear Cottage Children's hospice, Art therapy-Allied health, Sydney, Australia

\section{Introduction}

To discuss the extended family support that is required in children's palliative care, focusing on Grandparents, who sometimes may be seen as the 'forgotten mourners'.

Objectives

Utilizing art psychotherapy in a group setting to encourage peer support between ten grandparents (bereaved and non-bereaved) within our inaugural weekend Camp program.

\section{Methods}

Detailing one art therapy session the first morning of the Camp where each of the group chose a pebble to represent their grandchild. They were then invited to choose a different layer, from an extensive array of art materials, to represent each of the important people that wrap, swaddle, comfort, protect, support, and surround this child.

There was time dedicated to share their experience and/or artwork within the confidential safe space of the group. This allowed them to express some of the difficulties of having a grandchild with a life limiting illness and the dynamics within the extended family.

Results

Anonymous pre-and post-narrative evaluations were used to assess expectations prior to the Grandparents weekend and the effectiveness of the program.

Discussions within the group at the end of the session and throughout the rest of the weekend revealed that although it had been hard for some to open up, and at times emotional, it was therapeutic and helped form strong bonds within the grandparents.

\section{Conclusions}

It was a useful tool to share internally with the multi-disciplinary team, with added insight utilizing this creative medium. Would possibly like to evaluate more specifically the art therapy component of the program in future camps.

\section{eP653}

ADVANCED PRACTICE NURSING (APN) IN GYNAECOLOGICAL ONCOLOGY - DEVELOPMENT OF AN EVIDENCE-BASED CONCEPT FOR AUSTRIA AND SWITZERLAND

A. Kobleder $^{1,2}$, H. Mayer ${ }^{2}$, B. Senn ${ }^{1,3}$

${ }^{7}$ University of Applied Sciences St.Gallen, Institute for Applied Nursing Sciences, St.Gallen, Switzerland

${ }^{2}$ University of Vienna, Department of Nursing Science, Vienna, Austria

${ }^{3}$ University of Sydney, Sydney Nursing School, Sydney, Australia

\section{Introduction}

Internationally, there has been a significant development in APN roles, and their effectiveness could be demonstrated in several studies. However, in the Austrian and Swiss healthcare context, there is a lack of role clarity including uncertainty about how the role should be shaped and sustainably implemented. In the field of gynaecological oncology, the APN is considered as a chance to reduce gaps in current care practices. However, concepts, which provide key elements of an APN in gynaecological oncology are missing.

Objectives

The aim of this study was to develop an evidence-based APN concept in gynaecological oncology for the Austrian and Swiss healthcare context.

\section{Methods}

Evidence for the concept was generated by conducting four single studies representing (1) patient's perspective, (2) health care professionals' perspective, (3) current state of research regarding the effectiveness and (4) contextual factors. Data from these studies were synthesized by using a matrix, which was developed based on the internationally acknowledged frameworks PEPPA plus and NREM.

Results

Structure, process and outcome criteria and their relationships constitute the centrepiece of the developed APN concept in gynaecological oncology for the Austrian and Swiss healthcare context. Particularities in the field of gynaecological oncology are demonstrated in the APN's focus on psychosocial support and the value of its competence - "consultation" along the whole illness trajectory.

\section{Conclusions}

The developed APN concept provides a basis for further developing institution-based APN role descriptions. The common base might enhance APN's role clarity in gynaecological oncology in the Austrian and Swiss healthcare context.

\section{eP654}

\section{LEVEL OF DEATH ANXIETY AMONG NURSES WORKING IN ONCOLOGY SERVICE}

Z. Koç ${ }^{1}$, T. Kavalall Erdoğan ${ }^{1}$, T. Çınarlı ${ }^{2}$, S. Masat ${ }^{1}$, I.C. Koç ${ }^{3}$

${ }^{1}$ Ondokuz Mayis University Faculty of Health Science, Nursing, Samsun, Turkey

${ }^{2}$ Ondokuz Mayls University Health Services Vocational School, Emergency, Samsun, Turkey

${ }^{3}$ Hacettepe University, Faculty of Medicine Student, Ankara, Turkey

\section{Introduction}

Providing care for the individuals approaching death is usually a condition causing pain and sorrow for nurses

\section{Objectives}

This study was conducted as a descriptive study aiming to determine the level of death anxiety of the nurses working in the oncology clinic.

Methods

The research was carried out between 02.09.2017 and 24.09.2017. Seventy-two nurses responsible for the care of oncology patients constituted the study group while 72 nurses working in the internal and surgical units constituted the control group. The data were collected using a questionnaire form composed of 30 questions for determining the nurses socio-demographic, working characteristics and the Thorson Powell Death Anxiety Scale.

Results

It was determined that, of the participating nurses, $93.1 \%$ were female, $6.9 \%$ were male nurses, $58.3 \%$ had undergraduate degree, $67.4 \%$ loved their profession, $56.9 \%$ was partially satisfied with the clinic in which s/he was working and $66 \%$ were on shift work. The median score of the Thorson Powell Death Anxiety Scale of the study group nurses was found to be 54 while the median score of the Thorson Powell Death Anxiety Scale of the control group nurses was found to be 38.5. There was a 
statistically significant difference between the median scores of Death Anxiety of the study and control group nurses $(\mathrm{p}<0.001)$.

\section{Conclusions}

The level of death anxiety of the nurses working the internal and surgical units was found to be at low-level while that of the nurses working in the oncology clinic was found to be at moderate-level.

\section{eP655}

A PHASEII STUDY OF TOPICAL BOTANICAL LOTION FOR RECOVERY FROM CHEMOTHERAPY INDUCED ALOPECIA IN BREAST CANCER PATIENTS

Y. Kojima ${ }^{l}$, R. Yoshie ${ }^{l}$, A. Udagawa ${ }^{l}, K$. Tsugawa $^{l}$

${ }^{T}$ St.Marianna University School of Medicine, Breast and Endocrine surgery, Kawasaki, Japan

\section{Introduction}

Chemo Induced alopecia (CIA) is the most distressing and long-lasting side effect among breast cancer patients who underwent (neo)adjuvant chemotherapy. A national survey in Japan reported $98.4 \%$ breast cancer patients experienced hair loss by chemotherapy. By normalizing follicular cell apoptosis process in the scalp and attenuate micro-inflammation, a topical botanical lotion (CG428) was shown to improve hair regrowth from CIA in a small cancer patient cohort.

Objectives

Our study is a single institute, open-labeled trial for breast cancer patients who is planned to undergo (neo)adjuvant chemotherapy including both anthracycline and taxan, to evaluate the efficacy, safety of the CG428 and health related quality of life.

\section{Methods}

Patients start using CG428 prior to or from the first day of the first treatment cycle.

The primary endpoint is the rate of candidates who could take off their wig at twelve months after chemotherapy ends (wig-off rate). The secondary endpoints are duration of CIA and safety. We will also assess baldness by using Olsen/Canfield visual analog scale. Health related quality of life (HRQOL) during the trial will be collected prospectively.

It has been reported that the wig-off rate was between 68 and $76 \%$, including patients with one regimen. Therefore, we estimated that the wig-off rate under the null and the alternative hypothesis using onesided statistical test with significance level of 0.05 , the sample size of 81 was required to have $80 \%$ power for this study.

Results

Successfully, accrual rate of our study has achieved $50 \%$ without any severe adverse event.

Conclusions

The result of our study is awaited.

\section{eP656}

DISORDERED SLEEP IN PEOPLE WITH CANCER NEGLECTED NO MORE: INTRODUCING FORMAL SLEEP ASSESSMENT/MANAGEMENT NURSING PLANS FOR CANCER CARE

G. Kotronoulas ${ }^{1}$

${ }^{T}$ University of Glasgow, School of Medicine Dentistry \& Nursing, Glasgow, United Kingdom

\section{Introduction}

People with cancer are disproportionately affected by disordered sleep. Six in 10 of those recently diagnosed and/or receiving anti-cancer treatment suffer debilitating sleep loss. However, disordered sleep is still typically overlooked and poorly managed in cancer care.

\section{Objectives}

To introduce health professionals to formal sleep assessment/ management nursing plans as a systematic and coordinated approach to identify, evaluate and manage disordered sleep in people with cancer.

\section{Methods}

Integration of current research evidence on cancer-related sleep disruption, patient-reported outcome (PRO) measures use, supported self-management, and sleep management pathways.

\section{Results}

Formal sleep evaluation begins at pre-treatment with a screening PRO measure for multiple sleep disorders. The evaluation process continues at regular intervals (ranging from daily monitoring to specific transition points) depending on the outcome. If disordered sleep is detected, evidence-based sleep management pathways are used to collect multisource information to aid sleep specialist evaluation and implementation of a tailored nursing plan for supported self-management and treatment. Continuing evaluation allows modification according to a patient's changing circumstances.

\section{Conclusions}

Symptoms of disordered sleep (e.g. difficulty getting off to sleep, interrupted/restless sleep, unplanned early rising, daytime sleepiness) can be experienced as acute episodes. If untreated however, they may become chronic and adversely impact on key outcomes, including quality of life and survival. Nursing plans for disordered sleep enable multidisciplinary input on best treatment avenues, family caregiver involvement, and patient-centred care that acknowledges unique internal (e.g. comorbidity; fluctuating physical and psychological symptoms; lifestyle habits and sleep behaviours; potential for self-care) and external key factors (e.g. sleep environment).

\section{eP657}

\section{REVIEW OF THE NEURO-ONCOLOGY UNIT 2 YEARS AFTER ITS CREATION \\ C. Larible-Lefort ${ }^{I}$, E. Vauleon ${ }^{I}$, F. Lebrun $^{I}$, E. Pelotte ${ }^{I}$ \\ ${ }^{1}$ CLCC Centre Eugène Marquis, Supportive Care, RENNES, France}

\section{Introduction}

Medical oncology is a specialty, which by definition, takes into account the patient as a whole physical, psychic and social. Patients treated in neuro-oncology have in addition to this problem of tumoral pathology, its consequences on their life: appearance of a motor or cognitive handicap, modification of intrafamilial responsibilities with sometimes caregivers and relatives'dependence for everyday life.

\section{Objectives}

Therefore, we wanted to create a neuro-oncology unit (UNO) within the department of supportive oncology care (DISSPO) of the Center Eugène Marquis (CEM), to best meet these specific issues.

\section{Methods}

We organized a structure bringing together a neuro-oncology nurse practitioner, the DISSPO executive, the neuro-oncologists and radiation therapists.

Results

The UNO helped to structure the management of neuro-oncology at the CEM. The city-hospital liaison book has been updated. A multiprofessionnal meeting was created to discuss the problems encountered by the patients in the CEM but also with the city staff like homecare nurse. Weekly, the referent IDE neuro-oncology made telephone followup at home. Oncology support day hospitals were organized to best meet the needs of patients with the advice of at least 2 paramedics and a summary by the doctor. Finally, the AGAPE groups for caregivers have integrated the UNO.

\section{Conclusions}

The UNO has made it possible to structure the specific care of these patients, to facilitate communication between paramedical, medical, 
hospital and liberal professionals and to improve it by adapting the tools used.

\section{eP658}

REVIEW OF THE POSITION OF A REFERENT NURSE IN NEURO-ONOCOLOGY AT THE EUGENE MARQUIS CENTER. C. Larible-Lefort ${ }^{I}$, E. Vauleon ${ }^{I}$

${ }^{T}$ CLCC Centre Eugène Marquis, Supportive Care, Rennes, France

\section{Introduction}

During her daily work in a medical oncology department, a nurse from the Center Eugène Marquis (CEM) realized the difficulties experienced by patients treated for a brain tumor.

\section{Objectives}

Educate a referent nurse to facilitate care and follow up of these patients. Methods

She passed the nursing diploma of neuro-oncology. The CEM released $20 \%$ of its time to meet the needs of patients. She was able to carry out follow-up consultations with patients treated with chemotherapy. Patients have also a systematic accompaniment consultation by a radiotherapy manipulator in case of radiochemotherapy. She also performed telephone follow-up during the first three months of oral chemotherapy (TEMOZOLOMIDE, PCV). She participated in the meetings of the neuro-oncology unit, the AGAPE groups for caregivers and with the help of other professionals (physiotherapists, dieticians, psychologists ...) at supportive oncology day hospitals finalized by a medical synthesis.

\section{Results}

Between November 2015 and December 2016, the neuro-oncology referent nurse conducted 25 follow-up consultations, and a telephone follow-up of 44 patients out of the 75 new patients followed at the CEM. She has participated in most of the AGAPE workshops with caregivers, multidisciplinary paramedical consultation meetings, as well as 13 oncology support day hospitals.

\section{Conclusions}

A neuro-oncology nurse is an important pillar in the neuro-oncology unit. It can help to detect and anticipate the needs of patients by facilitating access to supportive care and / or alert the oncologist more quickly thanks to the relationship of trust established with the patient and the caregivers during the telephone follow-up.

\section{eP659}

3-YEAR ASSESSMENT OF THE AGAPE CAREGIVER GROUP C. Larible-Lefort ${ }^{I}$, E. Vauleon ${ }^{I}$, H. Bourien ${ }^{I}$, E. Pelotte ${ }^{l}$, F. Lebrun ${ }^{l}$ ${ }^{T}$ CLCC Centre Eugène Marquis, Supportive Care, RENNES, France

\section{Introduction}

The occurrence of glioblastoma exposes to a physical, emotional and cognitive decline. The appearance of such symptoms leads to dependence on loved ones. Several studies have highlighted the difficulties encountered by caregivers impacting their quality of life.

Objectives

Improve quality of life for patients and their caregivers

Methods

We have created a group of words: AGAPE. This exchange workshop brings together medical oncologist, nurse and the caregivers of patients followed for glioblastoma. During this group of speech, the symptoms presented by the patients as well as therapeutics are recalled. The actors being able to intervene throughout the specific and palliative oncological care management are presented with all possible resources. This workshop also allows each caregiver to share their difficulties and obtain answers adapted to their needs. In order to objectively evaluate the impact of this workshop; at each session, the caregiver completes a quality of life questionnaire: CareGiver Oncology Quality of Life.

\section{Results}

Since February 2015, 5 3-month sessions have been organized, allowing 22 families to participate. Each group of 3-4 families meets once a month for 3 months. While all caregivers seem to find an interest in the AGAPE workshop, the analysis of the CarGOQoL questionnaires shows that there is no deterioration in the quality of life of the caregivers when there is an alteration in the neurological functions of the patients.

\section{Conclusions}

The diagnosis of glioblastoma impacts both the quality of life of patients and caregivers. The integration of caregivers into medical care is essential, especially by allowing exchanges during AGAPE workshops.

eP660

\section{PHYSICIAN EXPERIENCE OF ADVERSE EVENT} ATTRIBUTION

J. Le-Rademacher ${ }^{1}$, E. Storrick ${ }^{1}$, A. Jatoi ${ }^{2}$, S. Mandrekar ${ }^{1}$

${ }^{T}$ Mayo Clinic, Health Sciences Research, Rochester, USA

${ }^{2}$ Mayo Clinic, Oncology, Rochester, USA

\section{Introduction}

Collection of adverse events (AEs) in clinical trials is required to ensure patient safety. Clinicians are also required to declare whether or not each $\mathrm{AE}$ is related to the treatment (known as $\mathrm{AE}$ attribution). However, prior research from our group demonstrates that attribution is unreliable. An overhaul of the current system is thus needed to ensure new drugs are safe for patients and that the development of promising cancer drugs is not halted for unfounded safety concerns.

Objectives

We conducted a survey to understand the current AE attribution process and to help formulate recommendations for an improved system.

\section{Methods}

An electronic survey was developed and conducted among 165 clinicians at the Mayo Clinic. It included 21 items that queried clinicians about their clinical practice and trial experience, their training in $\mathrm{AE}$ attribution assignment, and their recommendations for improving the current $\mathrm{AE}$ attribution system.

\section{Results}

$61(37 \%)$ clinicians responded to the survey. The median survey completion time was 3.7 minutes. $43 \%$ of responders were younger than 45 years of age and $28 \%$ were female. The median years of clinical practice experience was 15 and of trial experience was $12.89 \%$ had served as a trial PI. Only $15 \%$ of responders received formal AE attribution training. $66 \%$ suggested that formal training is the most effective mean of improving attribution accuracy.

\section{Conclusions}

Results of our survey of experienced clinical trialists from a large academic institution highlights the need for formal attribution training considering investigators in multi-center trials are typically from smaller institutions with less experience.

\section{eP661}

STABILITY OF SYMPTOM CLUSTERS AND SENTINEL SYMPTOMS DURING THE INITIAL PHASE OF ADJUVANT CHEMOTHERAPY

J. Lee I, M. Park', S.Y. Rha ${ }^{3}$

${ }^{T}$ Chungnam National University, College of Nursing, Daejeon, Republic of Korea

${ }^{2}$ Eulji University, College of Medicine, Daejeon, Republic of Korea

${ }^{3}$ Yonsei University, Colleve of Medicine, Seoul, Republic of Korea 


\section{Introduction}

Top priorities in symptom cluster studies include confirming stable groups of symptoms and identifying sentinel symptoms. Understanding the stability of symptom clusters and identifying sentinel symptoms would facilitate effective symptom management.

\section{Objectives}

This study aimed to evaluate the stability of symptom clusters and to identify sentinel symptoms during the initial phase of adjuvant chemotherapy (CTx).

\section{Methods}

A descriptive correlational study design was applied. A total of 209 cancer patients receiving adjuvant CTx completed symptom evaluation for the $1^{\text {st }}$ and $2^{\text {nd }}$ cycles. The severity of 20 symptoms were evaluated on a 0 to 10 numeric rating scale. Statistical analysis include principal component and hierarchical cluster analyses to identify symptom clusters, and principal variable analysis to identify sentinel symptoms.

\section{Results}

Four symptom clusters were identified in the $1^{\text {st }}$ cycle: a physicalpsychological (pain, dyspnea, sleep disturbance, anxiety, depression), a gastrointestinal (nausea, loss of appetite, taste change), a fatiguecognitive (fatigue, difficulty concentrating, drowsiness), and a urosexual (urinary problem, sexual problem) symptom cluster. In the $2^{\text {nd }}$ cycle, stable symptom clusters were identified, whereas the physicalpsychological and fatigue-cognitive symptom cluster were merged together resulting in 3 clusters. The identified sentinel symptoms were anxiety, loss of appetite and fatigue in the $1^{\text {st }}$ cycle, and loss of appetite, depression, and fatigue in the $2^{\text {nd }}$ cycle.

Conclusions

Symptom clusters were stable during the initial phase of adjuvant CTx. Symptom management needs to focused on managing sentinel symptoms. Managing emotional symptoms and improving appetite with an evidence-based approach is necessary. It is also important to inform and prepare patients about fatigue management.

\section{eP662}

IMPACT OF BRANDED VERSUS GENERIC DRUG USE IN CHRONIC DISEASE MANAGEMENT IN HONG KONG HEALTHCARE PROFESSIONALS' AND PATIENTS' PERSPECTIVES

V. Lee $^{l}$, F. Cheng ${ }^{I}$

${ }^{T}$ Chinese University of Hong Kong, School of Pharmacy, Shatin, Hong Kong S.A.R.

\section{Introduction}

Healthcare expenditure has been escalating in recent years and has become a global challenge. Generic substitution is one of the many ways to lessen healthcare expenditure. Therefore, it is not uncommon to adopt generic substitution in public hospitals although it is not legally required in Hong Kong.

\section{Objectives}

This study aimed to evaluate the understanding toward generic substitution of healthcare professionals and patients and identify their experience of undesirable clinical outcomes after substitution.

\section{Methods}

This was a cross-sectional descriptive study using a self-completed anonymous questionnaire from Mar 2015 to May 2017. The questionnaire covered the demographic data, knowledge on generic drugs, experiences of generic substitution and views on policy.

\section{Results}

A total of 2106 general public, 73 doctors, 22 nurses and 50 pharmacists responded the questionnaire. $41.1 \%$ of general public was aware that generic drug has the same active ingredients. "Efficacy" was ranked as the primary concern among all groups but a substantial of respondents reported experiencing adverse drug reactions upon generic substitution
(26.6\% in general public, $23.2 \%$ in doctors, $9.1 \%$ in nurses and $42.0 \%$ in pharmacists). At least half of general public, nurses and pharmacist agreed that patients should be given a choice for generic substitution. However, less than one-fifth of doctors and nurses and about one-third of pharmacists agreed to obtain patients' consent prior to generic substitution, compared to about two-third of general public.

Conclusions

The knowledge and perception of generic substitution remained low not only in general public but also among healthcare professionals.

eP663

BIOSIMILAR ISSUES IN CANCER MANAGEMENT IN HONG KONG

V. Lee ${ }^{I}$, F. Cheng ${ }^{I}$

${ }^{T}$ Chinese University of Hong Kong, School of Pharmacy, Shatin, Hong Kong S.A.R.

\section{Introduction}

Since the publication of "Guidance Notes for Registration of Biosimilar Products" by the Hong Kong Department of Health in 2016, it is expected that an increasing number of biosimilars will be registered in Hong Kong. Although biosimilars of simple proteins, such as epo or G-CSF, have already been used in Hong Kong for few years. Therefore, uncertainties for how these biosimilars shall be used in Hong Kong remains to be the challenges.

Objectives

The aim of this project was to evaluate the potential issues related to cancer management using biosimilars in Hong Kong.

\section{Methods}

The current project used the fact disclosing approach to evaluate key issues on "Naming \& Labeling", "Substitution", "Interchangeability" and "Pharmacovigilance \& Traceability" on biosimilars. Medication safety is vital for our patients. Their input and views are crucial for the development of guidance of biosimilar products. The implication of the WHO, EMA and FDA guidelines on the regulatory affairs of biosimilars in Hong Kong was evaluated.

\section{Results}

A lot more of the originator biological products will soon lose their patency in the next 5-10 years. Therefore, we will expect that more biosimilar products will be on the market for our patients. Nevertheless, healthcare professionals and patients have limited experiences with these biosimilar products. Continuous monitoring and research to ensure positive clinical, economic and humanistic outcomes should be reinforced.

\section{Conclusions}

In conclusion, multidisciplinary team efforts including patients, healthcare professionals, academics, policy makers, healthcare regulatory authorities and government are important to ensure medication safety of biosimilar products.

eP664

META-ANALYSIS OF STUDIES ON BREAST CANCER RISK AND MENTAL STATES: THE ROLE OF DEPRESSION, ANXIETY, AND STRESS

J.N. $\operatorname{Lin}^{1}$

${ }^{T}$ Dayeh University, Nursing Department, Changhua, Taiwan R.O.C.

\section{Introduction}

Breast cancer has become the leading cause of cancer mortality among women worldwide. Many studies have investigated various risks of breast cancer, but whether mental state increases the risk of developing breast cancer is still inconclusive. 


\section{Objectives}

This study aimed to conduct a meta-analysis to assess the association between three mental states (depression, anxiety, and stress) and the risk of breast cancer.

\section{Methods}

Relevant literature published from 1988-2017 was obtained from Pubmed, Airiti Library, and manual searches of reference lists of selected publications. Random-effect models were used to compute the pooled relative risks (RR) estimate. Begg's rank correlation test and Egger's linear regression test were utilized to evaluate publication bias.

\section{Results}

Of the 100 search results reviewed, 13 cohort studies and two case-control studies were included. RR of depression was 1.13 (95\% Confidence Interval; CI $=0.94-1.36$ ) from 11 studies, and odd ratio (OR) of anxiety was 2.17 (95\% CI $=1.00-4.68)$ from one study. Two studies revealed that participants with stress had about twice the risk to have breast cancer, compared to those without stress $(\mathrm{OR}=2.00 ; 95 \% \mathrm{CI}=1.10-3.61)$, but a British study showed no association between breast cancer risk and stress $(\mathrm{RR}=0.87 ; 95 \% \mathrm{CI}=0.78-0.97)$. Begg's test and Egger's test indicated no publication bias.

\section{Conclusions}

This analysis suggests that mental state may be associated with the development of breast cancer. However, available research evidence is insufficient to support the hypothesis that mental state is a predictor of breast cancer.

\section{eP665}

MEANING OF THE BREAST CANCER FOR WOMEN AGAINST THE DIAGNOSIS

A.P. Alonso Reis ${ }^{1}$, M. Sanches Panobianco ${ }^{2}$, P. Alexandria Pinto de Magalhães ${ }^{3}$, C. Valim Cortes Gradim ${ }^{4}$

${ }^{T}$ PhD student of College of Nursing of Ribeirão Preto - USP, Postgraduate Program in Nursing in Public Health, Ribeirão Preto, Brazil

${ }^{2}$ Prof Dr of College of Nursing of Ribeirão Preto - USP, Department of Maternal and Child Nursing and Public Health, Ribeirão Preto, Brazil

${ }^{3}$ National Service of Commercial Learning, Health and wellness, Catanduva, Brazil

${ }^{4}$ Prof Dr of Federal University of Alfenas, Department of Nursing, Alfenas, Brazil

\section{Introduction}

Introduction: In the Occident, female breasts symbolize femininity and sexuality. Thus, breast cancer becomes stigmatizing for women.

Objectives

Aim: To know the meaning of the breast cancer for women who have experienced the disease.

Methods

Method: It is a qualitative study, which used Symbolic Interactionism and Grounded Theory. The data collection occurred in January 2014. Thirteen women, who attended a Brazilian hospital, were interviewed through semi-structured interviews, recorded, guided by the guiding question: "What is the meaning of being diagnosed with breast cancer? Results

Results: Meanings: 1) Fear of death, feeling of sadness by the loss of breast and concern with alopecia. 2) Sequelae of treatment, such as difficulty in performing tasks, altered family structure, and autonomy deficit; the loss of hair (chemotherapy) referred to the image of a "naked body", "disfigured face", but also meant a passing and necessary event for healing. 3) Concern in making use of hormonal therapy, examinations, and medical consultation to follow the disease, and, with their daughters, because of the heredity of the disease. 4) Previous negative experiences related to cancer, such as death of an loved one, meant a more suffering coping of the current disease; positive experiences, such as experiencing the recovery of a person with cancer provided better coping of the disease.

\section{Conclusions}

Conclusion: To have breast cancer had a frightening meaning, and caused important changes in the daily lives of theses women. But, to have experienced a previous (positive) experience with cancer meant a better coping of the disease.

eP666

DERIVATION AND INTERNAL VALIDATION OF A READMIT SCORE AMONG PATIENTS WITH SOLID TUMORS

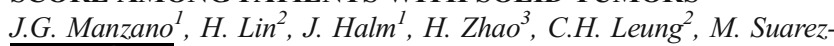
Almazor

${ }^{1}$ UT MD Anderson Cancer Center, General Internal Medicine, Houston, USA

${ }^{2}$ UT MD Anderson Cancer Center, Biostatistics, Houston, USA

${ }^{3}$ UT MD Anderson Cancer Center, Health Services Research, Houston, USA

\section{Introduction}

Unplanned hospitalizations and readmissions are common among patients with cancer. A valid readmission risk score to help focus interventions to patients who are high risk for readmission would help in safe transitions in this vulnerable population.

\section{Objectives}

We aimed to develop and internally validate a readmission risk score specifically for hospitalized cancer patients.

\section{Methods}

Multivariate logistic regression model was obtained to determine predictors of readmission. The model's discriminative capacity was evaluated through a receiver operating characteristic curve analysis and measured by area under the curve (AUC). The model was modified into a risk index that would allow for calculation of a total score. We validated the risk scoring system using the validation dataset by AUC.

\section{Results}

The derivation set included 4,503 patients. After multivariate regression, the factors associated with unplanned readmission included: Residence, ER visit within 30 days of index admission, stage of disease, length of stay of index admission, white blood cell count and type of cancer (all p-value < .001). An AUC of 0.67 indicated fair model discrimination. When the model was modified into a risk index, minimum score was -65 and maximum score was 130 . Validation of this model using the validation dataset $(n=2,217)$ resulted in an AUC of 0.64 .

\section{Conclusions}

We successfully developed a readmission risk scoring system for cancer patients with fair discrimination. Our READMIT score can be used to help providers identify high risk patients in order to prepare them adequately for discharge and facilitate post discharge care that can hopefully prevent cancer readmissions.

\section{eP667}

CANCER AWARENESS AMONG COMMUNITY PHARMACIST: A SYSTEMATIC REVIEW

K.B. mensah ${ }^{1}$, F. Oosthuizen ${ }^{2}$

${ }^{T}$ Komfo Anokye Teaching Hospital, Oncology Directorate, Kumasi, Ghana

${ }^{2}$ University of KwaZulu Natal, Discipline of Pharmaceutical Sciences, Durban, South Africa 


\section{Introduction}

The WHO recognises that community pharmacists are the most accessible healthcare professionals to the general public. Most patients regularly visit community pharmacies for health information and also seek advice from pharmacists with respect to signs and symptoms of cancer. As readily accessible health care professionals, community pharmacists are also in the best position to include cancer-screening initiatives into their practice. Pharmacists are therefore in a good position to raise awareness when they counsel people who buy over-the-counter medication for the control of possible cancer-related symptoms.

Objectives

To explore or assess

1) knowledge of community pharmacist on signs and symptoms of cancer 2) knowledge of community pharmacist on cancer screening.

Methods

EMBASE (ovid), CINAHL (EBSCOhost) and MEDLINE (EBSCOhost) were systematically searched for studies conducted between 2005 to July 2017. Studies that focused on knowledge of community pharmacist in cancer screening, signs and symptoms were included.

Results

A total of 1536 articles were identified from the search, of which 2 out of the 28 potentially relevant abstracts were included in the review. Findings of the selected studies revealed lack of sufficient knowledge on breast cancer screening, signs and symptoms. Both studies attributed knowledge limitation as the cause of reason for the key findings of their studies.

Conclusions

The selected studies focused on only breast cancer, which hinder the generalizability and transferability of the findings. Hence there is the need for more studies to be conducted in this area to draw a better conclusion.

\section{eP668}

MAIN CAUSES OF EMERGENCY ATTENTION IN PATIENTS WITH MALIGNANT SOLID TUMORS. EXPERIENCE FROM A SINGLE INSTITUTION IN MEXICO CITY

M.T. Nieto-Coronel ${ }^{1}$, A.D. López-Vásquez ${ }^{1}$, C.H. Arce-Salinas ${ }^{I}$

${ }^{T}$ Instituto Nacional de Cancerología, Medical Oncology, Mexico City, Mexico

\section{Introduction}

Patients with cancer are susceptible emergencies related to cancer or unrelated to it, which endanger their lives.

Objectives

To describe reasons for consulting to medical oncology emergency room. Methods

Prospective, observational study. We registered symptoms that cause the visit to emergency room at the first semester of 2017; patients included should have cancer diagnosis at any stage, and received any treatment. We analyze the prevalence of symptoms and its association with the treatment, stage, gender, socio-economic status. For the statistical analysis SPSS v.23 was used.

\section{Results}

246 patients were evaluated, average age was 54 years (18-91); $76.8 \%$ were women; main neoplasms attended were breast $24.4 \%$, ovarian $13.4 \%$ and cervical cancer $10.2 \%$; $71.4 \%$ of the patients were treated with chemotherapy. According with the initial evaluation, $15.5 \%$ was classified as an emergency and $84.5 \%$ as an urgency. $48.5 \%$ of patients reported a single symptom and $51.5 \%$ had several symptoms; the most frequent reason for consultation was pain $23.8 \%$, gastro-intestinal symptoms $23.2 \%$, followed by fever in $8.2 \%$. Overall in $39 \%$ of cases the symptom was tumor-related, $35.9 \%$ toxicity-related. None of the patients died in the emergency room and $26.7 \%$ were hospitalized. Factors lead to hospitalization were severity of symptoms, and manifestations related to unresponsive disease $(\mathrm{p}=0.000$ and $\mathrm{p}=0.033$ respectively).

\section{Conclusions}

Breast cancer represent the most common cause of emergency attention, the most common symptoms are pain and gastro-intestinal symtomps. One out of three patients required hospitalization. Factors associated with hospitalization were severity of symptoms and tumor-related symptoms.

\section{eP669}

TRIAL IN PROGRESS: A PILOT STUDY OF TACTILE CARE COMBINED WITH AROMATHERAPY IN PATIENTS WITH INCURABLE CANCER

Y. Oguma ${ }^{1}$, E. Tamai ${ }^{2}$, M. Kusuhara ${ }^{2}$, Y. Masuda ${ }^{2}$, S. Kazutoshi ${ }^{2}$, T. Shimoji $^{3}$

${ }^{1}$ Shizuoka Cancer Center, Division of Nursing, Shizuoka, Japan

${ }^{2}$ Shizuoka Cancer Center Research Institute, Region Resources Division, Shizuoka, Japan

${ }^{3}$ Empath Inc., Empath Inc., Tokyo, Japan

\section{Introduction}

Tactile care alleviates psychological distress and total pain of cancer patients. We hypothesized that aromatherapy might further enhance the efficacy of tactile care.

\section{Objectives}

The aim of this study is to evaluate feasibility of this strategy on incurable cancer patients. We will also exploratory assess psychological status and biomarkers before and after the procedure by questionnaire, heart rate variability, saliva test, and emotional evaluation of the voice.

\section{Methods}

Hospitalized patients with incurable cancer with written informed consent are eligible. Patients who have wounds in their soles, unstable psychological disorder, and difficulty in position change are not eligible. Registered therapist performs tactile care twice during hospitalization. Aromatherapy with essential oil of Citrus aurantium or Lindera umbellate were concurrently used according to patient's preference.

\section{Results}

Before and after procedure, we assessed subjective changes of pains, fatigue, and psychological status using Likert-scale. The primary endpoint is change in patient-reported outcomes. The secondary endpoints include changes in resting heart rate, coefficient of variation of R-R intervals, chromogranin A and amylase concentration in saliva, and the results of voice emotion analysis.

\section{Conclusions}

This study plans to recruit 70 patients in 5 years from April 2016 to Dec 2021. So far, 22 patients are recruited. No serious adverse events were reported.

\section{eP670}

INTERVENTION METHOD TO PREVENT THE DELAY OF HELP SEEKING BEHAVIOR IN CANCER PATIENTS: LITERATURE REVIEW

M. oshiro ${ }^{1},{\text { M. } \text { kamizato }^{2}}^{2}$

${ }^{T}$ Eötvös Loránd University, Health policy-planning and Financing MSc, budapest, Hungary

${ }^{2}$ Okinawa Prefectural College of Nursing, nursing, naha, Japan

\section{Introduction}

People who delay presenting with breast cancer have a reduced chance of survival.

\section{Objectives}

The aim of this study was to review the literature for the effectiveness of interventions to prevent the delay of help seeking behavior in cancer. We also aimed to get strategy to future research. 


\section{Methods}

We searched the literatures published in English which examines the interventions to prevent the delay of help seeking behavior in cancer. We searched the ProQuest, PubMed, MEDLINE, CINAHL from 1981 to May 2017 using the following key words; neoplasms, help seeking behavior, delay, and intervention. we also searched additional records identified through the reference of studies.

\section{Results}

We reviewed 6 studies. Five studies out of six were performed in UK, the other is performed in Indonesia. These study's concept framework is built based on the relation to health promotion. These studies show effectiveness to utilize multiple techniques which promote changing health behavior to prevent delay of help seeking behavior in cancer patients, such as expert consultation and using booklet builds on the information designed to raise people's awareness. However, the outcomes of intervention are different depend on the each country's characteristics related factors of delay of help seeking behavior.; UK study focus on the raising people's awareness. On the other hand, Indonesia study focus on how to get the social support.

Conclusions

Interventions using multiple techniques which are focus on the surrounding factors of delay may prevent delay of help seeking behavior, although the evidence is limited.

eP671

\section{IMPACT OF SYMPTOMS ON THE CAREGIVERS' WELL- BEING \\ E. Papastavrou ${ }^{I}$, A. Charalambous ${ }^{1}$ \\ ${ }^{T}$ Cyprus University of Technology, Nursing, Limassol, Cyprus}

\section{Introduction}

The increasing number of cancer patients worldwide combined with the chronicity and progressive nature of the disease, has increased the caring role of the family throughout the different stages of the cancer trajectory. Symptom management and response to side effects of treatment is a stressful experience that may have a negative impact on family caregiver's life.

Objectives

To discuss family care givers experiences in symptom management through an exploratory literature review and to briefly describe a comparative study exploring the caregiver coping, burden and depression in three different groups of care givers.

\section{Methods}

An exploratory literature review to answer the question of the impact of caring on family care givers of patients with cancer and a descriptive and correlation design.

\section{Results}

The results through the literature review showed that family caregivers provide emotional support, physical help including administration of medicines and feeding through "tubes", wound dressing, monitoring and assessment of symptoms and side effects. The one-way analysis of variance in the comparative study showed significant differences $(\mathrm{P}=.008, \mathrm{~F}=4.85)$ between the 3 caregiving groups in terms of depression, with the highest depression levels being for cancer caregivers.

\section{Conclusion}

Family carers are burdened to providing support to their relative and deal with the complexities of symptom and side-effect management that may result in negative outcomes for both the patient and the relative. The study findings support the necessity of family education to the management of cancer care.
eP672

SELF-REPORTED QUESTIONNAIRES IN LYMPHOEDEMA: SYSTEMATIC REVIEW OF MEASUREMENT PROPERTIES V.S. Paramanandam ${ }^{1,2}$, K. Boudreau ${ }^{3}$, M.J. Lee $^{2}$, D. Elizabeth ${ }^{2}$, S. Kilbreath $^{2}$

${ }^{I}$ Tata Memorial Hospital, Physiotherapy, Mumbai, India

${ }^{2}$ University of Sydney, Faculty of Health Sciences, Sydney, Australia

${ }^{3}$ University of Alberta-, Faculty of Rehabilitation - Physical Therapy, Edmonton, Canada

\section{Introduction}

The importance of patient-reported outcomes (PRO) is being increasingly recognized. This has led to development of PRO questionnaires specific to diseases, including for lymphoedema. For these questionnaires to be useful, their development needs to assess their psychometric properties including reliability and validity. Results of these studies, however, will largely depend upon their methodological quality. This systematic review was registered prospectively in PROSPERO, registration id: CRD42017064645.

\section{Objectives}

Primary objective of this systematic review was to review the methodological quality of the PRO questionnaires specific to lymphoedema.

\section{Methods}

A systematic literature search was performed in Medline, CINAHL, EMbase, Scopus, AMED, CENTRAL and PsycINFO databases to identify relevant studies that reported on the development of PRO specific to lymphoedema. Two reviewers independently screened these titles to ensure that the study reported on questionnaires developed specifically for use by lymphoedema patients. Following screening, the methodological quality of the studies was evaluated using the Consensus based Standards for the selection of health Measurement INstruments (COSMIN) checklist with four point scale.

\section{Results}

Initial data base search identified 6493 titles out of that, 23 studies on 17 PRO questionnaires. These include questionnaires in eight different languages addressing upper limb, lower limb, filarial, head and neck, genitourinary and gynecological lymphoedema. However, very few studies meet the COSMIN standard in limited number of measurement properties.

\section{Conclusions}

Although there are many PRO questionnaires are available to measure various outcomes related to lymphedema, further high quality studies related to their measurement properties are needed.

\section{eP673}

\section{DEVELOPMENT OF EVIDENCE-BASED GUIDELINES FOR NURSING HOME'S INFECTION CONTROL IN SOUTH KOREA(ENIK)

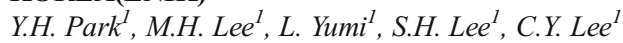 \\ ${ }^{T}$ Seoul National University, College of Nursing, Seoul, Republic of Korea}

\section{Introduction}

Older adults are vulnerable to infections because of immune dysfunction, functional impairment, comorbidities like cancer etc. Especially grouped living in long term facilities may provide unique opportunities for crosstransmission of pathogens.In South Korea, there is no infection control guideline for nursing homes, whereas another countries in worldwide has developed guidelines on this topic and evaluated effectiveness of them. Objectives

The purpose of this study was to deveolp an evidence based guidelines for nursing home's Infection control in South Korea(ENIK) for giving a theoretical foundation of infection control in nursing home and health and quality of life of older adults. 
Methods

The ENIK was developed based on international guidelines(SHEA/APIC guideline), systematic review(18 published articles) and focus group interview of nurses in nursing homes $(\mathrm{n}=11)$ from 2015 to 2016.

\section{Results}

The ENIK was composed of one-page algorithm and detailed guidelines. Algorithm pathway was organized in 3 parts, including screening at admission, prevention of infection, and control measure at early stage. The specific guideline included surveillance, outbreak control, hand hygiene and asepsis, isolation and precaution, resident care, resident health program, employee health program and facility. The experts verified the content validity.

Conclusions

Developed protocol will improve the quality of infection control in nursing homes as it can serve as a consistent and integrated standard for nursing care of nursing home residents

\section{eP674}

PALLIATIVE CARE IN GYNECOLOGICAL CANCER: RETROSPECTIVE ANALYSIS OF SYMPTOM MANAGEMENT AT THE NATIONAL CANCER INSTITUTE OF MEXICO

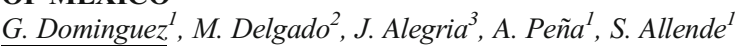

${ }^{T}$ Instituto Nacional de Cancerologia-México, Palliative care, Mexico city, Mexico

${ }^{2}$ MD Anderson Cancer Center, Palliative care, Houston- TX, USA

${ }^{3}$ Instituto Nacional de Cancerologia- México, Oncology, Mexico city, Mexico

\section{Introduction}

Gynecological cancer is a public health problem. Palliative Care (PC) has shown a beneficial impact on the quality of life and therapeutic modulation. In Mexico, information on the benefits of $\mathrm{CP}$ intervention in this population with cancer is limited

Objectives

Describe the sociodemographic characteristics and change the symptoms of patients with gynecological malignancies after entering a PC unit

Methods

Retrospective, analytical study, which included patients diagnosed with gynecological cancer, attended for the first time in the PC unit in one year. The sociodemographic, clinical and pathological characteristics and the modification of symptoms after the intervention were described by ESAS scale. The differences were established with the Wilcoxon test

\section{Results}

195 patients were included, with median of 55 years, predominance of residence in Mexico City (33.9\%), primary schooling (44.8\%) and Catholic religion $(89 \%)$. The median monthly income was US \$ 197.26. The predominant tumor was cervical cancer $(44.6 \%) .46 \%$ in clinical stage IV. The majority $(64 \%)$ entered by external consultation. The management by the CP team improved pain $(4 \mathrm{Vs} 2, \mathrm{p}=0.0004)$ and depression ( $1 \mathrm{Vs} 0 ; p=0.0002)$; there was a reduction in the 75th percentile of nausea $(\mathrm{p}=0.0025)$, anxiety $(\mathrm{p}=0.01)$ and insomnia $(\mathrm{p}=0.03)$ Conclusions

$\mathrm{CP}$ intervention significantly improved symptoms such as pain, depression, nausea, anxiety and insomnia. It is necessary to carry out interventions to improve the implementation of Palliative Care in a timely manner

\section{eP675}

SPIRITUALITY AND RELIGIOSITY AMONG LATINAMERICAN HEALTH PROVIDERS CARING FOR PATIENTS LIVING WITH ADVANCED CANCER AT THE MEXICAN NATIONAL CANCER INSTITUTE

G. Dominguez $^{1}$, J. Alegria' ${ }^{2}$, S. Allende ${ }^{l}$, M. Delgado $^{3}$
${ }^{1}$ Instituto Nacional de Cancerologia- México, palliative care, Mexico city, Mexico

${ }^{2}$ Instituto Nacional de Cancerologia- México, Oncology, Mexico city, Mexico

${ }^{3}$ MD Anderson Cancer Center, Palliative- Rehabilitation and Integrative Medicine, Houston- TX, USA

\section{Introduction}

Spirituality and religiosity might have a positive effects in patients with advanced cancer. Health care providers of these patients often neglect this important aspect of care.

\section{Objectives}

examine the differences in characteristics and intensity/importance of self-reported spirituality and religiousness between palliative care providers and oncologists

\section{Methods}

A retrospective file review was done of a voluntary and anonymous survey given to Latin American Health Care providers. All health care providers were taking care of patients with advanced cancer. Wilcoxon rank sum test was used

\section{Results}

A total of 84 health providers' data were included; 54 participants were female $(64.3 \%)$ and the mean age was 36.9 years $(\mathrm{SD}=+/-10.9)$. PCT were more likely to think spirituality and religion in their life important [8.5/10 (SD+/-1.7), IQR 2.0 vs 6.3/10 (SD+/- 3.1), IQR 4.0; p=0.0003], were more likely to consider spirituality or religiosity as a source of strength and comfort for themselves [8.9/10 (SD+/-1.8), IQR 1.0 vs 6.3/ 10 (SD+/- 3.4), IQR 4.0; $<<0.0001]$, and were more likely to think spirituality or religiosity help themselves [8.9/10 (SD+/-1.9), IQR 2.0 vs $6.2 /$ 10 (SD+/- 3.3), IQR 4.0; $<<0.0001$ ], their family or caregivers cope with problems or their illness [8.8/10 (SD+/-1.6), IQR 2.0 vs 7.4/10 (SD+/2.2), IQR 2.0; $\mathrm{p}=0.001]$, than ones in Oncology Team. Finally, PCT were more likely to think their spiritual or religious beliefs influence when they provide medical care to their patients than ones in oncology team $[8.1 / 10$ (SD+/-2.5), IQR 3.0 vs 5.7/10 (SD+/- 3.3), IQR 5.0;p=0.0002].

\section{Conclusions}

High percentage of health care providers of advanced cancer patients were spiritual and religious, more in frequent and intense in PCT. The importance attributed to spirituality and religiosity in their personal and professional life and how the care is provided might varies in this both population.

\section{eP676}

MODELS OF CANCER CARE - A COMPREHENSIVE RESEARCH PROGRAM IN THE MANAGEMENT OF CANCER AND TREATMENT-RELATED SYMPTOMS AND HEALTH-RELATED QUALITY OF LIFE

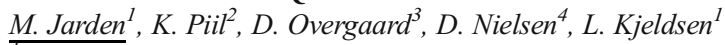

${ }^{T}$ Copenhagen University Hospital - Rigshospitalet, Hematology, Copenhagen, Denmark

${ }^{2}$ Copenhagen University Hospital - Rigshospitalet, Oncology, Copenhagen, Denmark

${ }^{3}$ Metropolitan University College, Nursing, Copenhagen, Denmark

${ }^{4}$ Herlev-Gentofte Hospital, Oncology, Herlev, Denmark

\section{Introduction}

This research program is an initiative that can guide future directions within disease and treatment-related symptom prevention, control and management across the cancer disease trajectory. Models of cancer care will increasingly incorporate user involvement, behavioral and biological aspects of symptom research to improve clinical outcomes.

\section{Objectives}

The aim of "Models of Cancer Care" is to examine the effect of applying different care and intervention approaches incorporating patient 
involvement in managing multiple symptoms and thereby reduce burdensome symptoms and enhance optimal treatment dosis/adherence and quality of life. Further, to improve symptom assessment and management skills of health care professionals and patients.

\section{Methods}

The "Models of Cancer Care" program includes five research projects

1. Patient Ambassador Support for newly diagnosed patients with acute leukemia during treatment - a feasibility controlled trial.

2. Patient Activation through Counseling, Exercise and Mobilization Engaging the older cancer patient - a randomized control trial.

3. Needs assessment and decision-making tool in clinical practice to enhance patient involvement in Head and Neck Cancer Rehabilitation a randomized controlled trial.

4. Nurse-led Family and Network Consultations - Addressing Symptoms and Concerns in Patients with High-grade Glioma and their families - a feasibility controlled trial

5. Models of Cancer Care - understanding and managing multiple symptoms and symptom clustersResults

To be published

Conclusions

This current research program $(2017$ - 2021) will provide new knowledge and innovative strategies for managing disease and treatment-related symptoms and thereby improve patients, families and caretakers' health-related quality of life during and after cancer treatment.

eP677

INCREASING ACCESS TO INFORMATION DURING THE CANCER TRAJECTORY WITH "MY COURSE APP" M. Poulsen ${ }^{I}$, R. skoett ${ }^{l}$, K. Dieperink ${ }^{l}$

${ }^{T}$ OUH, department of oncology, Odence C, Denmark

\section{Introduction}

Information and communication with patients and relatives is complicated with a risk of information overload or decline, which may lead to patients not coping efficiently.

Objectives

The purpose was to develop and implement modern access to communication for cancer patients and relatives about practical issues and patient specific tailored information about treatment and adverse effects during their cancer trajectory.

\section{Methods}

All material e.g. pamphlets about cancer diseases and treatments was reviewed by health professionals, and edited by a journalist to secure everyday language. Animations and podcasts about cancer and different treatments and practical guidelines as route directions were developed. An app was built with three informational parts: 1) Before, during and after treatment. This part is available for all patients (Nice to know). 2) My material. This part is tailored to each individual patient (Need to know). 3) FAQ, Frequently asked questions. Furthermore, the app can include Patients Reported Outcome measures. Patients and health professionals were interviewed about the implementation process. Results

The app is free and available at the internet or tablets/smartphones for lung- prostate and head/neck cancer patients. Patients report the app feasible and useful and they find it easy to find answers. Health professionals report that the app helps organize information and prevents reintroduction of already given information in the patient specific trajectory. Furthermore they report that patient and relatives seems to share information more.

Conclusions

"My Course" app is feasible but the implementation process has to be followed closely.
eP678

PROGNOSTIC FACTORS IN CRITICALLY ILL PATIENTS WITH SOLID CANCER ADMITTED TO MEDICAL INTENSIVE CARE UNIT

C. Baylot ${ }^{I}$, A. Francopoulo ${ }^{2}$, A. Daste ${ }^{1}$, O. Guisset ${ }^{3}$, G. Hilbert ${ }^{4}$, E.

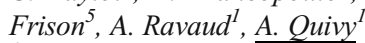

${ }^{I}$ CHU Bordeaux, Medical Oncology, Bordeaux, France

${ }^{2}$ CHU Bordeaux, Medical Information Department, Bordeaux, France

${ }^{3}$ CHU Bordeaux, Department of Medical Intensive Care Unit- Hôpital Saint-André, Bordeaux, France

${ }^{4}$ CHU Bordeaux, Department of Medical Intensive Care Unit- Hôpital Pellegrin, Bordeaux, France

${ }^{5}$ CHU Bordeaux, University of Bordeaux, Bordeaux, France

\section{Introduction}

Decision to transfer patients (pts) with solid cancer to intensive care unit (ICU) is still controversial and difficult. Few studies evaluated the outcome of these patients.

\section{Objectives}

The aim of this study was to identify prognostic factors 30-day mortality for pts with solid cancer admitted to ICU.

\section{Methods}

We conducted a retrospective cohort study of all consecutive pts with solid cancer admitted to ICUs of Bordeaux University Hospital, between January 2010 and December 2015. The study end point was 30-day mortality. Secondary end points were to describe characteristics and outcomes of pts and ethical practices.

\section{Results}

We included 235 patients with solid tumors. Most of them were in metastatic setting $(60 \%)$. The most common causes of ICU admission were sepsis (56\%) and/or respiratory failure (52\%). ICU, 30-day, 90-day mortality rates were $24 \%$, $36 \%$ and $50 \%$ respectively. After ICU stay, $44 \%$ of pts had restarting an antitumoral treatment. In multivariate analysis and after excluding SAPS 2 score, two or more organ failures $(p=.005)$ and being under non-curative care $(p=$ 0.028 ) were independent prognostic factors of 30-day mortality. Support person was designated in $81 \%$, advance directives expressed in $2 \%$ and collective decision reported in $21 \%$. Limitation of life-sustaining therapy was decided for $23 \%$ and $43 \%$ of pts before the admission and during the ICU stay, respectively. Conclusions

The number of organ failures is a variable quickly evaluable that can help oncologists and intensive care specialists in their decision. Support person is often designated but advance directives are still unusual.

eP679

MURAL THROMBUS OF ASCENDING AORTA SUCCESSFULLY MANAGED WITH ANTICOAGULATION IN A PATIENT WITH METASTASIC CANCER

A. Rodríguez

${ }^{T}$ HOSPITAL CLINIC DE BARCELONA, ONCOLOGÍA MÉDICA, BARCELONA, Spain

\section{Introduction}

There are few reports on acute aortic thrombosis (AAT) as a manifestation of cancer-associated hypercoagulability with optimal management remaining unclear (1)

\section{Objectives}

Here we present a patient with multiple thrombotic events during chemotherapy for metastatic cancer.

\section{Methods}

Description of clinical findings and patient outcome.

Results

A 70-year-old man, smoker, with a history of dyslipidemia, type 2 diabetes, and arterial hypertension underwent radical cystoprotectomy for 
bladder carcinoma T3N2M0 with adyuvant chemotherapy. In March 2017 he started palliative chemotherapy for cancer progression. On July $12^{\text {th }}, 2017$ he was admitted to hospital for acute arterial ischemia in the left leg secondary to a popliteal arterial thrombosis treated with embolectomy and anticoagulation.

On July 26th 2017 he was attended in the Hospital for a 7-day history of progressive shortness of breath. X-Ray and blood test results were normal.Angio-CT revealed a $12 \mathrm{~mm}$ floating mural thrombus in the ascending aorta (Figures 1A and 1B). Transthoracic echocardiography discarded signs of right ventricular overload, myocardial dysfunction or heart valve disease. Thrombophilia testingwas negative. The patient was managed with unfractionated heparin for 12 hours followed by weightadjusted LMWH, achieving a significant reduction in the thrombus to complete resolution (Figure 1C and 1D). He was discharged with LMWH treatment. A CT at one month showed disappearance of the AAT(1D) although chemotherapy was discontinued due to these events.

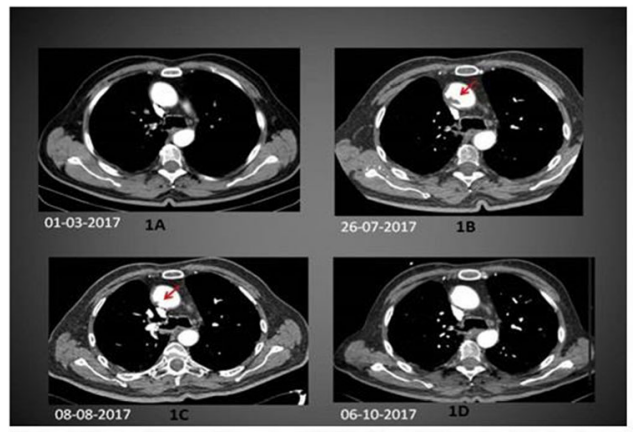

1A: basal CT, no evidence of thrombus. 1B: CT with aortic thrombus $1 \mathrm{C}$ : after 2 weeks of $L M W H$, reduction of the aortic thrombus 1D after 2 months of treatment, complete resolution of the aortic thrombus

\section{Conclusions}

This case illustrates the challenges associated with arterial thrombotic events. Conservative management with LMWH was effective and safe. Further studies are needed to assess the clinical impact and optimal management of cancer-associated arterial thrombotic events.

\section{eP680}

\section{SYMPTOMS DURING CHEMOTHERAPY IN COLORECTAL} CANCER PATIENTS

\section{K. Röhrl ${ }^{1}$, M.G. Guren ${ }^{2}$, M.C. Småstuen ${ }^{3}$, T. Rustøen ${ }^{4}$}

${ }^{7}$ Institute of Health and Society- Faculty of Medicine-University of Oslo, Department of Nursing Science, Oslo, Norway

${ }^{2}$ Oslo University Hospital, Department of Oncology and K.G.Jebsen Colorectal Cancer Research Centre, Oslo, Norway

${ }^{3}$ National Advisory Unit of Late Effects after Cancer Treatment-Oslo University Hospital, Oslo and Akershus University College of Applied Sciences- Faculty of Health Sciences, Oslo, Norway

${ }^{4}$ Institute of Health and Society- Faculty of Medicine- University of Oslo, Department of Nursing Science and Department of Research and Development-Division of Emergencies and Critical Care-Oslo University Hospital, Oslo, Norway

\section{Introduction:}

- Introduction. Colorectal cancer (CRC) patients experience a range of physical and psychological co-occurring symptoms, but little is known about symptom variation during chemotherapy cycles.

Objectives

- Objectives. The aims were (1) to describe the occurrence and severity of frequently occurring symptoms (worrying, lack of energy, numbness/tingling, nausea, and pain) at multiple time points during chemotherapy, (2) to analyse the differences in symptom trajectories between chemotherapy groups, and (3) to determine whether selected patient and clinical characteristics are associated with symptom severity throughout the treatment trajectory.

\section{Methods}

- Methods. In total, 120 curative or palliative CRC patients receiving chemotherapy completed the Memorial Symptom Assessment Scale, Karnofsky performance status (KPS) and Self-Administered Comorbidity questionnaire at eight time points, during and after two courses of chemotherapy. Data were analyzed using linear regression mixed models for repeated measures to assess the effects of selected variables on outcomes over time.

\section{Results}

- Results. Preliminary results show a peak in lack of energy, nausea and numbness/tingling in the first three to seven days after chemotherapy administration. Worrying and pain were the most occurring symptoms at enrollment, but declined during the treatment trajectory. Educational level, age, sex and KPS were associated with symptom severity throughout the course of chemotherapy.

\section{Conclusions}

Conclusions. CRC outpatients experience several symptoms over the course of chemotherapy. The peak in severity scores of nausea in the week following chemotherapy raises the question whether adequate antiemetic regimens have been prescribed. These preliminary results highlight the importance of paying attention to and assessing symptoms during and between chemotherapy cycles in CRC outpatients.

\section{eP681}

ENHANCING MEN'S AWARENESS OF TESTICULAR DISORDERS USING A VIRTUAL REALITY INTERVENTION: A ONE-GROUP PRE-POST STUDY

M. Saab ${ }^{1}$, M. Landers ${ }^{1}$, E. Cooke ${ }^{2}$, D. Murphy ${ }^{2}$, M. Davoren ${ }^{3}, J$. Hegarty $^{1}$

${ }^{T}$ University College Cork, School of Nursing and Midwifery, Cork, Ireland

${ }^{2}$ University College Cork, Department of Computer Science, Cork, Ireland

${ }^{3}$ University College Cork, School of Public Health, Cork, Ireland

\section{Introduction}

Three systematic reviews found that men's awareness of testicular disorders was lacking and their intention to seek help for testicular symptoms was low. Few studies promoted awareness of testicular disorders with none using innovative technologies.

\section{Objectives}

To enhance men's testicular awareness using a virtual reality-based intervention underpinned by a novel theoretical framework (Pre-Conscious Awareness to Action Framework, Figure 1).

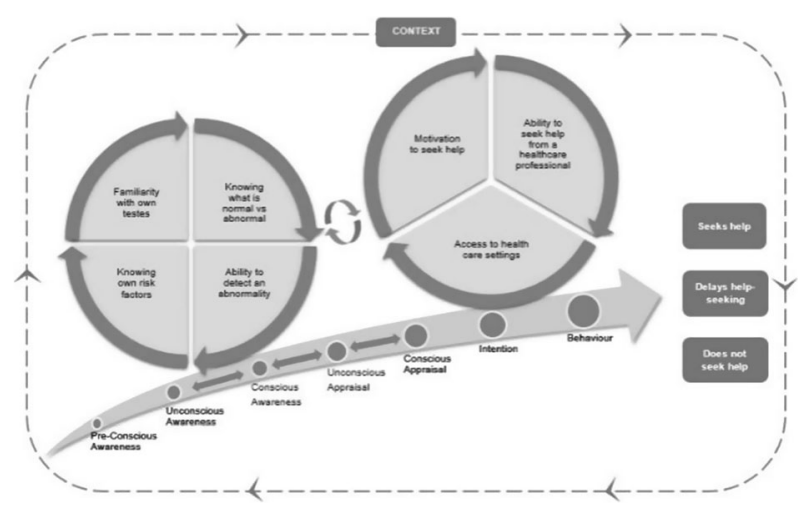




\section{Methods}

A one-group pre-post design was used. Men ( $\mathrm{n}=53)$ aged between 18 and 50 years were recruited using non-probability sampling and were asked to play a 3-level educational game using a virtual reality headset and controller. Knowledge, awareness, perceived risk, intention, and behavior (Table 1) were measured at baseline (T0), immediately post-test (T1), and one month posttest (T2). Data were analysed using descriptive and inferential statistics.

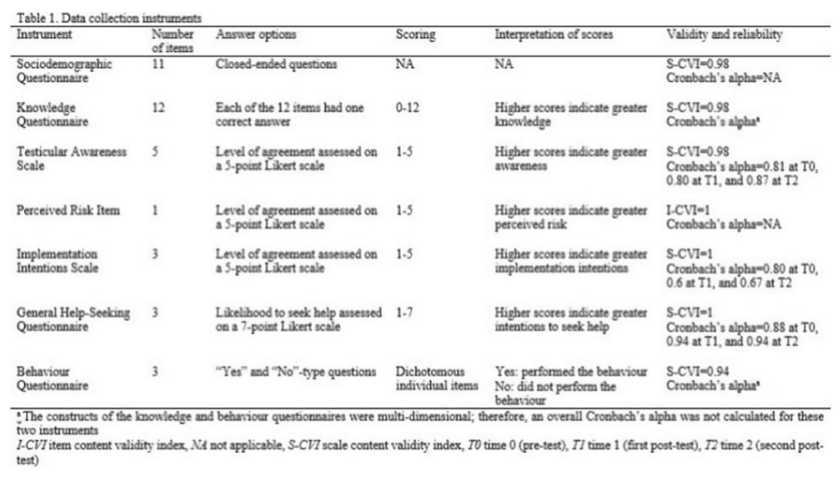

Results

Men's awareness of the normal testes and testicular symptoms and diseases; intentions to examine their testes; intentions to seek help for testicular symptoms; and behaviours pertaining to feeling their testes increased over time (Table 2). This increase was found to be statistically significant. Men who intended to feel their testes at T0 reported having done that at T2. In contrast, men's perceived risk for developing a testicular disorder was low at $\mathrm{T} 0$ and did not increase at $\mathrm{T} 1$ or $\mathrm{T} 2$.

\begin{tabular}{|c|c|c|c|c|c|c|c|}
\hline Outcome & $\mathrm{n}$ & $\begin{array}{l}\frac{\text { T1 is To }}{\text { Mean difference }} 195 \% \mathrm{CD} \\
\end{array}$ & & $\begin{array}{l}\text { T2 vs T0 } \\
\text { Mean difference }(95 \% \mathrm{CD})\end{array}$ & & $\begin{array}{l}\text { T2 vs T1 } \\
\text { Mean difference }(95 \% \mathrm{CD})\end{array}$ & \\
\hline Knowledge & 49 & $3.5(2.8,4.3)$ & $=0.001$ & $2.7(2,3.4)$ & $\frac{10 \text { paue }}{50.001}$ & $\begin{array}{l}\text { Metan dinterence } \\
-0.9(-1.6,-0.1)\end{array}$ & 0.014 \\
\hline Testicular Awreness & 49 & $0.2(0.01,0.4)$ & 0.038 & $0.4(0.3,0.6)$ & $<0.001$ & $0.2(0.01,0.4)$ & 0.033 \\
\hline $\begin{array}{l}\text { Implementation } \\
\text { lltentions }\end{array}$ & 48 & $0.6(0.3,0.9)$ & $\$ 0.001$ & $0.5(0.2,0.8)$ & 0.002 & $-0.2(-0.4,0.1)$ & 0.24 \\
\hline $\begin{array}{l}\text { Help-Seeling Intentions } \\
\text { Swelling }\end{array}$ & 49 & $0.3(0.1,0.5)$ & 0.001 & $0.4(0.1,0.7)$ & 0.01 & $0.1(-0.2,0.4)$ & 1 \\
\hline Help-Seeking Intentions & 49 & $0.3(0.1,0.5)$ & 0.003 & $0.3(0.02,0.7)$ & 0.04 & $0.1(-0.2,0.4)$ & 1 \\
\hline 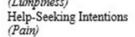 & 48 & $0.6(0.3,1)$ & $=0.001$ & $0.6(0.3,0.9)$ & $=0.001$ & $-0.04(-0.4,0.3)$ & 1 \\
\hline
\end{tabular}

\section{Conclusions}

The intervention proved successful in enhancing men's testicular awareness, implementation intentions, help-seeking intentions, and behaviors; therefore, full-scale testing with a larger sample size across different settings is warranted. Healthcare professionals can make use of virtual reality to educate men about different topics.

\section{eP682}

\section{REASONS FOR ACCESS TO A DEDICATED OUTPATIENT} SUPPORTIVE CARE SERVICE AND RESOLUTION RATE

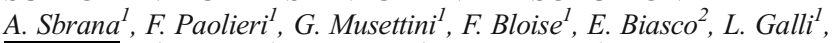
I.M. Brunetti ${ }^{I}$, S. Ricci ${ }^{1}$, A. Falcone ${ }^{I}$, A. Antonuzzo ${ }^{1}$

${ }^{1}$ Azienda Ospedaliero-Universitaria Pisana, Oncologia Medica, Pisa, Italy ${ }^{2}$ Azienda USL Toscana Nord Ovest, DH Oncologico Portoferraio, Livorno, Italy

\section{Introduction}

In April 2012 a dedicated outpatient supportive care service (OSCS) was created in our cancer center. Patients can access this service for any kind of problem related to their disease or to the treatments they are receiving.

\section{Objectives}

We wanted to analyze the reasons for access to the OSCS and to observe the resolution rate, that is the percentage of patients that resolved their clinical problems only through the access to the OSCS.

\section{Methods}

We analyzed all the patients that accessed to the OSCS during three months of activities in 2017. We registered the reasons why they access to the OSCS and we followed them up to see how many of them have their problems resolved through the therapies performed in the OSCS.

\section{Results}

194 patients performed 379 accesses to the OSCS during three months. $97 \%$ was on active treatment. The main reasons for access were: pain $(35.5 \%)$, fatigue $(23 \%)$, fever $(22 \%)$, anemia $(20.5 \%)$, and pleuropulmonary complications (19\%). 67\% resolved their problems through the access to the OSCS and only $13.5 \%$ of the patients needed to be hospitalised.

\section{Conclusions}

Our experience highlights that the most common problems in cancer patients on active treatment are pain, fatigue, fever, anemia, and pleuropolmonary complications. This experience also underlines how most of them could have their disease- or treatment-related problems resolved through the direct access to an OSCS and how this could avoid a high number of unnecessary hospitalisations.

\section{eP683}

COMPARISON OF OXALIPLATIN-RELATED SPLEEN ENLARGEMENT IN PATIENT WITH GASTRIC CANCER WHO RECEIVED S-1 VERSUS CAPECITABINE AS A COMBINATION PARTNER OF OXALIPLATIN

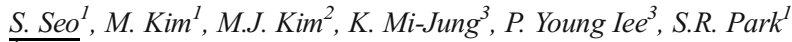

${ }^{T}$ Asan medican center, Department of Oncology, Seoul, Republic of Korea

${ }^{2}$ Research Institute and Hospital- National Cancer Center, Cancer for Colorectal Cancer, Goyang, Republic of Korea

${ }^{3}$ Research Institute and Hospital- National Cancer Center, Cancer for Gastric Cancer, Goyang, Republic of Korea

\section{Introduction}

Oxaliplatin (OX)-based chemotherapy is known to cause hepatic sinusoidal injury, resulting portal hypertension with splenomegaly.

\section{Objectives}

We compared OX-induced hepatopathy, SM, and their clinical significance according to combined oral fluoropyrimidines, S-1 vs. capecitabine $(\mathrm{X})$ in gastric cancer.

\section{Methods}

We analyzed patients from two prospective trials for gastric cancer, adjuvant XELOX (X $1000 \mathrm{mg} / \mathrm{m}^{2}$ bid on D1-14 + OX $130 \mathrm{mg} / \mathrm{m}^{2}$ on D1 q3w, 8 cycles; $n=52)$ and palliative SOX $\left(\mathrm{S}-140 \mathrm{mg} / \mathrm{m}^{2}\right.$ bid on D1-14 + OX $130 \mathrm{mg} / \mathrm{m}^{2}$ on D1 q3w, continuous [SOX-c, $\mathrm{n}=52$ ] vs. intermittent [SOX$\mathrm{i}$, discontinuing after $6^{\text {th }}$ and restarting on progression, $\left.n=53\right]$ ). Spleen volume was retrospectively measured by Rapidia software.

Results

Baseline sex, age, ECOG performance status, BSA, spleen vol, levels of platelet/liver enzyme/bilirubin and OX cumulative dose during 8 cycles did not differ in XELOX and SOX-c. After 8 cycles, the SOX-c had more splenomegaly hepatic enhancing heterogeneity, hyperbilirubinemia, and thrombocytopenia than the XELOX. The SOX-c was a risk factor for developing splenomegaly (adjusted OR, 4.7; 95\% CI, 2.0-10.8; $\mathrm{p}<.001$ ). When tracking serial spleen volume and platelet at baseline, $2^{\text {nd }}, 4^{\text {th }}$ and $6^{\text {th }}$ cycles in SOX-c and SOX-i, spleen volume significantly increased over time (mean, 160, 182, 216, $247 \mathrm{~cm}^{3} ; p<.001$ ), whereas platelet were decreased (mean, 290, 168, 132, $116 \times 10^{3} / \mathrm{uL} ; p<.001$ ). In SOX-i group, spleen volume decreased at $6^{\text {th }}$ cycles, $6,12,18$ and $24 \mathrm{w}$ after stopping SOX (mean, 194, 181, 167, 158, $150 \mathrm{~cm}^{3} ; p=.016$ ). 


\section{Conclusions}

S-1 seems to enhance OX-induced hepatic sinusoidal injuries than capecitabine in pts with GC with higher incidences of splenomegaly, thrombocytopenia, and hyperbilirubinemia.

\section{eP684}

THE SAFTY AND EFFICACY OF VASCULAR HEMOSTASIS

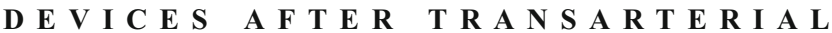
CHEMOEMBOLIZATION : A RANDOMIZED CONTROLLED TRIAL

S.M.SEOL ${ }^{1}$, S.Y. Chun ${ }^{2}$, M.Y. Lee ${ }^{2}$, K.M. Kim ${ }^{2}$, J.H. Shin ${ }^{2}$, P.H. Kim ${ }^{2}$, M.S. Lee

${ }^{1}$ Asan /Medical Cener, Radiology, Seoul, Republic of Korea

${ }^{2}$ Asan Medical Center, Radiology, Seoul, Republic of Korea

${ }^{3}$ InnoTherapy Inc, R\&D Center, Seoul, Republic of Korea

\section{Introduction}

This study was to compare the safety and efficacy of vascular hemostatic devices ( InnoSEAL versus Clo-Sur PAD) following Transarterial

chemoembolization through femoral artery access.

Objectives

This study was to compare the safety and efficacy of vascular hemostatic devices ( InnoSEAL versus Clo-Sur PAD) following Transarterial chemoembolization through femoral artery access.

Methods

The study was approved by the institutional review board and all patients gave written informed consent. In a randomized trial, InnoSEAL $(n=48)$ was compared with Clo-Sur PAD $(n=52)$ in patients after TACE with femoral artery approach (5-French sheath). Hemostasis time, time to ambulation, and access-site complications after hemostasis (Immediately after hemostasis and 7 days after TACE) were recorded.

Results

The averagetime to hemostasis was $5.6 \pm 1.0 \mathrm{~min}$ (InnoSEAL) and 5.3 $\pm 0.7 \mathrm{~min}$ (Clo-Sur PAD), and both groups achieved ambulation after 3 hours ofhemostasis. The immediate complication of access site was not different between the InnoSEAL and Clo-Sur PAD (Hematoma: 1.9\%, $2.1 \% ; P>0.05)$. The rate of delayed complication was lower in InnoSEAL group (4.2\%) than in Clo-Sur PAD group (Ecchymosis: 17.3\%; $p=0.036$ ). There was no statistical difference between the complicated groups and the uncomplicated groups for prothrombin time, platelet count.

\section{Conclusions}

Hemostasis time and ambulation time was not significantly different, andimmediate complications were rare(Hematoma: $1.9 \%, 2.1 \%$ ). However, InnoSEAL is better than Clo-Sur PAD regarding delayed complication such as ecchymosis $(\mathrm{p}=0.036)$.

\section{eP685}

\section{NATIONAL PALLIATIVE AND HOME CARE STRATEGIC} FRAMEWORK DEVELOPMENT IN JORDAN

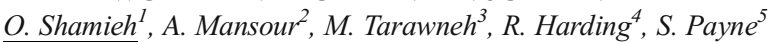

${ }^{T}$ King Hussein Cancer Center, Palliative Care, Amman, Jordan

${ }^{2}$ King Hussein Cancer Center, Radiology, Amman, Jordan

${ }^{3}$ High Health Council, Secretary General, Amman, Jordan

${ }^{4}$ King's College London- Cicely Saunders Institute, Department of Palliative Care-Policy and Rehabilitation, London, United Kingdom

${ }^{5}$ Lancaster University, International Observatory on End of Life CareDivision of Health Research, Lancaster, United Kingdom

\section{Introduction}

Palliative care in Jordan is developed better than most of its neighbors in the Middle East but lagging behind western countries. It is estimated that more than $70 \%$ of cancer patients who died in Jordan in 2016 didn't have access to palliative care . Currently, palliative care is only accessible by cancer patients. A national palliative care framework is needed to advance palliative care development.

Objectives

To describe the developmental process of designing and implementing a national palliative and home care strategic framework in Jordan. To describe barriers and challenges of advancing palliative care in Jordan

\section{Methods}

A systematic content analysis, SWOT analysis, multistage consensus procedure and national and international expert consultations were used to develop and revise the national strategic framework

\section{Results}

A detailed situational analysis was completed. The development of the framework took multiple steps, the first draft was developed by the national Palliative care committee, using the WHO framework, the draft was later revised by international experts, the second draft was revised in a national strategic multidisciplinary workshop of multi stakeholder experts, to reach a consensus. The final version was approved by the national and the international experts. The framework contains 6 domains; policy, finance, capacity building, service development and monitoring and evaluation.

\section{Conclusions}

The multistage process of development and the involvement of key stakeholders lead to significant revision of the framework and was successful in reaching a national consensus. The final draft will be endorsed by the Jordanian Ministry of health; a detailed implementation plan is underway.

\section{eP686}

A STATE-WIDE CANCER CLINICAL TRIALS NETWORK TO INCREASE THE REACH AND IMPACT OF CANCER CARE DELIVERY RESEARCH AND OPTIMISE CARE FOR PEOPLE AFFECTED BY CANCER

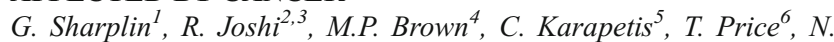
Singhal, M. Osborn ${ }^{8}$, D. Poprawski ${ }^{9}$, A. Brown ${ }^{10}$, J. Marker $^{11}$, C. Christensen $^{\text {II }}$, M. Eckert ${ }^{I}$, I. Olver ${ }^{12}$

${ }^{1}$ University of South Australia, Rosemary Bryant AO Research Centre, Adelaide, Australia

${ }^{2}$ Lyell McEwin Hospital, Medical Oncology, Elizabeth Vale, Australia

${ }^{3}$ Adelaide Oncology and Haemotology, Medical Oncology, North Adelaide, Australia

${ }^{4}$ Royal Adelaide Hospital, Haematology, Adelaide, Australia

${ }^{5}$ Flinders Medical Centre, Flinders Centre for Innovation in Cancer, Bedford Park, Australia

${ }^{6}$ The Queen Elizabeth Hospital, Haematology and Medical Oncology, Woodville, Australia

${ }^{7}$ Adelaide Cancer Centre, Medical Oncology, Kurralta Park, Australia

${ }^{8}$ Women's and Children's Hospital, The Michael Rice Centre, North Adelaide, Australia

${ }^{9}$ Country Health SA Local Health Network, Medical Oncology, Elizabeth Vale, Australia

${ }^{10}$ South Australian Health and Medical Research Institute, Wardliparingga Aboriginal Research Unit, Adelaide, Australia

${ }^{11}$ Cancer Voices SA, Cancer Voices SA, Adelaide, Australia

${ }^{12}$ University of South Australia, Sansom Institute for Health Research, Adelaide, Australia

\section{Introduction}

Cancer clinical trials networks (CTN) predominantly support pharmaceutical, surgical or device trials targeting cures or reducing disease impact. Cancer care delivery research (CCDR) examines how clinical, organisational, financial, technological, system, and human factors influence care quality, cost, access and equity and the health and well-being of 
consumers. CTNs have the capacity to link disparate trial sites to support large CCDR projects.

\section{Objectives}

To undertake large CCDR projects to optimise supportive care, treatment and healthcare outcomes for consumers using a state-wide CTN as the research platform.

\section{Methods}

A CTN formally linking all CT units across diverse settings in South Australia (the SACTN) will be a platform for improved CCDR. Researchers will develop, refine, and implement co-designed CCDR projects involving multiple stakeholders in its conduct; uses standardised healthcare quality measures; examines causal pathways and critical components of practice change.

\section{Results}

This novel program will increase the number, size, and scope of CCDR projects and support recruitment of consumer participants. CCDR outcomes will inform cancer service delivery at networked sites underpinning optimisation of healthcare delivery, consumer-centred care and clinical outcomes; key objectives of the SACTN strategy.

Conclusions

CCDR that leverages the SACTN will complement clinical trials by focusing on other components of cancer care across a diverse population to optimise the benefits of clinical intervention. The program will enhance supportive care, treatment and clinical research, and aligns to and supports the implementation of national and SA strategic initiatives like national cancer control indicators, optimal care pathways, the SA Cancer Plan and the SA Cancer Research Strategy.

\section{eP687}

BETA BLOCKERS USE IN CARDIO-TOXIC EFFECTS OF ANTHRACYCLINE-BASED CANCER CHEMOTHERAPY: A SYSTEMATIC REVIEW AND META-ANALYSIS

H. Singh ${ }^{1}$, R.P. Singh ${ }^{2}$

${ }^{T}$ Baba Farid University of Health Sciences, Pharmacology, Faridkot, India

${ }^{2}$ Baba Farid University of Health Sciences, Radiation Oncology, Faridkot, India

\section{Introduction}

Anthracyclines are commonly used chemotherapeutic agents with proven efficacy in such malignancies as breast cancer, Hodgkin, and nonHodgkin lymphomas. These agents are associated with irreversible accumulative dose-related cardiomyopathy. Many agents have been examined to reduce cardiotoxicity risk.

Objectives

We performed a systematic review and Meta-analysis to determine the efficacy of $\beta$-blockers to prevent early/late onset anthracyclines-induced left ventricular dysfunction and cardiac events in cancer patients on above said therapy.

Methods

Relevant articles were searched in PubMed, EMBASE and Cochrane database of systematic reviews up to August 2017. Eligible studies were limited to randomised controlled trials and prospective comparing the efficacy of cardio-protective $\beta$-blockers with control (either no treatment or placebo) in patients treated with anthracycline-based regimens.

\section{Results}

Pooled analysis showed an association of $\beta$-blockers with higher postchemotherapy left ventricular ejection fraction (LVEF) of $62.32 \%$ compared with $57.78 \%$ for control treatment. The mean difference estimate ( $95 \% \mathrm{CI}$ ) was $0.39 \%$ [0.24 to 0.55$], \mathrm{p}=0.00001$, with significant heterogeneity, $\mathrm{I}^{2}(95 \% \mathrm{CI})=90 \%$. The point estimate for the relative rate of cardiac events are significantly lower in the experimental arm (23/329) as compared to control group (53/389) and Heterogeneity: $\mathrm{Chi}^{2}=13.97, \mathrm{df}=$ $5(\mathrm{P}=0.02) ; \mathrm{I}^{2}=64 \%$.

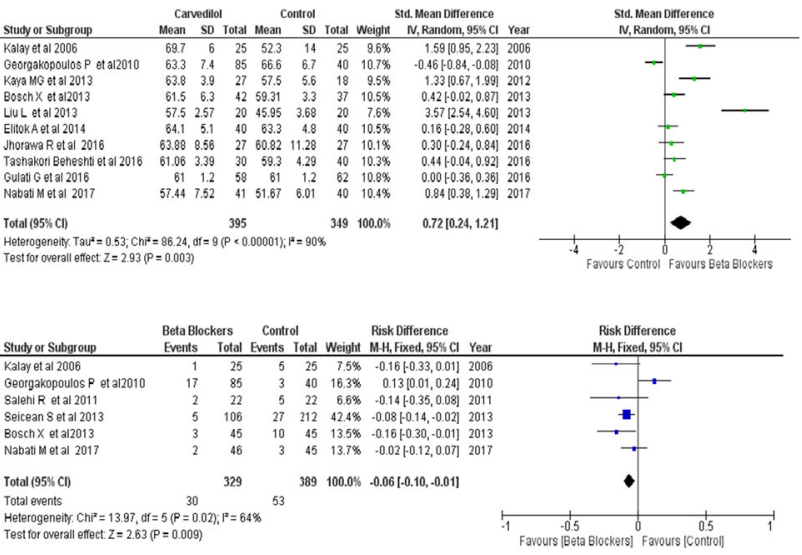

\section{Conclusions}

$\beta$-Blockers are associated with better LVEF preservation and significant lower cardiac events. This analysis provides some support for the routine use of $\beta$-blockers in patients undergoing anthracyclines treatment, especially when we expecting cardiac toxicity with higher accumulative dose.

\section{eP688}

A PATIENT DECISION AID FOR MEN WITH LOCALIZED PROSTATE CANCER: A COMPARATIVE CASE STUDY OF NATURAL IMPLEMENTATION APPROACHES

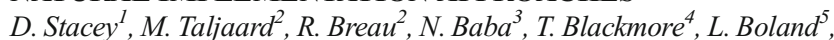

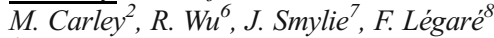

${ }^{1}$ University of Ottawa, School of Nursing, Ottawa, Canada

${ }^{2}$ Ottawa Hospital Research Institute, Clinical Epidemiology Program, Ottawa, Canada

${ }^{3}$ St. Paul's Hospital, Prostate Pathway, Saskatoon, Canada

${ }^{4}$ Saskatchewan Ministry of Health, Quality and Continuous Improvement- Acute and Emergency Services Branch, Regina, Canada

${ }^{5}$ University of Ottawa, Population Health- Faculty of Health Sciences, Ottawa, Canada

${ }^{6}$ University of Minnesota, Department of Surgery- Division of Colon \& Rectal Surgery, Minneapolis, USA

${ }^{7}$ The Ottawa Hospital, Ages Cancer Assessment Clinic, Ottawa, Canada ${ }^{8}$ Université Laval, Research Center $\mathrm{CHU}$ de Québec, Québec City, Canada

\section{Introduction}

There are multiple options for men diagnosed with localized prostate cancer. Patient decision aids (PtDAs) help empower individuals and reduce unwarranted practice variation but few are used in clinical practice. Objectives

We compared two programs implementing PtDAs for men with localized prostate cancer.

\section{Methods}

A comparative case study. Case 1 was a hospital prostate pathway and Case 2 was a provincial prostate pathway with two locations (2a, 2b). Nurses provided men with PtDAs and answered questions. Data sources: a) two years administrative data for men with localized prostate cancer; $b$ ) clinicians survey and interviews; and c) patients/spouses interviews. Analysis was within and across cases.

Results

The PtDA was used with $23 \%$ of men in Case 1 (95\% Confidence Interval (CI) $19.8-26.1 \%)$ and $98 \%$ Case 2 a (95\% CI 96.5-99.8\%). The pathway was not implemented in Case $2 \mathrm{~b}$. Men given the PtDA had positive experiences. Many clinicians supported the use of PtDAs, some adapted 
their discussions with patients, and others did not support the use of PtDAs. To increase use in Case 1, participants identified needing a Canadian PtDA available electronically and endorsed by all clinicians. Case $2 \mathrm{~b}$ needed to implement the provincial prostate pathway.

\section{Conclusions}

There was variable uptake of the PtDAs between the cases. Men who received the PtDA had positive outcomes. Several strategies were identified to increase or sustain PtDA use. Nurses have a key role in supporting men making decisions about prostate cancer treatment by providing PtDAs, answering questions, and advocating for men's preferences.

\section{eP689}

SYMPTOM PRACTICE GUIDES FOR HEALTH PROFESSIONALS TO USE ON THE TELEPHONE OR IN PERSON：EVIDENCE SYNTHESIS FROM THREE IMPLEMENTATION STUDIES

D. Stacey ${ }^{1}$, M. Carley ${ }^{2}$, E. Green ${ }^{3}$, D. Howell ${ }^{4}$, L. Jolicoeur $^{5}$, C. Kuziemsky $^{6}$, C. Ludwig ${ }^{1}$, T. Truant ${ }^{7}$, A. The COSTaRS Team ${ }^{2}$

${ }^{1}$ University of Ottawa, School of Nursing, Ottawa, Canada

${ }^{2}$ Ottawa Hospital Research Institute, Clinical Epidemiology Program, Ottawa, Canada

${ }^{3}$ Canadian Partnership Against Cancer, Person-Centred Perspective, Toronto, Canada

${ }^{4}$ Princess Margaret Hospital, Cancer Centre, Toronto, Canada

${ }^{5}$ The Ottawa Hospital, Cancer Centre, Ottawa, Canada

${ }^{6}$ University of Ottawa, Telfer School of Management, Ottawa, Canada

${ }^{7}$ BC Cancer, Education and Innovation, Vancouver, Canada

\section{Introduction}

The pan-Canadian Oncology Symptom Triage and Remote Support (COSTaRS) team developed 15 practice guides to enhance quality of symptom management. Each guide was based on a systematic review of clinical practice guidelines, met AGREE II rigour criteria, and used plain language.

Objectives

To build an effective and sustainable approach for implementing COSTaRS practice guides for health professionals to use for symptom support to patients undergoing cancer treatments.

Methods

Three implementation studies, guided by the Knowledge to Action Framework, included oncology nurse, homecare nurse, and radiation therapist participants. Teams of researchers and knowledge users: (a) assessed barriers to use; (b) adapted practice guides; (c) intervened to address barriers; (d) monitored use and assessed barriers influencing sustained use. Analysis was within and across studies.

Results

At baseline, participants rated guides positively but barriers were identified (poor awareness, learning curve, lack of time, clarify interprofessional roles). Adaptations included format for health record, add institutional logos, create pocket guide, identify trigger to use. To overcome barriers, we provided training, tailored documentation by location, and integrated into practice. Participants felt more confident with symptom management and using symptom guides post training $(p<.01)$. Chart audits revealed symptom guide use in $11 \%$ to $70 \%$ symptoms documented. Sustained use requires organizational alignment and ongoing leadership support.

\section{Conclusions}

Some increased use of practices guides; participants were encouraged to continue use. Use was higher when patients were flagged to use COSTaRS, practice guides filed on health record or integrated in flowsheets. Practice guides can be adapted for patients and health professionals globally.
eP690

RESEARCH PRIORITIES FOR THE PAN-CANADIAN ONCOLOGY SYMPTOM TRIAGE AND REMOTE SUPPORT (COSTARS) PRACTICE GUIDES: A MODIFIED NOMINAL GROUP CONSENSUS

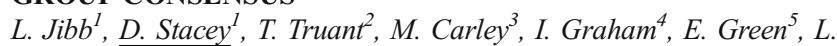
Jolicoeur ${ }^{6}$, C. Kuziemsky ${ }^{7}$, C. Ludwig ${ }^{1}, K$. Thavorn ${ }^{3}$, A. The COSTaRS Team $^{3}$

${ }^{1}$ University of Ottawa, School of Nursing, Ottawa, Canada

${ }^{2} B C$ Cancer, Education and Innovation, Vancouver, Canada

${ }^{3}$ Ottawa Hospital Research Institute, Clinical Epidemiology Program, Ottawa, Canada

${ }^{4}$ University of Ottawa, Faculty of Medicine- School of Epidemiology and Public Health, Ottawa, Canada

${ }^{5}$ Canadian Partnership Against Cancer, Person-Centred Perspective, Toronto, Canada

${ }^{6}$ The Ottawa Hospital, Cancer Centre, Ottawa, Canada

${ }^{7}$ University of Ottawa, Telfer School of Management, Ottawa, Canada

\section{Introduction}

Effective symptom management is a cornerstone of cancer care. To support symptom management, the pan-Canadian Oncology Symptom Triage and Remote Support (COSTaRS) team developed 15 evidenceinformed practice guides. Studies have demonstrated successful implementation in several settings. The next logical step was to determine subsequent research priorities for COSTaRS guides.

Objectives

To identify COSTaRS research questions and to reach consensus on research priorities.

\section{Methods}

A 1-day invitational working meeting. Participants $(n=36)$ included multidisciplinary clinicians from oncology and homecare, managers, cancer care policy makers, researchers, and a patient. Half the day involved presentations and discussion summarizing evidence from four funded COSTaRS studies and experiences with implementing COSTaRS in clinical practice. Participants proposed 5 broad categories for research and research questions within each category. A modified nominal group technique (NGT) involving item generation within small groups, round robin presentation of items, and 2 rounds of voting was used to reach consensus on research priorities.

\section{Results}

Five research priority categories identified were: symptom management throughout the cancer trajectory, measuring COSTaRS outcomes, immunotherapy, expanded use with interprofessional radiotherapy teams, and scaling-up in home care. Across these categories, the top three endorsed priority research items were: (1) how to improve healthcare provider adherence to guide use? (2) establish mobile solutions for real-time symptom management, and (3) adapted practice guides specific to immunotherapy.

\section{Conclusions}

Future COSTaRS research endeavors will be focused on identified priorities, which reflect the needs and perspectives of diverse stakeholders. Continued stakeholder collaboration will guide our research approaches for operationalizing this priority agenda.

\section{eP691}

STRUCTURAL BARRIERS FOR HEALTH CARE PROFESSIONALS TO PROMOTE PHYSICAL ACTIVITY TO CANCER PATIENTS

K. Steindorf ${ }^{1}$, A. Haussmann ${ }^{1}$, N. Ungar ${ }^{2}$, M. Gabrian ${ }^{2}$, A. Tsiouris ${ }^{3}$, M. Sieverding $^{2}, J$. Wiskemann ${ }^{3}$

${ }^{I}$ German Cancer Research Center DKFZ, Physical Activity- Prevention and Cancer, Heidelberg, Germany 
${ }^{2}$ University of Heidelberg, Institute of Psychology, Heidelberg, Germany

${ }^{3}$ University Clinic Heidelberg, Medical Oncology, Heidelberg, Germany Introduction

Despite the known beneficial effects of physical activity (PA) for cancer patients, health care professionals (HCPs) still do not promote PA routinely.

\section{Objectives}

The goal was to investigate whether PA promotion by HCPs (physicians and oncology nurses) depends not only on their attitude but also on external factors, such as structural barriers.

\section{Methods}

In a cross-sectional study, 918 HCPs (287 physicians working in outpatient care, 242 physicians working in inpatient care, and 389 oncology nurses) completed a comprehensive questionnaire. Nine items assessed structural barriers on a 4-point Likert Scale. Structural barriers and HCPs' PA promotion behavior were analyzed using descriptive statistics. Additionally, structural barriers were tested in their prediction on the PA promotion behavior using multiple ordinal regressions, adjusted for sociodemographic variables and HCPs' attitude.

Results

Across professional groups, more than $70 \%$ of HCPs indicated to promote PA to their cancer patients 'often' or 'routinely'. Highest ranked structural barrier was 'too less time per patient' $(M=2.85$; $\mathrm{SD}=1.02$ ), followed by 'lack of structured and reimbursed therapeutic programs' $(\mathrm{M}=2.68 ; \mathrm{SD}=0.94)$. The influence of structural barriers on the PA promotion behavior varied between professional groups: 'Too less time per patient' and 'lack of an expert contact person' was associated with a reduced PA promotion in two professional groups (all p-values less 0.05).

Conclusions

A large proportion of HCPs reported to frequently promote PA. However, our findings suggest that HCPs still perceive structural barriers. The perception and influence of structural barriers differed between professional groups, pointing to the importance to target them specifically.

\section{eP692}

RETROSPECTIVE EVALUATION IN EFFICACY AND SAFETY OF DIRECT ORAL ANTICOAGULANTS FOR TREATMENT OF VENOUS THROMBOEMBOLISM IN CANCER PATIENTS IN THAILAND

L. Suwannoi ${ }^{1}$, S. Chumnumwat ${ }^{1}$, J. Konmun ${ }^{2}$, P. Mitsantisuk ${ }^{3}$

${ }^{T}$ Faculty of Pharmacy Mahidol University, Pharmacy, Bangkok, Thailand

${ }^{2}$ Ramathibodi Hospital, Pharmacy, Bangkok, Thailand

${ }^{3}$ Police Hospital, Pharmacy, Bangkok, Thailand

\section{Introduction}

Venous thromboembolism (VTE) is a common complication in cancer patients and is considered as a major risk of death compared to patients without cancer. The current recommendations for treatment of VTE in malignancy is low molecular weight heparin; however, increased use of direct oral anticoagulants (DOACs) is practiced in clinical setting. Metaanalysis suggests DOACs may be an alternative for VTE treatment in this population; nonetheless, there is limited evidence to support this decision in cancer patients

Objectives

The primary aim of this study was to measure the incidence of VTE recurrences and bleeding among cancer patients who received DOACs that were available in Thailand. Secondary objective was to determine factors associated recurrent VTE and bleeding.

Methods

This is a retrospective cohort study conducted in tertiary care hospitals in Thailand. Data was collected from patients who had active cancer with new diagnosis of VTE.

\section{Results}

There were 32 patients recruited to this study. Among DOACs, rivaroxaban is the most frequent prescribed anticoagulant. We reported 4 cases $(12.5 \%)$ of recurrent VTE at 6-month. The overall bleeding appeared in 10 patients $(31.25 \%)$. Overall mortality rate at 12 months was $21.9 \%$. There was no associated factors to bleeding from our study.

Conclusions

DOACs may be effective to use in treatment of VTE in Thai cancer patients. This study also indicated that Thai patients may be more prone for bleeding than other population. However, robust studies are necessary to establish clinical use

eP693

NIVOMUMAB AS 2ND LINE THERAPY IN PATIENTS (PTS) WITH METASTATIC NON SMALL CELL LUNG CANCER (MNSCLC)

M. Vaslamatzis ${ }^{1}$, M. Pavlakis ${ }^{I}$, T. Tegos ${ }^{1}$, C. Vourlakou ${ }^{2}, N$. Alevizopoulos $^{\text {I, T. Bampouri }}{ }^{3}$, E. Vasili ${ }^{1}$, E. Gioti ${ }^{1}$, S. Benakis ${ }^{4}$, C. Zoublios $^{I}$

${ }^{1}$ Evangelismos General Hospital, Oncology, Athens, Greece

${ }^{2}$ Evangelismos General Hospital, Pathology, Athens, Greece

${ }^{3}$ Santorini Hospital, Public Health, Santorini, Greece

${ }^{4}$ Evangelismos General Hospital, Radiology, Athens, Greece

Introduction

Immunotherapy checkpoint inhibitors seem promising in the $2^{\text {nd }}$ line treatment of mNSCLC.

\section{Objectives}

THE AIM OF THIS STUDY is to record efficacy $\&$ toxicity of $2^{\text {nd }}$ line immunotherapy in mNSCLC after first line platinum-based chemotherapy.

\section{Methods}

Twenty-two pts, consecutively admitted in Our Department between 1/6/2015-31/12/2017, were included. Men/women ratio was $19(86 \%) / 3(14 \%)$ median age 68(47-86)years \& ECOG 1(0-3), stage IIIB 5(23\%) \& IV 17(77\%). Squamous histology had $7(32 \%) \&$ non-squamous $15(68 \%)$, all treated with $1^{\text {st }}$ line platinum-regimens. PD-L1 was tested in all pts. Nivolumab was administered at $3 \mathrm{mg} / \mathrm{kg}$ q2weeks, for 11(3-36)cycles.

Results

Median PD-L1 expression was $60 \%(15-100 \%)$. According to preliminary results: Complete Response(CR) was documented in 1/22(9\%), Partial Response(PR): 4/22(18\%), Stable Disease(SD): 9/22(41\%), PFS: $7(1+-28+)$ months, Non responders(NR's): 8/22(36\%)pts. The patient with CR was a 69 years, non-smoker woman with Stage IV adenocarcinoma, ECOG: 0, \& PD-L1 expression $60 \%$. After minimal PR with $1^{\text {st }}$ line Platinum- she relapsed with lung metastases $\&$ received Nivolumab for 12 cycles. Following 28 months, she remains in CR.

PR's had a median PD-L1 of $40 \%(30-75 \%)$ \& PFS $6(1-$ 36)months, while NR's $20 \%(15 \%-30 \%)$ respectively. Toxicities: No toxic death. Grade 2-3 adverse events: in 10(45\%)pts. Hypophysitis: 1(5\%), Pneumonitis: 7(32\%), Dermatitis: 2(9\%), Enteritis: 2(9\%), biochemical Hepatitis: 10(45\%). Patients treated symptomatically \& continue immunotherapy after a period of 30(15-40)days.

\section{Conclusions}

2nd line Nivolumab in our pts showed: 1) Satisfactory therapeutic results: RR $23 \%$ \& $\mathrm{CR}$ in one, 2) Correlation between PD-L1 levels \& clinical outcome, 3) Previous response to 1 st line platinum-regimens is not predictive of response to 2 nd line immunotherapy, 4) Manageable toxicity. 


\section{eP694}

CANCER-ASSOCIATED THROMBOSIS (CAT): MANAGEMENT OF THROMBOPROPHYLAXIS IN CANCER PATIENTS IN GREECE

N. Tsoukalas ${ }^{I}$, A. Mpokas ${ }^{I}$, A. Christopoulou ${ }^{I}$, A. Ardavanis ${ }^{I}$, G. Koumakis $^{I}$, C. Papandreou ${ }^{I}$, G. Papatsimpas ${ }^{I}$, P. Papakostas ${ }^{I}$, C. Andreadis $^{l}$, G. Aravantinos ${ }^{I}$, N. Ziras $^{I}$, C. Kalofonos ${ }^{1}$, E. Samantas ${ }^{I}$, M. Souggleri ${ }^{1}$, P. Makrantonakis ${ }^{1}$, G. Pentheroudakis ${ }^{1}$, A. Athanasiadis $^{1}$, I. Varthalitis $^{I}$, P. Papakotoulas ${ }^{I}$, I. Boukovinas ${ }^{I}$

${ }^{1}$ Hellenic Society of Medical Oncology HeSMO- http://www.hesmo.gr/ en - Athens- GR, Hellenic Society of Medical Oncology HeSMO- http:// www.hesmo.gr/en - Athens- GR, Athens, Greece

\section{Introduction}

Venous ThromboEmbolism (VTE) is a common cause of adverse outcomes in oncology. The risk of VTE varies with cancer type, stage, grade, therapy and other patients' characteristics.

Objectives

VTE prophylaxis should be provided to hospitalized cancer patients and high-risk outpatients according to Khorana score. Low-molecular-weight heparin (LMWH) remains the standard therapy for VTE in cancer patients.

Methods

This is an observational study conducted by the Hellenic Society of Medical Oncology (HeSMO) that aims to record and highlight the current clinical practice and management of VTE in Greek cancer patients.

Results

The 18 centers reported approximately 4300 cancer patients managed on monthly basis, where the majority (80\%) in outpatient setting. 340 patients with active cancer enrolled in this study: $53,2 \%$ males, mean age 64,3 years, PS 0-1:62,1\%, tumor types lung:22,3\%, pancreas:16,3\%, colon: $13,6 \%$, breast: $11 \%$, stomach:8,3\%, ovarian:6,5\% and other: $21,7 \%$. Moreover, 95,3\% received treatment while $21,3 \%$ were inpatients and 78,6\% outpatients. Among 340 patients, 254 had no VTE and 56,8\% had PS $0-1$. In the cohort of 254 patients, $72,8 \%$ were outpatients, $77,5 \%$ had metastatic disease and $95,7 \%$ were on treatment. Regarding cancer types $66,5 \%$ had high thrombotic risk cancers $(23,2 \%$ lung, $18 \%$ pancreatic, $10,4 \%$ colon and $8 \%$ stomach). Interestingly, the Khorana mean risk in this population was 2 and all patients received prophylactic antithrombotic treatment with LMWH.

Conclusions

Greek Oncologists are aware of Cancer-Associated Thrombosis (CAT), especially in high thrombotic risk cancer types and advanced disease stages. Of interest, the majority of patients who received thomboprophylaxis had good PS and the Khorana mean risk score was 2 .

\section{eP695}

PERIOPERATIVE TEAM THERAPY FOR THORACIC ESOPHARGECTOMY AND PHARYNGOLARYNGECTOMY WITH ESOPHAGECTOMY PATIENTS INCLUDING MANAGEMENT OF DELIRIUM AND NOURISHMENT

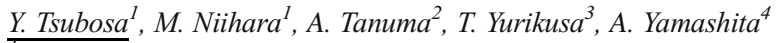

${ }^{T}$ Shizuoka Cancer Center, Division of Esophageal Surgery, Sunto-gun, Japan

${ }^{2}$ Shizuoka Cancer Center, Division of Rehabilitation, Sunto-gun, Japan

${ }^{3}$ Shizuoka Cancer Center, Division of Dentistry and Oral Surgery, Suntogun, Japan

${ }^{4}$ Shizuoka Cancer Center, Division of Nutrition, Sunto-gun, Japan

\section{Introduction}

We have practiced team therapy to prevent postoperative complications. In addition to the conventional practice, we have introduced counterplan for delirium prevention and management of tube-feeding nutrition after discharge, as new actions.

Objectives

Endpoints are the delirium incidence and body weight change.

\section{Methods}

The first of the new actions was prevention of delirium which includes (1) enforcement of abstinence, (2) prohibition of the benzodiazepine receptor agonist drug, (3) use of new type of sleeping agents (Ramelteon and Suvorexant) and (4) facilitation of the conference on delirium. We calculated the delirium incidence before $(n=24)$ and after the preventive action $(n=41)$. The second new action was 3-month tube-feeding management after discharge. We provided the education of maneuvering at-home tubefeeding, and carried out the tube-feeding of $300 \mathrm{kcal} / \mathrm{day}$ for about three months. We measured the body weight of no-feeding group $(\mathrm{n}=36)$ and home tube-feeding group $(\mathrm{n}=24)$.

\section{Results}

The incidences of delirium were $17.7 \%$ and $5.6 \%$ respectively before and after the introduction of the counterplan for delirium prevention. The average weights of the patients before surgery and 3 and 6 months after discharge were $58.8 / 52.1 / 51.5 \mathrm{~kg}$ and $57.2 / 53.4 / 51.8 \mathrm{~kg}$ respectively before and after the introduction of the 3 -month tube-feeding management, and weight reduction rates 3 and 6 months after discharge based on the weight before surgery were $11.4 / 12.4 \%$ and $6.6 / 9.4 \%$ respectively before and after the introduction.

\section{Conclusions}

We introduced counterplan for delirium prevention and nourishment management, as new actions. They are expected to bring profit to esophageal and hypopharyngeal cancer patients.

\section{eP696}

A SYSTEMATIC REVIEW ON THE WAYS THAT FAMILY CAREGIVERS MANAGE SYMPTOMS AND SIDE-EFFECTS OF PEOPLE AFFECTED BY CANCER AT THE HOME SETTING

H. Ullgren ${ }^{1}$, T. Tsitsi ${ }^{2}$, E. Papastavrou3 $3^{3}$, A. Charalambous $^{4}$

${ }^{7}$ Regional Cancer Center- Karolinska University Hospital, Department of Oncology, Stockholm, Sweden

${ }^{2}$ Cyprus University of Technolgoy, Department of Nursing, Limassol, Cyprus

${ }^{3}$ Cyprus University of Tehcnolgoy, Department of Nursing, Limassol, Cyprus

${ }^{4}$ Cyprus University of Technology, Department of Nursing, Limassol, Cyprus

\section{Introduction}

Cancer affects the patients and the whole family, especially when a member of the family assumes the role of the family caregiver. Family Caregivers (FCGs) of cancer patients are actively engaging in multiple tasks, for example coordination and symptom management. The focus of most studies on family caregivers and symptom management in the past, are mainly on FCGs experience and coping, not how or what they do.

\section{Objectives}

The aim of this systematic review was to provide evidence on how FCGs manage symptoms and side-effects at home, in adult cancer patients throughout the disease trajectory.

Methods

A search in PubMed, CINAHL, Web of Science and the Cochrane Central Register of Controlled Trials was performed with a combination of keywords and MeSH terms for informal caregivers, cancer, symptoms, side-effects and management. A total of 1270 articles were screened for inclusion and 20 studies were included in the analysis. A descriptive analysis was performed due to the heterogeneity of the findings. 


\section{Results}

FCGs were doing emotional work, such as supporting and motivating the patient, and physically helping the patients, for example with administration of medicine. Monitoring and assessing symptoms were also frequently reported. Further, FCGs were the decision maker - being alert, watching and waiting and decided when to act and when not to act.

Conclusions

FCGs are doing work similar to healthcare professionals, when managing symptoms and side-effects at home. Advanced tasks such as assessing, monitoring and deciding when and how to act are included in the daily routine of FCGs.

\section{eP697}

\section{ADOPTION OF PATIENT-CENTERED TOOLS BY CANCER CARE TEAMS: A CLOSER LOOK AT SURVIVORSHIP CARE PLANS AND PATIENT DECISION AIDS}

A. Glenn ${ }^{1}$, R. Urquhart ${ }^{2}$

${ }^{1}$ Dalhousie University, Medicine, Halifax, Canada

${ }^{2}$ Dalhousie University, Surgery, Halifax, Canado

\section{Introduction}

Moving new knowledge and tools into clinical practice is often a lengthy and challenging process, even when tools are strongly supported by research evidence. Understanding decision-making around knowledge/tool adoption is paramount to developing more effective strategies to supporting their use in real-world practice.

\section{Objectives}

To (1) illuminate the decision-making processes involved in the adoption of patient-centered tools by cancer care teams, including how research evidence is considered, and (2) identify additional factors influencing these decisions. We focused on two tools (survivorship care plans [SCPs] and patient decision aids [PtDAs]) due to their differing levels of research evidence and real-world adoption (SCPs = low RCT evidence; high adoption; PtDAs = high RCT evidence; low adoption).

\section{Methods}

Guided by the principles of grounded theory, we conducted semistructured interviews with clinicians, managers, and administrators of cancer care programs across Canada $(n=21)$. Data were collected and analysed concurrently, using a constant comparative approach. Data collection ended upon reaching theoretical saturation.

\section{Results}

Participants emphasized high-quality research evidence is often unnecessary when making adoption decisions around tools that are intuitively "good ideas." Six key factors contributed to adoption/non-adoption decisions around SCPs and PtDAs: 1) alignment of research evidence with clinical experiences, patient experiences/preferences, and local data; 2) perceived benefit to clinicians themselves; 3) endorsement by respected organizations; 4) existence of local champions; 5) ability to adapt to local contexts; and 6) ability to routinize across a large patient population. Conclusions

Many factors influence decisions to adopt patient-centered tools, including clinicians' experience and perceived benefits, and ease/reach of implementation.

\section{eP698}

\section{HEALTH LITERACY IN MEXICAN CANCER PATIENTS}

E. Verastegui-aviles ${ }^{I}$, P. González-Garza ${ }^{2}$, S. Allende-Perez ${ }^{2}, D$. Campos-Lopez ${ }^{2}$, A. Pena

${ }^{1}$ instituto nacional de cancerologia, cuidados paliativos, mexico city, Mexico

${ }^{2}$ Instituto Nacional de Cancerologia, palliative care, Mexico City, Mexico

\section{Introduction}

Cancer in Mexico is becoming a major public health problem. The poor impact of government actions to improve screening, and early diagnosis, results in presentation of advanced tumors with poor prognosis of a high percentage of patients. Health literacy (HL) is a construct associated with poor health outcomes. Low HL using different instruments has been reported in the Mexican population living in the US; however, no information is available for Mexico.

\section{Objectives}

The aim of the study was to evaluate the HL at the Instituto Nacional de Cancerologia (INCan).

Methods

Outpatient's patients being treated at INCan were invited to participate. HL was evaluated using the Short Assessment of Health Literacy for Spanish-speaking Adults (SAHLSA). Descriptive statistics, including median and inter-quartile range (IQR), frequencies and proportions (Stata Version 12).

\section{Results}

Four hundred and fifty patients were included. Median age $53.5 \mathrm{y} / \mathrm{o}$ (19$87 \mathrm{y} / \mathrm{o}) ; 60 \%$ females. Patients with all types of cancer were included; however, breast and gynecological cancers (29\%), gastrointestinal tumor (15\%), and Non-Hodgkin Lymphomas (12.4\%), were the most frequent diagnosis. Average HL was $42.8 / 50$, poor HL in $15,4 \%$ of the sample. Patients with the higher HL, had better income, education (college, more than $50 \%$ ) and were younger ( $48.7 \mathrm{y} / \mathrm{o})$. Poor HL was associated with low income, low education, and older age $(\mathrm{OR}=0.97)$. Cronbach's alpha was 0.91

\section{Conclusions}

In contrast to what has been reported in the literature, HL evaluation of patients seen at the INCan is acceptable. Several factors may have influenced these results; therefore, further research needs to be done.

\section{eP699}

DEVELOPMENT OF MICROSOFT ACCESS-BASED SOFTWARE FOR DOCUMENTING SIGNS, SYMPTOMS AND MANAGEMENT OF SUPPORTIVE CARE DELIVERY IN DEVELOPING COUNTRY SET-UP

R. Vinay Kumar ${ }^{1}$

${ }^{T}$ Kidwai Cancer Institute, Radiation Oncology, Bangalore, India

\section{Introduction}

Documentation of data is a problem in developing countries due to resource-constraint and over-crowding of health-care system in developing countries thereby hindering research on supportive care in developing countries. Hence development of supportive care documentation software based on access was need of the hour to document supportive care delivery and its effectiveness.

\section{Objectives}

Software development for recording of supportive care delivery in our set-up was aimed to supplement record-based documentation to facilitate analysis of data with respect to effectiveness of supportive care delivery and cost of the same.

\section{Methods}

Paper-based tool was developed for each of the anatomic region of body with signs and symptoms unique to that region of the body. Subsequently connected table and drop down list in microsoft access was prepared in order to ease the recording of symptoms, signs, investigations, treatment and response to treatment.

\section{Results}

Everyday recording of patients' supportive care issues, remedies and response was feasible and can substitute the need for paper-based documentation to collect and analyse the patients' data w.r.t to effectiveness of intervention and cost of intervention in our set-up. 


\section{Conclusions}

Microsoft access based supportive care data recording tool is effective in documenting, preserving and analyzing patients' data that was impossible in our set-up due to non-traceable patients' records

eP700

THE EXPERIENCE OF UNDERGRADUATE NURSING STUDENTS TOWARD PATIENTS' DEATH: A QUALITATIVE STUDY IN MAINLAND CHINA

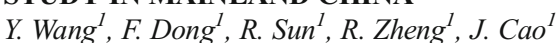

${ }^{T}$ Tianjin Medical University Cancer Institute and Hospital, Nursing Department, Tianjin, China

\section{Introduction}

Death education has gradually received global attention of nursing educators and researchers for decades, and it has largely promoted reforms of death-related education, training and programs. however, little is known about the experience and perspective of nursing students regarding the death of patients in China.

\section{Objectives}

To elucidate undergraduate nursing students' experience with patient death in Chinese cultural context.

Methods

Sixteen undergraduate students experiencing patients' death from three nursing universities were in-depth interviewed face-to-face with a semistructured interview guide. Thematic analysis was used to analyze the data.

\section{Results}

Four main themes emerged from the data: complex personal experiences, lacking of expertise and knowledge, coping strategies with patient death and impact of patients death on undergraduate students. Nursing students had intensive emotional reactions to patient death, such as fear, sadness and helplessness. Most students reported there was a huge difference between school education on death and clinical practice. Besides that, some stated that they were inadequate in end-of-life care issues, but hoped to get more chances of taking care of dying patients and assisting with body care. In addition, the nurses got personal and professional growth but most of them were left alone to cope with patient death.

\section{Conclusions}

Chinese undergraduate nursing students are challenged by care of dying patients. Lack of knowledge and skill was highlighted by this study in addition to the emotional context of care. Recommendations include the development of teaching and learning strategies and programs of support in addition to further research related to knowledge and skill development

\section{eP701}

\section{THE EFFECT OF CUSTOMER EXPERIENCE MANAGEMENT ON CANCER PATIENT JOURNEY}

Y.H. Won ${ }^{1}$, K. Jeong Hye $e^{2}$ K. Ji Hye ${ }^{3}$, K. Yeon Hee

${ }^{T}$ Asan Medical Center, 72 WardWest building, 05505, Republic of Korea

${ }^{2}$ University of Ulsan, Clinical Nursing, Seoul, Republic of Korea

${ }^{3}$ Asan Medical Center, 72 ward-Nursing, Seoul, Republic of Korea

${ }^{4}$ Asan Medical Center, Nursing, Seoul, Republic of Korea

\section{Introduction}

The customer experience is that oncology nurse is accompanied directly with the cancer patients and experiences the patient's position, including the admission, examination, surgery, chemotherapy and radiation therapy, Also, the high empathy ability of oncology nurse is known to have a positive effect on the recovery of cancer patient's health.

\section{Objectives}

The purpose of this study was to investigate the effect of customer experience management on nursing service satisfaction of cancer patients and their intent to revisit the hospital and also to identify empathy ability of oncology nurses.

\section{Methods}

Participants were 376 cancer patients and 190 oncology nurses from a tertiary hospital in South Korea. Cancer patients' satisfaction with nursing care and intent to continue using the hospital of were measured and empathy of oncology nurses was measured using Empathy Construct Rating Scale. Data were analyzed using descriptive statistics, $\chi^{2}$ test,t-test, one-way ANOVA and stepwise multiple regression analysis.

\section{Results}

Patient satisfaction with nursing care and intent to continue using the hospital were higher in patients who received the customer experience management program than those who did not. Oncology nurses' ability to empathize was high. Clinical career, participate in customer experience management and education level together explained $20 \%$ of variance in empathy ability of oncology nurses.

\section{Conclusions}

Findings from this study confirm that customer experience management affects patient satisfaction with nursing service and intent to continue using the hospital. Further research is necessary to reaffirm the empathy ability of the oncology nurses and to identify the related various factors.

\section{eP702}

\section{EMBELLISHING THE TOKUHASHI SCORE IN THE ERA OF NEWER SYSTEMIC THERAPIES}

V. Wong ${ }^{1}$, D. Quah ${ }^{2}$

${ }^{T}$ National Cancer Centre Singapore, Division of Palliative Medicine, Singapore, Singapore

${ }^{2}$ National Cancer Centre Singapore, Division of Radiation oncology and Division of Palliative Medicine, Singapore, Singapore

\section{Introduction}

The Tokuhashi Score is used to prognosticate patients with spine metastases and to stratify these patients to appropriate interventions. One parameter used in this score is the histology of lung cancer which in the original system resulted in an overall poor score and bad prognosis. However, in today's era of targeted therapies, patients with lung cancer can expect a long survival.

\section{Objectives}

Exploring if a modification of the Tokuhashi score is feasible by stratifying lung cancer patients according to their histology.

\section{Methods}

Retrospective case notes review of all patients with spine metastases undergoing radiotherapy from 2010 to 2014 were reviewed. Various parameters were explored, notably the histology and biochemical markers. These parameters were then correlated with their Tokuhashi score and their acutal survival.

\section{Results}

Patients with adenocarcinoma and mutations in Epidermal Growth Factor Receptors have a better prognosis. Final results will be presented at the conference.

\section{Conclusions}

The Tokuhashi score should be updated to reflect the better prognosis conferred by newer systemic therapies. 
eP703

\section{IMIQUIMOD ATTENUATES RADIATION-INDUCED PULMONARY FIBROSIS}

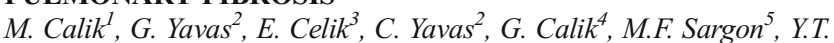
Sanli ${ }^{6}$, H. Esme

${ }^{1}$ Konya Training and Research Hospital, Department of Thoracic Surgery, Konya, Turkey

${ }^{2}$ Selcuk University, Radiation Oncology, Konya, Turkey

${ }^{3}$ Selcuk University, Department of Pathology, Konya, Turkey

${ }^{4}$ Necmettin Erbakan University, Department of Emergency Medicine, Konya, Turkey

${ }^{5}$ Hacettepe University, Department of Anatomy, Ankara, Turkey

${ }^{6}$ Onko Mersin Oncology Center, Department of Radiation Oncology, Mersin, Turkey

\section{Introduction}

Radiation-induced lung injury (RILI) is a major dose limiting factor during thoracic irradiation.Imiquimod (I) stimulates innate and adaptive immune pathways and induces cytokine production.

Objectives

We aimed to evaluate the impact of I RILI.

Methods

Sixty rats were divided into 6 groups: Group $(\mathrm{G}) 1$ control, G2 radiotherapy (RT) only, G3 and G4 5 and $10 \mathrm{mg} / \mathrm{kg} \mathrm{I}$; G5 and G6 RT plus 5 and 10 $\mathrm{mg} / \mathrm{kg}$ I groups respectively. A single dose of 15 Gy RT was given to lungs. I was applied intraperitoneally with daily doses, until animals were sacrificed 6 and 16 weeks after RT. Lung tissues were dissected for light and electron microscopy.

Results

The inflammation, fibrosis and transforming growth factor (TGF)- $\beta$ scores of study groups were significantly different at $6^{\text {th }}$ and $16^{\text {th }}$ week of RT ( $\mathrm{p}<0.001$ for all). At $6^{\text {th }}$ week of RT, pair-wise comparisons revealed that inflammation, fibrosis and TGF- $\beta$ scores of did not different in both RT and non-RT groups. By $16^{\text {th }}$ week of RT inflammation, fibrosis and TGF- $\beta$ scores were significantly different between G2 and G5 (p values were $0.033,0.041$, and $<0.001$ respectively), and G2 and G6 (p values were $0.043,0.002$, and $<0.001$ respectively). By $16^{\text {th }}$ week, there were vacuoles in alveolar epithelial cells and inter/pericellular edema in all RT groups. The vacuoles were larger and intercellular edema was more prominent in G2 than both G5 and G6; however there weren't any difference between G5 and G6.

Conclusions

Although I did not improve pneumonitis phase, I attenuated radiationinduced lung fibrosis.

\section{eP704}

MALIGNANT MELANOMA OF THE VAGINA: LONG-TERM REMISSION FOLLOWING RADIATION THERAPY

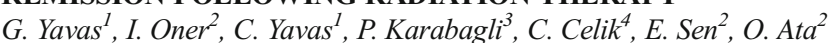

${ }^{T}$ Selcuk University, Radiation Oncology, Konya, Turkey

${ }^{2}$ Selcuk University, Medical Oncology, Konya, Turkey

${ }^{3}$ Selcuk University, Department of Pathology, Konya, Turkey

${ }^{4}$ Selcuk University, Department of Obstetrics and Gynecology-Division of Gynecologic Oncology, Konya, Turkey

\section{Introduction}

Vaginal malignant melanoma (VMM) is a rare form of mucosal melanoma, and accounts for only $<1 \%$ of all melanomas. The prognosis of VMM is poor, and the 5-year overall survival rate is $0-25 \%$. Since patients with VMM ultimately develop distant metastases regardless of primary treatment approach; patient preference and quality-of-life considerations are critical factors in determining initial management.

\section{Objectives}

We reported two cases of primary VMM who undergone curative radiotherapy

\section{Methods}

The first case was stage T4bN0M0 VMM who denied surgery therefore underwent definitive radiotherapy (External beam radiotherapy (EBRT) (pelvis+bilateral groin chains and vagina $50 \mathrm{~Gy} / 25$ fraction and vaginal brachytherapy ( $28 \mathrm{~Gy} / 4$ fractions). The second case was T4bN1M0 and underwent wide local excision with vaginectomy and bilateral inguinal lymph node dissection followed by adjuvant radiotherapy (50.4 Gy EBRT to the primary tumor and bilateral inguinal lymph nodes) and immunotherapy (30 MU interferon- $\alpha$ for 2 years).

\section{Results}

There weren't any unwanted treatment break. Both cases had acute grade 2 cystitis and diarrhea in addition to grade $2-3$ dermatitis which responded to medical treatments. The patients are still free of disease after 13 and 34 months of follow-up respectively.

\section{Conclusions}

There is no consensus regarding to optimal treatment modality of VMM. Although there is not enough evidence supporting an aggressive surgery, initial surgery seems to be the best option. Conservative surgery with adjuvant therapies including immunotherapy, chemotherapy and radiotherapy may be reasonable. Further studies with more patients and longer follow-up period are warranted in order to define the best treatment strategy.

\section{eP705}

RISK STRATIFICATION OF P16-NEGATIVE HIGH-RISK SQUAMOUS CELL CARCINOMA OF THE OROPHARYNX USING IMAGE HETEROGENEITY AND IRREGULARITY OF PRETREATMENT 18F-FLUORODEOXYGLUCOSE POSITRON EMISSION TOMOGRAPHY

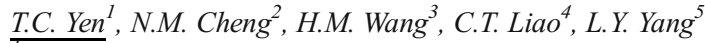

${ }^{T}$ Linkou Chang Gung Memorial Hospital and Chang Gung University, Nuclear Medicine, Taoyuan, Taiwan R.O.C.

${ }^{2}$ Chang Gung Memorial Hospital- Keelung, Nuclear Medicine, Keelung, Taiwan R.O.C.

${ }^{3}$ Chang Gung Memorial Hospital- Chang Gung University, Hematology/ Oncology, Taoyuan, Taiwan R.O.C.

${ }^{4}$ Chang Gung Memorial Hospital- Chang, Otolaryngology- Head \& Neck Surgery, Taoyuan, Taiwan R.O.C.

${ }^{5}$ Chang Gung Memorial Hospital, Biostatistics Unit- Clinical Trial Centre, Taoyuan, Taiwan R.O.C.

\section{Introduction}

Human papillomavirus (HPV)-negative oropharyngeal squamous cell carcinoma (OPSCC) has worse survival. Two groups with different outcomes have been identified based on smoking history and tumour stage. Objectives

We investigated the prognostic roles of image features of pretreatment ${ }^{18}$ F-fluorodeoxyglucose (FDG) PET in high-risk OPSCC patients who completed chemoradiotherapy.

\section{Methods}

We compared PET parameters derived from two segmentation methods: $42 \%$ of the maximum SUV (T42) and fixed value of SUV 2.5, to identify indices with high reproducibility. Disease-specific survival (DSS) and progression-free survival (PFS) served as outcome measures and were investigated with Kaplan-Meier and multivariate Cox proportional analyses.

Results

110 patients were enrolled.High reproducible indices, SUV-entropy, asphericity and high grey-level zone emphasis (HGZE), significantly associated with DSS and PFS. A scoring system based on those parameters were derived using T42 data. The worst survivals were observed for 
patients with both high asphericity and SUV-entropy. The c-statistics of the scoring system for DSS and PFS were 0.76 and 0.77 , respectively, which were significantly higher than AJCC stage and metabolic tumour volume. The prognostic impact of the scoring system was further validated using fixed SUV 2.5 data.

A

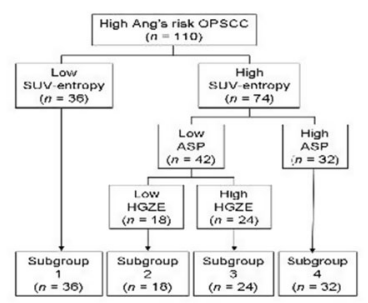

B

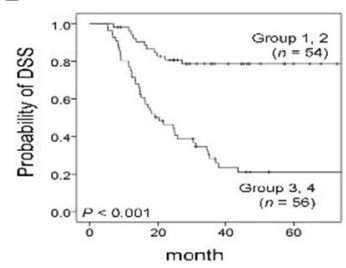

C

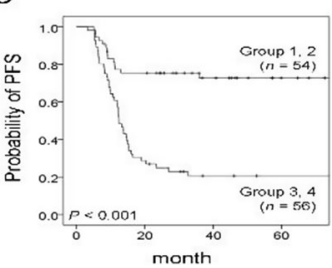

A

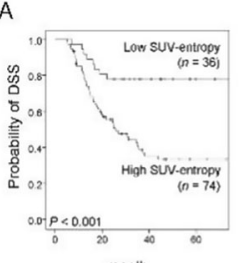

B

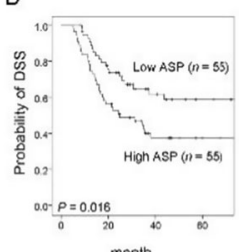

C

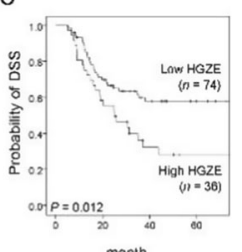

D
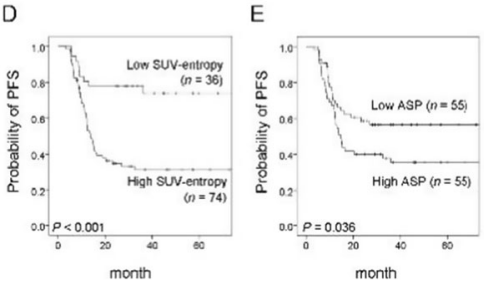

$\mathrm{F}$

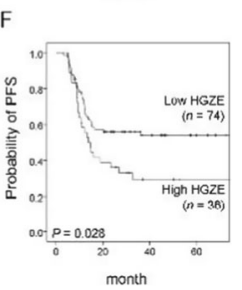

\section{Conclusions}

We concluded that SUV-entropy, asphericity and HGZE may improve the prognostic stratification of the high-risk OPSCC patients.

\section{eP706}

ORAL OPTION FOR THE TREATMENT OF VENOUS THROMBOEMBOLISM IN CANCER PATIENTS?

A. Young ${ }^{1}$, A. Marshall ${ }^{1}$, J. Thirwall ${ }^{1}$, O. Chapman ${ }^{2}$, A. Lokare ${ }^{3}$, C. Hill ${ }^{1}$,

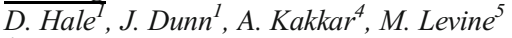

${ }^{1}$ University of Warwick, Warwick Medical School, Coventry, United Kingdom

${ }^{2}$ University Hospitals Coventry and Warwickshire NHS Trust, Haematology, Coventry, United Kingdom

${ }^{3}$ Heart of England NHS Foundation Trust, Haematology, Birmingham, United Kingdom

${ }^{4}$ Thrombosis Research Institute, Director, London, United Kingdom

${ }^{5}$ McMaster University, Oncology, Hamilton, Canada

\section{Introduction}

Cancer patients with acute venous thromboembolism (VTE) are at increased risk of recurrent thrombosis and anticoagulant-associated bleeding compared to non-cancer patients with VTE.

\section{Objectives}

The primary aim was to assess VTE recurrence in cancer patients treated with rivaroxaban or dalteparin. Secondary objectives were to assess bleeding and to assess extended anticoagulation beyond 6 months.

Methods

A randomised open-label pilot trial was conducted. Cancer patients with symptomatic or incidental $\mathrm{PE}$, or symptomatic proximal leg DVT were allocated to dalteparin (CLOT study doses) or rivaroxaban (licenced doses) for 6 months. Patients at higher risk of VTE could then be randomised to placebo or rivaroxaban for a further 6 months. The primary outcome was VTE recurrence. Secondary outcomes included major bleeds and clinically relevant non-major bleeds (CRNMB).

\section{Results}

406 patients were recruited over three years from 58 UK sites. Patient characteristics: median age 67 years (range 22-87); $53 \%$ males; $58 \%$ with metastases; $48 \%$ with symptomatic PE or DVT, and $52 \%$ with incidental PE. The 6-month cumulative VTE recurrence rate was $11 \%(95 \%$ CI $7-16 \%)$ for dalteparin and $4 \%(95 \%$ CI 2-9\%) for rivaroxaban (Figure 1). The 6month cumulative rate of major bleeding was $4 \%$ (95\% CI 2$8 \%)$ for dalteparin and $6 \%(95 \%$ CI $3-11 \%)$ for rivaroxaban. The corresponding rates of CRNMB were 4\% (95\% CI 2-9\%) and $13 \%$ (95\% CI 9-19\%).

\section{Conclusions}

In terms of individualised care for treatment of VTE, a careful discussion should occur between the patient and the clinician concerning the balance of the risks of recurrence and bleeding.

\section{W001}

\section{"DO"S AND "DO NOT"S IN PLANNING A CLINICAL TRIAL FOR GI MUCOSITIS \\ D. Keefe ${ }^{I}$ \\ ${ }^{1}$ University of Adelaide, Medicine, Adelaide, Australia}

In 2018, one would imagine that designing a clinical trial for GI mucositis should be pretty straight-forward; that one could just Google the 'how-to' manual, and up would pop the answer. Sadly, this is not the case. There are many considerations to be taken into account, and some key ones are listed below:

1. What question are we trying to answer?

2. Are we studying Radiation versus Systemic treatment, and why?

3. Chemotherapy versus tyrosine kinase inhibitor versus other targeted therapy versus immunotherapy?

4. Which patient population?'

5. Are we studying a particular cancer or a particular chemotherapy regimen?

6.What are the best regimens and therefore the best cancers?

7. Is the intervention used as prevention or treatment or both?

8. What trial design and which endpoints?

9.Inclusion and exclusion criteria and rationale?

And then there is the issue of how to interpret and present the results, and whether they actually answer the question we wanted to answer. It may be worth mocking up some results of the proposed trial and asking whether they have done what you intended. There are many trials in GI mucositis that have failed because these issues haven't been properly thought through. Ways to deal with these problems will be discussed. 


\section{W002}

\section{PREDICTING THE UNPREDICTABLE: MOVING FORWARD WITH PROGNOSTICATION RESEARCH}

\section{D. $\mathrm{Hui}^{1}$}

${ }^{T}$ University of Texas MD Anderson Cancer Center, Palliative Care, Houston, USA

Over the past 5 decades, palliative care has evolved from serving patients at the end-of-life to a highly specialized profession to delivering supportive care to patients with life-limiting illnesses throughout the disease trajectory. There is now a growing body of evidence to inform the key domains in the practice of palliative care, including symptom management, psychosocial care, communication, decision making, and end-of-life care. One of the most important roles of palliative care is to facilitate serious illness conversations to facilitate complex decision making, especially when patients are approaching the end-of-life. In the past few years, there has been a tremendous amount of new literature on this topic, demonstrating improved outcomes with prognostic discussions and care planning. In this presentation, we will review emerging prognostication tools to facilitate clinical practice, including (1) novel prognostic markers, (2) machine learning approaches, (3) internet based prognostic calculators, and (4) decision making tools. We will also discuss the need to standardize statistical analysis and reporting to move the field of prognostication forward.

\section{W003}

WHAT ABOUT CARERS?

Y. Kim $^{l}$

${ }^{T}$ University of Miami, Psychology, Coral Gables, USA

A substantial number of family caregivers go through bereavement due to cancer, but little is known about the effects of communications on bereaved caregivers' long-term adjustment. Despite the fact that death from cancer is statistically common, family members often encounter the death as an unexpected event. Caregivers who reported they were relatively unprepared have shown poor bereavement outcomes, in both elevated depressive symptoms and bereavement-specific distress, such as prolonged grief symptoms. A few studies have documented that caregivers who report that health care professionals managed the patients' pain and physical ailments well, provided clear information about the patients' prognosis, and were available for the family caregivers prior to the death of the patient have reported better adjustment during end-of-life care and bereavement. Key components of effective communications that help family members prepare for impending death of their loved one, which would also be associated with better longer term adjustment to bereavement, will be discussed. 


\section{Abstracts of the MASCC/ISOO Annual Meeting 2018}

Supportive Care
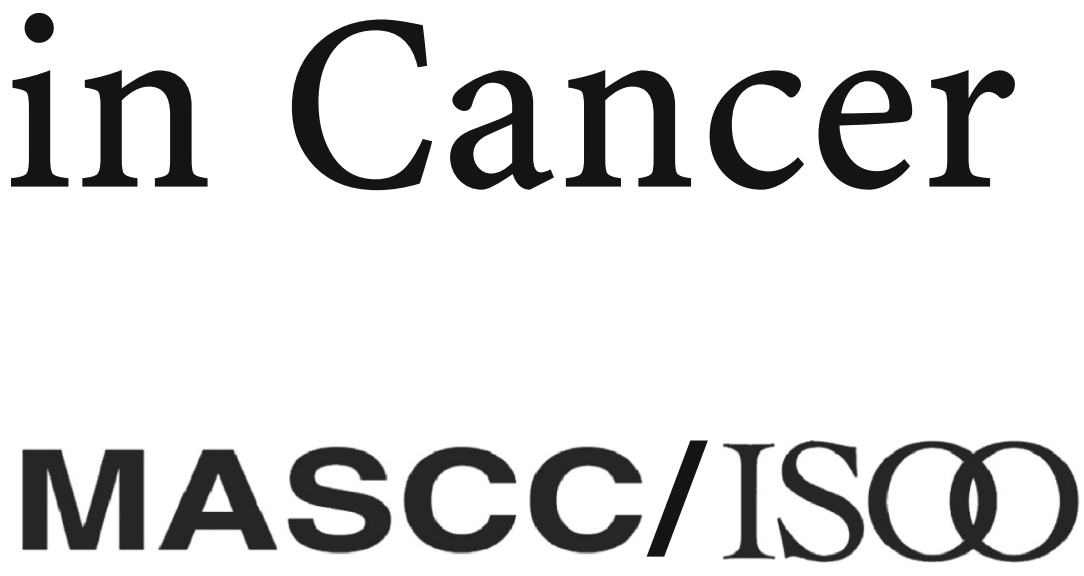

\section{ANNUAL MEETING ON SUPPORTIVE CARE IN CANCER \\ Vienna, Austria \\ 28-30 June 2018}
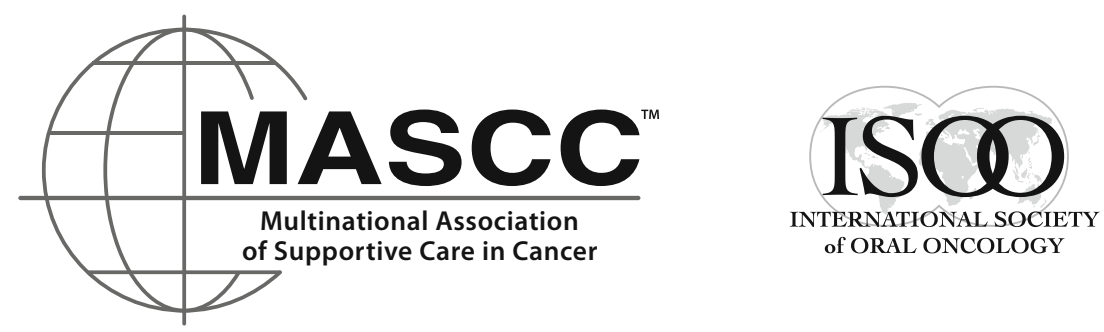

Supportive Care Makes Excellent Cancer Care Possible

This supplement was not sponsored by outside commercial interests. It was funded entirely by the publiher and the Societies 


\section{All authors}

Aaronson- $\mathrm{PhD}, \mathrm{N}$.

Abalo, R.

abbaszadeh, A.

abdeddaim, $\mathrm{C}$.

Abdelmegeed, D.

Abdelrahman, S.

ABDELSAMIE, $\mathrm{S}$.

Abdi, S.

\section{Presentation No.}

PS055

eP223

eP308, eP361

eP073

eP168

eP282

eP168

Abdul Azizi, A.F.

eP056

Abila, D.B.

eP157

Abildgaard, N.

eP477

Abrahm, J.

eP549

Abu Farsakh, F.

eP303

Abu Nasser, M.

eP526

eP526

Acharya, A.

eP489

Acharya, J.K.

eP304

Adam, S.

PS011

Adegboyega, B.

eP492

Adha, A.K.

eP622

Aditya, M.

eP037

Adnet, J.

eP507

Adonizio, $\mathrm{C}$.

eP327

Agajanian, R.

PS026

Agar, M.

eP062, eP320

Agarwal, E.

eP239

Agarwal, R.P.

eP304

Agasi-Idenburg- PT.- MSc, C. PS055

Aggarwal, G.

eP347

Aghmesheh, M.

eP008

Agulnik, J.

eP499

Aharon-Peretz, J.

eP211

Ahlgren, J.

eP558

Ahmed-Lecheheb, D.

eP576

Ahmed, M.

eP519

Ahmed, N.

eP566

Ahmed, S.

Ahmedzai, S.

eP451

eP566

eP640

eP643

eP640

Ahn, J.B.

eP526

Ajarmeh, S.

eP361

akbari sari, A

eP308, eP361

eP308

akbarisari, A.

eP483

Akbulut, $\mathrm{H}$.

eP386, eP409

Akgün Çıtak, E.

eP647

Akiko, T

eP647

Akira, F.

eP559

Akira, I.

eP091
Akyürek, S.

Al Haddad, A.

Al-Dasooqi, N.

Al-Hawamdeh, A.

Al-Omari, M.

Al-Rimawi, D.

Al-Sayed, T.

Al-Tabba', A.

ala dien, Y.

Alananzeh, I.

Alansari, A.

Alcantara, B.

Aldiss, S.

Alegria, J.

Alen, R.

Alessio, M.

Alevizopoulos, N.

Alexandra, $\mathrm{M}$.

Alexandre, J.

Alexandria Pinto de

Magalhães, P.

Ali, A.

Ali, N.

Ali, R.

Alibhai, S.

Alisa, K.

Alkan, A.

Alkebro, I.

Allam, A.

Allende-Pérez, S.

Allende, S.

Allgar, V.

Allsop, D.

Alm, M.

Aloia, T.

Alonso Reis, A.P.

Alparslan Atar, Ö.

Alqahtani, Q.M.

alsuhail, A.

Alves, B.

Alves, F.A

Alves, $\mathrm{P}$.

Amadori, F.

Amaral Mendes, R.

Amari, D.T.

Ameringer, $\mathrm{S}$.

Amin, Z.

Ammar, K

Anandarajah, G.

Anandi, P.

Anastasov, V.

Andersen, K.K.

Anderson, C.

Anderson, D.

Andrade, C.

Andrade, R.

André, $\mathrm{F}$.

Andreadis, C.

Angellier, E.

Angosta, J.

Anjum, S.

Anne, T.

Ansari, M.

Ansorge, S.
eP247

eP282

eP197

eP526

eP526

eP526

$\mathrm{PS} 025$

eP526

eP029

eP478

eP038

eP142

PS073

eP674, eP675

eP448

eP273

eP261, eP693

eP245

eP576

eP414, eP665

eP479, eP125, eP519

eP315

eP032

PS057

eP388

eP387

eP558

eP405

eP357, eP431, eP568, eP698, PS006, eP346, eP430

eP257, eP674, eP675

eP042, eP043

eP008

eP601

eP494

eP414, eP665

eP409

eP134

eP039, eP567

eP142

eP467

eP397

eP169, eP454

eP265

eP017

PS021

eP526

eP526

eP092, eP305, eP306

eP569

eP631

eP548

PS037

eP239, eP432

eP270

eP089, eP629

eP507

eP694

eP307

eP345

eP297

eP583

eP308, eP361

PS051 


\begin{tabular}{|c|c|c|c|}
\hline Anthony, L. & eP542 & Bachour, P. & eP171 \\
\hline Antic, D. & eP459 & Bachtold, S. & eP058 \\
\hline Antoni, M.H. & eP442 & Bacoanu, G. & $\mathrm{eP} 360$ \\
\hline Antoniou, F. & PS053 & Bae, D.S. & eP012 \\
\hline Antonuzzo, A. & eP235, eP236, eP237, eP682 & Bager, T. & eP626 \\
\hline Antunes, H.S. & eP170, eP266, eP270, eP271 & Bahar, Z. & $\mathrm{eP} 480$ \\
\hline Aoyama, T. & eP240, PS064 & Bai, J. & eP045 \\
\hline Appleton, R. & eP566 & Baid, J. & eP563 \\
\hline Arai, $\mathrm{H}$. & eP214, eP240 & Baillie, J. & eP376 \\
\hline Arai, I. & eP084 & Baird, J. & eP607 \\
\hline Arany, P. & $\mathrm{eP} 070$ & Bajic, J. & eP172, eP210 \\
\hline Araújo, C.M.M. & eP170 & Bakic, N. & eP095 \\
\hline Aravantinos, G. & eP694 & Bakouny, Z. & eP241, eP311 \\
\hline Araw, A.M. & eP184 & Balaam, S. & eP239, eP432 \\
\hline Arboleda, M.F. & PS031, PS034 & Balaji, K. & $\mathrm{eP} 447$ \\
\hline Arce-Salinas, C. & eP346, PS006 & BalajiSubramanian, S. & $\mathrm{eP} 447$ \\
\hline Arce-Salinas, C.H. & eP568, eP668 & Balasubramanian, A. & $\mathrm{eP} 455$ \\
\hline Ardavanis, A. & eP694, PS053 & Balcı Alparslan, G. & eP086 \\
\hline Arena, $\mathrm{F}$. & eP259 & Balik, A. & $\mathrm{eP} 267$ \\
\hline Argiles, J.M. & PS035 & Ball, I. & eP175, eP205, eP468, eP469, PS080 \\
\hline Ariello, K. & eP068 & Ball, I.A. & PS039 \\
\hline Arif, T. & PS028 & balti, M. & eP229, eP302 \\
\hline Arikan, F. & eP543 & Bambury, R. & PS069 \\
\hline Arluck, J. & eP045 & Bampouri, T. & eP261, eP693 \\
\hline Armstrong- Ph.D., M.L. & eP420 & Bana, M. & eP096 \\
\hline Armstrong, G. & PS082 & Bandla, A. & PS050 \\
\hline Arndt, V. & eP491, eP612, eP616, PS011 & Bando, E. & eP160 \\
\hline Aronowitz, T. & eP286 & Bando, $\mathrm{H}$. & eP452 \\
\hline Arora, R.D. & eP040, eP093, eP309, eP310 & Banerjee, $\mathrm{S}$. & $\mathrm{eP} 010$ \\
\hline Arrais Rodrigues-Silva, C. & eP198 & Bansal, S. & eP552 \\
\hline Arranz, J. & eP067 & Bar-Sela, G. & eP211, PS032 \\
\hline Arslan, Y. & $\mathrm{eP} 247$ & Barakat, A.O. & eP179 \\
\hline Arthur, J. & eP338 & Barasch, A. & PS038 \\
\hline Arua, S. & eP515 & Barber, C. & eP203 \\
\hline Arzate-Mireles, C. & eP346, eP568, PS006 & Barberet, C. & eP159 \\
\hline Asaba, K. & eP094 & Bardellini, E. & eP169, eP454 \\
\hline Asare, M. & PS043 & Barillari, M. & $\mathrm{eP} 276$ \\
\hline Ascencio-Huertas, L. & $\mathrm{eP} 430$ & Barnes, E. & $\mathrm{eP} 251$ \\
\hline Ashbury, F. & PS059 & Barni, S. & eP071 \\
\hline Asribabayan, Y. & eP388 & Barrett, J. & eP569 \\
\hline Assi, T. & eP311, eP241 & Barrett, M. & eP135, eP136, eP243, eP262 \\
\hline Assunção Caetano de Loyola, & eP414 & Barry, A. & $\mathrm{eP} 224$ \\
\hline E. & & Barry, C. & eP496 \\
\hline Asthana, R. & eP209 & Barton-Burke, M. & eP334 \\
\hline Astorga, E. & eP544 & Bartsch, R. & PS077 \\
\hline Ata, O. & eP619, eP704 & Basford, J. & eP547 \\
\hline Atasavun Uysal, S. & eP540 & Bashir, A. & $\mathrm{eP} 405$ \\
\hline Athanasiadis, A. & eP694 & Bastholt, L. & $\mathrm{eP} 466$ \\
\hline Atherton, P.J. & eP400 & Bataglion, C. & $\mathrm{eP} 276$ \\
\hline Atmaca Palazoğlu, C. & $\mathrm{eP} 411$ & Batten, L. & $\mathrm{eP} 604$ \\
\hline Augusta Novis, Y. & eP198 & Battersby, M. & eP595 \\
\hline Austin, A. & eP184 & Battiwalla, M. & eP569 \\
\hline Austin, T. & PS040 & Bautista, J. & $\mathrm{eP} 145$ \\
\hline Avogadri-Connors, F. & eP468, eP469, PS080 & Baylot, C. & eP678 \\
\hline Aya, F. & eP230 & Beaumont, J. & eP503 \\
\hline ayari, J. & eP229, eP302 & Beh, S.Y. & PS018, PS020 \\
\hline Azhar, A. & eP332 & Behm, B. & eP144 \\
\hline Azimi-Nekoo, E. & eP004 & Beijer, S. & eP130, PS084 \\
\hline Aziz, A.F. & eP597 & Beitler, J. & PS019 \\
\hline Baba, M. & eP242 & Bektaș-Kayhan, K. & eP267, eP035, eP027 \\
\hline Baba, N. & eP688 & Belhadj, Y. & eP395 \\
\hline Babovic, N. & eP458 & Belinha, J. & eP024, eP025 \\
\hline Babu, S.K.V. & eP644 & ben nasr, S. & eP229, eP302 \\
\hline Baca, E. & eP267 & Benakis, $\mathrm{S}$. & eP693 \\
\hline Bachani, D. & eP489 & Bender, J. & PS012 \\
\hline
\end{tabular}


Benhalla, K.N.

Bennett, $\mathrm{M}$.

Bensing, J.

Bentner, M.

Benzi, L.

Bergelt, C.

Berger, A.

Berger, K.

Bergmann, O.J.

Beriwal, S.

Berman, R.

Bernard, A.

Bernardi, M.

Bernareggi, A.

Bernier, C.

Bertetto, O.

Berton-Rigaud, D.

Bertram, $\mathrm{H}$.

Beutler, A.

Bezinelli, L.M.

Bharadwaj, J.

Bhasker, S.

Bhat, G.

Bhatnagar, S.

Bhattacharya, B.

Bhoo Pathy, N.

Bhoo Pathy, N.T.

Bhoo-Pathy, N.

Bhoo-Pathy, N.T.

Biancotto, A.

Biasco, E.

Biasotto, M.

Bibault, J.E.

Bidstrup, $P$.

Bigatti, S.

Bila, J.

Bilal, D.

Bilsborough, R.

Binder, G.

Bird, R.

Birnbaum, M.

Bisog, A.

Biswas, A.

Biswas, S.

Björkqvist, J.

Black, A.

Blackmore, T.

Blanchard, G.

blazkova, S.

Blijlevens, N.

Blijlevens, N.M.A.

Bloise, F.

Blomqvist, C.

Bluethmann, S.

Boakye, D.

Boanca, M.

boaventura da silva, Y.

Bobo, $\mathrm{H}$.

Bobrovskaya, L.

Boddy, J.

Boden, A.

Bødker, J.S.

Bodner, W.

Bødtcher, H.

Body, J.J.
eP283

eP042

eP418, PS075

eP080

eP273

eP407

eP137, eP144

eP173

eP190

eP529

eP341, PS025

PS045

eP481

eP312

eP149

eP030, eP399

eP576

eP491, eP612

eP227

eP178, eP177

PS026

eP340

PS026

eP060, eP059

eP010

eP157

eP157

eP597

eP597

eP569

eP235, eP236, eP237, eP682

eP181, eP194, eP195

eP319

eP583

eP623

eP459

eP288

eP624

eP017

eP245

eP518, PS017

PS014

eP340

eP010, eP192

eP545

eP262

eP688

eP624

eP029

eP201

eP268

eP235, eP236, eP237, eP682

eP517

eP256

eP570, eP625

eP360

eP538

eP593

eP172, eP210

eP604

eP041

eP190

eP518, PS017

eP626

eP034, eP035, PS054
Boerhof, J.

Bøgsted, M.

Boland, E.

Boland, J.

Boland, L.

Boltong, A.

bonneterre, J.

Bonomo, $\mathrm{P}$.

Boone, $\mathrm{M}$.

Bornstein, J.C.

Borodina, I.

Bosnjak, S.

Bossi, P.

Boudreau, K.

Boukovinas, I.

Bouleuc, C.

Bourgouin-Ariba, M.

Bourien, $\mathrm{H}$.

Bours, M.

Boussen, $\mathrm{H}$.

Bouterfas, N.

Boutroux, H.

Bowen, J.

Bowen, J.M.

Boyle, F.

Bozec, L.

Brady, B.

Brady, C.

Brandão, T.

Bravo-Ruiseco, G.

Brawner, E.

braz, K.

Breau, R.

Brennan, E.

Brennan, M.

Brennan, M.T.

Brenner, $\mathrm{H}$.

Brennock, J.

Brierley, M.E.

Bright, C.

Brill, J.

Brinkman, T.M.

Briscoe, K.

Brito-Dellan, N.

Bronkhorst, E.

Brosens, M.

Brouwers, P.

Brown, A.

Brown, J.

Brown, M.

Brown, M.C.

Brown, M.P.

Bruera, E.

Bruner, D.

Bruner, D.W.

Brunetti, I.M.

Bryan, G.

Buatti, J.

Buga, S.

Bugdayci Basal, F.
eP292

eP190

eP043, eP042

eP042, eP043

eP688

eP114

eP073

eP001

eP148

eP223

PS083

PS085, eP095, eP208, eP458, PS086,

PS088

eP154, eP259, eP523

eP672

eP694

eP307

eP041

eP659

PS084

eP395, eP510

eP283

eP009

eP468, eP469, eP175, eP197, eP202, eP204, eP205, PS080

PS039

eP062, eP453

eP307

eP135, eP136

PS069

eP438, eP439

eP176

eP212

eP538

eP688

eP334

eP191

eP268

eP491, eP570, eP612, eP625

eP026, eP044

eP587

eP515

PS037

PS082

eP008

eP097, eP456, eP457

eP201

PS016

PS082

eP686

eP366

eP058

PS012

eP686

eP047, eP057, eP143, eP158, eP159, eP164, eP332, eP337, eP338, eP381, eP418, eP552, eP562, eP600, PS062, PS075

eP045

PS019

eP235, eP236, eP237, eP682

eP284

PS037

eP348

eP078 
Bulens, $\mathrm{P}$.

Buller, $\mathrm{H}$.

Bulotiene, G.

Bundiwal, A.

Bundor Tarawally, J.

Buonaccorso, L.

Burns, K.

Burton, B.

Bussé, L.

Butler, T.

Buxo, E.

Buyinza, N.

Byker, W.

Byrne, A.

Cabral, C.

Cabras, $\mathrm{M}$.

Cadamuro, S.

Cai, Y.X.

Çakır Karabaș, H.

$\mathrm{Cal}, \mathrm{A}$.

Calaguas, M.J.

Calders, P.

Calik, G.

Calik, M.

Calman, L.

Campbell, F.

Campbell, G.

Campbell, K.

Campisi, G.

Campone, $\mathrm{M}$.

Campos-Lopez, D.

Candela, B.

Candiani, M.

Cao, J.

Capel, A.

Capela, A.

Capocasale, G.

Capra, M.

Cardinaels, N.

Cardoso, D.

Carhuapoma, L.

Carley, M.

Carnelli, L.

Carney, T.

Carregal, A.

Carrier, M.

Carrillo, C.

Carroll, C.

Carroll, J.

Carrow, D.

Cartagena, G.

Cartmill, B.

Carvalho, A.

Carvalho, D.L.C.

Carzaniga, F.

Castagno, J.

Castellani, P.

Castillo, D.

Cathcart-Rake, E.

Caunes-Hilary, N.

Cavaco, P.

Cavieres, N.

Çay Șenler, F.

Celakil, T.

Celik, C.
eP174, eP448, eP484, PS016

eP645

eP383

eP375

eP128

eP428

eP348

PS068

PS016

eP046, eP627

eP230

eP313

eP601

eP376, eP434

eP628

eP030, eP273

eP355

eP532

eP027

eP480

eP180, eP328

eP620

eP703

eP703

eP527, eP607

eP294

eP341, PS025

eP314, eP571, eP572

eP098, eP035

eP507

eP698

PS070

eP481

eP573, eP384, eP618, eP634, eP700

eP507

PS067

eP098

PS029

eP484

eP325

eP402

eP688, eP689, eP690

eP481

eP202

eP067

eP645

eP482

eP285, PS029

PS038

eP062

eP389, eP421

eP513, PS045

eP089

eP177

eP481

eP421, eP442

eP525

eP184

eP470, eP471

eP041

eP629, eP628

eP150

eP483, eP387

eP267

eP619, eP704
Celik, E.

Celik, Z.E.

Cella, D.

Celsi, F.

Censabella, S.

Çevik, B.

Chahine, G.

chaiviboontham, S.

Chakrabhandu, S.

Chambaere, K.

Champion, V.

Champlin, R.

Chan, A.

Chan, C.W.H.

Chan, K.C.

Chan, S.

Chander, S.

Chang-Claude, J.

Chang, C.L.

Chang, C.S.

Chang, C.T.

Chang, L.H.

Chang, M.

Chang, Y.P.

Chansriwong, $\mathrm{P}$.

Chao, F.H.

Chapman, O.

Charalambous, A.

Charles, V.

Chartier, L.

Charvet, E.

Chaudhuri, P.

Chauhan, A.

Chawla, U.

Chee, E.

Chee, W.

Chen, C.

Chen, H.L.

Chen, H.X.

Chen, J.S.

Chen, $\mathrm{M}$.

Chen, P.

Chen, S.C.

Chen, T.

Chen, W.T.

Chen, Y.J.

Cheng, C.Y.

Cheng, $\mathrm{F}$.

Cheng, $\mathrm{H}$.

Cheng, $\mathrm{K}$.

Cheng, L.

Cheng, N.M.

Cheng, S.H.S.

Cheng, T.C.

Cheon, J.

CHESHMEDZHIEVA, B.

Cheung, L.W.

Cheung, Y.B.

Cheung, Y.K.

Cheung, Y.T.

Chevalier, E.

Cheville, A.

Cheville, A.L.

Chi, C.C.

Chic, N.
eP703

eP281, eP619

eP535

eP194

eP174, eP448, eP484, PS016

eP409

eP241

eP359

eP352

eP366

eP535, eP608

eP167, PS058

PS081, PS018, PS020

eP083, eP394

PS050

eP068

eP340

eP570, eP625

eP541

eP505

eP316

eP532

eP138

eP139, eP485

eP486

eP298

eP706

PS002, PS023, eP671, eP696, PS003

eP190

eP630

eP345

eP490

eP542

eP569

eP590, eP591, eP592, PS022

eP591, PS022, eP590, eP592

PS061

eP539

eP532

eP505

eP569

eP023

eP546, eP508

eP264

eP450

eP269, eP390

eP592

eP662, eP663

eP402, eP495

eP574

eP047

eP705

eP069

eP139, eP485

eP162

eP631

eP487

eP380

eP069

PS082

PS072

eP547, eP575

eP400

eP450

eP230 


\begin{tabular}{|c|c|c|c|}
\hline Childs, D. & eP006 & Colleran, M. & eP344 \\
\hline Childs, R. & eP569 & Colson, D. & PS016 \\
\hline Chinchalker, G.G. & eP099 & Combis, C. & eP041 \\
\hline Chinot, $\mathrm{O}$. & eP148 & Como, M. & eP399, eP584 \\
\hline Chircop, D. & eP391 & Cong, $\mathrm{M}$. & $\mathrm{eP} 264$ \\
\hline Chisholm, G. & eP381 & Conner, $\mathrm{K}$. & eP092 \\
\hline Chiswell, M. & eP114 & Cook, D. & eP624 \\
\hline Chitapanarux, I. & eP352 & Cooke, E. & eP681 \\
\hline Chittem, M. & eP392, eP393 & Cooksley, $\mathrm{T}$. & PS004, PS025, eP341 \\
\hline Cho Contributed Equally, C. & PS079 & Cooper, B. & eP147, eP219 \\
\hline Cho, E.J. & eP290 & Corby, $\mathrm{P}$. & eP184, eP203 \\
\hline Cho, S.J. & eP650 & Cormie, $\mathrm{P}$. & PS056 \\
\hline Cho, V.W.C. & eP446 & Corner, J. & eP607 \\
\hline Cho, Y. & $\mathrm{eP} 290$ & Correa, L. & eP177, eP178 \\
\hline Choi, C.H. & eP012 & Costa, $\mathrm{T}$. & PS067 \\
\hline Choi, E.J. & eP632 & costello, V. & eP029 \\
\hline Choi, H. & eP590 & Coutant, C. & eP507 \\
\hline Choi, H.J. & eP640 & Cowie, K. & $\mathrm{eP} 002$ \\
\hline Choi, K.C. & eP394 & Cox, K. & PS049 \\
\hline Choi, Y.S. & eP650 & Crawford, J. & eP402, eP495 \\
\hline Chou, H.C. & eP532 & Crichton, M. & eP244, eP245 \\
\hline Chou, H.L. & eP532 & Crotty, A. & PS068 \\
\hline Chou, Y.J. & eP140, eP155 & Crouch, A. & eP577, eP228, eP535 \\
\hline Chow, E. & eP068, eP209 & Crovella, S. & eP194 \\
\hline Chow, K.M. & eP394, eP487 & Crowe, H. & $\mathrm{eP} 427$ \\
\hline Chretienne, M. & eP149 & Crucianu, L.N. & $\mathrm{eP} 360$ \\
\hline Christensen, C. & eP686 & Cubero, D. & $\mathrm{eP} 142$ \\
\hline Christensen, J. & eP583 & Cuffe, S. & eP135, eP136, eP153 \\
\hline Christiansen, I. & eP190 & Culakova, E. & eP145, eP603 \\
\hline Christopoulou, A. & eP694 & Cunha, Â. & PS067 \\
\hline Chu, S. & eP216, eP501 & Cunningham, E. & eP555 \\
\hline Chu, W. & eP528 & Cunningham, $\mathrm{M}$. & eP136 \\
\hline Chumnumwat, S. & eP692 & Currow, D. & eP320 \\
\hline Chun, S.Y. & eP684 & Curtis, A. & PS037 \\
\hline Chung, $\mathrm{H}$. & eP528 & Curvo, A.R. & $\mathrm{eP} 276$ \\
\hline Chung, O.K. & eP141 & Custódio, S. & PS067 \\
\hline Chung, O.K.J. & eP599 & Cutillar, E. & eP363 \\
\hline Chung, S. & eP410 & Cwiak, M. & PS069 \\
\hline Chung, W.H. & $\mathrm{eP} 450$ & da Silva Ferreira, A.R. & eP176 \\
\hline Ciorba, M. & eP204 & da Silva Vaz Paterra, T. & $\mathrm{eP} 414$ \\
\hline Ciotti, O. & eP193 & Daccache, K. & eP311 \\
\hline Cirrone, F. & eP184 & Dahiya, N. & eP489, eP111, eP291, eP596 \\
\hline Çınarlı, T. & eP654 & Dai, Z. & eP264 \\
\hline Çıracı Yaşar, Y. & eP100, eP317, eP318, eP488 & Daily, J. & eP518, PS017 \\
\hline Ckumdee, S. & eP486 & Dalakou, E. & eP110, eP500 \\
\hline Claes, S. & eP174, eP448, PS016 & Dalal, S. & eP047, eP143, eP338 \\
\hline Clare, $\mathrm{H}$. & eP341 & Dale, J. & eP566 \\
\hline Clarisse, B. & eP506 & Dale, W. & $\mathrm{eP} 348$ \\
\hline Clark, K. & eP320 & Dam, V. & PS031 \\
\hline Clark, M.M. & $\mathrm{eP} 400$ & Damani, A. & eP637 \\
\hline Clarke, K. & eP046, eP455 & Danaci, E. & $\mathrm{eP} 416$ \\
\hline Clarke, S. & eP008 & Daoud, N. & eP395, eP510 \\
\hline Clément-Zhao, A. & eP319 & Darlington, A.S. & eP528 \\
\hline Clinton, F. & eP285 & Darmani, N. & eP003 \\
\hline Cobb, $\mathrm{P}$. & PS026 & Dash, P. & eP050, eP258 \\
\hline Coelho, H. & PS067 & Daste, A. & eP678 \\
\hline Coffey, L. & eP579 & Datta, A. & eP490 \\
\hline Cohee, A. & eP608 & Dauchy, S. & eP507 \\
\hline Cohen, R. & eP151 & Daveau, C. & eP319 \\
\hline Cohen, V. & eP499 & Davidson, P. & eP062 \\
\hline Cohn, R. & eP429, eP587 & Davies, A. & eP356 \\
\hline Colak, E. & eP086 & Davis, M. & eP144 \\
\hline Colautti, N. & eP633 & Davison, N. & eP580 \\
\hline Cole, S. & eP603 & Davoren, M. & eP681 \\
\hline Coller, J. & eP175, eP197 & Dawod, M.A. & PS069 \\
\hline
\end{tabular}


De Angelis, C.

De Graves, S.

De Grazia, A.

de Jonge, M.

De La Cruz, M.

De la Cruz, M.G.

de Lima, M.H.A.

de Lima, V.C.C.

De Los Reyes, F.

de Macedo, L.

de Madariaga, M.

De Martino, I.

de Mattos-Pimenta, C.A.

de Menezes, J.

de Oliveira, C.

de Oliveira, M.A.

De Rauglaudre, G.

de Soet, J.

De Vleminck, A.

DeAngelis, C.

Deckert, A.

Dede, $\mathrm{M}$.

DeJesus, A.

Del Fabbro, E.

Del Giglio, A.

Delacroix, S.

Delarue, J.

Delattre, J.Y.

Delecroix, V.

Delgado-Guay, M.

Delgado, M.

Deliens, L.

DeMeyer, E.

Demir, M.

Demirbağ, B.C.

Demircioglu, B.

Demiri, M.

Demirkazık, A.

Dennert, G.

Dennis, K.

Dent, R.

Dent, R.A.

Deodhar, J.

Desideri, I.

Desjardins, A.

Desmond, D.

Despotova-Toleva, L.

Dessard-Diana, B.

Dev, R.

Devereux, L.

Dholam, K.

Dhollander, N.

Di Fede, O.

Di Lenarda, R.

Di Mattei, V.E.

Di Nisio, M.

Di Pede, $\mathrm{P}$.

Diallo, H.

Dias, E.

Dias, F.L.

Dias, M.

Diaz, P.L.

Dibaj, S.

Dibekci, S.

Diego, E.
eP209

eP131

eP020

eP534, PS076

eP164

eP158

eP467

eP467

eP184

eP276

eP067

eP273

eP220

eP423

PS062

eP354

eP576

eP185

eP321, eP322

eP068

eP601

PS053

eP047

eP212

eP142

PS043

eP252

eP148

eP576

eP158, eP633, eP143, eP431

eP159, eP674, eP675

eP321, eP322, eP366

eP122

eP027

eP317

eP032

PS053

eP483

eP074

eP528

PS020

PS018

eP099, eP353, eP637

eP001, eP528

eP226

eP579

eP101, eP631

eP319

eP143

PS029

eP028

eP321, eP322

eP098

eP181, eP194, eP195

eP481

eP645

eP154, eP259

eP148

PS067

eP170

PS067

eP209

eP338

eP543

eP529
Diel, I.

Dieperink, K.

Dieperink, K.B.

Dijk, D.J.

Dikman, A.

Dimitriadou, A.

Dimitrijevic, J.

Din, A.

Dinkel, A.

Dinney, C.

Dipasupil, J.P.

Dixit, N.

Djordjevic, F.

Dockter, T.

Doege, D.

Dogra, S.

Dohollou, N.

Dolan, M.

Domen, K.

Dominguez, G.

Dong, F.

Dorchin, M.

dos Santos, B.

Doucette, K.

Dowd, A.

dowling, M.

Downie, $\mathrm{P}$.

Drake, E.

dridi, $M$.

Droney, J.

Drost, L.

Drozd, Y.

Duarte, A.

Ducray, F.

Duger, $\mathrm{T}$.

duggan, $\mathrm{C}$.

Dugum, O.

Dukic, S.

Dunlap, N.

Dunn, J.

Dunne, S.

Dupuis, L.L.

Durasinovic, V.

Durdux, C.

Durutovic, I.

Dutta, P.

Duus, E.

Dworkind, M.

Dybkær, K.

Dzurillova, L.

Earp, M.

Eastman, P.

Eberle, A.

Eche, I.J.

Eckert, M.

EDGAR, A.

Edmunds, K.

Eduardo, F.D.P.

Edwards, B.

Edwards, T.

Eek, R.

Eicher, M.

Eid, A.A.

Ekinci, Y.

El Azzazy, S.
PS051

eP548, eP677

eP466

eP356

eP184

eP261

eP458, eP095, PS086

eP527, eP607

eP426

eP167, PS058

eP180

eP605

PS086, eP208

eP227

eP491

eP555

eP576

eP045

eP556, eP557

eP674, eP675

eP634, eP384, eP573, eP618, eP700

eP048

eP220

eP569

eP324

eP029

eP131

eP578, eP615

eP229

eP372

eP068, eP209

PS034

eP461

eP148

eP540, eP620

eP029

eP533

eP153

PS037

eP706

eP579

eP294

eP459

eP319

eP208

eP010, eP192

eP312

PS031, PS034

eP190

eP260

eP323

eP324

eP491, eP612

eP286

eP686

eP580, eP581

eP582

eP177, eP178

eP167, PS058

eP332

eP455

eP096, eP606

eP474

eP540

eP648 


\begin{tabular}{|c|c|c|c|}
\hline El Benna, H. & eP395, eP510 & Ferraris, K.P. & $\mathrm{eP} 328$ \\
\hline El Karak, F. & eP241 & Ferreira Ouchi França, A. & eP414 \\
\hline El Omri, H. & eP648 & Ferreira, C.E.D.S. & eP177 \\
\hline El Rassy, E. & eP241, eP311 & Ferreira, C.G. & eP170 \\
\hline Elad, S. & eP035 & Ferreira, E. & eP270, eP271 \\
\hline Elayan, A. & eP526 & Ferreira, E.M.S. & $\mathrm{eP} 170$ \\
\hline Eldridge, L. & eP372 & Ferreira, F. & eP325 \\
\hline Eldridge, R.C. & PS019 & Ferreira, M. & eP397 \\
\hline Eleanor, O. & eP579 & Ferreira, М.Н. & eP177 \\
\hline Elewah, M. & eP102 & Ferreira, P. & $\mathrm{eP} 355$ \\
\hline Elfeky, A. & eP179 & Ferrer Albiach, C. & eP067 \\
\hline ElHussein, A. & eP635 & Ferrer-Mileo, L. & eP326 \\
\hline Elizabeth, D. & eP672 & Ferrero, E. & eP584 \\
\hline Elmalik, H. & eP405 & Fieo, R. & eP151 \\
\hline Elsurer, C. & eP281 & Filbet, M. & eP072, eP159, eP398 \\
\hline Elswick- Jr., R.K. & PS021 & Fineza, A.M. & eP180 \\
\hline Elzembely, M. & eP125 & Finke, I. & eP616 \\
\hline Eng, L. & PS012 & Firdous, S. & $\mathrm{eP} 144$ \\
\hline Engels, $\mathrm{S}$. & $\mathrm{eP} 484$ & Flannery, M. & $\mathrm{eP} 327$ \\
\hline Epstein, J. & PS033, eP035, PS038 & Fliedner, M. & PS060 \\
\hline Epton, T. & eP392 & Floquet, A. & eP576 \\
\hline Erfe, R. & eP333 & Flores-Díaz, D. & eP568, PS006, eP346 \\
\hline Erickson, J.M. & PS021 & Flores, A.R. & eP180 \\
\hline Ernest, N.A. & $\mathrm{eP} 326$ & Flores, J.A. & eP180, eP328 \\
\hline Escalante, C. & eP107, eP108, eP109, eP627 & Follana, P. & eP576 \\
\hline Esme, $\mathrm{H}$. & eP703 & Fonseca, F. & $\mathrm{eP} 142$ \\
\hline Esparza-Duran, D. & $\mathrm{eP} 442$ & Fonseca, O. & $\mathrm{eP} 473$ \\
\hline Esperza-Duran, D. & eP421 & Font-Gonzalez, A. & eP294 \\
\hline Espín Gonzalez, I. & eP633 & Font, C. & eP230, eP627 \\
\hline Esposito, F.M. & eP230 & Fontillas, R. & eP551 \\
\hline Estilo, C. & PS054 & Fossella, F. & eP337 \\
\hline Eychmüller, S. & PS060 & Foster, C. & eP527, eP606, еР607 \\
\hline Ezendam, N. & eP583 & Fox, A. & eP184 \\
\hline Ezendam, N.P.M. & PS011 & Fox, J. & eP518, PS017 \\
\hline Ezeofor, S. & eP515 & Fox, $\mathrm{P}$. & eP008 \\
\hline Eziada, S. & eP405 & Francis, D.L. & $\mathrm{eP} 272$ \\
\hline F Javier, P.M. & eP326 & Francolini, G. & eP001 \\
\hline Fabbro, M. & eP576 & Francopoulo, A. & eP678 \\
\hline Fadipe, B. & eP396, eP492 & Frandsen, K. & eP005 \\
\hline Fadipe, Y.O. & eP396 & Freire, I. & $\mathrm{eP} 473$ \\
\hline Fagbule, $\mathrm{O}$. & eP103 & Friedlander - Barenboim, S. & eP036 \\
\hline Fakhoury, M. & eP241 & Friedman, $\mathrm{H}$. & $\mathrm{eP} 226$ \\
\hline Falcão, F. & eP629 & Friis-Hansen, L. & $\mathrm{eP} 233$ \\
\hline Falcone, A. & eP235, eP236, eP237, eP682 & Frison, E. & eP678 \\
\hline Fang, Y.Y. & eP609, eP610 & Fritz, G. & PS078 \\
\hline Fardell, J. & eP429, eP587 & Frølund, U.C. & eP549 \\
\hline Farquhar-Smith, $\mathrm{P}$. & eP058 & Frowen, J. & eP636, PS008 \\
\hline Farrell, H. & eP324 & Frydenberg, M. & $\mathrm{eP} 427$ \\
\hline Fasciolo, A. & $\mathrm{eP} 273$ & Fu, J. & eP512, eP552, eP562 \\
\hline Fatima, A. & eP246 & Fujimori co-primary author, M. & PS075 \\
\hline Favato, $\mathrm{P}$. & eP399, eP584 & Fujimori, M. & $\mathrm{eP} 418$ \\
\hline Fazekas, B. & eP320 & Fujisawa, T. & $\mathrm{eP} 250$ \\
\hline Fedunenko, V. & eP382 & Fujiwara, Y. & $\mathrm{eP} 460$ \\
\hline Felger, J. & PS019 & Fukuda, H. & eP011 \\
\hline fendri, S. & eP229, eP302 & Fukushima, T. & eP550, eP061 \\
\hline Fenlon, D. & eP527, eP606, еР607 & Fukuta, A. & eP163 \\
\hline Fern, L.A. & PS073 & Fung, H.K.S. & eP394 \\
\hline Fernandes, D. & eP560 & Furukawa, K. & $\mathrm{eP} 207$ \\
\hline Fernandes, L. & eP325 & Fusco, V. & eP030, eP273, eP399, eP584, PS054 \\
\hline Fernandez del Castillo, M. & eP602 & Gabinskiy, M. & eP203 \\
\hline Fernandez-Ortega, P. & PS041 & Gabrian, M. & eP691 \\
\hline Fernandez, C. & eP144 & Galeas, J.N. & eP004 \\
\hline Ferrara, E. & eP020 & Galiti, D. & eP034, eP035, PS054 \\
\hline Ferrara, M. & eP154 & Galitis, E. & eP188, eP502 \\
\hline Ferrara, M.L. & eP259 & Gallagher, P. & eP579 \\
\hline
\end{tabular}


Gallardo, L.

Galli, L.

Gallo-Hershberg, D.

Galvão, D.A.

Gambino, A.

Gamea, M.

Gameil, A.

Gan, Y.X.

Gandee, M.

Ganesan, P.

Ganesan, T.

Gao, Y.

Garces, Y.I.

Garcia-Erce, J.A.

García-Morillo, M.

Garcia, D.

Garcia, E.

Garcia, N.

García, R.

Gardner, S.

Garg, M.

Garg, S.

Garming Legert, K.

Garming-Legert, K.

Garssen, J.

Gascon, P.

Gasser, C.

Gathirua-Mwangi, W.G.

Gebreyohannes, E.

Gedye, C.

Geer, J.

Geng, Y.

Genot, M.T.

Gentry, M.

Gentzler, R.

George- M.D., T.

George, M.

Ghadirifard, A.

Ghazouani, H.

Ghosh, A.

Ghoshal, A.

Ghosn, M.

Gianesella, M.

Giannopoulou, C.

Giaquinto, A.

Gibbons, P.

Gibbs, $\mathrm{S}$.

Gibo, T.

Gibson, F.

Gibson, R.

Gibson, R.J.

Gibson, T.M.

Gifford, W.

Giguere, J.

Gilbert, A.

Gilliland, K.

Giordano, G.

Gioti, E.

Giotis, A.

Giraud, P.

Giuliano, C.

Glare, P.

Glaser, A.

Glenn, A.

Glynne-Jones, R.
eP494

eP235, eP236, eP237, eP682

eP209

eP582

eP030, eP273

eP329

eP405

PS018, PS020

eP122, eP358

PS015

PS015

PS061

eP400

eP071

eP230

eP645

eP514

eP348

eP493, eP514

eP184

PS017

eP111, eP489

eP268

eP191

eP200

eP071

eP525

eP608

eP049

eP008

PS043

eP167, PS058

PS072

eP400

eP402, eP495

eP420

eP494

eP016

eP648

eP192

eP637

eP241

eP482

PS051

eP193

eP213

eP199

eP274, eP280

eP284, PS073

eP175, eP197, eP202, eP205

PS039

PS082

eP585

PS043

eP528

PS043

eP071

eP261, eP693

eP209

eP319

eP013

eP320

eP231

eP697

eP528
Go, M.

Go, S.I.

Gobbi, M.

Gobbo, M.

Goddard, K.

Goel, V.

Gökay, E.Ö.

Gökçe, Ș.Ç.

Goldhirsch, J.

Goldman, S.

Goldstein, D.

Gomes, A.

Gonçalves, J.

Gong, J.

Gonzalez-Fernandez, R.

González-Garza, P.

Gonzalez-Melendrez, R.

Gooberman-Hill, R.

Goodall, S.

Goodman, M.

Goonan, J.

Gorken, I.

Goto, Y.

Gottumukkala, V.

Götz, A.

Gough, N.

Gouveia, E.

Graça, J.

Graham, I.

Graham, I.D.

Gralla, R.

Gralla, R.J.

Granata, R.

Granowetter, L.

Grassi, R.

Gray, L.

Green, E.

Grellard, J.M.

Griffin, $\mathrm{O}$.

Griffith, K.

Grimison, P.

Groen, A.

Groenvold, M.

Groschek, M.

Grosse Perdekamp, M.

Grosso, E.

Grosso, M.

Grummet, J.

Grunfeld, J.

Gruza, A.

Guan Zhen, C.

Guardado, B.

Guerrin, B.

Guglielmo, M.

Guideline Working Group,

O.B.O.T.P.I.C.W.C.

Guido, J.

Guillén-Núñez, M.D.R. eP051

Guimond, A.J. eP403, eP404

Guisset, O.

Gul, R.B.

gülhan, $\mathrm{S}$.

Gullett, J.

Gümüştepe, E.

gunawan, $\mathrm{S}$.
eP678

eP519

eP318

eP151

eP247

eP328

eP178

eP181, eP194, eP195

eP588

eP247

eP247

eP303

eP222, PS049

eP198

eP265
eP646

eP602

eP579

eP209

eP327

eP480

eP460

eP401

eP251

eP638, eP690

eP585

eP402, eP495

eP154

eP184

PS04

eP689, eP690

eP506, eP576

eP251

eP008, eP222

eP200

eP005

P234

eP399, eP584

eP645

eP427

eP639
eP268

eP104, eP105

PS087

eP154, eP259

eP294

eP287 


\begin{tabular}{|c|c|c|c|}
\hline Guo, F. & eP031 & Hasegawa, S. & eP557 \\
\hline Guo, H. & eP551 & Hashem, M. & eP288 \\
\hline Guo, Y. & eP551, eP552, eP562 & Hashimoto, H. & eP011 \\
\hline Gupta, A. & PS012 & Hashimoto, M. & eP557 \\
\hline Gupta, E. & eP552 & Hashizume, K. & eP061 \\
\hline Gupta, S. & eP489 & Haslbeck, J. & eP606 \\
\hline Guren, M. & eP528 & Hassan, A. & eP405 \\
\hline Guren, M.G. & eP680 & Hassan, H. & eP231 \\
\hline Gusev, L. & eP182 & Hasséus, B. & eP191, eP268 \\
\hline Ha, J.H. & $\mathrm{eP} 410$ & Hatake, K. & $\mathrm{eP} 238$ \\
\hline Habeebu, M. & eP492 & Hattori, S. & eP065 \\
\hline Haber, P. & eP008 & Häusermann, S. & eP364 \\
\hline Hablas, M. & eP331 & Haussmann, A. & eP691 \\
\hline Hacker, L. & eP341 & Haverman, T. & eP185 \\
\hline haddaoui, A. & eP229, eP302 & Haviland, K. & eP334 \\
\hline Hafez, H. & eP288 & Hayashi, R. & eP373 \\
\hline Hagedoorn, M. & eP166 & Hayden, C. & eP026 \\
\hline Hahm, B.J. & eP156, eP221, eP225, eP435 & He, W. & PS050 \\
\hline Hahn, C. & eP008 & Heckler, C. & PS043 \\
\hline Haider, A. & eP332, eP217 & Hedberg, A.M. & eP167, PS058 \\
\hline Haimovitz Friedman, A. & PS038 & Hegarty, J. & eP681 \\
\hline Hajj, G.N.M. & eP467 & Helissey, C. & eP523 \\
\hline Hakoyama, Y. & $\mathrm{eP} 274$ & Henninger, $\mathrm{C}$. & PS078 \\
\hline Haladuick, J. & eP601 & Henriques, G. & eP025 \\
\hline Halder, B. & PS042 & Heo, D.S. & eP118 \\
\hline Hale, D. & eP706 & Heo, S.J. & eP640 \\
\hline Hales, S. & eP369 & Herbert, B.' & eP623 \\
\hline Halfens, R. & PS060 & Herchenhorn, D. & eP170 \\
\hline Hall, R. & eP402, eP495 & Herlofson, B.B. & PS010 \\
\hline Halley, A. & eP372 & Hermelink, K. & eP426 \\
\hline Halm, J. & eP666 & Herndon, J. & $\mathrm{eP} 226$ \\
\hline Halmos, B. & $\mathrm{eP} 495$ & Herrstedt, J. & eP005, eP233, PS059 \\
\hline Halter, J. & $\mathrm{eP} 279$ & Herschbach, P. & eP426 \\
\hline Hamabe, Y. & eP214 & Herwansyah, P. & eP287 \\
\hline Hamajima, Y. & eP590 & Herz, S. & eP449 \\
\hline Hamano, J. & eP183 & Hesketh, P.J. & eP018 \\
\hline Hamauchi, S. & eP011 & Hess, $\mathrm{K}$. & eP338, eP465 \\
\hline Hamblin, M. & eP635 & Hetherington, $\mathrm{K}$. & eP587 \\
\hline hamdi, S. & eP229 & Hettinghouse, A. & eP031 \\
\hline Hamerschlak, N. & eP177, eP178 & Heußner, P. & $\mathrm{eP} 426$ \\
\hline Hamza, M. & $\mathrm{eP} 282$ & Heutte, N. & eP576 \\
\hline Han, N. & eP290 & Higashiguchi, T. & eP253 \\
\hline Hanai, A. & eP214 & Higashiyama, R. & $\mathrm{eP} 460$ \\
\hline Hanrahan, E. & eP153 & Higgins, $\mathrm{K}$. & eP251 \\
\hline hanriot, R. & eP538 & Higgins, K.A. & PS019 \\
\hline Hansen, A. & eP521 & Higuchi, T. & eP275 \\
\hline Hansen, O. & eP548 & Hilbert, G. & eP678 \\
\hline Hansen, S. & eP548 & Hill, C. & eP706 \\
\hline Haque, S. & eP333 & Hill, R. & eP587 \\
\hline Harada, T. & eP556 & Hilton, L. & PS025 \\
\hline Haraguchi, K. & eP472 & Hirashima, Y. & eP011 \\
\hline Harbeck, E. & eP239 & Hiratsuka, T. & $\mathrm{eP} 452$ \\
\hline Harder, S. & eP005 & Hirofumi, Y. & eP647 \\
\hline Harding, R. & eP685 & Hiroko, K. & eP531 \\
\hline Harding, V. & eP284 & Hjermstad, M. & eP042, eP043 \\
\hline Hargrave, D. & PS071 & Hlaing, S. & eP106, eP335 \\
\hline Harmsen, H. & eP200 & Hlalah, O. & PS026 \\
\hline Harmsen, H.J.M. & eP176 & Ho, C.L. & eP505 \\
\hline Harrison, M. & eP222, PS049 & Ho, H.K. & PS018, PS020 \\
\hline Harthoorn, L. & eP200 & Hoang-Xuan, K. & eP148 \\
\hline Harti, S. & eP149 & Hockenberry, M. & PL001 \\
\hline Hartmann, B. & eP187 & Hoffmeister, M. & eP570, eP625 \\
\hline Hartnett, E. & eP184 & Hohmann, D. & PS051 \\
\hline Hartog, A. & eP200 & Højsted, J. & eP220 \\
\hline Harutyunyan, L. & eP388 & Holleczek, B. & eP491, eP612 \\
\hline
\end{tabular}


Hollen, P.

Hollister, B.

Holm, K.

Holmlund, J.

Holst, J.J.

Holtom, N.

Hong, D.

Honkoop, A.

Horinouchi, H.

Horneber, M.A.

Horowitz, I.

Horrex, D.

Horvath, L.

Hosaka, M.

Hou, P.Y.

Housset, M.

Hovan, A.

Howard, C.

Howard, F.

Howard, N.

Howarth, G.

Howell, D.

Howell, R.M.

Hsiao, M.P.

Hsiao, W.L.

Hsieh, C.H.

Hsu, C.Y.

Hsu, S.C.

Hsu, S.P.

Hsu, T.H.

Hsuan-Ju, K.

Hsueh, K.C.

$\mathrm{Hu}, \mathrm{X}$.

$\mathrm{Hu}, \mathrm{Y}$.

Huang, C.C.

Huang, L.

Huang, T.W.

Hubbard, J.M.

Hueniken, K.

Hughes, P.

Hughes, R.

Hui-Hsuan, S.

Hui, D.

Hui, R.

Hulikal, N.

Hung, A.

Hunt, J.

Hussaini, M.

Husson, O.

Huszno, J.

Hutchinson, M.

Hutchinson, S.

Huth, J.

Huysmans, M.C.

Hwang, $\mathrm{H}$.

Hwang, I.C.

Hwang, S.H

Hwang, Y.J.

Hwu, P.

Hyman, P.

Idiga, E.

Ikeda, T.

Ikegame, K.

İlhan, T.T.
eP402, eP495

eP379, PS024

eP606

PS037

eP187

eP106, eP335, eP496

eP465, eP475

eP166

eP460

eP074, eP075

eP045

eP347

eP222, PS049

eP084

eP532

eP319

eP191, eP268

eP203

eP588

eP427

eP172, eP210, eP630

eP689

PS082

eP589

eP406, eP553

eP532

eP497, eP641

eP641

eP076

eP336

eP140

eP129

eP147

eP590, eP592

eP539

eP465

eP077

eP400

PS012

eP184

eP636, PS008

eP413

eP337, eP338, PS063, W002, eP143,

eP526, PS062

eP450

eP644

PS024

eP187, eP339

eP289

eP522

eP642

eP172, eP210

eP335

$\mathrm{PS} 024$

eP201, eP268

eP225, eP435

eP643

eP056, eP090

eP218

eP494

eP445, eP537

eP103

eP240

eP556

eP619
Iliescu, G.

Iloanusi, N.

Im, E.O.

Im, S.A.

Imai, $\mathrm{S}$.

Imataki, O.

Imhansoloeva, M.

Immanuel, A.

In-Fun, L.

Inamdar, M.B.

Inano, $\mathrm{T}$.

Inglis, J.

Inhestern, L.

Innocentini, L.M.

Inoguchi, $\mathrm{H}$.

Inomata, $\mathrm{M}$.

Inoue, A.

Inoue, J.

Inoue, $\mathrm{N}$.

Insel, $\mathrm{K}$.

Intorcia, M.

Inui, A.

Ionova, $\mathrm{T}$.

Isenring, E.

Ishida, $\mathrm{K}$.

Ishiguro, $\mathrm{H}$.

Ishii, K.

Ishii, S.

Ishikawa, $\mathrm{H}$.

Ishiura, Y.

Işıkoğlu-, B.D.

Ito, $\mathrm{S}$.

Ito, $\mathrm{T}$.

Ivanova, D.

Ivers, $\mathrm{H}$.

Iwasa, S.

Iwata, $\mathrm{H}$.

Izgu, $\mathrm{N}$.

Jackson-Cook, C.

Jacobsen, P.

Jaffar, N.

Jager-Wittenaar, H.

Jagoe, R.T.

Jahn, F.

Jahn, P.

Jain, N.

Jakkula, S.

Janelsins, M.

Jansen, L.

Janzic, U.

Jarden, M.

Jarosław, W.

Jatoi, A.

Jaulmes, $\mathrm{H}$.

Javed Khan, S.

Jean-Pierre, A.

Jean-Pierre, $P$.

Jefford, M.

Jehangir, W.

Jenewein, J.

Jenkins, N.

Jenkins, V.

Jeon, A.Y.

Jeon, J.Y.

Jeong Hye, K.
eP107, eP108, eP109

eP515

eP590, eP592, eP591, PS022

eP156, eP221, eP225, eP435

eP214

eP240

eP515

eP339

eP413

eP644

eP253

eP145, eP603

eP407

eP276

eP418, PS075

eP452

eP133

eP163, PS013

eP238

PL001

PS051

eP253

PS083

eP244, eP245

eP007

eP214

eP061

eP061, eP550

eP160, eP011

eP084

eP483

eP569

eP084

eP215

eP403, eP404

eP011

eP452

eP078

eP151

PS043

eP455

eP259

eP499

eP248

eP248, eP112

eP569

eP393

eP145, eP603

eP570, eP625

eP208

eP676, eP549

eP052

eP006, eP470, eP660

eP319

eP297

eP593

eP593

eP424

eP144

eP401

eP494

eP408

eP290

eP090

eP701 


\begin{tabular}{|c|c|c|c|}
\hline Jesús, G.B. & eP326 & Karelin, A. & PS083 \\
\hline Ji Hye, K. & eP701 & Karlsen, R. & eP583 \\
\hline $\mathrm{Ji}, \mathrm{X}$ & eP591 & Karthaus, M. & eP014 \\
\hline Jia-Mahasap, B. & eP352 & Kashiwagi, H. & eP647 \\
\hline Jibb, L. & eP690 & Kassem, N. & eP648 \\
\hline Jiménez López, A.J. & eP067 & Kassirian, S. & PS012 \\
\hline Jirajarus, M. & eP486 & Kasvis, $\mathrm{P}$. & eP498 \\
\hline Joaquim, A. & PS067 & Kasymjanova, G. & eP499 \\
\hline Johansen, C. & eP548, eP583 & Kataoka, T. & eP186 \\
\hline Johansson, J.E. & $\mathrm{eP} 268$ & Katherine, W. & eP051 \\
\hline Johnsen, H.E. & eP190 & Kato, R. & eP554 \\
\hline Johnson, C. & eP528 & Kattan, J. & eP241, eP311 \\
\hline Johnson, M. & eP042, eP043 & Kav, S. & eP409, eP386 \\
\hline Johnson, R. & eP529 & Kavalalı Erdoğan, T. & eP411, eP654, eP415, eP416 \\
\hline Johnston, L. & eP571, eP572 & Kawachi, H. & $\mathrm{eP} 061$ \\
\hline Jolicoeur, L. & eP689, eP690 & Kawagishi, R. & eP007 \\
\hline Joly, F. & eP506, eP507, eP576 & Kawaguchi, T. & eP183, eP531 \\
\hline Joos, S. & eP080 & Kawahara, M. & eP418, PS075 \\
\hline Josephine, R. & eP248 & Kawahara, T. & eP349 \\
\hline Joshi, A. & PS015 & Kawakami, T. & eP160 \\
\hline Joshi, R. & eP686 & KAWUBIRI, W. & eP649 \\
\hline Joshi, S. & eP054 & Kazama, T. & $\mathrm{eP} 250$ \\
\hline Joshi, U. & eP054 & Kazutoshi, S. & eP669 \\
\hline Joshi, V. & eP333 & Keefe, D. & W001, eP175, eP468 \\
\hline Jui-Chun, C. & $\mathrm{eP} 413$ & Keefe, D.M. & PS039 \\
\hline Jung, D. & eP156, eP221, eP225, eP435 & Kelly, D. & PS069 \\
\hline Jung, H.J. & eP216, eP501 & Kennedy, A. & PS029 \\
\hline Kaasa, S. & $\mathrm{eP} 042, \mathrm{eP} 043$ & Kennedy, M.J. & eP135, eP136 \\
\hline Kabarriti, R. & eP518, PS017 & Kenny, C. & eP249 \\
\hline Kacel, E. & eP421, eP442 & Kentaro, Y. & eP647 \\
\hline Kacel, E.L. & eP389 & Keogh, I. & eP579 \\
\hline Kaida, K. & eP556 & Kerins, $\mathrm{M}$. & $\mathrm{eP} 344$ \\
\hline Kajüter, H. & eP612 & Kerr, A. & $\mathrm{eP} 184$ \\
\hline Kakita, N. & eP242 & Kerr, R. & eP203 \\
\hline Kakkar, A. & eP645, eP706 & Kersu, O. & eP086 \\
\hline Kalbacher, E. & eP576 & Keskin, S. & eP027 \\
\hline Kalisch, A. & eP075, eP074 & Kettelhoit, N. & eP449 \\
\hline Kalnicki, S. & eP518, PS017 & Kevin, M.M. & eP326 \\
\hline Kalofonos, C. & eP694 & Keyur, M. & eP518 \\
\hline Kamath, J. & eP146 & Khanna, N. & eP045 \\
\hline Kamen, C. & PS043, eP327, eP603 & Khor, C.C. & PS018 \\
\hline Kamibeppu, K. & eP349 & Kiagia, M. & eP110, eP500 \\
\hline Kaminski, I. & PS016 & Kiely, F. & eP224 \\
\hline Kamizato, M. & eP670, eP611 & Kiernan, M. & eP222, PS049 \\
\hline Kamper, A. & eP166 & Kikuchi, Y. & $\mathrm{eP} 472$ \\
\hline Kampman, E. & eP130, PS084 & Kilbreath, S. & eP672 \\
\hline Kanamori, T. & eP055 & Kilgore, K. & eP337 \\
\hline Kanda, S. & eP460 & Kilgour, R. & $\mathrm{eP} 498$ \\
\hline Kane, J. & PS068 & Kim, A.J. & eP290 \\
\hline Kanellopoulos, P. & eP188 & Kim, C. & $\mathrm{eP} 410$ \\
\hline Kanelopoulos, $\mathrm{P}$. & eP502 & Kim, D.J. & eP087 \\
\hline Kang, H.J. & eP290 & Kim, D.Y. & eP118 \\
\hline Kang, J.H. & eP330 & Kim, H.K. & eP056 \\
\hline Kanodia, R. & $\mathrm{eP} 340$ & Kim, I.R. & eP598 \\
\hline Kanu, F. & eP515 & Kim, J.E. & eP650 \\
\hline Karaaslan Eser, A. & eP409 & Kim, J.H. & eP115 \\
\hline Karabagli, P. & eP704 & Kim, K. & eP501, eP216 \\
\hline Karabulut, A. & eP409 & Kim, K.J. & eP118 \\
\hline Karacan, Y. & eP032 & Kim, K.M. & eP684 \\
\hline Karadakovan, A. & eP120 & Kim, M. & eP683 \\
\hline Karambelas, I. & eP500 & Kim, M.G. & eP290 \\
\hline Karapetis, C. & eP175, eP686 & Kim, M.J. & eP290, eP683 \\
\hline Karapetis, C.S. & PS039 & Kim, M.K. & eP012, eP651 \\
\hline Karcı, E. & eP387 & Kim, N. & eP079 \\
\hline Kardamakis, D. & eP528 & Kim, P.H. & eP684 \\
\hline
\end{tabular}


Kim, S.

Kim, S.H.

Kim, T.Y.

Kim, Y.

Kim, Y.B.

Kim, Y.H.

Kim, Y.J.

Kimura, Y.

King, G.

King, J.

King, $\mathrm{T}$.

Kinoshita, T.

Kinsey, S.

Kiprian, D.

Kirby, A.

Kirkova, Y.

Kishi, R.

Kishore, J.

Kiss, $\mathrm{N}$.

Kissow, $\mathrm{H}$.

Kitano, S.

Kizawa, Y.

Kjeldsen, L.

Klafke, N.

Kleckner, A.

Kleckner, I.

Klein, D.

Klunklin, P.

Klunklin, P.D.A.

Knopf, K.

Knops, R.R.

Kober, K.

Kobleder, A.

Koç, İ.C.

Koç, Z.

Koch-Gallenkamp, L.

Koczwara, B.

Koerner, K.

Koh, H.D.

Koh, S.J.

Kohli, C.

Koishihara, Y.

Koizuka, S.

Kojima, Y.

Koklanaris, E.

Kokoreva, M.

Köksoy, E.

Kolberg, G.

Kolen, B.

Koller, A.

Kolosza, Z.

Komatsu, H.

Kondo, N.

Kong, Y.C.

Koning-van Zuilen- PT.- MSc., PS055

M.

Konishi, T.

Konmun, J.

Kono, Y.

Koom, W.S.

Koppelmans, R.G.A.

Korc-Grodzicki, B.

Korfage, I.

Korver, S.

Korver, S.K.
eP079, eP217, eP162, eP590

eP594, eP598, eP598

eP156, eP221, eP225, eP435

PS074, W003

eP079

eP115

eP337

eP611

eP136

eP652

eP222

eP418, PS075

eP231

eP196, eP367

eP008

eP218

eP033

eP291, eP596

eP636

eP187

eP452

eP183

eP676

eP080

eP327

eP145, eP603

eP534, PS076

eP352

PS042

eP555

eP292

eP147, eP219

eP653

eP411, eP415, eP416, eP654

eP411, eP415, eP416, eP654

eP491, eP612

eP595

PL001

eP640

eP162

eP111, eP291, eP596

eP559

eP250

eP655

eP569

PS083

eP387

eP449

eP130

eP112, eP113

eP642

eP007

eP452

eP597, eP157

eP033

eP692

eP452

eP079

eP268

eP165

eP366

eP175

PS039
Kosgeroglu, N.

Kothe, E.

Kotronoulas, G.

Kouloulias, V.

Koumakis, G.

Kouri, M.

Koutis, A.

Koutsikouri, E.

Kouvaris, I.

Kovacs, M.

Kremer, L.C.

Kreps, S.

Krizanova, K.

Kröner, A.

Kropf-Staub, S.

Krull, K.R.

Krumm, S.

Kruse, B.

Kubo, Y.

Kuipers, F.

Kumar, G.

Kumar, R.

Kumar, S.

Kung, S.

Kuo, D.Y.

Kuo, H.J.

Kurbacher, A.T.

Kurbacher, C.M.

Kurbacher, J.A.

Kurita, G.P.

Kurita, H.

Kurtz, J.E.

Kuruvilla, J.

Kusano, Y.

Kust, D.

Kusuba, Y.

Kusuhara, M.

Kuziemsky, C.

Kwok, A.O.L.

Kyrodimos, E.

Kyrtsonis, M.C.

L. Williams, J.

La Mola, L.

Labidi, S.

Lacey, J.

Lacouture, M.

Lacouture, M.E.

Laguerre, B.

Laheij, A.

Laheij, A.M.G.A.

Lai, F.M.

Lai, J.H.

Lai, J.S.

Lai, P.C.

Lai, Y.H.

Lai, Y.L.

Laigle-Donadey, F.

Lalic, N.

Lalla, R.

Lalla, R.V.

Lam, H.

Lam, L.

Lambrichts, I.
eP086

eP587

eP656

eP188, eP502

eP694

eP188, eP502, eP034, eP035, PS053,

PS054

eP110

eP500

eP188, eP502

eP645

eP292, eP293, eP294

eP319

eP260

eP401

eP096

PS082

PS068

eP494

eP250

eP176

eP137

eP340

eP580, eP581

eP400

eP532

eP155

eP449

eP449

eP449

eP220

eP274, eP280

eP576

eP601

eP238

eP208

eP061

eP669

eP689, eP690

eP069, eP263, eP446

eP188, eP502

PS053

eP381

eP341

eP395, eP510

eP081, eP082

eP227

eP453

eP523

eP185, eP199

eP268

eP497

eP077

eP503

eP504

eP412, eP129, eP589, eP609, eP610,

eP621

eP505

eP148

eP342

eP034, eP035

PS054

eP068

eP062

PS016 


\begin{tabular}{|c|c|c|c|}
\hline LaMola, L. & PS025 & LeVan, T. & eP137 \\
\hline Landers, M. & eP681 & Leverger, G. & eP009 \\
\hline Lane, $\mathrm{K}$. & eP114 & Levine, J. & $\mathrm{eP} 219$ \\
\hline Lange, $\mathrm{M}$. & eP506, eP507 & Levine, $\mathrm{M}$. & eP706 \\
\hline langin, $\mathrm{H}$. & eP073 & Lévy, C. & eP507 \\
\hline Langridge, J. & eP377 & Lhommé, C. & eP576 \\
\hline Lapid, M.I. & $\mathrm{eP} 400$ & Li, H.C.W. & eP599, eP141 \\
\hline Larible-Lefort, C. & eP658, eP659, eP657 & $\mathrm{Li}, \mathrm{J}$. & $\mathrm{eP} 264$ \\
\hline Larsen, R.F. & eP549 & $\mathrm{Li}, \mathrm{Q}$. & eP023 \\
\hline lartigau, E. & eP073 & $\mathrm{Li}, \mathrm{T}$. & eP222, PS049 \\
\hline Lasebikan, A. & eP515 & $\mathrm{Li}, \mathrm{Y}$. & eP022, eP023 \\
\hline Latifyan, S. & PS072 & Liakouli, Z. & eP188, eP502 \\
\hline Launay-Vacher, V. & eP523 & Liang, M. & PS012 \\
\hline Laurent, K. & $\mathrm{eP} 184$ & Liao, C.T. & eP705 \\
\hline Laviano, A. & eP259 & Licaj, I. & eP506, eP507 \\
\hline Lavoie Smith, E. & PS047 & Lighter, J. & eP184 \\
\hline Lawal, F. & eP103 & Lim, E. & eP574, eP574 \\
\hline Lawn, S. & eP595 & Lim, K.H. & eP057 \\
\hline Lawrance, I. & eP630 & Lim, L.H. & eP116 \\
\hline le deley, M.C. & eP073 & Lim, T. & eP381 \\
\hline Le Fel, J. & eP507 & Lin, C.C. & eP343 \\
\hline Le-Rademacher, J. & eP660, eP006, eP227 & Lin, C.Y. & eP505 \\
\hline Le, R. & eP569 & Lin, H. & eP666 \\
\hline Leanen, D. & PS072 & Lin, J.N. & eP664 \\
\hline Leão, I. & PS067 & Lin, J.P. & eP532 \\
\hline Leary, T. & eP335 & Lin, P.J. & $\mathrm{eP} 145$ \\
\hline LEBEDENCO-BOTEZ, M. & $\mathrm{eP} 232$ & Lin, Y.W. & eP189, eP277 \\
\hline LeBlanc, T.W. & eP017 & Linder, L. & PS021 \\
\hline Lebrun, F. & eP657, eP659 & Lindhart, C.L. & $\mathrm{eP} 233$ \\
\hline Lee, A. & PS037 & Lintzeris, $\mathrm{N}$. & eP008 \\
\hline Lee, $\mathrm{C}$. & PS037 & Lipp, E. & $\mathrm{eP} 226$ \\
\hline Lee, C.W. & eP650 & Lisy, K. & $\mathrm{eP} 424$ \\
\hline Lee, C.Y. & eP673 & Liu, B. & eP023 \\
\hline Lee, D.C. & $\mathrm{eP} 485$ & Liu, C. & $\mathrm{eP} 031$ \\
\hline Lee, G. & eP640 & Liu, C.Y. & eP541 \\
\hline Lee, G.E. & PS018, PS020 & Liu, D. & eP164, eP332, eP337 \\
\hline Lee, J. & eP661, eP347, eP410, eP435 & Liu, D.D. & eP057 \\
\hline Lee, J.B. & eP640 & Liu, G. & $\mathrm{PS} 012$ \\
\hline Lee, $\mathrm{K}$. & eP079 & Liu, J. & eP149, PS043 \\
\hline Lee, K.H. & eP079, eP156, eP221, eP225, eP435 & Liu, T. & eP045 \\
\hline Lee, K.M. & eP221, eP156, eP225, eP435 & Liu, W. & eP600, PS082 \\
\hline Lee, L.Y. & eP508 & Liu, X. & eP264 \\
\hline Lee, M.H. & eP673 & LIU, Y.H. & eP083 \\
\hline Lee, M.J. & eP672 & Livi, L. & $\mathrm{eP} 001$ \\
\hline Lee, M.S. & eP684 & Lloret, $\mathrm{T}$. & eP523 \\
\hline Lee, M.Y. & eP684 & Lo, C. & eP369 \\
\hline Lee, $\mathrm{S}$. & eP598 & Lo, Y.L. & eP532 \\
\hline Lee, S.C. & PS050 & Lobo, C.B. & $\mathrm{eP} 266$ \\
\hline Lee, S.H. & eP115, eP673 & Loeffen, E. & eP293, eP294 \\
\hline Lee, S.J. & eP592 & Loeffen, E.A. & eP292 \\
\hline Lee, S.N. & eP118 & Loew, J. & eP566 \\
\hline Lee, V. & eP662, eP663 & Logan, R. & $\mathrm{eP} 175$ \\
\hline Lee, Y. & eP591, PS022 & Logan, R.M. & PS039 \\
\hline Lee, Y.H. & eP413, eP139, eP485, eP589 & Loh, K.P. & eP603 \\
\hline lefebvre, G. & eP073 & Loh, K.W.J. & PS018 \\
\hline Légaré, F. & eP688 & Loh, W.J. & PS020 \\
\hline Léger, I. & eP507 & Lohning, A. & $\mathrm{eP} 245$ \\
\hline Leisenring, W.M. & PS082 & Lohrmann, C. & PS060 \\
\hline Leithold, C. & eP248 & Loi, M. & eP001 \\
\hline Lemonde, M. & eP555 & Loka, T. & PS071 \\
\hline Lenton, E. & eP601 & Lokare, A. & eP706 \\
\hline Leonel, A.P. & eP051 & Lolkema, M. & eP534, PS076 \\
\hline Lerebours, F. & eP507 & Lombard, J. & eP624 \\
\hline Lesser, M. & $\mathrm{eP} 402, \mathrm{eP} 495$ & Lommel, K.M. & PS082 \\
\hline Leung, C.H. & eP666 & Looker, S. & eP006 \\
\hline
\end{tabular}


Lopes Borges, M.

Lopes, C.

López Alarcón, M.D.

Lopez Saca, M.

López-Rojas, L.

Lopez-Rojas, L.A.

López-Vásquez, A.D.

Lopez, G.

Loprinzi, C.

Lorca Parraguez, L.A.

Lorenzo Cohen, L.C.

Lortholary, A.

Lorton, C.

Loscalzo, M.

Louw, S.

Lovati, E.

Lovell, M.

Lu, C.F.

Lu, C.W.

Lu, M.H.

$\mathrm{Lu}, \mathrm{Z}$.

Lucas, L.

Luckett, T.

Ludwig, C.

Lui, A.

Lundorff, L.

Luo, Y.

Luque, L.

Luthfi Kholisa, I.

Luu, M.

Luyten, D.

Lymn, K.

Lynch Kelly, D.

Lyon, D.

M'ghirbi, F.

Macedo, A.

Macpherson, C.F.

Madla, C.

Madureira, B.

Maes, A.

Mafodda, A.

Mafra, A.B.

Magarajah, G.

Magee, D.

Magony, A.

maguire, $\mathrm{A}$.

Mahler, C.

Mahon, C.

Mahore, R.

Mainali, A.

Maiya, A.

Majorana, A.

Makiura, D.

Makrantonakis, P.

Malaescu, D.G.

Malamud, D.

Mama, S.K.

Manalo-Igot, M.

Manalo, M.F.

Mandrekar, S.

Mangoni, A.

Mani, K.R.

Mann, S.

Manser, T.

Mansfield, P.
eP414

eP473

eP067

eP633

PS006, eP346

eP568

eP668

eP600

PS048, eP227, eP470, eP471

eP150

eP600

eP576

eP251

eP348

eP320

eP013

eP062

eP450

eP450

eP532

eP013, eP264, eP381

eP122, eP161, eP358, PS087

eP062

eP689, eP690

eP465

eP220

eP022

eP542

eP622

eP319

eP484

eP172

eP151

eP151

eP510

PS067

PS021

eP352

eP629

eP448, eP484, PS016

eP071

eP198

PS050

eP058

PS012

eP344

eP080

PS071

eP563

eP278

eP560

eP169, eP454

PS013, eP163

eP694

eP436, eP437, eP530

eP203

eP256

eP363

eP345

eP660

eP595

eP451

eP427

eP096

eP494
Mansour, A.

Mantik, M.

Manuela, E.

Manzano, J.G.

Manzullo, E.

Maragna, V.

Marc, K.

Marcusssen, M.

Marinescu, D.

Marinescu, I.

Marinho, J.

Marioli, A.

Marker, J.

marliot, G.

Maropoulos, G.

Marquette, S.

Marshall, A.

Marshall, S.

Marsiske, M.

Marten-Mittag, B.

Martin-Vasallo, P.

Martin, A.L.

Martin, D.

Martin, P.

Martins-Branco, D.

Martins, A.

Martins, B.

Martopullo, C.

Martucci, G.

Maruelli, A.

Marvin, D.G.

Marx, W.

Masakazu, A.

Masat, S.

Mashtoub, S.

Mason, $\mathrm{M}$.

Mastick, J.

Masuda, Y.

Masuzawa, Y.

Mathijssen, R.

Mathur, R.

Matkovic, S.

Matoba, M.

Matos, L.

Matos, P.

Matson, M.A.

Matsui, Y.

Matsumoto, S.

Matsumoto, Y.

Matsuoka, Y.

Matsuura, E.

Matthews, L.

Mattiucci, C.

Mattsson, $\mathrm{T}$.

Matuoka, J.

Matus-Santos, J.

Matus-Santos, J.A.

Matus-Santus, J.

Mauceri, R.

Maung, S.

Mauramo, M.

Mauries-Saffon, V.

May, S.

Mayer, $\mathrm{H}$.

Mayo, B.
eP685

eP287

eP525

eP666

PS044

eP001

eP323

eP034, eP190

eP436, eP437

eP436, eP437, eP530

PS067

eP110, eP500

eP686

eP073

eP188, eP502

eP484

eP706

eP244, eP245

eP442

eP426

eP602

eP507

eP468, eP469, PS080

eP320

eP419

eP325

eP422

eP323

eP428

eP428

eP430

eP244, eP245

eP011

eP415, eP416, eP411, eP654

eP210, eP630

eP408

eP219

eP531, eP669

eP084

eP534

eP304

eP458

eP132

eP325

eP438, eP439

eP312

eP007

eP132

eP373, eP460

eP418, PS075

eP061

eP408

eP020

eP233

eP220

PS006

eP568

eP346

eP098

PS020

eP279

eP041

eP408

eP113, eP653

eP197 
Mayo, S.

Mazzetti, M.

Mazzocato, C.

McAuliffe, P.

McCaffrey, N.

McCarthy, A.

McCarthy, $\mathrm{H}$.

McCrae, C.S.

McCutcheon, C.

McGregor, I.

McGuire, A.

Mckenna, N.

McKenzie, M.

McLellan, B.

McMahon, D.

McQuade, R.M.

McRobert, E.

Mead, K.

Meador, P.

Meador, R.

Mebis, J.

Megalakaki, A.

Mehdizadeh, M.

Mehmi, I.

Mehranfar, S.

Mehta, K.

Mehta, Z.

Mehvar, R.

Mei, Y.

Meiri, D.

Mejri, N.

Melo Cruz, F.

Members of the Study

Advisory Committee, S.A.C.

Mendoza-Galindo, L.

Meng, $\mathrm{R}$.

Mennillo, $\mathrm{H}$.

Mennillo, L.

mensah, K.B.

Mercieca-Bebber, R.

Mercuri, M.

Mersiades, A.

Mesleard, C.

Metcalfe, E.

Meyer, D.

Meyer, G.

Meyer, J.

Meza, J.

Mezni, E.

Mi-Jung, K.

Miaskowski, C.

Miccinesi, G.

Michalski, W.

Middleton, B.

Migliorati, C.

Miguéns, $\mathrm{M}$.

Mihaljevic, B.

Mikirova, N.

Miller, A.H.

Miller, R.

Minet, L.R.

Ming-Hsiou, L.

Minjeong, $\mathrm{P}$.

Minowa, C.

Mintus, $\mathrm{K}$.
eP601

eP481

eP525

eP529

eP062

eP239, eP432

eP339, eP648

eP421, eP442

eP638

eP008

eP239, eP432

eP344

eP588

eP518, PS017

eP117, eP262

eP223

eP379, PS024

eP175, PS039

eP046

eP045

eP484, PS016

PS053

eP016

PS026

eP509

PS017

eP144

PS070

PS066

PS032

eP510, eP395

eP142

eP527

eP346, eP568, PS006

eP595

eP306

eP305

eP667

eP587

eP645

eP008

eP507

eP086

eP427

eP645

eP507

eP137

eP510

eP683

eP147, eP219

eP428

eP196

eP347

eP034, eP035, PS054

eP325

eP459

eP511

PS019

eP542

eP548, eP549

eP444, eP564

eP332

eP417

eP623
Miranda-Silva, W.

eP198

Miranda, M.H

eP628

Mirmohammadirad, A. eP121

Mishima, Y.

Mishra, S.

Mitchell, L.

Mitnik, I.

Mitobe, Y.

Mitsantisuk, P.

Mitsuhashi, N.

Mitsunaga, S.

Mittal, D.

Miura, S.

Miura, T.

Miyaji, T.

Miyamoto, T.

Mizutani, Y.

Moawad, $\mathrm{H}$.

Mohammed, J.

Mohan, M

Mohandoussaid, A.

Mohile, S.

Molassiotis, A.

Mols, F.

Momo, K.

Monahan, $\mathrm{P}$.

Monceau-Baroux, L.

Mondrup, L.

Monreal-Carrillo, E.

Monreal, E.

Monsarrat, T.

Monteiro, L.

Montezari, $\mathrm{H}$.

Moon, D.

Moon, $\mathrm{H}$.

Mooney, S.

Moore, I.M.

Moore, $\mathrm{K}$.

Morales, M.

Moran, J.

Moran, S.

Morand, K.

Moreira Pinto, A.

Moreira, M.C.R.

Morgado, M.

Morgado, S.

Mori co-primary author, $\mathrm{M}$.

Mori, K.

Mori, M.

Mori, N.

Morikawa, A.

Morishita-Kawahara, M.

Morishita, A.

Morishita, S.

Morita, T.

Morrow, G.

Morton, R.

Motoo, Y.

Mouffak, S.

Mougeot, F.

Mougeot, J.L.

Mouhawej, M.C.

Mourão, A.M.

Mouri, T.

Moursi, A.
eP238

eP059, eP060

$\mathrm{PS} 012$

eP211

eP512

eP692

eP559

eP253, eP559

eP347

eP253, eP559

eP531

eP183

eP250

eP033

eP282

eP620

eP474

eP283

eP327, eP603

eP245

eP522, PS011, PS084

eP183

eP535

eP252

eP005

eP346, PS006

eP568

eP072

eP419

PS070

eP427

PS005

eP348

PL001

eP344

eP602

eP377

eP468, eP469, PS080

eP009

PS067

eP266

eP473

eP473

eP418

eP250, eP253, eP559

PS075, eP011

eP253

eP253, PS064

eP349

eP061, eP250

eP512, eP556, eP557

eP133, eP183, eP418, eP531, PS075

PS043

eP008

eP084

eP009

eP191

eP191

eP311

eP628

eP253

eP184 


\begin{tabular}{|c|c|c|c|}
\hline Moya, J. & eP203 & Ng, R.C.H. & PS018 \\
\hline Mpokas, A. & eP694 & Ngan, M.P. & eP013 \\
\hline Mrklas, $\mathrm{K}$. & eP638 & Ngelangel, C. & eP363 \\
\hline Muckaden, M. & eP099, eP637 & Ngo-Huang, A. & eP552 \\
\hline Muckaden, M.A. & eP353 & Nguyen Thi Kim, H. & eP119 \\
\hline Mueller, A. & eP080 & Nguyen, $\mathrm{K}$. & $\mathrm{eP} 332$ \\
\hline Muhammad Wali, R. & eP297 & Niazi, S.K. & $\mathrm{eP} 400$ \\
\hline Muhtadi, O. & eP526 & Nicolatou - Galitis, O. & eP502 \\
\hline Mukelabai, M. & $\mathrm{eP} 295$ & Nicolatou-Galitis, O. & eP035, PS052, eP034, eP188, PS053, \\
\hline Mukeshimana, M. & eP516 & & PS054 \\
\hline Mukhopadhyay, S. & eP010, eP192, eP152 & Nicolotti, M. & $\mathrm{eP} 273$ \\
\hline Mulder, R.L. & eP294 & Nielsen, D. & eP676 \\
\hline Mulyowa, I. & eP350 & Nieto-Coronel, M.T. & eP668 \\
\hline Munoz, M. & eP071 & Nightingale, K. & eP203 \\
\hline Murakami, H. & eP011 & Nigro, $\mathrm{O}$. & eP193 \\
\hline Murakami, S. & eP460 & Niihara, $\mathrm{M}$. & eP695 \\
\hline Murata, M. & eP373 & Nikitina, T. & PS083 \\
\hline Murielle, R. & eP398 & Nilmanat, K. & eP351, eP440, eP441, eP604 \\
\hline Murphy, B. & PS009 & Nishihara, A. & $\mathrm{eP} 238$ \\
\hline Murphy, D. & eP681 & Nishimura, N. & $\mathrm{eP} 238$ \\
\hline Murphy, R.T. & eP135, eP136 & Nisida, R. & eP611 \\
\hline Musch, R. & eP234 & Nixon, J. & eP513, PS045 \\
\hline Musettini, G. & eP236, eP235, eP237, eP682 & Niyomthai, N. & eP351 \\
\hline Mustian, K. & eP145, eP603 & Njoroge, C. & $\mathrm{eP} 243$ \\
\hline Mutluay Yayla, E. & eP085 & Noé, L. & eP484, PS016 \\
\hline Nabi, R.U. & $\mathrm{eP} 451$ & Nonoyama, M. & eP555 \\
\hline Nabiee, $R$. & PS070 & Nordly, M. & $\mathrm{eP} 220$ \\
\hline Näf, E. & eP096 & Nordman, I. & $\mathrm{eP} 624$ \\
\hline Nagaoka, $\mathrm{H}$. & eP183 & Nozaki, M. & eP611 \\
\hline Naing, A. & eP465 & Nozomu, M. & eP647 \\
\hline Naito, T. & eP253, PS036, eP084, eP559, PS064 & Ntokou, A. & PS053 \\
\hline Nakagawa, T. & $\mathrm{eP} 214$ & Nugent, K. & eP528 \\
\hline Nakai, M. & $\mathrm{eP} 242$ & Nukada, T. & $\mathrm{eP} 238$ \\
\hline Nakajima, K. & eP452 & Numico, G. & eP273, eP584 \\
\hline Nakajima, N. & eP254, eP255 & Nunes Marques, J. & $\mathrm{eP} 325$ \\
\hline Nakamichi, T. & eP557 & Nural, N. & eP100, eP317, eP318, eP488 \\
\hline Nakano, J. & eP061, eP550 & Nurgali, K. & $\mathrm{eP} 223$ \\
\hline Nakashima, K. & eP011 & Nwankwo, K. & eP515 \\
\hline Nakayama, T. & eP084 & Nwogu, C. & eP515 \\
\hline Nam, E.M. & eP118 & o leary, E. & eP029 \\
\hline Nangia, C. & PS037 & O' Leary, N. & $\mathrm{eP} 026$ \\
\hline Nangia, J.R. & eP453 & O'Brien, $\mathrm{H}$. & $\mathrm{eP} 224$ \\
\hline Nanton, V. & eP566 & O'Brien, $\mathrm{T}$. & $\mathrm{eP} 429$ \\
\hline Naqvi, S. & eP332, eP381 & O'Connor, B. & $\mathrm{eP} 153$ \\
\hline Nascimento, M. & eP419 & O'Gorman, A. & eP044 \\
\hline Nasr, F. & eP241 & O'Hare, P. & PS071 \\
\hline Nassairirad, S. & PS070 & O'Higgins, C.M. & $\mathrm{eP} 153$ \\
\hline Natal Jorge, R. & eP024, eP025 & O'Mahony, D. & PS069 \\
\hline Nates, J. & eP333 & O'Reilly, S. & PS069 \\
\hline Natsuzako, A. & eP550 & Oataway, K. & eP377 \\
\hline Navari, R. & eP017 & Oberoi, H.K. & $\mathrm{eP} 230$ \\
\hline Nayak, R. & eP563 & Obrecht, S. & $\mathrm{eP} 327$ \\
\hline Nazare, M. & eP461 & Ocampo, B. & eP357 \\
\hline Neergaard, M. & eP005 & Ochoa, $\mathrm{H}$. & eP514 \\
\hline Negami, R. & eP160 & Ochoa, M. & eP348 \\
\hline Negrini, F. & eP481 & Ogawa, $\mathrm{H}$. & eP556 \\
\hline Nelson, A. & eP376 & Oguma, Y. & eP669 \\
\hline $\mathrm{Neo}, \mathrm{S}$. & eP380 & Oh, G.H. & $\mathrm{eP} 225$ \\
\hline Newman, S. & eP334 & Oh, J.M. & $\mathrm{eP} 290$ \\
\hline Newton, R.U. & eP582 & Ohe, Y. & eP011, eP460 \\
\hline $\mathrm{Ng}, \mathrm{A}$. & eP552 & Ohlsson-Nevo, E. & eP558 \\
\hline Ng, C.H. & $\mathrm{PS} 020$ & Ohno, S. & $\mathrm{eP} 452$ \\
\hline Ng, C.W. & eP157 & Ohri, N. & eP518, PS017 \\
\hline Ng, D.K.H. & eP263, eP446 & Ohyama, T. & eP452 \\
\hline Ng, I.Y.Y. & $\mathrm{eP} 446$ & Ojeyinka, A.A. & $\mathrm{eP} 492$ \\
\hline
\end{tabular}


Ok, O.

Okawa, A.

Okayama, T.

Okere, $\mathrm{P}$.

Okita, M.

Okizaki, A.

Okoye, I.

Okui, R.

Okumura, M.

Okwor, C.

Okwor, V.

Olagunju, A.T.

Oldenmenger, W.

Olek, E.

Olga, T.

Oliveira, A.F.

Oliveira, A.R.

Oliveira, C.

Olsen, M.

Olver, I.

Omae, K.

Onchan, W.

Oner, I.

Ong, J.N.

Onishi, H.

Onitilo, A.

Ono, R.

Onyeka, T.

Oo, T.

Oomen-de Hoop, E.

Oosthuizen, F.

Oostra, D.L.

Orlu, N.

Ortner, P.

Orye, G.

Osborn, M.

oshiro, M.

Oskay-Oezcelik, G.

Ostermann, $\mathrm{H}$.

Ostrin, E.

Ostwal, S.

Otagiri, $\mathrm{H}$

Ottaviani, G.

Ottery, F.D.

Ottesen, $\mathrm{S}$.

Overgaard, D.

Ovsak Contributed Equally, G.

Owens, C.

Owosho, A.

Oyamada, S.

Özcan, İ.

Ozdemir, L.

Ozen, A.

Özençakır, H.

Ozkaraman, A.

Ozturk, E.

Öztürk, S.

Padron- B.S., A.

Padron, A.

Padula, G.

Paesmans, M.

Paik, E.S.

Paiva Fonseca, F.

Paiva, B.

Paiva, C.
eP598

eP094

eP559, eP253, PS064

eP515

eP550

eP531

eP515

eP021

eP163

eP515

eP515

eP396, eP492

eP534

eP468, eP469, PS080

PL001

eP024, eP025

eP439

eP419

PS068

eP008, eP686, PS059

PS064, eP253, eP559

eP352

eP704

eP328

eP512

eP227

eP163, PS013

eP515

eP627

eP534

eP667

eP256

eP120

eP521

eP484

eP686

eP670

eP014

eP173

eP107, eP108, eP109

eP353

eP274

eP194, eP195, eP181

eP259

eP626

eP676

PS079

eP285

eP103

eP183

eP027

eP078, eP085

eP086

eP409

eP086, eP120, eP533

eP086

eP100

eP420

eP421

eP603

PS072

eP012

eP198

eP354, eP355, eP422, eP423, PS062

eP354, eP355, eP422, eP423, PS062
Pal, S.

Pandya, C.

P348

Pang, G.

Pang, L.

Pannekoeke, L.

Pannekoeke, P.

Panta, S.

Pântano, N.

Panzarella, V.

Paolieri, F.

Papadopoulou, E.

Papakostas, P.

Papakotoulas, P.

Papandreou, C.

Papastavrou, E.

Papastavrou3, E.

Papatsimpas, G.

Pappot, H.

Paragas, J.

Paramanandam, V.S.

Pardon, K.

Park, J.

Park, J.C.

Park, K.E.

Park, K.U.

Park, M.

Park, S.

Park, S.R.

Park, Y.H.

Parsa, P.

Pascu, A.M.

Pasetka, M.

Passweg, J.

Pastrana Uruena, T.

Patel, M.

Patel, P.

Patel, S.D.

patnaik, N.

Patrick, $\mathrm{H}$

Pattamapaspong, $\mathrm{N}$.

Paul, S.

Pauline, U.

Paulo, C.

Paunero-Quezadas, M.

Pavlakis, M.

Pawar, C.

Payne, $\mathrm{H}$.

Payne, S.

Pedetti, V.

Pedrazzoli, P.

Peer, A.

Peguero, J.

Pei-Ling, K.

Peker, S.

Pelotte, E.

Peña-Nieves, A.

Peña, A.

Pentheroudakis, G.

Pepe, C.

Peppone, L.

Pereira- Ph.D., D.B.

Pereira, D.B.

Pereira, J.

Perera, R.

eP696
eP327

eP374

eP164

eP448, PS016

eP174

eP226

PS062

eP098

eP237, eP235, eP236, eP682

PS053, eP034, eP035, eP188, eP502, PS054

eP694

eP694

eP694

eP671, PS003

eP694

eP466

eP328

eP672

eP321, eP322

eP162

eP337

eP162

eP368

eP143, eP337, eP552, eP661

eP222, PS049

eP683

eP673

eP121

PS014

eP209

eP279

eP633

eP527, eP607

eP045

eP356

eP050

eP226

eP352

eP147, eP219

eP516

PS012

eP430

eP261, eP693

eP563

eP408

eP685

eP020

eP071

PS032

PS026

eP444, eP564

eP533

eP657, eP659

eP357

eP698, eP674

eP694

eP499

eP603, eP145

eP420

eP389, eP442

eP325

PS008 
Pérez-Camargo, D.

PEREZ, D.

Perloff, T.

Perna, M.

Pesantez, D.

Peters, K.

Peters, M.

Peters, $\mathrm{S}$.

Petersen, A.C.

Petit, A.

Petranovic, A.

petranovic, D.

Petrelli, F.

Petrone, M.

Petrucci, J.

Peyroteo, M.M.A.

Phillips, B.

Phillips, J.

Phillips, R.

phinitkhajorndech, N.

Phornphibul, A.P.D.P.

Piacenti, S.

Pichler, T.

Pietra, C.

Pigott, A.

Piil, K.

Pillay, B.

Pineda, B.

Pirscoveanu, D.F.V.

Pissarra, A.J.

Pitson, G.

Plotkin, E.

Podjamanpong, $\mathrm{P}$.

Poemmerl, M.

pokpalagon, $\mathrm{P}$.

Ponte, S.

Pontieri, J.

Poon, C.Y.

Popat, U.

Pophali, P.

Popovic, I.

Poprawski, D.

Porfir'eva, N.

Poroch, V.

Poropat, A.

Porter-Steele, J.

Posadas- Jr., R.

Postolica, R.

Postupack, R.

Postupack, R.A.

Potting, C.

Poulsen, M.

Pouymayou, J.

Power, D.G.

Prado, B.

Pralong, W.

Prapa, P.

Price, K.

Price, L.

Price, $T$.

Prinja, P.

Prityko, D.

Pritzkuleit, R.

Proctor, G.

Prosk, E.
eP568, PS006, eP346

eP257

PS087, eP161

eP001

eP230

eP226

eP424, eP200

eP096, eP525

eP223

eP009

eP425

eP425

eP071

eP481

eP507

eP024

eP231

PS046, eP062

PS028, PS073

eP359

PS042

eP481

eP426

eP013

eP513

eP676

eP427

eP345

eP530

eP325

eP324

eP122, eP358

eP351

eP521

eP359

eP628

eP184

eP263, eP446, eP069

eP167, PS058

eP569

PS088

eP686

PS083

eP360, PS014

eP181, eP194, eP195

eP239, eP432

eP345

eP360

eP442

eP421

eP201

eP677

eP041

PS069

eP381

eP149

eP110

eP333

eP537, eP445, eP565, eP617

eP686

eP152

eP182

eP612

eP201

PS031, PS034
Protonotarios, D.

Prudhomme-Hunter, J.

eP261

Prulhiere, K.

eP348

eP576

PS, S.

Psyrri, A.

Pundole, X.

Punt- M.D- PhD, C.

eP474

eP188, eP502

eP462

PS055

eP604

eP304

PS057

eP366

eP600, PS007

eP022

eP702

eP203

PS043

eP678

eP481

PS038

eP199

eP006

PS015, eP296, PS027, PS030

PS014

eP050, eP258, eP258

PS015

eP507

eP296, PS030, PS027, PS015

PS006, eP568, eP346

eP148

eP266

eP419

eP170

eP284

eP481

eP624

eP226

eP475

eP015

eP645

eP308

rassouli corresponder author,

M.

Rassouli, M. eP361

Rathi, N. eP333

Rattananont Ferris, P. eP161, PS087

Rau, K.M. eP505

Rauenzahn Cervantez, S. eP315

Ravaud, A. eP678

Read, A. eP062

Reddy, S. eP338

Reed, K. eP362

Reeves, P. eP582

Regan, J. eP249

Reilly, R.B. eP153

Remaley, A. eP569

Renner, P. eP074

Repousis, P. PS053

Restrepo, A. PS026

Reyes Donoso, M. eP633

Reyes-Habito, C.M. eP363

Reyna, R.N. eP051

Rezai, S. eP465

Rha, S.Y. eP661

Ribi, K. eP606

Ricard, D. eP148

Ricci, S. eP235, eP236, eP237, eP682 


\begin{tabular}{|c|c|c|c|}
\hline Riccio, I. & eP046 & Rummans, T.A. & $\mathrm{eP} 400$ \\
\hline Rice, A. & eP153 & Rumyantsev, A. & PS083 \\
\hline Richards, A. & eP175, PS039 & Rupel, K. & eP181, eP194, eP195 \\
\hline Richardson, A. & eP284, eP527, eP607 & Rustøen, $\mathrm{T}$. & eP680 \\
\hline Richardson, M. & eP107, eP108, eP109 & Ryan Wolf, J. & PS070 \\
\hline Rico, V. & eP068 & Ryan, A. & eP586 \\
\hline Ridgeway, J. & eP006 & Ryan, T. & eP184 \\
\hline Ried, K. & eP245 & Rybalka, E. & eP223 \\
\hline Rieder, E. & eP364 & ryoo, H.M. & eP087, eP368 \\
\hline Rietjens, J. & eP366 & Ryu, M.H. & eP115 \\
\hline Rigal, O. & eP507 & Rzepecki, A. & eP518, PS017 \\
\hline Rings, E. & eP200 & Saab, M. & eP681 \\
\hline Rios-Ochoa, L.A. & eP430 & Saarto, T. & eP517 \\
\hline Ripamonti, C. & eP259, eP428, eP034, eP154 & Saba, N.F. & PS019 \\
\hline Risendal, B. & eP606 & Sabry, N.M. & eP179 \\
\hline Risteski, M. & eP208 & Sacomori, C. & eP150 \\
\hline Rithara, S.M. & $\mathrm{eP} 123, \mathrm{eP} 365$ & Saeed, H. & eP297 \\
\hline Ritter, P. & eP150 & Safaeian, R. & $\mathrm{eP} 210$ \\
\hline Rivera, M. & eP357 & Sagar, T. & PS015 \\
\hline Riveros Rios, M. & eP633 & Sagar, T.G. & eP296, PS027, PS030 \\
\hline Robb, K. & eP565, eP537 & Sagawa, N. & $\mathrm{eP} 452$ \\
\hline Robertson, E. & eP429 & Sağlam, Z. & eP411, eP415, eP416 \\
\hline Robijn, L. & eP366 & Saha, U. & eP369 \\
\hline Robijns, J. & PS016 & saina, $\mathrm{C}$. & eP124 \\
\hline Robinson, M.E. & eP421, eP442 & Saito, T. & eP163 \\
\hline Robison, L. & eP623 & Sajjad, S. & eP519 \\
\hline Robison, L.L. & PS082 & Sakai, H. & $\mathrm{eP} 280$ \\
\hline Rocha, V. & eP198 & Sakai, Y. & eP163, PS013 \\
\hline Rodin, G. & eP369 & Sakamoto, J. & eP550 \\
\hline Rodrigues Fregnani, E. & eP198 & sakji, I. & eP073 \\
\hline Rodriguez Chamorro, A. & eP602 & Sakr, L. & eP499 \\
\hline Rodríguez-Mayoral, O. & eP430, eP431 & Sakurai, M. & eP011 \\
\hline Rodríguez, A. & eP047, eР679 & Salah, S. & eP282 \\
\hline Rodriguez, G. & eP605 & Salamat, A. & eP529 \\
\hline Roeland, E. & eP018 & Saligan, L. & PS079 \\
\hline Roeland, E.J. & eP017 & Saligan, L.N. & eP149 \\
\hline Roemer-Becuwe, C. & eP576 & Salins, N. & eP099, eP353, eP637 \\
\hline Roffel, S. & eP199 & Saltürk, Z. & eP488 \\
\hline Rogers, L. & eP344 & Samanta, B. & eP010 \\
\hline Roh, J. & eP079 & Samantas, E. & eP694 \\
\hline Röhrl, K. & eP680 & Samarasinghe, S. & $\mathrm{eP} 284$ \\
\hline Roine, E. & eP517 & Samoon, Z. & eP520 \\
\hline Rojas-Hernandez, C. & eP627 & Samuel, S. & eP560 \\
\hline Rojz, J. & eP482 & Sanal Yimaz, B. & eP619 \\
\hline Rolski, W. & eP196, eP367 & Sancakli, E. & eP267 \\
\hline Ron, M. & PS076 & Sanches Panobianco, M. & eP414, eP665 \\
\hline Ronan, G. & PS069 & Sanchez, S. & eP159 \\
\hline Roscoe, J. & eP566 & Sanderson, P. & $\mathrm{eP} 434$ \\
\hline Roseman, J. & eP305 & Sandvad, M. & eP220 \\
\hline Roshanaiee, G. & eP121 & Sanfilippo, N. & eP184, eP203 \\
\hline Rosin, F.C.P. & eP177, eP178 & Sanli, Y.T. & eP703 \\
\hline Ross, A. & PS079 & Santos, A.S. & eP629 \\
\hline Rossi, M. & eP584 & Santos, S. & $\mathrm{eP} 270$ \\
\hline Rosti, G. & eP071 & Sanz Yagüe, A. & eP067 \\
\hline Rostock, M. & eP075 & Sao, J. & PS008 \\
\hline Roulston, F. & eP251 & Sarangdhar, M. & $\mathrm{eP} 462$ \\
\hline Rowland, C. & eP393 & Sargon, M.F. & $\mathrm{eP703}$ \\
\hline Rozario, N. & eP191 & Sarraf, S. & $\mathrm{eP} 165$ \\
\hline Rozario, N.L. & eP268 & Sasaki, A. & eP007, eP275 \\
\hline Rozema, F. & eP185, eP199 & Sathiya, K. & eP447 \\
\hline Rozema, F.R. & eP268 & Satoshi, H. & eP647 \\
\hline Ruco, A. & eP369 & Sattar, S. & PS057 \\
\hline Rudd, J.A. & eP013 & Saunders, D. & PS037, PS054 \\
\hline Ruddy, K. & eP227, eP017 & Savard, J. & eP403, eP404 \\
\hline Rugo, H.S. & $\mathrm{eP} 453$ & Savina, S. & eP382 \\
\hline
\end{tabular}


Savoye, A.M.

Sayed, H.

Sbrana, A.

Scaife, J.

Scerri, J.

Scharll, M.F.

Schenker, M.

Schenker, R.A.

Schiavetti, A.

Schilling, J.

Schmidt, E.B.

Schmidt, F.

Schmidt, H.

Schmidt, S.

Schmitz, K.H.

Schneck, M.J.

Schneeweiss, A.

Schofield, P.

Schöley, J.

Schols, J.

Schoormans, D.

Schultz, G.

Schulze, P.

Schwartzberg, L.

SCOTTE, F.

Scotti, V.

Sebag-Montefiore, D.

Sebastiani, S.

Secombe, K.

Segers, A.

Seib, C.

Seiler, C.

Seki, Y.

Sekine, K.

Selway, C.

Sen, E.

Sengupta, A.K.

Senn, B.

Seo, S.

SEOL, S.M.

Seol, Y.

Serena, A.

Sete, C.

Setiyarini, S.

Seto, Y.

Seymour, J.

Shafquat, R.

Shahrokni, A.

Shalaby, L.

Shamieh, O.

Shand, M.

Shannon, V.

Sharma, B.

Sharma, D.

Sharp, L.

Sharplin, G.

Shaw, C.

Shaw, T.

Sheinman-Yuffe, H.

Shelton, B.

Shelton, J.

Shen, M.H.

Shi, M.

Shih-Chieh, S.

Shimizu, C.

\section{eP576}

eP125

eP235, eP682, eP236, eP237

PS045

eP391

PS072

eP436, eP530

eP437, eP530

eP020

eP014, eP521

eP370

eP606

eP528

eP315

eP256

eP465

eP080

eP424

eP233

PS060

eP522

eP421

eP200

eP019, eP018, PS026

eP523

eP001

eP528

eP019

eP197, PS080, eP468, eP469

eP645

eP432, eP239

eP327

eP084

eP460

eP172

eP704

eP524, eP451

eP653

eP683

eP684

eP371

eP525

eP142

eP622

eP472

eP366

eP519

eP165

eP288

eP526, eP685

eP434

eP463, eP464, PS065

eP063

eP489

eP579

eP686

eP372

eP062

eP211

PS068

eP045

eP539

eP645

eP444

eP418, PS075
Shimoji, T.

Shin, D.M.

Shin, H.Y.

Shin, J.H.

Shin, K.

Shin, S.J.

Shinozaki, T.

Shiow-Ching, S.

Shirren, J.

Shivananda, S.

Shrimpton, D.

Shu-Chiung, L.

Shueng, P.W.

Shum, W.M.

Shun, S.C.

Shwe, M.

Sibata, M.

Sidhu, R.

Sieverding, M.

Silva, C.C.

Silva, T.D.B.

Silvia, L.T.

Sim, S.H.

Simes, J.

Simon, J.

Simonin, M.

Singh Rana, S.P.

Singh-Carlson, S.

Singh, $\mathrm{H}$.

Singh, P.B.

Singh, R.P.

Singh, S.

Singhal, N.

Sinnarajah, A.

Sintonen, $\mathrm{H}$.

Sirachainan, E.

Sirilerttrakul, S.

Sisoix, C.

Siu, K.Y.

Sivell, S.

Sjøgren, P.

Skaare, A.

Skaczkowski, G.

Skeat, J.

skoett, R.

Skripekova, A.

Slaets, J.

Sloan, J.A.

Small, D.

Small, I.A.

Småstuen, M.C.

Smiljanic, M.

Smit, T.

Smith, A.

Smith, D.

Smith, E.C.

Smith, P.

Smits, C.

Smylie, J.

Sodergren, S.

Soga, Y.

Sohn, J.

Soivong, A.P.D.P.

Soliman, R.M.

Soluk Tekkeșin, M.
eP669

PS019

eP290

eP684

eP552

eP118

eP373

eP140

eP205, eP468, eP469, PS080

eP374

eP335

eP444, eP564

eP532

eP394

eP155

PS018

eP089

eP348

eP691

eP178

eP266

eP326

PS005

eP008

eP323

eP009

eP375, eP064

eP315

eP687

PS010

eP687

eP064, eP375

eP686

eP323

eP517

eP486

eP486

eP398

eP394

eP376

eP220

PS010

eP377, eP433, eP434

PS008

eP677

eP260

eP166

eP400

eP499

eP170

eP680

eP459

eP015

eP285

PS009, eP470, eP471

PS012

eP527, eP607

eP166

eP688

eP527, eP528, eP607

eP275

eP640

PS042

eP288

eP027 
Somers, V.

Son, K.L.

Sønderkær, M.

Song, J.

Sonis, S.

Soran, A.

Sorel Lazarovici, T.

Souggleri, M.

Spaeth, D.

Spahic, H.

Speksnijder, C.

Spence, D.

Spirig, R.

Spoelstra, S.

Sretenovic, A.

Sripan, P.

Srivastava, D.

Stacey, D.

Stapf, A.

Startkweather, A.

Stathopoulos, C.

Staudenmaier, T.

Steel, A.

Steer, C.

Stegenga, K.

Steindorf, K.

Steinmann, D.

Stensvold, E.

Stevens, J.

Stewart, G.

Stockler, M.

Stödter, M.

Stojanovska, V.

Storey, S.

Storniolo, A.M.

Storrick, E.

Stovicek, P.O.

Strasser, F.

Stringer, A.

Stroehlein, J.

Study Advisory Committee,

M.

Stuiver- PT- PhD, M.

Stump, T.

$\mathrm{Su}$, Y.C.

$\mathrm{Su}$, Y.T.

Suarez-Almazor, M.

Suarez-Almazor, M.E.

Subbiah, I.

Subbiah, V.

Subramaniam, S.

Sugiyama, M.

Sui, J.S.Y.

Sun, J.

Sun, M.

Sun, R.

Sundar, R.

Surinach, A.

Susnjar, S.

Sutton, C.

Suwannoi, L.

Syrigos, K.

Szecsenyi, J.

Szymanski, G.
PS016

eP435, eP156, eP221, eP225

eP190

eP107, eP108, eP109

eP171, PS037, PS079

eP529

eP036

eP694

eP523

eP593

eP561

eP595

eP401

PS057

eP459

eP352

PS082

eP688, eP689, eP690

eP075

eP151

eP261

eP173

eP072

eP455

PS021

eP691

eP075

PS010

eP523

eP251

eP008

eP248

eP223

eP608

eP623

eP660

eP436, eP437, eP530

eP370, eP253

eP197

eP167, PS058

eP607

PS055

eP535

eP609

eP610

eP666

eP462

eP465, eP475

eP465, eP475

eP597

PS064

PS069

eP264

eP167, PS058

eP646, eP384, eP573, eP618, eP634,

eP700

PS050

eP017

eP458, PS086

eP114

eP692

eP110, eP500

eP080

PS068
Tabea, M.

Tachibana, J.

Tagami, K.

Taha, T.

Taib, N.A.

Tajiri, H.

Tajiri, K.

Takahiro, T.

Takashi, S.

Takayama, K.

Takeshi, K.

Taljaard, M.

Talwar, V.

Tam, W.

Tamai, E.

Tamai, N.

Tamamyan, G.

Tan, A.

Tan, C.J.

Tan, D.

Tan, J.Y.

Tan, S.

Tan, T.J.Y.

Tan, W.

Tan, Y.Y.

Tanabe, M.

Tanada, D.

Tanaka, H.

Tanaka, T.

Tane, K.

Tanem, K.E.

Tang, C.

Tangteerakoon, P.

Tanikella, R.

Tanuma, A.

Tao, F.

Tao, Z.

Taranto, P.

Tarawneh, M.

Tashiro, T.

Tatematsu, N.

Tauber, D.

Tavakoli-Ardakani, M.

Tavares, R.

Taylor, R.

Tegegn, $\mathrm{H}$.

Tegos, $\mathrm{T}$.

Telzerow, E.

Temtap, S.

ten Berg, S.

ten Bohmer, K.

ten Hoopen, $\mathrm{S}$.

Tenner, L.

Tennison, J.

Teo, I.

Terakado, $\mathrm{H}$.

Terui, Y.

Thakor, N.

Tharavichitkul, E.

Thavorn, K.

The COSTaRS Team, A.

Thielens, S.

Thirunavukarasu, M.

Thirwall, J.

Thomas, R.
eP248

eP065

eP531

PS032

eP157, eP597

eP554

eP554

eP647

eP214

eP253, eP559

eP647

eP688

eP050, eP258

eP574

eP669

eP611

eP388

eP227

PS018, PS020

eP380

PS020

PS050

PS018

eP126, eP127, eP034, eP035, eP400

eP385

eP472

eP088

eP623

eP556, eP557

eP529

PS010

eP475

eP486

eP392, eP393

eP554, eP695

eP264

eP476

eP481

eP685

eP132

eP253, eP559

eP211

eP016

eP438, eP439

eP284

eP049

eP261, eP693

eP426

eP440, eP441

eP293

eP199

eP201

eP315

eP562

eP380

eP011

eP238

PS050

eP352

eP690

eP689, eP690

eP174

eP447

eP706

eP585 
Thong, M.

Thong, M.S.Y.

Threatt, S.

thu-kar, S.

thulkar, S.

Tian, $\mathrm{X}$.

Tiernan, E.

Timmermans, A.

Timmins, $\mathrm{H}$.

Timmons, A.

Timon, C.

Tissing, W.

Tissing, W.J.

Tissing, W.J.E.

Toffolatti, L.

Toftdal, S.

Tognela, A.

Toh, Y.L.

Tohme, A.

Toi, M.

Toledo Ahumada, G.

Tolentino, V.

Tolstrup, L.K.

Tomaszewski, K.

Tomoya, Y.

Torres, S.

Tourkantonis, G.

Tran, A.T.

Tran, J.

Tredan, O.

tresch, E.

Trichas, M.

Tricou, C.

Trinastic, L.

Trinder, D.

Trinidad Martín-Arroyo, J.M.

Tripathy, D.

Tron, L.

Truant, T.

Tryfonopoulos, D.

Tsae-Jyy, W.

Tsai, H.M.

Tsai, H.W.

Tsai, J.T.

Tse, D.M.W.

Tseitlin, G.

Tseng, T.W.

Tsiouris, A.

Tsitsi, T.

Tsoukalas, N.

Tsubaki, A.

Tsubosa, Y.

Tsuboyama, T.

Tsuda, M.

Tsugawa, K.

Tsuji, T.

Tsukanov, J.

Tu, P.

Tuke, J.

Turner, J.

Tuzi, A.

Uchitomi, Y.

Uchiyama, Y.

Udagawa, A.

Udchumpisai, M.
eP612, eP491

PS011

eP226

eP059

eP060

eP569

eP153

PS016

PS049, eP222

eP579

eP579

eP200

eP292, eP293, eP294

eP176

eP259

eP626

eP008

PS020, PS018

eP241, eP311

eP214

eP633

eP355

eP466

eP528

eP647

PS054

eP500

eP499

eP588

eP576

eP073

eP188, eP502

eP072, eP398

eP421, eP442

eP630

eP067

eP167, PS058

eP506

eP689, eP690

PS053

eP444, eP564

$\mathrm{PS} 022$

eP129

eP505

eP069, eP263, eP446

PS083

eP532

eP691

eP696

eP694, eP035

eP512

eP695

eP214

eP214

eP655

eP253, eP512, eP559

eP212

eP613

eP175, PS039

eP513, PS045

eP193

eP011, eP133, eP418, PS075

eP557

eP655

eP351
Udomvisatson, $\mathrm{R}$.

Ueberall, M.

Uehara, R.

Uehara, Y.

Ueno, J.

Ueno, $\mathrm{K}$.

Uğurlu, Z.

Uhlmann, L.

Ui Dhuibhir, P.

Ulfig, C.M.

Ullett, A.

Ullgren, $\mathrm{H}$.

Ullmer, C.

Ullrich, N.J.

Underhill, C.

Ungar, N.

Unverzagt, F.

Upton, J.

Urech, C.

Urquhart, R.

Ürün, Y.

Usta Yesilbalkan, O.

Utkan, G.

Uutela, P.

Uwayezu, M.G.

Uyar, Y.

Uzgor, F.

Vadalouca, A.

Valentino, $\mathrm{T}$.

Valero, V.

Valim Côrtes Gradim, C.

Valle, A.C.

Van Belle, S.

Van Bever, L.

Van Breda, E.

Van de Poll-Franse, L.

van de Poll-Franse, L.V.

Van De Werf, E.

van de Wetering, $M$.

van de Wetering, M.D.

van den Hurk, C.J.

van der Aa, S.

van der Biessen, D.

van der Burg, S.

Van der Heide, A.

van der Helm, $\mathrm{P}$

Van Draanen, J.

van Ee, B.

van Es, N.

van Godwin, J.

van Groningen, L.

van Leeuwen, S.

Van Poznak, C.

Van Sebille, Y.

van Teijlingen, E.

van Veen, $M$.

van Vliet, L.

Vandenberg, K.

Vandenhoucke, M.

Vander Woude, A.

Vanlemmens, L.

vanseymortier, $\mathrm{M}$.

Vansteelant, L.

Vardas, E.
eP441

eP066

eP559

eP531

eP559

eP061

eP409

eP080

eP243, eP262

eP442

eP588

eP696

eP187

PS082

eP455

eP691

eP535

eP614

eP606

eP615, eP697, eP578

eP483

eP120

eP483

eP279

eP443

eP488

eP533

eP188, eP502

eP354, eP423

eP167, PS058

eP665

eP089

eP321, eP322

eP174

eP620

eP583

PS011

eP448, eP174

eP293

eP294

eP453

eP200

eP534, PS076

PS076

eP366

eP534, PS076

eP209

eP166

eP645

eP376

eP201

eP201, eP268

eP034

eP202, eP204, eP205, eP468, eP469,

PS080

eP339

eP130, PS084

eP418, PS075

eP131

PS072

eP227

eP507

eP073

eP484 


\begin{tabular}{|c|c|}
\hline & PS053 \\
\hline Vargas, D. & eP544 \\
\hline Varrasso, G. & eP020 \\
\hline Varthalitis, I. & eP694 \\
\hline Vasconcelos, R. & eP203 \\
\hline Vasili, E. & eP261, eP693 \\
\hline Vasiliou, V. & eP528 \\
\hline Vaslamatzis, M. & eP693, eP261 \\
\hline Vasquez, R. & eP180 \\
\hline Vasseur, A. & eP307 \\
\hline Vauleon, E. & eP657, eP658, eP659 \\
\hline Veluswamy, S.K. & eP563 \\
\hline Verastegui-Aviles, E. & eP698, eP568, PS006 \\
\hline Verastegui-Avilez, E. & eP346 \\
\hline Verástegui, E. & eP430, eP431, eP357 \\
\hline Verhamme, P. & eP645 \\
\hline Verma, $\mathrm{H}$. & eP340 \\
\hline Victor, D.L.R.P. & eP051 \\
\hline Vidal, M. & eP378 \\
\hline Vidal, R. & eP150 \\
\hline Viégas, C.M.P. & eP170 \\
\hline Viegas, E. & eP629 \\
\hline Vigano, A. & eP498, PS031 \\
\hline Vigano, M. & PS034 \\
\hline Vijayakumar, V. & PS030, eP296 \\
\hline Villegas Estévez, F. & eP067 \\
\hline Vinay Kumar, R. & eP699 \\
\hline Vindrola, $\mathrm{C}$. & eP284 \\
\hline Vittiglia, A. & eP114 \\
\hline Vojin, V. & eP459 \\
\hline Von Ah, D. & eP228, eP535, eP577 \\
\hline von Bültzingslöwen, I. & eP191, eP268 \\
\hline von Hagens, C. & eP080 \\
\hline von Heymann-Horan, A. & eP583 \\
\hline Vongterapak, S. & eP486 \\
\hline Vourlakou, C. & eP693 \\
\hline Vranceanu, A.R. & eP360 \\
\hline Vyas, S. & eP304 \\
\hline Wagner, L. & eP548 \\
\hline Wahl, L. & eP398 \\
\hline Waits, M. & eP494 \\
\hline Wakasugi, T. & eP556 \\
\hline Wakefield, C. & eP429, eP587 \\
\hline Waldeyer-Sauerland, M. & eP491, eP612 \\
\hline Waldmann, A. & eP491, eP612 \\
\hline Walsh, A. & eP008 \\
\hline Walsh, D. & $\begin{array}{l}\text { eP262, eP117, eP135, eP136, eP153, } \\
\text { eP243, eP249 }\end{array}$ \\
\hline Walsh, T.D. & $\mathrm{eP} 251$ \\
\hline Walshe, K. & eP131 \\
\hline Walter, V. & eP570, eP625 \\
\hline Walters, C. & eP334 \\
\hline Waltimo, T. & eP279 \\
\hline Wan Ishak, W.Z. & eP157 \\
\hline Wan, A.B. & eP209 \\
\hline Wang, C.H. & eP505 \\
\hline Wang, H.M. & eP705 \\
\hline Wang, K. & eP068 \\
\hline Wang, M. & eP476 \\
\hline Wang, T.F. & eP645 \\
\hline Wang, T.J. & eP406, eP539, eP541, eP553 \\
\hline Wang, Y. & $\begin{array}{l}\text { eP700, eP022, eP384, eP573, eP618, } \\
\text { eP634 }\end{array}$ \\
\hline Wang, Y.T. & eP532 \\
\hline
\end{tabular}

Wannakansophon, N.

Wanner, K.

Wardill, $\mathrm{H}$

Warne, L.

Watanabe- Sumazaki, Y. eP531

WATANABE, $\mathrm{K} . \quad$ eP132

Watts, G. eP320

Waxenberg, L.B. eP442

Weathers, T. eP623

Weaver, R. eP555

Wechsler, A.

Wei Wei, C.

Wei-Wei, C.

Weijenberg, M.

Weiss, $\mathrm{H}$.

Weisser, L.

Weissman, T.

Weitz, J.

Weledji, E.P.

Westerman- PhD, M.

Wheelwright, $\mathrm{S}$.

White, J.

Whitefield, S.

Whitford, K.J.

Wierick, E.

Wignall, A.

Wilberg, P.

Wilder-Smith, E.

Wiley, G.

Wilkie, B.

Wilkinson, M.

Williams, J.

Williams, J.L.

Williams, N.

Wilson, C.

Winkels, R.M.

Winter, J.

Wiskemann, J.

Wojtowicz, M.

Wolff, B.

Wollner, M.

Wolschon, E.M.

Wommack, E.C.

Won, Y.H.

Wong- Ph.D., S.

Wong, J.

Wong, L.P.

Wong, $\mathrm{M}$.

Wong, N.

Wong, S.F.

Wong, V.

Wong, W.H.

Woo, K.W.

Wootten, A.

Woron, J.

Wowor, E.C.

$\mathrm{Wu}$, H.L.

$\mathrm{Wu}, \mathrm{J}$.

Wu, J.L.

$\mathrm{Wu}, \mathrm{R}$.

Wu, W.W.

$\mathrm{Wu}, \mathrm{Z}$.

Wuelfing, P.

Xiao, C.
eP486

eP205

eP204, eP205, eP197, eP202, eP468,

P469, PS080

455

PS007, eP494

eP564

eP444

PS084

eP542

eP616

eP036

eP645

eP536

PS055

eP527, eP607

eP580, eP581

eP036

eP400

eP014

eP205, eP468, eP469, PS080

PS010

PS050

eP445, eP537

eP580, eP581

eP227

eP058, eP332

eP057, eP158

eP205

eP377, eP433, eP434

eP256

eP527, eP607

eP256, eP691

eP327

eP149

PS032

eP612

PS019

eP701

eP420

eP379, PS024

eP157, eP597

PS018, PS020

eP008

PS070

eP702, eP385

eP574

eP069, eP263, eP446

eP427

eP053

eP287

eP129

PS001, eP164

eP532

eP688

eP298, eP299

eP017

eP014

PS019 
Xie, K.

Xie, W.

$\mathrm{Xu}, \mathrm{J}$.

$\mathrm{Xu}, \mathrm{W}$.

$\mathrm{Xu}, \mathrm{Z}$.

Xuecheng, L.

Yadav, B.

Yadav, R.

Yaganti, S.

Yahalom, R.

Yahoo, A.L.

Yamada, S.

Yamada, S.I.

Yamaguchi, T.

Yamamoto, K.

Yamamoto, $\mathrm{N}$.

Yamashita, A.

Yamatodani, A.

Yanagita, Y.

Yanai, T.

Yanan, L.

Yang, C.Y.

Yang, D.

Yang, G.

Yang, $\mathrm{H}$.

Yang, L.

Yang, L.Y.

Yang, P.

Yang, S.P.

Yang, Z.

Yano, I.

Yao, K.

Yap, Y.S.

Yarom, N.

Yassin, M.A.

Yasuda, M.

Yavas, C.

Yavas, G.

Yee, C.

Yeh, S.P.

Yehgambaram, P.

Yen, T.C.

Yennu, S.

Yennurajalingam, S.

Yeo, A.H.L.

Yeo, E.

Yeo, H.L.

Yeo, S.

Yeom, C.W.

Yeon Hee, K.

Yeung, .

Yeung, S.C.

Yildiz Kabak, V.

Yip, C.H.

Yokota, M.

Yokoyama, M.

Yom, S.K.

Yordanov, N.
eP023

eP031

eP618

PS012

eP385

PS034

eP206

eP552

eP617, eP537

eP036

eP538

eP274

eP280

eP011, eP133, eP183, eP253, eP418,

eP531, PS075

eP021

eP460

eP695

eP021

eP250

eP011

eP300, eP301

eP621

PS012

eP380

eP300, eP301

eP022

eP705

eP470

eP539

PS026

eP214

eP264

PS018, PS020

eP034, eP036

eP648

eP472

eP281, eP619, eP703, eP704

eP281, eP619, eP703, eP704

eP068

eP505

eP157, eP597

eP705

eP381, eP057, eP494

eP143, PS062

PS018

eP645

PS020

eP410

eP156

eP701

PS007

eP600

eP540, eP620

eP157, eP597

eP065

eP238

eP034

eP101
Yoshie, R.

Yoshihisa, M.

eP655

eP280

You, K.L. eP621

Youn, S. eP410

Young Iee, $\mathrm{P}$ eP683

Young, A.

Young, J.

yousfi, M.A.

Yu-Ping, L.

Yu, C.H.

$\mathrm{Yu}, \mathrm{M}$.

Yu, M.S.

Yu, P.

Yu, Q.

Yumi, L.

Yunakova, M.

Yurikusa, T.

Yusof, M.

Yusuff, S.M.

Yusuke, O.

Zacchigna, S.

Zachariah, F.

Zahariev, Y.

Zahrieh, D.

Zaki, H.

Zambrano, S.

Zaydiner, B.

Zeissig, S.

Zeissig, S.R.

Zemaitis, A.

Zenda, S.

Zeng, $\mathrm{X}$.

Zhang, G.

Zhang, J.

Zhang, L.

Zhang, X.

Zhao, H.

Zheng, R.

Zheng, R.S.

Zhong, W.

Zhu, J.

Zhu, S.

Zhu, W.

ZHU, X.

Ziegler, D.

Ziegler, V.

Zigogianni, A.

Zilic, A.

Zinkovskaya, A.

Ziras, N.

Zohrabyan, D.

Zou, H.

Zoublios, C.

zribi, A.

Zupin, L.

Zürcher, S.

Zwicker, J.

Zwisler, A.D.
eP706, eP453

eP571

eP229

eP444

eP541

eP023

eP090

eP023

eP542

eP673

PS072

eP207, eP695

eP597

eP451

eP647

eP181, eP194, eP195

eP348

eP631

eP470, eP471

eP282

PS060

eP382

eP612

eP491

eP383

eP133, eP011

eP023

eP645

PS066

eP018, eP209

eP167, PS058

eP666

eP573, eP618, eP634, eP700

eP384

eP003

eP022, eP023

eP586

eP264

eP385

eP429

PS078

eP188, eP502

eP208, PS088, eP095, PS086

PS083

eP694

eP388

eP646

eP693

eP229, eP302

eP194

eP096

eP645

eP466 